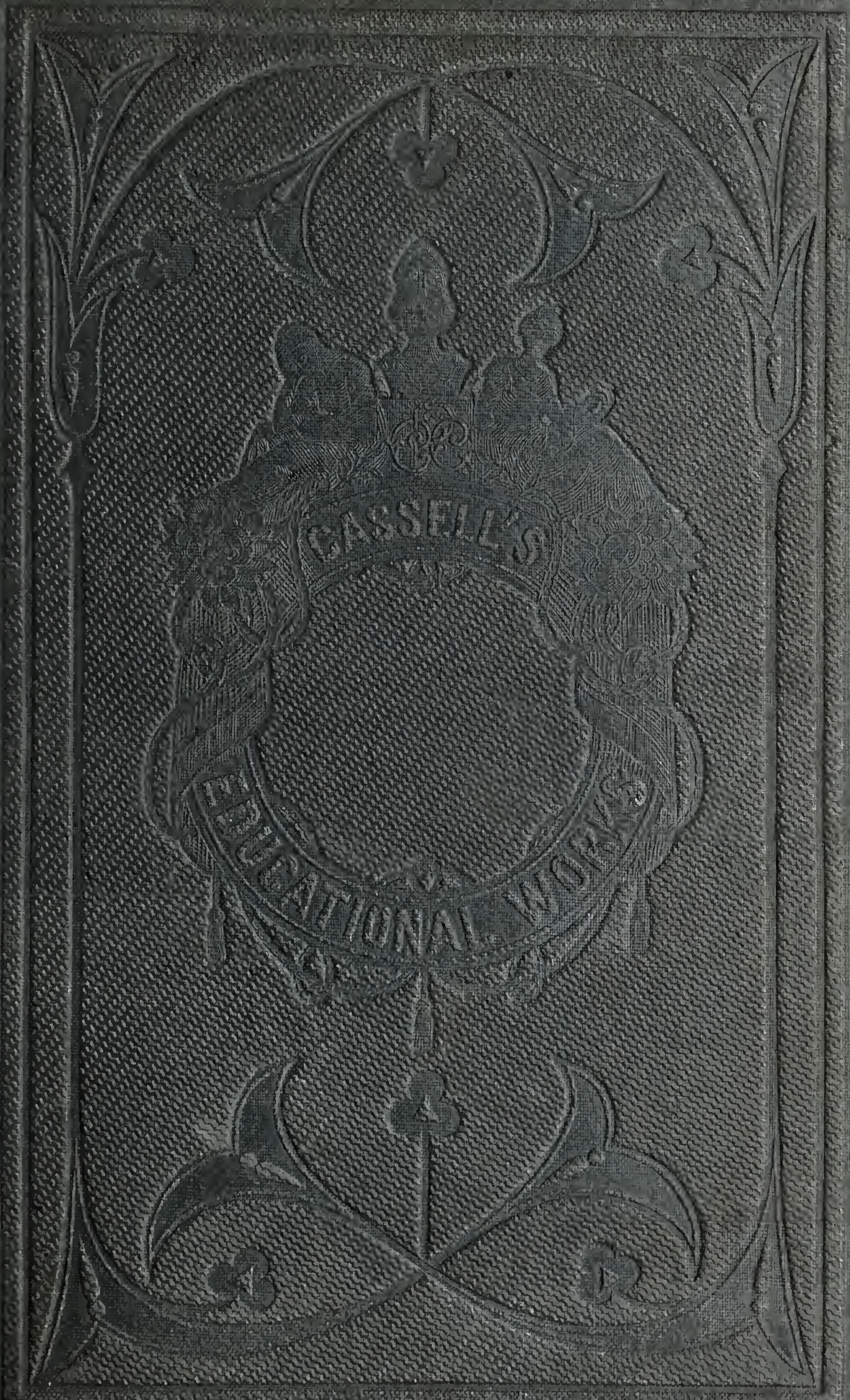


P1963591

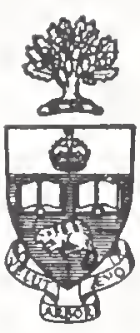

\section{Library}

of the

University of Toronto 




\section{S C I E N C E \\ POPULARLY EXPLAINED:}

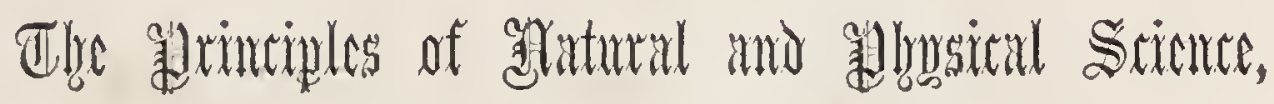

AND

THEIR PRACTICAL AND USEFUL APPLICATIONS TO THE EMPLOYMENTS AND NECESSITIES OF COMMON LIFE,

FAMILIARLY EXPLAINED.

ILLUSTRATED WITH UPWARDS OF TWO HUNDRED ENGRAVINGS.

BX

D A I D A. WELLS，A.M.

WITH ADDITIONS BY THE EDITORS.

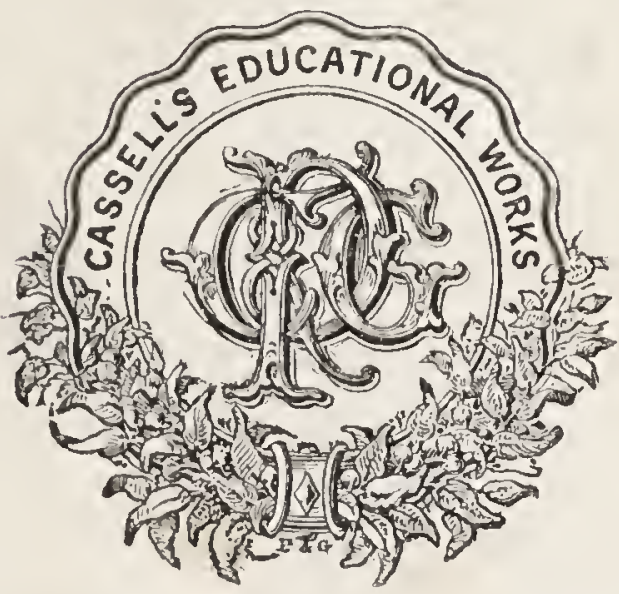

LONDON :

CASSELL, PETTER, AND GALPIN,

L A BEI,LE SAUVAGE YARD,

I.UIGA'TH HIII, F.C. 
Digitized by the Internet Archive in 2018 with funding from University of Toronto 


\section{PREFACE.}

ThE success which has attended the Educational Works already issued by the Editor, the circulation they have attained, and the popularity they have secured, have induced him to undertake the present Series. He is confident that such a Series is required; that self-education is one of the peculiar characteristics of the age; that the people are bent on obtaining information, and that those who render that information easy of access are doing good service. His object is to place within the reach of all an EDUCATTONat Libraki; to render it at once accurate in matter, attractive in manner, and cheap in price.

This, the First Volume of the Educational Course, is devoted to "Science Popularly Explained." The system of instruction which constitutes its marked and peculiar features embraces the whole circle of the Natural and Physical Sciences, so far, at least, as their elementary principles and practical applications are concerned.

Notwithstanding so many and such extensive departments of scientific knowledge are included within the limits of a single volume, the Editor is confident that a careful examination of the separate divisions will show that the Principles of each Science are carefully and distinctly set forth, and that many of their practical applications to familiar objects are illustrated and explained.

The Editor is aware that with some persons strong objections exist against any system of instruction based on the plan of Question and Answer; and some deprecate all attempts to popularise scientific truth-considering that science is degraded by clothing its principles in garments drawn from a homely wardrobe. He would urge in reply, that the extersive circulation and continued use of such works in England, France, and the United States, show that it meets a want not adequately supplied, and that this plan of instruction is both popular and practical. His aim is to familiarise the mind with the great principles of the various branches of science treated, and at 
the same time show how intimately they are connected with all the occupations of everyday life. And this communication of scientific truth he claims to be effected, so far especially as young persons are concerned, more perfectly by a well-arranged system of Question and Answer than in any other way; since a true system of instruction does not consist in presenting to the mind facts to be retained simply as such, but in making facts familiar, in pointing out their dependence on general laws, and illustrating their practical applications. The Question appeals directly to the curiosity of the learner; and this feeling, when aroused, concentrates the mind, and occasions a corresponding distinctness to all its perceptions. It appeals also to the reasoning faculties: to the mind, as an intelligent and investigating agent; and not to one single attribute-the memory. The knowledge which the pupil acquires in this way is fixed and permanent. It is continually recalled by the presence of the familiar objects treated of, which in their turn re-act, suggesting generalisations, and encouraging the deduction of other and more important inferences.

These remarks, howerer, apply only to the present volume. The plan of Question and Answer is not adopted in the volumes which immediately follow. With respect to these,- the "Treatise on the English Language," and also that on "Astronography,"-copious Questions are given at the end of each Chapter, which may be employed at discretion, the Editor being fully convinced of the utility of such a mode of examination.

London, September, 1856. 


\section{CONTENTS.}

chapter

LAWS AND PROPERTIES OF MIATTER.

PAGE

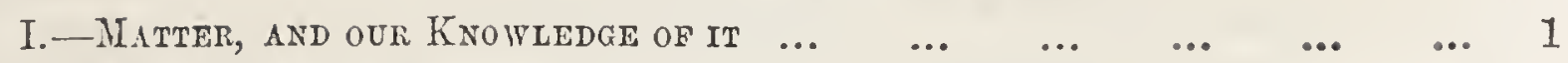

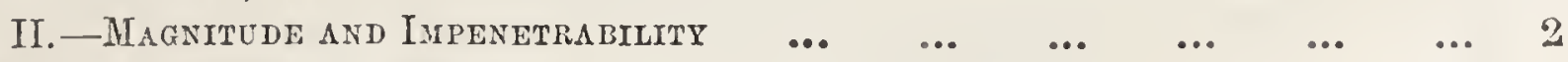

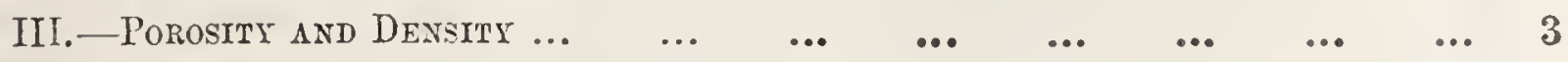

$\begin{array}{llllllllllll}\text { IV. }- \text { FORCE } & \ldots & \ldots & \ldots & \ldots & \ldots & \ldots & \ldots & \ldots & \ldots & \ldots & 6\end{array}$

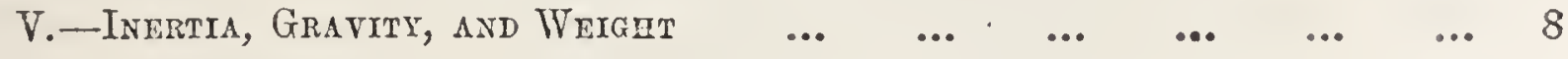

$\begin{array}{llllllllll}\text { SEcr. I.-Inertia } & \ldots & \ldots & \ldots & \ldots & \ldots & \ldots & \ldots & \ldots & 8\end{array}$

$\begin{array}{llllllllll}\text { II.-Gravity } & \ldots & \ldots & \ldots & \ldots & \ldots & \ldots & \ldots & \ldots & 10\end{array}$

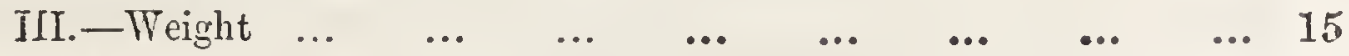

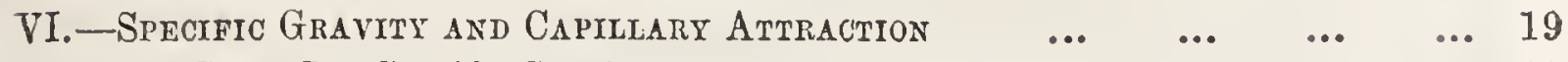

$\begin{array}{llllllll}\text { Sect. I._Specific Gravity ... } & \ldots & \ldots & \ldots & \ldots & \ldots & \ldots & 19\end{array}$

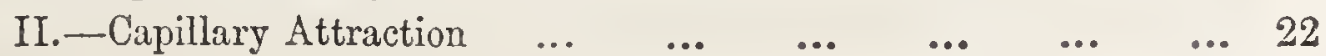

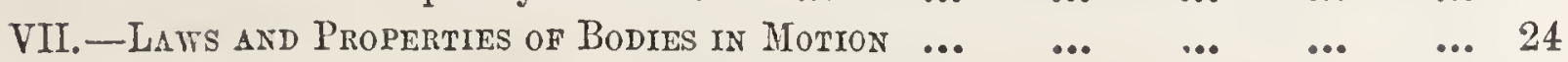

MECHANICS.

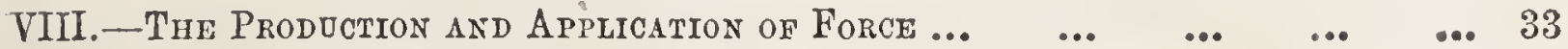

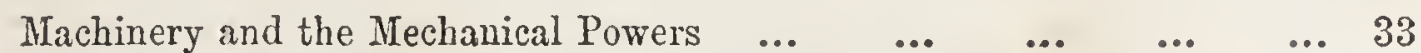

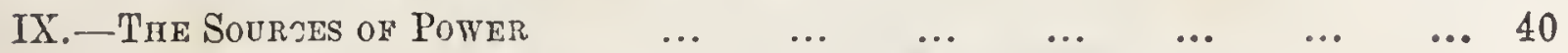

X. - On the Strexati of MLaterials used tr mate Arts, and their Afpuication

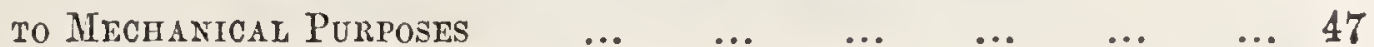

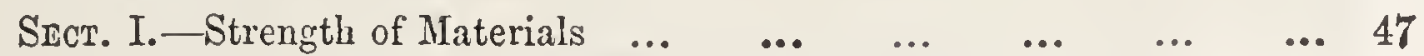

II.-Application of Materials for Architectural and Structural Pur$\begin{array}{llllllllll}\text { poses } & \ldots & \ldots & \ldots & \ldots & \ldots & \ldots & \ldots & \ldots & 48\end{array}$

THE PHENOMENA OF FLUIDS.

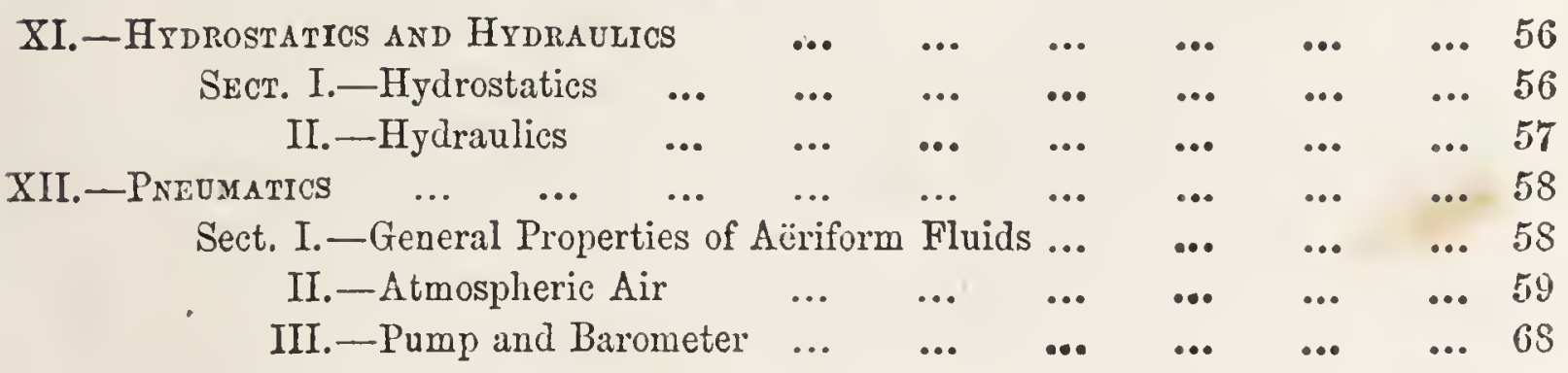

ACOUSTICS.

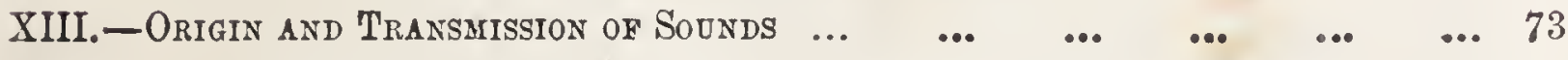

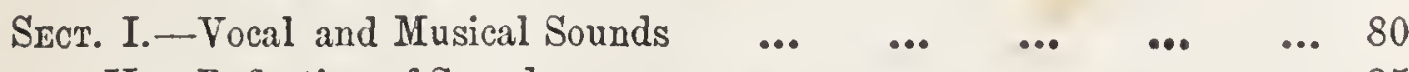

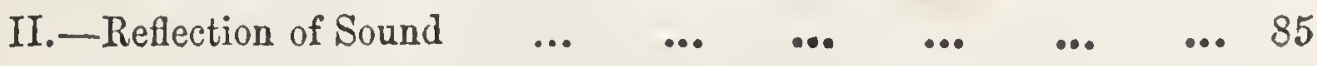

HEAT.

XIV.—NATURE AND SOURCES OF HeAT ...

SEcr. I. - The Sun considered as a Source of Heat $\quad \ldots \quad \ldots \quad \ldots \quad \ldots 0$

II. - The Earth and Electricity considered as Sources of Heat $\ldots 93$ 
CIIAPTEP

SECT. III.-Chemical Action considered as a Source of Heat...

IV.-Mechanical Action cousidered as a Source of Heat ...

V. - Vital Action considered as a Source of Heat _.. ... 99

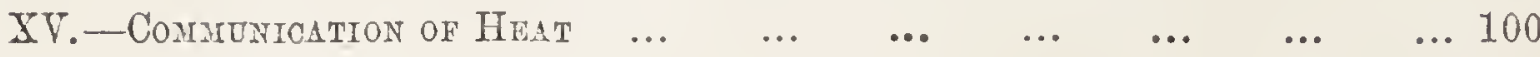

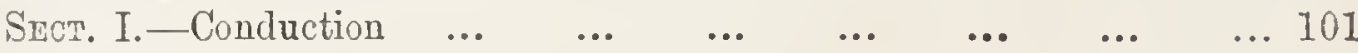

II. - Convection $\quad \ldots \quad \ldots \quad \ldots \quad \ldots \quad \ldots \quad \ldots . \quad \ldots \quad \ldots 108$

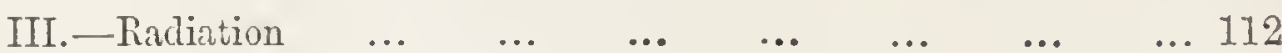

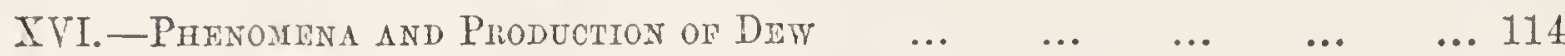

XVII. - Refuection, Absorption, and Transuission of Heat $\ldots . . \quad \ldots \quad$... 121

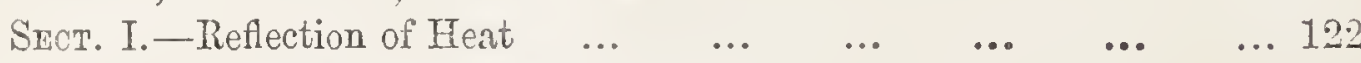

II. - Absorption of Heat $\quad \ldots \quad \ldots \quad \ldots \quad \ldots \quad \ldots \quad \ldots \quad \ldots \quad \ldots 123$

III._Transmission of Heat... $\quad \ldots \quad \ldots \quad \ldots \quad \ldots \quad \ldots \quad \ldots 127$

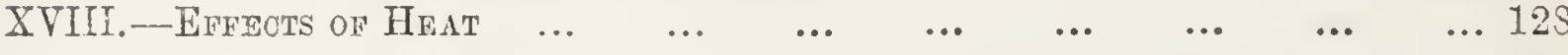

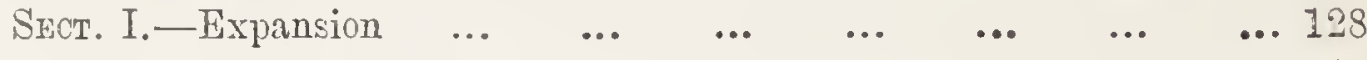

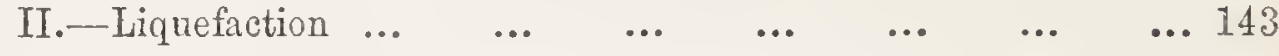

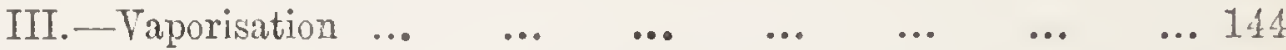

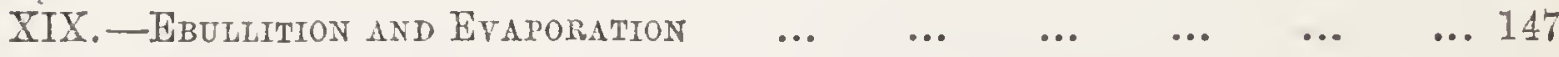

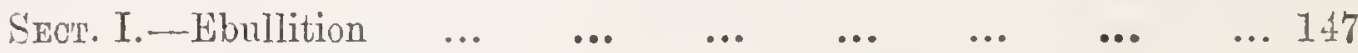

II. Evaporation $\quad \ldots \quad \ldots \quad \ldots \quad \ldots \quad \ldots \quad \ldots \quad \ldots \quad \ldots 150$

XX.-STEAM

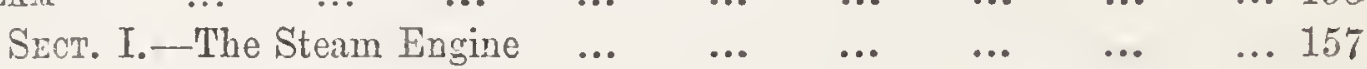

XXI.-VENTILATION...

XXIT._Solution and CRYstaluisatrov

METEOROLOGY.

XXIII.-The Atmospheri

XXIV.-WINDS

SECT. I.-Constant Winds

II.-Periodical Winds

III.-Yariable Winds

XXV.-CLoUDS

Szcr. I.-Modifications of Cloud

II. - Mist and Fog ...

XXVI.-RAIN, SNow, HaIL, Frose .

SECT. I.-Rain

II.-Snow $\ldots \ldots$

III.-Hail $\ldots \quad$, ... $\quad$...

IV._Frost ... … ...

XXVII.-Luninous Atmospieric Pheromena

SECT. I. -Meteorites

II. - Aurora Borealis

$\cdots \quad \cdots$

XXVIII. -POPULAR OPINIONS CONCERNING THE WTATHER

LIGHT AND ACTINISH.

XXIX.-LrGHT

XXX.-Reflection of Light

XXXI.-REFRACTION AND ABSORption OF Light

XXXII. - COLOUR

XXXIII.-The Organs of Vision

XXXIV.-DECEPTIONS OF VISION

XXXV.-Artiftoral LigHT 
XXxVi.-Double Refraction and Polarisation

\section{ELECTRICITY.}

XXXViII. - Nature and Production of Electricity

Electrical Machines ...

XXXIX.-AtMrospheric ELECTRICITY

SECT. I. - Lightning $\ldots \quad \ldots+\quad \ldots$

II.-Effects of Lightning

III.-Lightning Conductors

IV.-Thunder

\section{GALVANISM.}

XL.-Nature and Production of Galvanio Electrioity $\quad \ldots \quad$.. $\quad \ldots 232$

SEст. I._-Phenomena and Effects of Galvanic Electricity ... $\quad . .285$

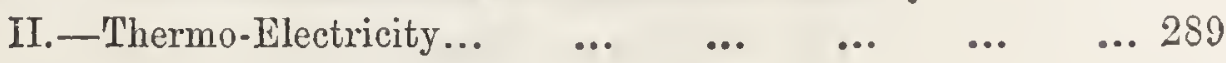

\section{MAGNETISM.}

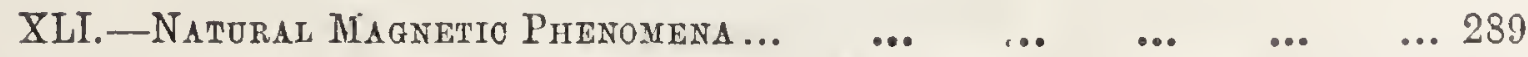

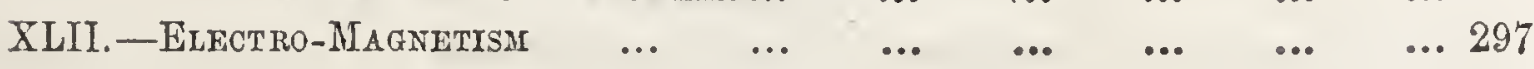

\section{CHENISTRY.}

Xlim.-Chemical Foroes and Agencies

XLIV.-OXIGEN

XLV.-HTDROGEN AND W TAER

$\cdots \quad \cdots$

$$
\text { II.-Water }
$$

\section{- XLVI.-Nitrogen AND ITS COMPounds}

SECT. I.--Nitrogen

II.-Composition and Chemical Properties of the Atmosphere

... $\quad$... $\quad \ldots \quad \ldots \quad \ldots 303$

XLVII.-CARBON AND ITS COMPOUNDS

Xlvimi.-Combustron, Respiration, and Nutrition

II. - Respiration ..

III.-Nutrition and Apinal Heat

XLIX.-Phospmorus And Sumpter

SECT. I.-Phosphorus

II.--Sulphur

... 308

... 311

... 311

... 312

... 317

... 317

317

... 319

... 322

... 330

... 330

... 347

... 352

... 356

... 350

... 357

L.-Chlorine, Iodine, Fluorine, Siliciom, Boron ...

SECT. I.-Chlorine

II.-- Iodine and Fluorine

III.--Silicium and Boron

LI. - The Mietalujo Elements ...

Srct. T.-The Alliali Metals...

II.-Netals of the Alkaline Earths

III.-Aluminum...

IV.-The Heavy Metals ...

$\cdots \quad \cdots$

$\cdots \quad \cdots \quad \ldots$

-.. $\quad$... $\quad \ldots$

$\cdots \quad \cdots$

$\ldots, \ldots$

\section{ORGANIC CHEMISTRY.}


CILAPTER

LV. - Animal ORganisation and Productions $\ldots . \quad \ldots \quad \ldots . \quad \ldots \quad \ldots 404$

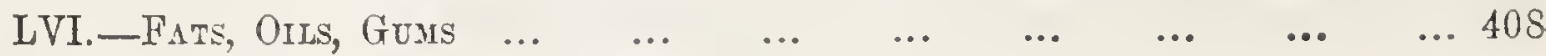

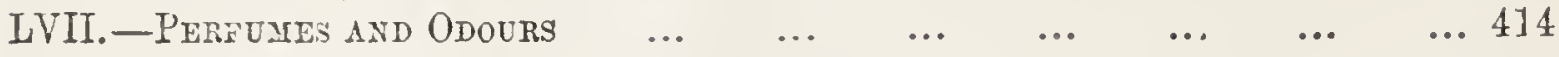

\section{PHILOSOPHY OF MIANUFACIULES.}

LVIII.-Nature of Fibrous Substances $\quad \ldots \quad$..

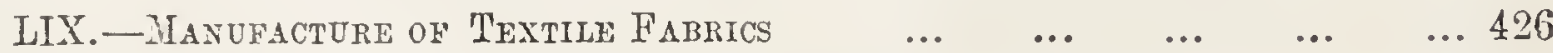

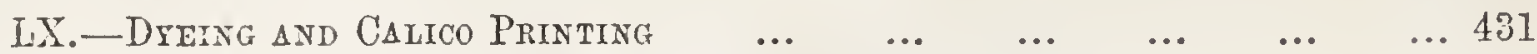

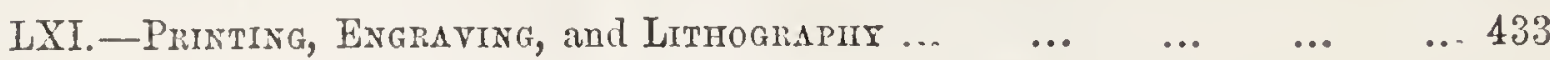

AGRICULTURAL AND RURAL ECONOMY.

LXiI.—Elementary Principles petitaining to Agriculture $\quad \ldots \quad \ldots$

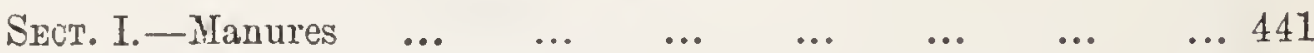

II. - Growth of Plants ... $\quad \ldots \quad \ldots \quad \quad \ldots \quad \ldots \quad \quad \ldots 443$

III.-Propagation of Fruit-trees ... $\quad \ldots \quad \ldots \quad \ldots \quad \ldots \quad \ldots 449$

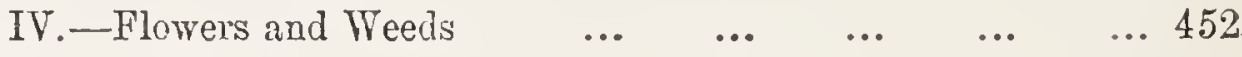

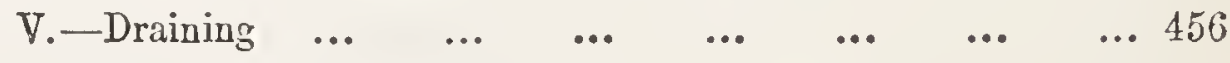

\section{GEOLOGY.}

LXIII.—Foryation and Structure of the Harth's Crust $\quad \ldots \quad \ldots$

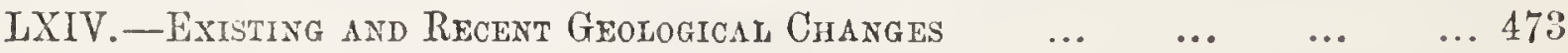

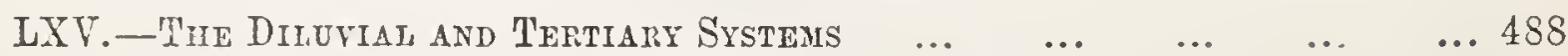

LyVi.—The Chalk, the Oolite, and the New Red Sandstone... $\quad \ldots \quad \ldots 491$

LXVII. - The Carboniferous, Old Red Sandstone, and Silurian Systems $\quad$... 494

LXVIII.-On the Progressive Drvelopyent of Animal Life upon the Surface

$\begin{array}{lllllllllll}\text { OF THE EARTH } & \ldots & \ldots & \ldots & \ldots & \ldots & \ldots & \ldots & \ldots & \ldots 00\end{array}$

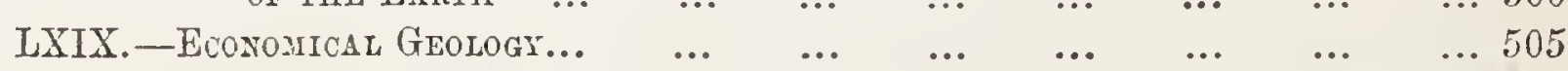

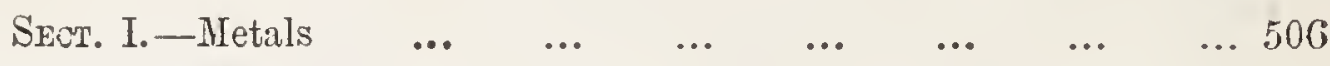

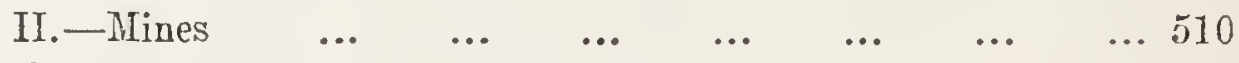

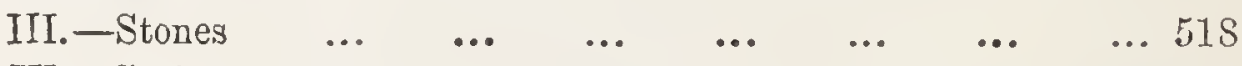

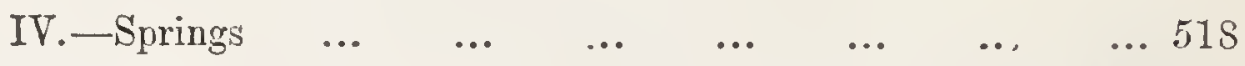

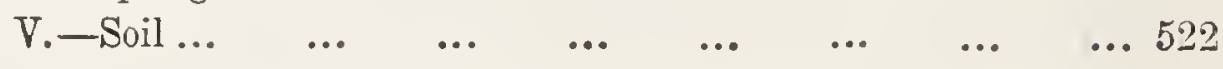

MINERALOGY.

LXX.—Characteristics and Classification of Minerals $\quad \ldots \quad \ldots \quad \ldots 523$

$\begin{array}{llllllllll}\text { LXXI.—CRTSTALLOGRAPHY } & \ldots & \ldots & \ldots & \ldots & \ldots & \ldots & \ldots & \ldots & \ldots 27\end{array}$

GEOGRAPHY OF PLANTS AND ANIMALS.

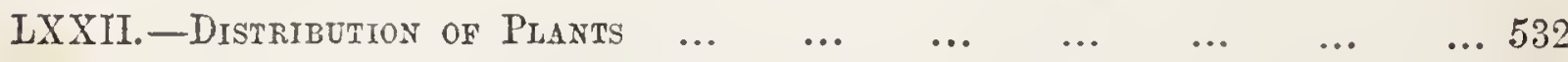

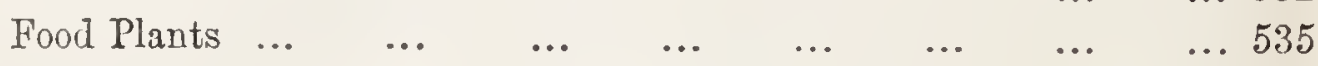

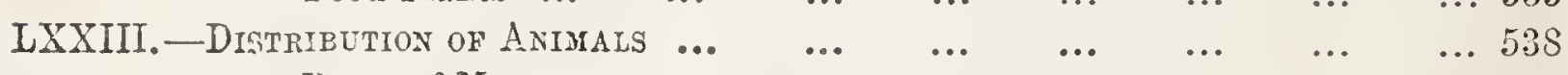

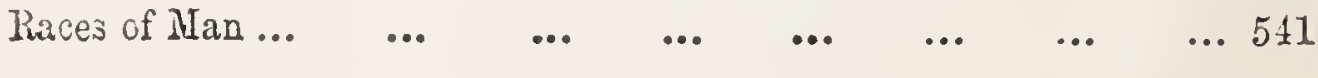




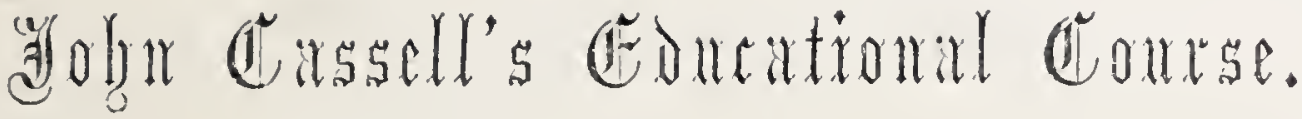

\section{SCIENCE POPULARLY EXPLAINED.}

LAWS AND PROPERTIES OF MATTER.

\section{CHAPTER I. \\ MATTER, AND OUR KNOWLEDGE OF IT.}

Q. What is MATTER?

A. Matter is the general name which has been given to that substance, which, under forms infinitely various, affects the senses.

Q. How do we know that anything ExISTS?

A. Because our senses give us evidence of the fact.

Q. What are the SENSES?

A. They are the instruments, or means, by which the mind is enabled to determine the existence, and take cognisance of the qualities of material objects.

Q. How Maxr serses are there?

A. Five: hearing, seeing, smelling, tasting, and feeling.

Q. Would a person deprived of all sensation, be conscious of any Materiat ExIstEXCE?

$A$. He would not; for all knowledge of the material world is lerived through the medium of the senses.

Q. Is the impression transmitted to the mind by each organ of sensation DIFFERENT ?

$A$. It is; each organ of sense is adapted to receive a particular influence of matter; and is designed to convey to the mind imme. diate rotice of some peculiar action. This is the more noticeable, when we consider that, however delicate its structure, each organ of sense is wholly insensible to every influence except that to which it is especially adapted; thus, the eye is never afrected by sound, or the car by light.

Q. What is meant by the term rody?

A. Any distinct portion of matter perceived by the senses.

Q. What do we mean when we speak of the rroperties or QUarrties of a body?

$A$. The powers belonging to the body, which are capable of exciting in our mind certain sensations.

Q. Are there any propenties or QUALITIES that belong alike to ali material bodies?

A. There are at least two, which are inseparable from matter, in whatever state, or in whatever form it may exist, viz., magnitude and impenctrabiitity. 


\section{CHAPTER. II. \\ MAGNITUDE AND IMPENETPABILITY.}

Q. What is ungNimudu?

A. The property of occupying space. It is impossible to conceive of a portion of matter so minute as to have no magnitude.

Q. What do we mean by the term SIZE of a body?

A. The quantity of space a body occupies.

Q. What is the SUREACE of a body?

A. The external limits of its magnitude.

Q. What is the $\Lambda \mathrm{REd}$ of a body?

A. The quantity of surface.

Q. What is IMPENETRABIITTY

A. That quality of matter which precludes the possibility of two bodies occupying, the same space at the same time. When bodies are said to be impenetrable, it is therefore meant, that one cannot pass .through another without displacing some, or all, of the component parts of that other.

There are many instances of apparent penetration; but in all these, the parts of the body which seem to be penetiated, are only displaced. Thus, if it needle be plunged into a vessel of water, all the water which previously filled the space into which the needle enters, will be displaced ; and the level of the water will rise in the vessel to the same height as it would by pouring in so much more water as would fill the space occupied by the needle.

Q. What is the FIGURe of a bocty?

$A$. Its form or shape, as expressed by its boundaries or terminating extremities.

Q. What is the volume of a body?

$A$. The quantity of space included within its external surface.

The figure and volume of a body are entirely independent of each other. Bodies having very different figures may have the same volume ; or, in like manner, bodies of the same figure may have different volumes. Thus, a globe, for example, may have ten times the volume of another globe, and yet have the same figure; or a globe and cylinder may have the same volume, with entirely different figures.

Q. What is an Amons of matter?

A. A particle so minute, as to admit of no division. Atoms are conceived to be the first principles or component parts of all bodies.

Sir Isaac Newton thus expresses his belief respecting the atomic condition of matter :"It seems to me," he remarks, "that God, in the beginning, formed matter" in a solis! mass of hard, impenetrable particles ; and that these primitire particles, being solids, are incomparably harder than any porous bodies compounded of them; even so very harel as never to wear, or break in pieces-no ordinary power being able to divide what Gar? made one in the first creation."

Q. Practically, do we kxow of any limit to the division of matter?

A. We do not; and yet many of the phenomena which the researches of those who have successfully examined the laws of natriv have developed, render it highly probable that all bodies are composed of elementary parts which are indivisible and unalterable.

An ounce of gold may be divided into four hundred and thirty-two thousand million parts. Each of these parts will retain all the characters and qualities which aro found in the largest masses of metal. It retains its solidity, texture, and colour; it resists ths aame agents, and enters into combination with tho same substances. 
Q. What is a MOLECULE of matter?

A. The word molecule is often used to signify componont parts of a body so small as to escape sensible observation, but not nltimate atoms; each molecule being supposed to be formed of several atoms, arranged according to some determinate figure.

Q. What is a PARTICLE of matter?

A. The term particle is also used to express small component parts of matter, but is generally applied to those which are not 100 minute to be discovered by observation.

\section{CHAPTER III. \\ POROSITY AND DENSITY:}

Q. What are the poris of a body?

$A$. No two particles of matter are supposed to be in actual contact with each other; and the openings or interstitial spaces between these particles are called pores.

Q. Are the pores of bodies ever VISTBLE to the eye?

A. In very many bodies they are easily recognised, as in a sponge, various animal and vegetable membranes (the skin, the rind of an orange), in wood, bread, \&c. In other bodies, as the metals, stone, \&c., they are not visible.

Q. What is the REASON that a sponge, a piece of wood or metal, can, by pressure, be made to occupy a smaller space than it did originally?

A. Because the particles of which the sponge, the piece of wod or metal, are composed, are by pressure brought more closely together, diminishing at the same time the pores and the space the bory occupies.

Q. What, then, is COMPressibiLite?

A. That quality of matter in virtue of which a body allows its volume or size to be diminished, without diminishing the number of atoms or material particles of which it consists.

Q. What reason have we for supposing that no two particles of matter are in ABSOLUTE CONTACT ?

A. Because all known bodies, whatever may be their nature, are capable of having their dimensions reduced without diminishing the amount of matter contained in them; hence the space by which the volume may be diminished must, before diminution, consist of pores.

Q. What is Dexsitr?

A. The proportion of the quantity of matter in a body to its magnitude. Thus, if of two substances one contains in a given space twice as much matter as the other, it is said to be twice as dense.

Q. What connection is there between the DENSITY of a body, and its POROSITY?

A. A body will be more or less dense according as its particles are no? r to or remote from each other; and hence it is evident that the 
greater the density the less the porosity, and the greater the porosity the less the density.

Q. Why do we call IIEAD heavy, and feathers IIGHT ?

$A$. Because the amount of matter contained in a quantity of lead occupying a given space is much greater than in a quantity of feather:s capable of occupying the same space. The original particles of matter which make up the composition of lead and feathers are also different, and in the lead are arranged in much nearer connection with each other than in the feathers.

Q. Gold and lead are metals of great density; their pores are not visible. Is there any Proof of their existence besides the fact that they can be compressed ?

A. Water can be forced mechanically through a plate of lead or gold without rupturing any portion of the metal. Mercury or quicksilver, confined in a dish of lead or gold, will soak through the pores, and escape at the bottom.

An interesting experiment was tried at Florence, Italy, nearly two centuries ago, which furnished a striking illustration of the porosity of so dense a substance as gold. A hollow ball of this metal was filled with water, and the aperture exactly and firmly closed. The globe was then submitted to a very severe pressure, by which its figure was slightly changed. Now, it is proved in geometry, that a globe has this peculiar property-that any change whatever in its figure necessarily diminishes its volume, or capacity. The result was, that the water oozed through the pores, and covered the surface of the globe, presenting the appearance of dew, or steam cooled by the metal. This experiment also proved that the pores of the gold are larger than the elementary particles of water, since the latter are capable of passing through them.

Q. How is water or any other liquid made PURE by filtering through paper, cloth, a layer of sand, rock, \&c.?

$A$. The process of filtration depends on the presence of pores in the substance used as a filter, of such a magnitude as to allow a passage to the liquid, but to refuse it to those impurities from which it is to be disengaged.

Q. Why is not the substance suitable for the filtration of ONE liquid equally adapted for the filtration of ALL liquids?

$A$. Because the magnitude of the pores in different substances and of the impurities in liquids is different; and no substance can be separated from a liquid by filtration, except one whose particles are larger than those of the liquid.

Q. Why do bubbles RISE to the surface when a piece of sugar: wood, or chalk is plunged under water?

A. Because the air previously existing in the pores becomes dis. placed by the water, and rises to the surface as bubbles.

Q. What occasions the SNAPPJNG of wood or coal when laid upon the fire.

A. Because the air or liquid contained in the pores becomes expanded by heat, and bursts the covering in which it is confined.

Q. What are the SPARES OF FIRE which burst from the WOOD?

A. Very small pieces "of wood made red hot, and separated from the $\log$ by the force of the air when it bursts from its confinement.

Q. Why does IIGIT, Porous wood make more snapping than any orür kind?

A. Because the pores are very large, and contain more air than wood of a closer grain. 
Q. Why does GReEN WOoD make LeSS SNAPPING than DRX?

A. Because the pores being filled with sap, contain very little air.

Q. Why does DRY wood make More SNapPING than green? instead.

$A$. Because the sap is dried $u p$, and the pores are filled with a $r^{\circ}$

Q. Why does DRY WOOD BURN more easily than GREEN or WET wood?

A. Because the pores of dry wood are filled with air, which supports combustion; but the pores of green or wet wood are filled with moisture, which extinguishes flame.

Q, When is a body said to be ELASTIC?

$A$. When, on being compressed by the agency of a mechanical power, it is capable of resuming its former dimensions with a certain force when relieved from the operation of the force which has compressed it. This property is called elasticity.

Q. Into how NANY classes may all natural bodies be divided?

A. All the bodies we meet with on the earth may be divided into three great classes; viz., solids, liquids, and gaseous or aeriform bodies; and each of these states in which bodies exist is called its stute of aggregation.

Q. What is a soLID?

A. A solid is a body whose particles of matter are so close or dense that they resist the impression or penetration of other bodies. Hence the parts of solid bodies are not movable or easily displaced like those of liquids.

Q. What is a LIQuid?

A. A liquid is a substance which, like water, manifests immediately to the touch but a very feeble resistance; but quite sufficient, however, to indicate its presence, even when in a state of repose. A liquid cannot be grasped between the fingers like a solid body; neither can it be collected permanently in a heap, or made to assume any particular figure except that of the vessel in which it is enclosed.

Q. What is a GASEOUS or AERIFORM body?

A. A gaseous or aeriform body is an elastic, and generally an invisible, fluid, which, like the air surrounding us, affords no evidence of its presence to the sense of touch when in repose. Gaseous or aeriform bodies may be confined in vessels, from whence they exclude liquids or other bodies, thus demonstrating their existence, though invisible, and also their impenetrability.

Q. In what respect does a gas DIFFer from a liquid?

A. A liquid, like water, milk, syrup, \&c., can be made to flow regularly down an inclined plane, but a gas cannot.

ฉ. Why is violent exertion NECESSARY in walking against a high wind?

A. Because the invisible particles of matter of which the atmosphere is composed press violently against us in an opposite direction, and require to be displaced before we can procecd.

Q. How does the wind cause a ship to sail through the water?

A. 'The particles of air are driven against the cancuss of which the sails are composed, and being unable to pass through, transmit their. 
notion to the sail and mast, and from thence to the hull of the vessel.

Q. When is a substance said to be PrASTIo?

A. When it possesses intermediate properties between a solid and a furd. Pitch is an example of a plastic body: it presents the appearance of a solid, but will be found to be continually changing its form by the morement of its particles, when left free to move.

Q. Then a sponge is placed in water, that liquid appears to penetrate it. Does the water really enter the solid particles of the sponge?

A. It does not; it only enters the pores or vacant spaces between the particles.

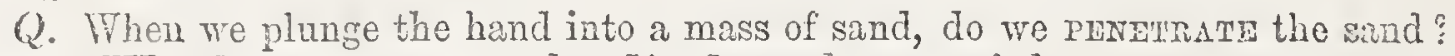

A. We do not; we only aisplace the particles.

Q. What is the science of Prysics?

A. The science of Physics comprehends those branches of human knowledge which treat of the conditions of matter not dependent on chemical combination, which we perceive around us, and of the operations of those agents which are ever active in producing those. conditions.

Q. What is rorce?

\section{CHAPTEP IV.}

FORCE.

A. Force is whatever produces or opposes the production of motion in matter. Force, in its merely mechanical signification, is whatever produces motion or pressure.

Q. How many EINDS of forces are recognised?

A. Two-external and internal forces. These are subdivided into Animal force, Mechanical force, Weight, Elasticity, Attraction, Repulsion, Polar forces, or Magnetism.

Q. What is the difference between an EXTERTAL and INTERNAL force?

A. External forces act upon matter at sensible distances; internal (or molecular) forces act only upon the particles or molecules of matter at insensible distances.

Q. Whiat is ATTRACTION?

A. It is the force manifested by the mutual approach or coliesion of bodies.

Q. What is Comesire attraction?

A. It is the force which holds together the atoms of bodies. Cohesion acts only between particles of matter of the same kind, and at diswances which are not measurable, or, as they are termed, insensible distunces.

Q. What are Poisin forces, or magnetism?

A. The forces which are exhibited by the mutual approach or adhesion of the locklstone and iron.

B. What is ripulsios?

A. It is the force manifest in the movement of bodies from each 
other. Thus, if a piece of glass, having been briskly rubbed with a silk-handkerchief, touch successively two feathers, these feathers, if brought together, will move asunder.

Q. What is ADErsion?

A. Adhesion is attraction between particles of matter of different kinds acting at immeasurably small distances only, and uniting the dissimilar particles into one mass.

Q. Why do two pieces of perfectly smooth material, as plate-glass or marbie, when laid upon each other, adhere with GREAT FOROE?

A. Because the force or influence of attraction binds the two surfaces together; other smooth suriaces present the same phenomena.

Q. Why is morTar used to fasten bricks together?

A. Because the adhesive attraction between the particles of the brick and the particles of mortar is so strong, that they unite to form one solid màss.

Q. Why is a bar of rRow stronger than a bar of wood of the same size?

A. Because the cohesion existing between the particles of iron is greater than that existing between the particles of wood.

Q. Why are the particles of a LIQUID more easily separated than those of a SOLID?

$A$. Because the cohesive attraction which binds together the particles of a liquid is much less strong than that which binds together the particles of a solid.

Q. Why will a small necdle, carefully laid upon the surface of water, FLOAT?

$A$. Because its weight is not sufficient to overcome the cohesion of the particles of water constituting the surface; consequently, it cannot pass through them and sink.

Q. Why can many light insects walk upon the surface of water without siNKING, or becoming WET?

$A$. Because their weight is insufficient to overcome the cohesion of the particles of water, and break through the surface. In like manner, a cat will walk safely upon the surface of thin ice, when a man would break through.

Q. If you drop water and laudanum from the same vessel, why will sIXTY drops of the water fill the same measure as ONE IUNDEED drons of laudanum?

A. The cohesion between the particles of the two liquids is different, being greatest in the water. Consequently, the number of particles which will adhere together to constitute a drop of water is greater than in the drop of laudanum.

Q. Why is the prescription of medicine ky Drops an unsafe method?

A. Because, not only do drops of fluid from the same vessel, and often of the same fluid from different vessels, differ in size, but also drops of the same fluid, to the extent of a third, from different parts of the lip of the same vessel.

Q. Why is it DrFrrculr to pour water from a vessel which has rot a projecting lip ?

$A$. Because, in consequence of the attraction between the water and the sides of the ressel, the fluid has a tendency to run down along the inclined outside of the ressel, and not at once to fill perpendicularly. 
Q. Upon what does the InADArss of a body depend?

A. Not, as is often supposed, upon the density of a body, but upon the force with which the atoms hold their places in some particular arrangement. Gold is much more dense than the diamond, yet the metal is soft, and the diamond the hardest body in nature.

Q. When is a body said to be BRITtLe?

$A$. When the cohesion among the atoms exists within such narrow limits, that a very slight change of position, or increase of distance among them, is sufficient to produce a rupture. Brittleness is the opposite of elasticity, and is a property of most very hard bodies. Some bodies, however, like glass, may be both brittle and elastic.

Q. When is a body said to be DucriLe?

$A$. When it is capable of being draun into wire. In ductile substances the atoms seem to have no more fixed relation of position than in a liquid, but yet they cohere very strongly.

Q. When is a body said to be PLIANT?

A. When the cohesion of the particles is not destroyed by a conlsiderable change of their direction. The most pliant substances are animal and vegetable fibres and membranes, as silk, bladder, hemp, \&c.

\section{CHAPTER V.}

\section{INERTIA, CRAVITY, AND WEIGHT.}

\section{SECTION I.-INERTIA.}

Q. What is INERTIA?

A. Matter is incapable of spontaneous change; and the term inertia (or inactivity) signifies the total absence of power in matter to change its state. A body endued with inertia cannot of itself, and independent of all external influences, commence to move from a state of rest; neither can it, when moving, arrest its own progress and become quiescent.

Q. When a CARRIAGE is in motion, drawn by Horses, why is the same exertion of power in the horses required to STOP IT, as would be necessary to BACK IT, if it were at rest?

A. Because the force required to destroy motion in one direction is equal to that required to produce as much motion in the opposite direction.

Q. If a body is incapable by ITSELF of changing its state, why will not a BALL fired from a cannon continuo to move on For EVER?

A. Chiefly on account of the resistance of the air or medium through which it passes, and the attraction of the earth.

Q. What do we mean by the term Frictios?

A. In mechanics, it signifies the resistance which a moving body meets with from the surface on which it moves.

Q. Is it possible to construct any machine, or arrangement of matter, which will resPETUALLY continue in motion?

A. It is not; because the operations of gravity, the resistance of 
the medinm through which the body moves, or the friction of the surfaces upon which the body rests, will, in a given time, destroy and teminate all motion. In addition to this, all materials which we employ in construction will, in the course of time, wear out by use, or decay by natural agencies.

Q. Do we know of any instances of perpetual motion in NATURE?

A. Yes; the various planetary bodies belonging to the solar system have been moving with undiminished velocity for ages past; and, unless prevented by the agency which governs all nature, will continue to move in the same manner for ages to come.

Q. If a coach, railway carriage, or boat, moving with speed, be suddenly STOPPED or IETARDED from any cause, why are the passengers, or the baggage carried, precipitated from their places in the DIRECTION OF THE MOTION?

$A$. Because, by reason of their inertia, they persevere in the motion which they shared in common with the body that transported them, and are not deprived of that motion by the same cause.

Q. Why will a prenson, leaping from a carriage in rapid motion, fall in the direction in which the carriage is moving at the MOMENT his feet meet the ground?

A. Because his body, on quitting the vehicle, retains by its inertia the motion which it has in common with it. When he reaches the ground, this motion is destroyed by the resistance of the ground to the feet, but is retained in the upper and heavier part of the body; so that the same effect is produced as if the feet had been tripped.

Q. Why are horses obliged to make a much greater exertion to sTukT a carriage, than afterwards to KEEP IT IN MOTION?

A. Because when a carriage is once put in motion upon a level road, with a determinate speed, the only force necessary to sustain the motion is that which is sufficient to overcome the friction of the rord ; but, at starting, a greater expenditure of force is necessary, iriasmuch as not only the friction is to be overcome, but the force with which the rehicle is intended to move must be communicated to it.

Q. If a body in motion strikes another body of equal mass at REST, the two will inove on together, but with only half the original velocity of the first. To what is this change due?

A. Because one-half of the velocity of the first body is expended in overcoming the inertice of the second.

"The language sometimes used to explain the property of inertia in popular works is eminently calculated to mislead the student. The terms resistance, and stubhornness to move, are faulty in this respect. Inertia implies absolute passiveness, a perfect indifference to rest notion. It implies as strongly the absence of all resistance to the reception of motion, as it does the absence of all power to move itself." - LALDNER.

Q. When the sails of a ship are first spread to receive the Foros or IMruLs: of the wind, why does not the ressel accuire her finll speed at once?

A. Because it requires a little time for the imnelling force to overcome the inertice of the mass of the ship, or its disposition to remain at rest.

Q. Why, when the sails are taken in, does the vessel continue to move for a considerable time?

A. Because the inerlice of the mass is opposed to a change of state, and the ressel will continue to move until the resistance of the water orercomes the opposition. 
Q. Why is a man standing carelessly in the sTeRs of a boat liable to fall into the water belind, when the boat begins to move?

A. Because his feet are pulled forward while the inertic of his body keeps it in the same position, and, therefore, behind its support. For a similar reason, when the boat stops, the man is liable to fall forwards.

\section{SECTION II.-GRAVITY.}

Q. What is Graviti?

A. The tendency of a mass of matter towards its central body. Gravitation is the tendency of all matter in the universe towards all other matter. Terrestrial gravitation is the attraction of a body towards the centre of the earth.

Q. In what respect does the $\triangle$ TrRACTION OF GRAVITATION differ from all other attractive force?

A. Because it is the common property of all bodies; since everything to which we can attach the idea of materiality is affected more or less by gravitation.

Q. Why does an APPLE loosened from the tree fall to the ground?

A. Because the earth attructs or draws it to itself.

Q. Since all bodies are attracted towards the earth, how does it happen that Surors and some other forms of matter display the contrary phenomena of ASOLLDDING from it?

A. Because the smoke is lighter than the air, buth for bulk, and Hoats upon it. It is unable to advance, however, in the most minute degree, without displacing or thrusting downward portions of the atmosphere equal to its own bulk.

Q. Why doas a conr pressed benoath the water rise and float on the surface?

A. Because the corl is lighter than an equal bulk of water, and is pressed up and sustained by it in the same manner that the particles of smoke are sustained by the particles of air.

Q. Why docs a Balioon rise in the air?

A. Because it is filled with a gas which is lighter, bulk for bulk, than the air.

Q. Ilow long will surows continue to float above the surface of the earth?

A. Until its particles, uniting, become heavier than the air, when they descend in the form of small flakes of soot.

Q. As GRavitativi atmRaction is the common property of all bodies, why is it that all bodies not fasteved to the earth's surface do not come in contact?

A. On account of the overpowering influence of the earth's attraction, the mutual attraction of particular bodies on its surface is neutralised or overcome.

In some instances, however, this mutual attraction exhihits itself.

Q. Why do the BUbBues in a cup of Tras range round the sides of the oup?

A. Because the cup attracts them.

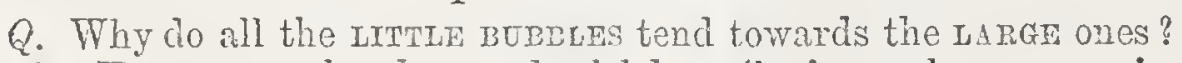
then.

A. Because the large bubbles (being the superior masses) attruct

Q. Why do the BUBBIFS of a CUP OF TEA FOLIOW a TEA-SPOONF?

A. Because the tea-spoon attracts them. 
Q. Why are the SIDES of a pond covered with zenves, while the MIDDLE of the pond is quite CLMAR?

A. Because the shore attracts the leaves to itgelf.

Q. Why can you fill a dry glass beyond the level of the brim?

A. Because the mass of water in the glass holds the overplus back by the attraction of its particles.

Q. Is the attraction of the EARTI the same at all distances from its SURFACE or CENTHE?

A. No; the attraction of the earth for a body varies inversely with the square of its distance from the centre.

Q. How can this be illustrated?

A. In the following manner:-If the earth attracts a body with a certain force at the distance of one mile, it will attract with fou times the force at half a mile, nine times the force at one-third of a mile, and so on in like proportion. On the contrary, is will attract with but one-fourth of the force at two miles, one-ninth of the force at three miles, one-sixteenth of the force at four miles, and so on as the distance increases.

Q. Do ALL BODISs attract each other equally?

A. They attract each other with forces proportioned to their masses.

Q. A FEATirer falls to the ground by the influence of the earth's attraction. Now, as all bodies attract each other, does the FEATHER ATTRACT OR DRAW UP THE EARTH in any degree towards itself?

A. It does, with a force proportioned to its mass; but as the mass of the earth is infinitely greater than the feather, the influence of the feather is infinitely small, and we are unable to perceive it.

Q. What would be the consequence if the FEATIIER DID No'T ATTRACT THE EARTH?

A. If any portion of the earth, however small, failed to attract another portion, and not be itself attracted, the axis of the earth avould be immediatcly changed, involving an alteration of climate, and the place of the ocean in its bed.

Q. Why is it more dangerous to fall from a lofty elevation than from a low one?

A. As the attraction of the earth varies inversely with the square of the distance, the force with which a falling body will strike the ground will increase in proportion to the height from which it has fallen.

Q. What do we mean by the CENTRI OE GRAVITS?

A. That point in a body about which, if supported, the whole body will balance itself.

Q. In what Posimios only can a BoDY rest?

A. Only when its centre of gravity is supported; and until this is accomplished the body will move, and continue to do so, until it settles into a position in which the centre of gravity cannot sink lower.

Q. Why does a PERson in rising from a chaIr bend forward?

A. When a person is sitting, the centre of gravity is supported by the seat; in an erect position, the centre of gravity is supported by the feet; therefore, before rising, it is necessary to change the centre of gravity, and by bending forward we transfer it from the chair to point over the feet. 
Q. Why does a QUADRUPED never raise both feet on the same side simultaneously?

A. Because, if it did, the centre of gravity would be unsupported, and the animal would fall over.

Q. Why is a Turiter placed upon its Bacis unable to more?

A. Because the centre of grarity of the turtle is, in this position, at the lowest point, and the animal is mable to change it; therefore it is obliged to remain at rest.

2. Why does a person carrying a Wergitr upon his BACK stoop forward?

A. In order to bring the centre of gravity of his body and the load over his feet.

If he carried the load in the position of the figure at $A$, he would fall backwards, as the direction of the centre of gravity would fall beyond his heels; to bring the centre of gravity over his feet, he assumes the position indicated by the figure $B$.

Fig. 1.

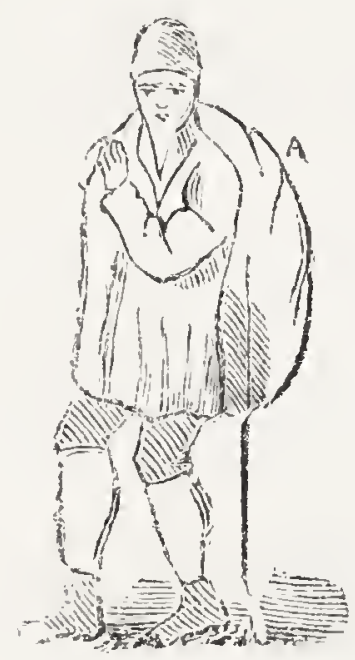

Fig. 2.

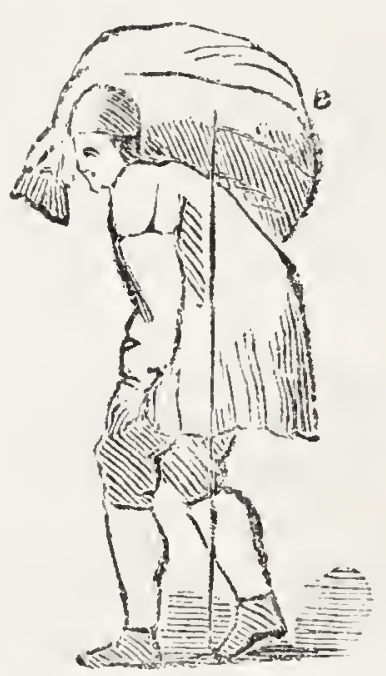

Q. Thy is it nore dificult to overtlurow a body having a broad base, than one resting upon an narrow basis?

A. Because a body camot fall over so long as a line directed from the centre of gravity rertically toward the surface upon which the body rests, falls within the figure formed by the base of the body in question. Hence, the broader the base of a body, the more securely it will stand.

Fig. 3.

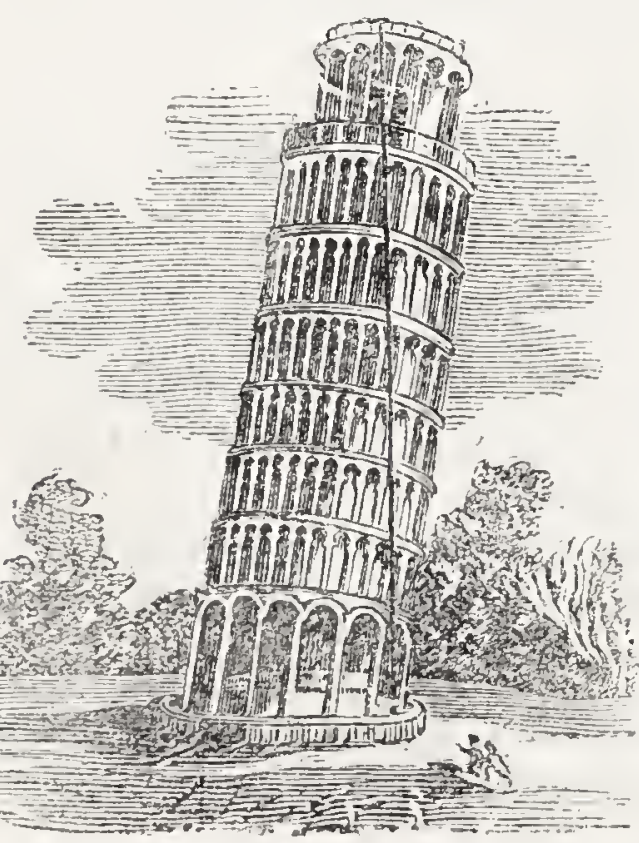

Q. How long will a wals or Tower staud securely?

A. So long as the perpendicular line drawn through its centre of gravity falls within its base.

The celebrated Leaning Tower of Pisa, 315 feet high, with an inclination from the perpendicular of 12 feet, is an example of this law. For instance, the line in fig 3 , from the summit to the ground, passing through the centre of gravity, falls within the base, and the tower stands securely ; but suppose an attempt was made to carry it a little higher, the perpendicular line passing through the centre of gravity would then fall without the base, and the structure could no longer support itself. 
Q. Why is a HIGn CARRIGe more liable to be overthrown by an irregularity of the road than a low one?

Fig. 4.

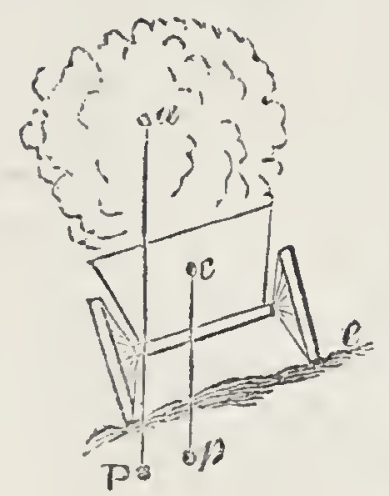

A. In a low carriage, as in the case of a truct: loaded with metal, the centre of gravity is low, and the perpendicular line from it falls much within the base, as is shown by the line $c p(f i g .4)$; but if the same truck be loaded with wool, the perpendicular let fall from the high centre of gravity would come very near to the wheel, as at a $P$, or altogether outside the wheels.

Q. Why is it more difficult to turn over a PYRAMDAL-SIIAPED STRUOTURE than a CYLINDRICAL ONE?

A. Before a structure will fall, its centre of gravity must be elevated, so that the perpendicular let fall from it will pass beyond its base. In a pyramid the centre of gravity is low, and the base broad; consequently, one side of the pyramid must be raised high up before it can be overthrown. In a cylinder the opposite conditions exist.

Q. What is the advantage of TURNIN

$A$. It increases the breadth of the base supporting the body, and enables us to stand more securely.

Q. Why do very FAT PEOPLE throw back their II with an air of apparent self-satisfaction?

A. In order that they may effectually keep the centre of gravity of the body over the base formed by the soles of the feet.

Q. Why cannot a MAN, standing with his HiELS close to a perpendicular wall, bend over sufficientily to pick up any object that lies before him on the ground, without falling?

A. Because the wall prevents him from throwing part of his body backward, to counterbalance the head and arms that must project forward.

Q. What is the reason that persons walkiug $A \mathrm{RM}-\mathrm{IN}-\mathrm{ARM}$ shake and jostle each other, unless they make the movements of their feet to correspond, as soldiers do in marching?

A. When we walk at a moderate rate, the centre of gravity comes alternately over the right and orer the left foot. 'The body advances. therefore, in a waving line; and unless two persons walking together keep step, the waving motion of the two fails to coincide.

Q. Where would the onNTRE of GrAvitr be in a whEFL made entirely of wond, and of a uniform thickness?

A. In the centre.

Q. Where would the centre of gravity be if a PAIT of the rin of the same wheel were made of IRON?

A. It would be changed to some point aside from the centre of the wheel.

Q. How can you find the centre of gravity in an IRREGULART-SIIAPED body?

A. First suspend it from a point, as at $a(f i g .5)$, and at the same point suspend a plumb-line $c$, when both board and line will hang in the position represented in the figure. Having marked this line, let 
it be suspended from another and different point, as in fiy. 6, and the perpendicular line indicated by the plumb again marked. The point where these two lines so marked cross each other is the centre of gravity of the body, as seen in fig. 7 .

Fig. 5.

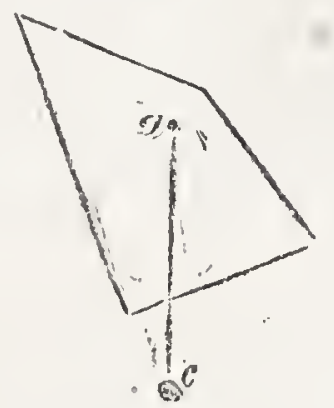

Fig. 6.

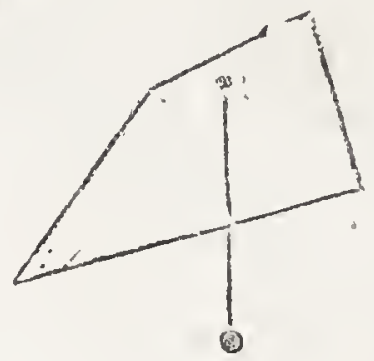

Fig. $\because$.

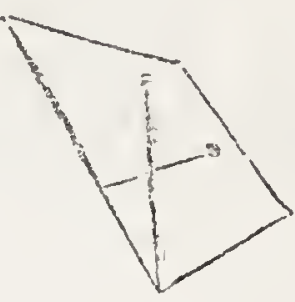

When two or more bodies are comnected together, the centre of gravity of the two is the point on which they will balance without falling. I'Thus, if two balls, each weighing four pounds, be connected together by a bar, the centre of gravity will be a point oir the bar equally distant from each. But if one of the balls be heavier than the other, then the centre of gravity will, in proportion, approach the larger ball. This is illustrater by reference to $f i g .8$, in which the centre of gravity about which the two balls support themselves, is seen to be nearest to the heaviest and largest ball.

Fig. 8.

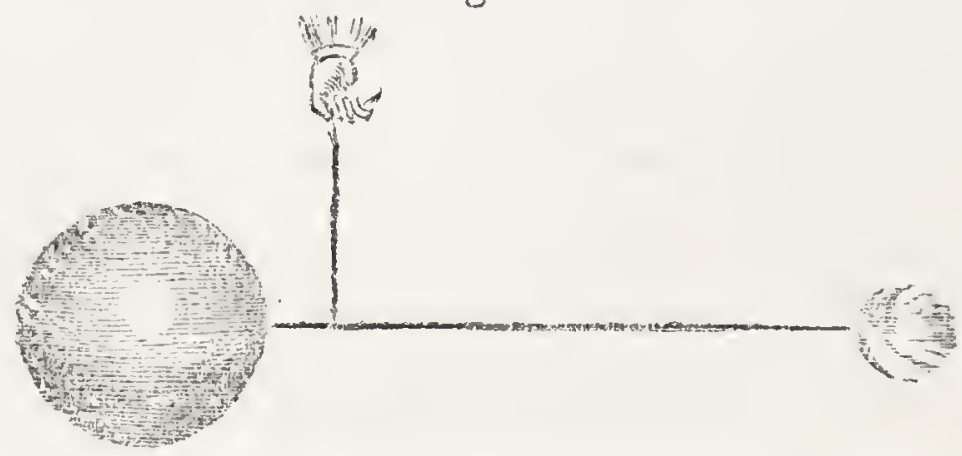

Q. What is a PLUMB-LINA or ILUMrET ?

A. It is a line with a weight attached to its end, used to determine a perpendicular, or to ascertain, by comparison, whether any other object is in a perpendicular position.

Q. When a LINS with a weight attached to its end is left to hang free, in what DIREOTION will its LINE OF DIRECTION be? earth.

$A$. Its line of direction will be towards the centre of gravity in the

Q. What is the PLUMB-rule used by BUILDERS, IIASONS, \&e.

A. It is a narrow board, having a plumb-line suspended from its top, and a perpendicular mark through its middle.

Q. How by the nise of the PLUMr-RULE can we ascertain whether the wall of a builaing is perfectly perpendicular?

$A$. If the wall be perfectly perpendicular and true, when the plumb-rule is placed against it, the plumb-line suspended from the top will coincide, or cover the perpendicular mark in the centre of tive board. If the wall is not perpendicular, the plumb-line will not coincide with the line, but deviate in some degree from it.

Q. In what does the art of balancing or waiking upon a Rops consist ?

A. In keeping the centre of gravity in a line over the base unon which the body rests. 
Q. What is the DASE upon which the HUISAN RODY rests or is supported ?

A. The two feet and the space included between them.

$Q$. Why is it a very difficult thing for cHILDREN to learn to walk?

A. In consequence of the natural upright position of the human body, it is constantly necessary to employ some exertion to keep our balance, or to prevent ourselves from falling, when we place one foot before the other. Children, after they acquire strength to stand, are obliged to acquire this knowledge of preserving the balance, by experience. When the art is once acquired, the necessary actions are performed involuntarily.

Q. Why do Young QUaDRUPEDS learn to walk much sooner than CHILDREN?

A. Because a body is tottering in proportion to its great altitude and nurrow base. A child has a body thus constituted, and learrs to walk but slowly because of this diffculty (perhaps in ten or twelve months), while the young of quadrupeds, having a broad supporting base, are able to stand and move about almost immediately.

Q. Are all the LMiBS of a rALI THEE arranged in such a manner, that the line directcd from the centre of gravity is caused to fall within the BASE of the TREE?

A. Nature causes the various limbs to shoot out and grow from the sides with as much exactness, in respect to lieeping the centre of gravity within the base, as though they had been all arranged witk the plumb-line. Each limb grows, in respect to all the others, in such a manner as to preserve a due balance between the whole.

\section{SECTION III.-WEIGIT.}

Q. What is WEIGHT?

$A$. It is the measure of force with which a body is attracted by the earth. In an ordinary sense it is the quantity of matter contained in a body, as ascertained by the balance.

Q. To what is the wergut of a body proportional ?

A. The veight of a body is always proportional to the quantity of matter contained in it.

Q. Why will a BALI of LEAD weigh more than a DALL of CoTTON of the same size?

A. Because the quantity of matter contained in the ball of lead is much greater than the quantity of matter contained in tho ball of cotton. The attraction of gravitation being proportioned to the quantity of matter, it follows that the lead ball will be drawn towards the earth with a greater force ( $i$. c. will weigh more) than the ball of cotton.

Q. A MAN of moderate weight upon the surface of the earth would weigh 'rwo TONS if transported to the surface of the sun: why would he weigh more upon the surface of the sun than upon the surface of the earth?

A. Because the attractive force of the sun, on account of its greater magnitude, far exceeds the attractive force of the earth.

Weight, it must be remembered, is the measure of the attraction of gravitition.

Q. Why will a yass of rnow weigh less on the top of a HIGI MOUNTAIN than at the LEVEI of the SEA?

A. Because the attraction of gravitation is less at the top of the 
mountain than at the level surface of the earth; weight decreasing or increasing as the attraction of gravitation increases or decreases, according to the squares of the distances.

A ball of iron, weighing a thousand pounds at the level of the sea, would be perceired to have lost two pounds of its weight if taken to the top of a mountain four miles high, a spring balance being used.

Q. Where will a DODY weigh the most on the surface of the EARTII

A. At the poles of the earth, for at these points the attractire power is greatest.

It must be remembered that the earth is not a perfect sphere, but flattened at the poles; consequently the poles of the earth are nearer the centre of attraction ( $i . c$. the centre of the earth) than at any other point on its surface.

Q. Where will a mony weigh the least on the carth's surface?

A. At the equator, for there the attractive power is less; the surrace at this point being the most distant from the centre of the earth.

Q. What would be the weight of a body carried to the crantre of the earth?

A. It would have no weight; for the attraction of gravitation, acting equally in every direction, no effect would be produced; and the body would be fixed as if sustained by a number of magnetic points.

Q. What twro sYSTEMS of weight are cmployed in Great Britain?

A. Troy weight and avoirclupois weight.

Q. What is T'Roy weight used for, and from whence does it derive its name?

A. Troy weight is used for weighing gold and silver. It derives its mme from the ancient designation of London, Troy Novant, or from Troyes, in France, where it was first adopted in Europe. It has existed in England from the time of Edward the Confessor.

2. What is Aroirdupois weight used for, and from whence does it derive its name ?

A. Avoirdupois weight is used for the weight of merchandise other than the precious metals. It derives its name from the French avoirs (averici), goods or chattels, and poids, weight.

Q. What is a GRAIr weight?

A. A grain veight is the smallest measure of weight made use of in the English system. By a law of England, enacted in 1286, it was ordered that 32 grains of wheat, well dried, should weigh a pennyweight. Hence the name grain applied to this measure of weight. It was afterwards ordered that a pennyweight should be divided into only 24 grains.

Q. How do we make a GRAIN WEIGHT for practical purposes?

A. By weighing a thin plate of metal of uniform thickness, and cutting out, by measurement, such a proportion of the whole as should give one grain. In this way, weights may be obtained for chenical purposes, which weigh only $\frac{1}{1000}$ th part of a grain.

Q. How is the IENGTH Or STANDARD of an inch, in the English standard of weights and measures, determined?

A. It has been found that a penduhum which, in the latitude of London, will vibrate or swing from the highest point on one side to the highest point on the other side in one second of time, will always, under the same circumstances, have a constant length. The lenyts of this pendutum is divided into 391,393 equal parts; and 10,000 
of these parts are called an inch, 12 of which make a foot, and 36 of them a yard.

Q. Are all the other measures determined from this STANDARD?

$A$. They are; and by referring to this standurd, we are enabled to verify or determine the accuracy of any particular weight of measure.

Q. What part of an Ixor is a IINE?

A. One-twelfth of an inch is designated as a line.

Q. How is the measure of a GaLion obtained ?

A. A cubic inch (accurately obtained from the pendulum) of distilled water, of a temperature of $62^{\circ}$ Fahrenheit's thermometer, is taken and weighed. This weight is divided into 252,458 equal parts; and of these 1,000 will be a grain. Ten pounds, or 7,000 of these grains of distilled water, at the same temperature, will be a gallon; which gallon will contain 277 cubic inches, and about onequarter of a cubic inch over.

Q. What is the most PERFECT STSTEM of weights and measures existing and in use?

A. The French system. This system is characterised by its great simplicity, all its divisions being made by 10 . Hence, it is called the decimal system of weights and measures.

Q. WHEN was this system of weights and mensures constructed?

A. In 1788 , the French government, feeling the necessity of having some standard by which all weights and measures might be compared and made uniform, ordered a scientific inquiry to be made; the result of which was the establishment of the present system of Irench weights and measures, which, from its perfect accuracy and simplicity, must ultimately take the place of all other systems.

Q. What did the French take as their UNIT or STANDARD?

A. The French standard is based on an invariable dimension of the globe, which is recognised in all countries; viz., a fourth part of the earth's meridian.

Q. How are the different localities on the earth defined?

A. By imaginary lines or circles drawn round it.

Q. What are those circles CALLED whicis run round the globe from EAST to WEST?

A. Parallels, or circles of latitude. The largest of these, as will be seen by reference to fig. 9 , is the equator. E. W.

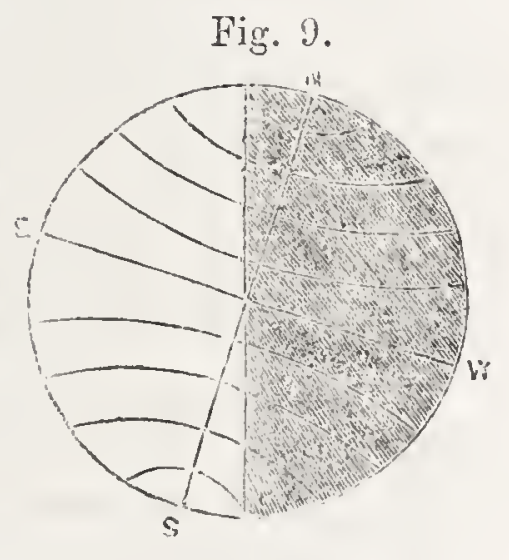

Q. What are those cIncles CaLied which run round the globe from son to to sourn?

A. Meridians, or circles of longitude. They run round the globe lengthwise, and mite with each other at the poles.

Q. How do the cricifs of Latitude differ from the cincres of rongitude, or the SIERIDIANS.

A.. The circles of latitude gradually become smaller as they approach the poles, while the meridians, on the contrary, are all of erual size. The circle NES IV N (fig. 9) represents a meridian. A fourth part of this circle, or, what is the same thing, a fourth part 
of the earth's circumference, as $\mathrm{N} \mathrm{E}$, is taken as the basis of the French system. inch?

Q. What is the uxir of length in the French system corresponding to the English

A. The metre, found by dividing one-fourth part of the meridian into 10,000,000 equal parts. A single ten-millionth part is a metre. It is equal to about 39 English inches.

Q. How are the SMALLER MEASURES produced from the MITRE?

A. By dividing by ten. The measures so obtained are designated by Latin terms: thus, a decimetre is the one-tenth of a metre; a centimetre is one-hundredth of a metre; a millimetre is one-thousandth of a metre.

Q. How are the LARGER MEASURES produced from the MEETRE?

A. By multiplying by ten. The measures so obtained are designated by Greek terms: thus, a decametre is 10 netres; a hectometre is 100 metres; a kilometre is 1,000 metres; a miriametre is 10,000 metres.

Q. How is the French system of WEIGITS derived from the MriAsures of Lenctir?

A. A cubical box was taken, measuring exactly on each of its sides the 100th part of a metre, or one centimetre. This was filled with water at its greatest density, and the weight of this water was called a gramme.

Q. How are the SMALLRR and IARGRR weights derived from the GRAMME ?

A. By multiplying it or dividing it by ten, designating the smaller weights by Latin terms, and the larger by Greek rerms. Thus, we have a centigramme, the one-hundredth part of a gramme, and the hectogramme, equal to 100 grammes.

Q. How is the standard for DRY MIELSURE obtained in this system?

A. A cube, measuring on each of its sides one metre, constitutes a stere, or the unit of dry measure. The larger or smaller measmes are obtained by multiplying or dividing this by ten.

Q. Wirker are the standards of woights and measures to be found in England?

A. At the Exchequer, in London, and in the keeping of the chief magistrates in the towns and cities of the kingdom.

Q. Are the standards of weights and measures in the United States the same wis in England?

A. They are essentially the same.

Q. In what countries is the Frexcri srs'rax adopted?

A. In Trance and Belgium; and by scientific men in all parte of the world. 


\title{
CHAPIER VI.
}

\section{SPECIFIC GRAVITY AND CAPILLARY ATTRACTION.}

\author{
SECTION I.-SPECIFIC GRAVITY.
}

Q. Why does ICE float upon WATER?

A. Because it is lighter than water.

Q. Why rloes IRoN sink in WATER?

A. Because it is heavier than water.

Q. If we put a piece of ICE in $\triangle L C O H O L$, it sinks; if we put a picee of Iros upon QUICE. sIIvEr, it floats: why is this?

A. Because the ice is heavier than the alcohol, and the iron is Tighter than the quicksilver.

Q. What do we mean when we say that ICE is linhter than Irox?

A. We mean that, taking equal buths of each, the former weighs less than the latter; and when we say that quicksilver is heavier than water, we mean that, in equal volumes, as a pint, for instance, the quicksilver has a greater weight than the water.

Q. How do we determine the SPEOIFIC WEIGHT or GRAVITY of spirits and iee, and iron and quicksilver; or; in other words, how do we ascertain how mueh LIGIIren the first two, and how much HeATIEn the last two are than WATrR?

A. By dividing the weight of a given bulk of each of these substances by the weight of an equal bull of distilled water.

Suppose we take five vessels, each of which would contain exaetly one hundred grains of water, and fill them respectively with spirits, ice, water, iron, and quicksilver. The following differences in weight will be found :-The vessel filled with spirits would weigh 80 grnins; with ice, 90 grains; with water, 100 grains; with iron 750 grains; with quicksilver, 1,350 grains. To facilitate the comparison of the numbers whieh indicate how much sreater the specific gravity of one is than that of another, water has been fixed upon as the standard or unit. T'herefore, in the above ease, the question is, how much lighter. than water are spirits and ice, and how much heavier than water are iron and quicksilver; or, in other words, how many times is 100 contained in $80,90,750$, and 1,350 ? The other numbers are therefore to be divided by 100 , the weight of the water; and there is found for spirits the veight $0 \cdot 80,1$-5 th lighter than water ; for the ice $0.90,1-10$ th lighter than water; for the iron $7 \cdot 50$, or $7 \frac{1}{2}$ heavier than water; for the quicksilver $13 \cdot 50$, or 13 $\frac{1}{2}$ times beavier than water. These numbers, therefore, are the specific gravities of the spirits, ice, iron, and quiclssilver.

Q. What, then, is SPECIFIC GMAviTr?

1. It is the weight of a body compared with the weight of an errual bulli of water.

Q. How does it differ from ondinART or ARSOLUTE WEIGIT ?

A. In absolute weight no regard is paid to the volume or buil: of substances. In specific weight, a given bulk or volume is compared with an equal volume or bulk of water.

$Q$. What kody has the GreatesT speeific weight?

A. Purified platina, which is 22 times heavier than an equal bulk of water.

Q. What substanee has the SxALIEST specific weight?

A. Hydrogen gas, being 0.00008 lighter than an equal bulk of watei.

Q. Why will an rag float in sTrONG BRINE, and not in FRESI WAYER?

$A$. Because the solution of a solid in any liquid increases its density 
or its specifu gravity; the addition of salt to the water renders the specific gravity of the brine greater than that of fresh water, or of the egg.

Q. How do cooks ascertain if their BRINe be SALT ENotGir for pickling ?

A. They put an egg into their brine. If the egg sinks; the brine is not strong enough; if the egg flocts, it is.

Q. Why will an EGG SINK if the brine be NoT STRoxc enough for pickling?

A. Because an egg will be the heavier; but if as much salt bs added as the water can dissolve, an egg will be lighter than the strong brine, and consequently float on the surface.

Q. Why is it more easy to SWIn in the SEA than in a River?

A. Because the specific gravity of salt water is greater than that of fresh, and, therefore, it buoys up the swimmer better.

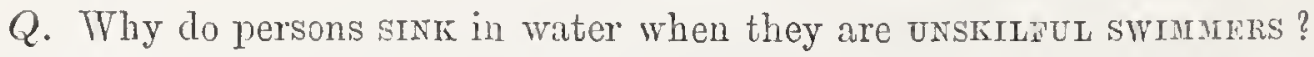

A. Because they struggle to keep their head out of water.

Q. EXPIALIN how this is.

A. When our head is thrown back boldly into the water, our mouth is kept above the surface, and we are able to breathe; but when the head is kept above the surface of the water, the chin and mouth sink beneath it, and the swimmer is suffocated.

This may be illustrated thus :- If a piece of wood be of such specific gravity that only t200 squcere incles can float out of water, it is manifest that if two other inches are raised out, the two former inches must be plunged in. The body (in floating) resembles this jiece of wood. If two square inches of our face float out of the water, we can breathe ; but if part of the back and croun of the head are raised above the water, the lower part of the face will be depressed beneath it.

Q. Why can QUADrupeDs swim More EASILY than MAN?

A. 1. Because the trunk of quadrupeds is fighter than water, and this is the greater part of them; and

2. The position of a beast (when swimming) is a natural one.

Q. Why is it MORE DIFEICULT for a MAN to swim than for a BEAST?

A. 1. Because his body is more heavy in proportion than that of \&l beast, and

2. The position and muscular action of a man (when swimming) differ greatly from his ordinary habits, but beasts swim in their ordinary position.

Q. Why can FA'T men swim more EasiLY than SPARE men?

A. Because fat is lighter than water; and the fatter a man is, the more buoyant will he be.

Q. How are Fisires able to ASCEND to the SURFACE of water?

$A$. Fishes have an air-bladder near the abdomen; when this bladder is filled with air, the fish increases in size, and (being lighter) ascends through the water to its surface.

Q. How are fishes alie tc Lirs in a minute to the Borron of a stream?

A. They expel the air from their air-bladder; in consequence of which their size is diminished, and they sink instant?.

Q. Why does the body of a Drowxan person rise and float upon the surface several dass ấtor cleath?

A. Because, from the accumulation of gas within the borly (cansed 
by incipient putrefaction), the body becomes specifically lighter than water, and rises and floats upon the surface.

Q. How are LIFW-BOATs prevented from sinking?

A. They contain in their sides air-tiglt cells, or boxes filled with air, which, by their buoyancy, prevent the boat from sinking even when it is filled with water.

Q. The suaves of the West Indies have a plan of steaing RuM from a CASK, by inserting the LONG NECK of a BOTTLE, full of water, through the BONG. IJow are they enabled in this manner to obtain the rum?

A. The rum is very much lighter than the vater; and as the heavy water falls out of the bottle into the cask, the lighter rum rises to take its place.

Q. Why does a sKY-rocket ascend when fired?

A. Because the lower part is always producing a large quantity of aeriform fuid, which, in expanding, presses not only on the air below, but also upon the rocket above, and thus lifts it. The ascent is also aided by the recoil of the rocket from the part of its substance which is constantly bursting downward.

Q. Why does CREA rise upon MILK ?

A. Because it is composed of particles of oily or fatty matter, which are lighter than the watery particles of the milk.

Q. Why do STALE EGGS float upon water?

A. Because, by keeping, air is substituted for a portion of the water of the egg, which escapes.

Q. Why does not a VESSEL constructed of IRos sink, as the iron is much HEAvier than the water?

A. Because the ressel is constructed in a concave form, and is thus rendered buoyant. Every substance becomes lighter in water, in proportion to the amount of water displaced. This is a law of nature: if it displaces less water than its weight in air, it sinks; if more, it floats. The ship, being concave, displaces a greater weight of water than the weight of the iron of which it is composed in the air.

A thick piece of iron, weighing half an ounce, loses in water nearly one-eighth of its weight; but if it is hammered out into a plate or ressel, of such a size that it occupies ejght times as much space as before, it then loses its whole weight in water, and will float, sinking just to the brim. If made twice as large, it will displace one ounce of water, consequently, twice its own weight; it will then sink to the middle, and can be loaded with a half an ounce weight before sinking entirely.

Q. What is a IIXDroneter?

A. An instrument for determining the specific gravity of liquills. It is particularly used for determining the strength of spirituous liquors, it being inversely as their specific gravities; thus the less specific gravity of a liquor, the greater its strength.

By this instrument, the amount of water added to a giren quantity of spirits can be determined with the utmost accuracy.

Q. Upon WHAT PRINCIPLE is the IYPROMETFr coustructed?

A. That the same body will sink to a greatcr or less depth in different liquids: leeper in the lighter ones, and not so deep in those which are denser. Advantage has therefore been taken of this, for constructing an instrument for determining the specific gravity of liquids. 
Q. Of what does the HYDRoxerER consist?

A. The hydrometer consists generally of a hollow glass tube, constructed, as is represented in fig. 10, wish two scales, one to represent the density $b$, the other a thermometer, to represent the temperature $a$. The end of the tube is blown ont into a bulb, which is loaded with quicksilver or shot, to cause it to float in a rertical position. 'The scale $b$ shows the depth to which it sinks in any liquid. There are varions instrunents of this kind especially adapted for detemining the density of spirits, oils, syrups, lye, \&c.

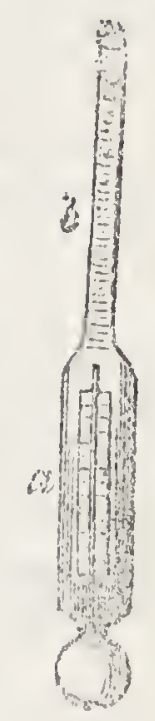

Q. Why are stonis, GRAVIr, and SAND so easily moved by WAVRs and CURRENTS ?

A. Because the moving water has only to overcome about half the weeight of the stone.

Q. Why can a STONE which on land requires the strength of two Ires to lift it, be lifued and carried in water by oNe MaN?

A. Because the vocier holds up the stone with a force equal to the weight of the rolume of water it displaces.

Q. Why will some kinds of WOOD which float in WATER, sink in OIL?

A. The wood, immersed either in oil or water, displaces exactly its own buth: of the liquid. In the water, the weight of the liquid displaced is yreater than the weight of the wood; consequently, the water holds it up. In the oil, the weight, of the liquid displaced is less than the weight of the wood; consequently, the oil is unable to sustain it, and the wood sinks.

\section{SECIION II.-CAPILLARY ATTRACTION.}

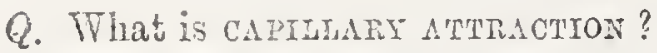

4. The power which very minute tubes possess of cansing a liquid to rise in them above its level.

"Ccepillary" from the Latin word "capillaris," (like a Their:; the tubes referred to are almost as fine and delicate as at hair. Water ascends through a lump of sugar or piece of spone, by coppillary attraction.

N.B. The smaller the tube, the higher will the liquid be attracted by it. Fig. 11 iliustrates the manner in which watci vill rise in tubes of aiferent diameters.

Q. Why dices WATtr MeLT SAL'?

Fig. 11.

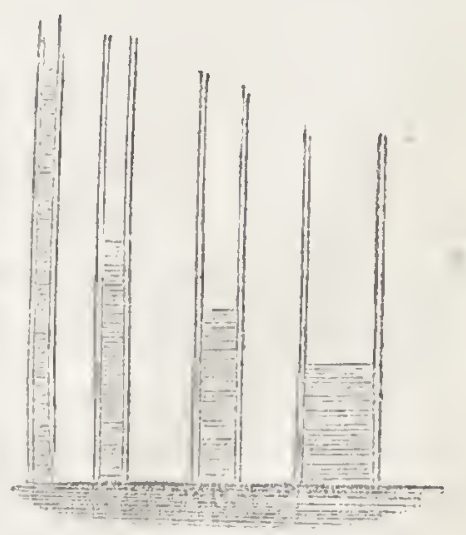

A. Because very minute particles of water insinuate themselves into the pores of the salt by capillary attraction, and force the crystals apart from each other. 
Q. Why does WATER MELT SUGAR?

A. Because very minute particles of water insinuate themsclves into the pores of the sugar by capillary attraction, and force the crystals apart from each other. ficll?

Q. Why is regetation on the Margin of a river more luxuriant than in an open

A. Because the porous earth on the bank draws up water to the roots of the plants by capillary attraction.

Q. Why do persons who water PIANTS very often pour the water into the saUdur and not over the plants?

A. Because the water in the saucer is drawn up by the mould (through the hole at the bottom of the flower-pot), and is transferred to the stem and leaves of the plant by capillary attraction.

Q. Why is cortox best adapted for LAMP-WICES ?

A. Because the arrangement of the fibres of the cotton-wick is sich, that the whole forms a bundle of minute tubes, in which the oil ascends and supplies the flame by capillary attraction.

Q. Why does brotTing PAPER absorb INE?

A. The ink is drawn up between the minute fibres of the paper by capillary attraction.

Q. Why will not WRITING or SIZED PAPER absorb INK?

A. Because the sizing, being a species of glue into which writing papers are dipped, fills up the little interstices between the fibres, and in this way prevents all capithary attraction.

Q. How does a SPONGE absorb WATER ?

A. The pores of the sponge constitute minute tubes in which the water rises by capillary attraction.

Q. What occasions the rise of water or other liquidis in SMALI SUBES?

A. The attraction between the liquid and the walls of the tube.

Q. Why does the water rise bigher in a tube of suAII DIANETER than in a LARGg one?

A. Because, in a small tube, the sides being nearer to the raised weter, attract it more powerfully. See fig. 11.

Q. If we GRFASE the INSIDE of a glass tube, will the water then rise in it?

A. It will not; because there is no attraction between the grease and the water.

Q. If we place a GLASS PLATE in water, will the liquid rise upon each side of it?

fig. 12.

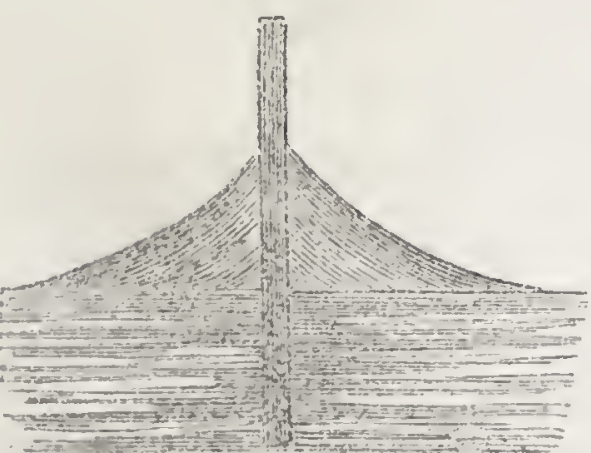

$A$. If we place a glass rod or plate in a vessel of water, we shall perceire, after the whole has been allowed to remain free of any disturbance, that the water will rise to a higner level on all sides of the glass, than over any part of its surace. (see fig. 
Q. If we place the SAME PLATE of glass in Mrercury, what will be the effect? Fig. 13.

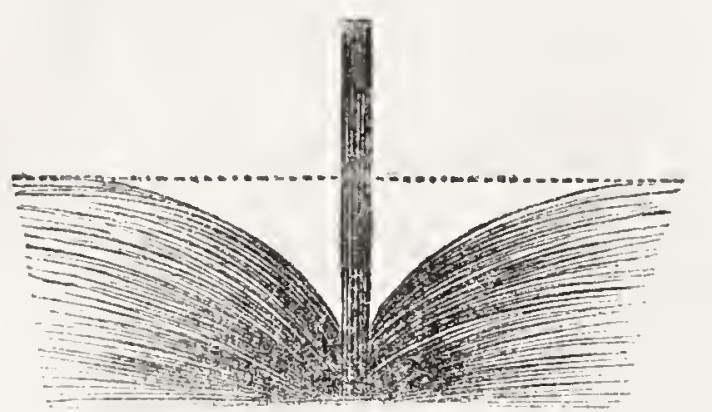

$A$. The mercury, instead of rising upon the sides of the glass, will be depressed (as shown in fig. 13), there being no attraction between the glass and the mercury.

Q. Why, in a Baroneter, is the top of the column of mercury convex or ovac? Fig. 14.

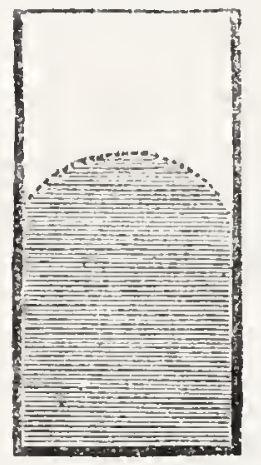

A. Because, owing to the repulsion between the glass and the mercury, the latter, when it comes in contact with the glass, is depressed; which leaves the central part of the top of the column of mercury the highest, as is shown in fig. 14.

Q. Why will WATER, INK, or oIL, coming in eontact with the EDGE OF A BOOK, soak further in than if spilled upon the sides?

A. Because the space between the leaves acts in the same manner as a small tube would-attracts the fluid, and causes it to penetrate far inward. The fluid penetrates with more difficulty upon the side of the leaf, because the pores in the paper are irregular, and not continuous from leaf to leaf.

Q. Why does DRY wood, immersed in water, SWELL?

A. Because the water enters the pores of wood by capillary attrac. tion, and forces the particles further apart from each other.

Q. Why may an mrmense masS, suspended by a DR ROPE, be raised a little way by merely wetTing the rope?

A. Because the moisture imbibed into the substance of the rope by capillary attraction, causes it to swell laterally, and to become shorter.

\section{CHAPTER VII.}

\section{LAWS AND PROPERTIES OF BODIES IN MOTION.}

Q. Wil you define мотток?

A. It is the term applied to the phenomena of the changing of place among bodies.

Q. What would be the state of things if no Motron existed?

A. The universe would be dead. There would be no rising and setting of the sun, no flow of water or of air (wind), no sound, light, or animal existence.

Q. The sUREACE of the EARTI at the EQUATOR moves at the rate of about a THOUGAND miles in an Houn: why are men not sensible of this rapid movement of the earth?

A. Because all objects about the observer are moving in common 
with him. It is the natural uniformity of the undisturbed motion which causes the earth and all the bodies moving together with it upon its surface to appear at rest.

Q. How can you easily see that the EARTI is in motion?

A. By looking at some object that is entirely uncomnected with it, as the sum or the stars. We are here, however, liable to the mistake that the sun or stars are in motion, and not we ourselves with the earth.

Q. Does the sUN really RISE and SET each day?

$A$. The sun maintains very nearly a constant position; but the earth revolves, and is constantly changing its position. Really, therefore, the sun neither rises nor sets.

Q. Why, to a PERSON SAILING in a BOAT on a smooth stream, or GOING SWIFTLY in a CARRIAGE on a smooth road, do the trees or buildings on the banks or roadside appear to move in an OPPOSITE DIRECTION?

A. The relative situation of the trees and buildings to the person, and to each other, is actually changed by the motion of the observer; but the mind, in judging of the real change in place by the difference in the position of the objects observed, unconsciously confounds the apparent and relative motion with that which is real and true.

Q. Upon what does the FoncE and IMPETUS of a BODX in MOTION depend?

A. Upon its mass and velocity.

Q. In what way may a SMALI BODY in motion be made to act with the SAME FOROr as another hody of much GREATER PROPORTIONS?

A. By giving to the small body a velocity as much greater than that of the large one, as the mass of the latter exceeds that of the former.

Q. How may a PIN-cusiron, weighing half an ounce, be made to produce as great an effect as a CANNON-BALL, weighing thirty-six pounds?

A. By giving to the pin-cushion 1,152 times the velocity of the cannon-ball; for 1,152 half-ounces being equal to 36 pounds, it must be apparent that the velocity of the pin-cushion would be just so much greater than the velocity of the cannon-ball, as the mass of the latter would be greater than that of the former.

Q. Why will a TALLOW CANDLE, fired from a gun, pierce a BOARD Or TARGET in the same manuer as a LEADEN BULLET will, under similar circumstances?

A. When a candle starts from the breech of a gun, its motion is gradually increased until it leaves the muzzle at a high velocity, and when it reaches the board or target, every particle of matter composing it is in a state of intense velocity: at the moment of contact the particles of matter composing the target are at rest, and as the density of the candle, multiplied by the velocity of its motion, is greater than the density of the target at rest, the greater force overcomes the weaticr, and the candle breaks through, and pierces a hole in the board.

Q. Why, with an Exorwous Pressure and sLow MoTION, can you not force a candle through a board?

A. Because the candle, on account of its slow motion, does not. possess sufficient momentum to enable the density of its particles to 
orercome the greater density of the board; consequently, the candle is itself mashed, instead of piercing the target.

Q. Why will a LARge SHIP, moving towards a whars with a motion lardly perceptibise, crush with great force a BOAT intervening?

A. Because the great mass and weight of the vessel compensate for its want of velocity of motion.

Q. Why will a bullet, fired against a door HaIf opres, pass through the wood without moring the door on its hinges?

A. Communication of motion from one body to another is never instantaneous, but requires some time : the velocity of the bullet is so great thas it penetrates the wood in a space of time too limitcd to admit of the momentum of the bullet being communicated to the door against which it strikes.

Q. How can lie decermine the velocity of a body moving uniformly?

A. By dividing the space passed through by the time consumed; thrus, the velocity of a steamboat going eighteen miles in two hours, will be found to be nine miles an hour.

Q. The VELOCITY of a BODY being known, how can we find out the DISIATUE passed over in a given time?

A. By multiplying the space by the time; thus, the steamboat, with a velocity of nine miles an hour, will of course run trvice nife miles in two hours, and forty-eight times nine miles in forty-eight locurs.

Q. In wriat IIRRCTion would a stone fill, if dropped on the deck from the masthead of a vessel in motion?

A. The attraction of gravitation would tend to draw the stone downwards in a straight line, the motion of the ressel would tend to carry the stone forwards horizontally: the line in which the stone would fall wonld be a mean between a straight and a horiwontal line, viz. "diagonal.

Q. What is CEXTIIIEUGA TOROS?

A. It is that force which causes a revolving body to fly from a contre.

Q. Why does a srons, discharged from a sling, MOVE FORWARDS, when the cord which zetained it is loosened?

A. Because of the centrifugal force it has acquired by the whirling of the sling previous to the discharge.

Q. Why do arINDSTONBS or WIEELLS, in rapid motion, not unfrequently break and fly to pieces with great violence?

A. Because the centrifugal force, generated by the rapid revolving motion, overcomes the cohesion of the particles, and thus causes them to separate and fly from the centre.

Q. Can almost all revolving bodies be broken by suficient rotative velocity?

A. Yes; for the centrifugal force increases with the rapidity of revolution, and finally becomes too strong to be resisted by the cohesive force which binds the particles of the body together.

Q. How MUCH TASTER would the EARTI be required to revolve in order to make the GinTRITUGAL FOREE equal to the ATTRACTION Of GRAVITATION?

A. Seventeen times faster, or in eighty-four minuies, instead of 
twenty-four hours: in this case, all bodies on the surface of the earth would be destitute of weight.

Q. What would the the consequence if the earth revolved around its wais in LESS TIMs than ErGITT-FOUR MINUTES?

A. Gravitation would be completely overpowered, and all fuids and loose substances would fly from the surface.

Q. When a vessiu containing water is whirled rapidly round, why does not the wamer. fall out when the VESSEL is UPSIDE DOWN?

A. Because the centrifugal force, tending to make the water fly from the centre, overcomes or balances the aitraction of gravitation, which tends to cause the water to fall out.

Q. Why does a IIAN or horse, in turning a Conzer rapidly, INGLINE INWATDS, Or IELM towards the corner?

A. Because the centrifugal force, produced by turning rapidly, tends to throw him away from the corner; therefore, he inclines inwards to counteract it.

Q. Why do Fatcr-dogs give a SEMrrotary zroveneNT to their skin to free themselves from water?

A. Because in this way a centrifugal force is generated, which causes the drops of water adherent to them to fly off.

Q. Why does a Forsa in a crrous ring lean toviarls the centre?

A. Because, when the horse moves round with the performer on the saddle, both the horse and the rider inctine continually towards the centre of the ring, and the inclination increases with the velocity of the motion: by this inclination their weights counteract the effect of the centrifugal force.

Q. Why does a sprNaring-rop stand?

A. Because, while the top is perfectly upright, its peg or point, being directly under its centre, supports it steadity, and, although turning so rapidly, has no tendency to move from the place; but if the top inclines at all, the sicle of the peg, instead of the very point, comes in contact with the floor, and the peg then becomes a little zoheel or roller advancing quickly, and with its touching edge describing a curve somewhat as a skater does, until it becomes directly under the body of the top as before. It thus appears that the very fact of the top inclining causes the point to shift its place, so that it cannot rest until it come again directly under the centre of the top.

Q. Why is the COURSE of rivers rarely STRAIGH, but SERPEMTINE and WINDING?

$A$. When, from any obstruction, the river is obliged to bend, the contrifugal force tends to throw awoy the water from the centre of the curvature, so that when a bend has once commenced, it increases, and is soon followed by others. Thus, for instance, the water being thrown by any cause to the left side, it wears that part into a curve or elbow, and by its centrifugal force acts constantly on the outside of the bend, until the rock, or higher land, resists its gradual progress; from this limit, being thrown back again, it wears a similar bend to the right hand, and after that another to the left, and so on. 
Q. If a cock at the Extremity of a PIPE be suddenly closed while water is running through, why is a rorse and sHock produced?

A. Because the forward motion of the whole body of the water contained in the pipe being instantly arrested, and the momentum of a liquid being as great as that of a solid, the water strikes the cock with as much force as if it were a long bar of metal, or a rod of wood having the same weight and velocity as the water. Then, as a fluid presses equally in all directions, a leaden pipe of great length may be widened, or even burst in the experiment.

Q. A Heavi coach and a LIGIIT WAgOn came in collision on the road. A suit for damages was brought by the preprietor of the wagon. How was it shown that oxe of the VEHICLES Was moving at an UNSAFE VELOCITY?

A. On trial, the persons in the wagon deposed that the shock, occasioned by coming in contact, was so great, that it theew them over the head of their horse; and thus lost their case by proving that the faulty velocity was their own.

Q. Why did the FACT that they were THROWN over the HEAD OF THE HORSE by coming in contact with the coach, prove that their velocity was GREATER than it ought to have been ?

A. The coach stopped the wagon by contact with it, but the bodies of the persons in the wagon having the same velocity as the wagon, and not fastened to it, continued to move on. Had the wagon moved slowly, the distance to which they would have been thrown would have been slight. To cause them to be thrown as far as over the head of the horse, would require a great velocity of motion.

Q. Why does a PERSON who is about to LEAP over a DITCH or CHASM, first make a run of a little distance?

A. In order that the impetus he acquires in running may help him in the jump.

Q. Why is a STANDING IEAP always shorter than a RUNNING ONE?

A. Because in the running leap, in addition to the force acquired by the contraction of the muscles, we have added the force of the motion acquired by rumning.

Q. Why do we KICK against the door-post to SHAkE the snow or dust from our GHOES?

$A$. The forward motion of the foot is arrested by the impact against the post; but this is not the case with respect to the particles of dust or snow which are not attached to the foot; but the motion imparted to them, equally with the foot, is continued, and causes them to fly off.

Q. Why do we BEAT a coat or carpet to ExpeL the dust?

A. The cause which arrests the motion imparted to the coat or carpet by the blow does not arrest the particles of dust, and their motion being continued, they fly off.

Q. Why is the UP-AND-DOWN motion of carriages and ressels often beneficial to invalids?

A. In the long, upright veins below the heart, when the body falls, the blood by its inertia, and the supporting action of the vessels, does 
not fall so fast as the body, and therefore really rises in the veins, and as there are valves in the vein preventing return, the circulation is thus quickened without any muscular exhaustion on the part of the individual. \&c.?

Q. Why are persons made SICK at SEA by the MOTION of the VESSEL; also by SWINGING,

A. Because the motion of the vessel or swing imparted to the body is not participated in by the organs and fluids of the body, they being thus caused to move in an involintary manner, produce nausea and sickness.

Q. Why do Birds fly?

A. Because they have the largest bones of all animals in proportion to their weight, and their bones are more hollow than those or animals that do not fly. Air-vessels also enable them to blow out the hollow parts of their bodies when they wish to make their descent slower, rise more swiftly, or float in the air. The muscles that move the wings of birds downwards, in many instances, are a sixth part of the weight of the whole body, whereas those of a man are not, in proportion, one-hundredth part so large.

Q. Why does FLYING differ from LEAPING?

$A$. Because flying is the continued suspension and progress of the whole body in the air, by the action of the wings. In leaping, the body is equally suspended in the air, but the suspension is only momentary. In flying, on the contrary, the body remains in the air and acquires a progressive motion by repeated strokes of the wings on the surrounding fluid.

Q. Why do BIRDs stretch out their NECKS when FLYING?

$A$. Because they may become like sharp points, dividing the air and diminishing the resistance.

Q. Why are the STRONGEST FEATIIERS of birds in the PINTons and TAIL?

A. Because, when the wing is expanded, the pinion-feathers may form, as it were, broad fans, by which the bird is enabled to raise itself in the air and fly, while its tail-feathers clirect its course.

Q. Why can a person safely skate with GREAT RAPIDITY over ice which would not support his weight if he moved over it MORE SLOWLY?

A. From the fact that time is required for proctucing the fracture of the icc, as soon as the weight of the skater begins to act on any point, the ice, supported by the water, bends slowly under him, but if the skater's velocity is great, he has passed off from the spot which was loaded before the bending has reached the point which would cause the ice to break.

Q. It sometimes happens when persors are rocked nown liy CArriages, that the WHLELS pass over them with scarcely any injury, though if the WEIGrim of the CARritage had rested on their body, EVEN FOR A rew sFCONDs, it would have crushed them to death. What explanation can be given of this fact?

A. The wheel moves with such rapidity that the weight has not time sufficient to cxert its full sffect. 
Q. In a nerouving BODY, what is the AXIS of nomion?

A. The line about which the body revolves.

Q. What is the CENTRE of NO'STON in a reVOLVING BODY?

A. The point about which it moves.

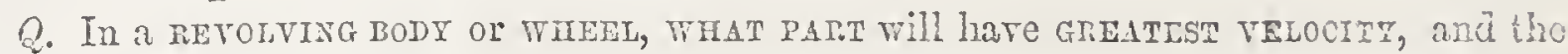
greatest CENTRIFUGAI TORCE?

A. The part farthest from the axis of motion.

Q. What is the iromexrour of a moving body?

A. The term momentum denotes the absolute force of a moving bolly with reference to the effect it is capable of producing.

Q. When Two pEnsors strike their medDs together, one being in motron and the other at REST, why are both equally hurt?

A. Because, when bodies strike each other, action and recuction are equal; the head that is at rest returns the blow with equal force to the head that strikes.

Q. When an elastic BALL is thrown against the side of a house with a OERTAIA FOJicr, why does it rebound?

A. Because the side of the house resists the ball with the scme foree, and the ball being elastic, rebounds.

Q. When the SAME BALL is thrown against a PANE of GLASS with the same force, it gocs through, breaking the glass: why does it not rebound as before?

A. Because the glass has not sufficient power to resist the full force of the ball: it destroys a part of the force of the ball, but the remainder continuing to act, the ball goes through, shattering tho glass.

Q. Why did not the MAN succeed who mdertook to make a FAIr WIND for his

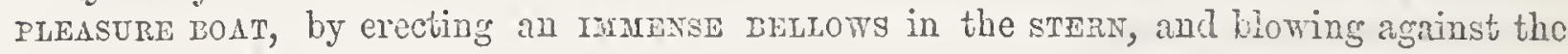
SAISS?

A. Because the action of the stream of wind and the reaction of the sails were exactly equal, and, consequently, the boat remained at rest.

Q. If he had blown in a conmranY DIREcrion from the sails, instear of against them, would the boat have moved?

A. It would, with the same force that the air issued from the bellows-pipe.

Q. Why cannot a Mad raise himself over a rexce ly pulling upon the STrupg of his BOOTS?

A. Because the action of the force exerted to raise himself is exactly counteracted by the reaction of the force which tends to keep him down. him?

Q. Why can a MAN raise himself in the AIR by PULLING upon a rores suspended above

A. Because the reaction of the force from the rope is not equal to the action of the force exerted in puiling by the muscies of the arms; consequently the body rises.

Q. Does a IIAN in rowing DRIVE the WATMR ASTERN with the SAME FORCE that he imply the BOAT FORWARDS?

A. He does: action and reaction being exactly equal.

Q. Why is it more dangerous to leap from a HIG WINDow than from a LOW TABLE?

A. Because the velocity of a falling body, and, consequently, the 
force with which it will strike the ground, increases with the clistane through which it falls. falls?

Q. Why does the relocity of a falling body MNCREASE with the distance throtigh which it

A. Orving to the inertic of matter, any force continuing to act upons a mass which is free to obey it, produces in it a quickening or accelerated motion; for, as the motion given in the frrst instant continues afterwards without any further force, merely on account of its inertin, it follows that as much more motion is added during the second instant, and as much again during the third; and so on. A falling body, therefore, under the influence of the attracion of gravitation, reccives cvery instant an increased velocity and momentum.

Q. Why, in a WATERTALL Or CATARACT, does the water at the EDGM of the FALI

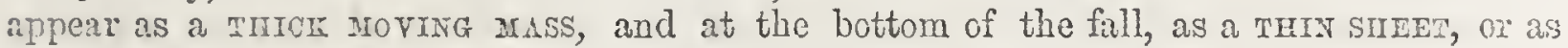
spray?

A. As the sheet of water descends, it becomes reduced in size, becanse it increases in volocity; the bulk diminishing from above downwards, in the same proportion as the velocity of the particles increases. Trus?

Q. How FAr will a kody fall through the influence of gravity, in one SEcord of

A. Sixteen feet.

Q. How far will is fall in two swcords?

A. Four times 16 feet, or 64 feet; in three seconds it will fall 144 fect; in four, 256 ; in five seconds, 400 feet, and so on.

Q. What is the ruser by which the height from which a body falls may be found, the time consumed in fulling being known?

A. Prulliply the square of the number of scconds of time consumen in falling, by the distance which a body will fall in one second.

2. If a sTONE is FIVE SECONDS in falling from the top of a precipica, Irow IiIGIi is the precipice?

A. The square of five seconds is 25 ; this multiplied by 16 , the number of fect a bocily will fall in one second, gives 400 , the height of the precipice.

Q. What is a PENDEITM ?

A. A pendulum is a heavy body, as a piece of metal, suspencled by as avire or corcl, so as to swing backwards and forwards.

Q. When is a PENDULCaI said to VIBRATE?

A. When it swings backwards and forwards; and that part of the circle through which it vibrates is called its $a r c$.

Q. What is a connon CLOCK?

A. Mcrely a pendutum, with wheelwork attached to it, to recori the number of vibrations, and with a weight or spring, having force sufficient to counteract the retarding effects of friction and the resistance of the air.

Q. How does the swrim of the pendulum mari trise?

A. The vibrations of the pendulum are numbered by a wheel having sixly iceth, which revolves once in a minute. Each toots. answers to one swing of the pendulum, and the wheel mores forward 
one tooth in a second. A luand fixed on the axis of this wheel, projecting through the dial-plate, will be the second-hand of the clock. The other wheels are so connected with the first, and the number of teeth on them is so proportioned, that one turns sixty times slower than the first, to fit its axis to carry a minute hand; and another, by moving twelve times slower still, is fitted to carry an hour-hand.

Q. How LoNa must a pendulum be to beat SECONDS?

A. About 39 inches.

Q. Why does a COMMON CLOCK go FASTER in WINTER than in SEMMFER ?

$A$. Because the pendulum-rod becomes contracted by cold in winter, and lengthened by heat in summer.

Q. Why does a cHANGr in the LENGTH of the PENDULUM cause a clock to go FASTER o. SLOWER?

$A$. The number of ribrations which a pendulum makes in a given time depends upon its length, because a long pendulum does not perform its journey to and from the corresponding points of its are so soon as a short one.

Q. How does a watOH differ from a CLOCK?

A. It has a vibrating wheel instead of a vibrating pendulum. This wheel, called the balunce-wheel, is moved by a spring, which is always forcing it to a middle position of rest, but does not fix it there, because the momentum acquired during its approach from either side to the middle position, carries it just as far past on the other side, and the spring has to begin its work again. 'The bulance-wheel at each vibration allows one tooth of the adjoining wheel to pass, as the pendulum does in a clock, and the record of the beats is preserved by the wheels which follow as already explained for the clock.

Q. A MAN receiving a HEAVT BLOW of a great HAMMER upon his cIIEST would be killed by it; but if a HEAVY $\Lambda$ NVIL be first laid upon the CHEST, and the blow then received upon the ANvis, the man bears it with impunity: WHY IS THIS?

$A$. The quantity of motion in the hammer being diffused, through the great mass of the anvil, produces but a trifling velocity, which the clasticity of the chest, in its slow yielding, easily overcomes.

Q. A LEADEN EULLET pressed slowly against a PANE of GLASS breaks it irregularly, where the strength happens to be the least; but the SAIIE BULIET, shot at it from a PISTOL, makes only a small round hole : EXPLAIN THE RFASON OF WHIS.

A. In the latter case, the force of the bullet is allowed to act. against or resist the force of cohesion in the glass for so short a time, ouing to its rapid passage, that it is not sufficient to affect any other part of the glass than the points against which it impinges. It has been amusingly said of such a case, by Dr. Arnott, "that the particles struck and carried away have not time to warn their neighbours of what is happening." In the first instance, a reverse action produces a contrary effect. 


\section{$M \mathrm{EC} \| \mathrm{A} \mathrm{N}$ I C S.}

\section{CIIAPTER VII. \\ THE PRODUCTION AND APPLICATION OF FORCE.}

SECTION I. - MACHINERT AND THE MECHAYRCAL POWERT.

Q. What is the Solknoe of Mrehanics?

A. Mechanics may be considered as the Philosophy of Machinory, or the science which investigates the laivs and effects of inotion.

Q. From what sources do we derive advantages by the use of Macunnes and MANUFACTURES?

A. From the addition they make to human power; from the economy they produce of human time; from the conversion of substances apparently common and worthless into valuable products.

Q. How do Machines make additions to human power?

$A$. They enable us to use the powers of natural agents, as wind, water, steam; they also enable us to use animal power with greater effect, as when we move an object easily with a lever, which we could not with the unaided hand.

$Q$. How do Hachines produce economy of human time?

$A$. They accomplish with rapidity what would require the hand unaided much time to perform. A machine turns a gun-stock in a few minutes; to shape it by hand would be the work of hours.

Q. How do MACHINes CONVERT objects apparently worthless into VALUABLE PRODUCTS ?

A. By their great power, economy, and rapidity of action, they make it profitable to use objects for manufacturing purposes which it would be unprofitable or impossible to use if they were to be manufactured by hand. Without machines, iron could not be forged into shafts for gigantic engines; fibres could not be twisted into cables; granite, in large masses, could not be transported from the quarries.

Q. What is a MACHINE?

A. By a machine we understand a combination of mechaniral porvers adapted to vary the direction, application, and intensity of a moving force, so as to produce a given result.

Q. What is the DIFFEREnce between A Machine and A TOoL?

A. The difference between a machine and a tool is not capable of very precise distinction. A tool is usually more simple than a machine: it is generally used by hand, while a machine is generally moved by some other than human power.

Q. Dues a Machine ever create power, or increase the quantity of power or Force anylied to it?

A. A machine will enable us to concentrate or divide any kind or 
quantity of force which we may possess, but they no more increase the quantity of force than a mili-pond increases the quantity of water flowing in the stream.

Q. How many kinds of motion are considered in machinery?

A. Two-rotary and reciprocating.

Q. What is RoTARY motion?

A. A rotary motion is that in which the moving parts revolve round an axis, as in a wheel, a crank, or a fly.

Q. What is RECIPROCATING Motion?

A. A reciprocating or alternate motion is that in which a body retraces its own pail, or moves alternately backwards and forwards in the same track, which may be curved, as in the beam of a steamengine, or straight, as in the piston.

Q. In the application of machinery, what THRER minas are to be considered?

A. 1. The force or resistance which is required to be sustained, opposed, or overcome.

2. The force which is used to sustain, oppose, or overcome that resistance.

3. The machine itself, by which the effect of this latier force is transmitted to the former.

Of whatever nature be the force or the resistance which is to be sustained or overcome, it is technically called the weight, since, whatever it be, a weight of equivalent force inay always be found. The force which is employed to sustain or overeume it is technically called the power.

Q. Into what THREE GREAT DIVISIONS may the mechanic powers be divided?

A. The Lever, the Multiplied Cord, and the Inclined Plane. 'To these some have added the Wheel and Axle, the Pulley, the Wedge, and the Screw, but these last are only modifications of the former powers.

Q. What MACHINES are included under the FIRST CLASS?

A. All which are composed of a solid body revolving on a fixed axis. In these the power and the weight are always supposed to be applied at right angles to the axis.

Q. What machines are included under the SRCond CrasS?

A. All those in which force is transmitied by means of flexible threads, ropes, or chains. Within this class are included all the various forms of pulleys.

Q. What MACHINES are included under the THIRD CLASS?

A. All those in which the weight or resistance is supported or moved on a hard surface inclined to the vertical direction; under this class are included the incined plane, the screw, and the wedge.

Q. What is a LEVER?

A. An inflexible bar turning on an axis.

Q. What are the ARMs of a LEVER?

A. Those parts of the bar which extend on each side of the axis.

Q. What is its Fulorum, or prop?

A. Simply another tern to desigriate the axis. 
Q. How many kinds of LevERS are there?

A. Three; divided according to the relative position of the pouver, the weight, and the fulcrism.

Q. What is a LEVER of the FIRST KIND ?

Fig. 15.

A. In a lever of the first kind (as in
and

Q. What are EXAMIPLES of this form of LEvER?

A. A crowbar, applied to elevate a stone, is an instance; the fricrum is another stone placed near that to be raised, and the pourer is the hand placed at the other end of the bar.

A poker applied to raise fuel is a lever of the first class, the fulcrum being the bars of the grate. The bratse or handle of a pump is also a familiar example.

Q. What OrDer of LEVER is a PAIr of SCISSORS, PINCERS, NITPERS, \&C. ?

A. Scissors, pincers, \&c., are composed of two levers of the fir st hind, the fulcrum being the joint or pivot, and the weight the resist muce of the substance to be cut or seized. The power of the figures is applied at the other end of the levers.

Q. What is a LEVir of the SECOND kins?

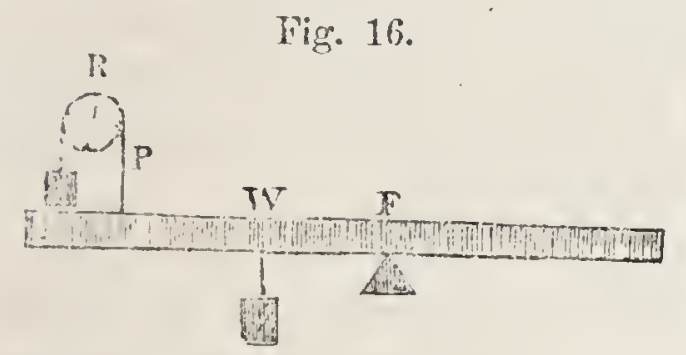

A. In a lever of the second kind (as infig. 16) the weight is between the fulcrum and the power. The power is represented as applied over the wheel R.

Q. What are ExAMPLES of TIIS TOM of LEY an?

A. Nut-crackers are two levers of the second kind; the hinge which unites them being the fulcrum, the resistance of the shell placed between them being the weight, and the hand, the poiver. An oar is a lever of the second kind, in which the reaclion of the vater against the blade is the fulerum; the boat, the weight; and the hand of the boatman, the pover. A door moved on its hinges is another example.

2. What sort of a LEYER is a WILELBARrow?

A. One of the second lind; the fuldrum being the point at which the wheel presses upon the ground; the barrow and its load, the veight; and the hand:; the power.

Fig. 17 represents the manner of using is liand-spike or bar as a lever of this tirst kind; fig. Is shows how a similar bar may be employed as a lever of the second kind. the ground against which the point of the lever is tixed being the fulcurn.

$$
\text { Fir. } 17 .
$$$$
\text { Fiin. } 19 \text {. }
$$
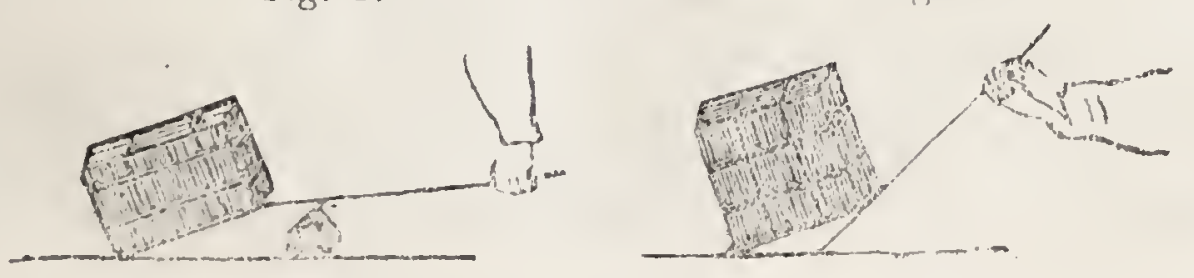
Q. What is a LEVER of the THIRD KIND?

A. In a lever of the third kind (as in fig. 19) the power is between the fulcrum and the weight.

Q. What are examples of this kind of lever" ?

A. The most striking example of levers of the third kind are found in the animal kingrom. The limbs of animals are generally levers of this description. The socket of the bone ( $a$, fig. 20 ) is the fulcrum; a strong muscle $b$ attached to the bone

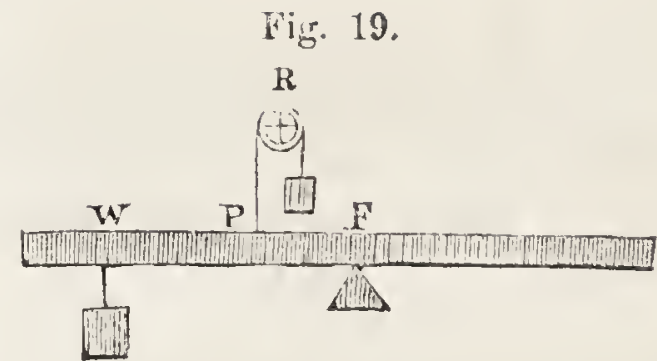

Fig. 20.

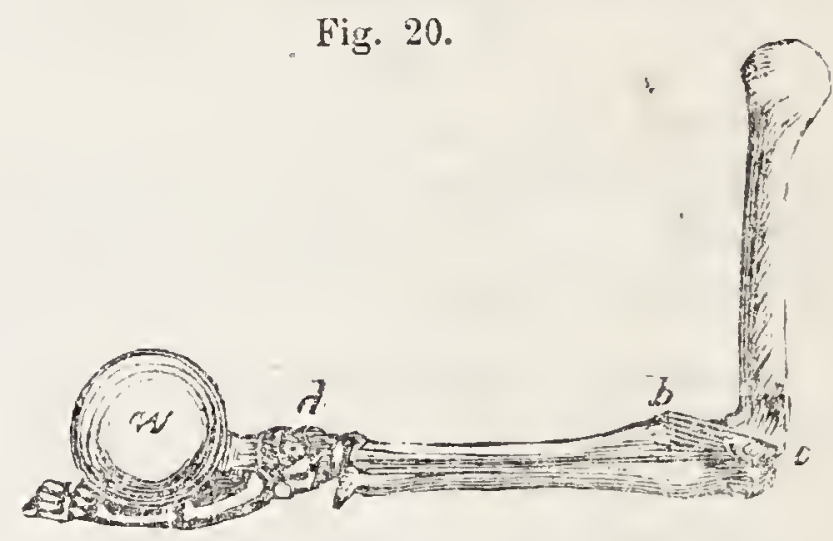
near the socket $c$, and extending to $d$, is the power; and the weight, of the limb, together with whatever resistance $w$ is opposed to its motion, is the weight.

Q. For what is this variety of lever generally used?

A. In cases where a quick motion is required, rather than great force: this effect is particularly, conspicuous in the motions of the arms and legs of the human body; a very inconsiderable contraction of the muscles at the shoulders and the hips giving the sweep to the limbs from which the body derives so much activity.

Q. What sort of levers is a pair of tongs?

A. Levers of the third kind, in which the power is the hand placed immediately beneath the fulcrum, or the point where the two levers are comnected. Shears used in shearing sheep, and the treadle of the turning-lathe, are other examples of levers of this order.

Q. Why do not BIRDS fall down in SLEEPING on their PEROH ?

$A$. Because such is the structure of their feet and legs, that the areater the weight upon the muscles, the more firmily the claws grasp whatever they lay hold of.

Q. What kind of a LEVER is the FOOT and LEG of a BIRD?

A. A lever of the third kind, the body being the weight; the muscle, the power; and the perch or roost, the fulcrum.

Q. A BOX who cannot exert a force of fifty pounds, may by means of a CLAW-HAMMER, which is a lever of the first class, draw out a nail which would support the weight of half a tOn: DOES NOT THE LEVER IN MHIS CASE CREATE POWER?

A. No; because the hand of the boy is required to move through perhaps one foot of space to make the nail rise one quarter of an inch. It has been already. shown that the force of a small body moving with great velocity may equal the force of a large body with a slight felocity. On the same principle, the small weight or power exerted by the boy on the end of the lever, moving through a large spaces 
with an increased velocity, acquires sufficient momentum to overcome a much greater resistance at the short end of the lever.

Q. In the ordinary arrangements for wEIGHING, what MECHANICAL POWERS are einployed?

A. Levers of the different orders, employed either singly or in combination. In the common balance, styled from its adventitious appendages a pair of scales, the beam, which is the essential part, is simply a lever of the first order, having equal arms, and turning freely on its fulcrum or centre of action.

Q. How in an ordinary balance may a FALSE WEIGHT be detected?

A. By changing the places of the weight and the article to be weighed; the equipoise before effected will then be destroyed.

Q. How are the so-called "HAY-SCALES" constructed?

A. They consist of a system of levers supporting a quadrangular floor. Four levers turning on their fulcrums, extend from the angles of a box beneath the floor towards its centre, where they are connected together, and also with another lever, extending across the middle of the box, and passing beyond its limits. This last lever acts upon a third, which presses on a spring or is connected with the arm of a balance, by which the amount of pressure on the whole floor may be ascertained.

Q. What circumstances Lisim the utility of the lever?

$A$. When a lever is applied to raise a weight or overcome a resistance, the space through which it acts at any one time is small, and the work must be accomplished by a succession of short and intermitting efforts. The common lever is, therefore, only used in cases where weights are required to be raised through small spaces.

Q. When a continuous мотіоN is to be produced, as in raising ore from the mine, or in weighing the $\triangle N C H O R$ of a ship, what contrivance do we adopt to remove the intermitting action of the lever, and reader it continual ?

A. That arrangement of the lever generally denominated the wheel and axle.

Fig. 21.

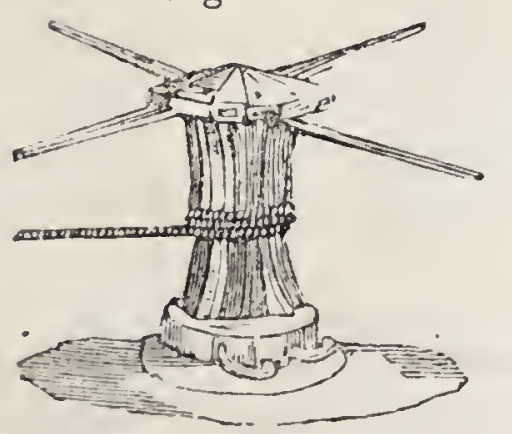

Q. What is the form of WHEEL AND AXLE called which is used for lifting the ANCHORS of ships?

A. A capstan. (Fig. 21.)

Q. Will you describe the construction of a CAPSTAN?

A. It consists of a vertical spindle or shaft fixed firmly, as in the deck of the vessel, but turning on its axis, and supporting a solid cylinder connected with it; the top of this cylinder being pierced with holes cut in its periphery, and directed towards its centre. It is then worked by long levers inserted in the holes, by men who walk 
In succession round the capstan, and thus make it revolve, while a rope or cable wound about the spindle may act with force sufficient to weigh a ponderous anchor, or warp a heary-laden vessel into harbour.

Q. In the various forms of wiEers, how is the Power applied?

$A$. In some cases by a rope coiled round the axle; in the common windlass, by a winch or handle applied to the axis; and in waterwheels, paddle-wheels, and tread-mills, by a weight applied to the circumference of the wheel. (See figs. 22 and 23.)

Fig. 22.

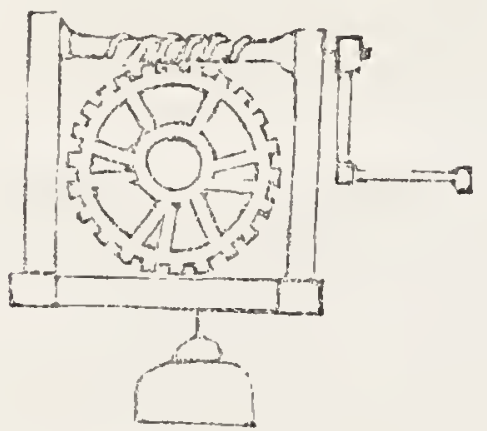

Fig. 23.

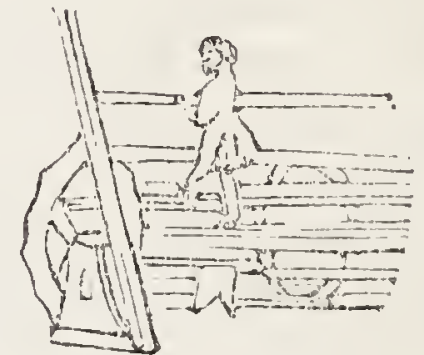

Fig. 24.

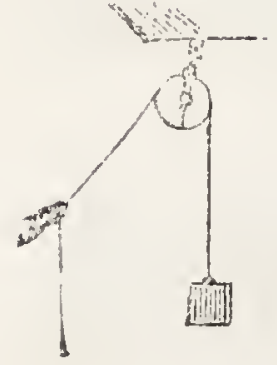

Q. What is a PULIEY?

A. A pulley is a smail wheel turning on añ axis, with a rope passing over it. It is used to change the direction of a weight, which may be raised to any height by a person not moving from his place. (See fig. 24.)

Q. What Trourics n. Nos is given to pulleys on board ship?

A. They are called Ulocks.

Q. What is an INCLINTD PuANE?

A. It is a hard plane surface, forming some angle with a horizontal phane; that angle not being a right angle. (Seefig. 25, a b c.)

Tig. 25.

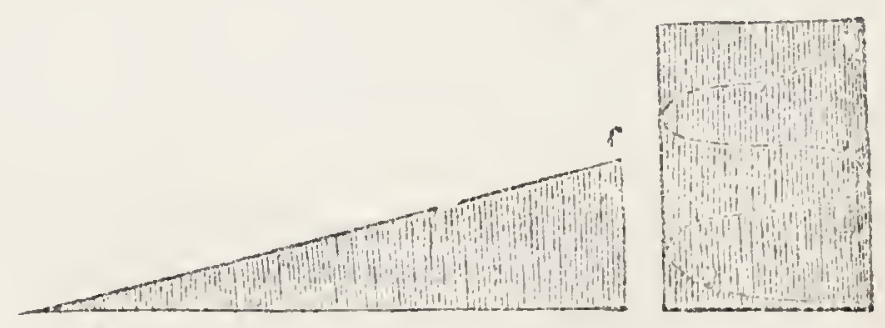

Q. What are FAMILAR MXAMPLES of the INCLINED PLAKE?

A. Roads formed on the sides of hills; a pritr of slicles, when in use for assisting in the removal of barrels, \&c., from a loaded vehicle to the ground.

Q. Why is it easier to roll a HoGSHEAD into a WAGON up an IXCIMED PIANT, than to lift it directly into the WAGON?

A. The gravity or weight of a body on an inclined plane may be divided into two forces; one drawing it to the earth, acting at right angles to the plane; the other, acting paralle! to the inclined plane, 
and forcing the weight down it. In lifting a body vertically from the earth, we have to overcome the full force of gravity acting directly on it: but by rolling it up an inclined plane, we are enabled to move great weights with comparative facility.

Q. Where a roAD rises ONE fnot in TWENTY, how much more labour is imposed on a horse drawing a loaded carriage, than when he draws the same load upon a level ?

$A$. On a level road the carriage moves when the horse exerts power sufficient to overcome the friction and the resistance of the atmosphere; but on a road rising one foot in twenty, besides these impediments, the horse is obliged to lift one-twenticth of the load.

Q. Why is it bad policy ever to construct a roAD directly over the summut of a HILI ?

A. Because hatf the power, with the increase of speed, exerted on a level road round the hill, would gain the same distance in half the time.

It shonld be remembered that the handle of a bucket is no longer when it lies donn than when it stands up.

Q. Do we use INCLINED PLANES in our HOUSES?

A. The stairs in our houses are inclined planes in principle; but being so steep, are cut into horizontal and perpendicular surfaces called steps, that they may afford a firm footing.

Q. Why does an INIFLLIGENT DRIVER, in ascending a stcep hill on which there is a broad road, wind from side to side of the road?

A. By so doing he diminishes the alruptness of the ascent, and favours the strength of his horses.

Q. What is a SCrew?

A. The screw is an inclined plane winding round a cylinder. This will be apparent if we take a rectangular piece of paper whose length is equal to the circumference of a glass rod (see fig. 25), and wind it round in such a manner that the horizontal side of the paper shall form the periphery $a b$ of the base of the cylinder; then the hypotenuse, as the line $a c$ is called, will wind around it in a uniformly ascending line, which line marks what is called the thread of the screw.

Q. What is a PFRPETUAL SCREW ?

Fig. 26.

A. A perpetual screw is the name given where a screw acts on the teeth of a wheel so as to produce a continued rotation of the wheel. (See fig. 26.)

Fig. 27. In practice, the serew is never used as a simple mechanical machine, the pow cr being always applied by means of a lever passing throngh the head of the scrow, or into a nut, which is another screw, through which the first one passes. The serew, therefore, acts with the combined power of the inclined plane and the lever.

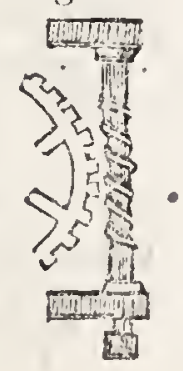

Q. What is a WRDGE?

A. A wedge (fig. 27) is composed of two inclined manes united at their bases. 
Q. For what purposes is the wevis used in the arts?

A. When enormous force is to be exerted through a very small space, as in the splitting of masses of timber or stone. Ships are raised in docks by wedges driven under their keels.

Q. What are FAMILIAR EXAMPLES of the use of the wedge in ordinary tools or instruments?

A. All cutting and piercing instruments, such as knives, razors, scissors, naits, pins, needles, awls, \&c., are wedges. The angle of the wedge is more or less acute in these instances, according to the purpose to which it is to be applied.

In tools intended for cutting wood, the angle is generally about $30^{\circ}$; for iron, it is from $50^{\circ}$ to $60^{\circ}$; and for brass, from $80^{\circ}$ to $90^{\circ}$.

\section{CHAPTER IX.}

\section{THE SOURCES OF POWER.}

Q. Is there any reason why the term MECHANIOAL POWERs should be applied exclusively to the contrivances explained?

$A$. There is no reason but old usage; since some of the forces which act mechanically with the greatest power are not included in che class of solid bodies, as steam, wind, water.

Q. Why are the SIMPLe Mechanical powens aids in physical exertion or labour?

A. Because they allow a small force to take its time to produce any requisite magnitude of effect. Thus, one man's efforts, or any small power, which is always at command, by working proportionally longer, will answer the purpose of the sudden effort of many men, even of a great number of men, whom it might be impossible to bring together.

Q. Why are so many attempts continually made to produce mechanical engines which shall generate PERPETUAL MOTION?

$A$. Because the projectors do not understand the great truth, that no form or combination of machinery can, under uny circumstances, increase the quantity of power applied.

Q. What is the object of a MAOHINE?

$A$. To receive and distribute motion derived from an external agent, since no machine is capable of generating motion or moving-power within itself.

Q. What are the PRINCIPAL SOURCES from whence POWER is obtained?

A. Men and animals, water, wind, steam, and gunpowder. The power of all these may be ultimately resolved into those of muscular energy, gravity, heat, and chemical affinity.

Q. Are there any other sources of POwER?

A. Yes; magnetism, electricity, capillary attraction, \&c.; but none of these are capable of being used practically for the production of motion. 
Q. How is MUSCULAR ENERGY exerted?

A. Through the contraction of the fibres which constitute animai muscles. The bones act as levers to facilitate and direct the application of this force, the muscles operating on them through the medium of tendons, or otherwise.

Q. What ANIysis possess the greatest amount of muscular power?

A. Beasts of prey. Some very small creatures, however, possess muscular power in proportion to their bulk incomparably greater than that of the largest and greatest of the brute creation. A flea, considered relatively to its size, is far stronger than an elephant or a lion.

Q. In WHAT METHOD can a man exert the greatest active strength ?

A. In pulling upwards from his feet; because the strong muscles of the back, as well as those of the upper and lower extremities, are then brought advantageously into action. Hence the action of rowing is one of the most advantareous modes of muscular action.

Q. What is the comparative effect produced in the different methods of applying the EORCE of a MAN ?

A. In the action of turning a crank, his force may be represented by the number 17; in working a pump, by 20; in pulling downwards, as in the action of ringing a bell, by 39 ; and in pulling upcuards from the feet, as in rowing, by 41 .

Q. What is the ESTIMATE of the UNRFORM STRENGTH of an ORDINARY MAN for the pexformance of daily mechanical labour?

$A$. That he can raise a weight of 10 pounds to the height of 10 feet once in a second, and continue the labour for 10 hours in the day.

Q. In what posimion will a Horse, or any orHer ANIMAL used for labour, exert its power to the best advantage?

A. When the line of draught is not horizontal, but inclines upuards, making a small angle with the horizontal plane.

Q. What is a "HORSE-POWER?" We say a steam-engine is of a certain horse-power; what is the meaning of the term?

$A$. The measure of " "horse's power," adopted as a standard for estimating the power of steam-engines, is, that he can raise a weight of 33,000 pounds to the height of one foot in a minute.

Q. What is the STRENGTH of a HORSE compared with that of MAN? men.

A. The force of one horse is considered to be equal to that of five

Q. What do we mean by "WATER-Power?"

$A$. The power obtained by the action of water,-applied genera!ly to the circumference of wheels, which it causes to revolve, either biy its weight, by its lateral impulse, or by both conjointly.

Q. What are the Four Forus of WATER-WHEEIS generally used for moving machinery?

A. The overshot wheel, the undershot wheel, the breast wheel, and the turbine wheel. 
Q. What is an OVERSHOT WHEEL?

Fig. 28.

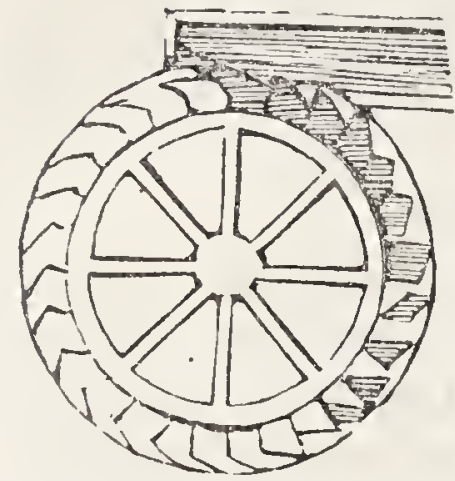

A. This is a wheel, the circumference of which is occupied by a series of cavities, commonly called buckets, into which the water is delivered by one or more spouts at the top of the wheel, and descends to the bottom. By fig. 28, it will be seen that the buckets on one side of the wheel are erect, and will consequently become loaded with water, while those on the other side are inverted, and, of course, empty. It follows that the loaded side will always preponderate, and, by descending, cause the wheel to revolve.

Q. What is an UNDERSHOT WHEEL?

A. One which acts by the impulse of a current of water flowirgs under it, and striking against paddles fastened to the circumference of the wheel. (See fig. 29.)

Fig. 29.

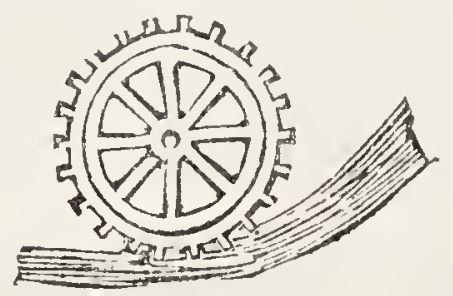

Fig. 30.

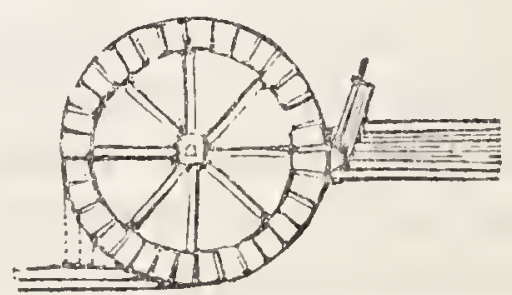

Q. What is a BREAST-WHEEL ?

A. One in which the water is received in buckets at about ralf the height of the wheel, as in fig. 30

Q. What is a TURBINE WHEEL?

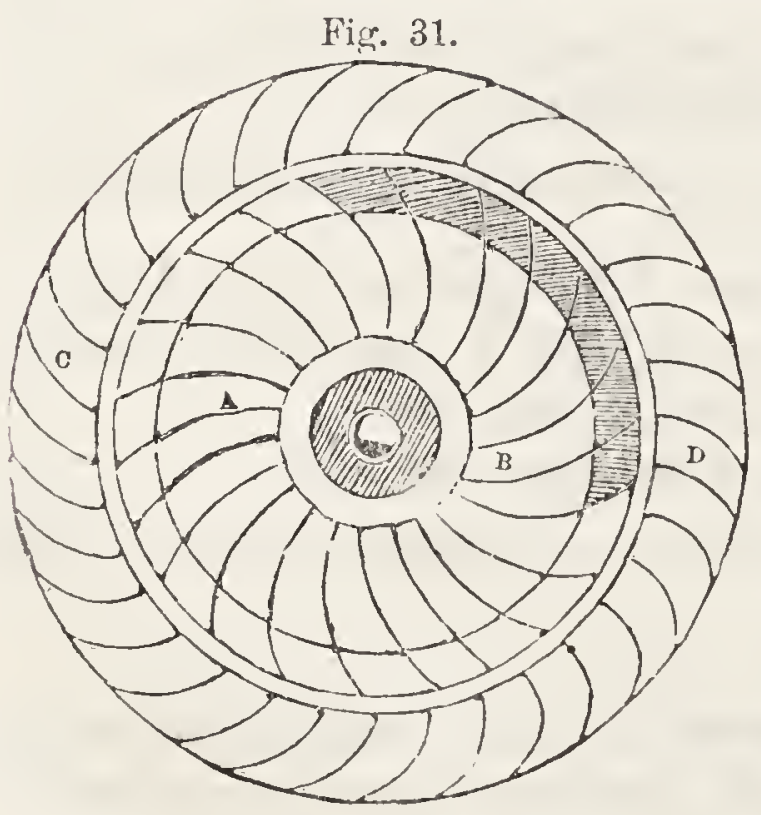

A. The turbine wheel ( $f i g .31$ ) consists of a fixed horizontal wheel, having curved channels, A B, in the centre of which the water enters; it diverges in every direction, and escapes at the circumference. Around the fixed wheel is a revolving circle, $\mathrm{CD}$, which, by a series of curves in an opposite direction to those on the fixed wheel, receives the water from the guide-curves of $\mathrm{A} \mathrm{B}$, and is driven round by the force of the afflux.

Q. With what VELOCITY will the TURBINE WHELL be impelled?

$A$. The velocity will be in proportion to the pressure of the water, which, in its turn, is in proportion to the weight of the vertical column that is sustained above the opening A B, fig. 31 . 
Q. What is the most economical WATFR-WHEEL? or, in other words, what form of water-wheel will accomplish the most work with the least expenci-iure of water?

A. The turbine. In some of the water-wheels, owing to friction and other causes, not one-half the estimated power of the water is obtained or applied to the driving of machinery.

Q. Upon what does the POWER of STEAM depend?

A. Upon the tendency which water possesses to expand into vapour when heated to a certain temperature.

Q. What is GuNPOWDER?

A. A solid explosive substance, composed of saltpetre or nitre, sulphur, and charcoal, reduced to powder, and mixed intimately with each other.

Q. Upon what does the POWER of GUNPOWJER depend?

$A$. When brought in contact with any ignited substance, it explodes with great violence. A vast quantity of gus, or elastic fluid, is emitted, the sudden production of which, at a high temperature, is the cause of the violent effects which this substance produces.

Q. Is the power produced in the EXPLOSION of POWDER ever used for PropaLLIN MACHNERY regularly?

A. It is not, on account of its expensiveness and the suddenness and violence of its action. It is chiefly applied to the throwing of shot and other projectiles, and the blasting of rocks.

Q. What is the estimated Force of GUNPOWDER when exploded?

A. At leust, 14,750 pounds upon every square inch of the surface which confines it.

Q. What are the essential properties of a GuN?

A. To confine the elastic furid generated by the explosion of the powdier as completely as possible, and to direct the course of the ball in a straight or rectilinear path.

Q. Why will a RIFLE send a BALL more accurately than a MUSKET, or ordinary gun?

$A$. The space produced by the difference of diameter between the ball and the bore of the gun greatly diminishes the effect of the powder, by allowing a part of the elastic fluid to escape before the ball, and also permits the ball to deviate from a straight line. The advantage of a rifle-barrel is chiefly derived from the more accurate contact of the ball with the sides of its cavity.

Q. In what consists the peculiarity and superiority of the "MinIE RIFLE?"

A. In construction of the ball, which, in the act of firing, is narle to completely fit the barrel or bore of the gun.

Q. How is this accomplished?

A. The shape of the ball is oblong, with a conical point, and an opening in its base, extending up for two-thirds the length of the ball. Into the opening of this internal cylinder there is placed a small concave section of iron, which the powder, at the moment of firing, forces into the leaden ball with great power, spreading it open, and cansing it 
to fit tightiy to the cavity of the barrel in its course out, thus giving it a perfect direction.

Q. On firing a Gun or CANwon, does the piece Recorl with as MOCH MOMENTUM as the BALL possesses in passing in an OPPOSITE DIRECTION?

A. It recoils with even more momentum than the ball has, for it suffers the reaction not only of the powder, but also of the ball.

Q. Why are not the effects of the REcorr equally evident with the effects of the BALr?

A. Because the momentum in the gun is diffused through a great mass, and is, therefore, easily checked; while in the ball the momentum is concentrated in a small compass.

Q. In some RIFLEs the BORE is TwIstivi : what is the advantage of this?

A. It is supposed to produce a rotation of the ball round an axis, in the direction of its motion, which renders it less liable to deviate from its path on account of irregularities in the resistance of the air.

Q. To what distance may a BALI be thrown by a TWENTYYTOUR POUNDER?

$A$. With a quantity of powder equal to two-thirds the weight of the ball, it may be thrown about four miles.

The effective range of a twenty-four pounder is, however, much less than this.

Q. How much further would the same ball go, were the resistance of the air removed ?

A. About five times the distance, or twenty miles.

Q. If a GUN be fired when the MUZZLE is STOPPED with SFow or CLAY, Or PLUNGED beneath WATER, why will it almost certainly burst?

$A$. Every force requires time to produce its effect; and if the time requisite for the elastic vapour (generated by the combustion of the powder) to force out the sides of the barrel is less than that in which the condensation of the air near the wadding is conveyed in sufficient force to drive the impediment from the muzzle, then the barrel must burst. It sometimes happens that these two forces are so nearly balanced that the barrel only swells, the obstacle giving way before the gun is actually bursted.

Q. Will a GUN recoil more with a charge of FINE SHOT than with a BULLET ?

If a gun is loaded with ball, it will not kick so much as when loaded with fine shot; and, among different kinds of shot, that which is the smallest causes the greatest recoil against the shoulder.

Q. What is the RXPLANATION of this?

$A$. With a ball the velocity is communicated to the whole mass at once, but with small shot or sand, the velocity communicated by the explosion to those particles of the substance immediately in contact with the powder, is greater than that received at the same instant by the outer particles; consequently, a large proportion of explosive force acts momentarily in an opposite direction.

Q. Why is GUNPOWDER always manufactured in LITTLE arains ?

A. In order to cause it to explode more quickly, by facilitating the passage of the flume among the particles.

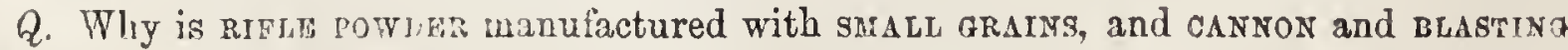
POWDER Of TARGE GRAINS?

1. Because vifle powder is required to be exploded more quickly 
and with greater force. This end, it is found, can be best attained by the employment of a fine grain. In the form of clust the particles would be too compact.

?. Are there any more explosive substances than gunpowder?

A. Very many; but all of them are too expensive or dangern. for practical use.

Q. What is the MosT EXPLosive substance known?

A. Chloride of nitrogen. A drop of this substance in an open saucer makes a hole in the bottom of the vessel and the support; because all its particles are decomposed at the same instant.

Q. What is FULiINATING PoTDER, or the material used in percussion caps?

A. Fulminate of mercury, or a compound of carbon, nitrogen, oxygen, and oxide of mercury.

Q. What is GUN-COTTON?

A. A product formed by immersing cleansed cotton in a mixture of sulphuric and nitric acids. By the action of the acids the vegetable fibre becomes changed into an explosive substance, known to chemists under the name of pyroxyline.

Q. Why is GUNPOWDER better adapted for explosive purposes than any other compound?

A. Because the affinities or attractions existing between the elements composing it are balanced in the best manner; not, like some explosive bodies, so loosely interwoven that the slightest mechanical force or the slightest elevation of temperature suffices to cause their explosion, rendering them totally unmanageable and inapplicable either to the purposes of war, mining, or engineering; but having its afinities safely interwoven - securely tranquil-yet ready to spring into action on the application of a definite amount of heat.

Q. Would it be possible to increase the RANGE and the EFFECT of a PROJECTILE fred from a gun or cannon, by using an explosive compound of GREATER FOWER than gunyowder?

$A$. It would not.

Q. For what REASON is it inipossible?

A. Supposing that the guns could be made indefinitely strong, and the gunpowder indefinitely powerful, the point would soon be reached where the resistance which the air opposes to a body moving very rapidly could balance the force derived from the explosive compound which drives the projectile forward. Beyond this point no increase of impulsive force would urge the projectile farther; and this point is considerably within the range of power that can be exercised by conmon gunpowder.

Q. What отпеR овJECTION is there to the employment of stronger explosive substances than gunpowder?

A. The strength of materials of which guns are made is limited.

Q. What is the LARGEST PIECr OF ARTILLER which can be cest perfect?

$A$. 'The largest mortar ever constructed was of iron, twenty-four inches in diameter, and was used by the French at the siege of 
Antwerp. The weight of this shell, when einpty, was 916 pounds; when loaded, it contained 99 pounds of powder, and was fired by a charge of 30 pounds. For ordinary purposes, mortars 13 inches in rliameter are used; but for special purposes, larger than 13 inches t.re occasionally employed.

Q. By what TERMS are CANNON of different sizes distinguished?

A. By the weight of the ball which they are capable of discharging. I'hus, we have 68-pounders, 24-pounders, 18-pounders, and the lighter field-pieces, from 4 to 12-pounders.

Q. What QUAFTITY of POWDER is generally used for the discharge of the several pieces?

A. For common brass and iron gums, one-third the weight of pouder to the ball, whatever the weight of the latter may be.

Q. What is the meaning of the triry used in GUNNERY, "POINT BLANE?"

A. When the camnon is so pointed that the ball goes perfectly straight towards the object aimed at.

Q. What is the meaning of Ricocher firing?

A. When the ball is discharged in such a manner that it goes bomnting and slipping along the surface of the ground. In this way a. ball can be thrown more effectively, and for a greater distance, than in any other way.

Q. In GENERAL WARFARE, what is the EFFECTIVE DISTAYCE at which ARTILLERY can be used?

A. From 500 to 600 yards, or from a quarter to half a mile. At the battle of Waterloo, the brigades of artillery were stationed about half a mile from each other. Cannon-balls and shells can be thrown with effect to the distance of a mile and a-half to two miles.

Q. What is a SHELL or BOMB?

A. A hollow globe of iron, which, being filled with gunpowder and fired from a mortar or howitzer, bursts into pieces during its flight or progress.

Q. In what respect do MORTARS and HowITZERS differ from ordinary cannon?

A. They are nearly in the shape of a cannon, but are larger in the bme or inside, and shorter in length.

Q. What bas been demonstrated by experiment to be the vELocitry and EFFECT of a SHOT discharged from an 18-POUNDER ?

A. It has been found that, by the firing of an 18-pound shot into a buitt or target made of beams of oak, when the charges were 6 pounds of powder, 3 pounds, $2 \frac{1}{2}$ pounds, and 1 pound, the respective depths of the penetration were 42 inches, 30 inches, 28 inches, and 15 incles; and the velocities at which the balls flew, were 1,600 feet in a second, 1,140 feet, 1,024 feet, and 656 feet.

a. By whom was GUNPOWDER supposed to have been discovered?

A. It is generally agreed that gunpowder was used by the Chinese many centuries before the Christian era. In Europe, its composition and properties were discovered by Berthold Schwartz, a Prussian monk, in the twelfth century. It was first used in battle in 1346. 


\section{CHAPTER X.}

\section{ON THE STRENGTH OF MATERIALS USED IN THE ARTS, AND THEIR APPLICATION FOR ARCHITECTURAL PURPOSES.}

\section{SECTION I.-STRENGTH OF MATERIALS.}

Q. When Materials are employed for Mechaxical pURposes, upon what does their POWER or STRENGTH, apart from the nature of the material, depend for RESISTING EXTERNAL FORCE?

A. Upon the shape of the material, its bearing, and the nature of the force applied to it.

Q. In what position will a BAR or BEAM sustain the greatest application of force?

A. When it is compressed in the direction of its length.

Q. What do we mean by STIFFNESS of a material ?

$A$. It is the resistance to the application of force tending to bend it.

Q. How much STIFFER is a BEAM supported at BOTH ENDS, than one of half the length frimly fixed at only ONE END?

A. 'Twice as great.

Q. In what FORM can a GIVEN QUANTITY of matter be arranged so as to oppose the greatest resistance to a bending force?

$A$. In the form of a hollow tube or cylinder.

Q. Why are the Bones of man and animals HoLlow and CYLINDRICAL?

A. Because in this form they can with the least weight of materiai sustain the greatest force. In man and animals, the hollow part of the bones is filled with an oily substance called marrow.

Q. Why are the BONes and QuiLLs of birds hollow and empty of marrow?

$A$. In order that they may possess the greatest strength, and by their lightness assist in flying.

Q. Why are the STEMS of SEEDS and GRAIN-BEARING plants HOLLOW TUBES?

$A$. Because this disposition of matter gives to the stalk its greatest strength, enables it to resist the action of the wind, and sustain, without breaking, the ripened ear of grain or seed.

Q. Is a COLUMN with RIDGES projecting from it, stronger than one that is perfectly smooth?

A. It is.

Q. Why is a HOLLOW TUBE of METAL stronger than the same quantity of metal as a SOLID ROD?

A. Because its substance standing farther from the centre, has a greater power of resisting a bending force.

Q. Of two bodies of similar shape, but of different sizes, which is proportionably the weaker?

A. The larger. That a large body may have proportionate strength to a smaller, it must have a greater proportionate amount of material; and beyond a certain limit, no proportions whatever will keep it together; but it will fall in pieces by its own weight. 
Q. Why cannot TREES attain an UNLIMITED HEIGHT Of TRURK?

A. Because, beyond a certain limit, the weight of the material will overcome the supporting strength of the material.

Q. Why is it impracticable to build sHYPs beyond a CERTAIN STZE?

A. Because the weight of the timber and other materials contained in thein tends to cause them to fall apart.

Q. Why does a PLANK bend and break more readily than a BWAM, and a BEAs resting on its SDGES bear a greater weight than if resting on its SIDE ?

A. Because the resisting lever is smaller in proportion as the beans is thinner.

When a single beam cannot be found deep enough to have the strength required in any particular case, as for supporting the roof of a house, several beams are joined together, and in a great variety of ways, as seen in house-raflers, dce, which, although often consisting of three or more pieces, may be considered as une very broad bearn, with those jarts cut out which do not contribute much to the strength.

Q. Why is a EEA when bent by its weight in the MIDDLw very liable to break ?

A. Because the destroying force acts with the advantage of a long lever, reaching from the end of the beam to the centre; and the resisting force or strength acts only with the force of a short lever from the side to the centre; at the same time, a little only of the substance of the beam on the under side is allowed to resist at all.

This last circumstance is so remarkable, that the scratch of a pin on the under side of a beam, resting as here supposed, will sometimes suffice to begin the fracture.

SECTION II.-APPLICATION OF MATERIALS FOR ARCHITECTURAL OR STRUCTURAI PURPOSES.

Q. What is ARCHITECTURE ?

$A$. In its general sense it is the art of erecting buildings. In modern use, this name is often restricted to the external forms or styles of building.

Q. To what cause do the DHFFEREMT VARIETIES of architecture owe their origin?

$A$. To the rude structures which the climate or materials of any country obliged its early inhabitants to adopt for temporary shelter.

These structures, with all their prominent features, have been afterwards kept up by their refined and opulent posterity. Thus, the Egyptian style of architecture had its origin in the cavern or mound; the Chinese architecture is modelled from the tent; the Grecian is modelled from the wooden cubin; and the Gothic from the bower of trees.

Q. What are the ESSENTIAL ELEMENTARY PARTS of a BUILDING?

A. Those which contribute to its support, enclosure, and covering.

Q. What is a PILE?

A. A beam of wood pointed at one extremity and driven forcibly into the earth, to serve as a support or foundation of some structure. It is generally used in marshy or wet places, where a stable foundation could not otherwise be obtained. 
Q. Why are LONG COLOMNS supporting GRTAT WEIGHTS made SMALLER at the TOP than at the воттом?

$A$. Because the lover part of the column must sustain not only the weight of the superior part, but also the weight which presses equally on the whole column. Therefore the thickness of the column shoukd gradually decrease from bottom to top.

(ใ. Why are STEEP ROOFs, or those constructed with considerable inclination, best alapted for houses in cold clinates?

$A$. In order that the snow may not be retained upon them, which otherwise would be liable to injure the building by its weight.

Q. In the construction of buildings various terms are employed to designate the method in which the timbers are fitted into each other: what do we mean by sorrisina?

A. Mortising is a method of insertion in which the projecting extremity of one timber is received into a perforation in another. (See fig. 32.)

Fig. 32.

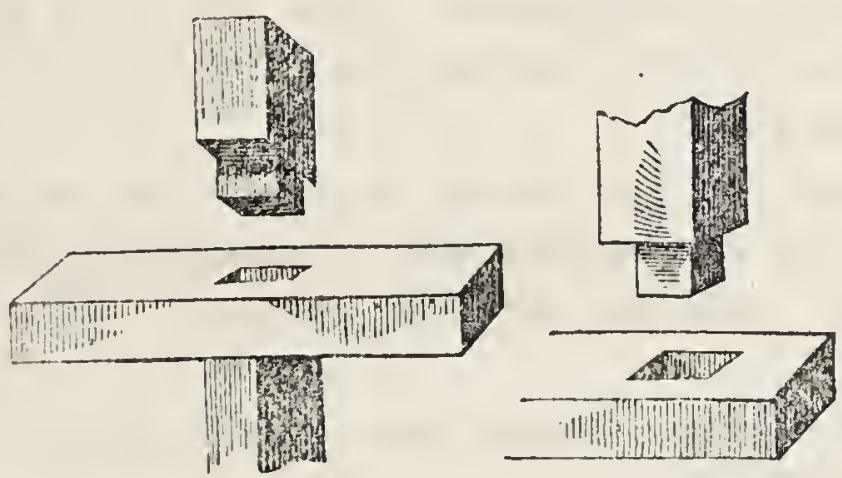

Q. What is a MORTISE?

$A$. The opening or hole cut in one piece of wood to admit the projecting extremity of another piece.

Q. What is a TENon?

$A$. The end of a piece of timber which is reduced in dimensions so as to be fitted into a mortise for fastening two timbers together.

Q. What is SCARFina and INTERLOCEING ?

A. It is that method of insertion in which the ends of pieces overlay each other, and are indented together, so as to resist longitudinal strain by extension, as in tie bearers and the ends of hoops. (See fig. 33.)

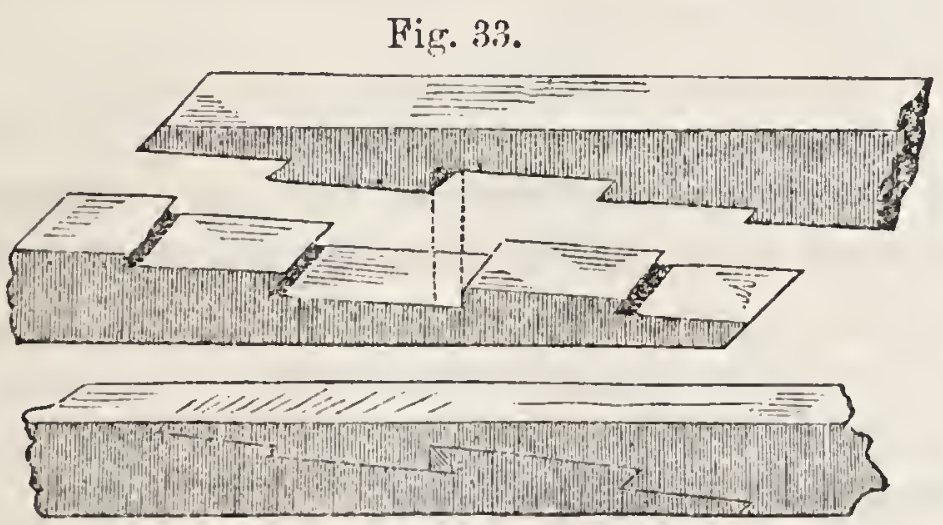

Q. What is tongueing and RABRating?

$A$. It is that method of insertion in which the edges of boards are wholly or partially received by channels in each other. 
Q. What is meant by Dovertaling?

A. It is a method of insertion in which the parts are connected by wedge-shaped indentations, which permit them to be separated only in one direction. (See fig. 34.)

Q. What is a CEMENT?

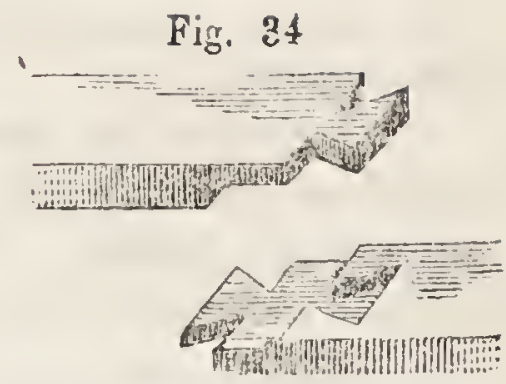

A. Cements are, for the most part, soft or semi-fluid substances, which have the property of becoming hard in time, and cohering with other bodies to which they have been applied.

Q. Of what are the ordinary cements which are called MORTAPs composed?

A. Of quicklime, sand, and water.

Q. What is QUICKLINIE?

A. Quicklime is principally pure lime, and is obtained from the limestone rock, ordinary marble, or shells, which are composed of carbonate of lime by calcination. The effect of the burning is tc drive off the carbonic acid, leaving the lime pure and uncombined.

Q. What is SLACKED LIME?

A. If quicklime obtained as above described be wet with water, it instantly swells and cracks, becomes exceedingly hot, and at length falls into a white, soft, impalpable product. This is denominated "slacked lime."

Q. Why should SLACKED LIME intended for MORTARs be excluded from the air, or used soon after it has been prepared?

A. Because if exposed to the air it absorbs carbonic acid, and becomes converted again into its former condition of carbonate of lime.

Q. Why does Mortar become HARD after a few days?

A. A portion of the water evaporates, and the lime, by a sort of crystallisation, adheres to the particles of sand, and unites them together. The lime also gradually becomes converted into carbonate of lime.

Q. What SAND is most suitable for the formation of MORTAR?

A. That which is wholly silicious and is sharp; that is, not haring its particles rounded by attrition.

Q. What are the PRoportions of lime and sand in good mortar?

A. The proportions are varied in different places: the amount of sand, however, always exceeds that of the lime. The more sand that can be incorporated with the lime the better, provided the necessary degree of plasticity is preserved.

Q. What is a WATER, HYDRAULIO, or ROMIAN OEMENT?

$A$. Those which have the property of hardening under water, and of consolidating almost immediately on being mixed.

Q. To what cause do the WATER-CEMENTS owe their property of becoming hard under water?

A. The cause is not satisfactorily known: all water-cements contain a portion of burnt clay, which probably absorbs immediately all 
superabundant moisture from the lime, and thus expedites its solidifi. cation.

This explanation.is rendered the more probable from the fuct, that if the clay is burnt suficient to vitrify it or cunvert it into brick, it ceases to form a water-cement.

Q. What are the CONSTITUENTS of a WATER-CEMENT ?

A. Quicklime, sand or silica, and a proportion of clay.

Q. What is stucco?

A. Stucco is composed of various ingredients, generally of " plaster of Paris," sometimes of white marble pulverised and mixed with plaster and lime.

Q. What is TERRA-COTtA?

A. Literally, baked clay, a name given to statues, architectural ornaments, vases, figures, \&c., modelled of potters'-clay and fine colourless sand, and afterwards exposed to a most intense heat.

Q. Why are BRICKS when burned usually of a RED COLOUR?

A. Because the iron contained in the clay is converted by the heat into the red oxide of iron, and acts in this state as red colouring material.

Q. Why are some Bricrs of a white or yellow colour?

$A$. Because the clay of which they are formed does not contain sufficient iron to colour them.

Q. Why did the CIIILDREN OF ISRAEL in making bricks desire to mix STRAW with the CLAY?

A. The bricks of the Egyptians were composed of clay simply baked in the sun, and not burnt. By using straw the clay was held together more firmly, and the brick rendered stronger.

Q. Why are the EGrptians enabled to dispense with the process of burning the bricks?

$A$. 'The extreme dryness of the climate in which they were used enabled them to dispense with the burning. Bricks from Egypt and Babylon, which have remained exposed to the open air uninjured for two thousand years, rapidly fall to pieces when transported to a moist cimate.

Q. Why do we mix HAIR with MORTAR ?

A. In order to render it more cohesive and stronger.

Q. What are TiLes?

A. Plates of burnt clay resembling bricks in composition and manufacture, and used for the corerings of roofs or floors.

Q. What is MASTICH, or MASTIC?

$A$. The name given to those cements which contain animal or vegetable substances in composition. Mastich used for the external decoration of houses often contains oil and a preparation of lead.

Q. What is an $\mathrm{ARCH}$ ?

A. It is a part of a structure or building suspended over a hollow, and concave towards the area of the hollow. 
Q. Is it known at WHAT TIME the ARCH was invented?

A. It is not; it does not appear to have been known to the ancients.

Q. Why is an $\triangle \mathrm{RCH}$ capable of resisting a greater amount of pressure than a lorizontal or rectangular structure constructed of the same inaterials?

A. Because the arrangement of the materials composing the arch is such, that the force which would break a horizontal beam or structure is made to compress all the particles of the arch alitie, and they are, therefore, in no danger of being torn or overcome separately.

Q. What beautiful application of the arch exists in the HUMAN STRUCTURE?

$A$. In the skull, protecting the brain. The materials are here so arranged as to present the greatest strength, with the least weight.

Q. Why is it difficult to break an $\mathrm{EGG}$ by pressing directly upon its ends?

$A$. Because the shell of the egg is constructed on the principle of the arch, and is therefore capable of resisting great pressure.

Q. Why is a DISHED or ARGHED WHEEL of a carriage much stronger for resisting all kinds of shocks than a FLAT WHERL?

$A$. In an arched or dished wheel, the extremity of a spoke cannot be displaced inwards, or towards the carriage, unless the rim of the wheel be enlarged, or all the other spokes yield at the same time; and it cannot be displaced outwards, unless the rim be diminished, or the other spokes yield in an opposite direction.

Now the rim, being strongly bound with a tire of iron, cannot suffer either increase or diminution, and the strength of all the spokes is thus conferred by it on each individually. In a flat wheel, a given degree of displacement, outwards or inwards, of the extremities of a spoke, would less affect the magnitude of the circumference, and therefore the rim of such a wheel secures it much less firmly.

Q. Why are the FORE WHEELS of carriages smaller than the MIND WHEELS?

$A$. Because they facilitate the turning of the carriage. The advantage of the wheel is proportioned to the magnitude; the smaller wheel having to rise a steeper curve.

Q. What is the ABUTMENT or SPRINGING of an ARCH?

$A$. The top of the wall or walls which receive the first archstones.

Q. What is meant by an ORDER in ARCHITECTURE?

$A$. By an architectural order, we understand a certain mode of arranging and decorating a column, and the adjacent parts of the structure which it supports or adorns.

Q. How many orders are recognised?

A. Five:-the Doric, Ionic, and Corinthian, derived from the Greeks; to these the Romans added two others, known as the Iuscan and Composite.

Q. What are the peculiarities of the Doric column?

A. The Doric is the earliest and most massive order of the Greeks. The column is always large, with a plain cap or capital. Its height, in the finest examples at Äthens, is six times its diameter; the true Doric column has twenty flutings, and no base stone. The best 
specimens of the Doric order are found in the Parthenon and Temple of Theseus at Athens. (See fig. 35.)

Q. What are the peculiarities of the IONIC ORDER?

$A$. The Ionic column is a lighter order than the Doric, being cight or nine diameters in height. It has a base, with twenty-four or more flutings upon the shaft. The capital has two double parallel scrolls, called volutes, occupying opposite sides, said to have been copied from ringlets of hair. The best ancient specimens of the Ionic order are to be found in the Temple of Erictheus at Athens. (See fig. 36.)

Q. What are the distinguishing peculiarities of the CoRINTHIAN ORDER?

A. The Corinthian was the highest and most decorated of the Grecian orders. Its base resembled that of the Ionic, but was more complicated. The shaft was often ten diameters in height, and was fluted. The capital was shaped like an inverted bell, and covered on the outside with two rows of the leaves of the plant acanthus, above which were eight pairs of small scrolls on volutes. (See fig. 3i.)

The origin of the Corinthian capital has been ascribed to the sculptor Calimachus, who is said to have copied from a basket accidentally enveloped in leaves of acanthus.

Q. What are the peculiarities of the TUSCAN ORDER?

$A$. The Tuscan order, derived from the ancient Etruscans, is not unlike the Doric in many respects. It is not, however, fluted on its shaft, and is supported by a base. There are no vestiges of this order among ancient ruins. (Sce fig. 38.)

Fig. 35.

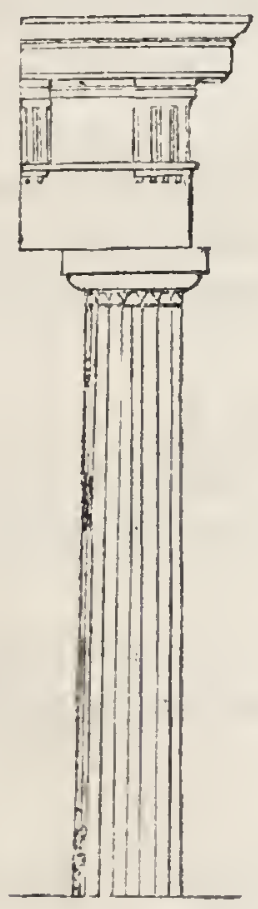

Fir. ¿6.

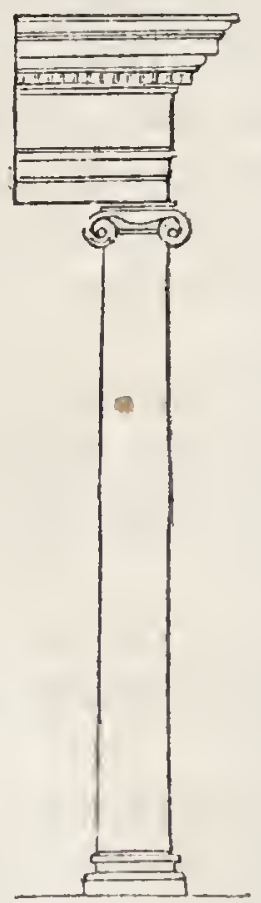

Fig. $37_{\text {a }}$

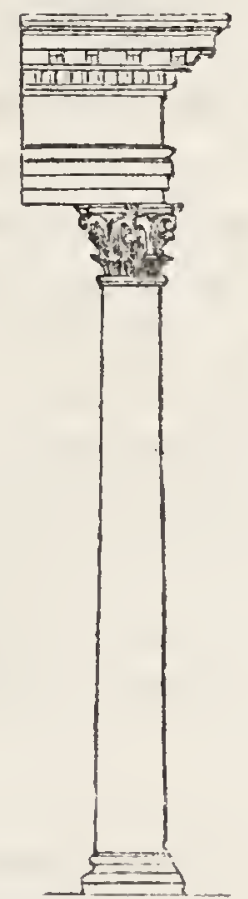

Q. What are the peculiarities of the composite orDER ?

A. 'This fifth order was made by the Romans out of the Corintrian, simply by combining its rapital with that of the Iomir. Its best example is found in the Arch of litus at Rome. (Sec fig. 39.)

Fig. 40 represents a modification of the Doric column, called the Roman Doric. 
Fig. 38 .

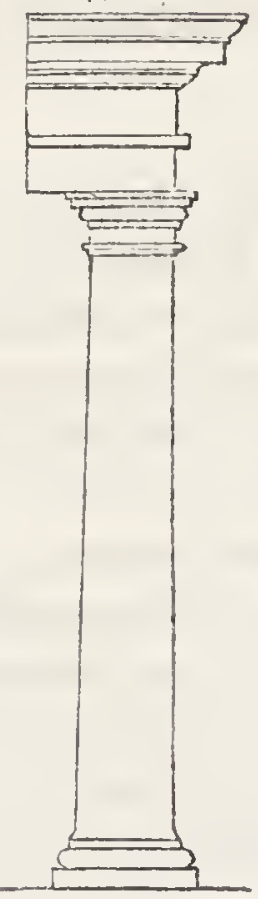

Fig. 39.

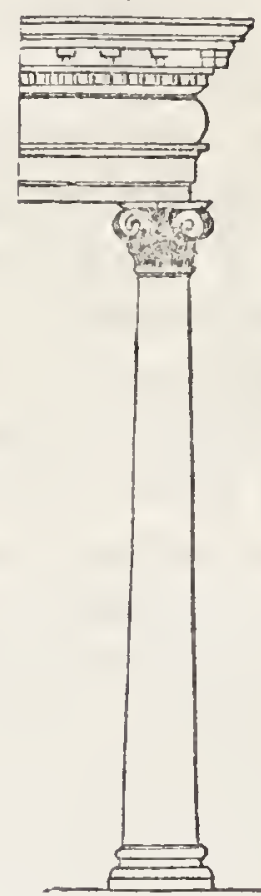

Fir. 40.

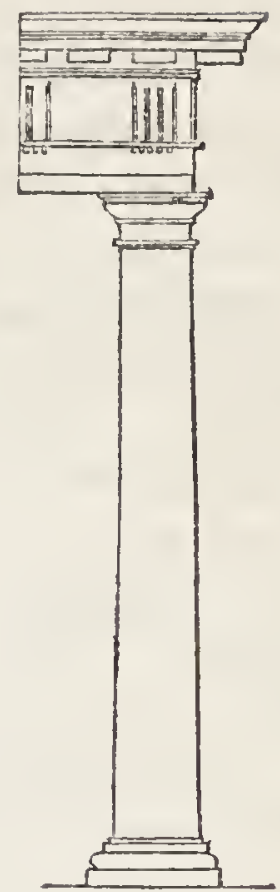

Q. How do pilasters differ from columas?

A. Only in their plan, which is square, as that of columns is round: pilasters are attached to walls.

Q. What is a portico?

A. A portico is a continued range of columns covered at the top to shelter from the weather. The portico of the temple at Palmyra was full four thousand feet long.

Q. What are BALUSTERS ?

A. Small columns, or pillars of wood, stone, \&c., used in terraces or tops of buildings for ornament; also to support railing. When continued for some distance, they form a balustrade.

Q. Where did the GothIO ORDER of ARCHITECTUR? originate?

A. Among the northern nations of Europe. After the destruction of the Roman empire, it was introduced to the exclusion of the Greek and Roman manner of architecture. It seems particularly adapted to religious edifices.

Q. What are the CIIARACTERISTics of the GotHic ARCHITECTURE?

A. Pointed arches, with greater height than breadth in the proportions, with profuse ornament, chiefly derived from an imitation of the leaves and flowers of plants.

Q. What is said to have been the rodel of the AISLE of A GOTHIC CATHEDRAL?

A. A group of tall trees, meeting at the top with interwoven branches.

Q. What TwO SPECIES of GOTHIC ARCIITEOTURE are there?

A. The Saxon, heavy, plain, massive, like the Tuscan; the Norman, like the Corinthian architecture, light and ornamental.

Q. Ought ARCHITECTURE to be considered as a FINE or a USFFUL ART?

A. As a useful art.

It is degrading the fine arts to make them entirely subservient to utility. It is out of taste to make a statue of Apollo hold a candle, or a fine painting stand as a fireboard. Onr houses are for use, and architecture is therefore one of the useful arts. In building, we should plan the inside first, and then the outside to cover it. 
Q. Why is it BAD TASTE to construct a DWELLING-Irouse in the form of a GRECIAN TEMPLE?

A. Because a Grecian temple was intended for external worship, not as a habitation or place of meeting.

Q. Had the Goths, who plundered Rome, anything to do with the invention of Goтнг ARCHITECTURE?

A. No; the name was introduced about two hundred years ago as a term of reproach, to stigmatise the edifices of the Middle Ages, which departed from the purity of the antique models.

Q. What is the FACADE of a building?

A. Its front.

Q. What is a PEDESTAL ?

A. The lower part or base of the column; a continued base, on which a range of columns is erected, is called a stylobate.

Q. What is the PLINTI ?

A. 'This term is applied to the lower part of the pedestal, or to any square projecting basis, such as those at the bottom of walls, and under the base of columns. The lower part of the pedestal being called the plinth, the middle part will be termed the die, and the upper part the cornice of the pedestal.

(See fig. 41.)

Fig. 41.

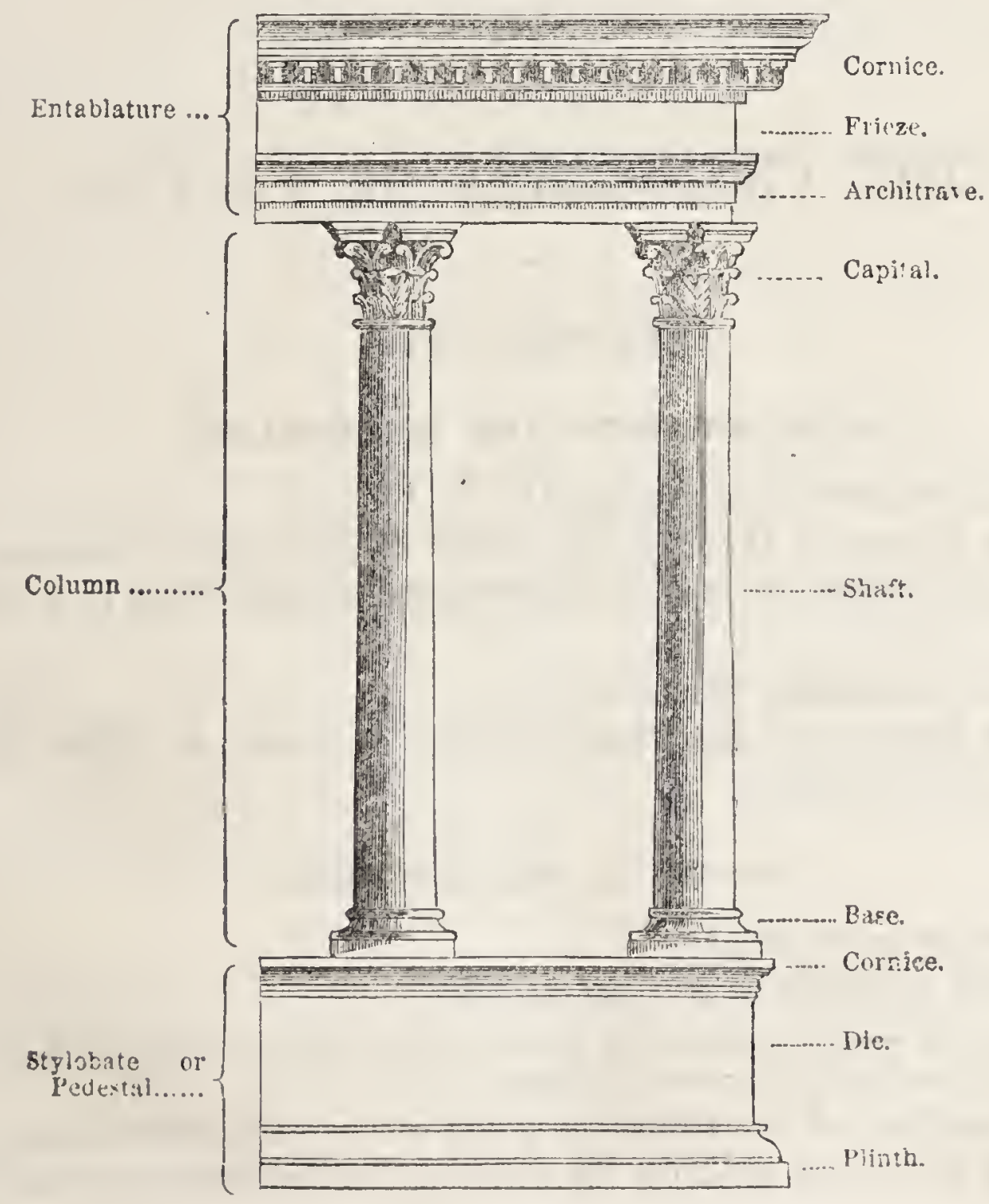


Q. What is the BASE of a COLUMIN?

A. The lower part, where it is distinct from the shaft.

Q. What is the sHAFT?

A. The middle or longest part of the column.

Q. What is the CAPITAL?

$A$. The upper or ornamental part resting on the shaft. The height of a column is measured in diameters of the column itself, always taken at the base.

Q. What is the entabiature?

A. The horizontal continuous portion which rests upon the top of a row of columns.

Q. What is the ARCHITrate?

$A$. The lower part of the entablature.

Q. What is the FRIEZE?

A. The middle part of the entablature.

Q. What is the ooR

A. The upper or projecting part of the entablature. (For illustration of these different terms, see fig. 41.)

\section{TIE PHENOMENA OF FLUIDS.}

\section{CHAPTER XI.}

\section{HYDROSTATICS AND HYDRAULICS.}

Q. What is the SCIENCE of Hydrostaitics?

A. The science of Hydrostatics treats of the weight, pressure, and equilibrium of water, or other slightly elastic fluids, when in a state of rest.

Q. What is the SCIENCE of Hydraulics?

A. The science of Hydraulics treats of water or other slightly elastic fluids in motion.

\section{SECTION I.-HYDROSTATICS.}

Q. Is water an ELASTIC body?

A. Water is elastic in a slight degree.

Q. Why does a PIECE of SLATE, when thrown nearly horizontal upon the suriace of lake, as in the game of ducks and drakes, REBOUND?

$A$. On account of the elasticity of the water; the water being compressed by the stone striking its surface, immediately recovers itself 
and acts as a spring against the surface of the slate, causing it to rebound.

Q. How does the PRESSURE of a FLUID differ from that of a solid ?

A. In solids the pressure is always downwards, or towards the centre of the earth's gravity; but in fluids, when confined, the pressure is exerted in all directions-upwards, downuards, sideways.

Q. What difference does the SHAPE or SIZE of a vessel make in respect to the pressure of a fluid upon its buttom?

A. The pressure exercised by a fluid from above, downwards on the bottom of the vessel in which it is contained, is quite independent of the form of the ressel, and is always equal to the weight of a column of the fluid, whose base is the bottom of the vessel, and whose height is the vertical distance from the bottom to the surface of the fluid.

Q. Why is the surface of a FLUID at REST always LEVEL ?

A. Because the particles are equally attracted towards the earth by gravity, and are all equally and perfectly movable among themselves.

Q. On what PRINCIPLE are we enabled to conduct WATER under GROUND and THROUGH IRREGULAR TUBES?

$A$. On the principle that water will always rise to an exact level in different tubes, pipes, or vessels communicating with each other. Thus, from a lake or reservoir, water can be conveyed in pipes under streets or buildings to any distance, and supplied to any height not exceeding that of its source.

Q. When a SHIP founders in sHaLLOW WATER, the wreck, on breaking to pieces, generally comes to the surface, and is cast upon the shore; but when a sHIP sinks in very DEEP WATER, it never rises: why is this?

$A$. The pressure of very deep water forces the water into the pores of the wood, and makes it so heavy that no part of the wreck is enabled to rise again.

Q. Can you sink a conk so deep that it will not rise to the surface again?

A. At a great depth the water forced by pressure into the pores of the cork renders it so heavy that it cannot rise.

Q. In the minds of many persons it is difficult to conceive how water can press UPWARDS as well as DOWNWARDS, and LATERALLY : how can this be rendered evident?

A. From the fact that, if in a fuid mass the particles below had not a tendency upwards equal to the weight or downward pressure of fuid over them, they could not support that fluid which entirely rests upon them. Their tendency upwards is owing to the pressure around them from which they are trying to escape.

\section{SECTION II.-HYDRAULICS.}

Q. How slight a DEcLivity is sufficient to give the RunNing Motion to water?

A. Three inches to a mile in a smooth, straight channel, gives a velocity of about three miles per hour. The river Ganges, at a distance of 1,800 miles from its mouth, is only 800 feet above the level of the sea. 
Q. In what waY ean WATER be DISCHARried most rapidly from a veGSEL ?-through a

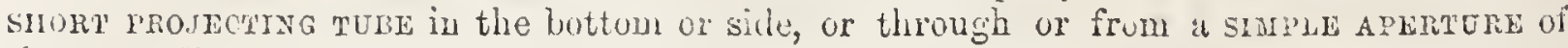
the same ilinensions?

A. From the short tube.

Q. In what part of a JET or STREAM of flowing water will the greatest relucity be found?

A. At the point where its dimensions are contracted to the greatest extent.

Q. In what PART of a RIVER does the water flow MIOST RAPIDty?

A. In the middle of the stream, at the surface. On the sides and bottom the velocity is diminished by the friection of the water against the banks, bars, \&c.

Q. Why will a PIPE Two INCHns in diameter give nore water than FIVE PIPES in the same position, of one inch in diancter?

A. Because the water flows with much grecter velocity in the large pipe than in the small ones, there being much less friction in the former than in the latter.

\section{CHAPTER XII.}

\section{PNEUMATICS.}

\section{SECTION I.-GENERAL PROPERTIES OF AERIFORM BODTES.}

Q. What is the object of the science of Preuratics?

A. To explain and illistrate those phenomena which arise fiom the ueight, pressure, or motion of cormmon air, or other Aluids possesising the same general properties.

Q. On what rests the distinction between LIQUIDS and GASEOUS BODIES?

A. The distinction between liquids and those more elastic fuids which we term air, gas, vapour, steam, \&c., depends principally on heat and pressure. Thus, water, according to the addition or subtraction of heat, may exist as a solid-ice; as a liquid-water; or as a vapour-steam.

Q. Under what pressure of the atmosphere is water converted into stras?

A. Under the ordinary pressure of the atmosphere, water is converted into stean at 212 degrees, Fahrenheit's thermometer; if this pressure is increased, it requires a proportionably higher temperature: if this pressure is diminished, the amount of heat required is proportionably less.

Q. How many kinds of ARRIYORM or GASEOUS noDIES exist in nature?

A. Those which, under common circunstances of temperature and gressure, are always in a gaseous state, as common air; and those which become gases chiefly at high temperature, as steam, or rapour of witer: 
Q. What is ATMOSPUIFRIC AIR?

A. A transparent fluid, composed mainly of two gases, oxiygen and nitrogen, the ultimate particles of which appear to be destitute of cohesion; hence air has a disposition not only to sink down and spread out laterally, like liquids when unconfined, but also to expand and rise upwards.

Q. Is the AIR Porous?

A. Yes; in a very high degree.

Q. How do we know this FACT?

A. Because air readily yields to pressure, and a great bulk of it may be forced to occupy a very small space.

Q. Is air also Impenetrable?

A. Yes; beyond a certain limit it cannot be comprassed.

Q. How much JiIGITTER is STEAMI than ordinary AIR?

A. Steam has but little more than hatf the weight of atmospheric air ; and hence it rises and floats in the air as a cork rises and floats in water.

\section{SECTION II.-ATMOSPHERIC AIR.}

Q. What do we understand by the ATMoSPHERE ?

A. The thin transparent fluid which surrounds the earth to a considerable height above its surface, and which, by its peculiar constitution, supports animal life by respiration, and is also necessary for the due exercise of the vegetable functions.

Q. Is the ATMOSPIIERe INTISIBHe?

A. It is generally, but crroneously, so regarded. The atmosphtere is not invisible.

Q. How can you prove that the ATMosphere is not INYisibie?

A. Because when we look upwards into the firmament on a clear diay, the vault appears of an azure or clear colour.

This colour belongs not to anything which occupies the spnec in which the stars or other celestial objects are placed, but to the mass of air through which the bodics aire bucit.

Q. If the air he an AZURE-Cotoursd body, why is not that portion which immediately surrounds us perceived to have this colour, in the same manner that a blue liquid contained in a bottle exhibits its proper laue?

A. There are certain bodies which reflect colour so faintly, that when they exist in limited quantities, the portion of coloured light they reflect to the eye is insufficient to produce sensation; that is, to excite in the mind a perception of the colour. Almost all semi-transparent bodies are examples of this.

if a glass tube of small bore be filled with sherry wine. or wine of a similar colour, and looked at through the tube, it will be found to have all the appearance of water, and he colourless. If viewed from above, downwards in the direction of its length, it will he found to possess its original colour. In the first instance there can he no doubt but that the wine has the same colour as the liquid of which it originally formed a part; but existing only in small quantity, that colour is transmitted to the eye so faintly as to by iusuficient in produciog pereoptiou. 
Q. Why do Distant mountains appear blue?

A. Not because it is their colour, but because it is the colour of the air through which they are seen. less?

Q. Why does the ocEan appear GREen, and a GLASS of the same WATER appear colour-

A. Because in the small mass of water the amount of colour is too small to be perceptible, while in the entire quantity it is sufficient.

Q. Has AIR WEIGHT?

A. It has; as well as lead, stone, or any other material substance.

Q. How can this be readily proved?

A. By weighing a vessel filled with air, and the same vessel after the air has been exhausted from it.

Q. Can the existence of air be known by the SENSE of TOUCH or FEELING ?

$A$. It can; since it opposes resistance when acted upon, and strikes with a force proportionate to the speed of its motion.

Q. Why do we always feel a BREEZE on the DECK of a STEAMBOAT in motion, even upon the calmest day?

A. Because our bodies forcibly displace the air as we are carried therough it.

Q. What is Wind?

\section{A. Simply air in motion}

Q. How are the WAVES of the OCEAN produced?

$A$. By the force of the air in motion, or wind striking upon the surface of the water.

Q. Why are waves calmed and subdued by pouring ord upon the surface of the water"?

$A$. The oil, from its inferior specific gravity, forms a floating film, which defends the surface of the water from contact with the currents of air; and the friction between the wind and the waves is greatly diminished, in the same manner that the friction between solids is by the application of unctuous matter. tion?

Q. Could a BIRD fly in a SPACE DEVOID of AIR, even if it could exist without respira-

$A$. It could not; as the bird rises simply by the resistance of the particles of air to the beating of its wings.

Q. How do we know that AIR is ELASTIC?

$A$. Because a volume of compressed air, the pressure being removed, immediately restores itself to its original bulk.

Q. When is AIR said to be RAREFIED?

$A$. When a given quantity of air is caused to expand and occupy a greater space, it is said to be rarefied.

When a part of the air enclosed in any vessel is withdrawn, that which remains, expanding by its elastic property, always fills the dimensions of the vessel as comple ely \&s before. If nine-tenths were withdrawn, the remaining one-tenth would occupy the same space that the whole did formerly.

Q. What is the HETGHT of the ATMOSPHERE abote the SURFACE of the EARTI ?

A.s It is supposed to be about 45 miles; the zone or shell of air 
which surrounds the earth to the height of nearly $2 \frac{3}{4}$ miles from its surface, contains one-half of the atmosphere; and the remaining half, being relieved of this superincumbent pressure, expands into another zone or belt of the thickness of 41 or 42 miles.

Some authorities suppose this last zone to have a wuch greater area.

Q. What is the WEIGHT of AIR compared with that of WATER? bullk.

A. Water is about 840 times the weight of air, taken bulk for

Q. What is the Estryated WEIGIT of the Whole atMosphere enveloping the globe?

A. To the weight of a globe of lead sixty miles in diameter.

Q. As air has weight, and as the mass of it extends at least 45 miles above the earth's surface, WIIAT AMOUNT of PRESSURE does it exert?

$A$. At the level of the ocean the atmosphere exerts a pressure of about 15 pounds for every square inch of surface.

Q. If the AIR were condensed so as to occupy no more space than the same WEIGHT of WATER, to how great an elevation above the earth would it extend?

A. To an elevation of thirty-four feet.

Q. In what DIRECTION is the PRESSURE of the ATMOSPHERE exerted?

A. It is the nature of a fluid to transmit pressure in every direction equally; therefore the air presses upwards, downwards, latercally, and obliquely, with the same force.

Q. How great a pressure is exerted by the air upon the body of a MIAN Or ANIMAL l.aving a surface of 2,000 square inches?

A. Not less than 30,000 pounds, or about 15 tons.

Q. Why is not the INDIVIDUAL CRUSHED beneath so enormous a loat?

A. Because the atmosphere presses equally in all directions, and our bodies are filled with liquids capable of sustaining pressure, or with air of the same density as the external air; so that the external pressure is met and counteracted by the internal resistance.

Q. How can this be illustrated experimentaLLY?

$A$. If we take a glass cylinder open at both ends, and over one end tie a piece of bladder, no pressure is perceived, because the air within the cylinder presses upon or exerts a supporting power equal to the weight resting upon it; but, if by an air-pump we remove the air under the bladder, the bladder will immediately exhibit the effects of the ressure, and eventually be torn asunder in consequence.

Q. Why are not the ANIMALS living at great DEPTHS in the OCEAN, and subjected to a pressure SIXTY or NINETY times GREATER than we sustain at the bottom of our ocean of air, CRUSIED, or at LESST IMPEDED, in their motions?

$A$. Because the pressure of the water is equal on all sides, and the bodies of these animals are equally pressed above, below, and around, and the fuids within the animal are also of similar density.

Q. What happens when FIsurs living at great depths of the sea are drawn to the surface?

A. They are almost insiuntly destroyed, by the expansion of the fluids within them on the removal of the pressure of the water. 
Q. What would be the effect upon a YIN or ANIMAL, if $A T$ ONCE RELIEVED of all atmospheric pressure?

A. All the blood and fuids of the body would ba forced by expansion to the surface, and the animal would burst.

Q. In what does the surgical operation of cupping consist?

A. In removing the atmospheric pressure from the part of the body submitted to the operation.

Q. How is this effected?

A. A vessel with an open mouth is connected with a pump or apparatus for exhausting the air. The mouth of the vessel is applied in air-tight contact with the skin; and by working the pump a part of the air is withdrawn from the vessel, and consequently the skin within the vessel is relieved from its pressure. All other parts of the body being still subjected to the atmospheric pressure, and the elastic force of the fluids contained in the body having an equal degree of tension, that part of the skin which is thus relieved from the pressure will be swollen out, and will have the appearance of being sucked into the cupping-glass. If the skin be punctured with lancets, the blood will be drawn or forced out.

Q. What do we mean by a vicuusis?

A. A space devoid of all matter; in general, we mean by a vacuum, a space devoid of air.

Q. Can a PHRFict vacuda be produced artirrotalis ?

A. No; but confined spaces may be deprived of air sufficientily for all experimental or practical purposes.

Q. Are there any instances of a VACUUM in NATURA?

A. There is no reason for supposing the interplanetary spaces to be filled with any material substance.

Q. What do we mean by the Dinsire of the AIR?

A. By the density of air we mean the quantity contained in a given bulls. Thus, when one portion contains twice as many particles of air in a given space as another, the first portion is said to possess twice the density of the second.

Q. Is there any LAT regulating the DENSTTY of the ATIOSPIIERE ?

A. The density of the air decreases with the altitude.

Q. To what is this owing?

A. To the diminished pressure of the air, and the decreasing foren Fig. 42. of gravity. Those portions directly incumbent upon the earth are most dense, because they bear the weight of the superincumbent portions; thus, the hay at the lower part of the stack bears the weight of that abore, and is therefore more compact and dense.

In the accompanying engraving this idea may be conveyed by the grarlual shading, which denotes the gradual diminution in the density of the atmosphere in proportion to its altitude. 
Q. Why do persons who ascen! in BALloons feel intense pain in their eyes and ears?

A. Because the air of the upper regions is more rarefed than that on the earth; and the air inside their bodies (seeking to become of the same rarity) bursts through their eyes and ears, producing intense pain.

Q. Why is it often PaINYuL and difficult to BREATII on a MOUNTAIN-TOP?

A. Because, owing to the extreme rarity of the air on the top of the mountain, a person, although expanding his chest as much as usual, really takes in only half as much air as he does when at the foot of the mountain.

Q. What effects are produced by the RARETACTION of Aris at LofTr elevations, as on the tops of high mountains?

A. The intensity of sound is lessened, respiration is impeded, and the minute veins of the body swell and open. Travellers, in ascending the high mountains of South America, have noticed the blood to gush from the pores of the body, and the skin in many places to crack and burst.

Q. Why do persons WHO DESCEND IN DIVING-BELLS Frig PATx in their eyes, and other sensitive parts of their bodies?

A. Because the pressure of the water upwards compresses the air in the diving-bell.

2. Why do we feel oppressed just previous to a storm?

A. Because the air is greatly rarefied by heat and vapour ; and the air inside us (seeking to become of the same rarity) produces an oppressive and suffocating feeling.

Q. Is the ExIsTrance of AIP. necessary to the production of sound?

A. It is; in a vacuum there can be no sound; and on the top of high mountains, where the air is greatly rarefied, as on Mont Blanc, the report of a pistol can hardly be heard.

Q. Can you Bor an EGG on the top of a very high mountain?

A. Travellers have found it impossible, since the temperature at which water boils is dependent on the pressure of the air; and in places where the pressure is very slight, the water boils and is converted into steam without becoming sufficiently hot to cook the eegr.

On the top of Mount St. Bernard, 7,688 feet above the sea-level, water boils at a temperature of $209^{\circ} \mathrm{F}$.

Q. If the rips be applied to the BACK of the maND, and the breath drawn in so as to produce a PARTIAL VACUUM in the MOUTI, why will the skin be drawn or sucked in?

$A$. Not from any force resident in the lips or the mouth drawirng the skin in, but from the fact that the usual external pressure of air is removed, and that the pressure from within the skin is suffered to prevail.

2. How is a Bor enabled to LIFT a stone by means of the cominon SUCkEn?

A. The sucker consists of a lisc of moistened leather, with a string by which it may be suspended with any weight attached to it. If its smooth, moist surface be pressed so closely against the flat side of a stunc or other body that the air cannot enter hetween them, the 
weight of the atmosphere pressing upon the upper surface of the leather makes it adhere so strongly, that a stone of weight proportioned to the extent of the disc of leather may be raised by lifting the string.

Q. How great a WEIGIT can be raised by the sUCKER?

A. The effect of the sucker arises from the exclusion of the air besween the leather and the surface of the stone, \&c. The weight of the atmosphere presses their surfaces together with a force amounting to 15 pounds on every square inch of those surfaces in contact. If the sucker could act with full effect, a dise an inch square vound shipyort a weiglit of 15 pounds; two square inches, 30 pounds, \&c. ; the practical effect, however, of the sucker is much less.

Q. Why do smooth plates of polished GLASS, MARBLE, or MIETAL stick together?

A. Because their surfaces are brought into such close contact that the air cannot insinuate itself between them; therefore the outside pressure of the atmosphere holds them together with a force corresponding to the extent of the surface of contact.

Q. How are Fuies, and other small insects, enabled to WaLK on crimings and surfaces presented downwards, or upon smooth panes of glass in an upright position?

A. Their feet are formed in such a manner that they act as small air-pumps or suckers, excluding the air between them and the surface with which they are in contact; and the atmospheric pressure keeps the animal in position.

2. How are SNAILS, LIMPETS, and other like animals enabled to ADHERE to the ROCKS ?

A. The surface of their shells at the openings are capable of being exactly fitted to any plane surface ; these animals have the power of producing a vacuum within their shells when thus fixed, and the atmosphere consequently presses upon them and holds them in their places.

Q. What causes some PEARI-DIYERS to be DEAF?

A. Because the membrane of the tympanum is ruptured by the pressure of the water. The deafness thus produced is, in many cuses, incurable.

Q. Why do the salls of a wiudmill turn round?

A. Because the wind, blowing against the oblique surface of the sails, pushes them out of the way, driving them from place to place in a restless round.

Q. Why in BREATHING do we first DRAW in the BREATII, as it is termed?

A. Because by so doing we make an enlarged space in the chest, and the messure of the external atmosphere forces the air in to fill it.

The air enters the lungs, not because they draw it in, but by the weight of the atmo. siphere furcing it into an empty space.

Q. How is the AIr caused to Bocape from the lungs?

A. Simply by means of its elasticity; the lungs by muscular action compress the air contained in them, and give to it by conpression a greater elasticity than the air without. By the excess of the elasticity it is propelled, and escapes by the mouth and nose. 
Q. Upon what does the ACTION and USE of the COMMON BELLOWS depend?

$A$. Upon the elasticity and compressibility of the air.

In the lower board of the bellows is a hole covered by a valve, consisting of a flat piece of stiff leather, movable on a hinge, and which lies on the opening, but is capable of being raised by a slight pressure. When the upper board of the bellows is raised, the internal cavity is suddenly enlarged, and the air contained in it is considerably rarefied. The pressure of the atmosphere forces in air at the nozzle, but this being too small to allow its admission with sufficient ease and speed, the valve covering the hole is acted upon by the atmosphere and raised, and air rushes in through the large aperture under it. When the space between the boards is filled with air in its common state, the upper board is depressed, and the air confined in the bellows is suddenly condensed. The valve covering the hole is thus kept firmly closed, and the air has no escape except through the nozzle, from which it issues with a force proportioned to the pressure exerted on the upper board.

Q. Why will not LIQUOR flow CONTINUOUSLY from a CASK after it has been tapped or pierced, unless another OPENING is made as a VENT-HOLE in the UPPER PART of the CASK?

$A$. Because the force of the air pressing upon the mouth of the opening, having nothing to counterbalance it, would support a column of liquor, if the cask was ail-tight, the height of which would be proportioned to the weight of the column of liquor.

Q. What is the effect when the air acts through the VENT-HOLE?

$A$. When the air is enabled to act through the vent-hole above, the pressure below is counterbalanced, and the liquor descends and runs through the opening by the effect of its own weight.

Q. What is the EFFECT if the lid of a tea-pot be air-tight?

A. If the lid of the tea-pot be air-tight, the liquid will flow freely for a short time, and then cease.

Q. Explain this.

$A$. By the escape of some portion of the liquid, the air under the lid expands in the enlarged space and becomes rarefied; the pressure on the surface of the liquid in the vessel is then so far diminished, that the atmospheric pressure on the outside resists its discharge at the spout.

Q. How is this REMEDIED?

$A$. By the hole in the lid, which allows the air to enter from witiout.

Q. Upon what principle does the construction of GAS-HOLDERS or TANKS and GASoMETERS depend?

\section{A. Upon the pressure of the atmosphere.}

Q. Suppose a vessel having its mouth upwards be completely filled with a liquid, the mouth then to be stopped by pressing a smooth plate of glass or metal against it, and the vessel to be INVERTED; the mouth being plunged into a cistern filled with the same liquid, would the liquid FLow OUT of the inverted vessel ?

A. If the height of the vessel in this case be less than the height of the column of the liquid which the atmospheric pressure would support, the vessel will continue to be filled even after the plate is removed from its mouth.

Q. Can you explain why the water will Nот FALI OUT of the inverted vessel after the plate is removed?

A. The atmospheric pressure acting upon the surface of the liquid 
in the cistern, will prevent the liquid contained in the vessel from falling out.

Q. What would be the EFFECT if air were introduced by means of a tube through the water, and suffered 's escape just UNDER the мо UTH of the inverted vessel ?

$A$. If air were introduced by means of a tube, and suffered to escape immediately under the mouth of the inverted vessel, it would ascend in bubbles, find its way to the highest part of the vessel, and expel the water from it.

Q. How MucH water can be expelled in this way?

$A$. The water will continue to be expelled as long as air is supplied, until all the water contained in the vessel is expelled, and the vessel is filled with air.

Q. Suppose the process to be CONTIN UED, what would be the effect?

$A$. If the process be continued, the air will begin to escape under the edge of the vessel, and rise in bubbles to the surface. (See fig. 43.)

Fig. 43.

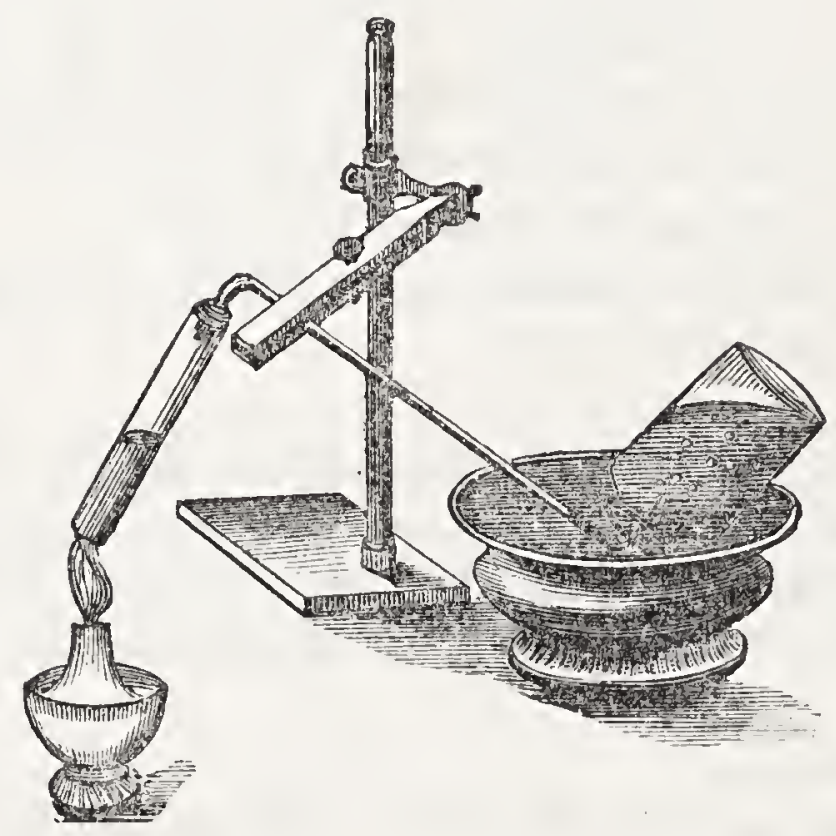

Fig. 44.

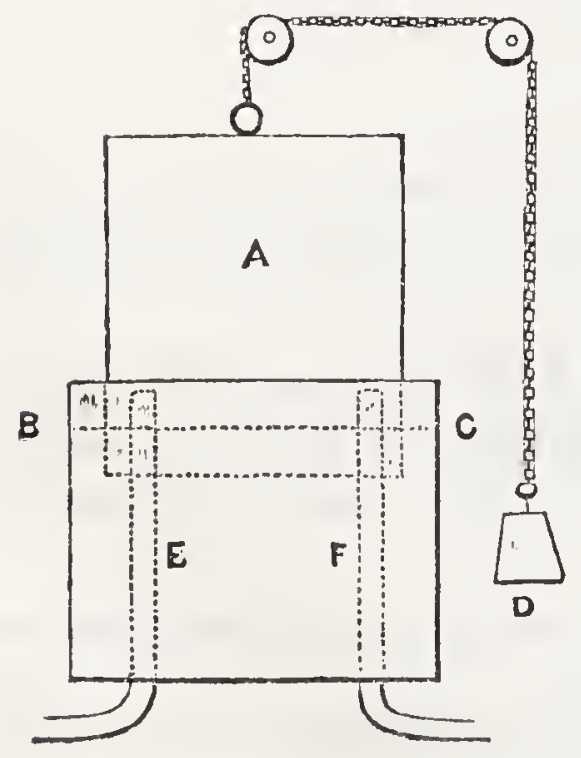

Large gasometel's, such as are necessary for the illumination of towns, are also constructed on the above principle. They consist of a large inverted vessel, A (fig. 44), made of iron, of a cylindrical form, and suspended over a reservoir of water $\mathrm{B} \mathrm{C}$, of a little larger size, by means of counterweights $D$. The gas is introduced by pipes, ascending from the bottom of the reservoir $\mathrm{E}$ and $\mathrm{F}$, and rising a little above the surfaco of the water. While the gasometer is filling with gas, $t$ gradually rises out of the water until it is filled, after which no more gas is admitted, and its contents are ready to be distributed through the pipes by which it is intended to be conveyed to the place to be illuminated by burning it. As the gas is forced out by the weight of the gasumeter and is burned, the gasometer gradually descends into the water till the whole of its contents are expelled, when it is again filled by the same process as befure. 
Q. Why does a BotrLE or JUG GURGLE when LIQUID is freely poured from it?

$A$. On account of the pressure of the atmosphere forcing air into the interior of the bottle. In the first instance, the neck of the bottle is filled with liquid, so as to stop the admission of air. When a part has flowed out, and an empty space is formed within the bottle, the atmospheric pressure forces in a bubble of air through the liquid in the neck, which, by rushing suddenly into the interior of the bottle, produces the sound.

Q. When will a BoTTLE CEASE to GURGLE?

A. So long as the neck continues to be choked with liquid, the gurgling sound continues; but as the contents of the bottle are discharged, the liquid, in flowing out, only partially fills the neck; and, while a stream passes out through the lower half of the neck, a stream of air passes in through the upper part. The flow being now continued and uninterrupted, no gurgling sound takes place.

Q. Does AIR EXIST in WATER?

$A$. Water, and most liquids exposed to the air, absorb a greater or less quantity, which is maintained in them by the pressure of the atmosphere acting on the surface.

Q. Why is BOILED WATER FLAT and INSIPID?

$A$. Because the agency of the heat expels the air which the water previously contained.

Q. Could FISHES and other MARINE ANIMALS LIVE in water DEPRIVED of AIR?

A. They could not, as they breathe the air contained in the water.

Q. Why do $A L E$, PORTER, and OIDER Froth, and CHAMPAGNE SPARKLE, when uncorked and poured into an open vessel ?

$A$. When these liquors are bottled, the air confined under the cork is condensed, and exerts upon the surface a pressure greater than that of the atmosphere. This has the effect of holding, in combination with the liquor, air or gas which, under the atmospheric pressure only, would escape. If any air or gas rise from the liquor after being bottled, it causes a still greater condensation, and an increased pressure above its surface. When the cork is drawn from a bottle containing liquor of this kind, the air fixed in the liquid, being released from the pressure of the air which was condensed under the cork, instantly makes its escape, and, rising in bubbles, produces effervescence and froth.

Q. Why do Botrles containing ALE, CIDER, PORTER, \&cc., frequently BURST ?

A. It is the nature of these liquids to produce gas or air in considerable quantities, the elastic force of which sometimes becomes greater than the cohesive strength of the particles of matter composing the bottle, which then necessarily gives way, or bursts.

Q. Why does ONE KIND of UIQUOR FROTH, and ANOTHER RIND Only SPARELE?

A. Those liquors only which are viscid, glutinous, or thick, froth, because they retain the little bubbles of air as they rise; while a thin liquor, like champagne, suffers the bubbles to escape readily. 
Q. What is the reason that CnAmpaane and other aerated liquors are so coos when first decanted?

A. Because the gas or air contained in them, in assuming its gaseous form, absorbs a large quantity of heat, and renders it latent or imperceptible.

\section{SECTION III.-THE PUMP AND BAROMETER.}

Q. Why, when we SUCK up a LIQUID with a TUBE or STRAw, does the LIQUID rise to the MOUTH?

A. One end of the tube being placed between the lips, the air is removed from the tube by the ordinary process of inhaling, when the pressure of the atmosphere compels the liquid to fill the space deserted by the air.

Q. Through how GREAT a LENGTH of TUBE could we RAISE a LIQUID by SUCTION?

A. About thirty-two feet.

Q. Why can we not raise it ABOVE THIRTY-TWO FEET ?

A. Because the atmospheric pressure will only support or balance a column of water or similar liquid of that height.

Q. How did the PHILOSOPHens of the sixteenth century ACCOUNT for the fiact that water would rise in a tube when the air was sucked out of it?

A. They declared that Nature abhorred a vacuum, and that she therefore compclled the water to fill the space deserted by the air.

Q. What REASON is said to have been given in explanation of the fact that water could not be raised by suction above thirty-two feet?

A. It was said that Nature's abhorrence of a vacuum extended only to the height of thirty-two feet, but that beyond this her disinclination to use empty space did not extend.

Fig. 45.

Q. How is the common pump CONSTRUCTED?

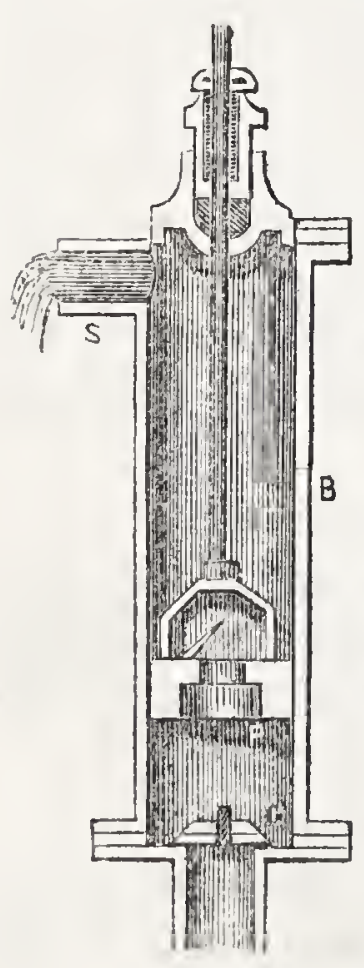

A. The common pump consists of a hollow tube, the lower part of which, descending into the water, is called the suction-pipe, and the upper part, b, fig. 45, the barrel or cylinder; of a spout, s, at the top of the cylinder; of an air-tight piston, which works up and down in the cylinder; and of two valves, both opening upwards, one of which, $g$, is placed at the top of the suction-pipe, and the other, $p$, in the piston.

Q. How does the common pump operate?

A. When the piston is raised from the bottom of the cylinder, the air above it is drawn up, leaving a vacuum below the piston; the water in the well then rushes up through the valve, g, and fills the cylinder; the piston is then forced down, shutting the valve, $g$, and causing the water to rise through the piston-valve, $p$; the piston is then raised, closing its valve, and raising the water above it, which flows out of the spout, $s$. 
Q. How High can Water be RAISEd by the COMnon SUCTION-PUMP?

$A$. As the action of this pump depends upon the pressure of the atmosphere, water cannot be raised by it from a depth of more than 34 feet below the upper valve, and in practice a much shorter limit is usually assigned.

Q. A tinman of Seville, in Spain, ignorant of the principles of science, undertook to construct a suction-pump to raise water from a well sixty feet deep; when the machine was finished, he was confounded at discovering that it had no power to raise water at all, and enraged at his disappointment, while some one was working the pump, he struck the suction-pipe with a hammer, or axe, so forcibly as to crack it, when, to his surprise and delight, the water almost immediately began to flow, and he found he had attained his purpose. How is this result to be ACCOUNTED for?

$A$. The explanation is as follows: the air pressed in through the slit, or aperture of the suction-pipe, and becoming mixed with the water in its ascent, formed a compound fluid far lighter than water alone, and therefore acted upon more readily by the atmospheric pressure; and thus produced the phenomenon described.

Q. How HIGH can WATER be raised in the suction-pump by resorting to the expedient above described?

A. About fifty-five feet, instead of thirty to thirty-four.

Q. To whom is the INVENTION of the common PUMP attributed ?

A. To Ctesibius, an Athenian engineer, who lived at Alexandria, in Egypt, about the middle of the second century before the Christian ela.

Q. When it is desired to raise water ABove thirty-four feet, as in FIRE-ENGINES, \&c., how is it accomplished?

A. By means of the forcing-pumn.

Q. In what manner is the Forcing-PUMP constructed ?

$A$. In the forcing-pump atmospheric pressure plays but a small part. There is no valve in the piston, $c$ (fig. 46), but the water raised through the suction-pipe $a$, and the valve $g$, is forced by each depression of the piston up through the pipe $e e$, which is furnished with a valve to prevent the return of the fluid.

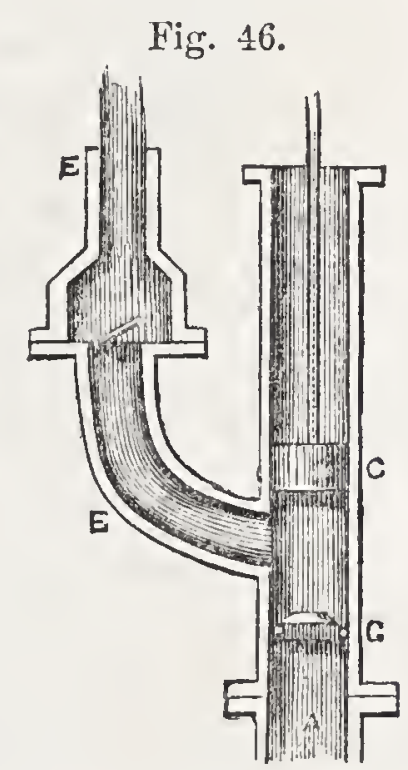

Q. Who first ascertained and demonstrated the REASON for the ASCENT of WATER in a TUBE by SUCTION, and in the COMMON PUMP?

A. Torricelli, a pupil of Galileo.

Q. How was he led to his conclusions?

$A$. He argued, that whatever be the cause which sustained a column of water in a common pump, the measure of that power must be the weight of the column of water; and consequently, if another liquid be used, heavier or lighter, bulk for bulk, than water, then the same force must sustain a lesser or greater column of such liquid. By using a much heavier liquid, the column sustained would necessarily be much shorter, and the experiment in every way more manageable. 
Torricelli verified his conclusions in the following manner:- - He selected for his experiment mercury, the heaviest known liquid. As this is $13 \frac{1}{2}$ times heavier than water,

Fig. 47. he argued, that the column of mercury which would

be sustained by a vacuum, must be $13 \frac{1}{2}$ times less
than the height of a column of water sustained in
like manner, or about 30 inches. He procured a
glass tube A B ( fig. 47) more than 30 inches in
length, open at one end A, and closed at the other
end B. Placing this tube with the open end up-
wards, he filled it with mercury, and applying his
finger to the end A, so as to prevent the escape of
the mercury, he inverted the tube, plunging the
end A into the cistern $\mathrm{C}$ D (fig. 48), containing
mercury ; the open end A being below the surface
Fof the mercury in the cistern, and no air having
been allowed to communicate with it. Upon re-
moving the finger, therefore, the mercury in the
cistern came in immediate contact with the mercury
in the tube. Immediately the mercury was ob-
served to subside from the top of the tube, and its
surface gradually to descend to the level E, about
30 inches above the mercury in the cistern. The
result was what Torricelli expected, and he soon
perceived the true cause of the phenomena. The
atmospheric pressure acting upon the surface F,
while the surface E was protected from the pressure
by the closed end B of the tube, supported the weight

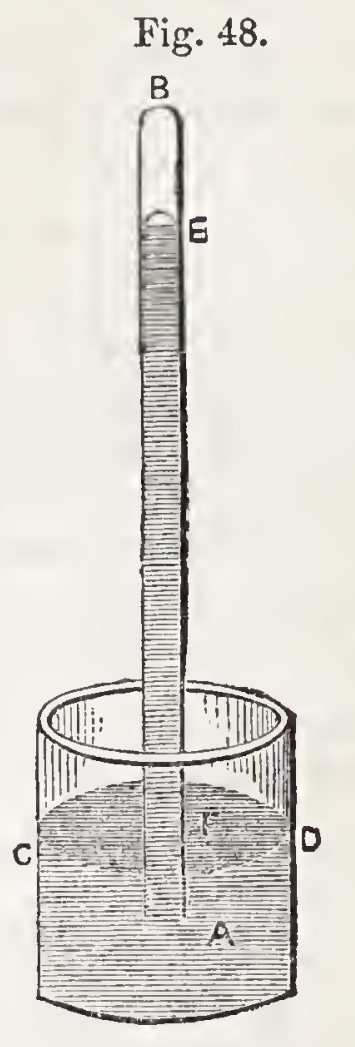

of the column E F. The pressure was transmitted by the liquid mercury in the cistern from the external surface $F$ to the base of the column contained in the tube.

Q. How was the fact that the column of mercury was sustained by the PRESSURE OF THE ATHOSPHER:, further verified?

A. By an experiment made by Pascal, in France. He argued, that if the cause which sustained the column in the tube was the weight of the atmosphere acting on the external surface of the mercury in the cistern, then, if the tube was transported to the top of a high mountain, where a less quantity of atmosphere was above it, the pressure would be less, and the length of the column less. This was tried and found to be the case.

Q. How did these experiments lead to the invention of the BARoMETER?

$A$. It was noticed that when the apparatus above described was kept in a fixed position, the height of the column fluctuated from day to day within eertain small limits. The effect was of course to be attributed to the variation in the weight of the incumbent atmosphere, arising from various meteorological causes.

This led to the use of the tube and cistern of mercury, arranged in the manner before described (figs. 47 and 48), for determining the changes in the atmosphere, and consequently the character of the weather.

Q. How cair the BARONETER be employed as a WEATHER GLASS?

$A$. By showing the variations in the air, it indicates the change in the weather: thus, when the air is light or moist, the mercury stands low ; when tre air is heavy and dry, the mercury rises in the tube.

Q. How may the Higight of MOUNTAINS be ascertained by the BaRoMETER?

A. As we ascend a high mountain the quantity of air above us becomes less and less every step we ascend, and requires less mercury 
to balance it; in consequence of which, the mercury in the tube of the barometer falls.

For general purposes, we may take this for a rule: for every one hundred feet of perpendicular height the barometer will fall one-tenth of an inch. If, therefore, the barometer has fallen one and a half inch, you know the mountain is fifteen hundred feet high.

Fig. 49.

Q. How is the common form of barometer, called the WHEEL barometer, constructed?

$A$. The barometer consists of a bent tube, filled with mercury, as represented in fig. 47 , the column being sustained by the pressure of the atmosphere upon the surface of the mercury in the shorter arm, the end of which is open. A small float of iron or glass rests upon the mercury in the shorter arm of the tube, and is suspended by a slender thread, which is passed round a wheel carrying an index. As the level of the mercury is altered, and the weight raised or lowered in the tube, the index moves; and as the divisions on the circumference of the circles within which it moves are much amplified, very slight changes are easily read off.

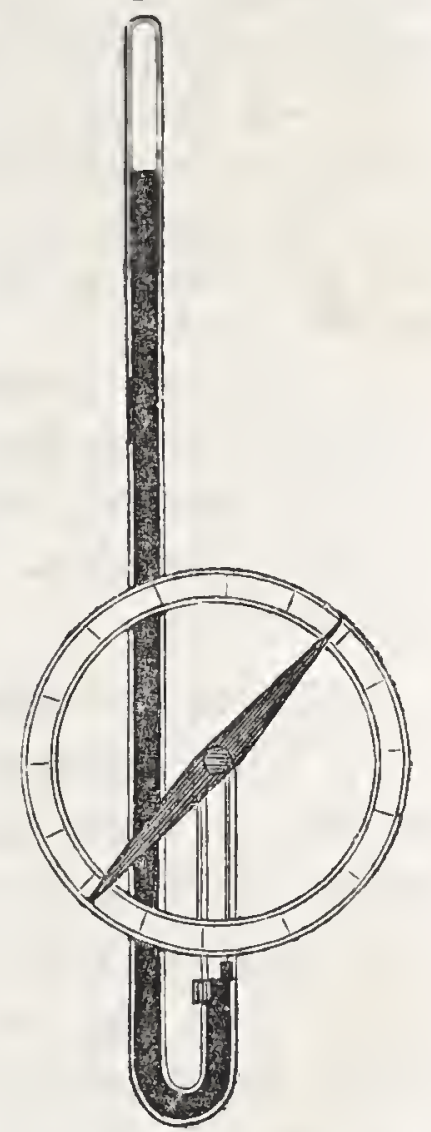

Q. Why is the ordinary use of the barometer on the LAND extremely limited and uncertain?

$A$. The height of the mercury in the tube at any time must depend partially upon the elevation of the place of observation above the level of the sea; and no correct judgment can be formed relative to the density of the atmosphere as affecting the state of the weather without reference to the situation of the instrument at the time of making the observation. Therefore, no attention ought to be paid to the words, "fair, rain, changeable," \&c., frequently engraved on the plate of a barometer, as they will be found no certain indications of the correspondence between the heights marked, and the state of the weather.

Q. What RoLEs, in observing by the barometer, may generally be relied on?

A. 1.-Generally the rising of the mercury indicates the approach of fair weather; the falling of it shows the approach of foul weather.

2.-In sultry weather the fall of the mercury indicates coming thunder. In winter the rise of the mercury indicates frost. In frost its fall indicates thaw; and its rise indicates snow.

3.-Whenever a change in the weather suddenly follows a change in the barometer, it may be expected to last but a short time. 'Thus, if fair weather immediately follows the rise of the mercury, there will 
be very little of it; and, in the same way, if foul weather immediately follows the fall of the mercury, it will last but a short time.

4.- If fair weather continues for several days, during which the mercury continually falls, a long succession of foul weather will probably ensue; and, again, if foul weather continues for several days, while the mercury continually rises, a long succession of fair weather will probably succeed.

5.-A fluctuating and unsettled state in the mercurial column indicates changeable weather.

Q. The inhabitants upon the elevated plains of South America have LARGER CHESTS than the dwellers in the plains below. What good purpose in the animal economy is apparent here?

$A$. The air upon these high plains is greatly rarefied; and a person, although expanding his chest as much as usual, really takes in at each inspiration only half as much air as he does when in a less elevated situation. This inconvenience is removed by an expansion of the chest.

Q. Explain the DIFFERENCE between the THERMOMETER and BAROMETER.

A. The mercury in a barometer is left exposed, and rises or falls according to the density of the air. In a thermometer the mercury is sealed up, and rises or falls as the air contracts or expands it.

Q. Why is the TUBE of a BAROMAETER left open?

$A$. That the air may press upon it freely; and, as this pressure varies, the mercury rises or falls in the tube.

Q. Why does the mercury in the barometer rise at the approach of FAIR WEATHER?

$A$. Because the air is becoming more dry, and the drier the air, and the more free it is from vapour, the greater the pressure.

Q. Why does the mercury sIN at the approach of Foul weather?

$A$. Because the air is laden with vapour or disturbed by wind.

Q. Why does VAPOUR in the air cause the mercury to sink?

$A$. Because air containing vapour is lighter than dry air; and its pressure on the mercury is therefore less.

Q. Why will there be so RAIN if the AIR be very DRY ? rain.

$A$. Because dry air will absorb moisture, and not part with it in

Q. Why will there be No RATN if the AIR be very COLD?

$A$. Because it is so mich condensed, that it has already parted with as much moisture as it can spare.

Q. When does the barometer VARY MOST ?

$A$. In winter-time.

Q. Why is there a greater VARIATION in the barometer in WINTER than in SUMMER ?

$A$. Because a greater disturbance is produced in the air, the difference of temperature in the temperate and torrid zones being greater in winter than in summer. 
Q. Why does the barometer VARX LESS in summer than in winter?

A. Because the temperature is more equal, being less disturbed by currents in the torrid and temperate zones.

Q. What effect have HEAT and COLD on the barometer?

A. Of themselves they have no effect: but because warm weather is often moist, the mercury sinks; because cold weather is generally dry, the mercury rises. The moisture in the atmosphere is the occasion of the changes indicated.

\section{A C O U S T I C S.}

\section{CHAP'TER XIII. \\ ORIGIN AND TRANSMISSION OF SOUNDS.}

Q. Of what PHenomera does the SCIENCE of Acoustics treat?

A. Of the causes and effects of sound, and of the manner in which it is perceived by the organs of hearing.

Q. How is sound produced?

$A$. Sound is heard where any sudden shock or impulse, causing vibrations, is given to the air, or any other body, which is in contact, directly or indirectly, with the drum of the ear.

Q. What is the DRUM Or TYMPANUM of the EAR?

A. A thin membrane which closes the aperture of the ear.

Q. How do the vIBRATIONS of the AIR, striking upon the DRUM of the EAR, give us the sensation of sound?

A. Behind the drum of the ear are various cavities and tubes in the bone which form the side of the head, in which the minute fibres of the auditory nerie are distributed. When the drum of the ear is made to vibrate freely by the action of the sonorous undulations of the external air, the vibrations are communicated by the action of minute bones, muscles, and fluids, contained in the cavities of the ear to the nerve, and from thence the impressions are conveyed to the brain.

Fig. 50

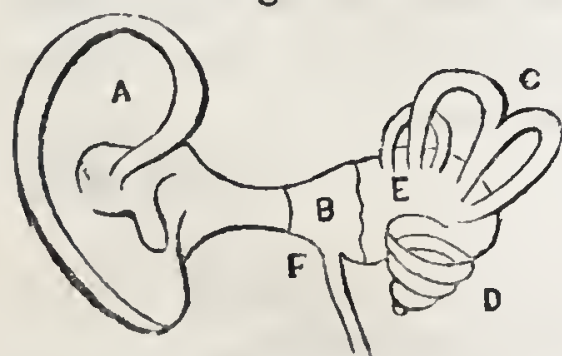

The Animal Ear (See fig. 50). - The parts of the ear and the progress of sound to the sentient nerve may be simply described as follows:-1. There is external to the head a wide-mouthed tube, or ear-trumpet, $a$, for catching and concentrating the waves of sound. It is movable in many animals, so that they can direct it to the place from which the sound ccmes.

2. The sound concentrated at the bottom of the ear-tube falls upon a membrane stretched across the channel, like the parchment of an ordinary drum, over the space called the tympanum, or dinu of the ear, $b$, and causes the nembrane to vibrate. That its motion may be free, the air con-

$3 *$ 
tained within the drum has free communication with the external air by the open passage, $f$, called the eustachian tube, leading to the back of the mouth. A degree of deafness ensues when this tube is obstructed, as in a cold; and a crack, or sudden noise, witl immediate return of natural hearing, is generally experienced when, in the effort of sneezing or otherwise, the obstruction is removed.

3. The vibrations of the membrane of the drum are conveyed farther inwards, through the cavity of the drum, by a chain of four bones (not here represented on account of their nimuteness), reaching from the centre of the membrane to the oval door or window leading into the labyrinth $e$.

4. The labyrinth, or complex inner compartment of the ear, over which the nerve of hearing is spread as a lining, is full of watery fluid; and, therefore, by the law of fluid pressure, when the force of the moving membrane of the drum, acting through the chain of bones, is made to compress the water, the pressure is felt instantly over the whole cavity. The labyrinth consists of the vestibule, e, the three semicircular canals, $c$, imbedded in the hard bone, and a winding cavity, called the cochlea, $d$, like that of a snailshell, in which fibres, stretched across like harp-strings, constitute the lyra. The separate uses of these various parts is not yet fully known. The membrane of the tympanum may be pierced, and the chain of bones may be broken, without entire loss of hearing. - AnNotr.

Q. Is AIr necessary to the production of sound?

$A$. No ; but most sounds owe their origin to the vibrations of the air. Sound can be produced under water, and all bodies are, in fact, more or less fitted to produce the sound vibrations; in many cases air is neither the quickest nor the best conductor of sound.

Q. Upon what does the LOUDNESS of sound conveyed by air depend?

A. Upon tho density of the air.

Q. Why does a bell rung in a receiver exhausted of air FAIL to produce sound ? tions.

A. Because no air is present to receive and transmit the vibra-

Q. Why is a whisper heard with greater distinctness in a DIVING-BELL?

$A$. Because the air in the diving-bell is greatly condensed by the pressure of the water, and receives and transmits the vibrations with great readiness.

Q. Why are Mountains NoIseless and quiet?

$A$. Because the air of mountains is very rarefied; and, as the air becomes rarefied, sound becomes less intense.

Q. How can we prove that liquids conduct sound?

A. A person diving under water would plainly hear the sound of a bell struck in the air at a moderate distance above. If both the hearer and the sounding body be immersed in the same mass of water, the sound will appear much louder than when passing through an equal extent of air.

Q. How is the propagation of sonorous vibrations in LIQ

$A$. By rubbing gently with a wet finger the edge of a drinkingglass half filled with water, sound will be produced, and the surface of the water will be covered with minute undulations.

Q. According to what circumstances does the Pow depend?

$A$. The intensity or loudness of sound in fluids appears to be augmented in proportion to their increase in specific gravity.

Thus, water being a fluid so much denser than air, sonnds procince a stronger effect in 
the former medium than in the latter. It is therefore a wise provision of the Greator, that the organs of hearing in fishes are much less perfectly developed, and consequently less sensible to the impressions of sound, than those of terrestrial animals.

Q. What are sovorous bodies?

A. Bodies which produce sound are called sonorous bodies.

Q. Why are some things sonorous and others кот ?

$A$. The sonorous quality of any substance depends upon its hardness and elasticity.

Q. Why are IRoN and COPPER sonorous?

$A$. Because these metals are hard and elastic; lead being neither elastic nor hard, is not sonorous.

Q. Of what is BELL-METAL made?

$A$. Of copper and tin in the following proportions:-In every five pounds of bell-metal there should be one pound of tin and four pounds of copper.

Q. Why is BELL-MrTAL composed of tin and copper?

$A$. Because the union of these metals produces a combination much harder and more elastic than could be obtained by simply using one metal alone.

Q. How does the conducting power for sound possessed by ELASTIC SoLIDS compare with that of other CLASSES of BODIES?

A. Solids, when they possess elasticity, convey sounds to the ear more readily and effectively than gases or liquids.

Q. In what manner may a person PARTIALLY DEAF be enabled to carry on a conversation readily?

$A$. If he places one end of an iron rod between his teeth while the other end rests on the edge of an open kettle, he will understand what is said by a person directing his voice into the kettle more distinctly than if the voice of the speaker passed through the air; so that he might converse with any one in this manner at a distance at which he would not hear under ordinary circumstances.

Q. What easy EXPERIMENT illustrates the transmission of sounds by solids?

$A$. When a stick is held between the teeth at one extremity, and the other is placed in contact with a table, the scratch of a pin on the table may be heard with great distinctness, though both ears be stopped.

Q. How does the EAR TAKE cognisance of sounds propagated in THIS MANNER?

$A$. There is a passage called the eustachian tube, which extends from the back part of the mouth to the cavity immediately behind the drum of the ear, through which the air passes. The sound vibrations are transmitted along this tube to the interior part of the organs of hearing.

Q. What plan do SAVAGES adopt to hear the APPROACH of an ENEMY Or BEAST OF PRET?

$A$. They place their ears to the ground, and by this means can distinguish clearly the approach of an enemy.

Q. What kind of SURFACES are best adapted for the TRANSMISSION of SOUND ?

A. Smooth surfaces, such as ice, water, or hard ground. 
Q. To what useful contrivance has the PRINCIPLE that solids convey sounds more perfectly than air been applied?

A. To the construction of the stethoscope, or chest inspoctor.

Q. What is a STETHoscope?

A. It is a wooden instrument which resembles a small trumpet. The wide mouth is applied to the body, and the other is held to the ear of the physician, who can hear distinctly the action of the lungs, and judge whether they be healthy or the reverse.

Q. Why has the peculiar kind of motion in budies which gives rise to the sensation of sound, been termed viBration?

$A$. Because a striking analogy may be traced between the tremulous agitation which takes place among the particles of a sounding body and the oscillations of a pendulum.

Q. How may the Nature of sonorous vibrations be illustrated?

$A$. By noticing the visible motions which occur on striking or twitching a tightty extended cord or wire. Suppose such a cord, represented by the central line in fig. 51 , to be forcibly drawn out to $\mathrm{A}$, and let go; it

Fig. 51.

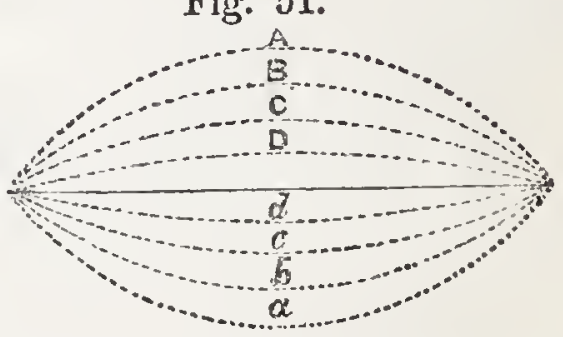
would immediately recover its original position by virtue of its elasticity; but when it reached the central point, it would have acquired so much momentum as would cause it to pass onward to $a$; thence it would vibrate back in the same manner to $B$, and back again to $b$, the extent of its vibration being gradually diminished by the resistance of the air, so that it would at length return to a state of rest.

Q. What purpose is subserved by the BODY of a STRINGED INSTRUMENT?

$A$. The string of an instrument when caused to vibrate, communicates the vibrations to the matter composing the body of the instrument and the surrounding air, and thus a tone or musical note is produced and rendered audible to the ear.

Q. How are AERIAL vibrations or pULSES communicated?

$A$. The air, encompassing sounding bodies on every side, conveys the sensation of sound in all directions; therefore the aerial vibrations, or, as they have been termed, "pulses," must be communicated successively and generally throughout the whole space within the limits of which they are capable of affecting the ear.

Q. To what have the SOUND, viBRations, or PULSATIons been compared?

$A$. To the waves spreading in concentric circles over the smooth surface of water.

When a stone is thrown into water, the liquid waves are propagated not only directly forward from the centre, but if they encounter any obstruction, as from a floating body, they will bend their course round the sides of the obstacle, and spread out obliquely beyond it. So the undulations of air, if interrupted in their progress by a high wall or other similar impediment, will be continued over its summit and propagated on the opposite sido of it. 
Q. Of what real naturc are the movements of the waves of air?

$A$. When the particles of air vibrate, the motion thus originated produces no permanent change of place among the particles of the sonorous mass, but simply an agitation or tremor, so that each particle, like a pendulum which has been made to oscillate, recovers at length its original position.

This motion may be best illustrated by comparing it to the motion produced by the wind in a field of grain. The grassy waves travel visibly over the field in the direction in which the wind blows; but this appearance of an object moving is only delusive. The only real motion is that of the heads of the grain, each of which goes and returns as the stalk stoops or recovers itself. This motion affects successively a line of ears in the direction of the wind, and affects simultaneously all the ears of which the elevation or depression forms one visible wave. The elevations and depressions are propagated in a constant direction, while the parts with which the space is filled only vibrate to and fro. Of exactly such a nature is the propagation of sound through air.

Q. What curious wave PHenomena may be noticed when a steamboat is passing very near the shore?

$A$. If a point is observed in advance of the boat, as a stone on the beach, or a plant on the bank, it will be seen, long before the boat arrives opposite that point, that the water recedes considerably, and that this recession increases as the boat passes.

Q. How is this EFFECT produced?

$A$. The paddle-wheels throw back the water, and form a trongh or hollow, into which the water in advance of the boat rushes; and this increases with the speed at which the paddle-wheels are driven.

Q. Why do LARGE WAYES always follow in the TRACK of an advancing STEAMrBOAT?

$A$. Because the levels of the water before and behind the boat are altered, and the effort to restore horizontality produces a series of large waves. These waves spreading, break at length against the bank, and submerge the point which had previously been dry. Where rushes grow in the water, these wave motions are rendered very striking by the movement of the plants.

Q. At what Rate does SOUND TRAVEL through the air?

$A$. At the rate of 1,142 feet in a second, or 13 miles in a minute. Light has a much greater velocity than sound, and therefore we see the flash of a gun before we hear the report.

Q. How is a KNOWLEDGE of the VELOCITY of SOUND made applicable to the MEASUREMENT of DistaAces?

A. Suppose a flash of lightning to be perceived, and on counting the seconds that elapse before the thunder is heard, we find them to amount to $3 \frac{1}{2}$ : then, as sound moves 1,142 feet in a second, it will follow that the thunder-cloud must be distant $1,142 \times 3 \frac{1}{2}=3,997$ feet.

Q. If from an eminence you look down upon a long coLUMN of soldiers mal'ching to a band of music in front, those in the rear will step a little IATER than those some distance befure them : can you explain the reason of this?

$A$. Each rank steps, not when the sound is made, but when in its progress down the column at the rate of 1,142 feet in a second of time, it reaches their ears. Those who are near the music hear it 
first, while those at the end of the column must wait until it has travelled to their ears at the above rate.

Q. Why do we use SPEAEING-TUBES for transmitting sodND?

$A$. Sound decreases in intensity from the centre where it originates, according to the same law as gravitation; that is to say, at double the distance it is only one-fourth part as strong; at three times the distance, a ninth; and so on. By confining it in tubes, which prevents its spreading, its force diminishes much less rapidly, and it will therefore extend to much greater distances.

Q. Why will a person at the extremity of a very long IRON PIPE or WIRE hear the same sound, made at the other extremity of the pipe or wire, twice?

$A$. Because the air is not so good a conductor of sound as the iron; therefore it passes along the metal almost instantaneously, but requires more time to travel the same distance through the air.

Q. What is the cause of WINDows Ratruing when carriages or carts are passing by ?

$A$. The air communicates a vibration to the glass, and the windowframe being shaken adds to the noise.

Q. Why do we HeAr a BelL if it be struck ?

$A$. The bell vibrates, and in its agitation compresses the air to a certain distance around it at each vibration. The compressed air instantly expands, and in doing so repeats the pressure on the air next in contact with it, and so on; as a pebble thrown into still water makes waves all around it, diminishing in force the more distant they are from the original stroke. The air thus agitated reaches the ear, where a similar impulse is given to the membrane, and the mind then receives the impression of sound.

Q. How can a bell which is solid be said to vibrate?

$A$. Although the metal of which the bell is composed is solid, yet it actually changes its form every time it is struck, and its particles are thrown into motion.

Q. Is the SOUND of a bell STOPPED by TOUCHING it?

$A$. The sound is stopped by the bell ceasing to vibrate; any weight stopping the vibrations of the bell.

Q. After striking a finger-glass, why is the SOUND SILENOED upon touching the glass with the finger?

A. Because the pressure of the finger stops the vibrations of the glass; and, so soon as the finger-glass ceases to vibrate, it ceases to make sound-waves in the air.

Q. Why is a hoarse sound produced by a SPLIT BELL ?

$A$. It is occasioned by a double vibration, which makes the sound-waves jar, and produces a discordant noise.

Q. Why are the BELLS of distant churches SOMETIMEs heard better than at отHERS?

A. It is owing to the state of the atmosphere; sound travelling much more rapidly when the air is cold and clear.

Q. Why can we hear distant cLocKS best in COLD WEATHER?

$A$. Because sound travels more rapidly under such circumstances: 
on the same account, voices of men can be heard for more than a mile in the cold air of the Arctic regions.

Q. Why are not SOUNDS heard more distinctly in FROSTY than in WARM WEATHER ?

$A$. 'Ihe air is more uniform, less rarefied, consequently a better conductor, and less liable to accidental currents.

Q. Why does a THIOK MIST or HAZE prevent the distinct transmission of distant souNDS ?

$A$. The air being laden with mist is of less uniform density, thus obstructing the sound-waves in their course.

Q. Why are SOUNDS HEARD better by NIGHT than DAY?

A. Night is more still, owing to the suspension of business, and the air is of more uniform density.

Q. Why is the air of more UNIFORM DFNSITY by NIGHT than it is by DAY?

$A$. Because it is less liable to accidental currents; inasmuch as the breezes (created by the action of the sun's rays) generally cease during the night.

Q. Why does the sea HEAve and STGH just Prevrous to a STORM?

$A$. Because the density of the air is very suddenly diminished, and (as the density of the air is diminished) its power to traismit sound is diminished also; in consequence of which the roar of the sea is less audible, and seems like heavy sighs.

Q. Why is the AIR so universally QUIET just PREVIous to a TEMPEST ?

$A$. Because the air is suddenly and very greatly rarefied; and (as the density of the air is diminished) its power to transmit sound is diminished also.

Q. Why does a railway train make more noise when it passes over a bridge, than when it runs over SOLID GROUND?

$A$. Because the bridge is elastic, and vibrates much more from the weight of the train than the solid earth; in consequence of which it produces more definite sound-waves.

The bridge acts as a sounding-board, and the water or earth below the bridge repeats the sound.

Q. Why can sOUNDS be heard (in a calm day) at a GREATER DISTANCE on the sea than on the land?

A. The plane surface of water, as a smooth wall, prevents the lateral spreading and dispersion of the sound-waves, although on only one side. The air over water, owing to the presence of moisture, is also generally more dense, and the density more uniform than over the land. Water, in addition, is a better conductor of sound than the earth.

Q. How should PARTITION WALLS be cunstructed so as to PREVENT the transmission of sound?

$A$. Shavings or sawdust should be put between the laths, so as to form several different media for the sound to pass through, and thus occasion as much obstruction as possible, and a consequent diminution of the strength of the sound-waves. 
Q. Why does sound seem LOUDER in a CAVE than on a plain?

$A$. Because the sides of the cave confine the sound-waves, and prevent their spreading; in consequence of which their strength is greatly increased.

Q. What soLIDS are among the BEST CONDUCTORS of SOUND?

A. Iron and glass; sound is transmitted by them at the rate of 17,500 feet, or more than three miles in a second; after these rank copper, several different linds of wood, silver, tin, \&c.

SECTION I.-VOCAL AND MUSICAL SOUNDS.

Q. What are MOSICAL SOUNDS?

A. Regular and uniform successions of vibrations.

Q. What is the difference between a MUSICAL sound and a mere noise ?

A. All mere noises are occasioned by irregular impulses communicated to the ear; but, in order to produce a musical sound, the impulses, and consequently the undulations of the air, must be all exactly similar in duration and intensity, and must recur after exactly equal intervals of time.

Q. What do we mean by toNe or PIтcH in sound?

$A$. If the sound impulses be repeated at very short intervals, the ear is unable to attend to them individually, but hears them as a continued sound, which is uniform, or has what is called a tone or pitch, if the impulses be similar and at equal intervals.

Q. Explain the production of Tose more fully.

$A$. If a wheel with teeth be made to turn and strike any elastic plate, as a piece of quill, with every tooth, it will, when moved slowly, allow every tooth to be seen, and every blow to be heard, separately; but, with increasing velocity, the eye will lose sight of the individual teeth, and the ear, ceasing to perceive the separate blows, will at last hear only a smooth, continued sound, the tone, the character, or pitch of which will change with the velocity of the wheel.

Q. When is a TONE said to be GRAVE or SHARP?

$A$. When the impulses are few in a given time, the tone is grave; when they are many, the tone is sharp.

Q. Why are SHARP sounds generally LOUD sounds?

4. Because to produce a sharp sound there must be quick vibrations, which are produced by forcible impulses; hence the sound is generally loud.

Q. Do ALL persons hear sounds aLike?

$A$. No; that faculty seems to depend upon the sensibitity of the auditory nerves.

"A Auditory"-having the power of hearing. 
Q. What are the BOUNDARIES Of HOMAN HEARING?

$A$. The whole range of human hearing, from the lowest note of the organ to the highest known cry of insects, as of the cricket, includes about nine octaves.

All ears, however, are by no means gifted with so great a range of hearing; many persons, though not at all deaf, are quite insensible to the highest notes of some insects.

Q. How many vibrations of a musical chord are necessary to produce a Definite SOUND?

$A$. It is generally believed that a vibrating chord will not produce a sound distinctly appreciable by the most delicate ear when it makes less than thirty-two vibrations in a second. It has, however, been asserted that a perceptible sound can be produced by a chord giving eight single vibrations in a second.

Q. How MaNy vibrations is the human ear able to appreciate?

A. The human ear is capable of appreciating as man y as twentyfour thousand vibrations in a second of time; and is, consequently, able to hear a sound which only lasts the twenty-four thousandth part of a second!

Q. Are vibrations more frequent than TWENTY-Four THOUSAND in a second of time produced in nature? exist:

A. Ceriainly; vibrations incomparably more rapid undoubtedly

Q. Why do we not hear sounds INDEFINITELY above this limit?

A. On accourt of the peculiar constitution of the human ear and nervous system.

While we have no proof that other animals are capable of perceiving sounds of which the human ear can take no cognisance, there are some reasons for supposing that animals, like the crickets or grasshoppers, whose powers appear to commence where ours terminate, may hear sharper sounds which we do not know to exist; and there may be insects hearing nothing in common with us, but endued with a power of exciting, and a sense that perceives, the same vibrations which constitute our ordinary sounds, but so different that the animal who perceives them may be said to possess another sense.

Q. How is it that some notes are TREBLE and others BASE?

$A$. The difference is attributable to the slow or quick vibration of the sound-wave; treble notes are produced by quick vibrations, base notes by slow vibrations.

Q. What occurs when of several sonorous bodies, near each other, and tuned to one accord, ONE is thrown into a state of vibration?

$A$. All others near it, capable of producing the same tone, will vibrate also.

Q. How are WEAK sounds affected by the occurrence of more powerful ones in their vicinity?

$A$. When one body is made to produce a certain note, its soniferous vibrations will be checked or interrupted by the emission of a more powerful or discordant sound near it. Hence weak sounds are generally drowned by loud ones.

Q. What is meant by "A MUSTCAL EAR?"

A. The faculty of distinguishing certain relations between sounds 
differing in tone-that is, sounds which are more or less grave or acute than others.

Q. What is meant by the terms CONCORD and DISCORD?

$A$. When two tones or notes sounded together produce an agreeable effect on the ear, their combination is called a musical concord; when the effect is disagreeable, it is called a discord.

Q. What is the GAMrUT Or DIATONIO SCALE of music?

$A$. It consists of seven notes, which are distinguished by the seven first letters of the alphabet, or by the seven syllables do, re, mi, fa, sol, la, si.

Q. Two waves of water may undulate in such a manner as To DESTROF EACH OTHER; can two sonorous waves be made to move in such a manner as to PRODUCE SILENOE ?

A. They can; if two volumes of sound are produced at the same time, they give rise to a sound double the strength of either separately; and, as in the case of water-waves, the undulations coincide, and meet at the point of greatest elevation, to swell into one note. Now, if we so regulate the vibrations of one sound that it is not in unison with another (the difference, however, between them being very slight), the quicker vibrations of the one will overtake the slower vibrations of the other; and, at a certain vibration, the depression of one wave will be filled with the elevation of the other, and no sound is heard; absolute silence results, and at intervals in the vibrations the absence of sound recurs.

Q. Into what Four CLASSES are MUSTCAL INSTRUMERTS divided?

A. Into stringed instruments, as the violin, harp, \&c.; pulsatory instruments, as bells, gongs, drums, \&c.; instruments in which the sound depends on the vibrations of elastic rods or plates, as the harmonicon, musical glasses, \&c. ; and wind instruments, as the trumpet, bugle, \&c.

Q. How are musical sounds produced by a PIANO-FORTE?

$A$. The keys of the instrument lift small hammers on being struck with the fingers, which hammers strike the strings of the piano, and by vibration set in motion the sound-waves.

Q. How is it that a DRUM is made to sound?

$A$. The blows of the drum-sticks on the parchment cause the air to vibrate, and consequently produce sound.

Q. How do Musical glasses, violins, and Flutes occasion sound?

A. On the same principle of vibration. In the flute the air is caused to vibrate by the breath of the performer, in the violin the bow being drawn across the strings, produces vibration. Musical glasses vibrate as soon as struck, and so agitate the sound-waves of the air.

Q. Why is an instrument FLAT when the STRINGS are UNSTRUNG ?

$A$. Because the vibrations are too slow; in consequence of which the sounds produced are not shrill or sharp enough. 
Q. Why do BIRDS alone, of animals, produce MUSICAL NoTES ?

$A$. Because they alone are gifted with a vocal organisation, which enables them to produce musical notes. In other animals, the larynx is placed wholly at the upper end of the windpipe; but in birds it is separated, as it were, into two parts, one placed at each extremity.

Q. Why cannot birds be so correctly said to SING as to WHISTLE?

$A$. Because nattiral singing is an exclusive privilege of man.

Q. Why do the notes of different species of birds vary?

$A$. Because, probably, of the structure of the organs of each species enabling them more easily to produce the notes of their own species than those of any other, and from the notes of their own species being more agreeable to their ears. These conditions, joined to the facility of hearing the song of their own species, in consequence of frequenting the same places, determine the character of the acquired language of the feathered tribes.

Q. Why does one sex only of birds sing?

$A$. Because, as Buffon supposed, of cheering his mate during the period of incubation; but this idea, gallant as it is, has but slight foundation in probability. The singing of most birds seems entirely a spontaneous effusion, produced by no exertion, or occasioning no lassitude of muscle, or relaxation of the parts of action. In certain seasons and weather, the nightingale sings all day and most part of the night; and we never observe that the powers of song are weaker, or that the notes become harsh and untunable after these hours of practice. The cuckoo is probably the only bird that seems to suffer from the use of the organs of voice.

Q. In the human system, what are the PARTS concerned in the production of SPEECH and Mosic?

A. They are the windpipe, the larynx, and the glottis.

Q. What is the WINDPIPE?

$A$. The windpipe is merely a cartilaginous canal through which the air issues from the lungs.

Q. What is the LARYNX?

$A$. The larynx is an enlarged continuation of the windpipe, formed, like it, of cartilage, or gristle, membrane, and muscle; it is, however, more complicated, terminating above in two lateral membranes which approach near together, leaving an oblong, narrow opening, called the glottis.

Q. How is SOUND produced by the ORGANS of VOICE?

$A$. The air expired from the lungs passes through the windpipe and out at the larynx, through the opening of the membrane called the glottis. The vibration of their membranes, caused by the passage of air, causes sound.

Q. How can the TONES of the VOIOE be made GRAVE or ACUTE?

$A$. By varying the tension of these membranes and the size of the opening. 
Q. What is the FORCE exerted by the HEALTHY CHEST in BLOWING?

A. To about one pound on the inch of its surface; that is to say, the chest can condense its contained air with that force, and can therefore blow through a tube the mouth of which is ten feet under the surface of water.

Q. What is the vocal ACTION of Cortaning ?

$A$. In coughing, the top of the windpipe or the glottis is closed for an instant, during which the chest is compressing and condensing its contained air; and on the glottis being opened, a slight explosion, as it were, of the compressed air takes place, and blows out any irritating matter that may be in the air-passages.

Q. Why does a POP-GUN make a loud report when the paper bullet is discharged from it?

A. Because the air confined between the paper bullet and the discharging rod is suddenly liberated, and strikes against the surrounding air; this makes a report in the same way as when any two solids (such as your hand and the table) come into collision.

Q. What is SNEEZING?

A. Sneezing is a phenomenon resembling cough; only the chest empties itself at one effort, and chiefly through the nose, instead of through the mouth, as in coughing.

Q. What is LAUGHING?

A. Laughing consists of quiclily repeated expulsions of air from the chest, the glottis being at the time in a condition to produce voice ; but there is not between the expirations, as in coughing, a complete closure of the glottis.

Q. What is HICCOUGH ?

A. Hiccough is the stopping of the commencement of a strong inspiration, by a sudden closing of the glottis.

Q. What is ORYING?

$A$. Crying differs from laughing almost solely in the circumstance of the intervals between the gusts or expirations of air from the lungs being longer. Shildren laugh and cry in the same breath.

Q. Why, in straining to LIFT weigirs, or to make any powerful bodily effort, do we COMPRESS OUT BREATH?

A. We shut up the air in the lungs in order to give increased steadiness and firmmess to the body.

Q. When is a PEnson suFfocated ?

$A$. When the windpipe becomes choled, or the supply of air to the lungs is in any way cut off.

Q. Why do birds sing comparatively LOUDER than man?

$A$. Because the strength of the larynx, and of the muscles of the throat, in birds, is infinitely greater than in the human race. The loudest shout of man is but a feeble cry compared with that of the golden-eyed duck, the wild-goose, or even the woodlark. 
Q. How are WINGED INSEOTS generally found to produce SOUND?

$A$. Generally, they excite sonorous vibrations by the fluttering of their wings, or other membranous parts of their structure.

Q. How does the Locust produce Sound?

$A$. They are furnished with an air-bladder, or species of bagpipe, placed under and rather behind their wings.

\section{SECTION II.-REFLECTION OF SOUND.}

Q. What is ECHO?

$A$. Echo is reflected sound.

Q. What is the CAUSE of ECHO?

$A$. When a wave of water strikes against a smooth wall or obstacle, it turns back; so that, at any distance after the reflection, it appears as it would have been at the same distance beyond the wall, only moving in an opposite direction; so the pulses or waves of sound are regularly reflected from flat surfaces, and produce what is called an echo.

Q. Why are caverns, grottoes, and ruins FAMOUS for ECHOES?

$A$. Because the sound-waves cannot flow fieely forward, but perpetually strike against the winding walls, and are beaten back.

Q. Why are BroUNTAINS and icebergs FAMOUS for ECHOES?

A. Because they present a barrier to the sound-waves, which they cannot pass, and are sufficiently elastic to throw them back.

Q. What BEAUTIFUL FICTION existed among the ancients relative to the production of ECHO?

$A$. They supposed that Echo was a nymph who dwelt concealed among the rocks, and who repeated the sounds she heard.

Q. At, what DISTANCE must the BoDY reflecting the sounds be situated in order to produce in EcHo?

A. It is requisite that the reflecting body should be situated at such a distance from the source of sound, that the interval between the perception of the original and reflected sounds may be sufficient to prevent them from being blended together.

Q. When the sounds become thus BLENDED together, what is the effect called?

1. A resonance, or resounding, and not an echo.

2. What is the SHORTEST INTERVAL of SPAOE and TIME requisite to produce an echo?

$A$. The shortest interval sufficient to render sounds distinctly appreciable by the ear, is about one-tenth of a second; therefore when sounds follow at shorter intervals, they will form a resonance instead of an echo; so that no reflecting surface will produce a distinct echo, unless its distance from the spot where the sound proceeds is at least $56 \frac{1}{2}$ feet; as the sound will in its progress forward and return through double that distance-113 feet-take up one-tentl of a second.

Q. Why do not the waLLS of a Room of ordinary size produce an echo?

$A$. Becanse the reflecting surface is so near the source of sound 
that the echo is blended with the original sound; and the two produce but one impression on the ear.

Q. Why do LARGE buildings sometimes REVERBERATE to the sound of the human voice?

$A$. The walls being too far off from the speaker for the echo to combine with the original sound, each is separately heard.

Q. Why do sons echoes repeat one syllable only and others two ?

A. The difference is caused by the distance of the echoing body. The farther it is removed the more sound it reflects. If near, it repeats but one syllable; if further removed, two syllables are heard.

Q. Are not TWO ECHOES sometimes heard?

$A$. Two or more echoes are heard in some places, because separate reverberating surfaces receive the sound, and in succession reflect it. The Killarney echo plays a second to any simple tune, if distinctly played on a bugle.

Q. What must be the CONDITIONS of the REFLECTING SURFACE in order to produce a PERFECT ECHO?

$A$. The surface must be smooth and of some regular form; for the wave of sound rebounds, according to the same law as a wave of water or an elastic ball, perpendicularly to the surface if it fall perpendicularly, and if it fall obliquely on one side, it departs with an equal degree of obliquity on the other side.

Q. What must be the EFFECT of an IRREGULAR SURFACE?

$A$. An irregular surface must break the echo; and if the irregularity be very considerable, there can be no distinct or audible reflection at all. For this reason an echo is much less perfect from the front of a house which has windows and doors, than from the plane end, or any plane wall of the same magnitude.

Q. Why have Halls for music PLANe BaSe WaLls?

$A$. Because the hard plane walls reflect the sound regularly, and increase the effect of the music.

Q. Why are Halls for SPEAKING, THEATRES, GHURCHes, \&c., generally ornamented on the wall, and furnished with pillars, curtains, \&c.

A. Because the ornaments, pillars, curtains, \&c., form irregular surfaces, which breat up and destroy the echoes and resonances.

Q. Why is a THIOK OURTAIN often placed behind a PULPIT or SPEAKING-DESE?

A. Because the material absorbs the sound, and by not reflecting it avoids the production of echoes and resonances.

If the room is not very large, a curtain behind the speaker impedes rather than assists his voice.

Q. In constructing a room for PUBLIO SPEARING, what ought to be the LIMIT of HEIGHT of the CEILING?

A. Not above 30 or 35 feet.

Q. Why not ABove this Limit?

$A$. If we advance towards a wall on a calm day, producing at each step some sound, we will find a point at which the echo ceases to be distinguishable from the original sound. The distance from the wall, or the corresponding interval of time, has been called the limit of 
perceplibitity. This limit is about 30 to 35 feet; and if the ceiling of a building for speaking be arranged at this limit, the sound of the voice and the echo will blend together, and thus strengthen the voice of the speaker.

Q. What will be the effect if the cEILINa be constructed HIGHER than the LIMIT of PERCEPTIBILITY ?

A. The direct sound and the echo will both be heard separately, and produce indistinctness.

Q. Why is it that we are enabled to hear for a great distance sound communicated through a tube?

$A$. Because the sides of the tube confine, or repress by a continued reflection, the advancing sound, which in the open air would quickly spread laterally and be dissipated.

Q. What is the construction and use of an EAR-TRUMPET ?

A. It is a tube wide at one end where the sound enters, and narrow at the other where the ear is applied. Its sides are so curved that all the sound that enters is reflected and brought to a focus at the narrow end. It thus increases many fold the intensity of the sound which reaches the ear through it, and enables a person who has become deaf to common conversation to hear tolerably well.

Q. Why do persons hold the HAND CONCAVE behind the EAR in order to hear more distinctly?

$A$. Because the concave hand acts in some respects as an eartrumpet, and reflects the sound into the ear.

Q. Why does an INFLATED BLADDER, when BURST OPEN WITH FORCE, produce a SOUND like the report of a pistol ?

$A$. Because sound, to some extent, always accompanies the liberation of compressed air.

Q. Why, when the sarore is projected from the lips of a rовACCO-SMOKER, does a LITTLE EXPLOSION usually accompany the PUFF?

A. This sound appears to be chiefly due to the sudden bursting of the film which connects both lips; and the nature of the sound is, in a great measure, dependent upon the state of the lips at the time, whether they be moist or dry.

Q. Why does a strman of Water, falling from one vessel into another, Fiow comparatively SILENTLY for a SHORT DISTANCE, and NOISILY if the distance BE INCREASED beyond a certain limit?

$A$. When the distance which the water falls is so limited that the end of the stream does not become broken into bubles or drops, neither air-bubbles nor sounds will be produced; but as soon as the distance becomes increased to an extent sufficient to break the end of the column into drops, both air-bubbles and sounds will be produced. The sound of falling water appears, therefore, to be in a great measure owing to the formation and bursting of the bubbles. 


\section{H E A T.}

\section{$-4$ \\ CHAPTER XIV. \\ NATURE AND SOURCES OF HEAT.}

Q. What is InAAT?

A. The sensation of warmth.

Q. To what CAUSE have different philosophers attributed the PHENoMENON of HEAT?

$A$. Some have ascribed the phenomenon of heat to be merely a species of motion among the minute particles of bodies generally, as sound is motion of another kind among the same particles; others have supposed that heat arises from the presence of a peculiar fuid or ethereal kind of matter, such as that which has been regarded as the cause of light.

Q. What great FACT is opposed to the idea that heat has a SEPARATE MATERIAL EXISTENCE as a fluid?

$A$. The fact that nature nowhere presents us, neither has art ever succeeded in showing us, heat alone in a separate state.

Q. Has heAT any PERCEPTIBLF WEIGIIT ?

A. No, if we balance a quantity of ice in a delicate scale, and then leave it to melt, the equilibrium will not be in the slightest degree disturbed If we substitute for the ice boiling water or redhot iron, and leave this to cool, there will be no difference in the result.

Count Rumford, having suspended a bottle containing water, and another containing spirits of wine, to the arms of a balance, and adjusted them so as to be exactly in equilibrium, he found that it remained undisturbed when the water was completely frozen, though the heat the water had lost must have been more than sufficient to have made an squal weight of gold red-hot.

Q. What other FAcTs are opposed to the supposition that heat has a SEPARATE EXISTENCE?

A. Heat can be produced without limit by friction, and irstense heat is also produced by the explosion of gunpowder.

Q. Are there any facts which tend to show that heat has a SEPARATE and ISOLATED EXIS'TENCE?

$A$. Yes; the fact that it can be communicated very readily through a vacum ; that it becomes instantly sensible on the condensation of any material mass, as if it were squeezed out of it: as when, on reducing the bulk of a piece of metal by hammering, we render it very hot (the greatest amount of heat being emitted with the blows that most change its bulk), and, finally, that the lanes of the spreading of heat do not resemble those of the spreading of sound, or of any other motion known to us. 
Q. What important PROPERTY distinguishes heat from all other agents or substances in nature?

$A$. The property of passing through and existing in all kinds of matter at all times; heat is everywhere present, and every body that exists contains it without known limit.

Q. Has IOE heat?

A. Yes, large quantities of it. Sir Humphrey Davy, by friction, extracted heat from two pieces of ice, and quickly melted them, in a room cooled below the freezing-point, by rubbing them against each other.

Q. How do we MEASURE the QUANTITY of heat in different bodies, or judge of its effects?

A. Only by the change in bulk or appearance which different bodies assume, according as heat is added or subtracted.

Q. According to WHAT LAW does heat diffuse or spread itself ?

$A$. Heat diffuses or spreads itself among neighbouring bodies until all have acquired the same temperature; that is to say, until all will similarly affect the thermometer.

Q. Why does a PIECE of IRoN thrust into burning coals become Hот among them ?

$A$. Because the heat passes from the coals to the iron until the metal has acquired an equal temperature.

Q. What is COLD?

$A$. Cold is a relative term expressing only the absence of heat in a degree; not its total absence, for heat exists always in all bodies.

Q. When the HAND touches a body having a HIGHER TEMPERATURE than itself, why do we call it нот?

$A$. Because, on account of the law that heat diffuses itself among neighbouring bodies until all have acquired the same temperature, heat passes from the body of higher temperature to the hand, and causes a peculiar sensation, which we call warmth.

Q. Under what CIRCUMSTANCES do we call a body CoLd ?

$A$. When we touch a body having a temperature lower than that of the hand, heat, in accordance with the above law, passes out from the hand to the body touched, and occasions the sensation which we call cold.

Q. What, then, really are the SENSATIONS of HEAT and COLD?

$A$. Merely degrees of temperature, contrasted by name in reference to the peculiar temperature of the individual speaking of them.

Q. When may a body feel Hот and COLD to the SAME PERSON at the SAME TIME?

$A$. The sensation of heat is produced by a body colder than the hand, provided it be less cold than the body touched immediately before: and the sensation of cold is produced under the opposite circumstances, of touching a comparatively warm body, but which is less warm than some other body touched immediately before. Thus, if a person transfer one hand to common spring water immediately after touching ice, to that hand the water would feel very warm; while the other hand 
transferred from warm water to the spring water would feel a sensation of cold.

Q. What is the RELATION between HEAT and LIGHT?

$A$. The relation between heat and light is a very intimate one. Heat exists without light, but all the ordinary sources of light are also sources of heat; and by whatever artificial means natural light is condensed, so as to increase its splendour, the heat which it produces is also, at the same time, rendered more intense.

Q. When is a BODY said to be INCANDESCENT or IGNITED?

$A$. When the body naturally incapable of emitting light is heated to a sufficient extent to become luminous.

Q. What is FLAME?

A. A luminous vapour issuing from a burning body.

Q. What is FIRE?

$A$. The appearance of heat and light in conjunction, produced by the combustion of inflammable substances.

Q. What CHARACTER was attributed to fire by the ANCIENT PHILOSOPHERS?

$A$. 'They used the term fire as a characteristic of the matter of heat, and regarded it as one of the four elements of nature.

Q. Enumerate the general PHYSICAL PROPERTIES and PRINCIPAL EFFECTS of heat.

A. It is invisible, without weight, elastic, with great tendency to expand, and is absorbed by all bodies. Its principal effects are expansion, liquefaction, vaporisation, and ignition.

Q. What do we understand by the term caloRIo ?

$A$. Caloric is the name given to the agent which produces the sensation of warmth; but heat is the sensation itself.

Q. Is CALORIC EQUALLI distributed over the globe?

$A$. No; at the equator the average temperature is $821^{\circ}$, while at the poles it is believed to be about $13^{\circ}$ below zero.

"Average Temperature"-that is, the mean or medium ternperature.

"ZERO"- the point from which a thermometer is graduated; it is $32^{\circ}$ below freezing, Fahrenheit's thermometer.

Q. How many SOURCES OE HEAT are recognised to exist?

A. Six.

Q. What are they?

A. 1. The sun; 2. The interior of the earth; 3. Electricity; 4. Vital action; 5. Mechanical action; 6. Chemical action.

Q. To the existence of what NATURAL FORCE is CALORIO continually opposed?

$A$. It is constantly and directly antagonistic to the force of cohesion.

\section{SECTION I.-THE SUN CONSIDERED AS A SOURCE OF IIEAT.}

Q. What is the PMIMCIPAL SOURCF of NATURAL IIEAT?

A. The sin.

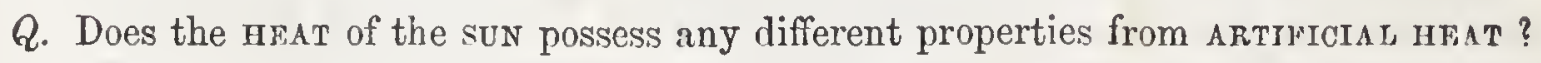

$A$. The heat of the sun pusses readily through glass, whereas this 
property is possessed by artificial heat in a very small degree. Solar heat also differs from artificial heat in some other minor points.

Q. What effect is produced when SUNBEAMS pass through a BURNING-GLASS ?

$A$. They are bent towards one point, the focus, when it is so concentrated as to set fire to natural substances.

Q. Is SUNSHINe detrimental to COMBUSTION?

A. By many it is supposed to be, though nothing is positively known upon the subject. Fires never appear so bright when the sun shines full upon them.

Q. In what WAY have philosophers attempted to account for the production of HEAT and LIGHT by the sUN?

A. Two opinions or theories have been entertained: one supposes that the sun is an intensely heated mass, which throws off its heat and light like an intensely heated mass of iron; the other supposes that heat is merely an uffection or state of an ethereal fuid, which occupies all space, as sound is an affection or motion of the air ; and that the sun may produce the phenomena of light and heat without waste of its temperature or substance, as a bell may constantly produce the phenomena of sound.

Q. What is the generally received opinion at present, in regard to the ACTUAI TEMPERATURE of the visible surface of the sun?

$A$. That the temperature of its luminous coating is much more elevated than any artificial heat we are able to produce.

Q. Have we any positive knowledge concerning the physical condition of matter at the SURFACE of the SUN?

A. Yes; experiments, conducted some years since by Arago, of France, settled the question to a most satisfactory extent.

Q. In Wha' MaNNER was he enabled to do this?

$A$. There are two states in which light is capable of existing - the ordinary state and the state of polarisation.* It has been proved that all bodies, in a solid or liquid state, which are rendered incandescent by heat, emit polarised light; while bodies that are gaseous, when rendered incandescent, invariably emit light in its ordinary state.

Thus the physical condition of a body may be distinguished when it is incandescent, by examining the light which it affords. On applying the test to the direct light of the sun, it was found to be in the unpolarised or ordinary condition of light. Hence it has been inferred by Arago that the matter from which this light proceeds must be in the gaseous state, or, in other words, in the state of flame.

From other experiments and observations, Arago was led to the conclusion that the sun was a solid, opaque, non-luminous body, invested with an ocean of flame.

Q. Why is the heat of the Sur always greater in some portions of the earth than at other's?

A. Owing to the position of the earth's axis, the rays of the sun always fall more directly upon the central portion of the earth than they do at the poles or extremities.

Q. Upon what does the succession of SPRING, SUMMER, AUTUMn, and WINTER, and the variations of the temperature of the seasons, depend?

A. Chiefly upon the position of the sun in relation to the earth.

* The term polarisation will be explained in a future cladpter. 
Q. When do we experience the greatest amount of HEAT from the RAYS of the SUN?

A. When its rays fall most perpendicularly.

Q. Why is the heat of the Sun greatest at roon?

$A$. Because for the day the sun nas reached the highest point in the heavens, and its rays fall more perpendicularly than at any other time.

Q. Why is it WARMEn in SUMMER than WINTER?

$A$. Because in summer the position of the sun is such that its rays fall more perpendicularly than at any other season. The sun is longer above the horizon in summer than in winter, and consequently imparts the greatest amount of heat.

Q. Why is it colder in winter than in summer?

$A$. Because in the winter the position of the sun is such that its rays fall more obliquely upon the earth than at any other season. The sun is also for a less time above the horizon in winter than in summer, and consequently imparts less heat.

Q. Upon what does the Heating Power of the sun chiefly depend?

A. Upon its altitude or height above the horizon; the greater its altitude, the more perpendicularly will its rays fall upon the earth, and the greater their heating effect; the less the altitude, the more obliquely will its rays fall, and the less their heating effect.

$Q$, Why should the DIFFErence in the INCLINATIon of the sun's rays falling upon the earth occasion a difference in their heating effect?

$A$. Because the more the rays are inclined, the larger the space over which they fall.

Fig. 22.

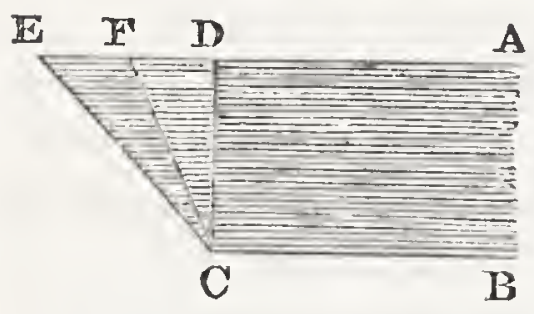

Let us suppose $A B C$ and $D$ to represent a portion of the sun's rays; let CD represent a portion of the earth's surface upon which the rays would fall perpendicularly; and let $\mathrm{CE}$ represent that portion upon which they would fall obliquely; the same number of rays would strike the surface C D and CE; but the surface C E being obviously greater than C D, the rays will necessarily fall more densely on the latter; and, as the heating power must be in proportion to the density of the rays, it follows that C D will be heated more than $C \mathrm{E}$, in just the same proportion as $\mathrm{C} \mathrm{E}$ is greater than $\mathrm{C} \mathrm{D}$. But if we would compare two surfaces, on neither of which the sun's rays fall perpendicularly, let us take $\mathrm{CE}$ and $\mathrm{CF}$. They fall on $\mathrm{C} \mathrm{E}$ with more obliquity than $\mathrm{C} \mathrm{F}$; but $\mathrm{C} \mathrm{E}$ is evidently greater than E F, and therefore, the rays being diffused over a larger surface, are less dense, and therefore less effective in heating.

Q. What is the greatest NATURAI HEAT ever observed?

$A$. On the west coast of Africa the thermometer (Fahrenheit) has been observed as high as $108^{\circ}$ in the shade; Burckhardt, in Egypt, and Humboldt, in South America, observed it at $117^{\circ}$ F.; and, in 1819, at Bagdad, the thermometer rose to $120^{\circ} \mathrm{F}$. in the shade.

Q. What is the LOWEST ATMOSPHERIC TEMPERATURE ever observed?

A. $53^{\circ}$ below zero, as observed by Dr. Kane; 58 , by Captain Parry ; $60^{\circ}$, by Captain Ross ; $71^{\circ}$, by Captain Back-all within the Arctic circle. 
Q. What is the greatest $\triangle$ RTIFICIAL COLD ever produced ?

A. $135^{\circ}$ below zero, which temperature was attained by Prof. Faraday. At this temperature, alcohol and ether did not freeze.

Q. What is the estimated temperature of the SPAOE ABOVE the earth's atmosphere?

A. $58^{\circ}$ below zero.

Q. At what TEMPERATURe does MERCURY FREEZE?

A. $38^{\circ} \cdot 6$ below zero.

Q. At what temperature does FRESH WATER FRELZE?

A. At $32^{\circ}$ above zero.

Q. At what temperature does SALT or SEA WATER FREIEZE ?

A. At $28^{\circ} \cdot 5$ above zero.

Q. Why does it require a GREATER AMOUNT of COLD to cause SEA WATER to congeal, than it does FRESH WATER?

$A$. Because sea water contains salt and other substances, which tend to prevent congelation.

Q. What is the average temperature at the EQUATOR ?

A. In America, $81^{\circ} .5$; in Africa, $83^{\circ}$.

Q. What is BLOOD-HEAT, or the vital temperature of the human body ?

A. $98^{\circ}$.

Q. At what TEMPERATURE does ALCOHOL boil ?

$A$. Under the ordinary atmospheric pressure at $173^{\circ} \cdot 5$.

Q. At what TEMPERATURE does WATER boil ?

$A$. Under the ordinary atmospheric pressure at $212^{\circ}$

Q. At what temperature does lead melt?

A. At $594^{\circ}$.

Q. At what heat does mercury boil?

A. At $661^{\circ}$ under the common atmospheric pressure.

Q. At what tEMPRRATURe does BRASS, COPPER, SILVER, and GoLd melt?

A. Brass at $1,869^{\circ}$; copper, $1,996^{\circ}$; silver, $1,873^{\circ}$; gold, $2,016^{\circ}$.

Q. At what TEMF artATURE does CAST-IRON melt?

A. At $2,786^{\circ}$.

Q. What is the greatest degree of ARTIFICIAL HEAT which we have been ENABLED TO MEASURE?

A. A furnace heat of $3,280^{\circ}$ : at this heat wrought-iron and platinum did not melt.

\section{SECTION II.-THE EARTH AND ELECTRICITY CONSIDERED AS SOURCES OF HEAT.}

Q. How far below the SURFACE of the EARTh does the influence of SOLAR HEAT extend $?$ $A$. The depth varies from 50 to 100 feet; never, however, exceeding the latter distance.

Q. How do we know that the EARTH is a SOUROE of HFAT?

$A$. Because we find as we descend into the earth, and pass beyond the limit of solar heat, that the temperature constantly increases. 
Q. At what RATE does the TEMPERAture increase?

$A$. About one degree of the thermometer for every fifty feet.

Q. Supposing the temperature to continue to increase according to this ratio, what would be its effects at DIFFERENT DEP'HS?

$A$. At the depth of two miles water would be converted into steam; at four miles, tin would be melted; at five miles, lead; and at thirty miles almost every earthy substance would be reduced to a fluid state.

Q. To what CAUSE may EARTHQUAKES and rorCANOES be attributed ?

A. Undoubtedly to the agency of the internal heat of the earth.

Q. What effect has the internat Heat of thr earth on the teMperature of the SURFACE?

A. No sensible effect: it has been calculated that it affects the temperature of the surface less than $\frac{1}{30}$ of a degree of the thermometer.

Q. Why, if so great an amount of heat exists in the INTERIor of the EARTH, does it not appear more manifest upon the SURFACE?

$A$. Because the materials of which the exterior strata or crust of the earth is composed do not conduct it to the surface from the interior.

Q. In what manner is ELECTRICITY a SOURCE of HFAT ?

$A$. When an electric current passes from one substance to another, the substance which serves to conduct it is very frequently heated; but in what manner the heat is produced we have no positive information.

Q How GREAT a DEGREE of HEAT is ELECTRICITX capable of generating?

$A$. The greatest known heat with which we are acquainted is produced by the agency of the electric or galvanic current. All known substances can be melted or volatilised by it.

Q. Has the hEAT generated by eLEOTRICITy been employed for any PRACTIOAL or ECONOMICAL purposes?

A. Not to any great extent; but for philosophical experiments and investigations it has been made quite useful.

\section{SECTION III.-CHEMICAL ACTION CONSIDERED AS A SOURCE OF HEAT.}

Q. What is CHEMICAL ACTION?

A. We apply the term chemical action to those operations, whatever they may be, by which the weight, form, solidity, colour, taste, smell, and action of substances become changed; so that new bodies, with quite different properties, are formed from the old.

Q. How does OHEMIOAL ACTION become a SOURCE of Heat?

$A$. Many bodies, when their original constitution is altered, either by the abstraction of some of their component parts, or by the addition of other substances not before in combination with them, evoive heat while the change is taking place.

Q. Furnish an iluustration.

$A$. 'Two cold liquids, as in the case of water and sulphuric acid, produce intense heat, if united. 
Q. Why does COLD WATER poured on LIME make it intensely HOT ?

A. Because heat is evolved by the chemical action which takes place when the cold water combines with the lime.

Q. Did HEAT exist in the cold water and lime before they were mixed together?

A. Yes, in a latent state.

Q. What do you mean by LAtent?

A. Latent, from the Latin lateo, to lie hid, is something that is not perceptible. Latent heat is heat not felt; such heat exists even in ice.

Q. Does cold iron contain HEAT?

A. Yes; everything contains heat; but, when a thing feels cold, its neat is latent.

Q. What is meant by LATENT heat?

A. Heat not perceptible to our feelings. When anything contains heat without feeling the hotter for it, that heat is called "latent heat."

Q. Does COLD iron contain latent HEAT?

A. Yes; and when the particles are compressed by hammering, latent heat is brought out, and the iron becomes red hot.

Q. How are we made conscious of the ExISTENCE of heat, if we cannot feel it?

A. By experiment; thus the temperature of ice is $32^{\circ}$, but, if melted over a fire, $140^{\circ}$ of heat being thereby absorbed, it will feel no hotter than before ; the $140^{\circ}$ degrees of heat, being hidden, is in a latent condition.

Q. What AMOUNT of heat may thus exist in a latent condition?

$A$. The quantity is variable $; 1,140^{\circ}$ may be in this condition, as $140^{\circ}$ of heat are latent when ice is melted by natural or artificial means, and $1,000^{\circ}$ of heat are secreted when water is converted into steam, so ice converted into steam contains $1,140^{\circ}$ of latent heat.

Q. By what simple experiment can we be made to feel the WARMTH of sNow ?

$A$. Salt added to snow makes it so intensely cold, that, after plunging our hands into the mixture, snow appears comparatively warm.

Q. Why does STEAM burn so much more severely than BoILING WATER?

$A$. Steam condenses as soon as it is exposed to the cold, and gives out all the heat by which it was produced; therefore, as one thousand degrees of heat become latent in steam, it gives out that amount when condensed, which is much greater than boiling water.

Q. Why is the AIR in the SPRING, when the ICE and sNow are melting, always very CHILLY and COLD?

A. Solid bodies, in passing to the liquid state, absorb heat in large quantities; when ice and snow are thawing, they absorb heat from the air, in consequence of which its temperature is greatly reduced.

Q. Why does the WeAtier always moderate on the FALL of SNow?

A. Bodies, in passing from the liquid to the solid state, give out heat; snow is frozen water, and in its formation heat is imparted to the atmosphere, and its temperature increased. 
Q. Why does the temperature of MELTING ICE and SNow never exceed $32^{\circ}$ ?

$A$. Because all the heat imparted to melting ice and snow becomes insensible until the liquefaction is complete.

Q. Who originated the doctrine of LATENT HEAT?

A. Dr. Black, of Edinburgh, during the last century.

Q. In WHAT MaNNer was he led to his conclusions?

$A$. He noticed that if a pound of water at $32^{\circ}$ be rmixed with a pound at $172^{\circ}$, the temperature of the mixture will be intermediate between them, or $102^{\circ}$. But if a pound of water at $172^{\circ}$ be added to a pound of ice at $32^{\circ}$, the ice will quickly dissolve ; and, on placing a thermometer in the mixture, it will be found to stand not at $102^{\circ}$, but at $32^{\circ}$.

In this experiment he noticed, therefore, that the pound of hot water, which was originally at $172^{\circ}$, actually lost $140^{\circ}$ of heat, all of which entered into the ice and caused its liquefaction, but did not affect its temperature. Hence it followed that a quantity of heat became insensible during the melting of the ice, sufficient to raise the temperature of an equal weight of water $140^{\circ}$.

Q. What CHanges did Dr. Buack suppose that the heat underwent during this transformation?

$A$. He conceived that heat, in causing fluidity, loses its property of acting on the thermometer in consequence of combining with the solid sulstance; and that liquefaction results because the compound so formed does not possess that degree of cohesive attraction on which solidity depends. When a liquid is cooled to a certain point, it parts with its heat of fluidity, which is set free or becomes sensible, and the cohesion natural to the solid is restored.

Q. Why do saLT and SNow mixed togetler produce INTENSE COLD?

$A$. The salt and snow are both solids, which, on being mixed, the salt causes the snow to melt by reason of its attraction for water, and the water formed dissolves the salt : so that both pass from the solid to the liquid condition, and a large quantity of heat is absorbed. This heat, being derived from that which previouslv existed in the solids themselves in a sensible state, causes the temperature necessarily to fall.

Q. How great a degree of cold can thus be generated by a mixture of salt and snow ?

$A$. About $32^{\circ}$ below the temperature of freezing water.

Q. What are the COLDEST MrXTUREs known?

$A$. Three parts of muriate of lime, with one part of snow. Five parts of diluted sulphuric acid, with four parts of snow.

SECTION IV.-MECHANICAL ACTION CONSIDERED AS A SOURCE OF HEAT.

Q. How does MeCHanical action produce heat?

$A$. By percussion, friction, and condensation.

Q. What is meant by Percussion?

$A$. The shock produced by the collision of two bodies. 
Q. Why does STRIRING IRON make it RED IIOT? heat.

A. It condenses the particles of the metal, and excites the latent

Q. Why are SPARKS produced by striking with FLINS and STERL ?

A. It disturbs the latent heat by compressing the particles of the bodies struck.

Q. How does the DISTURBANCE of this lavent heat SET TINDER ON FIRE?

A. A particle of red hot matter, either from the steel or flint, is struck off, and, faliing on the tinder, sets fire to it.

Q. What occasion these particles being RED HOT ?

$A$. The latent heat is rendered sensible by condensation, and is made red hot in some of its particles.

Q. When the percussion of the flint and steel is made to occur in a vacour, what takes place?

A. 'The heated particles are produced equally well as before, but are scarcely visible, from the absence of the atmosphere.

Q. What is Friction?

A. Friction is the act of rubling two things together.

Q. Cannot Fire be produced by Friction?

A. Yes; the Indians, for instance, take a flat piece of wood, and rub on it a sharpened, pointed piece, till a groove is made, and the dust catches fire, latent heat being evolved by friction.

Q. What SORT of WOoD is best adapted for this purpose?

A. Poplar, ivy, laurel, boxwood, and mulberry.

Q. Does not friction sometimes occasion CARRIAGE WHEELS to TALE FIRE ?

A. Yes; ignition being produced in consequence of the disturbance of latent heat.

Q. To what is this attributable?

$A$. It is owing to the wheels being too dry, too tightly fitted, or moving too rapidly.

Q. For what purpose are CART WHEELS GREASED?

A. Grease lessens the friction; and, when there is less friction, the latent heat of the wheels is less disturbed.

Q. What EFFECT is produced by RUBBING our HANDS and FACES?

A. They are made to feel warm, the latent heat being excited, and increased rapidity of 'circulation given to the blood. A person almost drowned is thus restored to animation.

Q. If pieces of ICE are RUBBED together, what is the result?

$A$. 'They melt, latent heat being excited by friction.

Q. Furnish some other instances of the RESULTS of FRICTION.

A. 'Tools become hot by being used; the borers, in boring cannon, blister the. hand. In all cases of this sort, latent heat is evolved by friction.

Q. What interesting experiment was made by Count RUMFord, illustrative of the offect of friction in producing Hest?

A. A borer was made to revolve in a cylinder of brass, partially 
bored, thirty-two times in a minute. The cylinder was enclosed in a box containing 18 pounds of water, the temperature of which was at first $60^{\circ}$, but rose in an hour to $107^{\circ}$; and in two hours and a half the water boiled.

Q. Is AIR necessary to the production of MEAT by FRICTION?

A. Air does not appear to be necessary to the production of heat by the attrition of solid bodies; heat is produced by friction within a vacuum.

Q. Does a BODY ever cease to give out HEAT by FRICTION?

A. No; however long the operation may be continued. fact?

Q. What conclusions respecting the nature of heat have philosophers drawn from this

A. That heat cannot be a material substance, but merely a property of matter.

Q. Is HEAT FVRr produced by the FRICTION of Liquids?

$A$. No; solids alone produce heat by friction; and no elevation of temperature takes place from the mere agitation of fluids with one another.

Q. Is the heat excited by friction in proportion to the HARDNESS or ELASTICITY of the bodies employed?

A. No ; on the contrary, a piece of brass rubbed with a piece of cedar-wood produces more heat than when rubbed with another pieco of metal; and the heat is still greater when two pieces of wood are employed.

Q. Why does the FuLminating composimion of a perctssion cap explode when struct witl a hammer?

A. Because the blow occasions compression of the particles, by which the latent heat is concentrated to such a degree as to cause an explosion.

Q. Why does a FRICTION IrATCH, drawn over sand paper or other rough substance, ignite?

A. When the match is drawn over sand paper or other rough sub stance, certain phosphoric particles are rubbed off, and beiny ccm. pressed between the match and the paper, their heat is raised sulficiently high to ignite them, and fire the match. If the match be drawn over a smooth surface, the compression must be increased, for the temperature of the whole phosphoric mass must be raised in order to cause ignition.

Q. Why is it easier to produce IIEAT from the friction of rovan SURFACrs than srootiI ONES?

A. Because in the friction of rough surfaces certain particles are rubbed off, which being smaill, are readily condensed, and made to evolve their latent caloric.

Q. Why, when you rub a smooth METALIIC SURFACE, as a BUTton for example, against a piece of plank, does the metal become more heated than the wood?

A. Because the caloric is forced out of the wood, as it were, by the compression of its parts, and the button receives most of the caloric, owing to a stronger attraction for than is possessed by the wood. 
Q. What is compression?

A. Compression is bringing various particles in closer contact.

Q. Is HEAT evolved by courpression?

A. Yes; even from common air. Thus a piece of German tinder, if placed at the bottom of a glass tube, and the air in the tube compressed, catches fire; the latent heat of the air being forced out, and thus setting fire to the tinder.

Q. Why does the Horis made by a shot or camnon ball in a wall ur timber, look as if it Tere BURAT?

$A$. Because the shot or cannon balls were so heated by the discharge, as actually to scorch the material into which they penetrated.

Q. Why are SHOT and CANNON BALLS HEATED by being discharged from a gun or cannon ?

$A$. Because the air is so rapidly condensed, when the charge is made, that sufficient latent heat is developed to make the shot or balls hot.

\section{SECTION V.-VITAL ACTION CONSIDERED AS A SOURCF OF HEAT.}

Q. What SINGULAr property have all living animal bodies?

A. 'The property of maintcining in themselves an equable temperature, whether surrounded by bodies that are hotter or colder than they are themselves.

Q. ImLustrate this fict.

A. The sailors of the Arctic exploring expedition during the polar winter, while breathing air that froze mercury, still had in them the natural warmth of $98^{\circ}$ Fahrenheit; and the inhabitants of India, where the same thermometer sometimes stands $115^{\circ}$ in the shade, have their blood at no higher temperature.

Q. Do vEGETALLES possess in ANY DEGREE THIS PROPERTY of maintaining a constant temperature within their structure?

A. Growing or fresh vegetables have this property to a certain extent.

Q. What, then, is VITAI MEAT? tuie.

A. The heat generated or excited by the organs of a living struc-

Q. What is the cause of ViTAL or ANMALI MEAT?

A. The cause of animal heat is not certainly known or determined; it is supposed to be due to chemical action, the result of respiration and nervous excitation.

Q. Has the power of animals to preserve a peculiar temperature any limits?

A. Yes; intense cold suddenly coming upon a man who has not sufficient protection, first causes a sensation of pain, and then brings on an almost irresistible sleepiness, which, if indulged in, proves fatal. A great excess of heat, also, cannot long be sustained by the human system. 
Q. Does FACH SPECIES of animal appear to have a peCULIAR temirerature?

$A$. Yes; each species of animal and vegetable appears to have a temperature natural and peculiar to itself, and from this diversity different races are fitted for different portions of the earth's surface.

Q. What EFFect does the PECUliarity of temperature have upon the Distribution and LOCATION of ANIMALS and PLANTS upon the earth's surface?

$A$. The different species are confined and circumscribed within certain districts, depending mainly on their relations to heat. 'Thus, the orange-tree and the bird of paradise are confined to warm latitudes; the pine-tree and the Arctic bear, to those which are colder.

Q. What cURTOUS FACT in relation to a SPECIES of wHALE illustrates the influence of temperature in defining locations?

$A$. It has been ascertained that at least one species of whale is precluded from migrating from the north to the south, from its inability to live in the heated waters of the equator.

Q. When animals or plants are removed from their peculiar and natural districts to. une entirely different, WHAT CHANGES take place?

$A$. They either cease to exist, or change their character in such a way as to adapt themselves to the climate.

Q. What curious illustrations do we find of this?

$A$. The wool of the northern sheep changes in the tropics to a species of hair. The dog of the torrid zone is nearly destitute of hair. Bees transported from the north to the region of perpetual summer cease to lay up stores of honey, and lose, in a great measure, their habits of industry.

Q. How has NATURe provided for the protectron of ANIMALS against the modifications of temperature?

A. By covering their bodies with a form of fur, or hair, or feathers, in the exact degree required, and to such an extent as to vary the covering in the same animal according to the climate and season.

Q. What oNe species of organised beings is fitted to live in ALL CLmatrs ?

A. Man alone is capable of living in all climates, and of migrating freely to all portions of the earth.

\section{CHAPTER XV. \\ COMMUNICATION OF HEAT.}

Q. In what THREE WAYS may HEAT be communicated?

A. By direct contact, by conduction and convection, and by radiution.

Q. How may HeAT be communicated by ConTACT?

A. When a hot body touches a cold one, the heat passes directly from one into the other, as when it enters a bar of iron put into the fire, or the hand immersed in hot water. 
Q. When is IEAT communicated by conduction?

A. When the heat travels from particle to particle of the substance, as from the end of the iron bar placed in the fire to that part of the bar most remote from the fire.

Q. When is Hest communicated by Rodation?

$A$. When the heat leaps, as it were, from a hot to a cold body throngh an appreciable interval of space, as when a body is warmed by placing it before a fire removed to a little distance from it.

Q. In what way does a HEATED BODY COoL itself?

A. First, by giving off heat from its surface, either by contact, or radiation, or both conjointly; and, secondly, by the heat in its interior passing from particle to particle by conduction through its substance to the surface.

Q. In what MANNER does a COLD BODY become heated?

A. First, by heat passing into its surface either by contact, or radiation, or by both conjointly; and, secondly, by the heat at its surface passing from particle to particle through its interior portions by conduction.

Q. What are the PRINCIPAL CONDITIONs which influence the COMMUNICATION of heat from one body to another by contact?

A. The degree of contignity, and the conducting power of the substances. The more perfect these relations, the more rapid the transfer.

Strictly speaking, the transfer of heat by direct contact is a case of radiation, in which the surfaces, though still separated by an interval, are at an insensible distance.

\section{SECTION I.-CONDUCTION.}

Q. Does HEAT pass through all bodies with the SAME VELOCITY?

A. No; some substances oppose very little impediment to its passage, while through others it is transmitted slowly.

Q. Into what Two CLASSES are bodies divided in respect to their CONDUCTION of HEAT?

$A$. Into conductors and non-conductors; the former are such as allow heat to pass freely through them; the latter comprise those which do not give an easy passage to it.

Q. Are DENSE and SOLID BODIES the best CONDUCTORS of HEAT?

A. Yes; such as metals. Of these, gold, silver, and copper are the best, and lead the most inferior.

Q. Are LIGHT POROUS BODIES gOod CONDUCTORS ? known.

A. No; hair, fur, wool, charcoal, are the worst conductors of heat

Q. On what principle do you account for SONE BODIES being COLDER than others?

$A$. Chiefly on account of their being better conductors, and drawing off heat from our bodies with greater rapidity than others.

Q. Why does a PIECE of WOOD blazing at ONE end NoT feel HOT at the OTHER?

4. Because wood is so bad a conductor that heat does not traverse 
freely through it; hence, though one end of a stick be blazing, the other end may be quite cold.

Q. Why does a poker resting on a fender feel colder than the HEARTH-ruG, which is farther off the fire?

$A$. Because the poker is an excellent conductor, and draws heat from the hand much more rapidly than the woollen hearth-rug, which is a very bad conductor: though both, therefore, are equally 'warm, the polier seems to be the colder.

Q. What is the CAUSE of IroN feeling so much CoLDER than wood in winter?

A. Iron is a better conductor than wood, and produces the feeling of cold by drawing off the heat from our hand more rapidly than wood.

Q. Is the IRON really COLDER?

A. No; it possesses exactly the same amount of heat, if exposed to the same temperature.

Q. Why does a stone PAVEMeNe feel colder than a carperted FLoor, though exposed. to the same temperature?

A. Because the stone is a better conductor than the carpet, and conveys heat away more rapidly from the human body.

Q. Does not the heartir-stome feel wararer than the Hearti-rog when the fire is kindled in the grate?

A. It does, being a better conductor, and giving forth heat more readily than the woollen rug.

Q. How does this account for its feeling W $\mathrm{WRAI}$ ?

A. Heat is communicated to our bodies so rapidly by the stone, that we are made immediately sensible of the change.

Q. Why are TOODEN HANDLES put to METAL TEAPOTS?

A. Because wood, being a bad conductor, does not convey heat so rapidly as metal, and therefore makes it more convenient, as a handle, to be taken hold of: thus, while the metal is quite hot, the wooden handle remains comparatively cold.

Q. Is WOOLLEN CLOTII a BAD CONDUCTOR?

$A$. It is ; and on this account we employ woollen kettle-holders, which prevent the rapid transmission of heat from the metal to our hands.

Q. Does a WORLLEN KETTLE-HOLDER entirely prevent the transmission of ALAT?

A. No ; but, being a bad conductor, it communicates heat too slowly to be felt, and never feels as hot, though, in reality, it may be as hot as the kettle itself.

Q. Is ATR or WATER the best CONDUOTOR of HEAT?

A. Water is a much better conductor than air, but no liquid is a good conductor.

Q. Furnish an inlustration of this principle.

$A$. If we plunge any part of our body into cold water, it produces a sensation of cold, because it rapidly draws off the heat, and makes us feel the loss. Air abstracts heat, but more slowly, consequently less perceptibly. 
Q. On what principle is WATER a better conductor than $\mathbf{A I R}$ ?

$A$. The conducting power of any substance depends on the closeness of its particles, and water being denser than air, is, in consequence, a better conductor.

Q. Are LiQuids good conductors of HEAT?

A. No; heat in liquids produces evaporation, and flies off in vapour; water may boil at the surface, and leave ice unmelted a short distance below the surface.

Q. Is $\Lambda \mathrm{TR}$ a good CONDUCTOR?

A. No; air is a very bad conductor.

Q. How is a ROOM WARMED by a STOVE?

A. The air nearest the fire is made hot first and rises; cold air then descends, is heated, and ascends in like manner; and this interchange goes on till all the air of the room is warmed.

$Q$. If $\triangle I R$ be a BAD ONDUCTor of heat, why should we not feel as warm wITHOUT clothing, as when we are wrapped in wool and fur?

$A$. Because the air draws off heat, and, by the ceaseless change of air, tends to decrease the warmth of that part of the body devoid of clothing. Thus, the air (which cases the body) absorbs as much heat from it as it can while it remains in contact; being then blown away, it makes room for a fresh coat of air, which absorbs more heat.

Q. Does the $\Delta I \mathrm{I}$ surrounding a body unclothed become as $W \Delta \mathrm{RII}$ as the body itself ?

$A$. The constant motion of the air prevents this: it would rise to the same heat if it remained without motion.

Q. What DENEFITS arise from AIR being a BAD CONDUCTOR?

$A$. If it were otherwise, and possessed as good conducting powers as metal or stone, the heat would be drawn off so rapidly from our bodies as to impede, if not destroy, animal and vegetable life.

Q. Why are Foor and FUR used for clothing in COLD WEATHER? and do they IMPART HEAT ?

A. Woollens and furs prevent the warmth of the body being drawn off, in consequence of being bad conductors. They prevent the escape of heat, but do not impart it.

Q. What is the CHIEF USE of Chothing in cold weather?

$A$. The prevention of the escape of animal heat, and the protection of the body from the cold air.

Q. How are these NECESSTTIES answered in the condition of the lower animals?

$A$. Beasts are covered with fur, hair, or wool; birds with down or feathers.

Q. What PURPose do these answer?

$A$. Being bad conductors, they serve to keep them warm, preserving animal heat and protecting from the cold air. Birds, which are the most delicate, have a thicker covering of feathers. Beasts in the frigid zone have coarser and warmer coats than those in tropical regions. 
Q. Why is moderately Loose clothing WARMER than that whiül fits TIGHTLY ?

A. Because air is a bad conductor; and the quantity of air confined between our bodies and clothing prevents, 1 . 'The heat of our bodies from es:aping; and

2. The external air from coming into contact with our bodies. But if our clothing is sufficiently loose to admit of a free circulation of air, we shall feel cold; and, on the contrary, if it fits very tightly, it impedes the free circulation of the blood, and we feel cold.

Q. How are Whales, SEALS, and other WAFM-BLOODED ANIMALs that live in the water, protected against the cold?

A. They are enveloped, beneath the stin, with a thick coating of "blulber", or fat, which, like fur, hair, and feathers, is a non-conductor of heat, and serves to protect them in a like manner.

Q. Why are BLANKETS and WARM WOOLLEN GOODS always made with a NAP Or PROJECTION OF FIBRES on the outside?

A. Because the nap or fibres retain air among them, which, from its non-conducting properties, serves to increase the warmth of the material.

Q. How does the corering of maIr, wool, and Feathers, serve to keep animals cool in HO'T WEATHER, as well as WARM in COOL WEATHER?

$A$. In warm weather the non-conducting medium will not allow the heat to enter the body from without; in cold weather, the heat of the body cannot escape from within.

Q. Why do we wrap up ICE in FLa NNEL to keep it from melting ?

$A$. Because the flannel, being a non-conductor, does not allow the heat of the atmosphere to penetrate to the ice.

Q. Does not the bad conducting power of air enable persons to judge whether an EGG be NEW Or STALE?

$A$. Yes; touch the larger end of the shell with your tongue ; if it feels warm, the egg is stale; if not, it is new-laid.

Q. Why will the SHELL of a STALE EGG feel warm to the tongue?

$A$. Because the thick end of an egg contains a small quantity of air (between the shell and the white); when the egg is stale, the white shrinks, and the confined air accordingly expands.

Q. Why does the expansion of air (at the end of an egg) make it feel WARM to the tongue?

$A$. Because air is a very bad conductor; and the more air an egg contains, the less leat will be drawn from the tongue when it touches the shell.

Q. Why will a NEW-LAID egg feel COLDER to the tongue at the thick end than a stale one?

A. Because it contains more white and less air ; and as the white of an egg is a better conductor than air, the heat of the tongue will be drawn off more rapidly, and the egg feel colder.

Q. Why are rooms much WARAER for being furnished with DOUBLE-DOORS and WINDows?

A. Because air is a bacl conductor; and the air confined between 
the double doors and windows opposes both the escape of warm air out of the room, and of cold air into the room.

Q. Why is a room Waraser when the WINDOW curTaINS are drawn or the SHUTTERS shut?

A. Because air is a bad conductor; and the air confined between the curtains or shutters and the window, opposes both the escape of varm air out of the room, and of cold air into it. The curtains and the shutters also are non-conductors, and, at the same time, prevent the circulation of air and the existence of currents.

Q. Is LINEN a better conductor than CoTTON?

$A$. Yes; therefore a linen shirt is much colder than a cotton one; and a cambric handkerchief cools the face, if wiped over it, far better than a cotton one.

Q. Is the soll a good conductor of HEAT?

$A$. No ; its particles are not sufficiently close together, and density of matter is essential to conducting heat.

Q. Is the SOIL PENetrated DEEPLY by the changes of SUMMIER and WINTER?

$A$. No; being a bad conductor of heat, the soil below the surface is cool in summer and warm in winter.

Q. What BENEFITS arise from this ?

$A$. If the soil was a good conductor, all vegetation would be destroyed; frozen in winter, and burnt up in summer.

Q. Is SNOW a GOOD CONDUCTOR Of HEAT?

A. No; therefore in winter time it protects vegetables and seeds from the frosts; and the Bible says, "God giveth snow like wool."

Q. How does SNow protect VEGETATION from the FROST?

'A. It prevents the heat of the ground being abstracted by the air.

$Q$. Why is swow a non-conductor of heat?

A. Principally because it contains a large quantity of air between its particles.

Q. Why is it coor under a SHADY tree in a hot summer's day?

A. 1. Because the overhanging foliage screens off the rays of the sun ;

2. As the rays of the sun are warded off, the air (beneath the tree) is not heated by the reflection of the soil; and

3. The leaves of the trees, being non-conductors, allow no heat to penetrate them.

Q. Why is the LID of a KETTLE intensely Hот when the water boils?

$A$. Because the bright metal lid is an admirable conductor; and therefore the heat from the boiling water pours into our hand tho moment we touch it.

Q. Why are ICEHOUSES lined with STRAW, and generally WHITEWASHED on the ortside?

A. 1. Because straw is a very bad conductor of heat, and therefore prevents the external heat from getting to the ice; and

2. The whitewashed roof and walls prevent the absorption of heat. 
Q. Why will a little ors on the surface of water prevent its FrEIZING ?

A. Because oil is a bad conductor, and prevents heat from leaving the water.

Q. A silver teaspoon becomes more heated by hot tea, than one of inferior metal (as German silver, pewter, \&.c.): why is this?

$A$. Because silver is a better conductor than German silver or pewter.

German silver is composed of twenty-five parts of nickel, twenty-five of zinc, and fifty of copper.

Pewter is, generally speaking, an alloy of tin and lead, sometimes with a little antimony or copper combined, in different proportions, according to the purposes for which it. is designed.

Q. Why does a MIETAI SPOON (left in a saucepan) RETARD the process of BOILING?

$A$. Because the metal spoon (being an excellent conductor) carries off the heat from the water, and (as heat is carried off by the spoon) the water takes a longer time to boil.

Q. Why does PAINT preserve WOoD?

A. 1. Because it covers the surface of the wood, and prevents both air and damp from penetrating into the pores;

2. Because paint (especially white paint) being a bact conductor, preserves the wood of a more uniform temperature; and

3. Because it fills up the pores of the wood, prevents insects and zermin from harbouring therein and eating up the fibre.

Q. Are BRIORS good CONDUCTURS?

$A$. No; they prevent the escape of heat, and are consequently used for building stoves or furnaces, where much heat is required.

Q. Would a BRICK STOVE in the middle of a room make the room WARMER thanan IRoN one?

$A$. No; bricks would retain the heat-iron give it forth; the iron stove would therefore make the room much warmer than a brick one.

Q. Why do CELLARS feel WARI in WINTER?

A. Because the external air has not free access into them; in consequence of which they remain almost at an even temperature, which (in winter time) is about 10 degrees warmer than the external air.

Q. Why do CELLARs feel COLD in SUMMrer?

$A$. Because the external air has not free access into them; in sonsequence of which they remain almost at an even temiperature, which (in summer time) is about 10 degrees colder than the external air.

Q. In what respect is $B \triangle A R E$ especially adapted as a covering for trees and shrubs?

A. Bark is composed of matter which is very slowly permeable by heat, and, like hair and fur in animals, is especially adapted for securing the teuperature necessary to vegetable life.

Q. What is the TEMPERATURE of the SAP of healthy trees during the SUMMER?

A. It is several degrees below that of the surrounding atmosphere. 
Q. What is the TEMPERATuRE of the SAP of a healthy tree in the winter?

A. Several degrees above that of the surrounding atmosphere.

Q. What occasions this DFFErETCE between the temperature of the sap of a tree and the temperature of the surrounding atmosphere?

A. The vital action of the tree.

It is also a noticeable fact that sap drawn from a tree will freeze at the same tempernture as water, while the sap circulating in the tree, under the influence of vital agency, will not freeze until reduced seventeen degrees below the freezing-point of water.

Q. Why in a frozen pond or lake is the ice always THINNer, and often HaTrutiY WANTING, in those parts where springs exist upon the bottom?

A. Because the spring water, coming from a point in the earth below the influence of the frosts, is elevated in temperature, and by imparting its heat prevents an accumulation of ice upon the surface above.

Q. Is there in reality any Posimrve varutr in the materials of clothing?

A. No; but we consider clothing warm or cool according as it impedes or facilitates the passage of heat to or from the surface of our bodies. The thick cloak which guards a Spaniard against the cold of winter, is also in summer used by him as a protection against the direct rays of the sun; and, while in temperate climates flannel is the warmest article of dress, we cannot at the same time preserve ice more effectually than by enclosing it in its softest folds.

Q. Are FINE or co.lRsE woollens the WARAIEST ?

$A$. Fine woollens are warmer than coarse ones, because the finer the cloth, the more slowly it conducts heat.

Q. Is siLK a bad. conductor of heat?

A. Yes, as it allows the heat of the body to pass off more rapidly than wool.

Q. The SHEETS of a bed feel COLD and the BLANIEETS WARM : is there any difference in the respective temperature of these articles?

A. No; the temperature of both the sheets and the blankets is always exactly the same.

Q. Why, then, does one feel corDER than the other?

$A$. Sheets feel colder than the blankets because they are $b \in t t e r$ conductors of heat, and carry off the heat more rapidly from the body; but when by the continuance of the body between them they acquire the same temperature, they will then feel even varmer than the blankets.

Q. In the summer a still, CALN ATMosphere feels warm, but if a wind arises, the same atmosphere feels cold : has there been any real CHANGE of temperature?

A. No; for a thermometer suspended under shelter and in a calm place will indicate the same temperature as a thermometer on which the wind blows.

Q. Why do we then consider that the AIr has grown CoLDER?

A. Because the air in motion by the wind conducts off the heat from our bodies faster than the same air at rest. 


\section{SECTION II.-CONVECTION.}

Q. What is CONVECTION of IIEAT?

A. Convection of heat is that by which heat is conveyed to some other thing or place. 'Thus, the lower particles of water in a vessel being heated by a spirit lamp gradually ascend, conveying heat to other parts of the water.

Q. Are LIquids good conductors of heat?

$A$. No; liquids are bad conductors, and are therefore made hot by convection.

Q. Why are LIQUIDS BAD CONDuctons of heat?

A. This peculiarity is referable to the mobility which subsists among the particles of all fluids, and to the change of size which is invariably produced by a change in temperature.

The constituent particles of solid bodies being incapable of changing their material position and arrangement, the heat can only pass through them from particle to particle, by a slow process ; but when the particles forming any stratum of liquid are heated, their mass, expanding, becomes lighter, bulk for bulk, than the colder stratum immediately above it, and ascends, allowing the superior strata to descend.

Q. Explain how WATER is made Hor.

$A$. When the heat enters at the bottom of a vessel containing water, a double set of currents is immediately established,-one of hot particles rising towards the surface, and the other of colder particles descending to the bottom. The portion of liquid which receives heat from below is thus continually mixed through the other parts, and the heat is diffused by the motion of the particles among each other.

These currents take place so rapidly, that if a thermometer be placed at the hottom and another at the top of a long jar, the fire being applied below, the upper one will begin to rise almost as soon as the lower one. The movement of the particles of water in boiling will be understood by reference to $f i g .53$.

Fig. 53.

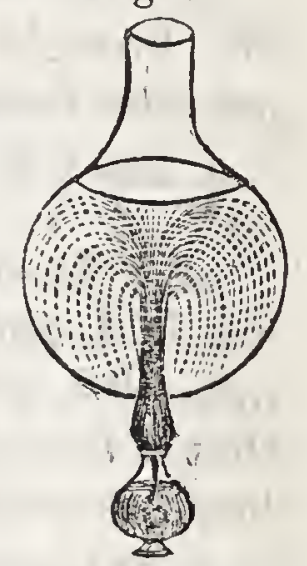

Q. What would be the EFFECT if the heat were applied to the ropinstead of the Botrour of a kettle containing liquid?

A. The particles of the liq id would maintain their mutual arrangement, and the transmission of heat would take place in the same manner as if the liquid were solia.

The heat is, in fact, conducted through the liquid. In this manner liquids have been klown to have very low conducting powers-so low, that for a long period they were supposed to be incapable of conducting leat.

Q. What EXPERIMLNT shows the inferior conducting power of liquids?

$A$. If a small quantity of alcohol be poured on the surface of water at $32^{\circ}$ Fahrenheit, and inflared, it will burn on the water. A 
thermometer, immersed at a small depth below the common surface of the spirit and the water, will, after a considerable lapse of time, fail to show any increase in temperature.

Q. Furnislı another ILLUSTRation of this principle.

$A$. If a bar of red hot iron be plunged into cold water, that part of the fluid which is immediately around the iron becomes boiling hot, the rest remaining cold as before.

Fig. 54 .

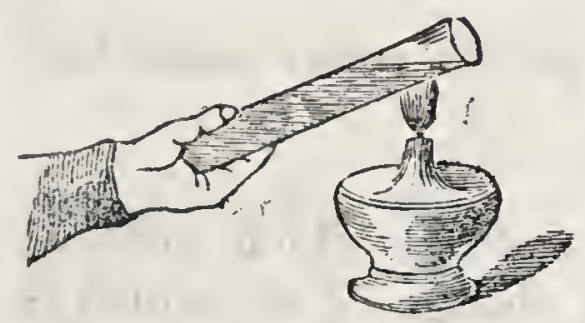

If a tube nearly filled with water is held over a spirit Iamp, as in $f g .54$, in such a manner as to direct the flame against the upper layers of the water, the water will be observed to boil at the top, but remain cool below. If quicksilver, on the contrary, be so treated, its lower layers will speedily become heated. The particles of mercury will communicate the heat to each other, but the particles of water will not do so.

Q. Why is BOILING WATER always in a state of comMotion?

$A$. The currents of hot and cold water rising and falling, together with the escape of steam, produce this effect.

Q. In what way do these ASCENDING and DESCENDING CuRRENTS of water act ?

$A$. The heated water rises through the centre, and the cold water rapidly descends at the sides.

Q. Is it the property of HEATED water to ascend to the surface?

$A$. Yes; and therefore if heat were applied to the top of a kettle, and not to the bottom, the water would never be made to boil.

Q. What is the best way of coolrng liquids?

$A$. By applying cold to the top of the liquid.

Q. On what principle?

$A$. The cold portions descend, and as the warmer ones ascend, they are brought into contact with the cooling substance, and their temperature lowered in consequence. hot?

Q. How is it, then, that a fire lighted in a grate makes the воттом of the stove red-

$A$. The difference results from the difference of substance. Water is a bad, and iron an excellent conductor ; water conveys heat with difficulty, metal readily; water will not diffuse heat in the same way that is done by heated metal; in iron, when one part is heated, the heat is conducted to every part.

Q. If heat be applied to boiling water, is its teMPERATURE INCREASED?

A. No, not if it be possible for steam to escape; the additional heat being carried away by the vapour of steam, and the water still remaining at boiling point.

Q. To what TEMPERATuRE can water exposed to the $\Delta \mathrm{IR}$ be heated under ordinary circumstances?

A. To about $212^{\circ}$ Fahrenheit; at this temperature water passes into steam or vapour.

Q. Can water be heated BEYond $212^{\circ}$ ?

A. Yes; if subjected to sufficient pressure, it can be heated to any extent without boiling. There is no limit to the degree to which 
water may be heated, provided the ressel is strong enough to confine the vapour; but the expansive force of steam is so enormous under these circumstances, as to orercome the greatest resistance which has ever been exerted upon it.

Q. On what principle does soup, or BRoTH, or WATER, thickened with starcis or flour, keep Hот LONGER than plain water?

A. The grease in soup or broth holds the heat for a longer time than plain water; while starch, flour, or any thickening substance, impede the ascending motion of the heated water, and thus retard the escape of heat.

Q. How is AIR heated?

A. By convective currents; that is to say, the air is heated as water is heated, one portion having received a certain portion of heat rising to give place to colder, which, in its turn, is heated, and ascends.

Q. What is the meaning of the word CONVECTIVE?

A. It is derived from the Latin cum vectus, carried with; and is employed to describe this branch of natural philosophy, because the heat is carried with the current.

Q. Do the RAYS of the SUN impart HEAT to the air through which they pass?

$A$. Not to any very great extent. They heat the soil, which communicates to the air the heat it has received; and thus the air is hotter on a sunny than a sunless day.

Q. Does heated Mital grow cold by exposure to the air?

A. Yes; both by radiation and convection.

Q. How by Radiation?

$A$. By giving forth in rays the heat it has received.

Q. How by CONvection?

A. By the air which rests on the iron being heated gradually ascending, its place supplied by cold air, which, in its turn, carries off more heat; and so on till the iron is cold.

Q. Is a liquid COOLED by being $\Lambda$ GTTATED or STIRRED $\Lambda$ BOUT?

A. Yes. Thus the stirring of hot tea brings to the surface more rapidly the hottest particles, thus bringing them in contact with the air, and producing convection with much greater rapidity than would have been the case had the liquid remained quiescent.

Q. How does BLOWING HOT FOOD make it cool?

A. It causes the air (which has been heated by the food) to change more rapidly, and give place to fresh cold air.

Q. If a shutter be closed in the daytime, the stream of light (piercing through the crevice) seems in CONSTLNT AGITATION : WHY is this?

A. Because little motes and particles of dust (thrown into agitation by the violence of the convective currents) are made visible by the strong beam of light thrown into the room through the crevice of the shutte:. 
Q. When Potatoes are boiled, why are those at the Top of the boiler COOKED sooner than those nearer the fire?

A. 1. Because the hottest particles of the vater rise to the top of the boiler, and the coldest particles sink to the bottom; and

2. Because the top of the boiler is always enveloped with very hot escaping steam; in consequence of which the potatoes on the top are subjected to more intense heat than those at the bottom of the boiler.

Q. Why does MILK BoII more QUICKLY than water?

A. Milk is a thicker liquid than water, and, consequently, less steam escapes through the thick liquid (milk) than through the thin liquid (water); therefore the heat of the whole mass of the milk rises more quickly.

Q. On what philosophical principle is an APARTMENT warmed by a STOVE?

$A$. The heat of the stove communicates itself to the surrounding air, which gradually rises, giving place to cold air, which is, in like manner, heated, and ascends. Warmth is thus, by degrees, caused all over the room. As heated air always ascends, the lower the position of a stove, the better adapted is it for effectually heating a room.

Q. Tould not the air of the lower part of a room be heated equally well if the fires were fixed higher up?

A. No; the heat of a fire has very little effect upon the air below the level of the fire; and therefore every fire should be as near to the ftoor as possible.

Q. Our FEET are very frequently cOLD when we sit close by a good fire: explain the reason of this.

A. As the fre consumes the air which passes over it, cold air rushes through the crevices of the doors and windows, and alony the bottom of the room, to supply the deficiency; and these currents of cold air rushing constantly over our fect, deprive them of their warmth.

Q. According to the foregoing illustrations, upon what does the PowEr of liquids to conduct heat DEPEND?

A. Upon the molitity of their particles, as their power to diffuse heat depends on their power of carrying, and not of conducting, it.

Any circumstances which impede the motion of the fluid particles among themselves diminish the diffusing power of the liquid.

Q. Why will sPIRITS boil more quickly than WATER?

A. Because the particles of the spirits are more free to move and concluct heat than the particles of water.

Q. Why does it require a higher temperature to boil oil, molasses, or syrups, than water?

A. Because the oil, molasses, \&c., are viscid fluids, and the particles do not move freely among themselves.

Q. Is the process of coourva in liquids influenced by the same law that regulates their BoILING temperature?

A. Yes; cooling being merely the reverse of heating; those liquids cool quickest whose particles have the greatest freedom of 
motion. Hence, all semifluid masses, such as tar, molasses, thin mush, \&c., retain their heat for a great length of time.

Q. How does the same law explain the fact that very porous Masses and powders, as charcoal, metal filings, sawdust, sand, \&c., conduct heat more suowur than denser masses?

A. Because their interstices are filled with air, which scarcely conducts heat, and which, by the structure of the substance, has no freedom of motion or circulation by which it might carry away the heat.

\section{SECTION III.-RADIATION์.}

Q. What is meant by RADIATION of MEAT?

A. The emission of rays of heat in all directions.

When the hand is placed near a hot body suspended in the air, a sensation of warmth is perceived, even for a considerable distance. If the hand be held beneath the body, the sensation will be as great as upon the sides, although the heat has to shoot down through an opposing current of air approaching it. This effect does not arise from the heat being conveyed by means of a hot current, since all the heated particles have a uniform tendency to rise ; neither can it depend upon the conducting power of the air, because aerial substances possess that power in a very low degree, while the sensation in the present case is excited almost on the instant. This method of distributing heat, to distinguish it from heat passing by contact or conduction, is called radiation.

Q. How do we designate HEAT so distributed?

A. As radiant or radiated heat.

Q. On what is Radiatios dependent?

A. On unevenness of surface, as in proportion to the number of points from which heat can escape, is the power of radiation increased.

Q. Is heat radiated from BURNING FUEL?

$A$. Yes; we feel hot on standing near a fire.

Q. Do not the FACE and HANDS feel the heat more than the rest of the body?

$A$. The face and hands being generally uncovered, the heat is felt by them more readily ; clothing, being in most cases a bad conductor, prevents the rapid transmission of heat to other parts of the shin.

Q. Are absonption and radiation of heat in any way CONNECTED ?

$A$. Substances which radiate the most heat, absorb the most heat ; and those which radiate the least, absorb the least.

Q. What other SUBSTANCES beside the SUN and BURNING FuEL radiate heat? heat.

A. Nearly all dull and dark substances are excellent radiators of

The action of a blackened surface of tin being assumed as 100 , it has been found that that of a steel plate was 15 ; of clean tin, 12 ; of tin scraped bright, 16 ; when scraped with the edge of a fine file in one direction, 26 ; when scraped again across, about 13 ; 2 surface of clean lead, 19 ; covered with a gray crust, 45 ; a thin crust of isinglass, 80 ; resin, 96 ; writing paper, 98 ; ice, 85 .

Q. What is meant by being a "bad RaDIutor of heat?"

$A$. To radiate heat is to throw off heat by rays, as the sun; a polished tin pan does not throw off the heat of boiling water from its surface, but keeps it in. 
Q. Why is a TIN BOX (filled with HOT WATER) employed as a FOOT-WARMER ?

A. Because potished tin (being a bad radiator of heat) lieeps hot a very long time, and warms the feet resting upon it.

Q. Why would the tin foot-warmer get CoLD SOoNER if the POLISH were INJURED ?

A. Because polished tin throws off its heat very slowly; but dull, scratched, painted, or dirty tin, throws off its heat very quickly.

Q. Why are TIN FOOT-WARMERS generally covered with FLANNEL ?

A. 1. That the polish of the tin may not be injured;

2. Because the flannel (being a very bad conductor) serves to keep the tin hot longer; and

3. Lest the conducting surface of the tin should feel painfully hot.

Q. Why does sNow (at the foot of a Tree or WALL) melt sooner than that in an open place?

A. Because the tree or wall melts the snow by radiating heat into it from beneath.

Q. How is hot iron cooled by Radiation?

A. While its heat is being carried off by "convection," the hot iron throws off heat (on all sides) by radiation also.

Q. Why should BLACK LEAD be employed to blacken the FLLES connected with stoves ?

A. As black lead radiates heat most freely, the heat is thereby. diffused more equally and more rapidly through the room.

Q. Is a DULL, BLACK TEAPOT, Or a BRTGHT METAL TEAPOT, the best for making TEA?

$A$. The brightly polished metal, which, being a very bad radiator of heat, keeps the water hot much longer.

Q. Why is boiling water EEPT HOT in a BRIGHT IETAL pot better than in an earthen vessit?

A. Decause hright métal (jeing a vad radiator) wili not throw off from its surface the heat of the boiling water.

Q. What is the DIFFERENcE in degrees in the radiating powers of a black stone-ware teapot, and a polished metal one?

$A$. The earthen teapot will radiate one lundred degrees of its heat in the same manner that the pot of polished metal will radiate only twelve degrees.

Q. Are bright DISIr-COVERS serviceable on the same account?

$A$. Yes; being bad conductors of heat, they prevent its escape, and thereby keep the cooked victuals hot much longer than would be possible without.

Q. What would be the result if these MFAT-COVERS were left DULI Or SCRATCIIED?

A. It would produce entirely the contrary effect from that which was intended, making the food cold instead of keeping it hot, by absorbing heat from the smoking victuals.

Q. Why should a SILVER MEAT-COVER be PLAIN, and not CHASED ?

A. Because a chased meat cover would rudiate heat derived from the food; and (instead of keeping it hot) make it cold.

Q. How could CHasing produce this effect?

$A$. Chasing makes the surface rough, and rough surfaces throw off the heat most readily. 
Q. Has courour any influence upon the radiating power of a surface?

A. It has generally been supposed to have a very sensible influence, but recent experiments have furnished conclusive proof that colour alone has no influence.

Q. Of what NATURE is the heat received from the sun?

A. It is all radiant heat.

Q. How does the heat radiated by the sun DIFrER from other radiant heat?

A. The heat of the sun passes through air, glass, water, and transparrent bodies generally very readily, while other radiant heat, though not obstructed by air, is almost totally intercepted or absorbed in passing through any of the various substances named.

Q. Is it necessary that there should be some MEDIUM of communication in order that heat shall be radiated from one body to another?

A. From the fact that radiant heat passes freely through a vacuum, it is inferred that the presence of a medium is not necessary to its passage.

Q. What two THEORIEs have been proposed to account for radiation?

A. Those of Pictet and Prevost, two eminent French philosophers.

Q. What is the theory of Pictet?

A. It supposes that bodies of equal temperature do not radiate at all; and when the temperature is unequal, the hotter gives calorific rays to colder bodies, till an equilibrium is established, at which moment the radiation ceases.

Q. What is the THEony of Prevost?

A. It supposes that radiation goes on at all times and from all substances, whether their temperature be the same or different from that of surrounding objects: therefore the temperature of a body falls when it radiates more heat than it absorbs; its temperature is stationary when the quantities emitted and received are equal; and it grows warm when the absorption exceeds the radiation. Of the two theories, that of Prevost is most generally adopted.

\section{CHAPTER XVI.}

\section{PHENOMENA AND PRODUCTION OF DEW.}

Q. What is DEW?

A. A watery vapour, which floating in the air is condensed into drops during the night.

Q. By what means is it CONDENSED? itself.

A. By coming into immediate contact with bodies colder than

Q. Does this account for the ground being COVERED with DFW?

$A$. It does: in the evening the earth is made so cold by radiation, that the warm vapours of the air are chilled and condensed into dew. 
Q. How do you account for the EARTH being colcier than the AIR after sunset?

A. On the principle that the air radiates less freely than the earth; thus the earth is colder than the air after sunset, though it may have been warmer than the air throughout the day.

Q. Why is the EARTH warmer than the AIR?

$A$. The earth absorbs the sun's rays, by which it is warmed; but the sun's rays pass through the air without effecting any perceptible difference in its temperature.

Q. When does the most DEw fall?

A. On calm and serene nights.

Q. On what principle do you account for this?

$A$. On a fine night the ground radiates more heat, and, being thus cooled, chills the vapour into dew; on a cloudy, murky night, the heat not escaping from the earth, the vapour in the air is not chilled, and consequently there is no dew.

Q. How do clouds ARREST or PREVENT the radiation of heat from the earth ?

$A$. The lower surfaces of the clouds turn back the rays of heat as they radiate or pass off from the earth, and prevent their dispersion into space.

Q. What is RAIN?

A. Rain is water taken up into the atmosphere in the form of vapour, and returned to the earth in liquid drops.

Q. What is the difference between RAIN and DEW?

$A$. Dew is condensed vapour on the surface of the ground; rain, liquid drops falling from a height.

Q. What is the cause of both DET and RAIN?

$A$. The condensation of vapour in the air.

Q. Why does $\triangle B U N D A N C S$ of $D E W$ in the morning indicate that the day will be FINE?

A. Because dew is never deposited in dull, clouly weather, but only in very clear, calm nights, when the cold currents of air are not mixed with those of a warmer temperature.

Q. When is DEW the most abundant?

A. In situations the most exposed. Thus, there is more dew in the country than the town; and thus, too, there is scarcely any dew to be found under a leafy tree.

Q. How is this to be accounted for?

$A$. Because any shelter arrests radiation from the earth, and prevents the surface cooling down sufficiently to chill the vapour into dew. day?

Q. Why, in travelling, do we experience less dust in the NIGHT-TIJE than during the

A. 1. Because the dews of night moisten the dust, and prevent its rising into the air ; and

2. As the surface of the earth is colder than the air after sunset, the curronts of the wind will incline downwards, and tend rather to press the dust down than to buoy it up. 
Q. Why, on a WINDY NIGHT, is there No DEW ?

A. Because the wind disturbs the radiation of heat, and thus diminishes the deposition of dew, and evaporates the moisture as fast as it is deposited.

Q. Why are substances which radiate the heat yost FREELY always the most TIICKLY COVERED With DEW ?

A. Because they are the coldest substances, and therefore condense vapour most readily.

Q. Are soxe bodies covered with DEW sooner than others?

$A$. Yes; because they are much cooler during the night, in consequence of radiating heat more freely.

Q. What circumstances farour the FORMATION of DEW"?

A. All circumstances that favour racliation equally contribute to the formation of dew. Any substance which is a good radiator will consequently be covered with dew.

Q. What bodies radiate IEAT most Freely?

A. Glass more than the metals; grass, wood, and vegetation generally radiate freely.

Q. Do ALL plants radiate EQUALLY WELL?

A. No; the holly-hock radiates better than the laurel.

Q. What BENEFICIAI RESULts ensue from this RADIATORY power of plants?

A. They receive thereby the moisture which is so essential to them, for by radiating heat they chill the vapours in the air, and convert them into dew.

Q. Then all substances SIMILARLY EXPosed to the fall of DEW are not EqUALLY AFFECTED?

$A$. No; they receive dew in proportion as they radiate heat. Thus, while a grass plot may be thick with dew, a gravel-walk, a piece of polished metal, or woollen cloth, may be almost dry.

Q. Does the fall of DEW depend ALONE on the RADIATING Pow ER of the FARTII?

$A$. No; it also depends on the amount of vapour in the atmosphere.

Q. What does a HFAYY FALL of DEW INDICATE?

A. It may be considered as a prognostic of rain, as it shows us the quantity of aqueous vapour which is in the air.

Q. Where does MoST DEW fall?

A. More dew falls on cultivated soils than on barren lands, as the loose, porous earth of cultivated soils radiates more freely, and condenses the vapour more rapidly.

Q. Why do our CLOTHES generally FEEL DAMP after walking in a fine evening in SPRING or $\triangle$ UTUMN?

A. Because the vapour (condensed by the cold earth) lights upon them like dew.

Q. When is DEW most coprousLy distilled?

A. After a hot day in summer or autumn, especially if the wind blows over a body of water. 
Q. Why is DEW distilled most copiousur after a not day?

$A$. Because the surface of the hot earth radictes heat very freely at sunset, and (being made much colder than the air) chills the passing vapour, and condenses it into dew.

Q. Why is there LESS DEW when the wIND blows across the uand than when it blows over a body of WATER?

$A$. Because the winds which blow across the land are dry and arid; but those which cross the water are moist and full of vapour.

Q. How does the DRYNESS of the wind PREVENT DEW-FALLS?

A. As winds (currents of air) which blow over the land are very dry, they imbibe the moisture of the air; in consequence of which there is very little left to be condensed into dew.

Q. How does the Morsture of the wind pronote dew-falls?

$A$. As winds which blow over water are saturated with vapour, they require very little reduction of heat to cause a copious deposition of dew.

Q. Does not $\Delta$ IR radiate heat, as well as the EARTH and its various plants?

A. No; the air never radiates heat; nor is the air made hot by the rays of the sun.

Q. Is the DEIT of EVENING INJURIOUS to HEALTH?

Yes; being laden with noxious vapours from the earth.

Q. Why is MEAT very subject to TAINT on, a roonurgrre night?

$A$. Because it radiates heat very freely in a bright moonlight night; in consequence of which it is soon covered with dew, which produces rapid decomposition.

Q. Why is the air in immediate contact with the earth, on a clear night, cooler than the air at a little distance from the surface?

A. Because it parts with its heat to the earth, which in turn loses it by radiation.

Q. Why are shrubs and low plants more liable to be frost-bitten than trees?

$A$. Because they do not rise far above the surface of the earth; and (as the air contiguous to the earth is colder than the air which is more elevated) therefore the low vegetation is often frost-bitten, when the foliage of high trees escapes uninjured.

Q. How can a thin covering even of suSLIN protect trees from FrosT?

$A$. Because any covering prevents the radiation of heat from the tree; and if trees are not cooled down by radiation, the vapour of the air will not be frozen as it comes in contact with them. For this purpose matting is used by gardeners.

Q. Why is the BASS or CANVASS itself (which covers the tree) always DRENCrned with DEW ?

A. Because it radiates heat both upwards and downwards; in consequence of which it is so cooled down that it readily chills the vapour of the air into dew.

Q. Why is there LESS DEW when the WIXD is EASTERLY than when the wind is IFESTERLY ?

A. Because easterly winds cross the continent, and (as they pass 
over land) are dry and arid; but westerly winds cross the Atlantic Ocean, and (as they pass over water) are moist and full of vapour.

Q. How does the DRYNESS of an easterly wind PREVENT DEW-FALLS ?

A. As easterly winds are very dry, they imbibe the moisture of the air ; in consequence of which, there is very little left to be condensed into dew.

Q. How does the DAMPNESS of a westerly wind Prosrote deposition of dew?

A. As westerly winds are saturated with vapour, they require very little reduction of heat to cause a copious deposition of dew.

Q. By what is MIST occasioned ?

A. Mist, or earth fog, as it is sometimes called, is the condensed vapour of the air, chilled with greater rapidity than can possibly be deposited in the dew, and which, consequently, settles down on the earth as a fog or mist.

Q. This MIST seems to RISE MIGHER and HIGHER, and yet remains quite as dense below as at first : explain the cause of this.

$A$. The air resting on the earth is first chilled, and chills the air resting on it; the air which touches this new layer of mist being also condensed, layer is added to layer; and thus the mist seerns to be rising, when (in fact) it is only deepening.

Q. Can the dew properly be said to "FALL?"

A. No; dew is always formed upon the surface of the material upon which it is found, and does not fall from the atmosphere.

Q. Does the colour of an object influence the deposition of dew?

A. It does to a considerable extent.

Q. How can this be shown?

$A$. If we take pieces of red, black, green, and yellow glass, and expose them when the dew is condensing, we shall find that moisture will show itself first on the yellow, and then on the green glass, but that none will appear on the red or black glass. The same thing will take place if we expose coloured fluids in white glass bottles.

Q. Is the cause of this phenomenon UNDERSTOOD?

A. It is not.

It is not explicable upon the idea of difference in mechanical arrangement, as it is quite independent of surface condition.

Q. In a clear summer night, when dew is depositing, what is generally the DIFFerExce of TEMPERATURE between a plat of grass and the air at some distance above it?

A. A thermometer laid upon the grass will sink nearly twenty degrees below one suspended in the air at some height above.

Q. Why is the deposition of dew rarely observed in the close and sheltered sTnEETs or oITIES?

A. Because there the objects are necessarily exposed to each other's radiation, and an interchange of heat takes place, which maintains them at a temperature uniform with the air. 
Q. What do we mean when we speak of the "DEW-POINT?"

$A$. It is the clegree indicated by the thermometer when dew begins to be deposited.

Q. Is this point a constant or INVARTABLE one?

A. No; dew is only deposited when the air is saturated with vapour, and the amount of moisture required to saturate air of high temperature, is much greater than air of low temperature.

Consequently, air, which contains moisture to its utmost capacity at a given temperature, must necessarily deposit some of it when its temperature is diminished.

Q. When will there be the GREATIST, and when the LEAST, difference between the temperature of the air and the dew-point?

$A$. If the saturation be complete, the least diminution of temperature is attended with the formation of dew; but if the air is dry, a body must be several degrees colder before moisture is deposited on its surface; and, indeed, the drier the atmosphere, the greater will be the difference between the temperature and its dew-point.

Q. How can we cause the production of dew in the DAYTIME at any season?

A. By bringing a tumbler of cold water into a warm room.

Q. How does this cause the production of dew?

$A$. The tumbler of water cools the air contiguous to it below the dew-point, and dew consequently forms upon it.

Q. When is dew converted into frost ?

$A$. If the temperature of the earth, or of the vessel, sink to the freezing point or below, the moisture will be deposited as before; but by freezing, it assumes the solid form, and is called frost.

Q. What occasions the ROUNDNESS of a DEWT-DROP ?

A. Every particle in the drop being equally balanced.

Q. Are not DEW-DROPS FLATTENED?

A. Yes; but this arises when two or more drops commingle, and so make one flattened drop.

Q. What is such a dew-drop Called?

A. A spheroid.

Q. Why is it that certain species of vegetation, as CABBAGE-PLANTS, PoppIES, and the PETALS Of a ROSE, REPEL WET ?

A. On account of their possessing some peculiar property which repels wet; the fine waxen porrder of the cabbage-plant, and the volatile oil of the rose, answer this purpose. On the same principle, aquatic birds dive under water without being wetted, on account of the oily secretion which covers their feathers.

Q. What is the FIGURE which water always assumes when unsupported, or supported on a surface having little attraction for it?

A. The figure of a sphere. This figure becomes more or less globular or spheroidal in its shape, as the attraction of the substances upon which it is received increases or diminishes. 
Q. What is the Forus of a drop of rain when descending in the air?

A. A sphere.

Q. Why should drops of water, resting upon surfaces which have no AFFinitr for them, assume a spheroidal shape?

$A$. Because such surfaces not having so great an attraction for the drops of water as the particles of water have for each other, the drops tend to preserve, as nearly as possible, the spheroidal form which they would have if entirely unsupported, as when falling as drops of rain.

Q. Is dew ever formed upon the SURFAcE of water?

$A$. The formation of dew upon ships which traverse the vast solitudes of the ocean has never been noticed; and it has been ascertained by experiment that even a small quantity of water gains no weight by exposure during a single night.

Although dew does not appear upon ships at a great distance from land, it is freely deposited on the same vessels arriving in the vicinity of terra firma. Thus, navigators who proceed from the Straits of Sunda to the Coromandel coast, know that they are near the end of the voyage when they perceive the ropes, sails, and other objects placed on the deck, become moistened with dew during the night.

Q. Why does not dew form upon the SURFACE of WATHR?

A. Because whenever the aqueous particles of the surface are cooled, they become heavier than those below them, and sink, while warmer and lighter particles rise to the top. These, in their turn, become heavier, and descend; and the process, continuing throughout the night, maintains the surface of the water and the air at nearly the same temperature.

Q. Does dew deposit upon surps at sea?

$A$. It appears, from the observations made by the United States. Exploring Expedition, and from other sources, that on the ocean heavy deposits of dew sometimes occur upon the decks of vessels.

Q. Why are the Exposed parts of the human body never covered with dew?

A. Because the vital heat, varying from $96^{\circ}$ to $98^{\circ}$ Fahrenheit, effectually prevents such a loss of warmth as is necessary to its production.

Q. Why is dew more abundant in the EARLY part of $\triangle$ UTUMN than at any other period?

$A$. The days being then warm, the nights cold, and the vapour in the air plentiful, the same degree of cold condenses a greater quantity of vapour than at any other period.

Q. In what cousrries are the dews most copious and abundant?

A. In tropical climates.

Q. What is the REASON of this?

$A$. Because in those countries there is the greatest difference between the temperature of the day and that of the night.

The development of vegetation is greatest in tropical countries, and a great part of the onocturnal coling is due to the leaves, which present to the sky an immense number of thin bodies, having large surface, well adapted to radiate heat.

The annexed figure, in which arrows denote the direction of heat, and the numerals the temperatures of the earth and air under different circumstances, will render the foregoing questions on the radiation of heat, and the production of dew and frost, more intelligible. 
Fig. 55.

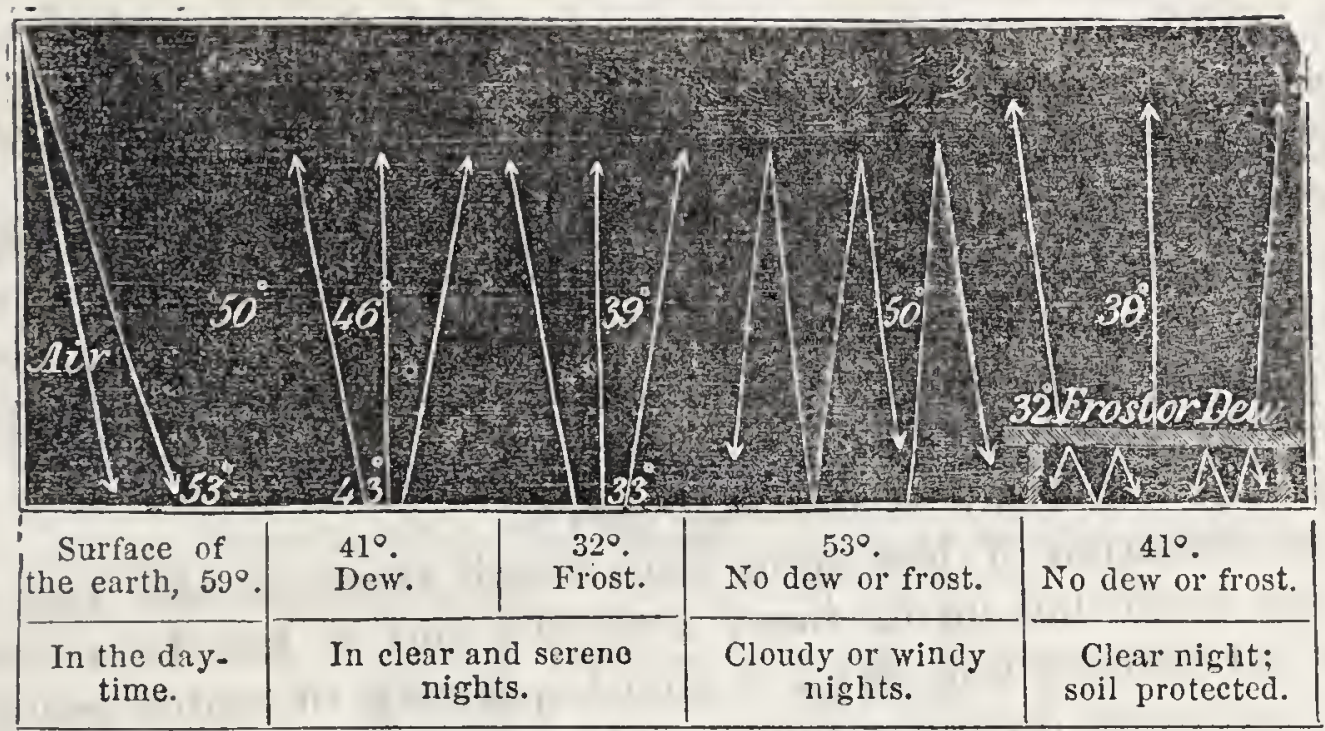

The figures in the middle of the above diagram represent the temperature of the air at a distance from the surface of the earth ; the figures in the margin, the temperature of the air adjoining the surface of the earth ; the figures below the margin, the temperature of the earth itself. The directions of the arrows represent the radiation and reflection of the heat.

Q. Does dew fall upon the islands of Pocynesia?

A. If at all, in very small quantities.

Q. What is the reason of this CURIOUS FACT ?

$A$. The air over the vast ocean in which these islands are situated, preserves a nearly uniform temperature day and night. The islands are comparatively of small extent, and the stratum of air cooled by the contact of the soil is warmed by mixing with the air that is constantly reaching it from the sea. This prevents a depression of temperature in the air sufficient to cause a deposition of dew.

Q. Who first originated the theory, and experimentally demonstrated the cause of the phenomena of dew?

4. Dr. Wells, an eminent English philosopher.

\section{CHAPTER XVI.}

\section{REFLECTION, ABSORPTION, AND TRANSMISSION OF HEAT.}

Q. In what MANNER is heat emitted from a body distributed ?

A. It passes from the surface in right lines, equally in all directions, like radii drawn from the centre to the surface of a sphere: so that a thermometer placed at the same distance on any side would stand at the same point, if the effect of the ascending current of hot air could be averted.

Q. When rays of beat fall upon the SURFACE of a solid or liquid substance, what is the effect?

A. They may rebound from its surface, or be reflected; they may pass directly through it, or be transmitted; ther may be received into its substance, or be absorbed. 


\section{SECTION I.-REFLECTION.}

Q. What is meant by the REFLECTION of heat?

A. Heat is said to be reflected when it is caused to rebound, or bo thrown back, from the surface of a reflecting body.

Q. In what MANNER is heat reflected?

$A$. In right lines, in the same manner that light is reflected.

This may be rendered evident by reference to the apparatus represented in fig. 56 .

Fig. 56.

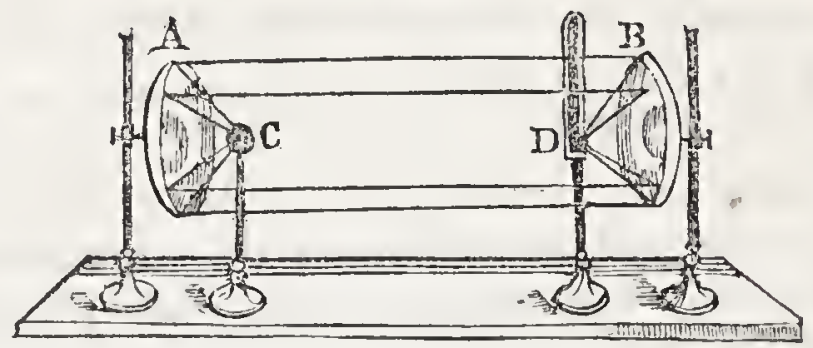

It consists of two concave mirrors, A and B, of planished tin or plated copper, abour one foot in diameter, and placed exactly opposite each other, at the distance of about ten feet. In the focus of one mirror, at C, must be placed a heated body, as a ball of iron; and in the focus of the other mirror, at $\mathrm{D}$, a thermometer. The rays of heat, then, impinging on the mirror $A$, are reflected through the air to the rnirror $B$, whence they converge to its focus at $D$, and produce an effect on the thermometer proportioned to the degree of heat of the iron ball or other heated body.

Q. Are the best ABSORBers the best REFLECTons of heat ?

A. No; all light colours and bright surfaces refiect heat best, but at the same time are the worst absorbers of it; the principles of absorption and reflection are so different that they cannot exist to any great extent in the same substance.

Q. What is the DIFEERENCE between REFLECTION and $\triangle$ BSORPTION?

A. Absorption sucks or draus in heat to itself; reflection throws off heat from itself; so that absorption and reflection cannot be going on at the same time in the same body.

Q. Is not METAL used as a REFLECTOR?

A. It is; polished metal being an excellent reflector of heat.

Q. Is not POLISHED metal also a conductor of heat?

$A$. Only when heat is brought into actual contact with it.

Q. What is MEANT by ACTUAL CONTACT?

$A$. Such as thrusting a piece of metal into the fire.

Q. By what OTHER MEANS is HEAT communicated to the metal ?

$A$. When polished metal is employed as a reflector the heat falls upon it in rays, and is reflected back again with an intensity proportionate to the brightness of the metal.

Q. Why should a REFLECTOR be kept very CLIAN, and free from all SCRATCHES ?

$A$. Because if a reflector were spotted, dull, or scratched, it would absorb heat instead of reflecting it; and consequently would be of no use whatever as a reflector. 
Q. Why will not a POLISHen tIN PAs bake bread as well as an IroN one?

$A$. Because the bright metal reflects the heat, and therefore will not brown the crust which surrounds the bottom and sides of the pan; consequently, the top of the bread would be burnt before the bottom and sides of the loaf were brown.

Q. Are LIGHT COLOURS good reflectors of HEAT ?

$A$. They are, and on this account white dresses are commonly worn in summer time.

Q. Are not WHITE DRESSES SO WARM as DARK ones?

$A$. No; dark colours absorb heat; light colours reflect it; therefore light colours are cooler to wear than dark colours.

Q. Why do not the solar rays, eren in the hottest day, scelT the srow upon the tops of high mountains, which are nearer to the sun than the level portions of the earth ?

$A$. Because they only heat those bodies which can absort their warmth, as the rough surface of the earth. The snow is indeed struck by the rays of the sun, but being a white and shining body, it refects them, and remains cold.

$Q$. How is it that the summits of lofty mountains, as the Alps, remain frozen even in the warmest weather?

A. Two reasons may be assigned: heat is absorbed by the air which flows up the sides of the mountain, and as the air is heated, not by the passage of the solar rays, but by contact with the earth, and as the summit of a lofty mountain presents a very limited space for such contact, the air remains intensely cold.

Q. Can we SEE the rays of reflected heat?

$A$. No; the rays of heat are invisible, and we know them only by their effects; yet they are governed in many respects by the same laws which belong to light, are reflected at the same angles, and may be concentrated in a focus.

Q. EnuMerate some of the best reflectors of heat.

$A$. Of 100 rays falling at an angle of $60^{\circ}$ from the perpendicular, polished gold will reflect 76 ; silver, 62 ; brass, 62 ; brass without polish, 52; polished brass varnished, 41; looking-glass, 20; glass plate blackened on the back, 12 ; and metal plate blackened, 6 .

\section{SECTION II.-ABSORPTION.}

Q. What is MeANT by condecting heat?

$A$. The transmission or conveyance of heat from one body to another.

Q. What is MEANT by ABSORBING heat?

A. The drawing in or sucking up of heat into any substance. Thus blotting-paper absorls ink.

Q. Are good $\triangle$ BSORBERS also good CONDUCToRs of heat?

A. No; black cloth absorbs heat, but does not conduct it; iron is a good conductor, but a bad absorber. A piece of metal, as a needle, 
heated at one end conducts heat to the other end; but a piece of black cloth, though intensely hot at one part, does not conduct heat to another part.

Q. If a piece of BROWN PAPER be submitted to the action of a BURNING-GLASS, it will eatch fire much sooner than a piece of WHITE PAPER would; explain the reason.

A. Because white paper reflects the rays of the sun, or throws them back; in consequence of which it appears more luminous, but is not so much heated as dark brown paper, which absorbs the rays, and readily becomes heated to ignition.

Q. Why is the TEMPERATURE of ISLANDS more EQUABLI than that of CONTINENTS?

$A$. Because the vater around the island absorls the extreme heat of summer, and gives out heat to mitigate the extreme cold of winter.

Q. ISLANDS are WARMER in winter than continents: explain the reason of this.

A. Unless the sea be frozen (which is rarely the case), it is warmer than the frozen land; and the warmth of the sea-air helps to mitigate the intense cold of the land air.

Q. How does the ceaseless CHANGE of air tend to DECREASE the WARMTII of a naked body?

A. The air (which cases the body) absorbs as much heat from it as it can while it remains in contact; being then blown away, it makes room for a frest coat of air, which readily absorbs more heat.

Q. Does the $\Delta I R$ which encases a naked body become (by contact) as wARM as the BODY itself?

A. It would do so if it remained motionless; but as it remains only a very short time, it absorbs as much heat as it can in the time, and passes on.

Q. Why does FANNing the face in summer make it coon?

$A$. Because the fan puts the air in motion, and makes it pass more rapidly over the face; and (as the temperature of the air is always lower than that of the human face) each volume of air carries off some portion of its heat.

Q. Why do persons FAN THEMSelves in hot weather?

A. That fresh particles of air may be brought in contact with their faces by the action of the fan, and as every fresh particle of air absorbs some heat from the skin, this constant change makes them cool?

Q. Does a fan coor the AIn?

$A$. No; it makes the air hotter by imparting to it the heat out of our face; but it cools our face by transferring its heat to the air.

Q. How does Fanning the face increase the HEat of the air?

$A$. By driving the air more rapidly over the human body, and causing it, consequently, to absorb more heat.

Q. If fanning makes the $\Delta I R$ HoTTER, why can it make a PERSON feel COoLER?

A. Because it takes the heat out of the face and gives it to the air.

Q. Why does WIND generally feel Coor ?

$A$. Because it drives the air more rapidly over our body, and this rapid change of air draws off a large quantity of heat. Every fresh 
gust of air absorbs a fresh portion, and the more rapid the succession of gusts, the greater the quantity of heat absorbed.

Q. If the AIR were HoTTER than our body, would the WIND feel COOL?

$A$. No; the air would feel insufferably hot, as it would add to the heat of our body, instead of diminishing it.

Q. Is the AIR EVER as HOT as the human BODY?

$A$. In some climates it is; and when that is the case, the heat is almost insupportable.

Q. Does SOOT absorb HEAT?

A. Yes; and by thus conducting the heat more quickly from the fire to the water, a kettle covered with soot boils faster than one which is perfectly clean.

Q. Is this the reason of an OLD KETTLE boiling faster than a NEW one?

$A$. It is ; the old kettle being covered with soot, and thus absorbing heat more rapidly.

Q. Why do we wear WHITE LINEN and a BLACK outer DRess if we want to be warm?

$A$. Because the black outer dress quickly absorbs heat from the sun, and the white linen (being a bad absorbent) abstracts no heat from the warm body.

Q. Which are the WARMEST colours?

A. Black is the varmest, and then those nearest to black, as dark blue and green.

Q. Why are DARE COLOURS SO much WARMER than LIGHT ones?

A. Because they absorb heat more abundantly than light ones. The order may be thus arranged: 1 , black (warmest of all) ; 2, violet; 3 , indigo ; 4 , blue ; 5 , green ; 6 , red; 7 , yellow ; and 8 , white (coldest of all).

Q. What SoRT of GLOTES are the Coolest for summer wear?

A. Lisle thread, on account of their absorbing the perspiration of the hands, and conducting away the heat.

Q. Why does HOARFROST remain on LIGHT-COLOURED STONES long after it has melted from GRASS and GRAVEL WALKS?

$A$. Because the stones (being white) do not readily absorb heat, like the darker grass and gravel; in consequence of which, they remain too cold to thaw the frost congealed upon their surface.

Q. Why does the BLACK skin of a NEGRO NEVER SUN-BURN or BLISTER with the hot sun?

$A$. Because the black colour absorbs the heat, conveys it below the surface of the skin, and converts it into sensible heat and perspiration. sun?

Q. Why does the WIITE EURopean SEIN BLISTER and BURN when exposed to the hot.

A. Because white will not ubsorb heat, and therefore the liot sun rests on the surface of the skin and burns it.

Q. By what SIMPLE EXPERIMENT can we ascertain that BuACE prevents the SEIN from lxing scorched or blistered by heat?

A. By putting on one hand a white.glove, and on the other a black 
glove : the latter will feel the warmer, but will not be sunburnt; the former will feel the cooler, but will be sunburnt.

Q. Why does a SAUCEP $\Lambda \mathrm{N}$ which has been USED boil in a shorter time than a NEW ONE?

A. Because the bottom and sides are covered with soot; and black soot rapidly absorbs the heat of the glowing coals.

Q. Why should the uID of a SAUCEPAN be clean and BRIGHT?

A. Because it cannot absorb heat, as it does not come in contact with the fire; and (being bright) it will not suffer the heat to escape by radiation.

Q. In what state should a SAUCEPAN be, in order that it may boil Mone QUTCKLY ?

A. All those parts which come in contact with the fire should be covered with soot, or be black, in order to absorb heat; but all the rest of the saucepan should be as bright as possible, to prevent the escape of heat by radiation.

Q. Why should Nor the воттом and sides of a kettle be cLEANED and PoLishred?

$A$. Because they come in contuct with the fire; and (while they are covered with black soot) absorb heat freely from the burning coals.

Q. Why should the UPPER PART of a saucepan or kettle be BRIGHTLY POLISHED?

$A$. Because thereby the heat is kept in, and not suffered to escape by radiation.

Q. What curious CHANGE takes place in the character of solar heat when it has been RADTATED from material substances?

A. Whe direct rays of solar heat produce less effect upon snow than those which are radiated from the trunks of trees, \&c.; and it can be shown that these secondary radiations are more abundantly absorbed by snow or white bodies than the direct solar rays themselves.

Q. What EXPERIMunT proves this fact?

A. If a blackened card be placed upon snow or ice in the sunshine, the frozen mass underneath it will be gradually ithawed, while that by which it is surrounded, although exposed to the full power of solar heat, is but little disturbed. If, however, we reflect the sun's rays from a metal surface, an exactly contrary result takes place; the uncovered parts are the first to melt, and the blackened card stands high above the surrounding portion.

Q. How can this phenomenon be explained?

A. The cause is not known. Science here discloses to us an intimate connection between the most ethereal and grosser forms of matter. "Heat, by touching the earth, appears to become more earthlike."

Q. Why do most of the animals inhabiting the frigid zones have wIIre fur, hair, or feathers?

A. Because white radiates and absorts but little heat.

Q. What RaLATION exists between the power of bodies to absorb and commuinicate heat?

A. Those bodies which absort heat freely, also part with it most rapidty; that is, they are sooner heated and more speedily cooled than other bodies. 
Q. At what TEMPERATURE do metals burn when handled?

A. Metals cannot be handled when raised to a temperature of more than one hundred and twenty degrees.

Q. At what temperature does water scald?

A. At one hundrec and fifty degrees.

Q. To what extent can the human system sustain the influence of beated air?

A. Workmen enter ovens, in the manufacture of moulds of plaster of Paris, in which the thermometer stands $100^{\circ}$ above the temperature of boiting water, and sustain no injury.

Q. Why is there so GREAT a DIFFERENCE between the burning temperature of metals and air?

A. The metals absorb heat quiclily, and part with it freely; the air absorbs heat very slowly, and does not readily part with it.

Q. What currous EXPERIMENT was made by Sir Joseph Banks in reference to the influence of heated air?

$A$. He entered an oven heated $52^{\circ}$ above the boiling point, and remained there some time without inconvenience. During the time, eggs, placed on a metal frame, were roasted hard, and a beefsteak was overdone. But though he could thus bear the contact of the heated air, he could not bear to touch any metallic substance, as a watch-chain, money, \&c.

\section{SECTION III.-TRANSMISSION OF HEAT.}

Q. What CLASS of BODIES interfere most with the transmission of heat?

A. Transparent media of great density, as the diamond, rockecrystal, gluss, and water. Heat passes freely through transparent bodies of little density, as the air, or the various gases.

Q. What SUBSTANCES permit the heat to pass most freely through them?

A. Rock-salt admits the passage of heat more readily than any other known solid; clear glass arrests more than half the incident heat. It is remarkable that transparent alum and pure water intercept more of the rays than the deepest coloured and even opaque glasses. Solids and liquids differ in transmissibility to rays of heat in the same manner as they differ in their action on light.

Q. Does heat, accompanied by light, pass MOKE READILY through dense, transparent bodies than heat without light?

$A$. Yes; the solar rays pass readily through glass, water, \&c., the heat and light being refracted in their passage, as is shown by the action of a burning-glass; but the result is very different when the heated body is not luminous. 


\section{CHAPTER XVIII.}

\section{EFFECTS OF HEAT.}

Q. What effect has HEAT upon substances generally?

A. It expands them, or enlarges their dimensions.

Q. Are the DIMEsions of every kind of matter regulated by heat?

A. They are; its increase, with few exceptions, separates the particles of bodies to a greater distance from each other, producing expansion, so that the same quantity of matter is thus made to occupy a larger space; the diminution of heat has an opposite effect.

Q. Is the For of bodies dependent on heat, and how is this shown?

A. The form of bodies is dependent on heat. This is seen by the fact that by the increase of heat, solicls are converted into liquids, and liquids are dissipated into vapour ; by its decrease, vapours are condensed into liquids, and these become solid.

Q. If matter ceased to be INFLUFNCED by heat, what would be the effect?

A. All liquids, vapours, and doubtless even gases, would becorne permanently solid, and all motion on the surface of the earth would be arrested.

Q. Does heat exert an Influence over most chemical phenomena?

A. Yes; there is scarcely any chemical action which is not in some degree modified by this principle. By its agency, bodies previously separate are made to combine, and the elements of compounds are disunited. An undue proportion of it is destructive to all organic and most mineral compounds.

Q. What are the THREE most apparent EFrECTS of heat, so far as relates to the form and dimensions of bodies?

A. Expansion, Tiquefaction, and vaporisation.

\section{SECTION I.-EXPANSION.}

Q. Is the AIR EXPANDED by HEAT?

A. It is, as may be seen by partially filling a bladder with air, tying it at the neck, and laying it before the fire.

Q. What EFEECT will he produced?

A. The air within the bladder will expand, until the bladder bursts.

Q. How is this expansion IFFECTED?

A. The particles of air are driven apart from each other; consequently, they occupy more room than before.

Q. Does heat Expand all matter?

A. Yes; every thing (that man is acquainted with) is expanded by heat. 
Q. Does this account for cuestruts Cracking when Roasted?

$A$. It does; the air contained within the chestnut cxpanils by heat, and, not having room to escape, bursts the shell.

Q. Dues this Bursting of the shell accasion the Norsw which we hear?

$A$. The noise is caused by the snapping of the shell, and by the report of the escaping air.

Q. How is this REPORT OCOLSTONED?

$A$. The sound of the report is the result of a violent impulse given to the air, which occasions rapid vibrations in the sound-wave.

Q. Cannot chestnuts be roasted without CRACKING ?

$A$. Yes; by making a slit in the shell.

Q. What PURpose does this answer?

A. It acts as a species of safety-valve, and enables the leated air to escape.

Q. Are the same EFFECTS caused by HEAT on other vegetable matter

$A$. Yes; as may very easily be perceived in the case of is rousted apple.

Q. What EFFEOT is produced?

A. The air within the apple, expanded by the heat of the fire, bursts the skin of the fruit.

Q. How much AIR is said to be contained within an apple?

A. The cells inside an apple, which resemble in appearance the honeycomb, contain sufficient air to fill a space forty-eight times greater than that occupied by the bulk of the apple.

Q. How can this be?

$A$. The air is condensed in the apple, but expanded by the force of heat.

Q. Are metals EXPANDED by heat?

A. Yes; but the degree of expansion differs. Platinum, glass, soft steel, cast iron, iron slightly hammered, steel tempered, pure gold, copper, bronze, brass, silver, tin, lead, zinc, are all more or less affected by the heat, but all in different degrees.

Q. Why does chestnut and the like kinds of wood make more sNappING than other woods?

$A$. Because the pores of these woods are larger, and contain more air than other kinds of wood.

Q. Why do SPARES of FIRE start with a crackling sound from sticks of woov laid upon a FIRE?

A. Because the expanded air forces its way through the pores of the wood, and carries with it that which resisted its escape.

Q. What are the SPARKS OF FIRE which burst from the WOOD?

A. Very small pieces of red hot wood, separated from the log by the force of the air. 
Q. Why does GREEN wOOD inake LESs SNAPriva than DRY?

A. Because the pores, being flled with sop, contain very litlle air.

Q. Why does DRT wOOD make Mort SNAPPiwg than green?

A. Because the sap is dried up, and the pores are tilled with air instead.

Q. What EFFECT has HEAT upon STONRs?

A. - It causes them to snop and $f y$ about.

Q. How is this?

A. The close texture of the stone preventing the esccope of aiv expanded by the heat, causes it to force " passage by bursting the stone and scattering the fragments.

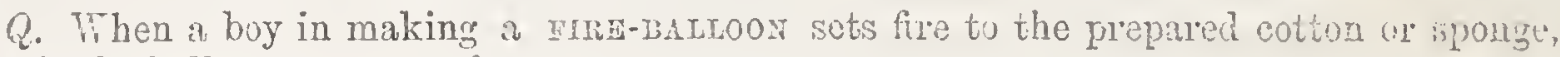
wby is the balloon INFIATED?

A. Because the atr of the balloon is expanded, by the hent of the flame, and fills the balloon to its utmost capacity.

Q. Why does the BALIOON IISE after it has been inflated by the expanded air ?

A. Because the same quantity of air is expanded and made so much lighter than common air.

Q. Why does sromi retsir up a chiner?

A. Because the heat of the fire expunds the air in the chimney, which (being thus made lighter than the air around) rises up the chimney, and carries the smoke in its current.

Q. Why will a rong chinney SMOEN, unless the RIRT be pretty Firron?

A. Because the heat of the fire will not be sufficient to rarefy all the air in the chimney.

Q. Why will the chimney sinoke, unless the fire be mwron enuagh to heat and the air in the OMMNAY-RIUE?

A. Becanse the cold air (condensed in the upper part of the flue) will sink from its own weight, and sweep the ascending smoke back into the room.

Q. Ilow are honses and other buildings heated with hot air?

A. The fire is kindled in a furnace which is erected in the cellar. this fre heats the air in contact with it in the air-chamber, as it is called, and as heated air always asconds, it is forced up into the different apartments of the building.

Q. What is an Arr-cirariber?

A. It is an enclosure around the grate or stove, with openings below to admit the cold air from the cellar to rush in to supply the place of the heated air which ascends into the rooms above. Sometimes the air-chamber is supplied with cold air by pipes, which conluct the cold air outside of the house into the air-chamber.

Q. How is it that heat can cause material substances to FXPiND?

A. If we assume that heat is a peculiar species of matter, whose particles are strongly repulsive of one another, we may readily conceive that the entrance of heat into a mass will have the eflect of 
removing the integrant molecules to greater distances from each other, and thus the body will be made to occupy a greater space, or expand.

Q. To what is the influence of heat always exposed?

A. To cohesion, or the force which tends to make the particles of matter approximate.

Q. In what bodies, therefore, should heat produce the GREATEST expansion?

$A$. In those which are least influenced by cohesion; thus the force of cohesion is greatest in solids, less in liquids, and least in aeriform substances; and while the expansion of solids is trifling, that of liquids is much more considerable, and that of gases is far greater.

Q. Why does heat change a sourd (like ice) first into a urquid and then into a GAs?

A. Because heat drives the component particles farther asunder; hence a certain quantity of heat changes solid ice into a liquid, and a further addition of heat changes the liquid into stecm.

Q. Do bodies continue to EXPAND so long as heat enters them?

A. Yes; the expansion of the same body increases with the quantity of heat that enters it, so long as the form and chemical constitution of the body is preserved.

Q. How can we prove that solins expand with heat?

A. If we take the exact dimensions in length, breadth, and thick ness of any substance when cold, and measure it again when strongl. heated, it will be found to have increased in every direction.

Q. Do bodies EXPAND with the increase of heat, and conrrsur upon itis withdrawal, with any degree of FORCE?

A. Yes; the force with which bodies contract and expand under the influence of heat is apparently irresistible, and is recognised as one of the greatest forces in nature.

Q. Do solids expand in any REGULAR araNiser by the addition of heat?

A. The amount of the expansion of solids, from the freeaing point of water to $122^{\circ}$, has been found to be equal to what takes place between $122^{\circ}$ and $212^{\circ}$, the boiling point of water.

Q. In what ratro do solids expand above $212^{\circ}$ ?

A. They do not dilate uniformly at high temperatures, but expand in an increasing ratio; that is, the higher the temperature beyond $212^{\circ}$, the greater the expansion for equal additions of heat.

Q. What prouliarity exists in the effect of heat upon the bulk of some fluids ?

A. That at a certain temperature increase of heat causes them to contract, and its diminution makes them expand.

Q. What cLASSES of LIQUIDS exhibit this peculiarity?

A. Those only which increase in bulk in passing from the liquid to the solid state, and this change remarked only within a few degrees of temperature above their point of congelation.

Q. What is a noted example of this rexcertios to the general laws of heat?

A. Water; ice swims upon the surface of water, and therefore 
must be lighter-a convincing proof that water in the act of freezing must expand.

Q. What отнеR FLUIDs besides water expand with a reduction of temperature?

A. Fused iron, antimony, zinc, and bismuth, are examples of such expansion. Mercury is a remarkable instance of the reverse, for when it freezes, it suffers a very great contraction.

Q. Why is the ICE produced by the freezing of SEA WATER al ways fresh and FREE from SALT ?

A. Because water, in freezing, if in sufficient quantity to allow freedom of motion to its particles, expels all impurities and colouring matters.

Q. If a solution of rNDIGo be frozen, why will the ice formed be clear and colourless?

$A$. Because the water in which the indigo was dissolved expels all the blue colouring matter while freezing.

Q. Why are blocks of ice generally filled with AIr-BUbBLES ?

$A$. Because the water, during the act of freezing, expels the air contained in it, and many of the liberated bubbles become lodged and imbedded in the thickening fluid.

Q. Is the Fonce created by the expansion of water in the act of freezing VERT GREAT ?

$A$. Yes; as an illustration the following experiment may be quoted :-Cast-iron bomb-shells, thirteen inches in diameter, and two inches thick, were filled with water, and their apertures or fuseholes firmly plugged with iron bolts. Thus prepared, upon exposure to the severe cold of a Canadian winter, about $19^{\circ}$ below "zero," at the moment the water froze, the iron plugs were violently thrust out, and the ice protruded, and in some instances the shells burst asunder, thus demonstrating the enormous interior pressure to which they were subjected by water assuming its solid state.

Q. What is the principal CAUSE of the rounded and weatherworn aspect of some rocks, especially the limestone and sandstone rocks?

$A$. The expansion of freezing water; water is absorbed into their fissures and pores by capillary attraction, and when it freezes during winter, it expands and detaches successive fragments, so that the original sharp and abrupt outline is gradually rounded and softened down.

Q. Why do trees often CRACI and SPLIT open in winter ?

A. The sap becomes frozen, and the expansion attendant upon such change is said to rend them asunder with a loud crackling or explosion. pipe?

Q. Why, in the winter, do we let the water RUN to prevent its freezing in the service-

$A$. Because the motion of the water prevents the crystals of ice from forming or attaching themselves to the sides of the pipe.

Q. Can a LENS be made of ICE capable of concentrating the rays of the sun, with sufficient intensity to INFLaMe substances?

A. Yes; a burning-lens can be formed of transparent ice, of power sufficient to produce effects nearly. equal to those of the glass lens. 
Q. Does ice possess gieat ANTISEPTIO POWER?

$A$. Yes; provisions packed in ice will keep fresh for a great length of time.

Antiseptic-opposed to putrefaction.

Q. Why will ice PREVENT meats from putrefying?

$A$. Because the cold prevents the chemical action necessary to putrefaction.

Q. What is "Ground ToE," or "ANCHOR ICE?"

$A$. Ice formed at the bottom of streams or rivers.

Q. Why does ice sometimes form first at the воттом of streams and rivers, the surface of the water being most exposed to the cold?

$A$. The formation of "ground" or "anchor" ice occurs only when the temperature of the whole bulk of the running waters is reduced to near $32^{\circ}$, and that of the atmosphere is considerably below such a point. The beds of rivers consist generally of rugged rocks and stones; these contain a certain degree of heat, and part with it, not by conduction, nor by convection, to the water, but by direct radiation through it to a cloudless sky; thus losing their heat, they quickly cool the water in their immediate contact, and suddenly freeze it in films upon their surfaces.

Q. Why is the ground or anchor ice only formed in CLEAR WEATHER?

$A$. Because a cloudless sky is essential for the radiation of heat to take place from the rough surfaces at the bottom of the stream through the water. The ice remains at the bottom so long as radiation and the cloudless sky continue, but radiation ceases when the clouds appear.

Q. Why is this ice seen floating upon the surface in the greatest quantities in cloudy weather?

$A$. When the sky clouds over, radiation ceases; the solid surfaces then communicate sensible heat to the ice, or the clouds may send forth radiant heat downwards through the water to the ice in a degree sufficient to cause thawing to take place. The ice then becomes detached from the bottom, and floats down the stream. Should the temperature of the air continue low, with a clouded sky, or become lower, the ground ice is not renewed, but the river is speedily frozen over at its surface in the usual manner.

Q. Upon what does the FORMATroN of ICICLES depend?

$A$. Upon the successive congelation of drops or slender streams of water.

Q. What is IOE?

A. Water congealed by cold, or frozen.

Q. At what temperature does this change take place?

A. When the temperature is reduced to below $32^{\circ}$ of heat.

Q. Can water be cooled BELow $32^{\circ}$, under any circumstances, without freezing?

A. If pure, recently-boited water, be cooled very slowly and kepr very tranquil, its temperature may be lowered to $21^{\circ}$ without the 
formation of ice, but the least motion causes it to congeal suddenly, and its temperature rises to $322^{\circ}$.

Q. Which is the LIGHTLS?, ICE Or WATER

A. Ice.

a. How is this ACCOUNTED IOR?

A. Water is expanded by frecaing, and the gravity is less in proportion as the bulk is increased.

Q. Does this ExPANSION of frozen water account for EARTIEN VESELS being BROKEN on a FROSTY NIGLT ?

A. Yes; the water cxpanded by the frost, and unable to escape at the top, in consequence of the ice there formed, bursts the sides of the vessel, just as water would do expanded by heat.

Q. Do not sTONEz very often SPLIT in WINTER?

A. Yes; and the reason of this is, that the moisture within them freezes, and the particles being thus expanded, split the stone into fragments.

Q. What other instance of the EFERCT of EXPAXSION by couD can you mention?

A. The bursting of water-pipes in frosty weather, and the cracking of the hard frozen ground. In both cases expanded moisture forces itself away, and bursts the particles asunder.

Q. Does not water expand by HEAT as well as by coLD?

A. Yes; it expands as soon as it is more than $42^{\circ}$, till it boils; aiter which time it flies off in steam.

Q. Explain the causs of water Expardirg when frozen?

A. It results from an entirely new arrangement of the particles of water. The crystallised moisture arranges itself in a sort of net-zork, lines crossing each other at right angles, and consequently occupies more space than when in a fluid state.

Q. When does this EXPANSTon of WATER from Comb begin to take place?

A. Water contracts with cold till it reaches a temperature of $40^{\circ}$, then it expands till it freeres.

Q. How may the expansion of water from cold be SHown?

A. Fill a flask with water at the temperature of $60^{\circ}$, and adapt to it a cork through which passes a glass tube open at both ends, about an eighth of an inch wide and ten inches long. After having filled the flask, insert the cork and tube, and pour a little water into the latter, until the liquid rises to the middle of it. On immersing the flask in a mixture of pounded ice and salt, the water at first contracts, and therefore descends in the tube, but soon after an opposite movement ensues, indicating that the mass of water, though growing colder, is expanding.

Q. What PART of a RIVER is FROZEN FIRST ?

A. Generally the surface.

Q. Why is this?

A. The surface of the river is in contact with the air, and what heat it has in it, is more readily carried away by the air. 
Q. Why is the BotTon of a river MALILY Frozen?

A. Water, as soon as it becomes colder than $42^{\circ}$, rises to the surface, and if frozen, flocts there till it thaws.

Q. Why docs it rLOAT ?

A. Because it is lighter than water; if it were heavier, it would sink, and the whole river soon become a solid block of ice.

Q. What UsBrum PURPOSB is answered by the ice FLodTng on the Tor?

A. The frowen surface of a river acts as a warm covering to the water below, and prevents the cold from freezing it.

Q. Docs this corfarna of ice Grow THrcker if the frost continues?

A. It does; in consequence of the leat of the water passing into the cold atmosphere, through the ice.

Q. Are not wrous nilfens sometimes converted into ICE ?

A. Seldom: the frosts, however severe, scarcely ever lasting long enough to effect that change.

Q. Is RUNMING water or STIL frozen the most RAPIDIY ?

A. Still water; because in runing water the heat of the water is excited by the motion, and distributed throughout the mass; and, because the motion of the water prevents the rapid formation of the crystals.

Q. Do ALI PATTS of a river FTEEZE AJIKE ?

A. No: incidental causes interrupt this; for instance, springs issuing from the bed of the river, thau the ice on the surface; and, by keeping up continued action, render it thinner than at other parts.

Q. What is meant by CRYsíaitisation?

A. The conversion of certain substances into regutar forms, occasioned by a loss of a portion of their caloric, or heat.

Q. Is SEA WATER TROZEN as well as FRESH?

A. But rarely; though instances of the kind have occurred; and in some places the water is ahoays solid.

Q. Why does not SEA WATIR FREEZE as readily as TRESH ?

A. 'Three reasons may be assigned-the saltness of the water; the $e b b$ and flow of the water; and the immense expanse of the water.

Q. Will not SALT WATER FREEZE as reddily as FRESEI ?

A. No: fresh water freckes when reduced to the temperature of $32^{\circ}$; salt water does not freeze till it is cooled down to $28^{\circ}$.

Q. How does the EBB and wrow of the water INTEREERE with its FREEZTing ?

A. By keeping the water in continual motion, it mevents the crystals forming a solid muss.

Q. How does the EXPANSE of water INTERFERE with its FREBZIIIG ?

A. The mass of water is so extcnsive and so deep that it requires a very considerable time before it can be reduced to 408 temperature, at which the surface begins to freene. 
Q. Where is the SEA Frozen?

A. In the Arctic regions, where masses of ice are met with eighteen hundred feet in height, and which never thaw.

Q. Will SALT DISSOLVE ICE?

A. Yes. Water freezes at $32^{\circ}$; salt and water at $28^{\circ}$; therefore if salt be added to frozen fresh water, it dissolves.

Q. Will ANYTHING ELSE DISSOLVE IOE?

A. Any acid will dissolve it.

Q. Which feels the COLDEST, a Frost or a THAW ?

A. A thaw always feels colder than a frost.

Q. How is this?

A. Frozen water absorls heat from the air as it thaws; the absence of that heat consequently makes the air colder. On the same principle, the air is much warmer during a frost, because, in being conrerted into ice, the water gives forth its latent heat to the air.

Q. Can water be Frozen by ARTIFICIAL MEANS?

A. Yes; in very many different ways.

Q. Hention one of them?

A. A bottle of vater, wrapped in cotton, saturated with ether, would soon fiecze; evaporation carrying off the heat of the water, and reducing it to the fieesing point.

Q. Why is a GLASS or EARTHEN VESSEL apt to break when HoT WATER is poured into it?

A. Because the inside of the glass is expanded by the hot water, and not the outside; so the glass snaps in consequence of this inequal expansion.

Q. Why is not the outsive of the GLASs expanded by the hot water as well as the INSIDE?

A. Becruse glass is a bad conductor of heat, and brealis before the heat of the inner surface is conducted to the outside.

Q. Why does a GLASS SNAP because the INAEI surface is HoTTER than the outER?

A. Because the inner surface is expanded, and not the outer; in consequence of which an opposing force is created, which breaks the glass.

Q. Why do persons DIP a RAZOr, in HOT WATER before shaving with it?

$A$. The edge of the razor is expanded by the heat of the water, and by that means is rendered more fine and sharp.

Q. Why does a cooper heat his noops RFD not when he puts them on a tub ?

A. 1. As iron expands by heat, the hoops will be larger when they are red hot; in consequence of which they will fit on the tub more easily; and

2. As iron contracts by cold, the hoops will shrink as they cool down, and gird the tub with a tighter grasp.

Q. Why does a WHEELWRIGIT make the TIRE RED HOT which he fixes on a wHFEL?

A. 1. That it may fit on more easily; and

2. That it may gird the wheel more tightly. 

กот?

Q. Why will the wheelwright's TIRE FIT the wheel MORE EASILY for being made Run

A. Because it will be expanded by the heat, and (being larger) will go on the wheel more easily.

Q. Why will the TIRE which has been PUT ON HOT, GIRD the wheel more FIRMLY?

A. Because it will shrink when it cools down, and therefore gird the wheel with a tighter grasp.

Q. What is the OAUSE of a stove making a CRACKING NOISE when the FIRE is VERY HOT, as well as when a large fire is PUT OUT ?

A. The crackling noise is caused by expansion and contraction. Heat expands the stove, and occasions the particles of which it is composed to rub against each other, and thus produce the sound mentioned. When the heat is removed, the stove contracts, and this second disturbance of its particles produces a similar sound.

Q. If the BoILER or KETTLE attached to a kitchen range be filled with cold water some time $\triangle F T E R$ the fire has been lighted, it will be very likely to crack or burst; why is this?

$A$. Because the heat of the fire has caused the metal of which the boiler is composed to expand; but the cold water very suddenly contracts again those parts with which it comes in contact; and as one part is made larger than the other, the boiler cracks.

Q. When the stopper of a DECANTER or smelling-bottle sticks, why will a clotlı wrung out of нот WATER, and wrapped round the NECK of the bottle, LOOSEN the stopper?

$A$. Because the hot cloth heats the neck of the bottle, causing it to expand, and consequently loosens the stopper.

Q. Why does the sTopper of a decanter sTicr fast if it be put in damp?

$A$. If the stopper be damp, it fits the decanter air-tight; and if the decanter was last used in a heated room, as soon as the hot air enclosed in the inside has been condensed by the cold, the weight of the external air will be sufficient to press the stopper down and make it stick fast.

Q. Why does the STOPPER of a SMELLING-BotrLe very often STicr fast ?

$A$. Because the contênts of a smelling-bottle are very volatile, and leave the neck of the bottle and the stopper damp.

If the smelling-bottle was last used in a hot room, as soon as the hot air and volatile essence inside the bottle have been condensed by the cold, the weight of the external air will be sufficient to press the stopper down and make it stick.

Q. Why are the NAILS in almost all old houses LOOSE and easily DRAWN OUT ?

$A$. Because the iron expands in the summer, and contracts in the winter, more than the stone or wood, and thus the opening is gradually enlarged after a lapse of time.

Q. Why does a piano give a HIGHER TONE in a cold than in a warm room?

$A$. Because in a cold room the strings are contracted and tighter.

Q. Why, in rooffing, are the metal plates made to overuap each other, instead of being mailed together?

$A$. In order that they may neither tear nor warp by alternate contraction and expansion. 
Q. Why do c'LOCKs go slower in summer and faster in winter?

A. Because the pendutums elongate in summer through the effects of heat, and consequently vibrate slower; while in winter they contract, become shorter, and vibrate more rapidly.

Q. How is this INEQUALTTY in the rate of motion in time-pieces obviated?

A. By what is called a compensating pendulum; that is, one constructed of two metals, possessing different expansive powers, in such a manner that the greater expansion of one bar in one direction equals the less expansion of other bars in a different direction, and thus maintain an invariable length of the pendulum.

Q. Does WOOD EXPAND under the influence of heat differently from metal ?

$A$. Yes; an iron bar expands and contracts equally in all directions, but wood expands and contracts more in breadth than in length.

Q. Why will a person buying oil, molasses, spirits, \&c., by the measure, get a GREATER WEIGIT of the same material in the same measure in the winter than in the summer?

A. Because these liquids contract and occupy less space in the winter than in summer; consequently it requires more of the same kind to fill the same space in winter than in summer.

Q. Glass chimueys, for lamps and gas-burners, are fiequently cracked and broken by irregular expansion when first heated by the flarne of the light: how may this liability to break be $\Lambda$ YOIDED?

A. By making a minute notch with a diamond in the bottom of the tube.

Q. How can heat be MKLSURED ?

A. Only by its effects : since the magnitude of any body changes with the heat to which it is exposed; and since, when subject to the same calorific influences, it always has the same magnitude, these dilations and contractions, which are the constant effects of heat, may be taken as the measure of the physical cause that produced them.

Q. What is the TMMPERATURE of a body?

A. It is the actual state of a body at any moment, determined by a comparison of its magnitude with the heat to which it is exposed.

Q. What is a CHANGE of temperature?

A. The change in magnitude which a body suffers by changes in the leat to which it is exposed.

Q. What are the INSTRUMents for measuring heat CALLED?

A. Thermometers and pyrometers.

Q. What is the DIFFERENCE between them?

A. A thermometer is used for measuring moderate temperatures; while the pyrometer is chiefly applied to determine the more elevated degrees of heat.

Q. What SUBSTANCES are besi adapted for measuring the effects of heat by their expansion and contraction?

A. Liquids, above all other substances. 
Q. Why are liquids BEST $\triangle D A P T E D$ for this purposo ?

$A$. Becauss in solids the direct expansion by heat is so small as to be seen or measured with difficulty; in air or gases it is too extensive and too liable to be affected by atmospheric pressure; but liquids are free from both disadvantages.

Q. Whiat LTQUID is generally used for the construction of ordinary therinometers?

A. Mercury or quicksilver.

Q. What MErat is distinguished from all others by its FLUIDITx at ordinary temperature?

\section{A. Mercury or quiclisilver.}

Q. Does mercury, like other metals, Expand by reat?

A. It readily expands or contracts with every variation of temperature.

Q. Why is mercury PREFERABJis to all othor liquils for the purposes of the thermometer?

A. Because it boils at a higher temperature than any other liquid, except certain oils; and, on the other hand, it freeses at a lower temperature than all other liquids, except some of the most volatile, such as ether and alcohol.

Thus, a mercurial thermometer will have a wider range than any other liquid thermometer. It is also attended with this convenience, that the extent of temperature included between melting ice and boiling water stands at a considerable distance from the limits of its range, or its freezing and boiling roints.

Q. Of what does the mercurial thermometcr consisr?

A. The mercurial thermometer consists essentially of a glass tube with a bulk at one extremity, and which, having been filled with mercury at a certain temperature, introduced through the open end, has been hermetically sealed while full, so that no air can afterwards enter it.

As the tube and mercury in it gradually bccome cosled, the cnclosed fluid contracts, and consequently sinks, lcaring above it a vacant space or vacum, through which it may again expand on the application of heat.

Q. As thermometers are constructed of different dimensions and capacities, how are they GRADUATED to indicate the same temperature under the same circumstances, as the frcezing point, for example?

A. The thermometers are first immersed in melling snow or ice. The mercury will be observed to stop in each thermometer-tube at a certain height; these heights are then marked upon the tubes. Now it has been ascertained that at whatever time and place the instruments may be afterwards immersed in melting snow or ice, the mercury contained in them will always fix itself at the point thus marked. This point is called the freeaing point of water.

Q. How is the boiling point ASCERTAINEI ?

$A$. It has been found that at whaterer time or place the instruments are immersed in pure water, when boiling, piovided the barometer stands at the height of thirty inches, the mercury will always rise in each to a certain height. This, therefore, forms another fixcd point on the thermometric scale, and is called the boiling point. 
Q. Huw are the INTERMEDIatr ronts determined?

$A$. In Fahrenheit's thermometer, the intervals on the scale, be. tween the freezing and boiling points, are dirided into 180 equal parts. 'This dirision is similarly continued below the freezing point to the place 0 , and each division upwards from that is marked with the successive numbers $1,2,3$, \&c. The freezing point will now be the $32 n d$ division, and the boiling point will be the 212th division. These divisions are called degrees, and the boiling point will therefore be $212^{\circ}$, and the freezing temperature, $32^{\circ}$.

Q. When and by whom was the thermometer INTENTen?

A. The thermometer was invented about the year 1600; but, like many other inventions, the merit of its discovery is not to be ascribed to one person, but to be distributed among many.

Q. Why is the thermometer in general use in England, the United States, and Holland, called FAHinnHiti's thermometer?

A. Because thermometers having a like graduation were first manufactured by Fahrenheit, a Dutch philosophical instrumentmaker. The employment of mercury as the most suitable fluid for' the thermometer is also usually attributed to him?

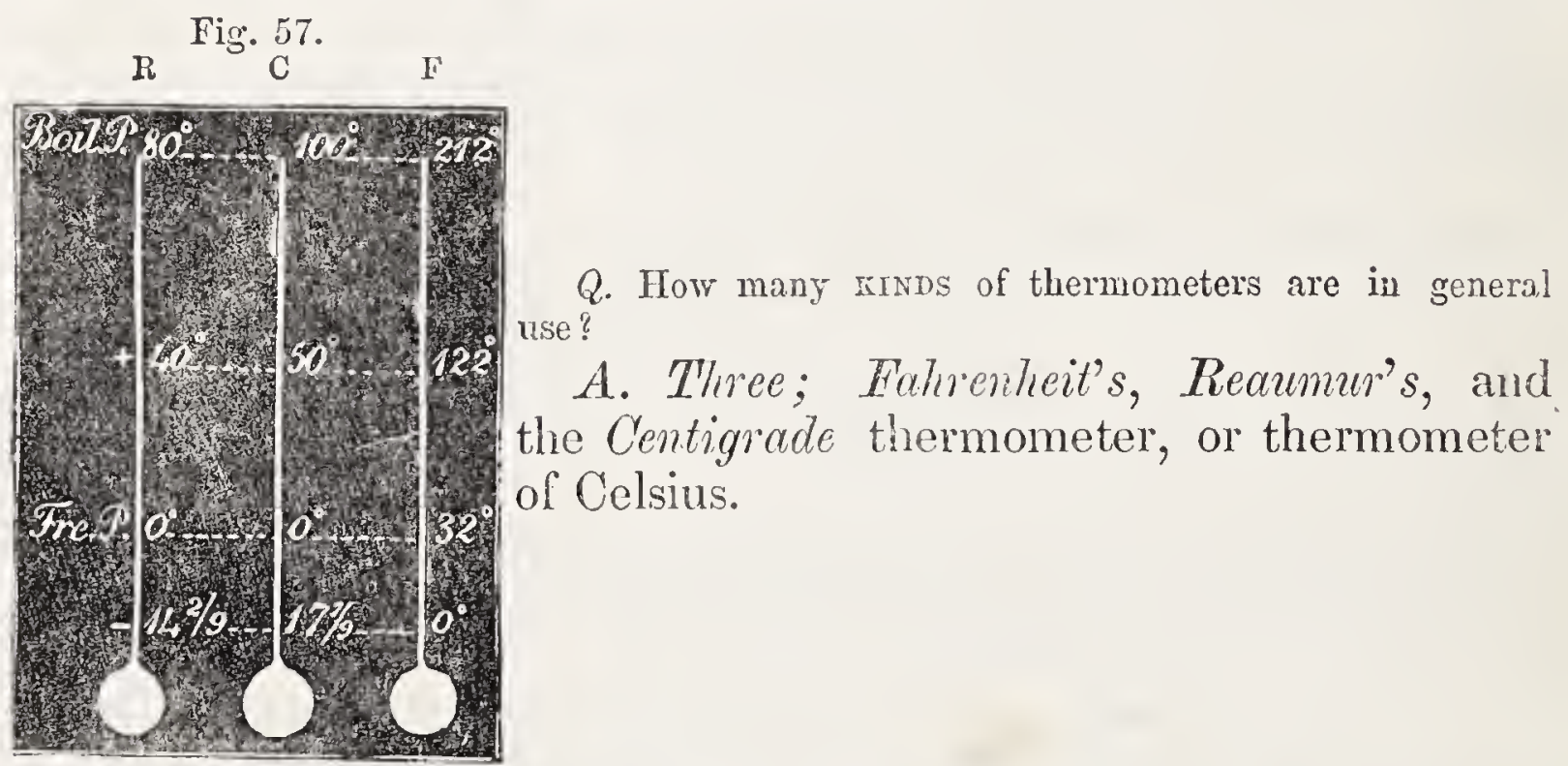

Q. What constitutes the DIFFERence between these instruments?

A. The differences of graduation between the freezing and boiling points of water. Reaumur's is divided into eighty degrees, the Centigrade into one hundred, and Fahrenheit's into one hundred and eighty. According to Reaumur, water freezes at $0^{\circ}$, and boils at 80 ; according to Centigrade, it freezes at $0^{\circ}$, and boils at $100^{\circ}$; and according to Fahrenheit, it freezes at $32^{\circ}$, and boils at $212^{\circ}$; the last, very singularly, commences counting not at the freezing point, but $32^{\circ}$ below it.

The differencss between these instruments can be easily seen by reference to $f i g .57$.

Q. In what couxtrirs are the Reaumur and Centigrade thermometers generally used ?

A. Reanmur is in general use in Germany, and the Centigrade in Trance; but for scientific purposes the Centigrade is almost universally adopted. 
Q. How may the different thormometers be COMPARED with each other?

A. "By remenbering that $4^{\circ}$ of Reaumur are as large as $5^{\circ}$ Centigrade, or $9^{\circ}$ Fahrenheit. In rerlucing Fahrenheit to Reaumur or Centigrade, if the degree be above the freezing point, $32^{\circ}$ must be subtracted, which process must be reversed in order to reduce the degrees of other scales to those of Fahrenheit. To the degrees above $0^{\circ}$, the sign + is prefixed; to those below, the sign -

Knowing the relation which exists between their degrces, it is easy to reduce the temperature expressed by one thermometer to that of another. Thus a degree of Fahrenheit'is scale is equal to 4 -9ths of one of Reaumur's, and to 5-9ths of a Centigrade degree; thercfore, to convert a degree of Fahrenheit into its equivalent on Reaumur's scile, we inust multiply by 4 and divide by 9 ; and the equivalent on the Centigrade is obtained by multiplying by 5 and dividing by 9 , always remembering to previously subtract $32^{\circ}$, becanse the zero of Fahrenheit is $32^{\circ}$ lower than cither of the others. The same process reversed enables us to reduce the degrees of the other scales to those of Fahrenheit-by adding $32^{\circ}$, multiplying by 9 , and dividing by 4 or 5 , as the case may be.

Fig. 58.

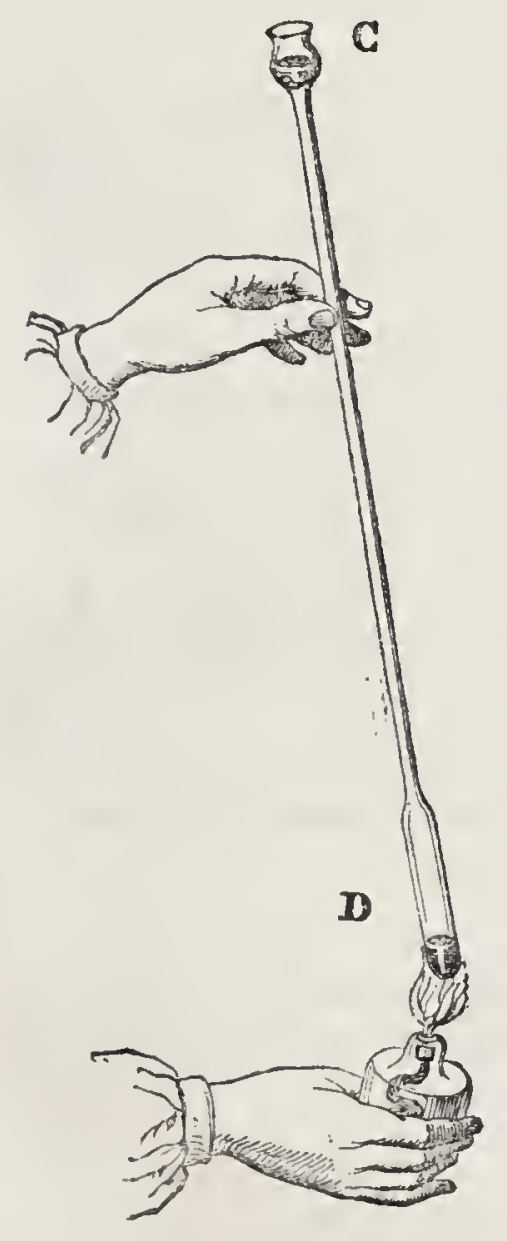

Q. How is Mercury introduccd into the tube of a thermometer?

A. In order to fill the bulb of the thermometer with mercury, there is blown to the upper end of the tube a funnel, $\mathrm{C}$, which is first filled with mercury; then, by inclining the tube a little to one side, and applying the heat of a spirit lamp to the bulb, the air within the latter is expanded, forced up" the tube, and expelled through the fumnel $\mathrm{C}$.

\section{Q, What is done next?}

$A$. The tube is then cooled, the remaining portion of heated air contracts, and the mercury, by atmospheric pressure, is forced into the bulb D. This process is conducted over and over again, until a very small quantity of air remains in the tube. In order to expel this, the mercury is heated to ebullition, and the vapour of the mercury forces out the air. The funnel $\mathrm{C}$ is then removed, and the extremity hermetically sealed.

Q. Is not some other process necessary before this is done?

A. Yes; before this is done, care must be taken to expel one-half or two-thirds of the mercury in the tube; otherwise, the mercury would expand and burst the thermometer.

Q. At what temperature dnes mercury FreEze?

A. At about $39^{\circ}$ below the zero of Fahrenheit's thermometer.

Q. How are degrees of cold more intense than this MEASURzD?

A. By using a thermometer filled with alcohol coloured red, as this fluid, when pure, does not congeal at $100^{\circ}$ Fahrenheit below zero. 
Q. At what Trarperaturir does mercury Bors?

A. At $656^{\circ}$ Fahrenheit.

Q. How are temperatures GRTATYR than this DETERMINED?

A. By means of the expansion of solids: and instruments founded upoin this principle are commonly called pyrometers.

Fig. 59.

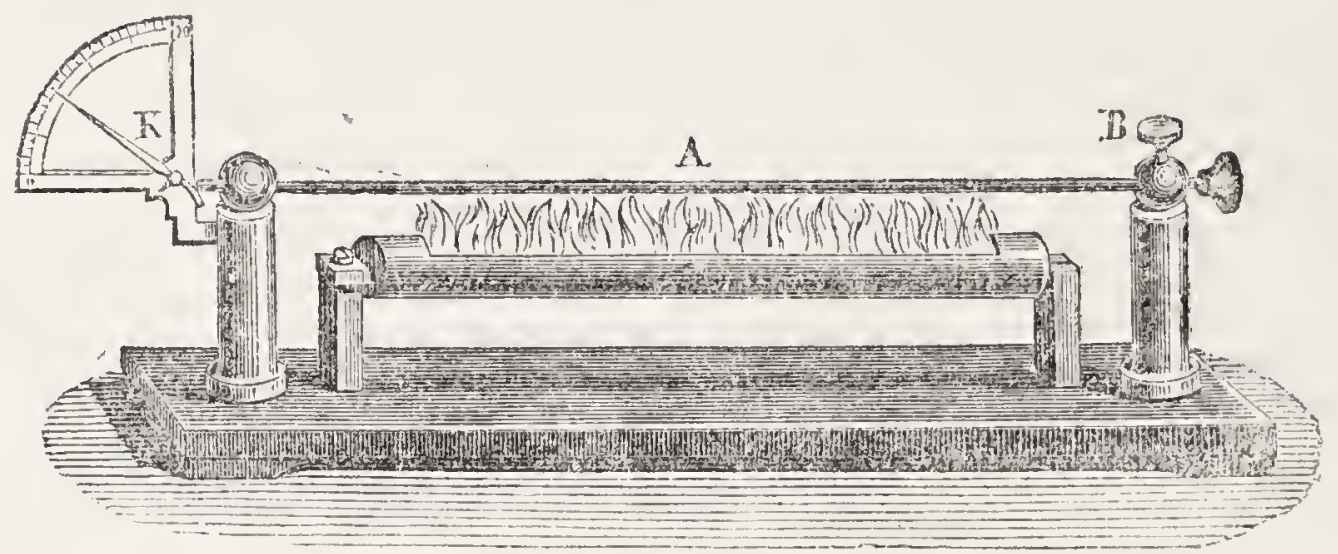

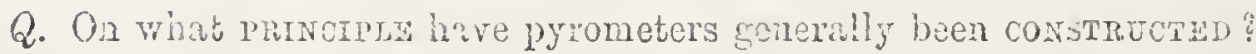

A. On the relative expansion of bars of iron, or some other metal. Several of these are constructed on the principle of that represented in fig. 59, in which a bar of metal, A, may be so placed, that when expanded by the heat of the furnace below, one extremity will press against a lever, and cause an index, $K$, to move along the graduated arc; and by means of such a pyrometer the effect of heat, applied in the same manner for a given length of time, to bars of different metals having the same length and diameter, may be ascertained.

Q. By what other mans can the cubic expansion of solids be ASCERTAINeD?

A. For this purpose, a small metallic ring is sometimes used, called the ring of Gravezande, a ring through which, at the ordinary temperature, a copper sphere having nearly the same diameter readily passes. When this sphere is heated by the flame of a spiritlamp, it can no longer pass through the ring.

Q. What OTHER KINDS of thermometers are employed?

A. A curious and useful thermometer was constructed by A. Brequet, of Paris; constructed on the principle of the unequal expansibility of metals.

Fig. 50 .

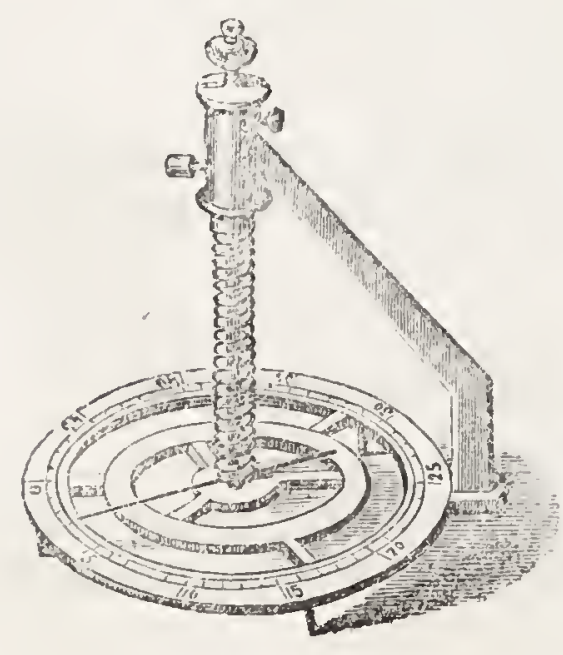

This instrument is composed of three equal slips of metal-platinum, gold, and silver. After being soldered and flattened into one thin metallic ribbon, they are wound in a spiral form round a support (sec diagram), and the upper extremity boing fixed to it, there is attached to the other extremity, which is free, a needle moving over a horizontal clial. On the raising of the temperature the metals begin to expand, the silver more than gold or platinum, and the spiral un. winds itself from left to right, as shown in the figure. 
Q. How do the manufacturers of porcelain and pottery sometimes Ascinials the HEAT of their furnaces?

A. By employing as a pyrometer a cylinder of fine porcelain clay, which contracts with some degree of regularity until melting or vitrefaction takes place.

Q. Does a thermometer inform us how nucrI IEAT any body contains?

A. No ; it merely points out a difference in the temperature of two or more substances. All we learn by the thermometer is whether the temperature of one body is greater or less than that of another; and if there is a difference, it is expressed numerically-namely, by the degrees of the thermometer.

It must be remembered that these degrees are parts of an arbitrary scale, selected for convenience, without any reference whatever to the actual quantity of heat present in bodies?

\section{SECTION II.-LIQUEFACTION.}

Q. After the expansion of solids, when acted upon by heat, what orHer EFrECr is next observed?

A. They change their aggregate state, become liquid, or melt. Many of them become soft before melting, so that they may be kneaded; for instance, wax, glass, and iron; in this condition, glass can be bent and moulded like wax, and iron can be forged or welded.

Q. What is IIQUEACTION?

A. Liquefaction is the conversion of a solid into a liquid by means of heat.

Q. Why is ICE MELTED by the HEAT of the SUN?

$A$. Because, when the heat cir the sun enters the solid ice, it forces its particles asunder, till their attraction of cohesion is sufficiently overcome to convert the solid ice into a liquid.

Q. Why does WAX become sorr before it turns LIQUID?

$A$. Because it absorbs heat sufficient to loosen the contact of its particles, before it has absorbed sufficient to liquefy the mass.

Q. Why does melted wax become mard when cold?

A. Because the particles collapse, and, being packed more closely together, form a solid.

The sole difference between a liquid and a solid is this : in a solid the particles are packed inore closely together than they are in a IIQUID. The tendency of heat is to drive the particles farther apart from each other, and thus to liquefy solids.

Q. Explain the PHILOSOPHX of MELTRD METAIS?

$A$. When the heat of the fire enters the metal, its particles are separated, until at last the solidity is entirely lost, and the metal becomes a liquid.

Q. Why will not wood usir like iron?

$A$. The heat of the fire, instead of melting wood, turns it to gas, smoke, and ashes.

Q. Why will How iron BEND more casily than cold?

A. Because it is not so sotid. The particles are driven farther 
apart by heat, and the attraction of cohesion is thereby weakened; therefore the particles can be made to move on each other more readily.

By a still further application of heat, the particles will be driven so far asunder from each other, that the solid iron will liquefy; in which state the particles will move on each other almost without resistance.

Q. In liquefaction, or the transformation of a body from a solid to a fluid state, what REMARTABLE CHANGE takes place in the character of the heat causing this transformation?

A. It disappears, or becomes insensible, during the process.

Q. The temperaturc of meltiug ice is $32^{\circ}$; if you pour just enough boiling water over the ice to melt it, will the TEMPERATURE of the water be INCrEAsEd?

$A$. No; the heat of the water is consumed in melting the ice; but pour boiling water on ice-cold water, and the temperature is immediately increased.

Q. What BECones of the heat which disappears during liquefaction?

A. It enters into the fluid, and becomes latent.

Q. Does this latent heat become SExsible again when the liquid is reconverted into a solid?

A. It does; and until this heat is withdrawn from the fiuid, it cannot become solid.

\section{SECTION III.-VAPORISATION.}

Q. What is VAPORISATION?

A. Vaporisation is the conversion of a solid or liquid into vapour.

Q. Why does Hor IRoN make a HISsING NoIss when plunged into water?

A. Because the hot iron converts into steam the particles of water which come in immediate contact with it; and as the steam flies upwards, it passes by other particles of water not yet vaporised; the collision produces very rapid vibrations in the air, and a hissing noise is the result.

Q. How is STEAsI produced by the application of HEAT to TATER ?

A. The globules of the liquid are separated by the leat of the fire, and, being made so much lighter than the air, fly off in steam.

Q. What IESIGNation has been given to the condition which water and other liquids of like character assume when dropped upon very hot surfaces?

A. The "spheroidal state."

Q. Why does the liquid ASSUMr this condition?

A. Because the heat which radiates from the heated surface produces, before the surface and the liquid can come into immediate contact, a quantity of vapour sufficient to support the liquid; and this vapour, being constantly removed, prevents the liquid from touching the surface. 
Q. Why does the liquid evaporate more stowly thau it would on a surfice less highly heated?

A. Because the layer of vapour between the hot surface and the liquid prevents the rapid transmission of heat by conduction.

Q. Why do the drops of the liquid assume in this condition a globular or spheroidal state?

A. The liquid rests upon a cushion of steam continnously evolved by heat from the liquid, and assumes a rounded shape as the result of the gravity of its particles towards its own centre.

Q. Jugglers sometimes perform the feat of plunging the hands without injury into molten lead or iron : how is this EXPLAINED?

$A$. The hand is moistened, and when passed into the liquid metal the moisture is vaporised, and interposes between the metal and the skin a sheath of vapour. In its conversion into rapour, the moisture absorbs heat, and thus still further protects the skin.

Q. Can any one dip their FINGErs with impunity into MELTED IRON?

$A$. Yes, for a few seconds; taking the precaution to first effectually moisten the hand.

Q. Will a RED-HOT IRON applied to the tongue BURN?

$A$. Not for some time; the moisture of the tongue passing into the "spheroidal condition," and protecting the skin.

Q. What is the tesperature of the bubble of liquid when in the sploroidal condition ?

A. Always below the boiling point.

Q. Can water be poured through the meshes of a moderately fine sieve, the wires of which are RED IIOT?

A. No; the water assumes the spheroidal shape, and is unable to pass through.

Q. To what substances do we apply the term "volatrle?"

A. To those which have a great tendency to assume the gaseous form.

Q. To what substances do we apply the term "FIXED" or "Nor-VoLATILE?"

A. Tho those in which the tendency to assume the gaseous form is small.

Q. Is it necessary that a solid should first become IIQUid before it can change into VAPOUR?

A. Mrost solids do become liquefied before they vaporise; but some pass at once, on the application of heat, from the state of a solid to that of vapour, without assuming the liquid condition.

Q. Do vapours occupy much more SPACE than the substances from which they were produced?

$A$. They occupy a much greater space; water, in passing from its point of greatest density into vapour, expands to sixteen hundred and ninety-six times its volume.

Q. Have vapours any ELASTIC FORCE?

$A$. Yes; and in consequence of this they act with a certain degree of pressure on the vessels which contain them. 
min: 61

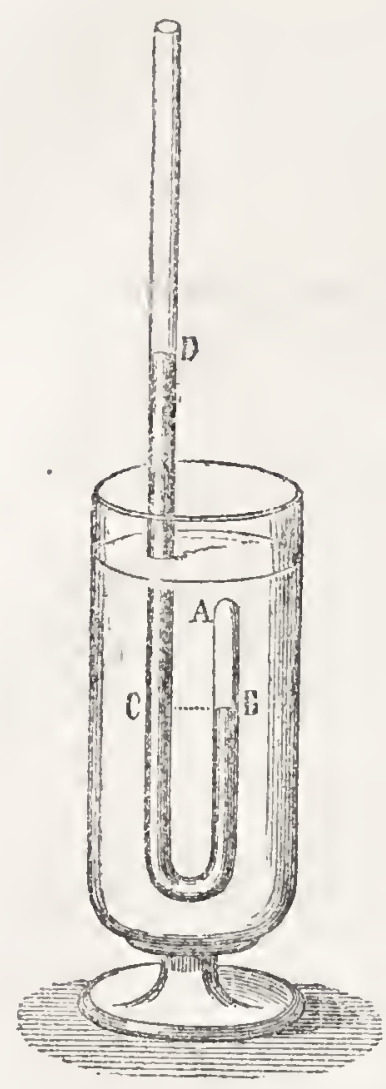

Q. How is this proved?

A. By experiment.

In order to ascertain the tension of vapours, a glass tube, of siphon shape, inverted, is half-filled with mercury (sce fig. 61) ; then a drop of ether is passed into a shorter branch, which is closed, and the tube is then immersed in a water bath about $45^{\circ}$ Centigrade. The mercury now sinks into the smaller branch, the space A B will be filled with a gas having entirely the appearance of air, and whose elastic force evidently balances the weight of the column of mercury $C D$, as well as the pressure of the atmosphere which acts on $\mathrm{D}$; this gas is the vapour of ether. If we cool the water in the vessel, or if we withdraw the tube from it, which will produce the same effect, the vapour which filled the space A B will rapidly disappear, and the drop of ether will reappear. If, on the contrary, the water-bath be leated more and more, the level of the mercury will sink below the point $B$, and thus the tension will be increased.

Q. Is not the PRESENCE of the AIR an OBSTACLE to vaporisation?

A. Yes; and, consequently, the experiment proceeds slowly. When the liquids are placed in a vacuum, the elastic force of the vapour meeting with no resistance is instantaneously developed.

Q. Why do wer clothes DRY in the air?

A. In consequence of the evaporation of the moisture which they contain.

Q. What is this CALLED?

A. Spontaneous evaporation. Thus a vessel full of water and open to the air gradually empties itself by evaporation; and thus, also, vapours rise from the seas, lakes, rivers, and earth, condense in the air, and fall again in showers of rain.

Q. What CADSES have an INFLUENCE on the rapidity of evaporation?

A. 1st. The temperature; 2nd. The quantity of the same vapour already in the surrounding atmosphere; $3 r$. The renewal of the atmosphere; 4th. The extent of the surface evaporated.

Q. How does the temperature AFFECT vaporisation?

A. By increasing the elastic force of the vapour it accelerates the evaporation.

Q. How does the CONDITION of the atmosphere $\triangle$ FFECT it?

$A$. If the atmosphere be already completely changed and saturated with the same vapour as that which we desire to evaporate, it is obvious that it can take up no more.

Q. What is meant by the RENEWAL of the atmosphere?

A. If the air is changed, vaporisation can, of course, procced more rapidly, on the principle last stated.

Q. How does the ExTENr of the surface evaporated AFFECT vaporisation?

A. The greater the surface from which the vapours can escape into the air, the more readily and rapidly will vaporisation be complete. 
Q. Under what TwO mEAns does the subject of vaporisation divide itself ?

A. Into ebullition and evaporation.

Q. What is the DIFrereace between EBULLITION and EVAPoration?

A. In ebullition the production of vapour is so rapid, that its escape gives rise to a visible commotion in the liquid; in evaporation the vapour passes off quietly and insensibly.

\section{CHAPTER XIX. \\ EBULLITION AND EVAPORATION.}

SECTION I.-EBULLITION.

Q. What is MEANT by EBULlition?

A. To the rapid production of vapour in bubbles of a larger or smaller size in the sume liquid, we give the name ebullition or boiling.

Iing. 62 .

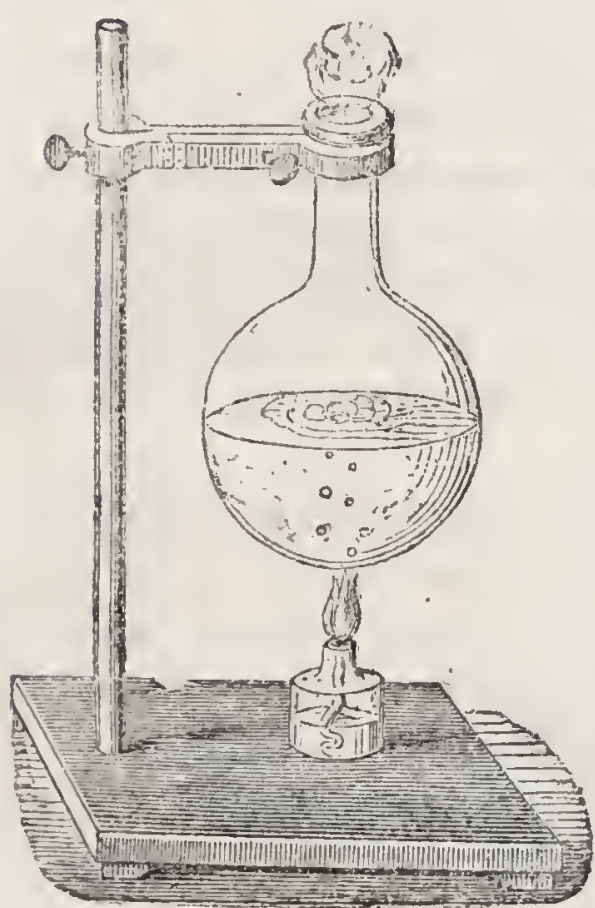

Q. What are the PHENOMENA of EBULLITION?

A. A liquid substance, heated from without, passes into the form of vapour chiefly in that part where the heat is most intense. When heat is applied to the bottom of a vessel, the vapour rises in bubbles through the water to the surface. (See fig. 62.)

Q. Does EbUlition commenco at a FIXed temperature?

A. Yes; but the temperature is different in different liquids.

Q. Does the temperature INCREASE as EBULhition iroceeds?

A. No. Whatever may be the intensity of the source of heat, the temperature remains stationary from the moment ebullition begins.

Q. What do we mean by the corling pornt of a liquid?

A. 'i'he temperature at which vapour rises with sufficient freedom to cause the phenomenon of ebullition is called the boiling point.

Q. Why does roILina Water STWELL ?

$A$. Because it is expanded by the heat; the heat of the fire driving the particles of water farther apart from each other, and making them, consequently, take up more room; in other words, the water swells.

Q. What is meant when it is said that " mest drives the PARTicuss of water farther APAF.T from each other ?"

A. Water is composed of little globules, like rery small grains of 
sand; the heat drives these particles away from each other, and (as they then require more room) the water swells.

Q. Why does Boiling Water BubBie?

A. Because the vupour (rising through the water) is diffused, and forces up bubbles in its effort to escape.

All the air of water is expelled at the commencement of its boiling.

Q. At what TEMPERature do LIQUids rom?

A. Ether, $100^{\circ}$; alcohol, $173 \frac{1}{2}^{\circ}$; water, $212^{\circ}$; water, with onefifth salt, boils at $219^{\circ}$; syrup, $221^{\circ}$; oil of turpentine, $314^{\circ}$; sulphuric acid, $472^{\circ}$; linseed oil, $640^{\circ}$; mercury, $656^{\circ}$.

Q. Is the boiling point of the same liquid always CoNsTANT?

A. Yes, under the same conditions; but it is liable to be afiected by various circumstances.

Q. Will water boil any QUTCKER in a METALLIC ressel than in a ressel of GLASS ?

A. It was observed by Gay Lussac that pure water boils precisely at $212^{\circ}$ in a metallic vessel, and at $214^{\circ}$ in one of glass.

Q. To what CaUse is this difference ascribed?

A. To the fact that the water adheres to glass more powerfully than to a metal.

Q. What effect does it have if water is boiled in a GiLSS vessel, the sides of which have been previously washed with SULPHURIO $\Lambda$ Cid ?

A. The presence of the acid so promotes the contact and adhesion of the glass and water, that the boiling point is raised to $221^{\circ}$.

Q. Why will boiling take place more uniformly, and at a somewhat lower temperature, if the sides of the ressel ke uneven, or when metallic filings, wire, \&c., are introluced ?

$A$. The formation of vapour in a mass of boiling liquid takes place principally at the edges of any solid bodies that may be contained in it ; and the introduction and presence of uneven substances facilitates its formation.

Q. What orIIER CAUSE has a powerful intuence in regulating the boiling point of liquids?

$A$. The pressure of the atmosphere. If the pressure be less than usual, then the boiling point of water and all other liquids will be lower than usual; if the pressure increases, and the barometer rises, the temperature of ebullition will be proportionably increased.

Q. If the atmospleric pressune be entirely REMoved, or if water be made to boil in a vacuum, at what TENPERATURE will EBULLITION commence?

A. At a point $140^{\circ}$ lower than in the open air.

Q. Thy is not a TIN vessel containing water DESTrOYeD BY FIRE, in the same way that a similar ressel would be, if placed upon the fire wITHo UT any water?

A. Because the water absorbs the heat communicated by the fire to the metal, and discharges it with the steam produced. The bottom of the tin vessel, so long as it contains water, can never exceed the temperature of the water; but if the water is absent, the inetal retains the heat, and the vessel is soon burnt or melted.

Q. What is distilitatios?

A. A process by which one body is separated from another by means of heat, in cases where one of the bodies assumes the form of 
vapour at a lower temperature than the other ; this first rises in the form of vapour, which is received and condensed in a separate vessel.

Q. How is the PROCESS of simple distillation EFEECTED?

A. A peculiar-shaped vessel, called a retort (fig. 63), is half filled with a volatile liquid and heated; the steam, as it forms, passes through the neck of the retort into a glass receiver contained in a vessel filled with cold water, and is then condensed.

Fig. 63.

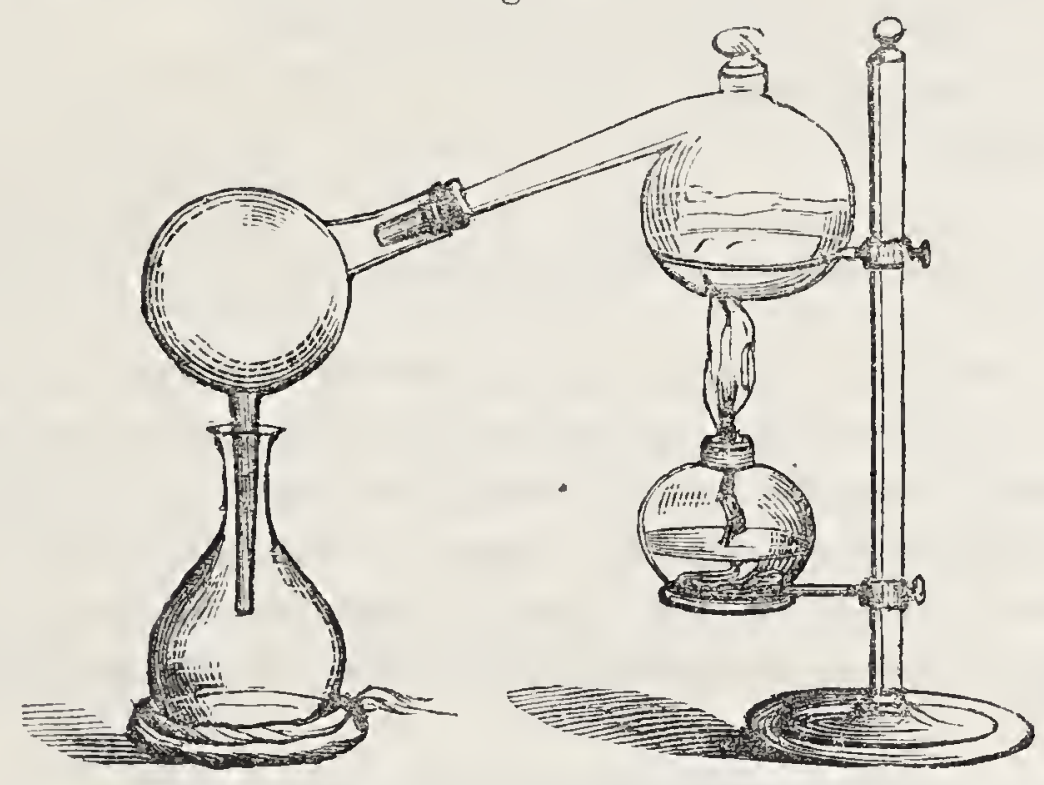

Q. When the ressel used for generating the vapour is VERY LARGE, what is it CALIFD?

A. A "still;" and for condensing the vapour, vats are constructed, holding serpentine pipes or "worms," which present a greater condensing surface than if the pipe had passed directly through the vat.

Fig. 64.

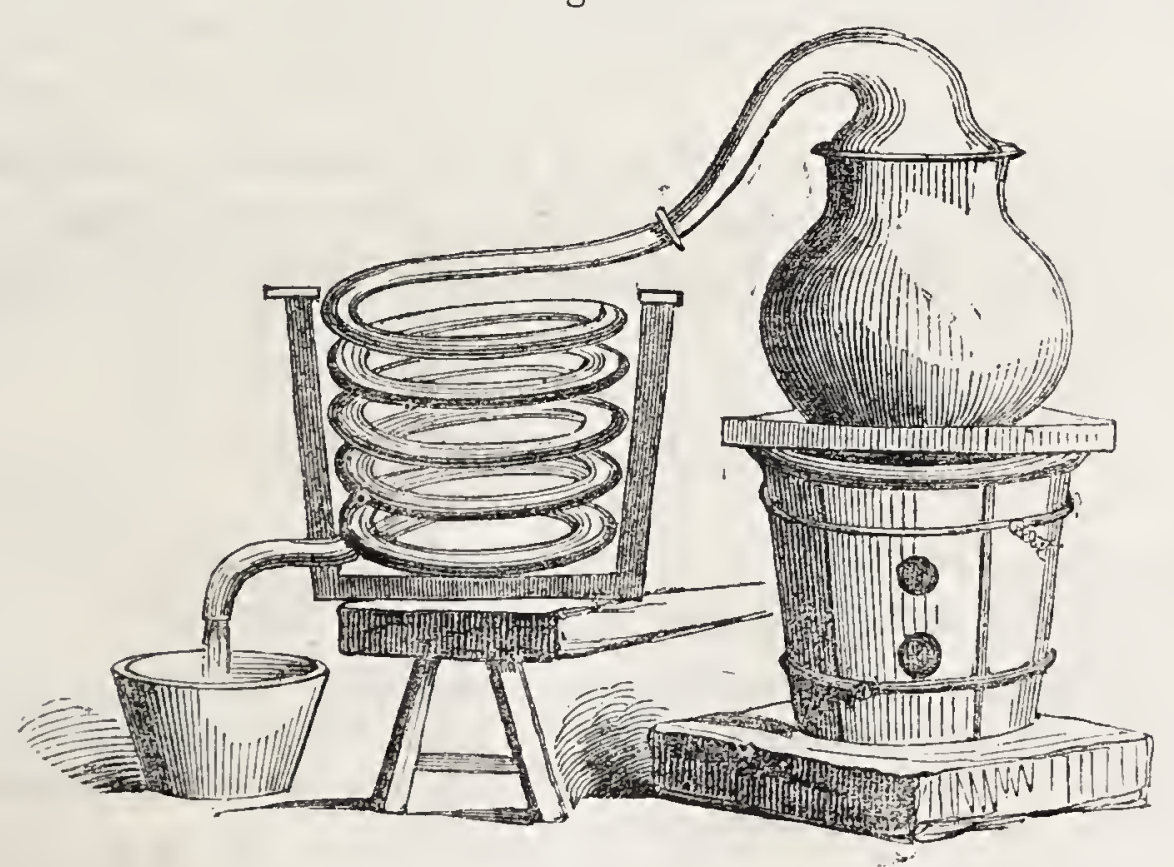

To keep the coil of pipe cool, the vats are filled with cold water. (See fig. 64.)

In this figure a furnace is represented, in which is fixed a copper vessel to contain the fluid. Heat being applied, the steam rises in the head, and passes through the worm, which is placed in a vessel of water-the refrigerator. The vapour thus generated is condensed in its passage, and passes out as a liquid by the external nipe into a receivel 
Q. Why is water obtained in this manner by distillation PURRR than spring water?

$A$. Because the non-volatile, earthy, and saline portions contained in all spring waters do not ascend with the vapour, but remain in the retort. By this means very volatile bodies can be easily separated from less volatile ones; as brandy and alcohol from the less volatile water which may be mixed with them.

Q. Do some substances deposit their vapours in a souid form?

A. Yes; sulphur, camphor, arsenic, \&c., when vaporised by heat, deposit their condensed rapours in a solid form.

Q. What is this Process termed?

A. Sublimation.

Q. What BEAUTIFUI APPLICATION in the arts has been made of the fact that liquids boil at much Lown TEMPERATUR: when freed from the pressure of the atmosphere than in the open air?

A. In the refining of sugar, if the syrup is boiled in the open air, the temperature of the boiling point is so high that portions of the sugar become decomposed by the excess of heat, and lost or injured; the syrup is therefore boiled in close vessels from which the air has been previously exhausted, and in this way the water of the syrup may be evaporated at a temperature so low as to prevent all injury from heat.

Q. What is the IFFERENCE between drying by heat and distilling?

A. In the one case, the substance vaporised, being of no use, is allowed to escape or become dissipated in the atmosphere; while in the other, being the valuable part, it is caught and condensed into the liquid form.

Q. What is the VAPOUR from damp linen?

A. The vapour from damp linen, if caught, would be distilled water.

Q. What is the VAPOUR given out by WHEAT BREAD during the process of baking?

$A$. The vapour given out by bread while baking would, if collected, be a spirit, like that obtained in the distillation of malt.

\section{SECTION II.-EVAPORATION.}

\section{Q. What is EVAPoration?}

\section{A. The conversion of a fluid into vapour.}

When vaporisation takes place only from the surface of a body, either because the heat has access to that part, or because the evolution of vapour takes place through the medium is a gas or air already present, the action can only be recognised by the diminution of the bulk of the body : this phenomenon is called evaporation.

Q. What EFFECTS are produced by evaporation?

$A$. The substance vaporised absorbs heat from the body whence it issues; and the body, deprived of a portion of its substance by evaporation, loses heat. 
Q. Why does WATER freeze under the RECEIVER of an AIR-PUMP when ETHER is present and the AIR exhausted?

$A$. Because evaporation is very greatly increased by the diminution of utmospheric pressure; and the ether, evaporating very rapidly, produces sufficient cold to freeze the water.

Q. Why is it customary, in very Hot countries, to sit in rooms separated by cur'raINS iustead of walls or doors, and to keep these curtains constantly sprinkled with WATER?

A. Because curtains are bad conductors of heat; and the rapid evaporation of water reduces the temperature of the room ten or fifteen degrees.

Q. Why does TATERINa the STREETS and ROADS COOL them?

$A$. Because they part with their heat to promote the evaporation of the water sprinkled on them.

Q. Why does a SHOWER of RAIN COOL the AIR in summer time?

$A$. Because the wet earth parts with its heat to promote evaporation; and when the earth is cooled, it cools the air also.

Q. Why does BREAD become HARD after it has been KEPT a few days ?

$A$. Because the vapour and gases escape, leaving the solid particles $d r y$, so that they collapse and become more solid and hard.

Q. Why are GLUE, GUM, STARCH, and PASTE, adhesive?

$A$. Because the water used with them rapidly evaporates, and leaves them solid; and they insinuate themselves so intimately into the pores of the substances with which they come in contact, that when the water evaporates the whole is one solid mass.

They lose their adhesiveness when dissolved in water, and therefore must always be siffered to become $d r y$ before they will hold with tenacity.

$Q$. Why will not lucifer matches ignite if they are DAMP?

A. 1. Because the cold, produced by the evaporation of the water, neutralises the heat produced by the friction of the match; and

2. Because the damp prevents the free accession of oxygen to the match, without which it cannot burn.

Q. Why does water in a very EXPOSED place freeze more rapidly than that which is under cover or in a place less exposed ?

A. 1. Because evaporation goes on more rapidly when water is exposed, and carries away heat from the general mass; and

2. Any covering will radiate heat into the water below, and prevent the mass from cooling down to the requisite temperature to cause congelation.

Q. Why does PAINT often BLISTER from heat?

$A$. Because the heat, penetrating through paint, extracts some little moisture from the wood, and turns it into vapour or steam. As this vapour requires room, it throws up blisters in the paint to make room for its expanded bulk.

Q. Why does the SUN Or FIRE WARP WOUD?

$A$. Pecause heat draws out the moisture from that part of the woor which faces it, and causes the heated surface to shrint; as, therefore, the heated surface of the wood shrinks, and is smaller than the other surface, it draws it into a curve, and the wood is warped. 
Q. How does STARCH assist in giving a smooth GLAZED SURFACE to linen?

$A$. It fills up the interstices between the threads, and makes the fabric of more uniform density.

Interstices between the thrcads-that is, the small groove or space between each thread.

Q. How does STARCH STIFFen linen?

A. By filling the interetices of the linen, by which means it is rendered more rigid and firm.

2. What are the CHIEF CIncusstances which influence the process of evaporation?

4. Extent of surface, and the state of the air as to temperature, dryness, stilness, and density.

Q. How does EXTTHA of SURFACE influence evaporation?

A. Evaporation proceeds only from the surface of fluids, and, therefore, cateris paribus, must depend on the extent of surface exposed.

Cueteris paribus -other things being equal.

Q. How does the teMperature of the air influence evaporation?

$A$. The effect of temperature in promoting evaporation may be illustrated by placing an equal quantity of water in two saucers, one of which is placed in a warm and dry, and the other in a cold and damp, situation. The former will be quite dry before the latter has suffered an appreciable diminution.

Q. How does the state of the air, as to DRyness or MOISTURE, influence evaporation?

$A$. When water is covered by a stratum of dry air, the evaporation is rapid, even when its temperature is low; whereas it goes on very slowly if the atmosphere contains much vapour, even though the air be very wàrm.

Q. Why are wet roads and footpaths, on some days, dried in a FEW HOURS, while on others they will continue wet, without any marks of drying?

$A$. In consequence of the state of the air in relation to the vapour with which it is charged. In the one case, it is undercharged, and therefore readily receives the evaporation from the roads and footways, which accordingly become dry; in the other case, it is at or near its point of saturation, and can receive no more vapour. The roads, therefore, remain wet, though no more rain falls.

Q. How does the sTiLINESS of the air influence evaporation?

A. Evaporation is far slower in still air than in a current. The air immediately in contact with the water soon becomes moist, and thins a check is put to evaporation. But if the air be removed by wind from the surface of water as soon as it has become charged with rapour, and its place supplied with fresh dry air, then the evaporation continues on without interruption.

$Q$. How does the DENSITY of the air influence evaporation?

$A$. Pressure on the surface of liquids has a remarkable influence over evaporation. This is easily proved by placing ether in the vacuum of an air-pump, when vapour rises so abundantly as to produce ebullition. 
Q. Is the process of evaporation contined to the SURFACE of the EARTII?

A. No; evaporation takes place from the surface of the sea as well as the surface of the soil, and from all animal and vegetable productions.

Q. "All the rivers run into the sea :" why is not the sea full?

A. Because the quantity of water evaporated from the surface of the sea is equal to the quantity poured into it by the rivers; therefore the sea is never full.

Q. Why is it frequently cooLer after rain?

$A$. Because water which falls from the atmosphere soon returns to it in the form of vapour, carrying with it, in the latent form, a large amount of heat taken from every object, thus moderating the temperature of the earth, and refreshing the animal and regetable creations.

Q. Does evaporation take place from the surface of swow and ICE ?

A. Yes, to a very considerable extent, even when the temperature of the air is below the freezing point.

Q. What very singular FACT has been noticed to exist in regard to the diffusion of vapours throughout the atmosphere?

A. That the vapours of some bodies arise in quantity and density into any space filled with air, in the same manner as if air were not present, the two fluids seeming to be independent of each other.

Q. Can AS MUCH vapour of water be forced into a vessel FULL OF AIR as into one from which the air has been Exrinusted?

A. Yes; precisely the same quantity.

Q. What, then, is the only way in which we can privent the dissipation of a volatile body by heat?

A. By enclosing it in an atmosphere composed of the substance of the body itself. 'Thus we can save camphor, musk, volatile oils, spirits, water, \&c., by placing them in closed bottles or vessels, in which, in addition to the air present, an atmosphere of their own substance is formed, involving the remaining masses with a pressure proportioned to their temperature and density.

\section{CHAPTER XX.}

STEAM.

Q. What is STEAM?

A. The vapour of boiling water.

Q. Is STFAar Invisible?

A. Yes; but as soon as it comes in contact with the atmosphere it condenses, and instantly becomes visible. 
Q. How can you ascertain that STEAM is INVISIBLE?

A. By observing the spout of a boiling kettle, from which steam is escaping; for the distance of about half an inch from the spout the steam is invisible, though it is afterwards clearly seen.

Q. How do you account for the STEAs being MNTISIBLE for half an inch?

A. As it first escapes, the air is not able to condense it; as it becomes more diffused, condensation takes place.

Q. Does air ever exist without STEAn or vapour of water?

A. Air without steam (theoretically called dry air) is not known to exist in nature, and is probably not producible by art.

Q. Is the visibie arATter, popularly called steam, really TRUE STEAM?

A. By no means, and should be carefully distinguished from steam proper, or the aeriform state of water. The cloud or smoke-like matter alluded to is really not an air or vapour at all, but a dust. like cloud of minute bodies of liquid water, wafted by a current either of true steam, or, more frequently, of mere moist air.

Q. Is it necessary to the production of steam that water should be raised to the BOILING TEMPERATUHE?

A. It is not; the surface of any watery liquid, about $20^{\circ}$ warmer than amy superincumbent air (however warm or cold that may be), rapidly gives off true steam, which is invisible, but which no sooner mixes with colder air than it is recondensed into water, and assumes the form of minute globules.

Q. What canses the visible WIITE APPRARANCE of condensed steam?

A. The myriads of minute globules of water into which the steam is condensed are separately invisible to the naked eye, but each, nevertheless, reflects a minute ray of white light. The multitudes of these reflecting points, therefore, make the space through which they are diffused appear like a cloudy body, more or less white according to their abundance.

Q. In what respects does steam agree with common air in its MECHANICAL and optiont properties?

$A$. Like ai:, steam always fills any artificial space, however large, that may be allowed it, and always presses equally in every direction against the whole of the enclosing surface, and with a pressure which (other things being equal) diminishes in the same ratio that the space is enlarged, and increases in the same ratio that the space is diminished. Although, like air, it has an appreciable and unalterable weight, it is always many times rarer than any liquid or solid, and when pure it is always invisible and odourless.

Q. In what manner is the production of steam in boiling water first manifested?

$A$. When steam begins to be produced, as in the process of making water boil, and the heat overcomes the atmospheric pressure on the surface, small bubbles are formed, adhering slightly to the sides of the vessel, as may be conveniently observed by using a Florence flask, or any other thin glass vessel. 
Q. In what parts of the boiler will its development be most conspicuous?

A. The bubbles are formed most rapidly at those points against which the flame is most strongly directed.

Q. How much LIGHTER is steam than water?

A. About 1,700 times; because a quantity of water yields nearly 1,700 measures of steam at $212^{\circ} \mathrm{F}$.

Q. How XIUCH STEA3r will a cubic inch of water furnish?

A. A cubic inch of water expands into about a cubic foot of steam at $212^{\circ} \mathrm{F}$., under the ordinary atmospheric pressure.

Q. Upon what does the power of steam DEPEND?

A. On the tendency which water possesses to expand into vapour when heated to a certain temperature.

Q. Water, when placed in a vessel over the fire, gradually attains the boiling temperature, or $212^{\circ}$; but afterwards, notwithstanding the heat continues to pass into it, it does not increase in temperature ; what becomes of the additional heat?

A. It passes into the steam, and becomes latent.

Q. If we immerse a thermometer in boiling water, it stands at $212^{\circ}$; if we place it in the steam immediately above, it indicates the same. Now, how do we kNow that steam contains MORE HEAT than boiling water?

A. If we mix an ounce of water at $212^{\circ}$ with five and a half ounces of water at $32^{\mathrm{Q}}$, we obtain six and a half ounces of water at a temperature of about $60^{\circ}$; but if we mix an ounce of steam at $212^{\circ}$ with five and a half ounces of water at $32^{\circ}$, we obtain six and a half ounces of water at $212^{\circ}$.

Q. How much MORE HEAT, therefore, does the ounce of steam at $212^{\circ}$ contain than the ounce of mater at the same temperature?

A. The steam, from which the increased heat is all derived, contains so much more heat than the ounce of water at the same temperature, as would be necessary to raise six and a half ounces of water from the temperature of $60^{\circ}$ to $212^{\circ}$, or six and a half times as much heat as would be requisite to raise one ounce of water through about $152^{\circ}$ of temperature.

This quantity of heat will, therefore, be found by multiplying $152^{\circ}$ by six and a half, which will give a product of $983^{\circ}$ - the excess of leat contained in an ounce of steam at $212^{\circ}$ over that contained in an ounce of boiling water of the same temperature.

Q. Why is steAx, when conveyed in pipes, well adlapted for warming dwellings, churches, manufactories, \&c.?

$A$. Because as it condenses it gives up nearly $1,000^{\circ}$ of heat to the adjoining objects.

Q. What is the most IMPORTANT Property of steam?

A. Its elasticity or pressure. By virtue of this property, when freed from the limits which confine it, steam will dilate into any space to which it may have access.

Q. If a quantity of pure steam be confined in a close ressel, in wHAT MaNNer will its pressure be exerted ?

$A$. It will exert on every part of the interior of the vessel a certain pressure directed outwards, and having a tendency to burst the vessel. 
Q. In what MANNER is the elastic pressure of steam DIMUNISHED by EXPANSION?

A. When the space it occupies is doubled, the temperature being preserved, its elastic pressure is equal to one half; and, in like man11er, in whatever proportion the space it fills be incrcased, its elastic pressure will be diminished in the same proportion.

Q. In what MANNer does the mLASTICITY of steam depend on the Pressure under which it is formed?

A. Steam raised from water, boiling under any given pressure, has an elasticity always equal to the pressure under which the water boils.

Thus, when water is boiled under the ordinary atmospheric pressure (when the barometer stands at 30 inches), the steam which is generated at the temperature of $212^{\circ}$ has an elastic pressure equal to that of the atmosphere. If water be boiled under a diminished pressure, and therefore at a lower temperature, the steam which is produced from it will have a pressure which is diminished in an equal degree.

Q. As water boils at different temperatures under different pressures, does the AMOUNT of HEAT rendered latent or absorbed at different temperatures and pressures YARY?

A. Yes; it has been found that the heat absorbed by vaporisation is always less the higher the temperature at which ebullition takes place. Thus, if water boils at $31^{\circ}$, the heat absorbed in ebullition will be less by $100^{\circ}$ than if it be boiled at $212^{\circ}$; and, again, if water be boiled under a diminished pressure at $112^{\circ}$, the heat absorbed in vaporisation will be $100^{\circ}$ more than the heat absorbed by water boiled at $212^{\circ}$.

Q. How great a pressure does steam, formed under ordinary circumstances, have to overcome before it can rise from the surface of the water?

A. That of one atmosphere-fifteen pounds on every square inch, or one ton on every square foot-a force equivalent to the strength of six hundred horses.

Q. What HATPENS when the temperature of steam, generated under ordinary pressures, is REDUCED BELOW $212^{\circ} \mathrm{F}$.?

\section{A. It is immediately condensed into water.}

Q. As steam sustains and elevates a weight occasioned by the pressure of the atmosphere, of fifteen pounds per square inch, what TAKES PLACE when a column of steam is MMEDIATELY CONDENSED?

A. The atmospheric weight will immediately fall with a force equal to that with which it was raised.

Q. How can steam be used to advantage for cooking vegetables, \&c. ?

A. In cookery, if steam raised from boiling water be allowed to pass through meat and vegetables, it will be condensed upon their surfaces, imparting to them the latent heat which it contained before its condensation, thus cooking them as effectually as if they were immersed in boiling water.

Q. Is the teMrperature of the water in working steam boilers ABOVE $212^{\circ}$ ?

A. Yes; water boils, under the ordinary pressure, at $212^{\circ}$. If the pressure be increased, the boiling temperature is likewise increased. The steam rising from the water in a close boiler 
presses on the surface of the water equally as on the surface of the boiler, and the temperature of the water rises.

If a boiler, containing water thus overheated many degrees beyond the boiling point, be suddenly opened, and the steam allowed to expand, the whole water is immediately blown out of the vessel as a mist by the steam formed at the same instant throughont every part of the mass. To use a common expression, "the water flasles into steam."

Q. What do we mean when we speak of HIGH-PRESSURE steam ?

$A$. High-pressure steam is merely steam condensed, not by withdrawal of heat, but by pressure, just as high-pressure air is merely condensed air. To obtain a double, triple, or greater pressure of steam, we must have twice, thrice, or more steam under the same volume.

Q. Is high-pressure steam, escaping from a boiler heated to $300^{\circ}$ or more, поттвк than low-pressure steam escaping from a boiler at $212^{\circ}$ ?

$A$. No; for the instant that high-pressure or condensed steam escapes into the air, it immediately expands and becomes low-pressure steam, and is greatly cooled down by its expansion.

Q. Does high-pressure steam, acting in a boiler at a high temperature, exert a GREATER MECHANICAL and CHENICAL POWER than low-pressure steam?

$A$. It does; high-pressure steam acting upon bones, breaks up and dissolves the whole mass, extracting all the glue and fat, when ordinary steam would dissolve nothing.

In the Western States of America, where large quantities of lard are manufactured, the whole hog is exposed to high-pressure steam, and the carcass reduced in short time to a fat fluid mass.

Q. Can high-pressure steam be raised to a very ELEVATED DEGREE of heat ?

$A$. It can; in some of the methods lately introduced for purifying oils, \&c., the temperature of the steam, before its application, is required to be sufficiently elevated to enable it to melt lead.

Q. Can steam be applied to the CARBONISATION of WOOD?

A. It has lately been applied to that purpose : ordinary steam is conducted through red-hot pipes, whereby it attains a very high degree of temperature. It is then allowed to pass into a vessel containing wood intended to be converted into charcoal. The heated steam, penetrating into the pores of the wood, drives off the volatile portions, the water, the tar, \&c., and leaves the pure carbon alone behind.

\section{SECTION I.-THE STEAM-ENGINE.}

Q. What is the STEMM-ENGINE?

$A$. The steam-engine is a mechanical contrivance by which coal, wood, or other fuel is rendered capable of executing any kind, of labour.

"Coals are by it made to spin, weave, dye, print, and dress silks, cottons, woollens, and other cloths; to make paper, and print books upon it when made; to convert corn into flour; to express oil from the olive, and wine from the grape; to draw up metals from the bowels of the earth ; to pound and smelt it ; to melt and mould it ; to roll it and fashion it into every desirable form; to transport these manifold products of its own labour to the doors of those for whose convenience they are produced; to carry persons 
and goods over the waters of rivers, lakes, seas, and oceans, in opposition alike to the natural difficulties of wind and water ; to carry the windbound ship out of port, to place her on the open cleep, ready to cominence her royage; to transport over the surface of the sea and the land persons and infurmation from town to town, and from country to country, with a speed as much exceeding that of the ordinary wind, as the ordinary wind exceeds that of a pedestrian."-LARUNeR.

Q. What SUBSTANCE furnishes the means of calling the powers of coal into activity?

A. Water.

Q. How arucre water will two ounces of coal evaporate?

A. About a pint.

Q. How MUCh STusar will this produce?

A. Two hundred and sixteen gallons.

Q. How much medunical Force can this steam exert? foot.

$A$. It can raise a weight of thirty-seven tons to the height of one

Q. What ANount of Force can a man exert when applying his strength to the best alsantage through the help of machinery?

A. It has been found by experiment, that a man working on a tread-mill continuously for eight hours, will elerate one and a half millions of pounds to the height of one foot.

Q. With Irow MUCIr COAL will a well-constructed steam-engine perform the same labour?

A. With the expenditure of a pound and a half.

Q. Huw muon cosis then would be equivalent to the average power of an able-bodied wan during his active life, supposing him to work for twenty years at the rate of eight hours per day?

A. The consumption of about four tons of coal would evolve in a steam-engine fully as much power.

Q. The great pyramid of Egynt is five hundred feet high, and weighs twelve thousand seven hundred and sixty millions of pounds. Herodotuss states that in construeting it one hundred thousand men were constantly employed for tiventy years. With how sucr CUAL could all the materials of this pyramid be RAIEED to their present position from the ground?

A. With the expenditure of four hundred and eighty tons of coal.

Q. What is generally considered the GrEATEST WoR recorded to have been performed by steam-engines?

A. The Cornish engines of Ingland have raised sixiy thousand tons of water a foot high with the expenditure of a bushel of coat.

Q. What is a Conisir steam-engine?

A. An engine modelled after the pattern of those employed in the tin and copper mines of Comuvall, for raising water from the mines -these mines being sunk to an immense depth in the earth.

Q. Why have they obtrined so great a CErEbitax as to make them sTANDArds of reference?

A. As Cornwall produces no coal, that invaluable fuel is enhancer in price by the cost of freight from other counties; the miners were hence led to extract from the coal all the heating power they possibly could; and hence has arisen the celebrity of the steam-engines made and used in Cornwall-a celebrity due to the fact that they get more power out of a bushel of coal than any other steam-engines. 
Q. When a miner or engineer wishes to calculate the relative efficiency of two engines, what STANDARD does he adopt?

$A$. He determines how much water can be raised to a height of one foot by the steam-power generated through the heat of one bushel of coal; and this he calls the "duty" of the engine.

Q. In what MANNER is steam rendered useful for mechanical purposes?

A. By its pressure.

Q. Why OANNo' steam, like wind and water, be. made to act advantageously by its impulse in the open air?

A. Because the momentum of so light a fiuid, unless generated in vast quantities, would be inconsiderable. The first attempts, however, to employ steam as a moving power, consisted in directing a current of steam from the mouth of a tube against the floats or vanes of a revolving wheel.

Q. In order to render the pressure of steam available in machiners, what condtrons are necessary?

A. The steam must be confined within a cavity which is air-tight, and so constructed that its dimensions or capacity may be altered without altering its tightness.

Q. When the steam enters such a vessel, what TiRES PLACE?

A. The steam by its pressure entarges the actual cavity, by causing some movable part to recede before it, and from this movable part motion is communicated to the machinery.

Q. How is such an arrangement of machinery EFEECTED?

A. By having a hollow cylinder with a movable piston accurately fitted to its base (as shown in fig. 65). The piston thus employed has a reciprocating motion, which is converted when necessary into a rotary one by appropriate mechanism.

Q. In a simple working engine, how may continuous motron be kept up by means of the piston and cylinder?

$A$. The pressure of the steam, admitted into the cylinder below the piston, causes the piston to rise, and the piston-rod also rising, communicates motion to a lever connected with it. When the piston is thus raised, if the steam be suddenly condensed or withdrawn from under it, a vacum will be formed, and the pressure of the atmosphere on the piston above will drive it down. It may then be raised anew by the admission of more steam, to be condensed in its turn, and in this manner the alternate motion may be continued indefinitely.

Q. What is a coNDENSIra steam-engine?

It is one in which the stean which has been employed in raising or depressing the ${ }_{4}$ piston is condensed, after it has accomplished its object, leaving a vacuum above or below the piston.

Q. In what IIANNER is the steam below the piston CONDENSED?

$A$. By opening at the proper time a communication between the cylinder and a strong vessel situated at a distance from it, called the condenser. Into this vessel a jet of cold water is thrown, which 


\section{instantly condenses the steam escaping from the bottom of the cylin- der into water.}

Q. In this engine, does the piston descend in the cylinder by the pressure of the ATMOSPHERE, in consequence of the vacum formed beneath it by condensing the steain ?

A. The piston would descend by the atmospheric pressure alone, and in the original atmospheric engines it did so; but in the improved engines, or the so-called double-action engines, steam is admitted into the cylinder above the raised piston at the same moment that it is removed below it; and thus the expansive force of steam is exerted in the returning as well as in the ascending stroke, and a much greater impetus is given to the machinery than by the old method.

In this arrangement the top of the cylinder is closed and rendered air-tight, the rod of the piston only passing through it.

The action of a condensing steam-engine may be further elucidated by reference to $f i g .65$, in which $s$ represents the steam-pipe proceeding from the boiler, and ly which steam is admitted to the cylinder. The piston $h$ works air-tight in the cylinder $g$, the rod of which passes air-tight through the stuffing-box $i$. The upper valve box a contains a single valve, which, when open, admits the steam into the cylinder; and also into the pipe which connects this with the lower valve box. The lower box contains two valves, $b$ and $c$; the valve $b$, when open, admits the steam to pass from the cylinder above the piston, by the connecting tube to the cylinder belor the piston; the valve $c$, when open, admits the steam to pass from below the cylinder down into the condenser d. This steam entering the condenser, meets the jet of water through the valve $d$, where it is condensed. The valve $e$, opening outwards, permits any steam which is not condensed, together with such atmospheric air as is accumulated, to pass away.

The valve $a$ is called the upper steam valve; $b$, the lower steam valve; $c$, the cxhausting valve; and $d$, the condensing valve.

The manner in which the alternate ascent and descent of the piston is produced is as follows:-

In the first place, all the air which fills the Fig. 65 .

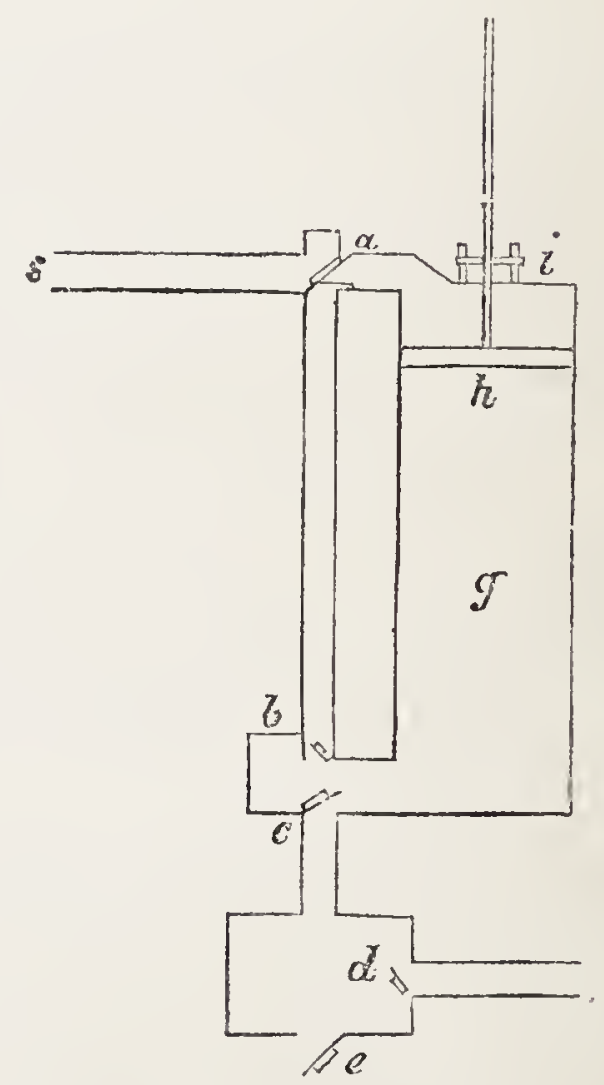
cylinder and tubes must be expelled. To do this, the valves $\alpha, b$, and $c$ must be opened. The steam will pass through the pipe $s$ into the upper part of the cylinder, and along the tube down through the valves $b$ and $c$ into the condenser $d$. After the steam ceases to be condensed by the cold of the apparatus, it will lush out, mixed with air, through the valve $e$, which opens outwards.

The apparatus is thus flled with steam, and all the valves are now to be closed; but in a few minutes a vacuum will be formed in the condenser by the cold surface of that vessel.

The apparatus being in this state, let the upper steam valve $a$, the exhansting valve $c$, and the condensing valve $d$, be opened. Steam will thus be admitted through $a$ to press upon the top of the piston, the steam being prevented from, circulating below the piston, by the valve $b$ being closed. But the steam below the piston will rush through the exhausting valve $c$ into the condenser, where a jet of cold water through the condensing valve $d$ will instantly condense it, and thus leave a vacuum below the piston in the cylinder. Into this vacuum the piston is instantly pressed by the action of the steam in the upper part of the cylinder.

When the piston has thus been forced to tlie bottom of the cylinder, let the valves $a$, 
$\therefore$ ami $d$ be closed, and let, the lower steam valve $b$ be opened. The effect of this will be, that the further ingress of steam will be stopped, and the further eondensation of stean will eease; and thus the steam which is shut within the apparatus will press equally (nn all sitles, so that the pressure on the upper and under sides of the piston will he equal. 'Lus there is no force to restrain the piston at the bottom of the eylinder, except its weight, which is more tban balancel by the weight of the pump-rod at the other end of the beam, and by the preponderanee of whieh the piston rises as in the atmospheric engine.

When the piston has arrired to the top of the eylinder, the raives $a, c$, and $d$ are again upened, when steam again presses vis the top of the pistun, while a racuur is formed below it into whieh the piston is driven, as already shown, and so on eoutinually.

Q. What oTHER NAME besides "eondensing engines" is given to engines working upon this minciple?

A. 'They are called low-pressure engines, because the stean employed in working them has a comparatively low temperature, and a moderately elastic force.

Q. What is a NON-CONDENSING or HIGH-PRESSURE stearn-engine?

A. In this engine the apparatus for condensing is dispensed with, and the steam is worked at a high temperature, and afterwards discharged into the open air. In this engine a part of the steam is expended in overcoming the pressure of the atmosphere, and the surplus only can be applied to drive machinery.

Q. With how GREAT A PRESSURE of the steam do the high-pressure engines generally work?

A. With a pressure of thirty to forty pounds on a square inch above the atmospheric pressure.

Q. Deseribe the Manner in whieh the high-pressure engine is made to operate.

A. The steam, in escaping from the boiler to the open air, is obliged to pass through the cylinder, the cavity of which is closed, except where it communicates through the valves. By the opening and shutting of these valves the steam is made to enter the cylinder alternately at each end, and escape by the opposite end. But in doing this its passage is always intercepted by the piston, so that before it can escape it must move the piston from one end to the other of the cylinder. The repetition of this movement gives motion to a beam, or other alternating point, from which it is communicater by a connecting rod or crank to a fiy-wheel and other machinery.

Q. In what siruations are the high-pressure engiries generally used ?

A. Where simplicity and lightness are required, as in locomorive engines; also in situations where a sufficient supply of water for condensation cannot easily be obtained.

Q. How do they COMPARE in SAFETY with the low-pressure or eondensing engines

$A$. They are less safe, yet as they cost less at the outset for cennstruction, they are often preferred.

Q. In a condensing engine, what USE is made of the warter used for condensing the excille steam?

A. The condensing steam renders the water very hot, and it is generally pumped back into the boiler's, thus effecting a great econumy of juel. 
Q. Hurv much greatuk poroe of steam is required in a high-ressure than in a low pressure engine?

A. In a low-pressure engine a vacuum (empty space) being formed on one side of the piston, the force of the steam required to move it is diminished by the amount of atmospheric pressure on the piston. But, in the high-pressure engine, the piston works in both directions against the weight of the atmosphere, and hence requires an additional power of steam equal to the weight of the atmosphere on the piston.

Q. What YARTS are dispensed with in the high pressure engine?

A. All the apparatus for condensing-nothing more being necessary than the boiler, cylinder, piston, and valves.

Q. Of wbat Two PARTS does every sterm-engine consist?

A. First, that which is employed to generate the steam, called the boiler; second; that in which the steam is amplied.

Q. What are the ESSENTIAL QuAlities of a steam-boiler?

A. It is requisite that a boiler should be of sufferent siremgth to resist the greatest pressure which is ever liable to occur from the expansion of the steam. It must also offer a sufficient extent of surface to the fire to insure the requisite amount of vaporisation.

In common low-pressure boilers, it requires about cight square feet of surface of the thiler to be exposed to the action of the tire and flime to boil ofi a cubic frot of water in an hour ; and a cubic foot of water thus converted into steam is equal to one-horse power.

Q. What is the BEST FOR for a boiler?

$A$. The strongest form for a boiler, and one of the earliest which was used, is that of a sphere or globe; but this form is the one which offers least surface to the fire. The figure of a cylinder is on many accounts the best, and it is now extensively used, especially for engines of high-preasure.

This form, usually given to the boilers of tixed engines, is scen in the acenopriging diagram.

jisig. 66.

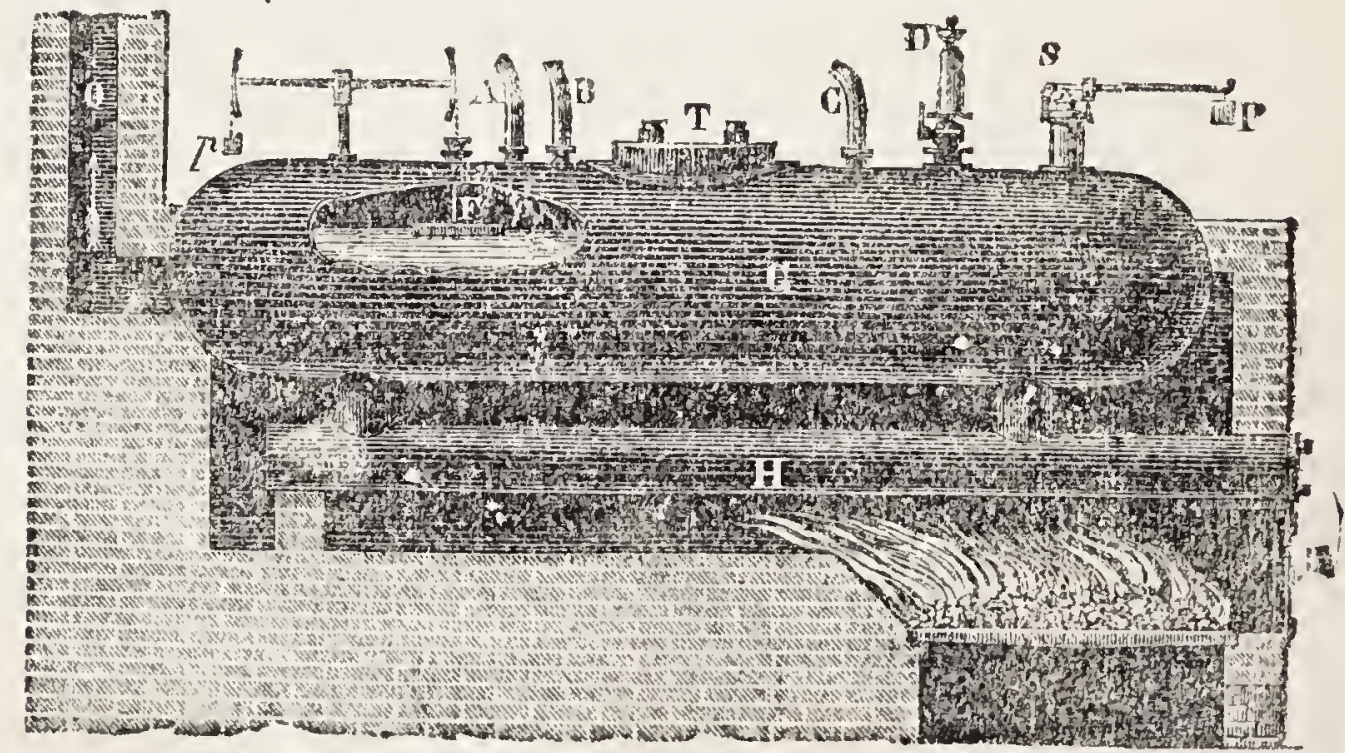

$A$, the trihe which conveys the stean to the valve-chest; $B$, the tu' e which conreys the steam to the BAROA ETER-GAUGR, or mannmetre, which indicates the pressure of steirn in the grisfrator; C, the tube which is used to carry water into the boiler, comm n'y

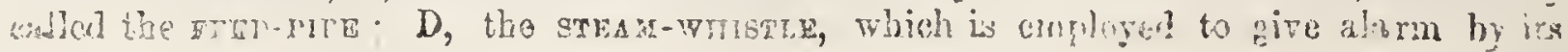


nnise when there is not water enough in the boiler. So long as the level of the water is not too low in the gencrator, the stcam does not eater the whistle, but if the level sinti: below a certain depth, a small float, not scen in the fi., closes the bott nn of the whisilu. descends, and the stea:n is almitted. The rusbing forth of the steam causes the shrory sonnd by striking against the edges of a metallic disc. F, the FLoAt empluyed to indients the level of the water in the generator; $G$, the cyliadrical GENERnTor, made of wanghi iron, and kept completely closed; $H$, the BorLER-TuBrs, placed side by sille; $O$, the PAscare to the chimney; $P$, the wergur which loads the sufety-valve; $p$, the counte:woight of the float; $R$, the noon of the fire-grate; $S$, the SAFNTr-VALVE; and I", lite wAX-IChe, or opening for the admission of the workmen to cleanse or repair the gerestut.

Q. What aDvantages has a cylinder boiler?

A. It has the advantage of being easily comstrueted firon sheets uf metal, and the form is of equal strength exeept at the ends. In snch a boiler the ends should be made thicler than the other parts. 'The furnace is so constructed that the flame and hot smoke may las under the whole length of the boiler, and afterwards around both it sides, before escaping to the chimney.

Q. How is the so-called "FLUE-BOILER" CONSTRUCtes?

A. In this a cylindrical fumace is placed within a cylindricalboiler. so that the fuel is surrounded by water on all sides, and communi cates to it nearly all its heat, except that portion which passes up the chimney.

Q. What is a SAFETY-VALNE?

A. The safety-valve is generally a conical or sufur-loaf lin fitted into the boiler, and opening outwards; it is kept down by a weight equal to the pressure which the boiler is capable of sustaining without daryer from the steam generated within. If the amount of steam at any time exceeds this pressure, it overcomes the resistance of the weight, lifts the valve, and allows the steam to escape.

Q. Notwitlstanding the use of the safety-valve, steam-boilers frecuent? 13 [ k w w th terrific explosions; how is the fact to be Accourvin Fon?

A. The explosion of steam-boilers, when the safety-valve is in good condition and working order, is sometimes inepplicable; but explosions often result from the engineer allowing the water to become too low in the boilers.

Q. How does the fact of allowing the water to becone too low in the boilers tead to YRODUCR FXPLOSIONS?

$A$. Some parts of the boiler, being devoid of water, become highty overheated; when then water is thrown into the boiler in this condition, it comes suddenly into contact with the intensely heated metal. and an inmense amount of sterm having great elastic force is at once generated. In this case the boiler may burst before the inertia of the safety-valve is overcome, and the stronger the boiler the grenter the explosion.

Q. An engine is sometines callod an expansive engine, in contradistinction to a simple ligh-pressurc, or a condeusing or low-pressure engive: what are wo to UNInnerAxn by the Trisa?

$A$. In some engines the steam from the boiler is not allowed to jness against the piston during the whole length of the stroke, but is cut off before the piston has fully completed its asecur or descrent in 
the cylinder. 'ihe stean already in the cylinder immecilately ex. pands, and completes the movement already begun, thus saving a considerable quantity of steain at each movement.

10 carry uth this plan to the hest advantage, the expansive force of the steam must le greatly increased by working it under a high pressure; that is, by having a greater amount of steam in the same space than in an ordinary low-pressure engine?

Q. What ADVANTAGE is it to use a large heavy wheel, called the FLY-WheEL?

A. This heavy wheel, when set in motion, revolves with a consiclerable momentum, and will continue to move after the force which has been imparted to it has ceased to act. This momentum causes the machinery to move uninterruptedly, even if the action of the steam be less at one point than at another.

Q. Do the $\Lambda$ NoIENTS appear to bave been acquainted with the power of steam?

A. They were unquestionably, to some extent, although but little is known on the subject. Whatever knowledge they did possess was confined to the priesthood.

Q. What is KNOWN of these DISCOVERIES?

A. Hero, of Alexandria, who flourished about 120 B.C., has recorded the principal facts then known regarding the vapour of water.

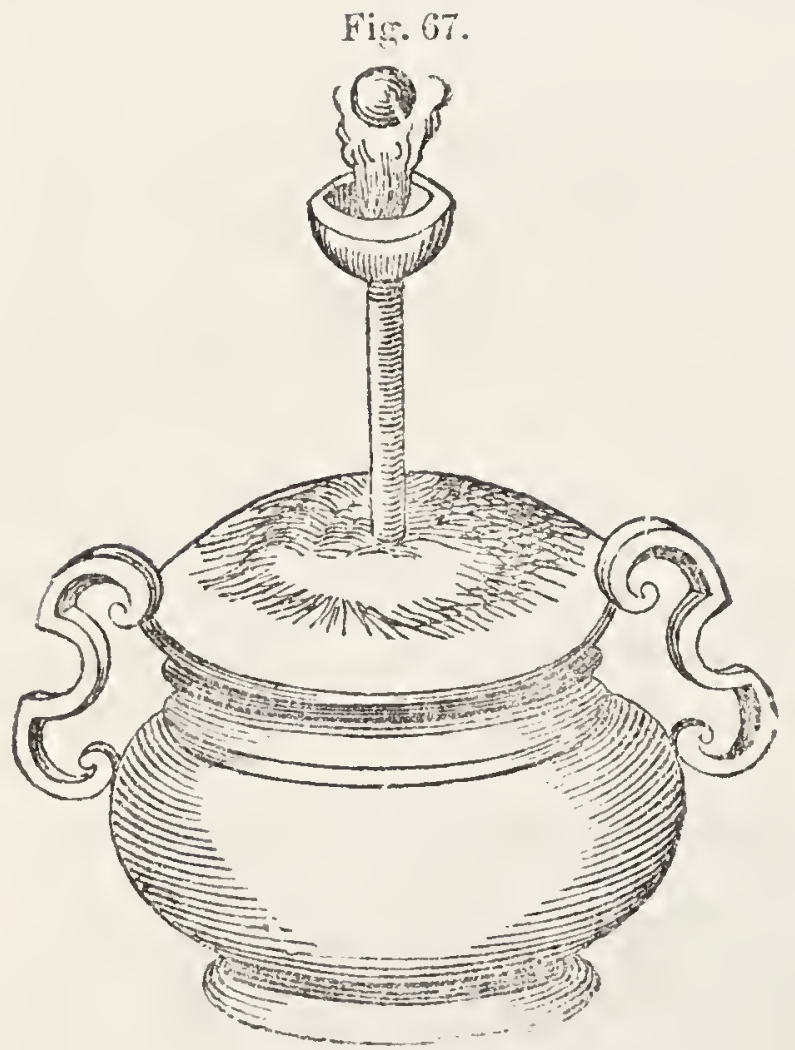

Q. Does he DESCRIBE any MACHINES muved by sTrair?

A. Yes; one of these consists of a pot with a lid, in to which is fastened an upright tube, terminating in a perforated cup, in which is placed a inovable ball. (Sce fig. 67.)

Q. What HEFEOT was produced by this apparatus?

A. Steam being generated in the pot, and suffered to issue through the tube, conmunicated motion to the ball.

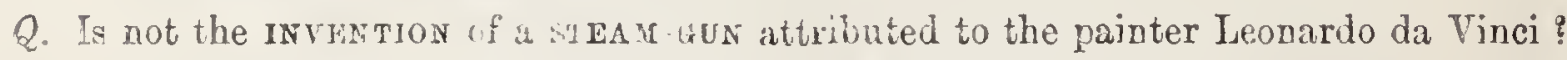

A. Ies; a description and sketch of a steam-gun was discovered mnongst his paintings, preserved in the library of the Institute of France.

Q. In modern times, who appears to have first Expermenten to any practical purpose in regard to using steam for economical purposes?

A. The power of confined steam acting by its pressure, was discowered by the Marrut of Worcester, and an account of its effect published by him in 1663 . He produced a steam power sufficient to burs, a cannon, and constructed a nachine capable of raising water 
to the height of forty feet. Ie has not, however, left any drawings or particular description of his machine.

Q. Who NEXT took up the subject?

A. Thomas Savery, an Englishman, who invented a method for raising water by steam in 1698 .

Q. What was the NATURE of Savery's engine?

A. It consisted of a boiler, a separate steam-vessel, and pipes furnished with valves. The steam from the boiler was first admitted so as to fill the steam-vessel. It was then condensed, and a vacuum being formed, the steam-vessel was filled with water which rose from the well or mine by the atmospheric pressure. The steam was then readmitted, and the water in the ressel was driven upwards by the pressure of the steam to the top of the pipes, and discharged. The opening and closing of the valves regulated the passage of the water and the steam.

Q. Who NEXT introduced material IMPROVEMENTS in steam apparatus?

A. Thomas Newcomen, a blacksmith, in the year 1705. He constructed a working steam-engine, which has since been called the atmospheric engine. It contained a cylinder and piston, and an alternating beam, which was applied to raise water by working a pump. The steam was condensed in the cylinder itself, and the valves were moved by hand, until an attendant contrived to make the machine move its own valves by attaching strings to the working beam.

Q. How tong did the engine of Newcomen continue to be used without material inprovements?

$A$. For more than half a century, or until 1769 , when the great improvements of James Watt, of England, made the steam-engine substantially what it now is.

Q. What were the GREAT IMPRoveMenrs introduced by Watt?

A. 1. He introduced the separate condenser;

2. He applied the double action of steam by closing the top of the cylinder, and admitting the steam alternately at each end; and

3. He converted to use the expansive power of steam by cutting off the current before the end of the stroke. He also invented the governor of the steam-engine, to regulate the supply of steam.

Q. What is the Governor of the steam-engine?

A. It consists (as in fig. 68) of two heavy balls, connected by jointed rods with a revolving axis; with every increase in the velocity of the engine they diverge or separate from each other, and draw downwards the jointed rods; while a slower motion of the axis causes the balls to approach each other, and the system of rods to be contracted laterally and ve extended upwards.

Fig. 68.

The grand effect produced by this means depends on making the ascending and de 
scending extremity of the jointed rods raise or lower the end of a bar which acts as a lever, and moves a ralve wichich regulates the supply of steam from tho principal steampipe

Eig: 6.

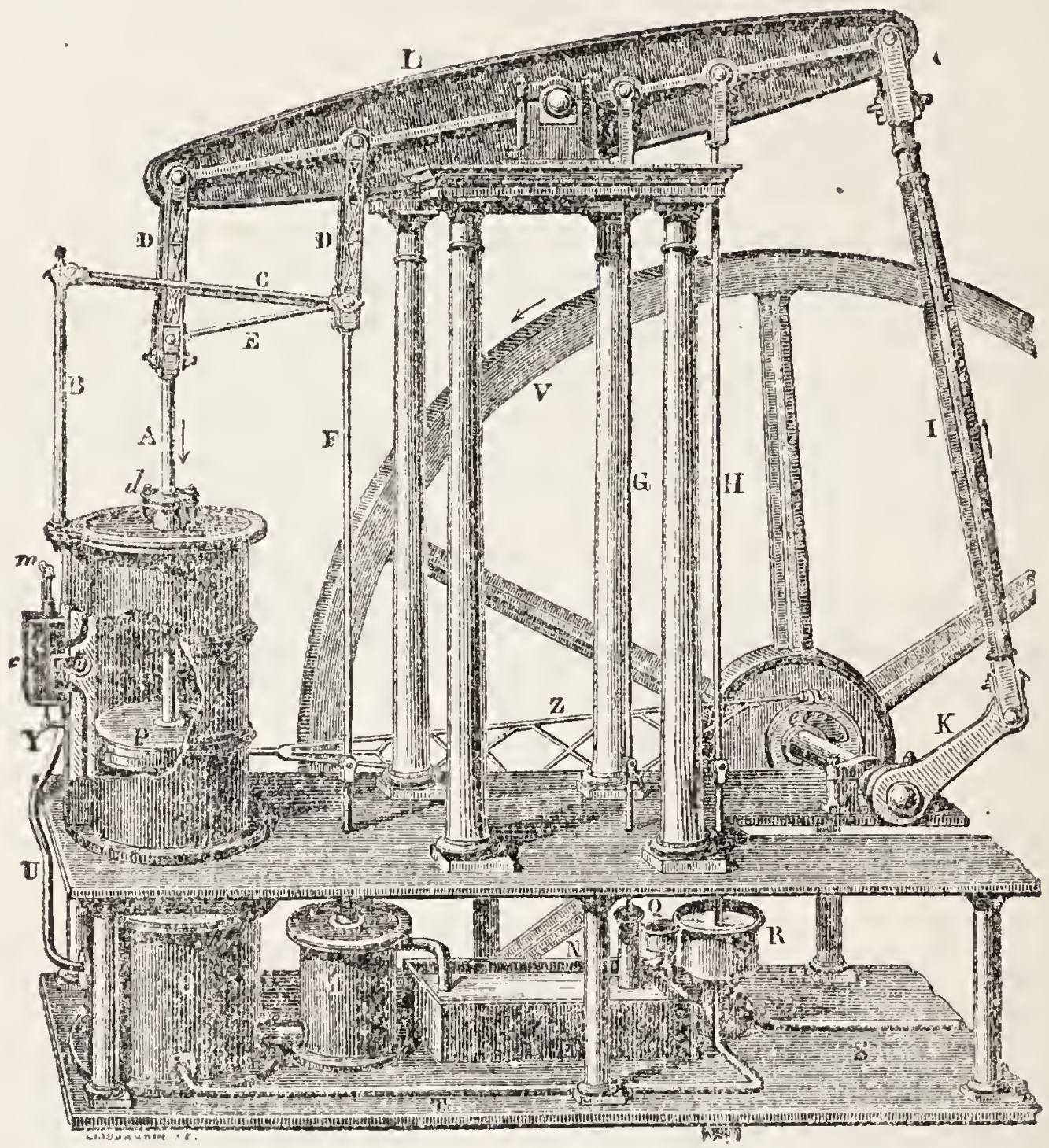

Fir. 69 illustrates the principal parta of a steam-engine, and ris mode of action.

Upon the left of the figure is the cylinder, which receives the steam from the boiler. A part of the side of the cylinder is cut away, in order to show the piston, which movez alternately up and down, according as the stcam is admitted above or below it. By the wo A the piston transmits its alteruating movewents to the ualking-beam $\mathrm{L}$, which is an cnormous lever accurately balanced on its centre, and supported by fomr columns, Whe walking-beam $L$ communicates its notion ly means of a comecting rod I to the crank $K$, by which a rotary movement is communicated to the wheel $V$; from this the power maj be applied by other wheels, or hy bands and pulleys, to efiect difierent operations.

At the left of the cylinder is an arrargement of valves and pines, by which the steam is allowed to act alternately above and helow the piston. After the sleam has completed its action $k$ for foring the piston to the extremity of the cylinder, it is necessary that it shouid be withdrawn, and a vacuum formed in its place. In order to accomplish this, the stestrn, after having acted, is caused to pass in to the cylinder 0 , which contains cold water, ant is termed the condenser. Here it is condensed, and a vacuum formed in the cylinder alrove or below the piston, as the silse may be.

As the cold water of the condenser becomes quickly heated by the condensed steam withdrawn from the cylinder, it becomes necessary to constantly withdraw the h.t water atud replace it by cold water, in order that the condensation of the steam may take place as rapidly as possible. This is effected by means of two pumps; the one, F NI, which is milled the "air-pump," which withdraws the hot water from the condenser, and with it aug air that may be present either in the cylinder or the condenser; the other, $H R$, culled the "cold water-pump," draws from a rell or river the cold water, to supply the phace of the heated water withdrawa from the condenser by the air-pums. There is also 
a third pump, G Q. which is cal!ed th? "supply" or "feed-pump," because it punpy into the boiler the hot water which the air-punp withdraws from the condenser, thus economising the consumption of fuel.

The various parts of the engine (as shown in fig. 09 ) are illustrated in detail by the fullowing descriptive explanation :-

A-Piston-rod connected with the walking-beam, and transmitting to it the alernating movement of the piston.

i3, C, D, E-Arrangements of levers and joints, intended to guide and preserve the piston-rod $\mathrm{A}$ in a perfectly rectilinear track during its up-and-down movements.

H-Arm or rod of the air-pump, which removes the hot water and air from the condenser.

G-Rod of the "supply" or "feed-pump," which supplies to the boiler the hot water withdrawn froin the condenser.

H-Rod of the cold-water pump, which supplies the cold water necessary for con. densation.

I - Connecting-10od, which transmits the motion of the walking-heam L to the crank K.

M-Cylinder of the air-punp in communication with the condenser 0.

O-Condenser filled with cold water, in which the steam after acting upon the piston is condensed.

P-Piston, movable in the cylinder ; it receives directly the pressure of the steam upou the upper and lower surface alternately, and transmits its novements by means if the rod A to the rest of the machinery.

- Pipe conducting the hot water withdrawn from the condenser to the boiler.

1'-Pipe discharging the cold water from the cold-water pump into the condenser $O$.

U-Pipe conducting the steam from the cylinder, after it has arted upon the pistin, into the condenser.

V-Fly-wheel.

7-Conuecting-rod, which transmits the movements of the eccentric $e$ throngh the lever $\mathrm{Y}$ to the valves $b$. The ecentric is a wheel fixed upon the crank-shaft, as seen it e. It is called an eccentric from the circumstance of the wheel not being concentric, or hatring a common centre with the crank-shaft unon which it is fixed. It becomes, therefire, a substitute for a short crank, and transmits a reciprocating movement to the rod $Z$, which is connected with the valves at $b$ by the lever Y. These valves being alternately opened and closed by the movement of the rod Z, admit the steam alternately above or below the piston.

Q. What is the difference between HIGH PRESSURE and LOW PRESSORE engines?

A. 'The difference exists in the elastic force of the steam; when the force of the steam is slight, the engine is said to be a low-pressure; when the force of the steam is great, it is said to be high-pressure.

Q. What is atMospHeric pRessure?

A. The pressure of the air, which amounts on an average to fifteen pounds on every square inch.

Q. How does the Pressure of the ATrospinere affect the STEAM-gNGINE?

A. As the piston of the non-condensing engine is subject to this atmospheric pressure, namely, fifteen pounds on every square inch, it naturally follows that the pressure of the steam by which that piston is moved exceeds the pressure of the atmosphere.

Q. To what Exthet does STEay pressure exceed that of ATMospheric pressore?

A. If the pressure of the atmosphere be fifteen pounds on every square inch, and the pressure of the steam be eighteen pounds on every square inch, that is, three pounds more than the ordinary weight of the air, the engine is said to be low-pressure. If the pressure of the steam be fifty-nine or sixty pounds on every square inch, which is fon tincs as much as the weight of the atmosphere, the engine is said to be high-pressure. 
Q. What is a conom:rsivi engine?

A. A condensing-engine is one which, being supplied with a condenser, liquefies the steam, after it has acted on the piston, instead of permitting it to escape into the air.

Q. What is a NoN-CONDENSING engine?

A. A non-condensing-engine, instead of condensing the steam after it has acted on the piston, allows it to escape into the atmosphere.

Q. What is meant by HORSE-POWER?

A. The term horse-power is used by engineers to indicate that mechanical power of a steam-engine which is equal to the labouring strength of a horse; thus, if a given amount of work could be accomplished by ten horses in a fixed time, and one steam-engine could accomplish the same work in the same time, the engine is said to be of ten-horse power.

Q. How did this SYSTEM of Calculation arise?

A. From the practice of employing horses to perform the labour now accomplished by steam, before the steam-engine was invented.

From repeated experiments, Mr. Watt found that a horse treading a mill path at the rate of $2 \frac{1}{2}$ miles per hour, or 220 feet per minute, will, on an average, raise 150 pounds weight by a cord lianging over a pulley, and suspended over a mine shaft; and this labour is equivalent to raising 33,000 pounds weight one foot high per minute. Mr. Watt's steam-engines were constructed so as to work at the rate of 44,000 pounds, raised one foot high, per minute, for each horse power ; but in his estimate of their actual effect, he reduced this rate to 33,000 raised one foot per minute for each horse power, considering the difference to be due to their loss of power by friction, and other causes.

Q. What is a LOCOMOTIVE ENGINE?

A. Steam-engines called locomotives, or locomotive-engines, are those which are worked on a carriage or a railway, and by transmitting their motion to the wheels, are moved from place to place.

Q. What is MEAN

A. The movement of vessels on the water by steam, instead of oars or sails.

Q. When was STEAM Navigation first introduced?

A. In the year 1801, Mr. William Symington made the first experiment in steam-navigation on the Forth and Clyde canals, which ended in the production of the first practicable steam-boat, named the "Charlotte Dundas."

Q. When was the FIRST STEAM-BOAT put in actual operation?

A. In 1812, when a steam-boat called the "Comet" began to ply for passengers between Glasgow and Gleenock.

Q. By whom was steam Navigation introduced into America?

A. Mr. Robert Fulton, who built the steam-boat "Clermont" in 1807, which plied between New York and Albany.

Q. When was STEAM POWER first applied to purposes of travelling on LAND?

A. Experiments were made for this purpose as early as 1785, by Mr. William Murdoch, and were tried upon the common roads in Cornwall. His machine is seen in the accompanying engraving. 
Fig. 70.

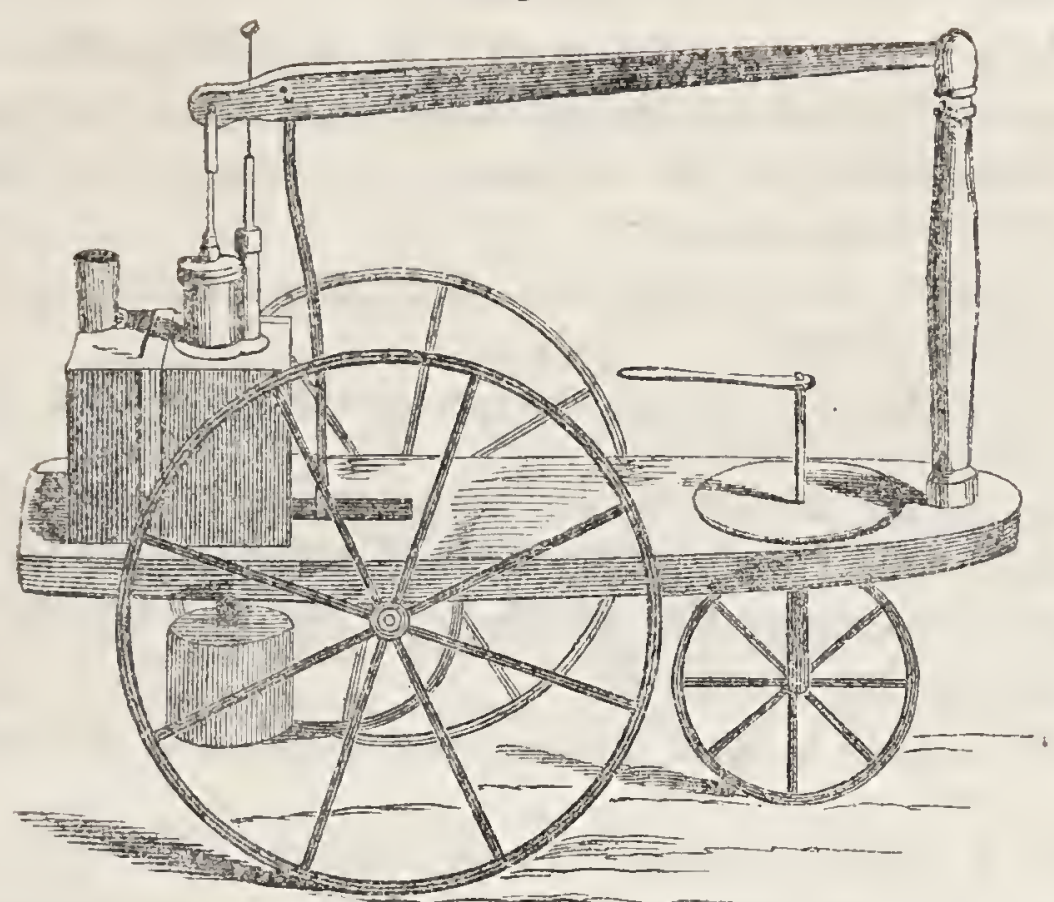

Q. When was the FIRST RAILW projected?

A. The Liverpool and Manchester railway was first projected in 1823, by Stephenson and others. On the opening of this line the benefits of the railway system became at once apparent.

Q. What is the estimated NUMBER of LOCOMOTIVE ENGINES employed annually in Europe?

$A$. The number of locomotive-engines employed on the various European railways is estimated at 7,500; of these 3,700 are employed and 5,000 were made in Great Britain.

Q. What is the estimated DISTANCE travelled by these Lovomotives every jear?

A. One husadred and twenty millions of miles.

Q. When did the first steam-vessel cross the Atlantic?

A. The first steam-vessel which crossed the Atlantic was the American shin Savannah, from Savannah, Georgia, in 1819.

\section{CHAPTER XXI.}

VENTILATION.

Q. What is VENTILATION?

A. The renewal of fresh air-a continual change of air.

Q. Is the air in a rooss constantly in motion?

A. Yes; there are always two currents of air in the room we occupy; one of hot air flowing out of the room, and another of cold air flowing into the room. 
Q. How do you kNow that there are these two currents of air in every occupied roos?

A. If I hold a lighted candle near the crevice at the top of the door, the flame will be blown outwards (towards the hall or passage); but if I hold the candle at the bottom of the door, the flame will be blown inwards (into the room.)

This is not the case if a fire be in the room. When a fire is lighted, an inward eurreut is drawn through all the crevices.

Q. Why would the flame be blown oUTWARDS (towards the HALI or PASSAGE) if a candle be held at the Top of the door?

A. Because the air of the room being heated, and consequently rarefied, ascends, and (floating about the upper part of the room) some of it escapes through the crevice at the top of the door, producing a current of air outwourds.

Q. Why would the flame be blown Inwards (into the roors) if the candle be held at the воттом of the door?

A. Because a partial vacuum or empty space is made at the bottom of the room, as soon as the warm air of the room has ascended to the ceiling or made its escape from the room; and cold air from the hall ruskies under the door to supply the void.

Q. What is moant by a Partial VACUUM being made at the Botron of the room?

A. A vacuum means a place from which the air has been talien; and a "partial vacuum" means a place from which a part of the air has been taken away. Thus, when the air near the fioor ascends to the ceiling, a partial vacuum is made near the floor.

Q. And how is the VACUUA FILLED UP again?

A. It is filled up by colder air, which rushes (under the door, and through the window crevices) into the room.

Q. Give me an intustration.

A. If I dip a pail into a pond, and fill it with water, a hole (or vacuum) is made in the pond as biy as the pail ; but the moment I araw the pail out, the hole is filled up by the water around.

Q. Show how this ILIOSTRamron applies.

A. The heated air which ascends from the bottom of a room is as much taken away as the water in the pail, and (as the void was instantly supplied by other water in the pond) so the void of air is supplied by the air around.

Q. Why is a room (even without a fire) generally WARMER than the OPEN AIR?

A. Because the air in a room is not subject to much change, and soon partakes of the same temperature as our bodies, when it no longrer feels cold.

Q. Why do we generally feel cotDen out-of-doors than in-doors?

A. Because the air (which surrounds us) is always chunging; and as fast as one portion of air has become warmer by contact with oup body, another colder portion surrounds us, to absorb nore heat. 
Q. Why is there a strong DRADGHT UNDFR the Door, and through the crevice on each side, or through the key-hole of the door?

A. Because cold air rushes from without, to supply the void in the room caused by the escape of warm air up the chimney, \&c.

Q. Why is there always a DHAUGHT through the WINDow crevices?

$A$. Because the external air (being colder than the air of the room we occupy) rushes through the window crevices, to sup- ply the deficiency caused by the escape of warm air up the chimney, \&c. sasll?

Q. By which means is a roou better ventritated ? by opening the upper or the lower

A. A room is better ventilated by opening the upper sash; because the hot air (which always ascends towards the ceiling) can escape more easily.

Q. Which is the Hortest PLACE in a church, chanel, or theatre ?

$A$. The gallery.

Q. How do you ACCOUN for this?

$A$. The heated air of the building ascends; and all the cold air keeps neur the floor till it has become heated.

Q. What EFFECT has RAREFACTION upon AIR?

A. It makes it lighter; and the lighter it becomes, the higher it rises, as a balloon rises in the common atmosphere, or a piece of cork rises in water.

Q. Does HOT AIR always rise?

$A$. Yes; and therefore the stores should be fixed as near the floor as possible, in order that they may properly heat the room.

Q. What arfans are usually employed to HEAT apartments ?

A. Open fire-places are commonly employed; but rooms are sometimes heated by hot air, by steam, or by hot water.

Fig. 71.

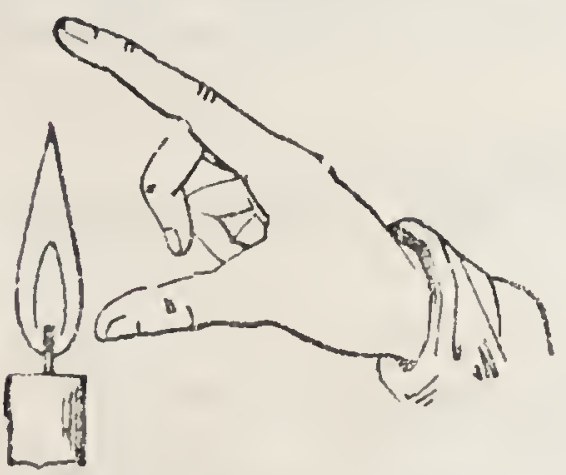

The method of heating by common fires may be under. stood by reference to the accompanying diagram. Here we sce that we can hold the finger or thumb at a short distance from the flame of a candle without experiencing any inconrenience; but when we hold the finger above the flarne, we are forced, by the increased intensity of heat in that direction, to remove it to a greater distance. An ordinary fire in a cormon stove, or open grate, is precisely the same in principle as the flame of a candle. The lesser amount of heat is thrown out in the firont, while the greater amount of heat escapes at the top. We thus learn that by the nsual method of heating ronms great waste of heat is occasioned, the heat ascends the chimney instect of uarming the room.

Q. What connection is there between WARMING and VENTILATING?

A. A fire cannot be made to burn wilhout air; the heat of the fire sets the air in motion. What is meant by ventiation, is a change of air; there is, consequenty, a close connection between waming and ventilating. 
Q. Is the AIR drawn TOWARDS the FIRE?

A. Yes; the mass of cold air in the apartment is immediately drawn towards the fire.

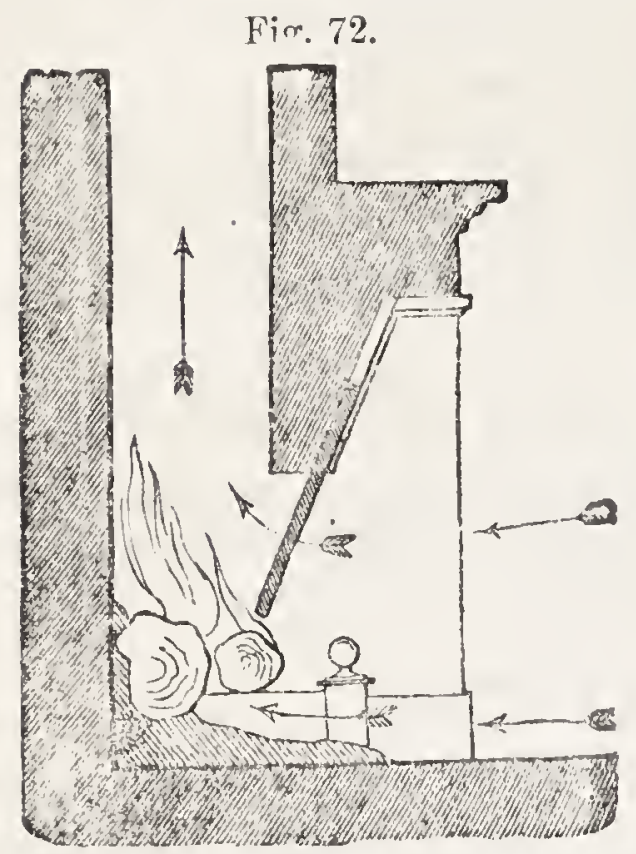

['The annexel diagran shows a section of a cornmon stove. The disection of the air is indicated by the arrows.]

Q. What is meant by the DRAuGur of a crimney?

A. By the draught of a chimney is meant an upward current, which is caused by the fire in the grate. When this current is rapir, we say the chimney draess well.

Q. What is smoke?

A. Minute particles of carbon, sepalated from the fuel, but not consumed.

Q. What occusions the suore to CrrR as it roes ul) the chimney?

A. The effect is produced by the ascending and descending currents of air, which oblige it to assume that form by driving it round and round.

Q. Why does the sMoke go up the cHmser?

A. It is carried up the chimney by the air, which is heated by the fire, and thus made to ascend.

Q. What is meant by a siory cimsun?

A. A chimney from which the smoke, instead of passing out into the air above, rushes out into the room.

Q. How is this OCCASIONED?

A. By the currents of air from above, which, descending the chimney, drive the smoke into the room.

Q. What is the cause of this?

A. It arises from the fact that fresh air is not supplied as rapirlly as it is consumed by the fire; the result is, that the air rushes in from above to supply the deficiency.

Q. Why do we open the DOoR or WINDow when a CMINAEX SAOKFs?

A. 'L'o supply the fire with a fresh current of air, and thus enabie the smoke to ascend the chimney.

Q. What is the FUNmin or FuUE of a chimney?

A. The part of the chimney through which the smoke ascends.

Q. Is a LONG Or SHORT FLUE the best?

A. A long flue.

Q. Why does a cminner suoke if the Funner be very shotit?

A. Because the drenght of a short flue is too slack to carry the smoke up the chimney.

Q. Why is the Draven's of a suont Flue more slack than that of a long one?

A. 1. Because the fire is always dull and shugish if the chimney be too short; 
2. Because the smoke rolls out of the chimney before it has acquired its full velocity or speed; and

3. Because the wind, rain, and air have more influence over : short funnel than over a long one.

Q. Why is the FIRE always DULL and SLUGGISH if the CHIMINEY-FLUE be very SHORT?

A. Because the draught is bad; and as the rarefied air presses very tardily up the chimney, fresh air flows as tardily towards the fire, to supply it with oxygen.

Q. Why does not s.Moke acquire its full veLocitr in a SHoRt funnel ?

A. Because the higher smoke ascends, for a limited distance (provided the fire be clear and hot, and the flue be unobstructed), the faster it goes; if, therefore, a funnel be very short, the smoke never acquires its full velocity. flue?

Q. Dues the DRAugir of a chimney depend on the SPEED of the SMOKn throngh the

1. Yes; the more quickly hot air flies up the chimney, the more quickly cold air will rush towards the fire to supply the place; and therefore the longer the flue, the greater the dranght.

Q. Why are the CHIMNEYS of MANUFACTORIES made so very LONG?

A. To increase the intensity of the fire. Because the drareght, being greater, more fuel is consumed in the same time, and, ot course, the intensity of the heat increased.

Q. Why do BLowers, when placed hefore a grate, tend to KINDLE the FIRE ?

$A$. Because the air (by passing through the fire) is made much hotter, and ascends the chimney more rapidly: and because the blower increases the draught; and the faster the hot air flies up the chimney, the faster will cold air rush towards the fire.

Q. If Two FIRES are kurning in one close room, the apartment is filled with SMOH s. why is this?

A. One or other of the two fires will exhaust the most air, and the remaining fire will smoke from the want of a sufficient quantity.

Q. What is a cows?

A. A kind of hood, fixed on the top of a chimney-pot, and which, turning like a weather-cock, constantly presents its back to the wind.

Q. What PURPose does it answer?

A. It is intended to prevent the wind descending the flue, and thrs making the chimney smoke, but it is not a perfect remedy.

Q. What is the BEST remedy to PREVENT a chimney SMOKING ?

$A$. To carry the flue higher than any surrounding building.

Q. Do not chimneys SMOKE when they require SWEEpIng?

$A$. Yes; as the soot accumulated in the flue prevents the free escape of the smoke. 
2. Besides the ordinary stoves, what other means are employed for heating rooms i

A. Hot air is sometimes employed for this purpose; steam is also usest, and hot water.

Q. How is HOT AIR employed in heating rooms?

A. The common air being heated by a calorifer, or hot-air stove, is conveyed through metallic pipes, and is thus made to heat any room w looms which may be desired.

Q. How is stens employed for this purpose?

1. Whe steam, being produced in a boiler, is driven through pipes, and thus conveyed to the room or rooms which are to be hitated.

Q. Is not the plan of heating apartments by поT WATER of ancient date?

A. Yes; the practice of heating houses by hot water was practised by the Romans.

Q. What systrus is Now adopted?

- Wuter, being heated in a boiler, is transmitted thrmigh a series of pipes, brought back again to the boiler, and so kept constantly in virculution.

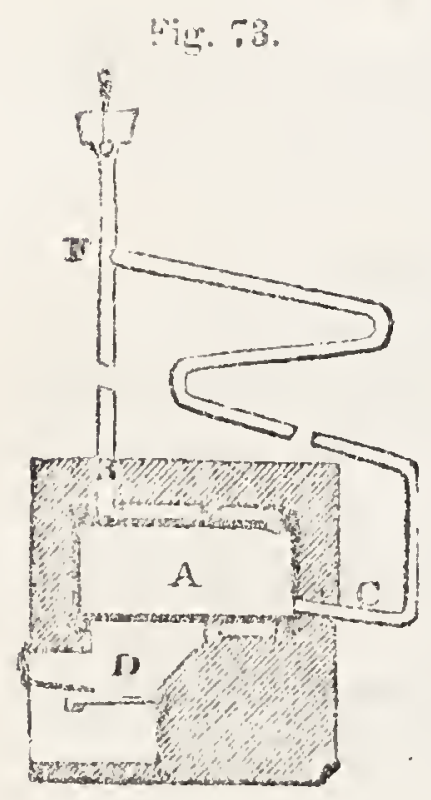

The diagram represents the plan of heating by the circulation of hot water. From the top of the boiler A, placed on a fire D, rises an upright tube $\mathrm{F}$. The hot water ascends towards $\mathrm{F}$; but at $\mathrm{F}$ is commenced a series of pipes through which the water passes, and successively heats the different parts of the building. During this passage it is cocled, and returns to the boiler at $\mathrm{C}$, when it enters at the bottom; and thrus a continued circulatiun is kept up in the direction A F C.

Q. Which would prove the warmest upon a bed-a BLANhTr or au oiled silk or India-rubber AIR-TIG⿴囗十 covering?

\section{A. 'The air-tight covering.}

Q. Why do we not ust oiled silk or India-rubber bed-corerings in the winter?

A. Because they prevent ventilation, and, by shutting in the insensible perspiration, soon produce clampness.

2. Is there a urRouldtion of the air through the bed-coverings at night?

A. Yes; from every part of the bed-clothes immediately over the person there is a constant outward oozing of warm air, and an oozing inwars of cold ail in lower situations around.

Q. What rmapraturn is most proper for keeping an apartment in a healtby and pleasant condition during the cold season?

1. From $60^{\circ}$ to $65^{\circ} \mathrm{F}$, with a free ventilation.

2. At what PERIOD were chimneys first used in England?

A. Not before the middle of the fourteenth century; and in the time of Queen Elizabeth they were considered as luxuries for the wealthy, and not nocessities. 


\section{CHAPTER XXI.}

\section{SOLUTION AND CRYSTALLISATION.}

Q. When SAL' is mixed with water and DISAPPEARS in the liquid, what is said to bave taken place?

A. The salt is said to have dissolved in the water, and the liquid is now a solution of salt.

Q. What, then, is a solotion?

A. A solution is the result of a combination between a solid and a fluid; and when a solid disappears in a liquid, if the compound exhibits perfect transparency, we have an example of a prerfect solution.

Q. When is a solution said to be SATURATED ?

$A$. When the fluid has dissolved as much of the solid as it is capable of doing, it is said to be saturated; or, in other words, the affinity of the fluid for the solid, that is, the power which inclines it to unite, continues to operate to a certain point, where it is overbalanced by the solid; it then ceases, and the fluid is said to be saturated.

Q. What is the difference between a solurrox and a Mrxture?

A. A solution is a chemical union; a mixture is a mere mechanical union of bodies.

Q. Why will WATER dissolve SUGAR?

A. Because there is attraction or affinity between the particles of the water and the particles of the sugar.

Q. Why will not WATER dissolve GRANITE or METALLIo iron?

$A$. Because there is not sufficient affinity or attraction between the particles of the water and those of the iron or granite.

Q. Are there ANY LIQUIDS that have sufficient affinity to dissolve iron and granite?

A. Yes; certain acids have so great an affinity for the iron and granite that they are enabled to dissolve them.

Q. Why will not WATER dissolve OIL?

$A$. Because there is no affinity or attraction between the particles of the two substances.

Q. Why will $\triangle \mathrm{LCOHOL}$ and ETHER dissolve oil ?

$A$. Because the attraction or affinity between the alcohol or ether and the oil is sufficient to enable them to effect a solution.

Q. What EFFECT has heat upon the dissolving power of liquids?

A. In most cases the addition of heat to a liquid greatly increases its solvent properties. Hot water will dissolve much more sugar than cold water, and hot water will also dissolve many things which cold water is unable to affect. 
Q. Why does a pIEce of sugar (held in a spoon at the rop of our tea) meit rery RAPIDLY?

A. Because as the tea becomes sweetened, it descends to the bottom of the cup by its own gravity, and fresh portions of unsweetened tea are brought constantly into contact with the sugar, till the lump is entirely dissolved.

Q. Why does the LUMP of SUGAR MELT more QUICKLY when STIRRED ABOUT?

A. Because fresh portions of unsaturated tec come in contact with the lump, and soon dissolve it.

Q. Why is a LUMP of SUGAr (left at the bottom of a cup) so LONG in MELTING?

A. Because (as it melts) it makes the tea above it heavier, and (so long as it remains at the bottom) is surrounded by tea fully saturated with sugar; in consequence of which the same portions of liquid will hold no more sugar in solution.

Q. Why does a WET SPONGE CLEAN A SLATE?

A. Because the water holds in solution the pencil-marks made on the slate; and the mechanical triction employed in wiping the slate detaches the particles of pencil-dust.

Q. What is SPONGE?

A. A well known substance, produced by very small animals which live in the sea; these animals are called polypi by naturalists.

Q. What do we mean by AFFivity?

A. Affinity is that kind of attraction by virtue of which bodies of a dissimilar nature combine together into a whole, which appears perfectly uniform to the senses, even when assisted by the most powerful optical instruments.

Q. If we evaporate some spring water in a vessel, the bottom of the vessel will generally be found to contain some EARTHY MATTER: why is this?

A. The spring water, as it comes through the ground, dissolves and retains some substances, which it leaves upon the bottom of the vessel when it is evaporated.

Q. How is SALT formed from sea-water?

A. The sea-water containing salt is collected in large vats or pans, and the water evaporated either by the heat of the sun or by fire. As the watel evaporates, the salt is left in the vessel.

Q. How MUCI SALT does a POUND of sea-water contain dissolved in it?

A. From one-half to five-eighths of an ounce.

Q. As heat enables liquids to dissolve and hold in solution a greater quantity of soluble substances than the same liquid is enabled to do when it is cold, what takes place when a HOT SATURATED SOLUTION is COOLED DOWN?

A. A part of the substance held in solution will be throun doun from the liquid and deposited.

Q. How much more SALTPETRE is boiling water enabled to retain than water having a temperature of only $32^{\circ} \mathrm{F} . ?$

A. One hundred parts of water at $32^{\circ}$ will dissolve only seven parts of saltpetre; the same quantity of boiling water will dissolve three mundred and thirty-five parts. 
Q. When a substance is thrown down from a solution, either by coolivs or EvaporaTION, in what MANNER is it deposited?

A. The particles sometimes join in an indiscriminate manner, and give rise to irregular, shapeless masses; but more frequently they attach themselves to each other in a certain order, so as to constitute solids possessed of a symmetrical form.

Q. When the substance depositer has no PARTroular SH.PFe, what is it called?

A. Amorphous; that is, without form.

Q. When it has a regular, sYMmetrical SHAPE, what is it called?

A. Crystallised br crystalline.

Q. What is ORYSTALLISATION?

A. Crystallisation is the effect of attraction among the atoms or particles of substances regulated by certain laws, according to which atoms of the same kind of matter unite in regular forms.

Q. How is this FACT shown by EXPERnEnT?

A. If we dissolve a piece of alum in pure water, the mutual attrattims of the particles are destroyed by the water; but if the water be exaporated, they unite, and form in uniting eight-sided figures called octahedrons. Fig. 74 represents crystals of alum.

Fig. 74 .

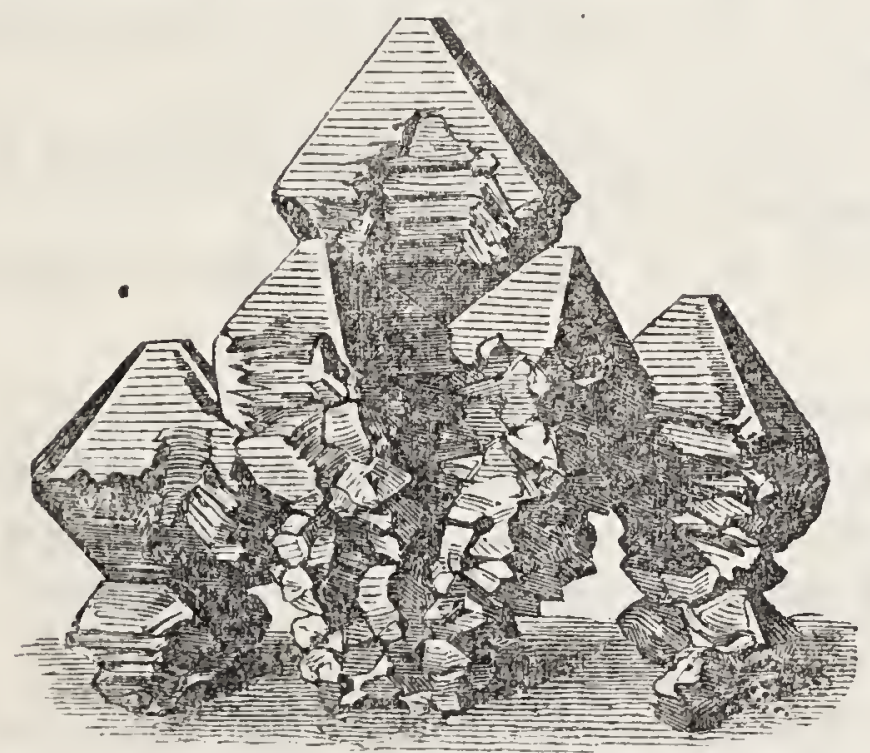

Q. Are most MINERALS and EARTHX substances capable of crystallisation?

A. Yes; under favourable circumstances.

Q. What CIRCUMSTANCES FAVOUR crystallisation?

A. When a fluid is slowly changing into a solid, the arrangement of the particles being at the same time undisturbed by motion.

Q. Are there many Foris of crystals?

A. The number of erystalline forms amounts to several thousands.

Q. Does the SAME cosrosition always crystallise in the same Forus?

A. Yes; unless the conditions of the crystallising body are altered. In accordance with this fact, we are often enabled to distinguish and pronounce upon the composition of a body merely from the form of 
its crystals, as the same body, under the same circumstances, always crystallises in the same manner.

Q. Explain, by EXAMPLE, the manuer of distinguishing a substance by the form of its crystals?

A. For example : fluor-spar always crystallise in cubes, and quartz in six-sided pyramids. These minerals, so far as colour and lustre are concerned, often closely resemble each other, but their forms are peculiar to them.

Q. What is the well-known " ROCK," or "SUGAR-CANDY?" "

1. Simply sugar suffered to crystallise under favourable conditions.

Q. What is the so-called "LOAF-SUGAR?"

A. In the formation of "loaf-sugar," the process of crystallisation is hastened or disturbed in such a manner that it commences at a myriad of points at the same instunt, and neither time nor space is allowed for the particles to expand to a large size and regular outline. Nevertheless, if we examine a small particle of loaf-sugar through a magnifying-glass, we shall find that it is distinctly crystallised.

Q. In what srats is the sugar which is contained in molasses?

A. Owing to a chemical change which takes place in the syrup during the process of manufacture, the sugar contained in molasses is uncrystallisable.

Q. Why, when we boil down molasses to make molasses-candy, do we always obtain a STICKY, GUM-LIKE sugar, and not a DRY, HARD sugar?

A. Because the sugar contained in the molasses is incapable of crystallising.

Q. How will you DEFINE a crystal?

A. The geometrical form possessed by a vast number of mineral and saline substances, whose particles combine with one another by the attraction of cohesion, according to certain laws.

Q. What are the ELENENTS of a crystal?

A. Every perfect crystal is bounded by plane surfaces; which are called its faces. The straight line formed by the intersection of two faces is called an edge; the meeting of three or more edges in a point forms a solid angle.

Thus, in the octahedron, fig. 75 , the bounding planes are the faces, the lines formed by their intersection the edges, the meeting of four of which in the same point produces a solid angle of the crystal.

Q. Into what Two CLASSES are crystals divided?

A. Into simple and compound.

Q. What are SIMPLE crystals?

A. A simple crystal has all its faces equal and similar to each . other. Thus figs. 75, 76, and 77 represent simple forms, fig. 75 being an octakedron bounded by eight faces, each of which is an 
equilateral triangle; fig. 76 , a cube bounded by six squares; while fig. 77 has for its faces twelve equal and similar rhombs.

Figr. 75.

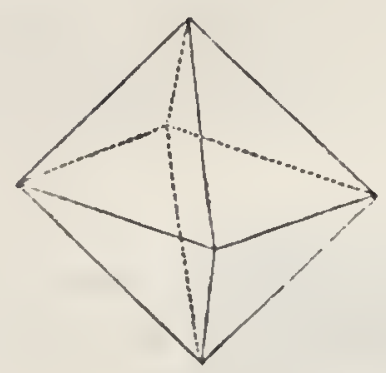

Fig. 76 .

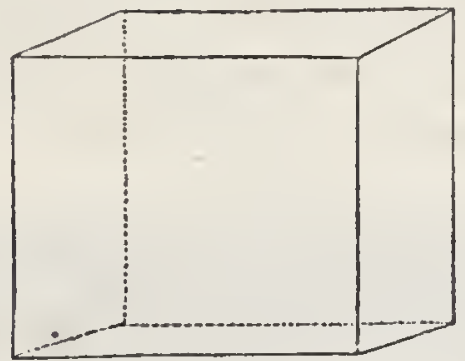

Eig. 75

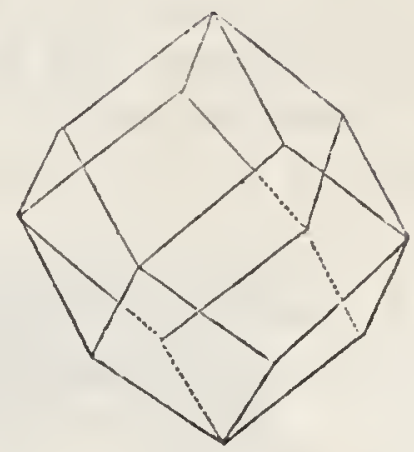

Q. What is a compound crystal?

A. A compound erystal is bounded by at least two different clusses of faces. Figs. 78, 79, and 80 represent forms and varieties of compound crystals.

Fig. 78.

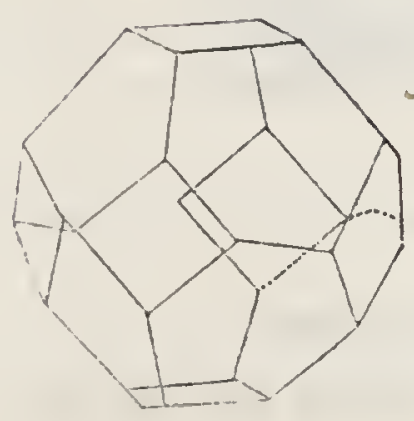

Fig. 79.

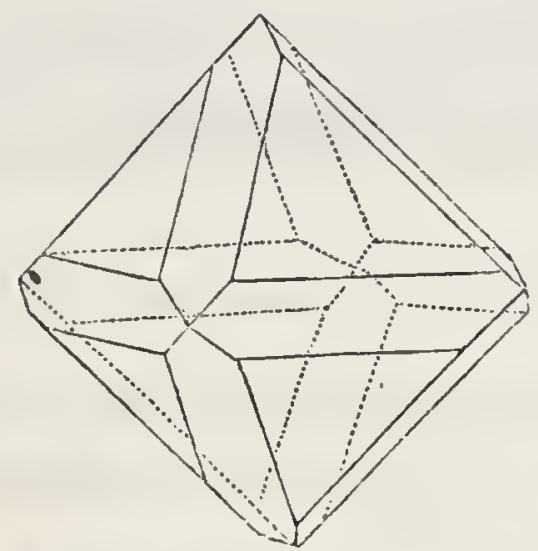

Fig. 80 .

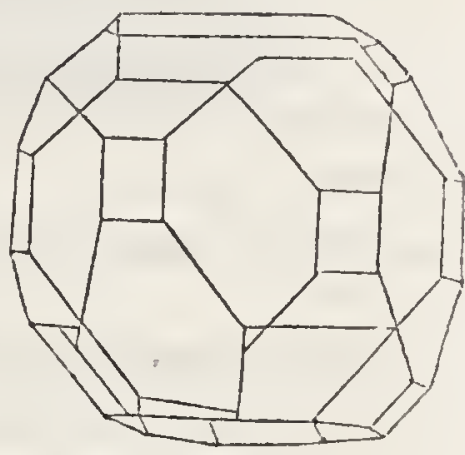

Q. How may two substances, dissolved together in water, be SEPARATen from each otiler by CRYATALLISATION?

1. If we dissolve salt and saltpetre together in warm water, and then suffer the liquid to evaporate, the two salts which are intimately united in the solution will, upon crystallisation, separate completely from euch other. The saltpetre separates into long prisms, containing "no trace of common salt, and the latter separates into cubes, entirely? fiee from saltpetre.

Q. Why did this SEPARATION of the two substances take place?

A. In accordance with the laws of crystallisation, which compelled each substance in crystallising to arrange its particles in a determinate and regular form, different from the other.

Q. Why do the figures of crystals VARY in regularity?

Because their regularity is influenced by the rapidity with which the liquid from which they are deposited evaporates: thus, if the mocess be slowly conducted, the particles unite with great regularity; if hurried, the crystals are irregular and confused. 'T'o obtain very regular crystals, the evaporation must be spontaneous, or that which takes place at common temperatures. 
Q. Why has strong salt and water a FILM or PELIICLE upon its surface?

$A$. Because the attraction of the saline particles for each other is becoming superior to their attraction for the water.

Q. Why will not salt CRYsTalise when dissolved in a considerable quantity of water?

A. Becanse the particles of the salt are too far asunder to exert reciprocal attraction; in other words, they are more powerfully attracted by the water than by each other.

Q. Why does salt crystallise upon Evaporation of part of the water?

$A$. Because some of the saline particles then gradually approach each other, and, according to the laws of crystallisation, form regular solids. Another portion of the salt will remain dissolved in the water which is left; this is usually called the mother liquor.

Q. Why do many chemical compounds (salts) when exposed to the air EFFLoresce, or fall to powder?

A. Because they lose their water of crystallisation; or, in other words, the water which they incorporate into their structure on assurring the crystalline form.

Q. Why do some chemical compounds (salts) EFFLORESCE more than others?

$A$. Because some salts thus completely lose their water of crystallisation, while others retain different quantities, according to the dryness of the air.

Q. Why do some salts DELIQUESCE (or become moist or liquid) by exposure to the atmosphere?

A. Because they attract moisture from the atmosphere.

Q. If two substances differently soluble in water are mixed together, how can they be PUR:FIED and SEPARATED one from another?

$A$. If a hot saturated solution of the mixed substances be made, and then suffered to cool, that substance which is least soluble in watel, or the one for which the water has the least affinity, will crystallise out and be deposited first, while the other substance, which is more soluble, will remain in solution, and may be drawn off, leaving the one first deposited tolerably pure. In this way saltpetre is refined from salt and other ingredients that may be mixed with it.

Q. Does water in FREEZING CRYSTALLISE?

A. Always. time?

Q. What is the cause of the pretty FrosTwork seen on bedroom windows in vinter-

A. The breath and insensible perspiration of the sleeper (coming in contact with the ice-cold window) are fromen by the cold glass, and, crystallising, form those beautiful appearances seen on a winter molning.

Q. Are all the figures of frostwork formed in accordance with certain FIXED LAWS ?

A. All these figures are limited by certain laws, and the lines which bound them form among themselves no angles but those of $30^{\circ}, 60^{\circ}$, and $120^{\circ}$. 
Q. If you fracture thin ice by allowing a pole or WEIGHT to fall upon it, will the lines of the fracture have anything of REGULARITY?

A. Yes; the fracture will generally present a star with six points equally distant or at angles of $60^{\circ}$.

Q. In what SUBSTANCE are the crystals of frozen water seen to the best advantage?

A. On snow, under the microscope. Fig. 81 illustrates the varied and beautiful forms of snow crystals.

Fis. S1.

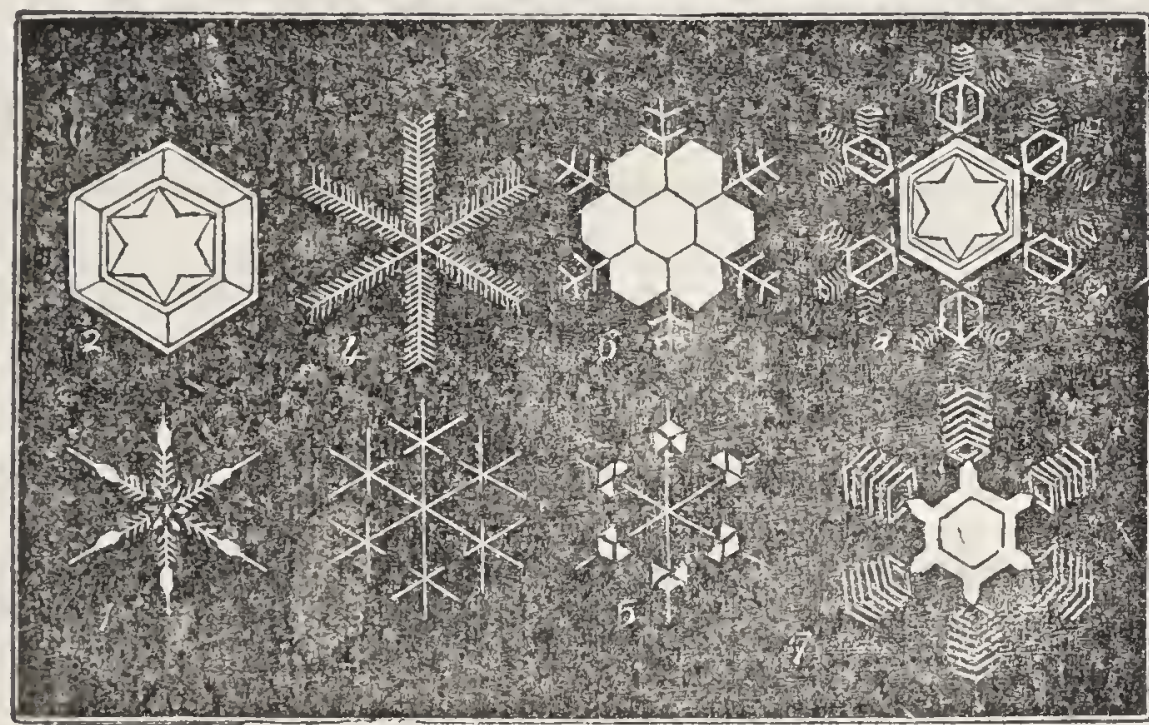

Q. Is much mise required for the furmation of large crystals?

A. Yes; many of the large crystals dug from the earth have undoubtedly required many years for their formation.

A crystal of quartz at Milan, in Italy, is three feet and a quarter long, five feet and a half in circumference, and weighs 870 pounds. Crystals of beryl have been found in New Hampshire that weigh over a ton.

Q. Is the formation of crystals accompanied with a DEVELOPMENT of heat?

A. Yes; heat is always liberated when a liquid passes into a soili state. If the crystallisation is rapid, heat is rapidly produced; and in some instances crystallisation is attended with the coolution of liglut.

Q. Dies a substance occupy MorE SPAcE when crystallised than when dissolved?

A. 'Most substances in crystallising expand, and occupy an enlarged space.

Q. What beautiful practical application has been made of this EXPANSIVE l'ROPERTY of crystallising substances?

A. Some fibres which are closely adherent to each other, are scaked in a saturated solution of an easily crystallisable substance, and then suffered to dry. The salt contained in the solution penetrates every part of the fibre with the liquid, and, as it dries, crystallises and expands. In this way the fibres are broken up, and forced apart from each other.

Q. Ilow ha crystallisation been taken alvantage of for SEPARATING SILver from IABI in MINING ?

A. Wuch of the lead mined from the earth contains silver, and the silver being of value only when separated from the lead, the followins method of parting them has been derised: the two metals are melteil 
and sufrered to cool slowly; the silver, forming into crystals more easily than the lead, crystallises first, when the lead left behind is poured off.

Q. When iron is subject to long-continued ribration, what cHange takes place in its composition and character?

A. Wronght iron has essentially a fibrous character, which gives it toughness and strength; but when subjected to long-continued vibration, especially under pressure, its tough, fibrous character becomes changed into a coarse, crystalline one. That is, certain of the fine crvstalline grains become enlarged at the expense of others, and thus its cohesive force (power of sticking together) is diminisher.

Q. What is the conditios of iron when it first comes from the furnace, and changes from a liquid to a solid state?

A. Its outermost particles, by a species of attraction, form crystals, and these crystals, drawn together by a like attraction among each other, constitute the entire mass.

Q. If you examine a piece of cast iron with a microscope, what APPEARANCE will it present?

$A$. It will be seen to be made up of a mass of crystals, each crystal having a regular form, and that the aggregation of the whole constitutes the mass of the metal.

Q. How is cast iron converted into Wrovghr mox?

A. The iron is freed in a great measure from the carbon* that is combined with it, and then subjected to blows of the hammer, and rolled between rollers, which elongate it.

Q. What FFFECT does this treatment have?

$A$. By thus working it, each of the crystals is gradually elonguted into a thread, so that wrought iron is an aggregation of fibres (fibrous iron, as it is sometimes called), or a series of threads kept together by the attraction of cohesion.

Q. What takes place when a bar of fibrous or wrought iron is made to viBRaTe by sirocks, either by the blow of a hammer, or by the rapping of any part of the machine, or by the contimned rolling and striking of a railway car-wheel upon the rails?

A. The little fine threads or fibres snap one by one, until the whole becomes a mass of crystals, and the metal loses its tenacity, and has to be recast or rewrought, before the aggregation of the particles can be restored.

Q. Why should all concussions and sudDex JARs in machinery be avoided as much as possible?

A. Because any one blow given on one part of a machine of metal must more or less vibrate through the connections to the other parts; and every shock which a piece of mechanism receives canses the ultimate particles to move upon each other.

Q. How was this curious fact FIRST DISCOYERnD?

A. An accident having occurred on a railroad in the breaking of an axle, by which many lives were lost, the attention of scientific men was called to the fact that the pieces of iron composing

* Carbon is pure charcoal. 
the axle when first used were strong and capable of staridng a test, but after use for a definite period, could be broken by a force far inferior to that by which they had been tested; many imaginary ideas were formed to account for this phenomenon, when, finally, one person took a series of rods about the size of pipestems, and with great patience allowed them to fall for hours and hours upon an anvil, producing rapid strokes and ribrations. After subjecting them for a long time to this treatment, he found that the rods could be snapped and broken as a pipestem of the same dimensions.

Q. Sone years since a large wrought-iron cannon exploded on board the United States ship Princeton, killing a number of distinguished men: what was supposed to be the UAUSE of the explosion?

A. The continued viluation of the particles of the iron, caused by the heary explosions, changed its nature from a fibrous to a crystalline character, and so weakened the cannon, that it finally burst.

Q. Are all large iron cannon LIABLE to Explote after they have been fired a certain number of times?

A. Yes; and, for this reason, a cannon which has been long in use, though apparently sound, is always condemned and broken up.

Q. WhiY are AXIES of railway carriages apt to BREAK after long-continued use?

A. Because the contimued vibration and strain upon the particles of iron of which they are composed, changes it from a fibrous to a crystalline siructure, and thus weakens it.

Q. Would a railway axle be almost cuRTAIn to break from this cause after long-contiuned use?

A. It is generally considered that they are certain to fracture trom this cause aiter a sufficient lapse of time and use.

Q. How may very LAKGF and PreFuct crystals of an easily crystallisable substanct he PORULD?

A. By repeatedly transferring a crystal from one saline solution to another of the same salt a little more concentrated, and evaporating at very moderate temperatures, or even spontaneously at common temperatures.

Q. In jewellery, are the SMaLt grims generally more pвRFECT and valuable than the large ones?

A. The most perfect crystais of gems met with in nature are only of a moderate size. The larger ones are less clear, and even opaque, and the faces lose their smoothness, and much of their lustre.

Emerajds sufficiently pure for jewellery do not often average lialf an inch iu length, and seldorn so much as this?

Q. D) some crystalline solids slowly change their interual structure SPONTANEOUSLY ?

A. They do; thus sugar, which has been rapidly boiled down to a solid consistence, as in bariey-sugar, is at first transparent, broken with difficulty, and presents a glassy fracture. After a short time it boemes nogque and almost friaine.

Q. HOW dOCS BRASS WIRE chinge?

A. Brass wire, when first manufactured, is strong and tenacious, but when exposed to damp air, or the fumes of an arid, becomes brittie. 
Q. What very curious chavaE takes place with the crystals of iodide of mercury ?

A. When they are first formed, they are of a bright red colour, but if scratched with a hard substance, as the point of a pin, the crystals turn over, or readjust themselves, and become bright yellow.

Fig. 8?.

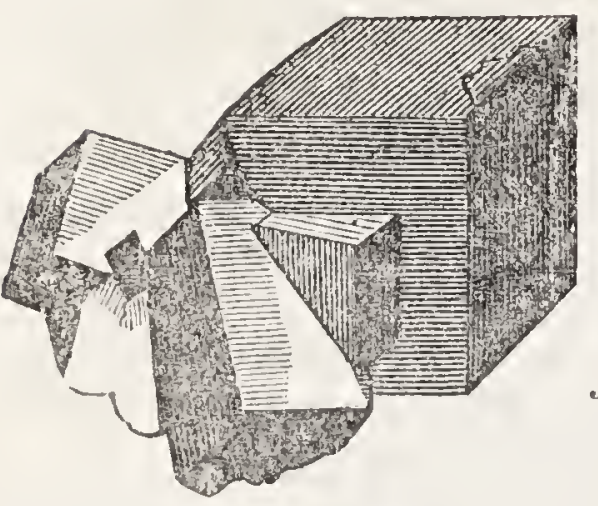

Q. In what form do sume of the varieties of IRUA URE crystallise?

A. The sulphuret of iron, so often mistaken for gold, crystallises very beautifully in cubes, which gold never does. (Sce fig. 82.)

Q. Does LIG HT appear to influence crystallisation?

A. Light has a powerful influence over the crystallisation of certain substances. Thus, in druggists' shops, the camphor contained in glass jars is arranged in beautiful crystals upon the side next to the light.

Q. What is meant by "ANNEALING ?"

A. Annealing is the process of softening and restoring the malleabitity of metals and the softness of glass, by heating them, and allowing them to cool very slowly. It consists in placing the articles while hot in an oven, where they are allowed to cool gradually.

Q. If glass is allowed to COOL VERY QUICKLY, what is the result?

A. It is very brittle, and breaks with the slightest blow ; whereas if it is allowed to cool slowly, its particles arrange themselves in such a way as to form a fibrous texture, elastic, and not easily broken.

Q. How are iron and stecl HARDENED?

A. By cooling them very rapidly after heating.

Q. How are iron and steel made soft?

A. By cooling them very slowly after heating.

$Q$. The hooked top of walking-sticks is made by Bolurva the end of the stick, and then liending it into an arch : why is a stick made FLEXIBLE by BOILING?

A. Wood contains many substances soluble in hot water, as starch, gum, \&c., and several others which are softened by it; as, therefore, several substances are dissolved and others softened by boiling water, the stick is rendered flexible.

Q. If you scrape a slip of paper with a knife, why will the paper cURL ?

A. Because the surface of the paper is contracted by scraping, which brings the particles closer together; this contraction of the surface bends the slip of paper into a curl or arch.

Q. If a crystal of alum, for example, is broken into fragments, and the fragments placed in a saturated solution, what TAKES PLACE?

$A$. Each fragment reproduces the form of the crustal from whence it was broken. 
Q. Have chemists been able to reproduce in their laboratories erystals of ans and other PRECIOUS STONES?

A. In som instunces they have been enabled to reproduce them most successfully.

Q. In what MANNER has it been ascomplished?

A. Sometimes through the agency of electricity, but generally by mixing the matter which enters into their composition, and submitting them to the long-continued action of intense heat. In this way crystals of the topaz, sapphire, garnet, and ruby have been pro-. duced of great beaity.

\section{METEOROLO G Y. \\ CHAP'TER XXII.}

THE ATMOSPHERE.

Q. What is METEOROLOGY?

A. Meteorology is that branch of natural science which treats of the atmosphere and its phenomena.

Q. What is the ATMOSPHERE?

A. A thin fluid which surrounds the earth on every side, and goes with it throughout both its daily and annual revolutions.

Q. Of WHAT is the ATMOSPHERE composed?

A. It is composed principally of two different sorts of gases-one termed oxygen, and the other nitrogen.

An aqueous (watery) vapour is also present in the atmosphere, and about ten parts of carbonic acid gas to every ten thousand parts of atmospheric air?

Q. In what PRoportion are oxygen and hydrogen present in the atmosphere?

$A$. If we represent the atmosphere at 100 , oxygen would be 21 , and nitrogen 79 ; that is, one part oxygen, and four parts nitrogen.

Q. What is MEANT by a GAS?

A. An elastic fluid, usually invisible and without colour, like common air.

Q. How is the air HEATED?

A. In two ways : first, by the luminous beams of the sun; secondly, by the radiation and conduction of heat from the earth.

Q. In what manner is the air heated by Radiation from the earth ?

A. By convection; thus, the sun heats the earth, and the earth heats the air resting upon it; the air thus heated rises, and is succeeded by other air, which is heated in a similar way, till the whole volume is warmed by "convective currents." 
Q. What is rivant by "convecrive curreits" of hot air?

A. Streams of air heated by the earth, which rise upwards, and carry heat with them.

Q. How is the AIr made colis?

A. The air resting on the earth is made cold by contect; this cold air makes the air above it cold; and cold currents (or winds) cause the whole to mix together, until all becomes of one temperature.

Q. What EFFEOT is produced upon $A$ IIr by CoLD?

A. It is condensed or compacted into a smaller compass; in consequence of which it becomes heavier, and descends towards the ground.

Q. Prove that air is condensed by coLD?

A. Lay a bladder half full of air before a fire till it has become inflated; if it be now removed from the fire, the bladder will colleipse again, because the air condenses into its former bulk.

Q. What effect has IIIST upin the AIR?

A. Heat rarefies the air, and causes it to expand.

Q. What is meant by the MFAN MALY MESM ERA TRE?

A. The mon or average temperature of the day is found by observing the thernometer at fixed intervals of time during the twenty-four hours, and then dividing the sum of the temperatures by the number of observations.

Q. How is the mean ANNULL temperature of a particular point ascertained?

A. By taking the average of all the mean daily temperalures throughout the year.

Q. How does temperature VanY with the Iatitune?

A. The average annual temperature of the atmosphere diminishes from the equator towurels either pole.

Q. Give manples of this variation.

$A$. At the equator, in Brazil, the average annual temperature is $84^{\circ} \mathrm{F}$.; at Calcutta, lat. $22^{\circ} 35^{\prime} \mathrm{N}$., the annual temperature is $82^{\circ}$ F.; at Savannah, lat. $32^{\circ} 5^{\prime} \mathrm{N}$, the annual temperature is $65^{\circ} \mathrm{F}$.; at London, lat. $5 \mathrm{l}^{\circ} 81^{\prime} \mathrm{N}$., the annual temperature is $50^{\circ} \mathrm{F}$.; at Melville Island, lat. $74^{\circ} 47^{\prime} \mathrm{N}$, the mean annual temperature is $1^{\circ}$ below zero.

Q How does the temperature vaIY with the ALTY'UUDF above the earth's stirface?

A. 'Temperature diminishes with the rllitude. As a general rule, a loss of heat occurs to the extent of $1^{\circ} \mathrm{F}$. for every 343 feet of elevation.

Q. How dies the gradual reduction of temperature as we ascend from the surface of the earth affect the MoIsture of the air?

A. In every latitude there is a point above the surfuce of the earth where moisture, once frozen, always remains congealed.

Q. Why are the Tops of very high mountains always corered with PERPETUAL SNow ?

A. Because, at the great elevation of their summit, the temperature of the atmosphere is so low that the congealed moisture which falls upon them never melts. 


\section{Q. What is a GLACIER?}

A. 'Whe glaciel only exists upon mountains whose summits ars corered with perpetual snow. 'The snow upon the higher parts becomen somewhat softened during the summer, and in the winter is agrin hardened nearly to ice. In the succeding summer, the action of the sun, and the internal heat of the earth, detach large masses loaried with recently deposited snow into the noighbouring valless, where, being accumulated, and the crevices filled with snow or water, which at last hardens to ice, they form huge seas of ice, or glaciers; in French, mers-de-ylace.

Q. Do the glaciers continue to INCREASE YRAR hy YaA?

A. Very many of them do; and in Switzeriand many valieys, once fertile, are now filled with glueiers. From the bottom of the glacier streams of water constantly issue, and it is from such sources that the vivers Rhine and Rume of Europe the their rise.

Q. How are the gigantic iceberg Forind whicin are found thuating at some seasons in the Atlantic?

A. They are portions of great glaciers formed in the northern lesions, which become detarhed and float in the sea.

Q. How Higi are icebergs simetimes seen ?

A. Sometimes exceeding 300 feet in height.

Q. At what sideviricx alove the surface of the earth, at the Equator, will water remain frozen?

A. At an elevation of about 15,000 feet.

Q. At what Elevatios in the STraits of Mageliak will water remiain frozen?

A. At about 4,000 feet.

Q. What is the POTYT where water remains frozen CAiLFD?

A. The line of perpetual snow.

Q. Why are not all places which lie unde: the same parallel of latitude of the sazu TEMTERATUR:?

A. Because various disturling circumstances telid to vary the mon tomperature.

Q. What IISTURBNG CrRcusstances affect the temperature oi particular situationa?

A. 1. The geographical position of the country.

2 . Its elevation above the level of the sea.

3. Its nearness to or distance from the sea.

4. The slope or inclined plane which it presents.

5. The position of mountain chails.

6 . The nature of the soil.

7. The degree of cultivation which the soil has received.

8. The prevalent winds.

9. The amnual quantity of rain that falls.

R. How does the geograpnical iosituriz of a country affect its crimate?

1. On account of the heat experienced from the sin's rays. In some comtries the heat is much more intense than in others. Africa is alwavs a liot climate; Cireenland always cold. 
Q. How does the elevation of IAND abore the SEA LEvGL allect its temperature?

A. The higher we rise, the colder the air becomes; consequently, lands considerably elevated above the sea level, are colder than those in lower regions.

Q. What EFFECT on climate has NEARNiss to, or RmMOTENESS from, the sea?

In cold climates the sea lessens the cold; in warm climates the saa diminishes the heat.

Q. How does the SLope of a country affect its climate?

A. By exposing it more or less to the direct rays of the sun.

Q. What EFFECTS have MounTAINS on climate?

A. Chains of mountains afford shelter from the winds, and thereby augment the temperature; mountains, however, which intercept south or west winds, lower the temperature, because these winds are warm.

Q. What EFFECT has the nature of the sorl on climate?

A. One soil acquires heat, keeps its acquired heat, or gives it forth in radiation more rapidly than another. All varieties of soil have different powers of radiation and absorption.

Q. What EFFECT has the cultivation of the sorl on the climate?

$A$. Wild and uncultivated land fills the air with noxious vapours; the draining of marshes and the felling of forests give free circulation to the atmosphere, and effect a wonderful change in the climate.

Q. Do the WINDS AFFECT the climate?

A. Yes; all other influences are modified by the prevalent winds.

Q. How is climate AFFECTED by the QUANTITY of RAIN which falls?

A. By the evaporation from the earth, and the moisture in the atmosphere.

\section{CHAPTER XXIV.}

\section{WINDS.}

Q. What is wind ?

A. Air in motion.

Q. What occasions those srovements of the air which we call wind?

A. The principal cause is the variation of temperature produced by the alternation of day and night and the succession of the seasons.

Q. How can winds originate through VARIATIONS of TEMPERATURE?

A. When through the agency of the sun a particular portion of the earth's surface is heated to a greater degree than the remainder, the air resting upon it becomes rarefied and ascends, while a current of cold air rushes in to supply the vacancy. Two currents, the one of 
warm air flowing out, and the other of cold air flowing in, are thus continually produced; and to these movements of the atmosphere we apply the designation of wind.

Q. Does the wind ALWAYS BLOW?

A. Yes; there is always some motion in the air; but the violence of the motion is perpetually varying.

Q. Does the Rotatron of the EARTH upon its axis effect the motion of the air?

A. Yes; in two ways: 1 . As the earth moves round its axis, the thin movable air is left somewhat behind, and therefore seems (to a stationary object) to be blowing in the opposite direction to the earth's motion; and

2. As the earth revolves, different portions of its surface are continually passing under the vertical rays of the sun.

Q. What is meant by "vERTICAL RAYs?"

$A$. When the sun is in a direct line above any place, its rays are said to be "vertical" to that place.

Q. Why is not the WATER of the sea made so пот by the vertical sun as the surface of the LAND?

A. Because the evaporation of the sea is greater than that of the land; the constant motion of the water prevents the increase of temperature at the surface; and water is a bad conductor of heat.

Q. Does the Motron of the SEA prevent its surface from being HEATED by the direct rays of the sun?

A. Yes; the rolling motion of the water prevents the surface being heated to a greater degree than the water below it; the evaporation also absorbs the heat in the generation of vapour, and carries it off into the air.

Q. Why are those winds which blow over LARGE CONaINENTS or TRACTS of LAND generally DRY?

A. Because in their passage they absorb very little water, as they do not blow over large oceans.

Q. Why do our HANDS and LIPS CHAP in frosty and windy weather?

A. 1. Because the wind or frost absorbs the moisture from the surface of the skin; and

2. This action of wind or frost produces a kind of inflammation on the skin.

Q. Do oLOUds affect the wIND?

A. Yes. As passing clonds screen the direct heat of the sun from che earth, they diminish the rarefaction of the air also; and this is wother cause why neither the strength nor direction of the wind is uniform.

Q. Would the wind blow REgularLy from east to west if all obstruotions were REROYED?

A. Without doubt. If the whole earth were covered with water, tho winds would always follow the sun, and blow uniformly in one direction. 
Q. Do winds ETER blow REGLIARLY ?

A. Yes; in those parts of the world which present a lurge suffuce of water, as in the Atlantic and Pacific oceans.

Q. With what valocirs do winds move?

A. Every graduation exists in the speed of winds, from the miliciest zephyr to the most violent hurricane.

Q. With what velocity does a wind which is HARDLY PERCEPTIBLE move ?

A. With a velocity of about one mile per hour.

Q. In a gentle wind, what is the veloctrx?

A. From four to five miles per hour.

Q. In a pleasant BRISK GLLE, what is the velusity?

A. From ten to fifteen miles per hour.

Q. In a very BRISK wIND, what is the velocity?

A. From twenty to twenty-jive niles per hour.

Q. In a very MIan WIND, what is the velosity?

A. From thirty to thirty-jive miles per hour.

19. What is the velocity of the winl in a stors?

A. From fifly to sixin mites per hour.

Q. In a IUR? IOANa, what is the estimated velocity?

A. From eighty to one hundred miles per hour.

Q. Why do we sometimes see crorns st one elevation muviug in ONE DIRECTRON, and at

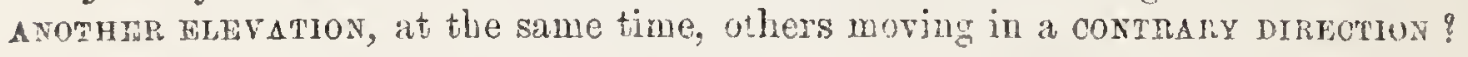

A. Because different currents of air exist at different elevations, moving in different directions, with different velocities.

In 1889, an English aer waut, at the height of 14,000 feet, enconntered a current that bure him along at the rate of five miles per honr; but, upon descending to the altitude of 12,000 feet, he met with $a$ contrary wind, blowing with a velocity of eigbty miles per hour.

Q. How is the Fons of the wind acertained?

A. By observing the amount of pressure that it exerts upon a given plane surface perpendicular to its oun direction.

If the pressure plate acts fieely upon spiral springs, the power of the wind is denoted hy the extent of their compression, and that weight will be a measure of their force, the adine as in weighing by the ordinary spring-balance.

Q. What is an INSTLUMENT for measuring the force of the wind CALLED ?

A. An Anemometer.

Q. Into what THREe crassess mily winds be diviled?

A. Into comstant, periodical, and variable winds.

\section{SECTION I.-CONSTANT WINDS.}

Q. What name is given to the constant winds which blow orer the ATrarrio and Panso Occars?

A. That of "trade winds."

Q. Why are they culled " Tradk-wines?"

A. Because they are very convenient to navigators who have to cross the ocean, as they always blow in one direction. 
Q. In what DIRection do the Trans-winds blow?

A. That in the northern hemisphere blows from the north-east; that in the southern hemisphere from the south-enst.

Q. Why do they not blow from the DIREct Nonth and sourn?

$A$. Because as the earth rolls every day once round itself from vest to east, air that has acquired slow movement at the poles finds the globe travelling too fast for it at the equator, and is obliged therefore to drop more and more behind.

Q. What is the CAUSE of these currants of air from the POLES to the EQUATOR ?

4. The heated air expands near the equator, rises and runs over towards either pole in two grand upper currents, under which there fiow from north and south two deluges of colder air to occupy the space vacated, and to restore the equilibrium.

Q. Is there an Upper as well as a Lowne currtart in the atmosphere?

A. Yes; the upper current is from the equator to the poles, where it is condensed, and thence returns as the lower current to the equalor.

Q. These Low explain the cause of this.

A. All the atmosphere revolves with the earth; but when a current of air from the poles flows towards the equutor, it comes to a part of the earth's surface which is moving fuster than ilse fr; in consequence of which it is left befind, and thus produces the effect of a current moring in the opposite direction.

Thus, to a persoil in a carriage, the hedges and trees seem to be ruuning in an orposite direstion.

As the circumference of the earth at the eruator is mon larger than the circuinference of the earth at the ples, therefure every spot of the anth's equatorial surface inust move much faster than the correspouding one at the poles.

As the eirth revolves on its axis from west 10 cast, therefore the air which is carried with it will seem to blow from the west. As, however, the current of air from the poles reems to blow in the opposite direction, it will seem to blow from the east, or to be an cisterly wird.

Q. By what MOMS are the north-east and south-east TRAJE-TINDS PRODUCED?

1. By a combination of the two motions of the polar currents, which produces the internediate directions of the north-east and south-east.

q. Are Born these motroys of the polar ements BaAL?

A. No; the motion from the edst to wost is only apparent. As the earth revolves from west to exst, the air carried with it will be a west wind; but the polar currents seem to blow in the opposite direction, merely because they have not acquired the same velocily.

Q. Do trade-winds blow from the north-east and sisth-east als the rear rovad?

1. Yes, in the open sea ; that is in the Atantic and Pacific Ocerns, for about $25^{\circ}$ each side of the equator.

Q. What io the north-eaterly and sulth casterly trade-winds pronvon when lieg XEE? nar the equator?

A. A region of calms and variabie winds, in which thick fogry air pevails, with sudden sinowers and thunderstorms. 
2. Is this region of calmns FLXED in its position?

A. No; it shifts its place according to the sun's distance and position in regard to the equator, being sometimes entirely to the north of the equator, and occasionally reaching as far as $2^{\circ}$ south of it.

Q. In the INDIAN OCEan do the trade-wints AlWAYs biow north-east and south-east?

A. No; from April to October a south,east wind prevails, and fiom October to April a north-east wind sets in.

Q. Why do not the trade-winds blow uninTterrupteder from pole to PoLE ?

$A$. The hot air that ascends from the equator and passes north, gradually cools, and becomes denser and heavier, running as it does over the cold current below. The cold air from the pole, too, gradually becomes warmer and lighter as it passes south, so that in the temperate climates there is a constant struggle as to which shall have the upper and which the lower position. In these regions, consequently, there are no uniform winds.

Q. Where do the trade-winds blow with UNIForM Forok and constancy?

$A$. In many parts of the Pacific embraced within the region of the trade-winds, a ressel may sail for a week without altering the position of a sail or rope.

Q. How does the MEEmING of the opposing winds produce a region of oALMS and VARI$\triangle B L E$ WINDS?

$A$. Because the forces of the opposing winds connterbalance one another, and the atmosphere becomes motionless. When their relative strength becomes changed, as it sometimes rapidly does, short and sudden gusts and squalls arise.

\section{SECTION II.-PERIODICAL WINDS.}

Q. What are Monsoons?

A. Monsoons are regular periodical winds which sweep over the Indian Ocean and the whole of Hindostan, as the sun moves into the northern or southern hemispheres; they are on this account called monsoons.

Q. Why is the term "Mrossoon" given to them?

$A$. The term is derived from a Persian word monsum, signifying "season."

Q. What are the Limirs of the Mossooss?

$A$. The south-west monsoon reaches from the east coast of Africa to the coasts of China; the north-east monsoon prevails from May to October within the same limits; the north-west extends from Madagascar to New Holland; and the south-east in the same limit from April to October.

Q. What is the smifres of these periodical winds called by sailurs?

A. The breaking up of the monsoon.

Q. By what PHENOMENA are these changes accompanied?

A. Generally by great and terrible storns. 
Q. Are the monsonns SFrYTOEABLE to sailors?

\&. Yes; as it often happens that a ship is wafted by a monsoon to is distant port, and enabled by their shifting to return in an olyosite direction.

Q. How is the CHANGE of the monsoons MARKED?

A. By an interval of alternating calms and storms.

Q. What is the CAusi of a SEd BREYHE?

A. When the land is more heated by the sun than the sea is, the lond air becomes hotter than that over the sea; in consequence of which the cooler sea air glides inland to restore the equilibrium.

Q. Why does a SEa Breeze feel coor ?

A. Because the sun cannot make the surface of the sea so hot as the land: therefore the air which blows from the sea is cooler then the air of the land.

Q. Is there a FRESH SEA BREEZE during the summer and autumn MORNINGS?

A. Yes; as the land air is more heated by the sun than that of the sea, the cool sea breeze glides inland to restore the equilibrium.

2. Are TROPICAL ISLANDS subject to a LAND BRERZR every evening?

A. Yes; as the surface of the land, after sunset, cools down faster than the surface of the sea, the cold air of the land is condensed, sinlis doun, and spreads itself into the sea air, causing the land breeze.

\section{SECTION III.-VARIABLE WINDS.}

Q. In what REGIoss do variable winds prevail ?

$A$. In what are called the extra-tropical regions.

Q. WHг do variable winds prevail mostly in the extra-tropical regions?

$A$. In the tropics, the great aerial currents known as the tradewinds exist in all their power, and control most of the local influences; but in the temperate zones, where the force of the tradewinds is diminished, a perpetual contest occurs between the permanent and temporary currents, giving rise to constant fructuations in the strength and direction of the winds.

Q. Why are EAST WINDS generally DRY?

A. Because they come over lurge tracts of land, absorb very litile water, and being thirsty, therefore lring dry weather.

Q. Why are NoRTH wiNDs generally coLD and DRY ?

$A$. Becalise they come from colder regions, and being warmed by the heat of our climate, absorb moisture from everything they touch; in consequence of which they are generally dry.

Q. Whiy are soUTH WINDs generally TARAI ?

A. Because they come over countries warmer than our own, where they are much heated.

R. Why are winss which blow over a vast BODY of watro generally rainy?

$A$. Because they come iaden with vapoir: if, therefore, they meet with the least chill, some of the vapour is deposited as rin. 
Q. Why do SOETH WINDS often bring IRAIN?

$A$. Because, coming from the torrid zone, they are much heated, and imbibe water very plentifully as they pass over the ocean.

Q. How does this account for the RAINY character of south winds?

$A$. As soon as they reach a cold climate they are condensed, and can no longer hold all their vapour in suspension; in consequence of which some of it is deposited as rain.

Q. Why are DRY WINDS in the spring months desirable and advantageous for agricul tural operations?

A. They dry the soil saturated with moisture of winter, breale up the heavy clods, and fit the land for the seed committed to it.

Q. What are the so-called "PONA" winds of South America?

$A$. They are winds coming from the snowy peaks of the Andes which sweep over the high table-lands of South America.

They are so extremely dry and cold, that they absorb the moisture of dead animal bodies with such rapidity as to prevent putridity. If a mule happens to die upon one of these plains, it is converted in the course of a few days into a mummy, even the entrails being free from the slightest evidence of decay.

Q. What is the "srroou?"

$A$. It is an intensely hot wind that prevails upon the vast deserts of Africa and the arid plains of Asia, causing great suffering, and often destruction of whole caravans of men and animals.

Q. How does the simoom originate?

A. Its origin is to be sought in the peculiarities of the soil, and the gengraphical position of the countries where it occurs.

The surface of the deserts of Africa and Asia is composed of dry sand, which the rays of the sun render burning to the touch. The heat of these regions is insupportable, and their atmosphere like the breath of a furnace. When, under such circumstances, the wind rises and sweeps over these plains, it is intensely hot and destitute of moisture, and at the same time bears aloft with it great clouds of fine sand and dust-a dreadful visitant to the traveller of the desert !

Q. What is a HURRICANe?

A. The hurricane is a remarkable storm wind, peculiar to certain portions of the world. It rarely takes its rise beyond the tropics, and it is the only storm to be dreaded within the region of the trade-winds.

Q. How are hurricanes especially DIsTraguished from other kinds of tempests?

A. By their extent, irresistible power, and the sudden changes that occur in the direction of the wind.

Q. Do any PARTICULAR PORTIONS of the Tropics appear to be especially visited with hurricanes?

A. In the northern hemisphere, hurricanes most frequently occur in the regions of the West Indies; in the southern hemisphere, they occur at the Manritius.

Q. Do the hurricanes occur at PARTICUIAR SEASONS ?

A. The West Indian occur from August to October ; the Mauritian from February to April.

Q. What have RECENT investigations shown the hurricanes to be ?

A. Extensive storms of wind, which revolve round an axis, either 
upright or inclined to the horizon; while at the same time the borly of the storm has a progressive motion over the surface of the ocean.

Q. ILuUSTRate more clearly the MaNNer in which a hurricane xroves?

$A$. It is the nature of a hurricane to travel round and round, as well as forvard, very much as a corkscrew travels through a cork, only the circles are all flat, and described by a rotatory wind apon the surface of ine water.

Q. What EFFEOT has this rotatory movement of the wind upon the water of the oceail and the surrounding atmosphere?

A. The rotatory wind blows the sea with it in a rotatory current; within the circle of the hurricane the air is calm, and its diminished pressure lifts the water up in a great storm-wave, which, advancing with the hurricane surrounded by its current, becomes a deluge if it strike upon a shore; but otherwise rolls on and on, while the wind dances round and round it, thus twisting circles while it marches on its main path-that main path being itself a grand curve.

Q. In what DiRsorions do the northern and southern hurricanes move?

$A$. Hurricanes ahways travel away from the equator. North of the equator, the great storm, revolving as it comes, generally rolls from the south-east to the north-west, until it has passed the northern tropic, when its course changes from south-west to north-east; the rotation of the storm being from right to left, contrary to the motion of the sun.

It always comes in that way; always describes in its main course the curve of an ellipse, which generally crosses the West India Islands, and presently, pursuing the ellipse, marches to the north-east from the coast of Florida, trealing the waves of the Atlantic. In the southern hemisphere, hurricanes come from the north-enst, and pursue a course away frum the equator precisely similar.

Q. In what MLANER can you sketch upon a MAP the course of a West India hurricane?

A. 'Take an egg, and place it on' an atlas map so that its small end shall be near the coast of Florida, and its lower edge rest on the Leeward Islands; take a pencil, and beginning eastward of these islands, trace the outline of your egg towards the west, turning its corner, and still tracing on towards north-east, as if travelling to Europe; leave off now, and you have sketched the ordinary elliptical path of a West Indian hurricane. wind ?

Q. In what DIRECTION would a ship revolving in the circles of a hurricane find the

A. As the ship revolved, she would in turn find the wind blowing from every point of the compass.

Q. What is known concerning the DISTANCE travelled by HURRTCANes?

$A$. The distance traversed by these terrible tempests is immense. The great gale of Angust, 1830, which occurred at St. Thomas on the 12th, reached the Banks of Northumberland on the 19th, having travelled more than three thousand noutical miles in seven days; the track of the Cuba hurricane of 1844 was but little inferion in length.

Q. What is kunwn of their PRogressive and Rotary Velooity?

A. Their prospesive velocity is from seventeen to forly miles per 
hour; but distinet from the progressive velocity is the rotary, which increases from the exterior boundary to the centre of the storm, near which point the force of the tempest is greatest, the wind sometimes blowing at the rate of one hundred miles per hour.

Q. How great is the BREADTH of the hurricane?

A. The surface simultaneously swept by these tremendous whirlwinds is a vast circle varying from one hundred to five hundred miles in diameter.

Q. How great is the SURFACr over which they PREvaIL?

A. The great Cuba hurricane of 1844 was estimated to have been not less than eight hundred miles in breadth, and the area over which it prevailed during its whole length was computed to be two million four hundred thousand square miles - an extent of surface equal to two-thirds of that of all Europe.

Q. What corious FAcr have mariners noticed when in the centre or vortex of the hurricane?

A. An awful calm evails, described as the lull of the tempest, in which it seems to have rested only to gather strength for greater efforts.

2. In a hurricane, how does the BaroNester RISE and FALL?

A. During the first half of the storm the barometer falls, and during the last half it rises.

Q. WHY does the barometer rise and fall in this manner?

A. Because the density of the air increases from the centre to the circumference of the storm, and even beyond its boundary; so that when a hurricane passed diametrically over a district, the atmospheric pressure would decrease, and the barometer continue to sink during the first half of the storm, but gradually rise as the last half jasced over.

Q. What is the CONDITIOX of the ATMOSPHRge included within the circle of the hurricane!

A. The hurricane being a vast whirlwind, the atmosphere constituting the body of the storm will be dricen outwards from the centre towards the margin, just as water in a pail which is made to revolve rapidy, fhies from the centre and swells up at the sides. But the pressure of the atmosphere beyond the whirl, checking and resiting the centrifugal force, at length arrests the outward progress of the mass of air, and limits the storm.

Q. In tropical climates, what indicatrons does the barumeter afford of the approach of a hurricane?

A. The mercury in the tube is depressed, or agitated in an extraordinary manner, for some time before any indications of a storn apnear in the horizon.

Q. What very cunvors Las"ance may be mentioned of the sailing of a ship caught in a hurriccae?

1. In 1845, a ship encountered a hurricane near Mauritius. The wind, as the ship saled in the circuit of the storm, changed fice 
times completely round in one hundred and seventeen hours. The whole distance sailed by the vessel was thirteen hundred and seventythree miles, and at the termination of the storm she was only three hundred and fifty-four miles from the place where the storm commenced.

Q. Wно discovered and demonstrated this theory of the hurricane?

A. 'The credit of making known these important facts belongs principally to Colonel Reid, of England, and Mr. William C. Rerbjield, of New York.

Q. How has a knowledge of the laws governing the movements of the hurricane been of PRACTICAL VALUR to the interests of navigation?

A. Ruties have been published by which a sailor may know the exact conrse of the hurricane he may happen to encounter, which enable him to steer his ship so as she can ride safely until the hurricane is gone.

Without such knowledge, puzzled by the changing wind, he perhaps drives before it, and is whirled round, circle after circle, dragged through the very road of danger ; or he escapes into the mildle of a circle, has a little breatbing time, and presently the crash returns; or he gets out of the inain course, and through ignorance encounters it again. Shipwrecks innumerable have been caused in this way. In the present day, though we have not yet established a full theory concerning hurricines, the sailor has been tangbt to step out of their path.

Q. What are the Accompantuents of a hurricane?

A. Thunder and lightning, and torrents of rain.

Q. What is the ryproox?

A. The typhoon is a storm of wind somewhat resembling the hurricane. It is met with only in the China seas, not so far south as the island of Mindanao, nor so far north as Corea, except upon the eastern borders of Japan.

Q. In what HESPECT does a TORNADO differ from a HURRICANE?

$A$. Tornadoes may be regarded as hurricanes, differing chiefly in respect to their continuance and extent.

Q. How IONG do they usually IAST ?

A. From fifteen to seventy seconds.

Q. What is their EXTENT?

A. Their breadth varies from a few rods to several hundred yards, and the length of their course rarely exceeds twonty miles.

Q. What PHENoxrasa generally attend them?

A. The tornado is generally preceded by a calm and sultry state of the atmosphere, when suddenly the whirlwind appears, prostrating everything before it. Tornadoes are usually accompanied with thunder and lightning, and sometimes showers of hail.

Q. What curious EFrECT does a tomado sometimes have upon fowls and birds overtaken by it?

$A$. They are often entirely stripped of their feathers.

Q. What currous THEORY was propounded some years since to account for this phenomenon?

$A$. The author of the theory supposed that in the vortex or centre 
of the tornado there was a vacuum, and the fowls being suadenly caught in it, the air contained in the barrel of their quills expanded with such force as to strip them from the body.

Q. What is supposed to be the origrs of tornadoes?

$A$. They are supposed to be generally produced by the lateral crtion of an opposing wind, or the influence of a brisk gale upon a portion of the atmosphere in repose.

Q. How are the EDDIEs, or whIRLPOols, produced which occur in water, and which, in their formation, resemble some tornadoes?

A. Eddies, or whirlpools, are most frequently formed in water when two streams flowing unequally meet. They may be seen at the junction of two brooks or rivers.

Q. How are the whirlwinds which we frequently see at the corrers of sTREETs in cities produced?

A. They are caused by a gust of wind sweeping round a corner of a building, and striking the calm air beyond it.

Q. Where does the WhIRL of the whirlwind appear to orIGINATE?

$A$. In the higher regions of the atmosphere; it increases in velocity as it descends, its base gradually approaching the earth, until it rests apon the surface.

Q. What is the EFFECT of EXTENSIVE FIRES in producing whirlwinds?

A. Great conflagrations sometimes produce whirlwinds, in consequence of a strong upward current, which is produced by the expansion of the heated air.

Q. What REMARKABLE EXAMPLE of this phenomenon is on record?

A. At the burning of Moscow, 1812, the air became so rarefied oy heat, that the wind rose to a frightful hurricane.

Q. What is a WATERSPoUt?

A. A waterspout is a whirlwind over the surface of water, and differs from a whirlwind on land in the fact that water is subjected to the action of the wind, instead of objects on the surface of the earth.

Q. When an observer is near, are any sounds perceptible?

A. A loud hissing noise is heard, and the interior of the column seems to be traversed by a rushing stream.

Q. What is the BREADTH and HEIGHT of waterspouts?

$A$. In diameter the spout at the base ranges from a few feet to several hundreds, and its altitude is supposed to be often upwards of a mile.

Q. What are its Successive APPEARANoES ?

A. At first it appears to be a dark cone, extending from the clouds to the water; then it becomes a column uniling with the water. After continuing for a little time, the column becomes disunited, the cone re-appears, and is gradually drawn up into the clouds.

Q. Is it supposed that water is SUCKED UP from the ocean or lake by the water-spout?

$A$. It is a common belief that water is sucked up by the action of 
the spout into the clouds; but there is reason to suppose that water rather descends from the clouds, as water which has fallen from a spout upon the deck of a ressel has been found to be fresh. There is no evidence, furthermore, that a continuous column of water exists within the whirling pillar.

Q. What are SAND-PILLARS?

$A$. They are gigantic columns of sand raised by whirlwinds in the deserts of Africa and other countries. They sometimes extend as ligh as the clouds, and move with various degrees of velocity, carrying destruction in their paths.

Q. Why does Wrind generally feel corD?

$A$. Because a constantly-changing surface comes in contact with our body to draw off its heat.

Q. What are the EFFEcrs of wind noticed in the Aretic regions?

A. Sir John Richardson, Dr. Kane, and other Arctic explorers inform us that in those regions when the thermometer ranges from $40^{\circ}$ to $60^{\circ}$ below zero, the cold of the external air is easily endurable, provided the air is calm, and the individual exercises freely; but if a wind arises at this temperature, the severity of the cold becomes too great for human endurance.

Q. It the winds should cease to blow over the ocean, what would be the EFFECT?

$A$. The water would undoubtedly become stagnant. Tempests and hurricanes also exercise a beneficial effect by agitating and purifying the atmosphere, and sweeping from it the seeds of pestilence and contagion.

\section{CHAPTER XXV.}

\section{CLOUDS.}

Q. What are cLouds?

$A$. Masses of condensed vapour floating in the air.

Q. From WHEMcE are they derived ?

$A$. They are evaporated, or drawn up from the earth.

Q. Are cLouds and FOGS the SAISE in their character?

$A$. They are caused in the same way, but while fogs cling to the earth, clouds rise above it.

Q. Why are CLOUDS HIGHER on a FINE DAY ?

A. Because they are lighter and more buoyant.

Q. Why are CLOUDS LIGHTER On a FINE DAY?

A. 1. Because the vapour of the clouds is less condensed; and

2. The air itself (on a fine day) retains much of its vapour in an invisible form. 
Q. How do you ACCount for cLouds Fu

A. By their lightness and the buoyancy of their composition.

Q. Of WHAT are they CoMposEi ?

A. The vapour of which they consist is formed into small globules or bubbles, which give lightness and buoyancy to the cloud.

Q. What are these Giobuties called?

A. Vesicles.

Q. What CIRCUMSTANCES occasion PARIETY in the appearance of the clouns?

A. Their colour, their weight, and their height above the earth.

Q. What is the cHIEF cause of fog and clouds?

$A$. During the daily process of evaporation from the surface of the earth, warm, humid currents of air, are continually ascending; the higher they ascend, the colder is the atmosphere into which they enter; and as they continue to rise, a point will at length be attained where, in union with the colder air, their original humidity can no longer be retained: a cloud will then appear, which increases in bulk with the upward progress of the current into colder regions.

Q. Are ALI countries alike subject to a CLOUDY atmosphere?

A. No; very great differences exist.

Q. On what do these DIFFERences chiefly depend?

A. On variable winds; thus the variable winds which prevail in England cause our atmosphere to be very cloudy. In Egypt, where the temperature is higher and more equal, the sky is seldon clouded.

Q. Do changes in the wind produce clouds?

$A$. Yes; a warm current of air disperses the clouds by absorbing the vapour of which they are composed; but a cold current of air, on the contrary, condenses the vapour in the air, and produces cloud and rain.

Q. What DISTANCE are the cLouds from the EARTH?

$A$. Some thin, light clouds are elevated above the highest mountain-top; some heavy ones touch the steeples, trees, and even the earth; but the average height is between one and two miles.

Streaky, curling clouds, like hair, are often five or six miles high.

Q. Does the HEIGHT of the CLOUDS VARY with the SEASON of the year?

$A$. Yes ; their height is much greater above the earth in summer than in winter.

Q. What is the ordinanY size of CLOUDS ?

$A$. The size of clouds is very variable; while some only measure a few square yards, others extend over many miles.

Q. Is it POSSIBLE to ascertain the THICKNesS of a CLOUD?

A. Yes; this is often quite easy, as the tops of some lofty mourtains rise higher than the clouds, so that travellers pass through them before they arrive at the summit. Balloons. also pass through and rise above the clouds. 
Q. Is there any rarietr in the SIIAPE of the CLOUDS?

$A$. An almost endless variety of shape is assumed by the clouds in the atmosphere.

Q. Is there any CLASSIFIOATION of these varied forms adopted by scientific inen?

A. Yes; they have been arranged in a classified order, which is now generally admitted.

Q. How is this VARIETY in the shape of the vLuvdo OCCASIONED?

A. By their origin, their electrical state, and the prevailing currents of air.

Q. Does, then, EiscTRICITY affect the SHAPE of CLOUDS?

A. It does; and, if one cloud be full of electricity and another not, they will be attracted to each other, and either mingle or disappear altogether.

Q. Why do clouds, when not continuous over the whole surface of the sky, appear JAGGED, ROUGH, and UNEVEN?

A. The rays of the sum, falling upon different surfaces at different angles, melt away one set of elevations, and create another set of depressions; the heat also which is liberated from below in the process of condensation, the currents of warm air escaping from the earth, and of cold air descending from above, all tend to krep the clouds in a state of agitation, upheaval, and depression. Under their various influences, the masses of vapour composing the clouds are caused to assume all manner of grotesque and fanciful shapes.

Q. What EFFECT have WINDS on the SHAPE of CLOUDS?

A. They sometimes absorb them entirely; sometimes increase their size and weight; and sometimes change the position of their parts.

Q. Has not the WIND at some periods an entirely OPPOSITE EFFECT on CLODDS from that which it bas at another?

A. This depends on whether the wind or current of air be hot or cold. If it be a warm, dry wind, it disperses or converts the cloud into invisible vapour; if it be a cold wind, it increases the bulk and density of the clouds.

Q. Why are MorNING CLOUdS generally of a RED tinge?

A. Because red rays are the least refrangible of all; and not being bent so much as blue and yellow rays, we see them sooner in the morning.

Q. Why is not the COLOUR of CLOUDS always ALIKE?

1. Because their size, density, and situation in regard to the sun are perpetually varying, so that sometimes one colour is reflected and sometimes another.

Q. Why are the LAST CLOUDZ of EVENING generally of a RED tinge?

A. Because red rays (being the least refrangible of all) are the last to disappear.

Let us suppose, as in fig. 83 , a ray of light proceeding from the sun $S$ to enter the earth's atmosphere at the poiut $P$. The red rays, which cumpose in part the solar beam, 
being the least refrangible, or the least deviated from their course, will reach the ere a spectator at the point $\mathrm{A}$; while the yellow and blue rays, being refracted to a greater degree, will reach the surface of the earth at the intermodiate points $B$ and $C$. They will, consequently, be quite invisible from the point $A$.

Fig. 83.

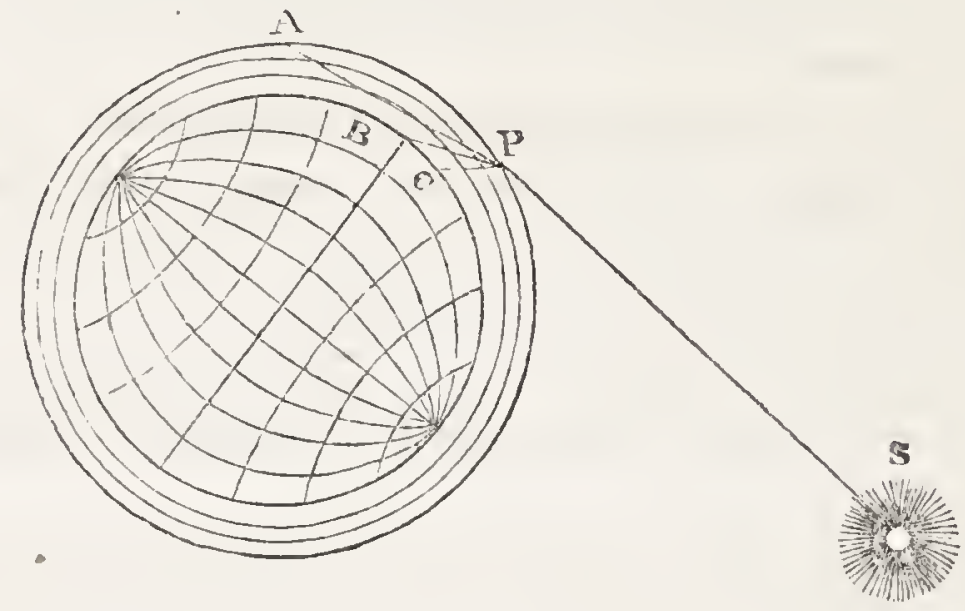

Q. What is meant by being "LESS KEFRANGIBLE?"

A. Being less able to be bent. Blue and yellow rays are more easily bent below the horizon through the action of the atmosphere, but red rays are not so much bent down, and therefore we see them later in the evening, as at $\mathrm{A}$, in fig. 83.

Q. What REGULATES the HoTION of the CLOUDS?

A. Principally the vinds; but sometimes electricity will influence their motion also.

Q. How do you know that oLOUdS Move by oTHER INFLUENCES besides wind?

A. Because (in calm weather) we often see small clouds meeting each other from opposite directions.

Q. How do you know that ELECTRICITY affects the yotion of the clouds?

A. Because clouds often meet from opposite directions, and having discharged their opposite electricities into each other, vanish altogether.

Q. What are the USES of oLOUDS?

$A$. They act as screens to protect the earth from the heat of the sun's rays; they are the great storehouses of rain, and carry the electric fuid.

Q. What is the CAUSE of a RED SUNSET?

A. The vapour of the air not being actually condensed into clouds, but only on the point of being condensed.

In the same manner, if light be transmitted through steam mingled with air, and therefore on the verge of condensation, it assumes a deep orange or red colour.

Q. Why is a RED SUNSET an indication of a FINE DAY to-morrow?

A. Because although watery vapour is present in the air, it is probably only on the verge of incipient condensution, and not sufficiently condensed to form rain clouds; and this slowly progressive transition of vast volumes of the air through the temperature of the 
dew point can only occur in serene weather at sunset, and not at sunrise.

Q. What is the CAUSE of a COPPERY, YELLOW SUNSET ?

$A$. The vapour of the air in the process of being condensed into clouds.

Q. Why do CONDENSED VAPOURS reflect YELLOW RAYS ?

$A$. Because the beams of light are refracted, or turned from their path, in a great degree by condensed vapour, in consequence of which those rays are bent down to the eye which are more refracted than the red, such as yellow.

Q. What is the CAUSE of a RED SUNRISE?

A. Vapour in the upper region of the air just on the point of being condensed.

Q. Why is a RED and LOWERING sky at SUNRISE an indication of a WET DAY ?

$A$. The red and lowering appearance of the morning sky, which indicates foul weather, probably depends upon such an excess of vapour being present in the whole atmosphere that clouds are actually forming in the higher regions, or upon the point of condensation, which the rising sun cannot disperse.

Hence our Lord's observation: "In the morning ye say, It will be foul weather today, for the sky is red and lowering." (Matt. xvi. 3.)

Q. Why is a GRAY MORNING an indication of a FINE DAY?

A. Because only the air contiguous to the earth is damp and full of vapour. There are no vapours in the higher regions of the air to bend down to the eye even the red rays of any beam of light.

Q. What DIFFERENCE (in the state of the air) is required to make a GRAY and RED SUNRISE?

$A$. In a gray sunrise, only that portion of air contiguous to the earth is filled with vapour; all the rest is clear and dry. But in a red sunrise, the air in the upper regions is so full of vapour that the rising sun cannot disperse it.

Q. Why is a GRAY SUNSET an indication of WET weather?

A. Because it shows that the air on the surface of the earth is very damp at sunset, which is a plain proof that the air is saturated with vapour, the vapour having accumulated during the day in opposition to the sun's influence; in consequence of which wet may be soon expected: hence the rhyme :-

"Evening red and morning gray

Will set the traveller on his way;

But evening gray and morning red,

Will bring down rain upon his head."

Q. Which is the most Transparent, dry or moist air?

$A$. Air moderutely moist is more transparent than very dry air.

Q. What is the CAUSE of the HAZINESS of the atmosphere during that portion of the autumn in America known as the "Indian Summer?"

$A$. It is undoubtedly due to several causes; partially to an exces- 
sive drymess of the atmosphere, and, in some degree, to the prevalence of smoke in the air, arising from burning forests. But it is also a fact, ascertained within a few years, that the constitution of the atmosphere is changed in the autumn, and that solar light at that season has less chemical influence than at any other portion of the year.

Q. Why dnes the sUr seen through a fing apnear Rro ?

A. Because the red rays of light have a greater pmier to pass through a thick, dense atmosphere, than any of the other coloured ravs.

Q. Why dnes VAPOUR sometimes form into OLOUDs, and sometimes rest upon the earth 9. MIST ar wan?

A. 'Lhis depends on the temperature of the air. When the surface of the earth is warmer than the lower air, the vapour of the earth (being condensed by the chill air) becomes mist or fog. But when the lower aiv is warmer than the earth, the vapour rises trirough the air, and becomes cloud.

Q. Why do elouds often hover around MounTaIN PEALS, when the atmosphere elsewhere is clear and free from clouds?

A. It is caused by the wind impelling up the sides of the mountains the warm humid air of the valleys, which in its ascent gradually hecomes condensed by the cold, and its excess of moisture becomes risible, and appears as a cloud.

\section{SECTION I.-MODIFICATIONS OF CLOUDS.}

Q. Into how MANY CLASSES are the different sorts of cLoons divided?

A. Into seven kinds; three original-viz., the cirrus, the cumulus, the stratus; and four formed by combination-viz., the cirro-cumulus, the cirro-stratus, the cumulo-straius, and the nimbus.

Q. What are cirRus cLorns?

A. They are distinguishable from other clouds by the lightness of their appearance, and the variety of forms they are capable of assuming.

The clouds of the cirrus are formed of the purest aqueous vapour, symmetrical in arrangement, delicate but decisive in their sharpness of edge, infinitely inultitudinous in their component parts, and of a vivid and unsullied white.

Q. What is their UsUat ror. ?

A. After a period of fine weather, the cimms clouds are seen stretching in white lines across the sky; at other times they resemble loose air; at others, a delicate, gauzy net-work.

Q. Why are these clouds called cirRus?

A. From the Latin word cirmus, a curl or lock of hair, on account of their appearance.

Q. Is the chrpes an RLEvated cloud?

A. Ies; it rises to a great height: higher, in fact than any other 
cloud. Its height at Halle, in Germany, was estimated at 21,300 feet.

Q. Of WHAT is it COMPOSED?

A. The philosopher Kaemetz declares it to be composed entirly of snow flakes, as the temperature in which it floats nust be far below freezing point.

Q. What are cuxulus clouds?

$A$. They are huge masses of vapour, which appear to be heaped or piled together, and look like mountains in the sky.

Q. Why are they called comulus? pile.

A. They derive their name from the Latin cumulus, a mass or

Q. At what PART of the DAY do these CUMULUS CLOULS mustly prevail ?

$A$. They prevail mostly in the morning, two or three hours before noon, and continue to the hottest part of the day, when they gradually disperse.

Q. How are they FORMED?

A. By the ascending currents of warm air caused by the rays of the sun.

Q. Why do the cumulus cluuds DISAPPaAr towards evening, and occur in the greatest number about noon?

$A$. At noon the currents of warm air are more buoyant, larger, and rise higher, and being condensed, form large masses of clouds; but when in the afternoon the solar heat diminishes, the strength of the currents abates, the clouds which are buoyed up by their force sink down into warmer regions of the atmosphere, and are either partially or wholly dissolved.

Q. How is the ROUNDED FIGURE of the cumulus produced?

$A$. The rounded figure of the cumulus has been attributed to its method of formation; for when one fluid flows through another at. rest, the outline of the figure assumed by the first will be composed of curved lines.

Q. How may the cumulus be IMITATED and ILLUSTRATED?

A. By suffering a drop of mill or ink to fall into a glass of water, the form of the cumulus can be imitated. The same thing is seen in the shape of a cloud of steam as it issues from the boiler of a locomotive.

Q. What SORT of clouds are those called STRATUS?

A. Stratus clouds, or, as they are sometimes called, full clouds, are creeping mists, which rise from low, damp places, and are always nearer to the earth than other clouds.

Q. How is the name DERIVED?

A. From the Latin stratus - that which covers or lays low. 
Q. What produces stratus clouds ?

A. Beds of visible moisture, formed by some chilling effects acting along the direct surface of the earth.

Q. What is a frequent VARIETY of the stratus clouds?

A. Those light and spreading mists which gather in meadows and vales in the evening of a warm summer's day, and which float like a reil a short distance above the surface of the ground.

Fig. 84 .

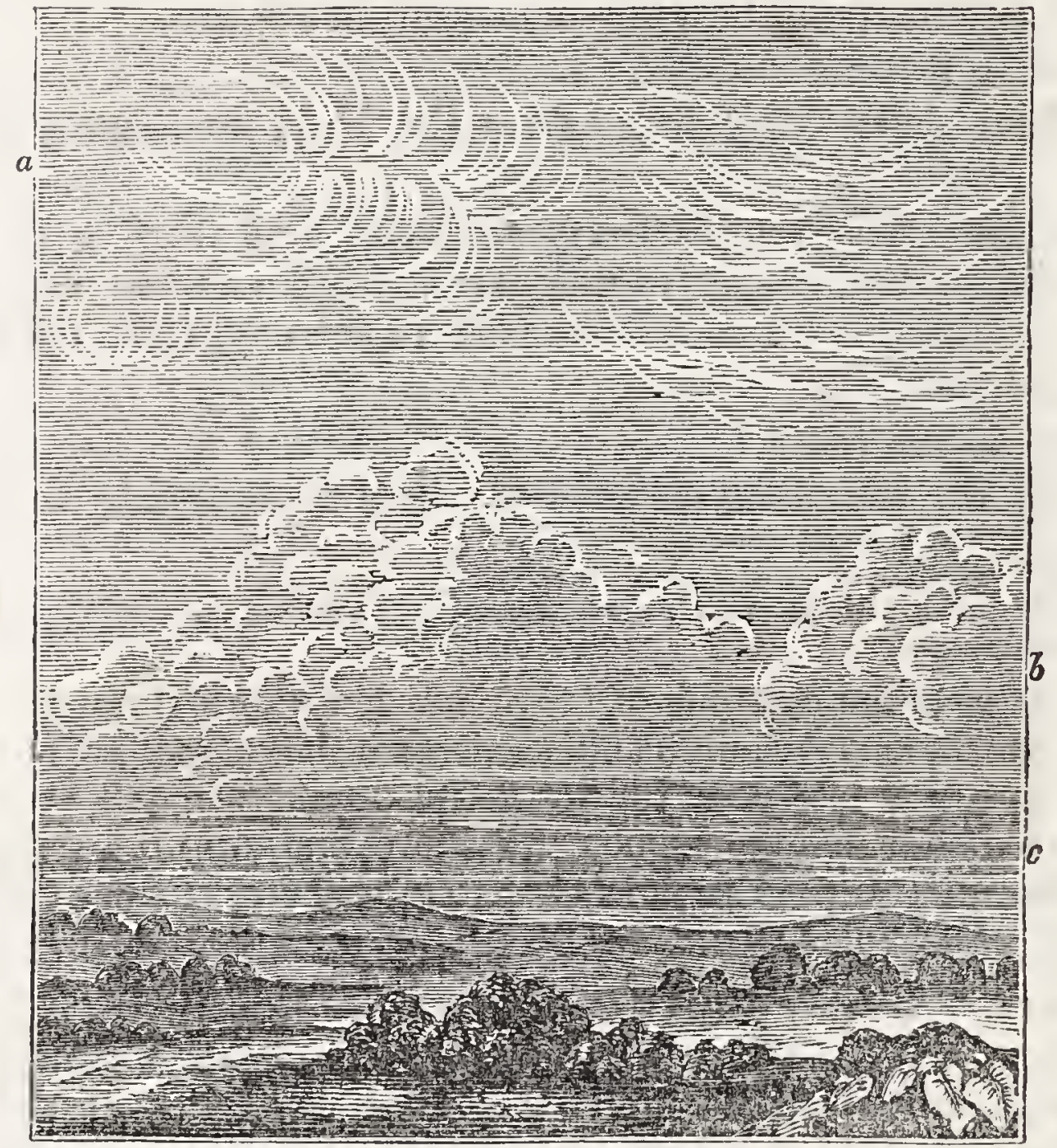

Cirrus, $a$; cumulus, $b$; and stratus, $c$.

Q. What are OIRro-COMULOS clouds?

A. Cirro-cumulus clouds are cirrus clouds springing from a massy centre, or heavy masses edged with long streaks, generally called "mares' tails."

A system of small round clouds may be called cirro-cumulus.

Q. What are oIRro-STRATUs clouds ?

$A$. The cirro-stratus, or wave-clouds, are so called because they resemble both the cirrus and stratus; they form what is generally called a "mackerel sky."

Q. What produces orrRo-sTrates clouds?

A. Cirrus clouds accumulating into denser masses, produce the intermediate class, called cirro-stratus. 
Fig. 85 illustrates the appearance of the cirro-stratus, the cirro-cumulus, the nimbus, and the cumulo-stratus.

Fig. 85.

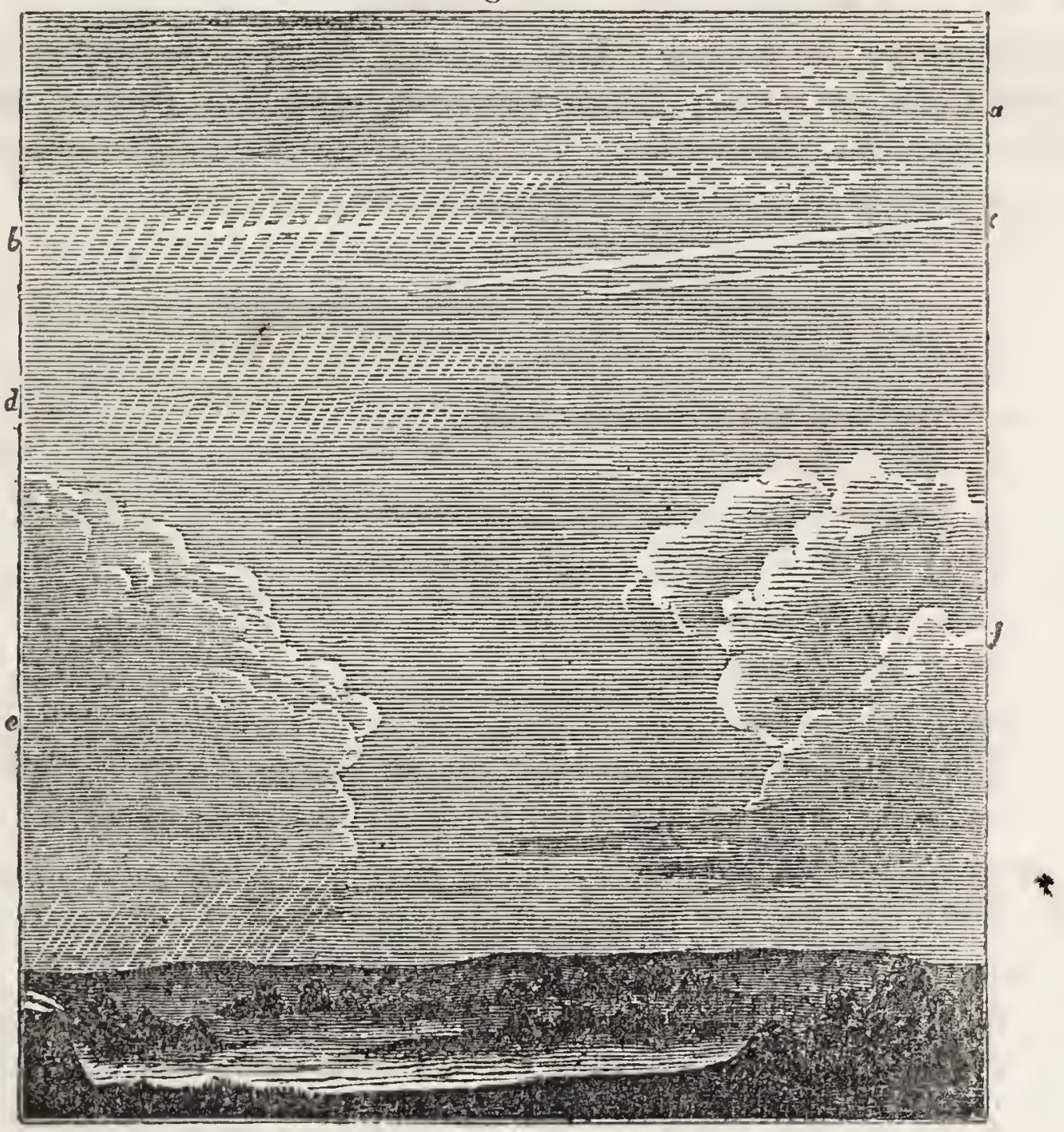

Cirro-stratus, $b, c, d$; cirro-cumulus, $a$; nimbus, $e$; cumulo-stratus, $f$.

Q. What is meant by condo-stratus clouds

A. Those clouds which assume all sorts of gigantic forms, such as vast towers and rocks, scenes of battle, and cloudy giants. This class of clouds is the most romantic and strange of all.

Q. WHEN is this FORII of cluud seen in PERFECTION?

A. Upon the approach of a thunderstorm, when the cumulus clouds, driven by the wind, are piled upon each other, and assume those peculiar forms known as "thunder clouds."

Q. What are सIMBUs clouds?

A. All clouds from which rain falls. Nimbus is the Latin word for "clouds which bring a storm."

Q. By what PARTICULAR CHARscter may the nimbus (or rain cloud) be at ouce DIsTINGOISHED ?

A. By the want of a defined outline; its edge is gradually shaded off from the deep gray mass into transparency.

Q. What 1 PPEARANOE takes place in the clouds at the approach of RAIN ?

A. The cumulus cloud becomes stationary, and cirrus streaks 
settle upon it, forming cumulo-stratus clouds; black at first, but afterwards of a gray colour.

Q. Why does a HAZE round the SUN indicate RAIN?

$A$. Because the haze is caused by very fine rain falling in the upper regions of the air; when this is the case, a rain of five or six hours' duration may be expected.

\section{SECTION II.-MIST AND FOG.}

Q. How are clouds, mists, and fogs PRODUEED?

A. By evaporation.

Q. Does moisture EVAPORATE at ALL temperatures?

A. Yes; even $i c e$ gives forth vapour.

Q. How is evaporation Produced?

A. By the solar heat.

Q. What is the RESULT?

$A$. The moisture from the land or sea being drawn up into the air, there condenses, and assumes the form of mist, fog, or cloud.

Q. Does it PETURN to the EARTH?

A. Yes; in the form of rain, snow, or hail.

Q. Is the AIR ALWAYS capable of receiving VAPOUR from the EARTH?

A. A certain quantity of air can only receive a certain quantity of vapour, after which it is said to be saturated, and can take up no more.

Q. How do you ACcount for the MIST which often covers windows at night?

$A$. The cold night air chills the window glass, condensing into mist the warm vapour of the room.

Q. How did this WARM VAPOUR arise?

$A$. The air of the room itself contains vapour; this vapour is increased by persons in the room and other incidental causes, such as the steam from hot victuals, or tea.

Q. Is not the GLASS of the WINDOW as Hor as the Room itself?

$A$. No; the glass cools down rapidly after sunset, while the room keeps at a warmer temperature.

Q. How does the PRESENCE of PEOPLE in the TOOM IROREASE its vapour?

$A$. Their breath and insensible perspiration add to the vapour of the room.

Q. What is INSENSIBLE PERSPIRATION?

A. Perspiration which is given forth constantly by the human body, but which is not perceptible, as is the case with violent perspiration.

Q. By what SIMPLE EXPERIMENT can this be aseertained?

$A$. By putting the naked arm into a clean, dry glass tube, when the perspiration will settle as a mist upon the glass. 
Q. Does not BREATIING on a GLASS produce the SAME EFHECT?

A. Yes; if we breathe on a piece of glass our breath is condensed, and forms a mist on its surface.

Q. Is our breath VISIBLE?

A. Only when it is condensed into vapour.

Q. When does this occur?

$A$. When the weather is intensely cold; then our breath is seen as a vapour, resembling the appearance assumed by steam.

Q. What are rogs?

A. Fogs are visible vapours that float in the atmosphere near the surface of the earth.

Q. What is the cause of fogs?

A. They originate in the same causes as rain-the union of a cool body of air with one that is warm and humid; when the precipitation of moisture is slight, fogs are produced; when it is copious, rains are the result.

Q. What DIstincrion is to be made between a mist and a fog ?

A. Mist is generally considered to be a fine ruin, while fog is vapour, not sufficiently condensed to allow of its precipitation in drops.

The term mist is also generally applied to vapours condensed on marshes; rivers, and lakes, while the name fog is often applied to vapours condensed on land, especially if those vapours are laden with smoke.

Q. Why does not the roo become dew?

$A$. Because the chill of the air is so rapid that vapour is con. densed faster than it can be deposited, and (covering the earth in a fog) prevents any further radiation of heat from the earth.

Q. When the earth can no longer RADIATE heat upwards, does it continue to conDENS the vapour of the air?

A. No; the air (in contact with the earth) becomes about equal in temperature with the surface of the earth itself; for which reason the fog is not condensed into dew, but remains floceting above the earth as a thick cloud.

Q. This FOG seems to RISE HIGHER and HIGHER, and yet remains quite as DENSE below as at first : explain the cause of this.

$A$. The air resting on the earth is first chilled, and chills the air resting on it; the air which touches this new layer of fog being also condensed; layer is added to layer, and thus the fog seems to be rising, when (in fact) it is only deepening.

Q. When is it that Fogs are MORE FREQUENT at NIGHT than in the DAY?

A. This occurs when the vapour taken up into the air has saturated the atmosphere, so that, as soon as the cold of night lessens its capacity for holding vapour, it returns the moisture to the earth in the shape of mist or fog.

Q. Where do roas mostly prevail?

A. In lowland, marshy places, and by river sides. 
Q. Whт is this?

$A$. Such places more readily give off vapour and saturate the air, which, at the least depression of temperature, returns the moisture as mist or fog.

Q. In London, fogs have an EXTRAORDINARY DENSITY and DARK COLOUR: how is this to be $\triangle$ CCOUNTED FOR?

$A$. It is owing to the smoke which mixes with the vapour, and gives to it the dark colour named.

Q. Why does VAPOUR sometimes form into OLOUDS, and sometimes rest upon the earth as MIST or FOG ?

A. This depends on the tempercture of the air. When the surface of the earth is warmer than the air, the vapour of the earth (being condensed by the chill air) becomes mist or fog. But, when the air is warmer than the earth, the vapour rises through the air, and becomes cloud.

Q. Why do HILls, \&cc., appear LARGER in WET weather?

$A$. Because the air is laden with vapour, which causes the rays of light to diverge more; in consequence of which they produce on the eye larger images of objects.

Q. Why do TREES, \&c., in wET weather appear FARTMER OFF than they really are ?

$A$. Because the fog or mist diminishes the light reflected from the object; and as the object becomes more dim, it seems to be fartier off.

Q. Are fogs common on a FROSTY MORNING:

$A$. No; on a cold frosty morning the vapour, which would otherwise become $f \circ g$, is turned to hoar frost on the ground.

Q. Why are Fogs more general in $\triangle$ UTUMN than in SPRING ?

$A$. The earth in spring is not so hot as it is in autumn; in consequence of which its vapour is not chilled into fog as it issues into the air.

Q. Are Foas more common in valleys than on Hills?

A. Yes; valleys containing more moisture than hills, and not being exposed to the wind.

Q. What is the cause of a BLACK MIST ? and why does it bring WET weather?

A. The mist is black, because it is overshadowed by dense clouds; and wet weather may be expected, because the air is saturated with vapour.

Q. Why is MIST sometimes WHITE? and why does a white mist indicate FINE weather?

$A$. The mist is white because no clouds blacken it with their shadow; and fine weather may be expected because the sky is cloudless.

Q. Why do fogs generally appear to hover about capes and HEADLANDS of the seacoast ?

$A$. Because the soil of these places becomes warmer in summer than the ocean that washes their shores, but in the winter colder; and the difference in temperature is usually sufficient to produce a constant succession of fogs. 
Q. Why do fogs appear over SHOALS and SANDBANES of the ocean ?

$A$. Because the water adjacent to these places is colder than that of the main ocean.

Q. Why do mists and fogs of great extent occur on the BANRS OF NRTFOUNDLAND?

A. A current of warm water, called the Gulf Stream, flowing frлm the tropics, encounters in this region a current of cold water flowing from the polar regions. The great differences in the temperature of the banks, the ocean, and the Gulf Stream, give rise therefore to almost constant fogs.

\section{CHAPTER XXVI.}

RAIN, SNOW, HAIL, FROST.

SECTION I.-RAIN.

Q. What is RAIN?

A. Rain is vapour condensed in the air, and precipitated to the earth in showers.

Q. In what MANNER is the vapour of the air CONDENSED so as to form rain?

$A$. When two or more volumes of humid air differing considerably in temperature unite, the several portions in union are incapable of absorbing the same amount of moisture that each could retain if they had not united. The excess of moisture, if very great, is precipitated as rain; if in slight amount, it appears as clouds, fogs, or mists.

Q. Upon WHAT LAW does this condensation of vapour and formation of rain depend?

A. Upon the law that the capacity of the air for moisture decreases in a greater ratio than the temperature.

Q. Why does RAIN fall in drops?

$A$. The particles of vapour, as they descend, unite with each other, and form into drops.

Q. What is the sIze of rain-drops?

$A$. The size is varied by the rapidity with which the vapours are condensed.

Q. Why do CLOUDS FALL in RAINY weather?

A. 1. Because they are heavy with abundant vapour; and

2. The density of the air being diminished, is less able to buoy the clouds up.

Q. How do you KNow that the DENSITY of the air is DIMINISHED in RAINY weather?

A. Because the mercury of a barometer falls.

Q. Does RAIN WATER possess any fertilising properties BESIDES that of mere MOISTURE? $A$. Yes; rain water contains an abundance of carlonic acid, and 
a small quantity of ammonia, to which much of its fertilising power may be attributed.

Anumonia is a compound of nitrogen and hydrogen. Common bartshorn is only ammonia and water.

Q. What EFFECT has RAIN upon the AIR?

A. It purifies it.

Q. In WHAT WAY?

A. By removing and dissolving injurious vapours, washing the air, setting in motion stagnant water, and mixing the air of the upper and lower regions.

Q. If paper is WETTED, it BLISTERS or PUCKERS : how is this?

$A$. The moisture (penetrating the paper) drives its particles farther apart, and (as the moisture is absorbed unequally by the paper) some parts are more enlarged than others; in consequence of which, the paper blisters or puckers.

Q. When the plants called treforl, dandeliox, prmpernei, \&c., fold UP their LEAYES, RAIN is always close at hand: explain this.

A. 1. The cloudy weather diminishes the light of the sun; and without the stimulus of sunlight, these flowers never open their leaves.

2. The vapour of the damp air, insinuating itself into the airvessels of these delicate plants, causes them to expand; in consequence of which, the leaflets contract and close.

All these plants close at sunset also.

Q. In which PART of the DAY does the MosT RAIN fall ?

$A$. More rain falls by night than by day, because the cold of night condenses the air, and diminishes its capacity for holding vapour in solution.

Q. When do we have the Most rain fall? in summer or WINTER ?

$A$. There are more rainy days from September to March, but the heaviest rains are between March and September.

Q. Can the air $\triangle B S O R B$ MOISTURE at all temperatures, and retain it in an invisible state?

A. It can; and this power of the air is termed its capacity of absorption.

Q. How much Morsture can a VOLUME of air at $32^{\circ} \mathrm{F}$. absorb?

A. An amount equal to the hundred and sixtieth part of its own weight.

Q. How does the OAPACITY of air for moisture increase with the temperature?

$A$. For every 27 additional degrees of heat, the quantity of moisture it can absorb at $32^{\circ}$ is doubled. Thus a body of air at $32^{\circ}$ F. absorbs the 160 th part of its own weight; at $59^{\circ} \mathrm{F}$, the 80 th ; at $86^{\circ} \mathrm{F}$., the 40 th; at $113^{\circ} \mathrm{F}$., the 20 th part of its own weight in moisture. 
Q. In what simustioss is the air always saturated?

A. Over the ocean, and upon the adjacent coasts.

Q. Where is the absolute HUMDITY of the atmosphere the greatest?

A. In the tropics, where the temperature of the air, and its con. sequent capacity for moisture, is the greatest.

Q. What do we mean by ABSOLUTE Hommit ?

A. When we speak of the absnlute humidity of the air, we have reference to the quantity of moisture contained in a given volume.

Q. What do we mean by RELATIVE IIUMINITY

A. The dampness of the atmosphere, or its proximity to saturation. Relative humidity is a state dependent upon the mutual influence of absolute humidity and temperature; for a given volume of air may be made to pass from a state of dampness to one of extreme dryness, by merely elevating its temperature, and this, too, without altering the amount of moisture it contains in the least degree.

Q. What do we call an Instrument designed for measuring the Homidir of the atmosphere?

A. A Hygrometer; that is, moisture measurer; from the Greek words which signify moist and measure.

Q. How may a sPonge be made a Mrgroneter?

A. If a large sponge be dipper in sea water, and then squeezed almost dry, upon being balanced $1 u$ a pair of scales suspended from a steady support, it will be found to preponderate or ascend according to the relative dampness or dryness of the weather.

Q. Why is it necessary, in order that the sponge should serve as a hygrometer, that it be previously dipped in S1:A or SALT water?

$A$. Because the salt left in it from the water is a ready absorbent of moisture.

Q. How may the BEARD of the WILD OAT serve as a bygrometer?

A. 'The beard of the wild oat tuists around, during atmospherical changes from dampness to dryness.

Q. How can a hygrometer be constructed of CATGUT ?

4. If we fix against a wall a long piece of catgut, and hang a l:pilibt to the end of it, it will be observed, as the air becomes moist or dry, to alter in length; and by marking a scale, the two extremities of which are determined by observation when the air is very dry, and also when it is saturated with moisture, it will be found easy to measure the variations. air?

Q. What oтfer instruments have been invented for measuring the moisture in the

A. Several kinds of hygrometers are employed: those instruments which lengthen when they are moist, and shorten when they are dry, are called absorption hygrometers. 
Fig. 86.

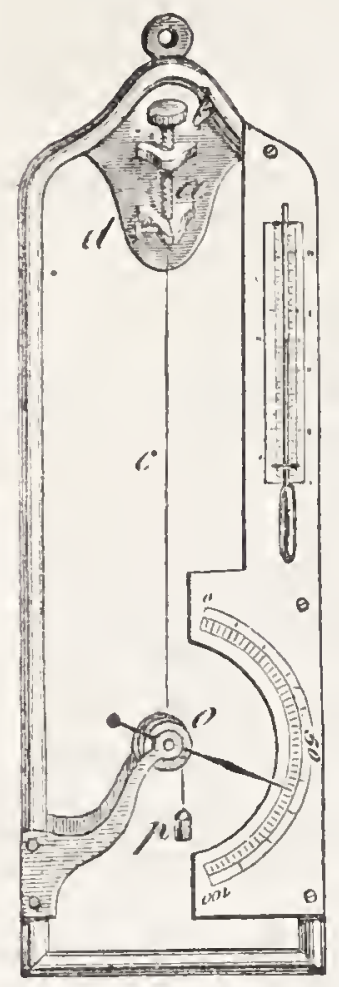

This instrument is made of a brass frame, on which a perfectly clean hair is stretched. This hair is supported at its upper end by a catch $(a)$, fastened by a screw $(d)$. The catch is raised or lowered by means of a screw (b). On its lower end it is wound on a pulley $(o)$, to which it is fixed. This pulley has two grooves, on one of which the hair is fastened ; and on the other, in a contrary direction to that of the hair, is a silk thread, which supports at small weight $(p)$. An index, or hand, is moved by the pulley over a graduated scale. The amount of humidity in the atmosphere affects the length of the hair; when the hair is shortened, the index, or hand, is raised; when it lengthens, the weight $(p)$ causes the index to descend. The graduated scile is marked from $0^{\circ}$ to $100^{\circ}$. The first represents a perfectly dry atmosphere, and the latter an atmosphere saturated with vapour.

Q. What OTHER CONTRIVANCES are used for ascertaining the amount of HOISTURE in the AIR?

A. An apparatus called a condensation hygrometer was invented by M. Regnault, and has answered its purpose extremely well.

Fig. 87.

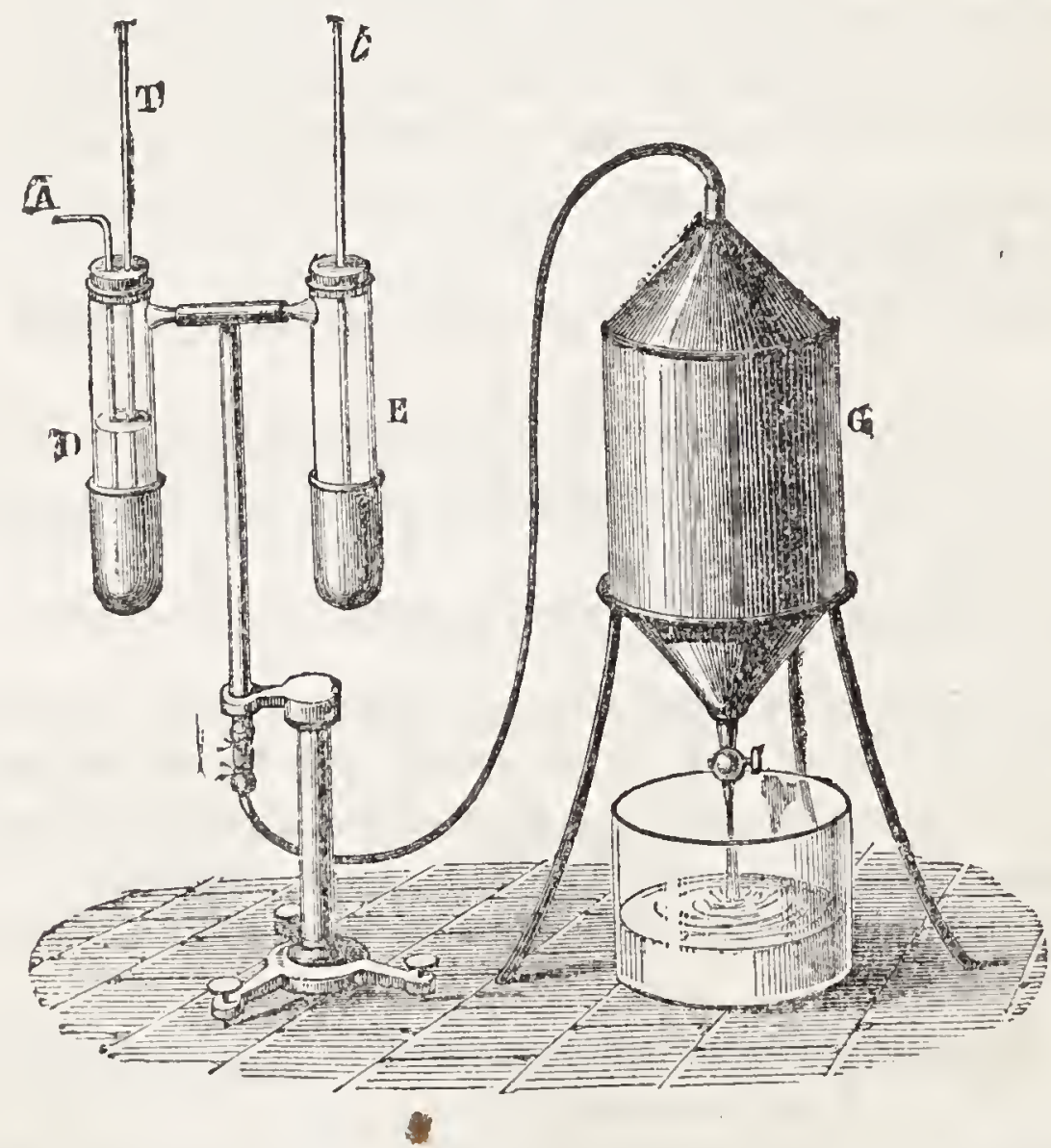

The apparatus in the diagram consists of two cups of polished silver, about two inches in height, and one across, or in diameter. In these cups two glass tubes are fixed, D and $\mathrm{E}$; each of these tubes contain a thermometer, fastened by means of a cork. $\mathrm{A}$ tube $\mathrm{A}$, passes through the cork in tube $\mathrm{D}$ : this tube is open at both ends, and is immersed in 
the cup to the bottom. By means of a leaden pipe, the tube is put in communication with a ressel, $G$, full of water, and called an exhauster. The tube $\mathrm{E}$ contains a thermometer, but does not communicate with the exhauster. Under this arrangement some ether is poured into the tube D, until it is about half full; then the stop-cock of the exhauster is opened, the water escapes, and the air in the tube is rarefied. In consequence of the atmospheric pressure, the air then enters by the tube $\mathrm{A}$; in doing so, passes through the ether, which it to some extent vaporises. When the process of cooling reaches the point at which dew is deposited, the thermometer $\mathrm{T}$ indicates the corresponding temperature, and we are then able to estimate the hygrometric state of the atmosphere.

Q. What is the CHIEF OBJECT answered by this apparatus ?

$A$. By reducing the temperature of the common air, it shows at what point the vapour which it contains would be sufficient to saturate it.

Q. Is the moisture in the air GREATEST in the SUMMER or WINTER?

A. It is greatest in the summer months, and least in the winter.

Q. Where does RAIN fall Most ABUNDANTLY?

A. Near the equator; the quantity decreases as we approach the poles.

Q. Hotr many INCHES of RAIN fall yearly at the city of Vera Cruz, Mexico?

A. About two hundred and seventy-eight inches.

Q. How great a DEPTH of rain, measured in inches, falls YEARLY in London?

A. About twenty-five inches.

Q. How do you ACCousn for the great amount of rain falling at Vera Cruz?

A. Vera Cruz, situated within the tropics, is backed by lofty mountains, whose summits are covered with perpetual snow; against these the hot, humid air from the sea is driven by the trade-winds, condensed, and its excess of moisture is precipitated as rain.

Q. In what LATITUDES do the greatest number of rainy days occur?

$A$. There are more râiny days in the temperate zones than in the tropics, although the yearly quantity of rain falling in the latter districts is much greater than in the former.

Q. About how MANY rainy days are there in a year on the coast of England ?

$A$. About one hundred and fifty-two, both on the east and west coasts.

Q. About how MANY rainy days are there in a year in the northern parts of the United States?

A. About one hundred and thirty-four; in the Southern States the number is somewhat less, being about one hundred and three.

Q. Why does it rain MORE FREQUENTLY in the TEMPERATE zones than in the tropics?

$A$. Because the temperate zones is a region of variable winds, and the temperature of the atmosphere changes often; while in the tropics the wind changes but rarely, and the temperature is rery constant throughout a great part of the year.

Q. How is the AMOUNT of rain MEASURED?

A. By means of a ruin-gauge.

Q. How is this Constructed?

A. The best form consists of a cylindrical metal vessel furnished 
with a float; the rain falling into the vessel raises the float, the stem of which is so graduated that the increase in depth can be very accurately measured.

Q. Why does it rain MORE upon the SEA-COAST than in the INTERIOR of a country?

$A$. Because the air adjacent to the ocean contains more moisture than the air inland.

Q. What is the average YEARLY FALI of rain in the tropics and temperate zones?

A. The average yearly fall of rain in the tropics is ninety-five inches; in the temperate zone only thirty-five.

The greatest rain-fall, however, is precipitated in the shortest time. Ninety-five inches full in eighty days on the equator, while at St. Petersburg the yearly rain-fall is but seventeen inches, spread over a great number of days. Again, a tropical wet day is not continuously wet. The morning is clear; clouds form about ten o'clock; the rain begins at twelve, and pours till about half-past four : by sunset the clouds are gone, and the night is invariably fine.

Q. In the tropics, huw are the seasons DIVIDED?

A. Into wet or rainy, and the dry season.

Q. When does the RAINY season of the tropics comurace?

A. When the trade-winds or monsoons shift.

The followiug is the received explanation of the pbenomena of the rainy seasons of the tropics: At a point $23 \frac{1}{2}^{\circ}$ north of the equator, at the tropic of Cancer, the vertical suu appears to stop when it is midsummer with us. As it moves southward our summer wanes; it crosses the equator, and appears to travel ou until it has reached $23 \frac{1}{2}^{\circ}$ on the other side of the line, the tropic of Capicorn; then six months have passed; it is mid. winter with us, and midsummer with people in the southern hemisphere. The sun turns back (and the word tropic means the place of turning), retraces its course over the equator, and at the expiration of a twelvemonth is at our tropic again, bringing us summer. Now the rainy season is produced between the tropics by the powerful activis of the sun, wherever it is nearly vertical, in producing vast quantities of vapour, which become condensed in the upper colder regions of the atmosphere, and dash to earth again as rain.

Q. Does the rainy season Follow the SUN? -

A. Yes; when the sun is at or near the tropic of Cancer, both before and after turning, all places near that tropic have their rainy season; when the sun makes a larger angle with their zenith, it has taken the rainy season with it to another place.

It is here obvious that a country between the tropics, and far from each, is passed ovel by the sun, in its apparent course, at two periods in the same year, with a decided. interval between them. It must have, therefore, and does have, two rainy and two dry seasons.

Q. Are there any ExCEPTIONs to this order?

A. Yes; the parts of Asia and Africa bordering on the Indian Ocean are exceptions to this rule. These regions being under the influence of the monsoons, and not the trade-winds, the rainy season is somewhat changed in its character and seasons.

Q. Are not some countries entirely DEsTITOTE of RAIN ?

A. Yes; in some districts beyond the tropics, it never rains at all: thus, no rains fall in the Sahara, the Arabian 'and Persian Deserts ; the great desert of Gobi; the shores of Peru and the arid regions of Nexico exhibit a similar character. 
Q. WIY are these cuuntries jestrutur of RaIN?

A. The cause of this scarcity is to be sought for in the peculiar conformation of the country.

In Peru, parallel to the cuast, and at a short distance from the sea, is the lofty range of the Andes, the peaks of which are covered with perpetual snow and ice. The prevailin: $w$ ind is an east wind, sweeping from the At!antic to the Pacific across the continent of South America. As it approaches the west coast, it encounters this range of mountains, and becoines so cooled by them that it is forced to precipitate its moisture, and passes on to the coast almost devoid of moisture. In Egypt, and other desert countries, the dry sandy plains heat the atmosphere to such an extent, that it absorbs moisture, and precipitates none.

Q. Are there some districts in which it may be said to ALWAYS RAIN?

A. In some portions of Guiana it rains for a great portion of the year. The fierce heat of the tropical sun fills the atmosphere with vapour, which returns to the earth again in constant showers, as the cool winds of the ocean flow in from the higher latitudes.

Q. Does it ever rinin WITHOUT Clouds?

A. Several cases are on record when this phenomena has taken place.

Q. What is the Explanation of this phenomena?

A. Rain is produced by the union of different portions of air at different temperatures. The first effect generally produced is the formation of small globules of vapour, which accumulate and become clouds; these, condensed, give us rain. Now, two volumes of air heavily charged with vapour may unite under such circumstances that the moisture will be at once condensed into drops too heavy to float as vapour, in which case they will at once fall as rain, and no cloud will be produced.

Q. How gREAT a QUANTITY of water is supposed to be ANNUALLY PRECIPITATED as

A. The amount is calculated to exceed seven hundred and sixty million of tons.

Q. Was this wHOLE $\triangle$ MOOUN raised by evaporation into the atmosphere?

A. Certainly; the daily amount of water raised by evaporation from the sea alone, amounts to no less than one hundred and sixtyfour cubic miles, or about sixty thousand cubic miles annually.

Q. What is the DAILY AMOUNT of evaporation from the sea between the Cape of Good Hope and Calcutta?

A. During the months of October and November, it is known to average three-quarters of an inch daily from the whole surface.

Q. Is the climate of New England and the Northern United States DRIER than that of England and Central Europe?

A. It is ; and this fact exercises an important influence upon many professions and callings. Painters find that their work dries quicker in New England than in Central Europe. Cabinet-makers in that country are obliged to use thicker glue, and watchmakers, animal instead of vegetable oil. 


\section{SECTION II.- SNOT.}

Q. What is sNow?

A. Snow is congealed rain.

Q. Is snow nothing more than OONGEILED RAIN?

A. It is both congealed and crystallised.

Q. Is there any GREAT VARIETY of Form, then, in the snow fiakes?

A. Yes; in the Arctic regions nearly a hundred different forms of snow hare been observed. (See fig. 88.)

Fig. 88.

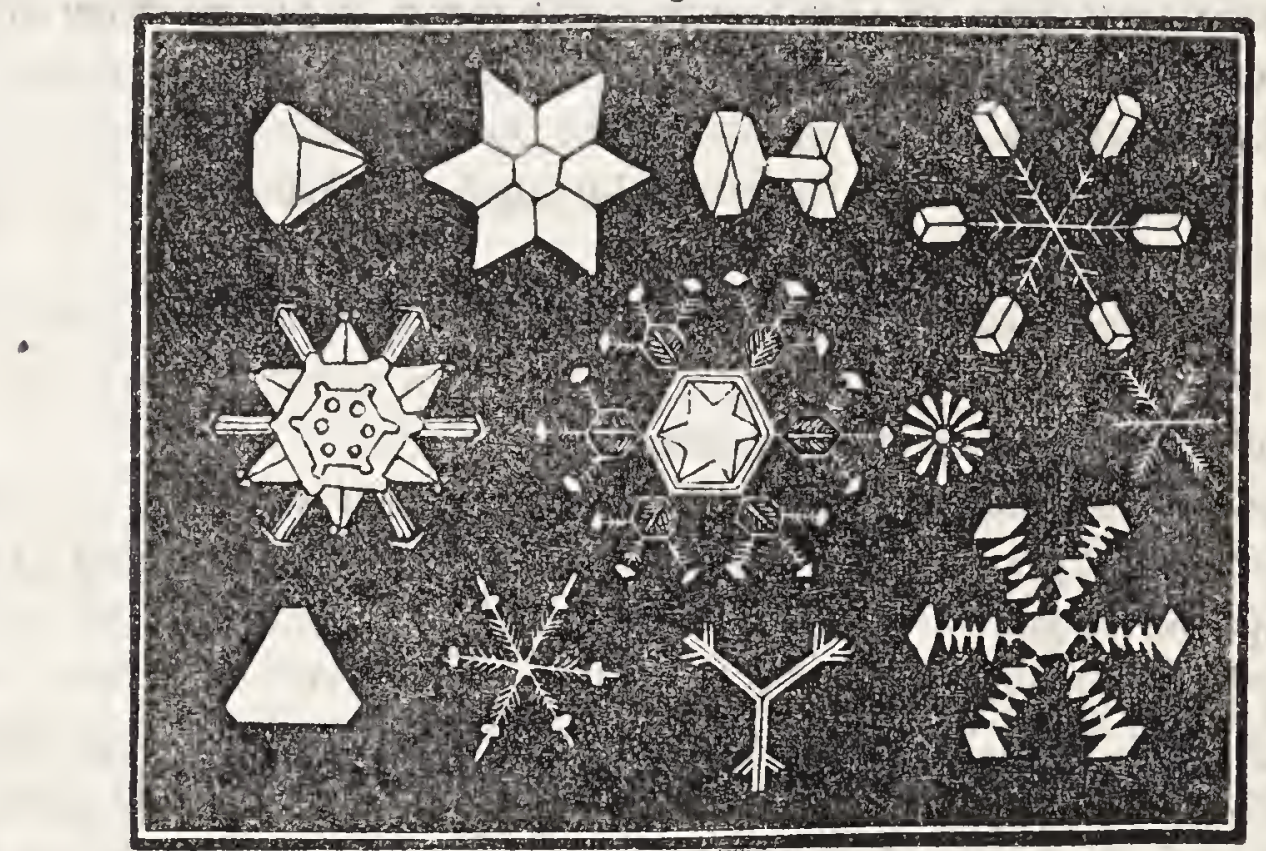

FORMS OF SNOW FLAKES MAGNIFTED.

Q. How is suow OCOASIONED?

A. By the cold air (air below freezing point) condensing the vapour in the air.

Q. Are SLEET and sNow the same?

A. Sleet is snow partially melted.

Q. How is snow partialix melted?

A. By passing through a warmer current of air as it descends to the earth.

Q. How does snow keep the surface of the earth WARM in WINTER time?

$A$. In consequence of its being a bad conductor.

Q. How does snow prove Benericial to the earth in the cold season ?

$A$. It keeps the surface of the earth warm, protects vegetation to a considerable extent from the cold, and acts as a fertiliser.

Q. Why is snow a BAD CONDUCTOR of heat and cold?

$A$. Because air is confined among the crystals, and air is a very bad conductor; when, therefore, the earth is covered with snow, it cannot throw off its heat by radiation.

Q. Why is there no snow in suMmri-Time?

A. Because the heat of the air adjacent to the earth melts it in its descent, and prevents it from reaching the surface of the earth. 
Q. Why is snuw White?

A. Because it is formed of an infinite number of very minute crystals and prisms, which refiect all the colours of the rays of light from different points, and these colours, uniting before they meet the ere, cause snow to appear white.

Q. Under what circumstances does snow fall in LARGE FLAKES, and when in SMALL?

A. 'The largest flakes are formed when the air abounds with vaponr, and the temperature is about $32^{\circ} \mathrm{F}$.; but as the moisture diminishes, and the cold increases, the snow becomes finer.

Q. What is the sNow FLane composed of ?

A. Regular and symmetrical crystals, having a great diversity of forms, as may be seen in $f i g .88$.

Q. Do we see the SAME crystals in ICE ?

A. They exist in ice, but are so blended together that their symmetry is lost in the compact mass.

Q. Is the BULK of snow GREATER than that of water?

$A$. The bulk of recently fallen snow is ten or twelve times greater than that of water.

Q. Does snow ever occur of any other appearance than wIITE ?

A. Yes; in the Arctic regions and on some mountains it is red, and occasionally green.

Q. What is the CAUSE of these appearances?

A. These singular hues are occasioned by little microscopic plants, which germinate and live in the snow. They consist of little globules from $\frac{1}{1000}$ of an inch to $\frac{1}{3000}$ of an inch. Each globule is divided into seven or eight cells, filled with a liquid, which gives a colour to the snow, and is sometimes green and sometimes red.

Q. What is HAIL?

\section{SECTION IIT.-HEAT.}

A. Frozen rain.

Q. What makes oNe part of the air colder than another?

A. It is frequently caused by electricity unequally distributed in the air.

Q. How can ELECTRICITY make air COLD?

A. Air, when electrified, is expanded, and expansion produces cold.

Q. When is hail most FREQUENT ?

A. In summer and autumn.

Q. WHY in SUMMrer and AUTUMN?

A. Because the air then is more electrical, and the vapours rising higher than at other times of the year, are in consequence much colder.

Q. Is not hail often PRECEDED or $\triangle T$ TTENDED by THUNDER?

A. 'Thunderstorms frequently accompany heavy falls of hail, the congelation of the water disturling the electricity of the air. 
Q. What is the FORM and sTRUCrURE of the hailstnne?

A. Hailstones are generally pear-shaped, and if they are divided through the centre, they will be found to be composed of altermate layers of ice and snow, arranged around a nucleus, like the coats of an onion.

"Nucleus"-the kernel, anything about which matter is gathered.

Q. What is the size of hail generally?

A. Hail varies in size from stones as small as a pea to those which are several inches in circumference.

Q. WhiRe do hail-storms most FREQUENTLY occur?

A. In temperate climes most frequently, and rarely within the tropics.

Q. In what LoCALImIES in the temperate zones do hail-storms nccur most frequentiy?

1. In the vicinity of high mountains, whose peaks are always covered with ice and snow. The south of France, which lies between the Alps and Pyrenees, is annually ravaged by hail; and the damage which it causes yearly to vineyards and standing crops is estimated at upwards of two millions of money.

Q. Is the occurrence and formation of hail OLEARLY UNDHRSTOOD?

$A$. It is not; much information exists upon the subject, but no theory has yet been formed which satisfactorily accounts for all the facts which have been observed.

\section{SECTION IV.-GLACIERS, ICEBERGS, AND FROST.}

Q. What are GLACIERS?

A. Huge masses of ice which are in the valleys of snow-capped mountains: such as the Alps, Mont Blanc, \&c.

Q. How are they produced?

$A$. By the alternate melting and freezing of the snow during the frequent changes of temperature.

Q. How Many of these glaciers are said to exist in the ALPS?

A. About four hundred between Mont Blanc and the Tyrol.

Q. What size are they?

$A$. Some of them are fifteen or eighteen miles long. One is estimated at a hundred square miles.

Q. What is the APPEARANCE of these glaciers?

$A$. They sometimes present the appearance of a field of ice; at others that of the waves of the ocean; at others that of rugged rocks.

Q. Are not sose glaciers covered with stones and earth?

$A$. Yes; these have been torn away from the surrounding declivities, and thus a wall of ruin is found on the edge of the glaciers.

Q. What are these Rurss called?

4. Morcines. 
Q. Are these Morarnes moved by the glacier?

A. Yes; these rocky masses are moved onward by the glaciers, and are finally deposited in what is called a terminal moraine.

$$
\text { Fir. } 89 .
$$

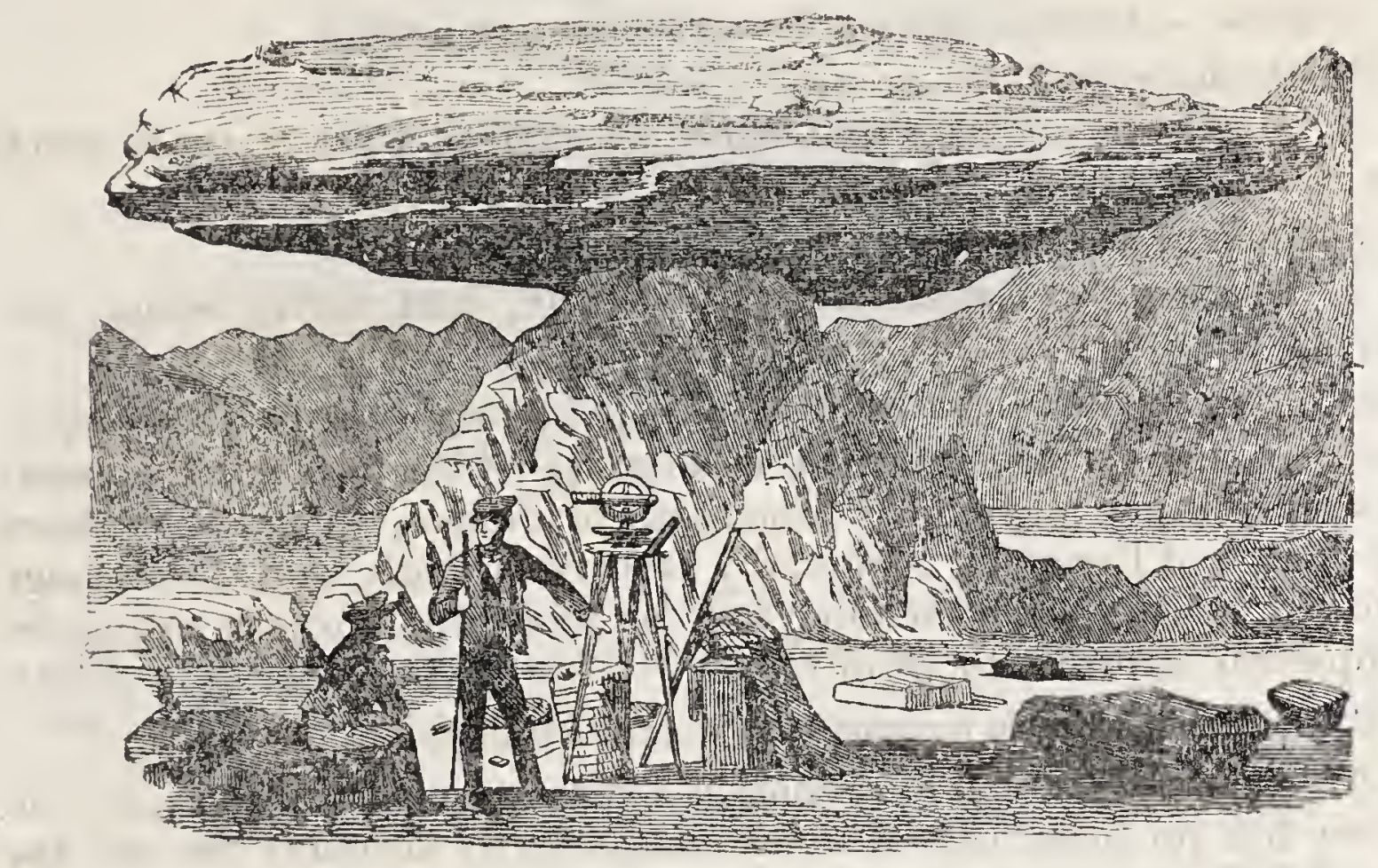

In the above engraving is represented what is called a glacier table, on the Mer de Glace of Chamouni. It is a magnificent slab of granite, measuring twenty-tbree feet in length, seventeen in breadth, and about three feet and a balf in thickness. It appears as if it had been elevated above the level of the glacier, and is supported by a rude pedestal of icy marble. It is probable that what appears as an elevation is only the result of circumstances. Once upon a time, the whole glacier bearing that huge piece of granite was at one level; but the rain and the sun have carried away the other parts of the glacier, and only that part which is protected by the stone from heat and wet has been preserved.

\section{Q. What is an IOEBERG?}

A. Masses of ice that float in the sea.

Q. How are these ICEBERGS FORMED?

A. They are supposed to be glaciers which have glided to the seashore from the mountains, and been drifted away by the waves.

Q. Would this $A$ coount for their VAST DIMENSIons?

A. These broken masses of glacier have been added to by the accumulation of snow and frozen rain, and are continually increasing in size.

\section{Q. How is it that they do Not MELT?}

A. 'The temperature where icebergs are found is rarely abore frcezing point, even in the height of summer.

Q What is the average nnght of icebergs?

A. Fuily formed icebergs vary in height above the water from 180 to 210 feet; but the mass of ice below the water is seven or eight times as great as that which is seen above. 


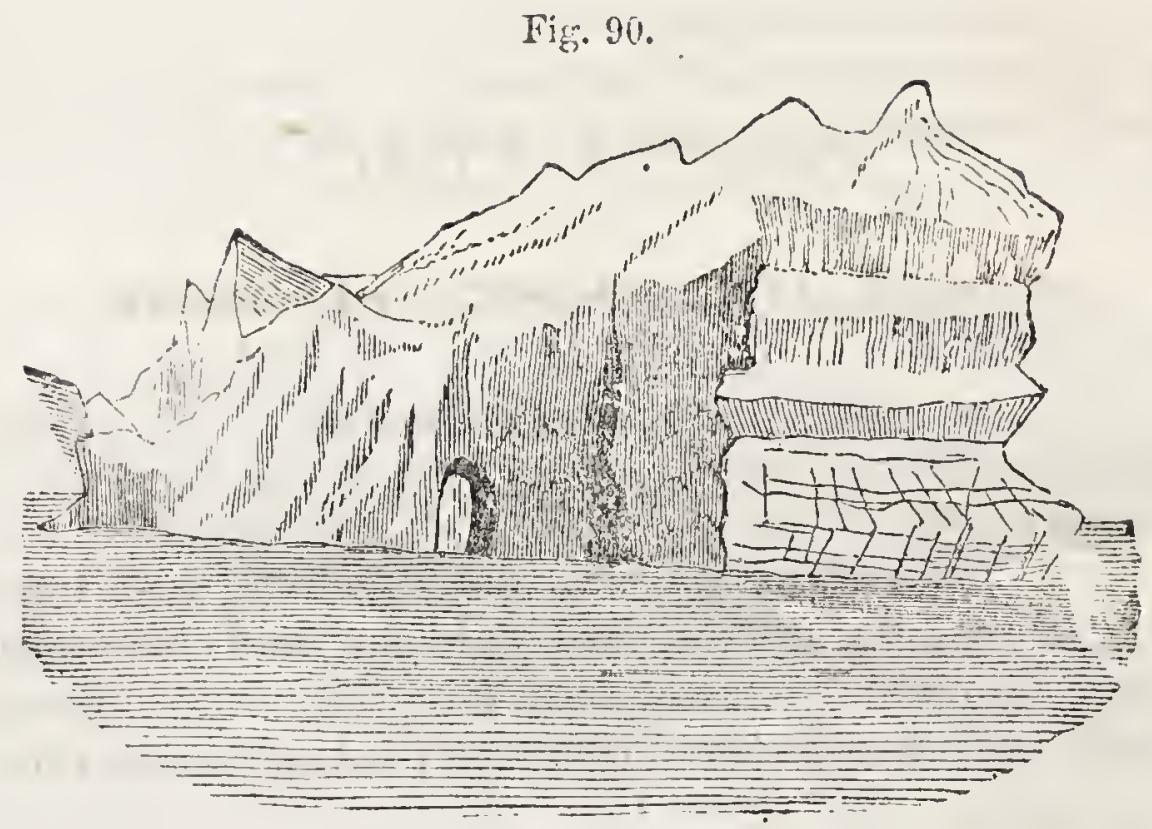

AN ICEBERG.

Q. Is there any DIFFERENCE in the description of HOAR FROST? frost.

A. Yes; frozen dew and frozen fog are both varieties of hoar-

Q. What is FROzEN DEW usually called?

A. Ground hoar-frost.

Q. How is it Producen?

A. By rapid radiation of heat from the earth.

Q. In WHAT WAY does this rapid ratiation cause HOAR-EROST?

$A$. It cools down the surface of the ground to so low a temperature, that it freezes the dew upon it.

Q. When is hoar frost rost oBsERVABLE?

A. After a very clear night.

Q. On what Accoun?

$A$. On a very clear night the radiation is more rapid, and the vapour or dew consequently freezes more quickly.

Q. What is the cause of that hoar frost which arises from Frozen foa ?

A. The thick fog which invested the earth during the night (being condensed by the cold frost of early morning) is congealed upon eiery viject with which it comes in contact.

Q. Why are not the crystals of frost and snow TRANSPARENT like ice?

A. The crystals of frost and snow are not solid, but they contain air; hence their brilliant whiteness : for the air, preventing the ready transmission of light through the crystals, the rays are copiously reflected from the mass of crystals.

Q. Why is the darkness of night DIMmisied by the presence of swow?

A. Because the snow reflects, instead of absorbing, like the bare ground, the laint light that proceeds from the sky. 


\section{CHAPTER XXVII.}

\section{LUMINOUS ATMOSPHERIC PHENOMENA.}

\section{SECTION I.-METEJRITES.}

Q. What are yETEORTES?

A. Meteorites are solid, luminous bodies which from time to time isit the earth, moving with immense velocity, and remaining visible but for a few moments. They are generally accompanied by a luminous train, and during their progress explosions are often heard.

Q. What is an AERourte?

A. The term aerolite is given to those stony masses of matter which are sometimes seen to fall from the atmosphere.

It is derived from the Greek words a $\rho$ (atmosphere), and $\lambda_{1} \theta 0$ s (a stone). A meteor is distinguished from an aerolite by the fact that it bursts in the atmosphere, and disperses in a vapour-like smoke; while the aerolite, which is supposed to be a fiagment of a meteor, comes to the ground.

Q. What is the WEIGHT of those aerolites which have been known to fall from the atmospbere?

A. 'Their weights vary from a few ounces to several hundred pounds, or even tons.

Q. At what HEIGHT in the atmosphere are meteors supposed to appear?

A. Their height above the earth has been estimated to vary from eighteen to eighty miles.

Q. With what velociry do they move?

A. The velocity of these bodies is somewhat more than three hundred miles per minute, though one meteor of immense size, which is supposed to have passed within twenty-five miles of the earth, mored at the rate of twelve hundred miles per minute.

Q. How can the HEIGHT of these bodies in the atmosphere be DETERMINED?

$A$. If a body in the atmosphere is seen by two observers at the same time from different stations, and its angular elevation taken at both stations, its height in miles and feet is easily ascertained by trigonometry, when the distance between the two stations is known.

Q. How can its SPEED be ascertained?

4. By dividing the length of its visible path (which is ascertained by noticing its points of appearance and disappearance) by the number of seconds during which the body is seen.

Q. What is the VALUE of such estimates?

4. Owing to the short time the meteor is visible and its great velocity, accurate observations cannot be made upon it; and all estimates respecting their distance, size, \&c., must be considered as only neas to the truth. 
Q. What is the general roRM and APPEaKANCE of rerolites?

A. Most of them have a pyramidal form, and appear covered with a black shining crust, as if the body had been coated with pitch. When broken, their colour is ash-gray, inclining to black.

Very many of the meteorites which have fallen at different times and in different parts of the globe, resemble each other so closely, that they would seem to have been broken from the same piece or mass of matter.

Q. What is their composition?

A. Great numbers of aerolites have been analysed, and found to contain nineteen or twenty different elementary substances. But for the most part they consist of malleable iron and nicliel. earth?

Q. Do the aerolites resemble in composition any oTHER BODIES upon the surface of the

A. They do not; malleable iron is rarely, if ever, found in earthy substances; and metallic nickel does not occur upon the surface of the earth naturally.

Q. What is PECULIAR to the composition of METEorIC iron?

A. It has a highly crystalline arrangement so peculiar that it is especially distinguished by it. This arrangement of its particles enables us to decide upon the meteoric origin of masses of iron which are occasionally found scattered up and down the surface of the earth.

Q. WHERE have such masses been found?

A. In the South of Africa, in Mexico, Siberia, and on the route orerland to California. Some of these masses are of immense weight, and undoubtedly fell from the atmosphere.

Q. How are meteorites supposed to originate?

A. Four suggestions have been advanced to account for the origin of these extraordinary bodies: 1. That they are thrown up from terrestrial volcanoes. 2. That they are produced in the atmosphere from vapours and gases exhaled from the earth. 3. That they are thrown from volcanoes in the moon. 4. That they are of the same nature as the planets, either derived from them, or existing independently.

Q. Which of these suggestions is regarded as MOST PROBABLE?

A. The fourth most fully explains the facts connected with the appearance of meteorites, and the third likewise has some strong evidence in its favour.

Q. In what respect do SHOoTING STARS differ from METEORS?

A. Their altitude and velocity are greater, they.are far move numerous and frequent, and are unaccompanied by any sound or explosion. Their brilliancy is also much inferior to that of the meteor; and no portion of their substance is ever known to have reached the earth.

Q. What do we know concerning their nfrair? ?

A. Owing to their greater number and frequency of occurrence, many careful observations have been made upon them; their height is supposed to vary from six to four hundred and sixty miles, the greatest number appearing at a height of about seventy miles. 
Q. What is their supposed velocitr? minute.

A. It is supposed to range from sixty to fifteen hundred miles per

Q. Are meteors and shooting stars at ALI TIMES EQUALLY ABUNDANT?

$A$. 'They are not; some may be seen occasionally on a clear night, but they appear to return at certain fixed times, when they descend literally in showers.

Q. What are the PErIoDs when they may be noticed most ABUNDANTLY ?

A. In the early part of August, and the middle of November.

Remarkable falls of aerolites were observed at Barbotan, in France, on the 24th of July, 1790 ; at Vienna, in Italy, on June 16th, 1794 ; in Connecticut, U.S., on the 14th of December, 1807 ; and at Ardèche, in France, on June 15th, 1821.

Q. How MANY very remarkable meteoric showers have been noticed ?

A. Four: in 1799, 1822, 1831, and 1833-all in the month of November. In the shower of 1833 , the meteors in many parts of the United States appeared to fall as thick as snow-flakes.

Q. Do the shooting stars appenr to emanate from any particular part of the heavens?

$A$. The majority seem to start from a point in the constellation of Leo, and undoubtedly far beyond the limits of our atmosphere.

Q. What тHEory has been proposed by Professor Olmsted to account for the origin and appearance of shooting stars?

$A$. That the source of these appearances is a body composed of matter exceedingly rare, like the tail of a comet, revolving round the sun within the orbit of the earth in a space little less than a year; that at times the body approaches so near to the earth that the extreme portions become detached and drawn to the earth, by virtue of its great attraction. It further supposes that the matter of which these bodies are composed is combustible, and becomes ignited on entering the earth's atmosphere.

Q. Supposing this theory to be true, with what veLociry would these bodies detached from the main mass enter our atmosphere?

$A$. The nearest point of approach of the central body to the earth is supposed to be about two thousand miles. Bodies falling from this distance would enter the atmosphere at a distance of at least fifty miles from the earth, with a velocity generated by the force of gravity alone of four miles per second-a velocity ten times greater than the utmost speed of a cannon ball.

Q. Why would the fragments necessarily TAKE FIRE on entering the earth's atmo* sphere?

A. When air is quickly compressed in a tight cylinder to the extent of one-fifth of its volume, sufficient heat is generated to ignite tinder. If we suppose that the fragments descend with such velocity as to compress the rarefied atmosphere at the height of thirty miles to such an extent only as to make it as dense as ordinary air, the temperature would be raised as high as $46,000^{\circ}$ Fahrenheit-a heat far more intense than can be generated in any furnace. 
Q. What OTHER THEORY has been proposed in relation to the origin of these bodies?

A. That of Chaldini, who supposes that, in addition to the planets and their satellites which revolve about the sun, there are innumerable smaller bodies; and that these occasionally enter within the atmosphere of the earth, take fire, or descend to its surface.

Q. What is the zodIACAI IIGHT?

A. It is a singulur luminous appearance seen in the horizon before sumrise and after sunset, most conspicuously in the months of April and May.

Observations made during the year 1855 seem to conclusively prove that the appearance known as the "zodiacal light" is occasioned by a ring of nebulous matter encircling and pertaining to the earth.

\section{SECTION II.-THE AURORA BOREALIS.}

Q. What are the NoRTHERN LIGHTS?

A. Flashes of light, of various colours, occasionally seen in the sky at night, and properly called aurora borealis.

Q. What is SUPPOSED to be the CAUSE of this phenomenon?

A. Electricity in the higher regions of the atmosphere.

Q. Is the aurora ever seen in OTHER PARTS of the heavens than towards the north ?

$A$. In the northern hemisphere it always appears in the north, but in the southem hemisphere it appears in the south : it seems to originate at or near the poles of the earth, and is consequently seen in its greatest perfection within the arctic and antarctic circles.

Q. What is KNown concerning the EXTENT of the aurora?

$A$. It is not local, but is seen simultaneously at places widely remote from each other, as in Europe and America.

Q. What ronus are generally assumed by the aurora?

$A$. It appears sometimes as a dark segment, as a corona or crown of light, as an arch, or in the form of luminous streamers.

Q. What CALCULATIONS have been made respecting the height of the aurora?

$A$. The height of the appearances varies from one to two hundred miles; they sometimes appear within the region of the clouds, and very near to the earth.

Q. Do any sounds ever attend the appearances of the aurora?

$A$. It has been often asserted, and on good authority, that sounds uave been heard attending the phenomena of the aurora, like the rustling of silk, or the sound and crackling of a fire. On this point, nowever, there is great difference of opinion.

Q. Do the auroras appear at any' PARTICULAR SEAsONS and times?

$A$. 'They appear more frequently in the winter than in the summer, and are only seen at night.

Q. Do they also occur in the DAY-TIME?

A. The aurowa is known to affect the magnetic needle and the 
telegraph; and as the effects upon these instruments are noticed by day as well a by night, there can be no doubt of the occurrence of the aurora at all hours. The intense light of the sun renders the auroral light invisible during the day.

Q. Of what UTILITY are the auroral appearances in the polar regions?

A. During the long polar night, when the sun is absent, the aurora appears with a magnificence unknown in other regions, and affords liglet sufficient for many of the ordinary out-door employments.

Fig. 91.

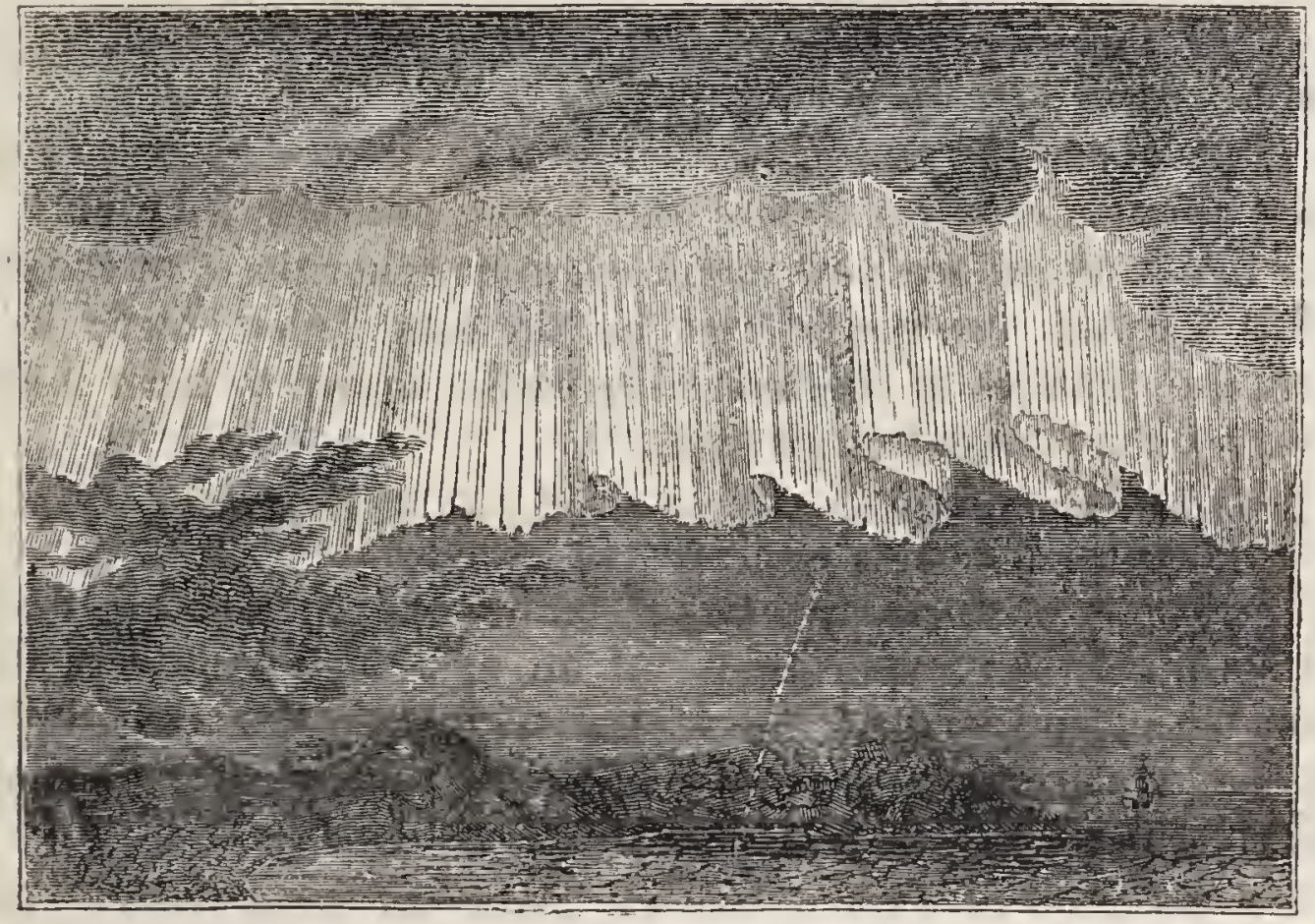

The accompanying figure represents one of the most beautiful of the auroral phenomena.

Q. Are the auroras subject to any other VARIATIoNs in their appearance?

$A$. They appear to be subjected to a variation extending through a cycle of years; thus from 1705 to 1752 the northern lights became more and more frequent; but after that, for a period, they were seen but rarely. In 1820 an increase in the frequency of these phenomena trok place, and of late years their brilliancy has been very great.

\section{CHAPTER XXVIII.}

\section{POPULAR OPINIONS CONCERNING THE WEATHER.}

Q. Do the general meteorological changes which take place almost daily, and which are designated as weather changes, occur in accordance with certain FIXED LAWS ?

$A$. There is no reason to doubt that every change in the weather is in strict accordance with some certain physical agencies, which are fixed and certain in their operations. 
Q. Why can we not, then, with certainty determine and foretell the oHARACTER of the weather for any particular time?

$A$. Because the laws which govern meteorological changes are as yet imperfectly understood.

Q. Is there any reason for supposing that the moon has any INFLOENCn upon the weather?

A. An examination of meteorological records, kept in different countries, through many years, proves conclusively that the popular notions concerning the influence of the lunar phases on the weather have no foundation in any well-established theory, and no correspondence with observed facts.

Q. Do meteorological records afford any support to the belief in the occurrence of mars at particular PHANEES of the moon?

$A$. There is some reason for supposing that rain falls more frequently about four days before full moon, and less frequently about four or five days before new moon, than at other parts of the month; but this cannot be considered as an established fact: in other respects the changes of the moon cannot be shown to have influenced in any way the production of rain.

Q. It is a current belief among many persons that all timber should be felled during a decline of the moon : is there any Foundation for this belief?

$A$. Some years since (this belief being one received in many different countries) an experiment on an extensive scale was made in France, by an eminent agriculturalist, to test the matter ; and it was found that there was no difference in the quality of any timber felled in different parts of the lunar month.

Q. Does the bright moonlight in any way hasten the PUTREFAOTION of ANIMAL or VEGETABLE SUBSTANCES?

$A$. It is generally supposed to do so; but the fact is, that on bright, clear nights, when the moon shines brilliantly, dew is more freely deposited on these substances than at other times, and in this way putrefaction may be accelerated. With this the moon has no connection.

Q. Is there any FOUNDATION for the belief that the appearance of the aurora borealis is followed by a CHANGE in the weather?

A. Meteorological registers conclusively show that there is no such connection, and that the appearance of the aurora is as often followed by fair weather as by foul.

Q. Is there any тRUTH in the traditional notion that a long and violent storm usually accompanies the period of the EQUINoxes?

$A$. The examination of weather-records for sixty-four years show that no particular day can be pointed out in the month of September (when the "equinoctial storm" is said to occur) upon which there ever was, or ever will be, a so-called equinoctial storm. The fact, however, should not be concealed, that taking the average of the five days embracing the equinox for the period above stated, the amount. of rain is greater than for any other five days, by three per cent., throughout the month. 
Q. Is there any REAson for believing that cold and warm seasons ALTERNatr

A. Meteorological records, kept for eighty years at the observatory of Greenwich, seem to show that groups of warm years alternate with cold ones in such a way as to render it most probable that the mean annual temperatures rise and fall in a series of curves, corresponding to periods of about fourteen years.

Q. Is it propable that some ANmals and insects are able to Fonetell changes in the weather before sas can perceive any indications of the same?

$A$. Of this fact there appears to be no donbt. Some varieties of the land snail only make their appearance before a rain. Some other varieties of land crustaceous (shelly) animals change theircolour and appearance twenty-four hours before a rain.

Q. What curious fact has been noticed in respect to the LEAVES of TREES indicating changes in the weather?

$A$. For a light, short rain, some trees have been observed to incline their leaves, so as to retain water; but for a long rain, they are so doubled as to conduct the water away. rain?

Q. What FACT has also been noticed respecting the changes in SPRINGS previous to a

$A$. The water of springs has been observed to rise and flow out in greater volume previous to a rain.

Most, if not all, of the popular proverbs respecting changes in the weather, the influence of the moon, of frosts, auroras, and the like, when tested by observation, will be found to be unsupported by facts, and unworthy of the slightest credence.

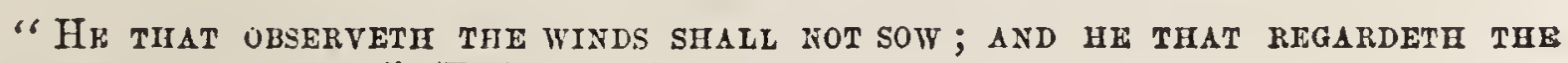
clouds SHald NOT' REAP." (Eccles. xi. 4.)

\section{LIGHT AND ACTINISM.}

Q. What is LIGHT?

\section{CHAPTER XXIX.}

\section{GHT.}

A. Light is supposed to be caused by the undulations or waves in, or vibrations of, an elastic fluid pervading all space, called ether. These undulations or vibrations reaching the eye, affect the optic nerve, and produce the sensation which we call light.

Q. What is that SCIENCE CALLED which treats of vision, and the properties and phenoinena of light?

$A$. The science of optics. This term is derived from the Greek. word which signifies seeing.

Q. What ANALOGY is there between the eye and the ear ?

$A$. The vibrations or undulations of the ether pass along the space. intervening between the visible object and the eye, in the same man-. 
ner that the undulations of the air, produced by a sounding body, pass through the air between this body and the ear.

Q. What is the THEORY which in this manner accounts for the origin of light called ?

A. The undulatory theony.

Q. Is there any oTIER THEOrT according to which the phenomena of light are explained and accounted for?

$A$. Some suppose that a distant object becomes visible to us by emitting yarticles of matter from its surface, which particles of matter, passing tarough the intervening space between the visible object and the eye, enter the eye, and, striking upon the nervous membrane, so affect it as to produce the sensation of light or vision.

Q. By what NAMr has this theory been designated?

A. The corpuscular theory: from corpuscle, an atom.

Q. What ANALOGI, according to this theory, exists between the eye and the organs of smolling?

A. We recognise the odour of an object in consequence of material partictes passing from the object to the organs of smelling, and there producing an effect. In the same manner, a visible object at any distance may be supposed to send forth particles of matter, which move to the eye, and produce vision, acting mechanically on its membrane in the same manner as the odour of a rose produces a physical effect on the organs of smelling.

Q. Which of these two theories is now generally RECEIVED by scientific men?

$A$. The first, or undulatory theory, because more of the phenomena of light can be satisiactorily explained by it than by the corpuscular theory. In some respects the undulatory theory is not satisfactory, and some phenomena cannot be perfectly explained by either theory.

Q. If the corpuscular theory be correct, how LARGE a SPACE will a common candle fill witl particles of luminous matter?

A. A common candle can fill for hours a space four miles in diameter, as it would be visible, under favourable circumstances, in every portion of this space.

$Q$. If we collect a large quautity of light in one point by means of a glass, and throw it upon the most sensitive balance, does it indicate any PERCEPTIBLE WEIGHT?

A. It does not, in the slightest degree.

Q. What are the chief sounces of light?

A. The sun, the stars, fire or combustion, electricity, and phosphorescence.

Q. How does consustrox make undulations of light?

A. The atoms of matter (set, in motion by heat), striting against the ether, produce undutations in it; as a stone thrown into a stream prorluces undulations in the vater.

Q. With what VELocixy does light move through space?

A. With a velocity of one hundred and ninety-two thousand miles $n$ a second of time. 
Q. Does ALu light travel equally fast?

A. Yes; the light of the sun, the light of a candle, or the light from houses, trees, and fields.

Q. How loNg a TIME does it require for light to pass from the sun to the earth ?

A. Eight minutes and thirteen seconds.

Q. How long in travelling from the planet Uranus to the earth?

A. About three hours.

Q. How much time is required for a ray of light to traverse the space intervening between the nearest FIXED STARS and the earth?

A. Nore than six years; and from the farthest cluster of stars, hundreds of years will be required.

Q. What, therefore, would be the consequence if one of the remote fixed stars were to-day "blotted from the heavens?"

$A$. Several generations of the earth would pass away before the obliteration could be known to man.

Q. In what MANmer does the moon, and the planets belonging to the solar system, GIVE LIGHT?

A. They shine only by means of the sun's light, which is reflected from their surfaces.

Q. Where does the LIGHit of HOUSES, TrEES, and FIELDS come from?

A. The light of the sun (or of some lamp or candle) is reflected from their surfaces.

Q. Why are SOME surfaces DULI and OTHERS BRILLIANT?

A. Those surfaces which reflect the most light are the most brillicint; and those which absorb light are dutl.

Q. BY wHOM, and in what MANNER, was the velocity of light first ascertained?

A. By Von Roemer, an eminent Danish astronomer, from observations on the satellites or moons of Jupiter.

Q. How would the InStantaneous transmission of light through space enable us to see the heavenly bodies in different parts of the earth's orbit?

A. If the transmission of light were instantaneous, it must be obvious that the reflected light of the sun would take up no more time in passing from any one of the planetary bodies to the earth, when they are farthest from us, than it does when they are nearest; and as the situation of the earth with respect to the planets is different in different parts of her orbit, the satellites of Jupiter, on emerging from the shadow of that planet, would be seen as quickly when the earth was in one part of her orbit as in another.

Q. Has the velocity of light been determined in oтHER WAYS?

A. It has; and the results uniformly agree with the velocity announced by Von Roemer.

Q. How does the velocity of light CoMrpare with the speed of a locomotive?

$A$. Light passes from the sun to the earth in about eight minutes; a locomotive engine, travelling at the rate of a mile in a minuie, would require upwards of one hundred and eighty years to accomplish the same journey. 
Q. How does the light of Sirius, one of the brightest of the fixed stars, compare with the light of the sun?

A. Dr. Wollaston has calculated that it would require twenty thousand millions of the brightest stars like Sirius to equal the light of the sun, or that that orb must be one hundred and forty thousand times farther from us than he is at present, to be reduced to the illuminating power of Sirius.

Q. How does the light of the FELL moon compare with that of the sun?

$A$. It is estimated to be three hundred thousand times weaker than sunlight.

Q. What did Von Roemer find to be the vacr in regard to Jupiter's satellites?

A. He found that the effect of the transmission of light was such, that when the earth is between Jupiter and the sun, the satellites, after being eclipsed, are perceived rather more than eight minutes sooner than they ought to appear according to the time as calculated by the most accurate tables; and when the earth is in the opposite part of her orbit, so that the sun is between this planet and Jupiter, the satellites emerge about eight minutes later than the calculated or mean time.

Fig. 92.

Hence it will be apparent that light takes up eight minutes in passing through a space equal to half the diameter of the earth's orbit, or the distance between the eartll and the sun, which is ninety-five millions of miles; so that it moves at the rate of nearly 200,000 miles in one second.

In the annexed diagram ( $f$ ig. 92 ) let $S$ represent the sun, $A$ and $B$ the earth in different parts of her orbit: J, Jupiter; $D$, his nearest satellite entering the shadow of that planet; and $\mathrm{C}$ the same satellite emerging from the shadow. Now the time of the commencement or termination of an eclipse of the satellite, as stated from calculation in tables, is the instant at which the satellite would appear to enter or emerge from the shadow, if it could be seen by an observer from the sun; and it is found, from repeated observation, that the eclipse takes place about eight minutes earlier than the calculated period, when the earth is in the nearest part of her orbit, as at $A$, and eight minutes later when she is in the opposite part of her orbit, as at B.

Q. What do we mean when we speak of light being neFLECTED?

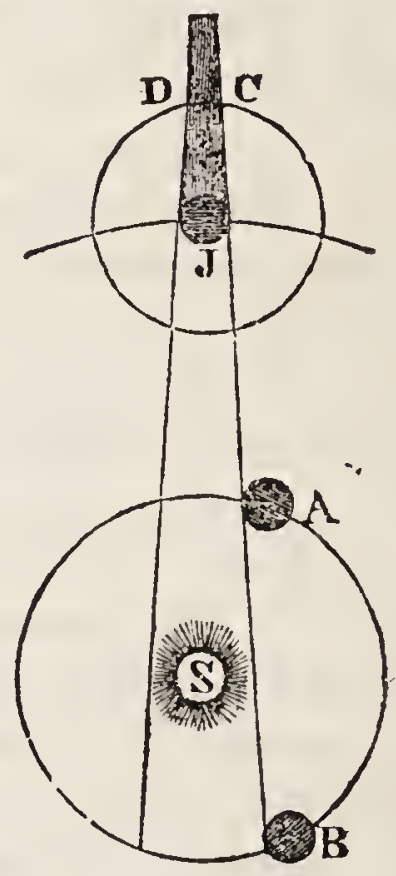

$A$. When a ray of light strikes against a surface, and is caused to turn back or rebound in a direction different from whence it proceeded, it is said to be reflected.

Q. When is light said to be ABSOREED ?

$A$. When its rays are retained upon the surface upon which they fall they are said to be absorbed; in consequence of which their presence is not made sensible by reflection.

Q. What curious EXPERIMENT has recently been made, tending to show that light is really a refined sort of matter?

$A$. By the aid of very delicate instruments, it can be shown that 
a ray of light is bent out of its right line wherever a solid body (as a. knife edge) is made to cut the edge of the beam.

Q. What is the CAUSE of strabismus, or squinting?

A. The inability of one eye to follow the motions of the other; this may arise from habit, imperfect power in one eye, or some defect in the muscular movements.

Q. In what manner is light PRopaGsted?

$A$. In right lines from every luminous point, every such line being called a ray of light.

Q. What do we mean by a PENCIL of light ?

A. A collection of radiating lines or rays, as seen in fig. 93 .

Fig. 93.

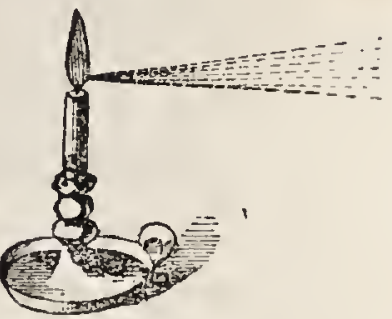

Q. Upon what CIRCOMSTANCES does the degree of illuminating power of any body depend?

A. Upon the intensity of the exciting power in the luminous body; the distance of the illuminating body; the amount of absorption which the rays undergo in traversing the intervening media; and the angle at which the rays fall upon the surface.

Q. In artificial light, upon what does the illuminating power DEPEND ?

A. Upon the quantity of solid matter which changes form. Pure hydrogen gas burns freely, but emits little light; if the vapour of turpentine, containing carbon, be added to it, it burns with great brilliancy.

Q. How is the INTENSITY of light proceeding from various sources MEASURED and COM. PARED?

A. By means of an instrument called a photometer.

Q. By what SIMPLe contrivance may the relative illuminating powers of different lights be COMPARED?

$A$. If we allow the shadows from a single object to fall upon a white screen, and then remove the sources of light from or towards

Fig. 94.

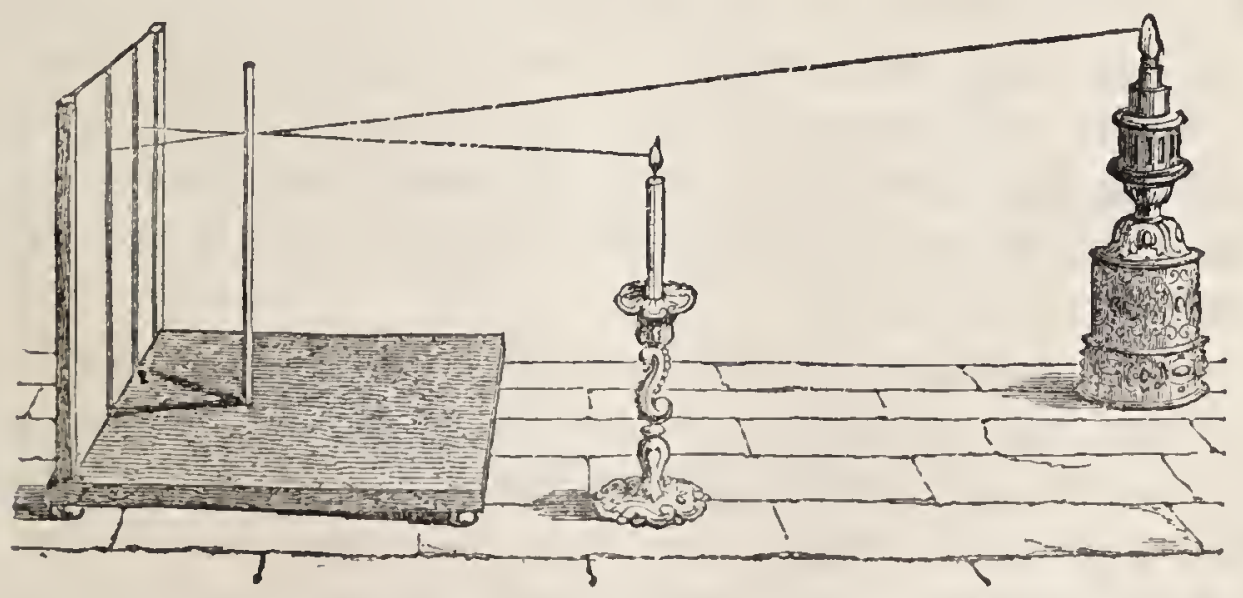


it until all the shadows are of the same intensity, the distances at which the illuminating bodies are from the screen will express the relative intensity of the light of each. (See fig. 94.)

Q. Can ANY NUMBER of rays of light CROSS each other in the same space without jostling or interfering?

A. They can.

Q. How can you prove this?

A. If a small hole be made from one room to another through a thin screen, any number of candles in one room will shine through this hole, and illuminate as many spots in the other room as there are candles in this, all their rays crossing in the same hole, without hindrance or diminution of intensity.

Q. What is DARENESS?

A. The absence of light.

Q. What is a SHADOW?

A. A shadow is the name given to the comparative darlaness of places or objects, which are prevented by intervening things from receiving the direct rays of some luminous body shining on the objects around.

\section{Q. What is the apparent darkness of a shadow PRoporTIONAL to?}

A. It is proportional to the intensity of the surrounding lights; thus, a finger held between a candle and the wall casts a shadow of a certain intensity; if another candle be placed in the same line from the shadow, the shadow will appear doubly dark, although, in fact, more light will be reaching it, and reaching the eye from it than before; it will be more dark only by comparison.

Q. Why cannot we see through a CrooKED TUBE as well as through a STraIGHT one?

A. Because light moves only in straight lines.

Q. What is the PHiLosopHy of taking aim with a gun or arrow?

A. In taking aim with a gun or arrow, we proceed upon the supposition that light moves in straight lines, and try to make the projectile go to the desired object as nearly as possible by the path along which the light comes from the object to the eye.

Q. Why does a carpenter look along the EDGE of a plank to see if it is STRAIGHT ?

$A$. If the edge be straight and uniform, the light from all points of the edge will come to the eye regularly and uniformly; if irregularities, however, exist, they will cause the light to be irregular, and the eye at once notices the confusion and the point which occasions it.

Q. When will the shadow of a body be LEss than the body casting it?

$A$. When the luminary is greater than the body casting the shadow, a cross section of the shadow thrown upon a plane surface will be less than the body; and less, moreover, the farther this surace is from the body, for the shadowed space terminates in a point. 
Q. Under what circumstances will the shadowed space be areater than the body producing it?

A. When the light-giving surface is smaller than the body casting the shadow, the shadow will be larger than the body (see fig. 95): thus the shadow of a hand held near a candle, and between a candle and the wall, is gigantic.

Fig. 95.

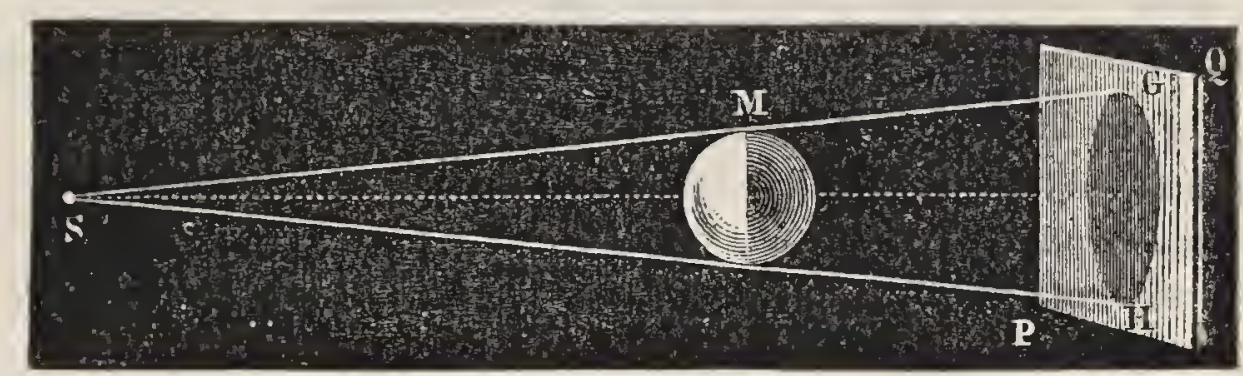

Q. What is meant by the $\triangle B$ ERrRATION of light?

$A$. By aberration of light is meant the difference between the real and apparent places of the stars. As the light is proceeding from them, the earth is moving onwards; consequently they appear to be rather more backward than they really are in the direction of the earth's annual motion.

Q. What is a MIRros?

A. Any substance reflecting light. The term is generally applied to glass covered on the back with quicksilver.

Q. What is a SPECULUM?

$A$. The term speculum is generally applied only to metallic mirrors, made usually of a mixture of tin and copper.

Q. How MANY KINDS of mirrors are there?

A. Three: plane, convex, and concave.

Q. What is a familiar EXAMPLE of a plane mirror?

A. A common looking-glass.

Q. What is a CONVEX MIrRoR?

A. A convex mirror is a part of a sphere or globe reflecting from the outside.

Q. How does the IMAGE always $\triangle P P E A R$ in a convex mirror?

A. Always smaller than the object.

Q. What is a CONCaTE Mrror?

$A$. The shape of a concave mirror is exactly like that of the convex mirror, the only difference between them being in respect to their reflecting surfaces. The reflection of a concave mirror takes place from its inside, or concave surface, while that of the convex mirror is from the outside, or convex surface.

Q. How do IMAGES $\triangle$ PPEAR in a concave mirror?

$A$. Concave mirrors magnify, or render the image larger than the object. 


\section{CHAPTER XXX.}

\section{REFLECTION OF LIGHT.}

Q. When light falls upon a body, in what THREE ways may it dispose of itself?

A. It may be reflected, refracted, or absorbed.

Like radiant heat, it will dispose of itself in one or more of these three different ways.

Q. What is the REFLECTION of LIGHT?

$A$. When a ray of light strikes a polished surface, it is thrown back, or, as we say, reflected.

Q. What are those POLISHED SURFACES which reflect light CALLED ?

A. Mirrors.

$Q$. In WHAT WAY are rays of light refiected from a mirror?

$A$. In the same way that a stone rebounds if thrown against a wall. Q. How is this DESCRIBED?

A. By the lines of incidence and reflection. The course taken by

Fig. 96. rays of light towards the mirror is the line of incidence; the course taken by those

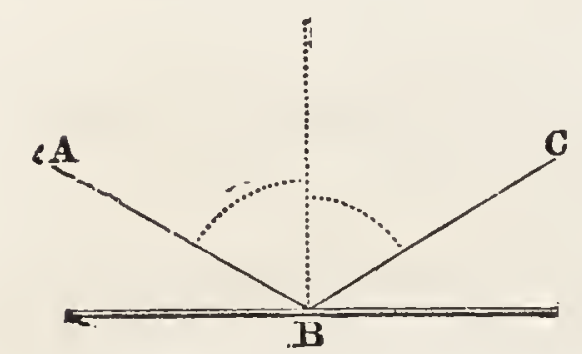
rays in rebounding from the mirror is the line of reflection.

In this diagram, if the line of incidence be $\mathrm{AB}$, the line of reflection is B C.

Q. How is it that the DISTANCE of any object from a looking-glass seems as GREAT BEHIND that glass as the real object is BEFORE it?

$A$. This effect is the result of the principle just stated, the line of reflection being always equal to the line of incidenee.

Q. If we look at any object in the water it appears INVERTED, or UPSIDE DOWN: why is this?

A. It is only another illustration of the same principle; the angle

Fig. 97.

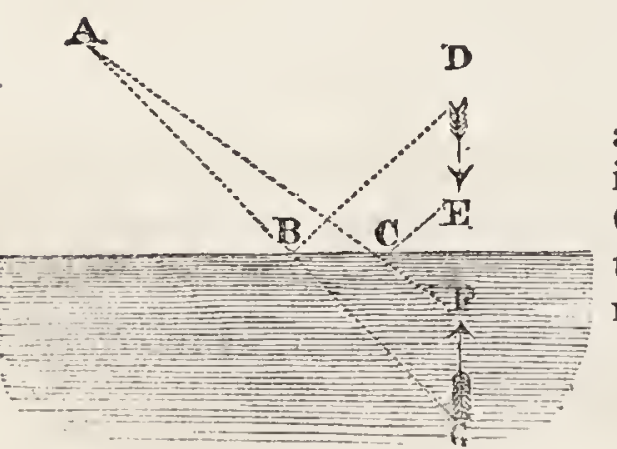
of incidence being equal to the angle of reflection.

In the accompanying diagram, the feather of the arrow $\mathrm{D}$ strikes the water at $\mathrm{B}$, while the reflection of it is seen at G. The barb of the arrow E strikes at $\mathrm{C}$, and is reflected at $\mathrm{F}$. If the spectator be supposed to stand at $A$, the reflection will have the appearance represented in the diagram.

Q. On a lake of water the nroon seems to make a PATH of light towards the eye of the spectator, while all the REST of the lake seems DARK : why is this?

$A$. The reason of this appearance is that every little wave, in an extent perhaps of miles, has some part of its rounded surface with 
the direction or obliquity which, according to the required relation of the angles of incidence and reflection, fits it to reflect the light to the eye, and hence every wave in that extent sends its momentary gleam, which is succeeded by others.

Q. Why can we NOT SER into the STREET Or ROAD when CANDLES are LIGHTED?

$A$. Because glass is a reflector, and throws the candle-light back into the room again; and the pupil of the eye (having become contracted by the light of the room) is too small, to collect rays enough from the dark street to enable us to see into it.

Q. Why do we often see the FIRE REFLECTED in the WINDow?

$A$. Because glass is a good reflector, and the rays of the fire (striking against the window-glass) are reflected back into the room again.

Q. How does LIGHT cause the pupil of the eye to contraOT?

$A$. The pupil of the eye is a round hole in the midst of a movable muscular curtain or screen, called the iris. When too much light falls on the nervous retina at the back of the eye, it irritates it; and this irritation is conveyed to the muscular rings composing the curtain by small nervous fibres, causing them to contract.

Q. What is the BALEIDOSCOPE?

A. An optical instrument, invented by Sir David Brewster. It consists of a tube, with narrow slips of looking-glass so placed on the inside, that small beads, pieces of coloured glass, and substances of a like kind, are reflected by them in regular and most elegant combinations of form. These combinations may be varied in endless succession, since every time the instrument is moved about or shaken a new figure is produced.

Q. In a long, straight STREET, why do the houses on the opposite sides seem to APPROACH NEARER together as they are more DISTANT?

$A$. Because the more distant the houses are, the more acute will be the angle which any opposite two make with our eye.

$Q$. Why does the MooN appear IARGER than the STARS?

$A$. Because the moon, which is in reality much smaller than most of the stars, being very much nearer to us than any of them, it has the appearance of being larger.

Q. What is the meaning of PERSPECTIVE?

$A$. The science of perspective teaches to draw on a plane surface true pictures of objects as they appear to the eye from any distance and in any position.

"Plane surface," a flat or even surface. The word perspective is from the Latin per (through), and specio (to look).

Q. An object in the SHADE is not so bright and apparent as an object in the sunshine : why is this?

$A$. Because objects in the shade are seen by reflected light reJlected; that is, the light is twice reflected; and, as the rays of light 
are always absorbed in some measure by every substance on which they fall, therefore some light is lost: 1. Before the second reflection is made; and, 2. In the object that makes the second reflection.

Parts of the rays are absorbed, and parts are scattered in all directions by irregular reflections; so that rarely more than half are reflected, even from the most polishod metals.

Q. Why is it light when the sky is corered with thick clouds?

$A$. Because the multiplied reflections of the sun in the atmosphere are sufficient to give light upon the earth, even when thick clouds obscure the sun.

Q. If a picture be highly VARNISHED, or covered with a GuASS, it cannot be seen in certain positions : why not?

A. 1. Because the glass or varnish is a reflector; and, whenever a strong light is reflected from the glass to the eye of the spectator, the glass or varnish becomes very luminous, and the picture remains in comparative darkness; and

2. When the spectator is so placed as to catch the rays of light reflected from the glass or varnish, his eye is dazzled, and cannot see the more faintly illuminated picture.

Q. What is the use of telescopes?

$A$. They gather together the rays of light, and a greater number are thus brought to the eye.

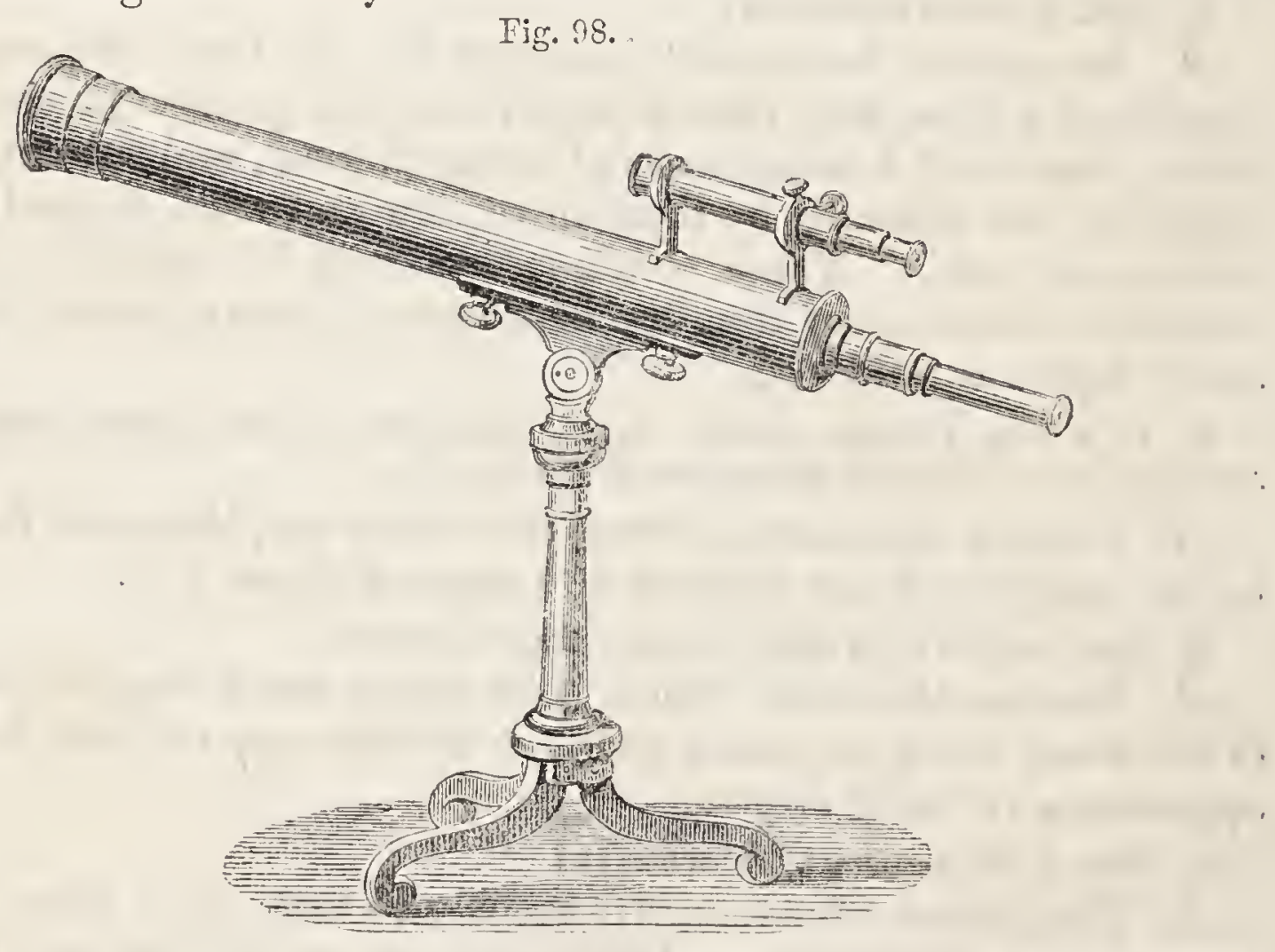

$\Lambda$ TELESCOPE.

Q. How can these rays be GATHERED TOGETHER?

$A$. Rays of light diverge-that is, spread out in all directionsfrom a luminous object. The number of these diverging rays which will enter the eye is limited by the size of the pupil. But, before they reach the eye, they may be received upon a glass lens of a convex form, which will have the effect of collecting them into a space 
less in magnitude than the pupil of the eye. If the eye be placed where the rays are thus collected, all the light will enter the pupil.

Q. Why do TELESCOPES enable us to SEE objects INVISIBLE to the naked eye?

$A$. Because they gather together more luminous rays from obscure objects than the eye can, and form a bright image of them in the tube of the telescope, where they are magnified.

Q. How MANY KINDS of telescopes are there?

A. Two: reflecting, and refracting or achromatic telescopes.

Q. What is a REFLECTIKG TELESCOPE?

A. One in which we look at the magnified image of the object reflected from a mirror or speculum.

Q. What is a REFRACTING telescope?

$A$. In a refracting telescope the use of a microscope is dispensed with, and we look directly at the object through a series of magnifying lenses. The common spy-glass and the opera-glass are examples of the refracting telescope.

Jijg. 99.

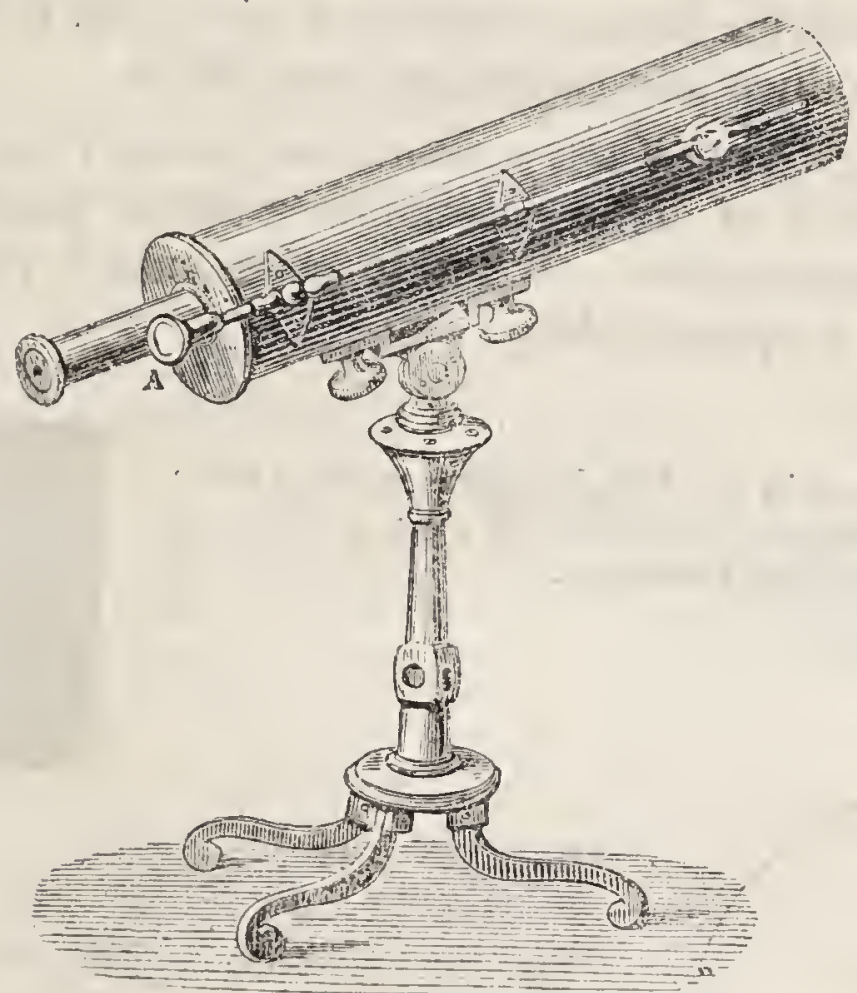

DR. GREGORY'S TELESCOPE, INVENMED 1650 .

Q. When a SMIP is approaching the shore, why are the MASTS scen before the IrULI?

$A$. Because the earth is round; and the curve of the sea hides the hull from our eyes after the tall masts have become visible.

Fig. 100.

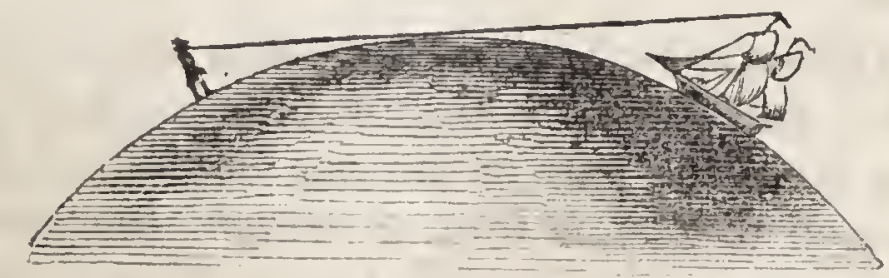




\section{CHAPTER XXXI.}

\section{REFRACTION AND ABSORPTION OF LIGHT.}

Q. What is meant by the REFrActron of light?

$A$. Light traverses a given transparent medium, such as air, water, or glass, in a straight line, provided no reflection occurs, and there is no change of density in the composition of the medium; but when light passes from one medium into another, or from one part of the same medium into another part of a different density, it is bent from a straight line, or refracted.

Q. Does AIr possess the property of REERACTING LIGHT?

$A$. Yes; the more dense the air, the greater is its refractive power. Consequently, that portion of the atmosphere at the earth's surface possesses the greatest refractive power; its density gradually diminishing according to its distance from the earth, till it becomes so rare as scarcely to produce any refraction upon light.

Q. How is a ray of light BENT as it passes from one medium to another?

$A$. When a ray of light passes into a denser medium, it is bent towards the perpendicular. When it passes into a rarer medium, it is bent from the perpendicular.

Let the incident ray, in fig. 101, be represented by $\mathrm{S} 0$; the line $\mathrm{OH}$ represents the refracted ray; $\mathrm{A} B$ shows the angles of incidence and refraction.

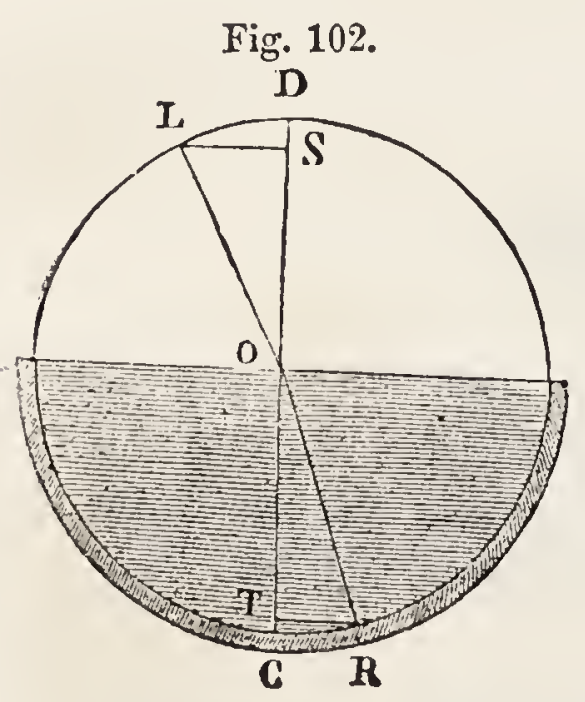

Fig. 101.

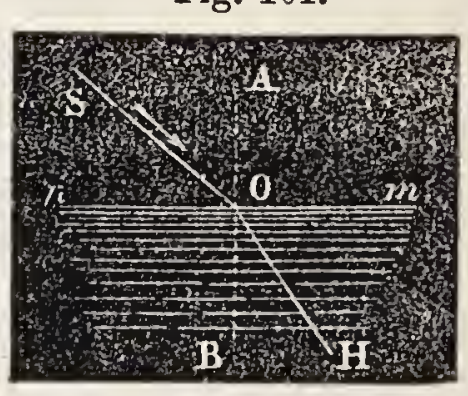

Q. Why does the part of an OAR immersed in the water appear BFNT or BROKEN?

$A$. The water and the air being of different densities, the rays of light proceeding from the part of the oar contained in the water are refracted, or caused to deviate from a straight line, as they pass from the water into the air; consequently that portion of the oar inmersed

Fig. 102 represents a globe half filled with water. $\mathrm{L} O$ is a ray of light, which, entering the denser medium of the water, changes its course to $R$. The different angles formed are evident. $D O L$ is the angle of incidence; $\mathrm{R} \mathrm{O} \mathrm{C}$ the angle of reflection. The angle is taken from the perpendicular line $\mathrm{S} T$. 
in the water will appear to be lifted $\imath p$, or to be bent in such a manner as to form an angle with the part out of the water.

As all rays of light are refracted more in their passage through water than in their passage through air, they will, at the intersection of the two mediums, tend to cross each other and form an angle.

Q. Upon what does the refracting power of bodies DEPEND ?

$A$. Chiefly upon their density. A mixture of sulphuric acid and water will refract or bend the ray of light more than simple water, and a plate of glass more than either.

Q. What CURIOUS PROPERTY have inflammable bodies in respect to the refraction of light?

A. All highly inflammable bodies, such as oils, hydrogen, the diamond, phosphorus, sulphur, amber, camphor, \&c., have a refractive power from ten to seven times greater than that of incombustible substances of equal density.

Q. What curious predicrion did Sir Isaac New ton make respecting the diamond, from its great refractive power?

$A$. Having noticed that the diamond refracted light powerfully, he predicted that it would from this circumstance be found to be inflammable. Many years afterwards this prediction was verified.

Q. What SIMPLE FXPERIMENT illustrates the effect of refraction?

$A$. Place a silver coin $m$ at the bottom of a basin, fig. 103. The rays $i$ i proceeding to the eye from the silver surface, render the coin visible. The point $a$, the eye, is then moved farther back, so that the edge of the basin obstructs the Fg. 103. direct rays, and of course the coin

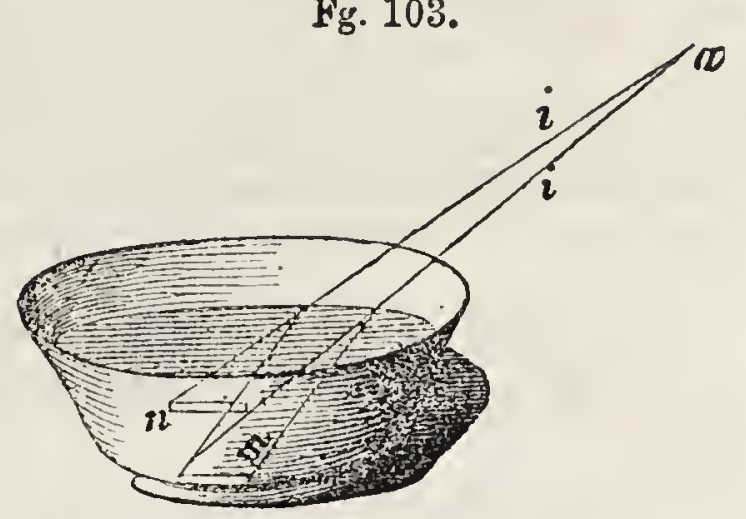
is no longer seen. If an attendant carefully pours water into the basin, so that the object is not moved, it will presently, as the water rises in the basin, become again visible. This arises from the refraction of the rays by the water, the image, indeed, appearing at $n$ instead of at $m$.

Q. How is this refraction of light ACCOUNTED for and EXPLAINED?

$A$. According to the undulatory theory of light, refraction is due to an alteration in the velocity at which the ray of light travels. According to the corpuscular theory, it is accounted for on the supposition that different substances exert different attractive influences on bodies coming within their influence; a highly attractive medium is supposed to exert a strong attractive force, while a weaker force is exerted by those in which the attractive power is less.

Q. What remarkable OPTICAL ETFHCT is produced by REFRACTION?

A. The mirage. 
Q. What is the MIRAGE?

A. An optical illusion, by which we appear to see distant objects inverted.

Q. How is this effect produced?

A. By the mequal density of the air.

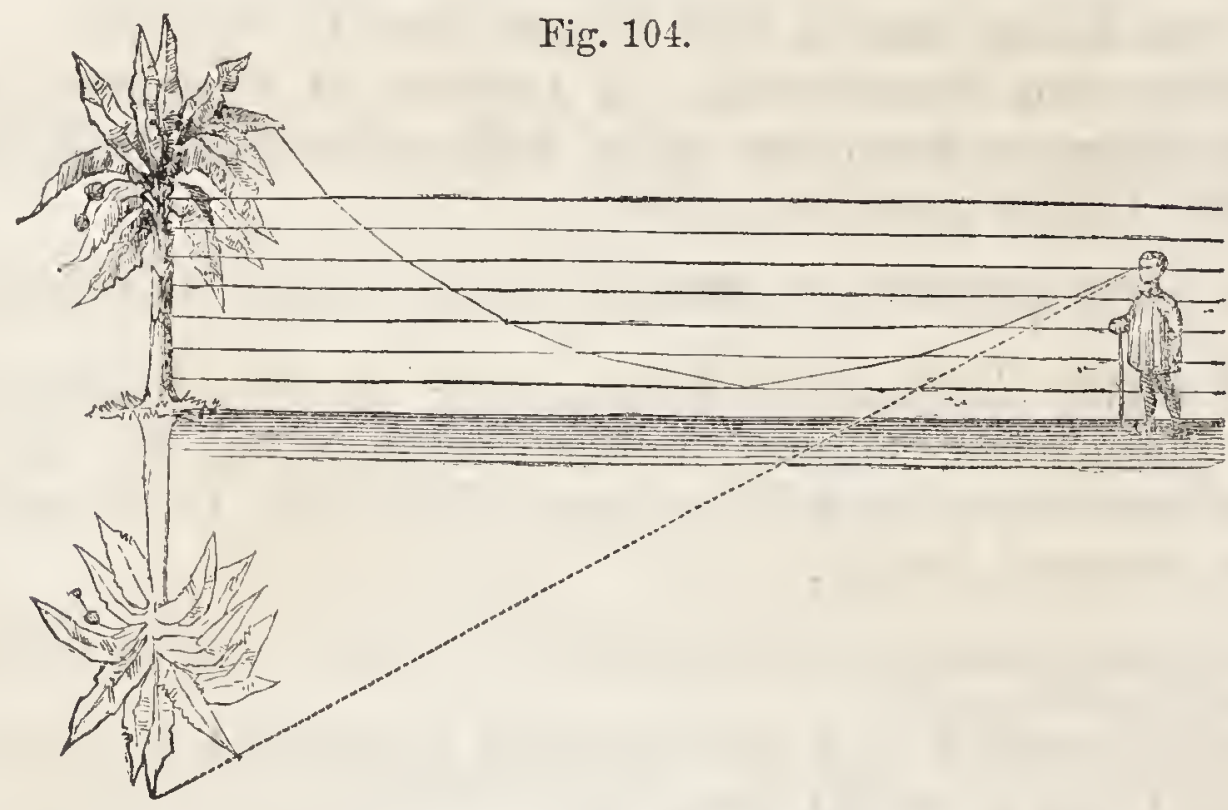

OPTICAL EFEECT OF THE MIRAGE.

The phenomena of the mirage is especially observable on the hot sandy plains of the desert. There the images of trees and water are often seen by travellers when neither are at all near. It arises from the unequal medium through which the light travels. Thus, as seen in fig. 104, the angle of incidence increases from one stratum to that which follows, and finishes by attaining an angle of limit, beyond which reflection succeeds to l'efraction. The ray is then elevated, undergoes another series of refractions, till it reaches the eye of the spectator, who observes an inverted image, as if it were reflected in water.

Q. Is a ray of white light SIMPLE or COMPOOND? light.

A. Every ray of white light is compouncled of other rays of coloured

Q. How is it known that a ray of light consists of several different colours?

A. A ray of light cast upon a prism, is divided into three colours, Red, Blue, and Yellow. These mixing, produce Orange, Green, Indigo, and Violet.

Q. What is a PRISM?

A. A solid piece of glass, triangular in form. (See fig. 105.)

Fig 105.

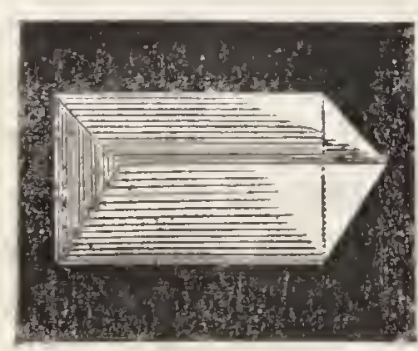

A PRISM.

Q. How does a PRISM separate a ray of light into SEVEN colours?

A. It is attributable to the fact of all these colours having different refructive susceptibitities. lied least, and blue the most; therefore, the blue ray will be bent to the top of the prism, and the red will remain at the bottom.

The separation of a ray of solar light into different colours, by refraction, ray be more accurately displayed by admitting a ray through an aperture in a window-shutter into a darkened chamber, and causing it to fall on a diaphanous prism A P, as represented in the following figure. A ray S thus entering, and suffered to pass unobstructed, would 
form on a plane surface a circular disk of white light; but the prism being so placed that the ray may enter and quit it at equal angles, it will be refracted in such a manner as to form on a screen I, properly placed, an oblong image, called the solar spectrum, and divided horizontally into seven coloured spaces, or bands, of unequal extent, succeeding each other in the order represented : red, orange, yellow, green, blue, indigo, violet.

Fig. 106

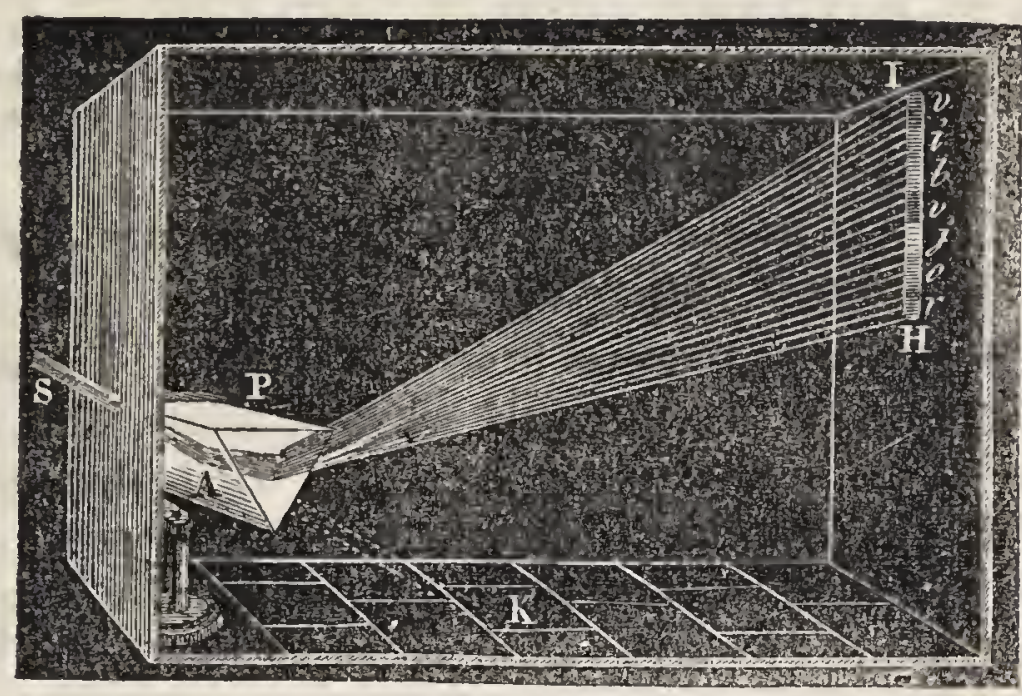

Q. Are the coloured rays, once separated and refiacted by the prism, capable of being: SEPARATED and REFRACTED again?

A. They are not, and are hence designated as primary colours.

Q. If the seven different colours as separated by the prism be again collected together, THAT will they form?

A. White light.

Q. What TERM has been applied to the seven different colours separated by the prism from a ray of light?

\section{A. The solar spectrum.}

Q. To what is the great BRILLIANCY of the diamond, and other precious stones, DUE?

A. To their power of refracting light; they are also artificially cut in such a manner as to form a series of prisms, which separate the rays of light falling on them into their component colourea? ruys.

Q. Why do BLUE and YELLOW powders, when mixed, form a GREEN powder?

A. Because of the mere effect arising in the eye from the intimate mixture of the yellow and blue light, separately and independently reflected from the minute particles of each; the proof of this is had by examining the mixture with a microscope, when the yellow and blue grains will be seen separately, and quite unaltered.

Q. Why cannot a SImiLAR separation be detected in liquid green, produced by agi. tating blue and yellow liquids together?

A. Because of the excessive minuteness of the parts, and their perfect intermixture; from the mixture of two powders, extreme patience would enable any one, by picking out with a magnifier, grain after grain, to separate the ingredients; but when liquids are mixed, no mechanical separation is practicable; the particles are so minute as to elude all search.

Q. What is a RuINeow?

A. The rainbow is a semicircular band or arc, composed of the 
different colours, generally exhibited upon the clouds during the occurrence of rain in sunshine.

If we take a glass globe filled with water, and suspend it at a certain height in the solar rays above the eye, a spectator standing with lis back to the sun will see the refraction and reflection of red light; if then the globe be lowered slowly, the observer retaining his position, the red light will be replaced by orange, and this in its turn by yellow, and so on, the globe at different heights presenting to the eye the seven primitive colours in succession. If now, in the place of the globe occupying different positions, we substitute drops of water, we have a ready explanation of the phenomena of the rainbow.

Q. What is the cause of the rainbow?

$A$. The rainbow is produced by the refraction and reflection of the solar rays in the drops of falling rain.

Q. What are the conditions NECESSARY in order that we may see a rainbow?

$A$. The rainbow can be seen only when it rains, and in that point of the heavens which is opposite to the sun. It is necessary also that the sun should not have too great an altitude above the horizon. Hence, within a certain interval each day, no visible rainbows can be formed, on account of the sun's high altitude above the horizon.

If the altitude of the sun exceeds forty-two degrees, no complete bows can be formed, the summits being depressed below the horizon.

Q. How do we know that the rainbow results from the decomposition of the solar rays by drops of water?

$A$. Because, in the case of cascades and vaterfalls, the spray and the drops of moisture dispersed over the grass and the spiders'-webs produce the same phenomena.

Q. What is the cause of morning and evening TwILIGHT?

$A$. When the sun is below the horizon, the rays which strike upon the atmosphere or clouds are bent down towards the earth, and produce a little light called twilight.

Q. Sometimes SHIPS are DISTINCTLY SEen by an observer on shore before they are astually above the horizon : explain this.

$A$. This is owing to the refracting power of the atmosphere at the time. The different strata of air being of unequal density, the rays of light from the ship to the eye of the observer are bent in a curve; so that the ressel is visible before it is really above the horizon.

It is owing to this refracting power of the atmosphere that the sun appears to us before he rises, and we see him after he has actually set.

Q. Why doez Mother-OF-peAri show so many colours?

$A$. Mother-of-pearl consists of a number of very thin half-transparent layers of unequal thickness, overlapping each other like the scales of a fish.

Where these layers terminate are very small grooves or streaks running in all directions, which act like prisms.

It is these strealings or grooves which cause the various and changing colours of mother-of-pearl.

The same thing may very easily be imitated, and is frequently done in what are callerl " iris ornaments," first invented by John Barton, Esq., of the Royal Mint. These iris ornaments are made of steel, and have about thirty thousand grooves per inch; they are used in court dresses, for buttons, sword-handles, \&c., and are very brilliant indeed. 
Mother-of-pearl may also be imitated, by taking impressions of it in wax, balsam of tolu, isinglass, or gum; these impressions will exhibit all the shades and colours of mother-of-pearl, merely because the impression will be streaked or grooved in a similar way.

Q. How is it that the stars appear to TWINKLE?

$A$. Because the inequalities and undulations in the atmosphere occasion unequal refractions of iight, which produce the twinkling appearance or irregular light of the stars.

Q. What is supposed to be the CAUSB of the refraction of light?

$A$. According to the undulatory theory of light, refraction is due to an alteration in the velocity at which the ray of light travels. According to the corpuscular theory, it is accounted for on the supposition that different substances exert different attractive influences on bodies coming within their influence.

Q. What is a LeNs?

$A$. A piece of transparent glass, bounded on both sides by polished spherical surfaces, or on the one side by a spherical, and on the other by a plane surface. Rays of light passing through it are made to change their direction, and to magnify or diminish the appearance of objects at a certain distance.

Q. How many VARIETIES of lenses are generally recognised?

A. Two: convex and concave.

Fig. 107.

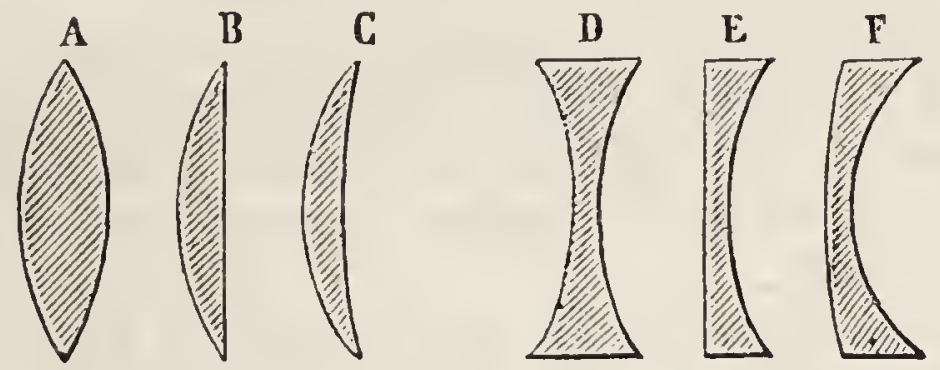

Among convex lenses are the double convex, A ( $f g .107)$, to which the appellation lens was originally applied from its resemblance to a lentil-seed (lens in Latin), being bounded by two convex spherical surfaces whose centres are on opposite sides of the lens; the plano-convex, $B$, having one side bounded by a plane surface, and the other by a convex surface; and the ineniscus or concavo-convex, $\mathrm{C}$, bounded on one side by a concave, and on the other by a convex surface : the former being a portion of a larger circle than the latter, and therefore the surfaces meet when produced.

There are also three principal varieties of concave glasses; as the double concave, $D$, bounded by two concave surfaces, forming portions of spheres whose centres are on opposite sides of the lens; the plano-concare, E, bounded on one side by a plane, and on the other by a concave surface; and convexo-concave $\mathrm{F}$, bounded by a convex surface on one side, and by a concave one on the other; but these surfaces, when produced, do not meet.

Q. What is a Focus of light?

$A$. When rays of light continually approach each other, as in moving to a point, they are said to converge, and the point at which the converging rays meet is called the focus.

Q. What sort of a lens is a common BURNIAG-GLASS?

A. A double convex lens. 
Q. What are Halozs?

A. Coloured rays seen round luminous bodies, as the moon or sun.

Q. Under what CIRCUMSTANCEs are they produced?

$A$. When the atmosphere contains moisture in such a condition that it refracts and decomposes the rays of light proceeding from the luminous body.

Q. What are transparnet bodies?

$A$. Those which do not interrupt the passage of light, or which admit of other bodies being seen through them.

Q. When is a body SEMI-TRANSPARENT?

A. When it allows other bodies to be seen through it, but only imperfectly and obscurely.

Q. When is a body opAQue?

A. When it entirely prevents the passage of light.

Q. Why are some thingS TRANSPARENT?

$A$. We cannot clearly explain what there is in the constitution of one mass of matter, as compared with another, which fits the one to transmit light, and the other to obstruct it; but the arrangement of the particles has undoubtedly much influence.

Q. Upon what QUaLITY does the transparency or opacity of substances especially DEPEND?

A. Upon their relative thickness or thimness. Thus gold can be beaten so thin as to transmit a soft green light.

Q. HorN is partially transparent : why are not horn sHaviNas the same?

$A$. Because the surface of the shaving has been torn and rendered rough; and the rays of light are too much reflected and refracted by the rough surface to be transmitted through the shaving so as to produce transparency.

If the parts of any substance are not pretty uniform, the rays of light are refracted and absorbed so frequently, that no part of them can emerge on the opposite side.

Q. Is there any body PERfectiy tranSPARERT ?

A. No; some light is evidently lost in passing even through space, and still more in traversing our atmosphere.

Q. How much of the sun's light is supposed to be INTrRarepted by the atmosphere?

$A$. It has been calculated that the atmosphere, when the rays of the sun pass perpendicularly through it, intercepts from one-fith to one-fourth of their light; but when the sun is near the horizon, and the mass of air through which the solar rays pass is consequently vastly increased in thickness, only one two hundred and twelfth part of their light can reach the surface of the earth.

Q. Why is cliarcoal BLAcK?

$A$. Because it absorbs all the light which falls upon it, and reflects none.

Q. What BECOMES of the light which is absorbed ?

$A$. This question cannot be satisfactorily answered. In all pro- 
bability it is permanently retained within the substance of the absorbing body. sun?

Q. How THICK must our atmosphere be to absorb all the light emanating from the

$A$. If our atmosphere in its state of greatest density could be extended rather more than seven hundred miles from the earth's surface, instead of forty or fifty, as it is at present, the sun's rays could not penetrate through it, and our globe would roll on in darkness.

Q. To what DEPTr is light supposed to penetrate the ocean?

$A$. It is calculated that sea-water loses all its transparency at the depth of seven hundred and thirty feet; but a dim twilight nust prevail much deeper in the ocean.

Q. If the objects of the material world had been illuminated with white light, all the particles of which possessed the same degree of refrangibility, and were equally acted upon by the bodies on which they fall, what would have been the APPEARANCE of nature?

A. All nature would have shown with a leaden hue, and the combinations of external objects, and all the features of the human countenance, would have exhibited no other variety than that which they possess in a pencil sketch or India-ink drawing.

\section{CHAPTER XXXI.}

\section{COLOUR.}

Q. What is the efficient $C \triangle$ USE of colours?

A. The action of light.

Q. How is this PROVED?

$A$. In the dark, bodies have no colour, and in the light their colours may be altered by subjecting to certain modifications the light by which they are rendered visible. Thus everything in a red light will appear red.

Q. Why is it that we find it difficult to distinguish colours by CANDLE-LIFET ?

$A$. Because we have modified the light upon which the full effect of the colour depends.

Q. What, then, is the true DEFinirion of colour?

$A$. The colour of a substance is the effect of light on a surface adapted to reflect its particular colour.

Q. How is it that ALI objects do not reflect the SAME colour?

A. On account of their different substances and chemical properties.

Q. Why are some things RED ?

$A$. Because they absorb all the yellow and blue rays, and reflect only the red.

Q. Why are some things BLACE ?

A. Because they absorb all the rays of light, and reflect none. 
Q. Is BLACK a colour?

$A$. It is not; it is the absence of colour.

Q. Why are some things whire? all.

$A$. Because they absorb none of the rays of light, but reflect them

Q. Why are clouds, snow, sugar, and salt wHrrs?

$A$. Because they reflect back unchanged the white light which strikes upon them.

Q. Why are the LEAVES of plants GREEN?

$A$. Because a peculiar chemical principle, called chlorophyl, is formed within their cells, which has the property of absorbing the red rays, and of reflecting the blue and yellow, which mixture produces green.

Q. Why do all things appear BLACK in the DARE?

$A$. In the dark there is no colour, because there is no light to be absorbed or reflected, and therefore none to be decomposed.

of course, in certain degrees of darkness, all objects are actually invisible. The question refers to that peculiar degree of darkness when the forms of objects may be seen, but not their hues.

Q. Why does the sky appear BLUE?

$A$. Because the atmosphere absorbs the red and yellow rays, and transmits the blue.

Q. Why are some PLANTS WHITE which are kept in the DARK ?

$A$. Because chlorophyl can be formed only by the agency of the sun's rays; and it is this peculiar principle which gives the green tinge to healthy leaves and plants.

Some plants are a yellowish green from the same cause.

Q. Why does the SUN most generally FADE artificial CoLOURS?

$A$. Generally the loss of colour arises from the oxidation of the substances used in dyeing; as tarnish and rust are an oxidation of metals. Sometimes, however, the ingredients of the dye are otherwise decomposed by the sun; and the colour (which is due to a combination of ingredients) undergoes a change as soon as the sun deranges or destroys that combination.

Q. What is meant by the term ACCIDENTAL COIOUR?

$A$. The accidental colour is the colour whick would be required to be added in order to make up white light: thus, the accidental colour of red is bluish green ; of orange is blue; of yellow is indigo; of green is reddish violet; of blue is orange red; of indigo is orange yellow; of violet is yellow green; of black is white; of white is black.

Q. What surfaces REFLECT LIGHT best,

A. Smooth and polished surfaces are the best reflectors of light.

Q. Glass is a smooth polished surface: is it a good reflector of light?

A. Glass is trunsparent, and therefore transmits light; but if one 
of its surfaces be covered with quicksilver, the light cannot pass through it, and is consequently reflected.

Q. Why do SANDY PLAINS DAZZLE from sunshine?

$A$. Because each grain of sand reflects the rays of the sun like a mirror.

Q. How is the origin and production of colour $\triangle$ CCOUNTED FoR, according to the cor puscular theory?

$A$. According to this theory, white light is an assemblage of atoms of various colours, which may be separated from each other by the action of a prism; and bodies, when exposed to the rays of the sun, display any given colour, because they are so constituted as to absorb all the atoms except those of the rays of their own peculiar colour; thus perfectly white substances absorb none of the atoms, but reflect the white or compound light unaltered; black substances absorb all the rays, and therefore yield no colour; and red, yellow, and blue substances respectively reflect those rays alone by which they are distinguished.

Q. Fow is the production of colour accounted for, according to the UNDULATORY theory?

$A$. The undulatory theory suppo:as that every simple colour depends on a certain number of vibrations, which are performed in a certain time. If, therefore, the rate at which a ray undulates is altered, a different sensation is produced upon the optic nerve.

Q. What analogy is there between the seven colours and the seven Notes in music?

$A$. The analogy between sound and light, according to the unduslatory theory, is perfect, even in its minutest circumstances. When a certain number of vibrations of a musical chord are caused in a given time, we produce a required sound; as the vibrations of the chord vary from a quick to a slow rate, we produce sounds sharp or grave. So with light. As the supposed ether varies the rate of its undulations, we have colours bright or dull.

Q. How NaYY times must a ray of light viBrate to produce red light?

A. Thirty-seven thousand six hundred and forty undulations in an inch, and four hundred and fifty-five billions in a second.

Q. How MANY vibrations are required to produce yelLow and BLUE light?

$A$. Yellow light requires forty-four thousand in an inch, and five hundred and fifty-five billions in a second; while the effect of blue results from fifty-one thousand one hundred and ten undulations within an inch, and sixhundred and twenty-two billions in a second of time.

Q. How are these facts KNOWN and Proved?

$A$. Newton proved, by a series of beautiful and satisfactory experiments, the number of vibrations of different coloured rays within the space of an inch. If now, from a star of a red colour, there proceeds a continuous line of waves, these waves will enter the pupjt of the eye, and stritie upon the retina. For each wave that thus strikes upon the retina, there will be a separate pulsation of the membrane. Its rate of pulsation, or the number of vibrations it 
makes per second, will therefore be known if we can ascertain how many luminous waves enter the eye per second.

Q. How can this fact be ASCERTAINED?

$A$. Light moves at the rate of about 200,000 miles per second. A length of ray amounting to 200,000 miles must therefore enter the eye each second; the number of times, therefore, that the retina will vibrate per second will be the same as the number of luminous rays contained in a ray 200,000 miles long.

In two hundred thousand miles there are in round numbers a thousand millions of feet, and therefore twelve thousand millions of inches. In each of these inches there are forty thousand waves of red light. In the whole lengtl of the ray, therefore, there are four hundred and eighty millions of millions of waves. Now, as this ray enters the eye in one second, the retina must pulsate once for each of these waves; and thus we arrive at the astounding conclusion that where we behold a red object, the membrane of the eye trembles at the rate of FOUR HUNDRED AND EIGHTY MIILLIONS OF MILLIONS of times between every two ticks of the common clock.-LARDNER.

Q. What remarkable correspondence is there between the GEOGRAPHICAL POSITION of a region and the coLours of its plants and animals?

$A$. In the tropics, where the sun shines longest and brightest, the darkest green prevails over the leaves of plants, the flowers and fruits are coloured brightly, and the plumage of the birds is of the richest description.

Q. What is the natural coloration exhibited in TEXPPERATE climates?

A. In temperate climates everything is of a more subdued variety; the flowers are less bright; the prevailing tint of the birds is brown; and the dresses of the inhabitants are sombre.

Q. How is this correspondence further exemplified in the AROTIC and ANTARCTIO regions?

$A$. Here there is little colour in natural objects; the few flowers are white or yellow; and the animals are almost uniformly black or quhite.

Q. In what part of the ocean do we find the BRIGHTEST shells and sea-weeds?

$A$. Near the shore, in shallow water, where the influence of light is greatest.

Q. What fishes are distinguished for the BRILLIANCY of their colours?

$A$. Those that swim near the surface; whereas those which live at greater depths are gray, brown, and black.

Q. What is the appearance of the sea-weeds and animals that live at great DEPTHS of the ocean?

A. They are nearly colourless.

Q. Why is grass growing under a COVERING of a white or yellowish white colour?

$A$. Because it is secluded from the light, whose presence and action is necessary for the production of the material whicl imparts to it its green colour.

Q. Of the various rays composing solar light, which are the Most visible to the human eye?

A. The yellow.

Q. Which have the greatest HEsting effect?

A. The faint red rays. 
Q. Why does a dress composed of cloths of DIFFERENT CoLOURS look well much LONGER, although worn, than one of only a SINGLE COLOUR, the character of the cloth in both instances being identical ?

$A$. It is owing to the effect of contrast between the colours. If a dress is composed of cloths of two colours, the one complementary to the other, as red and green, orange and blue, yellow and violet, they will mutually heighten the effect of each, and make each portion appear to the best advantage.

Q. Why will stains be less visible on a dress of DIFFERENT colours, than on one composed of only a SINGLE colour?

A. Because there exists in general a greater contrast among the various parts of the first-named dress, than between the stain and the adjacent part, and this difference renders the stain less apparent to the eye.

Q. Why can a coat, waistcoat, and trowsers of the SAJEE coLOUR be worn with advantage together only when they are NEW ?

A. Because as soon as one of them loses its freshness from having been worn longer than the others, the difference will increase by contreast.

Q. Give an ildustration?

A. A pair of new black trowsers, worn with a vest of the same colour, which is old and rusty, will make the tinge of the latter appear more conspicuous; and, at the same time, the black of the trowsers will appear more brilliant. White and other light-coloured trowsers would produce a contrary effect.

Q. What is the GENERAL IAW upon which the harmony of colours DEPENDS?

A. Every colour when placed beside another colour is changed, appearing different from what it really is; and it moreover equally modifies the colour with which it is in proximity.

Q. What EFFECT has rose-red upon a rosy complexion?

A. It causes it to lose some of its fresheness.

Q. For fair complexions, deficient in rose, which colour is Most Favourable?

A. A pale blue.

Q. What EFFECT has black drapery upon the colour of the skin?

A. It makes it appear whiter.

Q. What RULE should be observed in the grouping of flowers and the preparation of bouquets?

A. We must separate pink flowers from those that are either scarlet or crimson; orange from orange-yellow flowers; yellow flowers from greenish-yellow flowers; blue from violet-blue, red from orange, pink from violet; blue flowers from violet flowers.

Q. That is the optICAL EFFECT of dark colours and black upon the SIzE of the figure?

A. It causes it to appear smaller; therefore these colours are most suitable for stout persons.

Q. What EFFEOT does white and light-coloured dresses bave upon the SIZE of the figure?

A. They cause it to appear larger. 
Q. What EFFECT is produced by a large patterned dress?

$A$. It makes the figure look shorter.

Q. What is the EFFECT of narrow longitudinal stripes in dress!

A. They add to the apparent height of the figure.

Q. What is the EFFECT of horizontal stripes ?

$A$. The effect of horizontal stripes is opposed to that of longitudinal, and under every condition they are ungraceful.

Q. What colours are most cossprcuous in battle?

$A$. It has been found, by numerous observations, that red is the most fatal colour, and the least fatal is a light gray.

Q. What curious Provision for the protection of animals does nature appear to make?

$A$. She appears to have adapted the colour of the creature to its haunts in such a way as tends to preserve it from injury. Caterpillars and insects which feed on leaves are generally of the colour of the leaves. As long as they remain still, it is almost impossible to distinguish the grasshopper from the herbage on which it rests.

Q. What CURIOUS CIINAGE is noticed in the corour of animals inhabiting the Aretie regions?

A. During the snows of winter, foxes, hares, and some varieties of birds are white; when the ground is free from snow in summer, they are of a brown colour.

Q. How are Frogs found to escape observation and notice?

$A$. By varying their colour according to the mud or sand that forms the bottom of the ponds or streams which they frequent; and the tree-frog takes its specific name (Hyla vividis) from the colour of the leaves of the trees it inhabits.

\section{CHAPTER XXXIII. \\ THE ORGANS OF VISION.}

Q. Of what PArts does the eye consist?

$A$. The eye is of globular form, and is composed of three coats or membranes, called the sclerotic, the choroid, and the retina; and three humours, denominated the aqueous, the crystalline, and the vitreous.

Q. What is meant by the "RETINA of the BYr? ?"

$A$. The network which lines the back of the eye; it is composed of an expansion of the optic nerve.

Q. What is that portion of the eye called which in some persons is BLUE, in others GRAY Or MAZEL ?

$A$. It is called the iris.

Q. In the centre of the iris is a circular BLACK OPENING : what is this called ?

A. It is called the pupil. But this spot is not a black substance, but an aperture, which appears black only because the chamber 
within it is dark. It is, properly speaking, the window of the eye, through which light is admitted, which strikes on the retina.

Q. Does light admitted through the pupil to the RETINA produce visron?

A. Yes; provided the light enter in sufficient quantity.

Q. What is meant by the " conNes of the EYE?"

A. All the outside of the visible part of the eyeball.

The construction of the human eye may be seen from the accompanying diagram, which represents a transverse section of the eye. The eye itself, it will be seen, is shaped like a globe. It is composed of the following substances:-

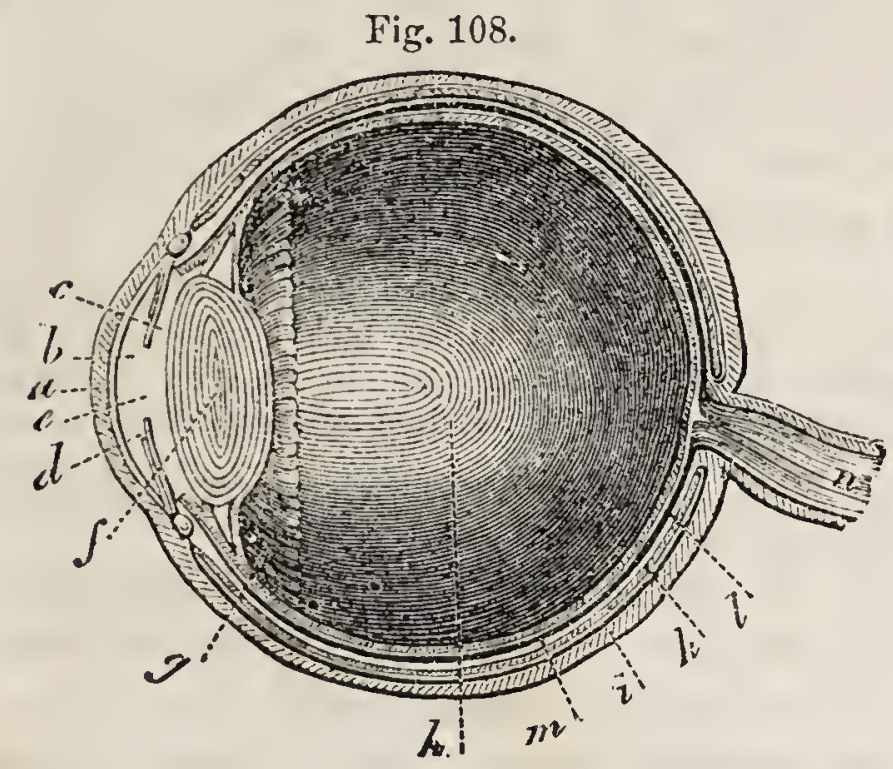

The CoRNeA (a), a transparent MEMBRANE, or COAT, in front of the ball of the eye.

The scLERotic (i) a membrane which, with the conNed, covers all the constituent parts of the eye.

The IrIs $(d)$, the delicate muscle which gives colour to the eye. This IRIs is pierced by an opening called the pupil. By the pupil the rays of light penetrate to the eye.

The AQUEOUS HCMour (e) occupies the spaces, chambers as they are called, marked $b$ and $e$.

The CRYSTALLINE LENS is placed behind the IRIS $(g)$.

The VITREoUs HUMOUR, which re. sembles in appearance the white of an egg, occupies the part of the eyeball behind the crystalline, marked $h$.

The RETINA $(m)$ receives the impression of the light, and transmits it to the brain by a NERVE, inarked $n$.

The cHLoroid MEMBRANe, between the reTiNa and the scherotic, is marked $k$. It absorbs all the rays of light which are not required for vision.

Q. What is the scLERoric?

$A$. That water, which, with the cornea, covers all those muscles which form the human eye.

Q. What is the IRIS ?

$A$. It is a very delicate muscle, which gives colour to the eye: blue, brown, gray, or black.

Q. What is meant by the Humours of the eye?

A. That matter which fills up the cavity or chamber of the eye, namely, the aqueous or watery, the vitreous or glassy, and the crystalline.

Q. What is the RETina?

$A$. The innermost coat of the eye, which, receiving the impression of light, transmits it to the brain.

Fig. 108 is a section of the human eye, seen from within. In this engraving we have represented, I, the coat of the eye; 2 , the pupil ; 3 , the iris; 4 , the ciliary process ; 5 , the border of the retiua.

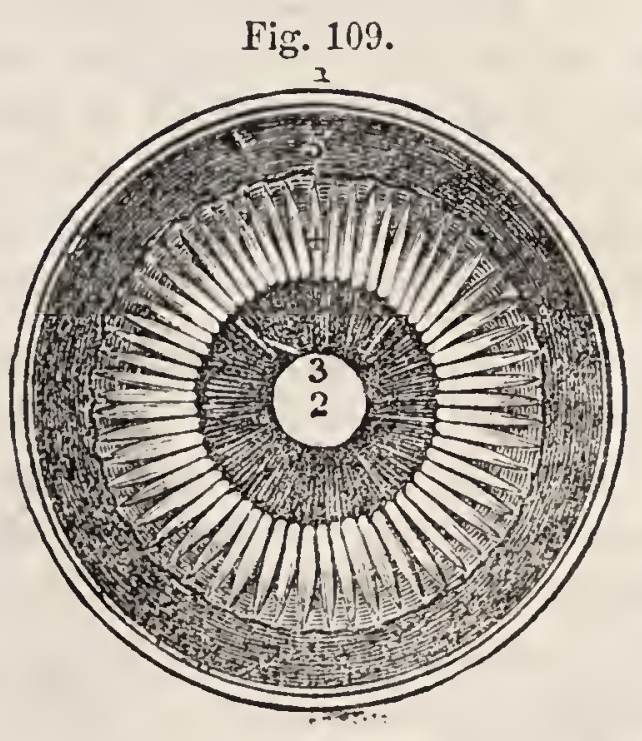


Q. What is the USE of the EYEBROWS?

$A$. The eyebrows defend the eyes from too strong a light, and serve to turn away substances which might otherwise fall into the eye.

Q. What is the use of the EYELASHuS ?

$A$. 'The eyelashes guard the eye from danger, and protect it from dust or insects floating or flying in the atmosphere.

Fig. 110.

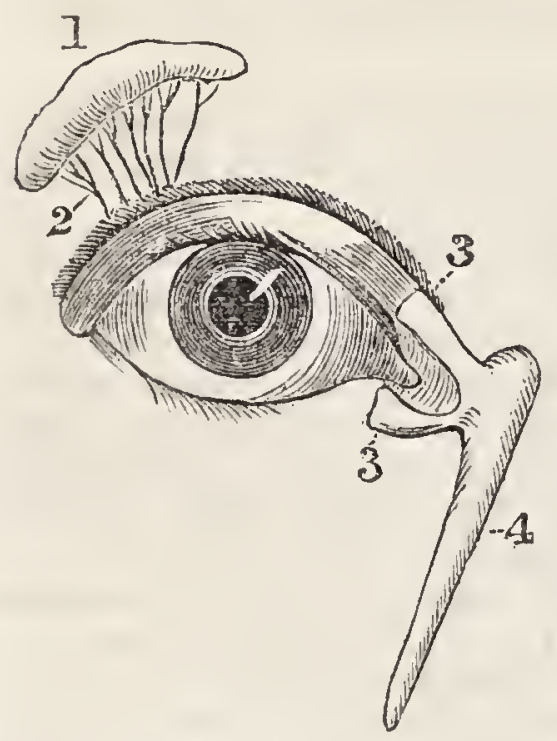

Q. What is the USE of the EYELID?

$A$. The eyelids protect the eye from too bright rays of light, and also from dust; they also distribute over the eye a watery fluid, produced in the tear-gland: this watery fluid flows to the eye by several small ducts.

In the cut (fig. 109) are represented, 1, the TEAR gland, or, as it is called, lachrymal gland; 2 , the ducts, passing from the gland to the eye ; 3 , ducts at the corners of the eyes; 4 , duct opening into the nose.

Q. What is the RELATIVE SPHERICITY of the eyes of land and aquatic animals?

A. In man, and other land animals, the eyeball is a mass nearly spherical, but somewhat flattened in front. Those animals that dwell in the water have eyes very much flattened, the eyeball in most fishes forming but half a sphere, and in the ray species it is but one quarter of the thickness of a sphere.

Q. What PECULIARITY is found in the eyes of birds that soar to great beights?

$A$. In those birds that soar to the higher regions of the atmosphere, the anterior part of the eye is sometimes flat, and sometimes in the figure of a truncated cone; the upper part forming a short cylinder, surmounted by a very convex eminence.

Q. What peculiar ARRANGEMENT exists in the eyes of flies, and some other insects?

A. 'They have eyes often very large in proportion to the bulk of the insect, and composed of a multitude of small facets, or plano-convex lenses, united into a hemispherical form, with their axes directed to a common focus.

Q. When we say we SEE an object, what do we in fact $D O$ ?

A. The mind is only taking cognisance of the picture or impression made on the retina.

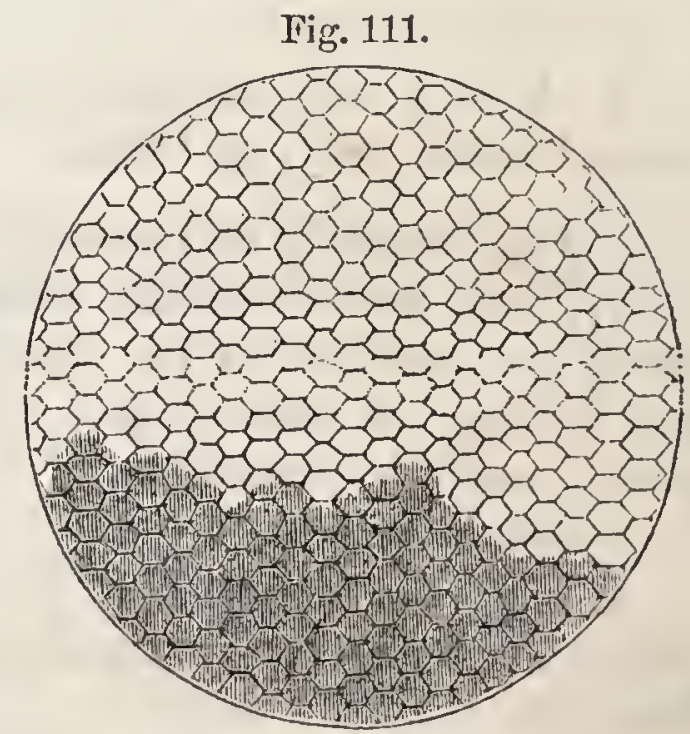

THE EYE OF A COMMON FLY.

Q. Why is it that when we PRESS SLIGHTLY upon the ball of either eye, while viewing an object, we see double?

A. Because the pressure of the finger prevents the ball of one cye 
from following the motion of the other, and the axis of vision in each eye being different, we see two images.

Q. Do persons who SQUINT SEe DOUBLE?

$A$. They do; but practice gives them power of attending to the sensation of only one eye at a time.

Q. Why do persons who are short-sighted in youth gradually have this failing corRECTED as they grow old?

$A$. They are short-sighted because the cornea of the eye is too globular; but as age advances the fluids are not secreted is before, the eye becomes flattened, and natural sight is again restored.

$Q$. If the mind, in seeing an object, sees in reality only a picture painted on the back of the retina, how is it enabled to JUDGE of MAGNITUDES, DISTANCES, \&c., the picture being on a comparatively flat surface?

$A$. It is only by experience. "I see men as trees walking," said the man born blind, when restored to sight.

Q. Would a person whose eyes, although perfect, had been covered up from infancy to maturity, be able TO SEE ?- - that is, comprehend any scene or prospect on which he first opened his eyes?

$A$. He would see the objects, but could no more understand them than a child understands the printed page on which it looks, although every word is clear and distinct.

Q. Are the images formed on the retina of the eye ERECT Or INVERTED ?

A. Always inverted.

Q. Why, then, do we not see objects always Upside Down?

$A$. It is difficult to satisfactorily answer this question. It is usually said, that although the images formed in the eye are inverted, they are conceived to be erect from the circumstance that everything has always been viewed under the same conditions.

Fig. 112.

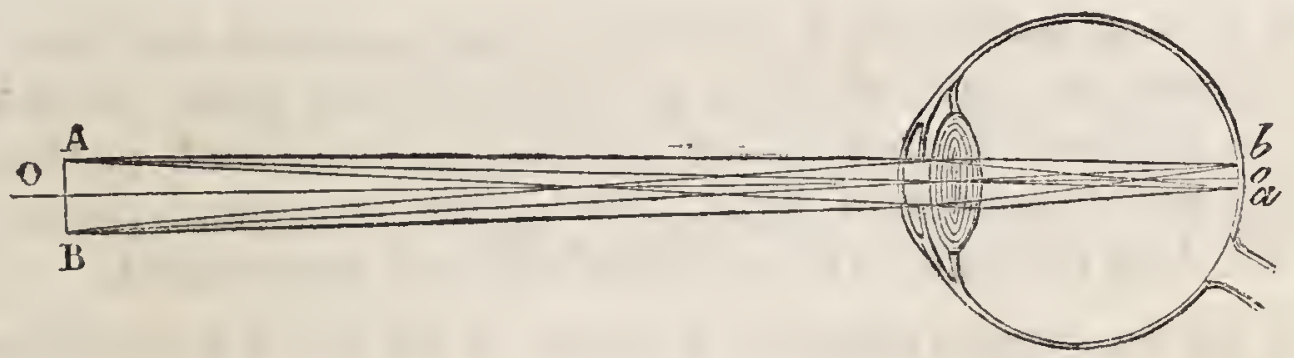

Various theories have been entertained respecting the reversing of images in the eye. It is the opinion of some that we perceive the real position of objects in the direction of the rays of light which they drive forth, and that as these lines cross each other in the crystalline lens of the eye, we perceive the points $A$ and $B$ in the direction $a$ and $b$ respectively, whence the object appears upright.

Q. Do we see the same lines and surfaces of an object ALIKE with EACII EYE?

$A$. We do not; and we may convince ourselves that we do not, by placing two candles, for example, in such a position, that when they are looked at with the right eye, one is made to cover the other; if now we close the right eye and look at them with the left, 
the most remote candle will be no longer screened by the front one, but will be seen about an inch to the left of it.

Q. What is a streroscope?

$A$. The stereoscope is an instrument invented by Professor Wheatstone, consisting of two mirrors, the backs of which are inclined to each other at an angle of $90^{\circ}$.

It will be obvious that if two pictures of the same object are placed at a little distance in front of these mirrors, we shall have two reflected images. If, however, we bring the nose close to the edge at which the mirrors are united, and thus look with an eye into either glass, the two will be resolved into one; and the resulting image, instead of appearing as a plane surface, appears as a solid, possessing length, breadth, and thickness. The result is very successfully obtained, if the precaution be taken of preparing the drawings so that they represent the object as seen by either eye.

Q. What is the disease called Cataract?

A. Cataract is the loss of transparency in the crystalline humour.

\section{CHAPTER XXXIV.}

\section{DECEPTIONS OF VISION.}

Q. Why cannot we count the posts of a fence when we are riding rapidly in a railroad train?

A. Every impression, according to the intensity of its effects, remains for a certain length of time on the retina, and a measurable period is necessary to produce the impression. The light from each post falls upon the eye in such rapid succession, that the different images become confused and blended, and we do not obtain a distinct vision of the particular parts.

Q. Why are More stars visible from the top of a HIGH MOUNTAIN than from a PLAIN?

$A$. Because the rays of light proceeding from them have to pass through a thinner stratum of air in the one case than in the other. Air being an absorbent of light, the higher we ascend in the atmosphere, the less the light will be absorbed and diminished.

Q. How can the apparent magnitude of the sun, at the time of his rising, and again at noonday, be measured?

$A$. This may be accomplished by extending two threads of fine silk, fastened in a frame, parallel to each other. The frame should be placed in such a position, and at such a distance from the eye, that when presented to the sun or moon in the horizon, the threads will exactly touch its upper and lower limb, or, in other words, be just sufficiently separated to admit of the disc of the sun or moon to appear between them, and touch. Now, if the sun or moon be viewed in the same marmer at noonday, it will be found that the threads are just far enough apart to admit of the disc between them, 
showing that the apparent increased magnitude at rising and setting is an optical deception, or rather an error in judgment.

Q. Is this illusion an optical one, or a mental illusion?

A. A mental one, since the organs of vision do not present to us a larger image of the moon or sun in the horizon than in the zenith.

Q. What do we MEAN by the HORIZON?

A. The circle or line where the earth and sky appear to meet.

Q. What do we mean by the zenitr?

A. The point or part of the heavens immediately overhead.

Q. Is the moon NEARER or FARTHER from us when upon the HORIzoN?

A. When the moon is on the horizon, it is about four thousand miles farther from us than when in the zenith; its apparent diameter, therefore, instead of appearing larger, ought to appear about a sixtieth part less.

Q. The number of stars seen on a cloudless night appears countless : is it REALLY so ?

$A$. It is an illusion: we do not see anything like the number of stars which we are accustomed to think we do.

On the entire sphere of the heavens there are not above twenty stars of the first magnitude, and it is seldom that as many as six or eight of these can be seen at once. The number of stars of the second magnitude does not exceed fifty, and of these, twenty can seldom be seen at any one time. The stars of the third magnitude may amount to about two hundred, half of which can only be at the same time above the horizon. The smaller stars are much more numerous, but they are discernible with difficulty, and do not produce upon the mind the idea of multitude that we conceive.

Q. Why are we so often mistaken in respect to the ACTUAL DISTANCE of a conflagration at night?

$A$. Light radiating from a centre rapidly weakens as the distance from the centre increases, being, for instance, only one-fourth part as intense at double the distance. The eye learns to make these allowances, and by the clearness and intensity of the light proceeding from the object, judges with considerable accuracy of the comparative distance. But a fire at night appears uncommonly brilliant, and therefore seems nearer than it really is.

Q. Why does the evening star rising over a hill-top appear as if situated directly oVER the TOP of the eminence?

A. Because we make brightness and clearness to depend on contiguity, as it ordinarily does; and as the star is bright, we unconsciously think it near us.

Q. How is it that after looking at a brightly coloured object for some time we appear to sce it even after the object is removed from our sight?

$A$. It is the effect of the brilliant complementary colours used, as red upon a green ground; the impression made on the retina lasting for an appreciable interval after the figure has been moved. 


\section{CHAPTER XXXV.}

\section{ARTIFICIAL LIGHT.}

Q. How is artigrcial light genemily obtained ?

A. By the combustion of inflammable matter.

Q. Wonld all bodies, if Sufficientiy Heated, become Luminous?

A. 'They undoubtedly would.

Q. When is a body said to be INCANDEScEnt?

A. When it is sufficiently heated to glow or shine.

Q. At what temperature do solids become rmcandEscrin?

A. The temperature at which solids begin to shine in the dark is bêween $600^{\circ}$ and $700^{\circ} \mathrm{F}$; but they do not appéar luminous in broad daylight until they are heated to about $1,000^{\circ} \mathrm{F}$.

Q. Is artificial white light found to contain the SAyE corouns as solar light?

A. It is; and the red, yellow, and blue rays may be separated from it by a prism.

Q. In what respect does ARTIFICIAL LIGHT differ from SOLAR LIGHT?

A. It contains heat in two different states.

It contains simple, RADYANT HFAT, like that radiated from a body not luminous, and which may be separated by transmission through a plate of moderately thick glass; but the light so purified still heats any body which absorbs it, and still possesses calorific rays, associated with its luminous rays, like those in solar light, and, like them, susceptille of refraction.

Q. Is LIGHT always AccompanIED by heat?

A. It is not; there is no heat recognisable by the most delicate instruments in the light of the moon.

Q. Why does a piece of old and decayed wood sonetimes appear LUMINous in the DARK ?

A. Because, during the process of decay and the transformation of its elementary particles, a chemical action is produced which evolves light.

Q. What TERM do we apply to such action and phenomena?

A. The term phosphorescence; and bodies exhibiting this peculiar light are called phosphorescent bodies.

Q. Is phosphorescence, when cccurring naturally, ACCOMPANIED by HEAT?

A. It is not.

Q. Does phosphorescence occur in organised, LIVING bodifs, as well as in dead, decaying substances?

A. It is most frequently noticed in living, organised structures; but the animals exhibiting it all belong to the lower classes, and are principally worms and insects, as the common fire-fly.

Q. To what is the plosphorescence or luminous appearance of the ocean, often observed, especially in the tropics, due?

A. To the presence of a multitude of little animals, known as meduse. 
Q. Do plants and flowers ever emit a phosphorescent or spontaneous light?

A. A sudden flashing light is often emitted on fine summer evenings, a little after sunset, by certain yellow flewers. Some tropical plants and flowers shine all night.

Q. How has this emission of light in plants been ACCOENTED for?

$A$. It has been supposed that they absorb light during the sunshine, and afterwards liberate it.

Q. Is the PUtREFactiox of animal bodies accoinpanied with phosphorescence?

A. In many instances: it exhibits itself most readily in putrid fish, although it has been observed in the decomposition of nearly all animal substances.

Q. WHY has this luminous appearance been called phosphorescence?

A. Because it resembles the light which a piece of phosphorus exhibits when exposed to the action of the atmosphere.

Q. How is it supposed that the fire-fly and other similar insects ARE ENABLED to produce luminosity?

A. They eliminate a peculiar and, in most cases, a liquid sulstance, containing phosphorus or some other element, which combines at common temperatures with the oxygen of the air, or of water containing air, producing a faint luminous appearance.

\section{CHAPTER XXXVI:}

\section{DOUBLE REFRACTION AND POLARISATION.}

Q. When is a ray of light said to be REFracted ?

A. When, in passing from one medium to another of different density, it is bent from its course, or from a straight line.

Q. When is a ray of light said to be DOUBLY REFRACTED?

A. If any transparent substance has an unequal constitution, or if we can produce in it an inequality of elasticity by mechanical pressure or by heat, the ray falling upon its surface undergoes two refractions; that is, the single pencil is divided into two pencils in passing through the medium.

Q. What substance shows most distinctly the phenomena of double refraction? lime.

A. A small crystal of Iceland spar, or crystallised carbonate of

Q. If we look at a small object, through such a crystal, how does it appear?

$A$. If we look at an object, as a dot, a letter, or a line, through a plate of glass, it appears single; but if a plate of Iceland spar be held over them, a doulle image will be perceived, as two dots, two letters, two lines, \&c. 
Q. What is POLARISED LigHT, and how was it DISCOVERED?

A. In 1808, Malus, a young engineer officer of Paris, was viewing through a double-refracting prism the light of the sun reflected from a glass window of one of the French palaces, and he observed some peculiar effects. The window accidentally stood open, like a door on its hinges, at an angle of $56^{\circ}$, and Malus noticed that the light reflected from this angle was entirely altered in its character, or became polarised.

Q. Explain how polarised light DIFFers from other light.

$A$. Suppose we have a cylinder with a mirror at one end of it, If we point this to the sun, and receive the image on a distant screen, we may turn the cylinder round on its axis, and the reflected ray will be found to revolve constantly with it. But if now, instead of receiving the ray direct from the sun, we allow a beam, reflected from a glass plate, at an angle of about $54^{\circ}$, to fall upon the mirror, and then be reflected on the screen, it will be found that the point of light will not have the same properties as that previously examined; it will be altered in its degree of intensity as the cylinder turns round; will have points where it is very bright, and others where it will be lost in shadow.

If we allow the pencil of light to fall upon a plate of glass at any usual angle, a part of it will pass through the glass, and a part of it will be reflected. If we place a second plate of glass over the reflected ray, a second reflection will take place on whatever side of the ray the reflecting glass may be placed. But if the ray falls upon the second glass at an angle of $56^{\circ}$ from the perpendicular, the light reflected by the first glass will be again reflected by the second, when the planes of the two glasses are parallel to each other ; but if, without altering its angle to the horizon, we turn the second glass to the quarter of a circle, the light will be transmitted, not reflected; if we turn it another quarter, the light will be reflected, not transmitted. By this we prove that light reflected at an angle of about $56^{\circ}$ has undergone some peculiar modifications.

Q. Why was light so modified called Poinarised light?

$A$. Because, when this curious property of light was first discovered, it was theoretically ascribed to a kind of polurity of such sort that each side of a ray of light was thought to have a character different from the two adjacent sides at right angles to it.

Q. To what has the beam of polarised light been COMPARED?

$A$. To a "long, flat, straight stick" having sides, the ordinary ray of light being regarded as cylindrical.

Q. Is light polarised by reflection from any oTHER substance than glass?

A. Light is polarised by reflection from many different substances, such as glass, water, air, ebony, mother-of-pearl, surfaces of crystals, \&c. \&c., provided that the light falls at a certain angle peculiar to each surface. This angle is called the polarising angle.

Q. How was Arago ENABLED to DEFINE the nature of the incandescent matter of the sun by means of the action of polarised light?

$A$. He ascertained, first, that light proceeding from such bodies as red-hot iron, glass, and liquids, under a certain angle, was polarised light; but the light proceeding, under the same circumstances, from an inflamed gaseous substance, such as is used in streetillumina- 
tion, is always in its natural state. Applying these observations to the sun, he discovered that the light-giving substance of the sun was of the nature of a gas, and not a red-hot solid or liquid.

Q. Does this phenomena of polarised light enable us also to tell whether the fixed stars shine by their OWN LIGHT, as the sun, or by REFLEOTED LIGHT, as the moon?

$A$. It does; all reflected light, come from whence it may, acquires certain properties which enable us to distinguish it from direct light; and thus the astronomer is enabled to determine with infallible precision whether the light he is gazing on (and which, from its distance, has taken thousands of years to reach the eye) be inherent in the body itself, or derived from some source without.

Q. Suppose we look at the shadow of a man on smooth water, through a polarising prism : by turning the glass, we shall find that in a certain position the shadow of the man will DISAPPEAR altogether, while the man himself is SEEN with all DISTINCTNESS : why is this?

$A$. It is occasioned by much of the light on the water being polarised by reflection; and therefore, as before shown, it is incapable of a second reflection by the prism, while the common light from the man himself admits of a reflection to the eye.

Q. It is well known that, by looking into the water from a great height, we can see farther into its depth than when looking from a convenient height above the surface: why is this?

$A$. It is owing to the fact that much of the light polarised by reflection from the water, and which obscures the vision when we view the water from a near position, is lost to the eye when at a considerable height above it.

\section{CHAPTER XXXVII.}

\section{ACTINISM, OR THE CHEMICAL INFLUENCE OF LIGHT.}

Q. What is the meaning of the term " $\boldsymbol{\Lambda}$ CTINISM?"

$A$. It is the name applied to the chemical agency of the sun's rays. ray?

Q. Besides heat and light, is there any oTHER distinct PRINCIPLE existing in the solar

A. There is; the chemical principle.

Q. What is the meaning of the term " Pнотоgraphy?"

A. Light drawing. The use of this term is most unfortunate, for light does not exercise that influence in producing what are called photographic pictures, which is usually supposed to be the case.

Q. How do we know that a SEPARATE chemical principle exists in the solar ray?

A. Because we can separate it from heat and light. There are certain media through which, if we pass a solar ray, one or two of its elements may be separated from the others; thus, a ray of light 
passes readily through a transparent plate of alum, but nearly all the heat is cusorbed. Certain dark-coloured bodies allow nearly all the heat to pass, and obstruct the light. A blue glass obstructs nearly all the light and heat of the solar ray, but allows the chemical principle to pass freely; while a yellow glass allows light and heat to pass, but obstructs the passage of the chemical influence.

Q. Th what may the constitution of the solar ray be CoMPARED?

$A$. To a bundle composed of three sticks: one representing heat, another light, and a third the actinis power-any one of which may be in a great degree separated from the other.

Q. When we decompose light by means of a prism, and throw the spectrum upon a kcreen, what are the RELATIVE Positions assumed by the three different principles?

$A$. All these principles will be refracted, but the calorific principle lrast of any. Its maximum point will be found but slightly thrown out of the right line which the solar ray wonld have traversed had it not been intercepted by the prism, and the heating action will be found to diminish with much regularity on each side of this line.

Q. What will be the posirion of the luminous principle?

$A$. It will be found to be subject to a greater refraction, its point of maximum intensity being in the yellow ray, lying considerably chove the point of greatest heat. The light diminishes on each side of it, producing orange, red, and crimson colours below the maximum point, and green, blue, and violet above it.

Q. What will be the Posirion of the chemical or actinic principle?

$A$. The radiations which produce the chemical change are more refrangible than either of the others, and the maximum of this power is to be found at the point where light rupidly diminishes, and where scarcely any heat can be detected.

Q. What curIoUS FACT has the study of the chemical principle of light elicited?

$A$. That no substance can be exposed to the sun's rays without undergoing a chemical change.

Q. Does DARKNESS appear to be ESSENTIAL to the healthy condition of inorganic matter as well as organised structures?

A. From numerous examples, it would seem that the change in the condition of bodies which the sunlight effects during the daytime is made up during the hours of night, when that excitement is no longer influencing them.

Q. What ILlustration can you give of this?

$A$. If a photographic impression be caused upon a sensitive surface at night, and no expedient for rendering it permanent be adopted, it fades away before morning, and we try to evoke it in vain.

Q. Why do colours FADE when exposed to the sunlight?

$A$. The materials of which the colours are composed become changed and altered through the action of the actinic principle of solar light. 
Q. Upon what PRINcrple does the process of forming the daguerreotype and other photographic pictures DEPEND?

A. Solely upon the actinic or chemical infuence of light.

Q. Wro made the first definite experiments which led to the discovery of the daguerreotype?

A. M. Niepce, a Frenchman of Chalons.

Q. What did he Drscover?

$A$. He ascertained that when a surface was covered with resin, and exposed to the solar rays, that the portion on which the bright light fell became much more solulle than those parts which had been influenced by the wealer rays, and could consequently be dissolved off before the other portion; thus an imperfect picture could be formed.

Q. Who PERFECTED the discovery thus made?

A. M. Daguerre, of Paris, from whom the process derives its name.

Q. What did he Drscover?

$A$. He ascertained that several compounds of silver were most easily affected by the action of light; those parts upon which the light fell brightly being quickly decomposed, while those portions protected by the shadow remained untouched. He also discovered a method of fixing the image thus drawn.

Q. What is a CAMERA OBSCURA?

$A$. In its original state it was nothing more than a darle room, with a very small opening, into which was fitted a lens.

If we make such an arrangement, and hold a sheet of white paper a few feet behind the opening, a very perfect image of any external object will be delineated upon it.

\section{Fig. 113.}

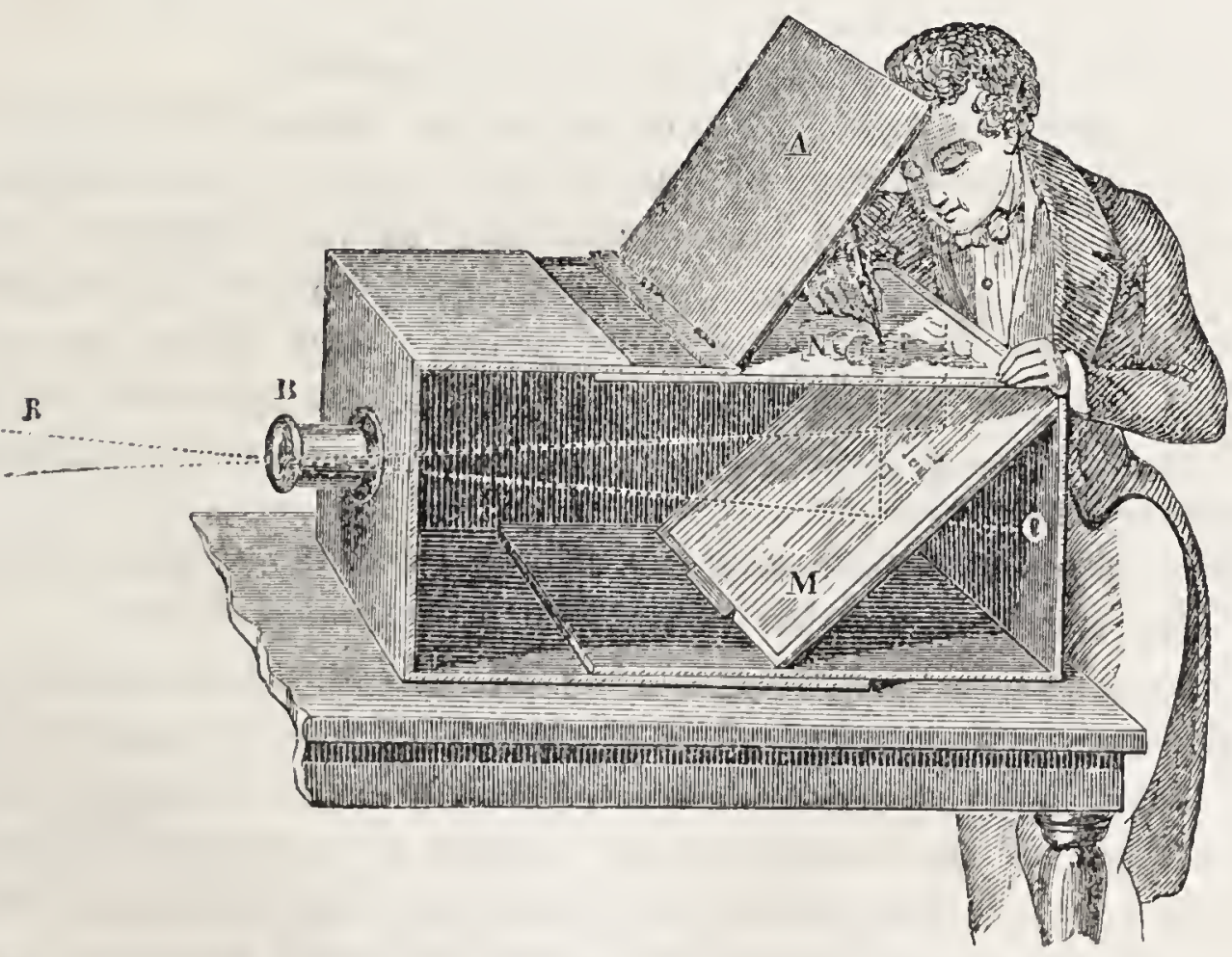

In $t$ is dingram (fig. 113) the onmera, or dark cbamber, is represented. It consists of a 
wooden box, into which the rays, $R$, enter through the lens, $B$, and form a picture on the opposite side, 0 . The rays of light, $\mathrm{R}$, encountering a glass mirror, M, change their direction, and the picture is formed on a screen of polished glass, N. By placing upon this screen a sheet of paper, we can make a true outline drawing of the picture. The screen, A, serves to intercept the light from the glass, $N$.

Q. What is the PRocess for taking a daguerreotype?

A. The production of a daguerrotype picture consists in preparing a silver plate by exposing it to the influence of iodine, and the vapour of bromine water. The plate is then exposed to the image formed by the lens of a camera obscura. Relatively, the quantity of light and actinism reflected from any object is the same; therefore, as the light and shadows of the luminous image vary, so will the power of producing change upon the plate vary, and the result will be the production of an image which will be a faithful copy of nature, with reversed lights and shadows; the lights darken the plate, while it is preserved white in the shadows.

Q. If the plate were then left without further care, what would HAPPEN to the image formed?

A. It would soon fade away, and leave no trace upon the plate.

Q. When the picture is taken from the camera, is there any IMAGE VISIBLE?

$A$. If the plate were allowed to remain a sufficiont time, the image would be visible; but in practice it is not allowed to assume this condition. After an exposure of a few seconds, the radiant influence is cut off, and no trace of an image can then be detected on the plate, even with the aid of a magnifying power.

Q. How, then, is the picture DEVELOPED, or brought out, and rendered PERMANENT?

$A$. It is exposed to the vapour of mercury; and this metal, in a state of very fine division, is condensed upon the surface of the plate.

Q. Is that condensation spread UNIFormLY upon its surface?

A. It is not: in exact proportion to the amount of chemical action produced, is the deposit of mercurial vapour, each layer being relatively to every other one, according to its thickness, an exact measure of the quantity of solar action. Where the shadows are deep there is scarcely a trace of mercury; and where the lights are strong the metallic globules are deposited of considerable thickness.

Q. What is the REASON that the mercury attaches itself only to those parts affected by the chemical influence of light?

$A$. We do not know how to account for it satisfactorily; in all probability, we have the involved action of several forces.

Q. Is it necessary that a surface should be CHEMICALLY PREPARED to exhibit this result

A. It is not; a polished plate of metal, a piece of marble, of glass, or even wood, when partially exposed to the action of light, will, when breathed upon, or presented to the action of mercurial vapour, shor that a disturbance has been produced upon the portions which were illuminated; whereas no change can be detected upon the parts kept in the dark. 
Q. Can a daguerreotype picture be taken in ABSOLUTE DARENESS?

$A$. It can, in the following manner:-A large prismatic spectrum is thrown upon a lens fitted into one side of a dark chamber; and as we know that the actinic power resides in great activity beyond the violet ray, where there is no light, the only rays which we allow to pass the lens into the chamber are those beyond the limit of coloration, and non-luminous; these are directed upon any object, and from that object radiated upon a highly sensitive plate in a camera. Thus a copy of the subject will be produced by the agency of radiations, which produce no effect upon the optic nerve.

Q. By what RAYS of the solar spectrum are VEGETABLE COLOURS most liable to be changed?

$A$. By the colours which are complementary to them.

Q. How do you illustrate this?

A. A red dye or colour fades under the influence of a green light, and a yellow under that of a blie light, much more speedily than when exposed to rays of any other colour.

Q. What SUBSTANCE is used in nearly all the preparations of INDELIBLE INES and washes for colouring the hair black?

A. A compound of silver: generally the salt known as nitrate of silver, or "lunar caustic."

Q. Wну is this substance especially used?

$A$. Because it turns black under the influence of light, and darliens whatever substance, it may be in contact with.

Q. Why does nitrate of silver TURN DARK when exposed to the action of light?

$A$. Because the chemical influence of light decomposes the nitrate of sitver, and leaves only the oxide of silver, which is black.

Q. Is there any reason for supposing that each of the three principles (light, heat, and actinism) included in a ray of solar light exercises a DISTINCT and PECULIAR INFLUENCE on VEGETATION?

$A$. There is no doubt of the fact; and also that solar light, at different seasons of the year, is in some way modified as respects the quantity it contains of these elements, in order to meet the wants of vegetation at different periods.

Q. What is supposed to be the Pecultar INFLUence exerted by the actinic element on vegetation?

A. It is supposed to be an active agent in exciting germination. Seeds exposed to its influence germinate with great rapidity, while if exposed to the influence of light deprived of its chemical element, they will not germinate at all.

Q. Is this principle found to be MoRE ACrIVE at any one portion of the year than at another?

$A$. It is in the spring, at which time solar light possesses an excess of this influence.

Q. What PART is light supposed to play in the development of vegetation?

$A$. It is by the influence of the luminous ray that carbon is secreted from the atmosphere, and the woody tissues of the plant built up. 
Q. Is this principle more active and abundant at oNE SEASON than at ANOTHER?

A. It is more abundant in the summer than at any other perion. Light and actinism together are essential to the production of the colouring matter of leaves and flowers.

Q. What PART is the peculiar calorific element of the solar ray supposed to exerti in the process of vegetation?

A. It facilitates the flowering of plants, and the production and ripening of seeds and fruits.

Q. Is this principle rore $A$ CTIVE at one seascn than at another?

A. In the autumn light and actinism both diminish, and calorific radiations are relatively to them the most extensive.

Q. How MUCH QUICG will seeds germinate under a blue glass than when exposed freely to the full action of light?

A. Seeds which ordinarily require ten or twelve days for germination, will germinate under a blue glass in two or three.

Q. Why will seeds germinate MORE READIIY under a BLUE glass than in any other condition?

A. Because the blue glass permits the chemical principle of light to pass freely, and excludes in a degree the heat and light.

Q. If we expose a seed under a STRONG LIGET, but with all the necessary conditions of warmth and moisture, will it germinate?

A. It will not; the action of the light prevents germination, for the full success of which shade or darkness is necessary,

Q. Why is it impossible to make seeds germinate under a FELLOW GLASS ?

A. Because a yellow glass excludes all the chemical infuence of the solar ray.

Q. How do plants acquire the CARBON of which their structure is in a great measure composed?

A. They obtain it from carbonic acid, which they abstract from the atmosphere under the influence of light.

Q. Why are plants grown in the dark colouriess?

A. Because they have no power to lay up chlorophyl, the green colouring matter of plants. This substance is principally composed of carbon. In the dark the plant still requires carbon for its further development; and, being unable to obtain it from the air, it removes it from the leaves, decomposing the chlorophyl, and thus supports its weak existence by preying on parts of its own structure, until at length, this being exhausted, it actually perishes of starvation. 


\section{E L E C T R I C I T Y.}

\section{CHAPTER XXXVIII.}

\section{NATURE AND PRODUCTION OF ELECTRICITY.}

Q. What is ELECTRICITY ?

A. Electricity is one of those impondercuble (that cannot be weighed) agents that appear to be diffused through all nature, existing in all substances without affecting their volume or their temperature, or giving any indication of its presence when in a latent state. When, however, it is liberated from this repose, it is capable of producing the most sudden and destructive effects, or of exerting powerful influences by a quiet and long-continued action.

Q. Was ELECTRICITY known to the ANCIENTS?

$A$. Yes; they knew that when amber (the Greek word of which is $\eta \lambda \varepsilon \kappa r \rho o \nu$, electron) is rubbed it acquires the power of attracting other bodies.

Q. Wно first noticed this property of amber?

A. Thales of Miletus; and it is from this Greek philosopher that we must date the commencement of our knowledge respecting this great natural agent.

Q. How may electricity be called into AcTIVITY ?

A. By mechanical power, by chemical action, by heat, and by magnetic influence.

Q. What is the most ORDINARY WAY of exciting electricity?

A. By friction.

Q. Do we know any REASON why the means above enumerated should develope electricity from its latent condition?

A. We are entively ignorant upon this subject, and do not know whether electricity is a material agent, a property of matter, or vibrations of an ether.

Q. When you rub a piece of paper with INDIA-RUBBER, why does it adhere to the TABLE?

A. Because the friction of the india-rubber against the surface of the paper developes electricity, to which this adhesiveness is mainly to be attributed.

Q. Does electricity present any appearance by which it can be known?

A. No; electricity, like heat, is in itself invisible, though often accompanied by both light and heat. 
Fig. 114.

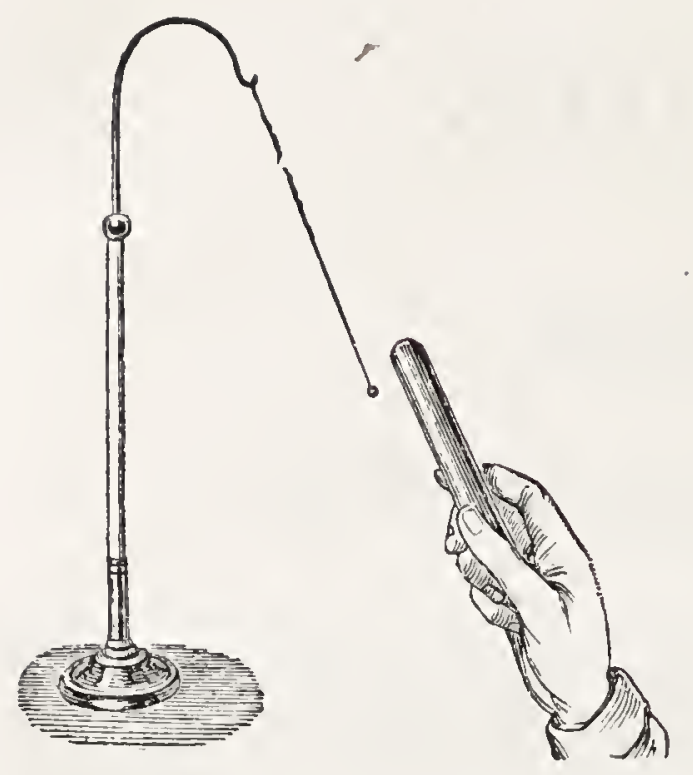

Q. How can we AScertain that a substance, whether by friction or other means, has been electrified?

$A$. This can be ascertained by means of small instruments, called electroscopes; the most simple of which is the electric pendulum.

This apparatus (see fig. 114) consists of a small pith ball, hung by a silk thread to a support with a glass foot. When an electrified body is brought near the ball, it is attracted, and then repelled immediately after contact.

Q. Is electricity accompanied with any ODOUR?

A. Yes; near a large electrical machine in good action there is always a peculiar sulphurous odour, which is ascribed to the production of a peculiar modification of oxygen, called "ozone."

Q. Has this peculiar odour, called "OZONE," been observed in THUNDER-sTORMS?

$A$. It has; especially in and about places and objects struck with lightning.

Q. Why are there DIFFerent colours in the aurora borealis, such as white, yellow, red, and purple?

$A$. Because it is supposed that the electric current producing the aurora passes through air of different densities. The most rarefied air produces a white light; the most dry air, red ; and the most damp produces yellow streaks.

Q. When a substance, by friction or by any other means, acquires the property of attracting other bodies, in what STATE is it said to be?

A. It is said to be electrified, or electrically excited ; and its motion towards other bodies, or of other bodies towards it, is ascribed to a force called electric attraction.

Q. Does an electrified body exercise any oTHER INFLUENCE than an attractive one?

A. It does; for it will be found that light substances, after touching the electrified body, will recede from it just as actively as they approached it before contact. This is termed electric repulsion.

Q. What is a NON-ELECTRIFIED body?

A. One that holds its own natural quantity of electricity undisturbed.

Q. What HAPPENS when an electrified body touches one that is non-electrified?

$A$. The electricity contained in the former is transferred in part to the latter.

Thus, on touching the end of a suspended silk thread with a piece of excited wax, the silk will be excited, as will be shown by its moving towards a book, piece of metal, or any other object placed near it, as already shown in fig. 114 .

Q. Do ALL BoDies conduct or allow electricity to pass through them equally well ?

A. Although three is so substance that can entirely prevent the 
passage of electricity, nor any that does not oppose some resistance to its passage, yet it moves with a much greater facility through a certain class of substances than through others. Those substances which facilitate its passage are called conductors; those that retard or almost prevent it, are called non-conductors.

Q. What SUBSTANCES are good CONDUCTORS of electricity?

A. The metals, charcoal, the earth, water, and most fuids (except oils), the human body, \&c. \&c., are good conductors.

Q. What substances oвsтrucr the passage of electricity, or are, as they are sometimes called, "non-conductors?"

A. Gluss, resin, oils, sill, sulphur, dry air, \&c. \&c., are nonconductors.

The old notion that some bodies were electrics or non-conductors, and others nonelectrics or conductors-that is to say, that some substallces were capable of becoming electrical, and that others were not-is incorrect. Everything in nature is capable of conducting electricity more or less; there is neither a perfect conductor nor one totally incapable of conduction. The difference is of degree, and not of kind.

Q. When a body capable of being easily electrified is surrounded or insulated from all other conductors by means of non-conducting substances, can electricity be ACCUMULATED in it?

$A$. It can to a very great extent; but as all substances conduct electricity more or less, the surplus quantity will gradually pass off.

Q. Does the SHAPE of the body have any influence in retaining electricity?

$A$. It does: it is more easily retained by a sphere than by a spheroid and a cylinder; but it readily escapes from a point, and a pointed object receives it with the greatest facility.

Q. Does there appear to be a DIFFERENCE between the kind of electricity excited by rubbing a piece of GLASS and that excited by rubbing a piece of WAX Or RESIN?

$A$. The electricities developed in these two ways are essentially different; and hence it has been inferred that there are two kinds or states of electricity-the one called vitreous, because especially developed on glass; and the other resinous, because first noticed on resinous substances.

Q. How does this DIFFERENCE MANIFEST itself?

$A$. If two substances are charged with vitreous electricity, they repel each other; two substances charged with resinous electricity also repel each other; but if one is charged with vitreous, and the other with resinous electricity, they attract each other.

Q. What THEORY is based upon these phenomena of attraction and repulsion by the different kinds of electricity?

A. The theory of Du Fay, or the theory of two fluids.

Q. What does this theory SUPPOSE?

A. It supposes an exceedingly thin and subtle fluid pervading the most compact bodies, which is composed of two ultimate elements, namely, the vitreous and the resinous electricities. Each kind is supposed to repel its own particles, but attract the particles of the other kind. 
Q. What OTHER THEORY has been propused to account for the phenomena of electricity?

$A$. The theory of a single fluid, or the Franklinian theory, so called from its having originated with Dr. Franklin.

Q. What does this theory SUppose?

A. It supposes the existence of a single, imponderable (that cannot be weighed), subtle fuid equally distributed throughout nature. Every substance, organic or inorganic, being so constituted as to retain a certain quantity of this agent, which is necessary to its physical condition, but when, in repose, offering no evidence of electrical presence. Any disturbance of the natural state of a body produces evidences of electricity.

Q. How does this theory explain the REASON of the differences in the electricity developed by GLASS and RESTN?

A. It supposes electrical excitation to arise from the difference in the relative quantities of this principle existing in the body rubbed and the rubber, or in their powers of receiving and retaining electricity.

Thus one body becomes overcharged by having abstracted this principle from the other.

Q. What NAMES did Franklin, in accordance with the theory, apply to the two different conditions of electricity, called by Du Fay vitreous and resinous?

A. Positive and negative, or plus and minus. Thus a body which has an overplus of electricity is called positive, and one that has less than its natural quantity is called negative.

Q. What theory has lately been proposed by Professor Faradax respecting the nature of electricity?

$A$. He considers it a mere power of matter, like what we conceive of the attraction of gravitation.

Q. Do light, heat, and electricity appear to have some properties common?

$A$. They do ; each may be made, under certain circumstances, to produce or excite the other. All are so light, subtle, and diffusive, that it has been found impossible to recognise in them the ordinary characteristics of matter. Some suppose that light, heat, and electricity are all modifications of some common principle.

Q. Why does. the FUR of a CAT sparkle and crackle when rubbed with the hand in cold weather?

$A$. Because the friction between the hand and the fur produces an excitation of positive electricity in the hand and negative in the fur, and an interchange of the two causes a spark, with a slight noise.

Q. Why does this experiment work best in very COLD WEATHER?

$A$. Because the air is then very dry, and does not convey away the electricity as fast as it is excited; if the air, on the contrary, were moist, the electricity would be conducted off nearly as fast as it was excited by friction, and its effects would not therefore be so manifest.

Q. With what vELocrTY is electricity transmitted throngh good conductors?

$A$. With a velocity so great that the most rapid motion produced 
by art appear's to be actual rest when compared to it. Some authorities have estimated that electricity will pass through copper wire at the rate of two hundred and eighty-eight thousand miles in a second of time-a velocity greater than that of light. The results obtained, however, by experiments with iron wire, show a velocity of from 15,000 to 20,000 miles per second.

Q. Is a perfect conductor $\triangle F F E C T E D$ by the passage of a current of electricity?

$A$. If it be of sufficient size to carry off the whole easily, it is not affected; but it is shivered into pieces in an instant if it happens to be too small: this also happens to a very bad conductor.

Q. What AGENTS are undoubtedly the most ACTVE in producing and exciting electri city in the operations of nature?

$A$. The light and the sun's rays.

Q. It has become the habit with mauy to ascribe to electricity the agency of phenomena in the natural world, the cause of which may not be appalent: is there any REASON for this?

A. There certainly is not; electricity is diffused through all malter, and is ever active, and many of its phenomena cannot be satisfactorily explained; but it is governed, like all other forces of nature, by certain fixed laws, and it is by no means a necessary agent in all the operations of nature.

It argues great ignorance to refer, without examination, every mysterious phenomena to the induence of electricity.

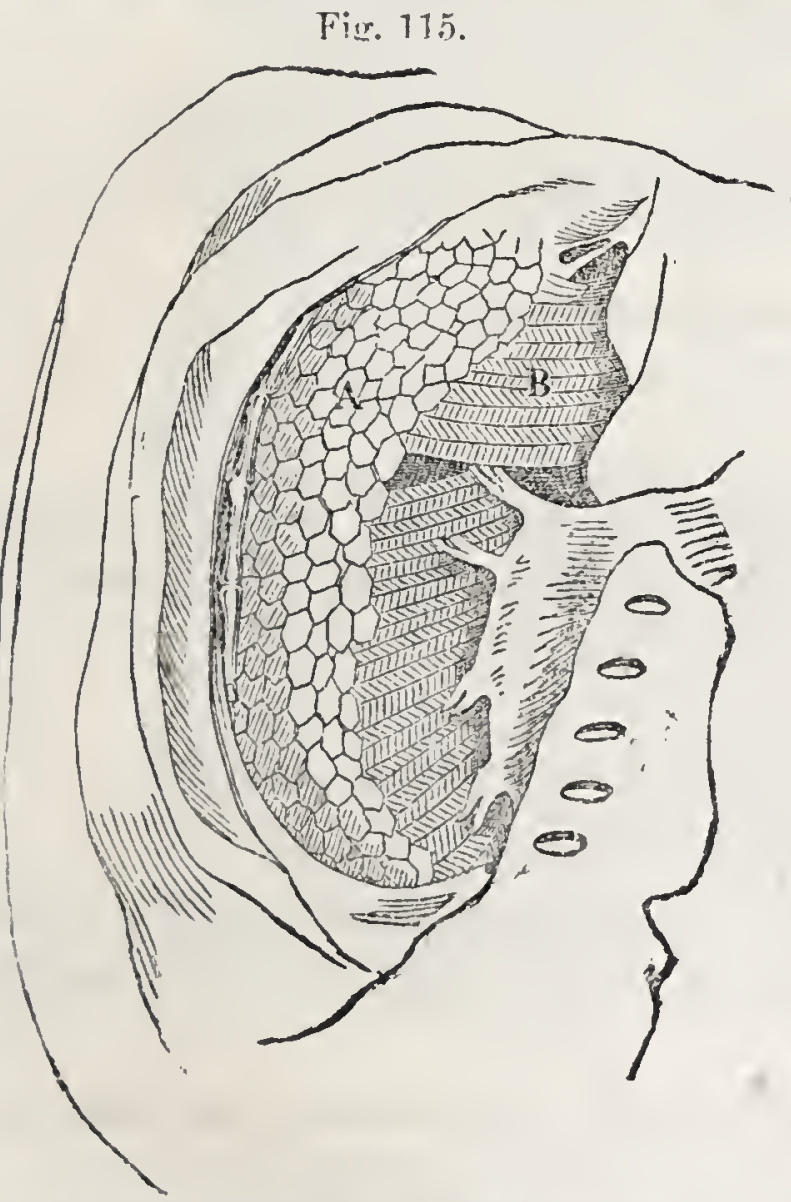

Fig. 116

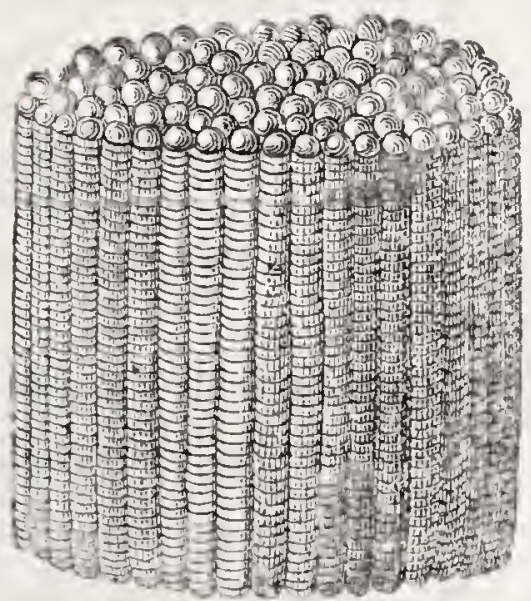

PERPENDICULAR SECTION Of PHISMS,

HEFCTRICAL ORRAN OF TIE TORPEDO.

A, trussuerse section; $B$, position of prisms, or seat of electricity. 
Q. Do some animals have the power of exciting electricity WITHIN THEMSELVES ?

A. There are certain animals which are gifted with the extraordinary power of producing electrical phenomena by an effort of muscular or nervous energy. Among these the electrical eel and thes torpedo are most remarkable. (See figs. 115, 116.)

Q. How powerful a charge of electricity can the ELECTRICAL EEL send forth when in full vigour?

A. Sufficient to knock down a man or stun a horse.

Q. Is the electricity generated by these animals the SAME as that occasioned by the ORDINARY ELECTRICAL MACHINE?

A. It $i$ s the same, and produces the same effects.

Q. Do vital action and muscular movements in man and animals give rise to electricity?

$A$. They do; and it can be shown by direct experiment that a person cannot even contract the muscles of the arm without exciting an electrical action.

Q. Does Change of Form or STATE in bodies generally produce electrical excitation?

A. Change of form or state is one of the most powerful methods of exciting electricity.

Water, in passing into steam by artificial heat, or in evaporating by the action of the sun or wind, generates large quantities of electricity. The crystallisation of solids from liquids, all changes of temperature, the growth and decay of vegetables, are also instrumental in producing electrical phenomena.

\section{ELECTRICAL MACHINES.}

Q. By whom was the FIRST ELECTRICAL MACHINE invented?

A. Otto de Guericke, who also invented the air-pump.

Q. How was it Formed?

A. It consisted of a ball of sulphur fixed to an axis turned with one hant, while the other rested on the sphere, and served to rub it.

Q. What was the Next IMprovement?

A. The ball of sulphur was exchanged for a glass cylinder.

Q. What IMPRovement was intreduced by WINKLER?

A. In 1740, Winkler, a German philosopher, substituted a horsehair cushion, covered with silk, instead of the hand, for rubbing the cylinder.

Q. When were such ELECTRICAL MACHINES as are now commonly used fIRST employed?

A. The present ordinary form of the electrical machine was introduced by Mr. Ramsden in London about 1766. It consisted of a glass plate rubbed by custions. 
Fig. 117 represents an electrifying machine. Between two wooden supports is a circular glass plate, $\mathrm{P}$, fixed at its centre to an axis, and turned by a winch. This plate is pressed by four leather pads, called RuBbers, F. Two brass tubes, called comes, face the plate, and are fixed to two larger tubes, C, called conductors, which stand on four glass legs. As the glass plate is moved round it is electrified positively, and the cushions negatively. These latter lose their electricity by communicating it to the earth through the wooden supports as soon as it is produced. The positive electricity of the plate acts on the conductors, and attracts the negative fluid, which combines with the positive electricity of the glass, and neutralises it. The conductors, which thus lose their negative electricity, therefore, remain positively electrified. The machine, being once charged, on placing the hand near it, a strong spark is elicited, which is repeated as long as the plate continues to be turned; for the spark being the result of the combination of the negative fluid of the hand with the positive fluid of the machine, the latter tends at each spark to return to the neutral state, but the influence of the plate excites it afresh.

Fig. 117.

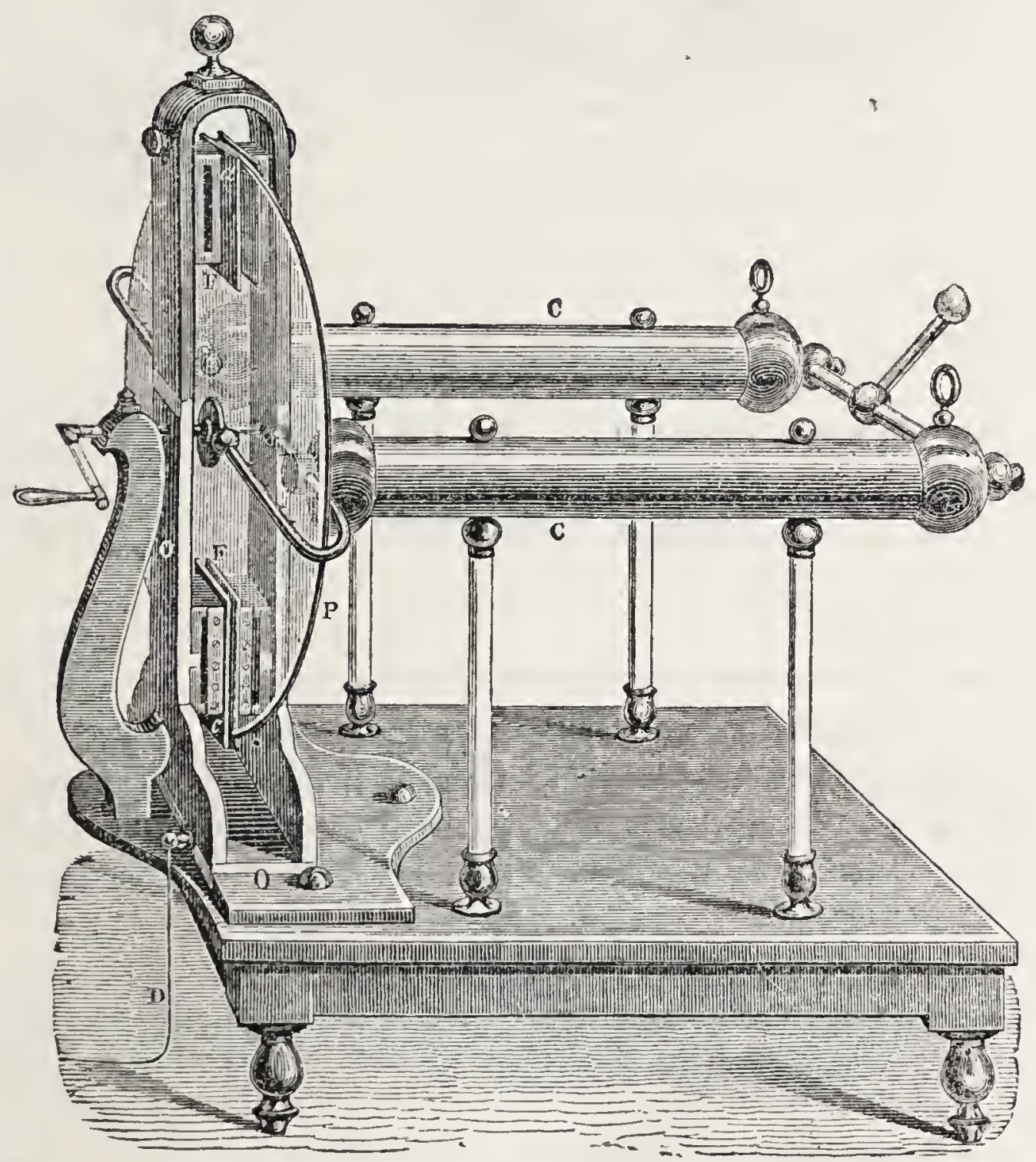

In fig. 118 we have represented Nairne's electrical machine. It is formed of two conductors, which do not touch one another; the one has a rubber, C, nade of leather, and stuffed with horse-hair; the other a comb, P, formed with several teeth. Between them is a glass cylinder. When turned, this glass cylinder excites electricity, negative 
and positive, given forth in a series of sparks at the copper balls ending the curved branches $\mathrm{D}$ and $\mathrm{E}$.

Fig. 118.

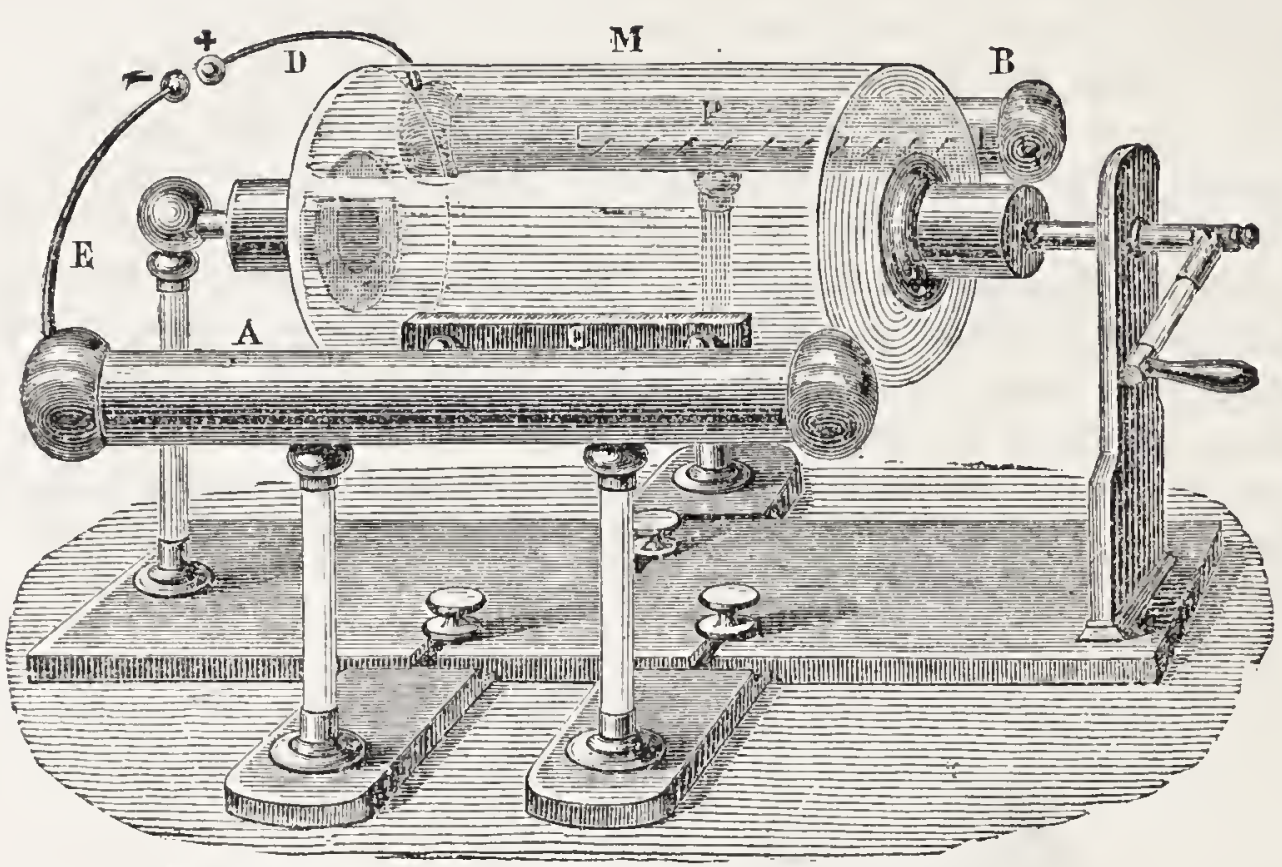

\title{
CHAPTER XXXIX.
}

\section{ATMOSPHERIC ELECTRICITY.}

\author{
SECTION I.-LIGHTNING.
}

Q. That is LIGHTNING?

A. Electricity accumulated in and discharged from the cloucts.

Q. By what means is this ELECTRICITY in the clouds produced?

A. By the evaporation from and the chemical changes which take place on the earth's surface, together with the expansion, condensation, and variation of temperature of the atmosphere and of the moisture contained in it.

Q. Does the amount of electricity in the air VARY at DIFEERFivT ALTITUDES?

$A$. The electricity of the air increases in strength with the altitude. It is sometimes different in the lower regions from what it is in the upper, being positive in one and negative in the other.

Q. How is the AMOUNT of electricity in the air DETERMINED?

$A$. By means of an electrometer.

Q. What is an ELECTroMETER?

A. It is an instrument which serves to indicate and measure clectricity. The one often used consists of a glass jar, surmounted by a 
pointed metallic rod, to the lower end of which, within the jar, two fine straws are loosely attached. The pointed rod collecting the electricity from the air, the two straws become similarly electrified, and recede from each other. The amount of divergence measures the quantity of the fluid.

Fig. 119.

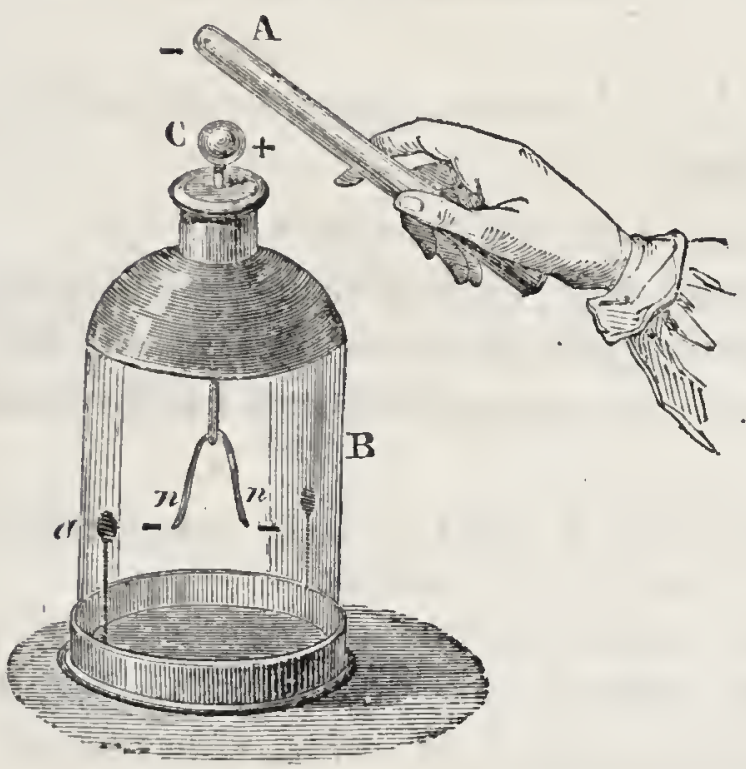

In the accompanying diagram (fig. $119)$ is shown what is called BenNerr's ELECTROMETER. It consists of a glass jar, B, resting upon a plate of copper, and baving its neck stopped by a cork. Through the cork passes a copper wire, having a ball, $C$, at one end, and tw: pieces of very thin gold-leaf at the other, inside the jar. When a body charged with electricity (negative) is brought near, this electricity acting upon the neutral fluid of the ball and the wire, the positive fluid in the ball is attracted, and the negative repelled, towards the pieces of gold-leaf, which, being charged with the same kind of electricity, repel each other. Thus we see that the substance $A$ is electrised. Different other experiments of the same nature may be made with this apparatus.

Q. What SORT of ELECTRICITY pervades the atmosphere, or in what condition is it generally?

$A$. In the ordinary state of the atmosphere its electricity is invariably positive.

Q. Under what CIRCOMSTANCES does the electrical condition of the atmosphere CHANGE ?

$A$. When the sky is overcast, and the clouds are moving in different directions, the atmosphere is subject to great and sudden variations, rapidly changing from positive to negative, and back again, in the space of a few minutes.

Q. When is the atmosphere MosT HIGHLT charged with electricity?

$A$. When hot weather succeeds a series of wet days, or wet weather. follows a succession of dry days.

Q. Is there more of electricity at ONE SEASON in the atmosphere than at another

A. There is more electricity in the atmosphere during the cold of winter than in the summer months.

Q. Is electricity ADDED to the atmosphere during the PRocess of COMBUSTION?

A. When a substance is burning, positive electricity escapes from it into the atmosphere, while the substance itself becomes negatively electrified. 'Thus the air becomes the receptacle of a vast amount of positive electricity generated in this manner.

Q. What CAUSES the DISCHARGE of an ELECTRIO CLOUD?

$A$. When a cloud overcharged with electric fluid approaches another which is undercharged, the fluid rushes from the former into the latter, till both contain the same quantity. 
Q. Is there any otHER CAUSE of LIGHTNING besides the one just mentioned?

A. Yes; sometimes mountains, trees, and steeples will discharge the lightning from a cloud floating "near, and sometimes electric fluid passes from the earth into the clouds.

Q. What is the ESTIMATED HEIGHT of LIGHTNING-CLOUDS above the earth ?

$A$. Their height varies from five miles to a few feet above the earth's'surface. The usual height in a storm is 700 yards.

Q. What is a THUNDER-STORM?

A. The disturbance caused in the air when successive discharges of accumulated electricity take place.

Q. How do thunder-storms occur, as regards LATITUDE and TIME?

$A$. They prevail most in the torrid zone, and decrease in frequency towards either pole. In the Arctic regions thunder-storms seldom or never occur. As respects time, they are most fiequent in the summer months.

Q. Why should the DISCHARGE of ELECTRICITY from cloud to cloud, or from a cloud to the earth, produce the VIVID FLASH of light which we call lightning?

$A$. When a portion of air is subjected to a very sudden and powerful compression, a spark is elicited. It can be proved that electricity produces such compression; and it is to the almost instantaneous condensation of the atmosphere before the electric fluid, as it darts from point to point, that the vivid flash we see is attributed.

Q. Into how MANY KINDS has lightning been divided?

A. Three.

Q. What ARE THEY?

A. The zigzag lightning, sheet lightning, and ball lightning.

Q. Why is lightning sometimes FORKED ?

$A$. Because the lightning cloud is at a great distance; and the resistance of the air is so great, that the electrical current is diverted into a zigzag course.

Q. How does the RESISTANCE of the AIr make the lightning Zigzag?

A. As the lightning condenses the air in the immediate advance of its path, it flies from side to side, in order to pass where there is the least resistance.

Q. Why is the FLASH sometimes quite STRAIGHT?

$A$. Because the lightning cloud is near the earth, and as the flash meets with very little resistance, it is not diverted; in other words, the flash is straight.

Q. What is SHEET LIGHTNING?

A. Either the reflection of distant flashes not distinctly visible, or beneath the horizon; or else several flashes intermingled.

Q. What oTHER FOrM does lightning assume?

$A$. Sometimes the flash is globular, which is the most dangerous form of lightning. 
Q. How is BALL LightNing supposed to be produced?

A. Philosophers have not yet been able to account for lightning of this description : it has, however, been supposed to arise from an uninterrupted discharge of electricity.

Q. Does a discharge produce a FLASH when it passes through GOoD conductors?

$A$. It does not, but passes quietly and invisibly.

Q. What is HeAt Lighiting?

$A$. Sometimes it is the reflection in the atmosphere of the lightnings of storms very remote, the storms themselves being so far distant that their thunders cannot be heard. This phenomenon is also occasioned by the play of silent flashes of electricity between the earth and the clouds, the amount of electricity developed not being sufficient to produce any other effects than the mere flash of light.

Q. When lightning flashes from the earth to the clouds, what is the flash CALLFid ?

$A$. It is popularly called the "returning stroke;" because the earth (being overcharged with electric fluid) returns the surplus quantity to the clouds.

Q. When does lightning pass FROM THE EARTH to the CLOUDS ?

4. When the clouds are in a "negative" state of electricity.

Q. When does lightning pass FROM THE CLOUDS to the EARTH?

$A$. When the clouds are in a "positive" state of electricity.

Q. What is generally meant by the clouds being in a "Positive STATE" of electricity?

$A$. When the clouds contain more electric fluid than they generally do, they are said to be in a " positive state of electricity."

Q. What is generally meant by the clouds being in a "NEGATIVE STATE" of electricity?

$A$. When the clouds contain less electric fuid than they generally do, they are said to be in a " negative state of electricity."

Q. Does the flash proceed from a NEGATIVE or Positive body?

A. Always from a positive body; that is, from one over-charged with electric fluid.

Q. How LONG is the DURATION of a flash of lightning?

A. Arago has demonstrated that it does not exceed the millionth part of a second.

Q. With what vecocity is lightning, or the electric fluid which gives rise to its appearance, supposed to rove?

A. Not less than two hundred and fifty thousand miles per second.

\section{SECTION II.-EFFECTS OF LIGHTNING.}

Q. Is there ANY DANGER attending LIGHTNING?

A. Yes; its effects are sometimes fatal.

Q. How are the EFFECTS of LIGHTNING fatal ?

A. By the electric fluid passing through the living body, and producing so violent an action on the nervous system that death ensues. 
Q. Will LIautwiva, then, pass through the LIVING Body?

A. Yes; if that body intercept its passage to the earth.

Q. What is the SAFEST thing a person can do to avoid injury from lightning?

A. Lie upon a bed in the middle of a room. A bed filled with feathers is an excellent non-conductor.

Q. Is there not generally a GREATER FEAR of the danger from lightning than experience would JUSTIFY?

$A$. The apprehension and solicitude respecting lightning are proportionate to the magnitude of the evils it produces, rather than the frequency of its occurrence. The chances of an individual being killed by lightning are infinitely less than those which he encounters in his daily walks, in his occupation, or even during his sleep from the destruction of the house in which he lives by fire.

Q. How are the MECHANICAL EFEBCTS of lightning displayed?

A. In piercing solid bodies with holes, in splitting them in pieces, and in projecting their fragments to great distances.

Q. How is it ACCOUNTED For that the mere passtge of a current of electricity, as developed in a flash of lightuing, can exercise such enormous mechanical power as to throw down buildings, shatter rocks, rupture masses of metal, \&c. \&c.?

A. We know that the passage of lightning along metallic conductors often produces suficient heat to melt them instantly.

Arago supposes that the heat of the lightning in passing through any substance may instantly convert all the moisture contained in it into steam of a highly explosive character, aud that the great mechanical effects observed are due to this ageut rather than to the direct effect of the electric current.

Q. How does Arago attempt to prove this?

$A$. He states that the temperature which could instantly render iron red hot would be sufficient to generate steam of such an elastic force as would overcome all obstacles.

If the water contained in the pores of bodies were therefore at once converted into steam of this character, its force would be capable of producing any of the mechrnical effects witnessed.

Q. What OTHER THEORY has been proposed to account for the effects of lightning?

A. A theory which supposes that the natural electricities of nonconducting bodies being forcibly decomposed by the presence of the electric fluid which forms the lightning, and which may be cousidered to have an almost infinite intensity, their violent separation would force asunder everything which might tend to confine thein or obstruct their" passage.

Q. Why does the lightning in its course down a building generally DART from porNT to POINT, and not follow a DIRECT PATH?

A. Because it always takes in its course the best conductors; and will fly both right and left in order to reach them.

Q. What EFFEct has a trunder-STorm on the ATMOSPHERE?

$A$. It purifies the air by producing nitric acid and ozone, which dispel noxious vapours, and, by agitating the air, stirs up fresh breezes, rendering it more pure and healthy. 


\section{SECTION III.-LIGHTNING CONDUCTORS.}

Q. What is a Lignining CONDUCTOR?

A. A metal rod, intended to conduct the lightning to the earth.

Q. Why should LIgHTAING CONLUOTORS be POINTED?

A. Because points conduct electricity away silently and imperceptibly.

Blarles of grass, ears of corn, and other pointed objects serve to withdraw electricity from the clonds.

Q. How Far does the influence of a lightning conductor extend?

$A$. It will protect a space all round four times the length of that part of the rod which rises above the building.

Q. Give me an EXAMPLE.

$A$. If the rod rises two feet above the house, it will protect the building for (at least) eight feet all round.

The area of protection, bowerer, varies according to conditions, " the protection being more or less complete according to the elevation of the rod, its size, its conducting power, the absence of other attracting objects in its vicinity, and some other circumstances. To demonstrate how small an area may be protected by a lightning rod, hy reference to what has occurred, the following example may be cited :-The mainmast of H.M.S. Eudymion was protected, the foremast not, and a flash of lightning striking the latter, shivered it to atums ; yet the distance between the two inasts was scarcely fifty feet."

Q. Can lightning conductors be productive of harm ?

A. Yes; if the rod be broken, the electric fluid, being obstructed in its path, will enter the building; and if the rod be not large enough to conduct the whole current to the earth, the lightning will fuse the metal and enter the building.

Q. Is not AIR a CONDUCTo ' of lightning?

A. No; dry air is not a conductor of lightning.

Q. By whom was the IDENTITx of lightning and electricity filst established?

A. By Dr. Franklin, at Philadelphia, in 1752.

The manner in which this fact was demonstrated was as follows :-

Having made a kite of a large silk handkerchief stretched upon a frame, and placed upou it a pointed iron wire connecter with the string, he raised it upon the approach of a thunder-storm. A key was a tinched to the lower end of the hempen string holding the kite, and to this one end of a silk ribbon was tied, the other end being fastened to a post. The kite was now insulated, and the experimenter for a considerable time awaited the result with great solicitude. Finally, indications of electricity began to appear on the string; and on Franklin presenting lis knuckles to the key, he raised an electric spark. The rain beginning to descend, wetier? the string, increased its conducting power, and vivid sparlis in great abundance flashed from the key.

Q. Why was the kime Insulated when Franklin fastened the key to the post with a SILK RIBEON?

A. Because the silk was a non-conductor, and would not allow the electricity received upon the kite to pass off by means of the string to the ground.

Q. Was this experiment one of GREAT. DANGER and RISK?

$A$. It was; because the whole amount of electricity contained in 
the thunder-cloud was liable to pass from it, by means of the string, to the earth, notwithstanding the use of the silk insulator.

Q. What HAPpenep when the experiment was subsequently repeated in France?

A. Streams of electric fire, nine and ten feet in length, and an inch in thickness, darted spontaneously, with loud reports, from the end of the string confining the kite. During the succeeding year, Professor Richman, of St. Petersburg, in making experiments somewhat similar, and having his apparatus entirely insulated, was iminediately killed.

Q. What PRAOHICAL RESULr flowed from Franklin's experiment?

$A$. The use of pointed rods to convey away electricity, and in this way to protect buildings, \&c.

Q. Which METAL is the BeSt to employ for a LIGHTNING CONDUCTOR ?

A. Copper.

Q. If a lightning rod is made of IRON, how IARGE should it be?

A. Not less than three-quarters of an inch in diameter.

Q. In what MANNER should the rod be erected?

$A$. The rod should be continuous from the top to the bottom, and an entire metallic communication should exist throughout its whole length. This law is violated when the joints of the several parts that form the conductor are imperfect, and when the whole is loosely put together.

Q. How should the conductor be FASTENED to the building?

$A$. By wooden supports. If there are masses of metal about the building, as gutters, pipes, \&c., these should be connected with the rod by strips of metal; for, unless this is done, the lightning may pass from the rod to the metal, and enter the building.

Q. How should the LOWER END of the rod be arranged?

$A$. It should be divided into two or three branches, and turned from the building. The end of the rod ought to extend so far below the surface of the ground as to reach earth that is permanently damp; or, if a mass of water be near, it is a good plan to extend the extremity of the rod into it.

Q. Why is it a good plan to BURY the end of the rod in POWDERE' CHARCOAI ?

$A$. Because it preserves the iron from rust, and facilitates the passage of the electricity.

Q. Have we any proof of the utility of lightning rods ?

$A$. The experience of a hundred years has shown that when all the necessary rules have been observed, the protection is perfect, as far as human effort can avail.

Q. Is a building more or less liable to be struck when furnished with a good LIGHTNING CONDUCTOR?

$A$. Lightning conductors do not, as many suppose, attract the lightning towards the building on which they are situated; they simply direct its course, and facilitate the passage of the fluid in the most 
direct way to the earth, only when a discharge must inevitably occur. There is no attraction, but the lightning takes the road which offers the least resistance.

Q. Are lightuing conductors prorectrve when even no visible discharge takes place?

$A$. They are; they possess a very great preventive power, and gradually and silently disarm the clouds by drawing the electric fluid from them; and this process commences as soon as the cloud has approached a position vertically over the rod.

Q. In what manner does Professor Faraday recommend that lightning conductors be ARRANGED?

$A$. He advises their location on the inside of buildings, rather than the outside.

\section{SECTION IV.-THUNDER.}

\section{Q. What is THONDER?}

$A$. It is a certain noise proceeding apparently from the clouds, which usually follows, after a greater or less interval, the appearance of a flash of lightning.

Q. How is it supposed to be occasioned ?

$A$. The usual explanation offered is a sudden displacement of the air, produced by the electrical discharges, in which the lightning is evolved. Others have supposed that the passage of the electric current creates a vacuum, and that the air rushing in to fill it produces the sound. No explanation that has yet been offered is altogether satisfactory.

Q. What occasions the RoLling of the thunder?

$A$. It has been ascribed to the effect of echo; but this, undoubtedly, is not the only cause. 'The rolling of thunder is heard as perfectly at sea as upon land; but there none of the causes which are generally supposed to produce echo, as mountains, hills, buildings, \&c. \&c., are present. Another and perhaps the true reason is, that the sound is developed by the lightning in passing through the air, and consequently separate sounds are produced at every point through which the lightning passes.

Q. Does THUNDER always Sound exactly the SAMIE?

$A$. No; sometimes it has a rolling sound, as already described; sometimes the sound is irregular; sometimes it is a loud crash.

Q. Is not the LIGHTNING SEEN before we HEAR the THUNDER ?

A. It is; light travelling nearly a million times faster than sound.

Q. Can we, then, aschrtain the DIsTaNom of the THUNDER-Cloud by noticing how LONG a TISB INTERVENES between the FLASH and the SOUND?

A. Yes; it will take a second of time for thunder to travel 380 yards; therefore, if three seconds elapse between the flash and the sound, the thunder-cloud must be 1140 yards distant. 
Q. What is a THUNDERBoLT?

A. The appearances which uninformed persons call thunderbolts are nothing more than the globular form assumed sometines by the electric fluid.

\section{G A L V A N I S M.}

\section{CHAPTER XL.}

\section{NATURE AND PRODUGTION OF GALVANIC ELECTRICITY.}

Q. What is GALVANISI?

$A$. It is the production of electrical disturbance by chemical action.

Q. How does galvanic electricity appear to DIFFER from frictional or ordinary electricity?

$A$. The electricity developed by friction from a glass plate, or the cylinder of an electrical machine, is a discharge with a sort of explosion, whereas that which is generated by chemical action is a steculy, fowing current.

Q. What is the most SIMPLE KANNER of illustrating the production of this electricity?

$A$. If we place a piece of silver on the tongue, and a piece of zinc underneath it, no effect will be produced as long as the two metals are kept asunder; but when their ends are brought together, a distinct thrill will pass through the tongue, a metallic taste will diffuse itself, and, if the eyes are closed, a sensation of light will be eviderit at the same moment.

Q. To wut is this result owivg?

A. To a chemical action developed the moment the two metals touched each other.

Q. What was this chemical action?

A. The saliva of the tongue oxydised a portion of the ainc, which excited electricity, for no chemical action ever takes place without producing electricity. Upon bringing the ends of the two metals rogether a slight current passed from one to the other.

Q. By шном was the production of galvanic electricity first noticed? 1790.

A. By Galvani, Professor of Anatomy at Bologna, Italy, in 


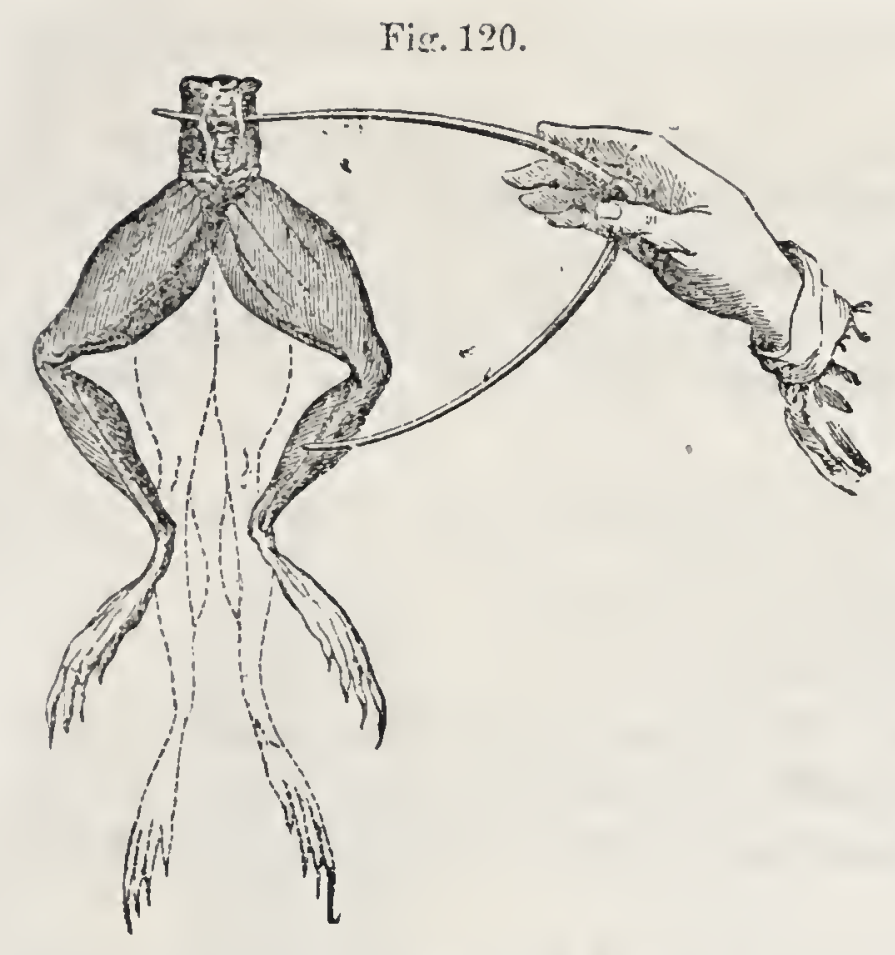

Q. How was his attention DIREOTED to the subject?

$A$. Having occasion to dissect several frogs, he hung up their hind legs on some copper hooks, until he might find it necessary to use them for illustration. In this manner he happened to suspend a number of the copper hooks on an iron balcony, when, to his great astonishment, the limbs were thrown into violent convulsions.

Q. On investigating the phenomena, to WEAT did he attribute the cause ?

$A$. He found that the mere contact of dissimilar metals with the moist surfaces of living muscles was all that was necessary to produce the convulsions. (See fig. 120.) He attributed the phenomena to electricity present in the muscles, and irnagined that the metals served only as conductors.

Q. What was the REAL CAUSE of the convulsions?

A. A weak chemical action, greatly quickened by the contact of dissimilar metals.

Q. What is the SMMPLEST WAY of exciting a current of GALVANIC ELECTRIOITY ?

$A$. By arranging a series of metal plates in a pile, placing them in pairs, with a wet cloth between them, it being necessary that one of each pair should be more easily oxydised than the other. The simple contact of these plates will produce a feeble and continued galvanic current.

Q. What is such an ARRANGEMIENT of PLATES for producing electrical currents CALLED ?

A. A galvanic or voltaic battery.

Q. Why are the terms " "GALVANIO" and "voltAIO" applied?

$A$. They originated in honour of Galvani and Volta, the Italian philosophers who first developed the phenomena of chemical electricity, and the means of producing them.

Q. Are there MANY METALs, or other substances, which, when brought together, are capable of producing galvanic action?

$A$. The number is large; among them we may enumerate the following:- -iinc, lead, tin, antimony, iron, brass, copper, silver, gold, platinum, blacklead or graphite, and charcoal.

Q. Will ANY Two of these brought together produce a galvanic current ?

A. 'They will; but they possess the power in different degrees; and the more remote they stand from each other in the order above given, the more decidedly will the chemical electricity be developed.

Thus zinc and lead will produce a voltaic battery, but it will be much less active than rinc and iron, or the same metal and copper, and this last less active than zinc aud platinum, or zinc and charcoal. 
Q. Does galvanic or voltaic electricity appear to consist of two kinds, Positrive and NEGATIVE, as in ordinary electricity?

A. It does; positive electricity always flows from the metal which is acted ripon most powerfully, and negative electricity from the other.

Q. What do we mean when we speak of a GAIVANIC CIRCUIT?

$A$. The connection of the two metals in the battery, so that the positive and negative electricities can meet, and flow in opposite directions.

Q. At what PorNT in the circuit will the manifestations of electricity be must apparent?

A. At the point where the two currents meet.

Q. What is meant by the PoLES of the battery?

$A$. The two metals forming the elements of the battery are generally connected by copper wires; the ends of these wires, or the terminal points of any other connecting medium used, are called the poles of the battery.

Thus, when zinc and copper poles are used, the end of the wire conveying positive electricity from the zinc would be the positive pole, and the end of the wire conveying negative electricity from the copper plate would be the negative pole. Faraday describes

Fig. 121.

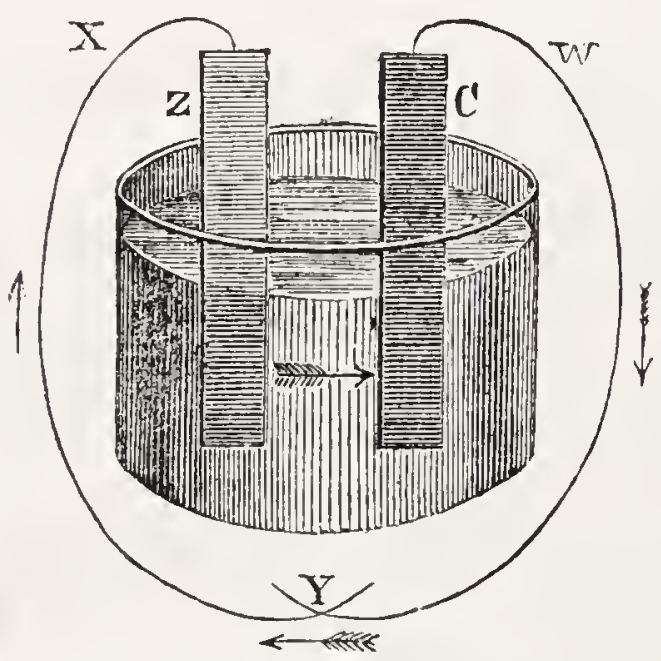

the poles of the battery as the doors by wh ch electricity enters into or passes out of the substance suffering decomposition.

A very simple, and at the same time an active, galvanic circuit may be formed by an arrangement as represented in fig. 121. $\mathrm{C}$ and $\mathrm{Z}$ are thin plates of copper and zinc immersed in a glass ressel containing a very weak solution of sulphuric acid and water. Metallic contact is made by means of the wires $\mathrm{X}$ and $\mathrm{W}$ soldered to the plates, the poles intersecting at $Y$. The current of positive electricity, when the circuit is closed, passes from the zinc, through the liquid, to the copper, and from the copper, along the conducting wires, to the zinc, as indicated by the arrows in the fgure. A current of negative electricity traverses the circuit also, from the copper to the zinc, in a direction precisely reversed.

Q. Are two metals ABsoldteli essential to the formation of a simple galvanic circle?

$A$. No ; a current is obtained from one metal and two liquids, provided the liquids are such that a stronger chemical action takes place on one side of the metal plate than on the other.

Q. Have galvanic batteries been formed wiтно UT resorting to the use of any metal ?

A. Dr. Baconio, of Milan, constructed a voltaic pile entirely of vegetable substances; using dises of red beet-root, two inches in diameter, and similar discs of walnut-wood, the latter deprived of their resinous matter by maceration in a solution of cream of tartar in distilled vinegar.

With such a pile, using grass as a conductor, he is stated to have produced contractions of the muscles of a dead frog. Other experimentalists bave formed voltaic piles wholly of animal substances. 
Q. By what CHEMical ACTion can the greatest abundance of galvanic electricins be dereloned?

A. By the oxidation of metallic sinc by weak sulphuric acid.

Q. The electricity developed by the action of a single pair of plates immersed in acid water is very feeble : how can it be INCREASED?

A. By increasing the number of the plates and the quantity of the iiquid, we increase the intensity of the electricity developed.

Fig. 122.

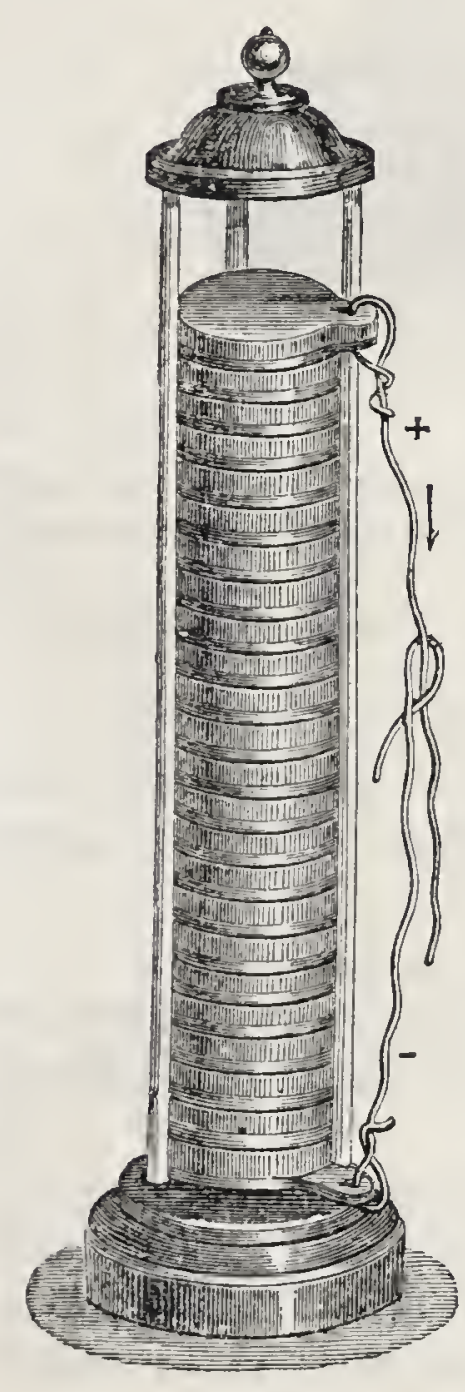

\section{SECTION I.-PHENOMENA AND EFFECTS OF GALVANIC ELECTRICITY.}

Q. What is the voltaIC PILE?

$A$. The term pile is generally applied to the apparatus which develops galvanic electricity. The voltaic pile takes its name from the inventor, Volta.

The voltaic pile consists of a series of plates piled one on the other, as seen in diagram 122. Thus, a copper disc or plate, a zinc disc, a piece of wet cloth ; then upun a copper disc a zinc disc, and another piece of wet cloth ; and so on throughout the whole extent of the pile.

Q. What is the RESULT of such an ARRANGEMENT as that adopted in the vOLTAIC PILE?

\section{$A$. Electrical action is excited and kept up.}

Q. What otHer SPECIES of GALPANIC PILES are used?

$A$. There are various modifications of the voltaic pile, but the principle of all is the same.

Fig. 123.

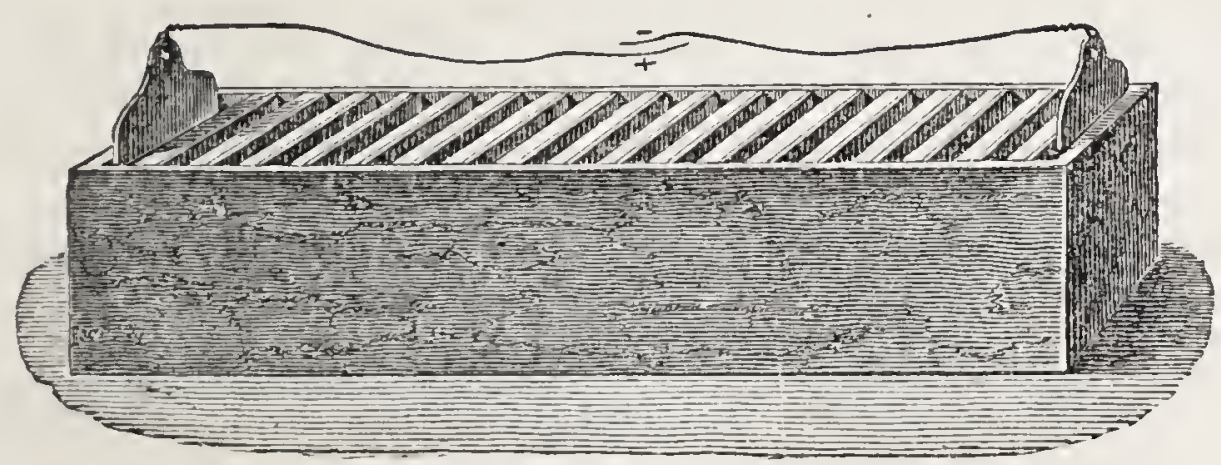

COMMON TROUGH PILE.

The trough pile (fig. 123) is the same as that of Volta, with the exception of its arrange ment. It is formed of a long square box, lined with cement or mastic. The rlates of zinc and copper are soldered together, and are then fixed in couples in the box, which is filled with a mixture of water and sulphuric acid. 
Fig 124

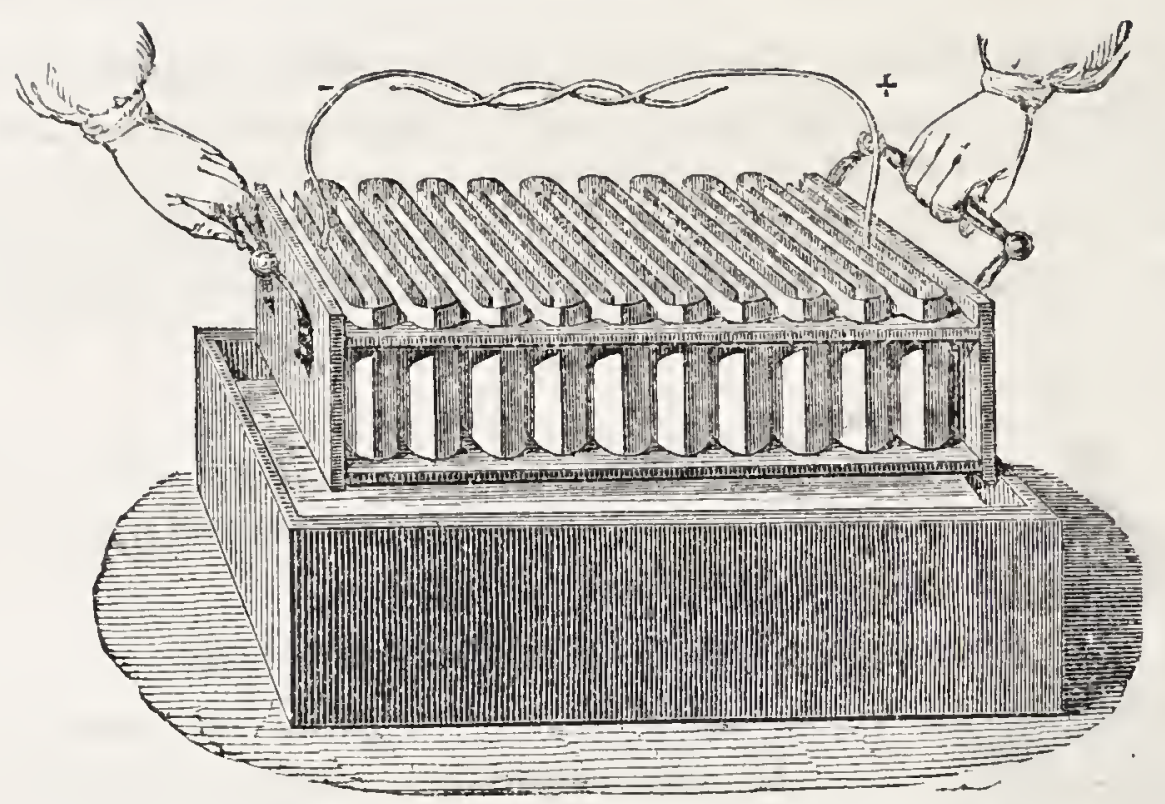

In the pile seen in fig. 124. all the plates of zinc and copper dip into the same wooden trough, filled with sulphuric acid and water. This pile, which occupies but a small space, produces very powerful effects.

Q. What are the most oRDINARY EFFECTS produced by the developed electricity of a large galvanic hattery?

A. The production of sparks and brilliant flashes of light, the heating and fusing of metals, the destruction of gunpowder and other inflammable substances, and the decomposition of w ater, saline sompounds, and metallic oxides.

Q. How may the most SPLENDid aRtificial Light known be produced ?

$A$. By fixing pieces of pointed charcoal to the wires connected with opposite poles of a powerful galvanic battery, and bringing them .nto contact.

Q. How does this light appear to DIFFER from all other varieties of artificial light?

$A$. It appears to be independent of combustion, since the charcoal points appear to suffer no change, and the light is equal ly strong and brilliant in such gases as do not contain oxygen, and even under water, where all other artificial lights would be extinguished.

To produce the electricity which develops the light, zinc, or some other metal, must be oxidised, or burnt up, as much as oil in our lamps, or coal in the gas-retorts, for roducing other species of artificial light.

Q. Can intense Heat be developed by the action of the galvanic battery, as well as intense light?

$A$. The greatest artificial heat man has yet succeeded in producing has been through the agency of the gatvanic battery.

Q. Is HEAT EVOLVED whenever galvanic electricity is sent along a conducting body?

$A$. It is; bad conductors, however, oi equal lenoth and thickness, becoming much hotter than good ones in the same lime.

Q. What REFRACTORY SUBSTANCES can be fused by the aid of the galvanic battery?

$A$. All the metals, including platinum, can be readily melted; quartz, sulphur, magnesia, slate, and lime are liquefied; and the diamond fuses, boils, and becomes converted into coal. 
Q. When Two different metals come into mutual contact, does the action which takes place between them favour chemical change?

A. It does in a marked degree; and it is to this action that we must ascribe many well-known phenomena, such as the more rapid cxidation or rust of certain metals when combined or in mere contact with other metals.

Thus, when sheets of zinc and copper immersed in dilute acid touch each other, the zinc oxidises or rusts more, and the copper less rapidly, than without contact.

Q. Why do rron NaILS, if used in fastening copper sheathing to vessels, rust so much QUICKER than when in other situations, not in contact with copper?

A. Because the contact of the two metals excites galvanic action, which causes the iron to rust speedily, but protects the copper.

Q. How did Sir Humphrey Davy attempt to TAKE ADVANTAGE of this fact in protecting the copper sheathing of vessels?

A. Copper, when immersed in sea-water, rapidly wastes by the chemical action of the oxygen dissolved in sea-water; but if it be brought in contact with zinc, or some metal that is more electropositive than itself, the zinc will undergo the same change, and the copper will be preserved. He therefore proposed to place at intervals over the copper small strips or wires of zinc or iron.

Q. How MUCH zinc was required to protect a given surface of copper?

$A$. A piece of zinc, as large as a $p e a$, was found adequate to preserve forty or fifty square inches of copper; and this wherever it was placed, whether at the top, bottom, or middle of the sheet, or under whatever form it was used.

Q. Was this plan found to WORK WELL practically, in preserving the sheathing of ressels?

A. It did not: the copper was protected at the expense of the zinc; but it was found, that unless a certain degree of corrosion took place in the copper, its surface became covered with sea-weeds and shell-fish, which impaired the speed of the vessel.

The corrosion or waste of the copper sheathing prevents these organisms from attaching themselves to its surface.

Q. Can GaLVANIC ELEOrRICITY be used in analysing or separating the constituents of bodies?

$A$. It is a powerful agent in chemical analysis. When transmitted through conducting fluids, it separates them into their constituent parts.

Q. Can WATER be decomposed by passing a current of gal vanic electricity through it?

A. It can; hydrogen being given out at the positive pole of the battery, and oxygen at the negative pole.

Q. What LAW exists respecting the quantity of electricity necessary to decompose a compound substance?

$A$. The electricity which decomposes, and that which is evolved by the decomposition of a certain quantity of matter, are alike. Thus, water is composed of oxygen and hydrogen; now, if the electrical power which holds a grain of water in combination, or which causes a grain of oxygen and hydrogen to unite in the right proportions to 
form water, could be collected and thrown into a voltaic current, it would be exactly the quantity required to produce the decomposition of a grain of water, or the liberation of its elements, oxygen and hydrogen:

Q. What GReat ohemical discovery was made by Sir Humphrey Davy through the decomposing powers of the galvauic battery?

$A$. The discovery that potash, soda, lime, magnesia, and other similar bodies, were not simple in their nature, as had been supposed, but compounds of a metal, with oxygen.

Q. What is RLectroty ping, or ELectro-aietalluray ?

A. It is the art or process of depositing, from a metallic solution, through the agency of galvanic electricity, a coating or film of metal upon some other substance.

Q. Upon what PRINorrLes is it accomplished?

$A$. The process is based on the fact, that when a galvanic current is passed through a solution of some metal, as a solution of sulphate of copper (sulphuric acid and copper), decomposition takes place; the metal is separated in a metallic state, and attaches itself to the negative pole, or to any substance that may be attached to the negative pole; while the acid or other substance before in combination with the metal, goes to, and is deposited on the positive pole.

In this way a medal, a wood engraving, or a plaster cast, if attached to the negative pole, may be covered with a coating of copper; if the solution had been one containing silver or gold, the substance would have been covered with a coating of silver or gold, instead of copper.

Q. How can the THIOKNESS of the deposits he REGULATED?

$A$. The thickness of the deposit, providing the supply of the metallic solution be kept constant, will depend on the length of time the object is exposed to the infuence of the battery.

In this way, a coating of gold thinner than the thinnest gold-leaf can be laid on, or it may be made several inches in thickness, if desired.

Q. Explain Nore Expricituy how the electrotype plate of the engraving is effected.

A. The engraved plate is plunged into a solution of copper, and the negative pole of the battery attached to it. The other pole of the battery is connected with the fluid solution. The solution is then decomposed; the copper resulting from it is deposited as a coherent mass, in a perfectly uniform manner, on the engraved plate, of which an exact copy in relief is obtained.

Q. What is meant by a copy IN RELIEF?

A. It is when the figure is formed by an elevation, and not by a depression of the surface. The lines constituting the figure map were cut into the plate in the original engraving; the deposit of copper filled up these depressions, and, when it was separated, the figure appeared to be raised on the surface.

Q. How, then, is an EXACT FAC SIMILE of the original plate obtained by the electrotype?

$A$. The electrotype itself is subjected to a repetition of the process, the result of which is that a reverse impression of it is obtained. 


\section{SECTION II.-THERMO-ELECTRICITY.}

Q. What is THERMO-ELECTRTCITY?

$A$. If a bar of metal is warmed at one enct, and lept cool at the other, an electric current circulates through it, and may be carried off by connection with any good conductor. Electricity thus generated or developed is called "thermo-electricity."

Q. What are its PRoperties?

A. 'The sume as those of common electricity.

Q. What METALS are iEST suited for showing the effects of thermo-electricity?

A. Bismuth and antimony.

By binding two bars of these metals together at one end, an electric current can be proved to circulate at the slightest variation of temperature. It is by means of such an instrument that some of the most delicate researches upon heat have been effected.

Q. To whAт may all the curious effects of electricity be ATTRIBUTED?

$A$. To an effort of the power or force to regain a position of stability when its equilibrium or balance has been disturbed. We have no evidence that electricity is an exciting power, but rather that it is one of those forces that tend to establish the balance of matter.

\section{A G N E T I S M.}

\section{$\rightarrow-$ \\ CHAPTER XLI. \\ NATURAL MAGNETIC PHENOMENA.}

Q. Is there any CONNECTION between MAGNETiSM and ELECTRICITY?

$A$. There is every reason to believe that magnetism and electricity are but modifications of one force.

Q. What is a LOADSTONE or a NatURAL MAGNET?

$A$. It is an ore of iron, known as the "protoxide of iron," or "magnetic oxide of iron," which is capable of attracting other pieces of iron to itself; and if suspended freely by a thread, and left to take its own position, it will arrange itself so that its extremities will point towards the north and south poles of the earth.

Q. What is the orrars of the terms " magnet" and "magnetism?"

$A$. The loadstone, or natural magnet, was first found at $M a g-$ nesia, in Lydia, Asia, whence were derived the names.

Q. What is the ATtractive INFLUENCE of the magnet called?

A. It is called magnetic power. Magnets do not possess the same magnetic force in every part. 
Fig. 125.

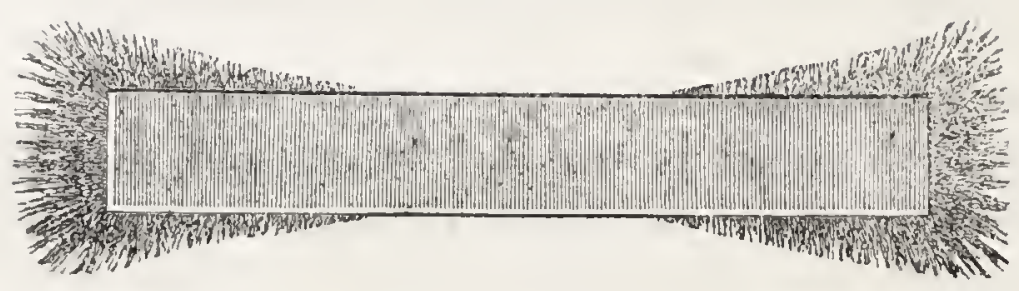

If a magnetic bar be rolled in iron filings, they will be found to stick to the ends or the bar, as seen in the diagram. The centre does not possess the same attractive power as the extremities. When the attractive power is altogether imperceptible, it is called the NEUTRAL LINE.

Q. Can a Natural magnet comudicate its attractive properties to other bodies by contact?

$A$. It can, and that, too, without any apparent loss of attractive strength.

Q. What BODIEs are capable of being magnetised by contact with natural magnets?

A. Iron and steel are the substances most susceptible of this influence, but brass, nickel, and cobalt can also become magnets.

Q. Are there any oTHER substances besides metals that are magnetic, or can have magnetism imparted to them?

$A$. This property is not confined to the metals: the emerald, the ruby, and various precious stones, as well as glass, chalk, bone, and wood, are more or less susceptible to the magnetic influence; and recent experiments would seem to show that the influence of magnetism is as pervading and wide extended as that of electricity.

Q. What is NEANT by magnetic polarity?

A. Two bodies endowed with the property of magnetism will attract each other at one part of their dimensions, and repel each other at another part. These contrary effects, belonging to opposite sides or ends, are called magnetic polarity.

Fig. 126.

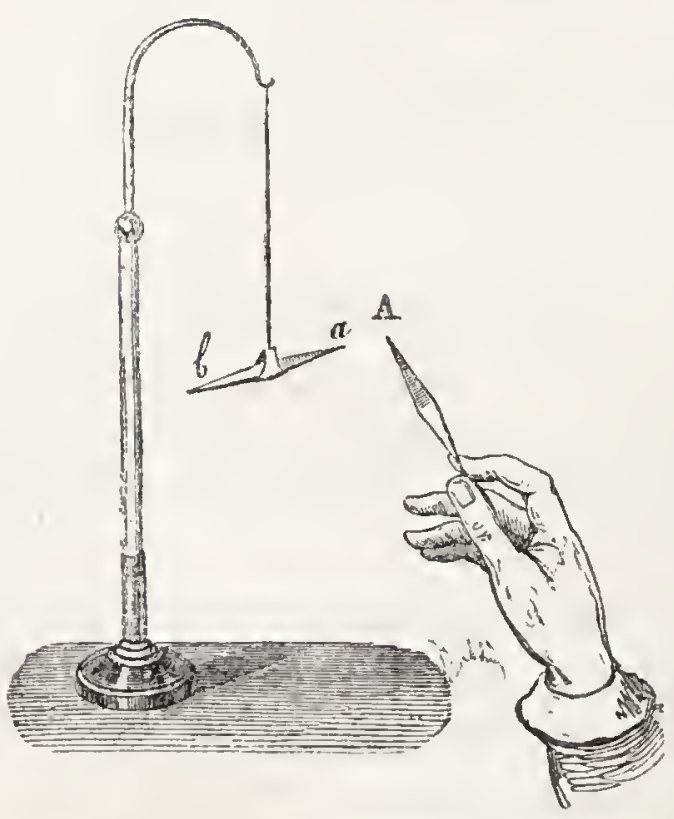

If we hang a magnetised needle, as seen in the figure, $a b$, by a fine thread, and bring the south pole, $A$, of another magnet near the south pole $a$ of the first magnet, we shall see a marked repulsion; if, on the other hand, we present the pole $A$ to the north pole $b$ of the suspended magnet, there will be a very observable attraction. We learn from this that the same poles in two different magnets repel each other, and that the opposite poles attract.

Q. Does the magnetism imparted to a piece of SOFT IRON, or steel, by contact with a natural magnet, remain permanent in their substances?

A. In the steel it does, but the soft iron loses its power as soon as it is removed from the magnet.

Q. Is it necessary that ABSOZUTE CONTACT should, take place between a magnet and a piece of soft iron to render the latter a magnet? 
$A$. No; every piece of soft iron brought near a magnet becomes, by induction, itself a magnet.

Q. What do you mean by INDUCrios?

$A$. It is the production of like effects in bociies near to one anzother. In electricity or magnetism it is the influence exerted by an electrified or magnetised body through a non-conducting medium, withou any apparent communication of a.current.

Q. What is meant by the DIREOTIVE POWER of the magnet?

$A$. It is that power which will cause a magnet, when suspended freely, to constantly turn the same part towards the north pole, and the opposite part towards the south pole of the earth.

Q. What are the poles of a magnet?

$A$. They are the ends of the magnet, and are denominated north and south poles, according as they point to the north or south poles of the earth.

Q. When is a magnet said to TRAVERSE ?

$A$. When it places itself freely in a norlt and south line.

Q. What are the Poles of the earth?

A. The extremities of the earth's axis, or the points on the surface of the globe through which the axis passes.

Q. Under what CIRCUMSTANCES do the north and south poles of two'magnets ATTr.ACT and REPEL each other?

$A$. The north or the south poles of the magnets repel each other, but the north pole of the one will attract the south pole of the other.

Q. What is a ISAGNETIO NEEDLE?

A. Simply a bar of steel which is a magnet, suspended in such a way that it can freely turn to the north or south,

Fig. 127.

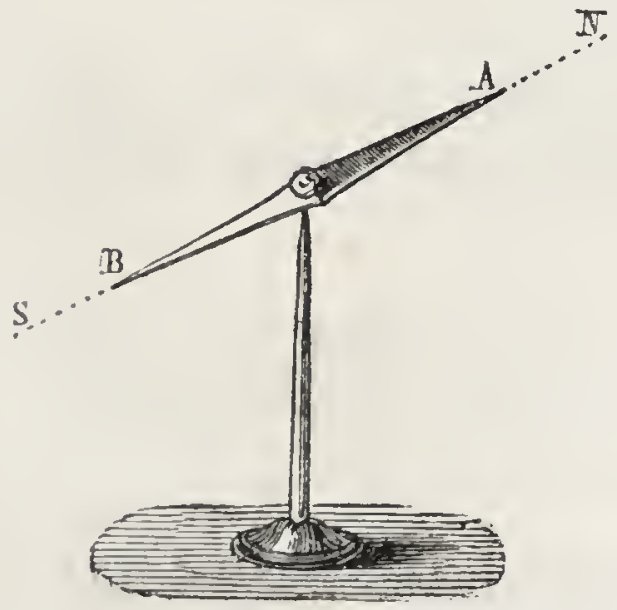

Q. What is the STMPLEST TIA of :onstructing a magnetic needle?

A. If we place a magnet on a piece. of cork, and allow it to float freely in vater, with no iron near it, it places. itself in the magnetic meridian.

A MARLNER'S NEEDLE.

Q. What is a MARINER's COMPASS?

A. It is a delicate steel bar or needle balanced upon a pivot placed beneath its centre of gravity in such a way that it can turn without obstruction. (See fig. 127.) This needle is usually enclosed in a 
box, upon the bottom of which is a card, with the various points--. north south, east, west, \&c. \&c., marked upon it. (Sec fig. 128.)

Fig. 128.

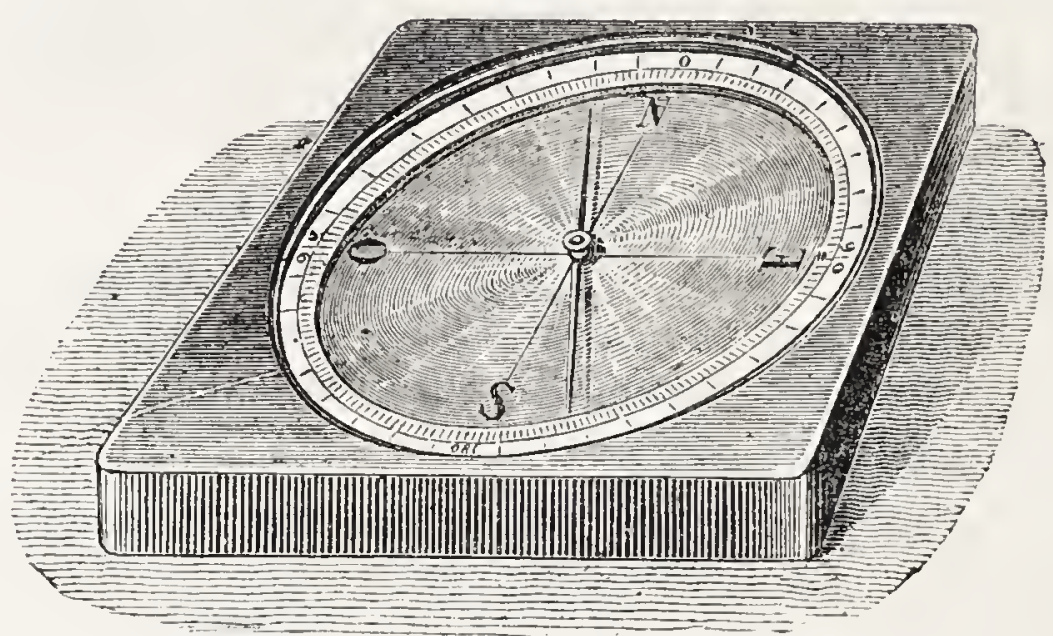

Such a needle, if the box containing it be placed on a level surface, will generally be observed to vibrate more or less, till it settles in such a direction that one of its extremities or poles will point towards the north, and the other, consequently, towards the south. If the position of the box be altered or reversed, the needle will always turn and vibrate again, till its poles have attained the same directions as before.

Q. Does the compass needle always point Ex.corrix north and scuth?

$A$. It does not; its natural direction is towards northern and sculthern parts, but it seldom points due north or south.

Q. What is the VARIATION or DECLINATION of the needle?

A. The deviation from the true north and south.

Q. Are there any points upon the earth's surface where the needle does кот VART, but points to the TRUE NORTH?

A. There are two lines upon the surface of the globe, along which here is no variation, called the eastern and western line.

Q. Are these lines REGUIAR?

A. They are very irregular, showing that the powers on which ierrestrial magnetism depends are regulated by causes which science t.as not yet reached.

Q. Where is the WESTERN IINE of no variation?

A. It begins to the west of Hudson's Bay, passes in a sonth Tirection through the American lakes to the West Indies and the extreme eastern point of South America.

Q. Where is the EASTERN LINE of no variation?

A. It begins on the north in the White Sea, makes a great semicircle, descending south, until it reaches the latitude of $71^{\circ}$; it then passes along the Sea of Japan, and goes westward across China and Iindostan to Bombay; it then bends east, touches Austratio, and goes south.

Q. If, instead of balancing the magnetic needle on a pirot beneath centre of gravity, we cause it to be sUSPENDED from its centre and carefully balanced, in what direction wiL it turn? 
$A$. If a simple bar of steel or ordinary needle were suspended from its centre as above described, it would hang horizontally, and show no inclination to dip from a horizontal line, either on one side or the other of the centre of suspension. But if the bar or needle be made a magnet, it will no longer lie in a horizontal direction, but one pole will incline downwards and the other upwards; the inclination in this latitude to the horizon being about $70^{\circ}$.

Q. What is such a needle CALIED ?

A. A dipping needle.

The construction of the dipping needle is represented by fig. 129 .

Fig. 129。

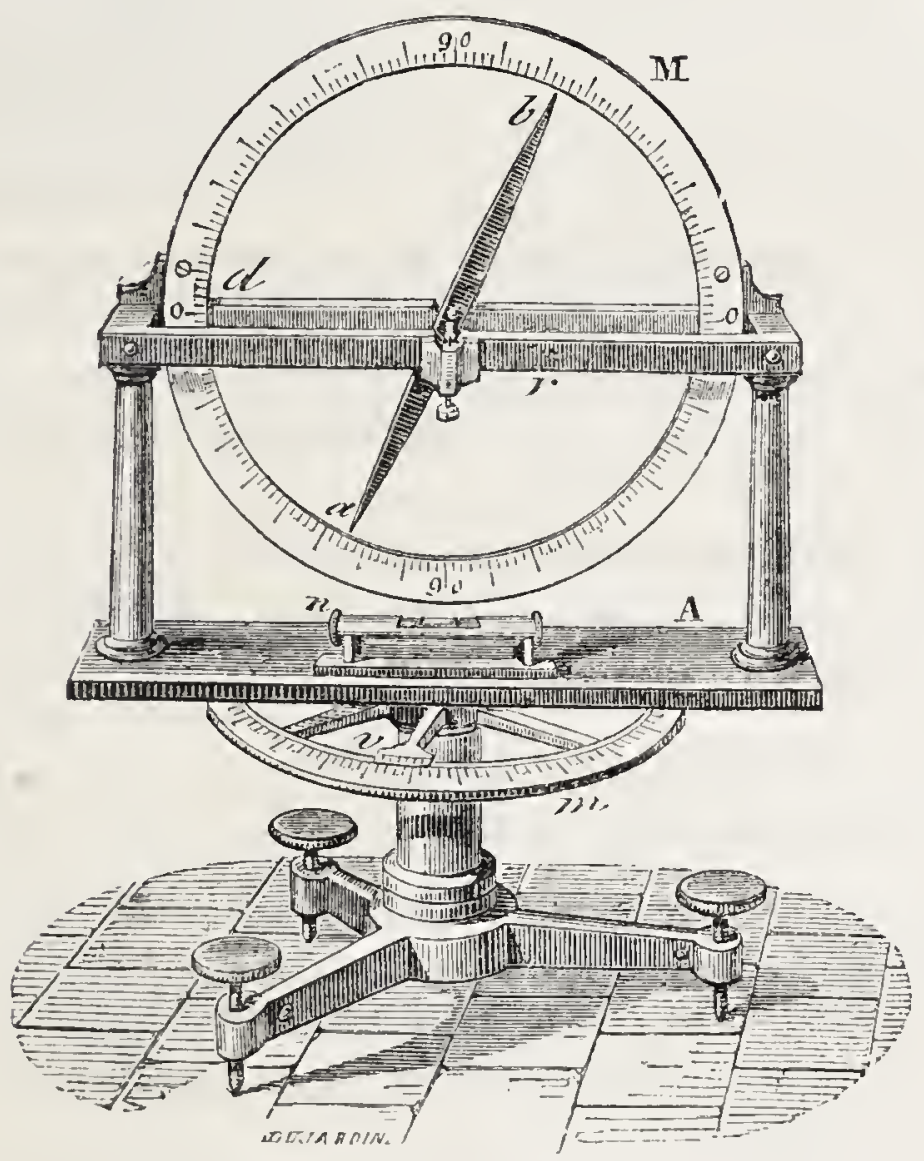

Q. As the ordinary compass does not point directly to the north or south pole of the earth, but to a point a little removed from them, what ways lias been given to trese points?

A. Whe magnetic poles of the earth.

Q. Is the exact position of these places known?

$A$. The position of one of these poles in the northern hemisphere is known, having been discovered and visited by Capt. Ross when attempting to discover the north-west passage around the continent, of America. The exact position of the southern magnetic pole is not known. 
Q. If the ordinary compass be carried to this point, in wrat manner is it affected ?

A. It appears to lose its power, and points indifferently in any direction.

Q. In WIAT MANNER would the dipping needle be affected if placed at the magnetic poie?

A. It would cease to dip, and stand perfectly upright.

Q. What position does the dipping needle assume at the equator?

A. It remains perfectly horizontal; as we go north or south, howaver, it dips more and more, until at the magnetic poles it is perpendicular, - the end which was uppermost at the north being the lowest at the south.

Q. If the magnetic needle is thus subject to variations, how can it be RELIED UPON?

A. The variations of the needle, except in the vicinity of the poles, are not sufficient to affect its general accuracy, and for all the ordinary purposes of the wanderer upon the ocean, the desert, or the wilderness, it may be considered as free from error; for more accurate purposes it is necessary to apply a rule of corrections.

Q. WHO FIRST DISCOVRRED the fact that a magnet would invariably point to the north and the south, and make use of this knowledge in constructing a compass?

A. It is claimed to have been discovered by the Chinese: it was known in Europe, and used in the Mediterranean, in the thirteenth century.

Q. How were the Coxpasses of that time constructed?

A. They were merely pieces of loadstone fixed to a cork, which floated on the surface of water.

Q. Is the EArTin itself stupposed to bo a IrAGNET?

A. It is undoubtedly a great maynet.

Q. Is IRoN, under certain circumstances, rendered MAGNerIC by the inductive action of the earth's magnetism?

A. Most iron bars and rails, as the vertical bars of windows, that have stood for a considerable time in a perpendicular position, will be found to be marnetic.

Q. If we suspend a bar of soft iron sufficiently long in the air, will it assume araGmeTIO PROPERTILS?

A. It will gradually become magnetis; and although when it is first suspended it points indifferently in any direction, it will at last point north and south.

Q. How may a bar of iron, such as a kitchen poker, be made IMMEDIATELr MAGNETic, without resorting to the use of other magnets?

$A$. If the bar devoid of magnetism is placed with one end on the ground, slightly inclined towards the north, and then struck one smart blow with a hammer upon the upper end, it will immediately acquire polarity, and exhibit the attractive and repellant properties of a magnet. 
Fing. 130.

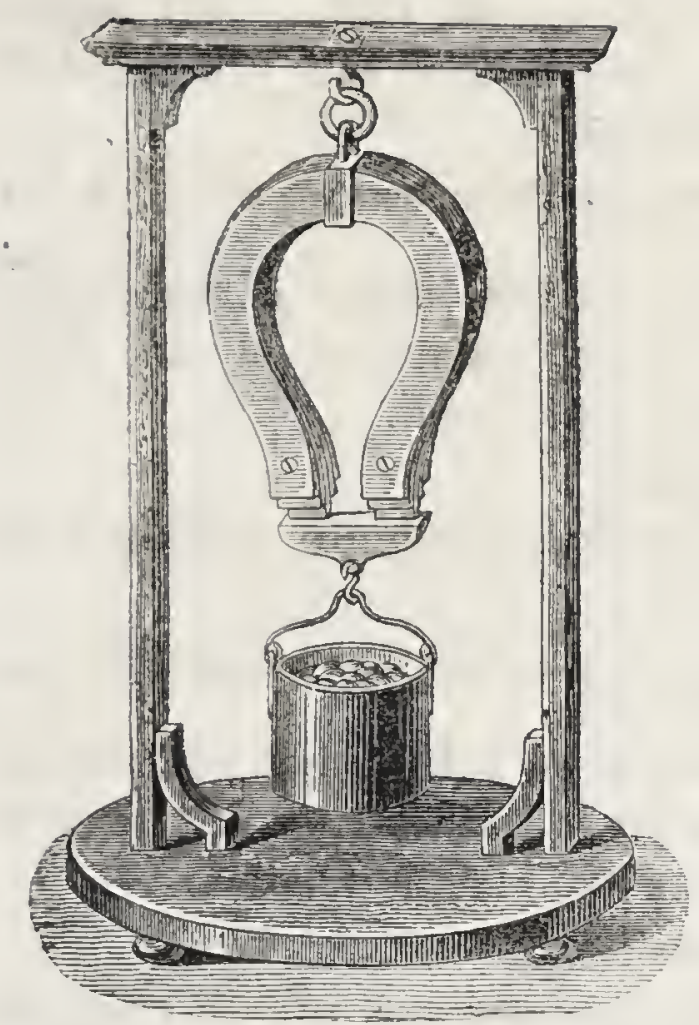

Q. What is a IORSE-SHOP MAGINET

A. It is a magnetic bar, bent into the form of a horse-shoe.

When a piece of iron, not magnetic, is brought in contact with a common magnet, it will be attracted by either pole; but the most powerful attraction takes place when both poles can be applied to the surface of the piece of iron at once. It is on this account that artificial magnets are often bent into the form of a horse-shoe, the north pole being usually marked by a line or point to distinguish it. Very powerful horse-shoe magnets are made by binding a number of magnetised bars togethør with a piece of soft iron, so as to form a compound horse-shoe, as is seen in fig. 130 .

Q. If we BREAK a magnet across the middle, What HAPPENS?

A. Wach fragment becomes converted into a perfect magnet; the part which originally had a north pole acquires a south pole at the fractured end, and the part which originally had a south pole gets a north pole.

Q. If we divide up a magnet to the extreme degree of mechanical fineness possible, will the pieces possess $M A G$ NETIC PowERs?

$A$. Each fragment, however small, will be a perfect magnet. air?

Q. Are the properties of the magnet at all $A F F E C T E D$ by the presence or absence of

A. They are not; its influence is as great in a vacuum (empty space) as in any other situation.

Q. What EFFECT has HEAT upon the power of a magnet?

A. It weakens it; and a white heat destroys it.

Q. How is the POWER of a magnet STRENGTHENED?

A. The gradual addition of weight to a magnet, kept in its proper situation, increases the magnetic power.

Q. In what Posirion should maguets be kept?

A. Magnets should always be kept with their poles pointing to the poles of the earth: this is said to be their proper position.

Q. Can two or more magnets communicate to IRON a power GREATER than either nf them possesses singly?

A. Two or more magnets joined together may communicate a greater power to iron or steel than either of them possesses singly.

Q. When an iron bar is rendered magnetic, where does the power appear to RESIDE: in it?

A. It appears to reside on the surface, and to circulate about it.

We are led to this belief from the circumstance of a stronger power, in proportion, being produced in thin plates of steel than in thick ones ; and from the acquired magnetism being diminished by rust, filing; or grinding, it apnears that the virtue communicater? is more external than internal. 
Q. How great a sustaining power can magnets exert?

$A$. The lifting or sustaining power of magnets varies very materially. The most powerful that we are acquainted with are capable of sustaining twenty-six times their own weight.

Q. Who first noticed the variation of the magnetic needle from the true north and south?

A. It was first noticed by Columbus in his first voyage of discovery. It was also observed by the sailors, who were alarmed at the fact, and urged it as a reason why they should turn back.

Q. Are the magnetic poles of the earth STATroNARY?

$A$. They are not, but change their position gradually during lo!ng intervals of time.

Q. What curious fact has been noticed in regard to the connecrion between the mingnetic poles of the earth and the points of GREATEST COLD?

$A$. The points where the greatest degree of cold is experienced, or where the yearly mean of the thermometer is lowest upon the surface of the earth, coincides with the location of the magnetic poles.

Q. Is the magnetic needle subject to a DAILY variation?

$A$. It is: this movement or variation commences about seven in the morning, when the north end of the needle begins to deviate towards the west; it reaches its greatest deviation about two o'clock in the afternoon, when it begins to return slowly to its original position.

Q. Besides this daily variation, is the needle subject to OTHER VARIABLE MOVEMENTS ?

$A$. It has a yearly movement; and a movement different in the winter months from that noticer in the summer months.

?. What is supposed to be the occasrov of these daily, monthly, and yearly variations of the magnetic needle?

A. Variations of temperature, depending upon changes in the position and course of the sun.

Q. Does the AURoRA BOREALIS have an effect upon the magnetic needle?

$A$. It does very sensibly, causing it to tremble and deviate from point to point.

Q. Do the RAYS of LIGHT have the power of exciting magnetism?

A. Light is generally believed to have the power of inducing magnetism in steel. Sewing-needles, it is said, when exposed to violet-coloured light, or when covered with a blue glass, become magnetic.

Q. Into what TWO GREAT CLASSES, according to the latest experimental researches, are all bodies divided as respects magnetic influences?

A. Into magnetic and diamagnetic bodies.

Q. What is meant when we call a body DraMagnerro?

A. The magnetic power is found to influence all bodies in two different ways; one class of substances act in relation to magnetism like iron, and arrange themselves along the line of magnetic force: these are called magnetic bodies. Another set, of which the metal 
bismuth may be taken as a representative, always place themselves at right angles to this line: these are called diamagnetic bodies.

Q. How do magnetic and diamagnetic bodies DIFFER as regards ATTRACTION and RE. PULSION?

A. The magnetic body is attracted by each pole; the diamagnetic body is equally repelled by either pole. Magnetism may be regarded as an attractive force, diamagnetism as a repelling one.

Every substance in nature is in one or the other of these conditions. "It is a curious sight," says Dr. Faraday, "to see a piece of wood, or of beef, or an apple, or a bottle of water repelled by a magnet; or taking the leaf of a tree, and hanging it up between the poles, to observe it take an equatorial position."

Q. To WHAT FORCE have some attributed the turning of the sunflower to the sun, the closing of the convolvuli, and the directions of spirals formed by twining plants?

$A$. To the diamagnetic property, which all plants have been found to possess. 'This idea is, however, a speculative one.

Q. Have GASES been ascertained to be influenced by the magnetic power?

$A$. Oxygen is strikingly magnetic; nitrogen is singularly the contrary. These two gases form our atmosphere; and both possessing different properties, the result, when united, is a compound, neutral in all its relations to matter.

\section{CHAPTER XLII.}

\section{ELECTRO-MAGNETISM.}

Q. What is ELECTRO-MAGNEMISM?

$A$. It is the magnetism developed through the agency of electrical or galvanic action.

Q. What were the EARLIEST PHENOMENA observed which indicated a relation between magnetism and electricity?

A. It was noticed that ships' compasses have their directive powers impaired by lightning, and that sewing needles could be rendered magnetic by electric discharges passed through them.

Q. What DIscovery, made by Professor Oersted of Copenhagen, established beyond doubt the connection of electricity and magnetism?

$A$. He ascertained that a magnetic needle placed near a metallic wire connecting the poles of a galvanic battery was compelled to change its direction, and that the new direction it assumed was determined by its position in relation to the wire and to the direction of the current transmitted along the wire.

Q. What oTHER IMPORTAXT DISOOVERY was made about the same time?

A. It was found that if a bar of soft iron, not possessing magnetic power sufficient to elevate a grain weight, be placed within a coil of copper wire through which a galvanic current is passing, it will 
become through the influence of the current a powerfut magnet; and will, so long as the current flows, sustain weights amonnting to inany hundreds of pounds.

Q. Is tre magnetic power of the bar found to be whoulr DEpradsan on the existence of the current?

A. It is; the moment the current stops, the weights fall arouy from the bar in obedience to the law of gravity.

Q. How GREAT WeIGH's have been lifted by magnets formed in this manuer?

A. An electro-magnet constructed by Professor Henry was capable of elevating and sustaining about a ton weight. (See fig. 131.)

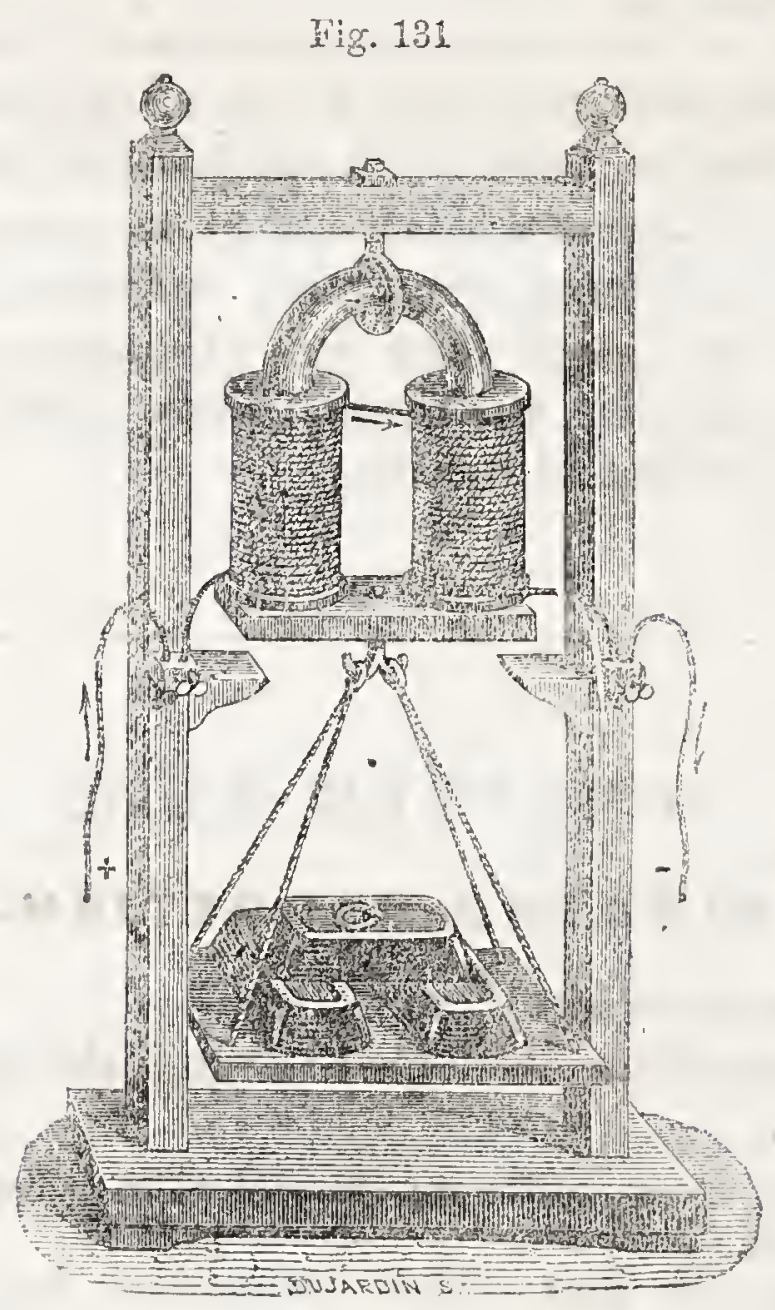

Q. What ormen Expmenrms have been made in the science of eletro-magnetism?

A. Professor Moll, of Utrecht, with a battery presenting a rine surface of eleven square fect, caused the magnet to lift seventy-fere pounds.

Q. Are an op'TrCaL EFFEoTs produced by electro-maguetism?

A. Yes; Professor Faraday discovered that a strong electromagnet had such a powerful effect on some transparent substances, that a polarised ray of light passing through them in the direction of the magnetic poles, the plane of polarisation, underwent a change, according to the direction of the magnetic influence.

In figure 132 is shown the apparatus employed by Professor Farnday to obtain

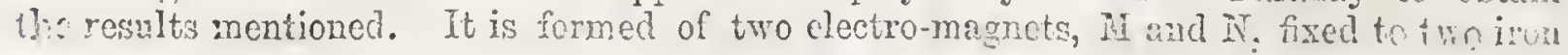


Fig. 132.

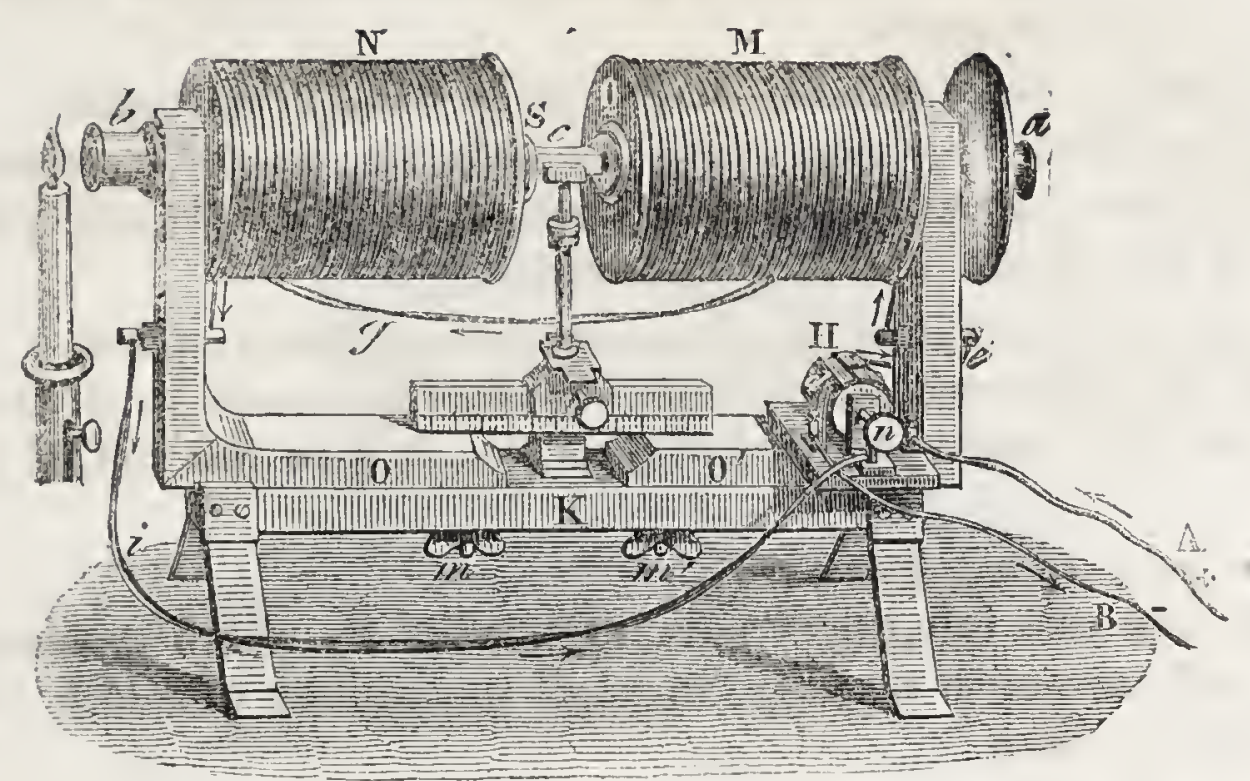

slides, $O$ and $O^{\prime}$, sliding on a support, $K$. The elechic current of a battery eiters at $A$, passes along to a cylinder $\mathrm{H}$, thence to the bobbin, $\mathrm{N}$; su : $:$ long the wire $g$ to $\mathrm{N}$, descends to $i$, reaches the cylinder $\mathrm{H}$, and goes ont at 3 . The iron cylinders, $\mathrm{S}$ and $\mathrm{Q}$, are pierend with holes, to allow the light to pass through. At $b$ and $a$ are two prisms, the former acting as a polariser, the latter as an analyser; thus the prism a completely extinguishes the light which passes through the prisin 7 , as long as the electric current does not pass ; but directly the communication is established, the light appears; and whichever way we turn the analyser, $a$, it still exhibits the various colours of the spectrum. This shows us the effects which are produced uin light by electro-magnetisin.

Q. Tas this enormous foree, developed and destroyed in an instant by making or breaking the chrrent, been employed for any IRuOrical PORPOSi?

1. The attempt has often been made to take advantage of it for nopelling machinery, but thus far all efiorts have failed to produce any pracical result.

Q. What is the RIASON why this enormons pover cannot be used with advantage?

A. Because the rate at which the power diminishes as we recede from the contact point of the magnets prevents our obtaining the full force of the magnets. Whus, a magnet whose force in contact would be sufficient to raise 250 pounds, would exert a force of only 30 pounds at the distance of $\frac{1}{5}-$ th of an inch, and of only 40 pounds at the distrance of $\frac{3}{50}$ th of an inch.

Q. What oxmse Diwroultx has presented itself?

A. It is found that, notwithstanding the loss of power with distance, a still greater loss takes place with motion. The moment any magnetic body is moved in front of either a permanent cr an electromagnet it loses power, and this loss increases very rapidly with the increase of velocity.

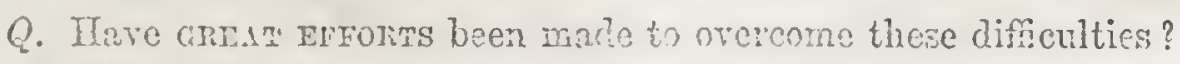

\&. Professor Jncobi, unter the rirection of the Russian government, expender uprards of $=30,000$, and then abundoned the enterprise as impreticticable.

Q. Can a span be obtained from an clectro-rnarnet as from an electrical rachine or gavauic bute $y$ ? 
A. Heat and light, by proper arrangements, can betin be obtained from the electro-magnet.

Q. Can COXSTANT and POWERFUL electro-currents be obtained from magnets?

A. A combination of horse-shoe magnets can be so arranged, that by causing them to revolve near to each other, a steady and powerful current of electricity can be generated.

Q. By what INFLUENCE is it supposed that the earth is rendered magnetic?

$A$. By the influence of currents circulating about it, in the same manner as they circulate around the iron bar in the coil of coner wire.

Q. What is the ELECTRic TELegraph?

$A$. The electric telegraph is an apparatus by which messages can be sent with immense rapidity, through the agency of voltcic currents along metallic wires.

Q. How LONG have such telegraphs been in USR ?

A. In $1814 \mathrm{M}$. Sommeny invented a telegraph based on the electrical theory; but the telegraph could not be made simple enough till Professor Wheatstone introduced the use of electro-magnets.

Q. What VARIETIES of the electric telegraph are used?

$A$. The principle in all is the same; the form only is varied.

Q. How MaNY DIFFERENT Forms are there?

$A$. We may mention three: the dial telegraph, the signal telegraph, and the writing telegraph.

Fig. 133.

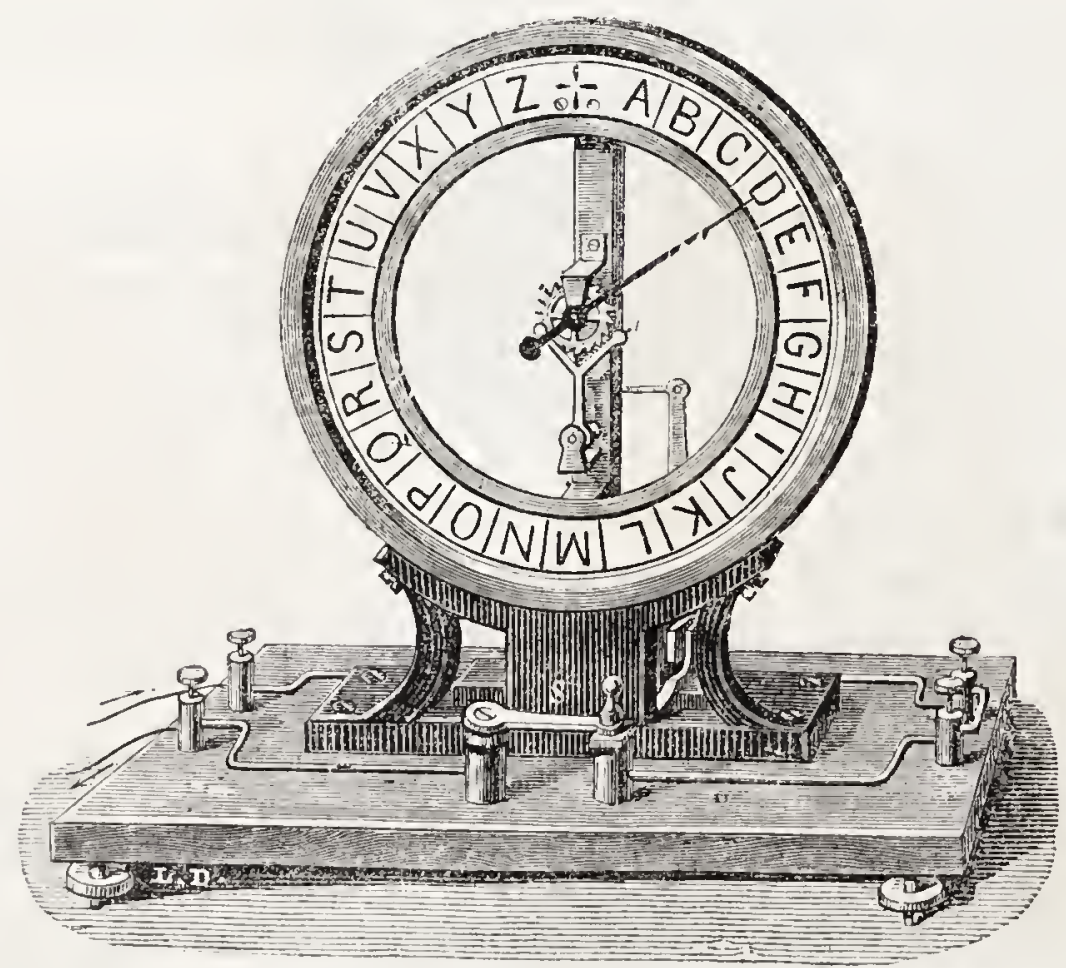

TIIE DIA PLATE OF AN ILECTRIC TELEGRARIS.

Two dial yiates are employed; one at the office from which the message is sent, one at the office at which the message arrives. The needle, or index, is moved by the hand of the operator to the different letters inscribed on the dial, until the word, or words, to 
be transmitted are speit. The electric current moves the hand on the lial at the other office in exactly the same way, so that the word, or words, are spelt there also; and thus a message is conveyed.

Fig̣. 134 .

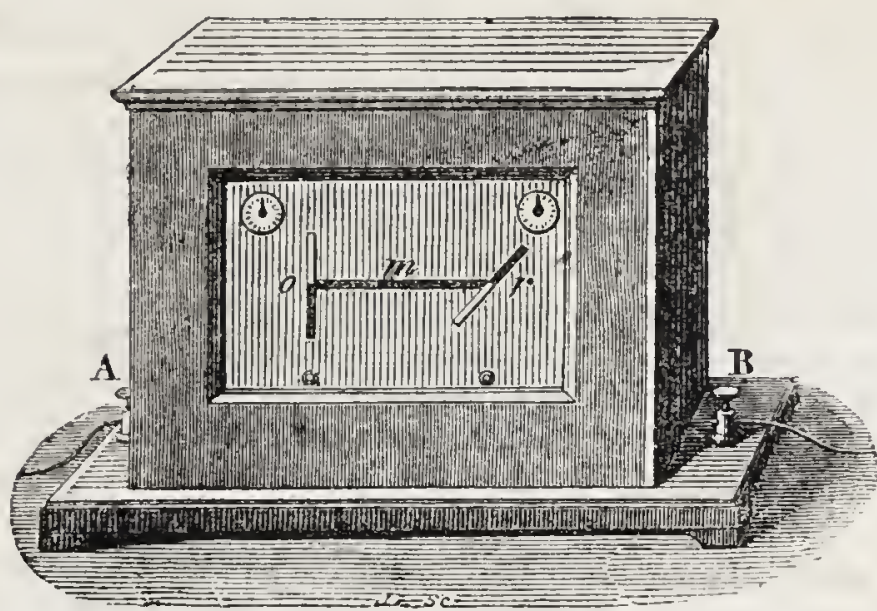

IMY RWCMYL GF A SIGNAL TELEGRAPIF.

The engraving shows the front of a box on which is placed a white plate, with a black line, $m$, having at its two ends movable radii $o, r$. The different angles assumed by these, in all forty-nine, convey different signals, previously arranged and understood. The motion of the indicator's is produced by clock-work, and an electric magnet in connection with the wire A B, which extends from station to station, and conveys the electric current.

Q. Upon What PRINciple does the construction of the writing magnetic telegraph depend?

A. Upon the principle that a current of electricity circulating about a bar of soft iron is capable of rendering it a magnet.

Fig. 135 .

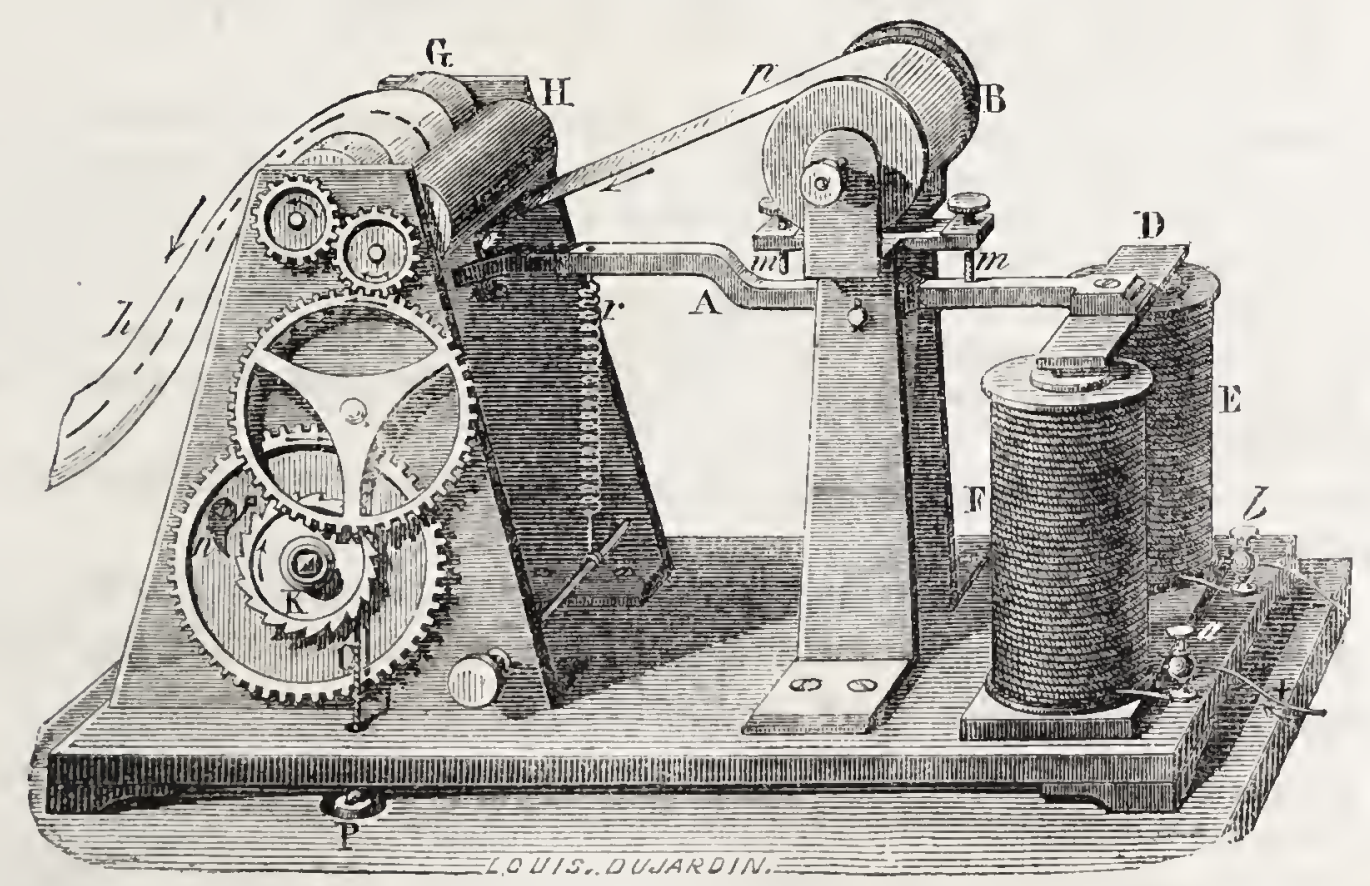

The arrangement by which this priuciple is mado avatilable in the construction and operation of this magnetic telegraph will be understood by reference to the accompanying diagram (fig. 135), which represents the construction and arrangement of this form of telegrapls. F and $\mathrm{E}$ are pieces of soft iron, surrounded by coils of wire, which are connected at $a$ and $b$ with wires proceeding from a galvanic battery. When a current is transmitted from a battery located one, two, or three hundred miles, as the case may ba 
it passes along the wires and into the coils surrounding the pieces of soft iron, $F$ and $\mathrm{E}$, thereby converting them into magnets. Above thoso pieces ef soft iron is a metalic bar or lever, A, supported on its centre, and having at one end the arm D, and at the other a small steel point, $o$. A ribbon of paper, $p h$, rulled on the cylinder, $\mathrm{B}$, is dirawn slowly and stendily off by a train of clock-work, $K$, moved by the action of the weicht, $P$, on the cord, C. This clock-work gives motion to two metal rollers, G aud H, letween which the ribbon of paper passes, and which, turning in opposite directions, draw the paper from the cylinder, B. The roller, H, has a groove around its circumference (not reprcsented in the engraving), above which the paper passes. The steel point, o, of the lever, $A$, is also directly opposite this groove. The spring, $r$, prevents the point from resting upon the paper when the telegraph is not in operation.

The manner in which intelligence is communicated by these arrangements is as follows: The pieces of soft iron $\mathrm{F}$ and $\mathrm{E}$, being rendered magnetic by the passage of a cirrent of electricity transmitted from the battery through the coils of wire surrounding them, attract the metal arm $D$ of the lever $A$. The end of the lever at $D$ being depressed, the steel point $o$ at the other extremity is elevated and caused to press against the paper ribbon and indent it. When the current from the battery is broken or interrupted, the pieces of soft iron $\mathrm{F}$ and $\mathrm{E}$, being no longer magnetic, cease to attract the arm $\mathrm{D}$. The lever $\mathrm{A}$ is therefore drawn back to its former position by the action of the spring $r$, and the steel point $o$ ceases to inclent the paper. In this way marks of any length can be made, and by a series or combination of dots and marks intelligense is communicated.

Q. Upon what PRINCrple is the chemical telegroph, commonly known as Bain's, constructed?

$A$. In this there is no magnet created, but a small steel wire, connected with the wire from the line, presses upon the roll of paper moved by clockwork. This paper, before being coiled on the roller, has been dipped in a nearly colourless chemical solution, but which becomes coloured when an electric current passes through it.

By sending a current through the wire resting on the paper, we can stain it, as it were, in dots and lines in the same manner as the last instrument embossed it in ciots and lines.

Q. What was the METEOD first proposed for COMMUNCATING ITELIEGNCE by electricity?

A. By turning a compass needle by causing a current of electricity to pass along its length.

The manner in which this turning of the needle would serve to communicatc intelligence would be as follows:- If at a given point we place a galranic battery, and at a hundred wiles from it there is fixed a compass needle, between a wire brought from, and another returning to the battery, the needle will remain true to its polar direction so long as the wires are frec from the excited battery; but the moment connection is made, the electricity of the whole extent of wire is disturbed, and the needle is thrown at right angles to the direction of the current. The motion of the needle may thus be made to convey intelligence.

Q. Why is it necessary, in conveying the telegraph wires, to SUPPORT them upon GLASS or EArTuren cylinders?

$A$. These are used for the purpose of insuring the perfect insulcation of the wires, since but for this the electricity would pass rlown a damp pole to the earth, and be lost.

Q. Is there any rRUTH in the idea that many persons have, that some PRINCipLI passes along the telegraphic wires when intelligence is transmitted?

A. This supposition is wholly crroneous; the word current, as something flowing, conveys a false idea, but we have no other term to express electrical progression.

Q. How can we gain an idea of what really takes place, and of the Naturis of the INFLUENCE transmitted?

$A$. The earth and all matter are reservoirs of electricity; if we 
disturb this electricity at London by voltaic influence, its pulsations may be felt in York. Suppose the telegraphic wire were a tube, extending from London to York, filled with water. Now, if one drop more is forced into it at London, a drop must fall out at York, but no drop was caused to pass fiom London to York. Something similar to this occurs in the transmission of electricity.

Formerly two wires were required in telegrapling: one conveyed tho current from the battery to the electro-magnet at a distance through which it passed, and then returned by another wire back to the battery. Now but one wire is used. It was found that the carth itself might be made to perform the function of the returning wire. All that is necessary is that one short wire from the galvanic battery at one end of a line, and lioun the electro-magnet at the other end, should be suak into the moist earth, and there connected with a mass of conducting 1netal, from which the electricity passes to complete the closed circuit.

Q. Can electricity be employed for Measuring tine?

A. It can; and by its agrency a great number of clocks can bo kept in a state of uniform comectness, which no other arrangement can effect.

Q. How is this Accompusmon?

A. A battery being united with the chief clock, which is itself connected by wires with any number of clocks arranged at a distance from each other, has the current continually and regulcorly intervipted iny the beating of the pendulum, which interruption is experienced by all the clocks included in the electric circuit; and, in accordance with this making and breaking of contact, the indicators or hands move over the dial at a constantly uniform rate.

\section{CHEMISTRY.}

\section{$\longrightarrow$ \\ CHAPTER XIIII.}

\section{CHEMCAL FORCES AND AGENCIES.}

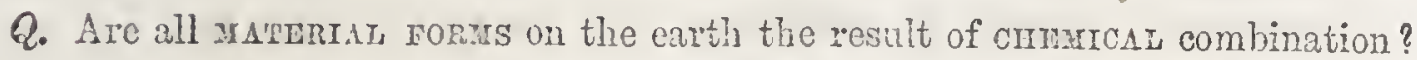

$A$. They are; and the chief object of the science of chemistry is to ascertain the composition of substances, and to determine the laws by which they are governed.

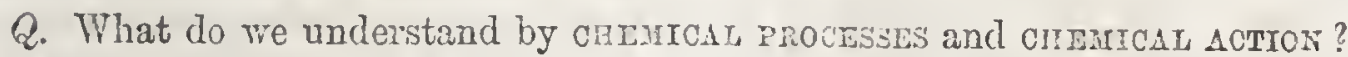

A. Such processes or action by which the weight, the form, the solidity, the colour, the taste, the smell, and the infuence of substances become changed, so that new bodies with quite different properties are formed from the old. 
Q. Are chemical processes and action CONTINUALLY GoING on in the bodies and systems of all animals and plants?

A. Yes; but so long as the animal or plant is endowed with rife, the chemical forces are controlled by a higher power, which we call vital force.

Q. When an ANimal or PLANT Dres, what becomes of these two forces?

A. The vital force cecses to exist, and the chemical forces, gaining the mastery, reduce all the component parts of the former living structure to their ultimate elements. "Earth returns to earth, dust to dust."

Q. Into what Two great CLASSES may all substances be divided?

A. Into simple and compound substances.

Q. What is a SIMPLE SUBSTANCE?

A. A body which cannot be divided, analysed, or decomposed, is called a simple substance or element: gold, silver, iron, oxygen, hydrogen, and nitrogen are considered simple substances or elements, because we are unable to decompose them, convert them into, $n$. create them from, other bodies.

Q. What is a compound substance?

A. One that can be divided, decomposed, or converted into other substances : air is a compound body, because it is composed of oxygen and nitrogen.

Q. With воW MaNY simple substances or elements are we acquainted?

$A$. The number now known to exist is sixty-four.

Q. Are these substances EquALI DISTRIBUTED over the surface of the earth ?

$A$. 'They are not; most of them are exceedingly rare, and some ten or twelve only make up the great bulk and mass of all the objects we see around us.

Q. The elementary bodies by uniting form compounds: into what Two great cLAssws may all the compounds of matter be divided?

A. Into organic and inorganic substances.

Q. Can all the ELEMENTs take part in forming organic and inorganic bodies?

A. All of them are capable of entering into inorganic combinations, but only a fcu are capable of forming organic compounds.

Q. What is an INoraAnic substance?

A. An inorganic substance is a product of the so-called inanimate world-a substance which is destitute not only of life, but of any organisation or properties derived either directly or indirectly through the agency of the vital force.

A crystal has a regular form and symmetry, and may present a beautiful and complex arrangement of parts; but this complexity of arrangement was not produced through auy vital agency, neitber were the particles of which it is composed ever endowed with life. Nost of the inorganic substances which occur in nature may be artificially imitated by combining together the materials of which they are composed.

Q. What is an ORGanic substance?

A. An organic substance or compound is one which has originated 
or been produced through the agency of life, or the instrumentaitity of the vital force.

The distinctive characteristic of inorganic bodies is rest; the aistinctive trait of organised bodies is motion, life. A rock or crystal once formed, and left undisturbed by foreign influences, never changes. An organic body, on the contrary, is limited in its duration, and will nltimately decompose, change, die, and its elementary constituents assume new and varied forms.

Q. Can we make ARTIFICIALLY an oRGANIC body?

A. Strictly speaking, we cannot; chemistry has, however, in certain cases, succeeded in forming certain compounds artificially, which occur as products of organic life; but all of these bodies require for their production the aid of an organic body.

With all their efforts, chemists have not succeeded in forming an organised tissite artificially, or any one of the compounds of which such tissues are made.

Q. Give some examples of inorganic and organic bodies.

A. Glass, sulphur, antimony, iron, \&c., are inorganic bodies; while sugar, alcohos, fat, glue, \&c., are organic bodies.

Q. What is DECAY, which we see in almost every form of matter ?

A. Simply chemical change.

Q. Can we ANNHHLATE any material substance?

A. We cannot; what appears to us as annihilation is only a chemical change. When we cause a $\log$ of wood to disappear by burning, there is no annihilation; all the matter formerly contained in the wood exists as much after the combustion as before, only its state and condition have been changed.

Q. If we heat a piece of iron to redness, it becomes covered with a SCALE or RUST; and if it is now weighed, it will be found to have increased in weight. It has absorbed oxygen from the air. By what name do we designate the force or attraction which caused the oxygen of the air to unite with the iron?

A. The force of affinity or kindred.

Q. How will you define chemical attraction, or AFFinity?

A. It is the peculiar disposition which one body has to unite with a different body, to the rejection of others which are dissimilar in their properties.

Q. Why will not o1 and WATER unite?

A. Because there is no affinity between the oil and the vater.

Q. Why will ALCOHOL and WATER readily unite?

$A$. Because there is a strong attraction between the elements of the alcohol and those of the water.

Q. If we adrl a little POTASH to the oIL and WATER, what is the effect?

A. The repulsion of the oil and water is overcome, and the there unite toyether to form soap.

Q. If to the soap we add an AOrD, what ensues?

A. The potash has so much stronger affinity for the acid than it has for the oil and water, that it quits both, and unites with the acid, leaving the oil and water to repel each other as before. 
Q. Are the different DEGREES of AFFINITY, Or ATTRACrION, which one body has for other substances constant in their action; or, in other words, may their attraction be always depanded on?

A. These elections are so constant, that chemists have been enabled to drau up tables for the purpose of showing the order in which they occur.

Q. Where one element unites with another, what is the Rosuturisg produor?

A. An acid, a base, or a neatral body.

Q. What are $\triangle$ CIDS?

A. Acids are substances which excite the taste of sourness when applied to the tongue; they change the blue juices of vegetables to red, and combine with allialies to form neutral compounds.

Q. What is an AIIKALI?

A. An allati is a body that possesses properties the converse of those of an acid. It has a highly bitter, acrid taste, changes the blue juices of vegetables green, or the juices of vegetables which have been changed red by an acid, back again to blue. Potash and soda are representatives of the alkalies.

Q. What is a NEUTRAL BODY?

A. One which possesses neither acid nor allatine properties.

Q. What is a BASE in chemistry?

A. A body which may be either an allati or a neutral subsiance which is capable of uniting with an acid. Thus, potash and soda, the oxide of iron, lime, \&c., are bases.

Q. Where ACIDS and BASES UNITE, what is the resulting compound called?

A. A salt.

Thus, carbonate of lime (chalk), composed of carbonic acid and lime, is a salt ; saltpetre, composed of nitric acid and potash, is another.

Q. Is the composition of all bodies FIXED and INVARIABLE?

A. It is; and a compound substance, so long as it retains its characteristic properities, must consist of the some elements united in the same proportions.

Q. When bodies combine chemically with each other, do they combine arbitrarily, or in some FIXED PROPORTIONS?

A. The proportions in which bodies combine chemically with each other are fixed and invariable.

Thus, water consists of one part of hydrogen united to eight parts (by weight) of oxygen. In whatever condition water may exist, whether in vapour, in springs, in the ocean, or as ice or snow, it is thus uniformly composed. When artificially prepared, by burning oxygen and hydrogen together, one grain, ounce, or pound of hydrogen is required for every eight grains, ounces, or pounds of oxysen. If one and a half ounces of hydrogem are taken, then a half ounce is left behind; if nine ounces of oxygen are taken, then one ounce will remain over uncombined.

Q. Into what two great CLASSES may the elements or simple substances be divided?

A. Into metallic and non-metallic bodies.

Q. To what ELEMents do we apply the term metallic?

A. To those which are good conductors of heat and electricitiy, which have a peculiar lustre, known as "metallic lustre," are 
opaque, insoluble in water, and are fusible through the agency of heat. All the metals, with the exception of mercury or quicksiver, are solid bodies.

Q. What are METALLOID, or Now-METALIIC elements?

A. Those elements which do not possess the provertiei and characteristics of the metals.

They are wanting in metallic lustre, and are bad conductors of heat and electricity; the metallic elements being, on the contrary, good conductors. Most of the metalloids combine with hydrogen, while the metals do not; most of them also combine with oxygen to form acids, while the majority of the inetals unite with oxygen to form bases. When compounds of tho metallic and non-rnetallic elements are decomposed by the galvanic battery, the metals appear at, or are attracted by, the negative pole, while the metalloids appear at, ol are attricted by, the positive pole. The non-metallic elements include solids, liquids, and gases.

Q. Name the Non-Metaldic ElFMerts.

A. Oxygen, hydrogen, nitrogen, carbon, sulphur, phosphorus, fluorine, chlorine, bromine, iodine, selenium, and boron.

Q. What are called the merats?

A. They are-potassium, sodium, lithium, barium, strontium, calcium, magnesium, lanthanium, didymium, cerium, yttrium, erbium, terbium, norium, glucinum, aluminum, thorium, zirconium, silicium, titanium, tantalium, niobium, pelopium, tungsten, molybdenum, vanadium, chromium, uranium, manganese, arsenic, antimony, tellurium, bismuth, zinc, cadmium, tin, lead, iron, cobalt, nickel, copper, mercury, silver, gold, platinum, palladium, rhodium. iridium, ruthenium, and osmium.

Q. Has one metal ever been CHANGED or transmuted into another?

A. No.

Q. What was it that the alchemists desired to EFFECT?

A. They endeavoured to transmute, or change, lead or some cheap metal into gold.

Q. Is there any reason for supposing that one metal can, or ever will be orANGED into, or manufactured from, ANOTHER MLTAL?

A. At present we have no good or sufficient reason for supposing that this will ever be the case.

There is, however, some reason for supposing that some of the bodies which we now call simple or elementary are in reality compound; but we have not been able as yet to verify or prove this supposition.

Q. What were the Four ELEMENTS of the ancients?

A. They supposed all things to be composed of fire, earth, air, and water.

Q. Are any of these ELEMENTS?

A. No: air, earth, and water are, however, significant of the forms in which all matter exists; everything with which we are conversant upon the surface of the earth being either solid, liquid, or aëriform. Fire is only a visible and perceptible indication of changes occurring within the so-called elements. 
Q. What do we mean by Chemical ANaLYSIS ?

A. The separation of the elements of a compound from one another, as when, by the aid of galvanism we decompose water into oxygen and hydrogen.

Q. What is SYNThesis?

A. The combining of the elements of a compound together, so as to form that compound; as when oxygen and hydrogen are united by the agency of electricity, and form water.

\section{CHAPTER XLIV.}

\section{OXYGEN.}

Q. What is OXYGEN?

A. A gas, colourless, tasteless, and odourless; it is heavier than atmospheric air, and is a non-conductor of electricity.

Q. Is oxygen a substance existing in GREAT ABUNDANCE ?

A. Oxygen is the most abundant of all known substances; it con. stitutes at least one-third of the solid mass of the globe, eight-ninths of all water, and nearly one-fourth part of the atmosphere; it also exists in most organic substances.

Q. What is the DIFFERENCE between OXYGEN and an OXIDE?

$A$. Oxygen is a gas, and an oxide is a compound formed by the mion of oxygen with other bodies.

Q. WHo gave this gas the NAME of OXYGEN? and what is the signification of the word?

$A$. Lavoisier gave it the name, which is derived from two Greek words ogvs (oxus), an acid, and yevvaw (gennao), I produce.

This name was given to it because it was then thought to be the sole acidifying principle. Modern discoveries have rectified this error by proving the existence of acids in the composition of which there is no oxygen.

Q. Is OXYGEN ever found in a LIQUID or SOLID state?

A. No: when pure, it is only known in the gaseous state; all efforts to reduce it to a liquid or solid condition by cold or pressure have completely iailed.

Q. Of what usr is Oxrgen in the $\Lambda$ TMrosphere?

A. It sustains animal life and supports combustion.

Q. If an animal were immersed in oxygen gas, would it IIVE LONGER than in an equal volume of confined air?

A. It would; at the same time, animal life could not be sustained for any great length of time in an atmosphere of pure oxygen.

Q. What is meant when it is said that the oxygen of the air "supports combusmion?"

$A$. It means this : That the oxygen of the air is essential to combustion. 
Q. How does the OXYGEN of the air make FuEL BUhis?

$A$. The fuel is decomposed (by heat) into hydrogen and carbon; and these elements combining with the oxygen of the air, produce combustion.

Q. What is meant when it is said that oxyGEN " sustaINS LIFE?"

$A$. It means this: If a person could not inhale oxygen, he would die.

Q. What GOOD does this inspiration of OXYGEN do?

A. 1. It gives vitality or life to the blood; and

2. It is the cause of animal heat.

Q. What are oxides?

$A$. The compounds formed by the union of oxygen with other bodies bear the general name of oxides.

Generally, when we speak of oxides, we mean simple substances, which are united to a smaller quantity of oxygen than is necessary to form acids.

Q. What is the meaning of the terms PERoxide and PRotoxide?

$A$. When a substance unites with oxygen in two different proportions, that which contains the greatest quantity of oxygen is called peroxide, and that which contains a less quantity a protoxide.

Q. What is a SUBoxIDE?

$A$. Many metals have the power of uniting with oxygen in more than these two proportions. In this case the combination which contains a less quantity of oxygen than the protoxide is called a suboxide, and the highest combination of the substance with oxygen is called a hyperoxide.

Q. What is RUST ?

$A$. The oxidation of iron in moist air.

"Oxidation," combination with oxygen.

Q. Why does Iron Rust?

$A$. Because water is decomposed when it comes in contact with the surface of iron; and the oxygen of the water combining with iron, produces an oxide, which is generally called rust.

Q. Does iron Rust in DRY air?

$A$. No; iron undergoes no change in dry air at common temperatures.

Q. Why does hot iron SCALE and PBEL OFF when struck with a HAMSIER ?

$A$. Because the oxygen of the air very readily unites with the surface of hot iron, and forms a metallic oxide (or rust), which scales off when struck with a hammer.

Q. Why does PAINTING iron prevent it from RUSTING ?

$A$. Because paint prevents the moist air from coming in contact with the iron.

Q. Why will BRIGHT IRON LOSE its POLISH by being put into a FERE?

$A$. Because the oxygen of the air rery readily unites with the 
surface of hot iron, and forms a metallic oxide, which displays itself in this case by a dull leaden colour instead of a red rust.

Q. Why does ornanental srrad (of a purple or LILAd colour) rust MORE READILY than polished wiIrese steel?

A. Because the lilac tinge is produced by partial oxidation, and the process which frims rusts has, therefore, already commenced.

Q. If DRY AIr contri.is OXYGur, why does it NoT RUST IRoN as well as rorst air?

A. Becanse moisture is always needed, in order to bring into action the affuing of oxygen for the iron at ordinary temperatures.

q. Whiy does copper taraisi?

A. Whe tarnish of copper is caused by its oxidation; that is, the orygen of the air combines with the surface of the copper, and (instead of rusting it) covers is with a dark tamish. air?

Q. Why does IE:D Losi its BrigritYiss, and become DULu by being exposed to the

A. The clullness of the lead is caused by the presence of a curbonate of the oxide. When the oxide is formed through the ageney of moist air, it attracts carbonic acid from the air, and (combining with it) produces a carlonate, which gives the chull tint to old lead.

Q. Why is it diffeult to keep SILVER Brignt?

A. Because the voxpour of the air oxidises its surface, and tamislies it.

Q. Is GoLD affected by the ATMOSPIIIRE?

A. It is not; gold will never combine with oxygen of itself (that is, without aid).

Q. What onHER METAL besides gold is capable of resisting the oxidising effests of air and moisture?

A. Platinum. Both gold and platinum, however, can be marie to unite with oxygen through chemical agencies.

Q. Why are gold and platinum often used for the construction of DELICLIE INSTREMENTS ?

A. Because they never oxidise, but retain their bright surfaces under all circumstrinces free from rust or tarnish.

Q. Can oxygen be made to combine with Ari the other elements?

A. It can be made to enter into combination with all the elementary bodies except fluorine.

There are several methods of gencrating oxygen gas. One of these methols is that which is detailed below, and for which it will be necessary to laive the following apparatus: A Iarge lest-tube, about threc-quarters of an inch in diameter, $a$ bent glass tube, a pneumatic trough ur basin, and a receiver.

The substance we shall require as the oxygcn-yielding material is a mixtme of two parts of chlorite of potash and one part of manganese, or rather the black oxidc of manganese. These two substances must be rubbed together in a mortar. Puar alout a tea-spoonful of the mixture into the test-tube, arrange the apparatus, and apply hent. Oxygen gas will come ovcr ranidly. The first portions are contaminated with the com. mon ain in the apparatzs. After this hus heen thrown away, we collect pure oxygen gas. 


\title{
UHAPTER XLV.
}

\section{HYOROGEN AND WATER:}

\author{
SECTION I.-IIIDROGEN.
}

\section{Q. What is IIYDROGH?}

A. It is an inflammable gas, one of the elements of which water is composed. The gas used in our streets is in great part hydrogen. Hydrogen, when pure, has neither taste nor odour.

Hydrogen dzrives its name from two Greek words viowo (udor), water, an? $\gamma \varepsilon z / z \alpha u$ (gennau), I produce.

Q. When was hydrogen gas discovined?

A. About the middle of the eighteenth century, and was tormed inflammable air.

Q. Does HXDRochan SUPPort IITE?

A. No; it destroys it, rather by exchuing oxygen than by its own injurious effects.

Q. Does kydrogen gas, like oxygen, supponer conbustron?

A. No; it is highly combustible, but does not support com?rustion; with oxygen, it forms water.

Q. What are the PECULIAR CHARACTERISTICS of hydrogen gas?

A. 1. It is the lightest of all known substances;

2. It will burn immediately on being ignited; and

3. A lighted candle (immersed in this gas) will be instantly extinguished.

The following experiment shows the extreme lightness of hydrogeil. Attach to one end of a thin slip of deal a glass or tumbler, and at the other end of the same slip of deal anything for the suspension of a courterpoise. Next support the slip by a fulcrum. Then, arranged under the susperded tumbler, let there be placed the bottle and pipe delivering hydrogen gas. The lightness of this gas is immediately proved by the tumbler being raised.

Q. For what usrs are hydrogen gas employed?

A. 1. Owing to its levicy, it is used to inflate balloons;

2. Burned with oxygen, it constitutes the oxy-hydrogen blowpipe; and, combined with carbon, it forms the ordinary illuminating ras; and

3. It is a powerful chemical agent.

"Chemical agent," a substance employed to efiect chemical changes.

2. What is a RLOWPIPE?

1. The blowpipe (see fig. 136) is a tube usually bent near the end, and terminated with a finely perforated jet or nozzle. This is used to

Fig. 136. 
propel a current or blust of air from the lungs, through the flame of $\alpha$ lamp or candle, upon a substance which it is desired to fuse. A small conical flame is produced in this manner, possessing an intense degree of heat.

Q. What is an OXY-HIYDROGEN, Or COMPOUND BLOIPIPE?

$A$. An instrument in which the current or blast is formed of the two gases, oxygen and hydrogen, instead of atmospheric air. These gases are made to unite at the orifice of the blowpipe at the moment when they are ignited. The heat produced by their combustion and union is sufficient to melt almost every substance upon which the blast is directed.

Q. Can you describe the Drummond Light?

$A$. It is the ignited flame of a mixture of oxygen and hydrogen? projected against lime; the lime becomes intensely luminous, and forms the well-known Drummond light.

SECTION II.-WATER.

Q. What is Water?

$A$. Water is a fluid composed of oxygen and hydrogen, in the proportion of eight parts of oxygen to one part of hydrogen.

Q. Why is WATER FLUID?

$A$. Water is fluid because its parts are kept separate by latent heat.

Q. Why is PUMP WATER HARD, or why is it called HARD WATER?

$A$. Because it contains matter which does not so quickly dissolve substances put into it.

Q. What is this HARDFNiNG MATTER?

A. Earth and minerals, which mix with the water as it filters through the earth.

Q. What is MEANT by a MINERAL SPRING?

$A$. Spring water which contains mineral matter.

Q. When is a mineral water called chaLybeate?

$A$. When it contains iron, in some form, dissolved in it.

Q. Mineral springs exist in all parts of our country: what is the NATURE of the suiv. stances contained in them?

A. The great majority are only impregnated with iron, suli, or sulplutr. Some few, however, contain many different substances.

Q. Why are springs containing iron in large quantities BENEFICTAL to some InVALids?

$A$. Because the iron contained in the water acts as a tonic; that is, it strengthens and invigorates the system.

Q. What QUANTTYY of mineral,matter is generally contained in comparatively pure natural water's?

A. Any water which contains less than fifteen grains of solid mineral matter in a gallon, is considered as comparatively pure. Some natural waters are known so pure that they contain only $\frac{1}{20}$ th of a grain of mineral matter to the gallon, but such instances are very rare. 
Waters obtained from different sources may be classed as regards comparative purity as follows :-

Rain water must be considered as the purest natural water, especially that which falls in districts remote from towns or habitations; then cones river water ; next, the water of lakes and ponds; next, spring waters: and then the waters of mineral springs. Succeeding these, are the water's of great arms of the ocean, into which immense rivers discharge their volumes, as the water of the Black Sea, which is only brackish ; then the waters of the ocean itself; then those of the Mediterranean, and other inland seas; and last of all, the waters of those lakes which have no outlet, as the Dead Sea, Caspian, Great Salt Lake of Utah, \&c. \&c.

Q. How much solid sutTer is ordinarily contained in a gallon of sea water?

A. From twenty-two hundred to twenty-cight hundred grains.

Q. How much solid Matrer is contained in a gallon of water from the Dead Sea?

A. From eleven thousand to twenty-one thousand grains, or nearly one-fourth part of its weight.

Q. Does AIr exist in all natural waters?

A. It does: fishes and other marine animals are dependent on the air which water contains for their existence.

Q. Would absolutely PURE WATER act as POISON to a fish ?

$A$. The fish would die of suffocation in such water.

Q. Where is the PUREST WATER to be found as a natural product?

$A$. The purest natural water that can be procured is obtained by melting freshly fallen snow, or by receiving rain in clean vessels at a distance from houses.

Q. Why is FLowING WATER not liable to become stagnant?

A. Because its currents carry awvay all contaminating substances to the sea.

Q. What makes water BUBBLE and SPARKLE?

A. The air or gas contained in it.

Q. Why is water fresh from the well or fountain more SPARKLING and REFRESHING than the same water after it has been for some time exposed to the air?

A. All spring and well waters contain atmospheric air, oxygen, and carbonic acid gases, dissolved in them.

The amount of these substances contained in water depends upon its tenperature, cold. water dissolving and retaining a larger quantity than warm or tepid waters. When cold waters from springs or fountains are exposed to the air, they become elevated in temperature, and the gases contained in them escape, rendering the water flat and insipid. The jrincipal agent in imparting a sparkle and freshness to water is atmospheric air, and not carbonic acid, as is often supposed and taught. The quantity of carbonic acid present in ordinary spring waters is generally inconsiderable.

Q. Why is it difficult to WASII with HARD water?

A. Because the water contains saline matters, which deprive the water of a part of its solvent power.

Q. Why is it difficult to wash with SOAP in salt water?

A. Because soap is insoluble in salt water.

Q. Why is RaIN WaTER SOFt?

A. Because it does not contain earths or minerals.

Q. Why is it MORE EASY to WASH with SOFT water than with MARD?

A. Because soft water unites freely with soap, and dissolves it; in 
hard water the soap is either insoluble or becomes decomposed. The solvent power of water increases also with its purity or softness.

Q. When we wash with soap in water, what CIImIrCAL ACTroN takes place?

A. The soap is resolved into a fatty substance and an alkali; the alkali dissolves most of the organic substances which constitute the dirt which we wish to remore, and the greasy matter effects by its lubricity an easy washing away of the dissolved matter from other substances.

Q. Why do wood ashes render IIARD water SOFT ?

A. Because they contain a powerful alkati-potash, which removes or neutralises those impurities in the water which rendered it hard and unfit for washing.

Q. What is a PETRIFACTIOY ?

A. An animal or vegetable substance converted into stone.

It frequently happens when an organic substance is saturated with water holding mineral matter dissolved or suspended in it, that as the particles of the substance gradually decay or become dissolved, their place is supplied by the mineral matter contained in the water ; until at last all of the organic particles disappear, and a mass of mineral matter is substituted, which resembles the original substance in form and structure, and not unfrequently in colour. It is a mistake to suppose, as is often done, that the original particles are converted into stone; the process of petrifuction is one of replacement, not of conversion, a particle of mineral matter of the same form being substituted for each organic particle.

Q. Why does Hor water dissolve sugar and salt more readily than cord water?

A. Because the heat of the water assists its solvent action, and opens for the water a passage through the particles of the substance.

Q. Why is the sea SALIT?

$A$. The sea has undoubtedly derived all its salt by washings from the land. The streams that have flowed into it for ages have been constantly adding to its quantity, until it has acquired its present condition.

Q. Why is NOT RAIN WATER SALT, aithough most of it is evaporated from the SEA ?

A. Because salt will not evaporate, and therefore, when sea water is turned into vapour, its sutt is left behind.

Q. Is there MORE Or IESS of SALT in every spring, river, or lake?

A. The saline (salt) condition of sea water is but an increase on that of all lakes, rivers, and springs; they all contain more or less of salt, but their contents are continually changing and discharging themselves into the sea; therefore the salt does not accumulate.

Q. Is every lake into which rivers flow, and from which there is no outlet except by evaporation, a SALT IAKE?

A. It is; and it is curious to observe that this condition disappears when an artificial outlet is provided for such waters.

Such lakes are the Dead Sea, the Caspian, the Sea of Aral, and the Grear Salt Lake of Jtah, the saltness of all of which exceeds that of the ocean.

Q. What GOOD PURPoses does the presence of so much salt in the ocean subserve?

A. It depresses the freezing point of the water many degrees, 
thereby diminishing the dangerous facility with which fields of ice are formed in the polar regions; it also aids in preventing the corruption of the water by the accumulation of animal and vegetable remains.

Q. What are the SUBSTANCES extracted from the earth which we find in sea water?

$A$. The most abundant substance is common salt; next, certain combinations of magnesia; then salts of lime, with small proportions of potash, iron, iodine, and bromine.

Q. Are these substances found in arost SPRINGs ?

A. With the exception of iodine and bromine, they may be found in small quantities in almost all springs and rivers.

Q. Are those substances which we call IMPT RITIES in water of any SERVICE to animal or regetable systems?

$A$. They give to water its freshness and sparkling properties; pure distilled water is very disagreeable to drink. These substances are also generally beneficial to the systems of plants and animals, and are absorbed by them with the water.

Q. Does water form PART of the conpositron of most bodies?

$A$. It enters directly into the composition of nearly all bodies which can be crystallised and most organic compounds.

Q. In what STATE does water exist in CRYSTAIS?

$A$. It is condensed into a solid form. Thus a crystal of alum contains one-fourth part of its weight of water, yet it is perfectly dry and solid. If we expose it to heat, the water soon boils and evaporates from it.

Q. If the waters of the ocean were not moved by winds, currents, and tides, what would be the EFFECr?

A. The water would become stagnant.

Q. Will water contaminated with animal and regetable matter, under some circumstances, PURIFY ITSELF?

$A$. Water contaminated with animal and vegetable matter, if kept for some time, undergoes a spontaneous purification, losing its offensive odour and colour, and depositing more or less sediment. Water for the supply of ships is well known to undergo this process of purification by fermentation; and the larger the quantity of destructible matter suspended in it, the more complete and rapid is its purification.

Q. What is a TIDE?

A. A wave of the whole ocean, which is raised to a certain height, and then sinks, after the manner of a common wave.

Q. What is the causs of tides?

$A$. The attraction of the sun and moon upon the waters of the ocean. The moon being nearest to the earth, her attraction is six times greater than that of the sun. This attraction of the moon raises the waters of the ocean as they come under her infuence by the motion of the earth on its axis. 
2. HOW MANY TIDES are there in a day?

A. Two in every lunar day-a period of 24 hours 49 miniates.

Q. What tides are the HIGHEST ?

A. The spring tides.

Q. Why are they higher than at other periods ?

$A$. Because the sun and moon are then in such a position that they exert their influence together. For every five feet of height in tide produced by the moon, the influence of the sun adds one foot.

Q. What are NEAP TIDES?

A. Low tides.

Q. Why are neap tides LowEr than other tides?

$A$. Because then the sun and moon have such positions that their attractive influence is opposed to each other ; and for every six feet of the moon's tide, the opposite attraction of the sun takes away one foot.

Q. How FAST does the tide wave move?

$A$. The rate of movement of the tide wave depends upon the nature and depth of the sea bottom. With a depth of one fathom, its rate is eight miles per hour; and with one hundred fathoms, eighty miles per hour.

Q. Does the height of the same tide VARY in different places?

A. The height of the tide in different places depends much on the configuration of the land; the same tide may rise in one place three inches, and in another place thirty feet.

Q. At what PERIOD during the day is it HIGH water?

$A$. When the moon passes the meridian - that is, when it is nearly. vertical over the place-the sea is elevated to the greatest extent, and it is said to be high water.

Q. WHEN is it Low water?

$A$. When the moon is upon the horizon, or about six hours after high tide. As the moon passes the meridian below the horizon, another elevation occurs, so that we have the ebb and flow of the tide twice every day.

Q. How MUch LATER does the tidal ebb and flow occur each day?

$A$. The tide becomes later every day by about fifty and a half minutes, which is the excess of a lunar day above a solar one: twenty-eight and a half minutes of the former being equal to twentyseven and a half minutes of the latter.

Q. What is the CAUSE of ordinary SEA WAVES?

$A$. The wind, pressing unequally on the surface of the sea, depresses one part more than another; every depression causes a corresponding elevation, and these undulations are called waves.

It must be remembered that waves have no other than a vertical motion, i.e., up and down. Any substance, as a buoy, floating on a wave, is merely elevated and depresser? alternately; it does not other-rise change its place. 
Q. What is the SURF?

$A$. When the shore runs out very shallow for a great exient, the breakers are distinguished by the name of surf.

Q. What do we know concerning the MAGNITUDE and VELOct:

$A$. On the Atlantic, during a storm, the waves rise to a height of about forty-three feet above the hollow occupied by the ship; the total distance between the crests of two large waves being 559 feet, which distance is passed by the wave in about seventeen seconds of ime.

Q. With WHAT VELOCITY is it estimated that such storm waves as above described travel ?

A. At the rate of about thirty-two miles per hour.

A wave is a form, and not a thing; the form advances, but not the subsiance of the wares.

\section{CHAPIER XLVI. \\ NITROGEN AND ITS COMPOUNDS.}

\section{SECTION I.-NITROGEN.}

Q. What is NITROGEN?

A. An invisible gas existing largely in atmospheric air, and in most animal and regetable substances.

Q. What are its principal characteristics?

A. 1. It is not combustible ;

2. It does not support animal life; and

3. It is the principal ingredient in the composition of atmospheric air.

Q. Is nitrogen capable of SUstainra combustron?

A. No ; nitrogen, like hydrogen, is incapable of sustaining combustion or animal existence, although it has no positive poisonous properties.

Q. Is there any DOUBT respecting the ELEMENTARY character of nitrogen?

A. There are some circumstances which have led to the belief that nitrogen is a compound body, and that its base is metallic. Nothing, however, is known with certainty concerning its real nature.

\section{SECTION II.-COMPOSITION AND CHEMICAL PROPERTIES OF THE ATMOSPIIERE.}

Q. What is atmospheric AIr?

A. Oxygen and nitrogen mixed together, in the proportion of seventy-nine parts of nitrogell and twenty-one of oxygen. 
Q. Is not the AIR we breathe almost wholly COMPOSED of NITROGE

$A$. It is; about four-fifths of the air is nitrogen, and the other one-fifth is oxygen.

Q. WHY is there so MucH nitrogen in the air?

$A$. The uses of nitrogen are in a great measure unknown. It has been supposed to act as a dituent to the oxygen, but it most probably serves some useful purpose in the economy of animals and vegetables, the exact nature of which has not been discovered.

Q. What would be the EFFECT if the proportion of oxygen in the atmosphere were INCREASED?

A. The inflammabitity (the quality of catching fire) of most substances would be increased, fires would burn out very quickly, and the functions of life would be called into such rapid action as to soon exhaust the powers of the system.

Q. Are the two gases, oxygen and nitrogen, existing in the atmosphere, CHEMrcaluY COMBINED, or merely mixed?

$A$. They are merely mixed, and not combined with each other.

Q. Does the atmosphere always contain any other INGREDIENTs besides oxygen and nitrogen?

$A$. There is always in the air, at all places, carbonic acid gas, in variable proportions, besides watery vapour.

Q. Is the air collected on the tops of high mountains, over marshes, in hospitals, and over deserts, the sAME in CHARACTER and COARPOSITION?

A. It is not found to vary, but is the same in all regions of the earth and at all altitudes.

Q. Are the component parts of air, oxygen, nitrogen, carbonic acid, and watery vapour, of different SPECIFIC GRAVITIES?

$A$. They are all different, carbonic acid gas being the heaviest.

Q. Then, as we have before stated that they are merely mixed, and not combined, why do they not ARRANGE themselves in the order of their DENSITIES, Or WEIGHTS, and FLOAT one upon the other, as oil and water do when mingled?

$A$. Because of a wonderful principle or law of nature, that when two gases of different weights are mixed together, they cannot remain separate, as fluids of different densities do, but diffuse themselves uniformly throughout the whole space which both occupy.

Q. Carbonic acid is twenty times heavier than hydrogen gas; if we fill the lower part of a tall jar with carbonic acid, and the upper part with hydrogen, will the two gases MIX?

A. After a few hours the two gases will be found equally mingled, as much carbonic acid being at the top of the jar as at the bottom.

Q. Does this law appear to be opposed to the principles of the law of gravitation?

$A$. It appears to be opposed to it; the only exception we are acquainted with in the natural world.

Q. How woch carbonic acid is estimated to exist in the atmosphere?

A. About one part in two thousand, by volume.

$Q$. If this were all collected in one layer over the surface of the earth, how gredr a THICKNESS would this layer or stratum have?

A. About thirteen feet. 
Q. Can we BREATHE carbonic acid?

A. No; any animal placed in it dies instantly.

Q. If, then, this singular law of the diffusion of gases did not prevail, would the surface of the earth be IIABITABLE?

A. It would not; carbonic acid would fill up all the valleys and lower levels, separating every hill and elevation by an invisible ocean of poisonous gas, as impassable as the barrier between the dead and the living.

Q. Is it owing to this law that we are enabled to enjoy and perceive at a distance the ODOUR of a FLOWER GARDEN, or the PERFUME which has been opened in an apartment?

$A$. It is by this law that a vapour, arising by its own elasticity from a volatile substance, is cansed to extend its influence and mingle with the surrounding atmosphere, until its effects become so enfeebled by dilution as to be imperceptible to the senses.

Q. If the oxygen and nitrogen of which our atmosphere is composed were combined together, instead of being merely mingled, what would the compound be?

A. A most deadly poison.

\section{- SECTION III.-COMPOUNDS OF NITROGEN.}

Q. Does nitrogen readily ENTER into CoMBINATIONS with other bodies?

A. Nitrogen enters into combination with but ferv bodies, and, when combined, has always a tendency to free itself.

Q. What CuRIOUS FACT illustrates the non-conducting power of nitrogen?

A. Through an average size iron blast-furnace it is estimated that six tons of air pass every hour; during this transit the oxygen part of the air is most active in forming combinations, yet the nitrogen, although subject to precisely similar conditions of heat and contact, emerges as it entered, uncombined.

Q. What is the principal CHEMrCal compound which nitrogen forms with oxygen?

A. Nitric acid, or aquafortis, composed of five parts of oxygen united to one of nitrogen.

Q. What are the PRopERTIES of nitric acid?

$A$. It is a liquid ; when pure, colourless, and highly corrosive; it attacks almost all dead, unorganised substances, and destroys living tissues.

Q. What is NITRE, or SALTPETRE?

A. Nitric acid, combined with potash.

Q. WHERE is saltpetre obtained?

A. It exists in the earth of many caves, and in the soil about barns and old buildings; in the Ecust Indies, from whence the greater part of the saltpetre of commerce is obtained, it is found in great abundance in the earth of certain districts.

Q. How is the nitre supposed to ORIGINAmE in the earth of these places?

A. From decayed animal and vegetable substances, which contain much nitrogen in their composition. In the chemical changes which take place when these substances decay, nitrogen is liberated, and 
nitric acid formed, which last, uniting with potash previously existing in the soil, forms saltpetre.

Q. Can saltpetre be also MaNUFACTURED?

A. It can, in the following manner:-Animal substances rich in zitrogen, such as hair, pieces of hides, flesh, \&c., are mixed with earth containing lime or potash, and suffered to putrefy ; during the putrefaction, by the chemical changes which take place, nitric acid is formed; this combines with the earth to form nitrates, which are afterwards extracted with water, and crystallised by boiling and everporation.

Q. In what SIMPLE WAY can you often determine whether a body contains nitrogen?

A. By the offensive smell which a body containing nitrogen alwavs produces when it is burnt; as, for example, the odorn of burnt hair, leather, flesh, bones, \&c.

Q. Do MANY of the offensive smells in nature result from the action of nitrogen?

A. Organic bodies which contain a large amount of nitrogen aluays emit a most offensive smell when they decay. The smell occasioned by the decaying of a dead human body, which contains much nitrogen, is one of the most offensive in nature.

Q. What is "LUNAR CAUSTIO ?"

A. A compound of nitric acid and oxicle of silver.

Q. Why, when lunar caustic is applied to the flesh, does it BURN and Des'roy it?

A. Through the agency of the nitric acid contained in it. Lunar caustic, when brought in contact with an organic substance, is readily decomposed, and the nitric acid set free to act upon the tissues.

Q. When nitrogen combines with hydrogen, what prodvcr is formed?

A. Ammonia.

Q. In what condrinion does pure ammonia exist?

A. As a gas, colourless, and of a strong, pungent smell, acting powerfully upon the eyes and nose.

Q. What are the PRoperties of ammonia?

A. It is a strong alliati, and combines with and neutralises the effects of acids.

Q. What is the SUDstaNCE used for the preparation of smelling salts, sal-volatile, \&c.?

A. A compound of ammonia and carbonic acicl-the carbonate of ¿tmmonia.

Q. Why is ammonia sometimes called "HARTSIORN" and "SPIRITS OF IARTSHORN?"

$A$. Because it was formerly obtained by distilling the horns of deer and harts, instead of bones.

Q. How is the aMroNia of COMMERUE PRODUCED

A. By distilling animal substances, especially bones. These substinces contain nitrogen and hydrogen, which, at the moment of being set free by heat, unite with each other, and form ammonia. 
Q. What is SAL-AMMONIAO?

A. A compound of chlorine and ammonium - the chloride of ammonium.

Q. From whLxoe did the compound of ammonia derive its NAME?

$A$. It was first manufactured in Africa, in the district of Ammonia, by the distillation of camels" dung; hence its name, "salts of ammonia."

Q. Is nitrogen an element in most EXPLOSIVE COMPOUNDS ?

A. Nitrogen being remarkable for its unstable combinations, is therefore an important element in most explosive compounds. It exists in gunpowder in the saltpetre, which constitutes nearly threefourths the weight of good powder.

Q. Will SALTPETRE of ITSELF EXPLODE when burnt?

A. It will not.

Q. What, then, has been the oocdsion of the fearful Explosions which have taken place when warehouses filled with saltpetre have been consumed by fire?

A. Saltpetre, when burning, liberates a large amount of oxygen; water, when thrown upon a hot fire, is frequently decomposed, and hydrogen is set free; hydrogen and oxygen form a terribly explosive compound. Again, the other products arising from the combustion of the different substances contained in the warehouses, united with the products arising from the decomposition of the saltpetre, would produce explosive compounds.

Q. Are there MANY OTHER compounds more explosive than gunpowder?

A. There are: the most explosive compounds known are produced by the union of nitrogen with iodine and chlorine. The iodide of nitrogen explodes when it is slightly jarred, or when its temperature is elevated in the slightest degree, shattering all solid bodies near it. A single drop of the chloride of nitrogen will also produce most destructive effects.

Q. Nitrogen, as we have shown, by uniting with oxygen, forms an acid; with hydrogen, an alkali-ammonia: what is the product formed when NITRoGEx unites with CARBON?

A. It is called cyanogen, which is the principal element in forming that most terrible and deadly poison, prussic acid.

Q. Why are EERNELS of PEACII STONES poisonous?

A. Because they contain prussic acid.

Q. What peculiar odour has prussic acid?

A. The odour of peach blossoms.

Q. What is the well-known LAUGHING GAS composed of?

A. One part of oxygen united to two of nitrogen. 


\section{CHAPTER XLVII.}

\section{CARBON AND ITS COMPOUNDS:}

Q. What is CARBon?

A. A solid elementary or simple substance, generally of a dark or black colour, well known under the forms of charcoal, lampblack, coke, \&c.

Q. Carbon occurs in nature CRYSTALLISED in two forms : what ARE THEY?

A. 'The diamond and graphite.

Graphite, known by the names of plumbago and blacklead, is used for making pencils for drawing and writing.

Q. What peculiar properties does the diamond possess?

$A$. It possesses a degree of hardness superior to that of any other mineral; it scratches all other bodies, but it is scratched by none; it acquires positive electricity by friction, but does not retain it; and it possesses either single or double refraction, according to its crystalline form.

Q. Can you give an EXAMPLE of carbon in its JNORYSTALLISED state?

A. Lamp-black, the soot produced by the imperfect combustion of oil or resin, is pure carbon in its uncrystallised or amorphous state.

Amorphous, "shapeless," "without form."

Q. What is CIAARCOAL?

A. Charred wood.

Q. Can all animal and vegetable substances, by partial burning, be changed into cosL?

A. They can.

Q. What is TINDER?

A. Charred linen.

Q. In the charring of animal and regetable substances, do we Form CHARCOAr, or did it exist there before?

A. The carbon or coal existed there previously, though in chemical combination with other bodies, which are principally driven off by heat, as is apparent from the fact that a charred body weighs much less than the original substance; animal and vegetable substances consist, therefore, in part of coal.

Q. How many Variesies of coal are there in the mineral kingdom?

A. Brown coal, sometimes called lignite, bituminous coal, and anthracite coal. Lignite is simply wood charred by the action of the earth in which it has for a long period been buried.

Q. What occasrons the DIFFerence between bituminous and anthracite coal ?

A. Bituminous coal contains a large amount of bitumen and other pitchy substances which readily ignite and burn with smoke and 
flame. In anthracite coal these substances have been driven out; it is therefore a purer variety of carbon, and burns without smoke or flame.

Q. What is Core ?

A. Coke is bituminous coal which has been heated apert from the air, until its volatile portions are in a great measure driven off.

Q. What is soor?

A. Coal in a state of minute division, which is deposited from the flame of bituminous or pit-coal, wood, oil, resin, \&c., when, during the combustion of these substances, there is an insufficient supply of air.

Q. What is ANIMAL CHARCOAL, IVORY-BLACK, Or BONE-BLACK?

$A$. The charcoal obtained by heating animal substances, such as ivory shavings, bones, \&c., in close vessels.

Q. How is CHarcoal PREPared?

A. By charring wood in mounds or pits, covered with turf or soil in such a way as to exclude in a great degree the admission of air, and thus prevent complete combustion. The finest kinds of charcoal, such as are used in the manufacture of gunpowder, are prepared by heating wood in close iron cylinders.

Q. How мuch charcoal can be obtained from a given weight of dry wood?

A. From one-third to one-fourth of the original weight of the wood.

Q. What singular chrmical propertirs does charcoal possess?

A. The property of absorbing the odoriferous and colouring principles of most animal and vegetable substances.

Q. Bу whат метиор is loaf-sugar freed from its original brown colour, and rendered so pure and sparkling white?

A. By filtering in solution through animal charcoal or bone-black.

Q. Does charcoal, after it has been used for removing impurities and absorbing colours, lose its oHeMical Properties?

$A$. In a great degree; they can, however, be restored by heating the charcoal.

Q. Why is a CHARCOAL fire IOTTER than a wOOD fire?

A. Because charcoal is very pure carbon; and as it is the carbon of fuel which produces the glowing heat of combustion, therefore the purer the carbon, the more intense will the heat of the fire be.

Q. Why will not stones do for fuel as well as COAL ?

A. Because they contain no hydrogen, and little or no carbon.

Q. How are the BEST BLACK-Litad pworis made?

A. The plumbago is ground to poucler, and subjected to extreme pressure simultaneously with the abstraction of all remaining traces of air by the air-pump. The result of this treatment is a block, which, when sawed, furnishes the little bars which, enclosed in a cedar case, constitute a pencil. 
Q. If you heat charcoal in a ressel, with an entire exclusion of the air, what craNaD takes place?

$A$. It seems to melt when subjected to intense heat, the air being excluded, and assumes a somewhat metallic appearance.

Q. How do we know that the diamond is simply CARBON CRYSTALLISED?

A. Because it is combustible, and can be entirely consumed by fire ; the products of the combustion being the same as those derived from combustion of pure charcoal.

Q. Have we any KNOWLEDGE of the MANNER in which the diamond is formed?

A. We have but little definite knowledge upon the subject. There is good reason for supposing, however, that the diamond was once in a fluid or semi-fluid state. Sir David Brewster inclines to the opinion that the diamond is simply a drop of fossilised gum.

Q. From what countries is the supply of diamonds chiefly derived?

$A$. Since the year 1720 , when diamonds were first discovered in Brazil, that country has been their chief source; before which period they were exclusively brought from the East Indies

Q. Who first succeeded in Bursing the diamond?

A. Luvoisier first proved it to contain carbon, by throwing the sun's rays, concentrated by a powerful lens, upon a diamond contained in a vessel of oxygen gas. The diamond was consumed entirely, oxygen disappeared, and carbonic acid gas was generated.

Q. The diamond being the hardest substance in nature, and capable of scratching all other bodies, in WHAT WAY are lapidaries enabled to cut and shape it?

A. It can only be cut or polished by portions of its own substance; hence diamond pouder is universally employed for that purpose; such stones as, on account of their inferior colour or their flaws, are valueless as gems, being broken down into powder for the purpose of cutting others.

Q. Into WHAT CLASSES are cut diamonds divided?

A. Into brilliants and rose diamonds. The former, which are the most beautiful and valuable, may be recognised by their fat summits, whereas the summits of rose-diamonds are peaked.

Q. How is the weight of the diamond rstrimated ?

A. In carats; $\mathbf{1 5 0}$ of which are equal to one troy ounce, or 480 grains.

Q. What is the LARGEST DIAMOND in existence?

A. The largest known diamond is one of the ugliest and most uninviting in its appearance. It belongs to the croun of Portugal.

This diamond, from its astounding value, which is set down at $£ 5,644,000$, has never been entrusted to any diamond-merchant to cut or polish. In size it resembles a large turkey-egg, with a piece notched out of the siele; it is semi-transparent on the surface, and weighs one thousand six hundred and eighty carats.

Q. What is the most VALUABLE cut diamond?

A. A diamond in the royal sceptre of Russia.

Its weight is seven hundred and seventy-nine carats, and its value, being without a 
flaw, and of very fine water, $£ 4,854,000$. Russia has another diamond worth $£ 297,000$, and France a sky-blue diamond, valued at $£ 150,000$. The Koh-i-noor diamond, which excited so much attention at the Industrial Exhibilion of 1551, weighed two hundred and eighty carats, and its worth was estimated at $£ 2,000,000$ sterling.

Q. In what situations is the diamond found?

A. The diamond is not found in rocks, but in sand and gravel in the beds of streams, or on the slopes of hills and mountains.

Q. How great a heat is required to effect the combustion of the diamond?

\section{A. About five thousand degrees Fahrenheit.}

Fahrenheit's scale of the thermometer is here referred to, his being generally used in England. When, in connection with degrees of temperature, we employ the letter $F_{0}$, it means by Fahrenheit's scale and measurements.

Q. What is STEEL?

A. Iron chemically combined with carbon.

Q. How is STEEL made from IRoN?

A. The iron is surrounded with charcoal, and placed, during six or eight days, in a furnace intensely heated; the carbon unites with the iron, and forms what is called "carburet of iron" (or steel).

Q. How MUCH CARBON is there contained in steel?

A. About two and a half pounds of carbon are contained in every hundredweight of steel. iron?

Q. What constitutes the DIFFERENCE between cast iron and wrought or malleable

A. Cast iron contains a large quantity of carbon, four or five pounds to the hundred, while malleable iron contains very little carbon.

Q. What is essentially the PROCESS for converting cast iron into wrought iron?

A. Depriving the cast iron of its carbon.

Q. In what STATE is carbon supposed to exist in STEEL?

$A$. There is some reason for supposing that carbon exists in steel in the form of the diamond.

Q. What CURIOUs THEORY has been proposed to explain the reason of the peculiar cutting properties of steel edges, or the so-called "hair edge" of a razor?

$A$. That the remarkable fine cutting properties of steel edges is due to a minute form of the diamond imbedded in the edge of the metal; and it has been supposed that by the dipping a razor in warm water the metal expanded and forced the sharp edges of the imbedded carbon into such positions as to cut with greater ease.

Q. Has the diamond been formed as yet ARTificially?

A. M. Despretz of Paris, in the year 1853, undoubtedly succeeded with a galvanic battery in fusing and crystallising carbon, thus producing a quantity of microscopic diamonds.

Q. What is CARBONIO ACID GAS?

$A$. It is formed by carbon and oxygen. Its chemical composition is one atom of carbon united to two of oxygen. 
Q. What GAS is generated by a lighted CANDLE or LAMP?

A. Carbonic acid gas, - formed by the union of the carton of the oil or tallow with the oxygen of the air.

Q. When does CARBon most readily Unite with oXIGEN?

A. 1. When its temperature is raised;

2. Carbon in the blood unites readily with oxygen during respiration; and

3. Carbonic acid is formed in large quantities during the chemical changes which we call fermentation.

Q. Is carbonic acid WHOLESOME?

A. $N_{0}$; it is fatal to animal life.

Q. When persons commit suicide by building a charcoal fire in a closed room, what ocCASIONS their death?

A. The intacation of carbonic acid, which is generated by the combustion of the charcoal.

Q. How can we ASCERTAIN if a pit or well contains CARBONTC ACID GAS ?

A. By letting down into it a lighted candle. Where a candle will burn, a man can live; but what will extinguish a candle, will also destroy life.

Q. Why does a CROWDED ROOM produce HEADACHE?

A. Because we breathe air vitiated by the crowd, deprived of its due proportion of oxygen, and loaded with carbonic acid.

Q. Is ALi the NitroGEN REJECTED by the lungs?

A. Yes; all the nitrogen of the air is always expired.

Q. IIow MUCH OXYGEN does a full-grown person consume per hour?

$A$. It is calculated that an adult of average size absorbs about $a$ cubic foot of oxygen per hour by respiration, and consequently renders five cubic feet of air unfit for breathing, since every five cubic feet of air contains one cubic foot of oxygen. It is also calculated that two wax or sperm candles absorb as much oxygen as an adult.

Q. To keep the air of a room pure, How MUCH FRESH AIR should be allowed to pass in per hour?

A. Five culic feet for each person, and two and a half cultic feet for each candle, should be allowed to pass in, and an equal quantity to pass out.

Q. Why is it dangerous for persons in a STATE of PERSPIRATton to expose themselves to a COLD CORRENT of air?

$A$. The heated surface of the body receives a chill, by which the integuments are caused to contract, and the pores of the skin become closed; the fluids, which ought to have been permitted to escape by cuticular transpiration, are driven back into the blood, producing, as an immediate or ultimate consequence, rheumatisms, colds, fevers, and, in more cases than are ever known or acknowledged, premature and ultimate death.

Q. Why do PERSONS who are much in the OPEN AIR enjoy the best HisatTiI ?

$A$. Because the air they inhale is much more pure than the air of close and confined rooms. 
Q. Do TREES and FLowers purify the air?

A. Yes; by absorbing carbonic acid, and giving forth oxygen.

Q. Whether is Town or couxtry aIR the best?

A. Country air is more pure than that of towns, because there are fewer people in the country, and they are not so closely packed as they are in towns; because the air is not vitiated by the sewers and drains; because the air can circulate more freely; and because vegetation absorbs the carbonic acid.

Q. What BEComes of the carbonic acid generated in crowded cities ?"

$A$. It is gradually diffused through the air, absorbed by vegetation and by water, and wafted by the winds to different localities.

Q. What is CHовE DAMP?

A. Carbonic acid gas accumulated at the bottom of wells and pits, which renders them noxious, and often fatal to life. It is called cholie damp, because it cholies (or suffocates) every animal that attempts to breathe it.

Q. Why is not this carbonic acid TAKEN OP by the AIR and DIFFUSED, as it is in cities?

A. Because (being heavier than common air) it cannot readily rise from the well or pit; and no wind can get to it to blow it away.

By the chemical law of diffusion, a portion of the carbonic acid which accumulates at the bottom of wells and pits, is removed; but in many cases the quantity removed is more than equalled by an increased supply.

Q. How MUCH CARBoN in the form of carbonic acid passes through the lungs of a healthy person every twenty-four hours?

A. The quantity would be very accurately represented by a mass of charcoal of the weight of three pounds.

The volume of carbon in the atmosphere, although it forms but one per cent. of the carhonic acid existing in it, cxceeds in amount all the carbon that is stored in the earth in the form of coal, or spread over its surface in the furm of animals or vegetables.

Q. What are the CHIEF SOURCES of CARBONIC $\triangle$ CID?

A. Combustion, respiration of men and animals, the decomposition of organic substances, and the exhalations of volcanoes. Carbonic acid also exists in large quantities in the atmosphere, in most waters, and combined with minerals in a solid state, as in marble, which consists of lime united to carbonic acid.

Q. From what SOURCE is the largest quantity derived?

A. From rotting vegetable or animal matter.

Q. How can we Hinder the ACCUMULATION of CARBonic ACID?

A. By throwing quantities of quicklime where such decay is likely to take place.

Q. What EFFEO' does this mroduce?

A. 'The quicklime absorbs the carbonic acid.

Q. What is the RESULT?

A. A combination called carbonate of lime. 
Q. Does rain act in the SAME WAY as quicklime?

$A$. It will prevent carbonic acid from accumulating, by dissolving it.

Q. Is volcanic action (the action of fiery mountains) a Prourfrc source of carbonic acid?

A. Large volumes of carbonic acid gas arise from volcanoes. At one place near Vesuvius, in Italy, it is said that six hundred pounds' weight are discharged every twenty-four hours.

Q. Why does TEAST canse bread to RISF?

A. Yeast produces fermentation in the dough ; fermentation produces carbonic acid, which, striving to escape, puff's up the dough and causes it to rise or become light.

Q. Why does wood DECAY ?

$A$. Because the oxygen of the air unites with the carbon and hydrogen of the wood, and forms carbonic acid and water.

Q. Why is QUICKLIMe formed by burning limestone in a KILN?

A. Because the carbonic acid (which was combined with the lime) is driven off by the heat of the kiln; and the lime becomes quict or caustic.

Q. What is Mormar?

A. Mortar is quiclitime, mixed with sand and water.

Q. Why does MORTAR become HARD after a few days?

A. Because the lime reimbibes from the air the carbonic acid which had been expelled by fire, and the water used in mixing the mortar also flies off, leaving a compound of carbonate and hydrate of lime.

Q. Wherein does LIMESTONE DIFFER in appearance from quicklime?

A. Limestone is a hard, rocky sulbstance; but quicklime is friable.

Q. Is Caribonio ACID a constituent of most spring and well waters?

A. Carbonic acid exists, either in a pure state or in combination with lime or magnesia, in most waters.

Q. From What sources do spring waters derive their supplies of carbonic acid?

A. Cold water absorbs carbonic acid from the atmosphere, and expels it again when the temperature of the water becomes elevated. Water, in trickling through the soil where organic substances are decaying, freely absorbs carbonic acid.

Q. Why should HARD WatrR (used for washing) be EXPOSED to the AIR?

A. Because it is made more soft by exposure to the air.

Most spring water holds lime in solution as a bicarbonate, in consequence of the pre. sence of abundant carbonic acid. Carbonic acid escapes by exposure to air, and the lime is, consequently, deposited as a carbonate.

Q. WHY is HARD WATER made more SOFT by exposure to the air?

A. 1. Because the mineral salts (which cause its hardness) subside; and

2. Because the carbonic acid of the water makes its escape into the air. 
Q. Can carbonic acid be made soun?

A. By means of intense cold and great pressure carbonic acid can be both liquefied and made solid. In the solid state it resembles sllow.

Q. Can the power of water for dissolving carbonic acid be increased?

A. Yes; carbonic acid may, by pressure, be forced into water. Effervescing draughts are made upon this principle.

Q. From what is the word EFFERVESCENCE DERIVED?

A. From the Latin word effervesco (to boil).

Q. What is the CAUSE of EFTERVESCENCE ?

$A$. The carbonic acid of the drink is forced into the tiquor by pressure, and absorbed by it; but when the cork (or pressure) is removed, some of the carbonic acid flies off in bubbles or effervescence.

Q. Why does SODA-WATER EFFERVESOE?

A. In soda-water there is forced eight times its oun bulk of carbonic acid gas, which makes its escape in effervescence as soon as the pressure is removed.

Q. Why does the EFFERVESCENOE of soda-water and ginger-beer so soon GO OFE ?

A. Because the carbonic acid (which produced the effervescence) very rapidly escapes into the air.

Q. Why does the cork of a champagne bottle FLY OFF the instant it has been LOOSENED from the neck of the bottle?

A. Because the quantity of carbonic acid gas contained in the liquor can no longer be confined, and, seeking to escape, drives out the cork with great violence.

Q. Why is IIARD WATER MORE AGREEABLE to DRINK than soft water?

A. Chiefly because it contains carbonic acicl.

Q. What occasions the pale BLEE FLAME which may be observed to play about ignited charcual, or at the lower edge of the flame of a gas-light or candle?

$A$. The presence of a gas, called carbonic oxide.

Q. What is CARBONIC OXIDE?

A. A compound of curbon and oxygen, containing one-half the quantity of oxygen that carbonic acid does.

Q. What are the pnopenties of carbonic oxide?

A. It is a deadly poison when inhaled, and is combustible. 


\title{
CHAPTER XLVIII.
}

\section{COMBUSTION, RESPIRATION, AND NUTRITION.}

\author{
SECTION I.-COMBUSTION.
}

Q. What is COMBUSTION?

A. Every species of combustion with which we are familiarly acquainted is the rapid chemical mion of the oxygen of the air with a combustible or explosive body, attended with light and heat.

Q. How may combustion, as we ordinarily see it, be REGARDED?

A. It may be regarded as simply a process of oxidation.

Q. What is the difference between combustion and explosion?

A. Simply the difference in rapidity of action in the two cases. In combustion, the combustible and the supporter of combustion are brought together by degrees, as in the flame of a lamp or candle; but in an explosion, the combustible and supporter of combustion are mingled together, and the whole action occurs at once.

Q. What becomes of the OXIDISED PRODUCTS of combustion?

$A$. They for the most part combine with oxygen to form gases and vapours. We apply to these products the general term smoke.

Q. What is an ESSENTIAL REQUISITE to every process of combustion?

$A$. That there should be a free supply of air, and that the products of combustion, or the smolie, should be conducted off.

Q. Why, when we burn a candle or a piece of wood in the air, does there always seem to be a LOSS of MATTER?

$A$. Because the results of combustion in these cases are either gases or vapours, the existence of which, not being apparent to common observation, requires to be made known by experiment.

Q. How is HEAT EVOLVED by combustion?

A. By chemical action. As latent heat is liberated, when water is poured upon lime, by chemical action, so latent heat is liberated in combustion by chemical action also.

Q. What CIrmical acrion takes place in combustion?

$A$. The elements of the fuel combine with the oxygen of the air.

Q. Is there not always an INCREASE in WEIGHT during the combustion of burning materials, rather than a loss?

A. The products of combustion always exceed the weight of the original substance burned, by an amount equal to the weight of the oxygen gas absorbed during the combustion.

Q. What is the TEMPERATURE required to induce the combination of oxygen with any substance called?

A. The burning point. 
Q. Is this point DIFFERENT for Different SUBSTANCES?

A. It is ; thus phosphorus will combine slowly at 77 degrees $\mathrm{F}$., but does not enter into rapid combustion until the temperature is raised to 104 degrees F. Charcoal burms slowly below a red heat.

Q. Is the QUANTity of HEAT given out, when a body combines slowly with oxygen, the same as when it combines rapidly with it, the relative quantilies of the combining bodies remaining the same in both cases?

$A$. 'The total quantity of heat given out is the same, whether the combustion takes place slowly or quickly; but in the case of slow combustion, the heat is much less intense, and often becomes insensible, because during the long time occupied in combination the greater part is carried away by conduction.

Q. Is the QUANTITy of Light given out the same whether the combustion be rapid or slow?

$A$. No; the quantity of light given out during the combination of oxygen with a given quantity of a combustible body varies greatly, according to the rapidity of the combustion.

Q. What is FLAME?

A. Burning gas or vapour.

Q. What does the Brigntwiss, or ILLUMinating PoWER, of flame depend on?

$A$. It depends on the degree of heat in part, but mainly on the presence or absence of solid particles in the flame, which may act as radiating points.

Q. How can you ILLUSTRATE this?

A. Pure alcohol, containing few solid particles, emits but a feeble light when burning, even if its temperature is the highest possible; but if we mix turpentine with it, which contains a large quantity of carbon, we have the ordinary burning fluid, which burns very brightly, its brightness increasing with the temperature to which the particles of carbon are raised.

Q. Are there solid particles in EVERY illuminating flame?

$A$. 'There are; and if we present a cold surface to the flame, they become deposited on it in the form of soot.

Q. When we say a lamp smorks, what do we mean?

$A$. That the solicl parts of the flame are passing off in an unconsumed state.

Q. When the flame burns properly, why does the smoke cELSE to be EMrTTED ?

A. Because the solid particles of carbon constituting the smoke are bumed up, or are completely united with oxygen, forming an invisible gas-carbonic acid.

Q. From what sourcr is the carbon, constituting the illuminating particles in the one case, and the soot in the other, derived?

A. It was originally a part of the burning or combustible body.

Q. When will a flame ssroxe?

$A$. When the supply of oxygen received from the air is insufficient to consume all the carbon which the heat separates from the combustible body in the form of soot. 
Q. What BENISIT arises from surrounding a flame with a glass cylinder or chimney open at the bottom and top?

$A$. When a flame burns without a chimney, the hot air radiates in all directions ; but when it is surrounded by a chimney, the hot air is confined within the walls of the cylinder; consequently, the hot air will issue rapidly from the top of the chimney, and cold air will enter equally fast at the bottom to replace it. In this way a constant current of fresh air is kept up through the centre of the flame causing a more perfect combustion, and a brighter and stronger flame.

Q. Why are lamp chimneys made conIC's, or caused to contract at a certain height above the burner, so as to form a shoulder?

$A$. By the contraction of the cylinder the draught of air is broken, and thrown in upon the flame at an angle.

Q. Is not the supply of air LESSENED by this contrivance?

A. It is; but the new direction given to the draught is a gain, since that part of the current which, in a straight cylinder, would pass along the inner surface without taking part in the combustion and at the same time cooling the flame, is almost wholly removed by contracting the cylinder.

Q. For what two purposes do we effect combustion?

A. For the sake of obtaining heat or light.

Q. Can we with advantage so regulate combustion as to obtain economically both HEAT and LIGHT at the same time, and by oNE operation?

A. The nature of the chemical process, and of the bodies employed, as well as in most cases the practical arrangements, render it impossible to attain both ends with advantage in the same time.

Q. What is FUEu?

A. Any substance which serves as aliment or food for fire. In ordinary language, we mean by fuel the peculiar substance of plants, or the products resulting from their decomposition, designated under the various names of wood, peat, and coal.

Q. What is wood FoRMED of ?

A. Carbon, hydrogen, and oxygen, combined together, make up the chief part of its bulk; all wood also contains water.

Q. At what PERIOD of the YEAr does wood contain the greatest amount of water?

A. In the spring and summer, when the sap flows freely, and the influence of vegetation is the greatest.

Q. Why is wood generally cut in the WINTER SEASON?

A. Because at that season there is but little sap in the tissues, and the wood is drier than at any other period.

Q. Why is it difficult to INFLANE COA I or hard wood with the blaze of a matc!n?

$A$. Coal and hard wood, on account of their density, are good conductors, and carry off the heat of the kindling substance, so as to extinguish it, before they themselves become raised to the temperatrue necessary for their combustion. 
Q. Why is it easy to ignite LIGHT FtEL with a small blaze?

A. Light fuel, being a slow conductor of heat, kindles easily, and from the admixture of atmospheric air in its pores and crevices, burns out rapidly, producing a comparatively temporary, though often strong heat.

Q. In recently-felled wood what PROPORTION of its WEIGHT is WATLR?

A. From one-fifth to one-half.

Q. After wood has been dried in the air for ten or twelve months, How MUCr WATER does it usually contain?

A. From twenty to twenty-five per cent.

Q. Why do we call some woods HARD, and others SOFT ?

$A$. This distinction is grounded upon the facitity with which they are wortied, and upon their power of producing heat. Hard woods, as the oak, beach, walnut, elm, and alder, contain in the same bulk more solid fibre, and their vessels are narrower and more closely packed than those of the soiter kinds, such as pine, larch, chestnut, \&c.

Q. Will the wood of a given species of tree growing in one situation be IENSwR than when growing in another situation?

A. High situations, much exposed to the wind, and a poor soil, cause the vessels of the wood to be less developed, and consequently more closely packed, than is the case with wood which is protected and growing on the more fruitful soil of valleys.

Q. Why is it Unprofitable to burn green wood or wet coal?

$A$. It is a well-known law of heat that the evaporation of liquids, or their conversion into steam, consumes or renders latent a great amount. of caloric. When green wood or wet coal are added to the fire, they abstract from it by degrees a sufficient amount of heat to convert their own sap or moisture into steam before they are capable of being burnt. As long as any considerable part of this fluid remains unevaporated, the combustion goes on slowly, the fire is dull, and the heat feeble.

Q. Is there any TRUTH in the remark often made, "that it is economy to burn GREEN WCOD because it is more durable, and therefore, in the end, more cheap?"

A. No; it is true that the consumption of green wood is less rapid than dry; but to produce a given amount of heat, a far greater amount of fuel must be consumed.

Q. What is PEAT?

A. Peat is composed of carbon, produced by the decay of mosses, grasses, and water-plants of various species, which in the course of time accumulate in the bottoms of bogs and swamps.

Q. In ordinary fuel, what THREE ELEMENTs enter into the process of combustion?

A. Hydrogen gas, carbon, and oxygen gas; the two former in the fuel, and the latter principally in the air which surrounds the fuel.

Q. Do BLAZING COAIS burn MORE RAPIDLX than red-hot coals?

$A$. They do; the inflammable gases of the coal which are then escuping making the coal itself burn much quicker than would otherwise be the case. 
Q. Does not a cLeAR RED FIRE burn a long time without going out?

A. Yes; it will burn much longer than a blazing fire, for the reason already stated.

Q. When a fire is RED Hот there is LESS SMOKE than at other times: how is this ?

A. As all the coals are burning, or in a state of combustion, there is but little carbon to escape in smoke.

Q. The flames of a fire are not all. of ONE COLOUR : how is this?

A. It arises from the difference of material which is burning.

Q. Are these DIFFrRENCES observable in coal fires?

A. Pure hydrogen produces a bluish flame; light carburetted hydrogen, a yellowish flame; heavy carburetted hydrogen, a white flame.

Q. When a coal FIRE is LIGHTED, why are paper and wood laid at the Botron against the GRATE?

$A$. That the flame may uscend through the fuel to heat it. If the fire were kindled from the top, the flame would not come in contact with the fuel placed below.

Q. Why will not TET MATEr.

A. Because the moisture prevents the oxygen of the air from getting to the fuel, and the heat of the fire is drawn off by the conversion of water into steam.

Q. Why do Two PIECES of wood burn better than one?

A. 1. Because they serve in some measure to intercept the heat of the passing smoke and thronv it on the fuel; and

2. The air, impinging against the pieces of wood, is throun upon the fire in a kind of eddy or draught.

Q. Why are CINDERS LIGHTER than COALS?

A. Because they are full of little holes or pores, from which gases and other volatile parts have been driven off by previous combustion.

Q. Is fire-light ALWAYS of the SAME intensity?

$A$. No; it varies very considerably, depending upon the degree of heat to which the carbon has been raised by combustion.

Q. Coals BLAzE, cinders do rот: WHy is this?

A. Cinders produce carbonic acid only, which does not give light; the flame of coal being caused by curburetted hydrogen.

Q. Where does the HunRogen Gas of a fire cone FRom? heat.

$A$. It is one of the elements of fuel produced by the action of the

Q. Why is it that the fire burns BRIGHTEST and CLEAREST during a FROST ?

A. Cold air contains more oxygen and is heavier than warm air; the result of this is that as the warm air from the fire ascends, the cold air very rapidly supplies its place, the fuel is more quiclily consumed, and the fire is thus made to burn bright and clear.

Q. AsHes or CINDERS are put over the fire to PREVENT its BURNING AWAY: can you tell the reason for thus Covering the fire?

$A$. The ashes or cinders prevent the oxygen of the air from gain- 
ing free access to the fire; and as fire will not burn freely without an abundant supply of oxygen, it keeps alive for several hours without being wasted.

Q. Why does RAREFIED AIR afford LESS NODRISHMENT to fire than cold air?

A. Because rarefied air contains comparatively less oxygen than the same volume of condensed air.

Inasinuch as the same quantity of oxygen is diffused over a larger volume of air.

Q. Why does the air flow to the fire MORE TARDIIY for being RAREFT:D ?

$A$. Because the greater the contrast between the external air and that which has been heated by the fire, the more rapid will be the current of air towards the fire.

Q. How is it that WATER EXTINGUISHES a FIRE?

A. Because, by forming a covering over the fuel, it keeps it from the air, and the conversion of the water into steam carries off the heat of the fire, and gradually puts it out.

Q. Why does a LITTLE WATER makes a fire FIERCER, while a LARGER quantity of water puts it опт?

A. Water is composed of oxygen and hydrogen ; when, therefore, the fire can decompose the water into its simple elements, they serve for fuel to the flame.

Q. How can WATER serve for FUEL to fire?

$A$. The oxygen and hydrogen of the water being separated from each other, unite with the carbon to form carbonic oxide and carburetted hydrogen, both of which are inflammable.

Q. Is the quantity of heat INCREASED by the addition of a little water to a hot fire?

A. The quantity of heat given forth is the same, whether carbonic acid is formed by the combination of carbon and oxygen, or carbonic acid from carbon, oxygen, and the elements of water. The access of water lessens the glow-fire, but produces a greater quantity of flame in consequence of the formation of inflammable gases.

If a sufficiency of water is thrown nn to canse, by its evaporation, a reduction of temperature below the point of combustion, the fire is put out.

Q. How do you explain the familiar experiment of drawing a bank-note over the head of a brass andiron, or the face of a flat-iron, and placing a burning coal upon it, and blowing the coal with a bellows until it is burned up, without scorching the paper of the bank-note?

A. The metal in close contact with the paper conducts off the heat so rapidly from the coal, that it prevents combustion.

Q. Why does a blast of air from a pair of bellows often ExTINGUISH a red-hot coal of anthracite?

A. Because the cold air absorbs the heat of the coal so quickly that it extinguishes it.

Q. Why can you not light a candle or lamp with a match so long as the SULPHor on the end of it is BURNING?

$A$. The chemical reason for this well-known fact is, that the sulphurous acid formed by combustion of sulphur in the air, surrounds the wick, and abstracts the oxygen from the air, by passing to a higher state of oxidation, and this heavy vapour hangs about the wick and excludes the air. 
Q. Cannot wood be made to BLAZE WITHOUT actual CONTACT with fire?

A. Yes; if a piece of wood be held near the fire for a little time it will blaze, even though it does not touch the five.

Q. WIIY will wood BLAzE, even if it does not touch the fire?

$A$. Because the heat of the fire drives out the inflammable gas of the wood, which gas is ignited by contact with red-hot coals.

Q. What causes the HEAT of FIre?

A. The cartun of fuel (when heated) combines with the oxygen of the air, and produces carbonic acid gas. Again, the hydrogen of the fuel, combining with other portions of oxygen, condenses into water; by which chemical actions heat is evolved.

Q. WHENCE does the HEAT of a DUNGHILL arise?

A. As the strurv, \&c., of the dunghill decays, it undergoes fermentation, which produces carbonic acid gas; and heat is evolved through a species of combustion.

Q. On what does the INTENSITY of FIRE depend?

A. The intensity of fire is always in proportion to the quantity of oxygen with which it is supplied.

Q. Why does STIRRIne a dull Fire serve to quicken it?

A. Because it breaks up the compacted cinders and coals, making a passage for the air into the very heart of the fre.

An anthracite coal fire should be stirred from the bottom, and not from the top.

Q. Why do LAMPS SMOKE?

A. Sometimes because the wick is cut unevenly; sometimes because it is raised up too high.

Q. Why does a LAMP SMoke when the WICK is COT UNEVENLY?

A. 1. Because the points of the jagged edge (being very easily separated from the wick) load the flame with more carbon than it can consume; and

2. As the heat of the flame is greatly diminished by these bits of wick, it is unable to consume even the usual quantity of smoke.

Q. Does a LAMP SMOKE when the WICK is TURNeI UP TOO HIGH?

A. Yes; more carbon being separated from the wick than can be consumed by the flame.

Q. Why is the flame of a candle ExTINGUISHeD when blown by the breath, and not made more intense, like a fire?

$A$. Because the flame of a candle is confined to a very small wick, from which it is severed by the breath, and (being unsupported) must go out.

Q. When a chimney with an open fireplace gets on fire, and burns so as to endanger the house, how may it at once be EXTINGUISHED?

A. By throwing a quantity of brimstone or sulphur into the fire, and closing up the fireplace with a fireboard or screen. The su? phurous acid soon fills the climney, and taking up all the oxygen from the contained air, extinguishes the fire. Even the fire, after it has 
extended into the woodwork of the house, may be extinguished by this simple method.

Q. Fire in a climney may be also extinguished by closing the top of the flue with a damper : How does this ExTINGusis the fire?

$A$. It cuts off the draught, and the carbonic acid generated by the combustion soon puts an end to the fire.

Q. What is meant by spontaneous CoMbustion?

A. Combustion produced without contact with fire or flame.

Q. Give me an ExAMPLE of spontaneous combustion.

A. Oiled cotton and rags imbued with any drying oil, when packed in mass in a barrel, take fire, after a time, at ordinary temperatures. Mixed lampblack and linseed-oil take fire at ordinary temperatures, if the lampblack is in excess, or a portion of it is dry.

Q. What is generally the CAUSE of sPonTANEOUS COMBUSTION?

A. The absorption of oxygen, which finally causes bodies to burst into a flame.

Q. Why do Goops packed in warehouses, in large masses and in a damp state, often OATCH FIRE?

$A$. Because the damp produces decay, and the great heat of the piled-up mass makes the decaying goods ferment. During fermentation great heat is produced by chemical action; a slow combustion ensues, till at length the whole pile bursts into flame. tity?

Q. Why is the HEAT of a LARGE MASS of goods GREATER than that of a smaller quan-

A. Because the heat produced cannot escape through the massive pile; and the temperature of the heap is therefore considerably raised. It is thus that haystacks sometimes catch fire of themselves, either because the hay was put up damp, or else because rain has penetrated the stack.

Q. Is PINE CHARCOAI capable of TAKING FIRE at an extremely LOW TEMPERATURE?

$A$. Porous bodies, like pine charcoal, when perfectly dry, absort oxygen rapidly from the air, and take fire at a temperature below 212 degrees Fahrenheit, or the boiling-point of water.

This has been proved by actual experiment, a piece of light pine charcoal taking fire on a surface of sheet iron heated below the boiling-point of water.

Q. In what Two WAYS is heat communicated to apartments by fires kept in them ?

A. By racliation and immediate contact.

The first portion passes through the air in diverging lines with great velocity. The second penetrates slowly through the substance of the densest bodies. To enjoy the full - effect of radiated heat we must be in the presence or sight of the radiating object. To receive conducted heat we must be in contact, either directly or through some intervening mediun, with the body that imparts it.

Q. Does a person who sits by a fire in the open air receive any IEAT by CGNDUCrton?

A. Very little; for the air which surrounds the fre, having nothing to confine it, ascends by its diminished specific gravity as fast as it is warmed, and its place is immediately supplied by strata of cold air from beneath. 
Q. Will a person sitting BEside a FIRE in the open air be exposed, upon the side of his body removed from the fire, to additional cold?

A. He will, because cold currents rush in from every side towards the fire.

Q. Why are OPEN FIREPLACES ill-adapted for cheaply heating apartments?

$A$. In an open fireplace the air flows from the room to the fire, becomes heated, and passes off directly into the chimney, without having an opportunity of parting with its heat for any useful purpose. In addition to this, a quantity of the air of the room, which has been warmed by radiation, is uselessly carried away by the draught.

Q. What is a "Reverberating Furnace?"

A. One provided with a concave covering, which reverberates or throus back the flame upon the substance to be heated or melted.

Q. What are the ADVANTAGES of a STOVE over an open FIREPLACE?

A. 1. Being detached from the walls of the room, the greater part of the heat produced by combustion is saved. 'The radiated heat being thrown into the walls of the stove, they become hot, and in turn radiate heat on all sides to the room. The conducted heat is also received by successive portions of the air of the room, which pass in contact with the stove;

2. The air being made to pass through the fuel, a small supply is sufficient to keep up the combustion, so that little need be taken out of the room; and

3. The smoke, in passing off by a pipe, parts with the greater part of its heat before it leaves the room.

Q. What are the DISAdvantages of stoves?

A. Houses containing them are generally ill-ventilated. The air coming in contact with the hot metal surfaces is rendered impure, which impurity is increased by the burning of the dust and other substances which settle upon the stove. The air is, in most cases, kept so dry as to render it oppressive.

Q. Upon WHAT PRINCIPLE are the common hot-air furnaces for warming houses constructed?

A. A stove, having large radiating surfaces, is enclosed in a chamber (generally of stone). This chamber is generally built with double walls, that it may be a better non-conductor of heat. A current of air from without is brought by a pipe or box, and delivered under the stove. A part of this air is admitted to supply the combustion; the rest passes upwards in the cavity between the hot stove and the walls of the brick chamber, and, after becoming thoroughly heated, is conducted through passages in which its lightness causes it to ascend, and be delivered in any apartment of the house.

Q. What are the ADVANTAGES of heating by steam?

$A$. The heat can be communicated for a great distance in any direction-upwards, downwards, or horizontally. As the temperature 
of the heating surfaces, when low-pressure steam is used, never exceeds 212 degrees F., the air in contact with them is never contaminated by the burning of dust or the abstraction of oxygen.

Q. In heating by steam, how such BoILER CAPACITY is required to heat a given space?

A. Under favourable circumstances one cubic foot of boiler will heat about two thousand cubic feet of suitably enclosed space to a temperature of 70 degrees to 80 degrees $\mathrm{F}$.

Q. In the construction and arrangement of a furnace for warming, what Two PoINTs are of special importance, so far as regards the economy of fuel?

A. 1. The perfect combustion of the fuel; and

2. The best possible transmission of all the heat formed into the air that is to pass into the rooms of the house.

Q. How is the first of these requisites OBTATNED ?

$A$. By having a good dranght and a fire-box which is broad and shallow, so that the coal shall form a thin stratum; by which arrangement the carbonic acid gas will be freely formed, and pass off without a previous production of carbonic oxide.

Q. How is the second of these requisites ATTAINED ?

$A$. By providing a great quantity of surface in the form of pipes, drums, or cylinders, through which the smoke and hot gases must pass on their way to the chimney, and to which their heat will be imparted, to be in turn delivered to the cold and pure air of the rooms of the house.

Q. Is DEOAY a species of combustion?

$A$. Decay is the act of gradual combination of the combustible elements of a body with the oxygen of the air. It is, therefore, a species of slow combustion.

Q. Why do AIR-TIGHT stoves sometimes explode?

$A$. It frequently happens that when air-tight stoves are closed very tightly, they become filled with a mixture of inflammable gases, chiefly carburetted hydrogen and carbonic oxide. If we admit air into the stove when thus filled with these gases, the instant flame is produced an explosion takes place, blowing a flash of flame and ashes out of the stove, and throwing open the valve in the funnel, if it is a proper turn-valve or disc. If, on the other hand, it is a slide-valve, and the air-hole of the stove is very small, the stove is blown asunder by the explosion.

Q. Why is the use of air-tight stoves injurious to the chimneys into which they open?

A. In an air-tight stove the wood undergoes a process of distillation, and a very abundant product of this distillation is wood vinegar, or pyroligneous acid. This acid acts upon the mortar of chimneys, and gradually removes the cement from between the bricks. The mortar of new houses, not fully hardened, is most readily dissolved out by the acids from air-tight stoves. New houses that have these stoves may be often known by the colour of the bricks on the outside 
of the chimneys, the oxide of iron having been dissolved out from the bricks quite through their mass.

Q. Why do not all flames give the same amount of light?

$A$. The light of a flame depends upon the presence of incandescent solid matter in it. Hence those gases and vapours can only become luminous which produce or deposit solid or liquid matter during their combustion.

Q. Upon what fact does the production of artificial light depend?

A. Upon the fact that at certain high temperatures all matter becomes luminous.

Q. In order that we may profitably use a combustible body for illuminating purposes, what is required of the products of the combustion?

$A$. That they should be able to fly off rapidly from the lightgiving centre.

Q. The product of all the ordinary forms of combustion is a GAS-CARBONIC $\triangle$ CID : what would have been the IiEsulT if the product of every combustion had been a permanent solid?

$A$. The world would have been biried beneath its own ashes.

Q. Phosphorus burns in the air with a beautiful dazzling light: why can we not use this substance as an ILLUMINATING AGENT?

A. Because when phosphorus burns in the air the product of the combustion is a solid-phosphoric acid, which forms a cloud of white vapour, which afterwards deposits itself on objects near at hand iike snow.

Q. Under what Two DIFFEritem ASPECTS does carbon appear when in the act of combustion, as in an ordinary candle-flame?

A. When it is giving forth heat and light, it is a solid; but immediately after it becomes a gas.

Q. Why is the FLAME of an ordinary fire YELLOW?

A. Because the heat is not sufficient to render the carbon white hot. Increase the intensity of combustion, and the colour of the burning bodies, or the flames, rise from red to yellow, and from yellow to white.

Q. A CAYMdLe BURNS when lighted: explain bow this is?

A. The heat of the lighted wick changes the tallow into its elementary parts, hydrogen and carbon. The hydrogen is first consumed as a gas by itself with an almost imperceptible light, but with a powerful heat: this causes the carbon produced at the same time to give out both light and heat.

Q. As more carbon is successively produced, what BEcomes of it?

A. The moment the carbon comes to the edge of the flame, it finds the oxygen of the air, unites with it, and becomes changed into the invisible gas, carbonic acid, while its place is occupied immediately by another portion of solid carbon.

Q. What if there is not stFFicient oXYGEN to consume the carbon?

A. It then passes off as soot, and we say the candle smokes. 
Q. When a CANDLE is BLOWN OUT, whence arises the OFFENSIVE SMILLL?

$A$. 'The tallow distils a substance in the smoke called acryle, which has a very offensive smell.

Q. Of what three parts does the flame of every lamp or candle consist?

A. The flame of every lamp or candle consists of three cones. The innermost cone consists of gaseous matter produced by the decomposition of the tallow; this is at a temperature below rediness. Around it is the luminous cone, consisting of burning hydrogen, in which the particles of carbon float at a white heat; and on the very outside, a thin, hardly-perceptible vail in which carbon is burning. The vail is of a blue colour, most plainly seen at the bottom of the flame.

Fig. 137.

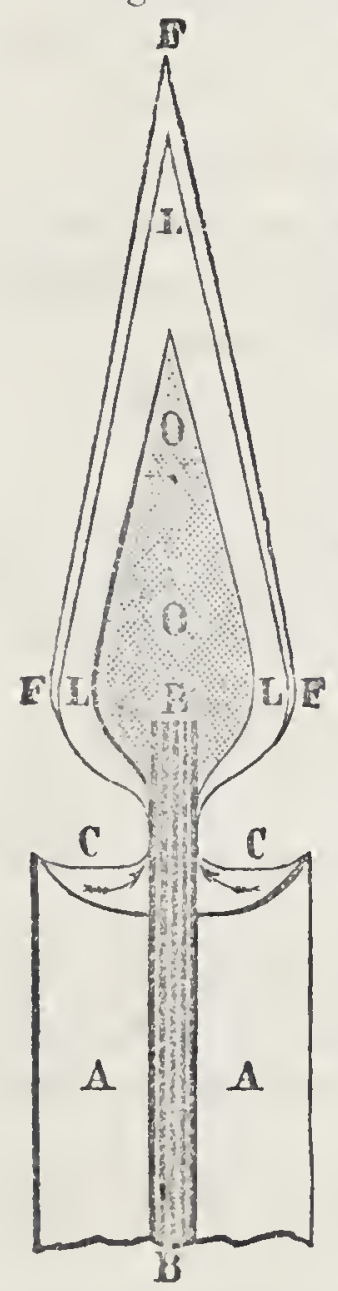

The accompanying figure shows the effect of combustion in a com. mon candle.

$\mathrm{A} \mathrm{A}$ is the candle, B B the wick of the candle. $\mathrm{C} \mathrm{C}$ is the cup. like bath of melted grease at the top of the candle, and around the lighted wick B B, the fibres of which, similar to small tubes, draw up the grease, and feed the flame. The flame itself, as already de. scribed, is composed of three parts - the gaseous matter, 00 , the light, L L, and the outside coating, F F.

Q. Which is the ноттеsт part of the flame?

A. The pale-blue flame; this marks the point where the combination of the oxygen, supplied from without, with the combustible matter evolved from the interior takes place.

Q. Why is the INsIDE of the flame Hotrow?

A. Because it is filled with vapour, raised from the candle by the heat of the wick, and not yet reduced to a state of combustion.

Q. Why does the FLANe of a candle POINT UPWLRDS?

A. As hot air always ascends, the air heated by the candle draws up the flame.

Q. Why is the FLAME of a candle POINTED at the top like a cone?

$A$. As the gas constituting the flame ascends, it will continue to diminish in diameter, on account of the constant combustion, until it entirely disappears. The speed of this diminution, together with that of the ascent (of the current of air), gives rise to the conical shape of the fiane.

Q. How is it that the FLAME of a candle can be BLown OUT?

$A$. It is parted from the wick, and goes out for want of support.

Q. Why is not the wick of a candle consumed?

$A$. The wick, although it is blackened by the heat, is prevented from consuming, merely because it is surrounded by inflammable vapour, so that the oxygen of the air has no access to it.

Q. A LONG Wrok is covered with what is called a flower at the top: what docs this ARISE FROM?

A. This knotty or flowery appearance arises from an accumulation 
of charred particles of cotton, which have not been completely burnt, because the oxygen of the atmosphere has been kept from them by the outer cone of the flame.

Q. Why do Cominon CANDLES require sNuFFINa?

$A$. The reason why the wick of a common tallow candle requires continual snuffing is, that if it is suffered to become long, it divides the blaze, and intercepts a part of the light. It also cools the flame by its radiation, obstructs the combustion, and thus causes the escape of smoke and the deposition of carbon.

Q. Why do WAX and SPERMACETI OANDLES never require snuffing ?

A. Wax and spermaceti, being less fusible than tallow, may be burnt with a smaller wick, which, if made sufficiently slender, bends out of the flame, and burns off so as not to require snuffing.

The wick of tallow candles (on the other hand) is made of coarse cotton, which is too substantial to be consumed by the heat of the flame, and must be cut off by snufiers.

Q. Why are the wicks of wax and sperm candles BRAIDED or TIVISTED strongly?

A. The braiding causes a tension of the fibres, which obliges the free part of the wick to bend considerably, and brings the end sufficiently soon out of the flame to be constantly reduced to ash.

Q. Why does a candle FLICKER, especially just previous to its being BURNT OUT?

A. Because it is unequally supplied with combustible gases.

When a candle is nearly burnt out, there is not sufficient tallow or wax to keep up the rezular supply of combustible gas ; in consequence of which the flame fickers-that is, blazes-when it is supplied with gas, and goes out for a moment when the supply is defective.

Q. How does an extinguisher put out a candle?

A. By exhausting the air round the flame.

Q. Why is the hydrogen arising from the decomposed elements of the candle mostly consumed in the MIDDLE CONE, and not upon the OUTSIDE?

$A$. Because the oxygen of the air has a greater affinity for hydrogen than for carbon, and that which penetrates to this part of the flame, not being sufficient to combine with both, unites with hydrogen to form water.

Q. Why will not the plan of braiding the wick, adopted in sperm and wax candles, answer in the manufacture of TALLOW CANDLES?

A. Because the bending of the wick, which causes part of the flame to project, would melt the tallow on one side too quickly, causing the candle to "gutter."

Tallow melts at a lower temperature than wax or stearine, which prevents the employment of this plan for consuming the wick.

Fig. 138.

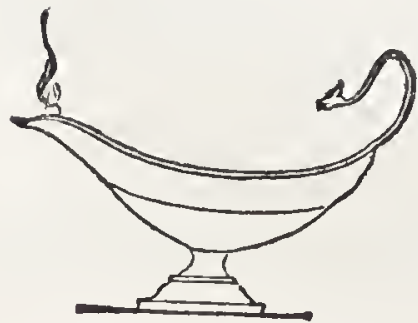

Q. What was the most ANCIENT Fora of lamp used by the Greeks and Romans?

A. It was simply a cup in which a wick was laid, the end of which projected from a sort of beak at the side, nearly on a level with the surface of the oil. (See fig. 138.) 
Fig. 139. Towards the close of the last century the pump, or spring lamp, was invented. The upper part of the lamp, seen in the diagrim, is movable, and can be mado to descend into the bath of oil by a spring. The oil is thus raised, and soaks the wick. By this means the oil is always kept at a proper lerel.

Q. Why does a lamp BURN INDIFFERENTLY if the oil in it is BELOW, or at a DISTANCE From, the flame?

$A$. Because the distance of the oil from the flame diminishes the capillary attraction of the wick, and the light fails through lack of illuminating material.

Q. Why do all closed lamps require a SMALI HoLt in the top?

$A$. 'To admit the air; otherwise the pressure of the atmosphere will prevent the oil from ascending the wick; if the hole be obstructed, the oil will sometimes overflow from the expansion of the confined ail.

\section{Q. What is a CARCEL or MECHANICAL IAMTP?}

$A$. One in which the oil is pumped up, by an arrangement of clock-vork, from the foot of the lamp to the wick, in such a mannel as to supply an minterrupted and equal quantity during the whole period of burning. (See fig. 140.)

\section{Q. What is an Argand ramp?}

A. This name is applied, afrer its. inventor, Argand of France, to all lamps with hollowe or circular wicks; the intention of the Argand burner is to furnish a more rapid supply of air to the flame, and to afford this air to the centre as well as to the outside of the flame.

An Argand lamp consists of two metallic cylinders, one witin the other; the space between them, which is closed at the base, contains the oil, and the cylindrically woven wick attached at the bottom to a movable ring; this ring is capable of being elevated or del ressed by means of a screw, so that the height of the wick may be varied to regulate the size of the flame. When the lanp is lighted, and a chimney adjusted over the wick, a powerful draught is caused ic pass up through the inner hollow cylinder, and the interior of the flame, and also upon the esterior; in this way a complete combustion is caused to take place, and the intensity of the light is increased.

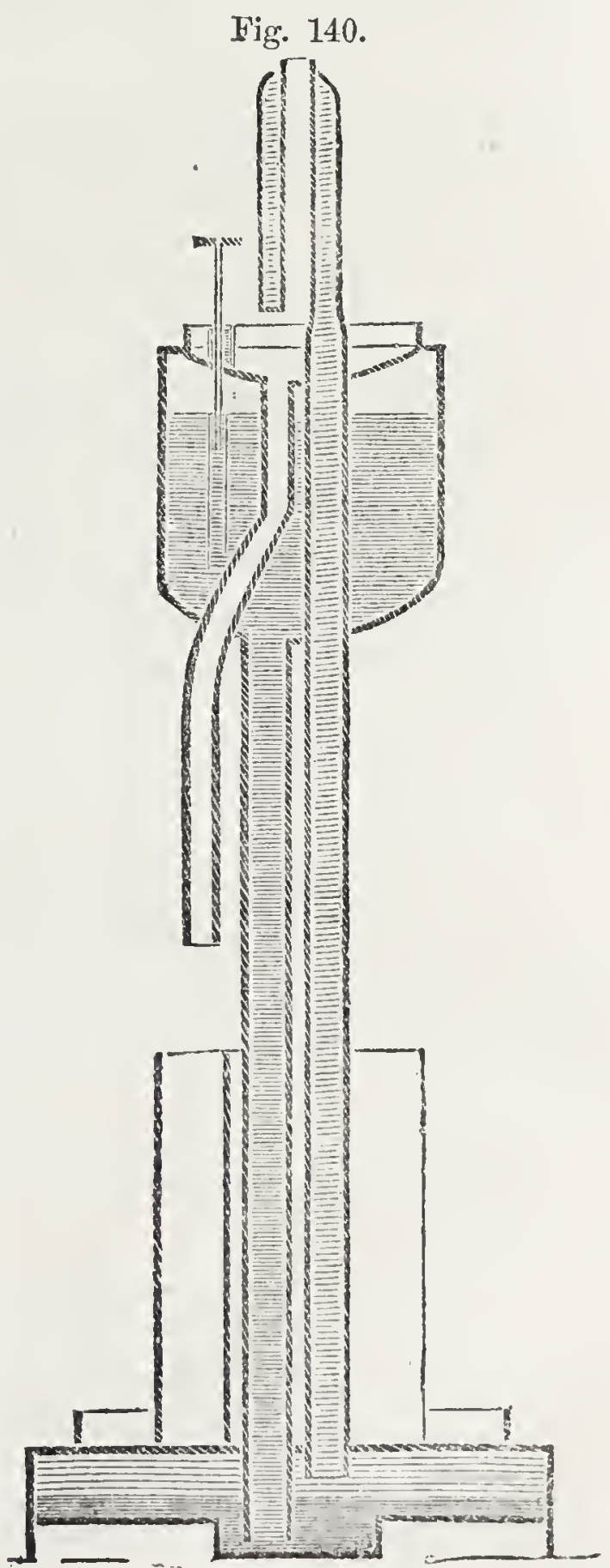




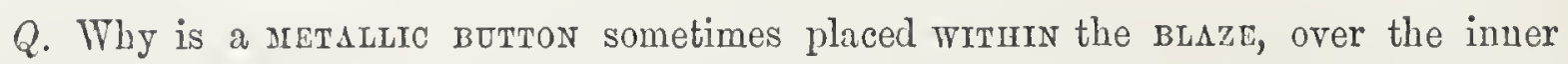
cylinder?

A. In order to deflect the current of air passing up the inner cylinder against the flame outward.

Q. Why do we use GrodND-GLASS globes for lamp shades?

A. To relieve the eye from the glare of light. Ground-glass shades have the effect of dispersing the rays by the numerous reflections and refractions which they occasion, until at length the light issues from all parts of their surface, and it appears as though the glass itself were the luminous body.

Q. Why are the shadows LESS DEEP and strong from a light surrounded with a ground glass, than from an open light, or one surrounded by a clear glass?

$A$. In the latter case the rays proceed in direct lines, without intermixture or crossing; but when the light proceeds from a ground glass, the rays are so broken and refructed, that they cross each other in all directions, and the objects illuminated by them can only throw short and indistinct shadows.

Q. Upon what MATHEMATICAL LAW is the measurement of the comparative illuminating power of different lights founded?

A. On the law that the amount of rays thrown on a given surface is inversely as the square of the distance of the illuminating body.

This law may be practically illustrated as follows:-

Place two lights which are to be compared with each other at a distance of a few feet from a white wall; on holding a small card near the wall, two shadows will be formed, the darker one by the interception of the brighter light, and the fainter shadow by the interception of the duller light. Bring the fainter light nearer to the card, or remove the brighter light farther from it, till both shadows acquire the same intensity. Measure now the distances of the two lights from the wall, and the squares of these distances will give the ratio of illumination.

Q. In what way should PICTURFS be HUNG in respect to a source of illumination, as a lamp or window, so as to secure for them a good light?

A. Paintings should not be hung opposite to lights, or in any situation in which a line drawn from the place intended for spectators will make the same angle with the surface of the picture, as a line drawn from a window or other illuminating point; the angle of reflection being always equal (as already explained) to the angle of incidence.

Q. As a general rule, when will a picture be always in a BAD LIGHT as regards the spectator?

A. Whenever the image of a vindow could be seen by him in a looling-glass, occupying the same place as the picture.

Q. In what respect does a gas light DIFFER from the light of an ordinary candle?

$A$. In the flame of a common lamp or candle the combustible matter is not burnt until it has first been converted into infummable vapour or gas. This gaseous matter is burnt as fast as it is formed 
in consequence of being brought in contact with atmospheric air, and is set on fire by the same heat that produces it. In the case of a gas light, the inflammable gas is distilled by heat from the illuminating substances in close vessels, and conveyed in pipes to the point where the illumination is required. In the one case, the gas is formed and burnt at the same time and place; and in the other, it is formed at one place and burnt at a different time and place.

Q. From what MALTERIALS is gas generally PRODUCED?

A. In most cases from lituminous cocl; it can, however, be readily produced from resins, oils, fats, wood, \&c.

Q. What is COAL GAS?

\section{A. Carburetted hydrogen.}

Q. What is CARBURETted HYDRogen Gas?

$A$. Carbon combined with hydrogen. There are two kinds of carburetted hydrogen-light carburetted hydrogen, and heavy carburetted hydrogen.

Q. What is the DIFFERENOE between LIGHT carburetted hydrogen and HEAVY carburetted hydrogen?

A. The latter contains twice as much carbon as the other; it is also known as olefiant gas. It is the common illuminating gas.

Q. What is MARSH GAS Or FIRR DANCP?

A. Light carburetted hydrogen gas accumulated on marshes, in stagnant waters, and coal pits. It is frequently called "inflammable air."

Q. WHY is light carburetted hydrogen gas called FIRE DAMP, or inflammable air?

$A$. Because, when mixed with air, it readily catches fire and explodes when a light is brought in contact with it.

Q. How may light CARBURETTED HYDRogen GaS be PROcURED on marshes?

$A$. By stirring the mud at the bottom of any stagnant pool, and collecting the gas (as it escapes upwards) in an inverted glass vessel.

Q. What gas is given forth by the wIOK of a burning CANDLE?

A. Ileavy carburetted hydrogen gas.

Q. What OCCASIONS the frequent ExpLosions in coal mines?

A. In most coal mines enormous quantities of light carburetted hydrogen are generated from the coal, and mingling with the air of the mines constitutes an explosive mixture. When the explosive compound is brought in contact with the flame of a lamp, ignition takes place, accompanied with the most fearful consequences. 
Fig. 141.

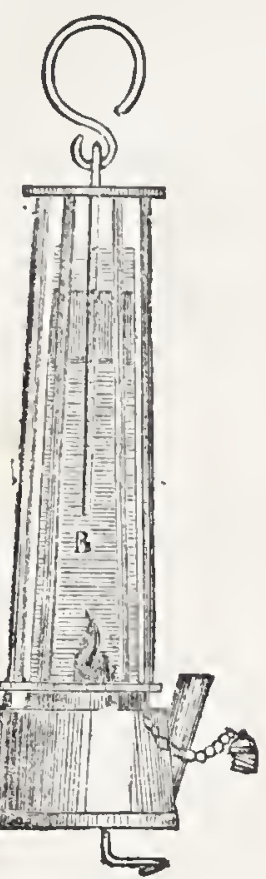

Q. If it is, therefore, dangerous to introduce a light into a coal mine, how are the miners enabled to PROSECUTE their OPERATIONS far below the surface of the earth?

$A$. Sir Humphrey Davy invented a lantern for the use of miners, called the "Safety Lamp,", which may be used without danger. It is a kind of lantern, covered with a fine gauze wire, instead of glass or horn, which prevents the flame of the lamp touching the gas of the mine. (See fig. 141.)

Q. When was coal gas FIRST USED for illuminating purposes?

A. It was first used in England in 1798, though it was not employed for street illumination in London until about 1812 .

Q. What is the process of manufacturing coal gas?

$A$. The coal is placed in iron retorts, which are subjected to a strong heut in a furnace. The gas is thus driven off, mixed with the vapour of tar, ammonia, and of several volatile oils, and in this state is conducted by pipes, first into a horizontal trunk of cast iron called the hydraulic main, and from thence into a condensing apparatus, where the various vapours are condensed and fall down, while the gaseous product is carried away, together with several other impure gases.

Q. How is the carburetted hydrogen SEPARATED from these gaseous impurities?

$A$ By passing it through a mixture of time and water, called mitle of lime, or over dry slated lime, which absorbs the remaining impurities. When thus purified, the gas is conveyed in pipes to the gasometer.

Fig. 142.

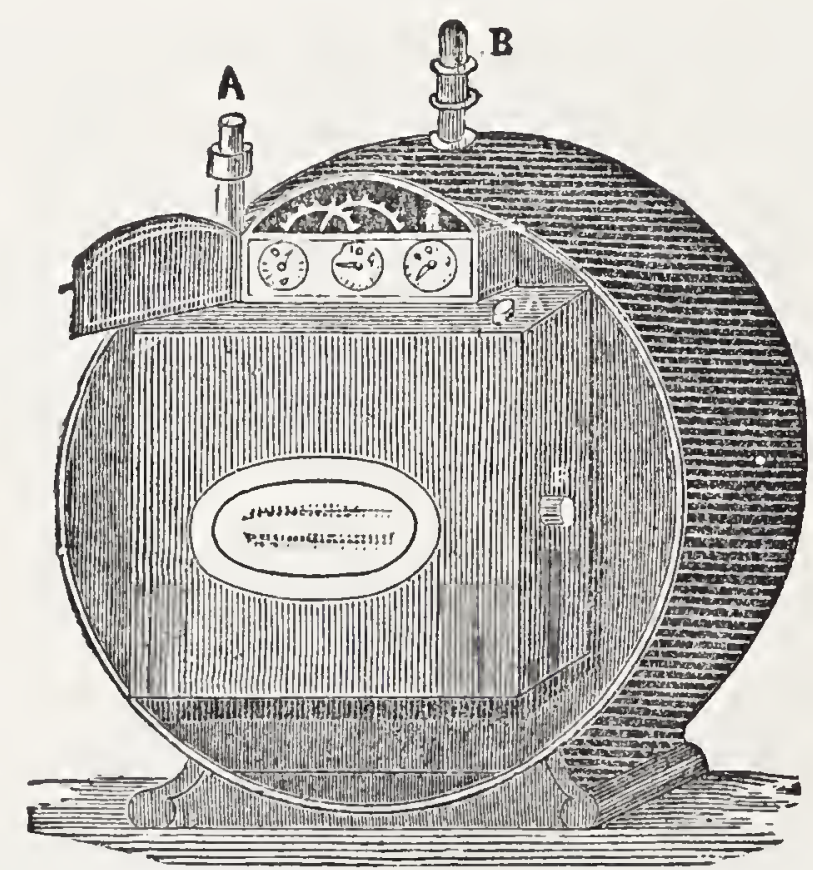

In the above diagram is shown the appearance of a gas-meter. The gas enters at $\mathbf{A}$, and goes out at $B$. 

coal?

Q. In what respect is gas manufactured from oil superion to gas manufactured from

A. It is richer in carburetted hydrogen, so that one cubic foot will afford as much light as two or three of coal gas. The latter generally contains sulphur and some other impurities, from which oil gas is free.

Q. What is the most EXPENSive Material for illuminating purposes?

A. Wax made into candles.

Q. Althongh we obtain light from coal, a product of the mineral kingdom, and from fats and oils derived from animals, yet are not THESE substances PRIMARILY DERIVED from the VIGETABLE kingdom?

A. 'They are; coal is a vegetable product, and animals derive their fats and oils from the vegetable substances on which they feed.

Q. Under this view, is not EVERY PLANT an APPARATuS for the manufacture of light and heat?

A. Every plant may be considered an apparatus for the absorption and concentration of light and heat from the sun's rays, and for the keeping of these forces during its passage through the subsequent stages of vegetable fuel.

Q. Who first DISTOVERED that combustion, as we generally see it, is merely the UNION of OXYGEN with a COMBUSTIBLE body ?

A. Lavoisier, an eminent French chemist, who was put to death during the reign of terror of the French Revolution.

Q. Before this discovery, how was combustion explained?

$A$. It was supposed that all combustible bodies contained a certain substance which was called phlogiston, to the presence of which was ascribed their combustibility. When a body burns, phlogiston was supposed to escape from it; and when a body had lost phlogiston, it became incombustible, or a burnt body.

Q. The advocates of this theory supposed that when a body was burned, something went out of it: how did Lavoisier OVERTHRow this supposition?

A. He weighed the body before combustion, and all the products of combustion afterwards, and found that, instead of the body losing weight, it gained; this gain being exactly equal to the amount of oxygen it had absorbed from the air.

\section{SECTION II.-RESPIRATION.}

Q. What is RESPIRATION?

A. The act of inhaling air into the lungs, and again expelling it.

Q. What is the oвJECT of drawing air into the lungs, and again expelling it ?

A. 'To oxidise the carbon and hydrogen of the blood.

Q. We receive into the lungs oxygen through the medium of the atmosphere, mingled with nitrogen : WHAT do we EXPEL from the lungs?

$A$. 'The nitrogen returns unaltered; the oxygen unites with the carbon of the blood to form a gas-carbonic acid, and with hydrogen to form the vapour of water. 
Q. Are not these the saye products of every ordinary form of combustion?

A. They are; therefore respiration or breatiing is but a form of combustion.

Q. It is estimated that a man receives into his system about eight hundred pounds of oxygen from the atmosphere in a year, but his weIGHT at the end of the year has in. creased but LITTLE, or NoT AT ALL : what BEcomes of all this oxygen?

A. No part of it remains in the body, but it is given out again, combined with carbon and hydrogen.

Q. How Mroch carbon passes out of the system of an adult man by the agency of respiration daily?

A. About three pounds.

Q. How is this great ABSTRACTION of material from the body MAADE UP ?

$A$. By the food which we eat.

Q. Are the organs by which respiration is performed the SAME in all animals?

A. The organs of respiration differ essentially in the various divisions and in the various subdivisions of the animal world.

Q. In what manner do the Lowest TXPes of the animal kingdom, as the polypes, or coral-building animals, respire?

A. Exclusively through the shin.

Q. Does man also respire, or absorb and expel oxygen and carbonic acid, in a degree THROUGH the SKIN?

A. He does; and this explains the fact that extensive burns on the surface of the body frequently produce diseases of the lungs.

Q. Why should extensive burns on the surface of the body tend to produce DISEASES of the LUNGS?

A. While in a condition of health, the skin aids the lungs in the expulsion of carbonic acid from the body; but the portion of the skin which has been scorched by an extensive burn, no longer being able to perform that function, the lungs are obliged to assume an extra duty, and suffer as a consequence of their exertion.

Q. If, by NEGLECT of WASHING, we suffer the skin to become covered with impurities, do we not disturb the healthy action of the system?

A. We do; there is no better-established law of health, than that the surface of the whole body should be kept clean and free from all impurities.

Q. What ANIMALS are provided with LUNGS?

$A$. Lungs are for the most part confined to animals that are possessed of vertebra or a back bone. Some animals, however, such as spiders and some species of snails, which are destitute of a back bone, have, nevertheless, regular lungs.

Q. How do INSECTS BREATHE?

A. By means of organs called trachece or windpipes-tubes which penetrate in various directions through their bodies, and terminate externally in open orifices called stigmata.

Q. Why, if we cover an insect with orr, does it speedily DIE?

A. Because the oil fills up the stigmata, respiration is suspended, and the insect dies of suffocation. 
Q. What are the LuvGs?

A. Lungs are made up of blood vessels imbedded in a fleshy substance which we denominate cellular tissue, and expanded over the walls of a series of chambers or cavities.

The organs of respiration are the lungs (lights), the trachen (windpipe), the bronchia (subdivision of the trachea), and the air-cells, or, as they are called, air vesicles.

Fig. 143 .

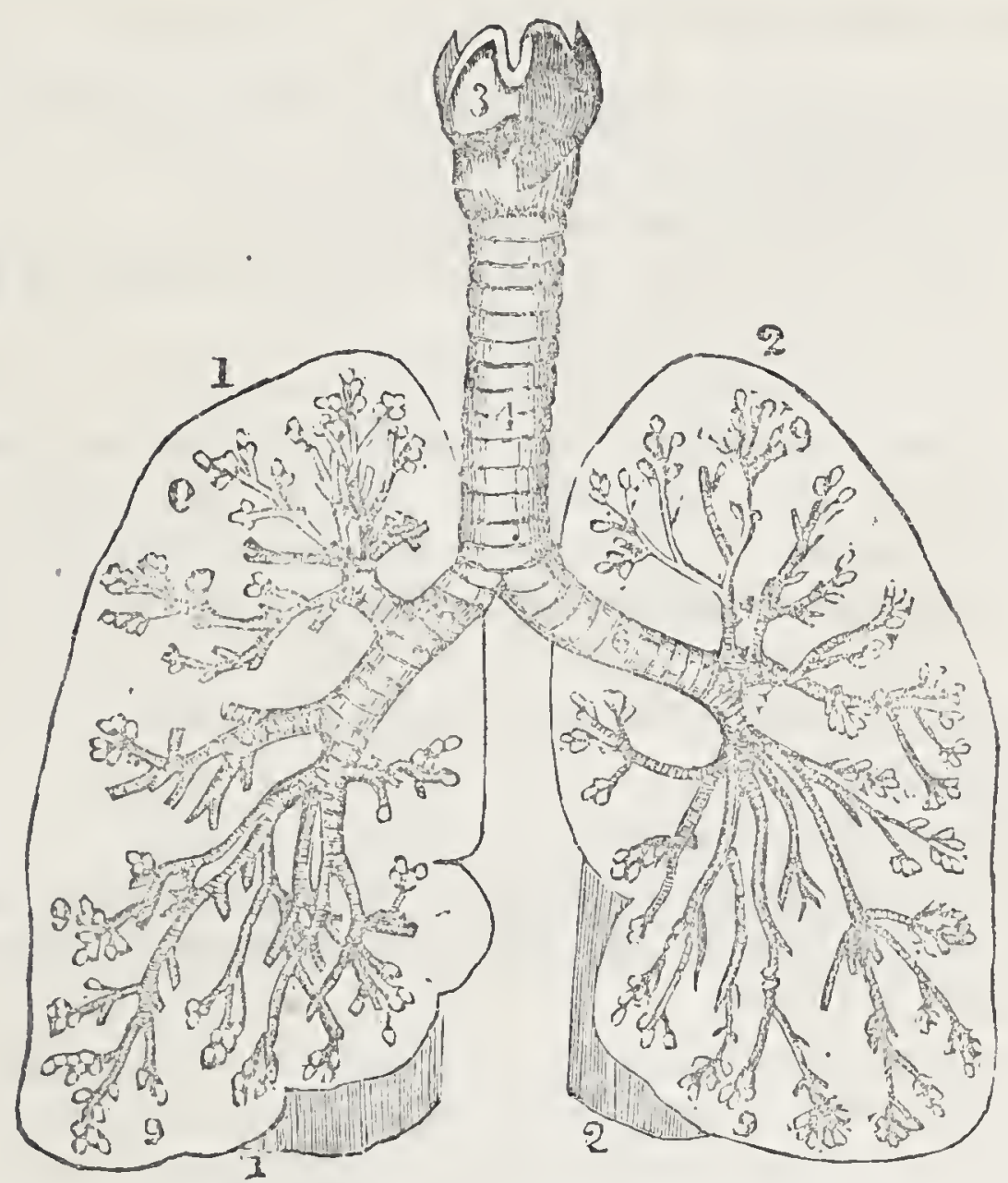

Representation of the larynx, trachea, bronchia, and air-cells.-1, 1, 1, an outline of the right lung; $2,2,2$, an outline of the left lung; 3 , the larynx; 4 , the trachea ; 5 , the right bronchial tube ; 6 , the left bronchial tube ; 7, 7, 7, 8, 8, 8, bronchial tubes of right and left lung; $9,9,9,9,9,9$, air-cells.

Q. To what may the MECHANisur of the lungs in respiration be COAPARED?

A. To the action of a bellows.

Q. In what respect do the gills of fishes differ from the Lungs of land animals?

$A$. Gills are a variety of lumgs, but in them the blood vessels are freed from all covering, and hang loosely in the form of a fringe, floating on the water; this form of lungs is best adapted for the extraction of the oxygen dissolved in the water.

Q. If the carbon taken from the system through the agency of the lungs be not restored, what is the CONSEQUENCE?

A. Starvaiion ensues.

Q. Does a person whose respiration is RAPID and ACTIVE, require MORE FOOD than one whose respiration is sluggish?

$A$. Two persons, who in equal times take up by means of the lungs 
and skin unequal quantities of oxygen, require in the same ratio unequal quantities of nourishment.

Q. A BIRD deprived of food dies on the THIRD day, while a SERPENT will live without food for TIIREE MONTHS or more: what is the reason of this?

A. A bird breathes quiclily, and rapidly consumes the carbon of its system, while the respiration of a serpent is very siuggish; consequently, the carbon of its system is wasted slowly.

Q. Why does a CHLD require food ofrener than an ADULT, and bear hunger less easily?

A. Because the organs of respiration in a child are more active than those of a grown person.

Q. How does the OXYGEN we inhale MINGLE with the BLOOD?

$A$. The oxygen of the air is taken into the lungs by the blood, which gives to it a bright red colour.

Q. How does OXYGEN convert the colour of blood into a BRTGIT RED?

$A$. The colouring matter of the blood is formed by very minute globules floating in it. The oxygen uniting with these globules changes their colour to a bright red. The blood contains iron, and this metal is supposed to play an important part in the colouration of the biood.

Q. What COLOUR is the blood BEFORE this change takes place?

A. A darli purple.

Q. Do plants Respire as well as animals?

$A$. They do; and their leaves may be regarded as performing for them similar offices as the lungs of animals. They are the breathing organs of plants.

Q. Is there any DIFHEREnce between the respiration of plants and animals?

A. The process of respiration in plants is exactly the reverse of that in animals. Animals absorb oxygen, and give out carbonic acid; plants, on the contrary, absorb carbonic acid, and return oxygen.

Q. It is estimated that the population of London adds to the atmosphere daily 4,500,000 pounds of carbonic acid: how is this immense quantity of deleterious gas REMOVED from the atmosphere?

A. Principally through the agency of plants, which absorb it.

"The carbonic acid with which to-day our breathing fills the air, to-morrow seeks its way round the workd. The date trees that grow round the falls of the Nile will drink it in by their leaves; the cedars of Lebanon will take of it to add to their stature; the cocor-nut of Tahiti will grow rapidly upon it; and the palms and bananas of Japan will change it into flowers. The oxygen we are breathing was distilled for us some short time ago by the forests of the Susquehanna, and the great trees tlat skirt the Orinoco and the Amazon; the giant rhododendrons of the Himalayas contributed to it, and the roses and myrtes of Cashmere, the cinnamon-tree of Ceylon, and the forest, older than the flood, buried deep in the heart of Africa, far behind the Mlountains of the Moon."

Q. Is animal hife dependent on that of vegerables?

A. Yes; animals require oxygen, and plants carbonic acid, to keep them alive. Plants give forth oxygen, which supplies the necessity of the animal creation; animals breathe out carbonic acid, which plants require. Thus the two great kingdoms-that of the animal 
and that of the vegetable world - are bound in a close alliance, and made dependent upon each other.

Q. Do plants DECOMPOSE CARBONIO ACID in the NIG ITT-TIME as well as during the day?

$A$. Plants require the influence of light to enable them to decompose the carbonic acid and liberate the oxygen. During the night, plants breathe carbonic acid, as before, but they return it, for the most part, again to the atmosphere, unchanged.

Q. Do WATER-PLANTS purify and free water from carbonic acid in the same manner that land-plants purify the atmosphere?

$A$. The respiration of fishes produces curbonic acirl, and unless this is removed from the water, animal life will cease to exist in it. Water-plants absorb the carbonic acid from the water, and restore the oxygen.

Q. During bright weather, the leaves of water-plants, it will be noticed, are covered with LITTLF BбBвLES : WHAT are these bubbles?

A. Oxygen gas, liberated by the organs of the plant.

Q. Is it good policy, in fountains and reservoirs of water, to free them wholiY from the PRESENCE of VEGETABLE and ANIMIAL organisins?

A. It is not: they are both dependent on one another, and the joint action of the two serves to keep the water pure and wholesome.

Q. Why is vegetation so much more vigorous and IUXURIANm in the Tropics than in the TEMPERATE ZONES?

$A$. In the tropics, the light of the sun is long-continued and brilliant. Plants are dependent upon light for their power of storing up carbon derived from the atmosphere; consequently, in those places where the action of light is most intense, the growth and increase of vegetation will be the greatest.

Q. Why do tropical plants, transported to northern climates and reared in greenhouses, always tANGUISII and INCREASE SLOWLY?

A Because they require the full, strong light of their native hathitots. We can impart to them the necessary heat and moisture, but the action of the light cannot be imitated.

Q. Do plants obtain OARBONIC ACID from any oTHER source than the atmosphere through their leaves?

A. They obtain considerable quantities from the soil.

Q. Do plants absorb any otHER sUBSTANCES from the atmosphere, or from the soil, becides carbon?

A. 'I'hey do: they are enabled to decompose water, retaining the hydrogen and liberating the oxygen; they also absorb ammonia, more or less of which substance is always present in the atmosphere and in rain-water.

Q. Animals, and the various forms of combustion, are continually producing large quantities of carbonic acid; all vegetation is continually liberating oxygen : does there appear to be any INCREASE or DISINUTION of these substances in the atmosphere?

A. There appears to be an exact balance or equilibrium kept up between the animal and regetable world, and we have no reason to believe that the great mass of the atmosphere ever changes its composition. 


\section{SECTION III.-NUTRITION AND ANIMAL HEAT.}

\section{Q. What is HeALTH in the animal body?}

A. Health, in the animal body, consists in the due batance or eyrilibrim between the oxidising or destructive agency of the atmosphere, and the process of nutrition, or feeding, by which the waste is made up.

Q. What is the CAUSE of animal heat?

A. The oxygen of the atmosphere, received into the blood in the lungs, and circulated throughout every part of the animal body, acting upon the food, is the chief source of animal heat.

Q. Why does OXYGEN received into the BLOOD produce HEAT?

A. Through the medium of the capillary vessels, oxygen absorbed from the atmosphere unites with carbon and hydrogen. This union is a species of combustion, and produces heat in the same manner as when oxygen unites with fuel in an ordinary fire.

Q. What are the CAPILLARY VESSELS?

A. The capillary vessels form a fine network all over the body. They cannot be discovered without the aid of a microscope, and the fimest needle cannot be introduced beneath the slin without wounding some of them.

Q. Does this combustion, and the consequent production of ANMAL HEAT, take place in EVERY PART of the body?

A. In the animal body, heat is produced only in those parts to which arterial blooct, and with it the oxygen absorbed in respiration, is conveyed.

Q. Why is there No HEAT developed in hair, wool, and feathers?

A. Because they receive no arterial blood, and therefore in them no heat is developed.

Q. What Two kiNDS of blood are there in the animal body?

A. Arteriul blood and venous blood.

Q. What is the DIFErRENce between the two?

A. The arterial blood going from the lungs conveys the oxygen which it has absorbed in the lungs to the capillary vessels. In these the combustion takes place, and the colour of the blood changes from a bright to a dark red colour.

Q. What BEcoures of the blood after it has given up its oxygen to the hydrogen and carbon in the capillary vessels?

A. It enters the veins, carrying with it the products of combustion. The venous blood passes to the lungs, throws off the products of combustion, absorbs more oxygen, becomes converted into arterial blood with a renewal of colour, and is again returned into the system. 
Q. What BECONES of the OARBONIO ACID GAS formed in the human llood?

$A$. The lungs throw off almost all of it into the air by the act of sespiration or breathing.

Q. Is the HEAT of the HUMAN BODY the SAME as the heat of a FIRE?

$A$. It arises from the same cause,-namely, the carbon of the blood combining with the oxygen of the air drawn in by the breath, and producing carbonic acid gas.

Q. Does the carbon of the food, converted into carbonic acid in the body, give out as IUCII MEAT as if it had been directly burnt in oxygen gas, or in common air?

$A$. The quantity produced is the same; the only difference is, the production of the heat is diffused over unequal times. In oxygen gas the combustion of carbon is rapid, and the heat intense; in atmospheric air it burns slower and for a longer time, the temperature being lower; in the animal body the combustion is still more gradual, and the heat is lower in proportion.

Q. If the HEAT of the HUMAN BODY is the SAME as the HEAT of a FIRE, how is it the human body is not burnt up?

$A$. It is; every portion of the human body wastes as a fire does; and as the fire would go out if fuel were not added, so the human body would be destroyed if food, the fuel of the body, were not supplied.

Q. Why does FlaNNeL, \&c., make us WARM?

A. Flannel and warm clothing do not make us warm, but merely prevent our body from becoming cold.

Q. How does FlanNel, \&c., prevent our body from becoming cold?

A. Flannel (being a bad conductor) will neither carry off the heat of our body into the cold air, nor suffer the cold of the air to come in contact with our warm body; and thus it is that flannel clothing keeps us warm.

Q. Why are FroGS and FISHES COLD-BLOODED animals?

$A$. Because as they consume very little air, combustion is too slow to produce much animal heat. All animals, strictly speaking, are warm-blooded; but in those only which possess lungs is the temperature of the body quite independent of the surrounding medium.

Q. In wHAT ANIMALS is the temperature the greatest?

$A$. Those animals which respire frequently, and consequently consume much oxygen, possess a higher temperature than others, which, with a body of equal size to be heated, take into the system less oxygen.

Q. Why is the temperature of the body of a child HIGHER than that of a man ?

$A$. Because a child consumes proportionally more oxygen, ant respires more frequently, than a grown-up person.

Q. Why do we Perspire when very bot?

$A$. Thie pores of the body are like the safety-valves of a steamengine; when the heat of the body is very great, some of the com- 
bustible matter of the blood is thrown off in perspiration, and the lieat of the body is thereby reduced.

Q. What is the MeAN TEMPERATURe of the human body?

$A$. In a healthy adult it is about 99 degrees $\mathrm{F}$.; the temperature of a healthy child is somewhat higher, 102 degrees $\mathrm{F}$.; the temperature of birds is from 104 degrees to 105 degrees $F$.

Q. Why, in fattening animals, do we compel them to REMAIN INACTIVE, by confining them in stalls and pens?

$A$. In order that the food which they consume may not be burnt $u p$, but retained and stored in the system as fat and muscle. Want of exercise, induced by their confinement, causes the animal to absorb but little oxygen; consequently, the system does not waste.

Q. Why is the flesh of WILD animals generally devoid of FAT ?

A. Wild animals exercise much, and the food they consume is employed in repairing the waste of the system consequent on their activity.

Q. Why do we feel LAZY, and averse to activity, in very hot weather ?

$A$. Because muscular activity increases the heat of our body by quickening respiration; and

2. The food we eat in hot weather (not being greasy) naturally abates our desire for bodity activity.

Q. How much MORE CARBON do we throw off from the system by respiration in winter. than in summer?

A. Full one-eighth more.

Q. Why is it easier to bear hunger for a longer time at the EQUATOR than in CouD OOUNTRIES?

A. As the quantity of oxygen introduced into the system varies with the temperature of the external air, the system wastes less rapidly from respiration in warm countries than in cold; consequently, there being less waste, less food is required in the one situation than in the other.

The temperature of the air at the equator being elevated, the temperature of the system does not diminish ; consequently, food is not required in great quantity for the production of animal heat.

Q. Why do the animals of the Anctic regions require more food than those of the torrid zones?

$A$. In consequence of the cold atmosphere with which the former are surrounded, they inspire an increased amount of oxygen, and the necessity for food containing carbon and hydrogen increases in the same ratio.

Food being the fuel of the animal body, by consuming large quantities, they obtain the most efficient protection against the piercing cold.

Q. Is WARM CLOTHING in winter an EQUIVALENT for a certain amount of food?

A. The more warmly we are clothed, the less urgent becomes the appetite for food, because the loss of heat by cooling, and consequently the amount of heat to be supplied by the food, is diminished. 
6. Why are persons whose lungs, or digestive organs, are impaired, BENEFITED by a residence in a SOUTHERN OLIMATE?

$A$. Because by a removal to a warm country the amount of inspired oxygen is diminished in so great a proportion that the diseased organs have sufficient power to supply the wants of the system, and are not overtaxed to perform their labour; hence an opportunity is afforded for recruiting, and an improvement in health follows.

Q. Does a CARNivorouz, or Flesh-bating animal, as a dog or cat, perspire?

$A$. They do not; their skin is destitute of perspiratory pores. They consequently require less food for their support than animals which feed on vegretable substances, and which perspire freely.

Q. Into what Two great DIVISrons may all the substances which man uses for food be divided?

A. Into those which are devoted to the repair and nutriment of the body, and those whose duty it is to furnish animal heat by their combustion in the blood.

Q. What sUBSTANCrS are adapted to furnish the hody with materials for the formation of muscles, nerves, brain, \&c. ?

A. Only those which contain nitrogen, such as animal flesh, and those grains and fruits which contain this element in their composition.

Q. What SUBSTANCES supply materials for producing animal heat by their combustion?

A. Those rich in carbon and hydrogen; as sugar, fats, starch, wine, beer, and spirits.

Q. Could LiFe be sustained for any great length of time by using as food only those Bubstances, like fats and sugar, which are DEsTiTUTE of NITROGEN?

$A$. Those substances only are capable of being employed in the renewal and repair of the body, which have the same chemical com position as the body itself.

The experiment has been tried of feeding dogs exchusively upon substances destitute of nitrogen ; starvation, within a limited period, was the result.

Q. What is SLEEP?

A. Sleep is a natural and healthy suspension of the intellectual functions of the brain.

Q. Why is the action of the senses, sucl as hearing, seeing, feeling, \&c., suspended during sleep?

$A$. Because the nerves of sensation are inactive and at rest.

Some of the phenomena of dreaming seem to show that the functions of the organs of sense are performed in sleep; the power of perception residing in the brain being, however, dormant, the intelligence conveyed by the nerves is not recognised.

Q. Why is sleep NECESSARY for the liealthy existence of the body?

A. During our waking moments, the vital force is expended in producing mechanical effects, as the labour of different kinds which we perform. During sleep, all labour of the body or the brain is suspended, and the vital force is employed in the formation of nevo parts, or in the repair of those which have been wasted. Sleep, therefore, is necessary to restore the equilibrium of the system. 
Q. Why is it necessary that an infaut should sleep the Grester part of the time?

$A$. In order that the vital force may be employed almost exclusively in building up and strengthening the system, and not be expended in producing mechanical action.

\section{CIAPIER XLIX.}

\section{PHOSPHORUS AND SULPHUR.}

\section{SECTION I.-PIIOSPHORUS.}

Q. What is phosphorus?

A. Phosphorus is a substance resembling wax, and of a pale yellow colour.

Q. What is the mearina of the word phosphorus?

A. The term is derived from the Greek, and means both to produce and to carry light.

Q. How is phosphorus COMmonly OBTAINed?

A. From bones, which are heated to a white heat, destroying both animal matter and charcoal, leaving behind what is called phosphate of lime.

Q. What is the PHosphate of Lime?

A. Phosphorus united to oxygen and lime; when this substance is treated with sulphuric acid, and the product mixed with charcoal and heated, phosphorus separates as a product of distillation.

Q. Is phosphorus INFLAMMAdie?

A. It is so exceedingly inflammable that it sometimes takes five by the heat of the hand; it therefore requires great care in its management, as a blow or " hard rub will often ignite it.

Q. Why will Friction MATCHes IGite by merely drawing them across any rougn SURFACE?

A. Because the phosphorus with which they are tipped has a great affinity for oxygen at a low temperature; so that the little additional heat caused by the friction of the match across a rough surface is sufficient to ignite it, and at the same time to ignite the sulphur which is combined with it.

Q. How is phosphorus PRESERved in its pure state?

A. Phosphorus, in its pure state, must be kept and handled under voater. It inflames in the air with great readiness, and the combustion produced by it is difficult to extinguish.

Q. Is phosphorus a porson?

A. Phosphorus is an excectingly violent poison; mixed with dough, it constitutes a poison much used for the destruction of rats and other vermin. 
Q. What is the composition with which matches are TIPPED?

A. $1 \frac{3}{4}$ parts of phosphorus, 4 of gurn arabic, 4 of water, 2 of nitre, and 2 of red lead. The ends of the matches are previously covered with sulphur.

Q. Why is NITRE and RED LE.LD used?

A. Both of these substances contain a large amount of oxygen, which the phosphorus abstracts at the moment of ignition; they therefore serve to increase the intensity of the combustion.

Q. What is PHOSHUURETED IIMTRGGe ?

A. Phospliuretted hydrogen is the gas formed by the union of phosphorus and hydrogen; it possesses a very disagreeable smell, is inflammable and destructive of animal life.

Q. Under what circumstances does phosphuretted hydrogen Occun in NATURE?

A. It is formed by the putrefaction of animal substances. Carburetted liydrogen is formed by the decay of vegetable substances. The very offensive smell of a dead body arises in part from the phosphuretted hydrogen.

Q. What is PHOSPHORIC ACID?

A. A compound of phospliorus and oxygen. United with lime, it forms the bones which constitute the framervork of the animal body.

Q. Does phosphate of lime alons compose the structure of bone?

A. Bones consist of about one-third organic malter-gelatine or glue, and two-thirds inorganic matter-phosphate of lime. It is the presence of gelatine in the bones which renders them supple and not liable to be easily broken.

Q. Why are the bones of old people FRAGILE and EASILY BROKEN?

$A$. Because they are dry and wanting in gelatine.

Q. How does phosphorus FIND ITS WAY into the SYSTEM?

$A$. It exists as phosphoric acid in the food we consume, especially in what are called the cereals-corn, wheat, barley, \&c.

Q. How does lihosphorus, in the form of phosphoric acid, Fin? ITS $W \Delta Y$ into the system of the GRAIN-BEARING PLANTS?

A. It is absorbed from the soil, in which it exists in a limited quantity.

\section{SECTION II.-SULPHUR。}

Q. What is Sulphur?

A. Sulphur is a yellow, britlle mineral, without taste, but emitting a peculiar odour when rubbed or lieated.

Q. In what STATE does it occur in nature?

A. It is found pure in large quantities in the vicinity of volcunoes; it occurs abundantly in combination with many metals, as silver, copper, lead, and iron; and is also a constituent, in small quantities, of many animal and vegetable substances. 
Q. Why has sulplur No TASTE?

A. Because it is not soluble in water.

Q. What are the rowses of SULPIUn?

A. When the metals or earth containing sulphur, or crude sulphur, are heated, the sulphur votailises, and, on cooling, is deposited from a raporous condition as a fine yellow powder. This powder has received the name of "flowers of sulphur."

2. What is Broustone?

A. IElted flowers of sulphur. When the sulphur is melted and poured into cylinders or tubes, it cools in the form of rolls, and has received the name of "roll brimstone."

Q. From witat covntrues is the supply of sulphur for commercial purposes chiefly derived?

4. From Naples, Sicily, and Southern Italy. From these districts large quantities are exported yearly.

Q. Why does the wHITE of an EGG tarnish a sILVER spoon?

A. Because it contains a little sulphur, which, at the temperature of an egg just boiled, will decompose the water or moisture upon the spoon and produce sulphuretted hydrogen gas, which will tarnish silver.

Both the white and the yolk contain sulphm; but the latter the most abundantly.

Q. What is SULPHURETTED HYDroGEN?

A. A gas formed by the union of sulphur and hydrogen. It possesses an offensive odour, and is very poisonous.

Q. How is sulphuretted hydrogen FORMED in NATURE?

A. Principally from the decomposition of animal substances, as blood, flesh, hair, \&c.; also from the decomposition of such vegetables as contain suiphur.

Q. What VRaEtadies especially contain SUlpuur as an element of composition?

A. Peas, beans, lentils, horse-radish, and onions.

Q. What is it that makes an OPwN or FouL SEWUr so destructive of health to any district in which it may be situated?

A. The evolution of sulphuretted hydrogen. When inhaled, it acts lirectly upon the blood, thickening it, and turning it black.

Q. Why do surfaces painted with laad paints, in the vicinity of sewers, soon turn black, or become discoloured?

A. Through the presence of sulphuretted hydrogen. The sulphur wintes with the lead to form sulphuret of lead, which is of a dark colour.

Q. Why do zINo paints in the same situations continue wHITE?

A. The sulphur unites vith the zinc in the same manner as with the lead, but the sulphuret of zinc is white, and not dark like the sulphuret of lead.

Q. When sulphur is burned in the air, what is the PRODUCT formed?

A. Sulphurous acid. 
Q. Why does a RED Rose exposed to the fumes of burning sulphur turn wHite ?

A. 'Ihrough the bleacting action of the sulphurous acid gas generated.

Q. Is the colour of the rose entirely DESTROYED?

A. It is not; the colouring principle may be again restored by immersing the rose in a weak solution of acid or alkali.

Q. How are STRAW BONNETS and HATS BLEACHED?

A. By exposing them to the fumes of burning sulphur.

Q. What causes the surfocating sMell of a lighted brimstone match?

A. The sulphurous acid generated by the combustion of the sulphur.

Q. What is sulphuric acid or OIL of VITRIOL ?

A. It is a compound of sulphur and oxygen, containing one-third more oxygen than sulphurous acid.

Q. What are the PROPERTIES of sulphuric acid?

A. As sold in commerce, sulphuric acid is a dense, oily liquid, of a brownish colour, emitting white vapours when exposed to the air. It is one of the most powerful acids and agents known in chemistry, and chars and destroys most animal and vegetable substances.

Q. Why does a piece of wood introduced into sulphuric acid become BLAck and reduced to CoAL, as if it had been exposed to the flame of a lamp?

$A$. The sulphuric acid seizes upon the oxygen and hydrogen of the wood, which combine to form water, and the carbon is left behind.

Q. What is the principal part of the sulphur of commerce USED FOR?

A. The formation and manufacture of sulphuric acid.

Q. What occasions the great DEMAND and consumption of sulphuric acid?

$A$. It is used in bleaching; in the refining of gold and silver; for the manufacture of soda, nitric acid, stecurine; the purification of oils; for the cleaning of iron; the preparation of phosphorus, chlorine, hydrogen, carbonic acid, and for many other purposes. It is able to expel and drive out all other acids from their combinations.

Q. In the so-called sulphur springs, WHAT IS IT that gives to the waters their peculiar nauseating odour and flavour?

A. Sulphuretted hydroyen, in most instances. 


\section{CHAPTER L.}

\section{CHLORINE, IODINE, FLUORINE, SILICIUM, BORON.}

\section{SECTION I.-CHLORINE.}

Q. What is CHLORINe?

A. It is a yellowish-green coloured gas, possessing a most disagreeable odour, and producing suffocation and great irritation of the throat and lungs, even when inhaled sparingly.

Q. Does chlorine exist in a FrFe state in nature?

A. Chlorine is not found free in nature; but, mited to other elements, is a large constituent of many organic and inorganic substances.

Q. In what FAMIIIAR SUBSTANCE does chlorine especially abound?

A. In common salt, which is a compound of chlorine and sodium.

Q. What is MURIATIC, or, more properly, HYDRoCHLORIC ACID?

A. A compound of hydrogen and chlorine usually prepared from salt. It is an acid much used in the arts.

Q. What REMARKABLE PROPERTY does chlorine possess, which renders it especially useful in the arts?

A. It bleaches and destroys all colour's derived from the animal and vegetable kingdoms.

Q. When a colour is removed by chlorine, can it be RESTORED again, as is the cuse in bleaching by sulphurous acid?

A. No; the colour is permanently destroyed, and can never be restored again.

Q. How does chlorine act as a DISINFEcting agent?

$A$. It not only destroys all colours, but it unites to and removes all the products generated by the decomposition and decay of animal and vegetable substances.

Q. In WHAT FORM do we use chlorine for bleaching or for disinfecting purposes?

A. Chlorine is a gas, troublesome to make, and dangerous to employ in its pure state. It, however, forms with lime and oxygen a compound, from which it is slowly disengaged when brought in contact with air or water. This compound is known in commerce as chloride of lime, or bleaching powder.

Q. When INFECTIOUS DISEASES prevail, how can the contagious matter be REMroved from bed-rooms, hospitals, houses, \&c.?

A. By using a solution of chlorine or of sulphurous acid, which will not only remove the contagious matter, but also the offensive odour of a sick-room.

Q. What is the process of bleaching cotton and linen cloths?

A. It mainly consists in immersing or steeping the goods in water containing chloride of lime dissolved in it. 
Q. Before chlorine was discovered, or its properties made known, now was the BLEACHING of fabrics EFFECTED?

A. By moistening them with water, and exposing them for a long period of time upon the grass to the influence of the sun and the weather. This process was called "grass-bleaching."

Q. Can you expuain how it is that chlorine is enabled to destroy all colouring materials and all noxious and offensive odours?

A. Chlorine has a great affinity for hydrogen, and very readily unites with it to form hydrochloric acid. All animal and vegetable substances contain hydrogen, which is taken from them by chlorine. But if a single chemical pillar falls, the whole chemical structure tumbles with it. By the abstraction of the hydrogen, the colouring matter becomes colourless, the odorous principle scentless, the infectious matter harmless, and insoluble substances are frequently rendered soluble.

Q. How was bleaching EFFECTED by exposing firbries to the action of the weather?

$A$. 'The oxygen of the air played the same part as the chlorine does; it united to the hydrogen of the colouring materials and formed water. In this action, the chemical influence of the sun's rays greatly assist.

Q. What REMARKABLE Property has common salt, as legards its solubility?

$A$. Common salt has the remarkable property of being equally soluble in hot and cold water. A larger quantity of almost all other salts is dissolved by hot than by cold water.

Q. What is " ROCK SALT?"

A. Salt which is obtained from the earth. In England, Poland, Austria, and other countries, rock salt in great purity is found in the earth in immense beds, from whence it is quarried or dug out, as coal or other minerals are.

Q. What is "BAY SALT?"

$A$. Salt formed by the evaporation of sea water, exposed in shallow tanks to the heat of the sun. $A$ pound of sea-water contains about one-half to five-eighths of an ounce of common salt.

\section{SECTION II.-IODINE AND FLUORINE.}

2. What is IODINE?

A. Iodine is a dart resplendent-looking solict of a bluish-blucti colour. As an article of commerce it has the form of crystalline scales. When heated, it evolves beautiful violet-coloured fumes.

Q. WIIEN was iodine DISCOVERED?

A. In 1812 , by $M$. Courtois, a manufacturer of saltpetre at Paris. In boiling soda prepared from the ashes of sea-weeds, he noticed that under certain circumstances his kettles were powerfully corroded, and an investigation into the canse of this corrosion led to the discovery of iodine. 
Q. Under what CIRConstances does iodine occur in nature?

$A$. It is very widely, though sparingly, distributed in nature. Its great source is sea water, of which it is always a constituent. It also exists in sea animals and plants, as in the oyster, sponge, and the common forms of sea-weed.

Q. Fow is iodine PREPARED?

A. The iodine of commerce is prepared from the ashes of seaveeds, ealled kelp, which are procured in large quantities on the coasts of Scotland, Ireland, and the Hebrides Islands. By crystallisation, treating with sulphuric acid, and heating, the iodine volatilises in a pure state, and is collected in receivers and cooled.

Q. For what purposes is iodine principally employed?

A. It is extensively used in medicine, and is an important agent in photographic operations.

Q. What is Fuvoring?

A. Fluorine is an elementary substance which has never been seen in its pure state. It forms with hydrogen a gas, which is known as hydrofluoric acid gas. This gas can be condensed to a liquidhydrofluoric acid.

Q. Why cannot fluorine be procured in a PURE STATE?

A. Because it unites, as soon as prepared, with whatever body it comes in contact.

Q. What are the properties of bydrofluoric acid?

A. Of all known substances, this is the most destructive of animal matter. When a drop of the concentrated acid of the size of a pin's head comes in contact with the skin, instant disorganisation ensues, and a deep ulceration of a very bad character is produced.

Q. Of what are the well-known orshneis Ts known as Dorbyshire spar composmo?

A. Of Auorine mited with calcium, the basis of lime. Fluorine exists in nature chiefly in this compound, which is known as "fluor spar."

\section{SECTION III.-SILICIUM AND BORON.}

Q. What is SILICrum?

A. An elementary or simple body, little known in its pure state, but; united with oxygen it constitutes a large part of the structure of. the globe. The compound formed from the union of silicium with oxygen is known as silica or silicic acid.

Recent investigations have proved, beyond a doubt, that silicium is a mctal ; for convenience, however, we have preferred to consider it, as formerly, among the non-metallic elements.

Q. Of what is the Common QUART rock composed?

A. Almost entirely of silica. A pure, transparent quartz crystal is pure silica. 
Q. Will you mention some of the otmer forsis in which silica is commonly met witla in nature?

A. The common flint, the jasper, the amethyst, opal, cornetian, chalcedony, and the agate are all varieties of silica. Their brilliant colours are owing to the small quantities of metallic oxides which they contain.

Q. What is the GLASS COATING which we see outside straw, cane, and the stems of rattan?

A. Pure silica, absorbed by the plants from the soil, and settled on the outside of their stalks or stems.

Q. What GOOD PURPOSE does this deposit of silica on the outside of straw, cane, rattan, \&c., serve?

A. Siticc is to these plants what bones are to men and animals. It is the substance to which the stalk owes its firmmess and stiffness. If the silica be wanting to the stalk, it will bend over.

Q. Of what is CONMON SAND composed?

A. Mainly of silica. It is rendered brown or yellow by the presence of oxide of iron (rust).

Q. What is GIASs ?

A. Glass is a compound substance, arificially produced by the combination of silica vith the allaties. These substances being melted together at a higl temperature, unite, lose their solidity, and are melted into a uniform mass, which, on cooling, has the properties of hardness, transparency, and brittleness.

Q. What are the MATERIALS which generally enter into the composition of glass?

A. First and most important is silica, which is used in the form of sand, poudered flints, or quart\%. An alkaline substance, either potash or soda, is the second substance in glass. Lime is often added to hasten the melting of the mass, and a metallic oxide, generally oxide of lead (red lead).

Q. Why is OXIDE of LEAD generally used in the manufacture of glass?

A. Oxide of lead has the effect of increasing the densit!l and britliancy of glass, and of rendering it more soft and manageable in the manufacture, when in a semi-fluid state.

Fig. 144.

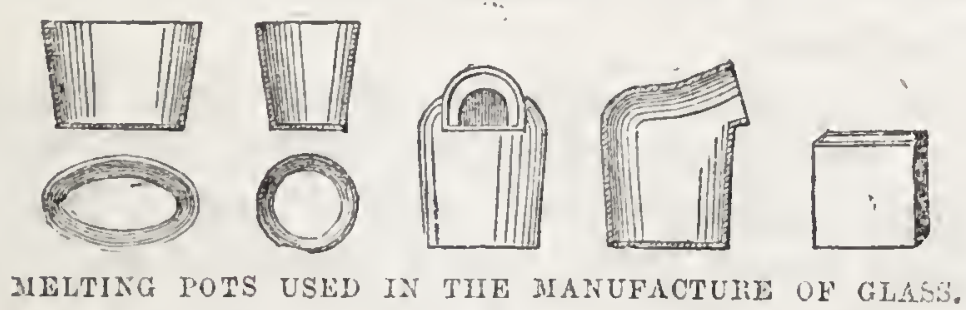

Q. Cannot silica be Metuted by ITSELF to form glass?

A. Silica is one of the most difficult substances to melt lonown, and resists the action of the most intense heat. When mixed with an alkali, and submitted to heat, the two unite, the silica playing the 
part of an acid; the result is a substance which will readily take the liquid form, and become transparent.

The fusibility or melting of glass depends upon the amount of alkali mixed with the silica; the larger the quantity the greater the fusibility.

Q. What is the Process of making glass termed?

A. Vitrification, and a half-fused, earthy substance is said to be vitrified.

Q. Upon what does the PURITY and quality of glass DEPEND?

$A$. The quality of glass depends on the proportion in which the silliceous matter and the allati are combined, on the temperature to which they are exposed, and on the skill with which they are previously mixed and with which the entire process is conducted.

Fig. 145.

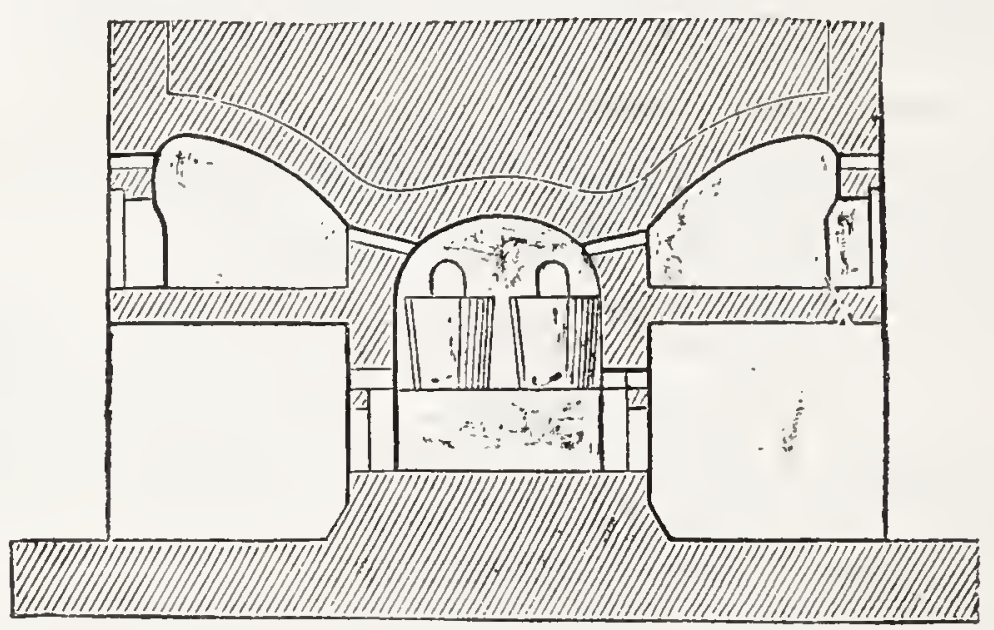

OVEN EMPLOTED IN GLASS BAKING.

Q. Into what THREE VARIETIES is glass divided?

A. Into crown ylass, plate glass, and flint glass, the varieties depending on the quantity and quality of the alkali, and the mode of manufacture.

Q. What is CRown GLASS?

$A$. The name of crown glass is given to the best kind of windorv glass, that which is hardest and most free from colour. It is made almost entirely of sand and alkali, with a little lime; but without lead or other metallic oxide. This variety of glass includes what is known as "Bohemian glass." This glass requires a greater heat to melt its ingredients than any of the other varieties.

Q. What is "FunNT GLASS?"

A. Flint glass, so called from its having been originally made of flints ground to powder, or pulverised, differs from window glass in containing a large quantity of red oxide of lead. It fuses at a lower temperature than crown glass, has a beautiful transparency, and a comparative softness, which enables it to be cut and polished with ease.

Q. From what DESCRIPrion of GLASS are tumblerg, omamental vessels, and lences fo: optical instruments manufactured?

A. From flint glass. 
Q. How is PLATE GLASS made?

$A$. It is cast on a flat metal table, and after careful annealing, it is ground and polished by machinery.

Q. For what punposes is plate glass used?

$A$. For mirrors and large window janes.

Q. How are MIRnoRs made?

A. They are made of plate glass, covered with an alloy of mercury and tin.

The alloy is formed of 30 parts mercury, 70 tin.

Q. Of what is the common green BоTтLE GLASS manufactured?

A. Of the cheapest and most ordinary materials; sand, with lime, and sometimes clay, mixed often with common wood ashes, or any other cheap alkaline material. The green colour is owing to impurities in the materials, but chiefly to oxide of iron.

Q. How is glass STAINeD and Coloured?

$A$. 'This is accomplisled by adding to the usual ingredients of glass, as already explained, the oxides or carbonates of certain metals, which have the quality of imparting to the structure of the glass a quality in virtue of which it absorbs certain constituents of the solar light, and transmits or reflects others.

Q. What METAL is used to impart a beautiful BLUE tint to glass?

A. Cobalt.

Q. What METAI gives to glass a RUBY Or ROSE COLOUR?

A. Gold; manganese imparts pink or violet shades.

Cut-glass ornamental articles, which exhibit different colours upon the same specimen, and at different depths in the thickness of the glass, are manufactured in the follow. ing manner : the object is first formed in white, transwarent, and colourless glass; then being allowed to cool, until it acquires solidity and consistency, it is dipped for a moment in a pot of coloured glass in a state of fusion, and being suddenly withdrawn, it carries away upon it a thin coating of coloured glass, which immediately hardens upon it and becomes incorporated with it. The article is then shaped by the processes of the glass. maker; and, if it be afterwards cut, those parts which are cut will disclose the clear transparent glass, the parts not cut remaining coated with the colour. It is by this process that all the effects which are seen in ornamental articles, which consist partly of coloured and partly of clear glass, are produced. Additional colours may be combined on the article after being coated, as already described, since glass of one colour may be dipped in the same manner in glass of another colour in a state of fusion, so as to talse up another coat of a different colour; and by cutting the surface of such objects to different depths, varieties of effects may be produced, in which two or more colours may be combined.

Q. What are ENAMELS?

$A$. Enamels are compositions of various substances which, when vitrified upon the surface of opaque bodies, communicate their colours, and produce the effect of painting. Enamels are executed on the surface of copper and other metals, by a method similar to painting.

Q. How are artificial GEMs, known as PASTE, prepared?

A. The basis of all artificial gems is the finest kind of flint glass. To this are added various metallic oxides, which impart colour and the requisite brilliancy. 
Q. When was giass FIIRT DISCOVERED and MANUFACTURED?

$A$. It is not linown when glass was first discovered. We find it in the oldest sarcophagi of the Egyptian tombs, and amid the ruins of ancient Babylon and Nineveh. Glass windows do not appear to have been used by the Romans at the commencement of the Christian era, although the art of glass-making had then reached a high degree of perfection.

Q. What is Borax?

A. Borax is a compound consisting of a simple substance called boron, united to oxygen and soda. Its chemical name is biborate of soda. It is chiefly obtained from the volcanic districts of Italy, and from Central Asia.

Q. What is borax USED For in the arts?

A. Chiefly as a fux for soldering or joining one metal with another.

Q. What do you understand by a FLux?

A. This name has been given to certain substances which assist fusion or melting, either by expediting the process or by protecting the substunce melted from alteration.

Q. Why does a blacksmith, in welding together two pieces of iron, often cover their SURFACES of contact with BORAX?

A. The borax prevents the formation of scale or oxide on the surfaces of the hot iron, or dissolves any that may previously exist there. In this way it hastens the union of the two metals, for metals only adhere to metals when they have clean, polished surfaces.

Q. Is the clean surface of a picce of iron Lost hy strongly Heatris it?

$A$. It is; because a layer of oxide is formed upon it by the oxygen of the air; but the bright strface is restored again by the borax, which when it melts dissolves the oxide formed.

Q. Do we use a FLUX in the smelting of ores of DIFFERENT METALS?

A. Always; in the case of the smelting of iron the metal occurs mixed with various siliceous substances, which are very infusible and difficult to separate from the pure iron. By mixing the ore with a flux, as potash, soda, or lime, the silica unites with it, forms an easily fusible glass, and floats on the top of the melted metal.

Q. What is the refuse, called "SLAG," which we see around iron smelting furnaces?

A. A glassy compound of the silica of the ore united to the flux used in the melting.

Q. Can slag be employed for any uSEFul PURPOSE?

A. Yes; it has recently been turned to good account by being made to acquire a texture ten times as durable as marble, and which can be polished as highly as agate or cornelian.

Q. For what purposes can it, then, be used?

A. Owing to its durability it can be employed as a luitcting material, while its beauty adapts it for purposes of ornament or decoration.

It is but recently that the refusc of the smelting furnaces has been employed at all. The worth of the rubbish was unknown. Now science has discovered the commercial 
value of slag; and by refuning, casting, pressing, rolling, moulding, and annealing, renders it at once more lasting and more bcautiful than marble. In the bands of the manufacturer this slag can be male to take any shape, assime any texture, or wear any colour. The cheapncss of the raw material is another recoinmendation, for it costs less than nothing, a beavy cxpesditure being now entailed by its removal from the furuace premises. In its preparation, also, cheapness is remarkable, it cosuing just about half the money to make slag blocks as it does to make clay bricks.

\section{CHAPIER II.}

\section{THE METALLIC E!.EMENTS.}

Q. In what condrion are the metals found in nature?

A. Most frequently in the state of ore; that is, combined with other substances in such a way that their metallic properies are in a great measure disyuised. When uncombined, or when combined only with another metal, they are said to be in a native state.

Q. What are the Most cominon states of combination in which the metalic ores are found?

A. As oxides and combinations of oxides with carbonic, sulphuric, hydrochloric, and phosphoric acid; also with sulphur, forming sulphurets.

Q. What metals possess the GREATEST DENSIT, or wcight, within a given volume?

A. Platinum, gold, mercury, and lead.

Q. What are the Ligriest metals?

A. Potassium and sodium, the metallic bases of potash and soda. These metals are so light that they float upon water.

Q. What metals possess the most MIALEABILITY?

A. Gold, silver, copper, tin, lead, iron, \&c.

Q. What is meant by MaLleability ?

A. The property which allows a metal to be beaten into thin leaves or plates by hammering. Gold surpasses all metals in malleability; one grain of it may be extended so as to cover about fifty-two square inches of surface.

Q. What is an Alior?

A. A combination of a metal with one or more other metals. Thus brass, type-metal, bronze, \&c., are alloys.

Q. What is GEFMAY SILTER?

A. German silver, or white copper, sometimes called argentan, $\mathrm{i}$ : an alloy of copper, zinc, and nickel.

The best is made of 50 parts copper, 25 zinc, and 25 nickel.

Q. What is BRASS ?

$A$. It is an alloy composed of copper and zinc.

Good brass contains about 2 parts copper to 1 of zinc. 
Q. What is BELL-METAL?

A. An alloy of copper and $t i n$. The proportions should be 78 of copper to 22 of tin. Large bells contain more copper than small ones.

Q. What is Bronze?

A. Bronze is an alloy of copper and tin, the proportion of tin being less than in bell-metal. Other metals are sometimes added, but they aie inot essontial. 'The metal of which the so-called brass camnon are constructed is bronze.

Q. What is PEWTER?

A. An alloy of tin and lead.

In the following proportions : 1 part lead, 20 parts tin.

Q. What is Britaninia metai, such as coffee and teapots, \&ce, are made of?

A. It is an alloy of tin with lead, copper, antimony, \&c., according tro its quality.

Q. What is TTPE METAL ?

A. An alloy of antimony and lead.

Q. What is the so-called "aALYANISED IRon?"

A. Iron covered with zinc. By plunging an iron plate into melted zinc an alloy is formed on the surface, which prevents oxidation or rust.

Q. What is an MNALGAM?

A. An alloy, of which mercury or quicksilver is a constituent.

Q. What is ASSAYING?

A. The term assaying is applied to the process of analysing metallic ores, so as to discover their component parts. The assaying of the precious metals is usually confined to ascertaining the quantity of gold or: silver in any alloy or compound, without regard to the other onstituents.

Q. What are the conponent parts of the Gold CoIns of the United Kingdom?

A. They are made of gold, sitver, and copper.

To 90 parts of gold, $2 \frac{1}{2}$ of silver, and $7 \frac{1}{2}$ of copper.

Q. What are the compoxent parts of the SILver corns of the United Kingdom?

A. Silver and copper.

To 90 parts silver, 10 of copper.

Q. What Two METALS possess the greatest degree of HARDNESS?

A. Titanium and manganese; they are harder than steel.

Q. What metals have the aREATEsT tenacity, or the power of resisting tension without breaking?

A. Iron, copper, platinum, silver, and goid, in the order enumerated.

Q. What metals are the most INFusible, or difficult to MELT ?

A. Platinum, iridium, titanium, chrominem, \&c.

Q. What metals are ESPECIALLY BRITTLE?

A. Antimony, arsenic, chromizm, manganese, bismuth.

Q. What is Hetaldurax?

$A$. The art of extracting metals from their ores. 
SECIION I.-TIIE ALKALI METATS.

Q. What are the ALFALI METALS?

A. Potessium, sodium, and lithium.

Q. Why are they called the ALKALr metals?

A. Because united to oxygen they form the non-volatile or fixed alkalies.

Q. What is the APPEARANCE of these metals?

$A$. They are of a silver-white colour, soft, and yield like wax to the pressure of the fingers. They cannot be preserved in a pure state exposed to the atmosphere, as they unite immediately with oxygen; they are therefore kept in some liquid, like naphtha, which contains no oxygen.

Q. When we throw a piece of potassium upon water or ice, what TAKES PLACE?

$A$. The potassium abstracts the oxygen from the vater, instantly catches fire, and burns with a bright flame; the product left is potash.

Q. What is the GREAT SOURCE from which we derive our supplies of potazh?

$A$. From the ashes of land plants. The plants obtain it from the soil, in which it exists, generally combined with silica. Potash is one of the constituents of granite.

Q. How is the CRUDE POTASH of commerce obtained?

$A$. In countries where wood exists in very great abundance, as in some parts of the United States, Canada, Russia, \&c., it is burned simply for the ashes. These are collected, placed in vats and heated with water; the water dissolves the potash, and is converted into ley; this, when boiled to dryness, gives the crude potash, or rather a compound of potash and carbonic acid-the carbonate of potash of commerce.

Q. Can we not also obtain potash from the ashes of Marine PLANTS?

$A$. No; the ashes of marine plants contain soda, and but little potash.

Q. What is the source from which we obtain our supplies of soda?

A. From common salt for the most part. Some soda is also obtained from the earth in a comparatively pure state, combined with carbonic acid. Until within about fifty years, however, nearly all the soda of commerce was derived from the ashes of marine plants.

Q. What is BARILLA?

A. The impure ashes of marine plants.

Q. How is soda OBTAINed from CoMmon SALt?

A. Common salt is a compound of chlorine and sodium. By treating salt, with sulphuric acid, we form sulphate of soda; this compound, heated in a furnace with lime and charcoal, is decomposed, and 
sulphate of lime and carbonate of soda are formed. 'This mass is then treated with water, the carbonate of soda dissolves, the sulphate of lime being insoluble, and the ley, evaporated down to dryness, gives carbonate of soda.

Q. By what CoMnoN NAME is this product known in commerce?

A. As soda-ush.

Q. Does potash exist MORE ABUNDANTLY in some portions of the tree, or plant, than in others?

A. 'The greatest quantity of potash is found in the fruits and their envelopes. The parts of a tree which yield the greatest quantity of ash are the limbs and branches; they contain more than the inside wood.

Q. Besides potash, what SUBSTANoss make up the bulk of wood ashes?

A. Sitica forms the principal substance, with small quantities of lime, magnesia, and iron.

Q. What is PEARLASH?

A. The common carvonate of potash.

Q. What is salemarus?

A. The bicarbonate of notash, or a mixture of poiash and carbonic acid, containing double the quantity of carbonic acid that ordinary pearlash does.

Q. What is " orpan of tartar?"

A. It is formed of potash and tartaric acid.

Q. What is TARTARIO $\triangle \mathrm{CID}$ ?

A. It is a vegetalle acid, which gives in part to surrel its sour taste, and imparts acidity to the branches of grape-vines, unripe grapes, \&c. It also exists in considerable quantities in the juice of ripe grapes.

$Q$. Why do not ripe grapes taste sour, if they coxtuin this $\Delta$ Ord ?

A. Because they also contain sugar, which nentratises the acid. The sour taste of the acid is thus concealed by the sweet taste of the sugar.

Q. How do we obtain " CREAM OF TARTAR?"

A. It is deposited in wine-casks, as a gray or reddish crust, from the wine or juice of the grape; this, refined and purified, constitutes "cream of tartar."

Q. Why are bodies containing potash valuable as MANURES?

A. Because potash is indispensable for the nourishment of certain linds of plants. Thus, on a soil destitute of potash, neither potatoes, turnips, nor grape-vines will flourish.

Q. How can we ascertain what MINERAL SUBSTANCES are essential to the nourishment of plants?

A. By burning the plant, and examining the ashes. The substances which are found, though their amount is generally very small, must be regarded as necessary to the life of the plant. 
Q. Is a soil DESTITUTE of potash adapted to the GROWTH of O'THER PLANTS ?

A. It $i$ s, unless it be entirely barren. Thus, in a soil from which potash has been exhausted, clover and peas will flourish if lime be present, for in these plants lime will take the place which potash fills in potatoes, turnips, \&c.

Q. Exprain what is meant by the rotation of crops.

$A$. If turnips are cultivated season after season upon the same field, the potash will finally be exhausted, and turnips will no longer grow there; the same thing will happen when peas are planted year after year upon the same land, as they will at last exhaust all the soluble lime from the soil. But turnips will flourish in this latter field, because it still contains potassa, and peas in the former field, where lime is still present. This explains the theory of the rotation of crops, which has now been everywhere introduced into agriculture.

Q. How does it benefit a field to Lin Faliow, or uncultivated, for a time?

$A$. Generally when we say that potash or lime has been exhausted from a field by cultivation, we mean that all of these substances, in a soluble state, or in a state adapted as food for plants, are wanting. The field still contains quantities which are united with sitica in rocks and earth, in an insoluble state. By the action of the elements, in time, portions are rendered solubte, and the land again acquires its former fertility.

Q. What is the popular aredicine known as "Grauder salts?"

A. Soda united to sulphuric acid, forming sulphate of soda.

\section{SECTION II.-ME'TALS OF THE ALIKALINE EARTIS.}

Q. What Metals united with oxygen form the alkaline earths?

A. Barium, strontium, calcium, and magnesium.

Q. Why are the oxides of these metals called ALK $\Lambda$ LINE LARTHS?

$A$. Because in appearance they resemble earth, and yet have altaline properties. Thus, lime, the oxide of calcium, neutralises acids, turns vegetable colours, and has a caustic or corroding action on animal and regetable substances.

Q. Are the metals which exist in baryta, strontia, lime, and magresia, easily obtained in a PURE STATE?

A. 'They are united so closely to oxygen, and have such an affinity for it, that it is almost impossible to separate them in a pure state. Thus far, only very small quantities of them have been prepared.

Q. What is their $\triangle$ PPEARANCE?

A. Barium is a dark-coloured metal, somewhat resembling castiron; strontium is similar in appearance to barium; calcium is a white metal; and magnesium is very brilliant, resembling silver. 
Q. What is CIIALK?

A. Lime united to carbonic acid, forming carbonate of lime.

Q. What is MARBLE?

A. Carbonate of lime, having the same composition as chalk.

Q. Of what are the SHELLS of oysters, and other marine animals, composed ?

A. Almost entirely of carboncte of lime. Pearl is also a carbonate of lime.

Q. Since all these substances have the same composition, how can you ACCoUNT for their very DIVERSE APPEARANCES?

$A$. Marble has been subjected, in the interior of the earth, to heat and pressure, and the carbonate of lime composing it has become crystalline; chalk is wanting in crystalline arrangement; and the lime of shells has its appearance in some degree changed by its connection with animal matter.

Q. When you pour an acid upon a piece of limestone, chalk, or marble, why does it ETFERVESCE?

A. Because the carbonic acid is liberated from its combination with the lime, and escapes.

Q. What is gypsum, or "PLASTer of PAris?"

A. A compound of lime and sulphuric acid-sulphate of lime.

Q. Can you explain how it is that the "plaster of Paris," used for ornamental figures and designs, HARDENS SO RAPIDLY when moistened with water?

$A$. The gypsum used for figures and designs is known as calcined plaster. The rock is first pulverised, and then strongly heated, until it loses one-fifth of its weight by the expulsion of moisture from its composition. When mixed with water, the anhydrous burnt gypsum again chemically combines with it, and passes into a solid state.

Q. What is ALABASTER?

A. A very fine compact variety of gypsum.

Q. Why is gypsum valuable as a $M \Delta \mathrm{NURR}$ ?

$A$. Because it affords a supply of lime to the soil, and it absorbs and fixes the ammonia of rain water and of decaying animal substances.

Q. Why is it difficult to heat water in a ressel COATED upon the INSIDE with "PLASTER of Paris?"

A. Because the plaster is so bad a conductor that the heat passes with great difficulty from the fire to the water.

Q. What PRACTICAL APPLICATION has been made of the non-conducting properties of plaster, as regards the transmission of heat?

A. By availing ourselves of the non-conducting properties of plaster of Paris we render metal safes fire-proof. The so-called fire-proof safes are constructed of double or treble walls of iron, with spaces between filled with plaster of Paris. This lining prevents the heat from passing from the exterior of the safe to the books and papers within. 
Q. Why is gypsum called "PLASTER of PARIS?"

A. Because immense beds or deposits of this substance are found in the vicinity of Paris, in which city it was first extensively used as a plaster or cement.

Q. Why is qaicklime called CAUSTIO LINE ?

$A$. Because, like potash and soda, it exerts a corrosive and destructive action upon most animal and vegetable substances.

Q. What is ordinary WHITEWASII ?

A. A mixture of slalied lime with water.

Q. What is SLAKED lime?

$A$. When quicklime is exposed to the air, it rapidly attracts water, and crumbles to powder. Lime which has thus united with water is termed slaked lime, or, in chemical language, hydrate of lime.

Slaked lime, if further exposed to the air, absorbs carbonic acid, and is converted into carbonate of lime. Slaked lime, if mixed with water, so as to form a paste, is mortar ; if more water is added, it becomes milk of lime, or whitewash; and when mixed with six hundred times its quantity of water, a clear solution is obtained-lime-water.

Q. Why should BEDRoons, COTtAGES, HOSPitals, and Stables be occasionally whiteWASHED?

A. Because the lime is very caustic, and removes all organic matters adhering to the walls.

Q. Why should we avoid MIXING caustic or slaked lime with MANURE that is already in a state of PUTREFACTION?

A. Because the manure in this state contains ammonia compounds, which would be decomposed by the lime, and the ammonia set free. The ammonia escaping into the air, deprives the manure of much of its efficacy.

Q. What is the SUBSTANCE used for producing the beautiful crimson flame of fireworks ?

A. Nitrate of strontia; a compound of strontia, oxygen, and nitric acid.

Q. What is it that gives to SOAPSTONE its SLIPPERY or GREASY feeling?

A. The presence of magnesia in its composition.

Q. What are Eps̃om SALte?

A. A compound of magnesia and sulphuric acid-sulphate of magnesia.

Q. What is MEersornaux, of which pipes are made?

A. A compound of magnesia and silica. Before being made into pipes it is boiled in a mixture of wax and oil.

\section{SECTION III.-ALUMINUM.}

\section{Q. What is ALUMINUM?}

A. A beautiful bright metal, very much resembling sitver, but lighter. It possesses the remarkable property of not becoming tarnished or oxidised by exposure to the atmosphere. Hitherto it has 
been obtained only in very small quantities: its value is about double that of silver. Common clay contains about one-fourth part by weight of this metal.

Q. What is common craY?

A. A compound formed by the union of aluminum and oxygen united with silica. The chemical name of clay is silicate of alumina.

Q. In what substances do we find PURE AIUMINa in nature?

$A$. The most costly red precious stone, the ruby, and the most costly blue precious stone, the sappliire, are crystallised alumina. Emery, that is used for polishing, is alumina, and when pure is the gem corundum.

Q. What is $A \mathrm{I}$ M M ?

A. A compound of sulphate of alumina and potash, with twentyfour parts of water.

Q. What is " FULLERS' EARTII?"

A. A snft variety of clay used in woollen manufactories for removing the grease and oil applied to wool in spinning.

Q. How is clay enabled to extract GREASE from woou and other substunces?

A. Clay possesses the property of alsorting oily and colouring matters when in solution, and retaining them within its composition.

Q. What is the most EFEECTUAL WAY of REMOVING GREASE or oil spots from carpets, woollen cloths, paper, \&c.?

$A$. Cover the oiled surfaces with a fine variety of clay, and allow it to remain in contact with them for several days. In this way a great proportion of the greasy matter will be absorbed and removed by the alumina of the clay.

Q. Why is a soil abounding in clay termed a coLD soIL ?

A. Because clay has a very great capacity for retaining water; this, slowly evaporating, causes the earth to remain for a long time cold and wet. A soil rich in clay is generally umproductive, since being compact and heary, it will not allow the roots of plants sufficient room to spread; it likewise prevents the free circulation of air and water among the particles of the soil.

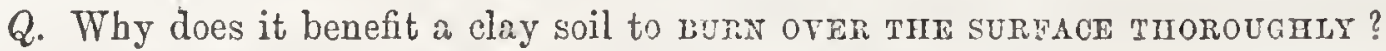

A. All clay and loam contain, combined in their composition, various proportions of potash, lime, soda, and silica. By burning the clay, these are to a considerable extent decomposed and rendered soluble, thus modifying and benefiting the character of the soil.

Q. Does alumina enter into the composition of PLAN's, and consequently into the structure of animals?

A. Although alumina exists in the soil in great quantities, it is not known as yet to cnter into the composition of any plant or animal. All the other constituents of the soil appear to be taken up by plants in a greater or less degree. 
Q. What are the PRoperties which render clay so eminently suitable for the manufacture of pottery, earthenware, porcelain, \&u. ?

$A$. Clay, when mixed with water, is capable of being moulded into various forms, and of acquiring, when exposed to the heat of a furnace, an almost stony hardness.

Q. What is PORCELAIN?

A. All kinds of china-ware, such as are used for dishes, cups, \&c., are denominated porcelain; some kinds are much finer and more beautiful than others.

Q. Of what is porcelain courposed?

A. The chief materials used in its manufacture are a certain clay derived from decomposed feldspar, calcined flints finely ground, together with a portion of feldspar reduced to powder.

"Feldspar", a species of mineral. "Calcined," heated irrensely hot so as to crumble.

Q. How are these materials MIXED TOGETHER?

$A$. They are put into a kind of mill, which is a large cylindrical vessel or tub, into which a small stream of water is constantly suffered to trickle; the mass is now ground or mixed into a kind of dough. This dough is kneaded or worked with the hands until the mass is quite smooth and of a uniform colour. Tt is now ready for moulding.

Fig. 146.

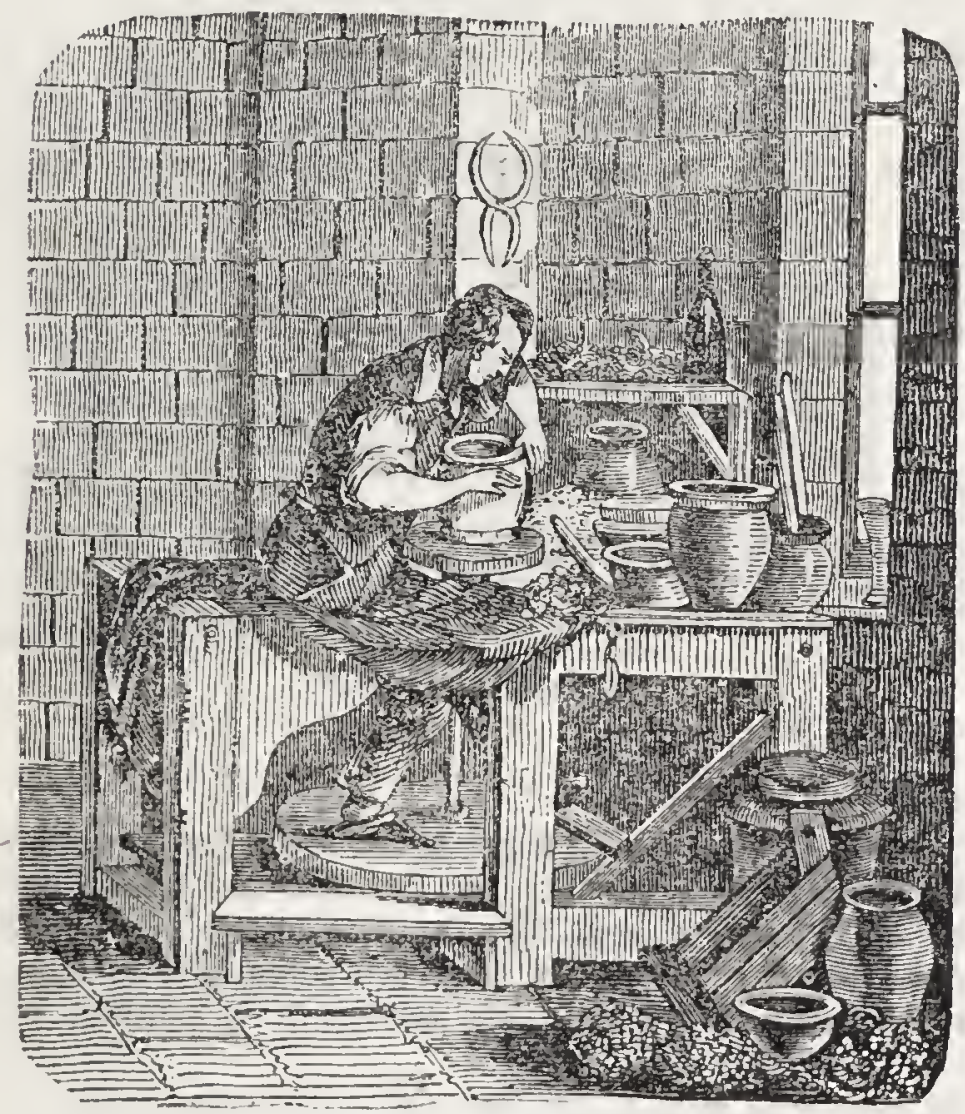

MOULDING PORCELAIN.

Q. What is Moulding?

$A$. Forming the dough or paste into the shape required, such as nowls, plates, cups, \&c. 
Q. How are these articles MoULDED?

$A$. The operation is performed on a machine called a potter's wheel or lathe. A small piece of the clay or dough is placed upon this lathe, and owing to the rapid rotary motion of the machine, the workman is able to shape a vessel by keeping his hands constantly wet; he moulds it to a proper size by means of tools and gauges. It is now suffered to dry partially; it is then placed upon another lathe, when it is shaped more evenly and accurately, and nicely smoothed and burnished with a smooth steel surface. The vessels are then put in a kiln and baked.

Q. How LONG is porcelain usually baked?

A. It requires forty hours or more.

Q. Though porcelain acquires, by baking, great hardness and solidity, jet it still remains so porous as to imbibe water, and also allows it to filter through its substance: how is this REMEIIED? -

A. By corering the ware with a vitreous coating, which is called a glaze.

Fig. 147.

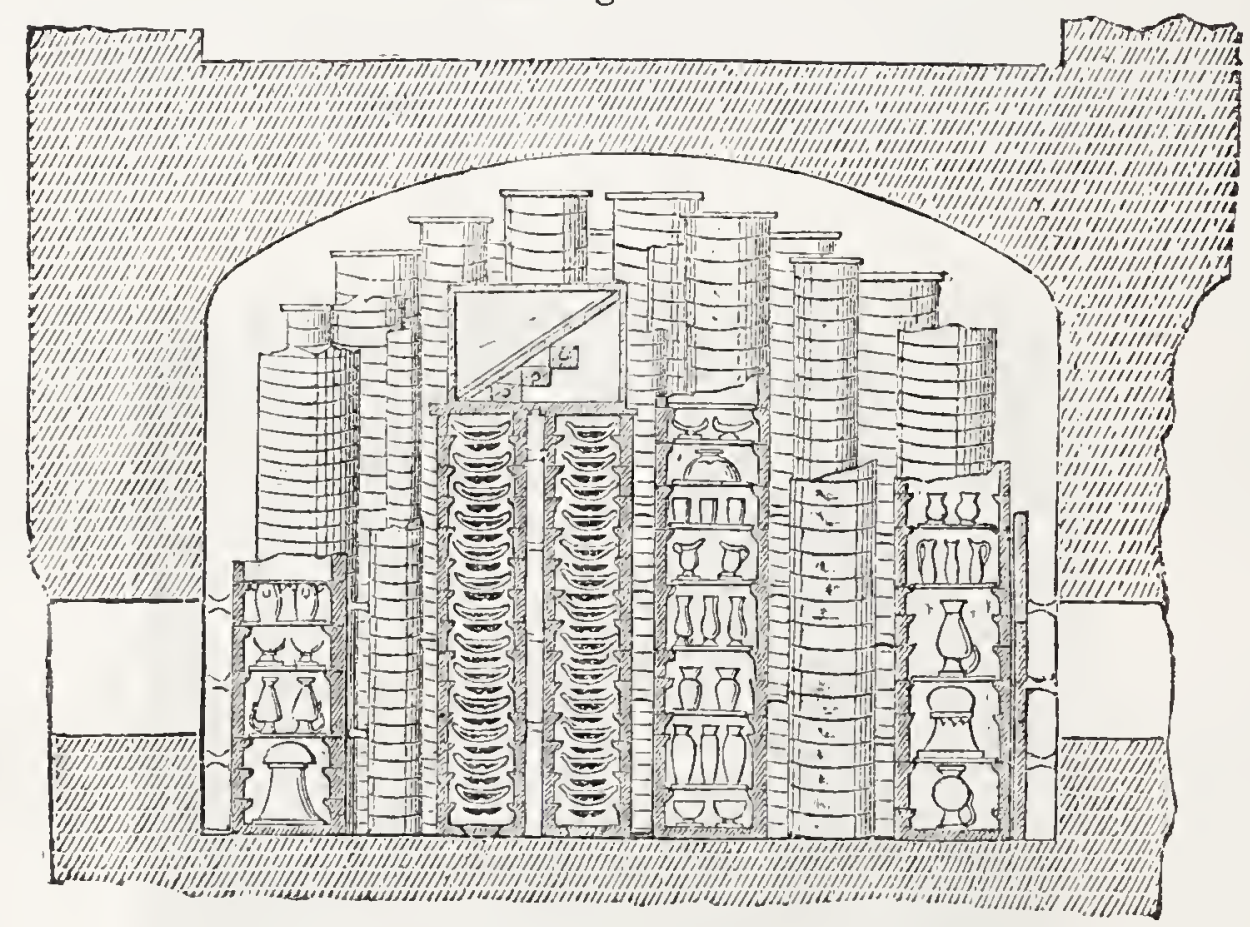

BAKING TORCELAIN.

This glazing is effected as follows :-

A mixture of oxide of lead and ground flints is generally used for glazing. These materials are first ground to an extremely fine powder, and mixed with water, to form a thin liquid. The ware is dipped into this fluid and withdrawn. The moisture is soon absorbed by the clay, leaving the glazing particles npon the surface. These are afterwards melted by the heat of the kiln, and constitute a uniform and durable vitreous coating.

Q. How is stoneware, such as is used for jugs, jars, \&c., made?

$A$. This is a very coarse kind of porcelain, made from clay contrining oxide of iron and a little lime.

Q. How is stoneware GLAZED ?

A. By throwing common salt into the hented furnace : this is 
volatilised and unites with the silica of the clay, of which the ware is composed. The mixture formed fuses over the surface of the ware, and gives a thin but excellent glaze.

Q. What is EARTHENIFARE?

A. This is composed of a species of clay mixed vith silica. - It is moulded in the same manner as porcelain, dried, and baked in a kiln; after that it is glazed with a mixture, which contains the oxides of lead and tin, after which it is reheated.

Q. How is porcelain GILDED?

$A$. The gold is reduced to a state of oxide, and ground up with oil of turpentine and some substance having the property of a flux. It is then printed upon the ware with a brush and exposed to heat. The oxygen of the gold is drawn off, and the metal, by the action of the flux, cemented to the porcelain.

Q. What is it that renders the finer varieties of porcelain so costuY and EXPENSIVE?

$A$. Not the value or quality of the materials which enter into their composition, but the labour bestowed upon their preparation and ornamental decoration. In richly-painted porcelain the articles enter the kiln four or five times.

Q. What is the composition of FIRE-BRICKS, used for the lining of furnaces ?

$A$. They are made of a variety of clay, which contains but a small proportion of the alkalies or alkaline earths or oxide of iron. These substances tend to make clay fusible, and their absence enables the clay to resist the action of heat.

Q. Why does the stem of a new tobacco-pipe ADHERE to the LIPS with considerable force when first used?

A. Tobacco pipes are composed of baked clay, which has a great atiraction for water, so that when a new pipe is put into the mouth, it alsorbs the moisture from the lips, and adheres with force to the skin.

Q. What is the material called "PARIAN," of which statuettes are manufactured?

A. A very carefully prepared variety of porcelain. It is called "Parian," from its resemblance to the celebrated Parian marble of Greece.

SECTION IV.-TIIE HEAVY METALS.

Q. In what respect does IRON DIFFER from all other metals?

A. It is the only metal which is not injurious to the health, the only inetal that always forms an element of the body, especially of the blood, and the only metal which is found everyuhere upon the earth, in almost all stones, rocks, soils, and in almost every plant.

Q. Is iron found PURE on the surface of the earth ?

$A$. The occurrence of native iron on the surface of the earth, except in meteoric masses, is exceedingly rare. 
Q. What is ocHre?

A. An oxide of iron; the colour is generally red or yellow. Ochres are much used as a cheap material for paints.

Q. What is the cause of the BEACTIFU FILA of colour which often invests the surface of stagnant waters?

A. It is due to a thin film of oxide of iron, which has become separated from the water in which it was formerly held in solution. A very thin film of oxide of iron gives a yellow reflection; a thicker film, a red or brown, and a still thicker one, a violet and blue reflection.

Q. Why are the stones in the beds of brooks and rivers generally of a BRoWN or XELLOW colour externally?

A. Because an oxide of iron has been deposited upon them from the water.

Q. What is CAS1 rRon?

A. Cast iron is the metal obtained by smelting the ore with carbon. It is by no means pure iron, as it contains carbon, silica, some aluminum, and generally sulphur and phosphorus. It is fusible at a glowing white heat, is brittle, and can neither be forged nor welded.

Q. What is Wrovght, MALLAALL, or BAR IRON?

A. Cast iron, deprived of its carbon and other impurities, is converted into malleable iron. It is not fusible at a white heat, and may be forged and welded.

The process of "puddling," or converting cast iron into wrought iron, is as follows :-

The cast iron is put into a reverberatory furnace, and when in fusion is stirred, so that every part may be exposed to the air and flames. After a time the mass heaves, emits a blue flame, gradually grows tongh, and becumes less fusible, and at length pulverulent; the fire is then urged, so that the particles again agglutinate at a welding heat, and are gradually wrought up into masses. In that state of intense heat the masses are passed successively through rollers, and converted into malleable bars. They are then cut into pieces, placed in parcels in a very hot reverberatory furnace, and again hammered or rolled into bars. In this way the excess of carbon existing in the cast iron, and most of the other impurities, is expelled from the metal, and the iron rendered nearly pure.

Q. Why does a drop of nitric acid, let fall upon steel, occasion a BLACK spot?

A. Because the iron is dissolved, and the carbon in combination with it exposed to view.

Q. Upon what PECULIARITY does the capacity of wrought iron for welding depend?

$A$. At a certain degree of heat, less than that of fusion, iron becomes soft like wax or glass, so that two glowing pieces may be cemented or welded into one.

Q. Is this property of weLDrac possessed by any of the o'THER metals?

A. None of the other metals, except platinum, can be welded. All the others become fluid instantaneously, as is the case with ice, without undergoing previous softening.

Q. What is understocd by the "TEMPERING of steel?"

A. The process of tempering gives to steel instruments a quality intermediate between hardness and ductility, which insures to them the proper degree of strength under the uses to which they are exposed. 
Q. In what MANNER is this effected?

$A$. Aiter the steel has been sufficiently heated, and hardened by cooling rapidly, it is partially softened, or let down to the proper temper, by heating it again to a less degree, or to a particular temperature suited to the hardness required, after which it is again cooled by plunging it into cold water.

Q. When steel is heated it assumes various colours, which succeed each other as the temperature increases ; frst yellow, then orange, crimson, violet, blue, and dark gray : what occusIoNs these colourations?

$A$. The cause of these colours is the same as that which produces iridescent appearances on the surface of standing waters containing iron.

A film of oxide of iron forms upon the steel through the agency of the heat; at first the film is thin, and has a ycllow appearance, but gradually it becomes thicker and also darker, as the heat increases. The final result, the dark gray coating, is iron scrles.

Q. Do these different colourations which steel assumes, on being heated, conRESPOND with a DEFINITE DEGIEE of hardness and elasticity?

A. They do; when the steel is covered with a yellow film it is hard and brittle; when it presents a bright blue appearance it is in its softest and most clastic condition. This bright-blue colour is especially to be noticed on swords and watch-springs.

Q. What is CopperAs, or GREen viTnou?

A. A componend of iron and sulphuric acid-sulphate of iron. It is principally manufactured from the sulphuret of iron-iron pyrites -by oxiclation. This is effected by exposing the sulphuret of iron to moisture and the action of the atmosphere.

Q. For WHAT IURPoSe is copperis, or green vitriol, chiefly used?

A. For rlyeing blacks or browns in calico printing, and for the preparation of inlis.

Q. As a solution of copperas has but little colour, how can it be used for DYEING BLACK?

A. When copperas in solution is brought in contact with gallic or tannic acid, a dense black fluid or precipitate is formed, the acid uniting with the oxide of iron to form gallate or tamnate of iron. If you dip a linen rag in a solution of gallic acid, and then into a solution of copperas, a black colour is formed in the fibre itself, and adheres so firmly that it cannot be washed out.

This is the general method of dyeing cloths, leather, and hair. Hats are also coloured black in a similar manner.

Q. What is GALLIC acid?

$A$. Gallic acid is a vegetable acid extracted from gall-nuts. The gall-nut is an excrescence produced upon an Asiatic species of oak, by the puncture of an insect.

Q. What is TANNIC acid?

A. Tannic acid (tannin) is the name given to that substance which exists in the barli of some trees, which imparts to them the well-known astringent taste. It especially abounds in the bark of the oak, hemlock, and birch. 
Q. How is the permanent BLAck colour given to leather of which brots and shoes are made?

$A$. The leather, containing tannic acid, is washed with a solution of iron; the tannic acid, uniting with the iron, gives to the leather its permanent black colour.

Q. How is common Writing Ink prepared?

A. All the common black inks are prepared from gallic and tannic acids and a salt of iron, generally copperas. The acids uniting with the oxide of iron produce a deep black precipitate; to prevent this from settling, and for thickening the fluid, a mucilage of gum-arabic is added.

Q. Why does the pale colour of fresh ink grow DARKER when exposed to the air upon paper?

A. The iron contained in the ink assumes an additional quantity of oxygen, and with it a darker colour.

Q. How can Mouldiness in ink be counteracted ?

A. By the addition of a small quantity of the oil of cloves, creosote, or corrosive sublimate; the latter in small amount is probably more efficient than all others, but it should be remembered that it is a deadly poison.

Q. How may the stains of ink prepared from tannic or gallic acid and iron be RENOVED from paper, cloth, \&c.?

A. By a solution of chlorine (bleaching powder), or by phosphoric or oxalic acids.

Q. How can FADED writings be RESTORED?

A. By washing the paper with a decoction of galls.

Q. What is the brilliant pigment called "Prussian BLuE?"

A. A compound of iron and cyanogen.

Q. How is BLUE INK prepared?

A. Blue ink is generally made by dissolving sulphate of indigo, or basic Prussian blue, in water, and thickening the solution with a little gum.

Common Prussian blue is rendered soluble in water by the addition of muriatic or oxalic acids. If the soluble blue be added to common black ink (îrom galls), the result is a black ink which cannot be removed from paper without destroying it.

Q. What is vHite vitriol?

A. Sulphate of winc.

Q. How is the oxide of zinc, or the "zinc white," used for paints PREPARED?

$A$. The ore of zinc mixed with coal is smelted in a furnace at a high temperature. The metallic zinc rises as a vapour; on coming in contact with the air this vapour unites with oxygen, and bursts into a flame; while the products of combustion, the oxide of ainc, fall in clouds as a beautiful white impalpable powder or dust.

Q. What is BLock mIN?

A. 'Tin purified by heat and run into moulds, which form blocks of great size. 
2. What is sheEt tin, such as is used in the manuficture of pans and other utensils ?

$A$. It is sheet iron dipped into melted tin, a portion of which adheres to the surface.

Q. From what countries do we obtain our supplies of tin?

A. From the mines of Cormuall; Malacca, in the East Indies; and a little from South America.

Q. Why are tin pipes PREFERABLe for the conveyance of water for household purposes to LEAD pipes?

$A$. Because the compounds which carbonic acid, and other substances contained in water, form with the tiul are not so injurious to health, if taken into the system with the water, as the compounds of lead are.

Q. What is the comparative Strengti of lead and tin pipe of equal dimensions for the conveyance of water?

$A$. The strength of tin pipe to resist the internal pressure of water is at least threefold greater than that of lead.

Q. What METAL stands next to iron in ABUNDANCE and cHEAPNESS ?

A. Lead; it is rarely found pure in nature, but in combination with sulphur it occurs in great quantities. The ore of lead and sulphur is called "galena."

Q. Is lead a poison?

$A$. Lead and nearly all its compounds are dangerous and secret poisons. When received into the system it frequently remains dormant for years, and then suddenly manifests itself in various forms of disease.

Q. What is the disease called "PAINTER's colic?"

A. A disease to which painters and others working in lead are liable, in consequence of receiving into their system, imperceptibly, portions of lead.

Q. Is it DANGEROUS to sleep in, or breathe the air of, a room newly painted with paints containing lead?

A. It is highly dangerous, since the air is filled with a vapour of the lead compound used as paint.

Q. Why are some WATER, when conveyed through LEAD pipe, Porsonous?

$A$. Waters which are very pure and contain much oxygen dissolved in them; waters which contain nitric acid compounds, such as those flowing from the vicinity of barn-yards, manure heaps, and those which contain common salt or organic matter, as water flowing from swamps and fields; waters containing soluble carbonates-all dissolve lead from the pipes through which they may be made to pass. Constant use of such waters, in the process of time, will introduce suffi. cient lead into the system to produce disease, which is often attributed to other causes.

2. What waters may be safely conveyed through lead pipes?

A. Those which contain sulphate of lime. The sulphuric acid acts upon the lead, and forms a compound of sulphate of lead on the interior of the pipe, which the water cannot dissolve. 
Q. What is IITHARGE, used extensively in the manufacture of glass, \&c. ?

A. A compound of lead with oxygen - the protoxide of lead.

Q. What is the so-called WHITE LEAD, used for painting?

A. A compound of oxide of lead and carbonic acid.

Q. What is SUGAr of lear?

A. A compound of oxide of lead and acetic acid-acetate of lead.

Q. What is ACETro acid?

A. The acid existing in vinegar.

Q. How are внот manufactured from lead?

A. The lead is melted at the top of high towers built for the purpose, and poured into a vessel which is perforated with numerous small holes in the bottom. The lead, after running through these perforations, immediately separates into drops, which cool in falling through the height of the tower, and are received in a reservoir of water at the bottom to break the force of the fall.

Q. How are shot so manufactured PROVED, and the different sizes SEPARATED?

A. By rolling them down an inclined board. Those which are irregular in shape roll off at the sides or stop, while the spherical ones continue to the end.

Q. What is the beautiful green pigment called VERDIaris composed of ?

A. Of copper united to oxygen and acetic acid. It is prepared on a large scale either directly from copper and vinegar, or indirectly by packing sheets of copper with the refuse of pressed grapes, the juice of which gradually passes into vinegar.

Q. Is verdigris a PoIson?

A. Verdigris, in common with all the compounds of copper, is very poisonous. The most efficacious antidotes for poisoning with copper are white of eggs and milk.

Q. When copper is exposed to the air and moisture, it gradually becomes covered with a greenish coating: to $\mathrm{WH} \Lambda \mathrm{T}$ is this appearance DUE?

A. To the formation of a carbonate of copper; carbonic acid being absorbed from the atmosphere and from water by the metal.

Q. In what respect does Mercury differ from all otber metals?

A. It is the only metal which is fuid at common temperatures.

For this reason, and also on account of its silver-white brilliancy, it has been called hydrargyrum (water silver, or liquid silver). This name is still retained in medicine. Subsequently, on account of its mobility, it was dedicated to Mercury, the most active of the heathen gods, and received his name.

Q. Does mercury ever become soms at natural temperatures?

A. In Siberia, and within the polar circles, mercury often solidifies through the intensity of the natural cold.

Q. If you make a stroke across a brass plate with a stick or brush dipped in solution of mexcury, and afterwards bend the plate at this place, it will break as though it had been cut: WHY is this EFFECT PRODUCED?

$A$. Because the mercury of the solution at once penetrates and combines with the brass, and renders it britle. 
Q. What is CALOMEL?

A. It is a compound of two parts of mercury united to one of chlorine, forming the subchloride of mercury. The preparation commonly known in medicine as "blue pill" is a preparation of calomel.

Q. What is CORRosIve SUBLIMATE?

A. A compound of mercury and chlorine united in equal proportions, forming the perchloride of mercury.

Q. Are both these compounds, calomel and corrosive sublimate, Porsons?

$A$. 'They are; corrosive sublimate, especially, is a most deadly poison. In cases of poisoning by it, the most effectual antidote is white of eggs.

Q. What is the process of PRESERving wood from decay, commonly termed "KYANISING ?"

A. It consists in saturating the fibres of the wood with a solution of corrosive sublimate.

Poisonous substances, and corrosive sublimate especially, have the property of protecting animal and regetable substances from decay. The skins of stuffed birds and animals, and the plants of a herbarium, may be protected from insects and decay by washing them with a solution of corrosive sublimate. It should not, howerer, be forgotten that these substances, by such treatment, become themselves poisonous.

Q. What is the beautiful red pigment called "VERMrmion?"

A. A compound of mercury and sulphur-sulphuret of mercury.

Q. In what STATE is mercury found in nature?

A. Generally as a compound of mercury and sulphur-forming a beautiful red mineral called cinnabar. In some places it is also found pure.

Q. From what countries do we derive our supplies of mercury?

A. From the mines of Idria in Austria, Almaden in Spain, California, and South America.

Q. Why are silver, gold, and platinum called NoBLe metals?

$A$. Because, when exposed to the action of air or moisture, they do not rust; when exposed to heat, they do not oxidise or volatilise; and they are of comparatively rare occurrence in nature.

Q. Why are silver and gold, when employed as money, or used in the arts, generally ALLOYED, or mixed with other metals?

A. Silver and gold in their pure state are very soft, and would in this state quickly wear out by using. By alloying them to a small extent with copper these metals are rendered harder without losing their lustre.

Q. When is silver called "FINE?"

A. In purchasing silver plate, \&c., the question is always asked, What is the proportion of pure silver in eight ounces? If it amounts to seven and a half ounces, the silver is said to be seven and a half ounces fine; if six, four, or two ounces of silver are contained in it, it is understood to be six, or four, or two ounces fine. 
Q. How fine is the silver commonly used for Piate or coins?

A. Six ounces fine; that is, eight ounces of the plate or coin contain three-fourths silver and one-fourth copper.

Q. How are ornaments of iron, such as carriage and harness trimmings, PLATED with sil rer?

$A$. The iron is first covered with a coating of tin, and upon this surface a thin plate of silver is applied. The whole is then submitted to a heat sufficient to melt the tin and form an alloy, the fusion being aided by the employment of a flux.

Q. What is the VALUE of SILVER?

A. About five shillings per ounce.

Q. What is the VALUE of GOLD?

A. About four guineas per ounce.

Q. How do we designate the PURITx of gold?

$A$. The quantity of pure gold contained in a mass is expressed by the word carat, the standard number not being eight as in silver, but twenty-four. Perfectly pure gold is said to be twenty-four carats fine. If gold is said to be eighteen carats fine, it is understood that the mass consists of three-fourths (eighteen parts) gold, and one-fourth (six parts) of alloy.

Q. Is there any soLvent for gold?

$A$. None of the acids alone can dissolve gold; chlorine is the only means of rendering it soluble. Commonly the chlorine is obtained for this purpose by mixing muriatic (hydrochloric) acid with nitric acid; in this mixture, known as aqua regia, the gold dissolves completely by sufficient heating, producing a brownish-yellow liquid (solution of gold).

Q. What is Buturon?

A. Gold and silver reduced from the ore, but not yet manufactured. At the mint, it means all gold and silver suitable for coining purposes.

Q. How is GOLD-LEAF manufactured?

A. Gold forged into plates is first rolled into a thin ribbon by means of steel rollers. This ribbon is divided into small squares, which are placed between leaves or sheets of gold-beater's skin (a thin membranous substance obtained from the intestines of animals), and the whole beaten with a heavy hammer. As the gold expands, it is divided and subdivided until the required thinness of leaf is obtained.

Q. What is PLATiNom?

A. Platinum is the heaviest known metal, possessing a lustre inferior to silver, and of a whitish colour. It is found only in the metallic state, generally in small grains, often mixed with gold.

Q. What are the Properties of platinum?

A. It is not affected by air or moisture, or oxidised by heating. It is tenacious, ductile, and can be welded like iron. It is infusible by the strongest furnace heat. Its value is intermediate between silver: and gold, and in Russia it has been coined into money. 
Q. Is there any solvent for platinum?

$A$. Platinum, like gold, only dissolves by heating it in aqua regia.

Q. What are the cHARACTERISTICS of the metals PALLADICM, IRIDIUM, RHODIUM, and OSMIUM?

A. They all resemble platinum, and are always found in very small quantities associated with it. Iridium, from its great hardness, is ased for pointing the tips of gold pens.

Q. What is ANTIMoNY?

A. A brittle metal, of a whitish colour, used in the composition of type-metal.

All the soluble preparations of antimony are poisons, and consequently the stomach violently exerts itself to remove froin it all such compounds introduced into it. This is effected by vomiting, and, for the reason of its emetic properties, antimony has become a very important medicine.

Q. What is the composition of the well-known "TARTAR EMretic?"

A. It is a compound of cream of tartar and tartaric acid, united to oxide of antimony. Dissolved in wine, it forms "wime of antimony."

Q. What is ARSENIC?

A. Metallic arsenic is an exceectingly brittle metal, of a steel gray colour. It vaporises, when heated, with a strong smell of garlic-a property not possessed by any other metal.

The substance used as poison, and sometimes known as ratsbane, is arsenious acid, a compound of arsenic and oxygen. Arsenious acid has the form and appearance of a fine white powder.

Q. What is the BEST REMEDY in cases of POISONING with arsenic?

A. A certain remedy is the hydrated peroxide of iron (iron rust). Before this remedy and the aid of a physician can be procured, it is well in cases of poisoning by arsenic, and in cases of poisoning generally by mineral substances, to administer milk, white of eggs, sugar, or soap-suds.

Q. Can we detect with certainty arsenic in the body, when it has been administered in poisoning?

$A$. 'There is no poison so liable and certain to be found as arsenic; and in almost every case of poisoning with mineral substances, science is enabled to detect the substance, even when life has been extinct for years, and the body nearly decomposed.

Q. How does the MANNER in which the metallic elements are DISTRIBUTED over the surface of the earth illustrate the wisdom and beneficence of the Creator?

A. All those metals which, either pure or compounded, are prejudicial or poisonous to the animal system, are comparatively rare, or exist in such forms that they are not soluble in water.

If the r.xides of copper or leat were as widely distributed as iron, the result to all animated beings would be most disastrous. Had carbonate of baryta been as abundant as carbonate of lime, all forms of life would have failed before its influence. 


\section{ORGANIC CHEMISTRY.}

\section{CHAPTER LII.}

\section{CONSTITUTION AND FORMATION OF ORGANISED BODIES.}

Q. What is ORGANIC OIIEMISTRY ?

A. Organic chemistry is that science which treats of the substances which form the structure of organised beings, and of their products, whether animal or vegetable.

Q. What are the ELEMENTS which compose oRGANIC SUBSTANCES generally?

A. Carbon, hydrogen, oxygen, and nitrogen, a little phosphorus and sulphur, and sometimes a small proportion of two or three alkaline and earthy compounds, are the sole materials which have been employed in the construction of all the countless multitudes of orders of living objects which people the earth and clothe it with beauty.

Q. What is the Force which governs and controls the elements existing in living organic structures?

A. Vital force; we recognise it as life only by its actions; of the nature of the force we are ignorant.

Q. Under what Fork does animal or vegetable life first manifest itself?

A. As a cell; under the guidance and direction of the vital force, all growth or increase in volume and weight in all animals and vegetables takes place by the introduction of new matter from without-namely, by the development of a multitude of little cells.

Q. What is a crut?

A. A cell is a little membranous bladder containing a fluid. When cells are once formed, they become modified in various ways, but their appearance always precedes the formation of circulating vessels, or any of the more complex forms of organic structure.

Q. What is the delicate GREEM sLIMI which is seen on the surface of standing water?

$A$. The lowest form of vegetable life; examined under the microscope, it is but a collection of cells.

Q. Wata TroNDERfur PROPERTY does the living cell possess?

A. The power of influencing chemical action. What is called "secretion" in animals and plants is the result of the use of this function: by it the body grows, for the cell secretes or prepares its jwn food.

Q. Do DIFEERENT KINDS of cells produce DIFTERENT SUBSTANCES, and possess different powers in this respect?

$A$. They do. For example, one kind of cell will decompose carbonic acid, reject the oxygen, and preserve the carbon within its walls or 
tissues; a second will produce out of the inorganic constituents of the air the sweet-smelling principle of the rose; a third will convert a portion of the blood into milk; and yet to the eye all are alike, "a collection of little wet bladders."

Q. Do we know why one portion of a plant should produce sugar, and another portion gum? WHY one portion of the animal system should produce blood, and another milk?

$A$. Under the influence of the vital or living force, the organs of animals and vegetables absorb oxygen, hydrogen, nitrogen, and carbon, and from these elements, in great part, are produced such products as the system or constitution of the animal or vegetable may require. Why this action takes place, we know not; neither can we combine these elements directly to form artificially any of these compounds.

The great principle of life has erer been a secret to mortals.

\section{CHAPTER IIII. \\ VEGETABLE ORGANISATION AND PRODUCTION.}

Q. Why do sEEDS GEnMINATE and sprout?

$A$. Through the agency of the vital force which slumbers in them until called into activity through the influence of heat and moisture.

Q. Of what are all the cELLS and Vessers of plants COMPOSED?

A. Of vegetable tissue.

Q. What is VEGETABLE TISSUE, or SKIN?

A. A compound of oxygen, hydrogen, and carbon. It is to plants what bones, flesh, and shin are to the animal body, forming the solid mass of all vegetable organs.

Q. Will you give sous EXAMPLES of the different forms of woody tissue?

$A$. In the bleached fibres of cotton and flox, we have examples of perfectly pure vegetable tissue or fibre; common cork, the stones of fruits, the leaves, stems, and flowers of plants, the soft pulp of the apple, peach, carrot, \&c., the hushs of grain (bran), and the hard indigestible structure of straw, wood, \&c., are all examples of vegetable tissue.

Q. What is VEGETABLE MOOLD, or humus?

A. It is the product of the decay of vegetab7e tissues. When vecretable tissues, as straw, wood, leaves, \&c., are exposed to the action of the common air, they absorb moisture, and become gra. dually brown and rotten. The chemical process which takes place is the same as when wood or other vegetable matter is burned; decay being a slow combustion.

Q. Why is the UPPER LAYER of earth in fields and gardens of a dark ERow or DLACK colour?

A. Because it is rich in tumms, or the products of the decay of 
vegetable tissues. As these decay, the oxygen and hydrogen pass off into the air, while the carbon, which is black, is in a great measure left behind, and colours the earth with which it is mingled black or brown.

Q. What is STARCH?

$A$. The name starch is given to a mealy substance which is deposited in most vegetables at the time of ripening, from the juices with which the cells of the plants are filled.

Q. What COMrON VEGETABLE especially abounds in starch?

$A$. The potato; which consists entirely of cells filled with starch and water.

Fig. 148.

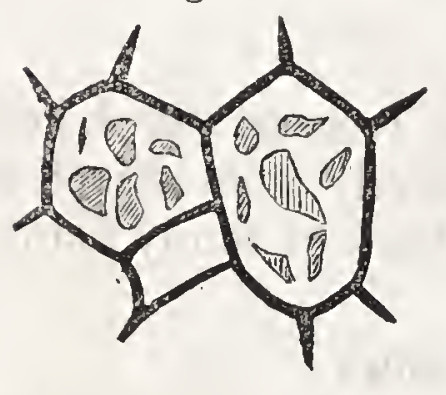

Q. When we examine starch under the microscope, what APPEARANCE does it present?

A. It is found to consist of a multitude of little transparent rounded grains or globules, giving one the idea of little eggs or seeds. The accompanying figure represents the starch globules of the potato.

Q. Why is it necessary to Borr starch before using it for stiffening linen, \&c. ?

A. The starch, consisting of little granules, is insoluble in cold vater; but when acted upon by hot water, the granules burst and allow their contents, which are soluble, to become mingled with the water. There results a kind of jelly, in which the small shreds of membranous matter composing the cell-walls float.

Starch is manufactured as follows:-

Potatoes, for example, from which most of the starch of commerce is manufactured, after being pared, are grated to a pulp. This pulp is put upon a sieve and stirred about, while, at the same time, a little stream of water is made to flow upon it. A milky liquid runs through the sieve, but the fibrous portion of the potato, the vegetable tissue, remains behind. This liquid, after a short interval, deposits a white powder, which is the starch. By the simple process of tearing up the vegetable tissue, and removing the enclosed starch by washing, this substance may be procured from a great variety of plants.

Q. Why do potatoes, beans, rice, and most of the common vegetables SWELL UP when boiled with water?

$A$. Because the starch absorbs water at the boiling temperature, which causes the cells to swell, thereby giving the vegetable a rounded appearance.

Q. What is the composition of WHEAT FLOUR?

A. Starch is one of the principal constituents of wheat flour, as well as all other kinds of meal. The other principal constituent is a gray, tough, clammy substance, called gluten.

Q. To what does PASTE, made of wheat or rye flour, owe its adhesiveness?

$A$. In some measure to the starch, but principally to the gluten contained in it.

Q. Can starch be converted into GUM and SUGAR?

A. It can. Fruits and plants effect this change naturally; we can 
also produce the change artificially, by boiling starch with a small quantity of sulphuric acid.

Q. Why are THAWED potatoes sweet?

A. Because by the freezing action the starch of the potato is in part turned into sugar.

Q. Why are apples, pears, grapes, \&c. in their UNRIPE state sour, and in their RIPE condition SWEET?

A. In the unripe fruits mentioned starch is present; in the ripe fruits it is absent; in the process of ripening the starch is changed into sugar, and the fruit, losing its sour taste, becomes sweet.

Q. Why are GREEN GOOSEbERRIES, CURRANTS, \&C., Hard ; and RIPE ones SOFT?

A. Because they contain a large number of little cells with thick walls; these become thinner from day to day, as the fruit ripens, until they break, when the fruit becomes soft.

Q. What is $G \mathrm{UM}$ ?

A. Gum is a vegetable substance, intermediate between starch and sugar. That variety of gum which is formed directly from starch is called dextrine; the gum which exudes from the bark of many plants has received the general name of resin.

Q. Why does CURRANT JUICE when boiled with sugar form a jelly?

$A$. Because the currant juice contains pectine - a gelatinous matter which abounds in many fruits. The consistence of currant and other fruit jellies is ascribed to this substance.

Q. Of what is SUGAR composed?

A. Of carbon, hydrogen, and oxygen.

The composition of sugar is identical with that of, starch and gum. These different substances are supposed to owe their different properties to a diverse arrangement of their atoms. In no other way can we account for the fact of substances having the same composition differing so greatly in their properties.

Q. Is sugar a VEGETABLE substance?

A. Yes; it is found in the juice of many plants, and in the sap of several trees; but it is extracted in the greatest abundance from the juice of the sugar-cane, which is cultivated for that purpose in the Southern States of America, the West Indies, \&c.

Q. Is sugar also an ANIMAL product?

A. It is; it exists in mill, imparting to it its sweet taste. In certain diseases of the body sugar is manufactured by the organs of the body from the food.

Q. What TWO GREAT VARIETIES of sugar are recognised?

A. Cane sugar and the sugar of grapes, or grape sugar. The first variety of sugar is obtained from the sugar-cane, the beet-root, and the sugar-maple ; the other variety is found in fruits, especially in the grape, from whence it derives the name of grape sugar.

The latter is much less sweet than the former, an ounce of cane sugar being equal, for sweetening, to two ounces and a half of grape sugar.

Q. What is the SUGAR which often gives to prunes and raisins their Write coating?

A. Grape sugar. 
Q. Does rolasses, or Treache, exist in the cane, or in the cane juice?

A. It does not, but is produced from the crystalline sugir by the presence of acids, and by the high temperature used in boiling: it is called uncrystallisable sugar.

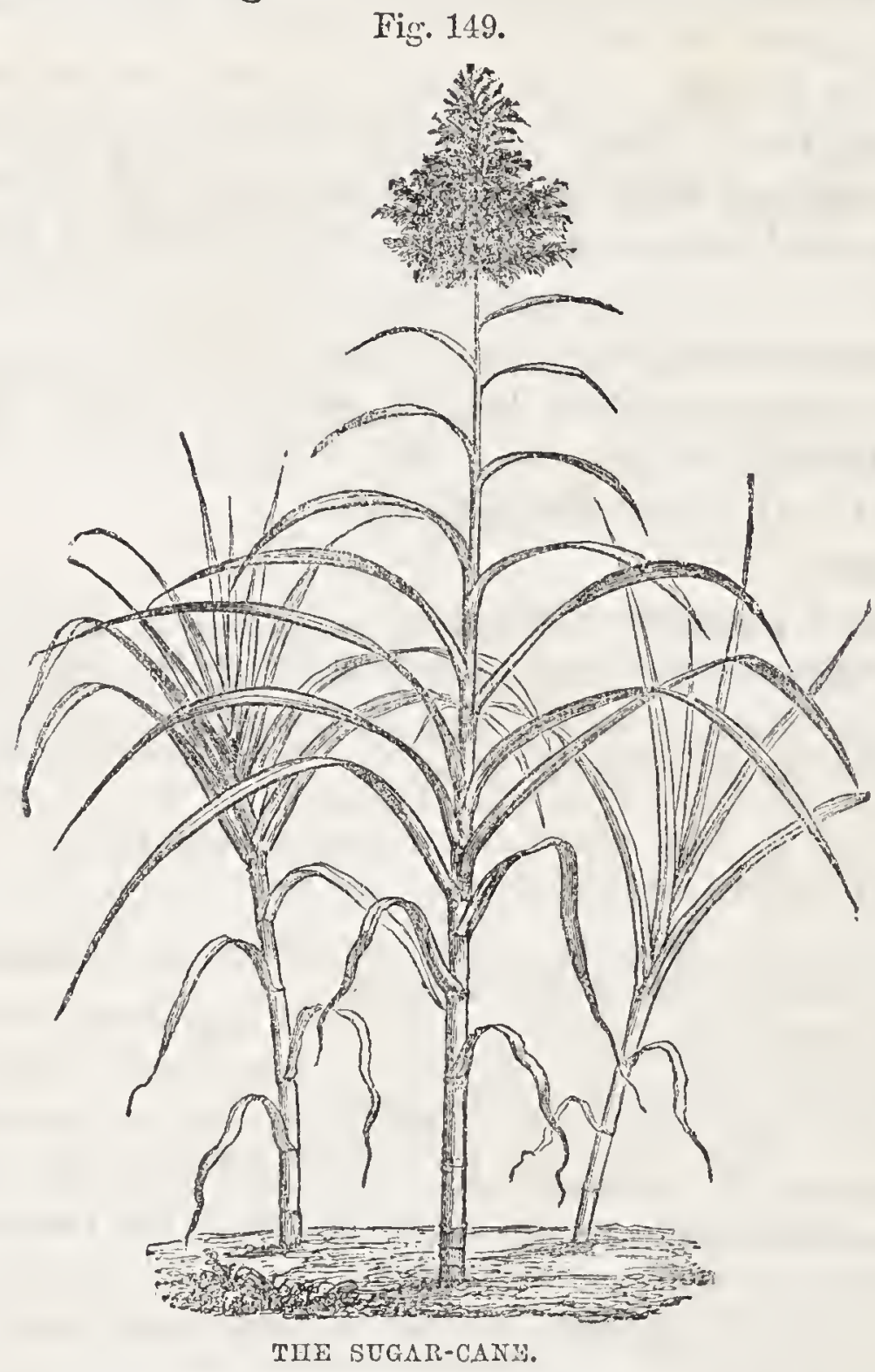

Q. What is the estimated annual production of sugar in all countries?

A. It has been estimated at four thousand five hundred and twentyseven millions of pounds.

Q. Why do the sweet juices of fruits, when boiled down, yield always a srrup, and never SOLID SUGAR?

A. Because.in the juices of fruits inorganic acids are always found, which convert the grape sugar into uncrystallisable sugar, or molasses.

Q. Do plants jroduce Acios?

A. Acids are formed in the vegetable lingdom in great abundance; they especially exist in unipe fruits, imparting to them a sour taste.

In the decay or destruction of veretable matter, acids are frequently formed which do not exist naturally in the substance of the plant from which they are clerived.

Q. Do we find in organised structures of plants a series of LOLDS and ALKALIES, or bases, answering to those in inorganic structures?

$A$. We do: the organic acids and allich are not, however, so powerfut as the inorganic. 
Q. From what sources are the organic acids mostly derived?

$A$. From the sap or juices of plants. Without being corrosive, they have a pure acid taste; and none, with the exception of oxalic acid, exert a poisonous influence.

Q. Why does tankizg hides convert them into leather?

$A$. Hides are steeped in water, with ground bark of the oak, hemlock, or other trees; these barks contain large quantities of tannic acid, which combines with the skin of animals, and forms a combination which is insoluble in water and not subject to putrefactionviz., leather.

Q. Is the process of tanning leather one requiring much Tims?

A. Some varieties of skins may be tanned in a ferv days or hours; but the best qualities of leather often remain in the vats containing the solution of bark for several months.

\section{Q. Why is VINEgar sour?}

A. Because it contains acetic acid.

Acetic, from the Saxon word ceed, "vinegar," whence also our word acid; that is, "like vinegar."

Q. If TINE, or BEER, be IMPERFECTLY CORIED, why does it rapidly turn sour?

$A$. Because air gets into the liquor, and the oxygen of the air, combining with the alcohol of the liquor, produces acetic acid, or vinegar.

Q. From THAT SOURCE are the vegetable or organic alkalies, or bases, DERIVED?

$A$. Certain vegetable substances, by their singularly bitter taste and remarkable effects upon the human system, have, from a very early period, attracted attention, and have been used as valuable remedies. With the progress of chemistry it was found that the whole of these did not possess the same useful qualities, but that the greater part of them consisted of useless substances, such as woody fibre, resin, gum, \&c., while the peculiar active constituents form only a small part of their weight.

Q. Why are these extractive substances called the ORG $\Lambda$ NIC ALRALOIDS ?

A. Because they resemble the well-known allati ammonia in many respects, and combine, like this body, with acids to form salts.

Q. To what does Peruviafi bark owe its efficacious qualities?

A. To the presence in it of a vegetable alkaloid-quinine.

Q. What is Morphis, or MorpHine?

A. It is the allialoid or active principle contained in opium.

Q. What are some of the PRINCIPAL ORGANIO ALEALOIDS?

A. Quinine, extracted from Peruvian bark; morpline, extracted from opium; strychnine, extracted from several poisonous fruits and barks of trees growing in South America, particularly the mux vomica, from which it is usually prepared; coneine, prepared from the hemlock; aconitine; nicotine, the poisonous principle of tobacco; veratryne; and many others less known. 
Q. What are the Genersu Properties of these substances?

A. They are colourless and odourless, but of extremely bitter taste. In water they are insoluble, but on the other hand they are snluble in alcohol and ether; even in very small doses they produce remarkable effects upon the animal system, most of them being powerful and active poisons.

Q. For what PURPoses are the organic alkaloids USED?

$A$. 'They are employed exclusively in medicine, in which they are of the greatest importance.

Thus formerly, in ague, and other diseases of like character, it was necessary to take many ounces of "bark" to effect a cure ; but now it is only required to take a few grains of quinine to eradicate the same disease. By using morphine, we obtain the soothing effects of opium, and at the same time avoid the deleterious effects of the other active principles which this drug contains.

Q. What is TEA?

A. Tea is the dried leaves of a plant which flourishes in China and Japan.

Q. When was tea FIRST INTRoducEd into England?

A. Tea was first introduced into England in 1610, by a Dutch merchant, who obtained it from the Chinese in exchange for dried sage.

Q. To what do tea and coffee owe their PECULIAR PRoperties?

A. Principally to the presence of two organic alkuloids contained in them-" theine" and "caffeine."

Q. Why do STRONG tea and coffee produce WAKEFULNESS?

A. Chiefly owing to the influence produced upon the nervous system by the allaloids which they contain.

Q. What is it that imparts to tea and coffee their PECULIAR A RoMA and FLAVOUR?

A. A volatile oil which is extracted from the tea and coffee by the hot water.

Q. Is the use of tea and coffee INJURIous or BENEFICIAL to the system?

$A$. When used in moderate quantities these beverages produce a beneficial effect upon the human system.

The effect of these beverages may be further explained as follows:-It is known that the animal body, while living, undergocs constant decay and renovation : the labours of life waste it; the food introduced into the stomach renews it; that which is wasted passes off by the lungs, or other excretory organs, and is rejected from the body of the animal. Now, the introduction into the stomach of a minute proportion of the regetable active principle, theine or caffeine (three or four grains per day), has the remarkable effect of sensibly diminishing the absolute quantity of wasted matter rejected daily from the system of a hcalthy pcrson, living on the same kind of food, and engaged in the same occupation, under the same circumstances. In other words, by using tea and coffee containing theine and caffeine, the waste of the body is diminishcd; and if the waste of the body is lessened, the necessity of food to repair it will be lessened in an equal proportion. By using tea and coffee, the health and strength of the body will be maintained in an equal degree upon a smaller quantity of ordinary food. These beverages, therefore, save food, or are substitutes for it, while, at the same time, they soothe the nervous system, and consequently act upon the mind.

Q. Why do the OrD and INFIR instinctively delight in Tes?

$A$. In an old person the system is enfeebled and worn out, and the stomach no longer digests enough of ordinary food to make up for the 
natural daily waste of the bodily substance. The size and weight of the body, therefore, begin to diminish more or less perceptibly. At this period, tea acts as a medicine to arrest the waste, to keep the body from falling away so fast, and thus enable the less energetic powers of digestion still to supply as much as is needed to repair the waste of the tissues.

Q. What is it that imparts to tea its PEcuLrar properties and taste?

A. The presence of tannic acid-the same acid which is present in oak and hemlock barks.

Q. Why is the coffee-bean VALUED in proportion to its $\triangle G E$ ?

$A$. Because the coffee ripens, as it were, by keeping; so that from old coffee there is a larger amount of the volatile oil obtained upon which the aroma of the infusion depends.

Q. Does this volatile oil exist as such in the GREEN COFFEE?

$A$. It does not, but is formed during the operation of roasting.

Q. What nute should be observed in the roasting of coffee?

$A$. The aroma of coffee is most agreeable when the heat to which it is exposed is not greater than is sufficient to impart a light-brown colour to the bean. When the roasting is carried too far, a disagreeable odour gradually mingles with the aroma, and lessens the value of the product.

Q. Why do some WATERS give a stronger and BETTER-FLAVOURED coffee than others?

A. Because they contain a slight quantity of alkali; hence it is recommended, to obtain a more uniformly strong and well-flavoured coffee, to add a little soda to the water with which the infusion is made.

The quantity of alkali (carbonate of soda) proper to be used for about two quarts of water, should not exceed the amount which can be taken up on the end of a penknife blade.

Q. What are the coco.As?

$A$. The beverages known as cocoas are prepared from certain oily seeds, which are first ground to a pulp by passing them between hot rollers, and for immediate use are diffused through hot water.

Q. What is CHOCOLATE?

$A$. 'The cocoa-bean ground to a fine paste, mixed with sugar', and flavoured with vanilla or other substances.

Q. What PECULIAR VEGETABLE PRoDUCT characterises the cocoas from tea or coffee?

A. The cocoas, like tea and coffee, contain an aromatic oil, and an active principle resembling theine and caffeine, namely, theobromine; but in addition to these they contain an abundant fatty substance known as cococt-butter, amounting to more than one-hait the weight of the bean. It is this substance which renders chocolate and the cocoas so nutritious and so rich an article of food.

Q. What oTHER important substances do we find in plants besides woody tissue, starch, gum, sugar, and the organic acids and alkalies?

A. Substances which contain nitrogen and sulphur, and which 
exercise neither a medicinal nor poisonous action upon the animal system, but are nourishing and healthiful.

Q. What Naxis have been given to these substances?

A. Vegetable albumen, which is particularly abundant in plants used in cookery, and in oily seeds, as flax-seed, almonds, poppyseeds, \&c.; vegetable caseine, which exists in large quantities in leguminous plants, as peas, beans, \&c.; and gluten, which is found in flour and meal.

Fig. 150.

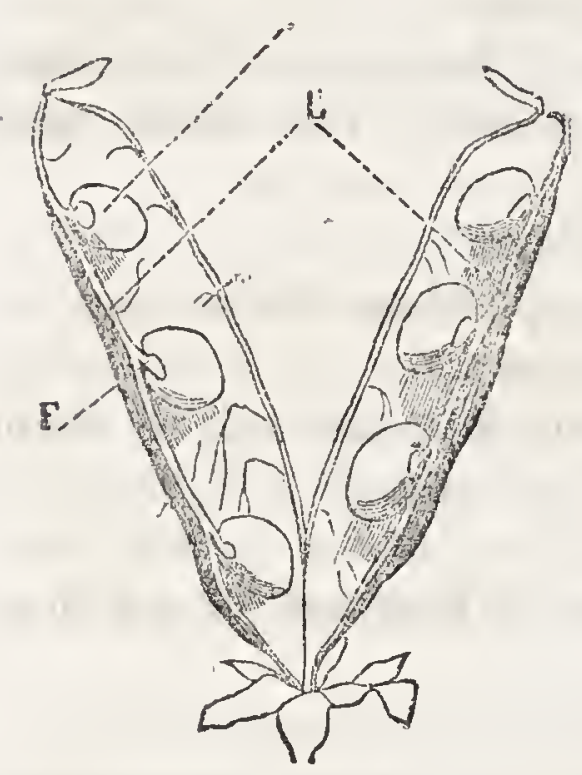

Q. What do you mean by LEGUnirous plants?

A. Those which have their seeds contained in a pod or vessel, arranged in the same manner as the seeds of peas or beans. The pea (see fig. 150) and bean are considered types of leguminous plants.

Q. What are the DISTINCTIV PRopenties of the vegetible substances which contain nitrogen and sulphur?

A. Being of a more complex nature than the other vegetable principles, they are especially liable to decomposition, fermentation, and decay. If, while in the act of decomposing, they come in contact with other organic substances, they cause these also to decay.

Q. Do alu vegetables contain one or more of these principles?

A. All vegetables contain, though not always in great quantity, one or more of these substances. Their office is undoubtedly to promote the growth and nourishment of the plant.

\section{CHAPTER IIV.}

\section{FERMENTATION, PUTREFACTION, AND DECAY.}

Q. What do we mean by the terms FEMIFNTATON, PUTREFACTION, and DECAX?

A. We mean those changes in forin and properties which compound organic bodies undergo when separated from the organism which prodnced them, and exposed to the infuence of moisture and a certain temperature.

Q. What are peculiarities of the process of decay?

A. Decay cannot take place without air, the oxygen of which is taken up by decaying bodies. Hence it is a process of slow combustion, in which heat is uniformly given forth, and sometimes even light. 


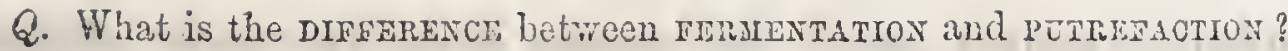

A. Fermentation is a change effected in the elements oj a borly composed of carbon, oxygen, and hydrogen, without nitrogen. Putrefaction is a change effected in the elements of a body composed of carbon, oxygen, hydrogen, and nitrogen.

Fermentation cannot take place, however, unless some decomposing body containing nitrogen is present. In fermentiotion, also, gaseous products are evolved which are destitute of odour ; in putrefaction, the gases which are emitted possess a nost offersive smcll.

Q. Cider and wine, when first made, are sweEr : why do they, in the course of tirne, BECOXIF SOUR?

A. All sweet vegetable juices abound in sugar and some substance containing nitrogen. This latter, becoming decomposed, acts as a ferment or agent, which induces decomposition in the sugar, and causes it to pass first into alcohol, and finally into vinegar.

Q. What is a FERAENT?

A. A ferment is a substance containing nitrogen in a state of decomposition, which is able to excite fermentation in solutions of sugar; old cheese, putrefying flesh, blood, \&c., are all of them ferments.

Q. What is YRAST ?

A. We apply the term yeast to a species of ferment, as the foan. of beer, produced by fermentation.

Q. Why is YEAST used in BREWTNa?

A. Because it consists of a nitrogenous substance (gluten) causing putrefuction, in a state which possesses the power of fermentation.

Q. Can you explain why it is, that a body in a state of fermentation or putrefiction should cause mulimited quantities of similar matter to pass into the SAME STATE?

A. We only know the fact: of the reason we are ignorant. The most minute portion of milk, paste, juice of grapes, flesh, or blood, in a state of fermentation or putrefaction, causes fresh milk, paste, grape juice, flesh, or blood to pass into the same condition, when in contac with them.

Q. In storing or packing fruit for future use, why is it necessary to carefully REMOVI every DECAYED SPECINEN?

A. Because the decayed portions of one specimen will quickly communicate decay to the fresh fruit in contact with it, and soon the whole mass of fruit will become roiten.

Q. If in a ressel, or any other structure, one tinber becomes decayed, what counss ought to be adopted?

A. It should be removed immediately, or the decomposition once commenced, will in time affect the whole structure.

Q. It sometimes happens that physicians, in dissection, are seriously poisoned by the slightest cut of a knife which has been used upon the dead body: wir is this?

A. The knife introduces to the healthy blood, through the wound, a minute portion of matter in the state of decomposition or putrefaction. This acts as a ferment, and causes the healthy matter in contact with it to pass into the same decomposed state. The action once com- 
menced, rapidly extends, until the whole body becomes affected, and death ensues. It is almost impossible to heal wounds of this character.

Q. Why is it ESPECIALLY DANGERous to eat fruit or meats PARTIALLY DECAYED?

$A$. Because the decayed portions of the substance eaten are liable to induce the same condition in the healthy organs of the stomach with which they may come in contact. Ferments of any kind appear to reproduce themselves, just as seeds reproduce seeds.

Q. What is vinous FERMENTATION?

$A$. The action or change by which sugar becomes converted into alcohol (spirit).

Q. When solutions of sugar are caused to ferment, what NEW CoMpounds are formed? A. Alcohol and carbonic acid. The alcohol is still further changed (unless the process be checked) into acetic acid or vinegar.

Q. What BECONES of the ALCOHOL which is thus formed by fermentation?

$A$. It mixes with the water, and forms the intoxicating part of the liquor produced, as beer, wines, \&c.

Q. What BECONiES of the CARBONIC ACID which is generated by fermentation?

$A$. It makes its escape into the air.

Q. What is ALCorror?

A. The spirit existing in wine, beer, cider, \&c., obtained in the process of fermentation.

Q. Of what is $\Delta \mathrm{LCOHOL}$ composed?

A. 4 parts are carbon, 2 oxygen, and 6 hydrogen.

Q. What is the oriarn of the term Proor SPIrIT?

$A$. It is derived from the old method of testing spirit, which was this: The spirit to be tested was poured over gunpowder, and ignited; if the powder exploded, the spirit was said to be above proof; it it did not explode, it was said to be below proof.

Q. What is meant at the PRESENT DAY by spirit ABOVE and BELOW PROOF?

$A$. If we say that spirit is ten over proof, we mean that one hundred gallons of it will require ten gallons of water to reduce the spirit to proof strength. So on the other hand, if we say that spirit is ten under proof, we mean that ten gallons of water must be taken from the spirit to raise it to proof strength.

The strength of spirit is now tested by the hydrometer.

Q. In what STATE does alcohol exist in wine, beer, cider, \&cc.?

$A$. It exists in wine, beer, cider, and other fermented liquors combined with a large quantity of water and some oily and other organic substances.

Q. What PROPORTION of alcohol generally exists in cider?

A. About nine per cent.; it thus resembles in strength the weak hock wines, or common ale.

Q. How is WINE MANUFACTURED?

$A$. The grapes are collected and pressed; the juice, which is called 
must, is poured into vats situated in cellars, where, as the temperature is low, the fermentation proceeds so slowly, that it is not completed until after some months. During the fermentation the impurities rise to the surface in the form of froth or yeast, or settle to the bottom of the vats (lees), so that the pure wine is finally drawn off clear, ready for use. tion?

Q. Why is it NoT needful to put YEAST into GRAPE juice in order to produce fermenta-

$A$. Because grape juice contains a sufficient quantity of a nitroyenised substance (like yeast) to produce fermentation.

Nitrogenised-that is, containing nitrogen.

Fermentation cannot occur unless the sugar be dissolved in a sufficient quantity of water.

Q. Why are some varieties of wine RED, and others YkLLOW, or colourless?

$A$. In the manufacture of red and coloured wines, the purple grapes are bruised, and then fermented, together with their skins and stalks; a red colouring matter is extracted from the skins, and tannic acid from the seeds and stalks, the last imparting to the wine a peculiar taste. In the colourless wines, the grapes from which they are manufactured are often colourless, and the pure juice is alone preserved and fermented.

Q. How is SPARKuing wine (Champagne) manufactured?

$A$. The fermentation is allowed to proceed in corked-up bottles, whereby the carbonic acid formed is retained in the wine.

The wines produced in Germany-the hock, \&c.-are possessed of a peculiar flavour, called the bouquet, and a sour taste ; while the wines of Southern Europe are sweet to the taste, but wanting in flavour. The cause of this difference has been explained as follows:-

The grapes growing in northern countries, as in Germany, contain so much nitrogenous matter (which produces the fermentation), that all the sugar is converted into alcohol, and a flavouring substance, called cenanthic ether, is also generated. In southern countries, in consequence of a higher temperature, the grapes are richer in sugar, but poorer in nitri)genous compounds. The latter not being present in sufficient quantity to decompose all the sugar, a part remains, and renders the wine sweet. Neither is there any of the peculiar flavouring material generated in these wines.

Q. How does the manufacture of strong beer and whisky DIFrer from that of wine?

$A$. Wine is manufactured from materials which contain sugar already formed, but the materials from which beer and whisky are produced contain no sugar in the outset, but starch.

Q. What are the MIATERIALS from which beer and whisky are manufactured?

A. Wheat, rye, potatoes, corn, barley, or any similar vegetable products,

Q. Can we manufacture alcohol by fermentation directly from STARCH ?

$A$. We cannot; it is necessary to first convert the starch into sugar, and from this we can produce alcohol by fermentation. This in the case of beer is effected by converting the barley used into what is called malt. 
Q. How is BARLET converted into MALT?

A. It is moistened with water, and heaped up; by which means great heat is produced, which makes the barley sprout.

Q. Whis is BARLEY MraLYed?

A. Because germination is produced by the artificial heat; and in germination the starch of the grain is converted into sugar.

Q. How is starch converted into sugar in the process of fermentation?

$A$. When seeds, and especially barley, begin to germinate, there is formen a peculiar nitrogenised substance called diastase, which possesses the remarkable property of converting starch into sugar. It is through the agency of diastase that the change takes place.

$Q$. Is starch converted AT oxcu into sugar?

A. No; it first becomes gum, and then changes to sugar.

Q. How is BARLIIY PREVENTED from Growing in the process of MALTING?

A. It is put into a kiln as soon as it sprouts, and the heat of the kiln checks or destroys the young shoot. The product as it comes from the kiln is called "malt."

Q. Why is not the BARLET suffered to Grow as well as SPRour?

A. Because plants in the germ contain more sugar than in any other state; as soon us the germ puts forth shoots, the sugar of the plant is consumed to support the shoot.

Q. How is raLT converted into BER?

A. It is mixed with water, and a sweet solution or infusion obtained, which is called wort. To the wort yeast is added, which produces fermentation, the result of the fermentation being a clear liquid, which we call beer.

Q. Does MALI contain GIUTEN?

A. Yes; the infusion of malt, called "sweet-wort," contains an abundance of gluten, and the yeast (which converts its sugar into alcohol) converts this gluten into yeast.

Q. Why are rops alded to beer during the process of manufacture?

A. Hops give to beer its peculiar bititer taste, and also impart body to it.

Q. How is whisky manufictured from corn, rye, or potatoes?

A. The grain or potatoes, boiled or mashed, are mixed with a portion of barley-malt and hot water, to form a paste, which is kept for a time at a high temperature. The diastase of the malt, acting upon the starch, converts it into sugar. To this mass yeast is added, and the whole left to ferment. When this fermentation is finished, the mass is placed in a still, and the spirituous principle distilled over by heat. I'his product is whisky.

Q. What is BRANDY?

A. Brandy is the product obtained from the distillation of wine. In wine countries, the lees remaining in the vats after the wine is 
drawn off are generally used for this purpose, as they retain mechanically a large quantity of wine.

Q. To WHAT does brandy owe its peculiar DARK BRown or red colour?

A. To burnt sugar, which is added for the purpose of imparting colour.

Q. How aUCH ALCOHOL is contained in strong brandy, rum, and whisky?

A. From sixty to seventy per cent.

Q. When rum, brandy, or whisky are distilled, what is the Producr?

A. A stronger liquor, called "spirits of wine," containing a larger proportion of alcohol.

Q. What is the PRODUCT obtained by distilling SPIRITS of WINE?

$A$. It is called rectified spirits of wine, and contains from eighty to ninety per cent. of alcohol, and the balance water.

Q. When the water is entirely separated from spirits of wine, wHAT is the Producr?

A. Pure alcohol, called anfydrous, or absolute alcohol.

It is difficult to remove all the water from alcohol; this can only be accomplished by mixing with it quicklime, or some other substance having a strong attraction for water, which absorbs it, and suffers the alcohol to distil over pure.

Q. What is Proof SPIRIT?

A. A mixture of equal volumes of rectified spirits of wine and water.

Q. What are CoRDILLS?

$A$. Cordials, for the most part, are made of $b$ randy, flavoured with what are called the essential oits of various plants, and afterwards sweetened.

Q. To WHAT does whisky owe its peculiar ODOUR?

A. To the presence of a volatile oily substance called fusel oil, formed from the potatoes or grain during the process of fermentation.

Q. Is fusel oil porsonous?

$A$. It is a very deadly poison, especially when inhaled into the system as vapour. It often exists in chloroform, and is the cause of the accidents which have sometimes happened on respiring the vapour of this agent.

Q. How are the FuATOURING Mrateriass, resembling the flavour of the APple, the PEAR, the ORANGE, \&c., which have been used in confectionery for flavouring the so-called "fruit-drops," and for other purposes, prepared ?

A. By mixing fusel oit with an acid, and distilling the product. A volatile substance is obtained, which dissolved in alcohol, constitutes these celebrated flavouring materials. By using a different acid, a different flavour is obtained. Thus, acetic acid gives the flavour of the pear; valerianic acid, the flavour of the apple; nitric acid, the flavour of the orange, \&c.

Q. Are these flavouring materials porsonous?

$A$. They are not, when used in proper quantities. Ordinary confectionery, properly favoured with them, is harmless; and there is reason to believe that these products, which we form artificially in 
the manner described, are identical with those which nature elaborates in the fruits themselves.

Q. Is alcohol a porson?

$A$. Strong alcohol acts as a poison, producing sudden death when swaliowed; when diluted with water, as in the ordinary spirituous liquors, it produces drunkenness and disease.

Q. What is ETHER?

$A$. Ether is a product obtained by distilling strong alcohol, and suipinuric acid. The product is called sulphuric ether, but it does not contain sulphuric acid, nor has it any sulphur in its composition.

Q. What are the PROPERTIES of ether?

$A$. It is an exceedingly light and inflammable body, producing insensibility when inhaled, and readily dissolving all fatty and oily bodies.

Q. Why will ether remove 'spors of oIL, PAINT, or GREASE from garments?

A. Because it dissolves all greasy, oily matters.

Q. What is VINEGAR?

A. Vinegar is a product formed from alcohol.

Q. What is ordinary TABLE VINEGAR?

$A$. An acid, called acetic acid, and water.

Q. How can brandy and wine be CHANGED into vINEGAR?

$A$. By adding yeust or some other substance which will produce fermentation, and exposing the mixture to the air at an elevated temperature. The alcohol gradually disappears, and, by combining with oxygen, is converted into vinegar.

Q. How may vinegar be made sTronger by FREEZING ?

$A$. When vinegar is exposed to the cold the water contained in it is frozen before the acetic acid; by separating the ice, we leave a stronyer solution of the acid behind.

Q. Why do fruit preserves frequently turn sour?

$A$. Because, owing to the action of some fermenting substance present either in the fruits themselves or in the air, the sugar used in preserving is converted into alcohol, and the alcohol into vinegar.

Q. Why does the housewife scald her preserved fruits to prevent their turning sour ?

$A$. Because fermenting substances and fermenting action are destroyed by a boiling temperature.

Q. Why is the FROTH on a jar of preserves an indication that FERMENTATION is going on?

$A$. The froth is occasioned by little bubbles of carbonic acid gas enveloped in the syrup; and the carbonic acid gas could only be present through the agency of fermentation.

Q. Why do we keep preserves, beer, cider, or other substances liable to turn sour, in a coou place?

A. Because as the temperature gets colder, it arrests fermentation, though it does not prevent its renewal when the temperature is increased. 
Q. Why does milk turn sour by KeEPING?

A. Because it undergoes a fermentation, during which "lactic acid" is formed, which turns the milk sour.

The lactic acid is formed from the sugar of milk by fermentation.

Q. Why does sour MILK OURDLE?

A. Milk consists of five ingredients: 1 , caseine, or curd; 2 , butter; 3 , sugar; 4, water; 5 , certain salts. The caseine, or curd of sweet 'milk, is like the white of an egg before it is hoiled; but the caseine, or curd of sour milk, is like the white of an egg after it is boiled. This caseine, or curd of milk, is thickened by acids. When milk is sour, the lactic acid of the sour milk, mixing with the caseine, thickens it; in consequence of which it separates from the water, and becomes an insoluble mass; or, in other words, the milk curdles.

Q. What is BREAD?

A. It is a kind of food prepared generally from the flour of wheat mixed to a dough with water, and submitted to the action of heat to bake. This kind of bread is called unfermented or unleavened bread.

Q. What is LEAVENED BREAD ?

$A$. It is flour mixed to a dough with water, to which is added a little leaven (or dough which has been fermented) or yeast.

Q. What EFFECr has the YEAST on the dough?

$A$. It assists in the fermentation of the dough, by which means carbonic acid is generated in the mass, and makes the bread porous and light. It is then placed in the oven, and the gas expanding by heat, raises the dough still more.

Q. Why does BAKING dough convert it into BREAD?

$A$. When dough formed of flour is baked, its starch is changed into a gum called dextrine.

A similar change is produced upon the farinaceous portion of the dough. The yeast (added to the dough) converts part of the starch and sugar into alcohol and carbonic acid; of these, the alcolol evaporates in the oven, and the carbonic acid forces the dough into bubbles, and, in its effort to escape, renders the bread light and full of holes.

Dextrine is a gummy matter, similar to that which composes the cells of wood (called cellulin), only it is soluble in cold water.

Q. Why is UNBOLTED FLOUR or meal more nutritious than the fine flour or meal ?

A. By bolting flour or meal, we separate the bran and those portions of the grain adjacent to it. These parts of the grain abound in nitrogen, and the nutritive value of food depends greatly upon the quantity of nitrogen it contains. flour?

Q. Why is unbolted flour or meal LESS DIGESTIBLE, though more nutritive, than bolted

$A$. Because the bran contains a large quantity of gluten, which is not easily soluble in water, and is difficult of digestion.

Q. Why is bread made of unbolted flour apt to be uEAvy and "SLACK BAKED?"

$A$. Because unbolted flour contains much gluten, which retains the water obstinately, and by its tenacity hinders the escape of the bubles of gas. 
Q. What substance is it that gives to dough its great nENACITY?

A. The gluten of the flour. About ten pounds of gluten are contained in every hundred pounds of fine flour.

Q. HoW MUCH WATER is contained in well-baked wheaten bread?

A. About forty-five per cent., or nearly one-half the weight of fresh bread, is water.

Q. Why does bread, a few days after baking, become STALE?

A. The change is merely in the internal arrangement of the particles of the bread.

It is generally supposed that the change which takes place in bread a few days after baking, whereby it becomes crumbly and dry, is occasioned by the gradual loss of water. This, however, is not the case : stale bread contains almost exactly the same proportion of water as new bread after it has become completely cold.

Q. What NeW COMPOUNDS are produced by the change called PUTREFACrIon?

$A$. The carbon, oxygen, hydrogen, and nitrogen of the original substance (being separated by decomposition) reunite in the following manner: 1. Carbon and oxygen unite to form carbonic acid; 2 . Oxygen and hydrogen unite to form water; and, 3. Hydrogen and nitrogen unite to form ammonia.

When bodies containing sulphur and phosphorus putrefy, the sulphur and phosphorus unite with hydrogen, and form sulphuretted and phosphuretted hydrogen gases.

Q. What BECOMES of these several products of putrefaction?

$A$. They are all elastic bodies, and escape into the air.

Water is elastic and gaseous when in the condition of vapour.

Q. What is the CAUSE of the OFFENSIVE SMELL which issues from putrefying bodies?

$A$. 'The evolution of ammonia, or of sulphuretted and phosphuretted hydrogen gases, all of which have pungent and offensive smells.

Q. Decaying vegetables are first of a brownish tint : why do they afterwards turn of a BLACKISII colour?

$A$. Because the hydrogen of the decaying vegetables is separated from the mass by the process of decay, and leaves a larger proportion of carbon behind.

Q. Why do BRICks turn GREeN on being exposed for some time to the weather, especially if deprived of the rays of the sun?

A. The "green" is a moss or lichen which grows on the bricks, and thrives better in the shade than in the sun. The seeds of these lichens are supposed to be scattered by the winds.

Q. Why does UNSEASONED WOOD DECAY much more RAPIDLY than wood well seasoned?

$A$. Because the albumen which the sap contains produces a species of fermentation, during which the cellular tissue and woody matter are converted into carbonic acid and water.

Q. Why will solutions of salts PREVINT the DECAY of wood steeped therein ?

A. Because the salts unite with the albumen of the sap, coagulate it, and prevent fermentation.

Q. Why is WOoD placed in a stream of running WATER to SEASOI it?

$A$. Because the running water washes away the sap, and thus prevents fermentation and decay. 
Q. Why do we pack eggs for preservation in LIME-TITER, SALT, SAWDUST, \&C. ?

A. In order to exclucle them from the action of the air. The air penetrates the egg through numerous minute holes or pores of the shell, by means of which it is conveyed to the young bird during the process of hatching. If these pores be closed up, by rubbing the new-laid egg with fat, or in any similar way, it will keep fresh for a long time.

Q. Why can animal and veretable substances be preserved by immersion in $\Delta$ rcoHoI (spirits of wine)?

A. Because the alcohol extracts the vatery fluicls contained in the substance, and prevents the access of atmospheric oxygen.

Q. What mHRE CoNDITIONS are requisite to produce putrefaction in animal and regetable substances?

$A$. It is necessary that they should be exposed to the combined influence of air, heat, and moisture.

Q. Why is a substance preserved from decry by DRYING, or by the EXCLUSIon of AIB from it?

A. Because by so doing we remove the moisture and air essential to the process of decay.

Q. Why does the sironING of FISH or FuEsI contribute to their preservation?

A. Because the volatile matters of the smoke, such as creosote, pyroligneous acid, and the like, effect a species of chemical combination with the fibre of the meat, and with the substances contained in the natural juices of the flesh, which combinations are less liable to decay than the substances themselves.

Q. What are the oHEMICA SUBSTANCES which thus retard decomposition called ?

A. Antiseptics.

Q. What are the best $\triangle$ GENTS for depriving putrid and decaying animal and regetable substances of their offensive odours?

A. Chloride of lime is the most effectual agent; and chloride of zinc and sulphate of iron (green vitriol) are also exceedingly efficient. On a large scale, as in the sanitary cleansing of towns, pulverised charcoal, burnt clay, and quicklime are to be recommended.

Q. What EFFECT does the use of perfumes or the burning of pastiles have upon offensive odours?

A. They merely disguise the odour, but do not remove or destroy it.

Q. By adopting what precautions may a person safely enter sick rooms, or visit, with. out risk, the most dangerous receptacles of filth?

A. By moistening a linen cloth with vinegar, and sprinkling over it finely-powdered chloride of lime. Air breathed through this, applied to the mouth and nostrils, will enter the lungs charged with a minute quantity of chlorine, which will effectually destroy any noxious vapours or miasms that escape from diseased bodies, or from decaying animal and vegetable subritunces. 


\section{CHAPTER L V.}

\section{ANIMAL ORGANISATION AND PRODUCTIONS.}

Q. What CHIEFLY Distinguishes the life of an animal from the life of a regetable?

$A$. During the life of the animal oxygen is incessantly inhaled, or drawn in; but during the life of the vegetable it is exhaled, or sent forth.

The food of all animals, in all circumstances, consists, with the exception of water and some salts, of organised matter; the food of vegetables is inorganic matter. Animals are distinguished from vegetables by the faculty of self-motion, and in general by the possession of senses.

2. Is the elementary matter from which the organs of the animal body are formed, the SAME as that which occurs in the vegetable kingdom?

A. It is exactly the same; namely, oxygen, hydrogen, carbon, nitrogen, sulphur, phosphorus, and chlorine; and the metallic substances, calcium, potassium, sodium, and iron. These must be introduced into the animal body in order that it may grow and live. The chief mass of vegetable matter consists of substances wanting in nitrogen, while animal substances particularly abound in this element.

Q. What is ALBUMEN?

A. Albumen is an animal substance as well as vegetable. It exists most abundantly, and in its purest natural state, in the white of an $\mathrm{egg}$, from whence it derives its name (album ovi), which is the Latin for the white of an egg.

The serum, or fluid portion of the blood (which, after exposure to the air, is separated from the more solid part), the vitreous and crystalline humours of the eye, the brain, spinal marrow, and nerves, all contain albumen.

Q. What is the rouk of an egg?

$A$. This also consists of albumen, but contains in addition a yellow oil, which imparts to it its colour. this.

Q. Why will MILK BURN very easily when boiled? water will not do so: EXPLAIN

A. 1. Because milk contains solid organic substances capable of burning, which water does not; and

2. Because the heat of the fire curdles the albumen of the milk, which falls to the bottom, and adheres to the boiler.

Q. Why are IAMB and VEAL more TENDER than beef and mutton?

$A$. Because they contain more albumen, and less muscular fibre.

Q. Why do LAMB and VEAL TAINT more quickly than beef and mutton?

$A$. Because they contain a large quantity of albumen, which is very liable to putrefaction.

Q. Why is meat TOUGH which has been BOILED TOO LONG ?

$A$. Because the albumen becomes hard, like the white of a hardboiled egg.

The best way of boiling meat to make it tender is this : Put your joint in very brisk 
boiling water; after a few minutes add a little cold water. The boiling water will fix the albumen, which will prevent the water from soaking into the meat, keep all its juices in, and prevent the muscular fibre from contracting. The addition of cold water will secure the cooking of the inside of the meat, as well as of the surface.

Q. Why is meat always rougr if it be put into the boiler before the water boils?

$A$. Because the water is not hot enough to thicken the albumen between the muscular fibres of the meat, which therefore runs into the water, and rises to the surface as scum.

Q. Why is the flesh of ond animals rovar?

A. Because it contains very little albumen, and much muscular fibre.

Q. Is SALTED meat as NUTRITIOUS as fresh meat?

A. No; because the albumen of the meat is sepurated from the flesh by the brine, as well as the alkaline phosphates, and some other substances of great value.

Alkaline phosphates are phosphoric acid combined with some alkali, such as soda, potash, magnesia, \&c.

Q. Why does SALT PRESERVE meat?

A. 1. Because it removes the water contained in the animal fibre, absorbing it, and leaving the meat dry;

2. Brine draws away the albumen from between the muscular fibres, which is especially subject to putrefaction;

3. The salt unites with the muscular fibre, and makes a new chemical compound much less subject to decay; and

4. It keeps the air, flies, \&c., from the meat.

Q. Fresh flesh, over which salt has been strewed, is found, after twenty-four hours, swimming in brine, though not a drop of water has been added: WHERE has the water come from?

$A$. The water has been yielded by the muscular fibre itself; and having dissolved the salt in immediate contact with it, and thereby lost the power of penetrating animal substances, it has on this account separated from the flesh.

Q. Why is water, containing much salt in solution, INCAPABLE of being ABSORBED by the tissues of the body?

A. On account of a remarkable property possessed by all animal membranes, skin, and tissues-namely, an inability of being soaked by any concentrated solutions of salt. It is only when these solutions are diluted to a great extent with water, that they are absorbed by animal tissues.

Q. Why is a sensation of THIRST excited by eating food containing much salt?

$A$. Because the salt extracts water from the stomach, and retains it. Hence a strong desire for water to restore that which has been taken from the system is experienced.

Q. Why does not the water of the ocean, when drank, ALLAY THIRST?

A. Because it is a solution of salt, and when drank absorts water from the system instearl of imparting any to it. 
Q. Why does the use of SALT LEEF produce Scurvy?

A. Because the soluble salts are removed from the beef by brine; in consequence of which it cannot restore to the human system those salts which are essential to meserve the blood in a healthy state.

Scurvy is a diseased state of the blood.

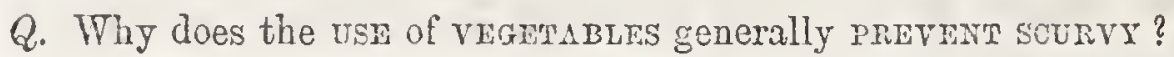

A. Because they contain the solutle salts removed from the beef by brine, which, being restored by the vegetables, preserve the blood in a healthy state.

Q. What is FiBring?

A. It is a componnd which abounds in both animal and vegetable substances; - the chief part of muscular flesh is formed of fibrine. It also exists in chyle, and enters into the composition of the blood.

Q. What is CASExNE?

A. It exists in mill: and constitutes the greater part of cheese made som skimmed mill.

Q. Does CASEINE exist also in VEgetabLES?

A. It is found in peas, beans, \&c. When these vegetables are crushed, mixed with water, and sirained, caseine is separated, which has all the characteristics of skimmed milk.

Q. What is GELATINE?

A. It is a jelly-like substance, formed by boiling animal membranes, skin, and bones. The well-known substance called isinglass and also calves'foot jelly are familiar examples of gelatine. Glue is a lind of gelatine dried in the air.

Q. What proporion of the beef we eat is somin substance?

A. Only about one-fourth. If a piece of beef be thoroughly dried, either in the sunshine or over boiling water, it will be so far diminished in bulk that four pounds will afford but one of dried flesh.

Q. What is the MAIN CONSTrident of dried flesh?

A. Fitrine : this substance closely resembles the gluten of plants in composition and properties, so that in comparing animal and regetable food, we may consider them as identical.

Q. What is the chemical DIFFERENGE betwcen DEEF and BRIAD?

A. Flesh does not contain starch, which is so large an ingrectient in vegetable products, and the proportion of fibrine in beef is about three times greater than it is in bread. Fibrine and gluten being the principal nutritious substances of beef and bread, it therefore follows that a pound of beefsteak is as nutritive as three pounds of bread. Lean fresh beef contains more water also than is found in an equal weight of biead.

Q. What is the ReLatrve Iv UTrimive Value of the different kinds of meat as food?

A. The relative nutritive value of the different meats for food is as follows: beef is the most nutritious; then clicken, multon, porti, and vecil. 
Q. What varieties of Fish are the most Nutnitious?

A. The salmon, the eel, the haddock, and the herring, in order.

Q. What VEGETABLE of ordinary consumption is the most nutritious?

4. The cabbage.

Q. Is there any REASON for the usual practice of cooking and eating pork and cabbage together?

A. The reason of eating these two articles of food together is that cubbage contains a large percentage of gluten, and little oily or. fatty matter; in pork, a reverse ortier exists. The mixture of the two causes a better digestion than when either are eaten alone.

Q. Why do we instinctively desire to eat BUTTER or cheese with bread, FAr with cabbage, and oIis with salad?

A. An article of food, to be in every respect nutritious, must contain gluien, starch, and some variety of fat. When the proportion of these substances in any article of food is too small, chemistry teaches, and experience and instinct direct, that an additional quantity of the deficient substance should be added in the process of cooking, or preparatory to eating.

Thus, bread contains little fat or oil, and therefore butter or cheese is eaten with it, in order that it may be more palatable, more digestible, and more nutritious. For the same reason we eat pork and cabbage, salad and vil.

Q. The peculiar character of all chemical compounds, when brought in contact with matter endowed with life, is an aptitude and power to enter into combinations and effect decompositions. When a chemical compound of simple constitution is introduced into the stomach, or any other part of the system, what must be its EFrecr?

A. It will tend to exercise a chemical action upon all substances with which it comes in contact. This action is opposed by the vital principle, and the result will depend upon the strength of the respective actions.

Q. When will the chemical compound be DIGESTED ?

A. When it is forced to yield to the superior power of the vital action, and exercises no chemical infuence upon the living organ.

Q. When will the chemical compound act MEDicinalLI ?

A. When it causes a change in the vital principle, without destroying it.

Q. When will the chemical compound act as a Porson?

A. When it obtains an ascendancy over the vital force, and destroys it.

Q. When will food act as a PoISoN, and produce DISE ASE ?

A. When it is able to exercise a chemical action by virtue of its quantity, or when either its condition or its presence retards, prevents, or arrests the motion of any organ.

Q. What is contagion?

A. We apply the term contagion to that subtle matter which proceeds from a diseased person or botly, and which commanicates the disense to another person or body. Contagion differs from miasm in being the product of disease, and in reproducing itself. 
Q. What is MIISM, or MIASMATA?

A. Miasm or miasmata is the product of the decay or putrefaction of animal or vegetable substances, and causes disease without being itself reproduced. Contagion occasions disease in the same way that yeast excites fermentation. Miasm often acts, by its chemical properties merely, as a poison.

Q. Why are contagious diseases sometimes communicated to individuals who merely APPROACH the VICINITY of diseased persons, but do not come in CONTACT with or even see them?

A. Because the air itself, which has been in contact with the diseased persons, carries with it the seeds or germs of infection, and thus communicates disease, sometimes at great distances.

Q. Why are not ALL persons affected alike when exposed to similar contagious diseases?

$A$. Contagious matter is not capable of producing disease, unless a compound is present in the system capable of being decomposed by contact with the exciting body.

Q. What do we mean by susoeptibiuity to contagion?

$A$. We mean that the blood of a person contains substances by the decomposition of which the exciting body or contagion can be reproduced. If these substances are not present, and if the system be perfectly healthy, contagion will fail to produce disease.

Q. When is disease called MALIGNANT, and when BENIGNaNT or MILD?

$A$. All disease is occasioned by changes or transformations, which take place in organs in a manner different from what occurs in ordinary healthy action. If these transformations are perfected on constituents of the body which are not essential to life, without other parts taking a share in the decomposition, the form of disease is mild; when these changes affect the organs essential to life, the disease is called malignant.

\section{CHAPTER LVI.}

\section{FATS, OILS, GU MS.}

Q. What is the CHEMICAL CONSTITUTion of a FAT or an oIL ?

$A$. All fats and oils consist of an acid united to a base. Every simple fat contains a peculiar acid, but the base in all fats and oils is the same. Thus stearine is composed of stearic acid united to glycerine; this combination makes up the great bulk of tallow; in oil we have oleic acid united to glycerine, the combination forming the bulk of the fixed oils.

Q. What is SOAP?

$A$. Soap is a combination of some one of the fat acids with an altiali. 
Q. How is SOAP manufactured?

A. The fat or oil is heated in the presence of potash or soda. These strong bases displace the weak base glycerine, and combine with the fat acids, forming combinations of fat acids with soda (soda or hard soap), or of fat acids with potassa (potassa or soft soap).

Q. Why is soap sorr when made with potash?

A. Because in soft soap the glycerine, dissolved in water, and formerly combined with the fat acid, remains mechanically mixed with the soap, and renders it soft and semi-fluid. In hard soap, made with soda, the glycerine and the water are separated from the soap by the action of common salt.

Q. How does Coмron SALT separate glycerine and water from soap?

A. Soap is insoluble in salt water. The soap-boiler takes advantage of this to make his soda soaps hard, by adding to the mixture of soap, glycerine and water, a solution of salt. The soap, not being soluble in salt water, immediately separates from the water and the glycerine dissolved in it, and floats upon the surface. In this situation it may be removed, and the waste lye, water, salt, and glycerine suffered to run to waste.

Q. What rwo important PROPERTIES do soaps possess?

A. 1. They dissolve fats and oils; 2. By mixing with much water, a part of the allali contained in them is set free, leaving an acid salt; that is, a combination in which the proportion of acid is greater than that of the base. The alliali dissolves most organic substances, and the fat acid causes the dissolved matter to wash away easily.

Q. How is the peculiar MOTTLED appearance of CASTILE soap produced?

A. By colouring the soap with oxide of iron.

Q. What is a LINIMENT?

A. Soap dissolved in alcoliol, forming a tincture of soap.

Q. Why is it impossible to WRITE on GREASY paper?

$A$. Because grease has no affinity for water or ink, and therefore will not mix with it.

Q. Why is МUTтON-FAT, \&c., solid, and Not liquid?

A. Because fat contains a predominance of solid stearine, and only a very small quantity of the liquid oily substance called oleine. On the other hand, oil contains more of the liquid oleine, and less of the solid matter called stearine.

Q. What is the DIFFrirence between a fat and an oil?

A. A fat is a compound of hydrogen and carbon which is solid at common temperatures; an oil is a compound containing the same elements which is fluid at common temperatures.

Q. Into what two great cLASSES do we divide fats and oils?

A. Into animal fats and oils, the products of animals; and into vegetable fats and oils, the products of the vegetable kingdom. 
Q. What constitutes the distinction between LARD and TALLOW?

$A$. We apply the term lard to those animal fats which at common temperatures have a soft, unctuous consistency, and tallow to those which remain hard.

Q. From the fats of what SPEOIES of ANIMALS do we extract IARD ?

A. From the fats of animals that are carnivorous, or such as will feed on fiesh; and also from the fats of birds.

Q. From the fats of what ArImaLS do we extract malLow?

A. From the fats of those animals which ruminate, or chew the cuct. The fats of vegetables are mostly liquid.

Q. In what PARTS of the vegetable are the fats most ABUNDANT?

$A$. In the fruit, particularly in the seeds of the plant. The olivetree oulv contains oil in the fleshy integuments of the fruit. As a rare exception, oil is sometimes found in the roots of plantis.

Q. What is TRAIN or FISI OIL?

A. The oil obtained from the $67 u b b e r$ of the right whate; also from seicts, porpoises, and other fish.

Q. Why does whale oil possess such a DISAGREEABLE ODOUR?

A. Whale oil, when melted out from the blubber at a moderate heat, has a yellow colour, and a slight odtour which is not disagreeable; but that which is obtained by strong heat, or from the blubber that has become putrid, is of a darli-brown colour, and has a very disagreeable odour.

The action of the heat, or the decomposition of blubber, generates a peculiar product, called "phocenic acid," which imparts to the oil its nauseous odour.

Q. Why do we apply the terms WINTER-STRAINED and SUMMER-STRAMNED oil to different varieties of fish oil ?

A. Oil extracted or clarified in cold weather is not so liable to congeal as that extracted during the elevated temperature of the summer months.

Q. What is SPERMACETI?

A. The cavities of the head of the sperm whale are filled with a peculiar fat, which, when taken out, immediately begins to deposit small crystalline particles-spermaceti, in large quantities. This, when pressed and purified, constitutes the spermaceti of commerce.

Q. What is "NEAT's-FOor" oil?

A. The oil extracted from the feet of oxen. It is chielly used by curriers in softening leather.

Q. Why do we employ Fars for greasing leather, harness, shoes, \&c. ?

A. Because they penetrate into the interior of these substances, and remain soft for a long period, thus rendering the leather supple and flexible.

Q. Why does oil become murch in WINTER-TIME?

A. 1. Because it is condensed by the cold, and rendered more solid; and

2. Because the "stearine," which is held in solution in warm 
weather, is separated by the action of the cold, and deposited as a thick, white, and almost solid substance.

Q. Why does CHURning cream convert it into BUTTER?

$A$. Cream is the fat or butter of mill: contained in little globular cases of albumen; by churning, this film or envelope of albumen is broken, and the butter or fat set free.

The globules are invisible to the naked eve, but may be distinctly seen floating about in milk by means of a tolerable microscope.

Q. Why do the fats and oils FLOAT upon water?

$A$. Because their specific gravity is less than water.

Q. Why does cream Rise to the surface of milk?

A. The cream of milk is composed of little globules of oil. The rest of the milk consists of water containing albumen, caseine, and sugar. The oily part, constituting the cream, having a less specific gravity, rises and floats upon the surface of the watery portion.

Q. Why does cream rise more freely from milk in a BROAD, FLAT dish, than from milk in a deep and narrow vessel ?

A. Because cream cannot rise through a great depth of milk. If, therefore, milk is desired to retain its cream for a time, it should be put into a deep, narrow dish; and if it be desired to free it most completely of cream, it should be poured into a broad, flat dish, not much exceeding one inch in depth.

Q. Why will cream rise upon milk more readily in How weather than in cold?

$A$. The separation of cream is hastened by a higher and retarded by a lower temperature. At the usual temperature of the dairy, 50 degrees $F$., all the cream will probably rise in thirty-six hours; but at 70 degrees it will rise in half that time. When the milk is kept near the freezing point, the cream will rise very slowly, because it becomes nearly solid.

Q. What is Rasin?

$A$. Under the term resin we include all the products of the sap of the trees belonging to the genus pine. The word resin generally indicates the sap of the ordinary varieties of white and yellow pine; the distinctive name for the white and yellow pine-tree sap is turpentine.

Q. What is oil or spirits of TURPENTINE?

A. The volatile oil obtained by distilling the resin of the pine.

Q. What is ordinary resser, such as is used in the manufacture of gas?

A. It is the product left after the oil of turpentine has been distilled from the sap or resin of the pine.

Q. What is campuine?

A. Pure spirits or oil of turpentine.

Q. What is the composition of BURNING FLUID?

$A$. Burning fluid is for the most part a mixture of spirits of turpentine and alcohol. Other oils and resins are not unfrequently mixed with it. 
Q. What is STEARINE?

A. Fats are not homogeneous (of the same nature) bodies, but are composed of a mixture of several more simple kinds of fat, into which, without being chemically decomposed, they may be divided. Most fats may, by chemical or mechanical action, be divided into a solid fat and a fluid fat. To the first we apply the term stearine, to the last, oleine.

Q. In what FATS does stearine especially PREDOMINATE?

A. In the hard varieties of tallow and lard. In mutton-tallow, the quantity of stearine is very great.

Q. In what FATS does oleine especially PREDONINATE?

A. In the oils; and it is to the abundance of this principle in their constitution that they owe their fluidity.

Q. Why do we call some oils DRYING oILS?

A. All oils absorb oxygen from the air, and become thereby thicker, but there is an essential difference between the various kinds; drying oil becomes perfectly hard and $d r y$, while an unctuous oil will always remain soft and sticky.

Q. What are some ExAMPLES of the drying oils?

A. Most vegetable oils are drying oils; the linseed, or flax-seed oil, the oil of the hempseed, poppy oil, castor oil, \&c.

Q. What is VARNISH?

$A$. Varnishes are divided into three kinds, according to the material in which a resinous substance is dissolved; these are spirit varnishes, in which the solvent is alcohol; essential oil varnishes, in which a volatile oil, commonly oil of turpentine, is used; and oil varnishes, which consist of a resin dissolved in a drying oil.

Q. What is OIL-CLOTH?

A. Coarse cotton or linen cloth covered with coloured varnishes.

Q. What is oIL-SILK?

A. Simply varnished sill.

Q. What is "JAPANNING?"

A. Varnishing in colours.

It is, therefore, a species of painting. It is most easily executed upon wood or metal, or such other substances as retain a determinate form, and are capable of sustaining the operation of drying the varnish. Japanning requires to be executed in warm apartments, and the articles are warmed before the varnish is applied to them. Ovens are also employed to hasten the drying of the work.

Q. What is "IACQUERING?"

A. Lacquering consists in the application of transparent varnishes to metals, to prevent their tarnishing, or to give them a more agreeable colour.

When the colour of the metal to be lacquered is to be changed, the rarnish is tinged with some colouring matter; but where preservation from rust or tarnish is the sole object, any of the transparent varnishes will answer, the best and hardest being used where the greatest durability is required. 


\section{Q. What is CAMPHOR?}

A. Camphor is a solid, white gum, obtained from the camphor-tree growing in Japan and the East Indies.

Q. What is LAC?

A. A resinons substance found on several different kinds of trees in the East Indies, and produced by the puncture of insects. Shelllac is refined lac.

Q. What is SEALING Wax?

$A$. Ordinary red sealing wax is a mixture of shell-lac, turpentine, and vermilion. The vermilion is only used as a colouring material, and, by using other pigments, other colours can be imparted to the wax.

Q. What is $A M B E R$ ?

A. A fossil resin. It is chiefly found in the earth, on the coast of the Baltic Sea, or floating on the water, and in some coal-mines. It is supposed to have been derived from the forests of a primeval age, which have been submerged by floods of water.

Q. What is ASPHALTUM?

A. A mineral resin, found in many parts of the world, particularly in the vicinity of the Dead Sea in Palestine, and in the West Indies. It is undoubtedly a product of coal, which has been acted upon in the interior of the earth by volcanic fires.

Q. What is NAPITIIA ?

A. A mineral oil, which flows from the ground. It collects upon springs and lakes, particularly in the United States.

Q. What is CAOUTCHOUC, Or INDIA-RUBBER?

A. A gum resin, which flows from various species of the Ficus (fig-tree), in South America and Africa. It hardens in the air into a white, elastic mass, insoluble in alcohol and water.

Q. Why has the India-rubber of commerce a dark or BLACK COLOUR?

$A$. Because it is dried in smoke, which penetrates the gum and discolours it.

Q. What substances DIssolve India-rubber?

A. Ether, oil of turpentine, naphtha, or oil of coal tar.

Q. What is COAI TAR?

A. Coal tar is a black, pitchy sulstance, which is formed in the condensing apparatus of gas-works. The gas formed from coal deposits, on cooling in the condensers, a variety of oily, resinous bodies, which collectively have received the name of coal tar.

By distillition, coal tar furnishes a number of volatile oils, one of which (benzole) is a powerful solvent for India-rubber.

Q. How is India-rubber vulCaNised?

A. By intimately mixing it with sulphur, a compound is formed which resists a high degree of heat, and is at the same time very elastic. 
Q. Why has the common BAR SOAP a YEILOW COIOUR?

A. Because it contains a certain amount of resin.

Q. Of what are the common WASHING FLUids composed?

A. Of solutions of the caustic allacilies.

\section{CHAPTER LVII.}

\section{PERFUMES AND ODOURS.}

Q. From what source do we derive most of the SUBSTANCES used in PERFUMERY ?

A. From the vegetable kingdom.

Q. Do we derive any perfumes from the ANIMAL KINGDON?

A. A few of the substances employed, either as perfumes or as ingredients of perfumery, are animal products; the principal of these are musk, civet, and ambergris.

Q. What is Muss?

A. Musk is a substance secreted by a ruminating animal of the deer family, inhabiting the mountainous portions of Central Asia. When fresh, it is a soft, reddish-brown mass; the musk of commerce is in the form of dark, rounded grains; it is one of the most penetrating, powerful, and lasting of odoriferous substances.

Q. What is CIVET?

A. A secretion, somewhat resembling musk, obtained from the civet cat, an animal found both in Asia and Africa.

Q. What is AMBERRGRS?

A. An odoriferous substance, which is found floating on the sece in some portions of the Indian Ocean, and also off the coast of South America. It is believed to be a secretion of the sperm whate, in which animal it has sometimes been found.

Q. What is the APPEARANCE of ambergris?

A. When fresh it is solid, grayish, streaked, or mottled in appearance, and somewhat soft. I' has a strong, agreeable odour, somewhat resembling musk, and a fatty taste.

Q. What DisTinguishing PRoperTy have all the animal odours?

A. They are remarkable for their permanence and durability. An article of dress, scented with ambergris, musk, or civet, will retain its odour even after it has been washed.

Q. Do we derive any pleasant smells, or odours, from the MINERAL kingdom?

A. The odours or smells purely derived from the mineral kingdom are more or less disagreeable.

Q. Into what TIREE CLASSES may the vegetable odours be divided?

A. Into the volatile oils, extracted from odoriferous plants; the fragrant resins, camphors, and balsams; and the volatile ether's. 
Q. To what is the ODOuR of most plants and flowers to be ASCRIBED?

A. To the presence of a volatile oil, which gradually evaporates.

Q. Does this volatile oil exist in any coNsIDERABLE QUANTITY in plants?

A. It is diffused and diluted in the system of the plant to a most incredible extent. Thus, in one hundred pounds of fresh roses, or orange-blossoms, there is scarcely one ounce of volatile oil

Q. How are the volatile oils OBTAINED from plants?

A. Generally by distillation. When the parts of a sweet-smelling plant are distilled with water, an oil passes over along with the steam, and floats on the surface of the water, which condenses in the receiver. In this way are obtained the oils of roses, lavender, cinnamon, peppermint, lemon, orange, wintergreen, \&c.

Q. What is ROSE-TATER, LAVENDER-WATER, and the like perfumed liquids?

A. Simply water, in which a portion of the volatile oil which is collected by distillation is retained.

Q. Is the odoriferous principle UNIFORMLI DIFrUSED orer the whole plant?

A. It is not: in the mint and thyme it is in the leaves and stem; in the sandal and cedar trees it is in the wood; in the cinnamon, in the barl; in the rose, violet, \&c., in the leaves of the flower; in the vanilla, anise, and caraway, it is in the seed; and in the ginger, sassafras, \&c., it is in the root.

Q. What is a poratum?

A. The name is given by the French to fats perfumed with the odour of flowers. The flowers are moistened with the fat, and, after lying a little while, are submitted to pressure; or they are put into hot water with the fat, and the mass well shaken together; after which the fat, rendered fragrant, is removed.

Q. What is the CHEMICAL COMPOSITION of the roustiLe oILS?

A. The volatile oils which constitute the odoriferous principles of most plants are composed of two elementary bodies, carbon and hydrogen. And what is very remarkable, many of them, which seem to be entirely distinct bodies, consist of these two elements united together in exactly the same proportion.

Q. What is the composition of orl of Terpextras?

A. One hundred pounds of oil of turpentine consists of eighty-eight and a fourth pounds of curbon, united to eleven and three-fourth pounds of hydrogen.

Q. What is the clemical composition of the oIL of Lmarons, ORANGE, ROSEMARY, ROSES, COIAIBA, QUETN OF THE MEADOW, and many other like oils?

A. The chemical composition of all these different oils is the sume. as that of oil of turpentine, vi\%. $88 \frac{1}{4}$ parts of carbon united to $11 \frac{3}{4}$ parts of hydrogen. So far as composition is concerned, they are all identically the same bodies.

Q. If these different volatile oils are all alike in composition, to WнАT can we ascribe their DIFFERENCE in PROPERTIES ?

A. It is supposed to be owing to the difference in which the minute 
molecules or atoms of carbon and hydrogen are grouped and arranged around each other.

Q. What KAME do we apply to bodies thus differing in properties, but agreeing in composition?

\section{A. Isomeric bodies.}

Q. Can any of the essential, odoriferous oils be formed arTifrcially ?

A. Oil of cinnamon has been manufactured artificially, and there is reason to expect that all the others will ultimately be formed or imitated by chemical art.

When benzole, an oily product of coal tar, is mixed with nitric acid, a product is formed which resembles, in odour and other properties, the oil of bitter almonds, and is now extensively used as a substitute in perfumery for the costly and genuine article.

Q. What is the CHemical composition of the camphors, balsams, resins, \&c. ?

A. They are principally composed of carbon and hydrogen, but differ from the volatile oils in containing a proportion of oxygen.

Q. What is it that gives to well-made HAY its peculiarly SWEET ODOUR?

A. The presence of a fragrant resin, called coumarin, which exists in a variety of sweet-smelling grass (anthoxanthum odoratum).

Q. What is the orrars of most of the unpleasant odours?

A. The greater number of the smells we dislike, as well as those which are agreeable, have their origin in the vegetable lingdom. The principal unpleasant odours of the mineral lingdom are sulphuretted hydrogen, sulphurous acid, hydrochloric acid gas, chlorine and some of its compounds, and some of the bituminous oils and compounds.

Q. What is it that imparts to the onroN and GARLrC their peculiar and disagreeable ODOUR?

A. A volatile oil, similar in composition to the fragrant oils, united to sulphur.

Sulphur always exists in the onion, and the odoriferous substance which this vegetable contains is called the sulpluret of allyle. The bad odour of the breath, after eating onions and garlic, is caused by a constant presence of a small quantity of this oil in the air we exhale from our lungs.

Q. What is ASSAFETIDA?

A. It is the juice or resin of an Asiatic plant. It contains the same smelling substance, the sulphuret of allyle, as the onion.

It is also a curious fact that the horseradish, the mustard, and several other plants of like character, contain the same oily compound which characterises the onion, the leek, the garlic, and assafoetida. Mustard, in particular, owes its peculiar penetrating odour, blistering properties, and burning taste, to the presence of the oil allyle, combined with cyanogen, as well as with sulphur.

Q. Will the organs of SMELL always gire us evidence of the presence of putrid, volatile, infectious matter in the atmosphere?

A. They will not give us this evidence, and cannot be relied on. 'The condor and the vulture will detect the presence of decaying matter in the atmosphere, emanating from a putrid substance many miles distant, when to man the air seems perfectly pure. Because we do not perceive an unpleasant odour from the vaults of churches 
and cemeteries, it is no evidence that the atmosphere is at any time free from infectious matter.

As an evidence of the effect which very minute quantities of volatile substances may rroduce in the air, Dr. Carpenter states "that a grain of musk lias been kept freely exposed to the air of a room, of which the doors and windows were constantly open, for a period of ten years, during all which time the air, though constantly cluanged, was completely impregnated with the oclour of musk; and yet at the end of that time the particle was found not to have sensibly diminished in weight."

Q. In preparing perfumery, is one SINGLE oIL, or odour, relied upon solely to produce the given effect?

A. No; it is seldom that a single oil, or the fragrant portions of a single plant, are relied on to produce any delicate perfume.

"The art of the perfumer consists in combining the odoriferous principles of many Howers, or in mingling together many volatile essences, iu sucl a inanner as to produce : more agreeable perfume than any single plant $n 0^{\circ}$ essence can be made to yield. In this way the huille de mille fleurs (oil of a thousand flowers) professes to be made; and the receipt for the popular 'Eau de Cologne,' called the perfection of perfumery, depends for. its excellency on the same principle."

Q. To what have the various odours been COMPARin ?

A. To notes in music.

"Some blend easily and naturally with each other, producing a harmonious impression, as it were, on the sense of smell. Heliotrope, vanilla, orange-blossoms, and the almond blend togerher in this way, and produce different degrees of a nearly similar effest. The same is the case with citron, lemon, vervaine, and orange, only these produce stronger impressions, or belong, so to speak, to a higher vctave of smells. Again, patchouls, sandal, and others, form a third class. It requires, of course, a nice or well-trained sense of smell to perceive this harmony of odours, and to detect the presence of a discordant note. But it is by the skilful admixture, in kind and quantity, of odours producing a similar impression, that the most delicate and unchangeable fragrances are manufactured. When perfumes, which strike the same key of the olfactory nerve, are mixed together for handkerchief use, no idea of a different scent is awakened as the odour dies away; but when they are not mixed upon this principle, perfumes are often spoken of as becoming sickly and faint, after they have been a short time in use." -Johnson.

Q. Can ART produce odours MOKE OFFENSIVE than any derived from NATURAL SOURCES ?

$A$. The researches of chemists have made known the composition of compounds which are inconceivably disgusting; so much so as to bear no comparison with any natural unpleasant odours. The element selenium forms, with hydrogen, a compound gas, one single bubble of which, in an ordinary sized room, produces on all that breathe it the usual symptoms of a severe cold and affection of the throat, lasting several days.

Q. What are examples of OFFENSIVE ARTIFICIAL ODOURS?

$A$. We are acquainted with a compound of arsenic, called kalodyle, which, when combined with cyanogen, the basis of Prussic acid, forms an odlour which is inconceivably foetid, and when inhaled produces instant death. Another substance, called mercaptan, is possessed of a most offensive, penetrating, and concentrated odour of onions, which adheres obstinately to all bodies with which it comes in contact. 


\title{
PHILOSOPHY OF MANUFACTURES.
}

Q. What is to be understood by the term MANưfactures?

$A$. Originally, this word signified the various products of handlabou" (from the Latin manus, a hand, and facere, to make); but in the changes of language it has come to signify the opposite of its real meaning, and now denotes every extensive product of art which is made by machinery, with little or no aid from the human hand.

Q. What is now considered the most PERFECY MANUFACTURE?

A. That which entirely does away with manual labour.

Q. What is the END and OBJECT of all manufacturing processes?

$A$. To modify the texture, form, or composition of natural objects by mechanical or chemical forces, acting either separately, combined, or in succession.
\end{abstract}

\section{CHAPTER LVIII.}

\section{NATURE OF FIBROUS SUBSTANCES.}

Q. Into what Two great CLASSES are the FIBRES used for textile purposes divided ?

$A$. Into fibres the produce of the vegetable lingdom, as cotton, flax, and hemp; and fibres the products of the animal lingdom, as silk, wool, and hair.

Q. Into what three varieties may vegetable fibres be distinguished ?

A. Into capsular fibres, cortical fibres, and foliaceous fibres.

Q. What are CAPSULAR FIBRES?

A. Capsular fibres are those obtained from pods or capsules, as the fibre of the cotton.

Fig. 151.

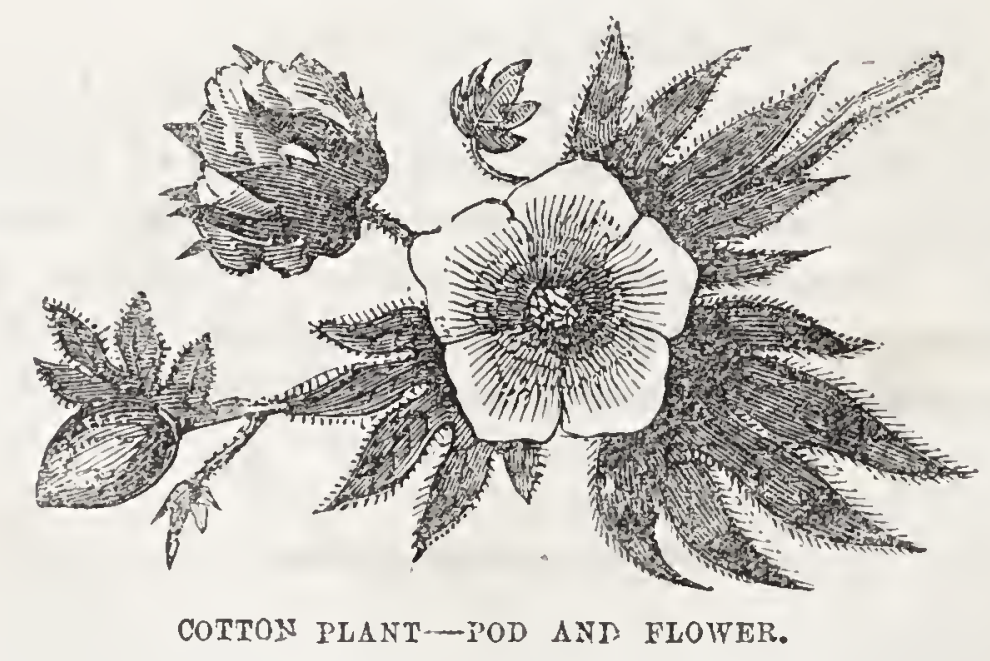


Q. What are CORTICAI FIBRES?

A. Fibres obtained from the cortex or bark of the stems of plants. Flax and hemp are the types of fibres of this character.

Q. What are FOLIACEOUS FIBRES?

A. Fibres obtained from the leaves (folia) of plants, and not, from the stalks or stems. The fibres known as manille and sisal hemp are the types of fibres of this character.

Q. Into what Two great cLASsrs may all flowering piants be divided?

A. Into endogenous plants and exogenous plants. Plants belong to one of these two classes, according to their manner of growth and the character of their stems.

Fig. 152.

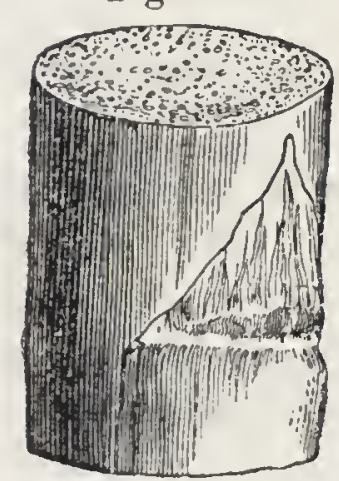

Q. What is an ENDGGENous plant

A. An endogenous plant (from two Greek words, endon, within, and gennao, I generate) receives its growth by matter deposited from within itself. Its stems are properly stalks, and are not covered with bark. Their leaves have also parallel veins. Fig. 152 represents a section of the stem of an endogenous plant.

Q. What are some EXAMPLES of endogenous plants?

A. The Indian corn, the sugar-cane, the common fay, the cactus: the lily, the pipe-apple, the palm, the agave, and the palmetto.

Fig. 153.

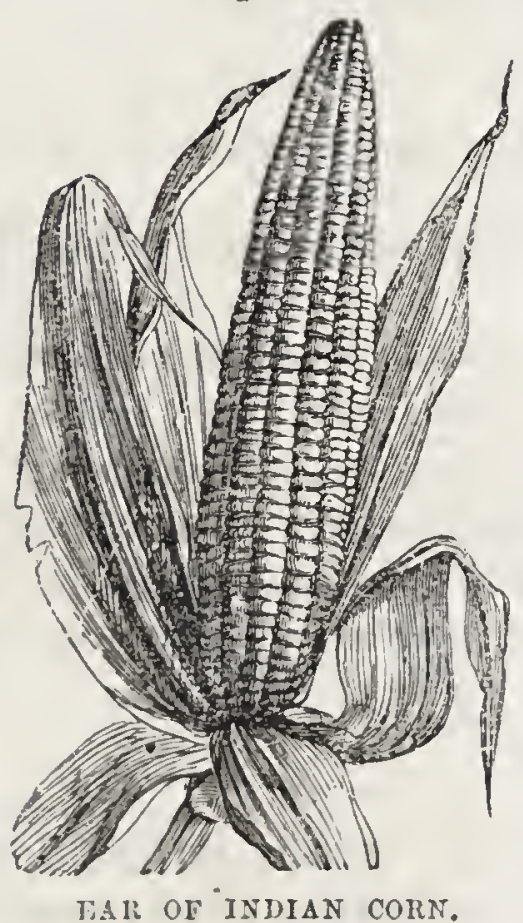

Fig 154.

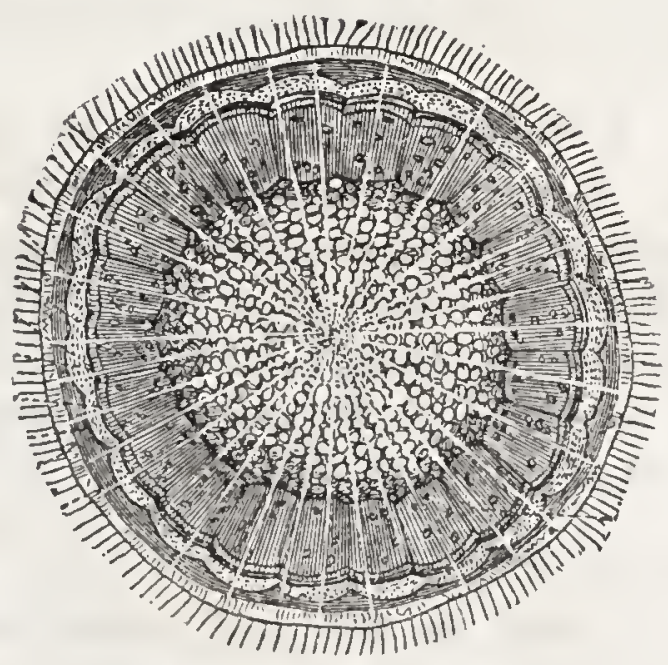

SECTION OF A BRANOH.

Q. What is an EXOGENOUS PLANT?

A. An exogenous plant (from exo, without, and gennao, I generate) is one that receives its growth by the deposition of woody matter on the outer surface. The stems of exogenous plants are properly trunks, conical and branched, and covered with bark. Fig. 154 shows the appearance of the section of aranch. 


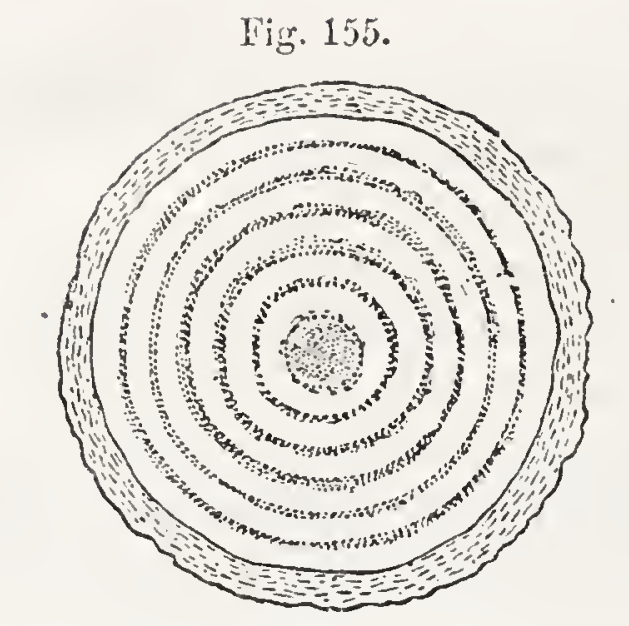

HORIZONTAL SECTION OF AN EXOGEN.
Fig. 156 .

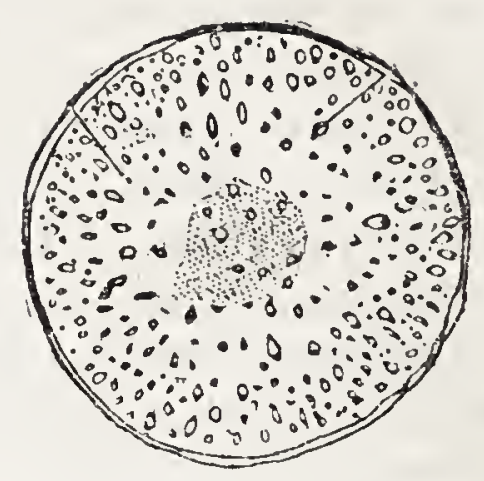

HORIZONTAL SECTION OF AN ENDGGEN.

Q. If you cut a trunk of an Exogenous plant across, what will be the APPEArance of the SECTION?

A. It will exhibit concentric circles of bark and wood, hardest at the centre, and softest at the circumference, with rays called "medullary rays," extending from the centre to the young external wood. In figs. 155 and 156 we have the representation of a section of the trunk of an exogenous plant and of an endogenous plant.

Q. If you cut a stalk of an ENDOGENous plant across, what will be the APPEARANCE of the SECTION?

A. It will exhibit a suface of porous materials, softest at the centre, hardest at the circumference, and without bark.

Q. That are exaxpies of Exogenoys plants?

A. Most of our forest trees, and the flax and hemp.

Q. How can the AaE of an fixogerous tree he learned from an examuation of a section of the trunk?

A. A section of the trunk of an exogenous tree, as is represented in the foregoing figures, exhibits concentric rings or circles. 'The distance between any two of these rings represents the growth of the tree during a single year; by counting the number of rings we obtain the age of the tree. It is to be remembered that each year a new layer of wood is deposited between the previous formation and the bark in all trees of this class.

Q. What is COTTON?

$A$. Cotton is the soft, white, tubular fitre which is found in the capsule of the gossypizm, or cotton-shrub, adhering to the seeds.

Q. When we examine a fibre of cotton under a microscope, what aPpearancri does it present?

A. It has the appearance of a flattened tube or ribbon, without joints, each fibre being perfect and entire in itself, with pointed or rounded ends. (See fig. 157.) 
Fig. 157.

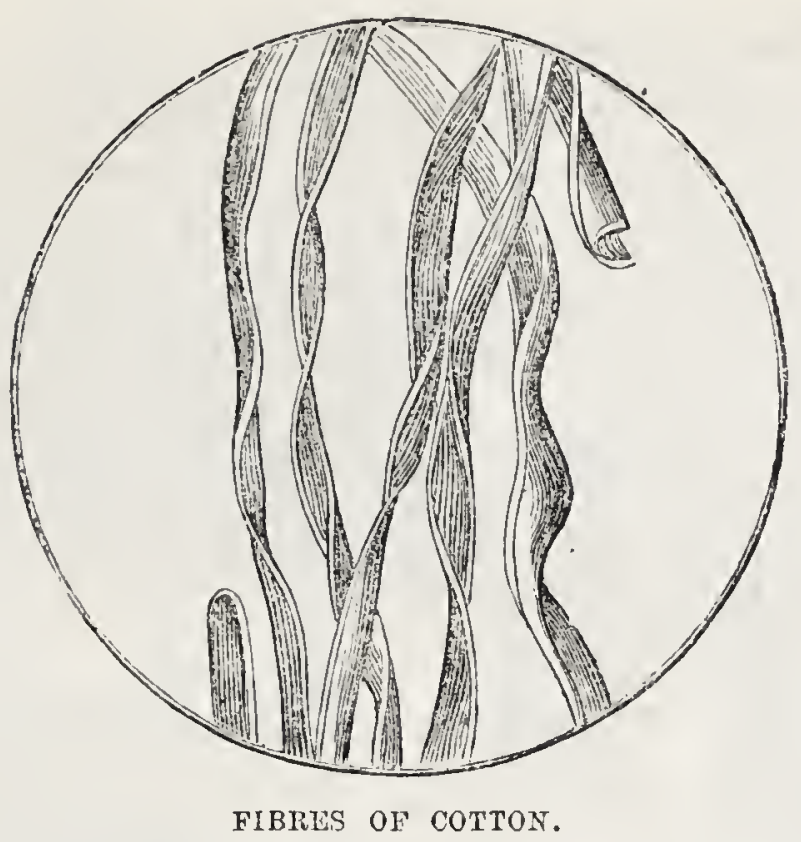

Q. When we speak of the STAPLE of cotton, what is to be understood?

A. By the staple of cotton we mean the length of the fibres. There are several species of cotton, in some of which the fibres are short, as the short-staple cotton; and in others it is long, as in the Sea-Island or long-staple cotton.

Q. Does the USE of cotton, as a material for textile purposes, appear to have been KNOWN to the ANCIENTS ?

A. Cotton appears to have been little known to the ancients, although the inhabitants of India have been accustomed to its use from very remote time. It was unkinom to the Egyptians, and also to the Jews. On the discovery of America the cotton manufacture was found to be in an advanced state among the original inhabitants of that country.

Fig. 158.

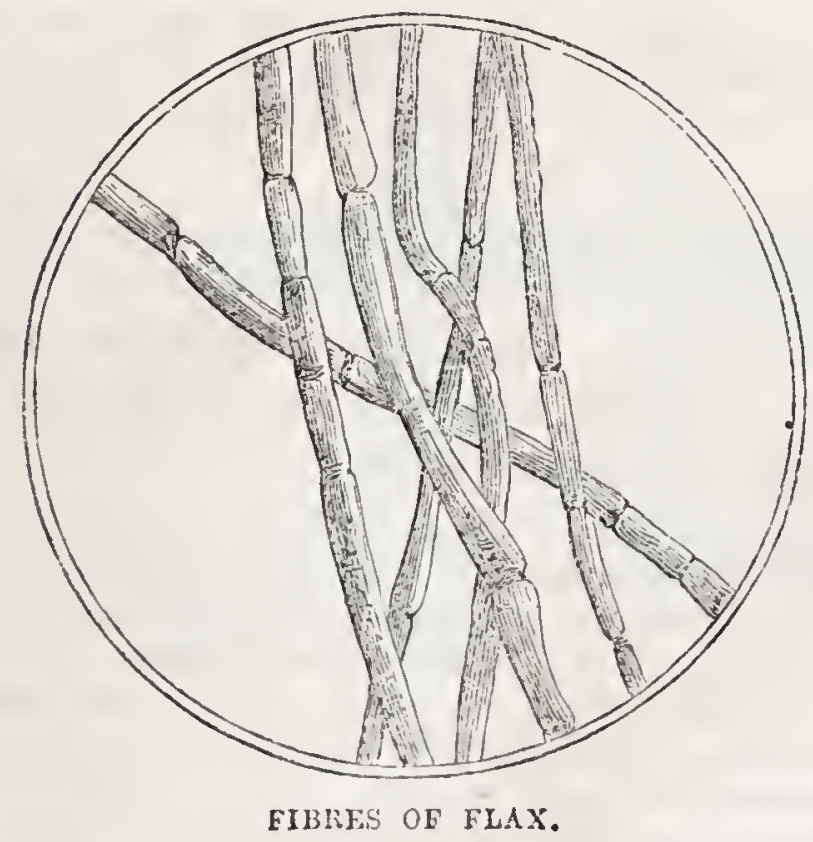


Q. What is the sost iscortant veatable FIBRE now used for the fabrication of textile producis?

A. Cotton. The amount consumed in England in a single year being upwards of eight hundred millions of pounds.

Q. How do the FIBRES of FuAx and maup differ from those of cotton?

A. The fibres of flax and hemp consist of long, capillary threads of woody texture, bundled together in parallel directions, which are easily separable into shorter and more minute filaments. When cxamined under the microscope, what to the eye alone appears to be a single flax fibre, is found to consist of a number of shorter fibres overlapping each other, and fastened together by rings of fibrous matter. (See fig. 158.)

Q. IIave flax and bemp any STAPLE?

A. They have not. The fibres are all of different lengths.

2. In what mannen are the fibres of flax and hemp arraged in the stems of their reapective plants?

A. The stems of the flax and hemp plants consist essentially of a woody core, with a sheath of fibrous matter surrounding it (the hemp and flax fibre), the whole cemented together by a peculiar sort of vegetuble glue, which binds the fibre and the woody core into one solid stem.

Q. In the preparation of flax and hemp fibres, what OBJECT is sought to be ATTAINED ?

$A$. In the preparation of flax and hemp, the great object to be attained is the removal of the vegetable glue, so that the fibrous part, being no longer bound together, may be easily separated from the woody part of the stem.

Q. How is this accomplisued?

A. Usually by steeping the stems of the flax and hemp in water, or by exposing them on the ground to the joint influence of air and moisture.

The vegetable glue is insoluble in water, but when steeped in that liquid, or exposed to moisture, it soon begins to ferment, or undergo a sort of slow putrefaction. When this is completed, the stems are dried, and the fibres separated from the brittle wool by beating, or "breaking."

Q. What is the operation of freeing the flax fibre from the glue which binds it to the wood called?

A. Retting, or rotting. That which has been steeped in water is called water-rotted flax and hemp; that which has been exposed to the air and moisture is called dew-rotted.

The Chevalier Claussen's process of preparing flax has excited considerable attention ; it is conducted in the following manner :- The flax being gathered when the sceds arc ripe, it is rippled; that is to say, it is drawn by liandfuls through an iron comb, which effectually separates the bolls, or seed-heads, from the stalks. It is then subritted to a breaker, which dirests the flax of all the refuse, which refuse is applicable as fond for cattle, as well as for manure. Brought from the breaker, it is boiled for four hours in hot caustic soda, or steeped in a cold solution of it for twenty.four hours. It is next washed in a weak solution of sulphuric acid, and then in pure water. 
Fig. 159.

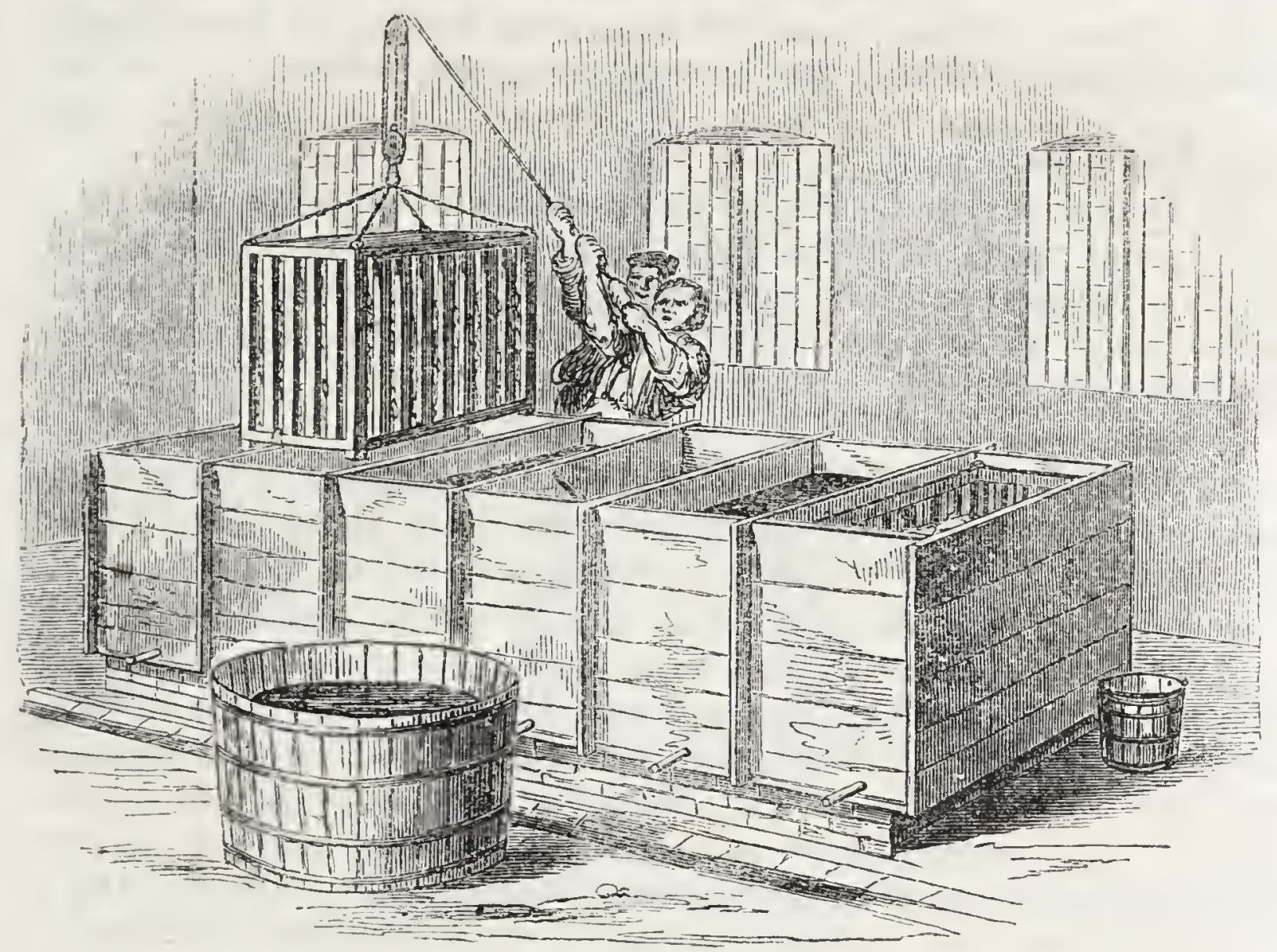

VATS FOR STLEPING THE FLAX.

In the engraving is represented the vats employed in the chemical process by M. Claussen. It shows the arrangement of the six vats, with the cradles in which the fibres are placed. One is being hoisted out, to be removed to the next vat. The first vat contains a solution of carkonate of soda; the second, a weak solution of sulphuric acid; the third, another vat of soda; the fourth, bleaching water-a solution of chloride of lime and sulphate of magnesia; the fifth, diluted sulphuric acid; sixth, pure water. The fibres, on being withdrawn, are taken to a machine sinilar to that used in chaff-cutting, when they are cut into lengths adapted for the purposes required.

Fig. 160.

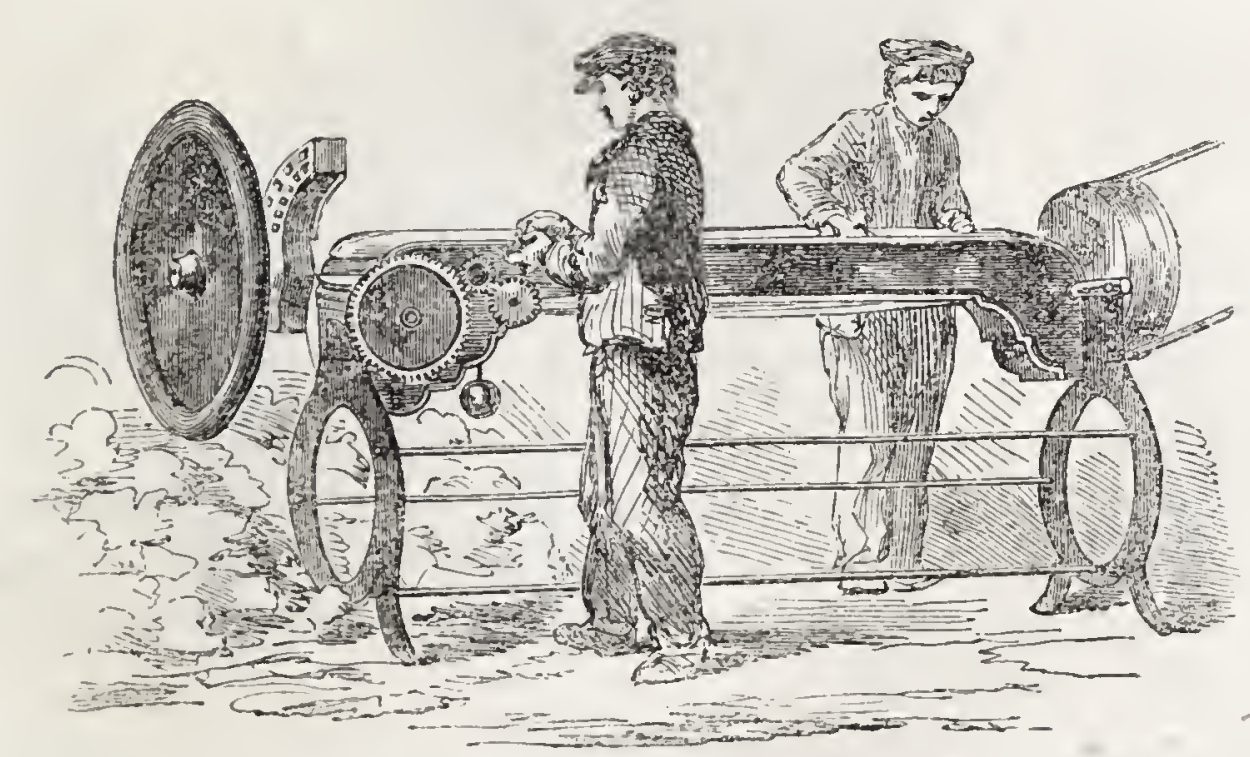


Q. What are the FABRIos manufactured from flax called?

A. Linens. Fabrics made of hemp are similar in properties to those manufactured from flax, except as regards fineness.

Q. Is the manufacture of flax and hemp attended with more Difficulties than that of cotton, wool, or silk?

A. It is. Owing to the irregular length of the fibres, and their rigidity and woody character, there are many difficulties in the way of spinning flax and hemp by machinery; the manufacture of the finest. threads used for lace and cambric is, for this reason, still performed by hand upon the ancient spinning-wheel.

Q. What is woou?

A. Wool is the peculiar sulstance which covers the skins of sheeps and some other animals, as the beaver, the llama, the goats of Thibet and Cashomere. Wool is simply a modification of hair.

Q. What is the APpEarance of WooL when examined under the Microsoope?

$A$. The fibres of wool, when examined under the microscope, are seen to possess a scaly surface, causing the edge of the fibre to have the appearance of a fine saw, with the teeth sloping in the direction from the roots to the points. Were a number of thimbles with uneven edges inserted into each other, a cylinder would result not dissimilar in outline from a filament of merino wool. (See fig. 161.) It is to this peculiar structure that wool owes its property of felting.

\section{Fig. 161.}

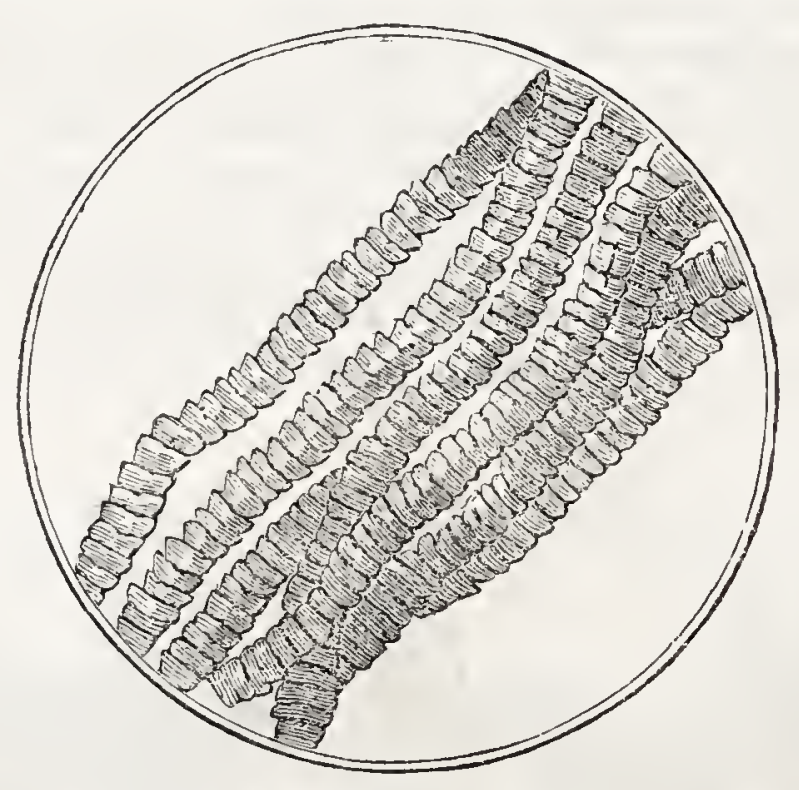

FILAJENTS OF MERINO Troor.

Q. How does the peculiar rovan surfice of wool cause it to FEL'T ?

A. The little imbrications or scales of the fibres lay hold and matck into each other; so that when a mass of wool is alternately compressed and relaxed, the filaments become enlarged and compacted into a solid tissue, which is called felt.

Q. Does hair also possess Felting properties?

$A$. Some varieties of hair, included under the term fur, have 
roughened surfaces, which causes them to felt. Both hair and wool, when heated or rubbed, have a tendency to curl up. This felting property of wool and fur is taken advantage of in the manufacture of hats.

Fig. 162 .

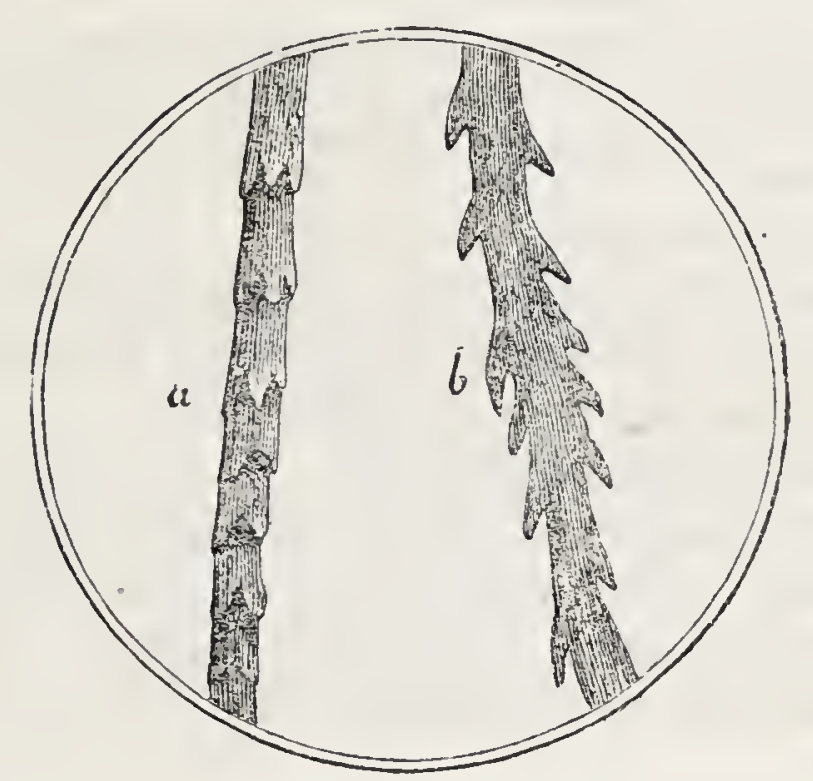

Fig. 162 exhibits the appearance of the hair of the seal $(a)$, and of a species of cater pillar $(b)$, when viewed under the microscope,

Q. What is SILK?

A. Silk is the secretion of a caterpillar, the larva of a species of moth, called the silk moth, originally found in China.

The changes which the silk insect undergoes preparatory to producing the silk are as follows:-This insect is subject to four metamorphoses, or changes. It is first of all an egg, produced by a species of moth, which the warmth of spring brings forth in the form of a caterpillar ( $a$, fig. 163). This caterpillar, at the end of twenty or thirty days, having attained maturity of size, commences spinning silk fibre, in which it envelopes

Fig. 163.

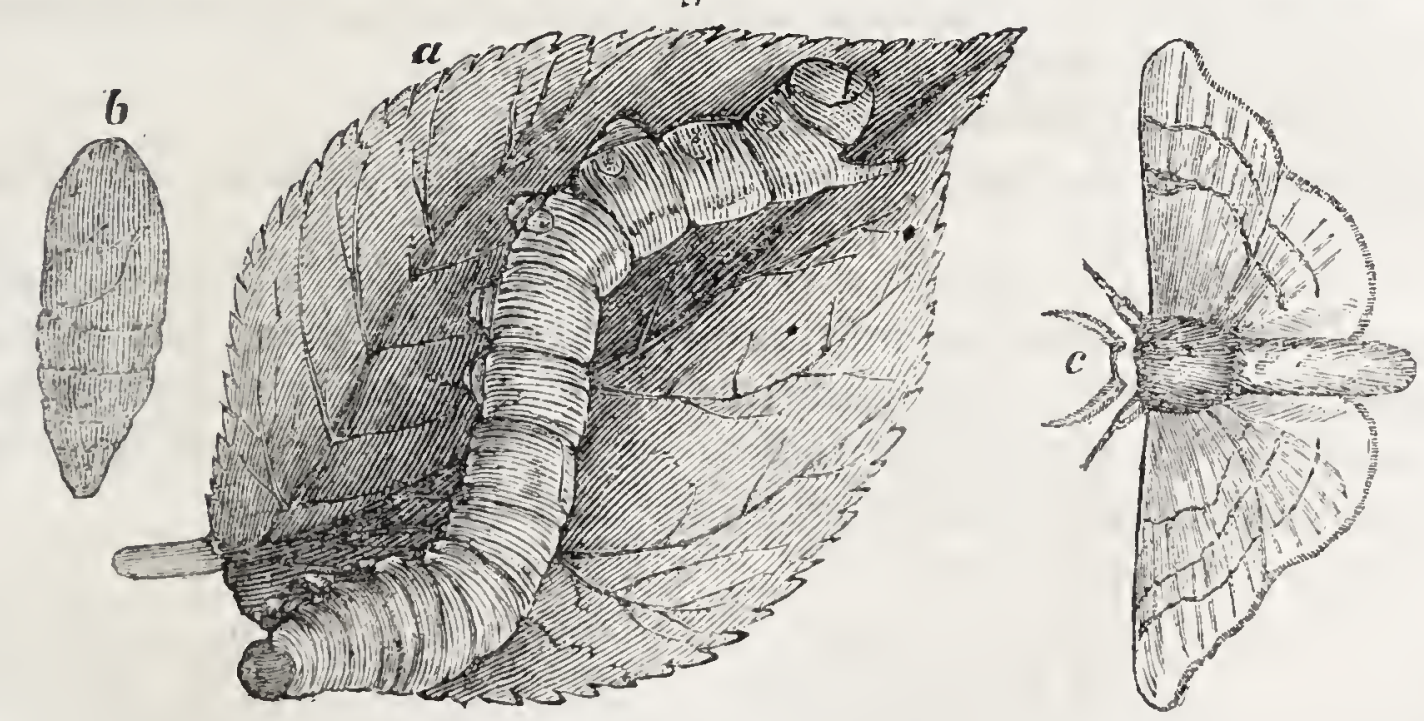

itself preparatory to changing to its third or chrysalis state (b), a sort of mummy condition, in which the enveloped insect lics apparcntly dead for fifteen or trienty days. A th end of this period it bursts through its shroud, and comes forth a butterfly, or moth (c). 
Iis. 164.

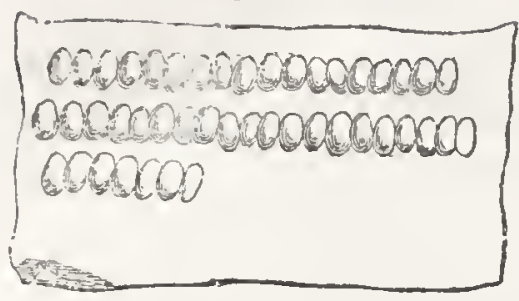

Q. What is the ENvELOPE in which the worm encases itself called?

A. A cocoon. (See fig. 164.)

Q. How is the silk obtarned from the cocoon?

A. By unvinding the fibres which com. pose it.

The original threads are too fine for manufacturing purposes, and therefore, in unwinding or recling them off from the cocoons, the ends or threads of several cocoons are joined together in lot water, which softens their natural gummy covering, and causes thein to cohere into a single thread.

Q. Of what is the silk COMPOSED?

A. Of a species of gummy matter secreted within the body of the worm. By dissecting the worm at the time it commences spinning the silk may be taken out as a separate mass.

Q. What is the APPEArance of the silk fibre?

$A$. The cocoon threads are twin tubes laid parallel by the worm in the act of spinning, and glued together with more or less uniformity by the varnish which covers their whole surface. Each filament of this thread is about the two thousandth part of an inch in diameter.

\section{CHAPTER LIX.}

\section{MANUFACTURE OF TEXTILE FABRICS.}

Q. Upon what does the STREnGtr of cordage, and the utility of flexible fibres which serve as clothing, depend?

A. Upon the friction or adhesion produced by the twisting and intermixture of the fibres.

Q. Is a TWISTED CORD aS ETRONG as the fibres that compose it, supposing the fibres and sord to be of the same length?

A. It is not as strong.

Q. Then what is the OBJECT of TWISTING fibres, as is done in the manufacture of textile falurics?

A. In order to connect successive numbers of fibres in such a manner that, besides the mutual pressure which their own elasticity causes them to exert, any additional force applied in the direction of the length of the aggregate, may tend to bring their parts into closer contact, and augment their adhesion to each other.

Q. What is spinnisa?

A. The twisting of filires into a thread or cord. Care is especially taken in spinning, that the fibres shall be intermixed, and their extremities engaged as much as possible in the centre of the twist, since it is obvious that if any fibre were wholly external to the rest, it could not be retained in the yarn. 
Q. In the spinning of a thread of wool, cotton, or flax, what is the FIRST ESSENTIAL OPERATION?

$A$. That of straightening the fibres, and laying them uniformly in a parallel direction. 'This, in the case of cotton and wool, is called carding; in flax and hemp, heckling. In cotton and wool, as the band or sheet of fibres comes from the cards, it is drawn by rollers through a hollow cone, or trumpet mouth, which contracts it into a narrow ribbon, which is called a sliver. The slivers are deliveren coiled up in tin cans, ready for the next operation.

Carding is a species of combing. If we were to take two combs, and pass the teeth of the one between those of the other, we should have an idea of the process. Carding is combing out, by means of a great number of wires acting in a contrary direction.

Q. What is the NExT STEP for forming a thread?

A. It is the elongating the slivers produced from the cards, by drawing out the ribbon of parallel fibres. The accomplishment of this by machinery was the great invention of Arkwright, which so wonderfully advanced the manufacture of cotton fabrics. The "sliver," elongated and drawn out, is called a "roving."

\section{Q. HOW is the DRAWING EFFECTED?}

A. It is performed by means of two pairs of rollers. The upper roller of the first pair is covered with leather, which, being an elastic substance, is pressed down by means of a spring or weight. The lower roller, made of metal, is grooved in order to keep a firm hold upon the fibres of the cotton. Another similar pair of rollers is placed near those which have been described. 'The second pair, moring with a greater velocity, pull out the fibres from the first pair of rollers. If the surface of the last pair move with twice or thrice the velocity of the first pair, the cotton will be drawn twice or thrice finer than it was before.

In pouring any thick fluid in a stream from one vessel to the other, it will be observed that the upper part of the strean is much larger and thicker than the lower part. In like manner, a sheet of water passing over a dam is much thicker at the top than at the bottom of the fall. In both these cases, the accelerated motion gained in falling causes the lower particles of the fluid to move faster than the uppermost ones; and for this reason the stream or sheet is drawn or thinned out at the base. In a like manner, by causing a ribbon of parallel fibres to pass through rollers revolving with unequal velocities, the fibres are drawn or thinned out at that end of the ribbon moving most rapidly.

\section{Q. What are the SUCCEEding OPERATIONS?}

A. The cotton is now one continuous cord, or "roving;" but in order to make it serviceable, it is necessary to twist it. This is effected in two operations, during which the cotton is farther drawn out by passing through pairs of rollers, and twisted by means of an arrangement called a flyer; after which, the thread, wound upon spools, called bobbins, is ready for the process of weaving.

The procass of forming wool and flax into thread or yarns is substantially the same as that of cotton-spinning. In silk it is, however, different, as the worm itself spins the threasl perfect and continuous.

All the different and beautiful maehines which have been invented during the last hundred years for the spinning of cotton are different contrivances for eficcting two 
objects: the first is the elongating the roving, until it contains in thickness exactly as many fibres as are necessary to produce the required size of yarns; and, second, the twisting of these yarns into a compact thread.

Fig. 165.

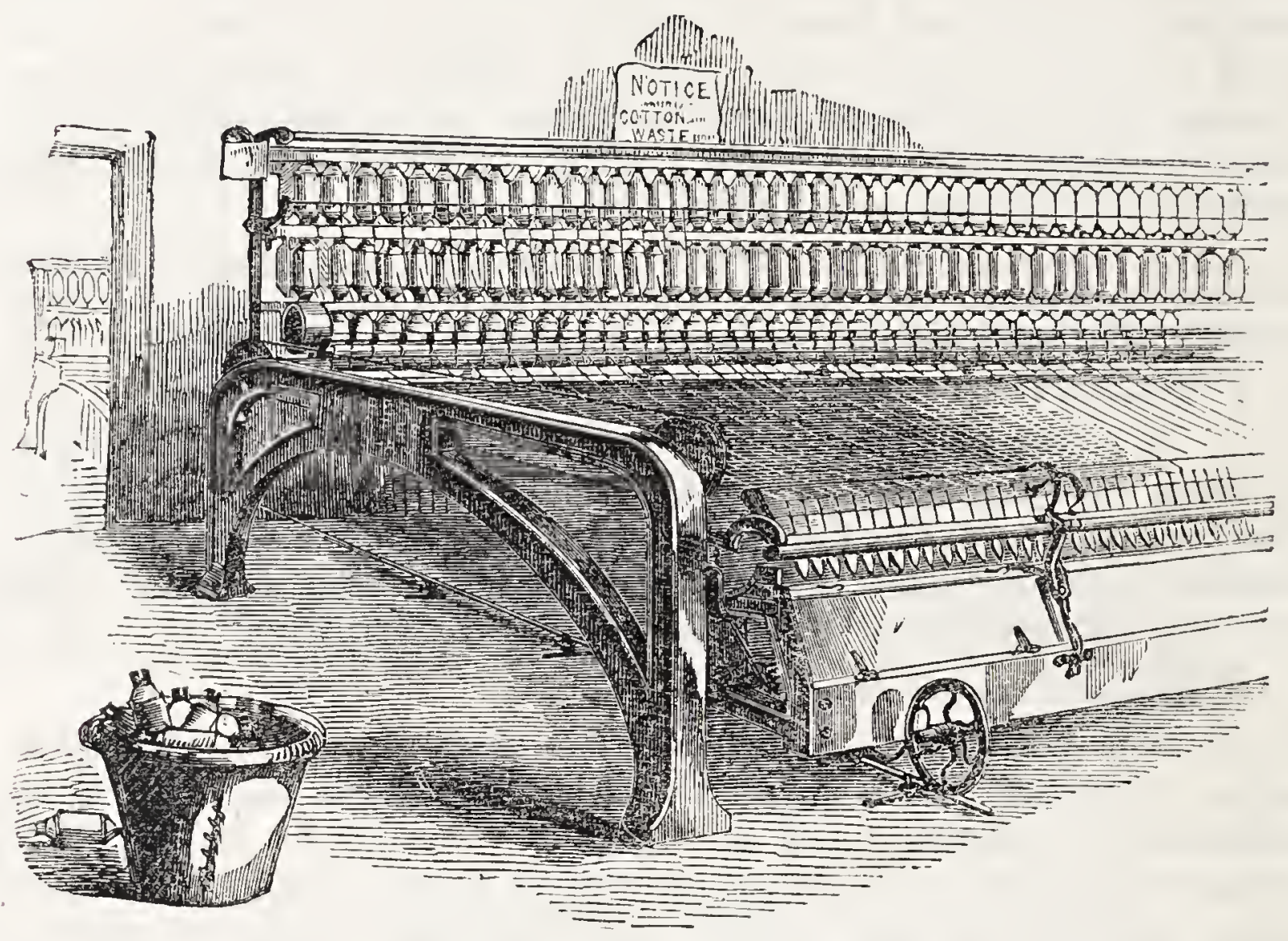

MULE, OR SPINNING JENNY.

Q. What is a SPINNING MULE?

A. It is a carriage some twenty or thirty feet long, travelling to and fro, and drawing out the most delicate threads through hundreds of spinules, whirling at a rate which scarcely permits the eye to trace their motions.

Fig. 166.

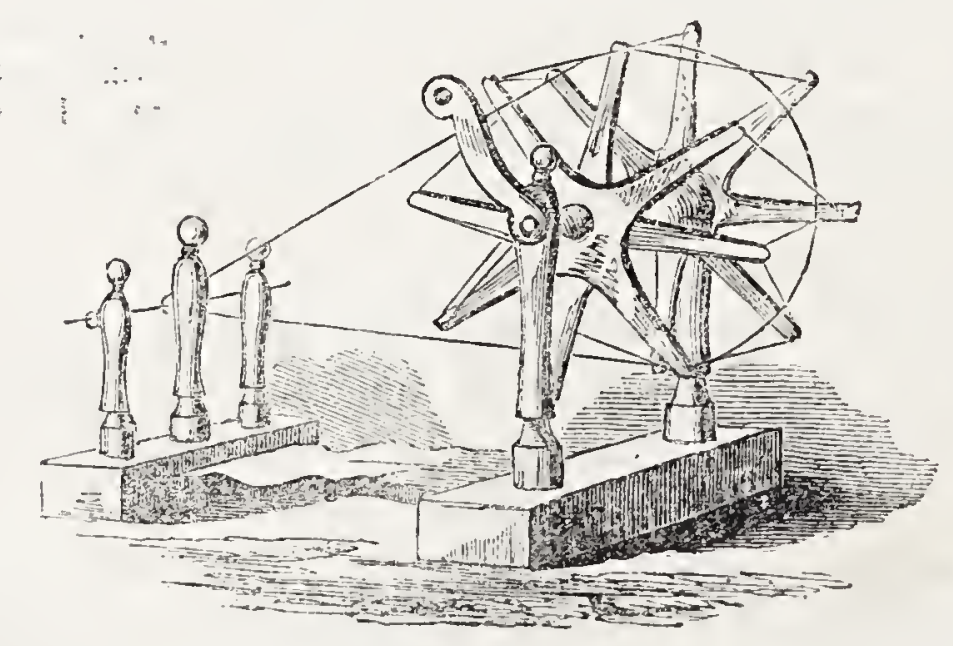

HINDOO SPINNING WIEEL。

Q. Can you give an illustration of the WONDERFCL EFFECT which the introduction of cotton-spinning machinery has had upon the production and cost of yarn and cloth?

1. At the present time, one man can attend to a mule containing, 
one thousand and eighty-eight spindles, each spinning three hanks, or three thousand two hundred and sixty-four hanks in the aggregate, per day. In Hindostan, where they still spin by hand, it would be extravagant to expect a spinner to accomplish one hank per day; so that, in the United Kingdom, we find the same amount of manual labour, by improved machinery, doing more than three thousand times the work.

Q. What is WEAVING?

A. Weaving is the art of combining threads, yams, filaments, or strips of different materials, so as to form a cloth or textile fabric.

Q. In weaving, the first step is to form the Warp: what is this ?

$A$. The term warp is applied to the threads which form the length, of the cloth. It consists of parallel threads continued through the whole length of the intended piece, and sufficient in number to constitute its breadth.

Q. What NaMe is applied to the tIIREADS which run transverseur across the cloth?

$A$. They are called the voof; sometimes, also, the weft and the filling. It consists of one unbroken thread, passed alternately over and under each thread of the warp, backwards and forwards. Plain wearing, therefore, is merely the process of making each single thread interlace with that next to it, the threads crossing at right angles.

Fig. 167.
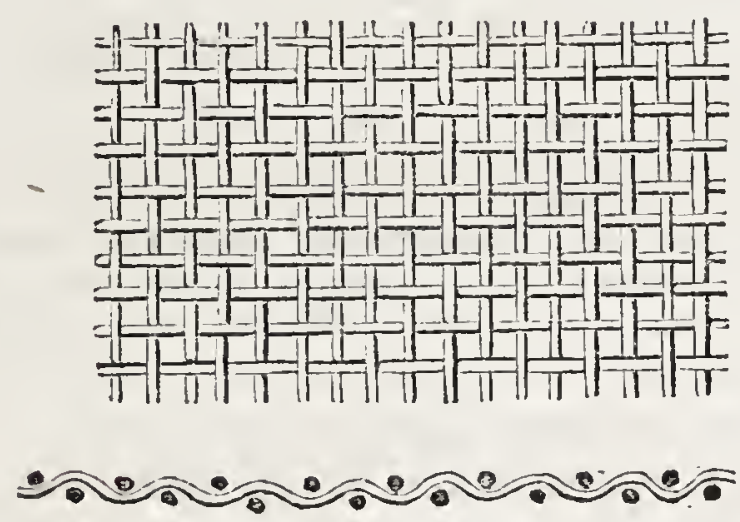

Fig. 167 represents the appearance of a piece of plain cloth seen through a microscope ; the alternate intersections of the threads are seen in the lower figure.

a. What is a shutrie?

A. A shuttle is a long, narrow box, pointed at both ends, containing a ball of thread or yarn; as this is passed through the alternate threads of the warp, the ball within it unwinds, and leaves the thread which constitutes the woof.

Q. How are twilled goods woven?

$A$. In these goods, the threads or tne warp and woof do not cross each other altemately, but only the third, fourth, fifth, sixth, \&c., cross each other. 
Fing. 168 a.

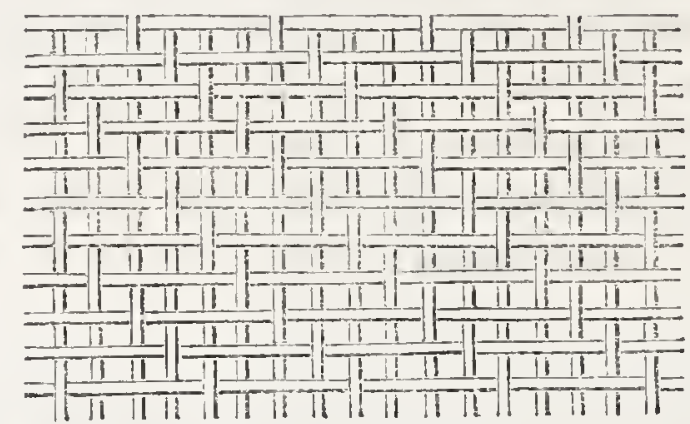

- Y O

Fig. $168 \alpha$ is an enlarged representation of a piece of twillest cloth, by which it will tho seen that the same thread of woof is separatel from the warp while passing over three threats, and is held down while passing over the fourth.

Q. How are Brussels and Wilton ourpers woven?

A. In these fabrics the basis is a warp and woof of strong linen thread; but to every two threads of linen in the warp there is added a parcel of about ten threals of woollen of different colours. The linen thread never appears upon the upper surface, but parts of the woollen threads are fiom time to time drawn up in loops, by being woven over wires of the breadth of the cloth.

Fig. 168

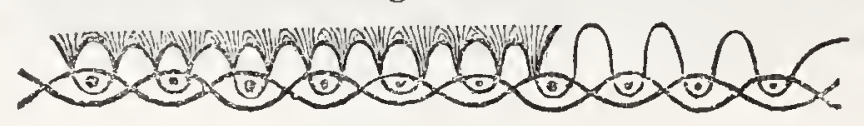

In Brussels carpets these wires are simply drawn out, and the loops left standing; but in Wilton carpets and in velrets they are cut out ky rassing a sharp knife through the loops. In this way a nap or pile is produced. (See fig. 168b.)

Brussels has long ceased to supply us with carpets, and carefully guards against our manufactures by heavy custom duties; orberwise, Kidderminster would supply carpets for Brussels. Rather let us say, the manufacturers of Kidderminster carpets would supply Brussels with carpets, for Kidderminster itself no longer makes the carpet that bears the name of that borough; and we depend on the manufacturers in the north of England and west of Scotland for that production, the Kidderminster carpet-makers having directed their attention to the higher qualities of carpet manufacture.

Q. Upon what does the PROCESS of PAPER-MAKING depend?

$A$. Upon the minute division of the fibres of the vegetable substances employed, and afterwards on their cohesion.

Q. How does paper intended for WRITING UPON differ from PRINTING papers?

A. Paper intended for writing upon is dipped in a thin solution of glue or getatine, obtained from the shreds and parings of animal skins. This process is termed "sizing."

Q. What is the BENEFrT of sizing paper?

A. It increases its strength; and, by filling the interstices between the fibres of the paper, prevents the inli from spreading among the fibres by capillary attraction.

Q. Why does not PRINTING INK SPREAD upon unsized paper as well as writing ink ?

A. Because printing ink is oily instead of watery, and has so much consistence, that it is not liable to run and spread. 
Q. What is PAPIER-MaCHÉ ?

A. 'The name papier-maché is given to articles produced either by pressing the pulp of paper between dies or moulds, or by pasting sheets of paper upon models. 'The articles, when dry, are varnished, japanned, and olnamented.

The old method of manufacturing papier-maché is as follows:-The paper for use is gray in colvur, but similar in texture to ordinary blotting-paper. Before using it the paper is well saturated with flour and glue, mixed with water, in about equal proportions, and is then laid on the mould of the article intended to be produced. These moulds are of iron, brass, or copper. The mould, coand with the first layer of paper, is then dried fi, twelve hours. A careful smoothing with a file follows; after which another deposit of paper is made. The processes of drying and smoothing are successively repeated with cach additional layer of paper, until the article assumes the required strength and thickiess. When the newly-formed article is taken from the mould, the several parts are plaiued, filed, and trimmed, so as to be quite correct and level. The process of stoving isen fullows, after which the varnish is laid on, and brought to a smooth, hard, brilliant surface. 'i'he article is then coated with several coatings of shell-lac varnish, coloured, which, after teing hardened, are scrared quite level. The fine surface is produced by hand polishing with rotteil stone and oil. Miny alterations have, however, been made in the process, and the paper is now usually reduced to pulp, and cast into the form required.

\section{CHAPTER LX.}

\section{DYEING AND CALICO PRINTING.}

Q. In what does the art of DYEring consist?

A. In impregnating cloths and other flexible fabrics with colouring substunces, in such a manner that the acquired colour may remain permanent under the conmon exposures to which the stuffs may be liable.

Q. How is this EFFECTED?

4. By producing a chemical union between the materials to be dyed and the colouring matter.

Q. Do JIGEERENT MATERILS present DIFFERENT ATTRACTIONS for dye-stuffs, and $\triangle$ BSORD colouring inatter in different proportions?

A. They do. Wool appears to have the greatest attraction for colouring substances; silk comes next to it-then cotton-and, lastly, hemp and fiax.

Q. Into what TwO CLASSES have colouring substances used in dyeing been divided ?

A. Into adjective and substantive colours.

Q. What is a SUDSTANTrve colour?

A. One that communicates its tint immediately to the subtances to be dyed, without the intervention of a third substance.

Q. What is an ADJECTrY colour?

$A$. One that requires the intervention of a third substance, that 
possesses a joint attraction for the colouring matter and for the substance to be dyed.

Q. To which class do MOST of the colours used in dyeing BELONG ?

A. 'To the adjective colours; if we except indigo, there is scarcely a dyestuff that imparts directly its own colour to goods; and the greater number of dye-drugs have so weak an affinity for cotton goods especially, that alone they impart no colour sufficiently permanent to deserve the name of a dye.

Q. How, then, does the dyer contrive to impart to oonron such beautiful colours by using substances which alone impart little or no colouring matter to the cloth ?

$A$. When the dyer finds there is no affinity between the goods and any colouring substance which is put in his possession, he endeavours to find a third substance which has a mutual attraction for the clotl and colouring matter; so that by combining the solution with the cloth, and then passing the cloth through the dyeing solution, the dyeing matter combines with the substance which is upon the goods, and constitutes a dye.

Q. What is this INTERMEDTATE SUBSTANCE called?

A. A mordant, from the French word mordre, "to bite," from an idea which the old dyers had that these substances bit, or opened a passage into the fibres of the cloth, giving access to the colour.

Q. Can you explain practically the manner in which a MORDANT ACTS in dyeing?

A. A solution of gallic acid is nearly colourless, and a solution of copperas (sulphate of iron) is in the same condition. Cloth, dipped in either of these solutions, will present no immediate change of colour; but if the eloth be first soaked in the solution of iron, and then passed into the solution of gallic acid, the acid and the base will unite, and form a beautiful black colour within the texture of the cloth.

Q. Why would a colour so produced be called a FAST COLOUR?

$A$. Because the colour is formed by the union of the gallic acid and the iron; and the iron having penetrated into the interior of the fibres, the colouring matter is developed there by the soaking in of the acid. Being thus formed in, and incorporated with, the very substance of the cloth, the colour will only disappear when the texture and fibre of the cloth is destroyed.

Q. How has the use of MORDANTS added greatly to the REsounces of the dyer?

A. Because a single colouring substance will impart very different colour's with different mordants; thus an extract of logwood will dye, with iron, black; with a solution of tin, violet; and by using other different mordants, this one vegetable extract will give all the shades of colour from a yellowish-white to a violet; from a lavender to a purple; and from a slate-brown to a black.

Q. What is the process of CALtCo PRINTIRG ?

A. The cloth is prepared by bleaching and other processes, which dispose it to receive the colour. 'The figures are then stamped or' 
minted, upon it from plates or rollers, which have been previously covered with different mordants, in the same way that ink is applied to types. The cloth is then passed through the solution of dye, and those parts which have been printed with the mordant seize upon and retain the colour. The cloth is afterwards washed, when all the colour not combined with the mordant disappears.

\section{CHAPTER LXI.}

\section{PRINTING, ENGRAVING, AND LITHOGRAPHY,}

Q. What is PRINTing ?

A. The word printing is commonly applied to the art of tating impressions of letters or type, with ink, upon paper.

Q. Who was the INventor of printing?

A. The honour of the invention is disputed. Some bestow it on - John Funst, others on John Guttenburg, others on Peter Schoeffer. All of them practised the art about the same time. The last-named invented metal types in 1452.

Q. How was printing effected befure the invention of METAL TYPES?

$A$. The letters and words were cut on blocks of wood, as is still the case in China, and impressions were taken from the block.

Q. Which was the FIRST Book ever printed in England?

A. Caxton's "Game and Playe of Chesse." It was printed in 1474. Caxton's printing office was a ruined chapel in Westminster Abbey.

Q. What book was first printed in the Exatrsm language?

A. The "Recollections of the History of Troy." Caxton translated it from the French; and he tells us in the preface, that he "had practised and learned at great charge and dispense to ordain the said book in print."

Q. What is the comson process of printing?

A. What is properly called printing consists but of one processnamely, tating impressions on paper from the types or woodcuts; but we commonly apply the phrase to the whole art-that is, to the setting up or arranging the type as well as that of taking the impression.

The letters, or types, are first arranged in a box, or case, every letter in its own alphabetical compartment. The compositor, in setting up lines of type, places the letters

Fig. 169.

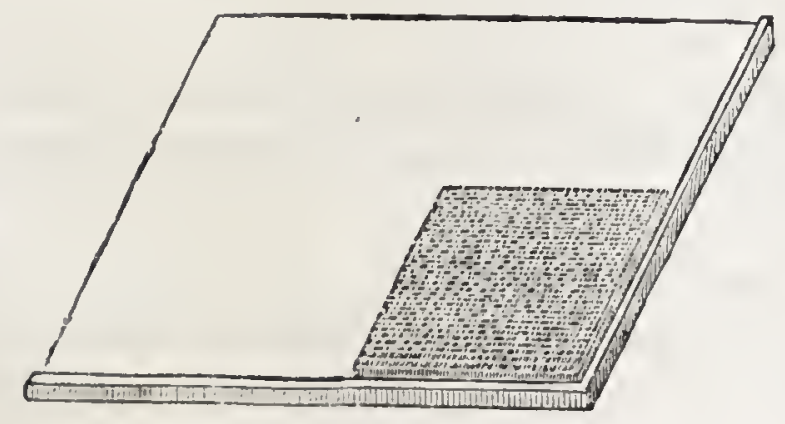

in a small frame, called a composing stick, which he holds in his hand, until, letter by letter, and word by word, the composin? stick is filled. He then transfers the matter thus set up to a gallcy. (See fig. 169.) Aftsi this the types are made up into pagee, which are arranged in a strong iron frama, called a chase, pieces of wood, or inctel (called fumiture), being placed between the pages, to keep them apart at proper dis. tances. The whole is then fastened or 
locked un, by means of small woalen wedges; and this when completed is called a form, which, after proper correction anu revision, is ready for the pressinan to take impressions from.

Q. What is the printing press?

A. The machine by which the impressions are taken. 'These are of various linds. Formerly, they were all worked by hand; mechanical appliances were afterwards employed; and steam-power is now applied to that purpose. The engraving below represents a Stanhope hand press.

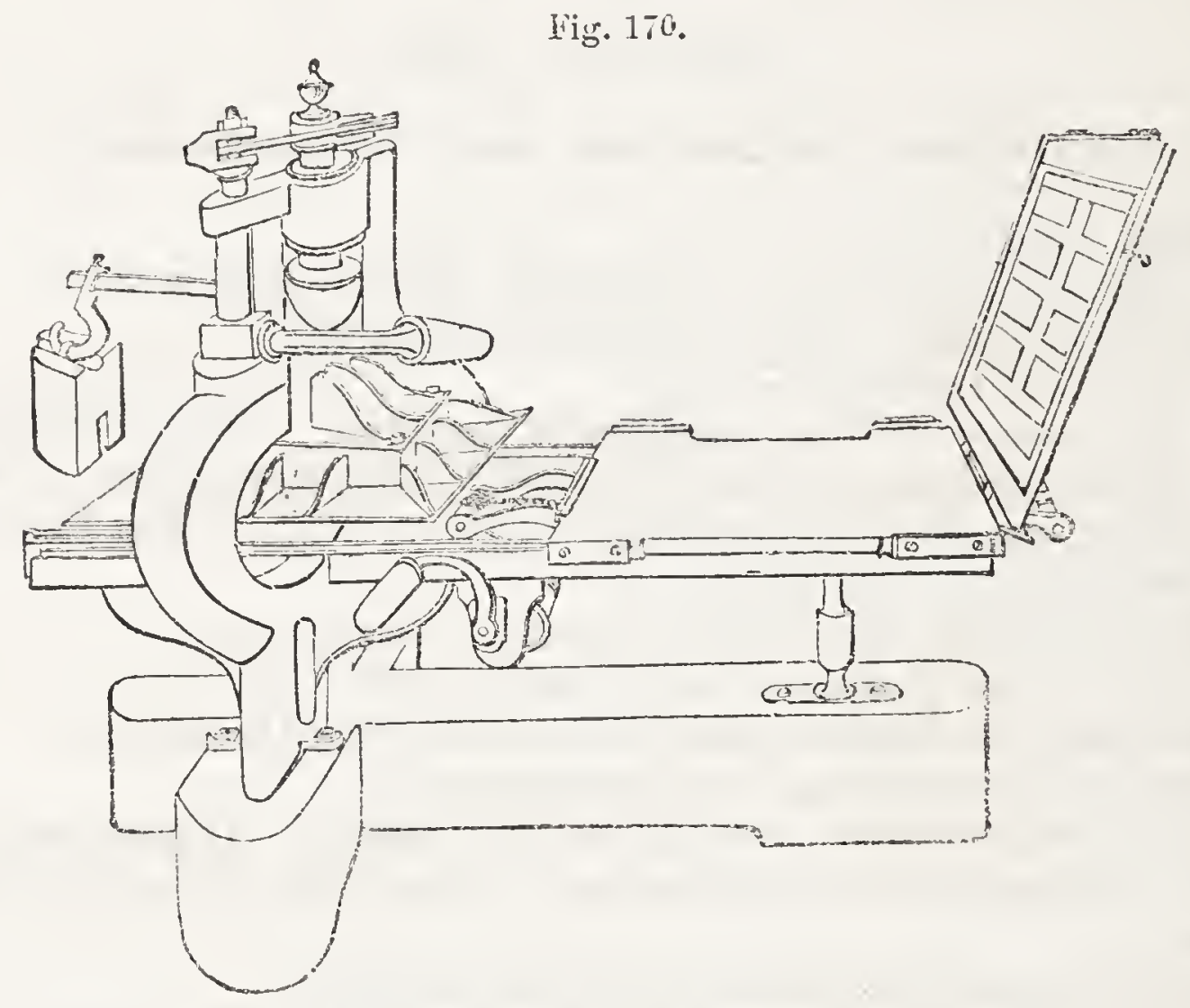

The following is a brief review of the progress of printing machinery. The first patent was obtained by Nicholson, in 1790, who then proposed placing both the types and paper upon cylinders, and distributing and applying the ink also by cylinders. In 1813, Messrs. Donken and Bacon introduced composition rollers. In 1814, Kœnig mad e the first working printing machine, and erected two of them at the Timcs office, each of which produced 1,800 impressions per hour. In 1816, $\mathrm{Mr}$. Cowper made a machine to print from curved stereotyped plates; and, in 1818, one to print books and newspapers from ordinary type, which machines are now in general use. In these machines he introduced the system of printing now so common. These machines printed from 2,000 to 2, 400 impressions per hour. The demand for greater speed continuing, Messis. Cowper and Applegarth, in 1827, conjointly invented the four-cylin!ler machine, which printed from 4,000 to 5,000 impressions per hour. The last, and most important, improvement was made in 1848; and the visitors to the Industrial Exhibition of 1851 had full opportunity of inspecting the apparatus.

Q. Is there MORE than ONN KIND of printing?

A. Yes; there are several methods. Sometimes movable letters are employed, sometimes solid pages, casts of the movable letters, called stereotype, are used.

Q. At what time was the STEREOTYPE Process first introduced? 1779 .

$A$. The present mode of stereotype was invented by $M r$. Tilloch, in 
Q. How does common ENGRAVING differ from PRINTING?

A. Common engraving differs from printing in having its subjects or devices cut into or below the surface of a metallic plate, instead of being elevated or raised above it, as in types or woodcuts.

Q. What are the principal raRIErIES or styles of engraving on copper or metal?

A. 1, Line engraving; 2, stippling; 3, etcliing; 4, mezsotint; 5, aquatint.

Q. What is LINE ENGRAVING ?

$A$. In this species of engraving, the surfaces and figures, the lights and shades, are produced by a multitude of minute lines cut into the metal, and approaching each other so nearly, that the inequality produced by the admixture black and white lines does not offend the eye, nor interrupt the harmony of the piece.

\section{Fig. 171 .}
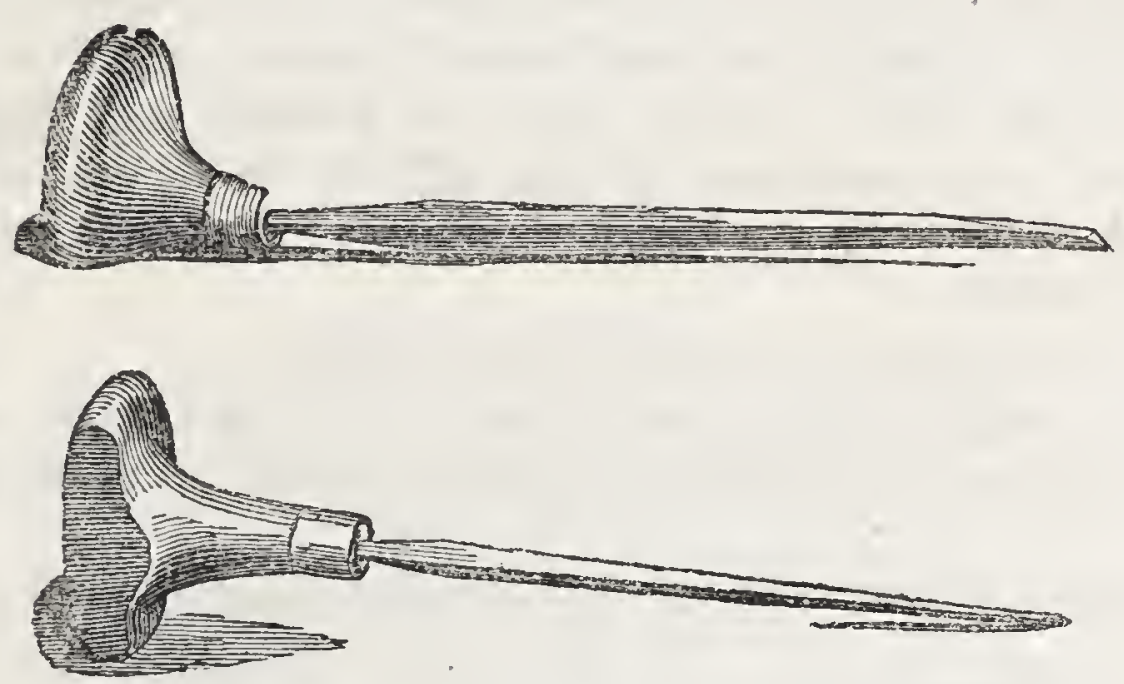

Grivirs.

2. By what years are these lines cut into the plate?

A. With a tool called a graver.

Q. What is a GRAVER?

A. The tool called a graver differs in size and shape according to the character of the line which it is intended to produce.

Fig. 172.

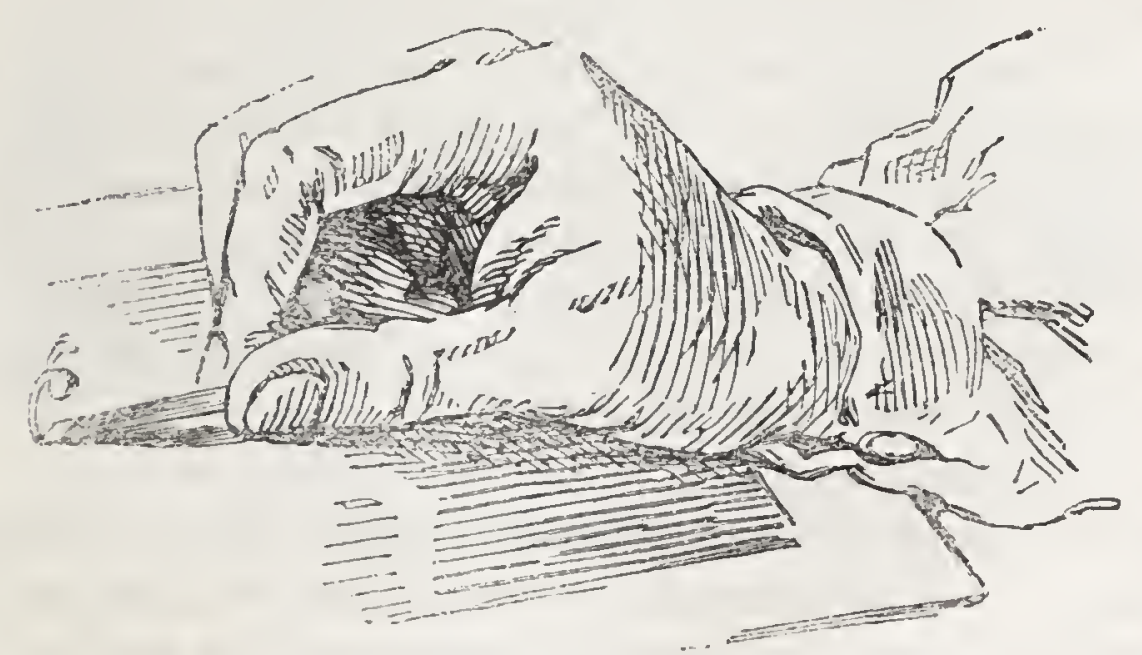

WA IN WHLUI 1 LINE IS TMACD BY THE GRATER. 
In making the incision, the graver is pushed forward in the direction of the line required, being held by the liandle at an angle very slightly inclined to the plane of the copper. It is requisite that the graver be well tempered, and great address is necessary in whetting it for use. The angle at the meeting of the two lower sides of the graver forms what is cilled its belly, and the breadth of the end is called its face. The two sides which form the belly are to be laid flat upon the oil-stone, and rubbed firmly until the belly slightly rises, so that if it were laid flat upon the copper the light could be seen underneath the point; otherwise it would be impossible to use it with freedom, as it would dig unequally deep into the copper. The face is next to be whetted, which is done merely by laying the face of the graver flat upon the stone, with the belly upward, and rubbing it steadily upon a moderate slope until it acquires a very sharp point, taking care that the stone be properly supplied with oil all the while. The gravers sold in the shops are commonly too hard for use, which is known by the frequent breaking of their points; when this is the case, they should be tempered by holding them on a red-hot poker, at a distance of half an inch from the point, until they acquire a faint straw colour; they should then be put into oil to cool ; or they may be tempered witl a candle, and cooled in the tallow. But it is best not to be hasty in tempering; for if the graver is only a little too hard, whetting alone will frequently bring it into good condition.

Q. How are GRADATIONS in LIGHT and SHADE effected in line engraving?

A. By the gradual and simultaneons tapering of all the lines which constitute the dark portions; and the softness and regularity with which this is accomplished greatly affects the beauty of the piecen Very dark shades are produced by lines crossing each other either i. squares or curves.

Q. What is that method of engraving calied strppling ?

$A$. It resembles line engraving in its processes, except that, instead of lines, it is finished by minute points or excavations in the copper.

The variations and progressive magnitude of these dots give the whole effect to stippled engraving.

\section{Q. What is ETCIING?}

A. In etching, the metal plate is covered with a varnish of wax, resin, \&c. The picture is drawn upon this coating with a needle, care being taken that the lines penetrate to the metal. Etching-points or needles are nearly similar in appearance to sewing-needles, but fixed into handles four or five inches long; some are made of an oval form, to produce broader lines with. Diy point is, in fact, nothing more than the common etching-needle brought to a very fine point. It is used to cut or scratch the more delicate lines with, such as skies, \&c. \&c. It does not, like the graver, cut the copper clean out, but throws it up on each side of the line produced by its progress through the metal: this is called the burr, which is removed by a scraper. This burr was left on by Rembrandt, until it wore away in the progress of printing, which it soon does; but by his management it added greatly to the effect of the etching, and impressions from his works with the burr on are much valued.

\section{Q. What is the NExr process?}

A. Acid is then poured upon the plate, when a chemical action takes place in all the lines or points where the copper is denuded by the strokes of the needle, while the rest of the surface is protected by the varnish. 'The picture is thus eaten or etched out of the metal 
by the action of the acid. Etched engravings never possess the smoothness and finish of mechanical work.

Q. What is Mezzorin'?

A. In mezzotint the whole surface of the copper plate is firs roughened, or covered with minute prominences and excavations, too small to be obvions to the naked eye; so that, if an impression be taken from it in this state, it has a uniform, velvety, black appearance. The picture is then produced by pressing down or rubbing out the rough. ness of the plate to the extent of the intended figure, obliterating the ground for lights, and leaving it for shades. Care is taken to preserve the insensible gradations of light and shade, upon which the harmony of the piece depends.

Q. What is AQUATINT?

A. This is done by a method partially mechanical, partially chemical. It consists in producing chemically a rough ground, covering the surface of the figure to be engraved, and afterwards introducing the lights and shacles by mechanical means.

Fig. 173.

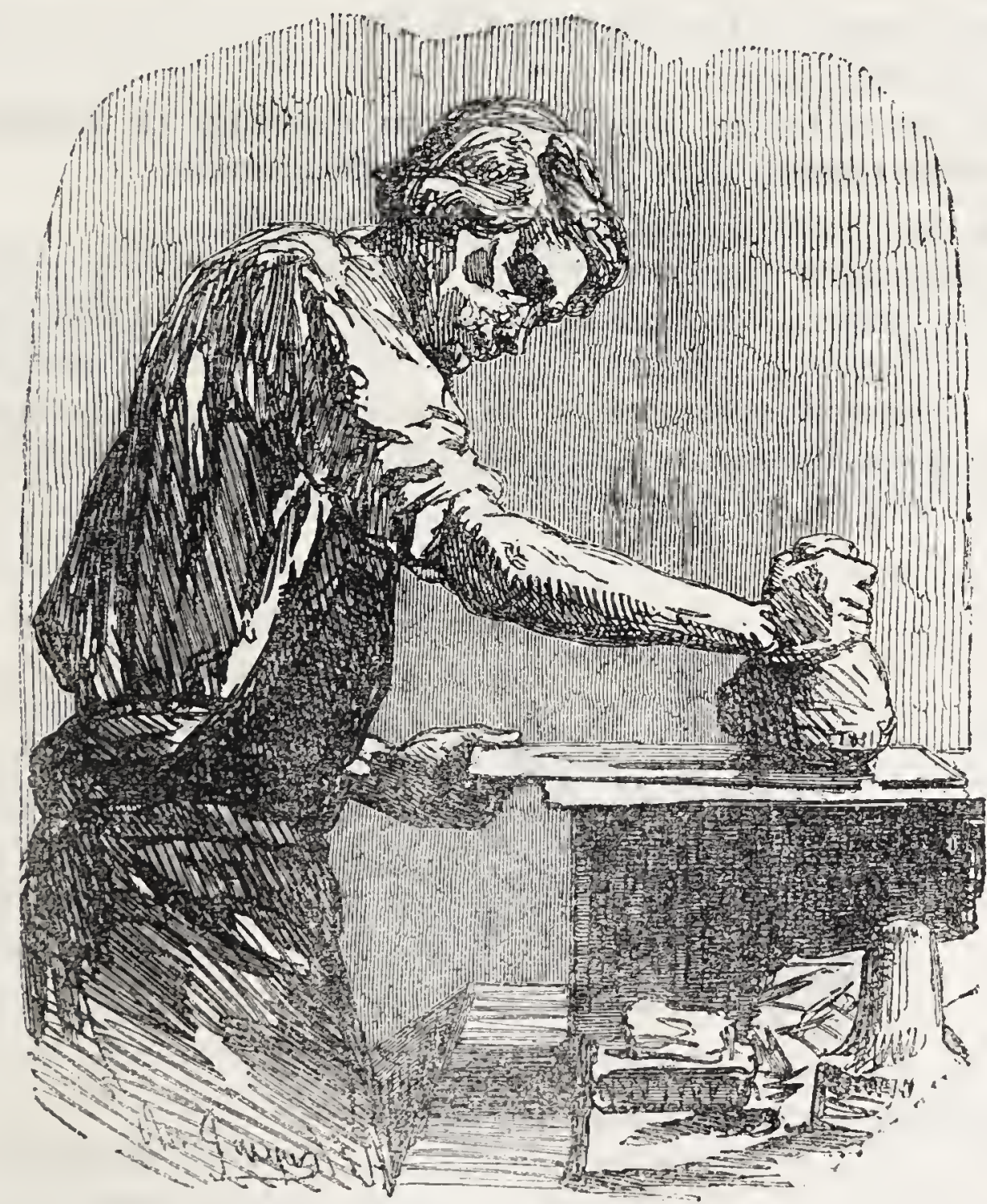

INEING TJE PIATE. 
Q. In what MAANNER is CopPerpiate Printing effected?

$A$. The cavities of the engraving are thoroughly charged with ink, and the smooth surface of the plate wiped as clean as possible. The latter part of the wiping is always performed by the palm of the hand. The ink, therefore, remains only in crevices of the engraving into which the hand does not penetrate in wiping the surface. The paper is then strongly pressed upon the plate, and portions of it are forced down into all the cavities of the engraving. The ink, or a portion of it, leaves the copper and adheres to the paper, giving an exact representation of the whole engraving.

Q. Upon what does the process of STEEL ENGRAVING DEPEND?

$A$. On the quality, which steel possesses, of being softened by losing a part of its carbon, and afterwards of being hardened by regaining it. The engraving is first made upon soft steel, and the plate is then hardened.

Q. What is the GREAT-ADVANTAGE of steel plates in engraving ?

A. On account of their hardness, they last for an indefinite time, and yield an almost unlimited number of impressions; whereas a copperplate wears out after two or three thousand impressions, and even much sooner, if the engraving be fine.

Q. What is LITHOGRAPHY?

A. The art of taking impressions from drawings or writings made on a peculiar smooth stone surface, without engraving.

Q. What sort of STONES are used for LITHOGRAPHIo printing?

$A$. Lithography owes not only its existence, but its possibility, to the fact, that several quarries in Bavaria, Germany, furnish a peculiar species of limestone, uniform in texture, apparently compact, yet having a somewhat open grain. A grained or smooth surface of this stone, therefore, having a porous structure, absorbs and firmly retains both water and oil, or inks made with oils or fats.

Fig. 174.

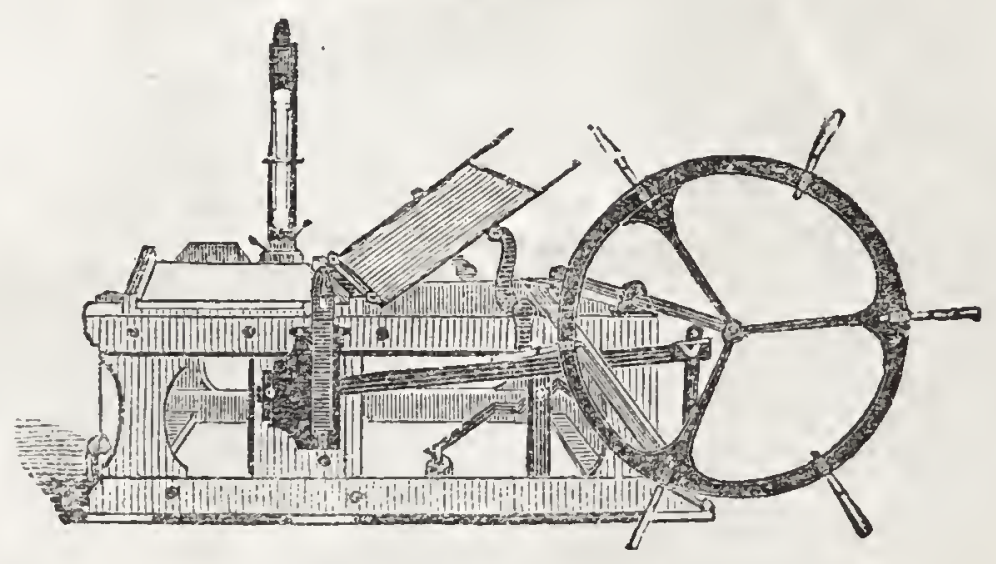

A LITHOGRAPHIC PRESS.

Q. What is the THEORY of LITHOGRAPHI printing?

A. If parts of the stone surface are covered with a drawing in oil or fut, the remaining surface may be wetted without wetting the inked prorts; and then if ink be rolled over the stone, it will be turned from 
the wet parts, and will adhere on the inked parts. Thus any drawing made with an oily ink on the stone, can have its lines duly charged with ink for printing, while all the rest of the stone continues clear.

Impression after impression upon paper can thus be taken off by wetting and inkin: the stonc after each printing. In this process the stone is merely the solid support for the ink and water to adhere to, and the whole manipulation has for its object to ink tho ink lines, and them only. If the stome be used when too dry, the whole surface takes the ink and prints, thus totally ruining the drawing.

Q. What is the so-called monsFer. Printing by the lithographic process?

A. The term transfer is applied to a stone on whose face the ink from an original drawing or writing, or from an impression on paper, has been so thrown down as to admit of printing in the same manner as if the drawing was first made on stone-thus reproducing the original indefinitely.

Q. What is PRINTING INK composed of?

A. Of oil, varnish, and fine lamplack.

\section{AGRICULTURE AND RURAL ECONOMY.}

\section{CHAPTER LXII.}

\section{ELEMENTARY PRINCIPLES PERTAINING TO AGRICULTURE.}

Q. What is GarJening?

A. Gardening is the art of cultivating flowers, fruits, and vegetables.

Q. Is this art called by any oTHER and LESS SISPLE name?

$A$. Gardening is also called horticulture, a word derived from the Latin, meaning garden culture.

Q. Is it an art EASTLY and QUTCKLY acquired?

$A$. The degree of skill necessary for raising common vegetables may be acquired without difficulty or long practice; but for the com1plete knowledge of the art, in its numerous branches, much experience, a good education, and some taste are requisite.

Q. What is the BEST SOIL for a garden?

A. The best soil for a garden is a rich and deep loam.

Q. What do you mean by LOAM?

A. By loam is meant a mellow, fertile soil, not stiff and greasy like clay, nor very loose and open like gravel and sand, but having these earths in proper proportions.

Q. What is TEGETABLE MOULD?

$A$. Vegetable mould is the darli-coloured soil we see in old, con. 
stantly-manured gardens. Vegetable or animal substances, rotted into earth, become vegetable mould, which contains rich nourishment for plants.

Q. Is it important to have a good SUBSoIL?

A. A good subsoil is very important. A very hard clay below, or gravel, if near the surface, are very bad subsoils, though in different respects.

Q. Why are they bad subsoils?

A. A hard clay bottom will not allow the rain which falls to pass through it; therefore, the upper soil is in a state of mud during the winter, and in spring and summer it hardens and cracks.

Q. What is the effect of Too oper a subsoil, such as sand or gravel ?

$A$. If a too open subsoil be near the surface, rain passes downwards too rapidly.

Q. What do you think of a soil resting on rock ?

A. A soil resting on rock is generally shallow, poor, and quickly dried up.

Q. What kind of subsoil is best?

A. 'The best kind of subsoil is a sandy or calcareous one, that will allow moisture to pass with moderate rapidity through it; such a subsoil, having no bad qualities in it, will nourish the roots of plants that strike deeply, instead of stopping or destroying their growth

Q. What is the BEsT SITUATION for a garden ?

$A$. The best situation for a garden is one facing the south, but slightly inclining towards the east, to receive the benefit of the morning sun; with perfect shelter from the north, and not exposed to winds that blow harshly and frequently.

Q. How should garden WaLKS be LAID OUT and made?

$A$. First, with a line, mark out the width of the walk or path; then dig the mould out of this space and spread it over the borders or beds, and cover the hollow with stones, brick-bats, or dry rubbish, on the top of which a thick coat of gravel should be spread. If the subsoil be perfectly dry, 10 dry rubbish will be necessary.

Q. If the soil contains too much clay, sand, or gravel, can the proportioxs of those earths be LESSENED?

A. Yes; a clay soil can be rendered less clayey (after deep draining in the first instance) by mixing with it sand, gravel, road-scrapings, ashes, building rubbish, or any other substances that help to separate the particles of clay. A sandy or gravelly soil may be made more solid by mixing with it a good deal of clay.

Q. Is it possible to reduce spongy PEat into garden soil?

$A$. Yes; the most spongy peat may be reduced to a solid and fertile soil by being mixed with other carths and with manure. 


\section{SECTION I.-MANURES.}

Q. What are MANures?

A. Manures are the substances employed for feeding and stimulating plants, and for exciting or altering the qualities of the soil by their union with it.

Q. Mention the General hends under which manures may be classed.

A. Manures may be divided into three classes: 1, animal and regetable manures; 2, mineral manures; 3, mixed manures.

Q. What are the most common $\triangle$ NIMAL and VEGETABLE manures?

A. Farm-yard manure, the manure of poultry, crushed or burned bones, guano, sea-weed, \&c.

Q. Are the excrements of ALL animals equally powerful as manures?

A. The excrements of animals fed on flesh are more powerful than those of animais fed on veyetubles alone; the excrements of animals fed on oily substances, such us oil-cake, are much more powerful than those of animals fed on straw alone. Even the manure of cattle fed on turnips and grain is far better than that of straw-fed cattle.

The manure of fat animals is of more power than that of lean ones. The manure of the pig, which consumes animal food, is preferable to that of the cow, which feeds on vegetables only; and the manure of the sea-fowl, which feed on fish, is more powerful than that of domestic [ow], fed on grain.

Q. Generally spealing, what is the most advastugeous metrod of applying the manure of stables, cow-Louses, \&c.?

A. 'The best mode of using stable manure, \&c., is in compost.

Q. What do you mean by a manure coMPoST?

$A$. By a manure compost is meant a mixture of fermented litter. from cow-houses, \&c., with mould, sods, peat, or any animal or regetable substances.

Q. What are the Nusres of the chief mineral manures?

A. Lime, gypsum, marl, sea-sand containing shells, phospluate of Time, common salt in some cases, \&c.

Q. By what DIstinguising Nane are those mineral manures, such as marl and shell sand, which contain portions of lime, called?

A. Mineral manures which contain lime in any form are called calcareous, which word signifies containing lime.

Q. Do mineral manures Exricir the soil?

$A$. Mineral manures can hardly be said to enrich the soil, but, by altering its defective qualities, and by preparing substances within it to feed plants, they are highly serviceable. Quicklime, in particular, acts powerfully on soil, by dissolving tough vegretable matter, such as peat, and by separating the particles of stiff clay.

Q. How do mineral manures act upon the sorr ?

A. The mineral manures loosen a stiff soil, improve its texture, and quicken its sluggish powers 
Q. Do not the mineral manures, in some degree, FEED plants?

A. 'I'he mineral manures, especially lime, enter into the substance of certain classes of plants. For instance, lime is found in the grains of wheat, yet it can hardly be said to nourish the growing wheat any more than the particles of lime which a laying hen picks up can nourish her, though it is necessary to her in forming the egg-shell. Yeast is requisite to make dough ferment, yet, though it assists in making good bread, it does not make it more nourishing.

Q. What is generally understood by the word LIME ?

A. By lime, farmers mean limestone or chalk burnt in a kiln; and quicklime means burnt lime before it has lost its caustic or burning power by exposure to the air or moisture.

Q. Is quicklime a GOOD INGREDIENT in a compost with manure?

A. Quicklime should never be mixed with manure; but marl, which contains lime in a very mild form, may be mixed with manure and earthy matter.

Q. Is quicklime USEFUn for any kind of compost?

A. Quicklime may be mixed with the surface sods of a peat bog, or any coarse vegetable and earthy matter, which it reduces by its caustic quality into fertilising mould.

Q. Wili the EFFECTS of lime LAST LONG?

$A$. The effects of lime will continue for six or seven years.

Q. Which is the LEAST VALUABLE of all the mineral manures?

A. Gypsum, or "plaster of Paris," as it is called, is the least valuable of the mineral manures for crops in general; but for topdressing plants of the clover tribe it is very useful, though it is doubtful whether it improves the soil.

Q. How should quicklime be APPLIED to ground in a fallow state?

A. Quicklime should be first reduced to a powdery state by slaking it with water, and then scattered over the ground, which ought to be in a perfectly dry condition, else the lime will form lumps of mortar, instead of being blended with the soil. It should be lightly raked or harrowed into the surface.

Q. Ought ANIMAL MANURE to be applied at the same time with IIME?

$A$. Animal manure should not be put on the ground for some weeks after the lime has been spread, because the lime would destroy the best qualities of the manure; and there are few cases in which it would be expedient to use lime in the same season on the same land. 


\section{SECTION II.-GROWTH OF PLANTS.}

Q. What is the SEED of a plant?

A. The seed of a plant is a living particle separating from it, and capable of growing into a new plant of the same kind.

Q. What ruakes a seed Grow?

A. Moisture, heat, and a free suppiy of air cause a seed to swell, burst, and push forth a root into the earth and a stem into the air.

Q. What More is necessary to make a seed thus break forth into life?

A. In general, it is necessary that a seed should be covered by earth, ol at least shaded from light, because light prevents a seed from undergoing a certain inward change which is necessary to make it start forth into life.

Q. What PART does a seed first put forth?

$A$. The part that first sprouts from a seed is the radicle; which soon becomes an active root, furnished with white threads called spongioles, which act as mouths to plants, by absorbing moisture and carrying it to the plant. The uses of the root are twofold: first, it enables the plant to take fast hold of the ground; secondly, it is also the means of carrying nourishment or food to the plant.

Fig. 175 .

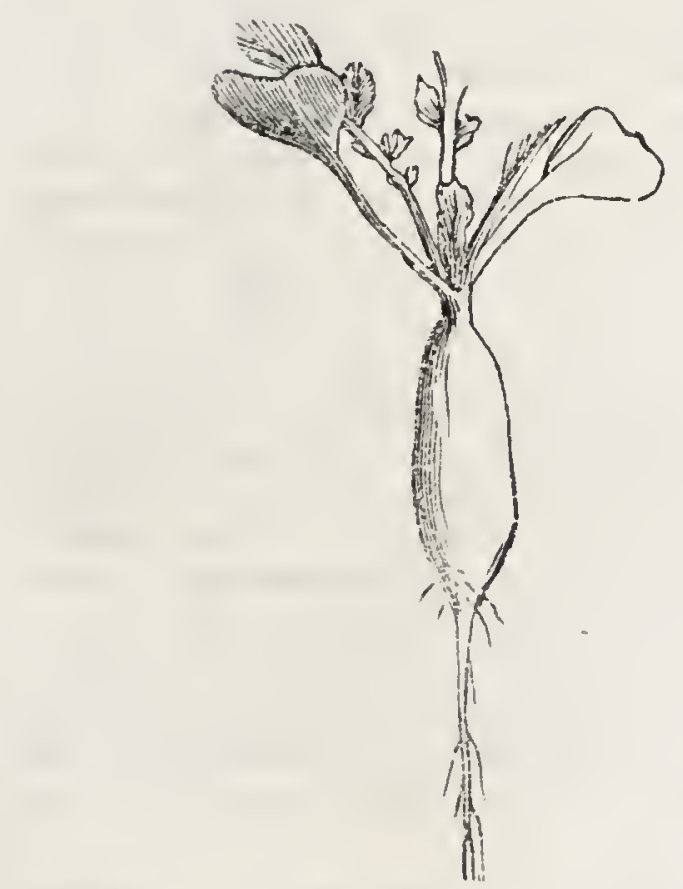

Fig. 176.

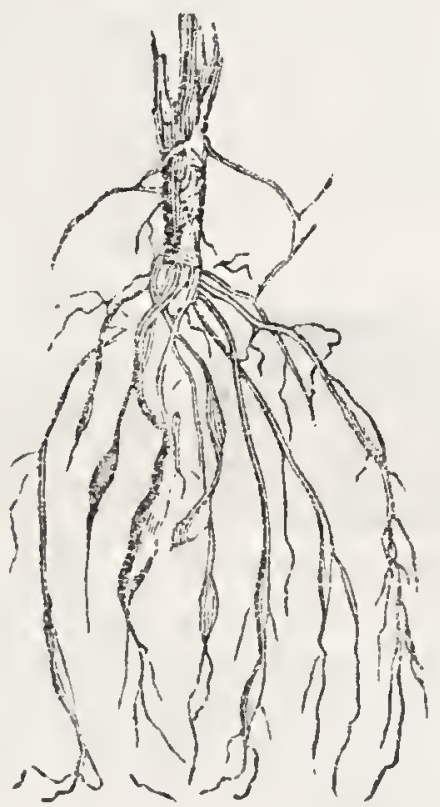

In the above engravings we have representations of various species of root. Fig. $17 \mathrm{~s}$ shows the radish, as a specimen of the spindle-shaped root; fig. 176 the fibrous root, as seen in the pasture grass.

In nearly all cases the end of the stem and the beginning of the root is most distirctly marked; the root seeks the ground, the stem seeks the air. But it occasionally happe is that stems become roots, and roots become stems. The Banian tree, for instanco, shoots down its stems till they reach the ground, and there take root, forming a great natural arbour, or temrle, in which hundreas of yeople may assemble. 
Fig. 177.

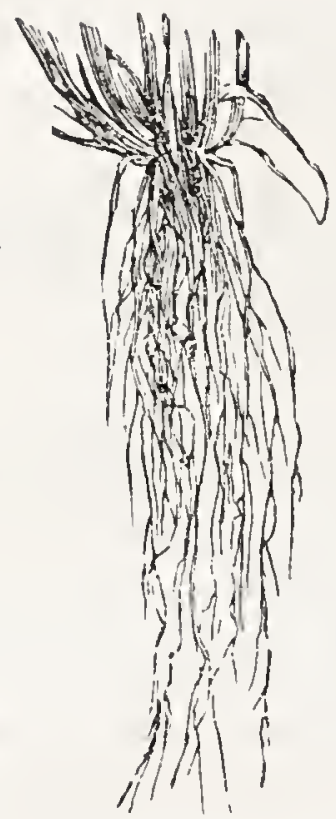

Fig. 173.

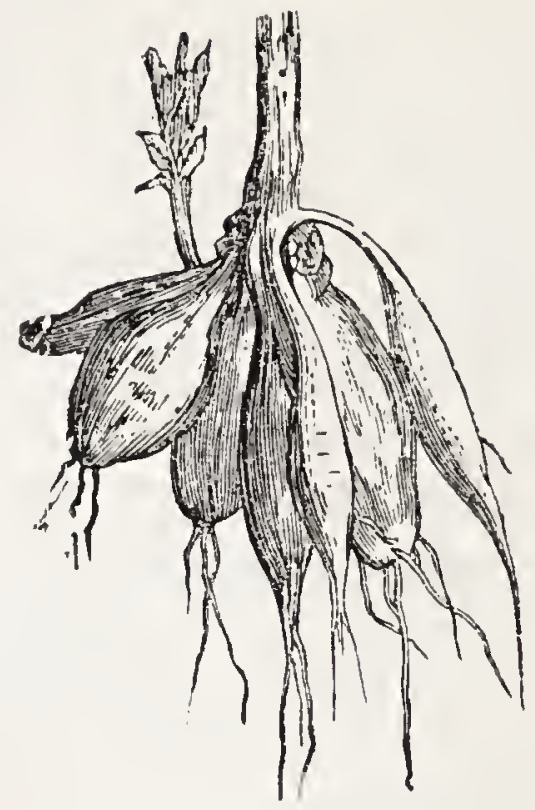

Fig. 177 shows the knotty root, the dropwort ; fic. 178 is a tuberous root, as seen in the dahlia.

Q. What is the SECOND part which a seed puts forth?

$A$. The second part which issues from the seed is called the plumule, which gradually lengthens into a stem furnished with leaves.

Iig. 179.

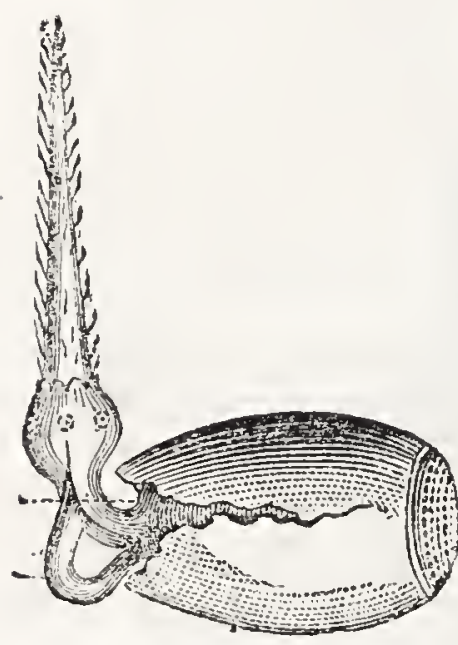

GERMINATION OF THF $\triangle \mathrm{CORN}$

The structure of the stems of vegetables is a very important point, and assists the botanist in separating flowering plants into two great divisions. "The acorn, on being planted in the ground, sends down its root and sends up its stem. At first this stem is a little tiny thing; year by year, however, it srows, until it at length becomes a large tree. If we now cut this tree across, and examine the structure of its section, or cut part, we shall find the appearance to be as follows:-In the first place, commencing our examination from without, we shall find the bark (cortex) separable into two distinct layers, the outer of which is termed cuticle, and the inner one liber. Passing onwards, we observe the woody fibre, and its central pitl. The woody fibre is apparently of two kinds; the outside softer, and termed alburnum; the inside harder, and termed by botanists duramen. Lastly, in the centre, comes the pith, or medulla. Regarding this section a little more attentively, we shall observe passing from the pith to the bark, and establishing a connection between the two, a series of rays, called medullary rays." Now, all these characters are found in the stem. They point out to us the true nature of the tree, and enable us to classify it correctly. A plant such as the oak is called an exogenous plant; the other great class are called endogenous. The meaning of these terms has been already explained. What we have to remember is, that these characteristics are ascertained by the stem.

Q. While this infant growth is adrancing, and before the roots are able to take up fool, how is the young plant rourismed?

A. The starch-like substance within the seed is changed into a species of gum and sugar, and is conveyed to the radicle and plumule by means of one or two seed-leaves called cotyledons, which act as nurses to the new-born plant until it can take its food without their help. 
2. What finally Becours of the SLED-Luaves?

$A$. When the true leaves shoot out from the stems, the seed-leaves perish.

Q. Of what do the TRUE LEAVES consist?

A. They consist of thin, flattened pieces of vegetable slin, enclosing a vascular or cellular tissue.

Q. What do you mean by vaScULAR TISSUE?

A. By vascular tissue is meant those little pipes or tubes which run through vegetables, just like veins and arteries through animal bodies, and which serve to carry juices from one part of the plant to another.

Fig. 180.

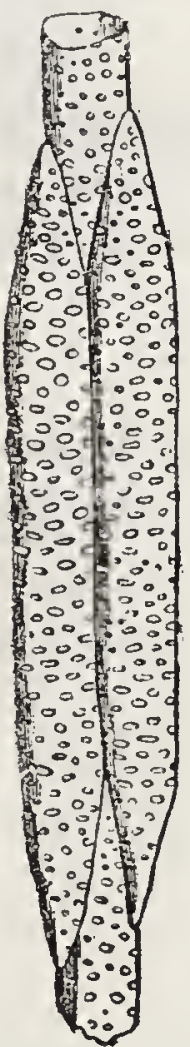

Fig. 181.

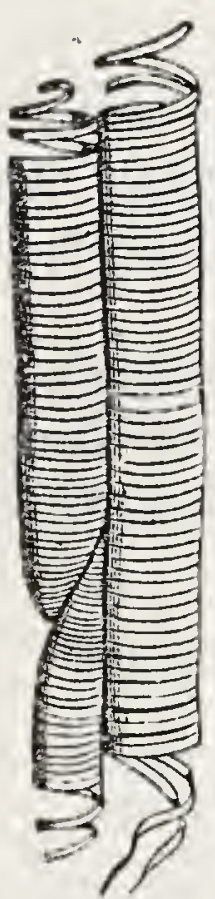

Fig. 182.

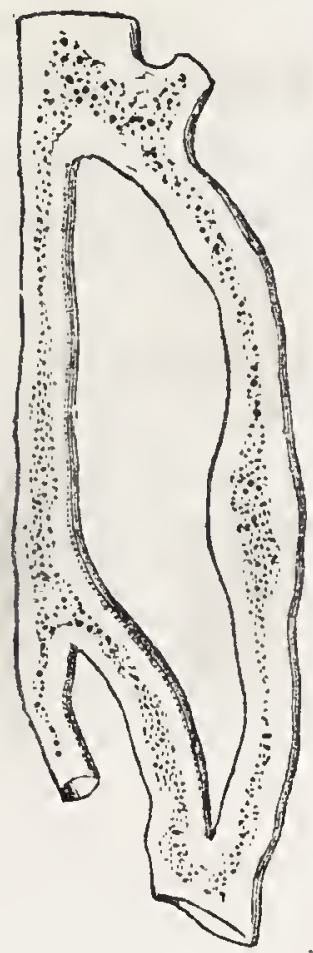

The forms of some of these vessels are seen in the above diagram. Fig. 1\&0 represents the dotted vessel of the clematis; fig. 181 the spiral vessels of the melon; fig. 182 the lactiferous, or juice-bearing vessels of the celandine. All these are magnified representations.

Q. What is meant by ceLLular TISSUE?

$A$. By cellular tissue is meant an assemblage of little cells. Their proper or natural form is oval, but this is often changed by their pressing against each other.

Fig. 183.

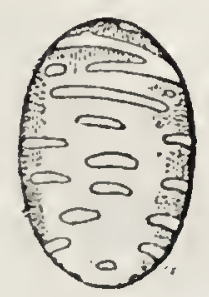

OVOID CELIS.
Fig. 184.

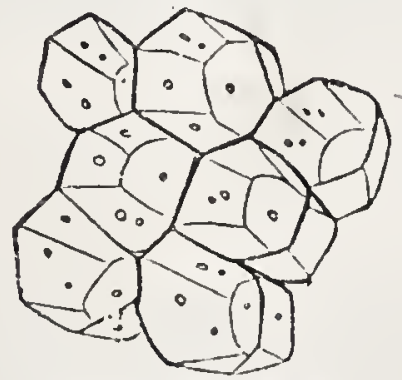

ANGULAR CELLS.
Fig. 185.

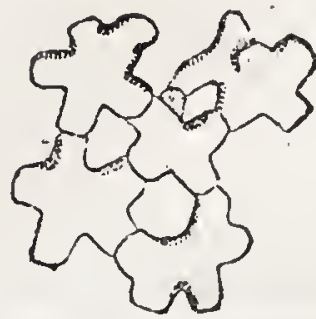

STAR-FORM CELLS. 
Q. What occasions the GREEN CoLOUR of leaves?

$A$. 'The presence of a colouring matter called chlorophyl, from twc Greek words, meaning yellowish-green and leaf. By a chemical change, this colouring matter turns to a red hue in autumn.

Q. What are the USES of leaves?

$A$. The uses of leaves are various; the principal use is that by which they act towards the plant as the lungs do for animals, and they may thus be considered the breathing apparatus of plants.

Q. What Varieties are there in leaves?

A. They vary in form, in the manner in which they are joined to the stem, in softness, hardness, thickness, thinness, and in many other ways.

Q. What are meant by the NERVES, or verns, of plants?

A. Nerves and veins in botany mean the same thing, and the terms are applied to those cord-like ribs which run under the surface of leaves.

Q. Are the nerves, or veins, of plants $\Delta$ LIKE in $\Delta \mathrm{LL}$ leaves?

A. No ; there are two great divisions, namely, those whose veins form a kind of network, and those whose veins run in straight lines without intersecting each other.

Fig. 186.

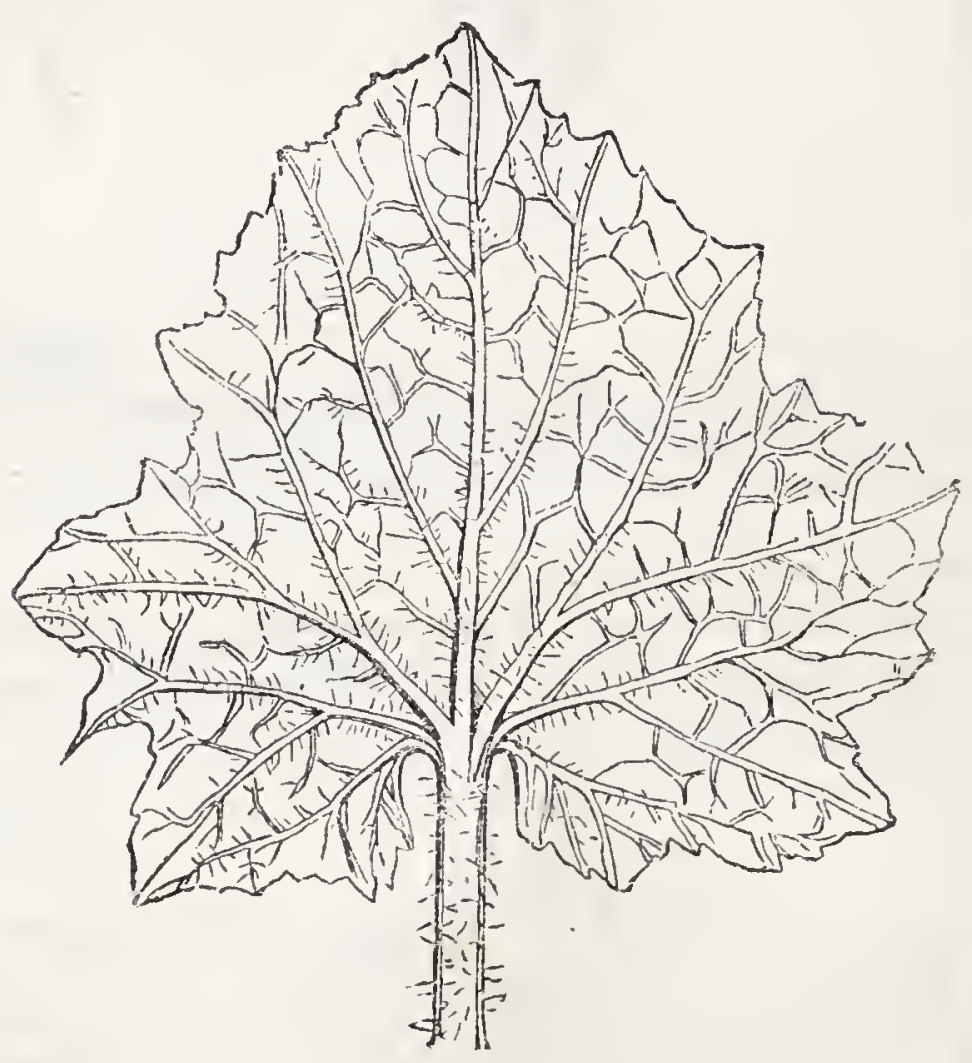

In the above engraving we have a representation of the melon leaf, which affords an excellent specimen of the net-work (reticulated) leaf; or those leaves in which the nerres and veins cross each other, and seem to spread over the leaf in every direction. 


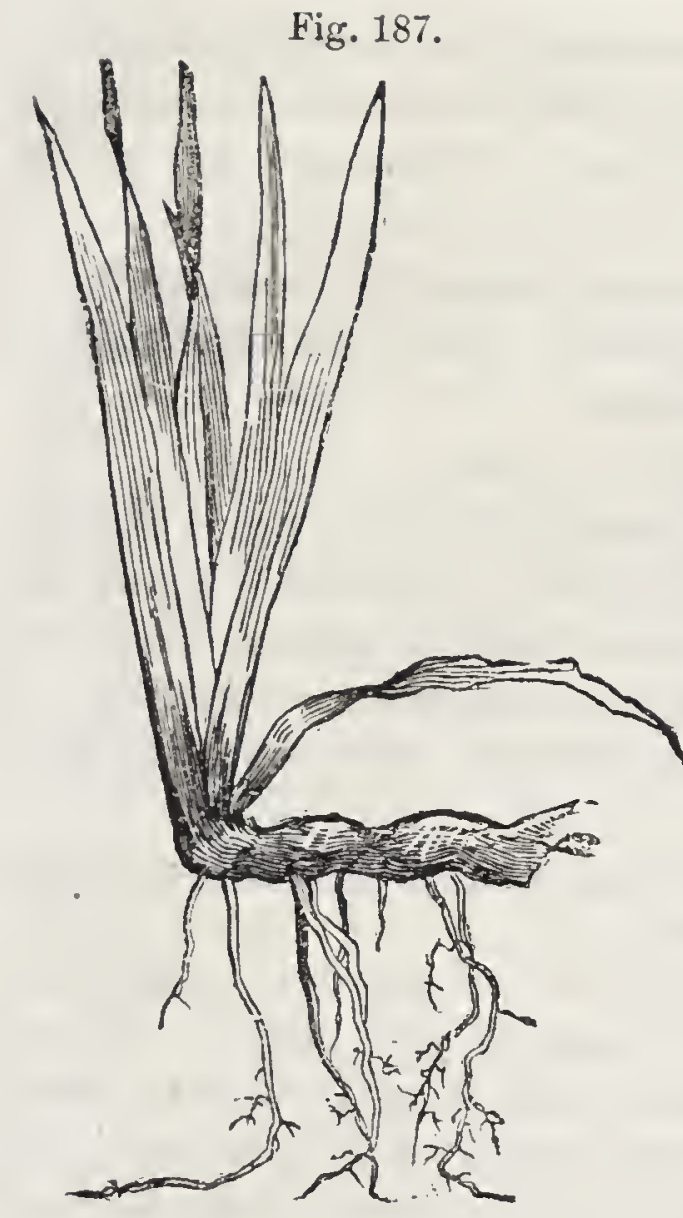

In the iris leaves we have an illustration of the other variety, namely, the straight-veined leaf. (Sce fig. 187.)

Q. Is there any GENERiL RULE as to the depth of covering necessary for seeds?

$A$. Experience teaches the gardener better than any rule what depth of covering is most suitable for seeds. A small, tender seed requires the merest sprinkling of sand or very light loam, barely enough to shade it and preserve moisture; whereas a bean, which has a very tough covering, will bear a heavy coat of clay, and will force its stem through it, when other seeds could not.

It is plain, that in a sandy soil seeds may bc sown deeper than in clay soils; an inch of clay might smother a seed which could bear an incls of sandy loam without injury or inconvenience.

Q. If the covering of seeds be necessary, how is it that so many seeds grow when SELF-SOWN on the surface?

$A$. Many self sown seeds, whether acorns or the light seeds of grasses, find sufficient shading from the light. An acorn, for instance, becomes buried under a covering of leaves, or falls into some chink in the soil, where it escapes from the light; and small seeds, if they have nothing else to cover them, are shaded for the necessary time by the seeds which lie over and around them.

Q. Is there any reason for supposing that a IIEAVY COVERING of seeds gives more depth and fixity to the roots than a light one affords?

$A$. It is a mistake to suppose that seeds must be well buried in order that the young plants proceeding from them may have a good hold of the ground, because a seed, when it begins to grow, plunges its roots downwards and throws its stem upwards from the seed; consequently, all the space between the surface of the soil and the seed is occupied by the base of the stem, and not by the roots.

Q. Is FRESHNESS of seed always to be DESIRED ?

$A$. Old seed is not generally to be depended upon; some seeds, particularly those of an oily quality, fail very soon after ripening; others, if kept in a dry state, retain their growing powers for a length of time.

The kidney-bean has been known to grow after one hundred years, and wheat-plants have grown from seed which had been taken from Egyptian mummies, and must have been thousands of ycars old. The soundness of seeds depends altogether, in many cases, on their having been kept in a dry state; dampncss, which mokes them grow if properly sown, causes them to rot if not sown.

Q. If the soundness of the secd be doubtful, what is the best way of TEsTriva it?

$A$. To test the degree in which seed is sound or otherwise, sow a 
certain number in a pan of earth. By counting the number of growing ones, the proportion of living to dead seeds can be ascertained, and the quality of seed to be sown calculated. Seeds are not to be rejected merely because they are old.

Q. How are the best seeds to be OBTAINED by persons who save their own seeds?

A. Those persons who save their own seeds should choose the best plants, whether fruits, flowers, or vegetables.

Q. In watering seeds and plants, what RuLes should be observed?

A. Neither seeds nor plants should be watered except when the sun has gone dorm, because the rays of the sun, acting suddenly upon the moistened plant, injure it; upon moistened ground, where the seeds have not yet appeared, the effect of watering in sunshine would be to draw forth the moisture from the place watered, and make it drier and harder than before.

Q. Why does Naturar RAIN in summer generally cause plants to arow more freely and with more vigour than the water from a watering-pot?

A. Rain water generally contains organic substances, ammonia, nitric acid, \&c., which invigorate and nourish the plant. When summer rain also is falling, the air becomes moist, and the sun, while its warmth is still acting, does not counteract, by his fiery rays, the good effects of that moisture, and the soil is then softened and disposed to the entrance of the genial rain.

Q. What KIND of WATen is the best for watering plants, seeds, \&c.?

$A$. The best water for using in the garden is that which contains the most nouristing substances, such as that of cesspools and ponds. Rain water also is good. When spring water alone can be obtained, it ought to be exposed some time to the air before it is used.

Q. What is the most convenient BREADTu of beds for all the close-growing plants, such as onions, and early parsnips, and carrots?

A. The most convenient breadth of beds for onions, parsnips, \&c., is four feet, with calleys between them; this breadth allows a weeder to weed and regularly thin the plants, and to loosen the soil about them, without treading on the beds.

Q. What kind of plants is it most difficult to TRAXSPLANT successfully?

A. Tap-rooted plants require the greatest care in transplanting; a light soil is most suited to them, and watering should be freely given to their roots.

Q. What is meant by the Roration of crops?

A. By rotation of crops is meant a regular succession of crops, so that the same kinds shall not follow each other immediately.

Q. Why is a CHANGE of crops to be RECOMMENDED ?

$A$. Since plants of the same sort consume the sarie elements of food contained in the soil, it follows that the continued cultivation of the same plants will, more or less, deprive the soil of the elements on which they feed.

Where frequent and abundant manuring is given, regard to rotations is of no great 
importance; but in a garden poorly manurel, and where, therefore, the dependencies on the soil itself for the nourishment of the crops, a rotation of them is absolutely necessary.

Q. Are there not, however, many perennial plants which must remain in the SAMIL GROUND for several years?

A. Such perennial vegetables as asparagus, rhubarb; and artichokes ought not to be displaced within less than eight or nine years, or until they begin to fail; but they are manured so amply every year that they do not depend entirely on the soil for their support: Crops. which strike deep and occupy the ground long, should be succeeded by plants which pierce but a little way under the surface.

Q. Does garden soil require REST betwcen the different rotations?

4. No soil to which manure can be applied ever requires what is called rest. The soil never becomes, as it were, tired of producing: all it requires is to be refreshed with those substances which may have been consumed by the plants it has nourished. If left unsown by the hand of man it will produce rotations of weeds rather than be at lest.

Q. Is it not desirable to have within the year as MaNY Rotations as possible?

$A$. The greatest profit is drawn from gardens in which the crops are raised and consumed in rapid succession. The market gardener, who can clear out a square of any sort of vegetables in a single week, and resow or replant it immediately with another kind of plants, makes more of his ground than the person who consumes his regetables very slowly, and yet does not break up a square until the whole crop on it has been cleared off. The season for a new crop is often lost by such delay.

\section{SECTION III.-PROPAGATION OF FRUIT-TREES.}

Q. How are FRUIT-TREES raised?

A. Fruit-trees are raised from seeds, by grafting and budding, by cuttings and layers, sucliers or offsets, and slips.

Q. Are fruit-trees GeveralLY raised from seeds?

A. Fruit-trees are seldom raised from seeds, except by nurserymen, when they want to obtain new varieties.

Q. Why are not fruit trees commonly raised from SEEDS?

A. Pecause there is no certainty that the seed of fruit-trees will produce a tree yielding the swme lind of fruit; and also because a ree is much longer growing from seed than from any of the other modes just mentioned; for instance, an apple-tree which would be many years in growing from a seed, may soon become a bearing tree from a graft.

Q. What is Grarimg?

' 1 . Grafting is the joining together of a cutting of one tree to mother, in such a way as that the tree on which the cutting is placed sends up its sap into the cutting, and makes it grow and become a tres 
Q. What is a cutting used in grafting CALLED ?

A. A "scion ;" and the stem on which this cutting is placed is talled a "stock."

Q. II

A. By no means: the stock and the scion may be different as to variety, but they must be of the same family of plants; for instance, peach and apricot trees are often budded upon a plum stock, which answers better than the natural stock in many cases. The new tree thus produced partakes of the hardiness of the plum-tree, without losing the delicate flavour of its fruit. The varieties of the cultivated apple are grafted on the wild crab-apple stock; and the most beautful varieties of roses may be budded upon the brier.

Q. What do we mean by BUDDING, in contradistinction from grafting?

A. In budding, the bud of one tree, with a portion of bark adhering to it, is introduced into the bark of another tree; but in grafting, the shoot is taken along with the bud and inserted in the stem; whereas in budding nothing but rind is taken with the bud to be inserted in the rind of the stock. The budded tree will be a year slower in its growth than the grafted tree. Grafting is performed in spring, and budding in summer.

Q. Since trees, then, are raised more quickly by grafting than by budding, why are they ever BUDDED?

A. Because stone fruits succeed better by budding than by grafting. Plants, also, of very slender stems will not bear the operation of grafting, though they will bear that of budding.

Q. Is there MORE than ONE METHOD of grafting?

$A$. There are several methods. The French reckon forty-three; but many of these are more curious than useful.

Fig. 188.

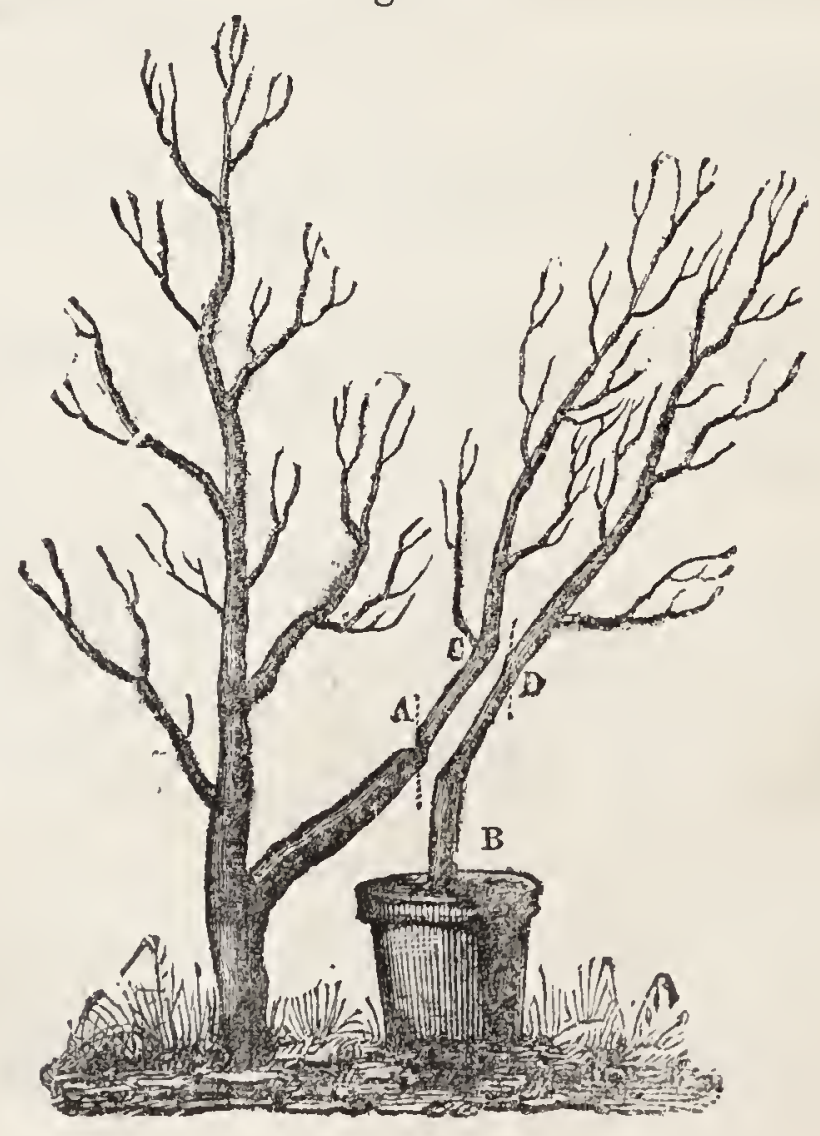


Q. What is Crown Grapting ?

A. Crown grafting is applied to large limbs with thick bark, or to large stems; in either case these are divided at right angles; the bark is raised by thrusting in a tapering, flattened piece of smooth hard ivood or irory between the wood and bark; this being withdrawn, the end of the scion, the plant to be grafted, is inserted and bound wits a ligature. Some idea of the common process of grafting may br found by observing the foregoing engraving (fig. 188), the scion A 6 being grafted on the stock, as $\mathrm{B} \mathrm{D}$.

Tig. 189.

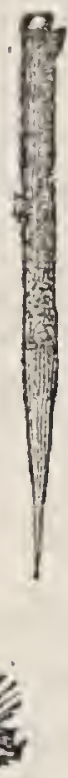

Q. What is CLEFT GRAFTING?

A. Cleft grafting is performed by splitting the stock; across the top, and inserting the scion cut like a wedge. (See fig. 189.)

\section{Q. What is SADDLE GRAFTING ?}

A. Saddle grafting is performed in a similar way to that of cleft grafting, except that the stock, instead of the scion, is formed like a wedge.

Q. What is meant by the sTock and the scion?

$A$. The stock is the plant on which the grafting is made; the scion is the stem which is inserted into the stock.

Q. What are cutrings?

A. Cuttings are small portions cut from a shoot of the preceding year, with a few leaf buds on them. The shoots should be cut smoothly across, below a leaf bud.

Q. How are cuttings made to Grow ?

$A$. Some cuttings are put into a moderate hotbed; others into any moist, loose earth mingled with sand, but covered with handglasses, and shaded from the light until they have struck root. Some do not require any glass covering.

Q. At what SEASON of the year should cuttings be taken?

A. Cuttings should be taken in early spring, before the sap has risen.

Q. What is LATERING?

A. Layering is the bending cioun into the earth of a branch (see fig. 190), after being cut about half way through, B, and lit upwards about half an inch from the cross cut, $\mathrm{C}$; it is then pegged down to the earth, A, in which it will take root at the wounded part, if that be properly covered with earth.
Fig. 190.

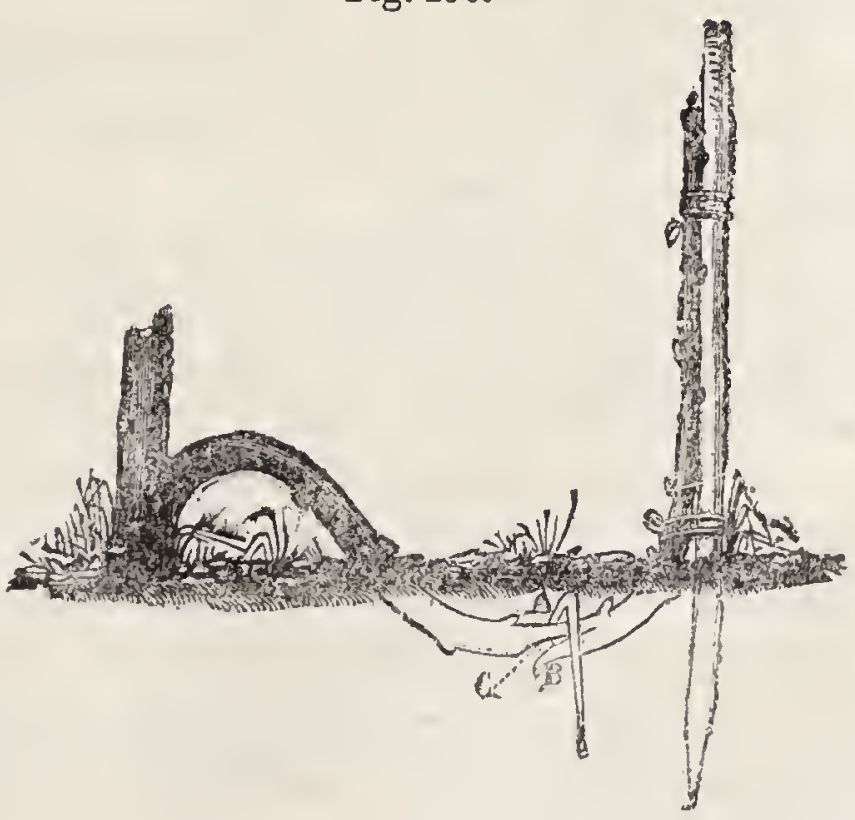




\section{SECTION IV:-FLOWERS AND WEEDS.}

Q. How are flower plants CLASSED in a general way?

A. Flower plants are distinguished by the terms bulbous, tuberous, and fibrous-rooted.

Q. How are flowers propsGated?

A. Flowers are propagated by seeds, buds, layers, suckers, offsets, cuttings, and by dividing. or parting.

Q. What KINDS of FLOWERS are always raised from seeds?

A. The flowers called annuals are always raised from seed.

Q. What are ANNusis?

A. Annuals are those which live during one year only.

Q. What are those plants called which are raised from seed ONE YEAR, bloom the 刃EXT, and then FAIL?

A. Such plants are called biennials.

Q. Have not $\Delta \mathrm{LL}$ plants fibres?

A. All plants have threadlitie fibres, which are, in fact, the mouths of the roots; but the plants we have last mentioned are especially distinguished as fibrous, because they have neither bulb nor tuber.

Q. What is a BULB?

A. A bulb is a ball consisting of either scales or coats, one within the other, which, when placed under ground, pushes forth a stem, roots, and all the other parts of a plant.

Bulbs are, in fact, underground buds : they. can be removed from one place to another without injury; they contain within themselves the elements of future plants; their roots perish with their leaves in winter; and bulbs in that state are incapable of drawing any nourishment from the earth?

Q. What is a toBer?

A. A tuber is a plain, solid root, like a potato; whereas a bulb is composed of scales or coats.

Q. How are tulips, and other bulbous-rooted flowers, propaGaTed?

A. Bulbs are usually propagated by offsets from the main bulb.

Q. What are ofFSETS?

A. Offsets are young bulbs formed on the old one, and which, if broken off and planted, will grow.

Q. Is there any RULE to be observed respecting the sIzEs of the pots in which plants wre to be kept?

A. Plants should at first be put into the smallest pots they will srow in. This rule is particularly to be observed in the potting and shifting of annuals.

Q. What is meant by INFIORESOENOE?

A. It means flowering; but by this term botanists understand, not coniy the flower itself, but the rarious parts which belong to, and are anited to it. 
Q. How is a flower ATTACHED to the stem of a plant?

A. By a small branch called a peduncle or pedicle.

Q. What is meant by the phrase DefINITE INFLORESCENCE?

A. It means that the branch or stalk ends in a flower.

Q. What is meant by INDEFINITE INFLORESOENCE?

A. A flower stalk which gives off, as it proceeds, little flower shoots on either side, but does not end in a flower.

Q. What are BRACTS?

A. Bracts are the small leaves, from the axils of which flower's grow.

Q. What do you mean by Axrus?

A. Axil, or axilla, is the angle formed by the junction of a leat stalk with the stem or branch. The literal meaning is arm-pit.

Bracts, although their.usual appearance is green, like a leaf, sometimes undergo very strange modifications. Thus the pineapple is, in reality, nothing more than an assemblage of fleshy bracts; and the scale of the fir cone is nothing more than hard leathery bracts. In proportion as bracts : are developed nearer to a flower, so does their natural green colour give place to the colour of the flower itself. Oceasionally, the flower actually springs from the upper surface of a bract, as is the case in the linden. (See fog. 191.)

Fig. 191

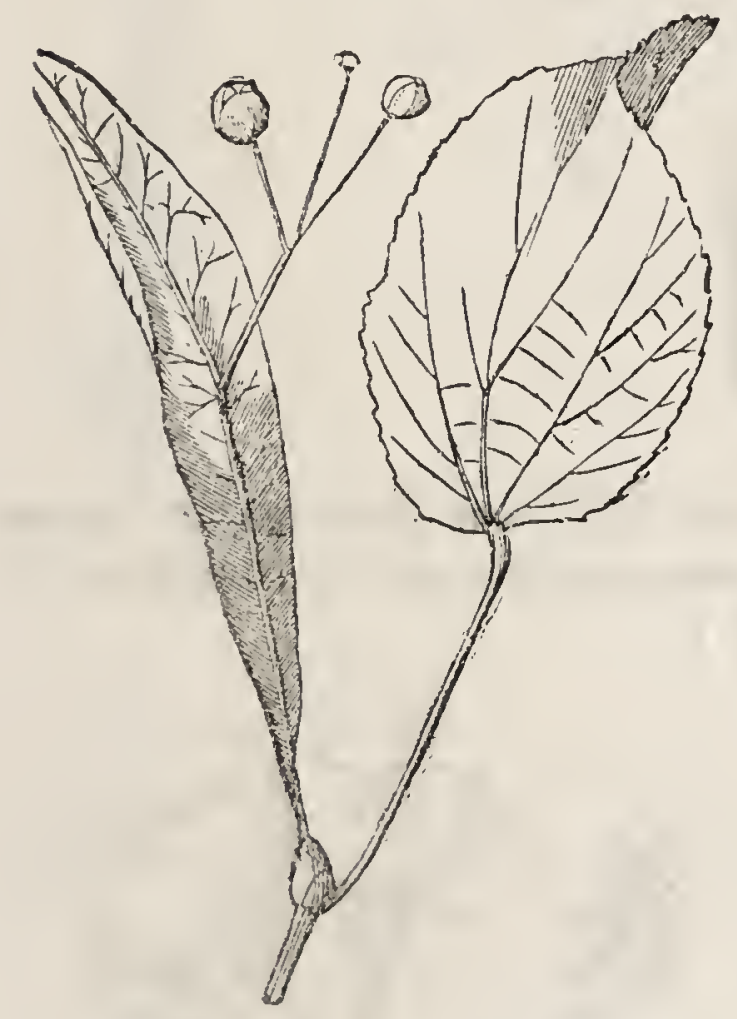

FLOWER OF TIIE LINDEN TREE.
Fig. 192.

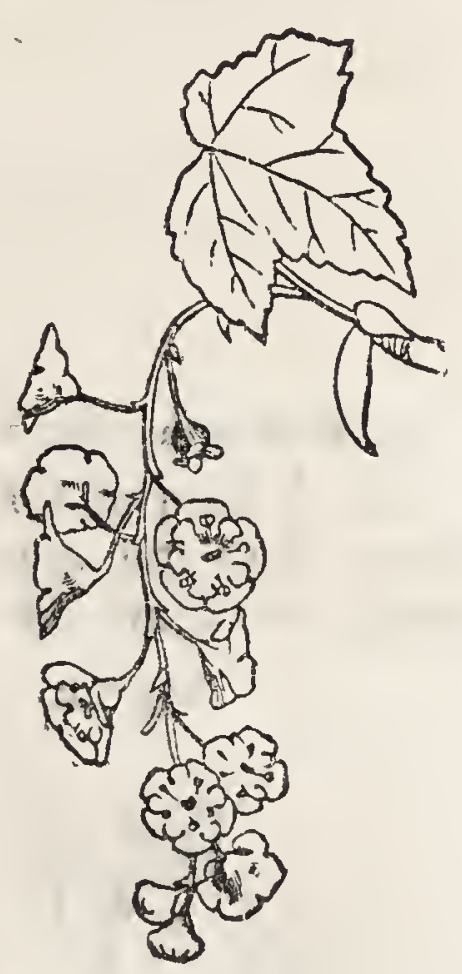

BACEME OF THE CURRANT:

Q. What is meant by a RACEME?

A. The term raceme is applied by botanists to those pedicles which are almost equal in length. Black, red, and white currant trees are examples of this kind. (See fig. 192.)

Q. What is meant by a COMPound RACEME?

A. 'The term is applied to that form of flowering where the pedicles grow from the stem; but instead of each bearing at once a flower at the end, ramify a third and even a fourth time. Of this we have an example in the horse-chestnut. (See fig. 193.) 
Fig. 193.

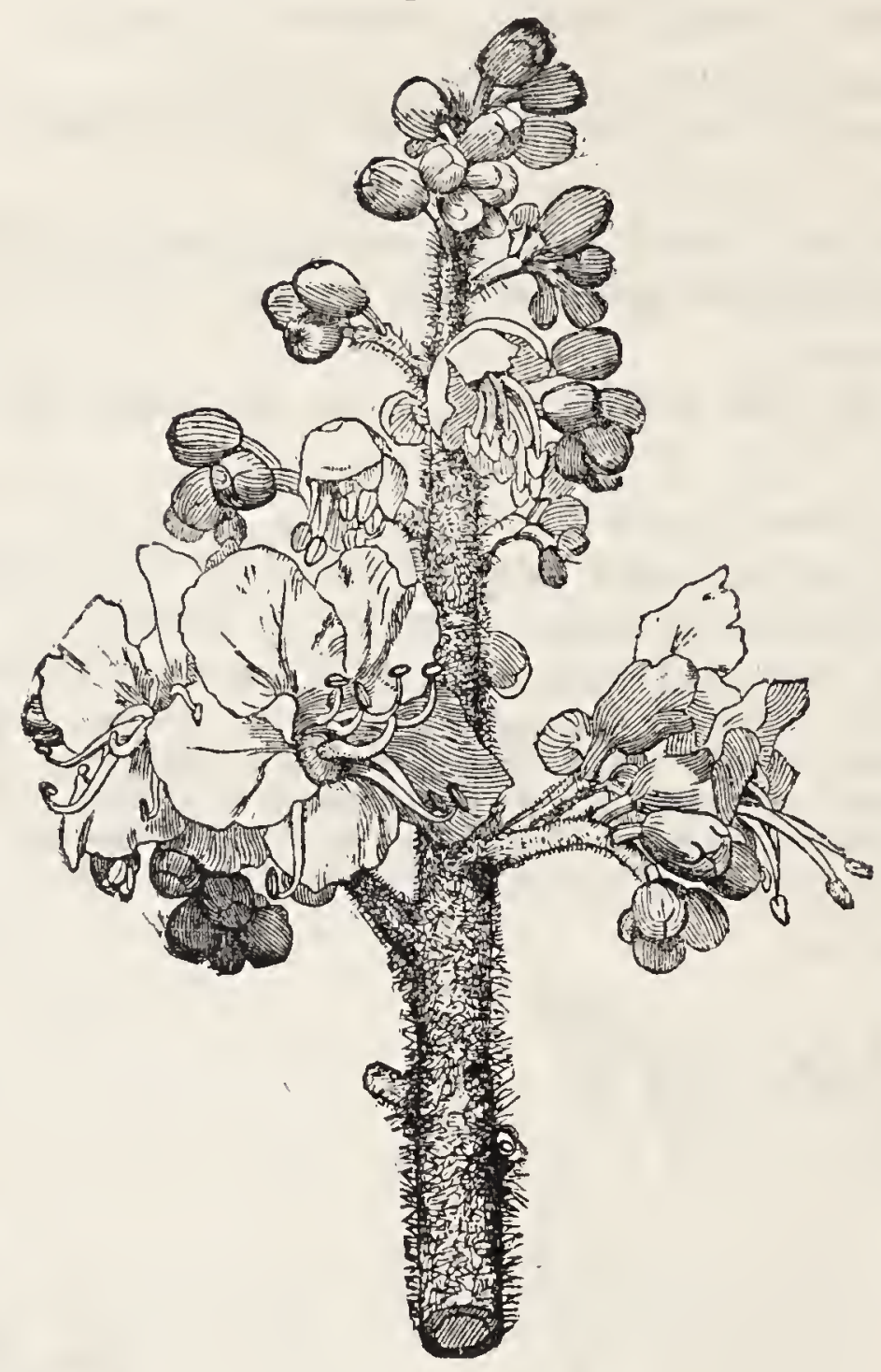

Q. What is the CORYMB?

A. A kind of inflorescence in which the lower pedicles are much longer than the upper ones; and, consequently, end at the same or nearly the same level. (See fig. 194.)

Fig. 194.

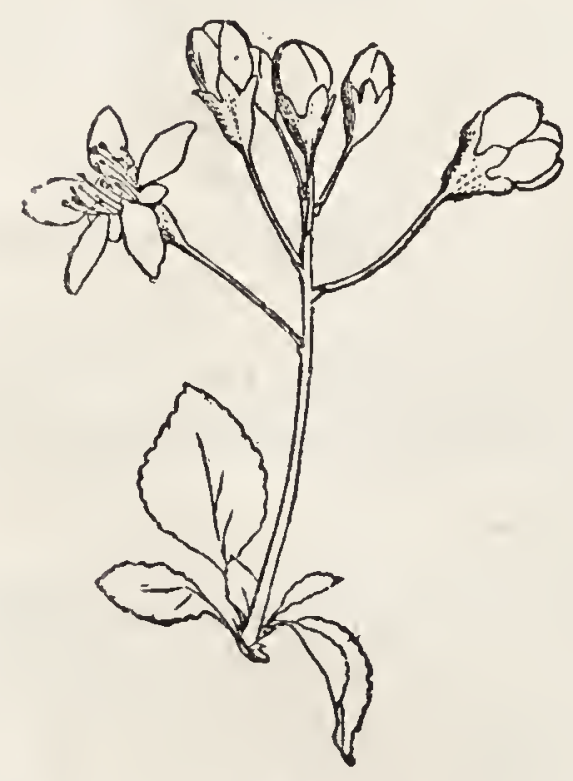

Fig. 195

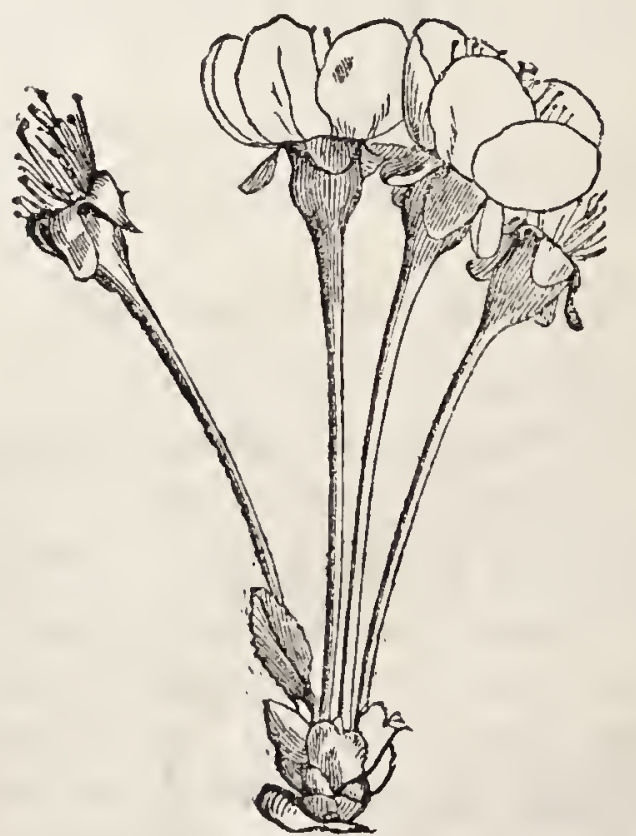

ORYIIB OF THE MOHAIEB CHERRY. SIMPLE UMBEL OF THE COMMON CHERRY. 
Q. What is meant by the UMrBeL?

A. It means that kind of infloresence in which the pedicles are of the sume length, and rise from the same part of the stem, stretching out like the ribs of an umbrella. (Sce fig. 195.)

Fig. 196.

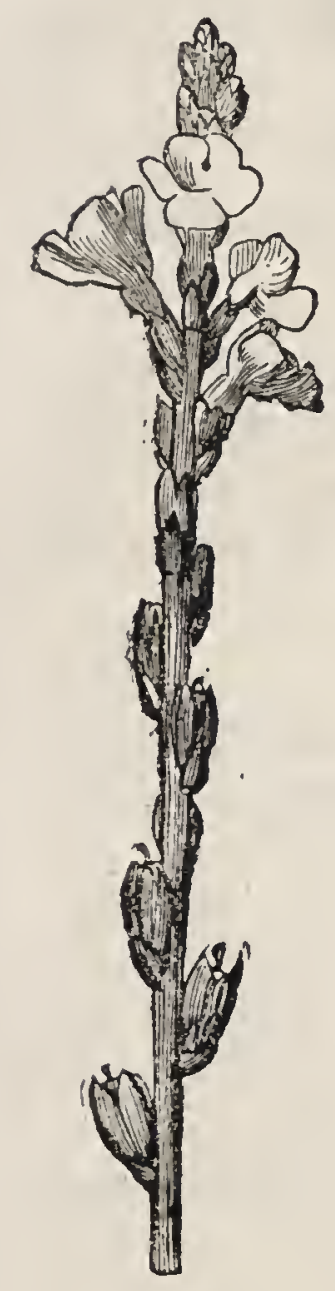

Q. What is meant by the SPIkE?

$A$. The term is applied to that species of inflorescence in which the pedicles are vanting, and the flowers accordingly are without stalls, as may be seen in the vervain. (See fig. 196.)

Q. Mention the various NAMES given by botanists to the different parts of a flower.

A. The whorls, calyx, petals, corolla, stamens, and pistils.

Q. What is meant by the term WHORL?

A. A circular row or round ring. In examining a flower it is found to consist of several circular rows of organs, and to these are given the name of whorl.

Q. What is a CALYX?

$A$. The calyx is the outermost whorl of a flower. It is formed of a number of parts called sepals, which are usually green.

Q. What are PETALS?

A. Small leaves, which together form what botanists call the corolla of a flower. (See fig. 197.)

Fig. 197.

Q. What are stumens?

A. The term stamen is given to those whorls or circular rows of organs vithin the corolla; they are the male part of the plant. They are divided into a filament, or threadlike portion, with an antler or head. This antler or head is covered with a dust called pollen.

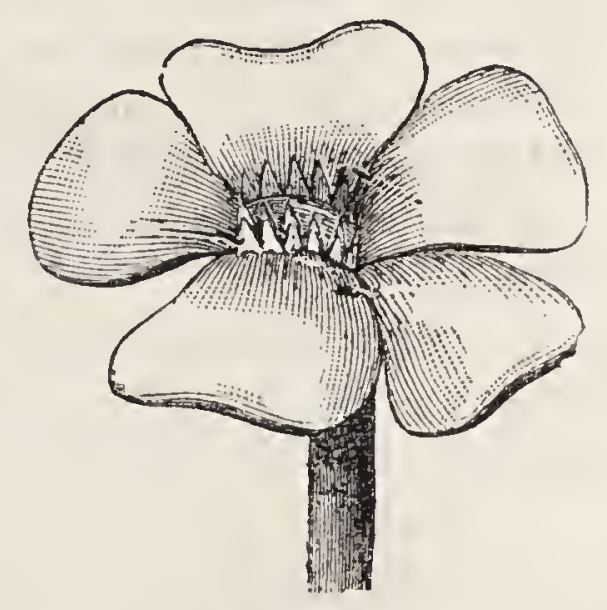

COROLLA OH THE RANUNCULUS.

Q. What are PIsTIIs?

$A$. Inner whorls or circuiar rows of organs which spring from what is called the ovary belon, and ending in what is called the stigma above: the intermediate part being called the style. The pistils, or carpels, as they are also called, are the female parts of the plant. 
Fig. 198.

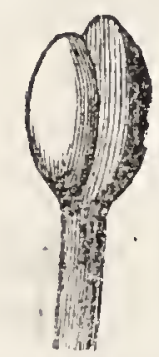

STAMEN OF THE RANUNCULUS.
Fig. 199.

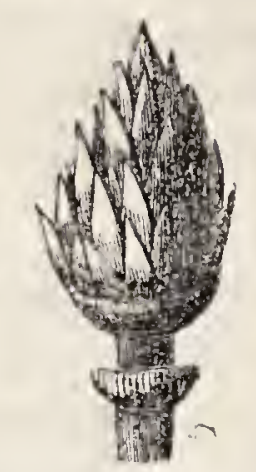

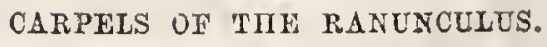

Q. What are to be considered as WEEDs in a garden?

A. Any plants that grow in a garden of themselves, and are not wanted there, may be called weeds; thus, a potato-plant, though not a weed, would be deemed and treated as such if it intruded itself into a bed of flowers or into a row of peas. The presence of real weeds should not be permitted among any cultivated crops.

Q. Why should not weeds be ALLOWED to GRow with the crops?

A. Because weeds rob the crops of much of the nourishment which the soil contains, and take up the room of valuable plants.

\section{SECTION V.-DRAINING.}

Q. What do you mean by the DrAINING of land ?

A. By the draining of land is meant the removal of the superabundant moisture by drains made in a regular manner.

Q. What are the COMMON CAUSES of excessive moisture in land?

$A$. The common causes of excessive moisture in land are the lodgment of rain water on a clay soil, and springs bursting from their confinement in the undersoil to the surface, by reason of the closeness of the undersoil, which will not let them pass downwards.

Q. What is the EFFECT of warm rain falling on loose or porous land, on which, of course, moisture does not rest?

A. Rain falling through warm air on porous land carries heat inio the free soil, through which every drop passes easily, and thereby forces the growth of plants.

Q. What DEPrn of drain is generally recommended?

$A$. The depth recommended for general drains is from three to four feet; the outlet drains, to receive the water which runs from the others, must, of course, be deeper than these.

Q. Why would not drains of a LisS DEPTI answer the purpose?

A. Shallow drains are less likely to intercept any underwater arising from land springs, and they do not afford sufficient depth of dry soil for the roots of such plants as grow deeply. The water 
which flows from shallow drains after heavy rains carries with it sand, which is so precious in a clay soil, and manures also; and shallow drains do not cause such warmth in the undersoil as deep ones.

Q. But when the surface moisture becomes warmed by the sun, will it not conduct the warmth Dowwing into the soil?

$A$. Surface moisture does not send down heat when it becomes heated by the sun, for its heat rises upwards in vapour, being lighter than the air, just as the steam of water boiling in a pot rises upwards. In fact, moisture lodged in the soil prevents heat from entering: into it.

\section{GEOLOGY.}

\section{CHAPTER LXIII.}

\section{FORMATION AND STRUCTURE OF THE EARTH'S CRUST:}

Q. What is GEOLOGY?

A. Geology is the naturat history of the earth. It comprehends. the investigation of its structure, and the character and causes of the changes which, since its creation, have been continually going on upor its surface, and in the organic and inorganic kingdoms of nature.

Q. What is the DeRIVATron of the word "geology?"

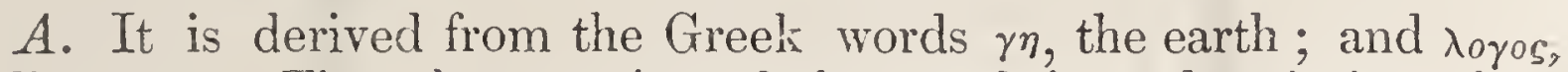
a discourse. Thus the meaning of the word is a description of, or discourse upon, the earth; that is to say, an account of the earth as regards its structure, and the different changes it has undergone since its formation.

Geology admits of division into descriptive geology; dynamic geology, which treats of the forces by which the surface of the earth has been modified; and into practical ant cconomic geology, embracing the application of geological science to mining, road-making, architecture, and agriculture.

\section{Q. What is the ronsr of the earth we inhabil?}

A. The earth may be described as a planet, about eight thousand? miles in cliameter, and in shape resembling an orange.

Q. What is the DENSITY, or WEIGHT, of the earth, compared witl the weight of ax EQUAT BULK of WATER?

A. It is five and a half times heavier than an equal bulk of watel.

Q. As tlic arerage density of all the materials upon the surface of the earth is only ahout three times greater than that of water, what INEERnNeE can we draw respecting the INTERTOR of the earth?

A. That it is composed of materials which are heavier than the materials which malie up the surfuce, probably metals. 
Q. What proportion of the earth's surface is covered by the ockAN?

A. About three-fifths.

Q. How MocI cf the earth's surface may be considered habitable by man?

$A$. Not more than one-fifth - a large part of the surface not covered by the ocean being occupied by vast bodies of fresh water, polar ice and snow, deserts, swamps, \&c.

Q. What is the diameter of the earth from pole to pole?

$A$. Seven thousand nine hundred and sixteen miles.

Q. How many square miles are embraced upon the surface of the earth ?

$A$. The surface of the globe is calculated at two hundred and eleven million square miles, of which about one hundred and fifty millions are covered with water, and sixty-one millions appear as land.

Q. What is that portion of the mass of the earth CALLED which man has been enabled to examine and study?

$A$. The crust of the earth.

The phrase "crust of the earth" involves many important principles in geology. The very word crust implies a surface that has undergone some process of hardening, and that retains beneath it some materials in a condition different from itself.

Fig. 200 .

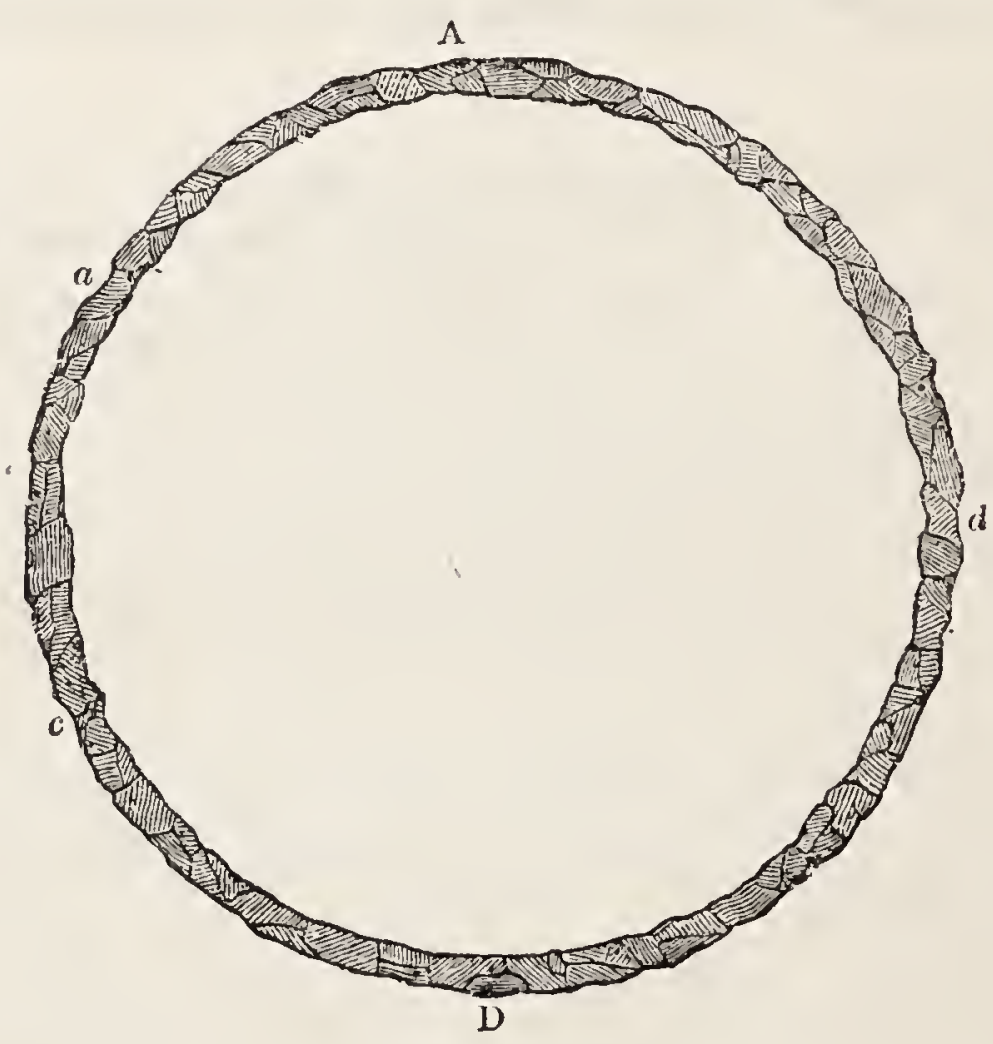

The above illustration represents the first hardening of the crust of the earth. The phrase crust, in geology, implies that the whole body of the earth was once in a state of fusion ; that it was once all in a melted state-glowing, burning, and flaming; and that it gradually cooled, until a crust, or surface, hardened all over it. The crust of the earth is at least four hundred miles in thickness. In observing this crust, as shown in the engraving, it will be observable that the outer lines are not even, but rugged. The onter line of the circle is full of dents and hollows, and of swellings and points. Every one of these indentations, marked $a, c, d$, represent deep hollows, or extensive valleys, some miles deep, and sufficient to form the bed of a gulf, or inland sea. The varied changes of weather and falls of rain would inaterially alter the form of these indentations; sea would be made to join sea, and oceans formed ; and land would be elevated by sand being deposited in layers at the bottom of the floods. 
Q. What is the THICKNEss of this crust?

$A$. The thickness of the earth's crust, from the highest mountain peaks to the greatest natural or artificial depths, is about 400 miles. As the entire thickness of the earth, from A to D (see fig. 200) is nearly 8,000 miles, that portion of its structure which man has explored is therefore insignificant compared with the magnitude oi the whole.

Q. What is the FIrST important geological fuct which it is necessary to learn?

A. That the earth was at first a mass of melted matter, which has gradually cooled at the surface, and is now covered with a crust.

Q. Has not all MELTED MATTER a tendency to EXPAND?

A. Yes; and the heat of the melted matter beneath the crust of the earth's surface has matericlly changed its form in many important. respects.

\section{Q. What cLAvGrs are thus effected?}

A. Mresses of matter are upheaved, and fissures or openings are made in the earth's crust.

Q. What ormer cranges take place in the earth's surface?

$A$. Other and important changes are produced by water in the formation of seas, oceans, \&c., and the carrying deposits of sand to lower levels.

Q. What is the nEIGHT of the most elevated mountain summit upon the earth's surface?

A. The mountain Dhwalagiri, the highest peak of the Himalayas, in Central Asia, is estimated at twenty-eight thousand feet above the level of the sea, being more than five miles of perpendicular altitude.

Q. What is the greatest XATURAL DEPRESSIox upon the earth's surface?

A. During the year 1853 soundings made in the South Atlantic Ocean showed a depth of seven thousund seven hundred fathoms, or about eight miles. This is the greatest depression known to exist upon the earth's surface.

Q. What are Rocks?

A. Rocks, in geology, are the mineral masses which compose the crust of the earth. The term rocks includes not only those masses which are in a solid state, as granite, limestone, \&c., but all those materials which are soft and yielding, as beds of clay and sand.

Q. What is the coxposition of rocks?

A. They consist principally of combinations of iron, lime, and silex or silica, the latter substance forming nearly one-half of all the materials composing the crust of the earth.

Q. Into what wwo great cuAsses are rccks divided?

$A$. Into stratified ruckis and unstratified rocks.

Q. When are rocks said to be stratiried?

$A$. When they are divided into beds, like the leaves of a book, or 
several books, or pieces of cloth, piled upon each other, they are said to be stratified.

Q. The stratified rocks are also denominated AQUEoUs rocks: Wr have they received this designation?

A. Because they were evidently deposited from vater, where, for the time, the substances of which they were composed were either chemically or mechanically suspended.

Q. When are rocks said to be UNSTRATIFIED?

A. When no traces of beds or layers can be detected, and the rock forms merely a great mass of mineral matter, it is said to be unstratified.

Q. If the mud and sand deposited by rivers, or other bodies of water, at the present time, were hardened into rocks, would the rocks so formed exhibit sTratrFicatiox?

A. They vould; water holding matter in suspension deposits the heaviest particles first, and afterwards the lightest. Bodies of water are sometimes charged with clay, and again with sand, owing to inundations or rains. These different substances would consequently be deposited in separate layers, one upon the other. Hence we infer that rocks consisting of layers, or strata of different thickness, and often of different materials, resting parallel upon each other, have been deposited from water.

Q. The unstratified rocks are also denominated "IGNEOUS or PLUTONIO rocks :" WHY have they received this designation?

A. Because they have evidently been in a melted or fused state through the action of intense heat.

Q. What are Exdsrpues of the igneous rocks?

A. Granite, the crystalline limestones or marble, trap, serpentine, quartz, lava.

Q. What IVIDENCr have we that these rocks were once melted?

A. They penetrate other rocks in the form of veins, they possess a crystalline structure, and are destitute of any layers or definite order of arrangement. They form great masses of mineral matter, without symmetry or shape.

Q. May not some of the so.called "igneous rocks" have been also DEPosimED from WATER, and been originally STRATIFIED RoCKS?

A. Many of the igneous rocks were zndoubtedly deposited from water originally in a stratified form, and have been subsequently changed, and caused to assume their present appearance through the agency of teat.

On the intensity of heat below the earth's surface would depend the thickness of the crust. All melted matter has a tendency to expand, or rise; and accordingly the melted matter within the eartl acquires an elevating force, which causes the mass that lies on its surface to swell out in the furm of a curve. In this process the heat produces many cracks and fissures. If we imagine that the crust of the earth were cut through, so that we could see the face of it, the cracks and fissures would then appear like those seen in the accompanying diagram; and the body of the crust would appear between the different fissures something like wedges whose sharp points had been cut off. The burning heat, 
Fig. 201.

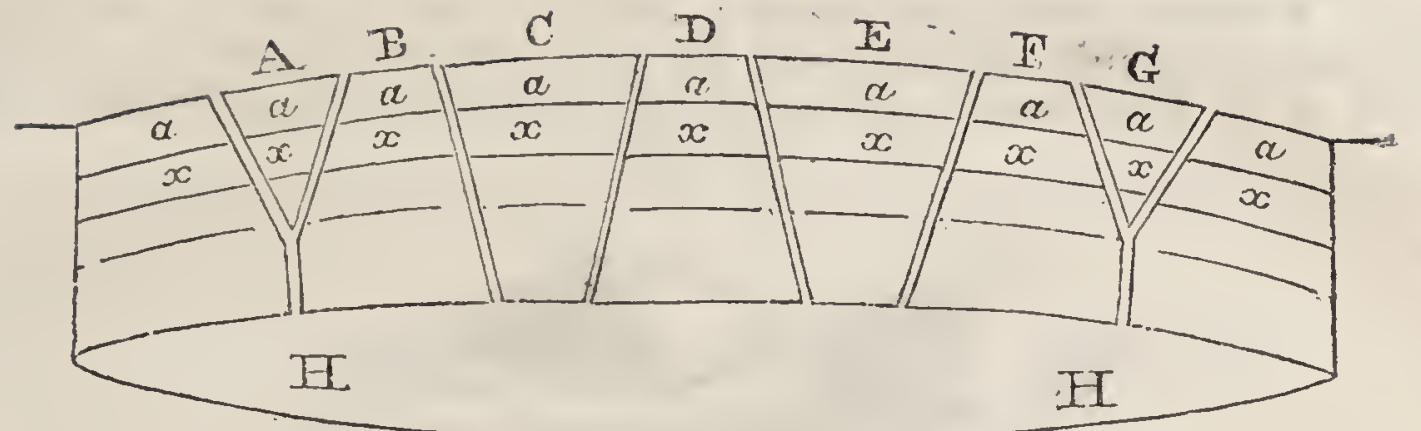

which has produced this wedge-like appearance, now applies its elevating force to the different masses. The wedges $\mathrm{B}, \mathrm{D}, \mathrm{F}$, with their strata $a x$, present larger surfaces to the fire $H$ than the wedges $A, C, E, G$, with their strata $a x$; therefore the elevating force of the fire will have greater power on B, D, F, G, than on A, C, E ; and, in consequence, will push them up above the others.

But, in studying the causes which have produced the earth's crust, and the varieties which it exhibits, we have not only to find out the cause of the first hardening of the earth's surface by cooling, and the action of subterranean heat in upheaving masses of matter on the surface; we have also to consider the action of water in dissolving and wearing away portions of upper rocks, and carrying down the sand to deposit it at a lower level.

Q. How does LAVA differ from other igneous rocks?

A. Lava is the product of melted rocks thrown out in a fluid or partially fluid state by volcanoes, within a comparatively recent period. It is not generally so compact and dense as the other igneous rocks.

Q. What is the "DIP" of stratified rocks?

A. The various rocky strata are not usually horizontal in their position, but inclined at various angles. The course in which water poured on an inclined stratified layer would descend, is called, ir. geological language, the fall or dip of the strata. The direction os railous strata is designated by the term strilie.

Q. When are strata said to CRor our?

\section{$A$. When they come to the surface of the earth.}

In fig. 202, the line $b d$ is the direction or dip of the strata, and the angle $a b d$, which the strata forms with the horizon, is called the angle of inclination. The exposed edge $b c$, where the strata come to the surface, is called the outcrop, and the line of outcrop $a b$, on a livizontal surface, is called the strike of the strata.

\section{Fig. 202.}

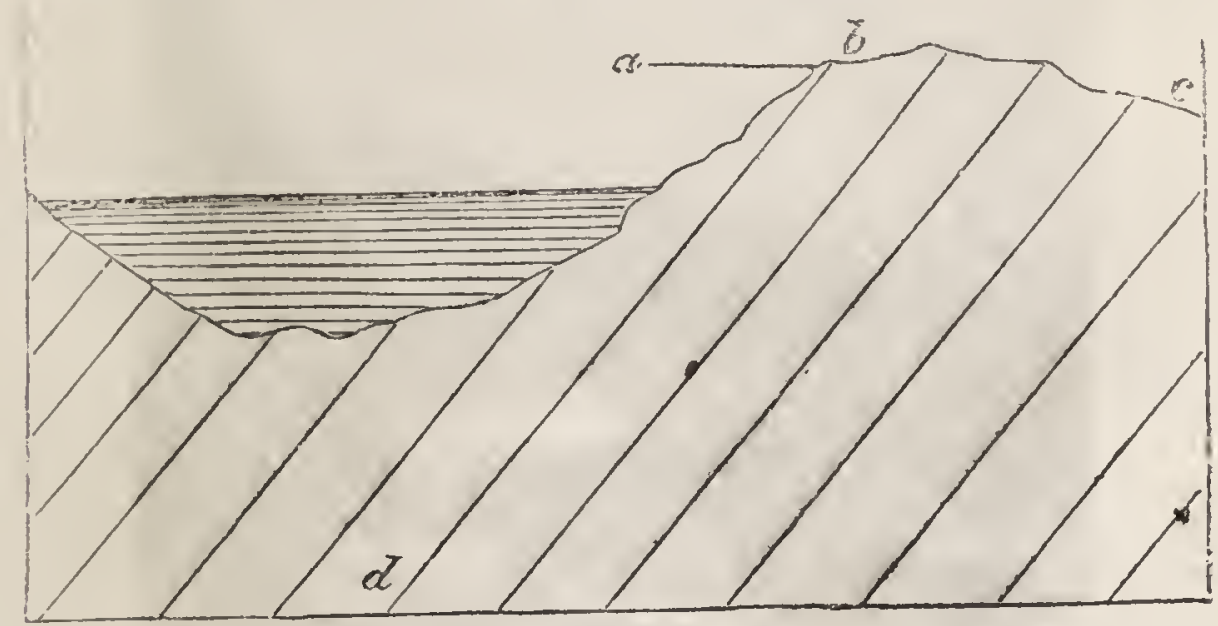

The dip and strike of the strata may be farther illustrated by reference to fig. 203 , in 
which $b$ represents a table on which some books, $a$, are made to rest in a slantiug position. by means of another book $c$, laid flat on the table. If we now consider the top of the table to represent a horizontal plane, and the books beds of rock, then the angle which the sides of the tilted books a make with the table $b$, would be called their dip, and

Fig. 203.

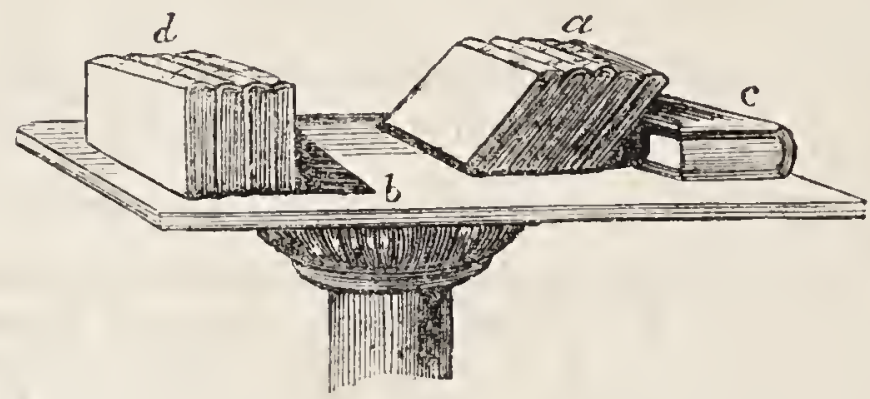

according to the amount of the angle would be the amount of dip-that is, the less the sides of the books sloped, the less the dip; and, conversely, the more upright they were, the greater the dip. The book resting flat upon the table would be said to be horizontal, and have no dip at all. Let $d$ be books standing upon the table with their backs upwards, their strata, or beds of rock, which occur in similar position, would be termed vertical.

The strike, or direction of the beds or strata, being a linc at right angles with their dip, the lines which the back of the books $a$ and $d$ would take towards the north, south, cr other points, as the case may be, would represent the strike, or direction. Let us farther suppose that the backs of the books $d$ are arranged parallel to those of the books $a$; then the strike, or direction of any beds of rock, situated as $a$ and $d$ are, would be the same, notwithstanding the beds at $d$ would be vertical, while those at $a$ would have a dip.

Q. What is GRINITE?

A. Granite is a rock frequently of a light-gray colour, composed of inree distinct minerals, quartz, feldspar, and mica, in various proportions, confusedly crystallised together.

Q. What is a trap rock?

A. Irap rocks are principally composed of feldspar and hornblende, with some iron and other mineral substances. 'It is a black or greenish-looking rock, and some varieties are known as greenstone.

Fig. 204.

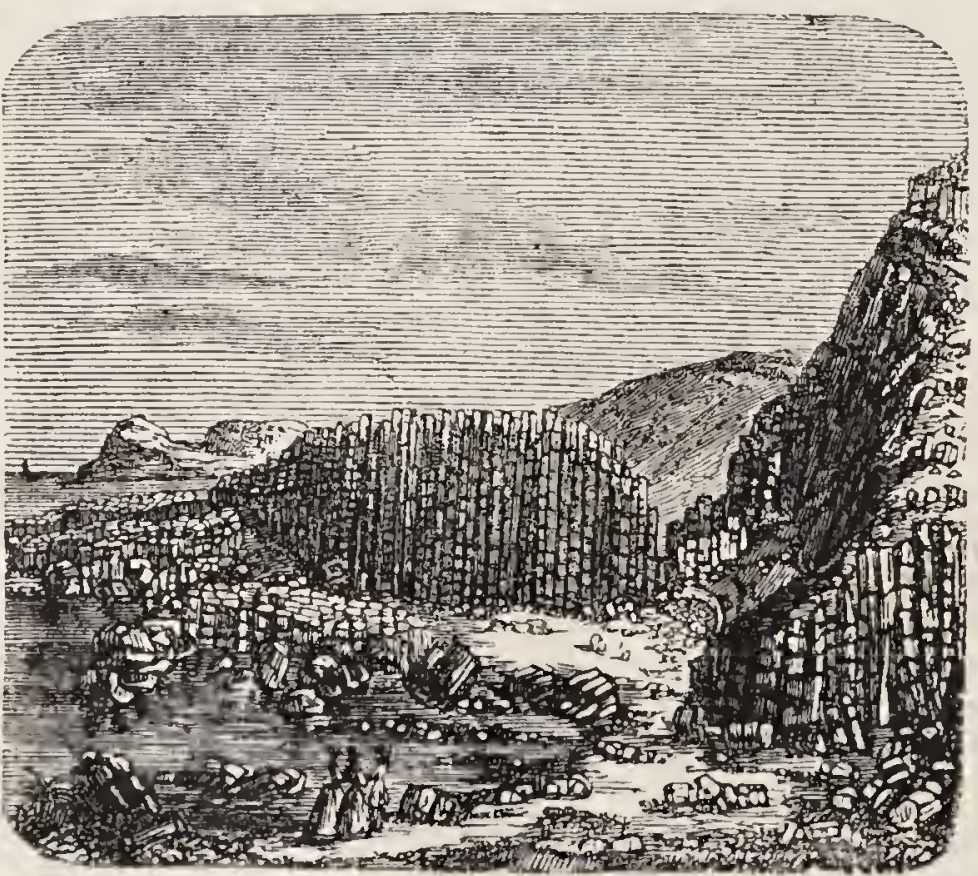

THE GIANTS' CAUSFWAT. 
Q. Can you give some No'LED EXAMPIES of trap rock?

A. 'The Giants' Causeway, on the north coast of Ireland, is composed of a variety of trap called basalt; the Palisades upon the Hudson, in America, are composed of trap.

This rock has a tendency to crystallise in large columnar masses, the appearance of which is represented in fig. 204.

Q. In WhAT PART of Ireland is the Giants' Causeway?

A. In the neighbourhood of Balimony, county Antrim.

Q. What is the Genreral appearance of the causeway?

$A$. The principal or grand causeway-there being several considerable and scattered fragments of a similar nature-consists of an irregular arrangement of many hundreds of thousands of columns formed of a black rock nearly as hard as marble.

Q. Are these columns EQUAL in HeIGHT?

A. No. They are of an unequal height and breadth; several of the most elevated, visible above the surface of the strand, are about twenty feet. How deeply they are fixed in the sand has never been ascertained.

Q. What is the EXTent of the principal causeway?

$A$. As it is visible at low water, it extends for nearly two hundred yards; the breadth of the principal causeway is from twenty to forty feet.

This is exclusive of the broken and scattered pieces of the same kind of construction, which are detached from the sides of the grand causeway, as they do not appear to have been contiguous to the principal arrangement, although these have been frequently comprehended in the width, which has led to such a diversity in the accounts of its dimensions. Its highest part is the narrowest, and there its width is not above twelve to fifteen feet. The columns of this narrow part incline from the perpendicular a little to the westward, and form a slope on their tops by the unequal height of their sides; and in this way a gradual ascent is made at the foot of the cliff, from the head of one column to the next above, to the top of the great causeway, which, at the distance of about eighteen feet from the cliff, obtains a perpendicular position, and, lowering from its general height, widens for nearly three hundred feet above the water. The tops of the columns, being ecual in height, form a grand and singular parade, which may be walked on safely.

Q. What is the PORM of the PILAars of the Giants' Causeway?

A. With some few exceptions, they are pentagonal, having five sides. They are not of one solid stone in an upright position, but composed of several short lengths joined together.

Q. What is a SANDSTONE?

A. A sandstone is a rock consisting of grains or small rounded fragments of any other rock, most frequently of silicious rocks. These particles are consolidated, sometimes without any visible cement, but often by an argillaceous (clay) or calcareous (lime) substance.

Q. When the rock consists of large rounded pebbles, or stones cemented together, what is it CALLED?

A. Conglomerate, or puddingstone.

Q. When the rock consists of fragments that are NOT ROUNDED, or ORMENTED together, what is it CALLED?

A. Breccia. 
Q. What is CLAY?

A. Clay consists of a mixture of silicious and aluminous earth. It is tough, easily moulded, and generally of a bluish colour.

Q. What is CLAY SLATE?

A. Clay hardened into roct. When the rock is partially solidified it receives the name of shale.

Q. How may all the varieties of ARGILLACEovs (clayey) rocks be DIsting UISHED?

1. When breathed upon they give forth a peculiar earthy smell.

Q. When is a rock said to possess a SLATY STRUCTURE ?

A. When it splits easily in one direction, in consequence of its component parts being arranged as laminæ in parallel layers.

Q. What is the BUILDING MATERIAL, so much used, which is called FrEEstone?

$A$. A fine variety of sandstone.

Q. What NAME is given to the confused masses of SAND, GRAVEL, and DETACHED ROCKS which occur heaped together upon the earth's surface?

\section{A. Diluvium.}

Q. What NAME is given to the fine coating of soil which overlies the coarser sand and gravel-that part of the earth which is capable of cultivation?

A. Alluvium, or calluvial deposits.

Q. What are BOULDERS?

$A$. Boulders are detached rocks, generally of a rounded form, confusedly scattered over the surface of the earth. The appearance of boulders is represented in fig. 205.

\section{Fig. 205.}

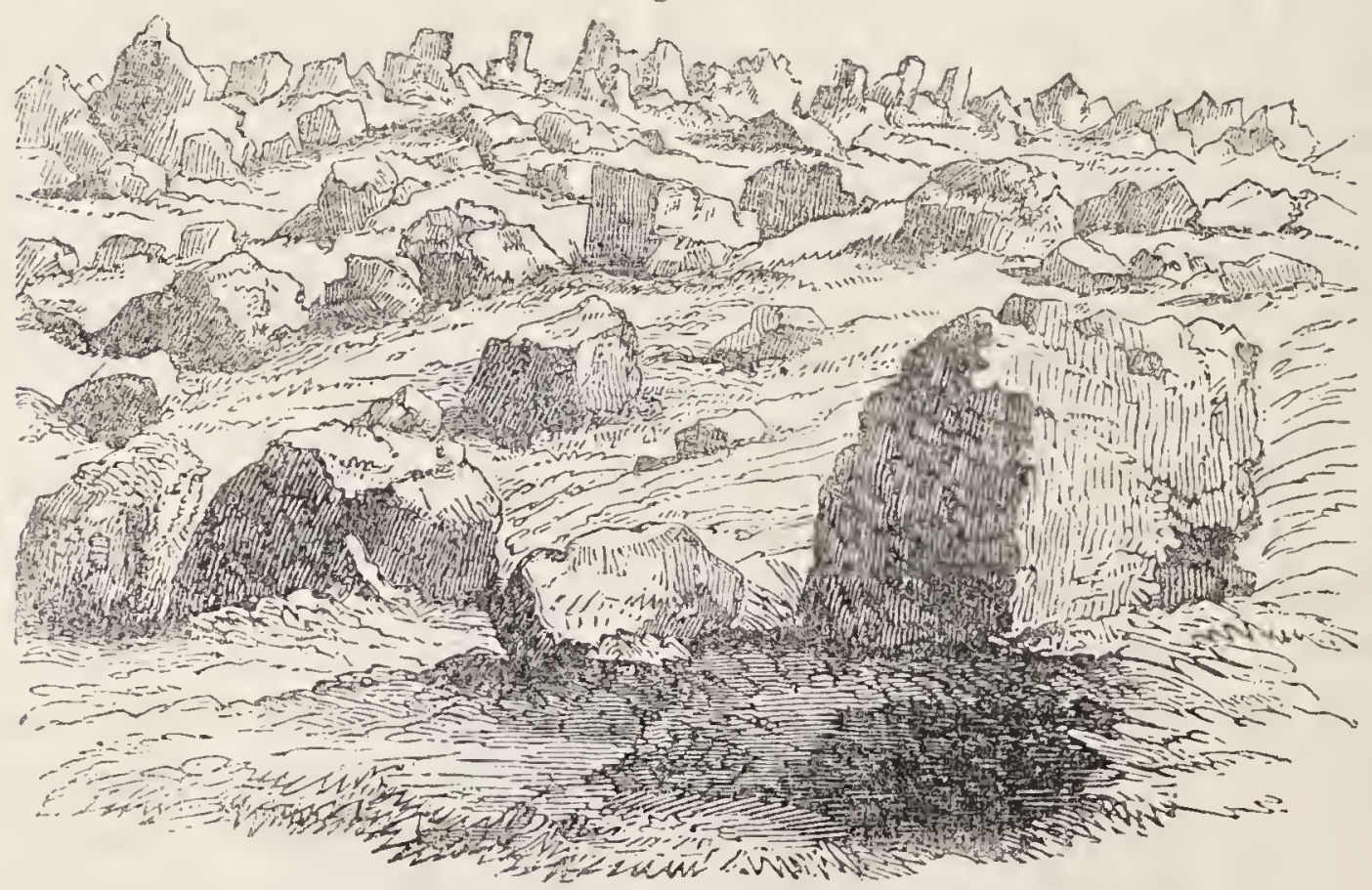

Q. Are BOULDERS found in ALL PARTS of the world?

A. They are found in Russia, Poland, Germany, Holland, England, Ireland, Canada, and North America, scattered over broad plains and mountain steeps.

Q. How do geologists 4 ccount for these boulders?

A. There can be no doubt that all the boulders have come from 
the North, for their course, both in Europe and America, is found to be either due north and south, or varying a few degrees to north-west and south-east.

Boulders, containing specimens of almost all known rocks, are found in the eastern cuanties of England. Some of these could have come only fiom Norway and Sweden; fur Sir Charles Lyell traced them from these two countries to Denmark, across the Elbe, through Westphalia, to the borders of Holland. "We need not," he says, "be surprised to find them re-appear on our eastern coast, between the Tweed and the Thamesregions not half so remote from Norway as are many Russian erratics from the source whence they came." As it is evident that very many of these boulders, in different parts of the world, are fragments of rocks which do not exist in those localities, the question, Whence came they? is most important and interesting. They have been torn from rocks far away-they are strangers in a strange land. How did they get there? Various answers have been given.

Q. By WIIT MEAxs have these boulders been distributed over the face of the earth ?

A. Amongst the answers which have been given to this questior, we may mention three:-

1. That they were swept away from the rocks and borne to foreign shores by the Great Flood-the universal deluge-in the time of Noah.

2. That they were transported at intervals by the recurrence of partial floods.

3. That icebergs were the means of their removal from place to place.

The icelerg theory has its difficulties, as well as the others; but it seems to come nearer the true eause of the phenomenon. It has been proved that all these boulders have come from the north. It lias also been ascertained that, at an early age in the history of our globe, the northern hemisphere was much colder than it is now, and that this intense coldness took place suddenly. Such a lowering of the temperature would fill the glens of the polar region with immense glaciers; these glaciers would start ont into the northern sea. Icebergs, detached or broken off from these glaciers, would convey to a distance huge masses of rock, which water alone could never have moved. As the lowe: surface of the icebergs would either be abraied by the action of the sea, or melted by the increased temperature in the south, the masses of clay, sand, gravel, and boulders, which they had brought down as glaciers or imbedded as coast ice, would drop down, and be scattered orer the bottom of the sea. Ages after the deposit of these rocks and sands, they, by volcanic action, would be elevated above the level of the sea, bearing on their edges and sides the boulders that had thus been deposited. Such is a bare outline of the ireberg theory.

Q. Does geology throw any light upon the oniars and Crestron of the earth ?

A. Respecting the origin and creation of matter geology affords no information; it teaches clearly, however, that there was a beginning; that there was a period when no vergetable or animal cxisted upon the surface of the earth; that the lowest forms of vegetable and animal life were first called into existence, which were gradually followed by other and higher organisations; and that, finally and lastly, man became an inhabitant of the earth.

Q. Can we form any IDEA of what is meant in revelation by the assertion that the earth at one time was "without Fors, and rorn?"

$A$. There is some reason to believe that the material of which the earth was composed was once in the conclition of the nebulce; that it flocted as a vapour in space; and that, through the influence of gravitation, cohesion, and chemical action, it was moulded in the form of a sphere. 
Q. What is the Appearance of nebula?

A. Some of the nebulæ seem to be mere clouds of thin vapour, without definite shape; others seem to be condensing into spleeroidal masses, and present a dense central nucleus of light, surrounded by a luminous halo; in short, a series of them may be traced from mere films of vapour to dense masses, which have the appearance of a star surrounded with a haze. Fig. 206 represents the appearance of rarious nebulæ, as seen through powerful telescopes.

Fig. 206.

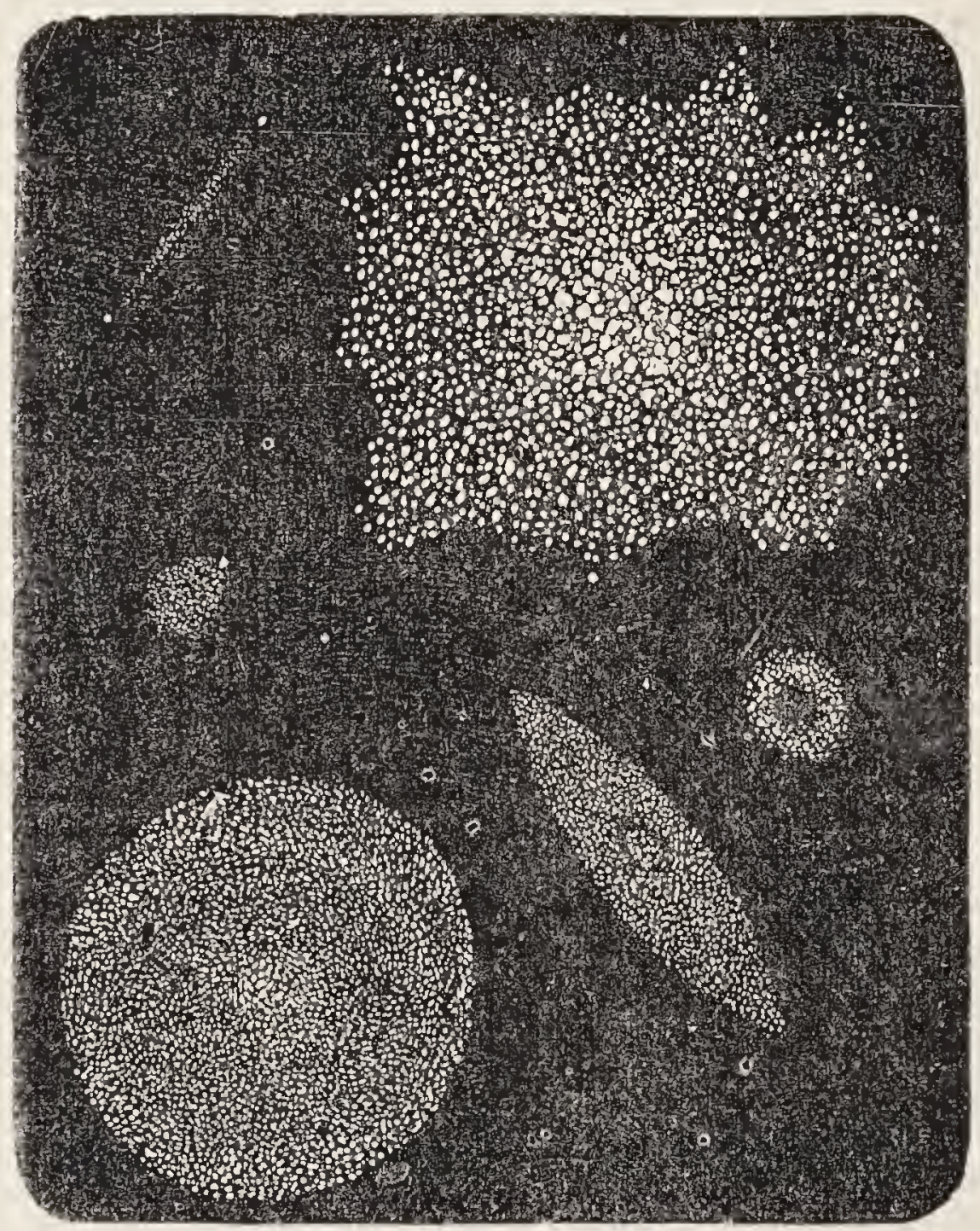

VARIOUS FORMS OF NEBULL.

-Q. What are the NEBULes supposed to BE?

A. Worlds in the process of formation.

Q. If the earth was once in the state of vapour, or nebulous matter, and this matter was gradually condensed, what CoNDITION would matter FIRST ASSUME in its progressive condensation?

A. A fluid conctition.

Q. Have we any REAT INIDENCE that the carth ever was FLUID?

A. Geology commences its revelations at this point, and teaches most clearly that there was a period when all matter comprising the earth was in a fluid or liquid conclition. 
Q. What are the Evidences which geology presents to us in relation to the former fluidity of the earth?

$A$. The fact that all the primitive rocks exhibit evidence that they were once in a melted condition; that the earth is a spheroid, and not a sphere; and that the centre of the earth is at the present time a great source of heat.

Q. What do we mean by the PRIMITIVe Rocks?

A. The lowermost rocks, those which constitute the foundations upon which all the stratified rocks rest. They also attain the highest elevations on the surface of the globe. Granite and its varieties are types of the primitive rocks.

Q. Why are they called primitive?

$A$. Because it was inferred, from the entire absence of any remains of vegetables or animals in them, that they had been formed before the creation of animals or vegetables; but it is now ascertained that granite and its associated rocks are of various ages, and in some instances have been formed from stratified rocks melted by intense heat.

Q. How does the fact that the earth is a SPHERorD, and not a sphere, show that it was ONCE FLUID?

A. A spheroid is the form which a fluid body will always assume when caused to revolve about its oun axis. The earth has exactly the form which it must have assumed had it been once a fluid in a state of revolution about its own axis. HEAT ?

Q. How do we know that the CENTre of the earth is at the present time a source of

A. The existence of a great number of volcanoes in all parts of the earth's surface-some of which are constantly sending forth melted rocks and fire-proves that the interior of the earth contains great reservoirs of heat, the sources of volcanic supply. It is also a fact that, as we descend into the interior of the earth, the temperature regularly increases; the existence of boiling springs in all countries also afford evidence of the central heat of the earth.

Q. Does the heat INCREASE as we descend into the earth?

$A$. After passing the first hundred feet the mercury in the thermometer begins to rise, and continues to rise the lower we descend.

Q. Are ALL SPRINGS that rise from a very great depth in the earth more or less WARM?

A. They are.

Q. If the theory of the central heat of the earth be true, what is the condrrion of the SURFACE upon which we live?

$A$. It is merely a hardened crust, resting upon a vast interior mass of melted fiery matter.

Q. Why has not the earth COoLED Dowr in the lapse of ages, and parted witil its heat?

A. The external portions of the earth doubtless cooled speedily, and, deprived of their heat, became solid. The crust so formed, consisting 
of earth and rock, on account of its bad conducting power, would effectually retain the heat of the central portions, and prevent its passing into space.

Q. Fow may the XatTLR composing the earth be supposed to have originally ACactren its HEAT?

A. Vapours, in passing into liquids, and liquids in becoming solid, develop large quantities of latent heat. The heat liberated during the condensation of the nebulous matter to a solid form would undoubtedly be sufficient to account for all the phenomena made known to us by geology.

Q. Does the xoov, when examined by a powerful telescope, present evidences of VOLCANIO or IGNEOUS action?

$A$. This satellite of the earth, which appears so plane and ever: becomes, under the telescope, studded with rough and ragged mountains, whose tops resemble the craters of volcanoes, or the sharp, conical peaks of the terrestrial volcmoes Cotopaxi, Etna, Vesuvius, \&c.

Fig. 207.

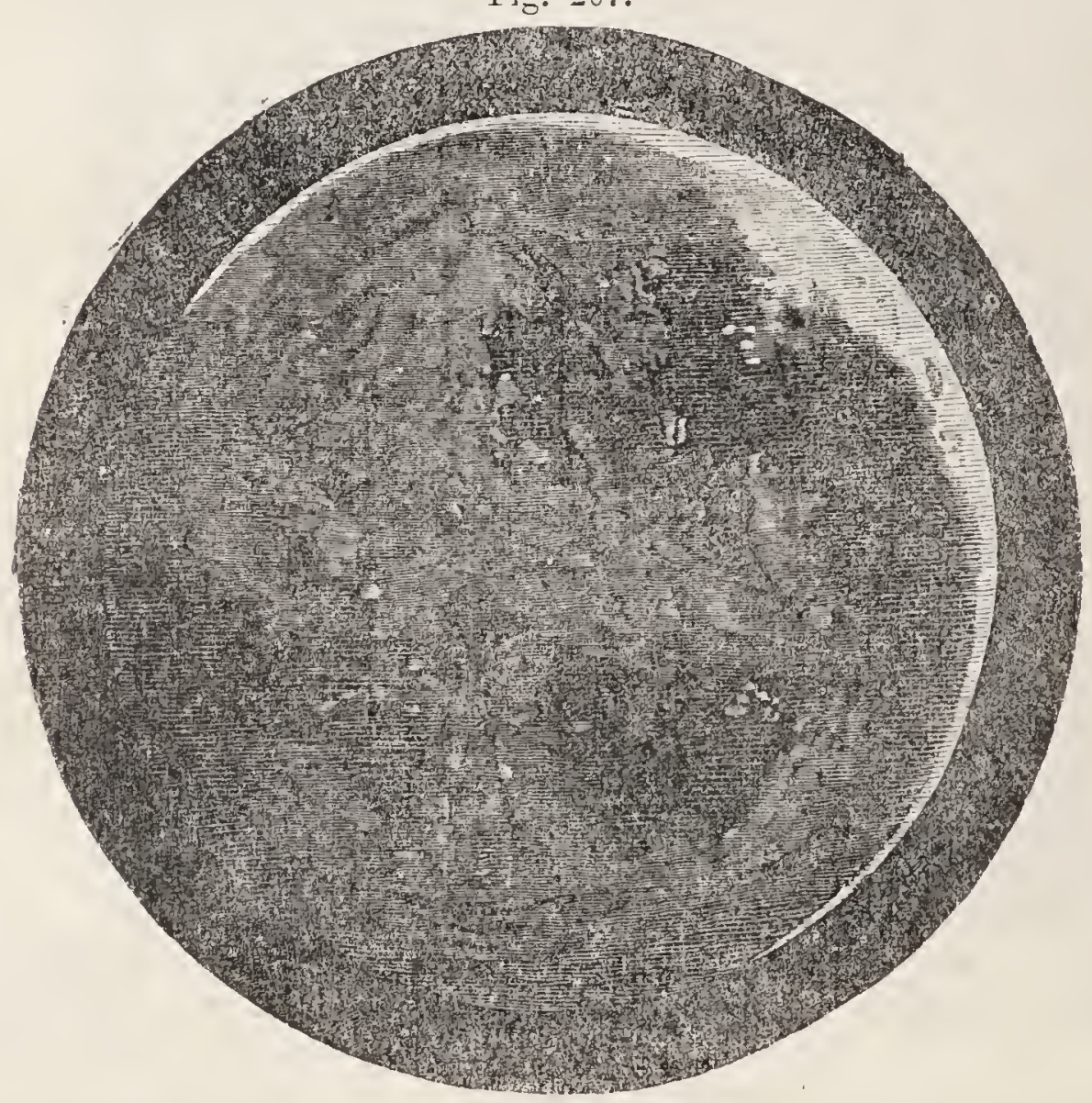

Fig. 207 represents the appearance of the lunar surface as seen through a large refracting telescope, when the moon was full. The moon is apparently but a smaller pattern of the earth, exhibiting upon its surface the effects of a more intense volcanic action: a fact which is probably due to the absence of water-an agent which, upon the earth, has so modified its surface, that its original igneous character is concealed in part beneath the sedimentary, aqueous, or stratified rocks.

Q. As we descend into the earth, what specins of Rocks do we uniformly find occupy * ing the LowEST positions?

A. The igneous or primary rocks-rocks which have the appearance of having been melted and fused. 
Q. Have we $A$ NY REASON for supposing that rocks of any different charactor lie BENEATH the igneous rocks?

$A$. The igneous rocks, occupying the lowest position in the crust of the earth, are undoubtedly the result of the solidificcition or cooling of the earth's surface in the first instance, and have remained unchanged and unaltered. We have, therefore, no reason for supposing that rocks, or materials of essentially different character, exist at any greater depth.

Q. What is the CIIARACTER of the rocks immediately overlying the primitive or igneous rocks?

A. We find immediately above them rocks which are stratified, and have resulted from the deposition from water of matter which has been worn away from the surface of the granitic or primitive rocks.

Q. Why do we suppose that these rocks were formed SUBSEQUENTLX to the granitic or primary rocks on which they rest? why may not both have been formed at the SAME TIME ?

A. Because they contain fragments, and are evidently made up of the ruins of granitic rocks; consequently, the latter must have had a prior existence. Again, it is natural to suppose, that when one layer of rock, of a certain composition, is superimposed upon a layer of rock of a different composition, that the latter must have been formed first.

Q. What is the CHARACTER of the rocks immediately resting upon the primary rocks?

A. They comprise the well-known rocks mica slate, clay slate, talcose slate, and gneiss, which last resembles granite, with the exception that it is stratified, and occurs in beds, which is not the case with granite. These rocks are of great thickness, are destitute of all organic remains, and appear to have been deposited in layers from water, and afterwards liardened by the action of heat.

Q. What Nasre has been given to rocks of this class?

A. They are called metamorphic, because they have been changed, and have lost their original character through the action of heat. They are also termed non-fossiliferous or azoic (wanting in life), and, by some, transition rocks, from their forming a connecting link between the primary and more recent formations.

Q. Rising in the series, what is the CHARACTER of the rocks SUCCEEDING and RESTING Lrov the metamorphic rocks?

A. The series of rocks resting upon and succeeding the metamorphic are all stratified, and contain fossits in great numbers and variety.

Q. What is a Fossir ?

A. Any organic substance. The remains or proctuct of an animat or regetalle, imbedded in a geologiral formation, is called a fossil. Fig. $\because 08$ represents the fossil remains of the chalk formation.

The most conspicuous of the fossils found in the chalk are shells of animals that 
Fing. 208.

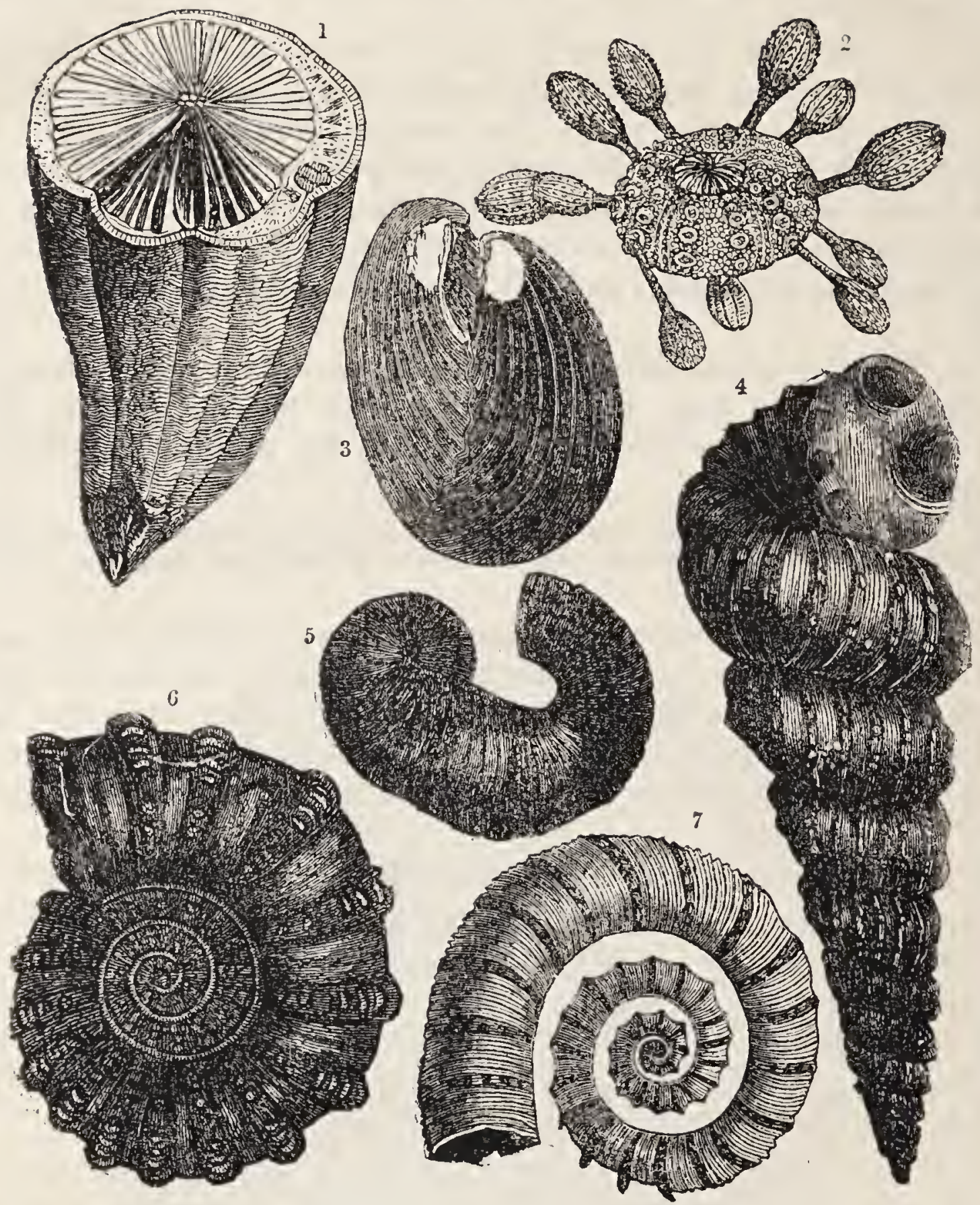

ORGANIC REMAINS OF THE CHALK FORMATION.

Eormerly lived at the bottom of the sea ; coral, and other species. The names they bear are as follows:-1. Hippurites organisans ; 2. Cidaris diadema, or Diadema rotulare (Agassiz) ; 3. Terebratula semiglobosa ; 4. Turrilites catenatus ; 5. Scaphites equalis ; i. Ammonite ; 7. Crioceras puzosianus.

Q. What is the study of fossils called?

A. The study of fossils is called Palceontology (from palaios, ancient, and ontology, the science of being). To become versed in the science requires an acquaintance with the principles and minute details of zoology and botany. Many of the general conclusions derived from the study of fossils may, however, be readily understood without this in nowledge.

Q. In WHAT MANNER have fossils been PRESERVED?

A. When any organic substance is imbedded in earthy material which is slowly changing into rock, it may either remain entive; it 
may be removed by the infitration of water, or other causes, so gradually, as to leave its form and even its most delicate markings in the rock; or some mineral substance may be substituted in place of the original animal substance, and fill the space it once occupied; sc that we sometimes find the organic substance itself, sometimes the mere impression of it, and again a cast of it.

Q. What NAME has been given to the rocks resting upon and succeeding the metamorphic rocks?

A. As a whole they are called the fossiliferous rocks; but they are divided into nine distinct and separate divisions, systems, or formations.

Q. What does the term "FoRMatiox" mean in geology?

$A$. We apply in geology the term formation to any portions of the earth's crust, of greater or less thickness, which were formed under the sume contemporary influence. Formations which, in consequence of their close proximity, stand in mutual relations to each other, are considered by the geologist as connected groups; the separate layers constituting a formation are called its members.

Q. Will you enumerate the NINE distinct sysTEMS, or Forvations, into which the IOSSILIFEROUS rocks are DIVIDED?

A. 1. The Lower Silurian: this formation is most extensive, no less than eight departments of which have been made out by geologists in North America, composed of various limestones and sandstones;

2. The Upper Silurian;

3. The Devonian, or Old Red Sandstone;

4. The Carboniferous or Coal formation;

5. The Triasic, sometimes called the New Red Sandstone, and sometimes the saliferous formation, from the fact that it contains in Europe the principal mines or deposits of rock salt.

6. The Oolitic formation;

7. 'The Cretaceous or Challi formation;

8. The Tertiary formation; and

9. The Diluvium or Drift, forming the most superficial deposits, and extending over a large portion of the northern countries of both hemispheres. Above the drift occurs the alluvial deposits-the soil we cultivate.

Each of the formations enumerated indicates an entirely new era in the eartli's bistory; while each of the layers that compose a formation indicates but some partial revolution, or change.

Q. How are these different rocks DISTINGUISHED from each other?

$A$. First, by the difference of their fossils; secondly, by their position; and, lastly, by the difference in the composition of their constituent materials.

Q. How are they distinguished by the difference of their Fossirs?

$A$. Each system represents a period of time, during which the rocks included in it were gradually deposited and formed at the bottom of 
an ocean. During this period races of animals and vegetables came into existence, lived, flourished, and became extinct, leaving, as the only memorials of their existence, forms impressed or embodied in the rocks.

Q. Does each system of fossiliferous rocks appear to contain remains of animals and vegetables PECUIIAR to ITSELF?

$A$. It does. Not one species of fossil occurring in the silurian rocks occurs in the carboniferous (coal); the fossils of the old red sandstone are totally distinct from those of the cretaceous (chalk) system; while none belonging to the latter are now living upon the surface of the earth.

Q. Can a geologist, conversant with fossils, TELL at ONCE from WHAT STSTEM a species is derived by mere inspection of it?

A. As respects the common species of fossils, he can readily determine at the first glance from what system of rocks they have been derived, no matter if the fossil may have been obtained in a remote portion of the earth; for the reason that the fossils of each system possess characters peculiar to themselves.

Q. How does the Position in which the various systems occur serve to IDENTIFY and DISTINGUISH them?

A. Each system maintains a certain invariable order and arrangement.' Unless the strata have been torn up and lifted by volcanic forces, we never find rocks of a lower system resting upon those of one higher in the scale.

Strata of the silurian system are never found naturally covering those of the carboniferous system ; neither do the rocks of the coal period rest upon those of the tertiary period.

Q. As these various systems rest one upon the other, is not the arrangement of the rocks, comprising the crust of the earth, smILAR to the ARRANGEMENT exhibited by the several CONCRNTRIC COATS of an ONION?

$A$. If the various systems extended uniformly over the whole surface of the earth, and had never been broken or disrupted, they would enclose one another in a manner similar to the coats of the onion; but they do not extend thus uniformly.

Q. What is the REASON that the various systems of the stratified rocks do not extend UnIFonum over the whole surface of the globe?

A. Because the agencies concenned in their formation did not operate at all places at the same time. The stratified rocks were formed at the bottom of an ocean from matter deposited by its waters. On those portions of the globe not covered by water at that period of time no such deposition could take place; and consequently we find particular systems restricted to particular portions of the earth's surface.

All the formations are not always found, or are not developel to the same exient, in all places. It is the same with the several strata of which they are composed. In other words, the layer's of the earth's crust are not continuous thronghont, like the coats of an onion. There is no place on the globe where, if it was possible to bore down to its centre, all the strata would be found. It is easy to understand how this must be so. Since irregularities in the distribution of water upon the land crust bave, necessarily, always existed to a certain extent, portions of the earth's surfice must have been left diy at 
erery epoch of its history, gradually forming large continents and islands, as the changes were multiplied. And since the rocks were mostly formed by the subsidence of sediment in water, no rocks would be formed except in regions then covered witl water. They would be thickest at the parts where most sediment was deposited, and gradually thin out towards their circumference. We may, therefore, infer that all those portions of the earth's surface which are destitute of a certain formation were dry.land during the epoch of the earth's history to which such formation relates, excepting, indeed, where the rocks have been subsequently removed by the action of water or other causes. - Agassiz.

Q. How does the DIFEERENCE of material constituting the different rocks serve, in some measure, to IDENTIFY them?

A. Because certain materials are found as exclusive constituents only of certain systems. 'Thus the occurrence of beds of chall characterises and gives name to the cretaceous system; and the occurrence of extensive beds of cocil indicates that the adjacent rocks belong to the carboniferous system.

\section{CHAPTER LXTV.}

\section{EXISTING AND RECENT GEOLOGICAL CHANGES.} DAY?

Q. Are GEOLOGICAL OHANGes taking, place on the surface of the earth at the PRESENT

A. Physical changes, often unappreciated by us, are continually going on upon the surface of the earth, which, operating on a large scale, and through an extensive period of time, are producing results of great magritude and importance.

Q. What are the EFFECTS of STREAMs and RIVERs upon the surface of the earth ?

$A$. They are continually wearing arvay portions of the dry land, and conveying it to the ocean.

Q. How much solid MATTER is annually brought down by the River Ganges, and carried into the ocean?

A. By careful experiments, it is estimated that the Ganges transports annually from the land to the ocean more than six millions of tons of solid matter.

Q. How much solid matter is annually discharged by the MIssissipPi RIVER into the Gulf of Mexico?

A. Upwards of twenty-cight billions of cubic feet.

Q. What BECOMES of all the SEDIMENT transported to the ocean?

A. It generally settles in layers to the bottom. If, by the agency of volcanic heat, or by chemical action, it should become hardened or solidified, it would exhibit all the appearances which the stratified rocks present.

Q. Would the rocks so formed contain Fossizs?

A. They would contain the greatest abundance and variety of organic remains of every description; the wrecks of ships, the bones of 
men, animals, and fishes, shells belonging to the ocean, and shells swept down from the land; trunks of trees, land-plants, and seaweeds, with works of art.

Q. Would not an examination of the STRATA forming at the bottom of the GuLF of IIEXICo, with the organro rakinss included in them, impart to us very exact INFormaITON respecting the PRESENT CONDITION of extensive portions of the earth's surface?

A. Such an examination would teach us the character of the animals and plants now living in the valley of the Mississippi; and the remains of man and the worlis of art, such as coins, earthen and glass ware, and other articles, would indicate the degree of civilisation which prevailed in countries adjacent to the Gulf of Mexico. In the same way, by examining the stratified fossiliferous rocks, we can tell the condition of the earth's surface at the time they were formed.

Q. Do we find the remains of $M A N$ in any of the FossLLIFErous rocks?

A. Neither the bones of man nor any worlis of art have ever been found in any of the fossiliferous rocks belonging to the various geological systems. It is only in the modern formations, the alluvial deposits, that the remains of man have been met with.

Q. What INFERENCE, confirming the TRUTH of REVRLATroN, can be rightfully deduced from this fact?

$A$. That man was the last created animal, and that a comparatively recent period only has elapsed since his first appearance upon the surface of the earth.

Q. Are great beds of LIMESTONE forming at the PRESENT DAY beneath the surface of the OOEAN?

A. The reefs built by the coral insect are calcareous, and extend over vast portions of the bed of the ocean. In the vicinity of New Holland a coral formation exists upwards of seven hundred miles in length. Upon the coast of Florida limestone rocks are in the process of formation, the action of the waves grinding the corals and shells to a fine powder, which is afterwards deposited and hardened into layers in quiet waters.

Q. Do rocks and stones GRow?

$A$. A rock or stone is an inorganic body, which can only increase in bulk by the addition of matter, not belonging to it, to its outer surface. Hence stones may be incrusted, or several may be changed into one, but they cannot grow.

Q. Cannot CRYSTALS be said to Grow?

A. They cannot. Crystallisation is the building up of a fabric by the adhesion of particles of matter to other particles already arranged in a geometric order; and we have easy methods by which we can greatly increase the size of any artificial crystals, by simply adding fresh coatings of the substances of which they are constituted; but they cannot be said to grow.

Q. How does ORGANIC growth DIFFER from CRYSTALLISATION?

$A$. In organic growth, through the influence of the vital or living 
phenomena, the inorganic particles dispose themselves into cells by collecting together, and these, again, by a similar process, into tissues. A crystal grows by gathering matter from without, by collecting solict particles on its solin faces; while a leaf grows by absorbing gaseous elements, and through the agency of the cells, assisted by light and heat, assimilates them into its own structure.

Q. What is a volcano?

A. An opening in the crust of the earth, through which five, smoke, and melted matier (lava) are ejected. Volcanoes are supposed to be connected with a great central mass of heated inatter occupying the earth's centre.

Q. What is the FISSURE in the earth's crust through which volcanic matter is conveyed called ?

$A$. It goes under various names; sometimes it is called the vent, sometimes the chimney, sometimes the chasm.

Q. WHAT PART of the volcano is called the CRATER?

$A$. The upper part of this vent or chimney; namely, that part which is considerably elevated above the surface.

Q. What matter is usually Expecied from the craters of volcanoes?

A. They send up boiling streams of lava: floods of hot mud, and showers of ashes and cinders.

Q. Do geologists make any DISTINCTION between the characteristics of these craters?

A. Yes. Some are called craters of eruption, others craters of elevation.

Q. What is meant by a CRATEI of ERUPTION?

A. A crater of eruption is one formed by the masses of boiling lava cxpelled from the earth through the fissure, vent, or chimney.

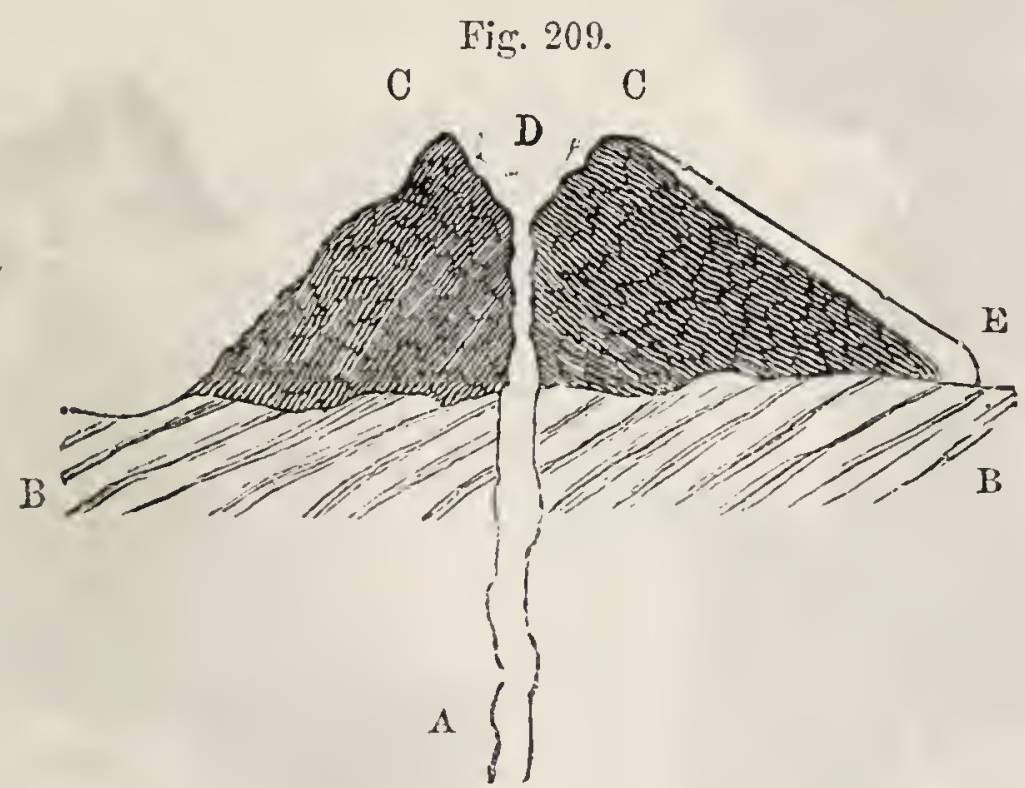

In the above diagram we have a representation of a crater of cruption. $A$ is the fissure, or vent, produced by the internal heat of the earth. $B$ B is the portion of the earth's crust through which it has penetrated. $\mathrm{CC}$ are masses of cinders and ashes, which have settled on the surface of the rock B B. D is the funnel-shaped crater. $E$ is a streain of lava flowing over the cinders. 
Q. What is meant by a CRATER of EIEVATION?

A. These craters are formed by the rupture of the flat sirata of the earth's crust. The beds of strata are tilted up and thrown into highly inclined planes.

Fig. 210.

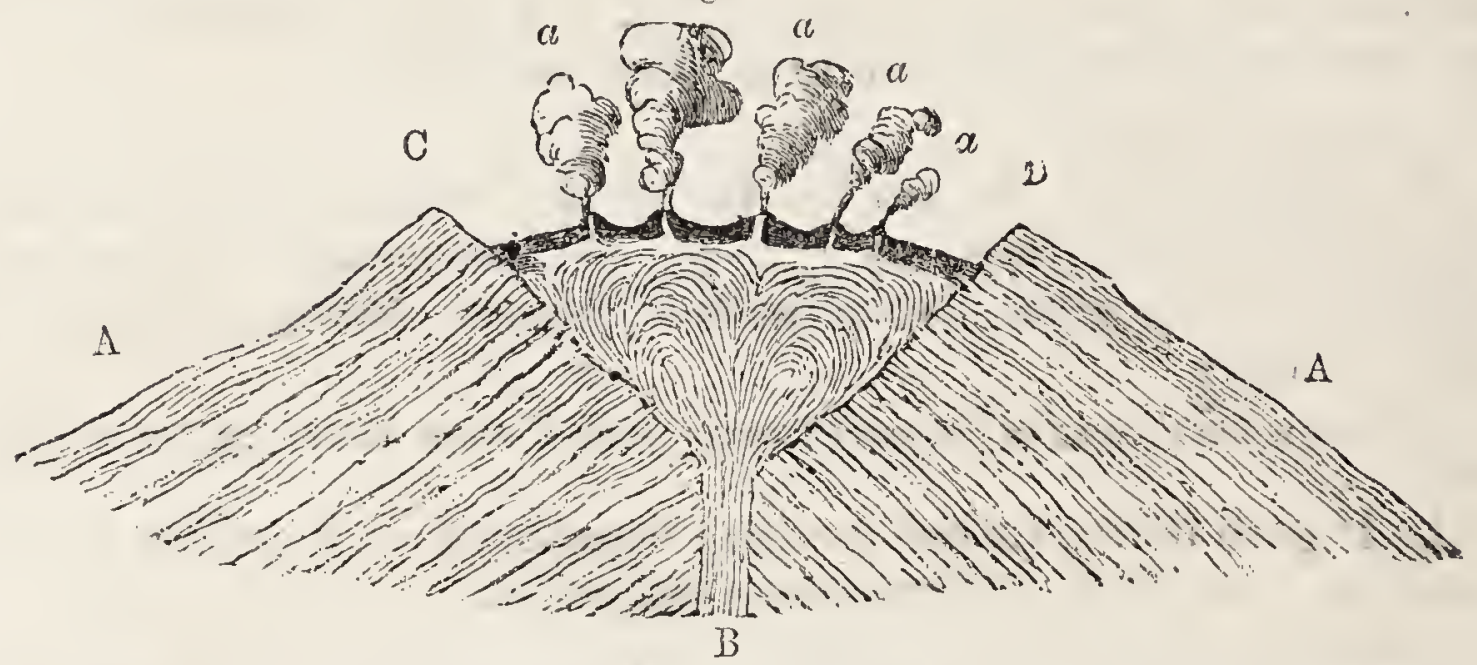

Here we have the representation of a crater of elevation. A A are rocks, which were originally horizontal, but which have been tilted up and snapped asunder by the force of melted matter, at B. The melted matter is not sufficient in quantity to flow over the edges of the crater CD; nor is the expansive power below strong enough to hurl it into the air : therefore it boils in the crater. Its surface is covered with a thin crust; and the volcanic materials escape only at the vents $a, a, a, a$.

Q. Has a crater only one VENT?

A. In the crater of a volcano, and on the sides of a volcanic mountain, there are generally a great number of smaller vents, emitting water and gas. Besides these, vents are sometimes formed by the fall of scorice or ashes.

Fig. 211.

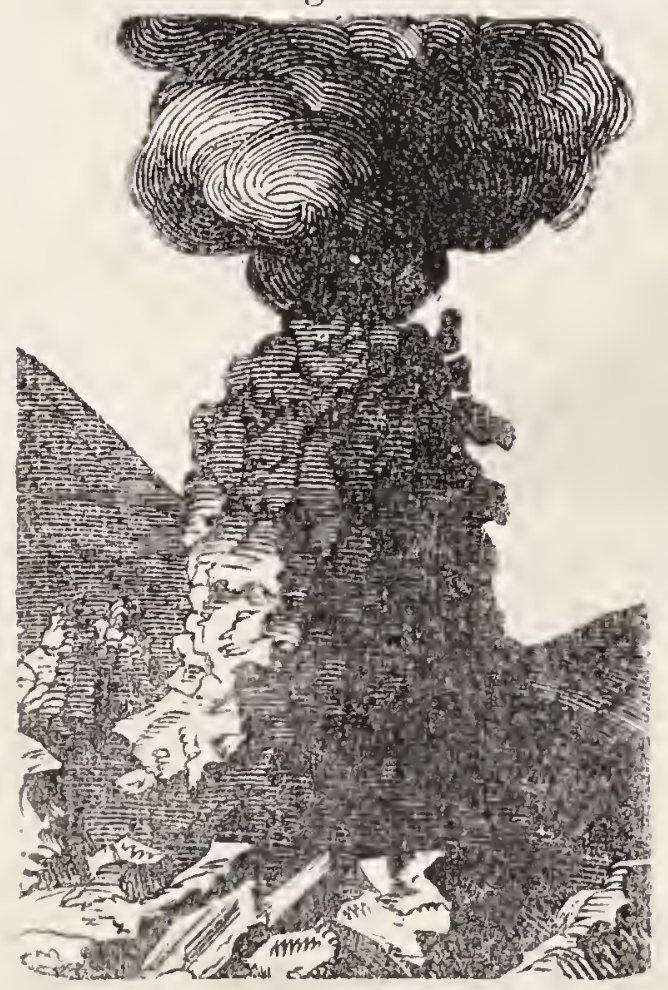

Fig. 212.

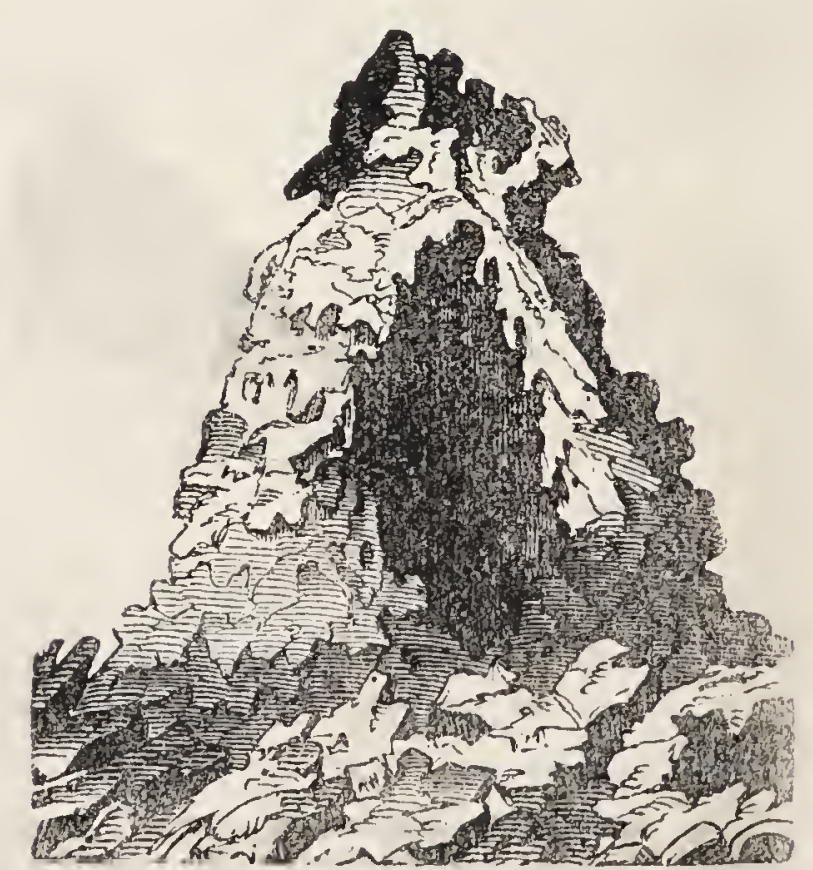

In the above figures are seen is conical vent, formed by the fall of cinders on Vesuvins ( $f y .211$ ) ; and the structure and appearance of a conical vent after volcanic action has ceased ( $f$ ig. 212). 
Humboldt affirms that it may be considered as a pretty well established law of volcanoes, that those which lave least elevation are characterised by the most unceasing activity. He proves this law by many examples, and explains it by the supposition, that a less internal force is sufficient to raise the molten masses to low than to high sumnits. He gives the following series of elevations of the craters of remarkable volcanoes:-

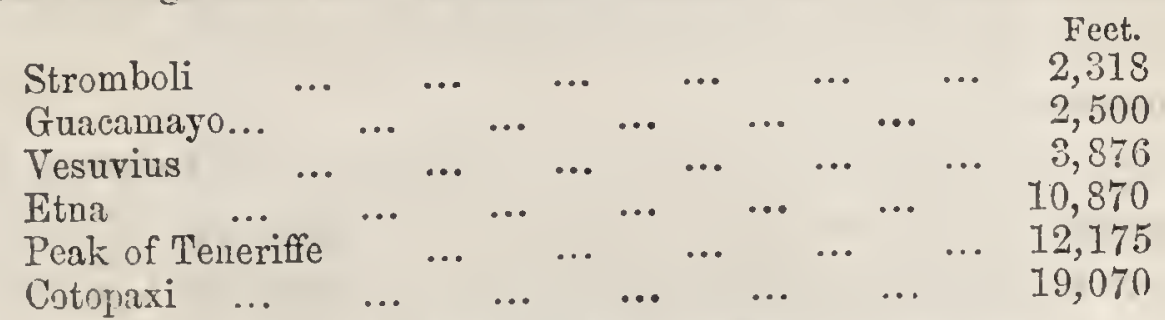

Now, of these, Stromboli has been in a state of activity from the Homeric age to the present; so unceasing, that it has served, and still serves, all the purposes of a stupeudous lighthouse to ships navigating that part of the Mediterranean. The entire island, situate off the nortl coast of Sicily, is of rolcanic formation.-Lardner.

Of all the volcanoes which I have seen in either hemisphere, the cone-formed Cotopaxi is at once the most regular and the most picturesque. Before each great eruption the sudden fusion of the snow which habitually invests its vast cone announces the coming catastrophe, even before the appearance of smoke issuing from its lofty crater. The sides of the cone acquire a glowing temperature, and the mass of the mountain assumes an aspect of most awful and portentous blackness, - Humboldt.

Q. Name the kind of matter thrown up by TOLCANIC ACTION?

A. Among the materials thrown up by volcanoes we may mention gases, watìry vapour, lava, minerals, scorice, ashes, sand, water, and muct.

Q. Are not sToxes thrown up by volcanoes?

A. Yes. The vent of the volcano passing through various strata wears away fragments from the sides of the rock, which are ultimately ejected at the cone. (See fig. 213.)

Fig. 213.

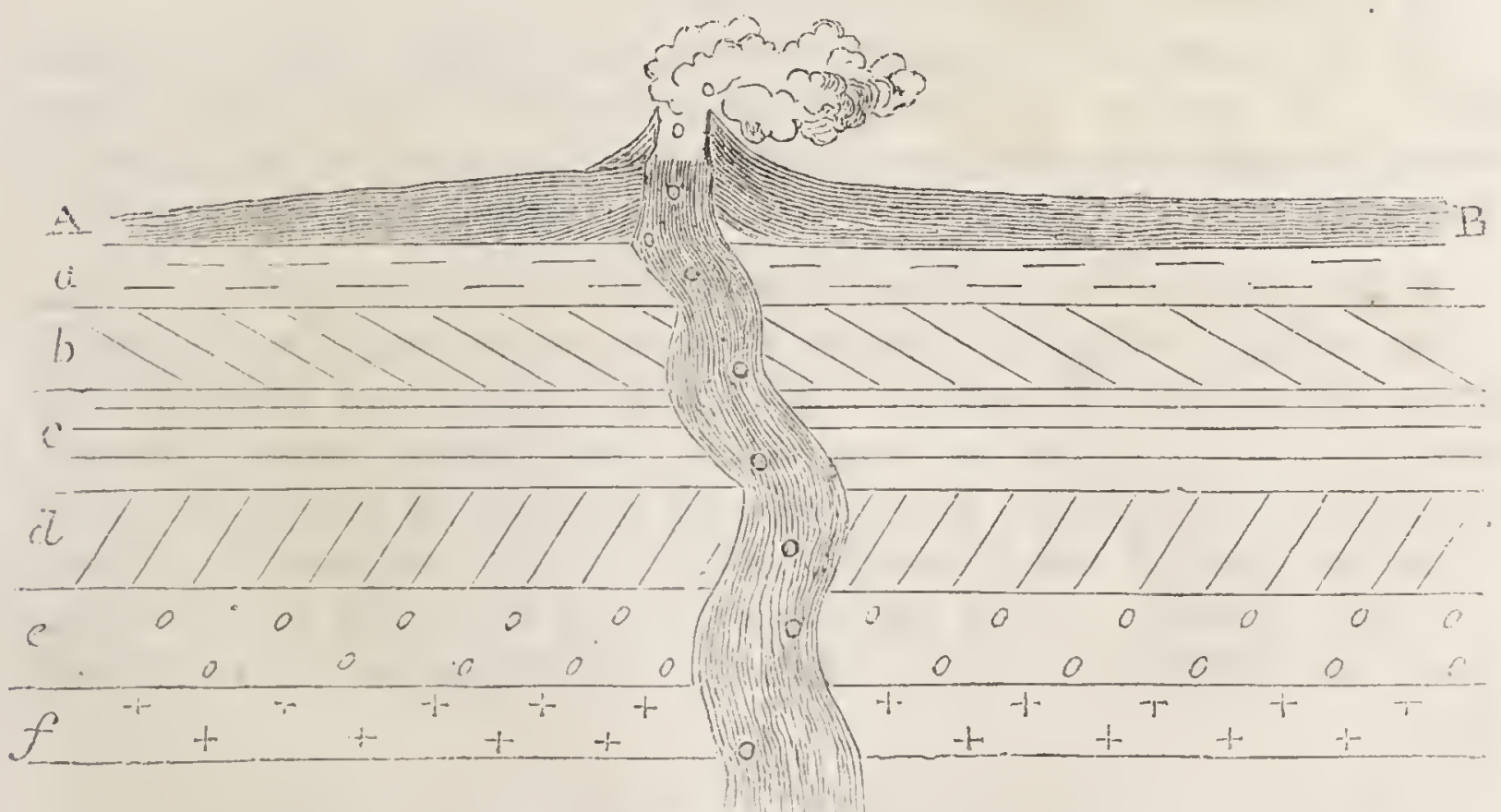

In the alove cirgram is secn how the vent of a crater passes through varinus beds of rock, such as $a, b, c, d, c, f$, some of which are fossiliferous, and others are of the more

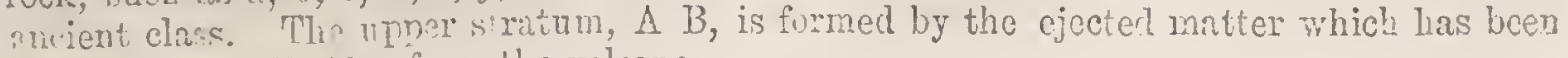
-Aviviz up on all si ies from the roleano. 
Q. What is LAVA?

A. Lava is a name given to any mineral matter melted in a volcano and ejected from the creter.

When the melted lava cools it receives fresh names, and is known as scoriæ, cinders, pumice, basalt, trachyte, \&c.

Q. What is SCORIE?

A. Scoria, or cinders, is the term applied to the refuse or stag of lava. Whether lava flows like a stream, or is thrown into the air like a fountain, it cracks, and becomes porous as soon as it is acted on by the air. The fragments, according to the effect of the air, assume a cellular appearance, that is to say, they are full of little round holes, as is seen in any piece of common pumice stone.

Q. Is it not recorded that, at the destruction of Pompeii, in the great eruption of Vesuvius, showers of ASHES DARKENED the air?

A. Yes. Volcanoes in eluption discharge immense quantities of dry, hot ashes, sometimes like impalpable powder, but very heavy as a mass.

Q. Do not some volcanoes EJECT WATFi? ?

$A$. Nearly all the vapour discharged by volcanoes is that of water. It is this vapour, when condensed by cold air, that forms the springs which are on the sides of volcanic mountains.

Q. Is MUD ejected by volcanoes?

A. Yes. This mud is called by the Italians lava d'aqua; and some geologists are of opinion that by this boiling mud Pompeii was destroyed.

Q. Are volcanoes IN GENERAL NEAR to the sea?

A. In nearly all instances volcanoes are situate near the ocean; and in the opinion of some geologists eruptions are caused by water being brought into contact with the heated material in the bowels of the earth.

Q. How can the ACCESS of WATER be EFFECTUAI in producing ERUPTIONS?

A. Water coming in contact with heated materials far in the interior of the earth is changed into steam. The steam suddenly rises, bursts open large fissures, and causes earthquakes, which generally precede an eruption. The increasing pressure of steam will at last force the glowing mass upwards, with the solid materials that cover it, until it finds vent at some opening, as the crater of a volcano, and an eruption of lava, cinders, and fragments of rock takes place.

Q. Are volcanoes, for the most part, distinguisheli by a PECULIAr ForM?

A. They are generally conical, being in fact gigantic air or steam vesicles, which have been upheaved from below, and finally elongated to an apex, at the termination of which the steam or gas broke though. (See fig. 214.) 
Fig, 214.

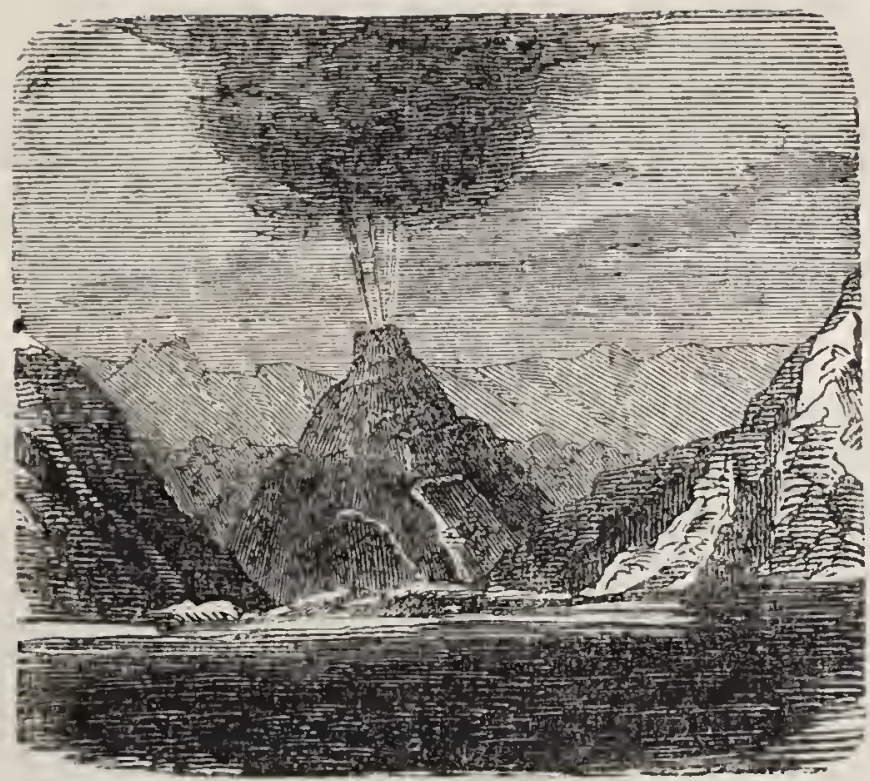

Q. How MANY volcanoes are there known at the present time?

A. Above three hundred; of these one hundred and fourteen are on the American continent or the adjacent islands.

Q. Are volcanoes situated chiefly on the MAIN LAND, or on ISLANDS?

A. In Europe and Asia they are, for the most part, on islands; in America they are chiefly on the main land.

The following is the volcanic distribution :-

\begin{tabular}{|c|c|c|c|c|c|}
\hline Europe-On the continent & & $\left.\begin{array}{ll}\cdots & 4\end{array}\right\}$ & Total & ... & \\
\hline "On islands ... & $\cdots$ & $\cdots$ & Lotal & $\cdots$ & $\bullet$ \\
\hline $\begin{array}{l}\text { Asia-On the continent } \\
, \text { On islands... ... }\end{array}$ & $\cdots$ & $\begin{array}{ll}\cdots & 16 \\
\cdots & 29\end{array}$ & Total & $\cdots$ & $\ldots \quad 46$ \\
\hline Africa-On the continent & $\ldots$ & 2 & Tatol & & \\
\hline " On islands $\ldots$ & .. & $\begin{array}{l}\ldots \\
0\end{array}$ & lotill & $\ldots$ & - \\
\hline $\begin{array}{c}\text { merica-On the continent } \\
,, \quad \text { On islands ... }\end{array}$ & & $\left.\begin{array}{ll}\ldots & 86 \\
\ldots . & 29\end{array}\right\}$ & Total & $\ldots$ & ... 114 \\
\hline Polynesian islands $\quad \ldots$ & $\cdots$ & $\ldots 108$ & Total & $\ldots$ & $\ldots 1$ \\
\hline
\end{tabular}

Q. What is KNOWN respecting IUUD VOLCANOES?

A. That they exist in different parts of the world; are supposed to originate with earthqualies; that their rise is accompanied with noises resembling the discharge of artillery, and with jets of flame.

Q. Describe ore of THESE volcanoes?

A. The mud volcano of Jolmali, in the Caspian Sea, was formed Fivember 27, 1827. Flames sprung up from the sea, and blazed to a great height. This lasted for about three hours. After this, for a whole day and night, it continued to burn about three feet above the newly-formed crater, from which great quantities of mud were ejected.

Humboldt describes a real mud volcano, near Carthagena, New Grenada, South America. There, in the midst of a thiclet of palms, is a marshy ground, called Los Volcancitos. The tradition of the inhabitants is, that this ground was once all in flames, but that the fire liad been extinguished through the good offices of a priest. Since then the fire roleano, so the people say, has become a watery one. The volcancitos are about fifteen or twenty in number, stand in cones, from nineteen to twenty-five feet high, and ...ensure round their bases from serenty-fire to eighty-fire feet each. On the top of each 
of these volcancitos is an aperture, or depression, from fifteen to thirty inches in diameter, and filled with water, through which air-bubbles are constantly esenping. In other parts of the ground there are apertures for such escapes of air; but which are not surrounded by cones. The cones have, no doubt, been raised by the clayey anud contained in the fluids : and the dull sound which precedes the boiling of the water in the cone indicates that the ground is hollow. It seems that each crater receives its supply of air and gas from separate channels. These little craters are.filled with water even in the driest. seasons. The temperature of the water is not higher than that of the atmosphere.

Fig. 215.

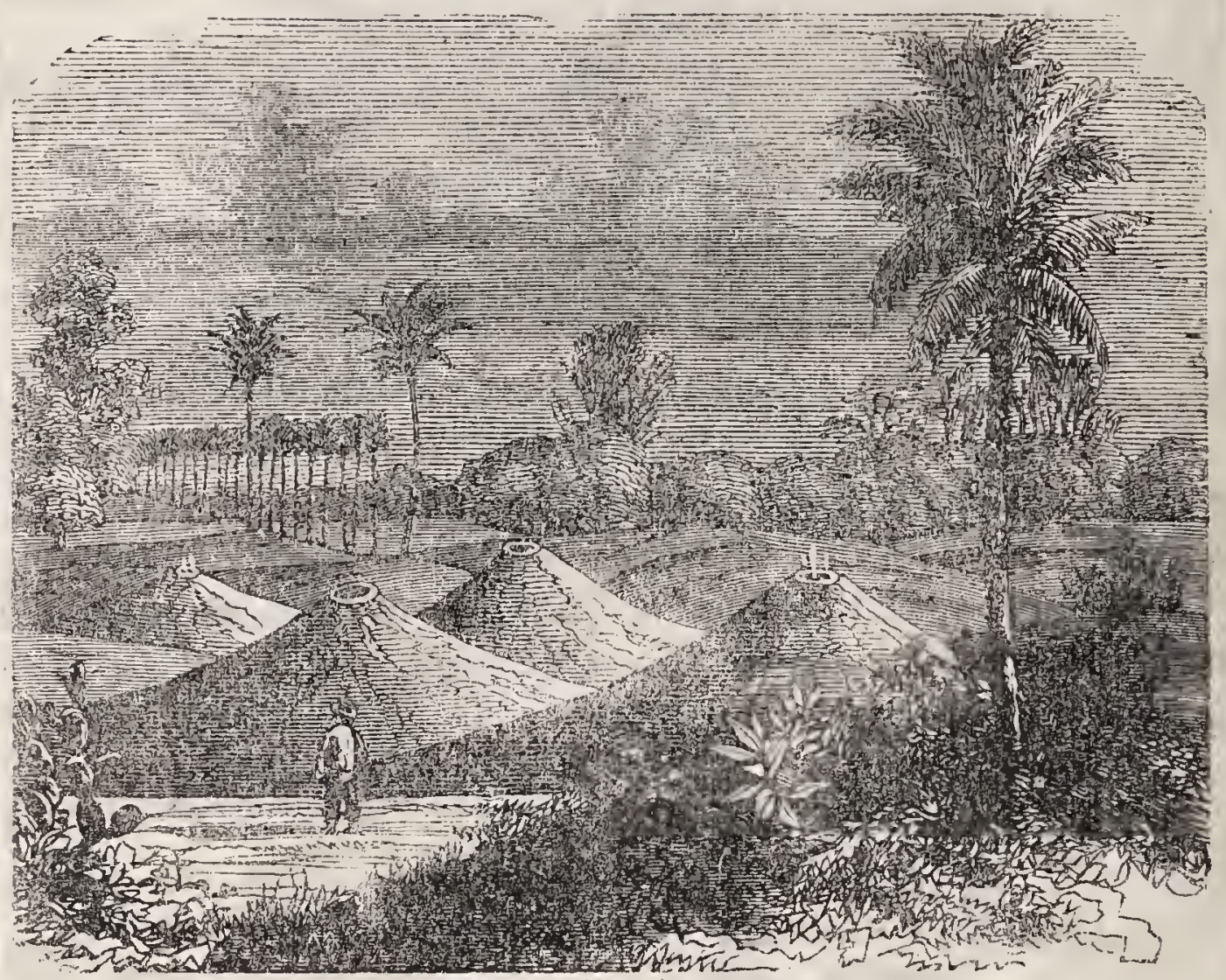

MIUD VOICANOES IN NTH GPEATAD.

Q. Are Hot WELIS, or THenMa SPrings, connected with volcanic action?

A. Of this there can be little doubt. It is evident that such springs are of a mixed charcuter, partly igneous (burning), partly uqueous (watery).

Q. Are mineral springs UseruL?

A. All mineral springs contribute essentially to the lenefit of animal and vegetable life.

Q. How do geologists ACCOUNT for mWrrmal, or Hot sprmas?

A. Sometimes on the theory that the water rises from a great depth, at which the rocks are intensely heated. Sometimes on the supposition that the internal liecat of the earth is forced upwards through fissures till it meets with water, to which it communicates heat. . It appears, indeed, most probable that these springs do receive their heat from the interior of the earth.

Q. Do these therruil'springs rise only from ONT IIND of rock?

A: No. Ther rise in all Trind of places, and from almost all kinds of rocts 
Q. What are the Gersens?

A. 'The Geysers are situated in Iceland, and throw up immense flumtities of stcam and boiling water. Within a circle of about two miles, one hundred of these Gevsers are found. They rise in a thich bed of lava, probably derived from Mount Hecla, which is thirty miles dinkint.

Fig. 216.

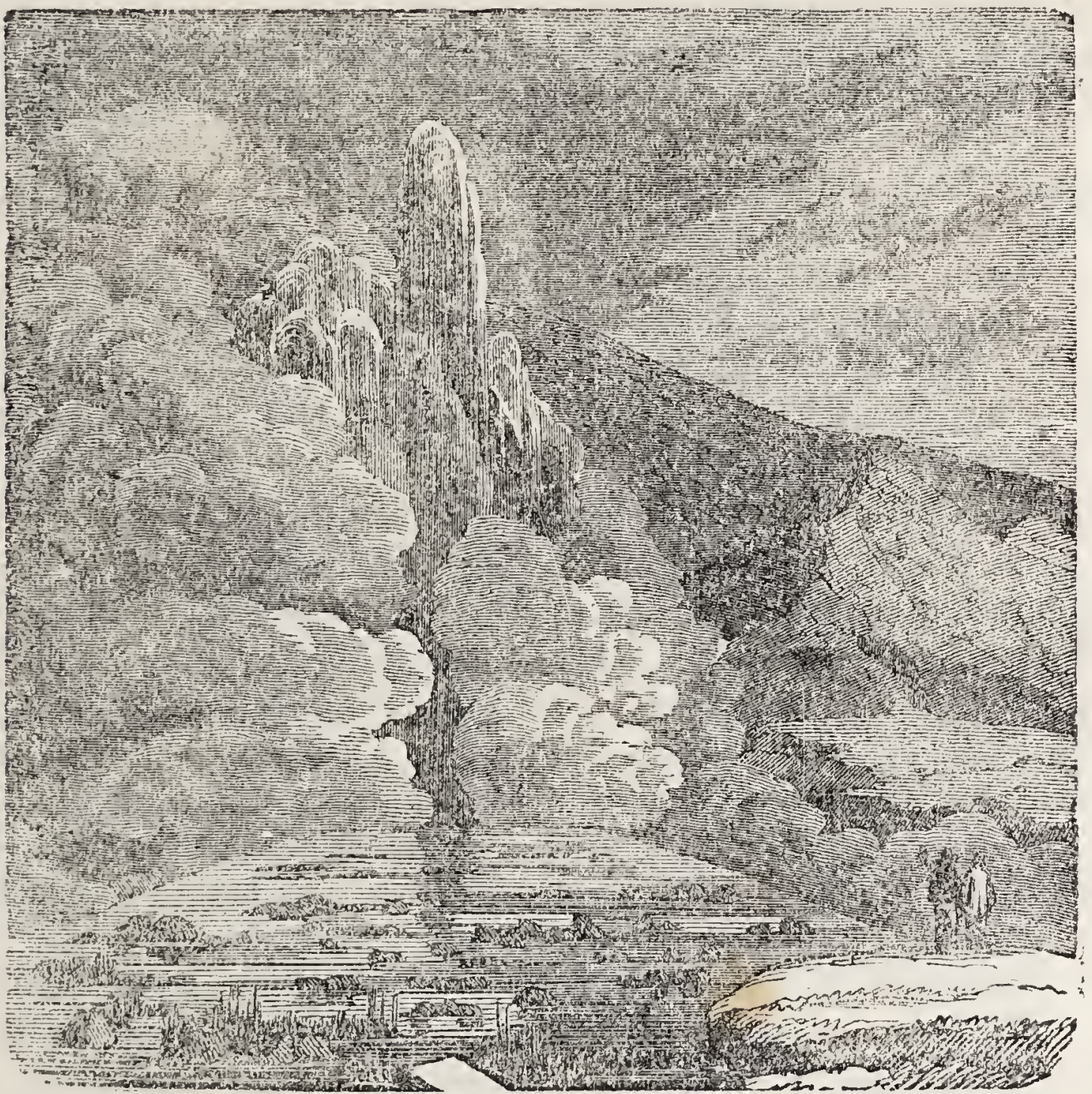

TIR GEYSER IN ICELAND。

In the above engraving we have a representation of what is called the Great Geyser. It rises out of a spacious basin, which surrounds a vent or a well. The basiu consists of a mound, which has been formed round the well by finty matter which has fallen from the spro,y of the water, and which has settled in a circular incrustation. The diameter of the whole basin is about fifty feet. In the centre is the well, shaft, or pire, which goes some twenty-seven feet deep. At intervals this rasin is quite cupty, but ubually it is filler? with water, which continues to boil like water in a kettle over a fire.

Q. What are EARTHQUAKRS?

4. The earthquake consists essentially of movements of the eurtis" crust or surface in the form of waves or undulations, which travel with great rapidity in all directions from the centre of the disturbance, that is to say, the place where the cause of the earthquals is situated 
Q. What is supposed to be the CaUSe of Earthquakes?

A. The same agency exhibited in volcanic action-internal heat-is undoubtedly the cause of earthquake phenomena. The cooling of the strata may cause contraction and movement of the earth's crust, or the pressure of gases generated by heat may cause violent movements and undulations of the strata.

Q. What is the INFLUENOE of earthquakes in changing the surface of the earth?

$A$. Earthquakes produce innumerable changes in the surface of the globe. Old lakes disappear, new lakes are formed; old islands are swallowed by the sea, new islands rise from its depths; portions of land are depressed, and others elevated; the relative positions of sea and land are changed; landslips occur on the sides of mountains; and rivers change their courses.

Q. What is the Acrion of an earthquake?

4. The action of an earthquake is threefold. It is vertical, and rises from the interior of the earth to the surface; it is horizontal, and follows the course of certain rocks or strata; it is circular, and appears to go round certain points in its course.

Q. What EFFECTS are produced by the VERTICAL ACTION of an earthquake?

A. It causes cracks and fissures in the outer crust of the earth; portions of the earth slip down, and are said to be swallowed up.

Q. What are the EFFEcTS of the HORIZONTAL AOTION of an earthquake?

$A$. It causes motions in the earth similar to that observed in the waves of the sea. They proceed in one direction, and when they encounter no resistance, are not considered dangerous. If, however, the course of this vibrating movement be checked by coming into contact with other vibratirig waves, the result of earthquake action, the effects are most frightful.

Q. What are the EFFECTS of the CIRCULA MOVFMmant of earthquakes?

A. What is meant by the circular movement is, that the earthquake power moves in a circle, large or small. Thus the great earthquake which destroyed Lisbon was felt in Spain, in North America, in the Alps, in Thuringia, in the North of Germany, in Sweden, in Loch Lomond in Scotland, in the Great Lakes of Canada, and in the West Indies.

Q. Nention some inst?nces of the SUBSIDENCE OF LAND by earthquakes.

A. In 1692 Jamaica was shaken by an earthquake. At Port Royal several large storehouses sunk below the level of the harbour, and some of them were forty-eight feet under water. A large tract of land and the town also sunk in less than a minute, and settled at the bottom of the sea.

2. Has any STRIKIna INSTANCE of the ELLEATION of land by the agency of an earthqualke vecurred within a RECENT period?

A. In 1822, an earthquake occurred in Chili, South America, which permanently elevated a line of coast over un area of about one 
humdred thousand square miles-a territory equal in extent to half the country of France.

Some geologists suppose that the whole country, from the foot of the Andes to a great distance under the sea, was thus elevated, for the greatest rise was at the distance of two miles inland from the shore. On the coast the rise was two or four feet; but a inile inland it was six or seven feet. This elevation has been disputed by severa, naturalists; but it has been verified by the Germian travellers, Dr. Neyen and M. Freyer, ass onr own Dirwin.

Q. What is the roost remarkable instarter of these phenomena on land?

$A$. Some very remarkable phenomena of this description have taken place in the Bay of Baice, to the north of Naples.

Fig. 217.

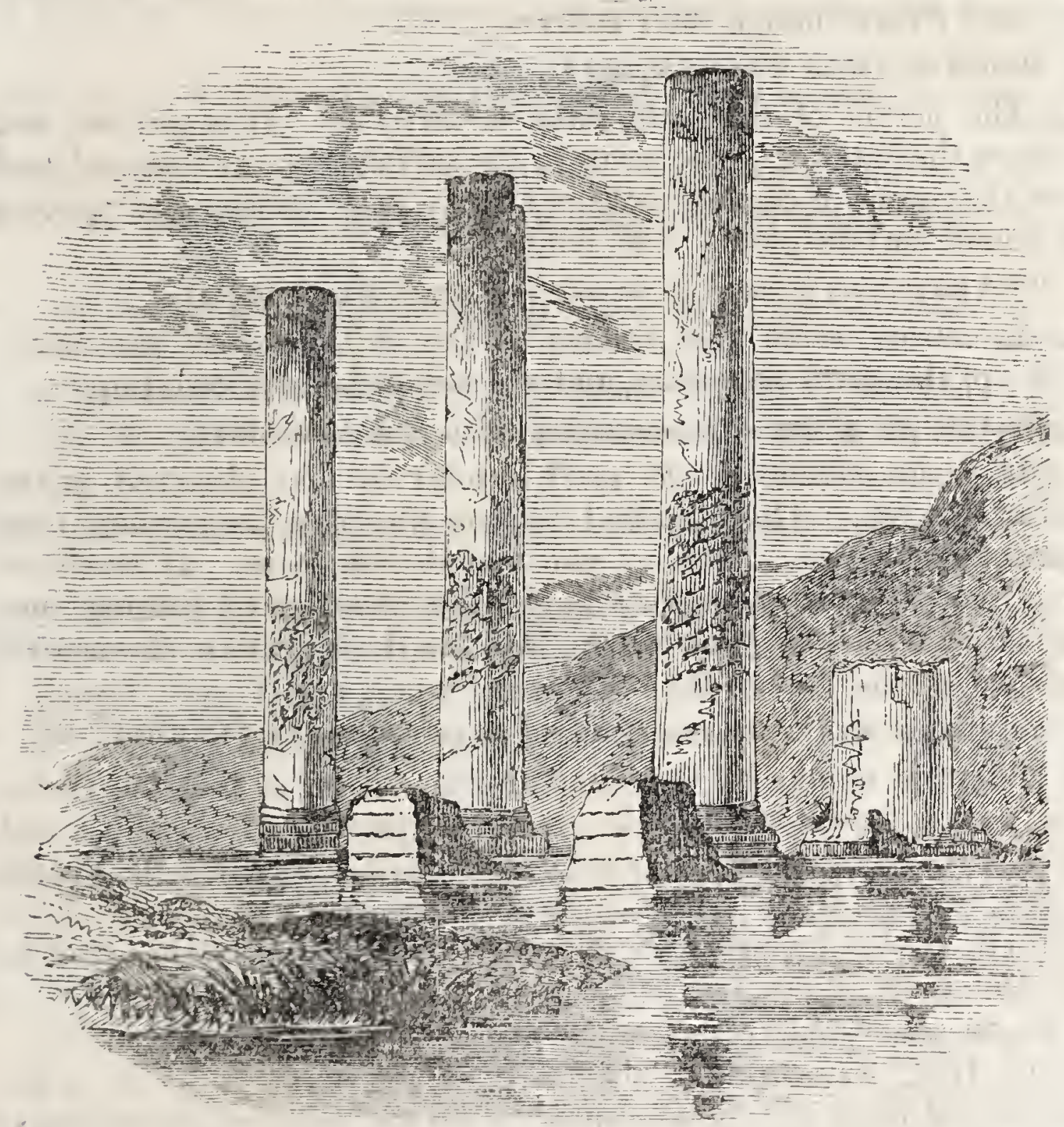

The abore illustration shows the rematius of a temple situated close to the sea shore, at Puzzuoli, in the bay of Baire. It is known as the temple of Serápis. The roof of the building was supported by forty-six pillars - twenty-four of granite, and twenty-twon of inarhle. Of these marble solumns three only remain : they are about forty-two feet hish. The surface of these columns is uninjured to the distance of twllve feet from the pedestal. Then a series of holes, or perforations, are seen in the marble, forming a regular band rouml the column, te the distance of nine feet. The surface then contisues smooth up iv the summit. The upper edge of the perforated base is now upwards of twenty-three 
feet above the sea level. This curious fact illustrates the effects of the elevation and subsidence of land. There is no doubt that these perforations are the work of marine animals. Shells of a species of mussel have been found in the perforations. What does this fact teach? That at some distant reriod, but since the erection of the edifice, the coast on which the temple is built must have suuk below the sea level; that this depression must have continued for a considerable time-time enough, in fact, for the per. firations to be made ; that the coast znust afterwards have been elevated; that both the depression and elevation of the land must have been so gradual as to permit the columns to maintain their erect position; and that the moveme:its downward and up again must hise been more than twenty feet. The time occupied by these changes has also to be borne in mind. They have taken zlace within less than eighteen hundred years. The Homperor Severus, who died A.D. 211, conferred on this building, then in a state of preservation, cortain embellishupus of uat.ble. In A. D. 1198, a subsidence of the coast trok place, and the temple sank with it. In 1530 it is well known that the whole of this coast was covered by the sea. In 1538, during an eruption of Vesuvius, the laud on which the temple stood was elevated to the height of twenty feet. A gradual sinking of the coast is now said to be perceivable, and the base of the pillars is often covered with water.

Q. Does the elevation and subsidence of land ALWAYS DEPEND on the action of earthyuakes?

A. No; land is sometimes elevated, and sometimes sulsides by the expansion of subterraneous matter lying deep in the interior of the eiditio.

Q. Are such clevations and depressions observed in districts in which earthquakes are חNTNOWN?

A. They are; and the instances are numerous. Sea beaches are discovered at a high elevation, and forests are found to be submerged.

Q. Can you furnish an instance of this sort which has taken place at a comparatively RECENT periud?

A. Yes. A large tract of land about a thousand miles in length, and fifty or sixty in breadth, situated on the northern shores of the izullic Sea, has been rising for many years, and is still rising. It ins already risen from one hundred to two hundred feet above the sea ierel.

Q. What do you know respecting suBMARINE Forests?

A. That on the shores of England, France, and North America, suamps, or thickets, or forests are found, in which roots, trunks, branches, and leaves of birch, fir, and oats are found intermixed with aquatic plants and shells; all of which evidence the fact that, at sorne distant period, these forests must have been covered by the octun.

Q. Are not SEA BEACHES fourd at a high elevation?

A. Yes: in England as well as in many other parts of the globe. These beaches, now very high above the sea level, consist of clays, muds, rounded pebbles, and shell deposits-such as are found in the neighbourhood.

In the following diagram is shown the elevatcd strata and bcach east of Kemptown, Brightoll. A B is the present sea-level. $\mathrm{C} x$ is a terrace of chalk, part of the incicut chalk rock, D D. E is a bed of shingle, which, when the sea was at that level, formed the beach. F is a stratum, or bed of loum and chalk, in which the bones of ele- 
Fig. 218.

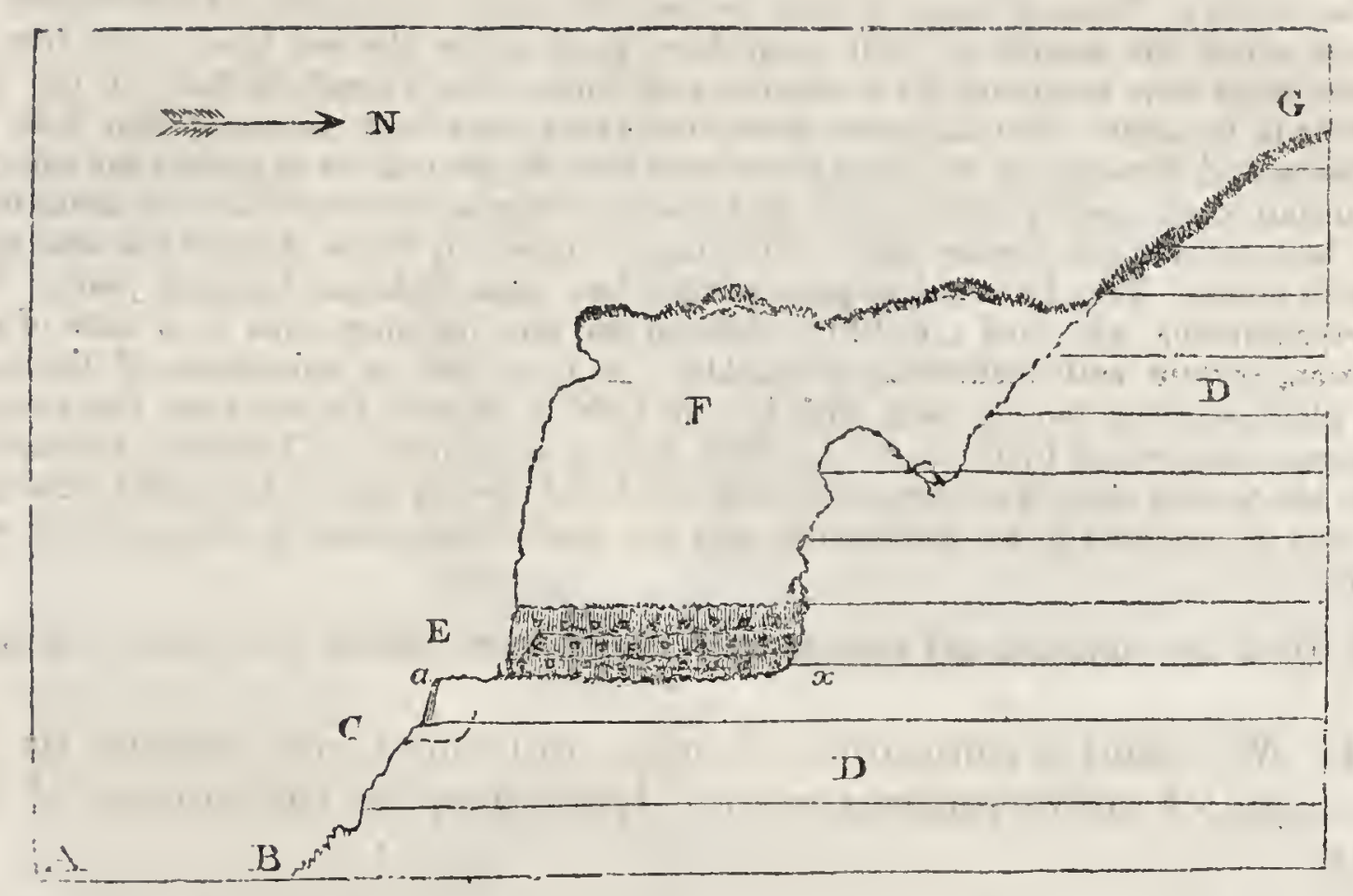

phants hare been discovered. Between the lower projection of the chalk $\mathrm{D}$ and the ved $\mathrm{F}$, there is the stratum $\mathrm{F}$. This, from its characteristics, proves to be an ancient sear beach. From careful observation of these phenomena, we learn the following facts :The entire chalk-rock D D was once the bottom of the ocean; that it afterwards began to rise, and during its progress was worn away in parts by the sea-current, as seen along the line $\mathrm{G} x a \mathrm{C} \mathrm{B}$. For ages the sea-level continued on the level of $a x$. The chalkbeds gradually arose, and the beach $\mathrm{F}$ was formed by the action of the waves on the chalkcliffs. After the formation of the shingle-beach, the whole rock sank again to such a depth as to allow the deposit of the thick stratum F. Then the entire chalk rock, the elephant bed, and the ancient beach, began to rise, and continued to rise, till the sea stjod at its present level, and formed the sea-beach as now seeu.

Q. Does rolcanic action take place UNDER THE OCKAN?

A. It does; as rocks and sea-beach are elevated by the fire under the earth's crust, so this same action under the bed of the sea canses many singular phenomena.

Q. How are these CHIEFLY DFTELOPED?

A. By the elevation of land from the depths of the sea, generally of a volcanic character.

Q. Furnish an INstance of this sort?

A. A well-known instance is that of St. Michael's, in the Azores. In 1811, after the shock of an earthquake had been felt in the neighbourhood, columns of ashes, accompanied with fire and smoke, were seen to rise from the sea to the height of several hundred feet. At the close of the eruption an island became visible, and gradually rose to the height of three hundred feet. It had at one end a deep crater, out. of which flames of fire were gushing under the water, and at the other end a summit in the form of a cone. The sea flowed into the crater, and the water there boiled as in a cauldron. After a few months the island santi and entirely disappeared. 
Fir 219

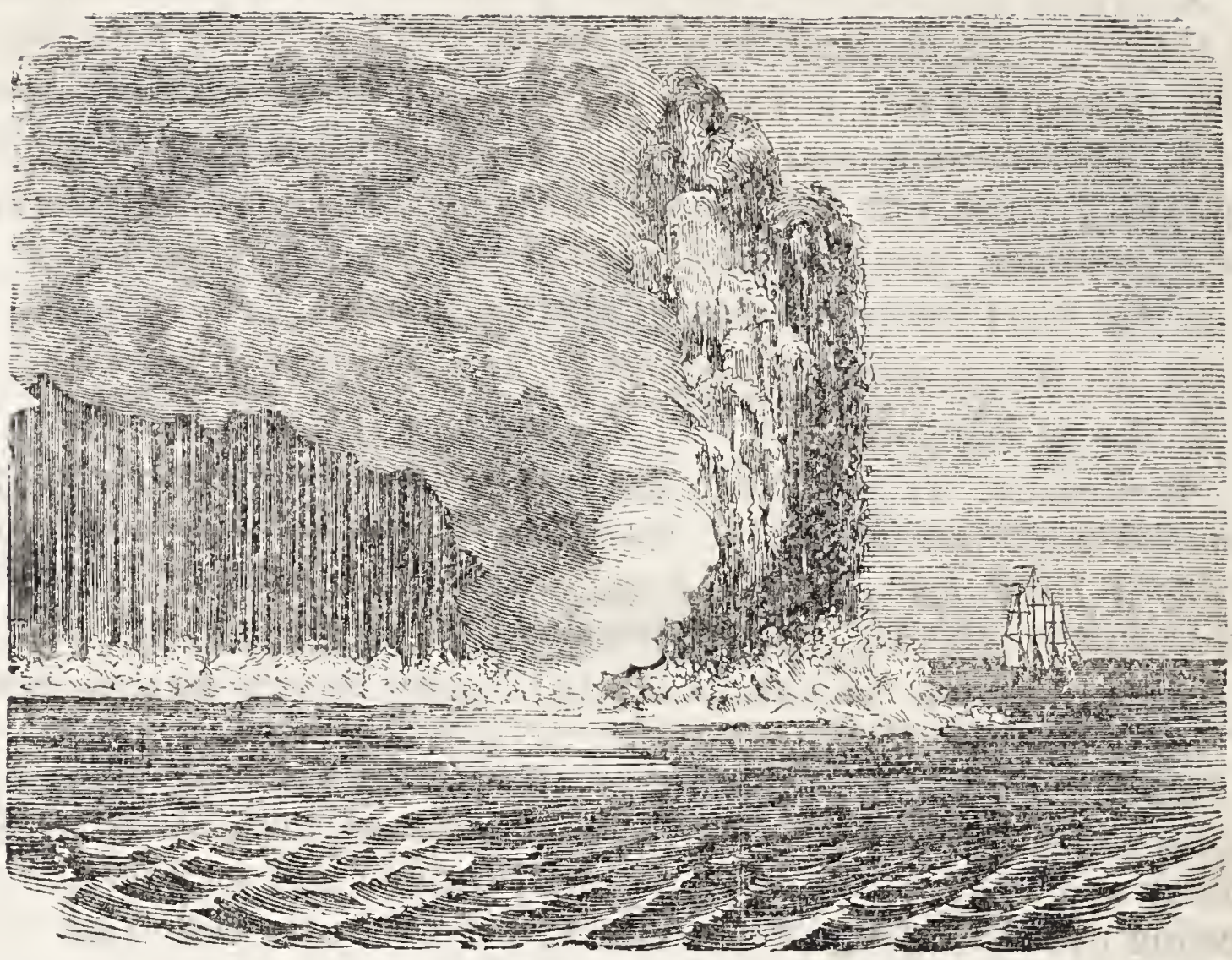

SUBHARINE LRUPTION, NEAK S'T. MICHAEL'S, IN THE AZORES.

Q. What is meaut by a voLCANIC DYKE?

A. A rock penetrating another rock is called a dyke. It means generally volcanic matter thrown into the fissures or crevices of other rocks.

Fig. 220

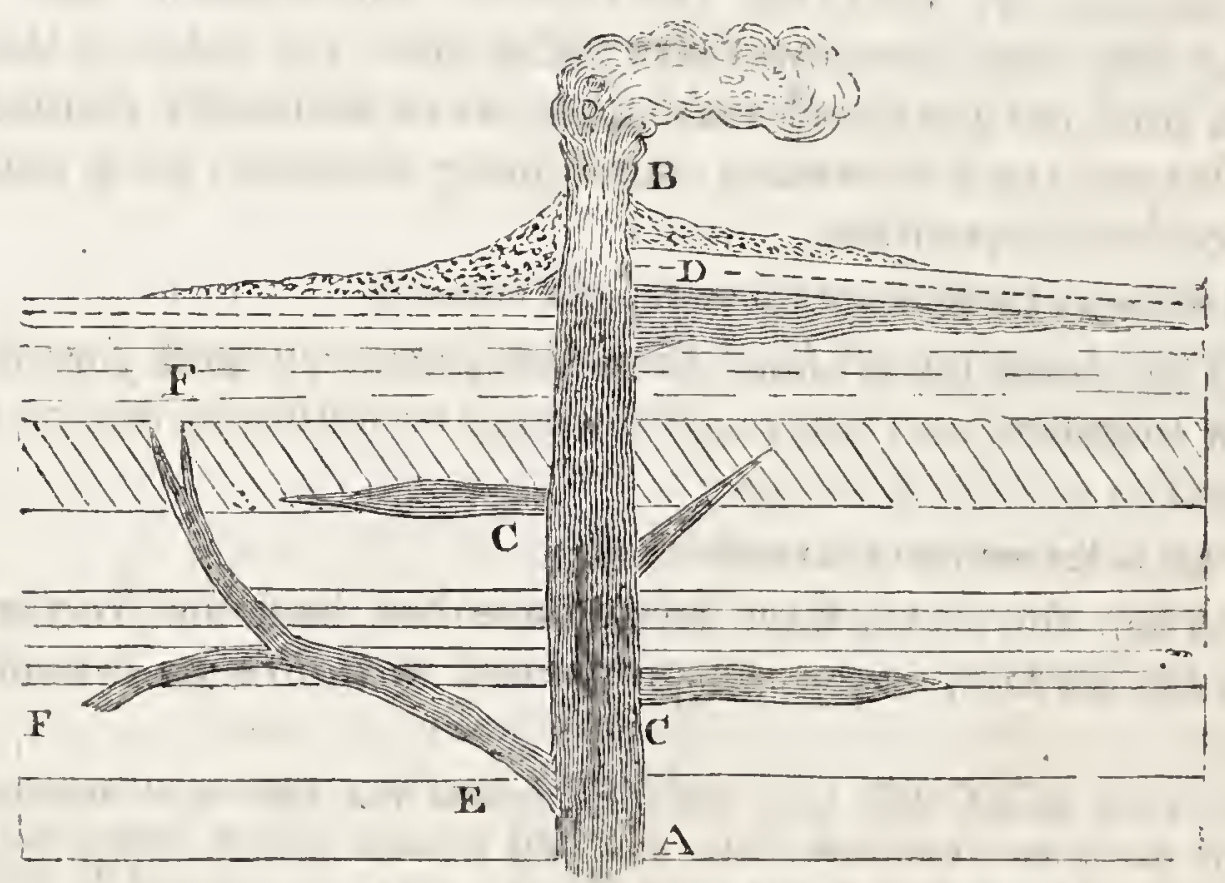

This figure represents, A B, a column of liquid lava, passing through horizontal strata. Finrly in its rise it produces the fissure $\mathrm{E} \mathrm{F}$; at C it enters between two beds of strata; at $\Gamma$ it divides the strata. This molten lava, afterwards cooling and hardening, produces those dykes, and otber rocks, which are cccasionally found in strata. 
Q. How do you $\triangle$ CCOUNT for the FISSURES in the rocks?

$A$. These fissures may have been produced in the process of cooling of the carth's crust, or afterwards by the shock of an earthquake.

Q. How is it that LAVA, or VOLCANIO MaTTER, could be thrown into these fissures?

$A$. The volcanic matter being in a molten state, and being forced upward, rushes into the fissures, in some cases produces them, and thus forms the mixture of rocks called dylies. (See fig. 220.)

Q. How have the MOONTAINS upon the earth been FORMED?

A. By the upheaval of portions of the solid crust of the earth.

Q. How do we know this?

A. Because we find that the stratified rocks of which they are sometimes composed, or the stratified rocks which are adjacent, are changed from their original horizontal position, and are. now tipped and tilted up, so that in many instances the strata are nearly vertical.

Q. What appears to have been the IMmediste $\mathbf{A E N C Y}$ by which the strata have been broken up and thrown into INCLINED POSITIONS?

A. Masses of granite or other igneous rocks have been, by the agency of the internal heat of the globe, forced upwards from below, lifting and breaking through all the stratified and sedimentary rocks resting upon them.

Q. Are all the ranges of MOUNTAINS upon the earth of the SAME $\triangle G E$ ?

A. Most of the great mountain ranges on the earth's surface have been elevated at distinct and different periods. Thus the Alps of Switzerland are of a comparatively recent origin, having been elevated during what is called the tertiary periods.

Q. How do we kNow that the Alps were elevated during the tertiary periods?

$A$. Because we find that the rocks of this system have been elevated by the same force, and now exist upon the sides of these mountains in inclined positions. As they were evidently formed in horizontal layers, their elevation must have occurred at a period subsequent to their deposition.

Q. How are RAVINES, GORGES, and VALLEYS formed?

$A$. The strata have been doubtless fractured and forced apart in the first instance, and afterwards eroded or hollowed out by the action of water.

Q. What is the ORIGIN of OAVERNS ?

$A$. When the strata have been fractured, and the fracture does not come to the surface, an opening is formed in the rocks, which we call a cavern.

Q. We have stated that each geological system has species of animals and plants peculiar to itself, and that these have gradually become extinct before the introduction and formation of a new system and series of rock: WHAT is supposed to have occasioned the extinction of WHOLE RACES of animals and plants in past ages?

A. The changes in temperature, and the rapid mutations of land and water were probably the principal agents of destruction. 
Q. How conld all the present races of animals and plants peculiar to the trupics be DESTROYED at ONCE, and the species rendered extinct?

A. By a sudden change in the climate, which should reduce the temperature to thirty-two degrees $\mathrm{F}$. or less.

Q. Is there any REASON to BELIFVe that such CHANGES have takeu place upon any portion of the earth's surffee?

$A$. There is reason to believe that the climate of Siberia and Northern America was once much warmer than it is at the present day. In Siberia we find the bones of the elephant, the lion, the tiger, and other animals which can only live in tropical clinates. Capt. Parry, at Melville's Island, within the Arctic Circle, found remains of plants similar to those now growing under the tropics.

Q. Have any species of aumals become ExTrNot within the MExonY of man?

A. Several species of animals, which have existed in considerable numbers within the memory of man, are now utterly cxtinct, and have disappeared from the surface of the earth.

Q. What are some EXampies?

A. The bird called the dodo and the apteryx, the first a native of Mauritius, and the last of New Zealand, are both extinct. A species of $e t l$, formerly common in Ireland, has disappeared.

Q. Do we know of any SPECIES of animals which are gradually DISAPPEARING?

A. Most wild animals which are hunted for their skins and furs are becoming more and more rare and uncommon. The beaver of North America and the fur seal of the Southern Pacific Ocean, once existing in great numbers, are now rarely met with.

\section{CHAPTER LXV.}

\section{THE DILUVIAL AND TERTIARY SYSTEMS.}

Q. In studying geology, is it NECESSARY to become acquainted with what is called the CLASSIFTOATION Of ROCKS?

A. Yes; it is as essential to the geologist as learning the alphabet to one who wishes to be able to read.

The geological section on page 489 shows the position and order of the different rocks, from the surface to the granite, supposed to be below the strata marked $\mathbf{v}$ in the diagram.

Q. Does the WHOLR CRUST of the earth resemble, in its arrangement, the section shown in the diagram.

A. No; it is certainly possible that in some parts of the earth's surface, if a shaft were made, it would pass through the whole series, from $\mathrm{A}$ to $\mathrm{v}$, but it is not at all probable. 
Fig. 221

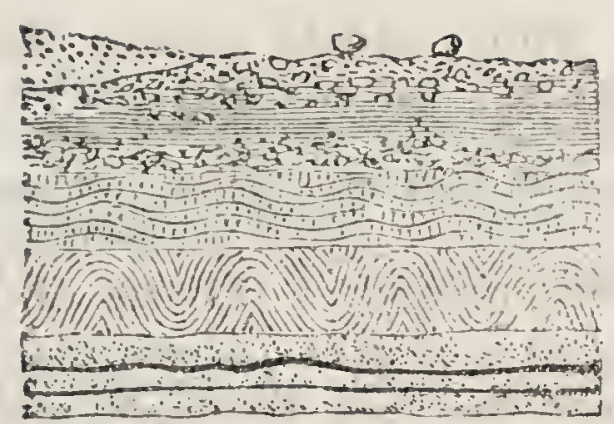

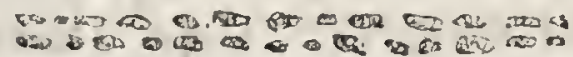

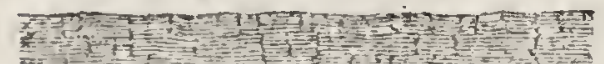

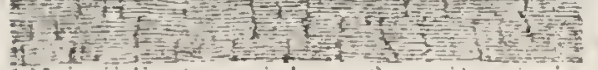
10

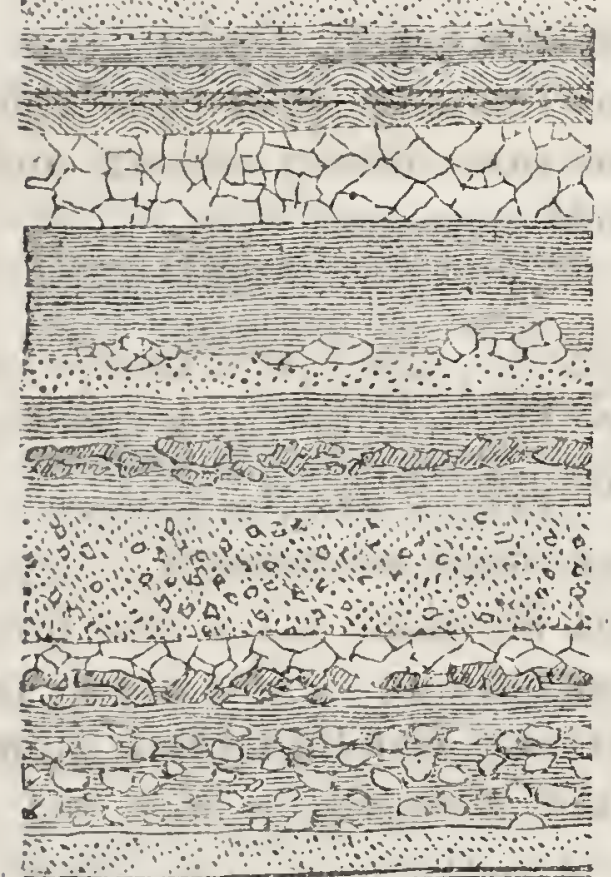

$1-5=0-10$

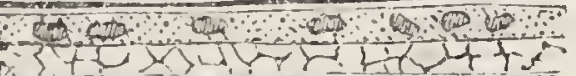

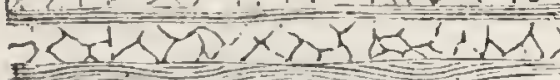

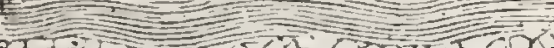

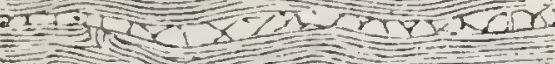
$+\mathrm{P}, \mathrm{N}$

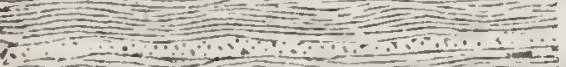

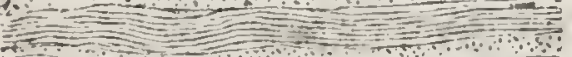

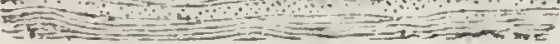

A. Alluvial Soil.

B. Erratic Blocks, and Iiil.

c. The Pleistocene.

D. The Pleiocene.

F. The Meiacene.

F. The Eocene.

G. The Chalk Forroation.

II. The Quadersandstein.

I. The Neocomien.

s. The Wealden.

k. The Oolite.

L. The Lias.

M. The Keuper.

s. The Muschelkalk.

o. The Buntersandstein.

P. The Zechstein.

Q. New Red Sandstone, or Rothliegendes.

ฉ. The Coal Measures.

s. The Mountain Limestone.

T. The Devonian, or Old Red Sandstone.

v. The Silurian.

v. The Cambrian.

AN IDEAL SECTION OF THE STRATIPIED ROCES, IN THEIF VERTICAL URDER OH PUSITION.

Q. What, then, is the USE of this clisssification?

$A$. It shows us that the strata marked $\mathrm{K}$ or $\mathrm{L}$ will never be above the strata marked $G$, and that $R$ will never be below $V$. In digging a shaft, any one of the rocks marked, or several of the rocks marked, might be missing, not occurring in that locality, never having been there upheaved or deposited; but their absence would not alter the accuracy of the classification, as, if they were there, they must occupy the positions marked. The entire series may never be found in one 
district: but the members of any series that are found to occur, will follow the order of sequence.

Q. What NAME do we give to those great accumnlations of SAND and COARSE GRAVEL which exist unon the surface of the earth, and, in geological order; are next beneath alla. vial deposits?

A. Dituvium or drift. They are considered by many geologists to constitute the upper member of the tertiary system.

Q. What is the gEOGRapHical RaNGE of the drift?

A. In America it is found from Nova Scotia nearly to the Rorky Momituins, and extending as far south as Pennsyluanice and the Ohio River. It also exists in the northern portions of Europe and Asia.

Q. How is this accumulation of sand, gravel, anl bonlders supposed to have onj A:NATRD?

A. It is supposed that during the epoch of the drift, the northern portions of the Eastern and Western continents were submerged and swept by an ocean carrent. Through the agency of this current and of icebergs, the rocks forming boulders were torn from their original beds, and the existing deposits of sand and gravel, formed by the wearing and denudation of the earth's surface, were produced.

Q. Do we find in these beds of sand and gravel any ANIMAL REMAIXs?

A. The quantity of animal remains found in the drift is immense. The epoch immediately preceding the drift agency was the period of the mammoth, the mastodon, and other huge ruminant animals, which probably became extinct before the creation of inan.

Q. What was the CHARActer of the mastodon and the mammoth?

A. They were animals allied to the elephant, and resembling it in form and structure, but larger. Skeletons of the mastodon have been found in various localities in the northern portions of the United States, some of them in the most perfect condition.

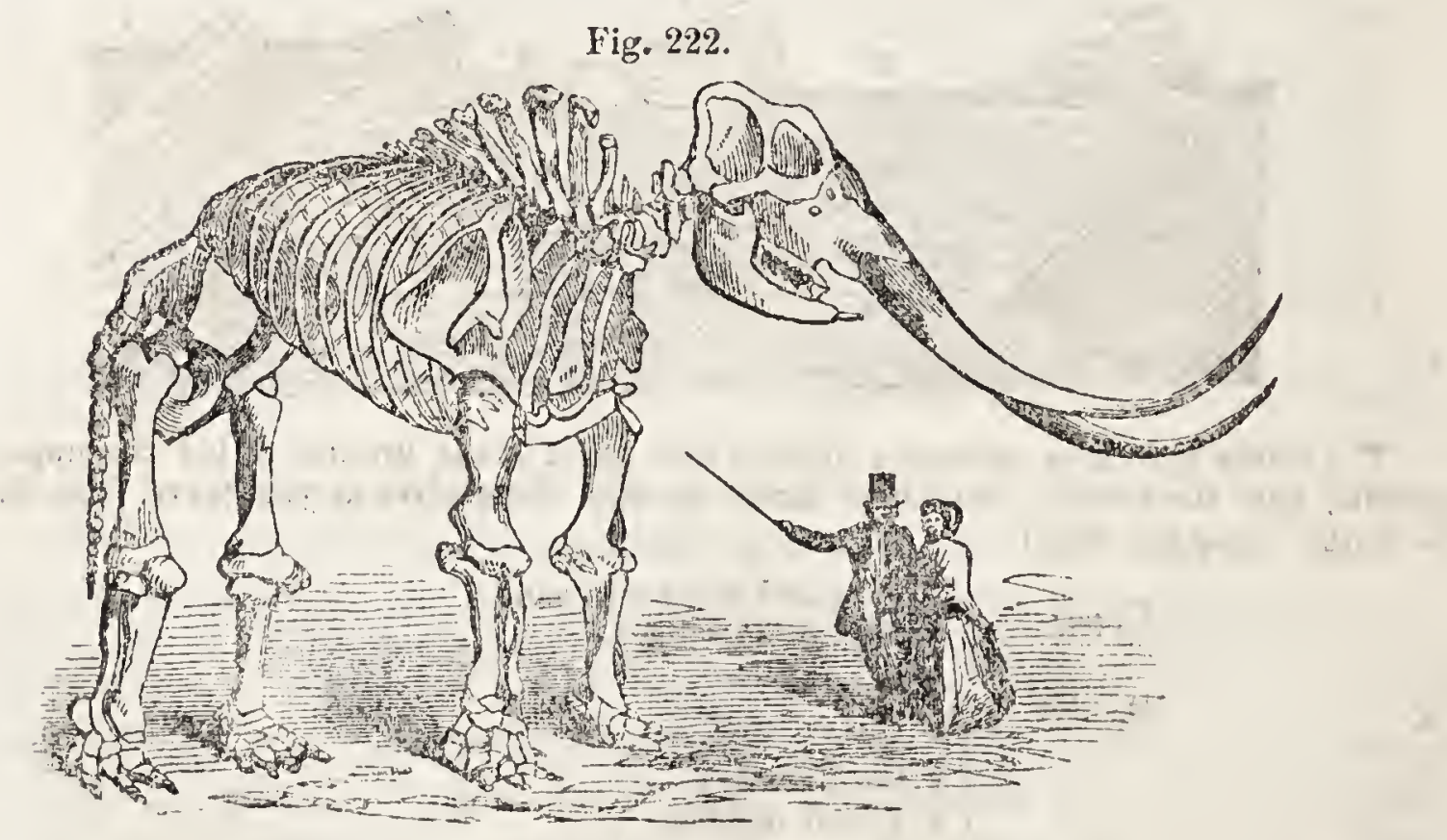

Pig. 222 represents the remains of is mastolon; one of the mast peifeci specimens ever discovered. 
Q. WHERE are the deposits belonging to the tertiary system found?

$A$. The tertiary system, though not generally so continuous ore? large areas as the older formations, yet constitutes the surface of a large part of Europe. In the United States it occurs along the seaboard from $N$ ew Jersey to Louisiana. It also covers large surfaces in South America and Southern Asia.

\section{CHAPTER LXVI.}

\section{THE CHALK, THE OOLITE, AND THE NEW RED SANDSTONE.}

Q. What SYsTEu of rocks succeeds the tertiary?

A. 'The cretaceous system, or the chall formation.

Q. What is CHALE?

A. Chalk is a carbonate of lime, which appears to have accumulated in immense beds or layers at the bottom of a tranquil sea.

Q. What is the character of the Fossrus of this system?

A. 'They are principally the remains of marine animals and plants in a beautiful state of preservation, such as corals, sponges, starfishes, \&c.

Q. Are the rocks constituting this system WHOLIX COMPOSED of chalk?

A. No. They consist of beds of clay, of greenish sand, and of limestone, with beds of pure chalk.

Fir. 223.

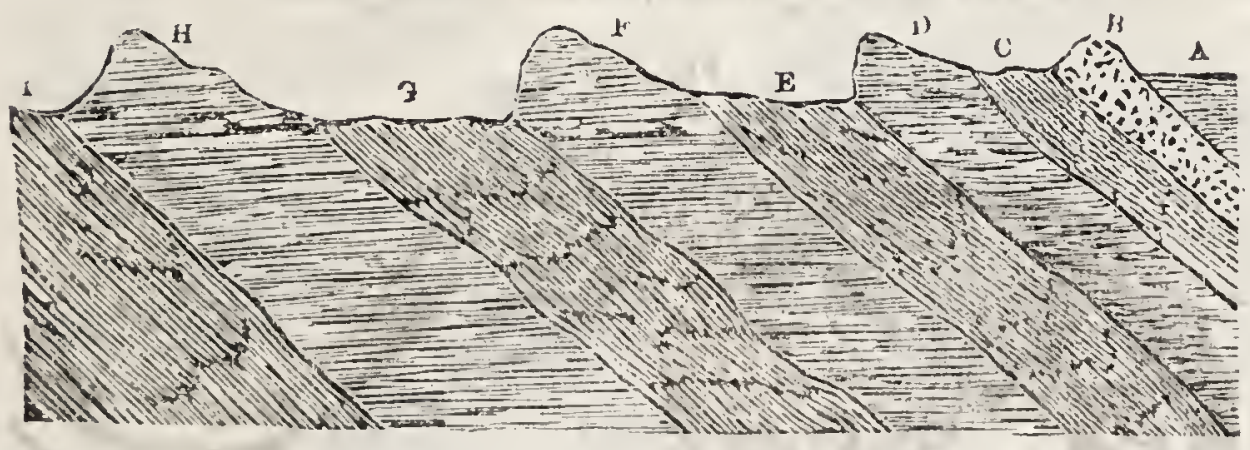

The oolitic system is generally divided into three great groups, called the upper, the middle, and the lower. All these strata develop themselves as you travel from London to Bath. (See fig. 223.)

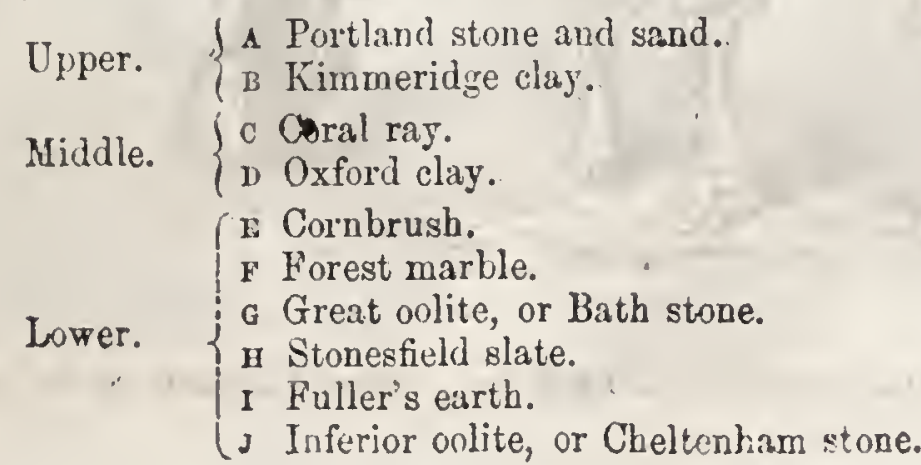


Q. From whar sources are the flint-stones, from which gun-fints are manufacturerl, abtained?

$A$. From the chalk formation; the flint exists in the chalk in the form of rounded masses or nodules. When these nodules are broken by the hammer. they furnish the ordinary gun-flint.

Q. What Grologrcal SYSTEM succeds the chalk, or cretaceous system?

A. 'The oolite; made up, principally, of beds of clay and limestone.

Q. Why is this system called the ootris ?

A. Because many of the limestones composing it have a curionis structure, consisting of little rounded particles, resembling the roe of a fish. The word is derived from the Greek owv, an egg, and $\lambda_{\iota} \theta 0$, a stone. (See fiy. 223.)

Q. What remarkable Fossins occur in this system?

$A$. The system was particularly characterised by the existence of reptiles of the saurian or lizard order. The most remarkable of these was the iguanodon, a lizard upwards of seventy feet in length, and proportionally large. It lived upon the land, and was herbivorous.

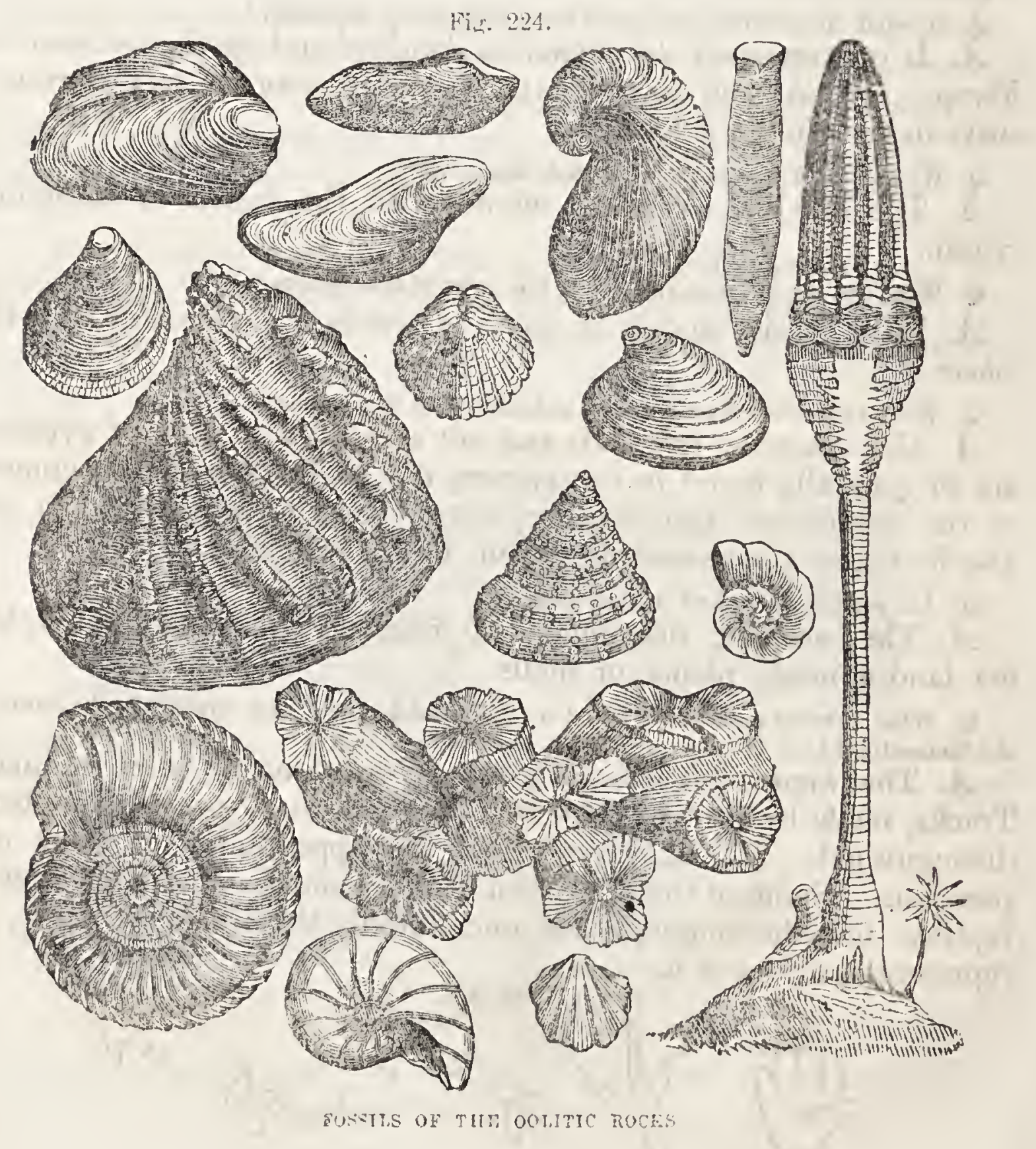


Q. What is an HERBIrorous animal ?

A. One that lives upon vegetable matier.

Q. What Two orHen remarkable animals lived at this period?

A. The ichthyosaurus and the plesiosaurus.

Q. What was the character of the ichthyosaurus?

A. It was a fish-liward, somewhat resembling the crocodile. It was a carnivorous animal of immense power and rapacity, with fins like a fish, paddles like a turtle, and eyes of unparalleled dimensions.

Q. What was the PLESIOSAURus?

A. The plesiosaurus had the head of a lizard, the neck of a bird, the body of a fish; and the tail of a crocodile. It appears to have lived in shallow waters, so that when moving at the bottom it couid lift its head above the surface for air or in search of food.

Q. What was the CHARACTER of the vegetation of the oolitic period?

$A$. It must have resembled, in a striking manner, the vegetation which at the present day clothes the islands of the South Pacific Ocein.

Q. In what Countries is the oolite most extensively developed?

4. It is extensively developed in England and on the continent of Europe. It has been doubted whether this system has any representative in the United States.

Q. What SYSTEM immediately succeeds the oolitic?

A. The new red sandstone, often called the triasic or saliferous system.

Q. What are the CHARACTERISTICS of the rocks of this system ?

A. They consist mainly of layers of sandstones, generally of a red solour.

Q. What valuable Mirmeat occurs extensively in this system ?

A. Common salt; salt beds and salt springs, with beds of gypsum, are so generally found in this system, that in Europe it is designated as the "saliferous (salt-bearing) system." The ores of copper are also found, to a considerable extent, in this formation.

Q. Are Fossils ABUNDANT in this system?

$A$. 'They are not; the remains of fishes are frequently found, but few land animals, plants, or shells.

Q. What currous Fossrus are found in the sandstones of this system in the valley of the Connecticut?

A. The impressions of the feet of birds and of some animals. 'Tracks, made by birds of more than thirty distinct species, have been distinguished. Fig. 225 represents the appearances of these impressions. Some of them, as seen at $c$, are impressions of the feet of reptiles, but the majority are undoubtedly the tracks of birds, as represented at $a$ and $b$.
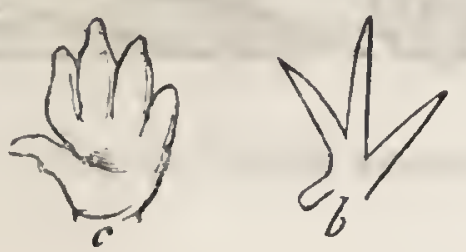

Fig. 225.

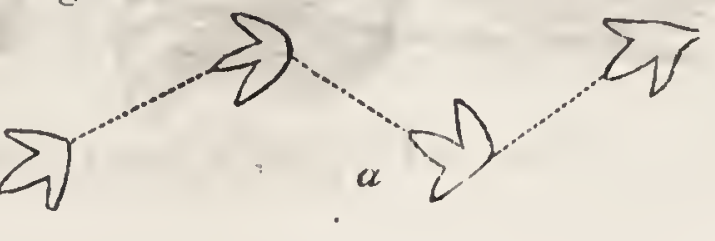


Q. How have these tracts been MADE and preserved?

A.' 'The surface upon which the track was formed was originally a soft mud. After the inpression was made, it hardened into rock, and thus a perfect cast or impression of the foot was preserved.

\section{CHAPTER LXVII.}

\section{THE CARBONIFEROUS, OLD RED SANDSTONE, AND SILURIAN SYSTEMS.}

Q. What STSTEM in order succeeds the new red sandstone?

A. The carboniferous system, or coal formation.

Q. Does this system contain ALL the great deposits of coal found upon the surface of the earth?

A. It does.

Q. Why is not coAL found to any extent in OTHER rocks, or systems?

A. Because the conditions under which coal could be produced appear to have been confined to one epoch of time-namely, the period of the formation of the carboniferous rocks.

Q. What is COAL?

A. Coal is the product of an accumulated vegetation which flourished during the geological period which is represented by the carboniferous formation.

Q. How does the coal occor in the earth?

A. In strata, varying from a few inches to several feet in thickness, enclosed between other strata of limestone, clay, or iron ore.

Q. How is the coal formation cuASSIFIED?

A. This series of rocks is divided into three classes,-

1. The Coal Measure;

2. The Millstone Grit; and

3. The Mountain Limestone.

Q. Describe those rocks which are specially called COAL MEASURES?

A. They consist of deep beds of gritty sandstone, layers of soft clay, seums of coal, bands of clay, rocks containing ironstone, and sand rocks. (See fig. 226.)

Fig. 226.

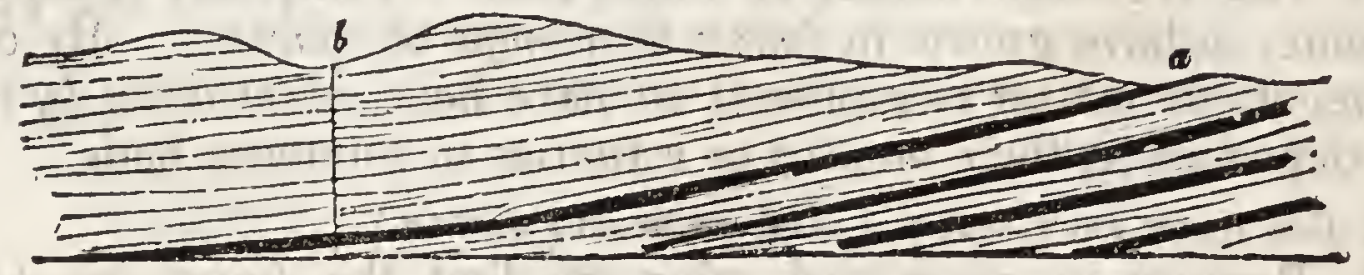

$a$, Butrance into the coal by adit, or level ; $b$, entrance through the grits by a shaft. 
Q. What is cosu ?

A. Coal is a pitchy mass of rotten vegetable matter, mineralised. The coal beds are found in seams, separated from each other by layers of hardened clay and sand rock. The appearance of these coal seams is indicated in the accompanying engraving.

Fig. 227.

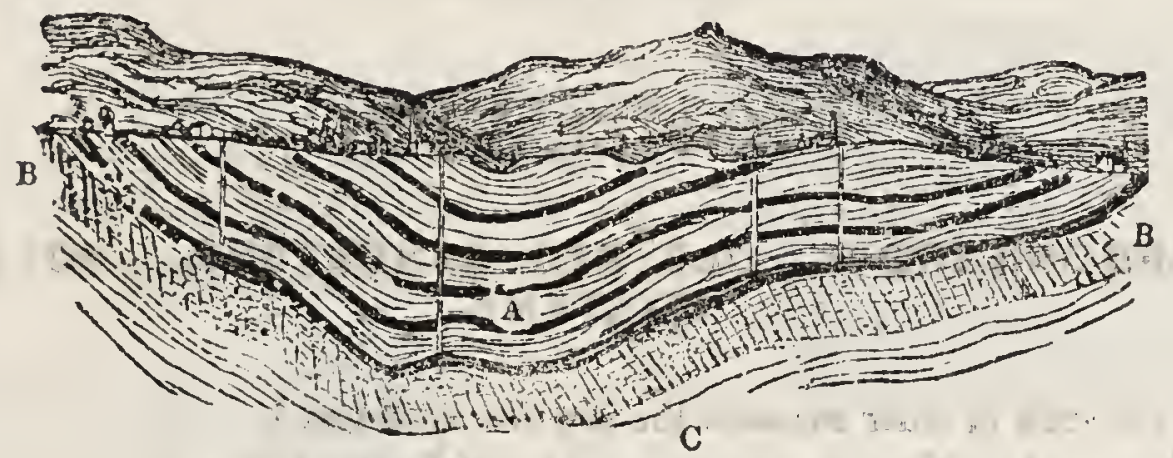

A, Seams of coal, shale, and sandstone; B, millstone grit ; c, mountain limestone.

Q. What is the FLOOR, or the BED, on which a coal seam rests, called?

A. The under clay. It consists of a sandy or gritty shale, sometimes called fire-clay, on account of its capacity to be formed, into bricks.

Q. What is the usual depth of these FLoors, or BEDS?

A. They vary from a few inches to ten feet. Sometimes they are but very slightly coloured with coal matter, at others they are completely blackened with it.

Q. What is the LAYER which goes above the coal called ?

A. The roof. This is generally of a slaty clay called shale... It abounds with leaves, branches, and fruits of plants.

Q. Does the Vegetable Matrer of which coal is formed alWays take one Forr?

A. No. It is capable of various modifications, forming peat, lignile, bituminous coal, anthracite, culm, steam coal, black lead, and the diamond.

Q. What is MILLSTONE GRIT?

A. Millstone grit is found beneath the sanclstone, coal seams, and shales of the coal measure; it is formed of thiclilayers of sandstones.

Q. What is the MounTaIN LIMESTONE?

A. It is the basis of the coal formation, and lies between the coal beds and the more ancient rocks-the old red sandstone.

Q. In what $M \triangle N N E R$ is it supposed that the great accumulation of regetable material of which coal is formed took place?

$A$. The vegetable matter of which coal is composed is supposed, by some, to have grown in immense suamps or marshes. By others, the vegetable matter is supposed to have been swept down by rivers, and deposited at their mouths or estuaries in immense beds.

Q. How do we kNow that coal is all VEGETALLE MATTER?

A. Because in every coal mine we find the leaves, trunks, and fruits of trees in immense numbers, many of them in a most perfect 
state of preservation; so much so, that the botany of the coal period can be studied with rearly as great ease and facility as the botany of any given section of the present surface of the earth.

Q. What is the APPEARAYCE of coal when examined uncer the microscope?

A. When coal has not been too much changed by heat or pressure, a thin layer exhibits all the ducts and vessels of the plant to which it originally belonged.

Q. What was the CHARAOTER of the regetation of the coal period?

A. 'The vegetation of the coal period was of a tropical character, consisting of ferns that attained the size and dimensions of our largest trees, of gigantic reeds and mosses, and a variety of trees which have no representatives now living.

Q. What KINDS and FORMS of VEGETABLES are chiefly found in the coal beds?

$A$. The varieties are very great, and contain the fossils of six hundred and eighty-three specimens of plants; in the whole group of the coal formation the ferns are the only plants that resemble plants of the present day. Among these ferns are included the embroidered fern, the nerved-leaf fern, the wedge-leaf fern, the fern stem, and the round leaf fern.

Q. What is the SIGLLLARIA?

A. The sigillaria is one of the most important plants in the coal formation.

The sigillaria derives its name from the beautiful impressions which are made upon it, and which appear as well defined as though they had been produced by a sigillum-the Latin word for seal.

Q. What is the SIZE and APPEÁANCE of this species of VEaETATION?

A. These sigillaria grew in lofty stems fifty or seventy feet high; the surfaces of their trunks futed, and generally without branches. They are now found in the form of long, flat, narrow slabs, their fluted surfaces bearing many curious impressions. (See fig. 228.)

Fig. 228.
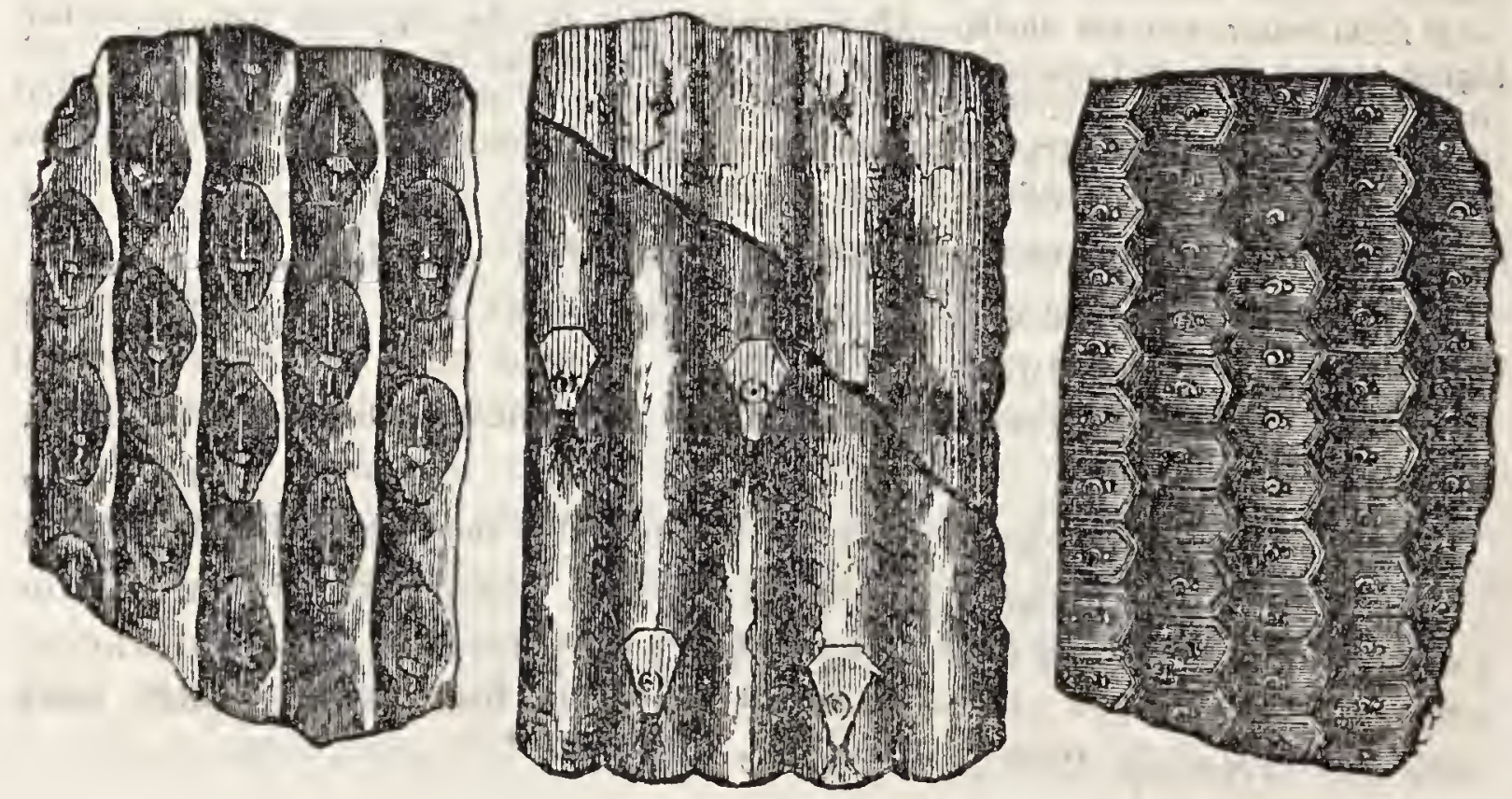
Q. What is meant by stigmaria?

A. Stiginaria are vegetable fossils, found in the clay which forms the bed of the coal seam. They are found, in their natural form, branching out in all directions.

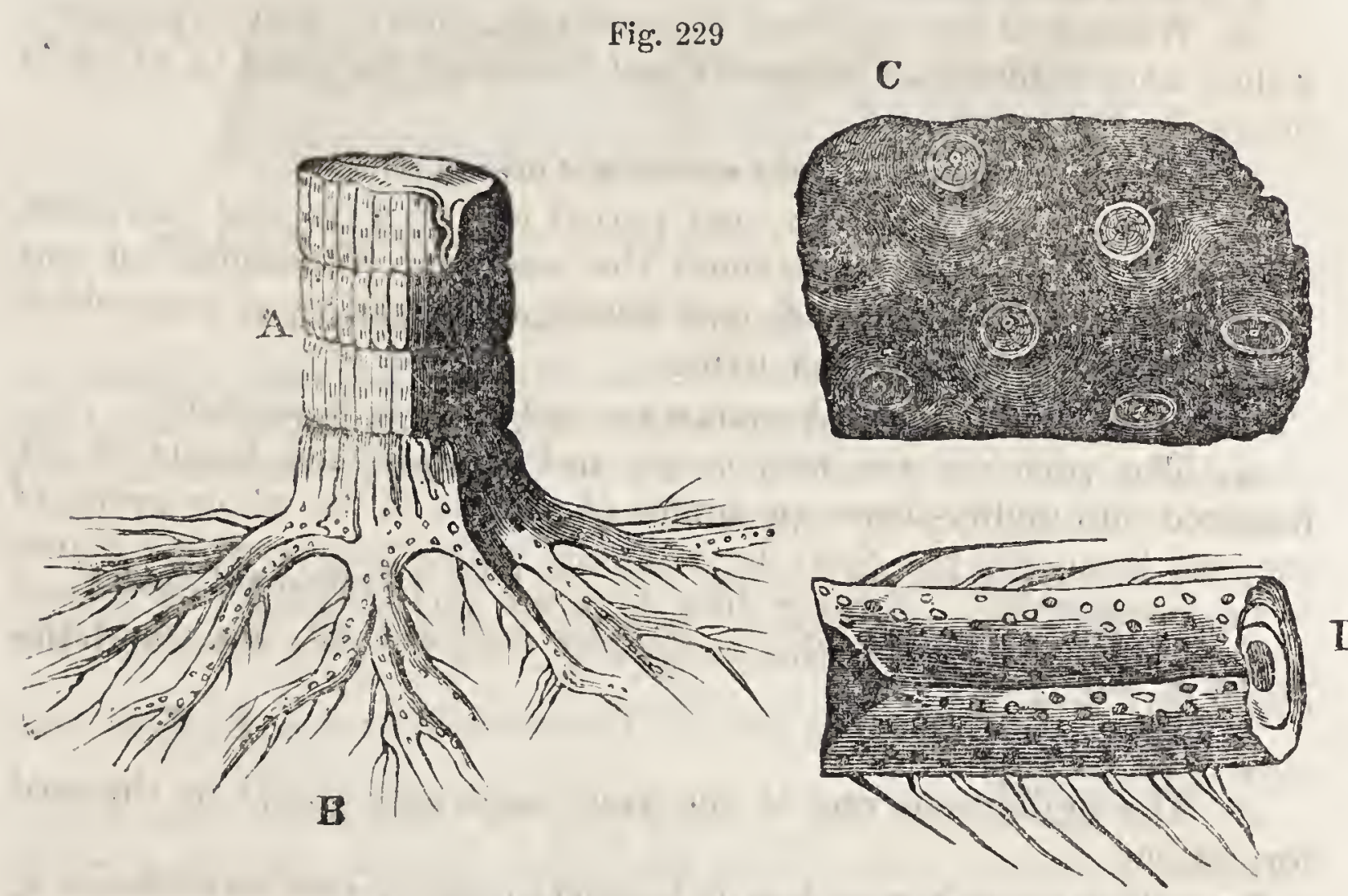

POSSIL VEGETABLES IN THE COAL FORMATIONS.

$\Delta$, The trunk of a Sigillaria in contact with B, the Stigmaria, as its root. C, A fragment of Stigmaria, with its eyelet markings, magnified. D, A piece of Stigmaria ficoides.

Q. Are shells, or FISHES, found in the coal formations?

A. They are. Many remains of this description have been discovered.

The mountain limestone is rich in zoophytes, or animal plants, and in coral of several species. Three hundred different kinds of shells have been also discovered, including both fresh water and sea shells. Until very recently it was supposed that no animal higher than a fish had existed at the coal period; neither frogs, tortoises, snakes, nor lizards having been discovered. In 1847 the skeletons of reptiles were, however, funnd in the coal works near Strasburg, and there appeared every reason for believing that these creatures properly belonged to the coal periud.

Q. How does BItUyinous coal DiFFER from aNTHRacite?

A. In anthracite coal the volatile bituminous part has been driven off by heat. The bituminous varieties of coal have not been changed by heat, and therefore retain most of their original vegetable constituents.

Q. Under what condrorioss do we find anthracite coal in the carboniferous formation?

$A$. Where the strata are broken up and disturbed, indicating the a tion of subterranean heat, we generally find the coal to be anthracite; whore the strata are andisturbed, the coal is, for the most part, bituminous. (See fig. 230.) 


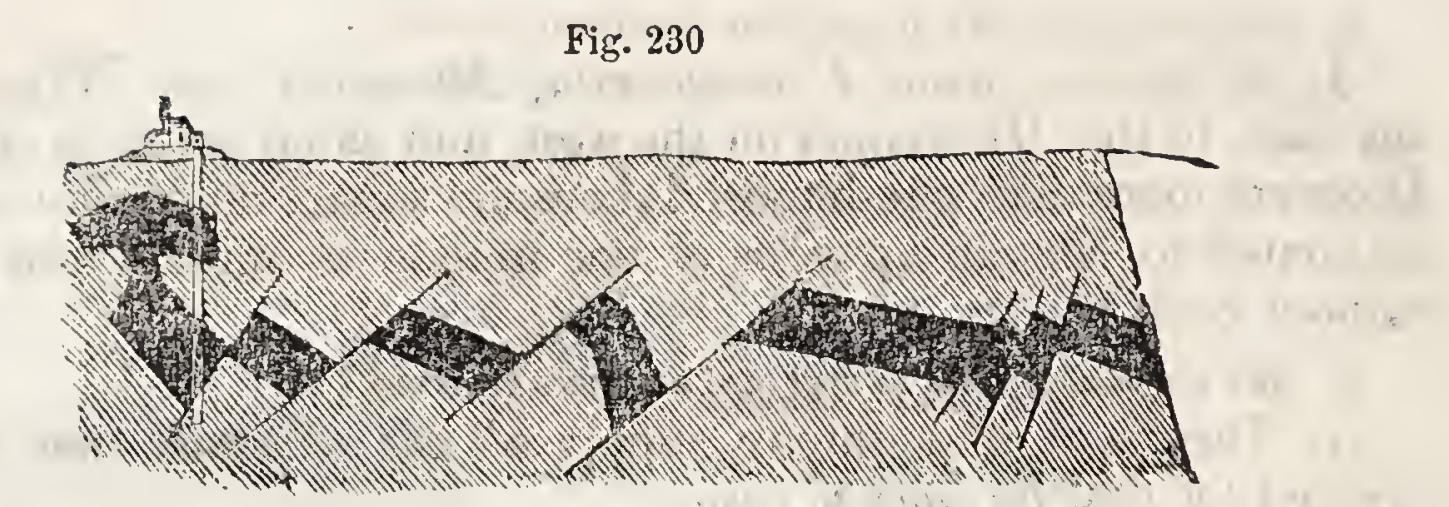

VARIOUS DISLOCATIONS OF $\triangle$ COAL-BED AT VIEILLE-POMPE, IN FRANOR.

Q. How is this Illustrated in the great coal-fields of Pennsylvania?

$A$. The Eastern Pennsylvania coal-fields, which are adjacent to the line along which the Appalachian chain of mountains have been elevated, furnish only anthracite, but in Western Pennsylvania, away from the mountains, the coal is bituminous.

Q. What is the GLNERA ARRANGEMENT of the coal-fields in England and Wales?

A. 1. The Great Northern District includes all the coal-fields north of the Trent;

2. The Central Districts include Leicester, Warwick, Stafford, and Shropshire; and

3. The Western Districts, which may be subdivided into NorthWestern, including North Wales, and South-Western, including South Wales, Gloucestershire, and Somersetshire.

There are 3,000 coal mines in Great Britain, which employ nearly 250,000 men, women, and boys, underground and above. The capital invested in working stock, tramways, shafts, and harbours, altogether exceeds $£ 30,000,000$ in value. The "get of coals" amounts to about $34,000,000$ tons annually, the estimated value of which, at the pit's mouth, is $£ 10,000,000$. One-third of this is worked from the Northern coal-field, in the counties of Northumberland and Durham, from whence, by the rivers Tyne, Wear, and Tees, the chief shipments of coals are made, both foreign and coastwise. The chief points of home consumption are in the iron works of Staffordshire, South Wales, and the West of Scotland; which, with the lesser works of North Wales, Shropshire, Yorkshire, and Derbyshire, consume nearly one-third of the whole. The Lancashire coal-field pro. duces about 4,0C0,000 tons annually; Wigan, 2,000,000 tons annually; Bolton, 1,000,000 tons annually; St. Helens, 1,000,000 tons annually. The cost of "getting" the coals appears to average much the same in Lancashire as in Durham and Northumberland; the reason of the latter obtaining such a heavy preponderance in exporting appears to arise from the increased vigour with which the trade has been pushed there, and the increased facilities that have been created in railways, staiths, \&c. ; the operation of loading a vessel with coal at Newcastle, Sunderland, Shields, Hartlepool, Middlesbro', \&c., being performed in about as many hours as it takes days fur the same task in Liverpool.

Q. Is the carboniferous formation of GREAT EXTENT?

A. It is. It exists in all the great divisions of the earth. Large deposits of coal occur in the United States, in Pennsylvania and the Valley of the Mississippi.

Q. What $\triangle$ MOUNT of coal is supposed to be ANNUALLY mined in EUROPE?

A. An amount exceeding seven hundred millions of hundredweight; of which England alone contributes four hundred and fifty, and Germany about forty millions. 
Q. What is the Extent of the great American coal-field?

A. It extends from Pennsylvania, Maryland, and Virginia on the east, to the Mississippi on the west, and as far south as Alabama. It covers more than one hundred thousand square miles, and has been estimated to contain upwards of one million of million tons of bituminous coal.

Q. May the coal deposits be regarded as INeXHa ustible?

$A$. They will undoubtedly supply all the demands that may be created for coal for ages to come.

RELATIVE AMOUNTS OF PRODUCTION OF MINER.AL COMBUSTIBLES IN THE SIX PRINCLPAL COAL-PRODUCING COUNTRIES OF THE WORID.

\begin{tabular}{|c|c|}
\hline Grent Britain-A & $31,500,000$ \\
\hline Belgium. & $4,960,077$ \\
\hline France & $4,141,617$ \\
\hline United States of America :- & \\
\hline Anthracite. ............ & $2,650,000$ \\
\hline Bituminous ............ & $1,750,00$ \\
\hline Prussia,$\ldots . . \ldots \ldots \ldots \ldots \ldots$ & $3,500,000$ \\
\hline & 700,000 \\
\hline
\end{tabular}

THK SUPERFIOIAL COAL AREAS OF VARIOUS COUNTRIES.

(From Taylor's "Statistics of Coal.")

United States of America :-

Bituminous coal area

Anthracite coal of Pennsylvania

British America :-

Bituminous coal area

France, ditto

Belgium, ditto

Spain, ditto

Great Britain and Ireland :-

Anthracite and culm coal area.........

Bituminous
Square Miles. 133,132

437

18,000

1,719

518

3,408

3,720

8,139

Q. What SYSTEM SUCCEEDS the carboniferous?

A. The old red sandstone, also called the Devonian system.

Q. What is the CHARAOTER of this system?

A. 'This fomation consists almost entirely of sandstones of a red or gray colour. It is extensively developed in England, and to some extent in the United States. The Catskill Mountains are made up of rocks belonging to this system.

Q. What are the PEouliar Fossins of the old red sandstone ?

$A$. The fossils of this system are a few shells, a small number of vegetable species, and in some localities the remains of fishes occur in great abundance.

Q. What is the DISTINGUISHIN G CHARACTERISTIC of the FISHES of this system ?

$A$. The fishes of this system have a skeleton composed of cartilage (gristle) covered with plates of bone. 'The jaws, consisting of solid bone, were not covered with integuments, and the exterior bony covering seems to have been the true skeleton, as is, in part, the case with the tortoise. (See fig. 231.) 


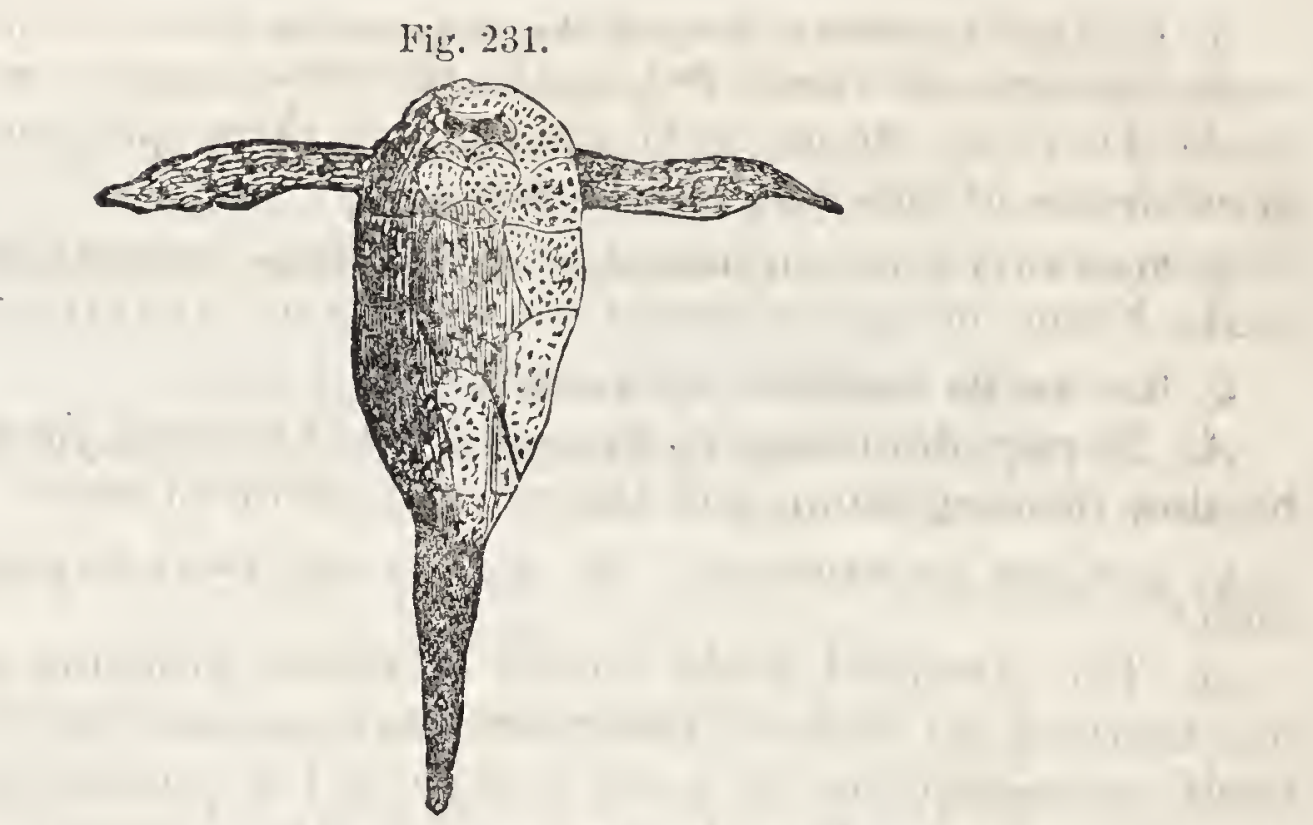

THE PTEROPTHYS, OR WINGED FISH, UPPER SIDE.

Q. What SYSTEM SUcceeds the old red sandstone?

A. 'The siturian, divided into the upper and lower silurian formations.

Q. Is this system EXTHNSIVELY DEVELOPED?

A. 'The silurian system of rocks covers a greater portion of the curth's surface, and is better known and studied, than any of the other yrological formations.

Q. WhERE do the silurian rocks occur in the United States?

A. The silurian rocks occupy a large portion of the territory west of the Alleghany Mountains, from Canada, through New York and the other States to Alabama, and extending westward far beyond the Mississippi. Most of the rocks in the State of New York belong to the silurian system.

Q. What is the OHARACTER of the FOSSILS of this system?

$A$. The fossils of the silurian rocks are very numerous, but consist mostly of the lower forms of animal life, of marine origin. They are shells, corals, and some articulata, as worms and crablike animals.

\section{CHAPTER LXVIII.}

\section{ON THE PROGRESSIVE DEVELOPMENT OF ANIMAL LIFE UPON THE SURFACE OF THE EARTH.}

Q. What FACT is revealed to us by the study and examination of the stratified rocks of sur globe?

$A$. That as regards animal and vegetable life, there has been a continuous and gradual progression in the acts of creation, from the lime when the first organised being was created up to the period of man's appearance upon the earth. 
Q. Are there any srass by which the geologist can determine the antiquity of the earth, or the time which has elapsed since the sedimentary deposits commencus?

$A$. There are no means of determining these questions; yet a very great deyree of antiquity can be shown.

Q. From Whexce have the materials which compose the stratified rocks been obrainfil ?

A. From the destruction of the igneous or mimitive rocks.

Q. How was the destruction accompursuer ?

$A$. In part, doubtless, by the operation of volcanic forces, but mainly by slow disintegration, and the eroding action of water.

Q. How does the ExIsTENCE of the stratified rocks Prove the great antiquity of the earth?

A. The stratified rocks consist of layers, probably not averaging one tenth of an inch in thickness; and yet each layer is distinct in itself, in consequence of some change in the conditions under which it was deposited. The time required for these depositions must have been very great, as the thickness of the metamorphic and fossiliferous rocks is estimated to be at least eighty thousand feet.

Q. What does the cxasination and study of the igneous rocks, constituting the fundation of the earth's crust, TEACH?

A. That there was a period when animal and vegetable life did not exist, and the surface of the globe was one homogeneons mass.

Q. In what STPATA and SIstim do we find the first evidences of animal and vegetable life on the earth's surface?

$A$. In the oldest and lowest strata of the silurian system.

Q. What is the CIIARACTER of the fossils of these strata?

A. 'The fossils of these strata consist of one or two species of wormlike animals, a few shells and sea-weeds, of the lowest orders of animal and regetable life; these fossils are supposed to be the remains of the first organised structures that were created.

Q. As we ascend in the series of rocks, what is the CHARAOTCR of the fossils belongiug to the strata of the MIDDLE SERIES of the silurian system?

$A$. The number and variety of shells increase; corals and curious articulated and jointed animals called crinoids and trilobites make their appearance. The remains of fiskes also have been found in these strata.

Q. What is the CrARACTEP of the fossils of the upper silurian rocks?

A. In these rocks fossil shells in immense numbers, exhibiting an almost infinite variety of form and structure, exist. Fishes appear in greatei numbers during this epoch. Corals are abundant, and vegetable life has also expanded. All these forms, however, are of the lowest orders of organised existence.

It was once believed that animals were successively created in the order of their relative perfection, so that the most ancient formations contained only animals of the lowest urarle, sucl as the polyps, and echinoderms, to which succeeded the mollusks; then the urticulated animals; and last of all the vertelratce. This theory is now, however, untentile, since frissils lelunging to ench of the four departments bave been found in the tossiliferous rocks of every age.-Agussiz.

By this it is to be understood that the four orders of animal life have representative fossils in the rocks of the lower silurian formation. Whether they commenced their existence at a sinultaueulus period we have no evidence; in the oldest strata of the low ar 
silurian formation they do not occur together. All the furms of animal and regetable existence belonging to those older rocks are alon exreedingly low in the scale of organisation, and during this whole perivd no air-breathing auimals existed.

Q. What progresstre development occurs in the next system of rocks-the old red sandstone?

$A$. The period represented by the strata of the system has been called the "reign of fishes." In the silurian system fishes made their appearance during the formation of the lower strata, but were few in number and low in organisation. In the period of the old red sandstone, they existed in immense numbers, and possessed a highly organised structure.

Q. Did any LAND ANTMASS Or PLANTS exist at this period?

A. We have no evidence that they did; the ocean probably covered the greatest portion of the earth's surface.

Q. What is the CHARACTER of the ORGANIC REMAINS found in the carboniferous rocks?

A. During the carboniferous period, vegetable life was developed in an extraordinary manner, and under a great variety of forms. Many of the plants of this period belonged to the class cryptogamia (plants that never flower); the great mass of the coal, however, appears to have been derived from the structure of endogenous plants, and from trees allied to the pine and fir.

It is exceedingly rare to find remains of plants or trees of high organisation allied to the oak, the elm, or maple during the carboniferous epoch.

Q. During what EPOCH do we FIRST find the remains of land animals?

$A$. In a few rare instances, indications of the existence of land animals of low oryanisation have been first observed in the rocks of the carboniferous epoch.

Q. Was the earth, at the poriod of the formation of the carboniferous rocks, in a FIT OONDITION for the HABITATION Of MAN and LAND ANIMALS?

A. A large portion of the earth's surface at that period was undoubtedly but little elevated above the surface of the vater, and was low and swampy. There is also reason to believe that the atmosphere.was charged with carbonic acid gas to such an extent as to render it irrespirable by any of the higher orders of animals. Fig. 232 represents the fauna and flora of the carboniferous period.

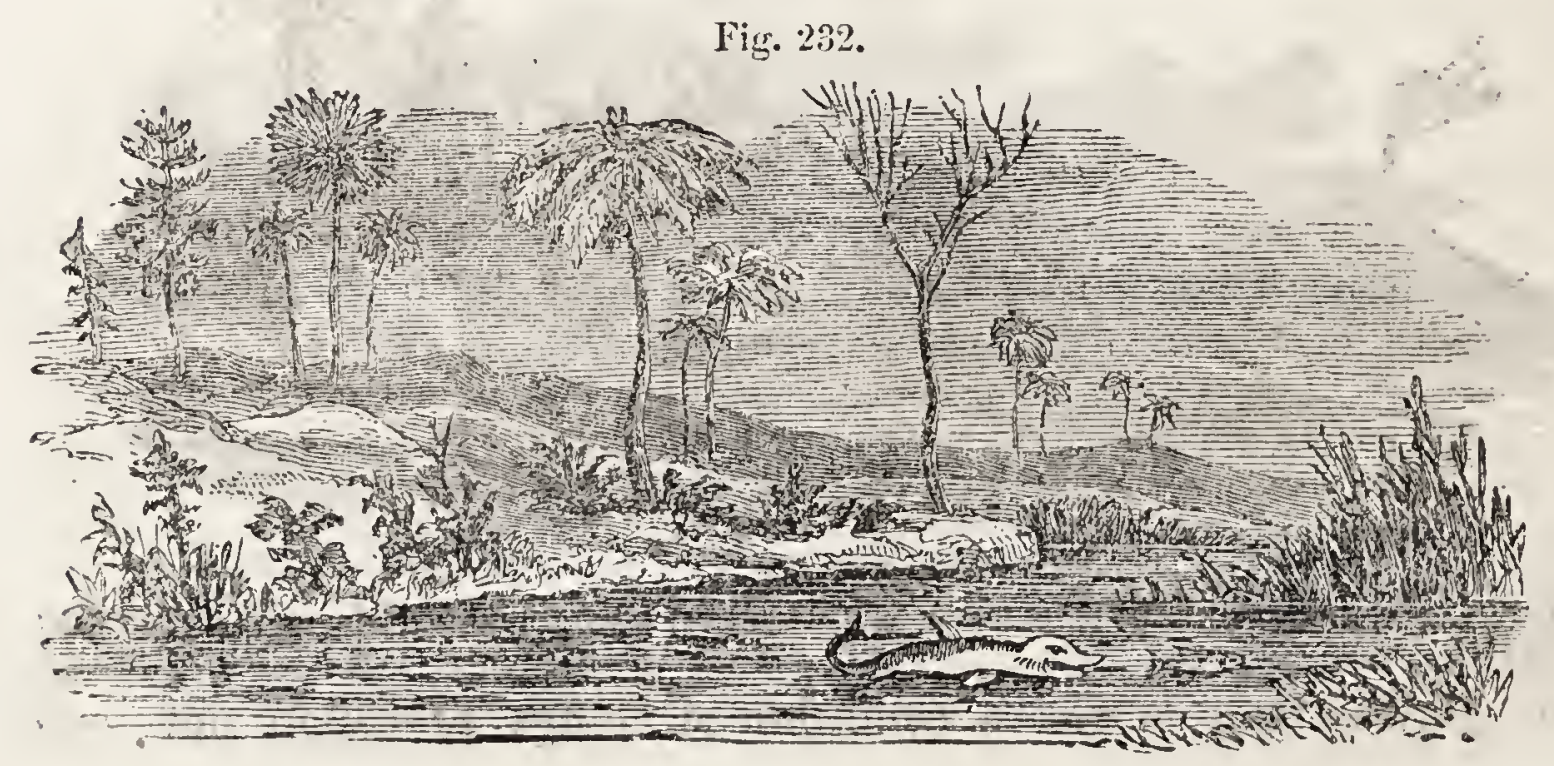


Q. Does there appear to be sTRIKINa EvidRnces of DESIGN in the extraordinary de: : zopment of vegetation at this period?

A. The design of this abundant growth was prospective. The plants of the ccal period were not fitted for the nourishment or support of animal life, but for fuel; and these remains have been stored up until the era of man, when this mighty supply of carbonaceous matter has become subservient to his happiness and necessities.

Fig. 233.

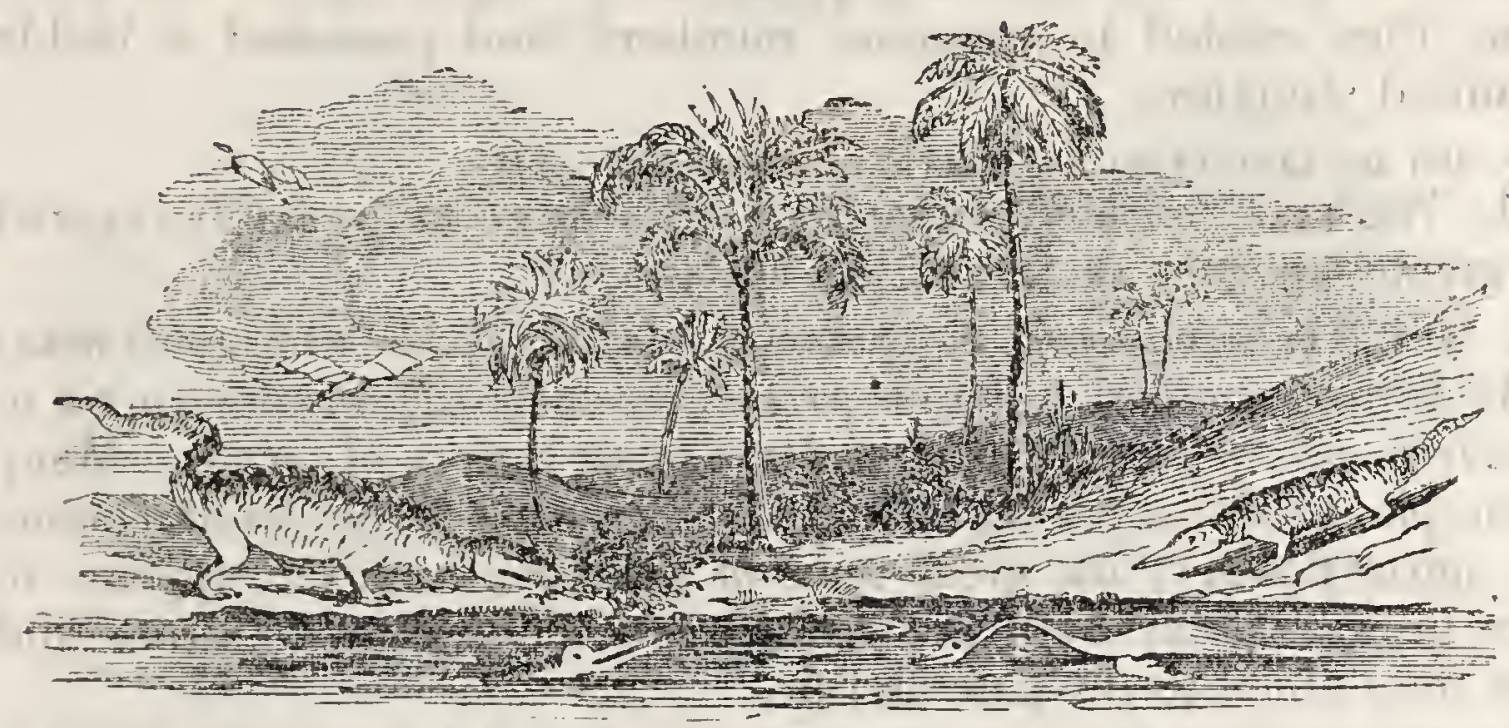

Fig. 233 represents the appearance of the fanna and flora of the oolitic perind?

Q. What CIIANGes in animal and vegetable forms take place in the three succeeding systems, the new red sanditone, the oolite, and the cretaceous?

$A$. In these three systems large reptiles prevail to an extraordinary degree; so much so, that the period has been designated as the "reign of reptiles." Birds first appear in the period of the new red. sundstone. Many land animals also for the first time came into existence, and vegetation was greatly modified, the peculiar plants of the cual period having become extinct, and exogenous trees having become more common.

Fig. 231.

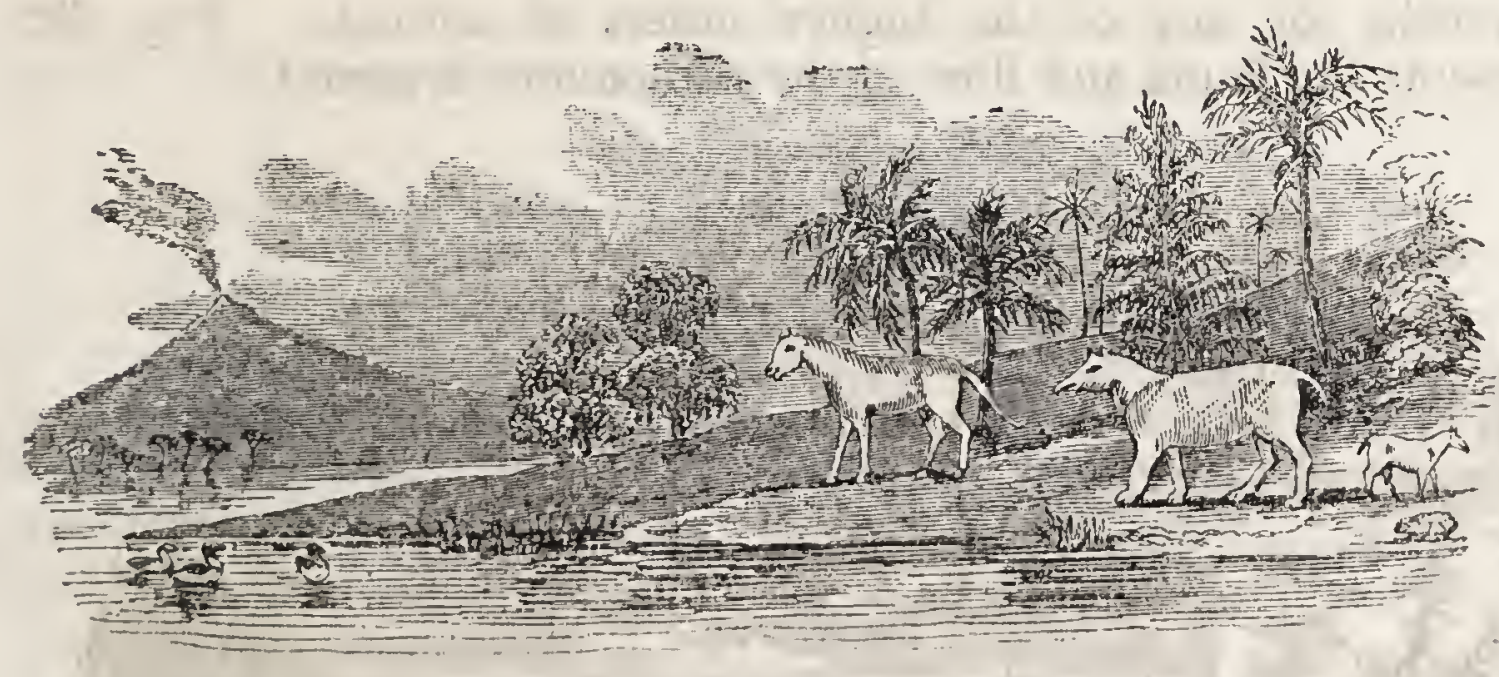

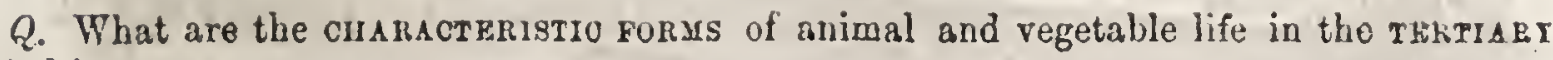
iod?

A. During this period a condition of things prevailed not very 
dissimilar to that of the present period. The huge reptiles of the former eras have disappeared, and have been succeeded by gigantic land animals - the mastodon, the mammoths, elephants, various species of the rhinoceros, \&c.

A class of animals allied to the hippopotamus and the tapir, now extinct, existed also at this epoch. All the existing orders of animals, including the monkey family, were represented in this period. Figs. 234 and 235 represent the fauna and flora at the comruencement and close of the tertiary period"

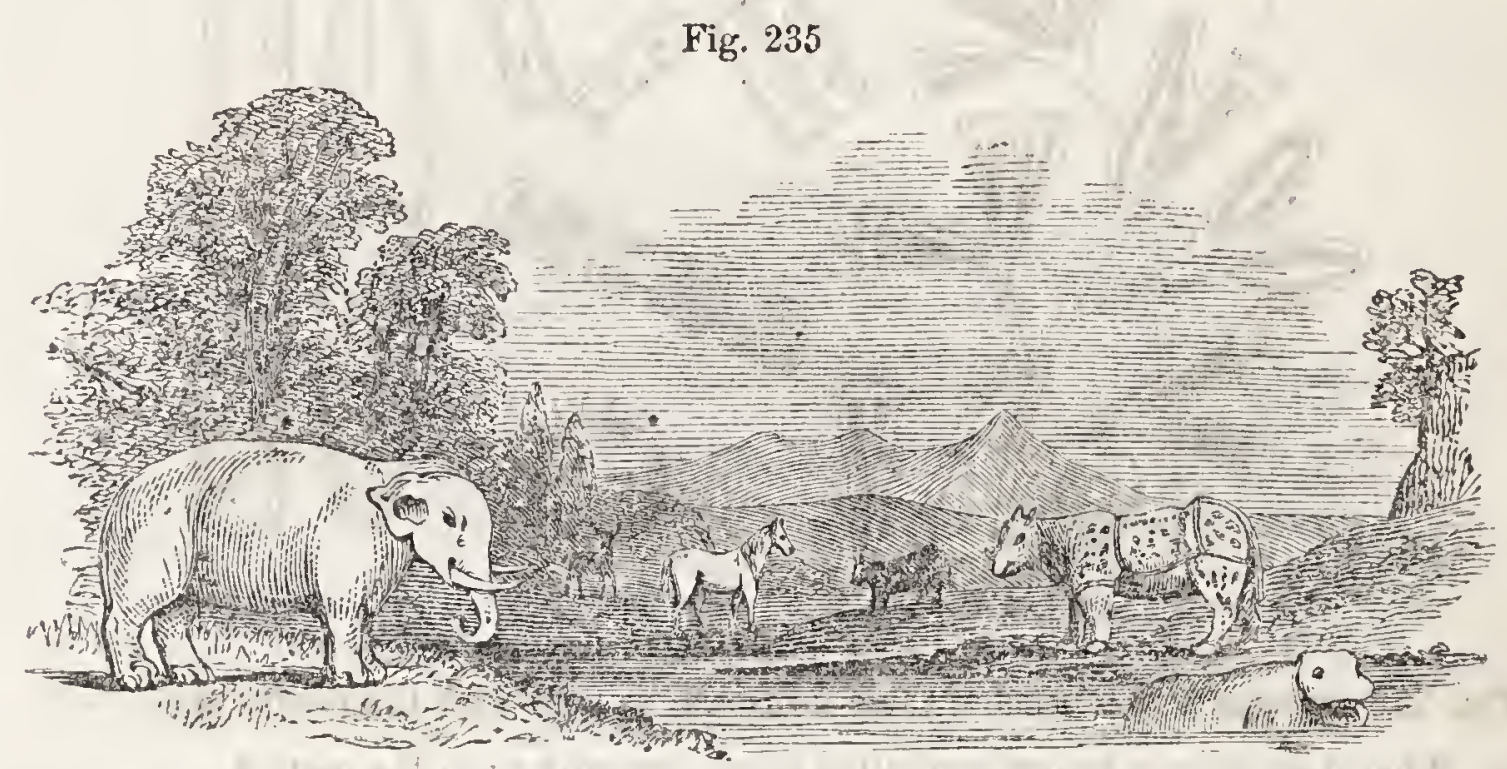

Q. Is there reason for believing that the climate of the globe during the tertiary period was WARMER than at the present?

A. We find the remains of tropical animals and plants imbedded in strata of this period in extreme northern countries. Hence we infer that the climate of these countries was at that period of a tropical character.

Q. Why do we suppose that MAN did NoT EXIsT at this period?

$A$. Because although we find remains of all the animals and plants that then flourished, we have never met with the bones of man or any of the works of his hands in any of the rocks found during this period.

Q. WHEN did man make his appearance upon the earth ?

A. At the termination of the tertiary period, and after the formasion of the drift or diluvium.

Q. What FAMILY of PLANIS appear to have come into existence contemporary with man?

$A$. Geologists have never found any fossil remains of the plants belonging to the family of the rose, which includes not only many of the most beautiful flowers that are known, but also the richest fruits, as the apple, the pear, peach, strawberry, cherry, \&c. Hence it is inferred that the introduction of this family was coeval with, or subsequent to, the creation of man, to whose comfort and happiness they seem especially designed by a wise Providence to contribute.

Q. Do beds of ROCK or EARTH exist, EXCLOSIVETI COMPOSED of the remains of arrernsCOPIO ANIMALS?

$A$. There are extensive beds of rock and earth wholly composed of the 
semuins of minute animalcule, called infusoria, the shells or coverings of which, being formed of silica, are nearly indestructible.

The rock, or stone, known as tripoli, or polishing slate, consists entirely of the remains (f these animals, so minute that a cubic inch contains upwards of thirty-fire thousand willions of inclividuals.

Fig. 236 ,

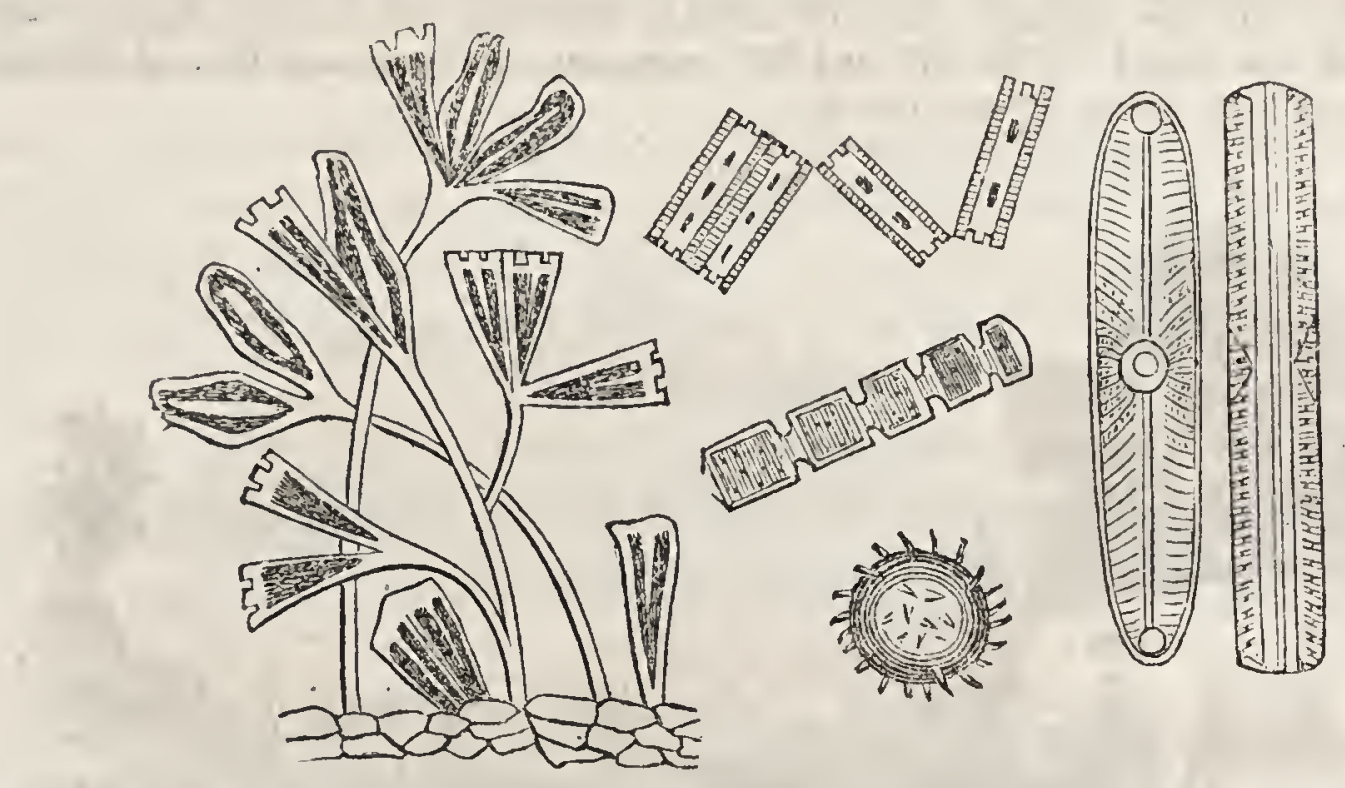

Fig. 236 represents the forms of these infusoria, as seen with the microscone.

Q. What does geology TEACH respecting man's position upon the earth?

$A$. It teaches us that his position is one far in advance of all other and former creations. It shows that since the work of creation commenced upon the earth, there has been a progressive improvement of its surface, all with a design of fitting it at last for the abode of the human race.

Q. Is it Probable that any MATTER has been added to the bulk of the earth since its first creation?

$A$. If we except the small masses, called meteorites, respecting whose origin we are ignorant, no grain or matter has been added to our planet since its creation, neither can any be removed.

\section{CHAPTER LXIX.}

\section{ECONOMICAL GEOLOGY.}

Q. If No geological OHANGES had taken place, what would have been the PRESENT CoN Dirior of the earth's surface?

$A$. It would have been an almost uniform plain, ill adapted for the abode of man.

Q. What ResulTs have been Producen by the violent disruption, upheaval, and change of the earth's crust?

$A$. The division of the general surface of the earth into land and water, as well as the diversified form of the land, the existence of 
mountains and lowlands, and the consequent modifications of climate; the water-falls, river-courses, and the drainage of continents,-are all the results of upheavals of the earth's crust.

Q. Should we have known of the EXISTENOE of GRANITE if the strata bad not been LIFTED UP ?

A. We should not, as granite was formed below the sedimentary rocks, and covered by them to the thickness of many thousand feet.

Q. How have MiNeral veins, Beds of MARBLe, SAlt, \&c., become ACCESSIble to us?

$A$. The strata containing these substances have been forced up towards the surface of the earth; whereas, if they had remained in their original position, it would have been necessary to have sought for them far beneath other overlying rocks.

Q. How do the oRES of different metals ocouR in rocks?

$A$. They sometimes occur in beds or layers, as iron ore, sometimes in veins, and occasionally as masses of pure metal.

Q. What is a MINERAL VEIN?

A. A mineral vein is a separation in the continuity of rocks of a limited width, but extending indefinitely in length and depth, and filled to a greater or less extent with metallic or mineral substances, of a different nature from that of the rocks they traverse.

M E TALS.

Q. Are mineral veins confined to any PARTIOULAR CLASSES of rocks?

$A$. They are not confined to any particular epoch of formation, nor to any portions of the earth's crust, although they are most frequent in beds that form mountain elevations, and in the oldest rocks.

The minerals are not distributed on the earth according to climate, like animals and vegetables ; but, by the kindness of Providence, those which are most necessary to man are found in almost all countries; and others are more or less abundant according to their importance. It is remarkable, also, that the most barren portions of the earth are often most plentiful in mineral treasures.-Hughes.

Q. In some instances how does the vein or fissure in the rock appear to have been FILLED with METALLIC ORES ?

$A$. The metallic substance oftentimes seems to have been forced up in a melted state from the interior of the earth, and, flowing into all the little fissures and cavities, to have hardened and crystallised. In such veins the deeper we penetrate into the crust of the earth the richer they become.

Q. Do veins or beds of metalliferous ore ever appear to have been formed by ELECTROCHEMIOAL AOTION?

$A$. In certain cases the mineral and metallic particles appear to have been collected from the adjacent rocks, and deposited in a particular locality by the action of electro-chemical forces, in somewhat the same way as the metal is deposited at the terminal pole of a galvanic battery in the process of electrotyping. 
Q. What is the ORDINARY COLOUR of METALS ?

A. There is little variety in the colour of metals. Gold is the only yellow metal, copper and titanium are reddish, all the rest are different shudes of white.

Q. What do you mean by DIFFERENT SHADES of WHITE?

A. Variations from the pure dead white of silver to the bluish-gray of lead.

Q. Which is the MOST PRECIOUS of all metals?

A. Gold.

Q. How and WHERE is it usually found?

A. It is usually found in grains or gold-dust, in a pure state; but nearly three-fourths of the gold in use is obtained by washing the sands of rivers.

\section{Q. WHERE is GOLD found?}

A. This metal is widely diffused over the surface of the earth, and there are few parts of the world but have yielded it in some proportion.

Q. Mention Some of the countries in which it has been found.

A. It is found in most parts of Europe, but only in very small quantities, as in the sands of the Danube, the Rhine, the Rhone, the Garonne, the Tagus, \&c. The mines of importance are at Kremintz, in Austria, and in the Ural Mountains. Africa furnishes large quantities of gold: it is specially abundant on the coast of Zanguebar, Western Africa, and the Niger. It is also found in Japan and the East India Islands. Large quantities are produced from the alluvial sands of Brazil.

Q. Where of late has it been OHIEFLY Found?

A. In California and Austratia.

Q. Where, in California and Australia, has gold $h \cdot$ n inund?

A. In California it has been found in the valleys of the River Sacramento. In Australia it has been found lying to the eastward of the Blue Monntains, in the neighbourhood of Bathurst; through the valleys of the Macquarie River; and, to the south, in the province of Victoria.

CoRIods FACTS. - The 100,000th part of a grain of gold may be seen by the naked eye; and a cube of gold whose side is but the 100th of an inch has $2,433,000,000$ of visible parts. If a silver wire be covered with gold, it may be drawn out into wire of still greater fineness, and still retain this coating of gold; and one grain of gold will in this way coat a surface of wire about two miles and three-quarters in length. The malleability of gold exceeds that of all other metals. Gold leaf can be reduced to the 300,000th of an inch, and gilding to the 10,000,000th. Lace gilding is 1,000,000th of an inch thick, tinfoil 1,000th of an inch, and gold leaf the 200,000th; so that 200 gold leaves are only equal in thickness to one of tin-foil. One grain of gold will cover $7 \frac{1}{2}$ inches each way, or 52 square inches; or be 1,500 times thinner than ordinary writing-paper. Its tenacity, however, is greatly inferior to that of other metals. A wire one-thirteenth of an inch thick will not support a greater weight than $150 \mathrm{lbs}$.; whereas an iron wire of the same diameter will sustain a weight of $560 \mathrm{lbs}$. It is not an opaque body, like all other metals, for gold leaf transmits a green light, as may be conveniently observed by laying a leaf between two thin plates of culourless glass, and holding it between the eye 
and a strong light. It is less fusible than silver, and more so than copper. It is the most perfect conductor of heat-that is to say, if heat be applied to one end of a rod of gold, it will le transmitted from particle to particle, and become sensible at the other extremity of the rod nore quickly than through any other substance in nature. Gold snay be exposed for ages to air and moisture without undergoing any alteration; and a quintity of it has been kept for thirty weeks in a melted state in a glass-house furnace without the losa of a single grain, and without any change in its nature. But if a small portion of it be intensely heated by electricity, or by the oxy-hydrogen blowpipe, it burus with a greenish blue flame, and is dissipated iu the form of a purple powder.

Q. Is not gold ALtoYen BEFOnE it is USED for purposes of COIMAGE?

A. Yes. Gold is too sift for purposes of ornamentation or coinage without alloy. The base metal used as an alloy is generally copper, though occasionally silver and zinc are used.

Q. What is PLATINum?

A. Platimum is a grayish-white metal, more rare, and on some account more valualle even than gold. It occurs chiefy in South America.

Q. When was this metal FIRST INTroducED into EUROPE?

A. In the middle of the last century. It derives its name from the American Spaniards, who called it platina (little silver).

Q. What are the PECULIAR Properties of PLA'TINUM?

A. Its extreme density, it being the heaviest of all known bodies, the impossibility of melting it with the strongest furnace heat, and the resistance which it offers to the solvent power of acils.

Q. Where and in WHAT STATE is sILver generally discovered?

A. Like gold, silver is a very widely distributed metal, and is found more or less in nearly every part of the world. Sometimes it is found pure, or native silver; when in combination, it is found with sulphmr, as sulphide of silver; with sulphur and lead, as the mineral galena; or, in union with chlorine, as chloride of silver.

Q. What are the CHARACTERISTICS of silver?

A. Pure silver is harder than gold, but is still a soft metal; when polished it is more brilliant than any other metal; it does not tarnish unless attacked by the vapour of hydro-sulphate of ammonia or hydrosulphuric acid gas. Next to gold, it is the most ductile and malieable of all metals.

Silver leaf can be reduced to the 170,000 th part of an inch. It can be put into plates of which 110,000 make an inch. It may be dritwn out into wire more slender than the finest human hair; and in this way a single grain may be stretched out to 400 feet. Itis tenacity is inferior to that of iron, copper, or platinum; its specific gravity is somewhat lighter than lead; it is elastic, and, when hardened by alloy, is highly sonorous.

Q. Which is the MOST USBFur of all inetals?

A. Iron.

Q. Is iron obtained in a PCRE STATE?

A. No. 'This very important and widely diffused metal is scarcely ever obtainea in a pure state.

Q. Where is iron CHInfLY FOUn! ?

A. The ore of iron is found abundantly in all countries. The 
principal ores are the magnetic ore and ironstone of mountainous regions, and the bag iron and iron earth of alluvial districts.

Iron is said to form two per cent. of the crust of the earth. Grent Britain and France contain the most extensive mines in the world. Sweden has large mines of magnetic ore, which produce the best of bal iron. The mines of Denmark are particularly celebrated. A: Gellivara, in Swedish Lapland, is a momntain of ore three miles in length. Norway, Russia, Spain, Germany, and Austria have extensive mines. The island of Elba contains one of the most cnrious known, remarkable for the beautiful play of colours on the oie. In the United States there are numerous and inexhaustible beds of iron ore, particularly glong the Allechany and Appalachian mountains.-Hughes.

Q. What are the PRINCTPAL VARIETIES of irnn employed in COMMERCE?

A. Cast iron, wrought iron, and steel.

Q. What is STEEL?

A. A peculiar preparation of iron. The common plan adopted for converting iron into steel is that called cementation. This is done by stratifying bars of wrought iron with charcoal, and exposing them to furnace heat; the union between carbon and iron takes place, and steel is the result.

Q. What yetaL ravls mext to iron?

A. Copper.

Q. Is COPPER as AEUNDANT as irou?

A. Copper, though less abundant then iron, occurs in many parts of the world. It is found in pure metallic masses, sometimes of very considerable extent. The copper mines of Comwall produce more than all the rest in Europe.

Q. What are the other Principal Coppiro arimas?

$A$. Considerable copper mines are wrought in the middle and rorth of Europe, among the Ural Mountains, Russia, and in Siberia. Copper is also furnished from Greenland, Iceland, Tartary, and Japan, Barbary, Southern Africa, and the southern part of South America, the United States, Canada, and Australia.

About half the total amount of copper annually manufactured is produced in South Wales, from the ores brought from various parts of the world, on account of the immense coal-fields of that district. "The practice of Welsh copper smelting," says an eminent writer, "is complex and ingenious ; the principle which it involves is, however, simple, and mity be rencered intelligible by the general statement, that whilst iron, one of the immurities associated with the greater number of copper ores, has a tendency to unite with oxygen when strongly heated, cupper unites, noder similar circumstances, with sulphar. This mixed product leing heated in contact with siliceous matter, silicate of oxide of iron furms, melts, and rises to the surface, whilst sulphuret of copjer, still nudecomposed, sinks below. When the iron slag (silicate of oxide of iron) bas teen remored, the furnace heat is raised sufficiently to effect the decomposition of sulphuret of copper into sulphuric acid, which escapes, and metallic copper, slightly contaminated with oxide of copper, remains. The metal holding this small portion of oxide is deficient in mallesbility: hence, the oxygen of the oxide must be removed. This is accomplisher by the I:"ness of copper-refining, as it is termed. The roun material being liquefied by finsion, powdered charcoal is added, and the whole stirred by a green wooden pole. It is almost unnecessary to remark that the pole, thus circumstanced, burus, evolving oxygen, the rernoval of which is consequently effected." 
Q. What are the CHARACTERISTICS of copper?

A. It is reddish in colour, soft, mulleable, fusible at a strong red heat. It is tarnished by moist air, resulting in oxide of copper or verdigris.

Q. IS ZINC WIDELY DIFFUSED over the earth?

A. Not to so great an extent as iron or copper; but in those places where it exists it is found in abundance.

Q. What are the CHARACTERISTIC PROPERTIES of zinc?

A. The properties of zinc materially alter in proportion to the degree of heat to which it is raised. After being cast, if permitted to cool down to the temperature of the common atmosphere, zinc is brittle, and is comparatively easily broken. This brittleness may be greatly increased by raising the temperature. At four hundred degrees F., zinc is so brittle as to be easily powdered.

Q. Where are the PRINCIPA TIN Mines of Enrope?

A. In Cornwall. These are of very ancient date, and are also very extensive. They are said to have been worked by the Phœnicians.

Q. In what OTHER COONTRIES besides England are tin mines found?

A. The mountains of Germany, and Gallicia, in Spain, produce tin; it exists in Mexico, Chili, the United States, the East. Indies. The purest is obtained from Malacca.

Q. IS LFAD WIDELY DIFHUSED?

A. Yes. It is found more or less in all conntries; in the Ural Mountains and in Peru, it is, however, extremely rare. 'This very abundant and useful metal is rarely found pure. The presence of particles of silver and other matter deteriorates its value for many important purposes where pure lead is absolutely necessary.

Q. In WHAT ROCKS are the PRECIOUS STONES most ahundant?

A. Most of the precious stones occur in the igneous rocks, especially in granite, or in the sand and gravel formed by the destruction of these rocks.

M I N E S.

Q. For WHAT PURPoses are MINES EXCAVATED?

A. 1. For the working of veins containing the ores of metuls; and

2. For the working of such minerals as generally lie in beds, such as coal mines, ironstone mines, or limestone mines.

Q. How is the nperation of mining, in a COAL MINE, for instance, COзMLACED?

$A$. This is done by boring the earth, in order to ascertain the exact position of the strata or beds of coal before any great operation is undertaken. 
Fig. 237.
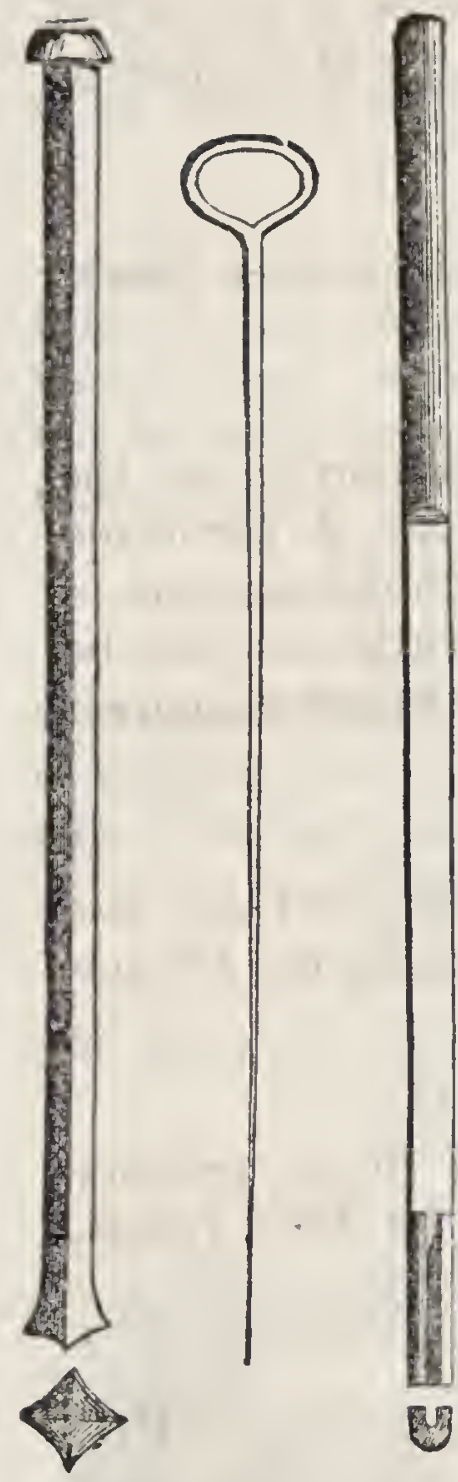

Q. Describe the sort of Instruments used in BORING ?

$A$. The rods commonly used are made of the best Swedish iron, about an inch and a quarter square. Each rod is three feet in length, having screws at either end. 'The chisels for boring are eighteen inches in length, and about three inches in breadth. There are besides a number of other instruments, called wimbles, sludgers, rounders, \&c.

When bores are but a few fathoms in depth, the whole operation is performed by manual strength; but when a very deep bore is to be made, a set of woodeu triangles, with a pulley, crane, or windlass, is used. There are professional bore masters in the coal districis, who undertake to put down bores for coal, and to register the strata passed through, at the rate, when no uncommon difficulties oceur, of six shillings a fathom for the first five fathorns, and an increase of six shillings a fathum for every additional five fathoms.

Q. Does the work of the miner simply consist in EXCAVATING the Roor?

A. No; the work of the miner is of two sorts-excavating the ground when soft, blasting it when hard. 'Tools called the borer, or jumper, and hammer, are used for this latter

TOOLS EMPLOYED IN MINING OPERATIONS.

Q. What SORT of tool is the BORER, Or JUMPER?

A. It is an iron rod or circular bar about two feet in length, and formed into a flat, sharp edge at the end, which is driven into the rock and made to revolve in the process so as to expose the cutting edge to different surfaces of the rock. The matter thus pulverised is drawn out from time to time; and when the hole is of a sufficient depth, it is charged with powder. A piece of copper wire having a loop at the end, is then introduced-the charge is then rammed down-the wire withdrawn-and a train of powder inserted in its place. The train is then fired by a slow match, and the explosion takes place; the rock is blasted, and the miners' work greatly facilitated.

Q. In the incavation of a MINE, what imPORTANT CONTINGENoIEs have to be taken into consideration?

A. One of the first, and, at the same time, one of the most important, is that of drainage: water rapidly filters in, and must be worked out. 
Q. What means are USUALLY EMPLOYED to answer this purpose?

A. In the first place, this may be done by what is called a horse "whim" or a "gin;" but this is only serviceable when the mine is not very deep, and when the quantity of water to be drained off is but trilling.

Q. What is a "WHIMSEY," Or " aIN?"

A. An application of machinery by which iron baskets are liept continually ascending and descending the shaft, one basket being emptied at the mouth while the other is filling at the bottom of the pit. It is worked by horses, and its application on a large scale is enormously expensive.

Q. What OTHER MEANS are adopted for the drainage of mines?

A. The best and cheapest in the mining 'districts of England is that of the stean-engine. In our coal mines the steam-engine is the only power employed for drainage, and in deep and extensive works the motive power is employed for extraction also.

Q. What is the NEXT POINT to be considered in the working of a mine?

A. The support to secure the excavations, which will depend very much on the nature of the soil.

Fig. 238

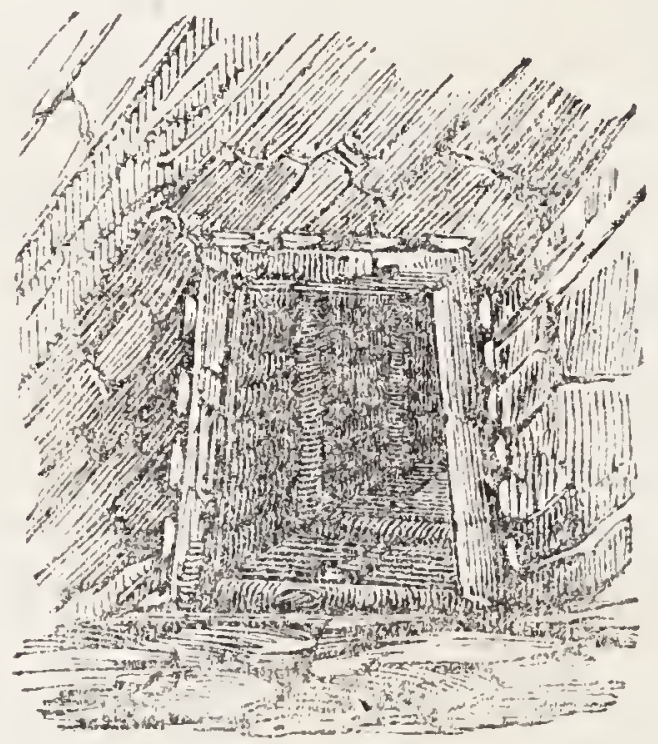

L.FIEL.
Fig. 239.

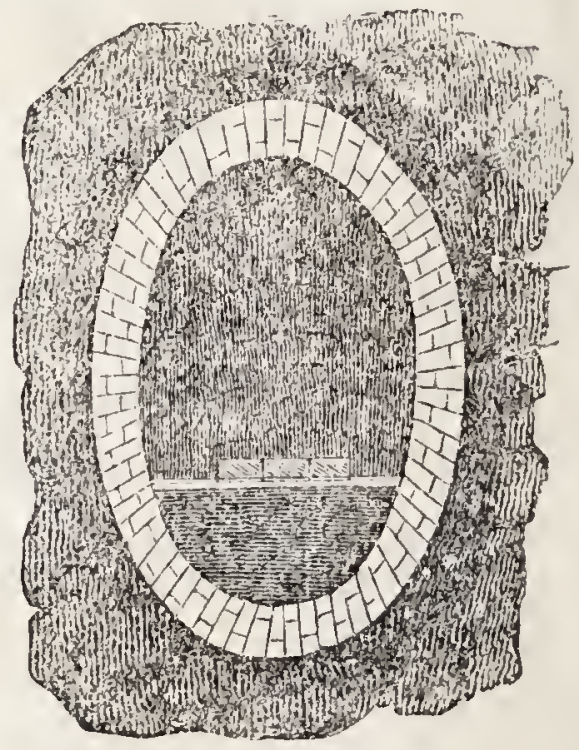

CIRCTI.AR GALITERT.

Q. What inode of SUPPORT is generally employed?

A. There are three linds of supports used in the excavation of mines: 1 , by leaving pillars of the original rock; 2, by wailing with brick or stone; 3 , by timbering.

In fig. 238 is shown what is termed a level, and which consists of three pieces of timber, arranged something like a doorway, narrower at the top than at the bottomi. Levels and shafts are also, in some cases, supported by walling of brick or stone work, is indieaterl in 6 ig. 239. 
Q. What means of ventiLation are adopted in mining operations?

A. The ordinary arrangements of the works and the communications continually kept up with the surface, in most cases, furnish sufficient air without adopting any mechanical means of supplying it. An apparatus, known as the cylindrical cxhauster, was invented some years ago, which, being placed at the mouth of a shaft, drew up the foul air, and consequently the pure air rushed in to supply its place, but such applications are not generally necessary.

In coal mines the ventilation is much more difficult than in ordinary mines. It is cfiecterl by two shafts, called the down-cast and up-cast. The up-cast shaft has a large

Fig. 240.

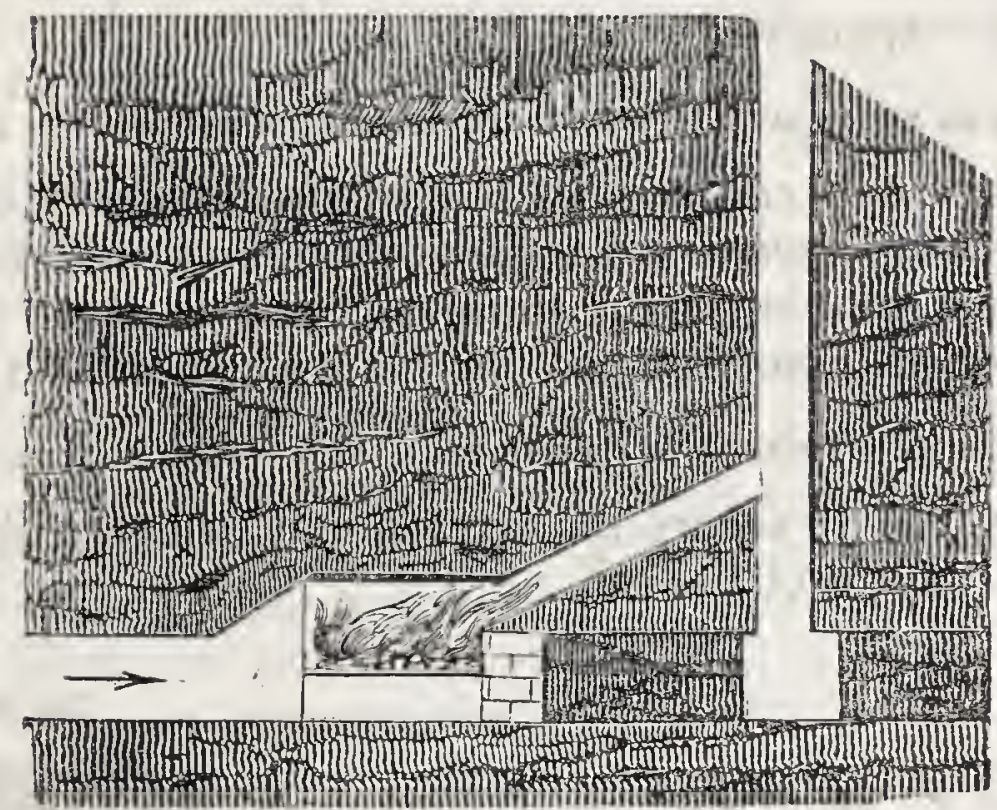

VENTILATING APPARATUS.

furuace continually burning near the bottom of it (see fig. 240), and the air being con. siderably rarefied at this point, an ascending current is formed, which passes upward with great relocity, and causes a descending current to pass through the down-cast shaft, to supply its place.

Mine Shafts. - Shafts of extensive dimensions are usually divided into several compartments, which are devoted to different purposes. Thus fig. 241 represents the plan of is

Fig. 211.

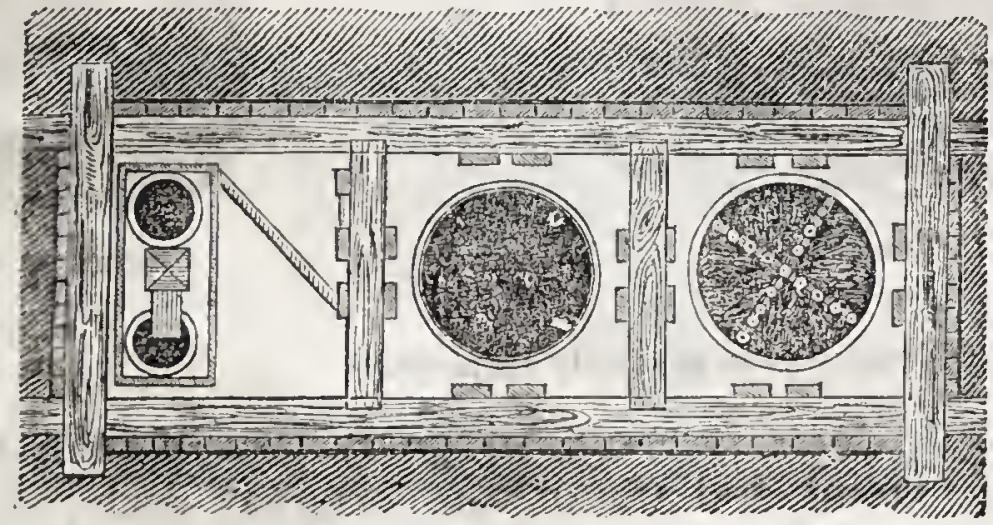

shaft divided into three compartments, of which two are devoted to the purposes of extrac. tion, while the third serves as a passage for drainage and as an entrance for the workmen.

Q. How is the PRODUCE of a MINE brought up to the SURFACE of the ground?

A. This is very simply effected by adapting an efficient motive power to a convenient form of winding apparatus. 
Q. What Motive power is commonly employed?

A. Steam.

rijg. 242.
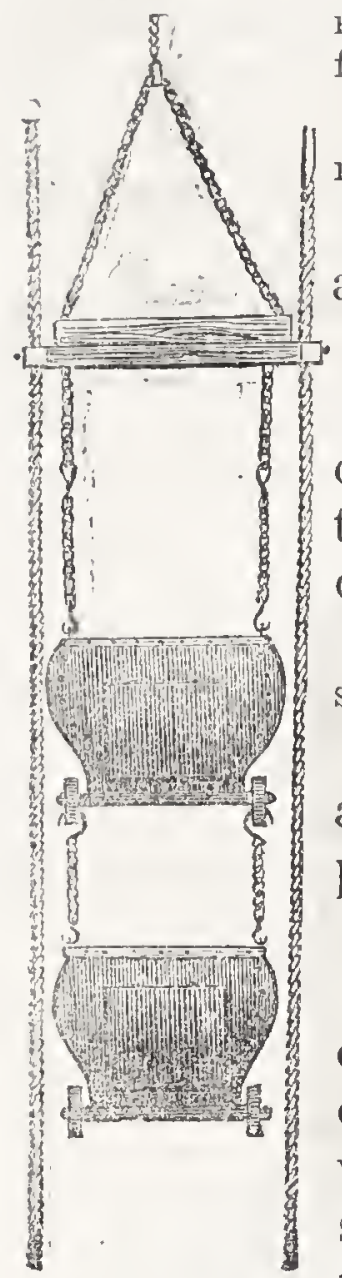

Fig. 242 represents two mining baskets, one above the other, connected by strong chains, and supported by cables, which carry them from the bottorn to the month of the shaft.

Q. Is similar apparatus employed in the ASCENT and DESCXNT of miners to and from the pit? above.

A. Yes; they are lowered by a motive power from

Q. Is there not considerable DANGER attending such transit?

A. Mining operations are altogether of a very perilous character, but much has been done by scientific investigation and mechanical contrivance to lessen these dangers.

Q. Has any Safett apparatus been invented for ascending and de. scending mines?

A. Fourdrinier's invention has this object in view, and has been severely tested in several mines, and proved to afford a great amount of safety to the miner.

Q. What is the character of this invention?

A. It consists of a cage or basket, which carries tubs of coal attached to guide rods or chains from the side of the shaft. Supposing that the cable or chain by which the whole is supported snaps, the self-acting springs or arms forming levers, attached to the top of the cage, are freed, and these wedging themselves securely against the guide-rods, its descent is immediately averted.

In an experiment made at Usworth colliery, Durham, the cage containing two tubs loaded with coal, the whole weight of the cage, trbs, and coal being forty-eight hundredweight, when the rope was disengaged the apparatus instantaneously took effect, and the whole mass was firmly fixed to the guides. On another occasion, at the same colliery, the cage, with a total weight of fifty hundred-weight, was safely arrested in its descent, which but for the safety apparatus must have been precipitated to the bottom of tho

Fig. 243

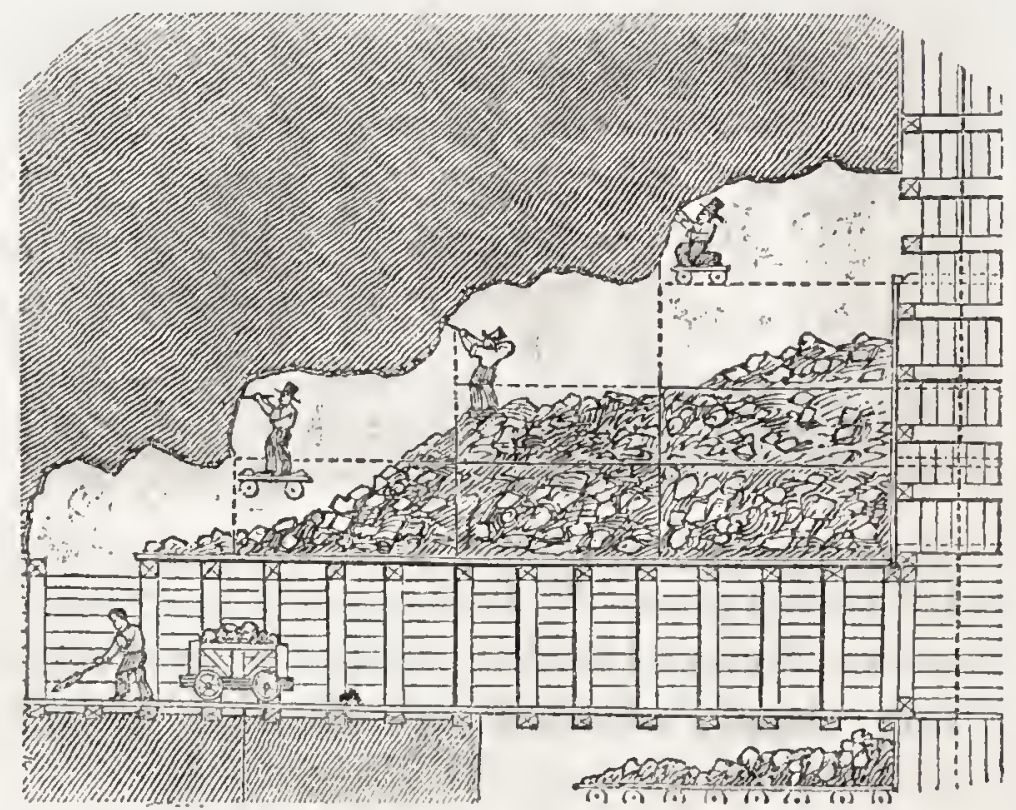


shaft, a thousand feet below. But this was not all. Two of the workmen then placed themselves in the cage, and by a touch of their hands stopped this weight of tity hundredweight, and themselves in addition.

Fig. 244.

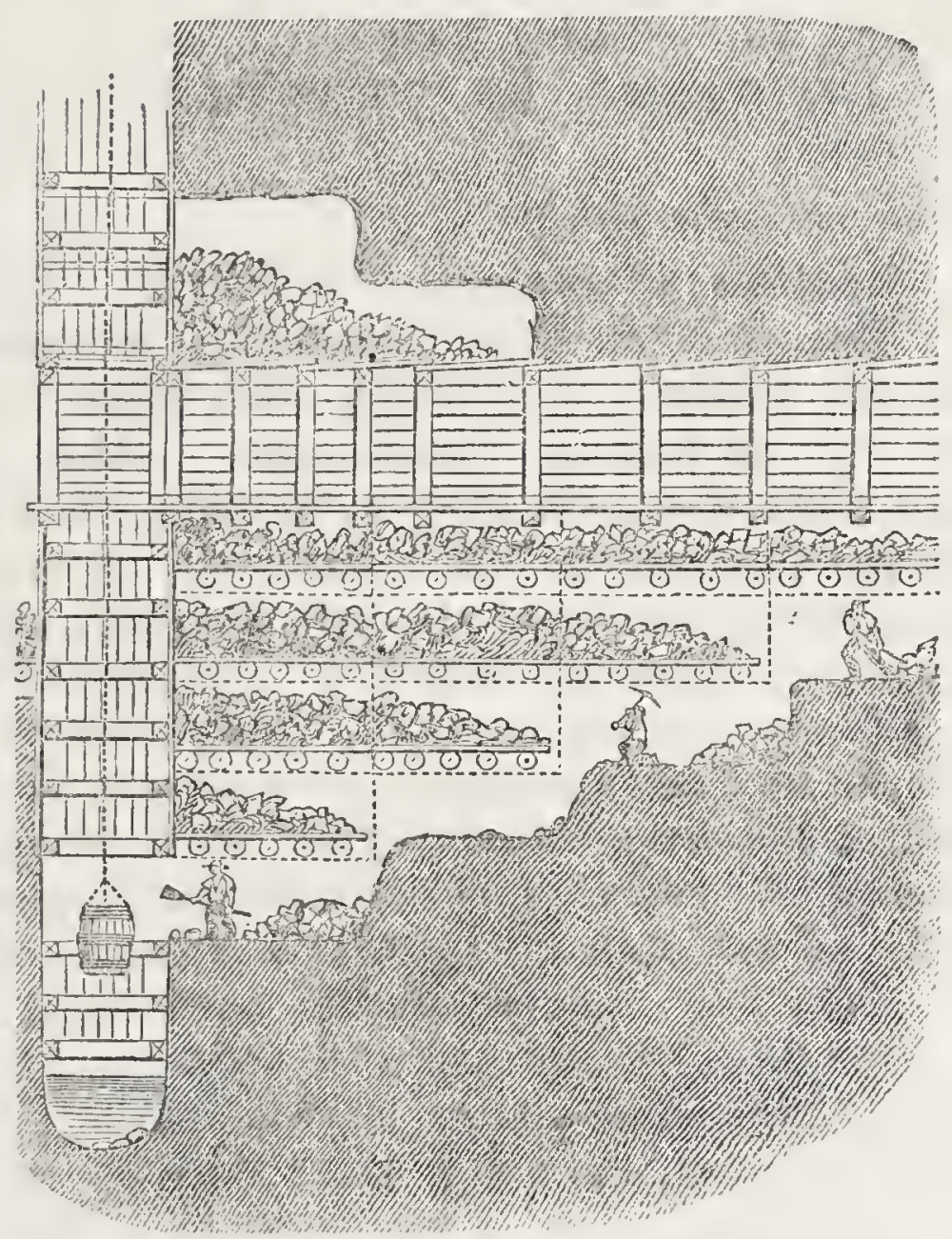

The preceding diagrams (figs. 243,244 ) represent two of the methods adopled in working a mine-the first from below, the second from above.

Q. Does the ore of a mine undergo any MECHATICAL PReparation from the hands of the miner?

A. It does; and such mechanical preparation is technically known as dressing.

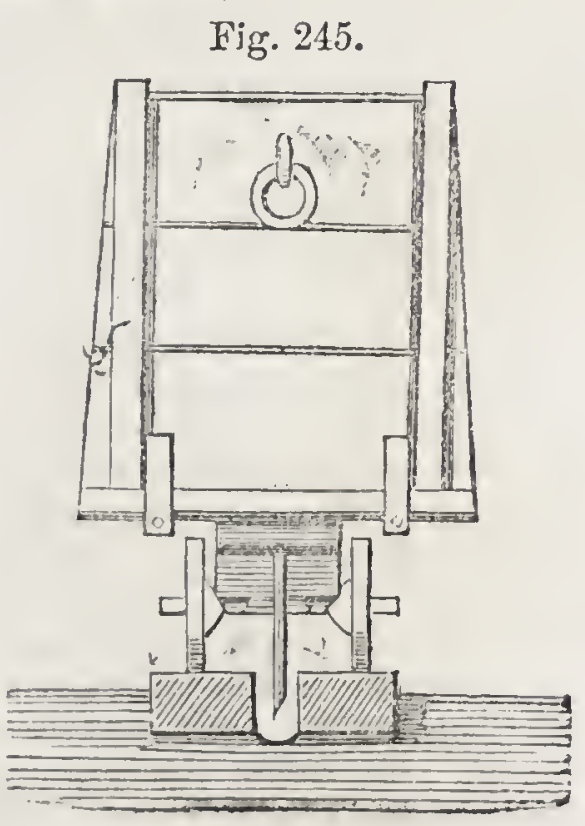

Q. What is the PURPOSE of this preparation?

A. The operation termed dressing is, in fact, separating as far as possible the earthy matter from the metallic ores.

Q. Upon what PRINorple is the operation conducted?

A. The operation is conducted on the principle of the difference of specific gravity between earthy and metallic matter.

Q. Describe the ordinary process.

A. The dressing-floors of a mine are always placed near the principal shaft, and are always provided with a channel of water. The floor is paved, and there are side ranges of sheds for the labourers to work in. The ore is conveyed to them by a small railway, in an apparatus similar to that which is seen in fig. 245 . 
Q. What is the Finst operatios which the ore undergoes?

A. The first operation is that which is technically described as picking. The ore, brought from the mine in rough, irregular masses, is broken into smaller pieces. When the sparry matter is picked out and rejected, the remaining portion of the ore is comparatively pure.

Q. Describe the succeeding Processes.

A. These processes consist of "crushing", "jigging," and "stamping." "The first of these operations is performed by a machine called the grinder or crushing mill, which consists of iron rollers moved by mechanical contrivance. Above them is a "hopper," into which the ore is thrown, through which it passes, and is crushed by the iron rollers. The pure and impure matter, the metallic and the earthy particles, are still as much mixed as ever, and another process is rendered necessary. It is therefore transferred to the brake sieve, or jigging machine, which consists of a wooden frame, open at the top, and provided with a sieve at the bottom. By a peculiar motion, this sieve containing the ore is plunged again and again into a cistern of water; a movement which causes the heavy metallic particles to settle at the bottom, and throws the lighter or earthy matter to the top. The separation, however, is not complete. The earthy matter thrown to the top still contains metallic ore, and to extract this from the refuse matter, several processes are employed, such as grinding, washing, stamping, \&c. Thus, by repeated washing and grinding, the metallic ore is separated to a great extent from earthy matter; but it still contains many impurities, for, when afterwards placed in the furnace, these impurities become fused, and are known as slag.

Q. Were mining operations conducted at an EARIY PERTOD of the world's history?

$A$. They were, according both to sacred and profane histories. Thus Moses ascribes the first use and manufacture of metals to Tubal Cain; and metallurgy was known both to the Egyptians and Greeks-such as gold, silver, copper, tin, and iron.

Sucli facts as these, however, do not, it has been very justly observed, "imply any great knowledge of mining, properly so called; as it is well linown that metalliferous deposits are often found near the surfaee, frequently in a state of extreme purity, as gold and silver, for example; and in early ages, when they had been so much less ransacked by the miner, these superficial deposits must have been muel more abundant than at present, and probabiy formed a large proportion of the metallic produets of those times."

MIost of the mines of antiquity were probably of a similar nature to the stream works of Cornwall; and it appears from Strabo that the Pbonieians at that early time used to trade to Cornwall for tin and lead. In early times the demands for the metals could not have been rely great; their use was then either as instruments of luxury or war, and thus eonfined to a limited elass; so that the quantity found near the surfice was, in all probability, fully adequate, leaving but little indueement for deeper and more laborious research. There is, however, evidence enough to show that operations similar to those in modewa mining were earried on by the nations of antiquity. Herodotus observes that

mountain in the island of Thrasos was eompletely burrowed by the Phcenicians, in their seareh for the preeious metals; and the curious fragment of Agatharchides, preserved in Dioclorus, shows that the art of forming shafts and passages for exploring mines and pro- 
curing the metals was well known in Egypt. The silver mines of Laurium, in Atticn, were worked by the Athenians, to some extent at least, as early as the beginning of the fourth century e.c. Under the Romaus, the quicksilver mines of Almadeu, in Spain, were extensirely worked.

Q. Was not the art of mining in England NEGLEOTED foraa long time?

A. Yes; during what is called the Saxon period the mines were much neglected. Before and during the Roman conquest they were a source of considerable wealth to the country; but after the establishment of the Saxons in England the troublous state of the land drew away attention from them, and up to the time of the Norman invasion they were suffered to continue in neglect.

Q. What occurred with respect to British mines SUBSEQUENTLY to this date?

A. After that period the mines were, for a considerable time, worked by the Jews; but the subject was, to a greist extent, still suffered to remain in neglect; and in the reign of Queen Elizabeth a number of German miners received every encouragement to settle in England, in order that this most important commercial undertaking might be revived.

It was by the wealth of the lead mines of Cardiganshire that Sir Hugh Middleton derired much of his large fortune; a fortune which was so nobly spent in the formation of the New River.

Q. When was the process of BLASTING WITH GUNPOWDER in mining operations FIRST INTRODUCED?

A. It is probable that gunpowder was first employed in England for the purpose of blasting mines in the reign of James $I$.

"The application of gunpowder to the purposes of mining first took place in Hungary, or Germany, about the year 1620 ; and it was first introduced into England at Ecton, in Staffordshire, about the year 1620, by some German miners, brought over by Prince Rupert. It was in use in Somersetshire about 1684 ; and it was not till after this period probably that the Cornish miners became acquainted with this powerful assistant in their operations. Its importance may be judged by the amount of the present consumption in the mines of Cornwall alone, which has been calculated at an annual value of about forty thousand pounds sterling." -Taylor.

Q. Had the invention of the STEAM-ENGINE any CONSIDERABLE INFLUENCE on the progress of mining?

A. It had, as the introduction of Savery's engine into our mining districts completely altered the principles of this important art, and very considerably extended its utility. It was first applied to the drainage of mines.

Q. Who was the FIRST IMProver of Savery's engine?

A. Thomas Newcomen, of Dartmouth, in Devonshire, who, having had occasion to witness experiments made with Savery's steam-engine in the tin mines of Cornwall, and to observe the danger and difficulties attending its application to the purposes of drainage, was led to the invention of the atmospheric steam-engine.

Q. When were the GREATEST IMPROVEMENTS introduced?

$A$. The greatest improvements in the appiication of steam power to the art of mining were attributable to the discoveries of Watl. Besides many other adrantages, they were far more economical than those of former time. 


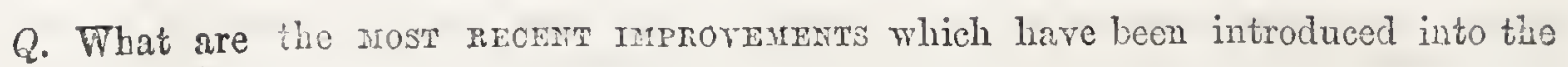
art of mining?

$A$. They have been chiefly directed to the sufety of the miners, or to the mechanical tregtment of the ores.

\section{STONE.}

Q. In selecting a stone for ARCHITECTURAT purposes, how may we be ablo to form an opinion respecting its DURABILITY and PERMANENCE?

A. By visiting the locality from whence it was obtained, we may judge from the surfaces which have been long exposed to the weather if the rock is liable to yield to atmospheric infuences, and the conditions under which it does so.

"For example, if the rock be a granite, and it be very uneven and rough, it may be inferred that it is not very durable ; that the feldspar, which forms one of its component parts, is more readily decomposed by the action of moisture and frost than the quartz, which is another ingredient; and therefore that it is very unsuitable for building purposes. Moreover, if it possesses an iron-brown or rusty appearance, it may be set down as highly perishable, owing to the attraction which this metal has for oxygen, causing the rock to increase in bulk, and so disintegrate."

Q. Why are the SANDSTONES, termed FREE stones, ILL ADAPTED for the external portions of exposed buildings?

A. Because they reculity absorb moisture; and in countries where frosts occur, the freezing of the water in the wet surface continually peels off the external portions, and thus, in time, all ornamental work upon the stone will be defaced or destroyed.

Q. Why do some species of rock become HARDER when taken from the quarry and exposed to the atmosphere?

A. This quality, in some species of stone, arises from the fact that the water contained in it, when forming part of the natural rock, evarorates, and the stone, becoming dryer, becomes harder.

Q. Why do some stones, although hard when first quarried, become Fr.IABLE, and fall to pieces, when exposed to the atmosphere?

A. Because they contain clay or alumina in such a state as to readily absorb moisture from the atmosphere; and through the agency of the moisture the particles lose their colesion and fall apart.

\section{S.P P INGS.}

Q. What is the oRIGIN of springs?

A. The water falling upon the earth sinks downwards through the sand and porous materials, until an impervious bed of clay or rock is reached. Here the water accumulates, and finally bursts out at some point where the impervious bed or strata comes to the surface in consequence of a valley or excavation.

Q. Why does not the water OOZE OU' everywhere along the LIN of JUNCTION of the two formations-the gravel and the rock or clay-so as to form one conTINUOUS IAND soak, instead of a few springs only, and these far distant from one another ?

A. For two reasons: first, on account of rents and fissures in the strata, which act as natural drains; secondly, the existence of 
incquatities in the upper surface of the impermeable stratum, which lead the water, as valleys do on the external surface of a country, into certain low levels and channels.

The water-tight rocks of the earth are of four different kinds, each kind giving rise to a different form of spring. They are :-

1. Where the underlying water-tight rock is a ridge that bends down on each side.

2. Where the rock is an inclined plane in one direction.

3. Where the sides of the rock cul'e up on both sides, like the inside of a basin.

4. Where rocks have been dislocated by what is called a fault.

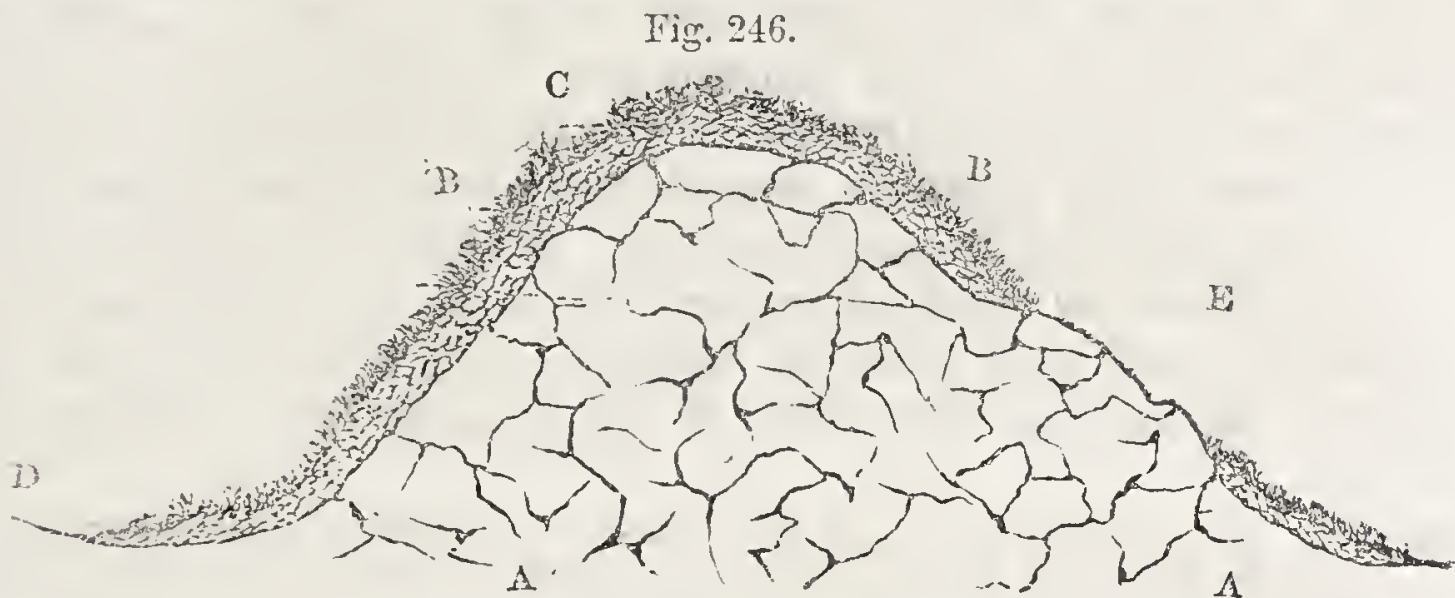

The above diagram represents the first instance of these varieties, and shows the concave curve of a water-tight rock. A represents the mountain mass of a water-tight rock, $B$ a gravelly bed, formed from the rock $\mathrm{A}$, and resting on it. $\mathrm{C}$ the vegetable mould, which covers $A$ and $B$. From $D$ to $E$ is a well, formed at the bottom of the mountain; and $\mathrm{E}$ is another spring, bubbling at a high eleration, on one of its sides.

Fig. $21 \%$.

$\mathrm{D}$

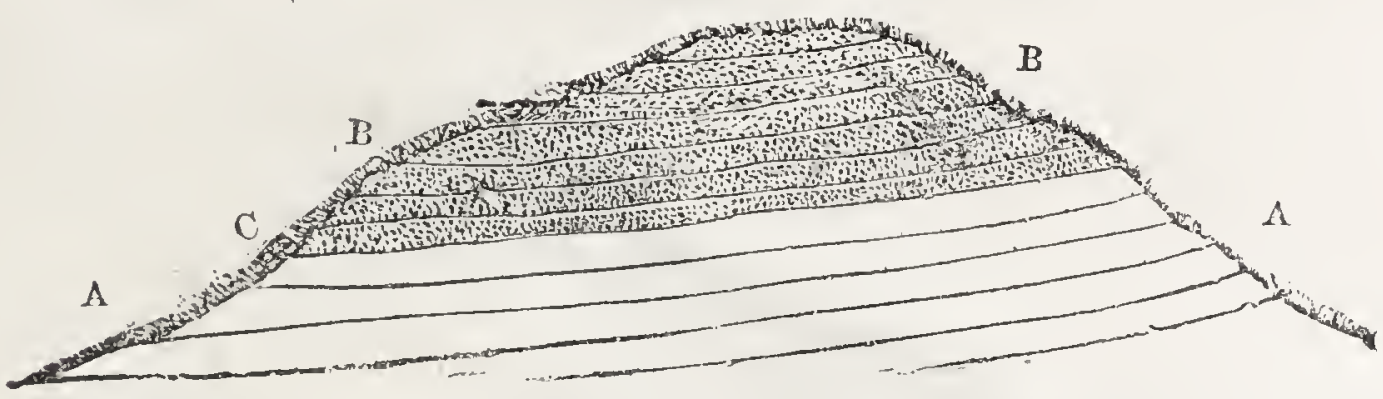

In this diagram is shown a series of inclined beds-the second instance indicated of water-tight rocks-and forms the base of a hill. A is the series of inclined beds; $B$ the strata of grarel, or sand, resting on the inclined beds; $C$ the point of junction of the porous bed of sand, or grarel, with the water-tight strata, in which the permeating vater will appear as a spring at a high eleration on the side of the hill. No spring of water can, under the circumstances, appear at D. The copious and continual flow of such a spring must depend on the deptr and extent of the beds B B.

Q. Why does the water coLIECT in an ordinary WeLL?

A. An ordinary well consists of an excavation continued until a stratum is reached that is permanently saturated with water. These wells are not commonly supplied with springs, but merely by the draining of the water, which exists within the circuit of a few yards, into a carity.

Q. Why do WELIS and springs FAIL oftentimes in DRY WEATHER?

A. Because they are supplied by the water falling as rain, which percolates from the surface of the earth. 
Q. What is an Artesine well?

A. Water is sometimes obtained by boring into the eccrth with a species of auger, until a vein or sheet of water is found, which rises to the surface through the cylindrical excavation. Such excavations are called Artesian wells.

Q. How do you $A$ ccount for the water RIsing to the surface in the Artesian well ?

A. Strata which are pervious frequently alternate with others which are not so; or may form a basin, the area of which is partially filled with clay, through which water cannot pass; in such a case it is obvious that the bed of sand beneath the clay, fed by the rain which descends on the uncovered margin of the basin, must form a reservoir where the water will gradually accumulate beneath the central layer of clay, through which it cannot escape. If the bed of clay be penetrated by natural or artificial means, the water must necessarily rise to the surface, and may even be thrown up in a jet to an altitude which will depend on the level of the fluid in the subterranean reservoir.

Thus, if a sandy straturn, acting as a filter, occupies an inclined position between the two other strata impervious to water, such as clay, the water being absorbed by the superficial parts of the strata (which may be of very great extent) will penetrate through its whole depth, and, finding no egress below, on account of the basin-like form of the stratum, or from its resting at the lower termination upon a compact rock, will accumulate. The porous strata, therefore, becomes a reservoir to a greater or less extent; and if, by boring through the superincumbent mass, we form an opening into the stratum, the water will rise in it, and flow over in a jet proportional to the height of the water accumulated in the stratum from whence it flows.

Fig. 248.

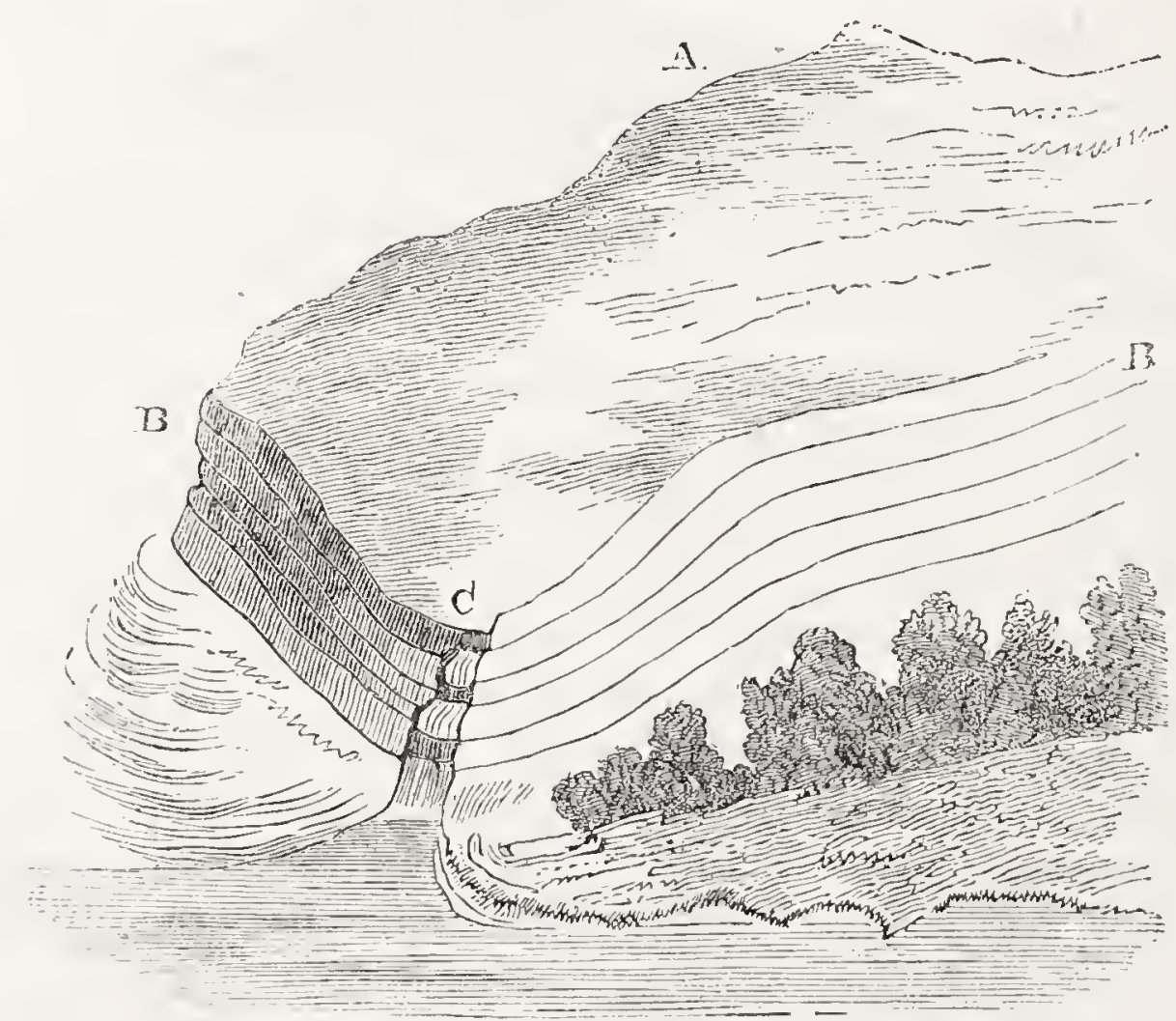

The above engraving represents a spring in a mountain side in Switzerland. The upper part of the hill $A$ is formed of a large, deep mass of gravelly soil. Through this mass rain-water and melted snow penetrates, till it reaches the impervious stratum, B B. 
A kind of trough is formed (C) by the carrying upward of these beds, when the waters gush out into a powerful spring.

Q. Are the Artestan welis of Modern invention?

A. Such wells were lnown in China from a very remote period

Q. Whr are they called Artesian?

A. From the French province called Artois; or, anciently, Artesium; it being in this district that wells of this description were first formed in Europe.

Q. Describe the meaning of the terms ARENACEOUS and ARGILlAOEOUS.

$A$. 'The term arenaceous, or sandy, is given to such soils and rocks as easily absorb or drink in the water that falls upon them. Argillaceous, or clayey, denotes such soils and rocks as retain the water, or through which water penetrates with great difficulty

Q. How does the water PASS THROUGH these porous beds?

$A$. It descends by its own gravity lower and lower, until it finds a stratum of clay, or rock, which is impervious--that is to say, through which it cannot pass.

The principle here involved has been already explaincd. When the impervious rock curves upward, the water produces wells on each side of the hill; when the surface is an inclined plane, the water flows along the slope in one direction, and appears as a well on one side only of the mountain. It is, therefore, evident that when water reaches an impervious stratum it must accumulate as a reservoir, and will then ooze out by any outlet which it can effect.

Q. How is it that the water does not ooze out along the WHoLe IINE of JUNCrion between the porous stratum and the impervious rock?

A. 1. Because, in loose, gravelly beds, there are always to be found fissures which act as drains to the water.

2. Because the dents and curves in the hard rock cause the warer to accumulate more in one part than another, and so overflow.

Fig. 249.

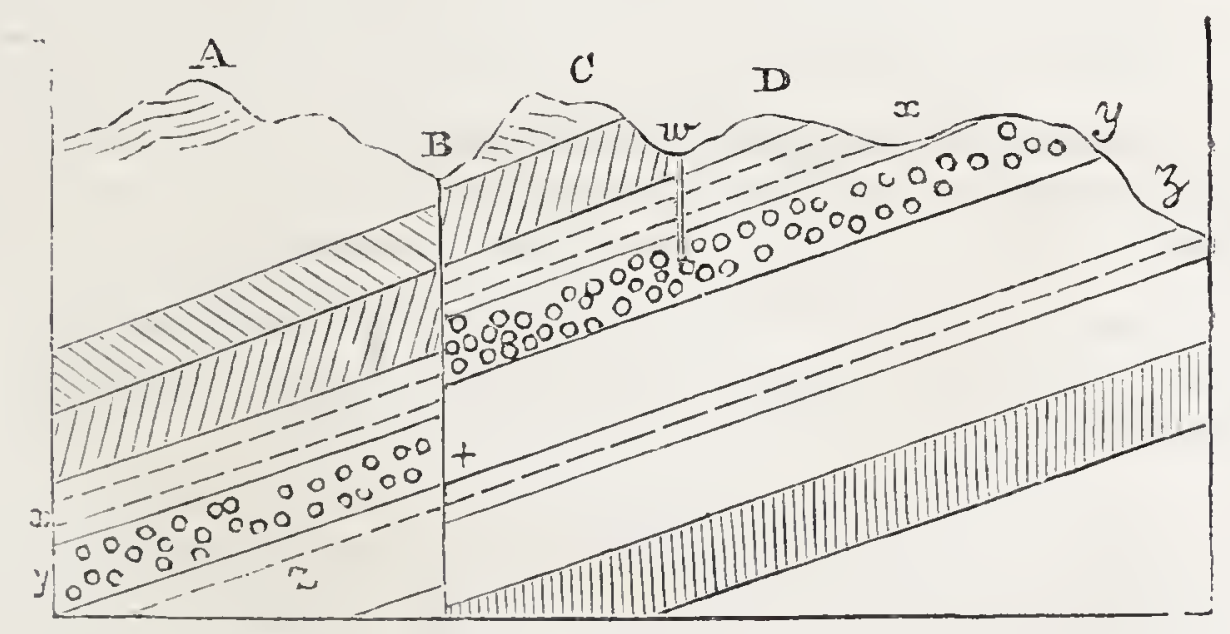

In fig. 249 , let $A, B, C, D, x, y, z$, represent the section of several milcs of country, with three different rocks resting on each other, $x, y, z$. The rocks $x$ and $z$ are impervious to water; the rock $y$ is porous; thcrefore the rain enters between the rocks $x$ and $z$. It will be noticed that the beds are shown as fractured by some dislocation from beneath. This is called by geologists a fault. It has produced a fissure, or crack, with an outlet at B. Now, the water which has entered the bed $y$ is stopped by this fissure, which has broken the continuance of the bed, and the water is met by an impervious rock. 
Then lct the rock? be tapped by the Artesian process, and the water inmediately rises at $x$. What is thus accomplished by artificial mcans at $w$, is effected by the natural fissure at B. There the water rises as a permanent spring. The differcnce between the two is this: the artificial boring is a mere puncture, as to extent; but the effects of the "fault" arc felt for miles downwards. On this account, springs produced by faults are more permanent than others.

Q. What is a NATURAL sorm?

SOII.

A. Natural soils are mercly decomposed parts of the subjacent rocks, mixed with the decomposed portion of regetable substances which have grown or fallen upon it, with some animal substances.

"Whererer the surface of the earth is not covered with water, or is not naked rock, there is a layer of earth more or less mixed with the remains of animal and regetable substances in a state of decomposition, which is commonly called the soil. The nature and composition of the soil, and consequently its ercater or less aptitudc to the growth and maturity of regetable productions, depcnd chiefy on the proportion and mechanical strucure of the various substances of which it consists. When the soil is favourable to the chemical action by which the elements are combined to form regetable substances, and admitting the quantity of air and moisture, without which this chemical action cannot take place in any given climaic or temperasurc, vegetation goes on rapidly, and all the plants which are suited to the climate grow in the sreatest perfection, and bear abundant fruit."

Q. What is the NAMr given to the vegetable and animal products mixed with the mineral ingredients of a soil?

A. Humus.

"Organic mattcr is, no doubt, essential to great fertiliby in a soil ; but some soils require more of it than others. Frumus, which is the form which organic matter naturally comes to by slow decomposition in the earth, gives out ccrtain elements which the roots can take up in their nasccnt statc, and from which they obtain the carbon which is so abundant in all vegetable productions. But organic matter, in every stagc of its spontaneous decomposition, keeps the pores of the soil open, and admits, if it does not even attract, air and moisture to the fibres of the roots. In all rich soils, which have been long cultivated, especially in gardens, thcre are particles, of a darls colour and fibrous texture, which in the microscope appenr like minute logs of cherred wood. These keep the soil open, and supply carbonic acid, when the air reaches them, or they are slowly trans. formed into liumus, which rcmains inert as long ais it caunot imbibe oxygen and form carbonic acid by a specics of slow combustion. Hzmus is undoubtedly one of the chief causcs of fertility."

Q. How are soils DISTINGUISHED ONE FROM ANOTHER?

A. By a comparison of their texture as to the size and character of the particles, and tracing their original formation.

Q. How can you ASCERTAN the sIze and CHARACTER of the PATTCLES of soil?

A. By drying it thoroughly, and then slightly bruising it with a wooden pestle in a mortar.

Q. What BENEFICIAL EEFECT do loose stones and rocks have upon dry, porous soils?

A. They retain moisture in the soil by preventing the evaporation which would otherwise take place. In high lands they serve to condense fogs and low clouds, and thus add to the moisture of the subjacent soil.

Q. What is DratiL?

A. Any rock which contains a considerable portion of carbonate of Time, and which rapidly disintegrates on exposure to the atmosphere, is called marl. 


\section{E E A L OGY.}

\section{CHAPTER LXX.}

\section{CHARACTERISTICS AND CLASSIFICATION OF MINRRALS.}

Q. What is Mineralogy?

A. Mineralogy is that branch of natural history in which minerat substances are classified and distinguished, their uses made known, and their modes of occurrence in the earth pointed out.

Q. What Constitutes a mineral species?

A. A mineral species is a natural inorganic body, with a definite chemical composition, and a regular determinate form or series of forms.

Q. Into what Four cLASSES may mineral substances be divided?

A. Into the silicious and earthy minerals, the saline or salts, the infammable, and the metals.

Q. What are the sILICIous and EARTHY minerals?

$A$. The silicious and earthy minerals comprise all those species which derive their principal characteristics from silica and the earths. In this class would be included most of the common stones, and the constituents of all ordinary roctis.

Q. Will you give some Examples of the silicious and earthy minerals?

A. Those in which silica predominates, as the flints, quartz; those in which alnmina, lime, and magnesia form essential constituents, as common clay, slates, soapstone, mica, marble, limestone, spar, \&c.

Q. What suestances would be included in the SALins class?

A. Most of the combinations of the acids and chlorine with the cllkalies which exist in the mineral kingdom; as saltpetre or nitrate of potash, common salt or chloride of sodium, and sal ammoniac or chloride of ammonium.

Q. What mineral SUBSTANCES would be included within the class of INFLAMMABLES or COMBUSTIBLES?

A. All the mineral combustible bodies, except the metals; such as sulphur; coal, amber, bitumen, \&c.

Q. What minerals are included in the class of motals?

A. All those mineral substances which are composed either entirely of metals, or of which metals constitute the most considerable part.

Q. How Mari mineral species are known?

A. About fou hundred mineral species are known; the varieties of these species are almost infinite. Thus carbonate of lime, a mineral species, occurs as marble, as chalk, as Iceland spar, as dogtooth spar, and in many other forms, more or less common. 
Q. What two mineral species occur in the GREATEST ABUNDANCE upon the surface of the earth?

A. Quurtz and carbonate of lime.

Q. What is QUARTz?

A. Quartz is nearly pure silex, or oxide of silicum. When pure, it is transparent or translucent, and has a vitreous or glassy appearance. It then receives the name of rock-crystal.

Q. What is AMETHYST ?

A. When the rock-crystal is of a rich purple colour, it is called amethyst; when the colour is faint red or pink, it is called rose quartz.

Q. What oTHER precious stones are included under the varieties of QUARTZ ?

A. The jasper, the carnelians, the opal, the agate, and the like.

Q. What is the CoMposition of the RUBY and the SAPPHIRE?

A. The ruby and the sapphire consist principally of aluminous carth, with a little silex and oxide of iron.

Q. How do these gems appear to have been ForMED?

A. They appear to have been crystallised through the action of heat in the rocks in which they are found. This supposition is rendered almost certain by the experiments of French chemists, who have succeeded by a similar method in producing artificial rubies, in every respect resembling the natural gems.

Q. What is FELDSPAR?

A. Feldspar, a constituent of all granite rocks, is composed of silex, alumina, and potassa. It is by the decomposition of this mineral that much of the potash existing in the soil, and absorbed by plants and trees, is furnished.

Q. How is the Potash extracted from the feldspar, and made accessible to the roots of jlants and trees?

$A$. When the feldspar decomposes, the potash, being soluble, is washed out, acted upon by the atmosphere, and imbibing carbonic acid, always present in the air, becomes converted into carbonate of potash. The carbonate thus formed is disseminated throughout the soil, and is then taken up by the roots of plants.

Q. What is MICA?

A. Mica is composed of nearly the same ingredients as feldspar, together with oxide of iron. It is often colourless, but frequently green, black, or smoky. It is well known from its capability of division into exceeding thin, transparent, elastic plates. From its ability to resist the action of heat, it is much used for the doors of stoves and furnaces.

Q. What is SOAPSTONE?

A. A compound of magnesia and silica. It is generally of a lightgray colour, earthy texture, and unctuous to the touch.

Q. What is SERPENTINE?

A. Serpentine has much the same constitution as soapstone. It is 
generally of a greenish colour, variegated with spots of green of different shades. With a mixture of carbonate of lime, it forms the verd-antique marble.

Q. When feldspar decomposes, and its potash is washed out, what NAME is given to the compound formed by the two remaining constituents?

\section{A. Porcelain clay.}

Q. In what MANNER are minerals, and particularly metallic ores, DISTRIDUTED in the earth?

A. In layers or masses; in lodes or large veins running parallel with, or traversing, the general course of the stratification of the rock; in nests or pockets; in nodules, which are concretions or accumulations of minerals of small extent; and in small veins, which are either branches of heary veins, or an expansion of a large vein, ramifying the rock in all directions.

Q. How are minerals DISTINGUISHED from each other, and classified?

4. By their characteristic differences; these are principally-1, their form; 2, their physical; and, 3 , their chemical properties.

Q. What is that DEPARTMENT of mineralogy CALLED which treats of the Form of minerals?

\section{A. Ciystallography.}

Q. What do we UNDERSTAND by the PHYSICAL CHARACTER of a mineral?

A. Its cohesion, specific gravity, colour, and its relation to light, electricity, and magnetism.

Q. Are there any Liquid or. FLUID minerals?

A. One or two species of minerals, as native mercury, naphtha, \&c., are liquid.

Q. What is the CLEAVAGE of a mineral?

$A$. When its particles are arranged in such a manner that they exhibit less colesive power in one direction than in another, it is said to possess cleavage, in the same manner as wood is more easily cleavable in the direction of its fibre than across the grain. All minerals possessed of a crystalline structure are cleavable. Some, however, exhibit this property more strikingly than others; thus, mica is clearable into the thinnest laminæe or plates.

Q. Does the Fracture, or manner in which a mineral breaks, serve, in a measure, to DISTINGUISI it ?

A. It does. It may be even or uneven, or conchoidal; as, for example, in the case of flint. It may be splintery or earthy, as in the case of chalk.

Q. Does the HaRdNESS of a mineral serve to oHARAOTERISE it?

A. In the description of a mineral particular attention is always paid to its hardness. Many minerals are sufficiently hard to resist the best files, while others are so soft as to admit of being scratched by the finger-nails. Of two minerals, that, of course, is the hardest which will scratch the other without being itself scratched. 
2. What is the SCALE of HARDNESS?

A. A scale of hardness has been constructed, consisting of ten well-known minerals, so arranged that each one will scratch that which precedes it, and may be itself scratched by all those which follow it in the scale. Thus ten degrees of hardness are obtained between the softest mineral, which is talc (a mineral composed principally of magnesia), and the hardest-viz., the diamond.

These degrees are represented in the following table by the corresponding bers: they are as follows:-

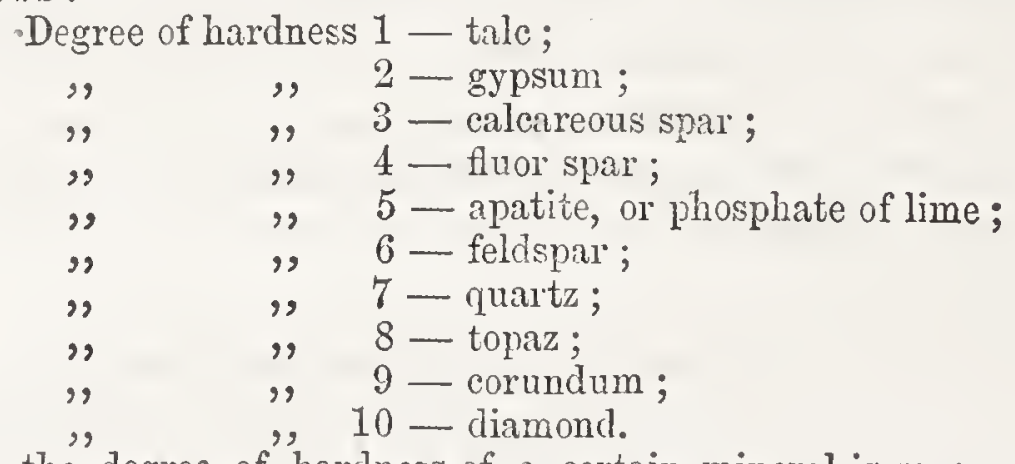

If, therefore, the degree of hardness of a certain mineral is seven, we know it to be equal to that of quartz. The best way of testing the hardness of a mineral is to rub it on a fine hard file, in comparison with the minerals that constitute the scale.

Q. Upon what does the LUSTRE of a mineral depend?

$A$. On the nature of its surfaces; thus we distinguish minerals as having a metallic lustre, a vitreous or glassy lustre, a waxy lustre, a pearly lustre, and a silky lustre. Some minerals are also described as resplendent, shining, glistening, glimmering, and dull; those of earthy fracture are distinguished by the latter term.

Q. What is the STREAK of a mineral?

$A$. It is that colour which appears on rubbing or streaking a mineral on a white body, or on scratching it with a harder substance. "The streale of a mineral is a very remarkable characteristic.

Q. What minerals possess tASTE?

A. Those only which are soluble in water, of which there are comparatively few.

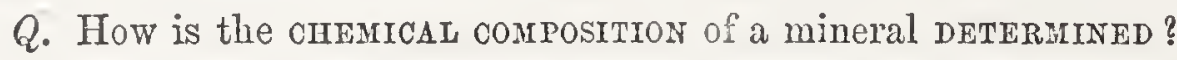

A. By analysis, or by heat and the use of the blowpipe.

Q. Does the Fusibility of minerals vaRY in any great degree?

A. Some fuse at a gentle heat in the flame of a lamp; others are fused only by the application of most intense heat. The products of fusion are also exceedingly multifarious, and serve to point out the character and composition of the mineral in question. 


\section{CHAP'TER LXXI.}

\section{CRYSTALLOGRAPHY.}

\section{Q. What is CRYSTALLOGAPIY ?}

A. Crystallography is the science which teaches the mathematical moperties and classification of crystals.

Q. What are the COMnoN Forus of CRYSTALS?

A. They are of all kinds of forms: some very simple; others, exceedingly complex.

Q. Can crystals be produced by ArTifrotal means?

A. Yes ; most of the salts, and other substances soluble in water, deposit crystals as they are subjected to evaporation. Crystals of sulphur may be obtained by cooling from a state of fusion; by evaporation of its solution; and by sublimation. Bismuth and some other metals assume the crystalline form in passing from the fluid to the solid state.

Q. By what terns is the form of a crystal described?

A. By faces, edlges, angles, and axes.

Q. What is meant by the term FACES?

A. The plane surface of a crystal.

Q. What is meant by its EDGES?

A. The lines formed by the mion of two faces.

Q. What is meant by its ANGLES?

A. The union of more than two faces.

Q. What is meant hy the term Axes?

A. The imaginary lines draun through a crystal for the purpose of describing it geometrically.

Q. What is meant by the SECONDARY FORIS of crystals?

A. These are said to be produced by the supposed truncutions of the solict angles or edige of the more regular forms. They are not of any regular form.

Q. How are TWIN CRYSTALS produced?

A. These are produced by the anion of two or more crystculs, according to some regular plan.

Q. Are there ANY OTHER CLASSES Of IRTEGULARLY FORIED crystals?

A. Yes; one termed epigene, and the other, pseudomorphous; the first is where a crystallised mineral has undergone a chemical change, without in any way changing its form; the second appears as if it 
had been produced in moulds, resulting from the destruction of the original and the subsequent filling up with a new mineral of the space thus left vacant.

Q. Are these secondary forms accidental ?

A. No; they may in all cases be accounted for on definite principles and laws.

Q. Are the DIFFERENT Forars of crystals dirided into classis ?

$A$. They are classified into six systems, namely; the cubical; the mismatic or pyramidical; the rhombohedral or hexagonal; the prismatic or rhombic; the oblique; and the anorthic or doubly oblique.

Fig. 250.

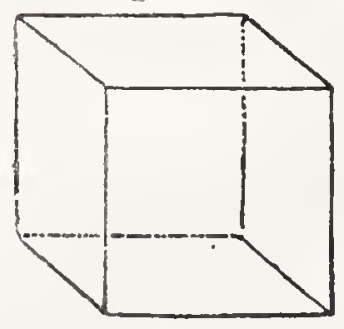

Q. Which is the FIRST SXSTEII ?

$A$. 'That of the cube, which is at once the simplest and most regular. Fig. 250 shows the cube or square block.

Q. What are the conners of the cube called?

A. The corners of the cube are called its solid angles. A solid angle or edge cut off so as to produce a new surface or plane is said to be truncated.

2. How is the CODE described?

A. It has six faces, twelve edges, eight angles; and faces, edges, and angles are all equal.

Q. What MODrFications can this form undergo?

A. Under what is called the cubical system is included-the octahedron, the rhombic dodecahedron, the three-faced octahedron, the twenty-four-faced trapezohedron, the four-faced cube, the six-faced actahedron, \&c. \&c.

Fig. 251.

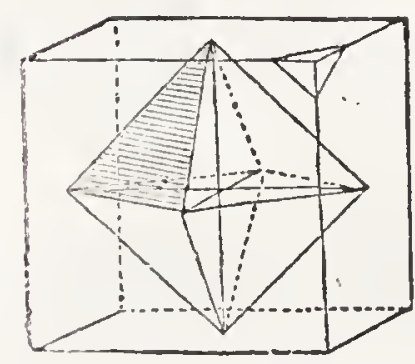

Iiig. 252.

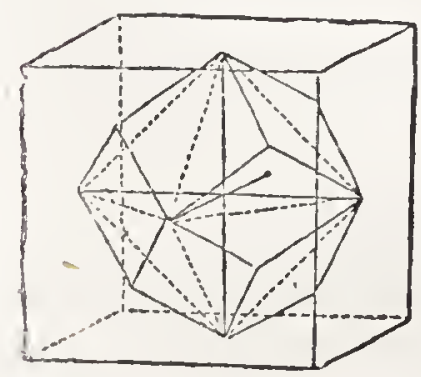

Q. Describe sone of these forms.

A. The octahedron (fig. 251), has eight equal faces, has twelve equal edges, and six angles. The rhombic dodecahedron (fig. 252), is a solid with twelve equal faces, called rhombs; it has twentyfour edges, six four-faced solid angles, and eight three-faced solid angles.

Q. Is not the RIOMBIO DODECAIEDRON sometimes called by another name?

A. Yes; it is sometimes called the garnutoedron, on account of its form resembling the garnet.

Q. What oTHER FORMS are included in the FIRST SYSTEM?

$A$. The hexatet:2hedron (fig. 253), consisting of twenty-four triangular faces; and the trapie- 
zohedren (fig. 254), consisting also of twenty-four faces, each of that form known as the delloid, or traperium.

Fig. 253.

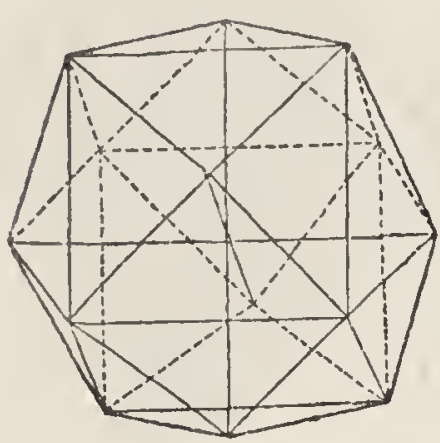

Fig. 254.

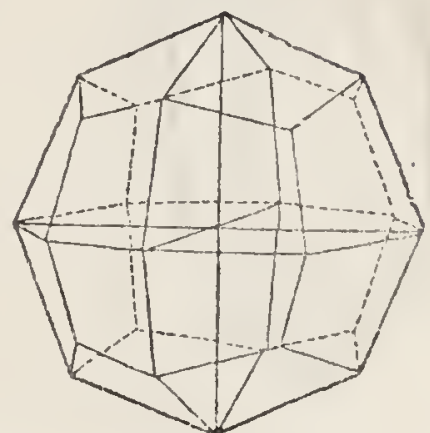

Q. What is the form known as TERRAHEDRON?

A. Its form is derived from the twenty-four-faced trapezohedron, by the development of half its faces. (See fig. 255.)

Fig. 255.

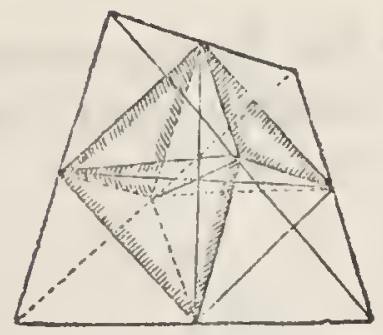

Fig. 256.

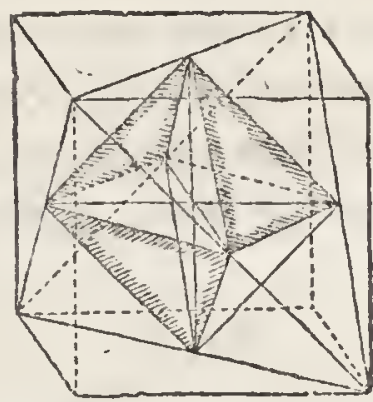

In the following figures are seen other forms belonging to the cubic system.

Fig. 257.

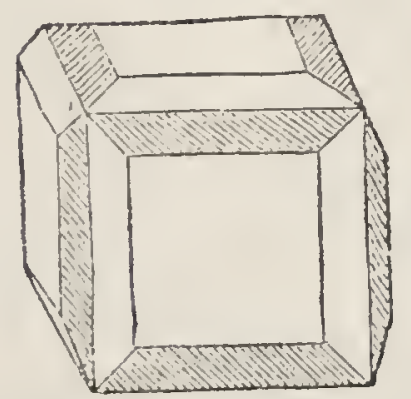

Fig. 258.

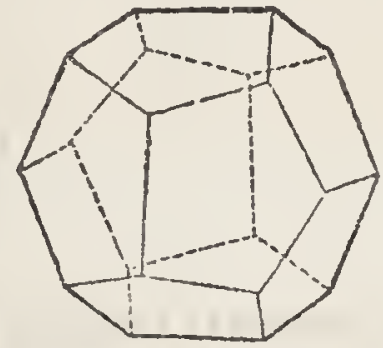

Fig. 259.

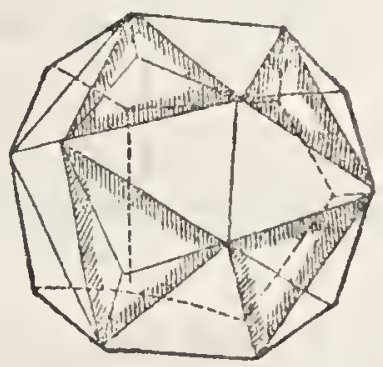

Q. Nention sove of the MINerals whose crystals occur in the form of the CuBE, $o t^{\circ}$ MODIFICATIONS of the CUBE.

A. Alum, bromine, copper, diamond, fluor, garnet, gold, iron, muriate of silver, sulphuret of cobalt, magnetic iron ore, platinum, sulphuret of iron, sal armmoniac, salt, silver, \&c.

Q. What is the SECOND SYSTEM, Or OLASSIFLCATION, of minerals?

A. The pyranidical, or prismatic.

Fir. 2 .

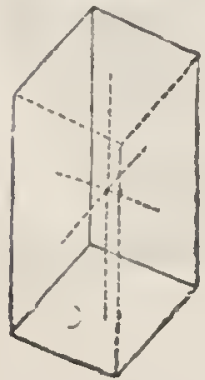

Q. As the cube is to the cobical syster, what is the leading figure in the PYRAMIDICAL SISTEM?

A. The square prism (fig. 260.) It has six fuces, four of which are oblongs or rectangular parallelograms; the ton and bottom are square; it has twelve edges and eight angles. Modifications of this form are seen in figs. 261, 262. 
Fig. 261.

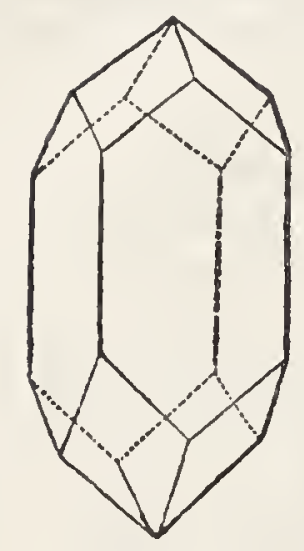

Fig. 26,2.

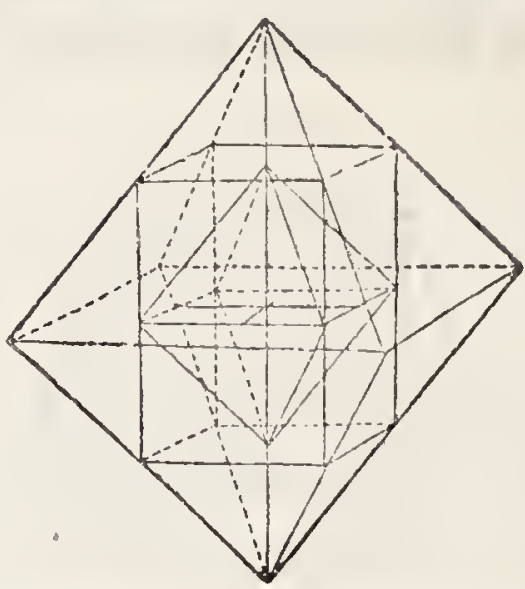

Q. What MINERAL ORTSTALIISATIONS are classified under this SECOND SYSTEM?

A. Among them are: braunite, calomel, gehlenite, pyramidical garnet, oxide of titanium, carbonate of cerium, tungstate of lead, tin, \&c.

Q. What is the THIRD OLASSIFICATION?

A. The rhombohedral or the hexagonal; the former when its forms are derived from the rhomboid (fig. 263), trate latter when derived from the hexagonal prism. (See fig. 264.)

Fig. 263.

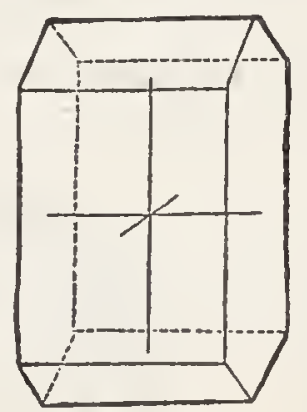

Fig 264.

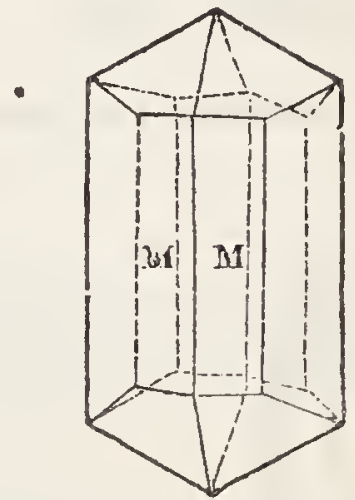

Q. Mention some of the Minerar CRYSTALs belonging to this third system.

A. Antimony, arsenic, bismuth, calamine, emerald, graphite, nickel, arsenite of lead, nitrate of soda, quartz, tellurium, \&c.

Q. What is the FOURTH SYSTEM, or CLASSIFICATION?

Fig. 265.

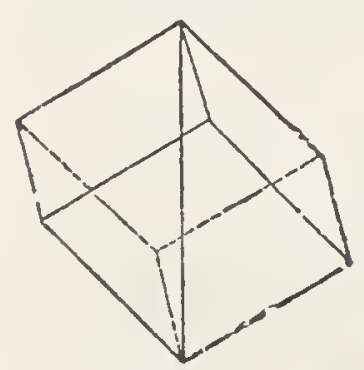

$A$. The rhombic or prismatic. The rhomboid (fig. 265) has six equial faces; all of which are rhombs -namely, four-sided figures with equal sides and opposite angles; but the angles are not all equal. As in the case of the cube, this figure gives rise to numerous and complex variations. Some of these forms are indicated in figs. 266, 267, 268.

Q. Inumerate SOME of the ORXSTALIISATIONS included under this fourth division.

A. Sulphate of lead, carbonate of lime, sulphate of barytes, phasphate of iron, nitrate of potash, sulphur, topaz, \&c. 
Fig. 266.

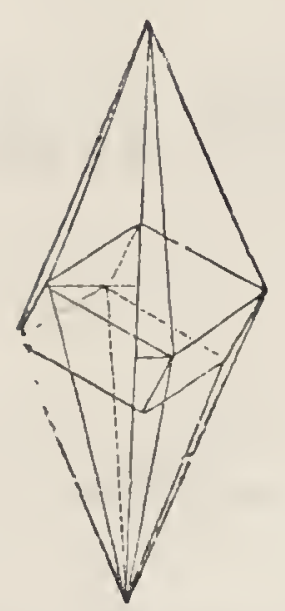

Fig. 267

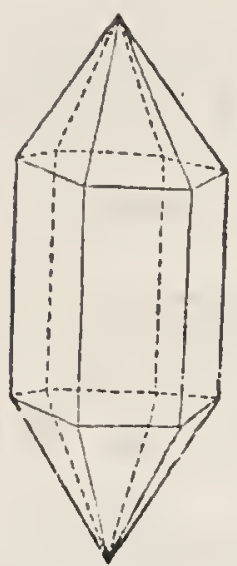

Fig 268.

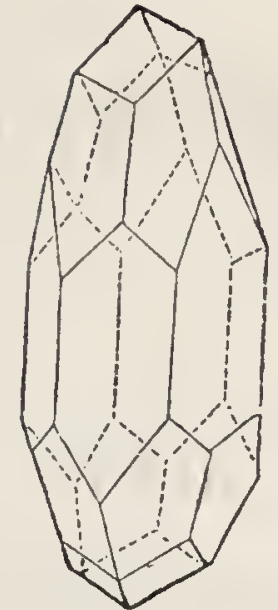

Q. What is the FIF'II SYSTEM?

A. That which is known a the oblique. Some of its modifications of form are indicated in the lollowing figures.

Fir. 271.
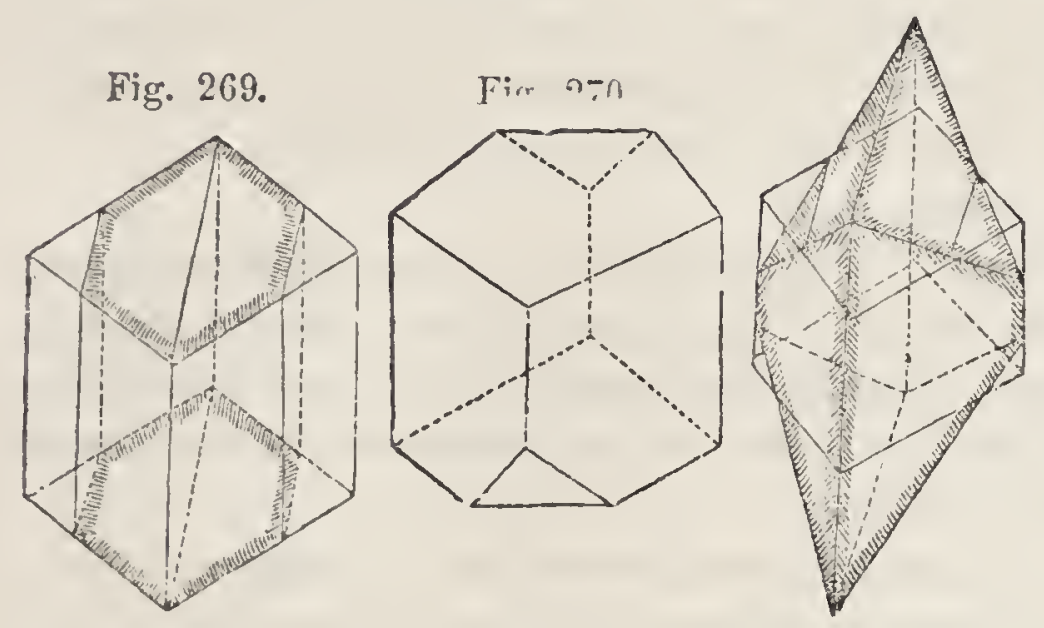

sig. 272.

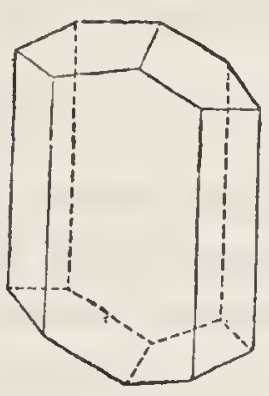

Fir. $2 \div 3$

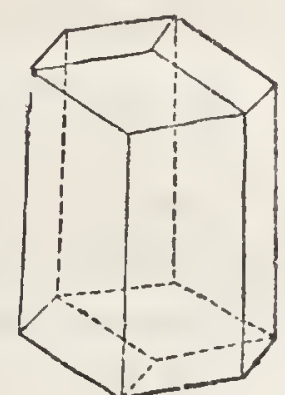

The substances incladed in this classification are felspar, glauberite, gypsum, red antimony, sulphate of lead, phosplate of copper, mica, \&c. \&c.

Q. What is the SIXth CLASSIFICATION?

A. The sixth classification is known as the doubly oblique, and sometimes the anorthic, from the want of symmetry in its form. In this division all crystals are included which cannot be put under any other classification. Figs. 274 and 275 represent forms of this system.

Fig. 274.
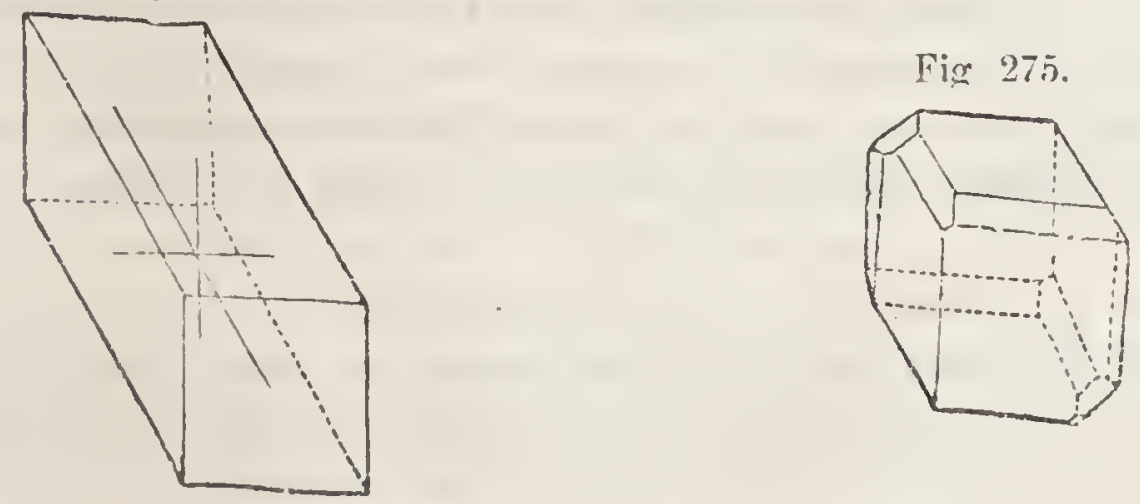


\section{GEOGRAPHY OF PLANTS AND ANIMALS.}

\section{CHAPTER IXXII.}

\section{DISTPIGUTION OF PLANTS.}

Q. What is MEANT by BOTANICAL GEOGRPHY?

A. That branch of natroul science which treats of the distribution of plants throughont the world, and shows how the various parts of the eroth's surface are characterised by distinctive forms of veyeiation.

Q. Is VEGTATION found in ALL PARTS of the world?

1. Yes. But the greatest varieties are observable, not only in the direct and positive contrasts, such as are afforded by the plants rif the arctic and tropical regions, but in varieties of a finer and more delicate character, which are still obvious to the naturalist.

2. Is vegetation proulis to the land ontr?

A. The waters as woll as the land exhibit an almost endless variety of vegetalle life. Vast meadows of gulf weed Hoat in the ocean, submarine forests exist in its depths, every marsh and pool has a sor't of slime of peculiar charicter, and the bed and surface of rivers bear indications of veretable existence.

Q. What scimsin mens are empluyed in treating of botanical geography?

A. Two of the most common terms user with respect to the geographical classification of plants are hrbitution and station. By habitation is meant that particular part of our globs to which the plant in question is peculiar-where, in fact, the plant has its habitation-its home. By station is meant the peculiar nature of the locality, the character of the home as regards climate, soil, moisture, light, elevation above the sea, and other circumstances which affect the growth and full development of the plant.

Q. What is meant by VEGFTBLE zONES?

A. It having been ascertained that certain plants are only to be frund in certain places, that is to say, that a peculiar species of vegetrition distinguishes one part of the giobe from another, naturalists have classitied these divisions, and given them the name of vegetuble zones.

Q. Into How MANY VEGETAJE zONES is the earth dividen?

A. Ijight divisions called by this name are recognised, namely:-

1. The equatorial zone, chiefly remarkable for pahns and bananiss.

2. The tropical zone, of figs and fern trees. 
3. The sub-tropical, of laurel and myrtle.

4. The warm temperate zone, of erergreens.

5. The cold temperate zone, of European trees.

6. The sub-arctic zone, of conifers.

7. The arctic zone, of rhododendrons.

8. The polar zone, including the various forms of Alpine plants.

Q. Then we are to understand that the GFograpHIOAL IISTRIBUTION of PLANTS is rerulated by PARTICULAR LAWS, and that each PARTICULAR IISTRICT is distinguished by its own PUCULIAI SPECIES of vegetation?

A. Such is, undoubtedly, the fact. The phenomena which control this appear to be climate, humidity, light, and soil.

Q. What are the EFFLCTS of TEMTERATURe on vegetation?

A. The most luxuriant vegetation is in a great measure attributable to an increased temperature; thus, under the brilliant sun of the tropics, the variety and the beauty of the vegetation surpass all that is ever seen in the temperate zones. If, again, we contrast the beautiful productions of the temperate zones with those of the polar, nothing can be more evident than that the influence of temperature upon vegetation is most powerful.

Q. Does the Mannir in which a given amonnt of meat is communicated at all DIVEnSIFY the effeets produced?

A. It does; as, for instance, in those districts where a long winter is followed by a hot, brilliant, but short summer, the vegetation is torally different from that of those other districts where the temperature is more equable. This is the case when the same amount of heat is the mean annual temperature of both.

Q. What are the EFFECTS of Morsture upon regetation?

A. Moisture has a very marked and decided effect on vegetation. The characteristics of those plants which are more exposed to it than others is very different from those others; as, for instance, the deep valleys of the American rivers present a striking contrast to the American prairies.

Q. What INFLUENCE has the sorl upon regetation?

A. An influence the most important. Any ordinary agriculturist acquainted with the common principles of farming will be able to bear testimony to this fact, even in a small district. We have only to extend this evidence; what is true of one locality will be true of another; and if the artificial varieties engendered in soils produce such marked effects, what still more remarkable effects may be safely predicated of the varieties of soil which extend over the wide surface of the earth.

Atmosphisic Pressure. - "Atmospheric pressure would at first seem only a very suburdinate influence upon vegetation. But comparing Alpine vegetation with that of bigher latitudes, which, from their situation, must have climates otherwise very similar, we shall be lerl to the conchasion that atmospheric pressure has its share in bringiug about the dirersity of plants; for, though analogous, the flora of the ligh north is by no means identical with that of the most elevated Alpine ridges over which vegetation continues to 
extend. The infuence of atmospheric pressure seems to be particularly evinced in the great, the prevailing number of Alpine species endowed with a volatile fragrance, which adds so much to the sweet and soothing influence of mountain rambles; whilst the northeru species, however similar to those of the Alps, partake more or less of the dull. ness of the heary sky under which they flourish."-Hughes.

\section{Q. Has ELECTRICITY any INFLUfance on vegetation?}

$A$. The rapidity of growth seen in all vegetation after the thunderstorms of spring, shows that electricity has a marked influence on vegetable life. Light also exerts a wonderful influence, some plants thriving best in shaded places, others under the direct rays of light.

Q. Has the POSIIION and FORM of a country any INFLUENCE of this kind?

A. 'The form of continents, their mountain ranges, the mean level of their great plains, all have an influence on vegetation; that of land on the coast also, or that of islands, differs materially from that of inland parts.

"However active," says a recent writer, "these physical agents may be, it would be very unphilosophical to consider them as the source or origin of the beings upon which they shed su extensive an influence. However powerful may be the degree of heat, be the air ever so dry or ever so moist, the light ever so moderate or ever so bright, alternating ever so suddenly with darkness, or passing gradually from one condition to the other, these agents have never been observed to produce anything new, or to call into existence anything that did not exist before. Whether acting isolated or jointly, they have never been known even to modify, to any great extent, the living beings already existing, unless under the guidance and influence of man, as we observe among domesticated animals and cultivated plants. This latter fact shows, indeed, that the influence of the mind over material phenomena is far greater than that of physical force, and thus refers our thoughts again and again to a Supreme Intelligence for a cause of all these phenomena, rather than to so-called natural agents."

Q. What are the cHIEF PRoducnions of the tropical regions?

$A$. In the tropical regions vegetation is seen in the greatest luxuriance, the fullest energy, the most marvellous diversity, and most dazzling splendour. The grasses, which in our climate we only know under the humble forms they put on in our fields and pastures, rise into the elegant and majestic bamboo, to the height of sixty or seventy feet. The tropical regions fiurnish more than thirteen thousand species of plants. The palm, the baobab, the sugar-cane, \&c., are remarkable; while geraniums of every kind, myrtles, roses, and other plants, are as common as weeds.

Q. What are the PECULIARITIES of Arctic vegetation?

$A$. In the polar and frozen regions life seems almost extinguished by the rigorous cold of a perpetual winter. A colourless and stunted regetation, a few creeping shrubs, and endless plains covered with mosses and lichens, are alone beheld.

In the order of nature, and at the first approach, we cannot deny to the tropical regions a marked superiority. The most powerful spring of physical life, the most active source, that which surpasses all others, is the heat of that life-giving orb which the aucient nations, forgetting God, the only true Creator of all things, adored as the parent of Nature. But, in virtue of the spherical form of the earth, each district of the surface receives an unequal portion. Slanting, scattered, and feeble in the regions near the poles, but assuming more strength and falling thicker in the middle regions, in those of the equator only they gain all their intensity, all their splendour 
Q. What are the CHARACTERISTICS with respect to vegetation of the temperate regions?

$A$. Those countries which approach nearest to the torrid zone have a climate which in many particulars resembles it; as you advance farther and farther north it becomes colder; those countries which lie near to the polar circle are almost as cold as those within it. But the peculiarities of the temperate regions are seen in evergreen woods, in rich grassy meadows, in varieties of trees, and many kinds of grain and fruit.

$$
\text { F } 00 \text { D P L A N T S. }
$$

Q. What is meanl by FOOD PLANTS, and what are their PRINCIPAI VARIETIES?

$A$. Food plants are those plants which form the vegetable part of the food of man; they are barley, oats, rye, wheat, millet, olive, date, palm, banana, rice, maize, potatoes, arrow-root, sugar, coffee, tea, wine, cocoa, \&c.

Q. Are these food plants WIDELY DISTRIBUTED?

$A$. They are; the staple food of one country differing very widely from that of another. Barley is one of the most widely spread, bein:

Fig. 276.

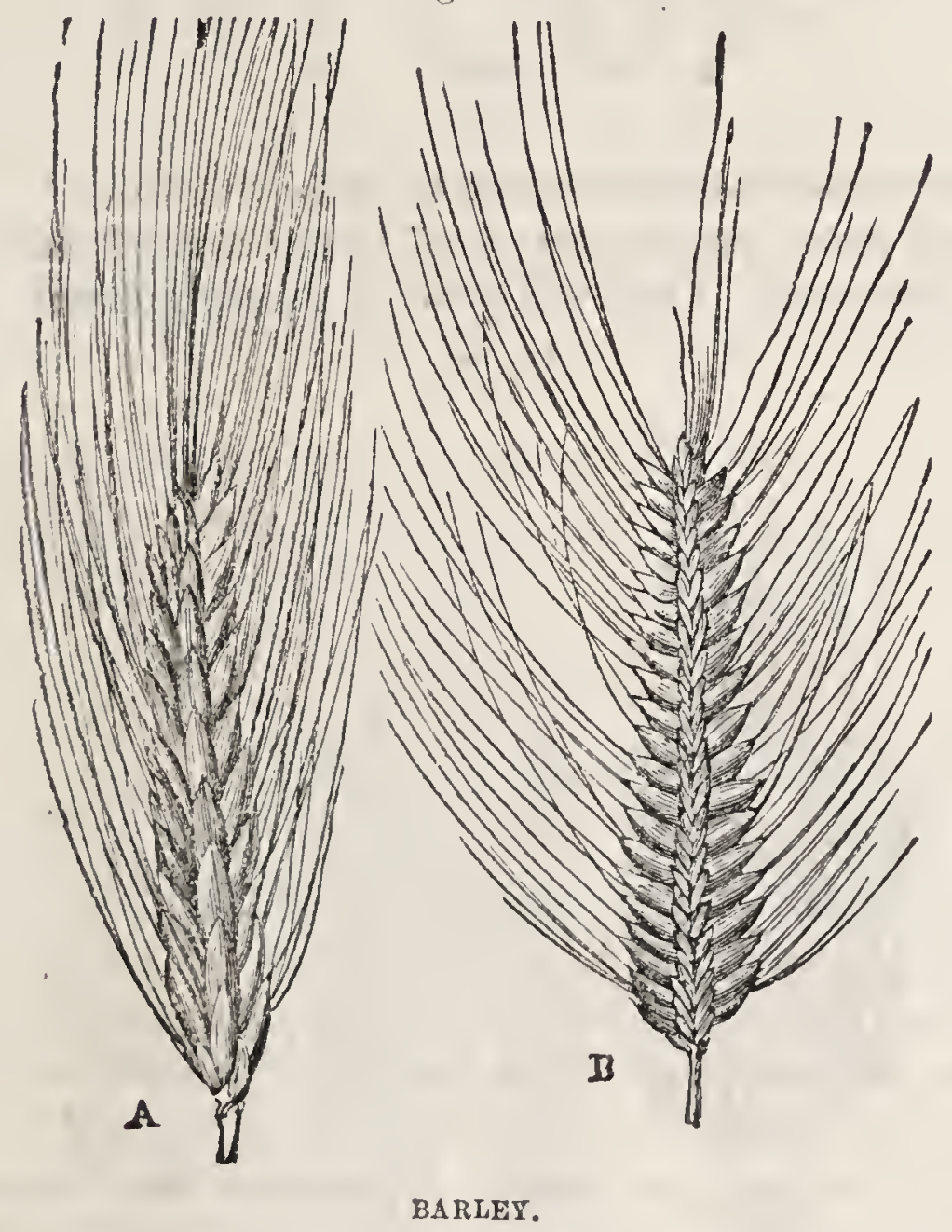

cultivated from the limits of Lapland to the herghts beneath the equator. In the East barley is the staple grain for making bread.

In the above illustration (fig. 276), A represents the common barley, B the fan barley. 


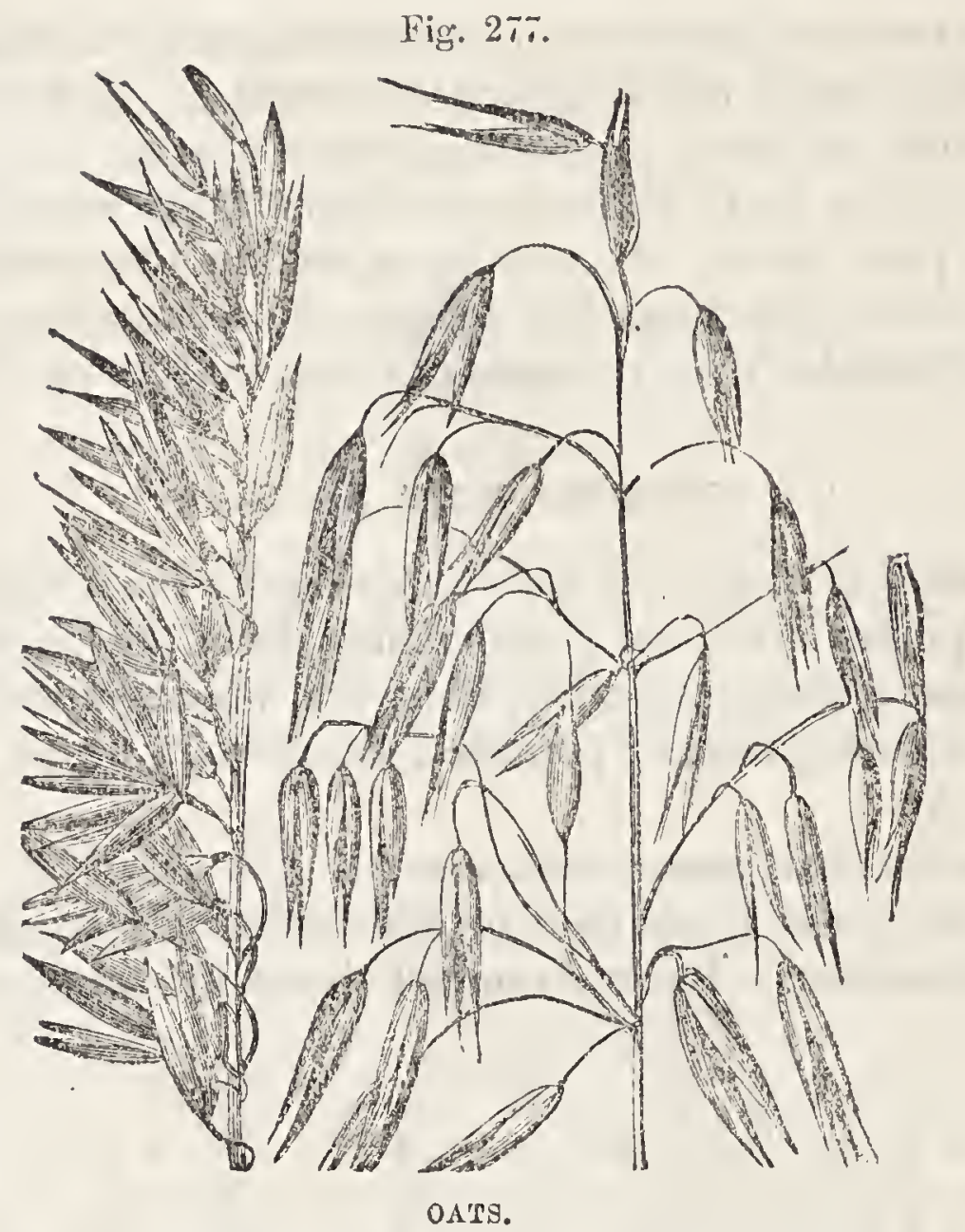

Q. What GRAIN is employed as BREAD CORN in the north of Europe?

A. Rye and oats form the principal bread corn of Russia, Finfand, Norway, and Sweden. Oats will grow in soils which will bear neither

T:₹. 278.

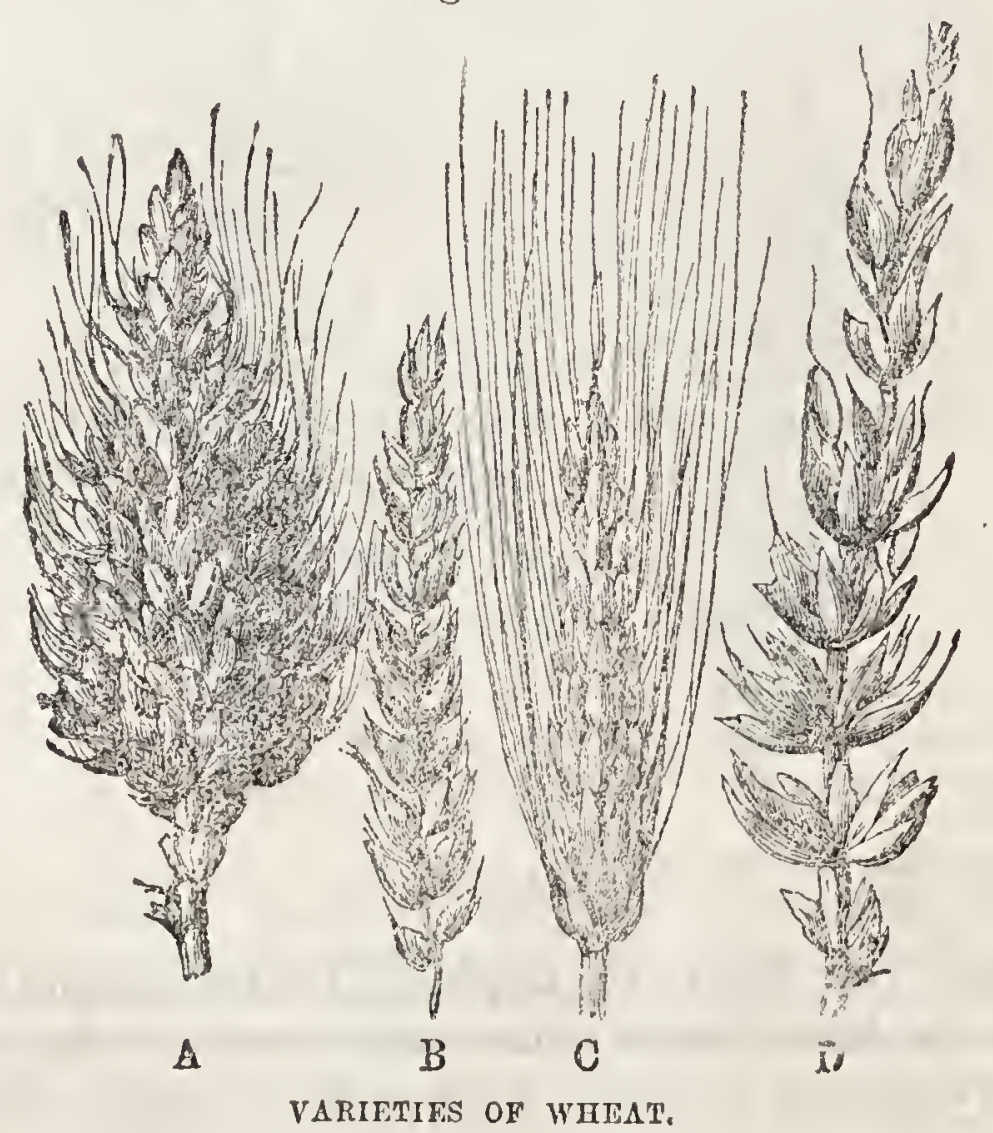

A, the giant species; $B$, the common winter wheat $C$, the bearded wheat; $D$, the Talavera wheat. 
wheat nor barley, and in situations not adapted to other grain. In the hilly districts of Scotland and Derbyshire it is almost the only grain cullivated.

\section{Q. Where is wheat PrinctPaLIY orown?}

A. Wheat is cultivated extensively throughout Great Britann, France, Germany, and towards the East; it is also cultivated to a great extent in North America. It flourishes not only in our temperate climate, but in the extremities of heat and cold. It is found in Lapland, and was seen by Humboldt, under a burning sun, growing with coffee, plantains, and sugar-canes. It is the plant most necessary to mankind, and, therefore, most widely diffused.

Q. What is MAIzi ?

A. Maize, or Indian corn, known also by the name of Turkey corn, is the largest and handsomest of all the grasses cultivated for food. When full grown, it attains a height of from five to six feet; while its broad leaves, springing from its thick, straight stem, and its elegant spike of flowers at the surnmit, present a form which is rarely surpassed. Next to rice, it supplies food to the greatest number of the human race. It forms the staple crop in North America, where the farmers make it answer a great number of purposes besides supplying their families with brearl. It is also cultivated in Mexico, and, to a limited extent, in Germany and France.

Q. Is rice EXTENSIVELY CULTIVATED?

A. Yes: it is the staple food of millions of people in the East, including those of the Indian Peninsula, China, Japan, and the East Indies.

Q. What is the MOST USEFUl FOOD PLANT of the tropical regions?

$A$. The palm tree. It yields the cocoa-nut, the date, oil, sago, sugar, and a juice which is easily converted into wine; while its leaves, if boiled, form an excellent substitute for a cablage.

Palm trees are now found growing native in Europe, Asia, Africa, America, and Australia; but, with the exception of two dwat species, the chamcerops humilis, in Europe, and the chamorops palmetta, in North America, they are all denizens of tropical lands, and their region may be considered as bounded by the thirty-fifth degree of morthern, and the fortieth of southern latitude. Nevertheless, one species at least, the date-palm, lias been so far naturalised in certain localities of Southern Europe, especially Andalusia and Valencia, that it grows to maturity and produces fruit-though far inferior to the dates of Africa. The greatest authority on palm trees is Herl Von Martius, a German botanist, who, with a view of studying their characteristics, devoted three years to a travelling excursion in Brazil-a region more rich in palms than any on the face of the globe. This botanist considers that there are existing at this time upwards of a thousand species of palms. If the opinion be correct, future botanical explorers have a rich field of investigation yet untrodden, inasmuch as no more than 175 species have yet been individualised and described : of these, 119 belong to South America, 42 to India, and 14 to Africa. It is a very curions fact, not satisfactorily accounted fur, that the cocoa-nut palm will not flourish at any great distance from the sea: hence, islands are best adapted to their culture, and in Central Africa there are none. Botanists are inclined to refer this predilection for the sea-shore to the tendency which these trees have to take up salt; and the idea is partly confirmed by the known fact of their partiality, if the tcrm may be allowed, for alkaline food. 
Q. What is the BANANA, Or PLANTAIN ?

$A$. This plant, a native of Southern Asia, is now generally found in all tropical climates. The fruit is very valuable, and at the same time economical. The saine space which will bear five hundred pounds of potatoes will bear twenty-two thousand pounds of bananas.

Q. Mention SOME OTHER FOOD PLANTS, and the countries to which they belong.

A. The bread-fruit tree is found among the Friendly and Carolina Islands; the yam is cultivated throughout the inter-tropical zone of the Old and New Worlds; the potato is a native of Chili, and was introduced into Britain by Sir Walter Raleigh, in the reign of Queen Elizabeth; the sugar-cane extends all over the regions of the torrid zone; coffee is a native of Africa, and was introduced from Ethiopia to Arabia and the West India Islands; tea is cultivated throughout China and Japan.

Q. Are not some species of plants confined to VERY LIMITED DISTRICTS?

A. They are: as, for instance, the Irish yew, which appears to grow naturally nowhere but in Ireland. The Scotch fir, on the contrary, is met with in various other regions having a similar climate.

Q. Does not the variation in vegetation agree with the degree of latitude in one direction, and the elevation above the sea level in the other?

$A$. It does. The natural productions of any given latitude bear a striking resemblance to that at a given height above the sea level. Thus polar vegetation and that of lofty mountains is almost the same.

"Changes in vegetation, similar to what are found in passing from the equator to the poles, occur from the base to the summit of mountains, which is strikingly exhibited in Etna, the Peak of Teneriffe, the Alps, Pyrenees, Andes, and Himalaya mountains. Each plant has its limit in elevation, as in latitude. On Teneriffe Humboldt found vegetation in about five zones; the region of vines, from the shore to an elevation of about 640 yards ; the region of laurels ; the region of pines, from 1920 to 2770 yards ; then follows a zone characterised by a species of broom; then the region of the grasses. Above these are a few cryptogamic (flowerless) plants."-Reid.

Q. How are the VARIETIES of VEGETATION distributed over the wide expanse of the globe to be ACCOUNTED FOR?

$A$. Humboldt makes the geography of plants part of the theory of the earth; and, including the whole in one intelligent glance, shows that the distribution of plants on a larger scale, as well as on a smaller scale, depends on the physical qualities of our earth.

\section{CHAPTER LXXIII.}

\section{GEOGRAPHICAL WISTRIBUTION OF ANIMALS.}

Q. What is the OBJECT of ZOOLOGICAL GEOGRAPHY ?

A. Zoological geography treats of the distribution of animals, as they at present exist, as well as the varieties of animal life considered in relation to external circumstances. 
Q. In what does it DIFFER from zooLOGY ?

$A$. Zoology describes the animals of our world in their distinctive characters; zoologic geography teaches their place on our world, the particular species which are common to particular countries, and shows us that they, as well as plants, have their natural stations and habitations.

Q. What is meant by the term FAUNA ?

$A$. The term fauna is applied to all species of animal life-both of land and water-which belong to a particular country or district. Such a collection is called the fauna of the country, as the plants peculiar to that country are called its flora.

Q. What PRINCIPAL DIVISIONs are usually made in describing the distribution of animal life?

$A$. The fauna are distributed into three great divisions: the temperate, arctic, and tropical.

Q. Does the ANIMAL LIFE of any country, or district, depend on the VEGETABLE PRoDucTioss of that district or country?

$A$. It does to a certain extent; but to a certain extent onty. The vegetable productions are mainly dependent on climate; the animal life is less dependent on it, as the fauna of any locality includes not only the terrestrial animals, but those of the sea.

Q. Has not the FOod of animals an IMPORTANT BEARING on their distribution?

$A$. The herbivorous (herb-eating) animals are naturally confined to those localities where such regetation is abundant. The carnivorous (flesh-eating) animals have a wider distribution, as they can find their food in all parts. The same remarks apply to birds of prey and to grainivorous (grain-eating) birds.

Q. The ocean being unIFons in its nature, are not the animals which dwell there all of ONE CLASS, or all classes in all places?

A. No. The animal life of the ocean is distributed into particular localities, as much as are the terrestrial animals or the vegetation of the different zones. In order rightly to understand this, we must remember that the coast line of the earth, and not the open sea, is to be considered in the position of local fauna.

Q. Does the DEPTH of the water affect the distribution of AQUATIC ANIMALS?

A. Yes; the fishes generally found near the surface differ from those which live at a considerable depth. Their colour, in particular, is obviously different.

Q. Are not SOME ANIMALS found only in ONE LOCALITT, while others are distributed in GRo UPS in similar climates?

A. Yes; as the Irish yew tree belongs essentially to the flora of Ireland, so the kangaroo is peculiar to Australia.

Q. Do the animals which are found at very great distances apart bear an EQUAL DISProportion in form and character?

A. No; not as a rule. Very similar faunas are often very widely separated. Very similar faunas are found in Europe and in the United States of America. 
"Animals are endored with instincts and facilities corresponding to the physical character of the countries they inbabit, and which would be of no service to them under other circunstances. The monkey, which is a frugivorous animal, is organised for living in the trees, from which he obtains his food. The reindeer, on the contrary, whose food cunsists of lichens, lives in cold regions. The latter would be quite out of place in the torrid zone, and the morkey would perish with hunger in the polar regions. Animals which store up provisions are all peculiar to temperate, or cold climates. Their instincts would be uncalled for in tropical regions, where the regetation presents the herbivora witly an abundant supply of food at all tines."-Hughes.

Q. What are the CHARACTHISTICS of the FAUNA of the TEMPERATE REgions?

$A$. The temperate regions abound with terrestrial animals, which, if less in size and brilliancy of appearance than those of the tropics, are nevertheless infinitely superior in form, life, and colour to those of the arctic zones. There is a great similarity existing between the faun of the temperate regions, both in the northern and southern hemispheres. Of these we may mention the horse, deer, ox, hoy, squiriel, hare; many of the birds are identical ; and among fishes may be counted, trout, sturgeon, pike, cod, maclierel, herring, \&c.

Q. Are there not animals PFCuliar to America which are not found in Europe?

A. Yes; in the temperate regions of America are found the opossum, some species of moles, and the species of tortoise known as the snapping turtle.

Q. What species are PECULIAR to the temperate regions of Europe?

A. The wild boar and the mouse.

Q. Do not consmerabie CHANGes take place in the fauna of the temperate regions?

A. The variations of climate produce many apparent chunges in the animal world, especially among insects and some species of birds.

Q. Are there not sone specres of animals which are found extending from one extreme of the temperate zome to the other in both the American and European continents?

A. Such is the case, as we find different kinds of birds of prey, with the jlying squirrel, the deer, the bison, \&c.

Q. What are the CHIEF peculis pities of the animals of the tropics

A. They are distinguished by their numerous variety and brilliant coloniving.

Q. Does this variety and beauty extend to $\Delta$ LL CLASSES of animal life?

A. Yes; both terrestrial and aquatic animals, birds and insects, are remarkable for an increased size and brilliancy.

Q. Neution some of these animals.

A. In the tropical regions are the largest species of montrys, the elephant, himpopotamus, chiropotamns, giraffe; the largest of the cat tribe, such as the lion, tiger, \&c. Crocodiles and huge tortoises are counted amongst its reptiles; parrots and macaws, amongst its birds. Above three hundred species of humminy birds, and islands covered with coral reefs fomed by the polymi.

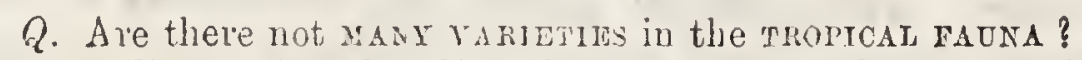

A. The aninals ristributed over what are known as the tropical regions vary in diflerent localities: thus, in Braril we find an im- 
mense number of insects and enormous reptiles, with many peculiar varieties of humming birds.

Q. What are the CHIFF CFIIIACTiRISTICS of the animals belonging to the AReTre or POLAR REgIONS?

A. Their principal and most prominent characteristic is $u n i$ formity. The species are few, but the individuals of each species are very numerous: this is especially remarkable with the birds and fishes. Among the former are a large number of gulls, cormorants, petrels, and other aquatic birds; among the latter is the salmon. The mammaila consist of the white bear, the moose, the rein-deer, the musk-ox, seals, lemming, and the whale: the whale, indeed, is the most important.

\section{T H E A CES O F M A N}

Q. While animal and vegetable life, of a peculiar and distinctive character, marks the different localities of the globe, is not MAN the SAME in every counti'y and climate?

A. The olganisation of animal and vegetable life is adapted to particular and distinct climates and country; but man, the chief being in this world-the lord of creation,-has physical capacities which enable him to dwell in every climate, soil, and situation.

Q. Is there, then, No VARIETY in the Human Race?

A. On the contrary, there are very great varieties in the human family, although it is perfectly identical, and in every important particular essentially the same.

Q. Has this identity of the human race erer been Drsputwo ?

A. Notwithstanding the direct testimony of Scripture that we are the offspring of common parents, it has been asserted that some of the people of uncivilised countries are so low in the scale of intellect as to be really an inferior race. Such has been said of the Negro and native Australian.

Q. Is there ANY Truth in such an assertion?

A. None whatever. Man is the same everywhere, and only requires the facilities afforded by civilisation and refinement to develop tinse faculties the non-existence of which has been so boldly asserted.

Q. How is the human family Jistracisnev?

1. Naturalists commonly divide it into races.

"The expressiun races, however, applied to man, involves something abhorrent from his high urlifted spirit, and debasing to his native dignity. This diversity of races amonis men no one vumbt to cxagserate in a manner so as to raise dovilits as to the idientity of their origin; for, according to a general organic law, which, indees, is allowed to fuld geri in the natural history of animals, races canable of a prolific union nust be cont. sidercd of the :anse origin, and, in constintion, the same species. Even the aprorent critus of differcut langnages may ie classed in to kindred families, which, though se araned

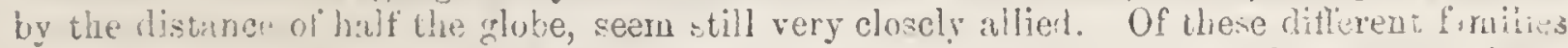
of tonesnes, the first and nost eminent are thise which, by their internal betuly, and the noble spirit hreathing through them, and apparent in their whole construction, denote, fur the most part, a higher origin, and divine inspiration; and, much as all the langunire 3

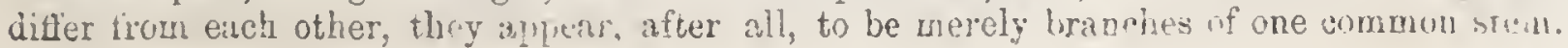


When man had once fallen from virtue, no determinate limit conld be assigned to his degradiation, nor how far he might descend by degrees, and approximate even tu the level uf the brute."-Schlegel.

Q. Into How MANY RACES has the human family been divided?

A. The three leading divisions are the Caucasian, the Mongotian, and Ethiopian, to these may be added the Malayan and American.

Fig. 279.

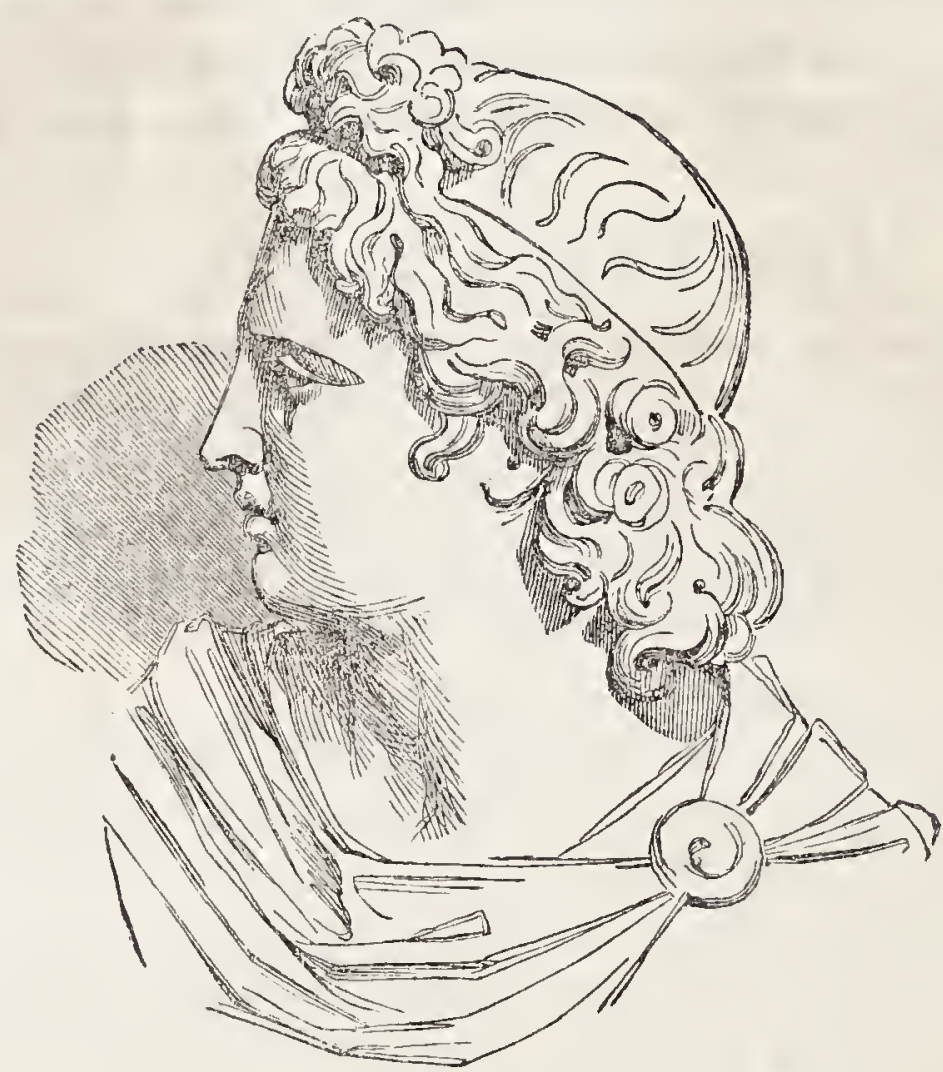

THE CAUCASIAN RACE : APOLLO BELVEDERP.

Q. What nations are comprised under the CAUCASTAN RACE?

A. It includes the European nations, with the exception of the Lapps and Finns. It also occupies some part of Africa and Asia.

This class is, indeed, very extensive, including the ancient and modern Furopeans and Western Asiatics, such as the Assyrians, Babylonians, Medes, Persians, Scythians, Parthians, Arabs, Jews, Syrians, Turks, Affghans, and Hindoos. To these must also be added the European cilonists who have settled in America and other parts of the world. Our engraving (fig. 279) shows the finest specimen of the race, taken from the ancient sculpture of the Apollo Belvedere.

Q. What are the DISTINGUISHING CHARACTERISTICS of the race?

A. The complexion is usually fair ; the face oval; the forehead broad; the hair of various colours, soft and flowing, or slightly curled; the eyes varying in colour; the nose rather thin and straight; the mouth rather small; and the chin full and round. The whole figure and aspect of the race is handsome and agreeable.

Q. What are the CHARACTERISTICS of the Mongolian race?

A. The head is squarer than that of the Caucasian; the nose flat, with distended nostrils; the eyes drawn up at the outer cornels; the 
cheek bones projecting; the complexion of a yellowish, dirty hue; and the height of the figure rather shorter than that of the European.

Fig. 280.

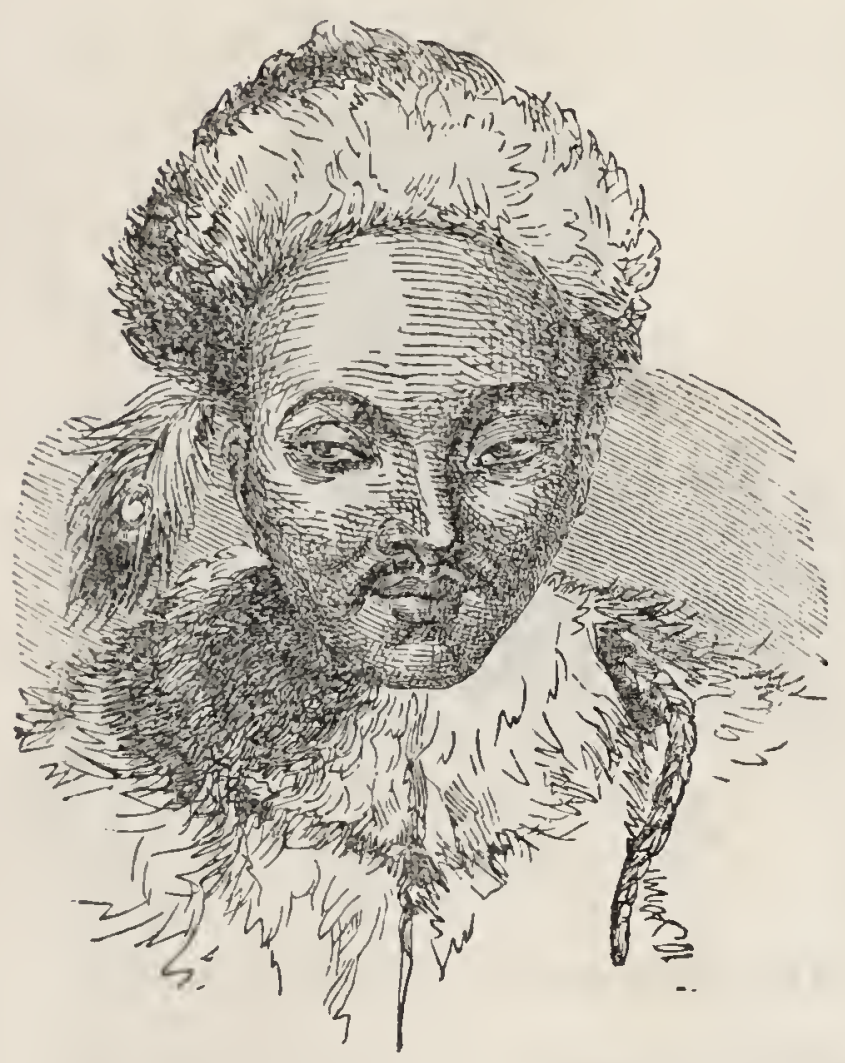

THE MIONGOLIAN RACE : A CHINFSE.
Fig. 281.

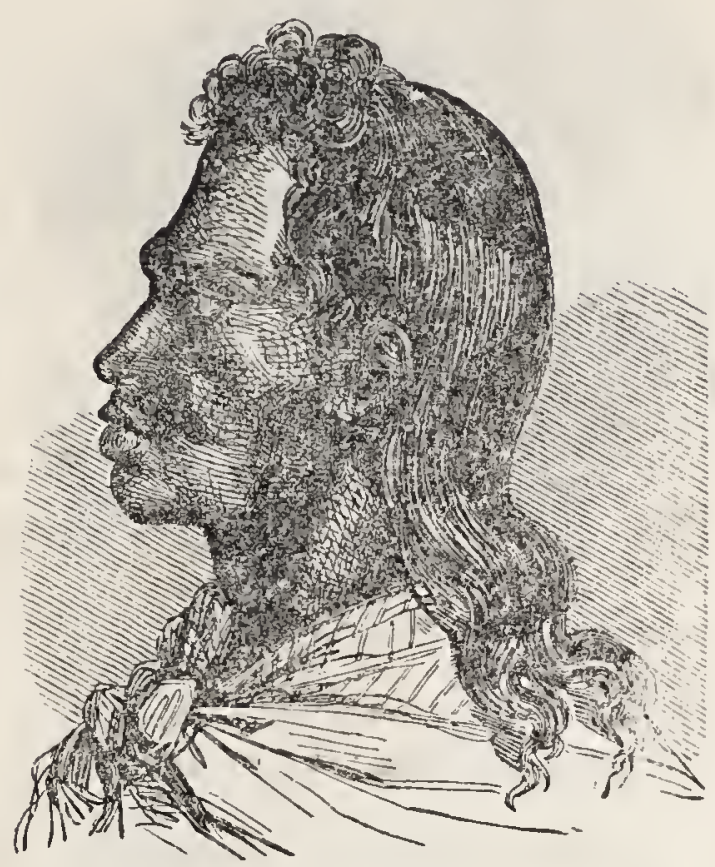

THE MALAYAN RACE : A TAHITIN.

Q. Over what countries does this race extend?

A. It includes the Lapps and the Finns, with some of the Hungarians in Europe; all the Asiatics north and east of the Caspian Sea ; the Chinese and Japanese; and the Esquimaux who reside on the shores of the polar sea, in America and Greenland.

Q. What are the PHYSTCal PECULIarities of the. Malayan race?

$A$. The Malayan race has the upper part of the head slightly narrowed; the forehead arched; the face narrower, and the features more prominent than those of the Negro; the complexion brown; and the hair black and abundant.

Q. What DISTRICTS are included in this division?

A. The islands of the Indian and Pacific Oceans.

Q. What are the characteristics of the natrve Amerroan races?

A. They approach in many respects those of the Mongolian race. The face is broad; the features prominent; the skin red, or copper colour; the hair dark. 'This division includes the whole of the native American tribes.

Q. Has not the maturat separation of the continent of America into two parts AFFHCTED the DISTRIBUTYON of the race?

A. It has led to the subdivision of the Annerican race accortling to their position in the North American nations; among which are 
the Iroquois, the Cherokees, the Sioux, the Columbians, and the Californians; and the South American nations, including the 1 eruvians, the Patagonians, the Caribbees, \& c.

Fig. 282.

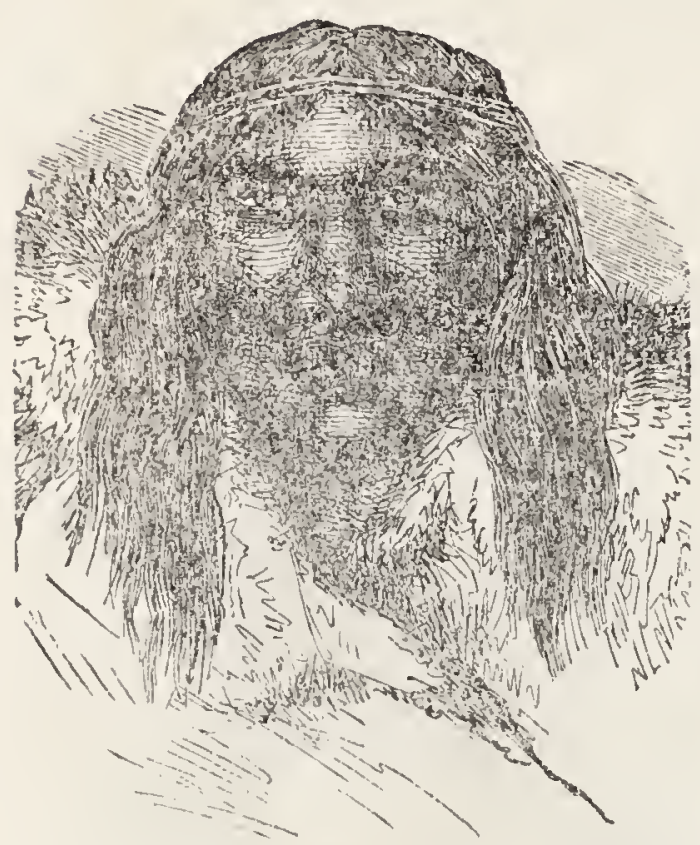

THE AMEPICAN RACE : A PATAGONIAN.
Fir. 283.

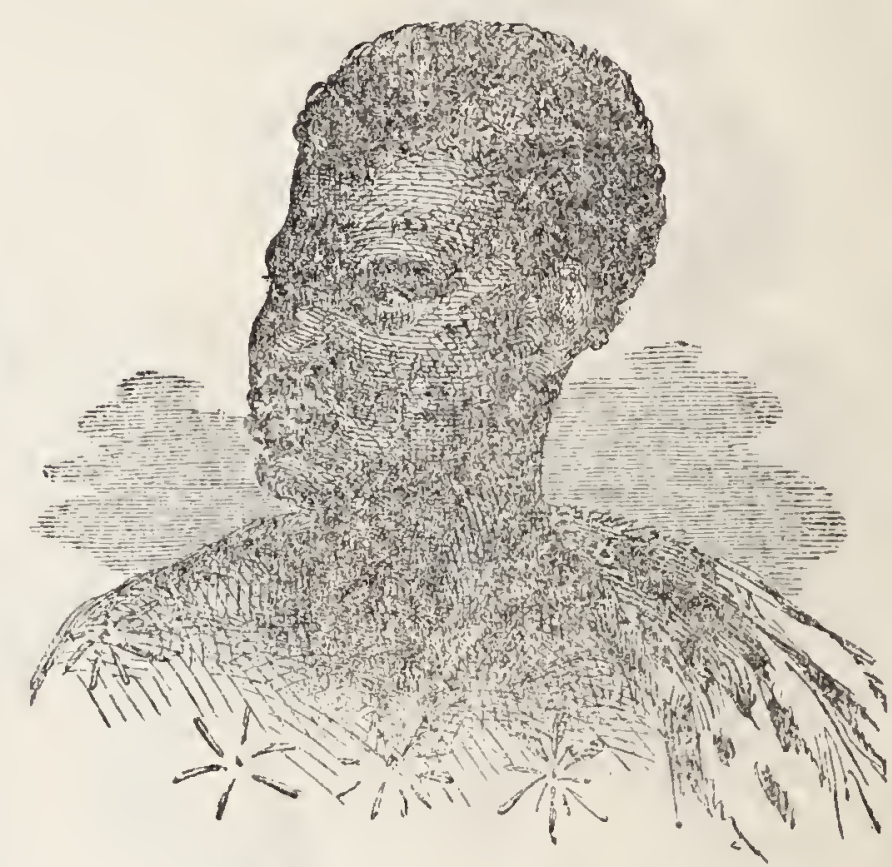

THE LCHOPIAN RACL : A NATVE OH MOZAMLIQUE.

Q. What are the cedRAorenistros of the Ethiopian race?

A. A black skin, black woolly hair, low forehead, projecting jaws, thick lips, and a retreating chin. The type of the race is fully seen in the Negro. T'o the Ethiopian race are connected the Hottentots and Kaffirs. To the same class are referred the Negroes of Oceanica, who are distinguished from the African race in several particulars, and who, under the name of Papouas, are the native inhabitants of Australia, New Caledonia, Van Diemen's Land, New Guinea, \&c.

Q. What GREAT FAOT is established by a CLOSE ExaMINATION of the DISTnotive PECULARETES of the races of man?

A. That, notwithstanding all the variations of colour and appearance which are observable in the different races of man, the most positive identity exists among them all, and that GOD HATI MADE TF ONE LLOOD ALL NATIONS OF MEN. 


\section{N D E X.}

Arsolute humidity, 213

Absorption and conduction, 123 of heat, examples of, 123,150

Accidental colour, 248

Acetic acid, 391

Acid, every fat contains an, 408

Acids, 306

dissolve, 136

produced from plants, 391

Aconitine, 391

Acoustics, science of, 73

Acorn, 444

Actinism, what is, 261

Action and reaction equal, 30

Adhesion, definition of, 7

Adjective colour, 431

Aerolites, 223 ignite, why, 225

Affinity, 176

force of, 305

Agassiz, Prof., 472

Aggregation, state of, 5

Agriculture and rural economy, 439

Air, a bad conductor of heat, 103

a conductor of lightning, 279

always saturated, where, 213

atmospheric, 59

how heated, 110

how made cold, 186

of what composed, 185

chamber, 130

change in, how diminishes the heat of the body, 124

circulation of, through a bed at night, 174

colour of, 59

currents of, in a room, 169

density of, law regulating, 162

influence on evaporation, 152

more unif rm at night than by day, 79

does not radiate heat, 117

drawn towards the fire, 172

appears blue, 59

effect of rarefaction, 171

effects of cold on, 186

earth's motion on, 189

hent on, 186

elasticity of, 60

electrified, when, 275
Air exists in water, 313

expanded by heat, 128

expansion of, 128

heated, influence upon the human system, 127

Sir Joseph Banks's experiment, 1.2

hot as the human body, 125

how caused to escape from the lungs, 64

how heated, 110,185

impenetrable, 59

in an apple, 129

in cities less pure than in the country, 327

in contact with the earth, why colder, 115

in motion absorbs heat quickly, 124

in spring, why damp and cold, 95

in water, 61

its electricity varies, 275

motion of, tends to abstract heat, 110

moist, most trausparent, 203

not heated by sun's rays, 110

necessary for production of sound, 63,74

not invisible, 59

porous, 59

rarefied, 60,186

previous to a storm, 63

rarity of, on high mountains, 63

seeks an equilibrium, 191

refractive power of, 244

vibrations of, produce sound, 73

waves, nature of, 77

what composed, 185

weight of, 60

compared with water, 61

where of uniform temperature, 118

why quiet before a tempest, 79

Alabaster, 372

Albumen, 404

vegetable, 394

Alchemists, 307

Alcohol, 396

and ether dissolve oil, 175

and water readily unite, 305

boiling point, 93

Ale, porter, \&c., why froth, 67

Alkali, 306 
Alkali metils, 369

Alloy, 3067

Alluvium, 464

Altitude of shooting-stars, 224

Alum, $17 \%$

Aluminum, 373

Amber, 267 electric properties of, 267

Ambergris, 414

Amalgam, 368

Ammonia, 320

Amorphous bodies, 177

Amethyst, 524

Anemometer, 190

Angle of incidence, 236 of reflection, 236

Animal bodies, temperature of, 99, 100 heat, cause of, 352

life distinguished from vegetable, 404

life, development of, 500

odours, 414

organisation, 404

Animals and plants, distribution of, 100 peculiarities of, occasioned by temperature, 100

Arctic, have white fur, 125

flesh of old, tough, 405

living deep in the ocean, 61

what varieties possess the greatest muscular power, 41

Annealing, explanation of, 184

Annual plants, 452

Antimony, 289

Antiseptics, 403

Apple, why falls to the ground, 10

Aquatint, 437

Arago, his experiments, 91, 260

Arch, abutment or spring of, 52

application of, in the human structure, 52

strength of, 52

what is an, 51

when invented, 52

Architecture, definition of, 48

orders of, 52

origin of the different varieties of, 48 useful art $t, 54$.

definition of, 48

Area of a body, 2

Argand lainp, 343

Argillaceous rocks, 464

Arms and legs, levers of the third kind, 36

Arsenic, 385

poisoning with, 385

Arterial blood, 352

Artesian wells, 520, 521

Artificial diamonds, 325

light, 258

how obtained, 258

of what colours composed, 258

Artillery, effective distance of, 46

Arts, strength of materials used in, 47

Assafoetida, 416
Atmosphere, 185

charged witl electricity, 274,275

height of, 60

pressure of, $61,150,167$

renewal of, 146

thickness of, necessary, 247

weight of the whole, 61

Atmospheric air, elements of, 59,317 engine, 167

Atom, definition of, 2

Atomic c ndition of 'matter, Newton's belief, 2

Attraction between particles of bodies, 6 capillary, definition of, 22

cohesive, 6

definition of, 6

of the earth, law governing it, 8

Aurora borealis, 226

Aurora does not affect the weather, 228 not local, 226

where seen, 226

Auroras, appearance of, periodical, 226 more frequent in winter, 226

occur in daytime, 226

their use, 226

Avoirdupois weight, definition of, 16

Axles of railroad carriages, why liable to break from long-continued usage, 183

BACoNio, his voltaic pile, 284

Ball, distance thrown by a twenty-four pounder, 44

elastic, does not rebound from a pane of glass, 30

Balls, elastic, rebound when, 30

Ball fired from a cannon, why not for ever move, 8

Ball-lightning, 277

Balloon, how inflated, 130

Balloons, 130, 311 ascend, why, 10, 130

Balusters, definition of, 54

Barilla, 369

Barium, 371

Bark adapted as a covering for trees, 106

Barley, how converted into malt, 398

Barometer and thermometer, 72 cause of its changes, 196 effect of heat and cold on, 73 invention of, 70

mercury in tube of, convex, 24 rises in fair weather, 72

sinks in foul weather, 52

principle of, 70

tube left open, why, 72

rules for observing by, 71

use as a weather-gึlass, 70

for measuring heights, 70

on land, limited, 71

varies most, when, 72, 196

wheel, construction of, 71

Base in chemistry, 306

of a column, 56 
Base of support for the human body, 13, 15

Battery, Ealvanic, 253 ivoles of, 284

Beari. bent in the middle, liable to break, 48

Beasts, why corered with wool, hair, \&c., 104

Bed, circulation of air through the coverings of, 174

Bell, cracked, sound of, 78 , made to vibrate, how, 78 metal, 75,368

Bells henrd farther at one time than another, 78

Bellows, explanation of the action of, 65

Biennials, 452

Pirds do not fall from their perch when asleep, why, 36

feathers of pinions and tail strongest, 29

fly, why, 29

notes of, vary, 83

of prey see very far, 254

only one sex sings, 83

sing louder than man, 83

stretch out their necks in flying, 29

whistle rather than sing, 83

Bituminous, 497

Black absorbs heat, 125

lead, why used for blackening stovepipes, 113

not a colour, 248

Bladder, inflated sound produced by the bursting of, 87

Blankets and woollens made with a nap, 104

feel warm, why, 104

Blocks, pulleys on board ship, 38

Blood, circulation of, how affected by friction, 97

Blond-heat, definition of, 93

Blowers help to kindle a fire, 173

Blowing food cools it, 110

force exerted by the chest in, 84

Blowpipe, 311, 312

Blue, why sky appears, 248

Boats, life, how prevented from sinking, 21

Bodies, natural, classes of, 3

Body, area of, 2

cool, becomes heated, how, 101

definition of, 1

figure of, 2

heated, becomes cool, how, 101

hot and cold at the same time, 89

position in which it rests, 11

qualities or properties of, 1

size of, 2

voluine of, 2

Boiler, best form of, 162

Brilers, bursting of, accounted for, 163

liable to break, when, 137

of a liquid, what is, 147,148

of thermometer, 140
Boiling water bubbles, why, 148

swells, why, 147

Bomb-shells, construction of, 46

Bones hollow and cylindrical, 47

Book, water, oil, \&c., spilled unon, 24

Bottles, containing ale, cider, \&c., liable to burst, 67

gurgle when liquor is poured out, 67

Boulders, 464

Brandy, 398

Brass, 367

Brass wire, how liable to cliange, 183

Bread, 457

becomes stale, why, 151

not bake well in polished pans, 123

Breath visible in winter, 209

Breathing difficult on high mountains, 63

on glass dulls it, 209

operation of, 62

Bricks, children of Israel used straw in the manufacture of, why, 51

of Chicago, why yellow in colour, 51 red, when burned, 51

Brimstone, 356

Brine, for pickling, strength of, ascertainer, 20

Britannia metal, 368

Brittleness, definition of, 8

Bronze, 368

Broth cooled by blowing, 110

by convection, 110

by exposure to the air, 110

Bubbles, in a cup, follow the spoon, 10 in a cup of tea, 10 origin of, 4

Budding, 450

Building, essential parts of, 48

Bulb of plants, 452

Bullion, 384

Burning-point, 330

glass, 245

CABBAGR very nutritious, 407

Calcareous manures, 442

Calm in the vortex of a hurricane, 196

Calms, 196

Calomel, 383

Caloric, definition of, 90 distribution of, 90

Calyx, 455

Camera-obscura, low formed, 263

Campline, 411

Candle burns, how, 341

tallow, when fired from a gun pene. trates a board, 25

Candles, image of, reflected in the windows, 237

several reflections produced, how, 237

Cane-sugar, 390

Cannon, effect of continual firing, 183

Cannon, recoil of, 44

varieties of, 46

Capital of a column, 56 
Capstan, description of, 37

Capsular fibres, 418

Carbon and its compounds, 322

Carbonate of lime, 327

Carbonic acid, in the air, 326 chief sources of, 327

fatal to life, 326

gas, 325

of close rooms, how generated, 326 weight of, 330

Carbonic oxide, 329

Carboniferous or coal formation, 494

Carbonisation of wood, 157

Carburetted hydrogen, 345

Carcel, or mechanical lamp, 343

Carding, 427

Carnivorous animals do not perspire, 355

Carpets, Brussels and Wilton, 430

Carriage, high, liable to be overthrown, 13

Caseine, 406

Cask, phenomena of liquor flowing from, 65

Cast iron, 378

Cataract, cause of, 256

Cat, fur of, sparkles when rubbed, 270

Carerns, famous for echoes, 85

Caves, sound louder in, than on a plain, 80

Ceiling, limit of height for speaking, $86^{\circ}$

Cellars cool in summer, 106 warm in winter, 106

Cellular tissue, 445

Cements and mortars, composition of, 50

Cement, definition of, 50 hydraulic, definition of, 50

Centimetre, definition of, 18

Centrifugal force, definition of, 26

Chair, person rising from, bends over, 11

Chalybeate, 312

Champagne sparikles, why, 68

Charcoal, 322

black, why, 246

fire hotter than wnod fire, 323

Chemical action, a source of heat, 94

forces and agencies, 303

analysis, 308

attraction defined, 305

forces and agencies, 303

Chestnuts, crack when roasted, 129

Chimney-cowl, use of, 173

Chimneys, draught of, on what depends, 172

first used in England, 174

of manufactories, why made high, 173

raised high above a roof, 173

long, apt to smoke, 130

smoke, remedy for, 172

sometimes smoke, why, 172

Chinese discovered the properties of the magnet, 294

Chladni, liis theory, 226

Chloride of lime, 360

Chlorine, 360

Chocolate, 393
Choke-damp, 327

Cinders lighter than coals, 334

Circuit, galvanic, 281

Cirro-cnmulus clouds, 206

Cirro-stratus clouds, how produced, 206

Cirrus clouds, 204

Cleavage of a mineral, 525

Clock and watch, difference of, 32 conmon, 31 slower in summer than in winter, 138

Clothes, fine, warmer than coarse, 107

Clothing, loose, warmer than tight, 104 use of, in winter, 103

Clouds, 199

affect the wind, 189

around mountain peaks, 201

cirrus, 204

cirro-cumulus, 206

cumulus, how initater, 205

dissipated suddenly, 232

distance of, 200

electricity of, 210

float, why, 200

higher on a fine day, 199

illustration of, 238

modifications of, 200

motion of, affected by clectricity, 202

not always the same colour, 201

prevent the radiation of heat, 115 , 201

red in the moruing, 201

in the evening, 202

size of, cirro stratus, 206

cumulo-stratus, 207

stratus, how produced, 205

variety in, 200

Coal, 158

amount of, mined in Europe, 499

bituminous and anthracite, 497

fields of England and Wales, 493

floors and beds, 495

formation, classification of, 494

mines in Great Britain, 498

in America, 499

occurs in strata, 494

relative amounts of production, 499

shells and fossils, 497

sigillaria, 496

stigmaria, 497

varieties of, 322

vegetation of, 496

Cocoa butter, 393

Cocoas, 393

Cocoon, 426

Coffee, rule for roasting, 393

Coke, 323

Cold, greatest natural, 92

what is, 89

Colour, accidental, 248

complementary, 75

cause of, 247

definition of, 247

influence of, on radiation, 111 
Colour of animals in arctic regions, 250

Colours, effect of contrast between, 251

law of the halmony of, 251

of animals suited to their habits, 250

of plants aud animals on the globe, 250

optical effect of, 251

primary, 242

vegetable, by what rays of light most affected, 265

warmest for dresses, 125

Columns, Corinthian, 53

Doric, 52

Ionic, 53

Combustion, 230

respiration and nutrition, 330

Compasses, how constructed formerly, 343

Complexions, effect of colours on, 300

Composite order of architecture, 53

Compasition of aerolites, 223

Compost, 493

Compounds of nitrogen, 317

Compressibility, definition of, 3

heat produced by, 99

Compression, definition of, 99

Concave and convex, definition of, 292

Concave mirror, 275

Concord and discord, 82

Condensing steam-engine, 159, 160

Conduction of heat, 101

Conductor, lightning, influence of, 279 its use, 279

Conductors do not attract lightning, 280 how productive of harm, 273

of lightning, 279

pointed, 279

Conglomerate, 463

Constant winds, 190

Contact, absolute, non-existence of, in matter, 3

Convection, meaning of, 108, 185

Cooling, ]rw of, 109

Cooper, why heats his metal hoops before

Copper, 509 placing upon a tub or barrel, 136

and iron, why sonorous, 75

sheathing of vessels, decay of, 287

the best conductor, 279

Copperas, 379

Copperplate printing, 438

Cordials, 399

Cork, why, floats upon the surface of water, 10

Cornea, 253

Curnice, definition of, 56

Cornish steam-engine, 158

Corpuscular theory, 230, 249

Cortical fibres, 419

Cotton, 421

best adapted for lamp-wicks, 23

known to the ancient Indians, 422

staple of, 421

unknown to Jews and Egyptians, 422

Coughing, explanation of, \&4
Countries least cloudy, 200

most clondy, 200

Country air, 327

Courtois discovered iodine, 361

Cowl, its use on chimneys, 173

Cream rises to surfitce of milk, 21

Cretaceous formation, 492 system, 492

Crop out, strata said to, 461

Crops, rotation of, 371

Crown glass, 364

Crust of the earth, 458

Crying, explanation of, 84

Crystalline bodies, 177

Crystallisation, 134

develops heat, 181

influence of light on, 184

how most easily effected, 184

of sugar, 178

substances capable of, 178

Crystallising substances expand, 131

Crystallography, 527

anorthic, or double oblique, 531

common form of, 527

cubical, 528

definition of, 527

faces, edges, angles, axes, 527

how described, 527

irregularly formed, 527

oblique, 531

produced by artificial means, 527

pyramidical, 529

rhombic, 530

rhombohedral, 530

secondary forms of, 527

twin crystals, 527

various systems, 528

Crystals, 178

defined, 178

elements of, 178

faces of, 179

figures vary, 179

forms of, 177

how formed, 181

how used for distinguishing minerals, 178

of the same substances similar, 177

two classes of, 178

Cumulus clouds, 205

how imitated, 205

Cumulo-stratus clouds, 207

Cupping, operation of, 62

Currents of air, 186

of air from the poles, 186

Curtain, why placed behind a speaker, 86

Cyanogen, 321

Cylinder boiler, its adrantages, 159, 163

DAGUerreotype developed, how, 264

discovered, when, 263

furmed, how, 264

principle of, 264

taken in darkness, 265 
Darkness defined, 234

Dary's, Sir H., attempt to protect the sheathing of vessels, 281

Davy's lamp, 346

Decanter stopper sometimes stichs, 137

Decay, 305

a species of combustion, 305 of wood, 305

Deceptions of vision, 255

Decimetre, definition of, 18

Density and porosity, connection between, 3 definition of, 3

of air in rainy weather, 211

Deserts, their effect on winds, 192

Deviation of needle, 292

Devonian system, 471

Dew, abundance of, in the morning, 115 abundant on cultivated land, 116 and rain compared, 115 cannot be said to fall, 118 defined, 114

deposited freely, when, 115 on woollen cloth, 116

does not cover the exposed parts of our bodies, 120

Dr. Wells's theory of, 121

evening, injurious to health, 117

ground covered with, 114

in Polynesia, 121

influence of winds on, 117

on colours, 118

under a leafy tree, 115

more abundant in autumn, 116

most abundant, when, 115

copious after a hot day, 116 in tropical climates, 120

none on a windy night, 116

not deposited readily on polished metal; 116

not formed on the surface of water, 120

on ships, 120

point expliined, 119

produced at any time, how, 119

rare in large cities, 118

rarely deposited on rocks, 142

round, why, 119

Diamagnetic bodies, 297

Diamond, 322

brilliancy of, 243

properties of, 322

iuflammable, 241

largest known, 324

weight of, 324

Diamonds, where found, 324

Diluvial systems, 488

Diluvian or drift, 471

Diluvium, 464

Dip of rocks, 461

Dipping needle, 293

how affected at the magnetic pole, 294 position of, at the equator, 297

Directive juwer of the nagnet, 295
Distance, 257

Distillation and evaporation, 150

definition of, 149

simple, how affected, 150

Diurnal variation of the needle, 296

Diving bells, phenomena accompanying the descent in, 63

Divers, pearl, frequently deaf, 64

Dodo, 488

Dogs, why, shake their skins to free themselves from water, 27

Doors, double, use of, as a protection against cold, 105

Domble vision, how produced, 255

Dovetailing, definition of, 50

Draining, 456

land, how promotes warmth, 456

Draught, inwards, through the erevices of windows, 171

Dresses, white, why worn in summer, 123 not worn in winter, 123

Drift, 471

Drops, the difference of bulk in different liquids, 7

prescription of medicine by means of, unsafe, 7

Drowned persons, why does the body tloat after some days, 20

Drum produces sound, why, 82

Dry air does not exist, 154

Ductility, definition of, 8

Dust, how we free our clothes of, by agitation, 28

less rises at night, why, 115

Duty of an engine, 159

Dwellings warmed by steam, 155

Dyeing and callico-printing, 431

EAR TRUMPET, construction of, 87

musical, definition of, 81

tympanum or drum of, 73

Earth absorbs solar heat, 93

Earth, a spheroid, 467 attracted by a feather, 11

density of, 457

effect of an increased rapidity of its revolution, 26

effect of the rotation of, on the air, 189 extent of surface, 458

form of, 457

once fluid, 466

radiates heat, 115

when colder than the air, 115

when warmer than the air, 115

Earthen and porcelain vessels burst, when, 134

Earthenware, 377

Earthquake:, 482

and volcanoes, probable cause of, 94

Ebb and flow of sea, effects of, 135

Ebullition and evaporation, difference between, 147

explanation of the phenomena of, 147 
Echo, origin of, 85

situation of bodies for the production of, 85

not produced in an ordinary-sized room, 85

Echoes, remarkable, 85

Economical geology, 505

Effervescence, 329

Efflurescence, definition of, 180

Egg, difficult to break by pressing directly upon its ends, 52

floats in brine and not in water, cause of, 19

Eggs cannot be boiled on the top of very high mountains, 63

stale, why do they float, 21

Elasticity, 145

Electrical eel, 272

Electricity, 267

a source of heat, 94

accumulation of, 267

animal, 272

communication of, 269

conductors of, 269

connection with light and heat, 271

cools air, when, 270

current of, how generated, 267, 274

definition of, 267

developed, 267

Du Fay's theory, 269

electric asd non-electric, fallacy of, 269

electric properties of amber, 267

employed for measuring time, 303

experiment with pitlı ball, 268

Franklin's theory, 270

how excited, mechanical power, chemical action, \&c. 267

influence of the shape of a body in retaining, 269

invisible, 267

kinds of, 269

known to the ancients, 267

not the cause of all unexplained phenomena, 271

odour of, $26 \mathrm{~s}$

ordinary mode by friction, 267

positive and negative, plus and minus, 270

produced by change of state in bodies, 270

torpede, organs of, 271

velocity of transmission, 271

vitreous and resinous, 269

what is, 267

Electrical machines, 272

Nairne's, 273

Otto de Gucricke's, 272

Ramsden's, 272

Winkler's, 272

Electric telegraphs, 300

cliemical (bian's), 302

dial, 300
Electric telegraphs, invention of, 300

signal, 301

varieties of, 300

Wheatstone's discoveries in, 300

writing, 301

Electro-magnetism, 297

definition of, 297

phenomena connected with, 297

Professor Henry's experiment with, 293

Moll's experiments with, 298

Faraday's experiments with, 298

Electrometer, what is, 274

Electrotyping, 288

principles of, 288

process of, 288

Elements, four, of the ancients, 307

Endogenous plants, 419

Engine, atmospheric, 167

condensing, 168

expansive, 167

low-pressure, 167 .

non-condensing, 168

Engraving and lithography, 433

Entablature in architecture, 56

Equator, average temperature at, 93

Fquinoctial storm, 228

Etching, 436

Evaporation, 150, 208

effects of, 150

how carried on and produced, 150

how productive of cold, 153

not confined to the surface of the earth, 153

spontaneous, 146

Evening star appears near, 257

how accommodates itself to darkness and light, 237

how caused to contract by intense light, 237

when struck, we see light, 253

parts of, 252

pupil of, what is, 252

why pained by sudden light, 271

Eyes of flies, 254

two, use of, 255

Eyelashes, use of, 254

Eyebrows, use of, 254

Eyelid, 254

Expansion, 128

of solids, liquids, and gases, 131

ratio of, 132

Expansive engine, 163

Explosive sulsstances, 45

Exogenous plants, 4.19

Exogenous tree, agc of, 420

Explosions of boilers explained, 163

FAgAdE of a building, 55

Falling bodies, rule for calculating the height, 31

Fanning cools the face, 124

increases the heat of the air, 124

Fan, use of, dces not cool the air, 124 
Faraday's electric theory, 297

his arrangement of conductors, 281

his observations on magnetism, 298

Fahrenheit's thermometer, 140

Fast colours, 432

Fat and oil, difference between, 409

people throw back their heads in walk-

Fats, 408

$$
\text { ing, } 13
$$

and oils float on water, 411

of vegetables, 410

used for greasing leather, 410

Fauna and flora of the oolitic period, 503

Feather, falling, attracts the earth, 11

Feet, why often feel cold near a good fire, 111

Feldspar, 375

Felting of wool, 425

Ferment, 395

Fermentation and putrefaction defined, 394

Fermentation, vinous, 396

Fibre of cotton magnified, 421

Fibres, capsular, 421

cortical, 419

foliaceous, 419

of flax and hemp, 422

Fibrine, 406

Fibrous substances, nature of, 421

Figure of a body, 2

Filtration dependent on porosity, 4

Fine silver, 305

Fire burns well in frosty weather, 334

covered, why, 334

damp, 345

effect of, to produce wind, 335

explanation of, 90

fly, light of, how produced, 258

intensity of, 334

places, 171

produced by friction, 97

Fires placed near the floor of a room, 111

Fishes enabled to ascend to the surface of water, 20

unable to live in water deprived of air, 67

Flame, 331

definition of, 90

hottest part of, 341

not equally luminous, $3 \div 0$

yellow, 340

Flannel keeps us warm, 353

preservation of ice, 104

Flax and hemp, fibres of, 422

have no staple, 422

preparation of, 422

Flea, relative strength of, 41

Flies, enabled to walk upon ceilings, \&c., 64

Flint and steel, production of sparks by, 97 sparks in a vacuum, 97 glass, 364

Flowers, how propagated, 452

Flue boiler, construction of, 163

Flues, use of black-lead in blackening, 113
Fluids and solids, difference in the pressure of, 57

effect of heat on, 132

phenomena of, 57

pressure of, 57

surface of, at rest always level, 57

Fluorine never been fuund pure, 362

Flux, 366

Flying, difference of, from leaping, 29

Fly-wheel, advantage of, 164

Focus of light, what is, 245

Fog and mist, their difference, 209

cause of, 204, 208

does not become dew, why, 209

Fogs, 210

more general in autumn, 210

occur at night, when, 209

over the banks of Newfoundland, 211

over shoals and sandbanks, 211

round capes and headlands, 210

Food, 349

more required in cold weather, 354

Force and impetus of a body, on what depends, 9,30

defined, 6

production and application of, 34

Forces, external and internal, 6

Forked lightning, 276

Form of aerolites, 224

of the earth, 457

Forins of aurora, 2.6

Fossil, 469

Fossiliferous rocks, 471

Fossils, how preserved, 470

of upper silurian rocks, 500

Franklin, electric theory of, 270

his discoveries, 279

Franklin's experiment, 279 kite, 279

Freestone, 5, 8

Fresh air, how much required in a room per hour, 326

Friction, definition of, 8,97

heat produced by, 97

influence of, in exciting electricity, 267 matches, 98

Frieze, definition of, in architecture, 53

Frogs and fishes cold-blooded, 353

Frost and snow, crystals of, 181

Frostwork figures, always limited by certain angles, 180

on windows, origin of, 180

Frosty weather, less chilly than a thaw, 136

Frozen dew, 222 fog, 222

Fruit-trees, propagation of, 450

Fruits, hard when unripe, 389 juices of, yield syrup, why, 390

Fuel, 332

burns, why, 309

Fuller's earth, 374

Funnel of a chimney, definition of, 172 
GaLLerY of a public building, why the hottest place, 171

Gallic acid, 379

Gallon, standard, how obtained, 17

Gitl vani, 282 his observations on electricity, 283

Ga!vauic action influences chemical change, 285

pattery, 83

circuit, 284

current, how excited, 283

electricity, 282

trough pile, 285

voltaic pile, 285

Galvanism, 282

Gamut, definition of, 82

Garden, best situation for, 440

Gardening, 439

Garien walks, how laid out, 440

Garlic, odour of, 416

Gas, definition of, 5, 185

difference of, from a liquid, 5

light, 344

produned, how, 345

Gaseous bodies, varieties in nature, 58

Gases and liquids, difference between, 5, 58 expanded by heat, 128

Gasometers, construction and principle of,

Gelatine, 406

$$
65,66,364
$$

Gems, 183 small, more perfect than large, 183

Geological changes, 500

Geology, 457

changes, how effected, 459

changes in, existing and recent, 473

definition of, 457

derivation of the word, 457

economical, 505

metals, 506

mines, 511

first gieat fact of, 459

formation of, 471

of boiling springs, 480

of earthquakes, 481

of stratified rocks, 489

of submarine eruptions, 486

of the origin of caverns, 487

of volcanic craters, 475

rocks in, 460

scientific division of, 457

what it teaches respecting the origin of tive earth, 465

German silver, 367

Giant's Causeway, 462

Glacier, definition of, 187, 220

Glaciers, increase of, 187

Glass, 364

and earthen vessels break, when, 136, 184

chimneys of lamps, 138, 332

crown, 364

fint, 364
Glass, plate, 364

reflector, 248

Glasses, burning, why ignite substances, 97

Glaze, 376

Globular lightning, 377

Glottis, definition of, 83

Glowworms and fireflies, why shine in the night only, 258

Glue, gum, and starch, why adhesive, 151

Gold, 384

large masses of pure, 384

leaf, 334

not affected by the atmosphere, 310

proof of the porosity of, 4

solvent for, 384

value of, 384

Gorges, how formed, 487

Gothic order of architecture, origin of, 54 peculiarities of, 54

two species of, 54

Governor of steam-engine, 165

Graduation of thermometers, 140

Grafting, 450

Grain weight, definition of, 16 how made, 16

Gramme weight, definition of, 18

Granite, 175

Grape-sugar, 389

Graphite, 322

Gravity, 10

centre of, 11

centre of, in irregular-shape-bodies, 13

Gravitation, definition of, 10

common property of all bodies, 10

Grass devoid of colour, when, 249

snow, 219

Grindstones in rapid motion, why liable to burst, 26

Ground colder on a clear than cloudy night, 115

covered with dew, 114

ice, 133

surface of, hardened by the sun, 186

Growth of plants, 494

Gum, 445

Gun, recoil of, when loaded with fine shot, 44

Gun-cotton, 45

essential properties of, 43

Gunpowder best adapted for explosive purposes, 45

compounds more explosive than, 321

described, 43

discovered, when, 46

explosive force of, 43

manufactured in little grains, 44

Gypsum, 372

called plaster of Paris, why, 372

HuIr, what is, 219

cause of, 259

Hailstunes, shape of, 220 
Hailstorms, occur where, 220

Falls for music, construction of, 86

Haloes, explanation of, 246

Hand, held concave behind the ear assists the hearing, 87

Handkerchiefs, linen cooler than cotton, 105

Handles of metal, why warmer than wooden, 102

Hands and faces, why made warm by friction, 97

and lips chap, when, 226

Hardness of a body, on what depends, 8

Hard water, 312 how made more soft, 314

Hartshorn, 320

Hay, injured by heating, 337 odour of, 416 scales, how constructed, 37

Haystacks catch fire, 337

Haze round the smu indicates rain, 208

Haziness, cause of, 203

Headache produced by a crowded room, 326

Health, 355 pure air necessary for, 326

Hearing different in different persons, 60 human, boundaries of, 81

Hearthstone, cold to the feet, 102

Heat, absorbed most freely by what substances, 116

absorbers of, not good reflectors, 122 absorption and communication of, 123 and cold, sensations of, 89

and light, relations between, 90

best reflectors of, 123

black absorbs, 118

clouds prevent the radiation of, 121

communicated by contact, 100 radiation, 101

communication of, 100

conductors of, 101

definition of, 88

derived from electricity, 94

differs from other agents, how, 89

diffusion of, 89

distributed, how, 148

earth radiates, 114

effect of, on a magnet, 295

on dissolving power of liquids, 131 on fluids, 131

effects of, 94

enumeration of the best reflectors of, 123

evolved by chemical action, 330

expands air, 128

form of bodies dependent on, 128

greatest artificial, ever measured, 93 natural, 92

in ice, 89

internal, of the earth, 94

intense, how measured, 138

instruments for measuring, what callod, 138
Heat, its tendency to liquefy solids, 131

latent, 95 phenomena of, $8 \mathrm{~S}$

measured, how, 138

melts metals, why, 143

nature and sources of, 88,90

not produced by friction of liquils, 98

of the sun different from all other

heat, 90

passes from a body in right lines, 121

produced by chemical action, 94

compression, 99

easily by the friction of rough surfaces, 98

friction, 98

produced by mechanical action, 96 vital action, 97

applied to rooms, 171

rays of, 121

reflected, not visible, 123

reflection of, 122

reflectors, why made of polished metal, 122

solar; changed by radiation, 126

soot absorbs, 125

transmission of, 121, 127

weight of, imperceptible, 88

what bodies permit it to pass freely, 127

Heary metals, 377

Hectometre, definition of, 18

Height of aurora, 226

of meteors, 223

of tides varies, 316

lightning clouds, 276

Henry, Prof., his electro-magnet, 298

Herbivorous animals, 493

Hiccougli or hiccup, what is it, 84

High-pressure engine, 161 operates how, 161 parts dispensed with, 162

engines, when used, 161

safety of, compared with low pressure, 161

steam, 161

its mechanical power, 161

Hoarfrost, 125, 222

Holders, paper or woollen, why used, 102

Horizon, 257

Horn, transparency of, 246

Horse-power, definition of, 168

Horse-shoe magnet, 295

Hot air, houses heated by, 174

Humus, 387, 522

Hunger produced by work, 408

Hurricane, 194

Hurricanes travel from the equator, 195 breadth of, 196

surface of, 196

velocity of, 195

Hydraulics, definition of, 56

Hydrochloric acid, 361

Hydrofluoric acid, 362 
Hydrogen and water, 312

discovered, when, 311

gas, characteristics of, 311,345

IIydrumeter, 21

IIydrustatics, science of, 56

Hygrometers, different kinds of, 213

Hyperoxide, 310

ICE, 133

anclior or ground, 133

crystals of, 219

filled with air-bubbles, 132

floats upon water, 19, 134

fiacture of, angular, 181

heat in, 97

lighter than water, 132

melted by heat of the sun, why, 143

melting, tcmperature of, 96

of running water rough, 132

possesses antiseptic power, 133

preserved in tlannel, 104

sea-water, fresh, 132

Icebergs, how formed, 187, 221 size of, 187, 221

Icicles, how formed, 133

Ichthyosaurus, 493

Identity of lightning and electricity, 276

Irveous rocks, 460

Illuminating power of light, 233

Image in a mirror, 235

in water, 236

Impenetrability, definition of, 2

Incandescence, 90, 258

Inch, standard, how determined, 16

Incidence, angle of, 236

line of, 236

Inclined plane, definition of, 38 familiar examples of, 38

Indian Ocean, trade-winds in, 192 summer, 192

India-rubber, 413 bed-coverings, why not used, 174

Induction, 290

Inertia, force necessary to overcome, 89 what is, 8

Inflammable bodies, refractive power of, 241

minerals, 523

Ink, blotting-paper absorbs, 23

for writing, preparation of, 380

Wlue, from what prcpared, 380

stains of, extracted, how, 330

Iodine and fluorinc, 361

Inorganic chemistry, 304 substances, 304

Insects, winged, how produce sounds, 85 light, why able to walk upon water, 7

Instrument, sounds flat when unstrung, 82

Instruments, stringeu, purpose subserved by the body of, 76

Insulation of telesraphic wires, 302

Intensity of light measured, 233

Iridium, ن̈68, 385
Iris, 252

Iron, 377

and granite, dissolved, how, 175

and steel, hardened, how, 184

made soft, 184

rendered magnetic, 293

becomes hot in a fire, 89

colder than wood, 102

cast, crystallisation of, 182

melting point, 93

cast and wrought, 182

effects of continued vibration on the structure of, 182

bot, beuls more easily than colit iron, 143

by hammering, 97

hisses when immersed in cold water, 144

made cold by convection, 110

mass of, weight on the top of a high mountain, 15

rendered masnetic, 290

rusts, why, 309

sinks in water, 19

sulphuret of, form of crystals, 182

wrought and cast, 378

fibrous condition of, 182

ships, why do they not sink, 21

Islands, temperature more equable than continents, 124

warmer in winter than the main land, 124

Isomeric bodies, 416

$\mathrm{J}_{\triangle \mathrm{COBI}}$, Prof., his experiments, 299

Japanning, 412

KAKODYLE, 417

Kaleidoscope, description of, 237

Kane, his observations in Arctic regions, 199

Kettle, bottom and sides should not be polished, 126

effect of heat at the top, 108

lid of, why hot when the wate boils 105

Kilometre, definition of, 18

Kyanising, 383

LAC, 413

Lacquering, 412

Lamb and veal contain albumen, 404

Lamps, ancient, 342

argand, 313

mechanical (Cąrcel's), 343

pump or spring, 343

Lamp-wicks, cotton best adapted for, 23

Lund animals, first remains of, 490

breeze cool, why, 193

on tropical islands, 193

Lard and tallow, difference between, 410

manufactured by high-presivure steam, 
Larynx, definition of, 83

Latent heat, 95,144

theory of, by whom originated, 96

Laudanum, sixty drops equal to one hundred of water, 7

Laughing gas, vocal action of, 84

Lava, how different from other igneous rocks, 461

Lavender water, 415

Lavoisier first burnt the diamond, 347

Layers, 197

Lead, 381

and cotton, weights of, compared, 15

poisonous, 381

when exposed to the air becomes dull, 310

why called heavy, and feathers light, 4

Leap, standing, shorter than when running, 28

Leaping, difference between flying and, 29 use of running in, 28

Leather, black colour of, 380

how manufactured, 391

Leavened bread, 401

Leaves, green, 248

of trees affected by weather, 229

turn brown, why, 295

why attracted to the shore of a pond, 11

Ie :uminous plants, 394

Lens, definition of, 245

Lenses, varieties of, 245

Lever, arms of, 34

circumstances, which limit the utility of, 37

definition of, 34

Levers, effects of, in accumulating power, 36

of the first kind, examples of, 35

second kind, examples of, 35

third kind, examples of, 36

Life, first manifests itself in what form, 442

Light and actinism, 229

and heat, relations between, 90

absorption of, definition of, 232

absorbed, what becomes of it, 246

artificial, 258, 286

illuminating power of, 233

Light, cause of the refraction of, 240 chemical principle of, 261

position of, in the spectrum, 243

depth to which it penetrates the ocean, 247

excites magnetism, 296

focus of, 245

how polarised, 259

galvanic, 386

influence on crystallisation, 184

intensity of, measured, 233

moves in straight lines, 234

not al ways attended by heat, 258

no weight in, 230
Inight polarised, what is, 260

phenomena of, 260

pencil of, 233

propagated, how, 233

radiating from a centre, effect of, $306^{\circ}$

ray of, bent, 240

compound, 242

how divided, 242

rays of, how gathered by a telescope, 238

refined sort of matter, 230

reflected by a mirror, 236

by mirrors, 236

reflection of, 232,237

refraction of, 240,259

smooth surfaces reflect it best, 248

sources of, 230

velocity of, 230,250

compared, 231

vibration of, 249

Lightning, causes of, 274

conductors, 279

follows the best conductors, 276

how divided, 276

mechanical effects of, 278

more common in surnmer, 275

passes down a man, how, 278

rod, how erected, 279

travels faster than thunder, 277

what is, 274

Lime, 442

carbonate of, 491

caustic, why made hot by the applica-

tion of cold water, 95

effects of, as manure, 442

slaked, definition of, 50 why excluded from the air, 50

Limestone, 326

Line of incidence, 236

of reflection, 236

Linen, why cooler than cotton, 105

Linens, 424

Liniment, 409

Liquefaction, 143

Liquid and solid, difference between, 143

boiling-point of, what is, 147

definition of, 5

difference of, from a gas, 5

Liquids and gases, the difference between, 58

boiling points of, always constant under the same circumstances, 148

cooling of, how best effected, 110

expanded by heat, 131

heat not produced by the friction of, 98

height to which they can rise in tubes by suction, 68

impart no additional heat after they boil, 147

not good conductors of heat, 103, 108

power of, to conduct heat, 111 
Liquids, power of, to conduct sound, 74 rise in tubes by suction, the reason of, 68

the "spheroidal" shape, 144

temperature of, when in the spheroidal state, 145

why well adapted for measuring changes of temperature, 139

Lithography, 438

Lithographic printing, 439

Loadstone, 289

Lnaf-sugar, how refined, 372

Loam, 439

Locomotives, $16 \mathrm{~S}$

Locusts, how produce sounds, 85

Longitude, meridians of, 17

Low-pressure engine:, 161 water, 316

Lower silurian formation, where situated, 500

Luminous bodies, 258

Lunar caustic, 320

Lungs, 349

MAOBINERT, concussions and vibrations in, to be avoided, 182

Nachine defined, 33 object of, 40

Nachines add to human power, $3 \dot{3}$ and manufactures, sources of advantage of, 33

do not create power, 33

make cbjects apparently worthless valuable, 33

produce economy of time, 33

Magellan, Straits of, elevation at which water remains frozen, 187

Magnet, a natural, 289

directive power of, 291

horse-shoe, 295

retains its magnetic property when broken, 293

Magnetic effects of lightning, 278

meridian, 291

needle, 291 reversed, 291

phenomena, 289

polarity, 290

poles, greatest cold felt there, 296 of the earth, 291

variable, 292

power resides, where, 293

Magnets, proper position for, 295

Magnitude and impenetrability, 2 defined, 2

Malignant disease, $40 \mathrm{~S}$

Malt, how converted into beer, 398

Mammotl, 490

Mall, alone fitterl for all slimates, 200 ordinary strength of, 41

races of, 541

American, 543

Caucasian, 542
Mall, races of, Ethiopian, 544

Nalayan, 543

Nongolian, 543

distinctions of, 542

identity disputed, 541

Schlegel, quotation from, 541

varieties of, 541

respires, how, 349

Manganese, 365

Manufactures defined, 418

object of, 418

philosonhy of, 418

Manure of different animals, 441

Manures, 441

calcareous, 440

common animal and vegetable, 441

kinds of, 441

mineral, 441

Narble, 272

Mariner's compass, 291

Marl, 522

Marsh gas, 345

Mastic or mastich, definition of, 51

Mastodon, 490

Matches, why not ignite when damp, 151

Materials, application of, for architectural purposes, 48

Natter defined, 1

evidence of its existence, 1

limits to the division of, 2

molecule of, 3

particle of, 3

Mauritius, region of hurricanes, 194

Meat covers should be plain, and not chased, 113

rendered tough, how, 405

subject to taint on monnlight nights, 117

Mechanic powers, three great divisions of, 34

science of, 33

Mechanical action, a source of heat, 96

force, 158

powers, how do they assist labour, 5

Nedicine, the prescription of, by drops, unsafe, 7

Meerschaum, 373

Men, fat, swim easier than spare men, 20

Mercaptan, 417

Mercury, 383

boils at a high temperature, 92,142

contracts in freezing, 132

freezes at what temperature, 93,1$\} 1$

iodide of, change in the crystals of, 184

used for thermometers, 139

Meridians, definition of, 17

Metal, hot, why seem warmer than hot wool, 102

fused by lightning, 279

Metallic bodies, 306

elements, 383

Metalloids, 307 
Metallurgy, 384, 506

Metals best adapted for showing thermoelectricity, 289

cannot be changed, 307

colour of, 507

copper, 509

electro-chemical action on, 506

gold, 507

iron, 507

lead, 509

melted by heat, why, 143

of alkaline earths, 371

ores, 506

platinum, 507

silver, 507

steel, 507

temperatures at which they burn, 127

their number, 307

tin, 50 ?

zinc, 509

Meteoric iron, 224

showers, 225

Meteorites, 223

their origin, 223

Meteorology, definition of, 185 imperfectly understood, 228

Netre, definition of, 18

Mezzotint, 437

Miasma, 408

Mica, 524

Microscopic plants in snow, 219

Milk, boils more readily than water, 111 quicker than water, reason of, 111 curdles when sour, 401.

turns sour by keeping, 401

Millimetre, definition of, 18

Mineral, fracture of, its utility, 525

its lustre depends on what, 526

manures, 441

species, constituents of, 523 their number, 523

springs, 312

vein, 506

Minerals, their fusibility, 526

scale of hardness, 525

which possess taste, 526

Mineralogy, 523

Mining, blasting with powder, 517

introduction of steam power, 51$\}$

mechanical preparation of the ore, 515

motive power commonly employed, 514

neglected in $\mathrm{E}$ gland, 517

operations known at an early period, 516

ordinary process, 515,516

produce of, brought to the surface, 513

recent improvements, 518

safety apparatus, 514

shafts, 513

ventilation, 513

Mines, 510

blasting of, 511

boring for, 511
Mines, drainage of, 511

purpose of working them, 510

supports of, 512

tonls employed, 511

Mirage, 242

Mirror, 235

concave, 235

convex, 235

light reflected by, 236

plane, 235

Mirrors, how made, 365

reflect light, 236

Mist and dew, 208

and fog, 208

their difference, 209

vanish, when, 118

cause of, 118, 208

and dew, 118

phenomena of, 118

sometimes white, 210

Modifications of clouds, 204

Moisture on the hand, 145

Molasses, 390

candy, why sticky and gum-like, 178

oil, \&c., occupy less space in summer than in winter, reason of, 138

Molecule of matter, definition of, 3

Momentum of a moving body, definition of, 30

Monsoons, 192

change of, how marked, 193

their limits, 192

useful to the mariner, 193

Moon appears larger than the stars, 237

as seen through a telescope, 468

farther off when in the horizon, 257

how makes a path of light in water, 236

light of, compared with sun, 231

Moonlight nights, 117

no effect on putrefaction, 228

Mordants, 432

Morning, sray, indicates a fine day, 203

Mortar, 328

becomes hard, 50

proportions of lime and sand in, 50

use of hair in, 51

why user, 7

Mortars and howitzers, 46

Mortising, definition of, 49

Moscow, burving of, 198

Motion, accelerated in falling bodies, 30 apparent, of distant objects to a person riding or sailing, 25

axis of, in a revolving body, 30

centre of, in a revolving body, 30

communication of, never instantaneous, 26

definition of, 24

diffused effects of, 32

effects when suddenly imperied, 9

low commuricated to steam-engine, 159 
Motion of the earth, 24

perpetual, 9, 40

rotary and reciprocating, definition of, 34

state of things if none existed, 24

Mouldiness in ink obviated, 380

Moulding, 375

Nountains, distant, why appear hlue, 60 high, always covered with snow, 186 noiseless and quiet, 74

Mountainous countries rainy, 215

Muriatic acid, 360

MIuscular energy, how exerted, 41

Mlusical chord, number of vilurations necessary to produce sound, 81 glasses, why produce sounds, 82 instrument, four classes of, 82 sounds, definition of, 80 and mere noises, difference between, 80

Musk, 414

Mislin, how protects plants from frost, 117 Mustard, its composition, 413

Mutton, solid, why, 409

NaILS in old houses, why loose, 137

Naphtha, 413

Nature and production of electricity, 267 of fibrous substances, 413

Navigation, steam, originated where, 169

Neap tides, 316

Neat's-foot oil, 410

Nebulæ, 466

Needle, dipping, 293

floats upon water, 7

magnetic, 291

of compass, points how, 292

Negative electricity, 270

Negro's skin never blisters, 125

Network of ice, how formed, 134

Neutral body, 306

Newcomen, his experiments, 165

Newton, his prediction of the diamond, 241

Nimbus clouds, $20 \tau$

Nineveh, glass found there, 366

Nitrate of strontia, 373

Nitre, 319

N:tric acid, 319

Nitrogen and its compounds, 317 chloride of, 45

defined, 317

not magnetic, 297

Non-condensing engine, 161

Non-metallic bodies, 306

North wind generally cold, 193

Nutcrackers, what kind of levers, 35

Nutrition and animal heat, 352

Nutritive value of meats, 405,406 various fishes, 407

OAR appears bent, why, 240

Objects inverted, 255

Ocean affects climate, 188
Ocean, depth to which light penetrates, 247

green, or blue colour, 60

how much of the earth covered ky, 458

Ochre, 378

Odoriferous principle of plants, $\mathbf{4 1 5}$

Odours, animal, 414

artificial, offensive, 417

compared to notes in music, 417

origin of, unpleasant, 416

Oersted, his discoveries, 297

Offsets, 452

Oil, 408

and water will not unite, why, 305

cloth, 412

neat's-foot, 410

of cinnamon, 416

on water, prevents freezing, reason of, 106

silk, 412

thick in winter, 410

water will not dissolve, 175

whale, 410

Oils, drying, 412

volatile, 415

Oldest strata, 501

Old red sandstone, 499

Oleine predominates in oils, 412

Olmsted's theory of shooting-stars, 225

Onion, odour of, 416

Oolite system, 492

Oolitic period, fauna and flora of, 503

Opaque bodies, what are, 246

Opinions concerning the weather, 227

Optical delusion, 257

Optics, 229

Orders of architecture, 52

Ore, nietals generally found in, 367

Ores, occur how, 506

Organic chemistry, 386

substances, 304

Organs of vision, 352

Origin of colour, 247

of meteorites, 224

Osmium, 385

Oxide of iron, 378 of lead, 363

Oxides, 309

Oxygen, 308

and oxide, their difference, 308

its use, 308

magnetic, 297

Oysters, shells of, of what composed, $3 \% 2$

PAINT, blisters through heat, 151

$$
\text { preserves wood, } 106
$$

Painters' colic, 381

Painting iron prevents its rusting, 309

Palæontology, 470

Palladium, 385

Paper, curls wlien scraped, 184 
Paper for writing and printing, 430 ignition of brown, 124

making, 43

puckers when wet, 212

Papier maché, 431

Parallels of latitude, definition of, 17

Parian, 377

Particle of matter, definition of, 37

Peachstones, kernels of, poisonous, 321

Pearl así. 370

"mother of," cause of colours in, $\because 44$

Peat, 333

Pedestal, definition of, 55

Pencil of light, 233

Pendulum, compensating, 138

definition of, 31

length of, effect on the rate of the clock, 32

marks time, how, 31

seconds, length of, 32 use of, 16

Perceptibility in sound, limit of, 87

Perfumery, preparation of, 417

Perfumes and odours, 414 use of, 403

Periodical winds, 192

Peroxide, 309

Perpetual motion, 8, 9

Perspective, meaning of, 237

Perspiration, cause of, 353 insensible, 208

Petrifaction, 314

Pewter, 368

Phenomenon of dew, 114

Philosophy of manufactures, 418

Phosphate of lime, 356

Phosphorescence, 258

Phosphoric acid, 357

Phosphorus, 356

and sulphur, 356

derivation of, 356

Phosphuretted hydrogen, 357

Photographic pictures, principle of formation, 262

Photography described, 261

Photometer, 233

Physics, definition of the science of, 6

Pianos, effect of temperature on, 137 produce musical sounds, why, 82

Pictet's theory of radiation of heat, 114

Pictures, not seen well in certain positions, 238,344

Pilasters, definition of, 54

Pile, definition of, 43

Pin-cushion, how made as effective as a cannon ball, 25

Pipe, comparative strength of lead and tin, วิ81

Pisa, Leaning Tower of, 12

Piston, steam, 159

Plank, why more liable to break than a beam, 48
Plants acquire the carbon of their structures, low, 265

endogenons, 419

exogenous, 419

fold their leaves before rain, 212

growth of, 443

in snow, 219

nou rishment of young, 444

respire, 350

rules for watering, 448

Plaster of Paris, 373

non-conducting properties of, 373

Plastic, definition of, 6

Plate glass, 365

Platina, specific weight, 19

Platinum, 310

and gold never oxidise, 310

solvent for, 385

Plesiosaurus, 493

Pliant, when is a body, 8

Plinth, in architecture, 55

Plumb line, 14 rule, 14

Plummet, 14

Plumule of the seed, 444

Plutonic rocks, 460

Pneumatics, science of, 58

Point-blank, definition of, 46

Poker, why feels colder than the hearthrug, 102

Polarisation of light, 259

Poles of the earth, 291

Polypes respire, how, 401

Pomatum, 415

Pop-gun, why occasions a loud report, 84

Porcelain, 375

clay, 525

Pores, existence of, in gold and lead, 4 of a body, definition of, 3

Porosity and density, 3

Porous bodies, conduct heat slowly, 112

Portico, definition of, 54

Pusitive electricity, 270

Potasb, crude, of commerce, 369

whence principally derived, 369

Potassium, 367

Potato abounds in starch, 388

Potatoes at the top of a boiler, 111 sweet, why, 389

Power of magnets, 291 sources of, 40

Precious stones, 185

Primary colours, 243

Primitive rocks, 467

Printing, conmmon process of, 433

copperplate, 438

definition of, 433

ink, its composition, 439

invention of, 433

lithographic, 438

machine, history of, 434

paper, 430 
Printing press, 434 stereotype, 434

Prism divides a ray, 242

Prevost, theory of, 114

Projectiles, 45

Proof spirit, 399

Propagation of fruit-trees, 449

Protoxide, 309

Pudding-stone, 463

Pulley, definition of, 38

Pump, construction of common, 68 forcing, 69

handles cold in winter, 102

height of water raised by, 68

invention of, 69

Puna winds, 194

Pupil, 352

Pure water poison to fish, 313

Putrefaction, 228

Pyramid, difficult to overturn, 13

Pyrometer, how constructed, 142 the Gravezande, 142

used to ascertain the heat of furnaces, 143

SUADRUPED never raises both feet on a side simultaneously, 12

Quadrupeds learn to walk sooner than children, 15

swim easier than man, 20

Quartz, 362

Quicklime, application of, to fallow ground, 441

as manure, 442

called caustic lime, 373

desoribed, 50, 328

Quills of birds, why hollow and cylindrical, 47

Quinine, 391

Radiation and reflection of heat, 123, 185 ceases in cloudy weather, 115

influence of colour on, 114

of heat, 112

on what depends, 112

two theories of, 114

Radiators, good absorbers of heat, 112 of heat, what substances are bad, 113

Radicle of the seed, 443

Railway carriage, first, 169 trains, why produce more noise in crossing a bridge than upon the ground, 79

Rain, amount of annual precipitation, 215 cooler after, 153

cools the air, 151

drop a sphere in form, 120

falls continually in some countries, 217 in drops, why, 211

more abundantly at the equator, 216

gauge, 215

more falls at night, 212
Rain, more falls in winter, 215

on the sea coast, 216

never falls in some countries, 216

none when the air is very dry, 72

purifies air, 212

quantity which falls at Vera Cruz, 215

snow, liail, frost, 211

some countries destitute of, 216

water not salt, why, 314 soft, 313

what is, 211

without clouds, explanation of, 115, 217

Rainbow, 213

how produced, 244

Rainy days, more in temperate zone, 213

Ratio of expansion of solids, 131

Rays, calorific, 121

of light diverge, why, 242

Ray of light, 240, 271

compound, 242

divided, how, 242

Razors dipped in hot water before using, 136

Reaumur's thermometer, 140

Redfield, his estimate of a hurricane, 197

his theory of hurricanes, 197

Red rays heating, 250

Reflection, angle of, 236

line of, 236

of heat, 122

of light, 236, 248

Reflectors, necessity for keeping clenu, 122 the best, 12?

Refraction of light, 240

double, what is, 259

explained, 241

illustrated, 241

phenomena of, 245

Refracting power depends on what, $24 \hat{\mathrm{i}}$

Reid, his theory of hurricanes, 197

Reign of fishes, 502

of reptiles, 503

Repulsion, definition of, 6

Resin, 270, 411

Resins, fragrant, 414

Resonance, 85

Respiration, 347 a form of combustion, 348

Retina, 252 images formed on inverted, 255 retains impressions, 254

Retort, what is, 149

Retting or rotting of flax, 422

Reverberating furnace, 338

Richardson, his observations in Arctic regions, 199

Ricochet firing, definition of, 46

Rifles, hore in some, twisted, 44

Rifle, "Ninie," peculiarities of, 43 more accurate in shooting than a musket, 43

Rivers and streams, effects of, 473 
Rivers generally of a serventine course, 27 velocity of, 58

Roads, constructed over tlie summit of a hill, bad policy of, 39

dried in a few hours, 152

effect of elcration of, in increasing labour, 39

Rocket ascends when fired, 21

Rock salt, 361

Rocks, 459

and stones, increase how, 474

clissification of, $45 S$

distinguished, 471

once in a state of fusion, 362

split in winter, why, 132

vertical order of position, 489

watertight, 519

weatherbeaten, 132

Roemer discorered velocity of light, 231

Roofs, steep, why best adapted to cold climates, 49

Poom, air in, without a fire, why warmer than out of doors, 170

two fires in, apt to smoke, 173

Rope-walling, art of, 14

Rose diamouds, 324

red, its effect on complexions, 251

water, 415

Rotation of crops, 448

Rotatory movement of wind, effect of, 195

Ruins and mountains, why famous for ecloes, 85

Rum, 399

method of stealing in the West Indies, 21

Rurst, 309

\section{S_Fetr lamp, 346}

valve, 163

Sal-ammoniac, 321

Salt, amount of, in sea water, 176 and snow colder than snow, 196 crackles when thrown in the fire, 181 crystallises, 180

dissolves ice, $135^{\circ}$

formed from sea water, 176

in chemistry, 306

in springs, 314

in the ocean, 314

lakes, 314

water freezes, when, 135

Saltpetre, 319

of itself, will not explode when burnt, 321

quantity of, retained in boiling-water, 176

Sand of deserts, of what composed, 363

pillars, 199

variety most suitable for mortar, 50

Sandstone, 463

Sandy plains dazzle from sunshine, 249

Sap of a healthy tree, temperature of, in winter, 109
Sapphire, 526

Saturation, 175

Saucepan, blackened with soot, boils quicker than a bright one, reason of, 126

Savages, method adopted by, for distinguishing the approach of an enemy, 75

Savery, his experiments, 165

Scale in music, 82

Scarfing and interlocking, definition of, 49

Scion, 450

Scissors, pincers, levers of what order, 35

Screw, definition of, 39

perpetual, 39

Sea breeze, cause of, 193 cool, why, 193

on tropical islands, 193

evaporation of, greater than the land, 153,189

salt, 314

salt in, 176

sickness, cause of, 29

water, freezing point of, 135 rarely frozen, 135 solid matter in, 315 substances contained in, 315

Sealing wax, 41 ?

Seed, freshness of 4.47

how made to grow, 443

leaves, 445

mode of testing, 447

of a plant, 443

one hundred years old, 447

Seeds germinate, low, 144 self-sown, 447

Seeing described, 252 double, why not, with two eyes, 255

Sensation, different impressions transmitted by each organ of, 1

effect of deprivation of, 1

Senses, number of, 1 what are they, 1

Serpentine, 524

Serpents live? ? ? without food, 350

Shadow, defirwod, 234

Shaft of a $\mathrm{co}^{\mathrm{l}} \cdots \mathrm{mn}, 56$

Shavings obstruct the transmission of sound, 79

Ship in a lurricane, 197

Ships, impracticable to construct, beyond a certain size, 48

Shell-lac, 413

Shells, brightest near shore, 250

Sheet-lightning, 276

tin, 381

Sheets of a bed, why feel cold, 107

of linen, why cooler than cotton, 105

Shooting-stars, 224

Shot and cannon-balls, why heated by their discharge, 99

manufacture of, 382

Shuttle, 429

Silicious and earthy minerals, 523 
Silicium and boron, 362

Silk, 425

a bad conductor of heat, 107

composition of, 426

oil, warm, 174

Silver, 383

difficult to keep bright, 310

niitrate of, turns black, 265

plating, 384

spoous, more heated in tea than pewter ones, 106

ralue of, 384

Simoom, 194

Simple and compound substances, 304 substances, number of, 304

Sizing of paper, its utility, 430

Shating swiftly over thin ice, effect of, 29

Slag, $360^{\circ}$

Slate, why rebounds upon water, 56

Sleep, 355

Smell, organs of, 416

Smoke, 331

ascends a chimney, 10, 130, 173

definition of, 172

little with a red-hot fre, 334

rises up the chimney, why, 172

wreaths of, origin of, 172

Saapping of wood, coal, \&ce., 4

Sneezing, definition of, 84

Snow, a bad conductor, 218

a non-conductor of heat, 105

at the foot of a tree or wall, melts readily, 113

beneficial to the earth, 218

crystals of, 131

evaporation of, 153

flake, 219

green, 219

keeps the earth warm, 218

on the tops of high mountains, 123

perpetual line of, 186

protects vegetables from cold, 105

red, 219

white, why, 219

what is, 218

Soap, 409

Castile, 409

how manufactured, 409

properties of, 109

Soapstone, 524

Soda, obtained where, 369

Sodium, 369

Soils, bad conductors of heat, 105

best for a garden, 439

cooler in summer below the surface than the surface, 105

natural, 522

warmer in winter beluw the surface than the surfice, 440

Solids converted into liquids, how, 131 expansion of, 131 power of, to conduct sound, 75

Solution and crystallisation, 175
Solution and mixture, their difference, 175 effects of heat on, 175

of salt, 175

Sonorous bodies, definition of, 75 vibrations, nature of, illustrated, 74

Soot, absorbs heat, 125

influence of, on cooking utensils, 125

prevents the escape of smoke, 175

Sound, air not necessary for its production, 74

attends the appearance of aurora, 226

communicated through a tube, 87

effect of irregular surfices on, 86

interruption of, in camp weathes, to

loudness of, on what depends, 54

more distinct by night, $T 9$

in cold weather, 79

perceptible near: a water-spout, 193

produced by organs of voice, 83

reflection of, 86

sharp, generally loud, so

transmission of, 75

velocity of, 77

Soup, why retains heat longer than water, 110

South magnetic pole, position, 291

winds generally warm, 193

Spar, Iceland, 259

Sparks fiy from burning wood, 129

how produced, 129

of fire, origin of, 4

Speaking tubcs, why used, 78

Specific gravity, definition of, 19

detcrmination of, 19

difference of, from ordinary neight, 19

Spectrum, 262

Speculum, 235

Spermaceti, 410

Sphcroidal state of liquids, explanation of, 144

Spinning, 426

mule, 428

Spirit sbove and below troof, 399

Spirits, boil quickcr than water, 111

Sponge, 170

absorbs water, 6, 23

wet, clcans a slatc, 170

Spontaneous combustion, 387

Spoons, metal, retard the proces: of boiline, 106

Spray of waterfalls causes rainhow, 244

Spring tides, 316 water contrins earthy matter, 312 always cool in summer, 05

Springs, indicate change of veather, 220

origin of, 518

warm, when, 480

Squinting causes to sec doulle, $23 ?, 255$

Stairs, common, varieties of incined p'anes, 39

Staple of cotton, 421 
Standard for comparing steam-engines, 168 Starch, 388

boiled before using it, why, $3 s s$ imparts a glazed surface to cloth, 152 manufactured, how, 388

stiffens linen, 152

Star-light, nature of, determined by polarised light, 261

Stars, number of, 257 twinkle, 245

Steam adapted for warming dwellings, 155 advantages of heating by, 174 appearance of, condensed, 154 at what temperature produced, 154 available in machinery, 157

burns more than boiling water, 95 compared with common air, 154 condensed into water, 156

contains more heat than boiling water, 155

cooking by, 156

defined, 153

engine, 157

condensing, 159

consists of two parts, 162

governor of, 165

greatest work of, 158

illustration of, 158

bigh-pressure, 157

in boiling-water first manifested, 154

invisible, 153,154

its most important property, 155

its pressure how diminished, $\mathbf{1 5 6}$

how exerted, 155

lighter than water, 155

mechanical force of, 157

power of, 43

weight of, compared with air, 59 compared with water, 155

Stearine, 412

Steel, 325

engraving, 438

of a purple colour rusts easily, 310

Stems of grain-plants, why hollow tubes, 47

Stère, or the French unit of dry measure, 18

Stereoscope, 256

Stethoscope, construction of, 76

Stiffness of a material, 47

Stigmaria, 497

Still, definition of, 149

Stippling, 436

Stock, 450

Stone, durability of, how ascertained, 518 used for lithographing, 438

Stones, effect of loose, on soil, 522 gravel, and sand, why easily moved by waves, 22

snap when heated, 130

Stones, the precious, 524

Stove, rooms warmed by, how, 103

Stores, advantages of, 338 ancient water, 174

bot air, 174
Stoves, known to the Romans, 174 manner of warming rooms by, 111 snap, when the fire is extinguished, 137 a fire is kindled in them, $13 \mathrm{t}$

St. Petersburg, quantity of rain at, 216 Strabismus, or squinting, 233

Strata at the bottom of the Gulf of Mexico, 473

crop out, when, 461

Stratified, 459 rocks, 469

Stratus, 204 clouds, how produced, 205

Straw, why used in ice-houses, 105

Strengtb, greatest active, of a man, 41 of materials, effect of shape upon, 47

Streets, watering, how cools tbem, 151

Strontium, 371

Strychnine, 391

Stucco, what is it, 51

Sublimation defined, 150

Suboxide, 309

Subsoil, important to have a good, 439 best kind of, 439

Substances, differently soluble, how separated, 304 simple and compound, 304

Substantive colour, 431

Sucker, common, operation of, 63 weight raised by, 64

Suffocation, explanation of, 84

Sugar, a vegetable and animal substance, 389 candy, 178

Sugar, composition of, 389 loaf, 178

melts in water and tea, 175,176

method of refining, 150

of lead, 382

varieties of, 389

Sulphur, 357

Sulphuretted hydrogen, 358

Sulphurous acid, 359

Summer warmer than winter, 92

Sin, a source of heat, 90

does not actually rise and set, 25

heat of, and artificial, compared, 90 greater in some parts of the earth, 91

greatest at noon, 92

rhysical condition of, 91

power of, 92

production of heat and light, 91

rays of, when fall perpendicularly, 92 temperature of, 91

nature of, how determined by polarised

light, 261

red through a fog, 204

Sunrise, cause of a red, 203

Sunset, cause of yellow, 203

gray, indicates wet weather, $20{ }^{\circ}$

Sunshine, effects on combustion, 91

Surf, 317 
Surfaces, brilliant, 248

extent of, affects evaporation, 152

Sweet-wort, 398

Swimmers, unskilful, why liable to sink, 20

Swimming, why easier in salt than in fresh water, 20

Synthesis, 305

TALLOW, what animals furnish, 410

Tannic acid, 379

Tap-rooted plants, 448

Tartar emetic, 385

Tartaric acid, 370

Tea and coffee, properties of, 392 cools quicker for stirring, reason why, 134

old people generally fond of, 392 pot, dull-black, does not make good tea, 113

effect of air-tight lids, 65

lids, hole in the top, use of, 65

Telescope, reflecting, definition of, 239

Telescopes, how enable us to see objects otherwise invisible, 239

varieties of, 239

use of, 238

Temperature, change of, 138 circumstances which influence, 187

defined, 138

disturbing causes of, 187

cultivation, 188

distance from the sea, 188

elevation of land, 188

geographical position, 187

mountain chains, 188

rain, 188

soil, 188

the slope, 188

winds, 188

effect of, on the distribution of animals and plants, 100

influence of the configuration of land on, 188

mountains, on, 188

sea, on, 188

soil, on, 188

mean, daily and annual, definition of, 186

mean daily, of a particular locality, how ascertained, 186

not the same at all places under the same latitude, 187

of the body, 353

of wind, effect of change of, $18 \mathrm{~S}$

proper for a room in cold reather, 174,208

reduction of, how affects the moisture of the air, 152

varies with altitude, 186

with latitude, 186

i'empering of steed, $37 \mathrm{~S}$

Terrib-cotta, what is it, 51
Tertiary formation, 471

pcriod, animals of, 472

Textile fabrics, manufacture of, 418

Theine, 392

Theo bromine, 393

Thermo-electricity, 288

Thermometer and pyrometer, difference between, 138

by whom invented, 140

centigrade, 141

construction of, 139

Fahrenheit's, 140

Reaumur's, 140

Thermometers, 138

comparison of, different, 140

graduation of, 139

mercury used for, 139

Thirst created by eating salt food, why, 405

Thunder clouds, 281

and lightning accompany hurricanes, 197

cause of, 281

its distance calculated, 281

purifies the air, 278

rolling of, 281

sound of, 281

storm, what is, 276

where most frequent, 276

Tide wave, velocity of, 316

Tides, 315

Tiles, what are, 51

Tin, 380

box, why used for foot-warmer, 113

foot-warmers, why covered with flannel, 113

Tinder, 322

Tissue, vegetable, 387

Toes, advantage of turning out, in walking, 13

Tone or pitch in sound, definition of, 80

when grave or sharp, 80

Tongs, pair of, levers of the third kind, 35

Tongue, hot-iron applied to, momentarily, does not burn, 145

Tongueing and rabbeting, definition of, 49

Top spinning, 27

Tower, circumstances under which it will stand securely, 12

Tornado, curious effect of, 197

Tornadoes, 197

their origin, 198

Torpedo, 271

Trade-winds, 190

blow all the year round, 191

do not blow direct north and south, 191

effects near the equator, 191

how produced, 191

in the Atlantic, 191

in the Indian Ocean, 192

motions of the Polar currents, 191

occasioned by, 191 
Trade winds, region of calms, 192

uniform foree, 192

upper and lower currents, 191

Train oil, 410

Transfer priuting, 439

Transmission of heat, 12 T

Trasisparent bodies, 246 semi, 246

Transparency and opacity, 246 not perfect in any substance, 246

Trap rock, 462

Traverse, when a magnet is said to, 291

Tree, shady, why cool in the summer, 105

Trecs, arrangements of the limbs of, in reference to the centre of gravity, 15 crack with frost, 132

vital action of, 107

why unable to grow mnlimitedly, 48

Triasic, 471

Tropical islands, land kreeze on, 193 sea breeze on, 193

Tropics, rainy season of, when commence, 216

Tropical wet day, 216

Turpentine, 411 oil of, 415

Turtle, why unable to move when placed on its back, 12

Tuscan order of architecture, 53

Twilight, cause of, 244

Twilled goods, how woven, 429

Twisted cord, strength of, 426

Type-rnetal, 386

Typhoon, 197

Uxiolted flour untritious, 401

Undulatory theory, 230, 249

Unstratified rocks, 160

Upper silurian, 471

Vacuers, definition and illustrations of, 170

in nature, 62

opcration of, 62

perfect, cannot be made artificially, 62

Tapours, how condensed into liquids, 128 occupy more space than the substances

from which they are produced, 145

Vaporisation, 144, 145 explanation of, 144

Tariable winds, 192 prevail, where, 193

Tariations of the magietic needle, 292

Varnishes, 412

Tegetable life dependent on animnl, 350

mould, 387

organisation, 387

tissue, 387

zones, the arctic, 534

the cold temperate, 533

the equatorial, 532 .

the polar, 593

the sub-arctic, 533
Vegretable zones, the sub-tropica], 533

the tropical, 532

the warm temperate, 533

Vegetalles, decaying, 394

Vegctation, in tropical climates, 351

in arctic regions, peculiarities of, 531

influence of solar light upon, 265

effects of atmospheric pressure on, 533

of electricity on, 584

of moisture on, 533

of position of country on, 534

of soil on, 533

of temperature on, 533

temperate, 534

tropical, 534

Vein, mineral, 473

Velocity being known, to determine the distancc passed over, 26

of a body moving uniformly, how detcrmined, 26

of a falling body increases with the distance passed through, 31

of liglit, 231

of shooting stars, 225

of wind, 190

in a hurricane, 190

in a storm, 190

unsafe, instance of, how determined, $2 S$

Ventilation of a room, how best effected, 171

what is, 169

Veratryne, 391

Verdigris, 382

Vermilion, 383

Vertebrated animals have lungs, 348

Vertical rays, 189

Vibrations, aërial, how communicated, 76

danger of, 182

necessary to produce ycllow light, 249

to produce red light, 249

of sound, number capable of apprecia.

tion by the human ear, 81 sonorous, how made visible, 76

Vinegar, 391

sour, why, 391

Vinous fermentation, 396

Vision, deceptions of, 256 organs of, 252

Vital action, a source of heat, 99, 272

Vital force, 386

forces cease to exist, when, 201

Vitrifaction, 364

Voice, tones of, how made grave and acute, 83

Volatile bodies dissipated by heat, 153

definition of, 145

cthers, 415

oils, 415

Volcanocs, 475

form of, 475

number of, active, 479

mobable sause of, 94 
Volta, 285

Voltaic kattery, 285

Volume of a body, 2

Vulcanised India-rubber, 413

WaLking arm and arm, philosophy of, 13

Walls, construction of, for obstructing sound, 79

Warmth, definition of, 103 produces breeze, 193

Warming and rentilating, 171

Warp, wood, 151

Watch and clock, difference of, 32

Water, 312

a bad conductor of heat, 103

air in, 67

amount daily raised by eraporation, 217

an elastic body, 56

ascent of, in tubes by suction, discovery of the reason of, 62

boiled, why flat and insipid, 67

boiling, ordinary temperature of, $14 \mathrm{~S}$ temperature increased by pressure, 148

boiling-point, 93

of, in a vacuum, 148

boils quicker in a metallic vessel than in one of glass, 148

can never be heated above the boilingpoint, 147

cements, why become harder under water, 50

congelation of, effects of, 218

contracts, 134

converted into steam under what pressure, 58, 158

currents produced in boiling water, 109

declivity sufficient to give running motion to, 57

discharge of, from vessels, 58

dissolves sugar, why, 175

drop of, why rolls upon very hot metal, 45

expanded by lieat, $3 \pm$

expands in freezing, 3,31

into steam, 144

expansion of, when freezing, 132, 151

extinguishes a fire, 333

falling, influence of gravity upon, in waterfalls, 31

freezing-point of, 93, 133

gas contained in, 334

how converted into steam, 154

frozen by rapid evaporation, 136 , 150 made hot, 108

ice lighter thau, 132

in freezing always crystallises, $21 \mathrm{~s}$

may be lieated beyond two hundred and twelve derrees F., 109

obtained by distillation purer than any other, 150
Water pipes, why they burstin frosty weatter, 131

plants, 351

power, definition of, 41

purified, how, 4

purifies itself, when, 315

rise of, in small tubes, 23

rotted flax, 422

running, effect of closing a pipe of, suddenly, 2s

its appearance, 198

temperature at which it scalds, 127

two ounces of coal evaporate a pint, 158

undulations or waves of, how made to

destroy each other, 82

wheels, four forms of, 41

where found purest, 313

why in motion when boiling, 109 melts salt, 22 sugar, 23

Waters, flowing, 313

Watt, his improvements, 165

Wave, phenomena occasioned by the prssage of a boat, 77

Waves of sea, cause of, 131

of water, effect of oil on, 60

how produced, 60

Wax becomes hard when cold, why, 143

soft before it melts, why, 143

Weather foretold $b_{y}$ animals and insects, 229

popular opinions of, 227

cold, 270

Weaving, 429

Wedge, definition of, 39

uses of, in the arts, 40

Weeds, 453

Weinht, 15

avoirdupois, definition of, 16

defuition of, 15

false, method of detecting, 37

of aërolites, 228

of a man on the surface of the sun, 15

Troy, definition of, 16

Weights and measures, the French system of,, 17

Wells, 510

when adopted, 17

Artesian, 520

$D_{r}$., on the theory of dew, 121

West Indies, region of hurricanes, 195

Wet clothes dry in the air, 146

Whales, seals, \&c., how protected against cold, $10 . t$

Wheatstone invented the stereoscope, 256

Wheel, advantage of fly, 164

and axle, 38

centre of gravity of, 13

water, breast, 42

overshot, 42

turbine, 42

undershot, 42 
Wheelbarrow, a lever of what kind, 35

Wheels, axles of, why greased, 97 of carriages, fore wheels why smaller than the lind wheels, 52 why arched or disc-shaped, 52 tires of, fitted hot, 136

Wheelwrights, why fit tires red hot, 136

Whirlpools, 198

Whirlwinds, 198

White, cause of, 248

light, 242

vitriol, 380

White lead, 382

Wick of candle not consumed, 341

Wild animals not fat, 354

Wind, action of, in sailing, 5

affects the shape of clouds, 200

always blows, 189

clouds affect it, 189

definition of, 60,189

feels cold, why, 199

force of, how ascertained, 190

pipe, construction of, 80

temperature of, changes its direction, 189

velocity of, 190

why generally feels cool, 199

would blow regularly, when, 189

Winds, cause of, 188

constant, 190

do not always blow one way, why, 189

easterly, how prevent dew-falls, 115 why dry, 117

north, generally cold, 193

periodical, 192

south, generally warm, 193

trade, 190

variable, 193

where prevail, 193

westerly, favour the deposition of dew, 118

why moist, 118

which are dry, 194

are rainy, 194

Windows, why made to rattle by the passage of vehicles in the streets, 78

Wine, manufacture of, 396,397

or beer turns sour, when, 391

sometimes red or yellow, 397
Wood, constituents of, 332

decay of, 328

dry, buras better than green, 130

why swells in water, 24

expands under the influence of heat

differently from inetal, 138

how made flexible by boiling, 184

light and porous makes the most snapping, 4

snapping of, during combustion, 4

unseasoned, decays, 402

why not melt witlı heat like inetals, 143

preserved by paint, 106

some varieties float, and others sink, 22

Wool, 424

warped by heat, 151

fur and hair, why a protection against heat, 103, 104

has great attraction for colouring substances, 431

seen under a microscope, 424

Woollens and furs do not impart heat, 102. why used for clothing in cold weather, 103

Woof, 429

Worcester, experiments of Marquis of, 164

Writing paper, 430

YEAST, 395

causes bread to rise, 328

Yellow light, how prevents germinatioc of seeds, 266

rays most visible, 250

Yolk of an egg, 404

ZеNith, 257

Zig-zag lightning, 276

Zinc, 287

sulphate of, 380

Zodiacal light, 226

Zoological geography, 538

definition of, 538

difference from zoology, 538

fauna, 539

definition, 539

dependent on vegetation, 539

temperate, 540

tropical, 540

arctic, $54 \%$ 



